\title{
Antimicrobial Effectiveness of Citrus Essential Oils and Organic Acids against Salmonella and Listeria monocytogenes
}

\author{
Karina Walker
}

Follow this and additional works at: https://researchrepository.wvu.edu/etd

\section{Recommended Citation}

Walker, Karina, "Antimicrobial Effectiveness of Citrus Essential Oils and Organic Acids against Salmonella and Listeria monocytogenes" (2015). Graduate Theses, Dissertations, and Problem Reports. 6888.

https://researchrepository.wvu.edu/etd/6888

This Thesis is protected by copyright and/or related rights. It has been brought to you by the The Research Repository @ WVU with permission from the rights-holder(s). You are free to use this Thesis in any way that is permitted by the copyright and related rights legislation that applies to your use. For other uses you must obtain permission from the rights-holder(s) directly, unless additional rights are indicated by a Creative Commons license in the record and/ or on the work itself. This Thesis has been accepted for inclusion in WVU Graduate Theses, Dissertations, and Problem Reports collection by an authorized administrator of The Research Repository @ WVU. For more information, please contact researchrepository@mail.wvu.edu. 


\title{
Fine-Particle Charging-Rate-Limit Modification to Grain Dynamics in ABRUpt and GRAduAl Inhomogeneities
}

\author{
Jeffrey J. Walker
}

Dissertation submitted to the Eberly College of Arts and Sciences at West Virginia University

In partial fulfillment of the requirements for the degree of

Doctor of Philosophy

in

Physics

\author{
Mark E. Koepke, Ph.D., Chair \\ Paul Cassak, Ph.D. \\ Maura McLaughlin, Ph.D. \\ Earl Scime, Ph.D. \\ David Lewellen, Ph.D. \\ Department of Physics \\ Morgantown, West Virginia \\ 2015
}

Keywords: dusty plasma, charging models, non-stationary charging

(C)2015 Jeffrey J. Walker 


\title{
Abstract \\ Fine Particle Charging Rate Limit Modification to Grain Dynamics in Abrupt and Gradual Inhomogeneities
}

\author{
Jeffrey J. Walker
}

Gyro-phase drift is a guiding center drift that is directly dependent on the charging rate limit of dust grains. The effect of introducing a gyro-phase-dependence on the grain charge leads to two orthogonal components of guiding-center drift. One component, referred to here as grad-q drift $\left(\boldsymbol{v}_{\nabla q_{e q}}\right)$, results from the time-varying, gyro-phase angle dependent, in-situ-equilibrium grain charge, assuming that the grain charging is instantaneous. For this component, the grain is assumed to be always in its in-situ-equilibrium charge state and this state gyro-synchronously varies with respect to the grain's average charge state. The other component, referred to here as the gyrophase drift, arises from any non-instantaneous-charging-induced modification of the grad-q drift and points in the direction of $-\nabla R_{L, d}$ (where $R_{L, d}$ is the grain gyro-radius) i.e., the direction associated with increasing magnitude of in-situ-equilibrium charge state. Gyro-synchronous grain charge modulation may arise from either abrupt or gradual inhomogeneity in plasma conditions. In the abrupt inhomogeneity, $q_{1}$ is the $i n$-situ-equilibrium charge on one side of the inhomogeneity, $q_{2}$ is the $i n$-situ equilibrium charge on the other side, $q_{1}<q_{2}<0$, and the the capacitive effects of charging and discharging of the dust grain can result in a modification to the particle trajectory, i.e., gyro-phase drift. Abrupt inhomogeneity may arise in the wakes and shadows of planets, moons, and other airless bodies. In the gradual inhomogeneity, a plasma parameter such as electron temperature or relative ion flow varies during a dust grain gyro-orbit, and the finite, non-zero charging time of dust grains can also result in gyro-phase drift.

In this work, a single-particle trajectory tracker was developed using the option of either a modified Boris scheme or iterative leapfrog scheme with fixed timestep for grain motion and an adaptive time step for grain charging. This single-particle trajectory code resolves dust grain motion for abrupt or gradual inhomogeneity in the plasma profile. A semi-analytical method was also developed to specifically analyze the abrupt inhomogeneity by modeling the charging and discharging of the dust grain as a capacitor. This semi-analytical method was used to find the gyrophase drift magnitude and direction of a dust grain in abrupt inhomogeneity for a wide range of dust and plasma parameters, charge model choice (orbit motion limited, Patacchini-Hutchinson, and Gatti-Kortshagen), dust-neutral collisionality, and choice of drifting Maxwell-Boltzmann or monoenergetic ion distribution function. The semi-analytical technique developed in this dissertation is particularly useful as compared to simulations for the abrupt inhomogeneity because it allows predictions for many orders of magnitude. For the abrupt inhomogeneity, the simulation and semianalytical results agreed to high precision, validating the semi-analytical approach. For the gradual inhomogeneity, Northrop's analytical results are confirmed by the single-particle trajectory tracker for the conditions analyzed by Northrop. The three grain-charging models were compared with each other and with the single-particle trajectory tracker and found to predict distinctly different trajectories depending on the treatment of neutral drag and flowing ions. Northrop's prediction for the case of ions flowing faster than the ion thermal speed in the frame of dust grain is correct within a factor of two. The effect of the ratio of the gyro-period to the charging time $\left(\tau_{g} / \tau_{c}\right)$ on the magnitude and direction of the gyro-phase drift for abrupt and gradual inhomogeneity is evaluated using the semi-analytical technique and simulations, and it is found that the gyro-phase drift is largest when $\tau_{g} \approx \tau_{c}$. Additionally, the analysis for abrupt and gradual inhomogeneity demonstrates that gyro-phase drift is a sensitive indicator of sheath mechanisms and ion distribution function. 
Applications to the Auburn Magnetized Dusty Plasma Experiment (MDPX) and Enceladus were investigated. For MDPX, gyro-phase drift from gradual inhomogeneity is predicted to be undetectable due to the small degree of inhomogeneity of the plasma across a typical dust gyro-orbit. The thresholds for detecting gyro-phase drift from abrupt inhomogeneity in MDPX are evaluated, and it is found that large UV photoelectron fluxes $\left(f_{u v} /\left(n_{e} v_{t h e}\right)>0.01\right)$ and low neutral gas pressures (less than one mTorr) are necessary for distinguishing between the Patacchini-Hutchinson and the Gatti-Kortshagen charge models. Near Enceladus, gyro-phase drift is predicted to not be responsible for structuring, observed or not observed, in the orbiting dust population. 



\section{Acknowledgements}

I would like to take this opportunity to recognize the many people who helped me in my journey to complete this dissertation. I want to thank Mark Koepke for offering me the chance to work on this project and serving as the chair of my dissertation committee. His enthusiasm for basic science and his eternal optimism are inspiring. I want to thank Mike Zimmerman for helping me get started with the simulation component of this project, and also for his help in tackling some of the difficult challenges faced in its early stages. I want to thank Paul Cassak, David Lewellen, Maura McLaughlin and Earl Scime for agreeing to serve on my graduate committee, reviewing my dissertation, and offering their suggestions to improve this work. I would like to thank Dimitris Vassiliadis for always being available to provide valuable input and advice on nearly any question I could come up with, Herbert Gunell for introducing me to plasma simulation, Vladimir Demidov for his willingness to share with me his expertise on probe theory, and Siegfried Bleher for our useful discussions. I would like to thank all of the PPL-K group members, past and present, who provided companionship and assisted me along the way, including Catalin Teodorescu, Eric Reynolds, Sean Finnegan, Paul Miller, Mattias Törnquist, Sam Nogami, Jim Franek, Jonathon Tucker, Matthew Flaugh, Theodore Lane, and Tommy Steinberger. I want to extend my thanks to Doug Mathess, Tom Milam, Phil Tucker, Nathan Carver-Daniels, and Carl Weber for all of their technical expertise or help in the physics machine shop. I also want to thank Siobhan Byrne, Devon Clelland, Valerie Burgess, Viola Bryant, Vanessa Baker, and Amy Matuga for their help in the office, and Greg Puskar for his help with the physics laboratory curriculum. Special thanks to Sherry Puskar for helping me with the fine details regarding coursework and the dissertation process and always being helpful and friendly to me.

I want to thank my family, especially my mother, father, and grandmother for their unflagging love and encouragement. When I was growing up, my mother, father, and grandmother were my heroes. They still are. I am thankful that my family has always been there for me. I want to thank Nathan Bancroft, Jeannine Fallon, Josh Miller, Stephanie Sears, Jon Blessington, David Wise, Ryan Yeager, William Booth, John McKee, and Audrey and Aaron Holsclaw for their friendship;

truer friends would be hard to find. Most of all, I would like to thank Kelly Pisane. I don't think I could have handled the last leg of this journey without her love and support. 


\section{Table of Contents}

$\begin{array}{ll}\text { Abstract } & \text { ii }\end{array}$

Acknowledgements $\quad$ v

List of Tables

List of Figures $\quad$ xiii

List of Symbols $\quad$ xxxvii

List of Acronyms and Initialisms xli

I Introduction $\quad 1$

A Multi-Phase Case of Solid Particulates in Ionized Gas . . . . . . . . . . . . . . 1

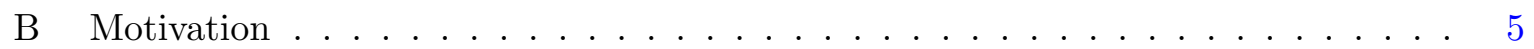

1 Applications of Dusty Plasma . . . . . . . . . . . . . . . . 5

2 State of Knowledge of Grain Charging . . . . . . . . . . . . . 5

$3 \quad$ Utility of examining nonstationary charging effects $\ldots \ldots \ldots \ldots$

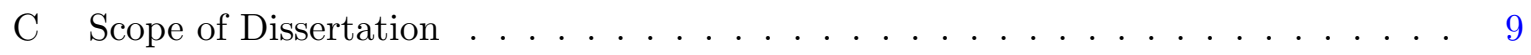

II Description of Plasma $\quad 11$

A Parameters of Homogeneous Plasma . . . . . . . . . . . . . . . . 11

1 Debye Length . . . . . . . . . . . . . . . . . . . 13

2 Number of Electrons in a Debye Sphere . . . . . . . . . . . . . . . . 15

3 Electron Plasma frequency . . . . . . . . . . . . . . . . . 16 
4 Mean Free Path and Knudsen Number . . . . . . . . . . . . . . . . 17

5 Electron, Ion Gyro-frequency and gyro-radius . . . . . . . . . . . . 18

6 Mach numbers. . . . . . . . . . . . . . . . . . . . 19

B Inhomogeneous Plasmas . . . . . . . . . . . . . . . . . 20

1 Planar Sheaths . . . . . . . . . . . . . . . . 20

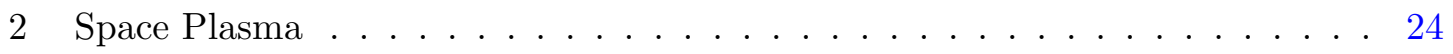

3 Basic parameters of Inhomogeneous Plasma . . . . . . . . . . . . 25

III Description of Dust in Plasma $\quad 29$

A Distinction between Dust in Plasma and Dusty Plasma . . . . . . . . . . . . 29

1 Unmagnetized-orbit and Magnetized-orbit Dust grains . . . . . . . . . . 30

B Physics of the Grain Sheath in Dusty Plasma . . . . . . . . . . . . . . 30

1 Debye-Huckel Grain Sheath Potential Profile . . . . . . . . . . . . . . . . 30

2 Non-monotonic Grain Sheath Potential Profiles . . . . . . . . . . . . . . 31

3 Ion-Neutral Charge-Exchange Collisions in the Dust Grain Sheath . . . . . . 31

4 Grain Capacitance and Dimensionless Surface Potential . . . . . . . . . . 32

C Charging of Dust Grains in the Plasma . . . . . . . . . . . . . 32

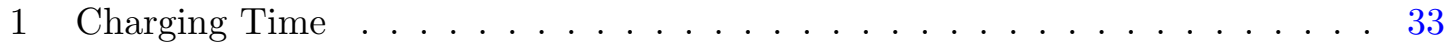

2 Specifics of OML Model . . . . . . . . . . . . . . . . 35

3 Specifics of Gatti-Kortshagen Model . . . . . . . . . . . . . . . . . . . . 41

4 Specifics of Patacchini and Hutchinson Model . . . . . . . . . . . . . . 44

5 Photoemission and Grain Work Function . . . . . . . . . . . . . . . . 46

D Forces acting on a Solid-State particle in Plasma . . . . . . . . . . . . . . . 47

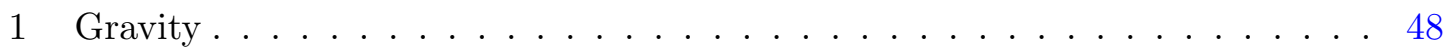

2 Electric Force . . . . . . . . . . . . . . . . . . . . 48

3 Magnetic Force . . . . . . . . . . . . . . . . . . . 49

4 Neutral Drag Force . . . . . . . . . . . . . . . . . . 50 50

$5 \quad$ Ion Drag Force . . . . . . . . . . . . . . . . . 51

6 Thermophoretic Force . . . . . . . . . . . . . . 53

7 Photo-electron Recoil and Radiation Pressure Forces . . . . . . . . . . . . . 54 
E Gyration and Charge Model Parameter Space . . . . . . . . . . . . . 55

IV Methodology for Test Particle Investigation $\quad 59$

A Numerical Treatment of Forces . . . . . . . . . . . . . . . . . . . 60

1 Modification to Boris Algorithm . . . . . . . . . . . . . . . . . 61

2 Iterative Velocity Solver . . . . . . . . . . . . . . . . . 64

3 Adjustments for Non-Inertial Reference Frames . . . . . . . . . . . . . . . . 66

4 Adjustments for Ion Flow along Magnetic Field Direction . . . . . . . . . . 67

B Numerical Treatment of Charging . . . . . . . . . . . . . . 68

C Gyro-averaged Quantities . . . . . . . . . . . . . . . . 69

D Benchmarking Simulations to Analytical Examples . . . . . . . . . . . 71

$1 \boldsymbol{E} \times \boldsymbol{B}$ Drift in slab Geometry . . . . . . . . . . . . . . 72

$2 \quad \boldsymbol{F} \times \boldsymbol{B}$ Drift in slab Geometry with Epstein Drag . . . . . . . . . . . 72

$3 \boldsymbol{F} \times \boldsymbol{B}$ Drift with Flowing Ions $\ldots \ldots \ldots \ldots \ldots$

$\begin{array}{lll}\text { V Analytical and Simulation Results } & 77\end{array}$

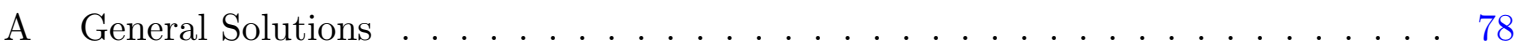

B Abrupt Inhomogeneity . . . . . . . . . . . . . . . . . . 81

1 Charging Model Comparisons with Sheath Mechanisms . . . . . . . . . . . 93

C Gradual Inhomogeneity . . . . . . . . . . . . . . . . . . 98

$1 \quad$ Linear Profile . . . . . . . . . . . . . . . . . . . . . . . . . 99

2 Cylindrical Geometry . . . . . . . . . . . . . . . . . . . 104

3 Gradual versus Step-Function Inhomogeneity . . . . . . . . . . . . 105

$\begin{array}{lll}\text { VI Applications } & 107\end{array}$

A Application to Auburn Magnetized Dusty Plasma Experiment . . . . . . . . . . 107

1 Accessible Parameter-Space Regimes in MDPX . . . . . . . . . . . . . 109

2 Abrupt Inhomogeneities in Auburn MDPX . . . . . . . . . . . . . . . 110

3 Gradual Inhomogeneities in MDPX . . . . . . . . . . . . . . 116

B Application to Enceladus . . . . . . . . . . . . . . . . . 121 
A Optimizing the Detection of Gyro-phase Drift in MDPX . . . . . . . . 125

B Model Uncertainty . . . . . . . . . . . . . . . . . . . . 129

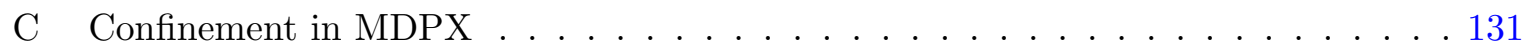

D Gyro-phase Drift in Space . . . . . . . . . . . . . . . 133

$\begin{array}{ll}\text { VIII Conclusions } & 135\end{array}$

$\begin{array}{ll}\text { Appendices } & 139\end{array}$

$\begin{array}{llr}\text { A Derivations } & 141\end{array}$

1 Orbit-motion Limited Charge Model derivation _ . . . . . . . . . . . . . 141

$2 \boldsymbol{E} \times \boldsymbol{B}$ Drift in Slab Geometry . . . . . . . . . . . . . . . 146

$3 \boldsymbol{E} \times \boldsymbol{B}$ Drift with Linear Drag in Slab Geometry . . . . . . . . . . . . . . 150

4 Northrop's Adiabatic Approximation to Guiding Center Motion . . . . . . . . . . 152

i Adiabatic Approximation to Guiding Center Motion in Arbitrary Geometry . 153

ii Adiabatic Approximation to Guiding Center Motion for Time-dependent Grain

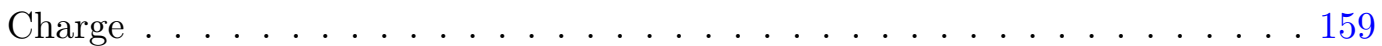

B Computer codes $\quad 163$

1 Grain Motion . . . . . . . . . . . . . . . . . . . 164

i Main Simulation Routine . . . . . . . . . . . . . . . . . . . 164

ii Position and Velocity Advancement . . . . . . . . . . . . . . . 205

iii Abrupt Inhomogeneity Theory Code . . . . . . . . . . . . . . . 237

2 Specifying the Space or Laboratory Plasma Profile . . . . . . . . . . . . . . . 278

3 Gyro-phase Analysis Code . . . . . . . . . . . . . . . . . . . 334

4 Charging Algorithm Codes . . . . . . . . . . . . . . . . . 355

i Specifying Charging Model . . . . . . . . . . . . . . . . . 362

ii Charging Model Selection . . . . . . . . . . . . . . 366

iii Bisection Method . . . . . . . . . . . . . . . . . 397

iv Dimensionless Charging Equation . . . . . . . . . . . . . . . . . 421 
Tables

Figures 


\section{List of Tables}

1.1 Parameters for laboratory dust experiments and other regimes with magnetic fields. 461

1.2 Dimensionless Parameters for laboratory dust experiments with magnetic fields. Even experiments with large magnetic fields $(B>1 \mathrm{~T})$ do not necessarily produce magnetized orbit dust grains due to the presence of neutral drag, which is represented in this table by the $\omega_{c d} / \nu_{d n}$ parameter. Parameters that depend on the gyro-radii quoted here correspond to the mean gyro-radius $R_{L s}=\sqrt{\frac{\pi k_{b} T_{s}}{2 m_{e}}} / \omega_{c s}$. The dust gyro-radius $R_{L d}$ quoted in this table does not include the enlarging effect of an applied electric field that is perpendicular to the magnetic field. Dust grains are levitated in a planar sheath where the ions have a fluid drift $v_{i}=\sqrt{\frac{k_{b} T_{e}}{m_{i}}}$ directed along the sheath dimension, which means that the Debye length is given by $\lambda_{D}=\sqrt{\frac{\epsilon_{0} k_{b} T_{e}}{e^{2} n_{i}}}$ (Carstensen et al., 2012). The dust-neutral collision frequency $\nu_{d n}$ is calculated assuming Epstein drag and is given by equation 3.59 . . . . . . . . 462

2.1 Summary of retarding and attracting conditions for electrons and ions. . . . . . . . 462

6.1 Selected plasma parameters throughout the Saturnian system, from Khurana et al. (2008); Richardson (1998). . . . . . . . . . . . . . . . 463 


\section{List of Figures}

2.1 For a small spherical conductor $a \ll \lambda_{D}$, the Yukawa, or Debye-Huckel potential is compared to a Coloumb potential. . . . . . . . . . . . . . 4 466

2.2 Space potential, electron and ion densities, and ion fluid velocity along the sheath direction for a collisionless and weakly collisional Child-Langmuir sheath, represented by the top and bottom panels, respectively. For the same plasma parameters, the sheath is larger in the weakly collisional case. . . . . . . . . . . . . 467

2.3 Example grain trajectory around Saturn in the corotating reference frame. The $a=0.025 \mu \mathrm{m}$ grain is launched from Enceladus at the Kepler velocity. On the left side of the figure, the thick red line in the center shows the extent of Saturn, the larger, concentric cyan circle shows the radius for geosynchronous orbit, and the thin black line corresponds to the grain trajectory. A dipole magnetic field is assumed, and the parameters in the corotating plasma are $n_{0}=4 \times 10^{7} \mathrm{~m}^{-3}, T_{e}=5 \mathrm{eV}$, $T_{i}=10 \mathrm{eV}$, with ions having $20 \mathrm{amu}$. On the right side of the figure, the radial excursion of the grain as a function of time is plotted. The grain drifts radially inward.468

3.1 Knudsen capture parameter $K n_{R}$ as a function of the gyration parameter, $\omega_{c d} / \nu_{d n}$. The number product is expressed as $\frac{9 \sqrt{\pi}}{8(1.22)} \sqrt{\frac{m_{i} T_{i}}{m_{e} T_{e}}} \beta_{e} \frac{n_{i}}{n_{n}} K n_{a}^{3}\left(\frac{T_{i}}{T_{e}}+\frac{n_{e}}{n_{i}}\right)^{-1} \ldots \ldots . . .469$

3.2 Figure from Merlino (2006) which shows how dimensionless surface potential $\chi_{e}=$ $e V_{d} /\left(k_{b} T_{e}\right)$ depends on the ratio of electron to ion temperature. $\ldots \ldots \ldots$ 
3.3 Figure from Gatti and Kortshagen (2008). This shows the normalized particle potential $\chi_{e}=q_{d} /\left(C_{d} T_{e}\right)$ as a function of the capture radius Knudsen number $K n_{R 0}$ for a particle with a diameter of $500 \mathrm{~nm}, n_{i 0}=10^{10} \mathrm{~cm}^{3}, T_{e}=2.5 \mathrm{eV}, T_{i}=0.025$ $\mathrm{eV}$, and $n_{d} / n_{i 0} \approx 0$, meaning the plasma is treated as dust in plasma rather than dusty-plasma. Also plotted are the probabilities of performing zero $\left(P_{0}\right)$, one $\left(P_{1}\right)$, and more than one collision $P_{>1}$ inside the capture radius sphere. . . . . . . . 471

4.1 This diagram shows the relationship between the velocity vector and the gyro-phase angle $\phi$ for an example grain trajectory. . . . . . . . . . . . . . . . . . 472

4.2 A $1 \mu \mathrm{m}$ diameter grain starts at the origin, gyrates, and drifts in a magnetic field $B=4 \mathrm{~T}$ and an electric field $E_{x}=100 \mathrm{~V} / \mathrm{m}, E_{y}=100 \mathrm{~V} / \mathrm{m}$. The grain has $\approx 1400$ electrons on its surface. . . . . . . . . . . . . . . . . . 473

4.3 The effective gyro-radius can be accomodated in a laboratory vacuum vessel only for small magnitude of radial electric field and small grain radius. A: Effective dust grain gyro-radius as a function of a constant, ambient electric field is plotted for grain diameters $a=1 \mu \mathrm{m}, a=0.1 \mu \mathrm{m}, a=.015 \mu \mathrm{m}$, and $a=0.01 \mu \mathrm{m}$. B: Effective gyro-radius as a function of grain radius is plotted for constant, ambient electric field values of $100 \mathrm{~V} / \mathrm{m}, 10 \mathrm{~V} / \mathrm{m}$, and $1 \mathrm{~V} / \mathrm{m} . \ldots \ldots \ldots \ldots \ldots$

4.4 A $1 \mu \mathrm{m}$ diameter grain starts at the origin and moves in a magnetic field $B=4$ $\mathrm{T}$, an electric field $E_{x}=100 \mathrm{~V} / \mathrm{m}, E_{y}=100 \mathrm{~V} / \mathrm{m}$, a neutral gas pressure of 1 mTorr, with the neutrals flowing at $v_{x n}=-100 \mathrm{~m} / \mathrm{s}$, which produces a drag force of magnitude $\approx 10^{-15} \mathrm{~N}$. By comparison, the magnitude of the magnetic force on this grain $\approx 10^{-18} \mathrm{~N}$. The grain has $\approx 1400$ electrons on its surface, $\omega_{c d} \approx 2 \mathrm{~s}^{-1}$, $\nu_{d n} \approx 2 \times 10^{-2} \mathrm{~s}^{-1}$ so that $\omega_{c d} / \nu_{d n} \gg 1 \ldots \ldots \ldots \ldots$. . . . . . . . . . . . . . . . . .

4.5 A $1 \mu \mathrm{m}$ diameter grain starts at the origin and moves in a magnetic field $B=4$ $\mathrm{T}$, an electric field $E_{x}=100 \mathrm{~V} / \mathrm{m}, E_{y}=100 \mathrm{~V} / \mathrm{m}$, a neutral gas pressure of 1 mTorr, with the neutrals flowing at $v_{x n}=-100 \mathrm{~m} / \mathrm{s}$ which produces a drag force of magnitude $\approx 10^{-15} \mathrm{~N}$. By comparison, the magnitude of the magnetic force on this grain $\approx 10^{-18} \mathrm{~N}$. The grain has $\approx 1400$ electrons on its surface, $\omega_{c d} \approx 2 \mathrm{~s}^{-1}, \nu_{d n} \approx 2$ so that $\omega_{c d} / \nu_{d n} \approx 1$ and the grain does not make a complete gyration. . . . . . . 475 
4.6 A $1 \mu \mathrm{m}$ diameter grain starts at the origin and moves in a magnetic field $B=4 \mathrm{~T}$, an electric field $E_{x}=-100 \mathrm{~V} / \mathrm{m}$, a neutral gas pressure of $0.01 \mathrm{mTorr}$, with the neutrals flowing at $v_{x n}=-100 \mathrm{~m} / \mathrm{s}$, which produces a drag force of magnitude $\approx 10^{-15} \mathrm{~N}$. By comparison, the magnitude of the magnetic force on this grain $\approx 10^{-18} \mathrm{~N}$. The grain has $\approx 1400$ electrons on its surface. . . . . . . . . . . . . 476

4.7 A $1 \mu \mathrm{m}$ diameter grain starts at the origin and moves in a magnetic field $B=4 \mathrm{~T}$, an ion density of $10^{12} \mathrm{~m}^{-3}$, with the ions flowing at $v_{x i}=-200 \mathrm{~m} / \mathrm{s}$, which produces a drag force of magnitude $\approx 10^{-17} \mathrm{~N}$. By comparison, the magnitude of the magnetic force on this grain $\approx 10^{-18} \mathrm{~N}$. The grain has $\approx 1400$ electrons on its surface. . . . 477

5.1 Grain trajectory for $E_{0 y}=0, E_{0 x} /\left(B v_{\perp}\right)=-1, \lambda_{D} / a=10^{3}, \lambda_{i} / a=10^{5}, m_{d} / m_{e}=$ $10^{11}, q_{1} / q_{2}=1.74, \omega_{c d} / \nu_{d n}=10^{4}$, and $\tau_{g} / \tau_{c}=5$. In this case, the instantaneous gyro-radius must be specified by the more general $v_{\perp}(t) / \frac{\mathrm{d} \phi}{\mathrm{d} t}$, rather than $v_{\perp}(t) / \omega_{c d}(t)$, where $\phi$ is the gyro-phase angle. The dotted line represents the guiding center position.

5.2 Grain trajectories for $a=0.05 \mu \mathrm{m}$. The dashed and solid lines represent the trajectories of $\tau_{g} / \tau_{c}$ ratios for $\tau_{g} / \tau_{c} \rightarrow \infty\left(n_{0}=n_{e}=n_{i}=10^{16} \mathrm{~m}^{-3}\right)$ and $\tau_{g} / \tau_{c}=19.204$ (for $n_{0}=10^{13} \mathrm{~m}^{-3}$ ) respectively. Squares and diamonds indicate the gyro-averaged guiding centers for $\tau_{g} / \tau_{c} \rightarrow \infty$ and $\tau_{g} / \tau_{c}=19.204$ respectively. . . . . . . . . . 479

5.3 Example of a drag affected trajectory overlaid with a non-drag affected trajectory. The ratio $\omega_{c d} / \nu_{d n}$ is 10 for the drag affect trajectory and $10^{4}$ for the non-drag affected trajectory, with all other parameters equal. The drag affected trajectory has an inherent drift component along the $\hat{x}$-direction. For this plot, Argon ions are assumed, $q_{1} / q_{2}=1.74, T_{e} / T_{i}=200, a / R_{L e}=0.1, \lambda_{D} / a=10^{5}, \lambda_{i} / a=5, N_{D e}=10^{3}$, $m_{d} / m_{e} \approx 10^{12}$, and $\tau_{g} / \tau_{c} \approx 20$ (non-instantaneous charging). . . . . . . . . 480

5.4 Charge evolution for $a=0.05 \mu \mathrm{m}$ grain in the OML and capacitor approximation. A: Charge evolution of a dust grain as it transitions from the UV-absent to UV-present region. The UV flux is $4 \times 10^{18} \mathrm{~m}^{-2} \mathrm{~s}^{-1}$, which yields a photo-electron current of $5.03 \times 10^{-15} \mathrm{~A}$. The capacitor model reaches $\frac{1}{\mathrm{e}}\left(q_{2}-q_{1}\right)$ at the same time as the OML charge model, although it is a different function of time. B: Charge evolution of a dust grain as it transitions from the UV-present to UV-absent region. . . . . . . . . 481 
5.5 Example trajectory of a grain that starts at the origin, but does not transition back to the $x<0$ or $q_{1}$ region due to excessive drag. In this example, $\omega_{1} / \nu_{d n}=3$, so when the grain goes from $x<0$ to $x>0$ when $t \approx 0$, this ratio is barely above unity. For this plot, Argon ions are assumed, $q_{1} / q_{2}=1.74, T_{e} / T_{i}=200, a / R_{L e}=0.1, \lambda_{D} / a=10^{5}$, $\lambda_{i} / a=5, N_{D e}=10^{3}, m_{d} / m_{e}=10^{20}$, and $\tau_{g} / \tau_{c} \approx 20$ (non-instantaneous charging).

5.6 Simulated example trajectory of a grain that starts at the origin, transitions to the $x<0$ half-plane, and does not re-enter the $x>0$ region. This situation generally occurs for small values of $\tau_{g} / \tau_{c}$. Argon ions are assumed for this drag-absent simulation plot, and $a=10^{-7} \mathrm{~m}, \rho_{d}=90 \mathrm{~kg} \mathrm{~m}^{-3}, n_{0}=10^{14} \mathrm{~m}^{-3} T_{e}=5 \mathrm{eV}, T_{i}=0.025$ $\mathrm{eV}$. The dimensionless numbers for this trajectory are $q_{1} / q_{2}=1.74, T_{e} / T_{i}=200$, $a / R_{L e}=0.1, \lambda_{D} / a=10^{3}, \lambda_{i} / a=10^{5}, N_{D e}=10^{3}, m_{d} / m_{e}=10^{12}$, and $\tau_{g} / \tau_{c} \approx 20$ (non-instantaneous charging).

5.7 This figure shows the possibility of dust grains re-entering the $x=0$ region when drag is present. This is shown clearly in the solid blue trajectory $\omega_{c d} / \nu_{d n}=10$, while it does not occur for the drag-absent trajectory with $\omega_{c d} / \nu_{d n}=10^{4}$. Other than $\omega_{c d} / \nu_{d n}$ ratios, both cases have identical parameters; Argon ions are assumed, $q_{1} / q_{2}=2, T_{e} / T_{i}=200, a / R_{L e}=0.1, \lambda_{D} / a=10^{5}, \lambda_{i} / a=5, N_{D e}=10^{3}, m_{d} / m_{e}=$ $10^{22}$, and $\log \left(\tau_{g} / \tau_{c}\right)=5.3$. When drag is present, this situation generally occurs for larger values of $\tau_{g} / \tau_{c}$.

5.8 Guiding center drift magnitude dependence on the gyration parameter $\omega_{c d} / \nu_{d n}$ for the parameters $T_{e} / T_{i}=200, q_{1} / q_{2}=1.74, a / R_{L e}=0.1, \lambda_{D} / a=10^{5}, \lambda_{i} / a=5$, $N_{D e}=10^{3}, m_{d} / m_{e}=10^{12}$, and $\log \left(\tau_{g} / \tau_{c}\right)=1$. The abscissa is mislabelled; $\tau_{g} / \tau_{c}$ should be $\omega_{c d} / \nu_{d n} \ldots \ldots \ldots \ldots \ldots \ldots \ldots \ldots$. . . . . . . . . . . . . . . . . . . .

5.9 Guiding center drift magnitude and direction dependence on the initial gyro-phase angle $\theta_{0}$. In this figure, drag is absent $\left(\omega_{c d} / \nu_{d n}=10^{4}\right)$, UV illumination is the source of abrupt inhomogeneity, $q_{1} / q_{2}=2$, Argon ions are assumed, $T_{e} / T_{i}=200$, $a / R_{L e}=0.1, \lambda_{D} / a=10^{3}, \lambda_{i} / a=10^{5}, N_{D e}=2 \times 10^{4}$, and $m_{d} / m_{e}=10^{12}$. The top panel shows the guiding center drift magnitudes, the middle panel shows the guiding center drift angle relative to the $x$-axis, and the bottom panel shows the $\tau_{g} / \tau_{c}$ ratio as a function of the initial gyro-phase angle. 486 
5.10 Gyro-phase drift magnitude and direction for the first gyro-cycle as a function of the ratio $\tau_{g} / \tau_{c}$ for the abrupt theory and Northrop's prediction. The absolute values of the velocities are plotted in the topmost plot, while the direction is supplied by the bottom panel. In this figure, UV illumination is the source of inhomogeneity, Argon ions are assumed, $T_{e} / T_{i}=200, a / R_{L e}=0.1, \lambda_{D} / a=10^{3}, \lambda_{i} / a=10^{5}, N_{D e}=2 \times 10^{4}$, $q_{1} / q_{2}=2$, and $10^{10} \leq m_{d} / m_{e} \leq 10^{20}$. The grain drifts in the negative $\hat{y}$-direction

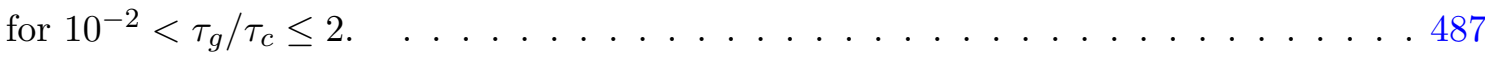

5.11 This figure shows examples of fitting a sinusoidal charge variation to $q(t)$ in order to obtain the gyro-phase drift vector using Northrop's method for the case of abrupt inhomogeneity. In this figure, the dimensionless surface potential $\chi_{e}(t)=$ $e q(t) /\left(C_{d} k_{b} T_{e}\right)$ is shown instead of $q(t)$. Each plot shows $q(t)$ and a sinusoidal fit to a selected data point from figure $5.10 \ldots \ldots \ldots$. . . . . . . . . . . . . . . . . .

5.12 Radial distance from the origin is the gyro-radius as a function of gyro-angle in meters. The grain size is $a=0.05 \mu \mathrm{m}$. In the semi-analytical model, $q(t)$ is continuous, while for the simulations, $q(t)$ is discrete. Despite this major difference, the semianalytical model and the simulation closely agree. The quantitiy $\frac{\mathrm{d} q}{\mathrm{~d} t}$ is discontinuous at $0^{\circ}$ and $\approx 80^{\circ}$ because the current to the grain abruptly changes at both of these

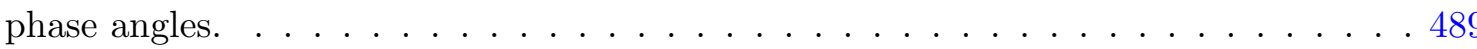

5.13 Gyro-phase drift magnitude and direction for the first gyro-cycle as a function of the ratio $\tau_{g} / \tau_{c}$ with an electric field. In this figure, drag is absent $\left(\omega_{c d} / \nu_{d n}=10^{4}\right)$, UV illumination is the source of abrupt inhomogeneity, $q_{1} / q_{2}=2$, Argon ions are assumed, $T_{e} / T_{i}=200, a / R_{L e}=0.1, \lambda_{D} / a=10^{3}, \lambda_{i} / a=10^{5}, N_{D e}=2 \times 10^{4}$, and $m_{d} / m_{e}$ is swept from $10^{10}$ to $10^{20} \ldots \ldots \ldots \ldots$. . . . . . . . . . . . . . . . .

5.14 Gyro-phase drift magnitude and direction for the first gyro-cycle as a function of the ratio $\tau_{g} / \tau_{c}$ for different values of $q_{1} / q_{2}$. 
5.15 Gyro-phase drift magnitude for the first gyro-cycle as a function of the ratio $\tau_{g} / \tau_{c}$ for different values of $m_{e} / m_{i}$ when $q_{1} / q_{2}=2$ is held constant. The top panel corresponds to the guiding center velocity along the $\hat{x}$-direction and the bottom panel corresponds to the guiding center velocity along the $\hat{y}$-direction. The values of $m_{e} / m_{i}$ chosen correspond to Hydrogen, Nitrogen, Argon, and Xenon plasmas in ascending order. Evident in this figure is that all of the plots have exactly the same shape and same values, but lower values of $m_{e} / m_{i}$ allow access to smaller values of $\tau_{g} / \tau_{c}$. For this plot, $\lambda_{D} / a=10^{3}, \lambda_{i} / a=10^{5}, N_{D e}=10^{4}, a / R_{L e}=10^{-1}$, and $T_{e} / T_{i}=200$

5.16 Gyro-phase drift magnitude for the first gyro-cycle as a function of the ratio $\tau_{g} / \tau_{c}$ for different values of $m_{e} / m_{i}$ when the normalized photo-current $f_{u v} /\left(n_{e} v_{t h e}\right)=0.25$ is held constant. The top panel corresponds to the guiding center velocity along the $\hat{x}$-direction and the bottom panel corresponds to the guiding center velocity along the $\hat{y}$-direction. The values of $m_{e} / m_{i}$ chosen correspond to Hydrogen, Nitrogen, Argon, and Xenon plasmas in ascending order, which produce $q_{1} / q_{2}$ values of 1.4 , $1.61,1.74$, and 1.93 respectively. Like in figure 5.15 , lower values of $m_{e} / m_{i}$ allow access to smaller values of $\tau_{g} / \tau_{c}$, but in these plots the lower values of $m_{e} / m_{i}$ also produce greater drift amplitudes. For this plot, $\lambda_{D} / a=10^{3}, \lambda_{i} / a=10^{5}, N_{D e}=10^{4}$,

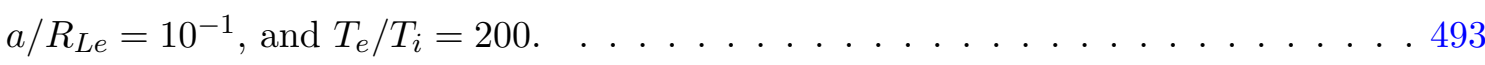

5.17 Gyro-phase drift magnitude for the first gyro-cycle as a function of the ratio $\tau_{g} / \tau_{c}$ for different values of $T_{e} / T_{i}$ when $q_{1} / q_{2}=2$ is held constant. The top panel corresponds to the guiding center velocity along the $\hat{x}$-direction and the bottom panel corresponds to the guiding center velocity along the $\hat{y}$-direction. The temperature ratios $T_{e} / T_{i}=$ $10^{-1}, T_{e} / T_{i}=10^{0}, T_{e} / T_{i}=2 \times 10^{1}$, and $T_{e} / T_{i}=2 \times 10^{2}$ are shown in ascending order on the $\log \left(T_{e} / T_{i}\right)$ axis. Higher values of $T_{e} / T_{i}$ allow access to smaller values of $\tau_{g} / \tau_{c}$, in a much more dramatic way than seen in smaller values of $m_{e} / m_{i}$ in 5.15. For this plot, hydrogen plasma assumed, $\lambda_{D} / a=10^{3}, \lambda_{i} / a=10^{5}, N_{D e}=10^{4}$, and

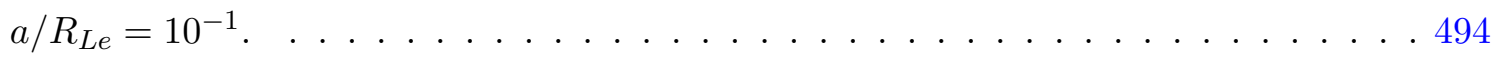


5.18 Gyro-phase drift magnitude for the first gyro-cycle as a function of the ratio $\tau_{g} / \tau_{c}$ for different values of $T_{e} / T_{i}$ when $f_{u v} /\left(n_{e} v_{t h e}\right)=0.25$ is held constant. The top panel corresponds to the guiding center velocity along the $\hat{x}$-direction and the bottom panel corresponds to the guiding center velocity along the $\hat{y}$-direction. The temperature ratios $T_{e} / T_{i}=10^{-1}, T_{e} / T_{i}=10^{0}, T_{e} / T_{i}=2 \times 10^{1}$, and $T_{e} / T_{i}=2 \times 10^{2}$ are shown in ascending order on the $T_{e} / T_{i}$ axis, and these temperature ratios produce $q_{1} / q_{2}$ ratios of $2,1.94,1.57$, and 1.4 respectively. Higher values of $T_{e} / T_{i}$ for constant UV photo-current allow access to smaller values of $\tau_{g} / \tau_{c}$, in a much more dramatic way than seen in smaller values of $m_{e} / m_{i}$ in figure 5.16. For this plot, hydrogen plasma assumed, $\lambda_{D} / a=10^{3}, \lambda_{i} / a=10^{5}, N_{D e}=10^{4}$, and $a / R_{L e}=10^{-1} \ldots \ldots \ldots$

5.19 Gyro-phase drift magnitude for the first gyro-cycle as a function of the ratio $\tau_{g} / \tau_{c}$ for different values of $N_{D e}$ when $v=f_{u v} /\left(n_{e} v_{t h e}\right)=0.25$ is held constant. The top panel corresponds to the guiding center velocity along the $\hat{x}$-direction and the bottom panel corresponds to the guiding center velocity along the $\hat{y}$-direction. Higher values of $N_{D e}$ allow access to smaller values of $\tau_{g} / \tau_{c}$. For this plot, hydrogen plasma assumed, $\lambda_{D} / a=10^{3}, \lambda_{i} / a=10^{5}, T_{e} / T_{i}=200$, and $a / R_{L e}=10^{-1}$.

5.20 Gyro-phase drift magnitude for the first gyro-cycle as a function of the ratio $\tau_{g} / \tau_{c}$ for different values of $N_{D e}$ when $f_{u v} /\left(n_{e} v_{t h e}\right)=0.25$ is held constant. In the top panel, solid curves correspond to $\left|v_{x g c}\right|$, the guiding center velocity long the $\hat{x}$-direction, and the dashed curves correspond to $\left|v_{x g c}\right|$, the guiding center velocity along the $\hat{y}$ direction. The plots of $\left|v_{x g c}\right|$ and $\left|v_{x g c}\right|$ overlap and are identical for different values of $N_{D e}$, showing that the gyro-phase drift magnitude and direction is not affected by the $N_{D e}$ parameter. The bottom panel shows the guiding center drift angle with respect to the $x$-axis in the dust grain trajectory configuration space. The guiding center drift is calculated for electron magnetization parameter values $a / R_{L e}=10^{-2}$, $10^{-1}, 10^{0}, 10^{1}$, and $10^{2}$, and the $q_{1} / q_{2}$ ratios are given by $1.74,1.74,2.57,2.57$, and 10.14 respectively. The $q_{1} / q_{2}$ ratios are increasing because the current collection regime changes at certain values of the $a / R_{L e}$ parameter. Higher values of $N_{D e}$ allow access to smaller values of $\tau_{g} / \tau_{c}$. For this plot, hydrogen plasma assumed, $\lambda_{D} / a=10^{3}, \lambda_{i} / a=10^{5}, T_{e} / T_{i}=200$, and $a / R_{L e}=10^{-1} \ldots \ldots \ldots 79 \ldots$ 
5.21 Gyro-phase drift magnitude for the first gyro-cycle as a function of the ratio $\tau_{g} / \tau_{c}$ for different values of $a / R_{L e}$ when $f_{u v} /\left(n_{e} v_{t h e}\right)=0.25$ is held constant. The top panel corresponds to the guiding center velocity along the $\hat{x}$-direction and the bottom panel corresponds to the guiding center velocity along the $\hat{y}$-direction. For this plot, Argon plasma assumed, $\omega_{c d} / \nu_{d n}=10^{4}, N_{D e}=10^{4}, \lambda_{D} / a=10^{3}, \lambda_{i} / a=10^{5}$, and $T_{e} / T_{i}=200$. The OML and Patacchini-Hutchinson charge models produce the same guiding center drift magnitudes for these parameters. . . . . . . . . . . 498

5.22 Gyro-phase drift magnitude for the first gyro-cycle as a function of the ratio $\tau_{g} / \tau_{c}$ for different values of $\omega_{c d} / \nu_{d n}$ when $f_{u v} /\left(n_{e} v_{t h e}\right)=0.25$ is held constant. The top panel corresponds to the guiding center velocity along the $\hat{x}$-direction and the bottom panel corresponds to the guiding center velocity along the $\hat{y}$-direction. For this plot, hydrogen plasma assumed, $N_{D e}=10^{4}, \lambda_{D} / a=10^{3}, \lambda_{i} / a=10^{5}, T_{e} / T_{i}=200$, and $a / R_{L e}=10^{-1} \ldots \ldots \ldots \ldots \ldots \ldots \ldots \ldots \ldots \ldots \ldots$

5.23 Gyro-phase magnitude and direction dependence on $\tau_{g} / \tau_{c}$ for the three different charging models for several values of the Knudsen number $\left(\lambda_{i} / a\right)$. Solid (dashed) red line corresponds to the Patacchini-Hutchinson model guiding center drift $v_{x g c}\left(v_{y g c}\right)$, solid (dashed black line corresponds to the OML model guiding center drift $v_{x g c}$ $\left(v_{y g c}\right)$, and the solid (dashed) cyan line corresponds to the Gatti-Kortshagen model guiding center drift $v_{x g c}\left(v_{y g c}\right)$. The other parameters chosen for an Argon plasma include $f_{U V} /\left(n_{e} v_{t h e}\right)=0.25, \omega_{c d} / \nu_{d n}=10, N_{D e}=10^{4}, a / R_{L e}=0.1, \lambda_{D} / a=10^{3}$, and $T_{e} / T_{i}=200$. The OML and Patacchini-Hutchinson charge models predict the same guiding center drift for these parameters. . . . . . . . . . . . . . 500

5.24 Gyro-phase magnitude and direction dependence on $\tau_{g} / \tau_{c}$ for the three different charging models for several values of the Knudsen number $\left(\lambda_{i} / a\right)$. The abscissa is $\tau_{g} / \tau_{c}$, not $\log _{10} \frac{\tau_{g}}{\tau_{c}}$. Solid (dashed) red line corresponds to the Patacchini-Hutchinson model guiding center drift $v_{x g c}\left(v_{y g c}\right)$, solid (dashed black line corresponds to the OML model guiding center drift $v_{x g c}\left(v_{y g c}\right)$, and the solid (dashed) cyan line corresponds to the Gatti-Kortshagen model guiding center drift $v_{x g c}\left(v_{y g c}\right)$. The other parameters chosen for an Argon plasma include $v=f_{U V} /\left(n_{e} v_{t h e}\right)=0.25, \omega_{c d} / \nu_{d n}=10$, $N_{D e}=10^{4}, a / R_{L e}=1.1, \lambda_{D} / a=10^{3}$, and $T_{e} / T_{i}=200 \ldots \ldots \ldots \ldots 1$ 
5.25 Gyro-phase magnitude and direction dependence on $\tau_{g} / \tau_{c}$ for the three different charging models for several values of $a / R_{L e}$. Solid (dashed) red line corresponds to the Patacchini-Hutchinson model guiding center drift $v_{x g c}\left(v_{y g c}\right)$, solid (dashed black line corresponds to the OML model guiding center drift $v_{x g c}\left(v_{y g c}\right)$, and the solid (dashed) cyan line corresponds to the Gatti-Kortshagen model guiding center drift $v_{x g c}\left(v_{y g c}\right)$. The other parameters chosen for an Argon plasma include $f_{U V} /\left(n_{e} v_{t h e}\right)=0.25, \omega_{c d} / \nu_{d n}=10, N_{D e}=10^{4}, \lambda_{i} / a=10^{5}, \lambda_{D} / a=10^{3}$, and $T_{e} / T_{i}=200$. The OML and Patacchini-Hutchinson charge models produce the same guiding center drift magnitudes, producing overlapping plots except for the values $a / R_{L e}=1$ and $a / R_{L e}=10$. In the limit of very large or very small values of $a / R_{L e}$, the OML and Patacchini-Hutchinson models predict the same guiding center drift

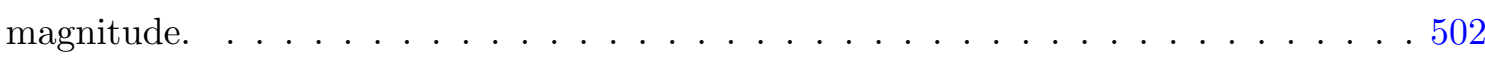

5.26 Guiding center drift magnitude dependence on $\tau_{g} / \tau_{c}$ for the three different charging models for several values of the parameter $\lambda_{D} / a$. Solid (dashed) red line corresponds to the Patacchini-Hutchinson model guiding center drift $v_{x g c}\left(v_{y g c}\right)$, solid (dashed black line corresponds to the OML model guiding center drift $v_{x g c}\left(v_{y g c}\right)$, and the solid (dashed) cyan line corresponds to the Gatti-Kortshagen model guiding center drift $v_{x g c}\left(v_{y g c}\right)$. The other parameters chosen for an Argon plasma include $v=f_{U V} /\left(n_{e} v_{t h e}\right)=0.25, \omega_{c d} / \nu_{d n}=10, N_{D e}=10^{4}, a / R_{L e}=0.1, \lambda_{i} / a=10^{5}$, and $T_{e} / T_{i}=200$. The OML and Patacchini-Hutchinson charge models predict the same guiding center drift for all parameters, and the Gatti-Kortshagen charge model predicts the same guiding center drift as the OML and Patacchini-Hutchinson models

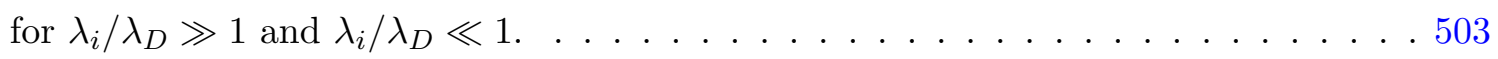


5.27 Guiding center drift magnitude dependence on $\tau_{g} / \tau_{c}$ for the three different charging models for several values of the parameter $\lambda_{D} / a$. Solid (dashed) red line corresponds to the Patacchini-Hutchinson model guiding center drift $v_{x g c}\left(v_{y g c}\right)$, solid (dashed black line corresponds to the OML model guiding center drift $v_{x g c}\left(v_{y g c}\right)$, and the solid (dashed) cyan line corresponds to the Gatti-Kortshagen model guiding center drift $v_{x g c}\left(v_{y g c}\right)$. The other parameters chosen for an Argon plasma include $v=$ $f_{U V} /\left(n_{e} v_{t h e}\right)=0.25, \omega_{c d} / \nu_{d n}=10, N_{D e}=10^{4}, a / R_{L e}=1.1, \lambda_{i} / a=10^{5}$, and $T_{e} / T_{i}=200$. The OML and Patacchini-Hutchinson charge models predict the same guiding center drift for these parameters. . . . . . . . . . . . . . 504

5.28 Guiding center $x$-component drift magnitude dependence on $\lambda_{D} / a$ and $\tau_{g} / \tau_{c}$ for the three different charging models as a function of the parameter $\lambda_{D} / a$. In the leftmost plot, the abscissa is actually $\lambda_{D} / a$ instead of $\log _{10} \frac{\lambda_{D}}{a}$, and in the rightmost plot, the abscissa is actually $\tau_{g} / \tau_{c}$ instead of $\log _{10} \frac{\tau_{g}}{\tau_{c}}$. Here, a grain with $a=10^{-7}$ $\mathrm{m}$ made out of carbon has a mass ratio $m_{d} / m_{e}=10^{13}$, and it is the variation of $\lambda_{D} / a$ that produces the $\tau_{g} / \tau_{c}$ variation of the guiding center drift. Solid red line corresponds to the Patacchini-Hutchinson model guiding center drift $v_{x g c}$, solid black line corresponds to the OML model guiding center drift $v_{x g c}$, and the solid cyan line corresponds to the Gatti-Kortshagen model guiding center drift $v_{x g c}$. The parameter $\lambda_{D} / a$ is swept while all other parameters are held constant. The other parameters chosen for an Argon plasma are $v=f_{U V} /\left(n_{e} v_{t h e}\right)=0.25, \omega_{c d} / \nu_{d n}=10, N_{D e}=10^{4}$, $a / R_{\text {Le }}=1.1, \lambda_{i} / a=10^{5}$, and $T_{e} / T_{i}=200 \ldots \ldots \ldots \ldots$ 
5.29 Guiding center $x$-component drift magnitude dependence on $\lambda_{D} / a$ and $\tau_{g} / \tau_{c}$ for the three different charging models for several values of the parameter $\lambda_{D} / a$. In the leftmost plot, the abscissa is actually $\lambda_{D} / a$ instead of $\log _{10} \frac{\lambda_{D}}{a}$, and in the rightmost plot, the abscissa is actually $\tau_{g} / \tau_{c}$ instead of $\log _{10} \frac{\tau_{g}}{\tau_{c}}$. Here, a grain with $10^{-7}$ m made out of carbon has a mass ratio $m_{d} / m_{e}=10^{13}$, and this is held constant while $\lambda_{D} / a$ is swept. Solid red line corresponds to the Patacchini-Hutchinson model guiding center drift $v_{x g c}$, solid black line corresponds to the OML model guiding center drift $v_{x g c}$, and the solid cyan line corresponds to the Gatti-Kortshagen model guiding center drift $v_{x g c}$. When $\lambda_{D} / a=10^{2}$, the other parameters are $a / R_{L i}=0.12, \lambda_{i} / a$, and $m_{d} / m_{e}=10^{13}$, and these parameters are swept consistently throughout the values of $\lambda_{D} / a$ shown. The other parameters chosen for an Argon plasma include $v=f_{U V} /\left(n_{e} v_{t h e}\right)=0.25, \omega_{c d} / \nu_{d n}=10, N_{D e}=10^{4}$, and $T_{e} / T_{i}=200 . \ldots 506$

5.30 Gyro-phase magnitude and direction dependence on $\tau_{g} / \tau_{c}$ for the three different charging models with drifting Maxwellian ions for several values of the Mach number $M_{i}=v_{i} / v_{b}$, which is the flow speed normalized by the Bohm speed $v_{b}=\sqrt{\frac{k_{b} T_{e}}{m_{i}}}$. Solid (dashed) red line corresponds to the Patacchini-Hutchinson model guiding center drift $v_{x g c}\left(v_{y g c}\right)$, solid (dashed black line corresponds to the OML model guiding center drift $v_{x g c}\left(v_{y g c}\right)$, and the solid (dashed) cyan line corresponds to the GattiKortshagen model guiding center drift $v_{x g c}\left(v_{y g c}\right)$. The other parameters chosen for an Argon plasma include $v=f_{U V} /\left(n_{e} v_{\text {the }}\right)=0.25, \omega_{c d} / \nu_{d n}=10, N_{D e}=10^{4}$, $a / R_{L e}=0.1, \lambda_{i} / a=10^{5}, \lambda_{D} / a=10^{3}, T_{e} / T_{i}=200$, and $10^{8} \leq m_{d} / m_{e} \leq 10^{22}$. The OML and Patacchini-Hutchinson charge models predict the same guiding center drift for these parameters. . . . . . . . . . . . . . . . . . . . . . . . 
5.31 Gyro-phase magnitude and direction dependence on $\tau_{g} / \tau_{c}$ for the three different charging models with drifting Maxwellian ions for several values of the Mach number $M_{i}=v_{i} / v_{b}$, which is the flow speed normalized by the Bohm speed $v_{b}=\sqrt{\frac{k_{b} T_{e}}{m_{i}}}$. Solid (dashed) red line corresponds to the Patacchini-Hutchinson model guiding center drift $v_{x g c}\left(v_{y g c}\right)$, solid (dashed black line corresponds to the OML model guiding center drift $v_{x g c}\left(v_{y g c}\right)$, and the solid (dashed) cyan line corresponds to the GattiKortshagen model guiding center drift $v_{x g c}\left(v_{y g c}\right)$. The other parameters chosen for an Argon plasma include $v=f_{U V} /\left(n_{e} v_{\text {the }}\right)=0.25, \omega_{c d} / \nu_{d n}=10, N_{D e}=10^{4}$, $a / R_{L e}=0.1, \lambda_{i} / a=10^{5}, \lambda_{D} / a=10^{3}, T_{e} / T_{i}=200$, and $10^{8} \leq m_{d} / m_{e} \leq 10^{22} . \quad \ldots 508$

5.32 Gyro-phase magnitude and direction dependence on $\tau_{g} / \tau_{c}$ for the three different charging models with mono-energetic ions for several values of the Mach number $M_{i}=v_{i} / v_{b}$, which is the flow speed normalized by the Bohm speed $v_{b}=$ $\sqrt{\frac{k_{b} T_{e}}{m_{i}}}$. Solid (dashed) red line corresponds to the Patacchini-Hutchinson model guiding center drift for mono-energetic (drifting Maxwellian) ions, solid (dashed black line corresponds to the OML model guiding center drift for mono-energetic (drifting Maxwellian) ions, and the solid (dashed) cyan line corresponds to the GattiKortshagen model guiding center drift for mono-energetic (drifting Maxwellian) ions. The other parameters chosen for an Argon plasma include $v=f_{U V} /\left(n_{e} v_{\text {the }}\right)=0.25$, $\omega_{c d} / \nu_{d n}=10, N_{D e}=10^{4}, a / R_{L e}=0.1, \lambda_{i} / a=10^{5}, \lambda_{D} / a=10^{3}, T_{e} / T_{i}=200$, and $10^{8} \leq m_{d} / m_{e} \leq 10^{22}$. The OML and Patacchini-Hutchinson charge models predict the same guiding center drift for these parameters. . . . . . . . . . . . 509

5.33 Gyro-phase magnitude and direction dependence on $\tau_{g} / \tau_{c}$ for the three different charging models with mono-energetic ions for several values of the Mach number $M_{i}=w_{i} / w_{b}$, which is the flow speed normalized by the Bohm speed $v_{b}=\sqrt{\frac{k_{b} T_{e}}{m_{i}}}$. Solid (dashed) red line corresponds to the Patacchini-Hutchinson model guiding center drift $v_{x g c}\left(v_{y g c}\right)$, solid (dashed black line corresponds to the OML model guiding center drift $v_{x g c}\left(v_{y g c}\right)$, and the solid (dashed) cyan line corresponds to the GattiKortshagen model guiding center drift $v_{x g c}\left(v_{y g c}\right)$. The other parameters chosen for an Argon plasma include $v=f_{U V} /\left(n_{e} v_{\text {the }}\right)=0.25, \omega_{c d} / \nu_{d n}=10, N_{D e}=10^{4}$, $a / R_{L e}=0.1, \lambda_{i} / a=10^{5}, \lambda_{D} / a=10^{3}, T_{e} / T_{i}=200$, and $10^{8} \leq m_{d} / m_{e} \leq 10^{22} . \quad .510$ 
5.34 Gyro-phase magnitude and direction dependence on $\tau_{g} / \tau_{c}$ for the three different charging models for different values of the Mach number $M_{i}=v_{i} / v_{b}$, which is the flow speed normalized by the Bohm speed $v_{b}=\sqrt{\frac{k_{b} T_{e}}{m_{i}}}$. This figure shows a direct comparison between mono-energetic and drifting Maxwellian ions. All solid lines correspond with mono-energetic ions, while all dashed lines correspond to drifting Maxwellians. The colors black, red, and light blue correspond to the OML, Patacchini-Hutchinson, and Gatti-Kortshagen charge models respectively. The other parameters chosen for an Argon plasma include $f_{U V} /\left(n_{e} v_{t h e}\right)=0.25, \omega_{c d} / \nu_{d n}=10, N_{D e}=10^{4}$, $a / R_{L e}=0.1, \lambda_{i} / a=10^{5}, \lambda_{D} / a=10^{3}, T_{e} / T_{i}=200$, and $10^{8} \leq m_{d} / m_{e} \leq 10^{22} . \quad . .511$

5.35 Comparison of simulation and theory results for the gyro-phase magnitude and direction dependence on $\tau_{g} / \tau_{c}$ for the three charging models. The colors black, red, and light blue correspond to the OML, Patacchini-Hutchinson, and Gatti-Kortshagen charge models respectively. Other parameters chosen for an Argon plasma include $f_{U V} /\left(n_{e} v_{\text {the }}\right)=0.25, \omega_{c d} / \nu_{d n} \approx 10$, drifting Maxwell-Boltzmann ions with $M_{i}=v_{i} / v_{b}=10, N_{D e}=10^{3}, a / R_{L e}=0.1, \lambda_{i} / a=10^{5}, \lambda_{D} / a=10^{3}, T_{e} / T_{i}=200$, and $10^{8} \leq m_{d} / m_{e} \leq 10^{13}$. The ratios of the in-situ equilibrium grain charge on the shadowed side and the illuminated side of the abrupt inhomogeneity are $q_{1} / q_{2}=2.65$, $q_{1} / q_{2}=2.65$, and $q_{1} / q_{2}=1.38$ for the OML, Patacchini-Hutchinson, and GattiKortshagen charge models respectively. The OML and Patacchini-Hutchinson charge models predict the same guiding center drift for these parameters. . . . . . . . . . 512

5.36 Linear profile used for simulations. All of the grain trajectories modelled using this inhomogeneity do not include the effect of the electric field that would be produced by this inhomogeneity. The electron and ion densities, $n_{e}, n_{i}$, are normalized to $n_{0}=10^{16} \mathrm{~m}^{-3}$. The ratio $n_{e} / n_{i}$ is also plotted, as is the dimensionless quantity $q_{e q}(x) / q_{e q}(x=0)$, which is proportional to the number of electrons on the grain. The discrete steps of $q_{e q}(x) / q_{e q}(x=0)$ correspond to an addition or subtraction of one electron. The abscissa is scaled to the gyro-radius corresponding to the equilibrium charge of a $0.015 \mu \mathrm{m}$ radius grain at $x=0$, which is 43 electrons $\left[R_{L d}(t=0)=0.572\right.$

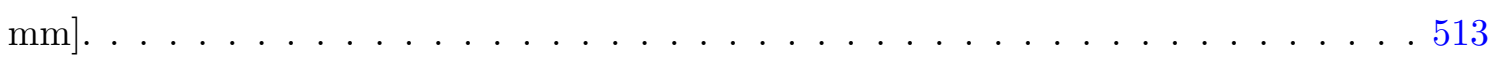


5.37 Grain trajectories for $a=0.015 \mu \mathrm{m}$ and $\boldsymbol{B}=B \hat{z}$, where $B=4 \mathrm{~T}$, using the profile from figure 5.36 and the effects of an electric field are not included in the trajectories. The dashed and solid lines correspond to a charging rate parameter of $\alpha=1$ and $\alpha=0.0105$, respectively. Squares and diamonds indicate the gyro-averaged guiding centers of the trajectories for $\alpha=1$ and $\alpha=0.0105$, respectively. The instantaneous guiding centers are represented by the solid $(\alpha=1)$ and dashed $(\alpha=0.0105)$ helical

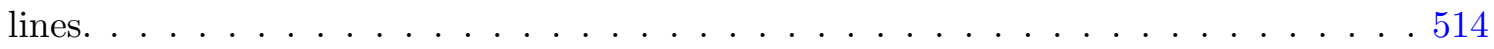

5.38 Linear fit to the in-situ equilibrium grain charge as a function of the inhomogeneous coordinate $x$. The solid line represents the in-situ equilibrium grain charge, which has a step-like appearance because it changes in increments of $1 e$. The dashed line shows the linear fit to $q_{e q}(x) \ldots \ldots \ldots \ldots \ldots \ldots$

5.39 Northop fit to $q(t)=q_{0}+q_{1} \cos \left(\phi-\phi_{1}\right)$. The quantity $q_{0}$ represents the in-situ equilibrium grain charge at the gyro-averaged guiding center, and $q_{1}$ represents the amplitude of charge modulation. The solid black line shows $q(t)$, the dashed gray line shows the Northrop style fit to $q(t)$, and the circle indicates the gyro-phase angle where the grain is most negatively charged during its gyro-orbit. . . . . . . . . 516

5.40 Radial distance from origin is grain charge normalized to the instantaneous in-situ equilibrium grain charge $\left(q_{0}=-43 e\right.$, in this case) as a function of gyro-angle for $\alpha=1$ (solid line) and $\alpha=0.0105$ (dashed line). In this figure, the effects of the electric field produced by the density gradient are not included. Lines appear thickened because multiple gyro-periods are displayed and gyrophase angle at which single-electron charging events occur are not unique and because the thickness reflects charge fluctuation $+1,-0$ electron. The gyro-angle $\phi=0$ refers to the $+\hat{x}$ axis here. 517 
5.41 Gyro-phase drift magnitude and direction dependence on the adjustable charge-rate parameter $\alpha$. In this plot, the abscissa is actually $\alpha$ instead of $\log _{10} \alpha$. In this figure, the effects of the electric field produced by the density gradient are not included. A: The magnitude is normalized by the perpendicular velocity, $v_{\perp}=11 \mathrm{~m} / \mathrm{s}$. B: The angle $\theta_{\text {drift }}$, in degrees, is relative to the $\hat{x}$-direction. An angle of $180^{\circ}$ corresponds to a drift direction that is entirely along the $-\hat{x}$, and an angle of $90^{\circ}$ corresponds to a drift direction that is entirely along the $\hat{y}$-direction. Above $\alpha=0.02$, no gyro-phase drift occurs for this case. Below $\alpha=10^{-4}$, neither gyro-phase nor grad-q drift occurs

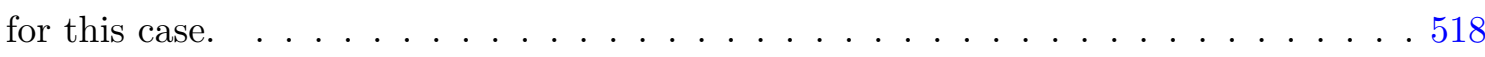

5.42 Gyro-phase drift magnitude and direction dependence on $\tau_{g} / \tau_{c}$. In this figure, the effects of the electric field produced by the density gradient are not included. This figure uses the same data as figure 5.41, but has been recast in terms of $\tau_{g} / \tau_{c}$ instead of the adjustable charge-rate parameter $\alpha . \ldots \ldots \ldots$. . . . . . . . . . . . . . . . .

5.43 Gyro-phase drift magnitude and direction dependence on $\tau_{g} / \tau_{c}$ for $a=1.5 \times 10^{-8}$ $\mathrm{m}$ grain in the linear profile. In this plot, the abscissa is actually $\tau_{g} / \tau_{c}$ instead of $\log _{10} \frac{\tau_{g}}{\tau_{c}}$. In this figure, the effects of the electric field produced by the density gradient are not included. Electron and ion number density varies between $n_{0}=10^{9} \mathrm{~m}^{-3}$ and $n_{0}=10^{20} \mathrm{~m}^{-3}$ to produce $\tau_{c}$ variation. The large disparity between Northrop's theory and the simulation near $\tau_{g} / \tau_{c}<10^{-1}$ is a result from taking the arctangent of two numbers very close to zero. . . . . . . . . . . . . . . 520

5.44 Gyro-phase drift magnitude and direction dependence on $\tau_{g} / \tau_{c}$ for $a=5 \times 10^{-7} \mathrm{~m}$ grain in the linear profile. In this figure, the effects of the electric field produced by the density gradient are not included. Electron and ion number density varies between $n_{0}=10^{9} \mathrm{~m}^{-3}$ and $n_{0}=10^{20} \mathrm{~m}^{-3}$ to produce $\tau_{c}$ variation. . . . . . . . 521

5.45 Gyro-phase drift magnitude and direction dependence on $\tau_{g} / \tau_{c}$ for $a=1.5 \times 10^{-6} \mathrm{~m}$ grain in the linear profile. In this figure, the effects of the electric field produced by the density gradient are not included. Electron and ion number density varies between $n_{0}=10^{9} \mathrm{~m}^{-3}$ and $n_{0}=10^{20} \mathrm{~m}^{-3}$ to produce $\tau_{c}$ variation. $\ldots \ldots . \ldots 522$ 
5.46 Gyro-phase drift magnitude and direction dependence on $\tau_{g} / \tau_{c}$ for $a=10 \times 10^{-6} \mathrm{~m}$ grain in the linear profile. In this figure, the effects of the electric field produced by the density gradient are not included. Electron and ion number density varies between $n_{0}=10^{9} \mathrm{~m}^{-3}$ and $n_{0}=10^{20} \mathrm{~m}^{-3}$ to produce $\tau_{c}$ variation. $\ldots \ldots . \ldots 53$

5.47 The ratio of charge variation to equilibrium charge $q_{1} / q_{0}$ dependence on $\tau_{g} / \tau_{c}$ for $a=10^{-6} \mathrm{~m}$ grain in the linear profile. This plot of $q_{1} / q_{0}$ corresponds to figure 5.46. The ratio of dimensionless surface potential variation amplitude to the in-situ equilibrium dimensionless surface potential at the gyro-center $\chi_{e}^{(1)} / \chi_{0 e}$ is also shown. Electron and ion number density varies between $n_{0}=10^{9} \mathrm{~m}^{-3}$ and $n_{0}=10^{20} \mathrm{~m}^{-3}$ to

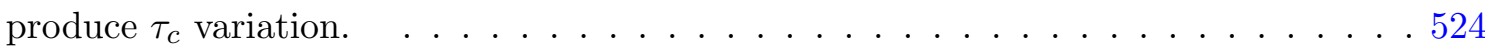

5.48 Grain trajectory in a linear-profile for drag-absent conditions in cylindrical geometry with constant charge. The coaxial blue circles correspond to the limits of the linear profile; outside this region, the plasma is quasi-neutral. The electric field is $100 \mathrm{~V} / \mathrm{m}$, directed radially-inward, and is not consistent with the density gradient. . . . . . .525

5.49 Grain trajectory in a linear-profile for drag-absent conditions in cylindrical geometry with instantaneous charging. The coaxial blue circles correspond to the limits of the linear profile; outside this region, the plasma is quasi-neutral. The electric field is $100 \mathrm{~V} / \mathrm{m}$, directed radially-inward, and is not consistent with the density gradient.

5.50 Grain trajectory in a linear-profile when drag is present in cylindrical geometry with non-instantaneous charging. The coaxial blue circles correspond to the limits of the linear profile; outside this region, the plasma is quasi-neutral. The electric field is $100 \mathrm{~V} / \mathrm{m}$, directed radially-inward, and is not consistent with the density gradient.

5.51 This shows a comparison between the guiding center drift for abrupt and gradual inhomogeneity for the $\lambda_{D} / a$ parameter. The data shown for the abrupt inhomogeneity use the parameters from figure 5.29. For the gradual inhomogeneity, the effects of the electric field are not included. . . . . . . . . . . . . . . . . 528

6.1 Spectrum of Osram Xeradex lamp used in the experiments of Dove et al. (2012), and used to make predictions for guiding center drift in an abrupt inhomogeneity. . . 529

6.2 Photo-electron yield as a function of wavelength for selected Carbon allotropes. Taken from Feuerbacher and Fitton (1972). The inset shows the reflectance. . . . . 530 
6.3 Photo-electron yield as a function of wavelength for Zirconium. Taken from Eastman (1971).

6.4 Photo-electron yield as a function of wavelength for Platinum. Taken from Lin et al. (1971).

6.5 Guiding Center drift for spherical Platinum grains, assuming an initial gyro-phase of $-90^{\circ}$ and the UV spectrum shown in figure 6.1 but with 10 times as much power output. Additional parameters include $a=200 \mathrm{~nm}, T_{n}=0.025 \mathrm{eV}, T_{e}=5 \mathrm{eV}$, $\omega_{c d} / \nu_{d n}=4$, drifting Maxwellian ions flowing at the Bohm speed, and an Argon plasma. The solid black, red, and cyan curves correspond to the results for the OML, Patacchini-Hutchinson, and Gatti-Kortshagen charging models respectively. There is virtually no difference between the OML and Pattacchini-Hutchinson charging models. The smaller dotted lines indicate the upper and lower bounds of the guiding center drift, assuming that $T_{e}=5 \mathrm{eV}$, with an uncertainty of $\pm 0.5 \mathrm{eV} \ldots \ldots 53$

6.6 The $v=f_{u v} /\left(n_{e} v_{t h e}\right)$ parameter as a function of plasma density used in figure 6.5. The smaller dashed lines indicate the upper and lower bounds of $v$, assuming that $T_{e}=5 \mathrm{eV}$, with an uncertainty of $\pm 0.5 \mathrm{eV} \ldots \ldots \ldots \ldots \ldots \ldots$

6.7 The ratio of in-situ equilibrium surface potentials $\chi_{e}^{(1)} / \chi_{e}^{(2)}$ corresponding to figure 6.5. The smaller dashed lines indicate the upper and lower bounds of $\chi_{e}^{(1)} / \chi_{e}^{(2)}$, assuming that $T_{e}=5 \mathrm{eV}$ with an uncertainty of $\pm 0.5 \mathrm{eV} \ldots \ldots \ldots \ldots$

6.8 Guiding Center drift for spherical Platinum grains, assuming an initial gyro-phase of $-90^{\circ}$ and the UV spectrum shown in figure 6.1 but with 10 times as much power output. Additional parameters include $a=200 \mathrm{~nm}, T_{n}=0.025 \mathrm{eV}, T_{e}=5 \mathrm{eV}, \omega_{c d} / \nu_{d n}=$ 4, mono-energetic ions flowing at the Bohm speed, and an Argon plasma. The solid black, red, and cyan curves correspond to the results for the OML, PatacchiniHutchinson, and Gatti-Kortshagen charging models respectively. There is virtually no difference between the OML and Pattacchini-Hutchinson charging models. The smaller dotted lines indicate the upper and lower bounds of the guiding center drift, assuming that $T_{e}=5 \mathrm{eV}$ with an uncertainty of $\pm 0.5 \mathrm{eV} \ldots \ldots \ldots$ 
6.9 The ratio of $i n$-situ equilibrium surface potentials $\chi_{e}^{(1)} / \chi_{e}^{(2)}$ corresponding to figure 6.8. The smaller dashed lines indicate the upper and lower bounds of $\chi_{e}^{(1)} / \chi_{e}^{(2)}$, assuming that $T_{e}=5 \mathrm{eV}$ with an uncertainty of $\pm 0.5 \mathrm{eV} \ldots \ldots \ldots . \ldots . \ldots 57$

6.10 Guiding Center drift for spherical Zirconium grains, assuming an initial gyro-phase of $-90^{\circ}$ and the UV spectrum shown in figure 6.1 but with 10 times as much power output. Additional parameters include $a=200 \mathrm{~nm}, T_{n}=0.025 \mathrm{eV}, T_{e}=5 \mathrm{eV}$, $\omega_{c d} / \nu_{d n}=4$, drifting Maxwellian ions flowing at the Bohm speed, and an Argon plasma. The solid black, red, and cyan curves correspond to the results for the OML, Patacchini-Hutchinson, and Gatti-Kortshagen charging models respectively. There is virtually no difference between the OML and Pattacchini-Hutchinson charging models. The smaller dotted lines indicate the upper and lower bounds of the guiding center drift, assuming that $T_{e}=5 \mathrm{eV}$ with an uncertainty of $\pm 0.5 \mathrm{eV}$. . 538

6.11 The $v=f_{u v} /\left(n_{e} v_{t h e}\right)$ parameter as a function of plasma density, used in figure 6.10. The smaller dashed lines indicate the upper and lower bounds of $v$, assuming that $T_{e}=5 \mathrm{eV}$ with an uncertainty of $\pm 0.5 \mathrm{eV} \ldots \ldots \ldots \ldots \ldots \ldots$

6.12 The ratio of in-situ equilibrium surface potentials $\chi_{e}^{(1)} / \chi_{e}^{(2)}$ corresponding to figure 6.10. The smaller dashed lines indicate the upper and lower bounds of $\chi_{e}^{(1)} / \chi_{e}^{(2)}$, assuming that $T_{e}=5 \mathrm{eV}$ with an uncertainty of $\pm 0.5 \mathrm{eV} \ldots \ldots \ldots \ldots$

6.13 Guiding Center drift for spherical Zirconium grains, assuming an initial gyro-phase of $-90^{\circ}$ and the UV spectrum shown in figure 6.1 but with 10 times as much power output. Additional parameters include $a=200 \mathrm{~nm}, T_{n}=0.025 \mathrm{eV}, T_{e}=5$ $\mathrm{eV}, \omega_{c d} / \nu_{d n}=4$, mono-energetic ions flowing at the Bohm speed, and an Argon plasma. The solid black, red, and cyan curves correspond to the results for the OML, Patacchini-Hutchinson, and Gatti-Kortshagen charging models respectively. There is virtually no difference between the OML and Pattacchini-Hutchinson charging models. The smaller dashed lines indicate the upper and lower bounds of the guiding center drift, assuming that $T_{e}=5 \mathrm{eV}$ with an uncertainty of $\pm 0.5 \mathrm{eV}$. . . 541

6.14 The ratio of $i n$-situ equilibrium surface potentials $\chi_{e}^{(1)} / \chi_{e}^{(2)}$ corresponding to figure 6.13. The smaller dashed lines indicate the upper and lower bounds of $\chi_{e}^{(1)} / \chi_{e}^{(2)}$, assuming that $T_{e}=5 \mathrm{eV}$ with an uncertainty of $\pm 0.5 \mathrm{eV} \ldots \ldots \ldots \ldots 42$ 
6.15 Measured profiles from an ECR plasma (Nunomura et al., 1997). . . . . . . . . 543

6.16 An example of an equilibrium profile assuming a gaussian electric field. In this figure, the electric field is consistent with the density gradient. Compare these modelled profiles with experimental data from figure $6.15 \ldots \ldots . \ldots . \ldots 544$

6.17 A possible equilibrium profile, assuming a gaussian electric field, using the profiles from figure 6.15 as a model. The electric field is consistent with the density gradient and it is centered at $r_{0}=5 \mathrm{~cm}$, with a FWHM of $4 \mathrm{~cm}$. In the bottom plot of this figure, the red line represents the in-situ-equilibrium charge for the PatacchiniHutchinson and OML models, while the cyan line represents the in-situ-equilibrium charge as calculated using the Gatti-Kortshagen model. . . . . . . . . . . . . . 545

6.18 Trajectory of a zirconium grain with $a=0.05 \mu \mathrm{m}$, started at the dust thermal speed in the $x$-direction at $(x, y)=(0.1,0) \mathrm{m}$ for the profile shown in figure 6.17 ; the grain charge is kept constant at $q=-695 e$. This trajectory includes the effects of an electric field that is consistent with the density gradient. The solid red circle indicates the boundary of the vacuum vessel, the smaller, solid magenta circle indicates the uniform magnetic field region, and the black line shows the grain trajectory. . . . . 546

6.19 Radial excursion of the grain and grain charge corresponding to the trajectory in figure 6.18 when the grain has a constant charge $q=-695 e$. The radial excursion includes the effects of an electric field that is consistent with the density gradient. . 547

6.20 Trajectory of a zirconium grain with $a=0.05 \mu \mathrm{m}$, started at the dust thermal speed in the $x$-direction at $(x, y)=(0.1,0) \mathrm{m}$ for the profile shown in figure 6.17 and the grain is forced to charge instantaneously. The trajectory includes the effects of an electric field that is consistent with the density gradient. The solid red circle indicates the boundary of the vacuum vessel, the smaller, solid magenta circle indicates the uniform magnetic field region, and the black line shows the grain trajectory. . . . . 548

6.21 Radial excursion of the $a=0.05 \mu \mathrm{m}$ grain and grain charge corresponding to the trajectory in figure 6.20 when the grain is forced to charge instantaneously. The radial excursion includes the effects of an electric field that is consistent with the

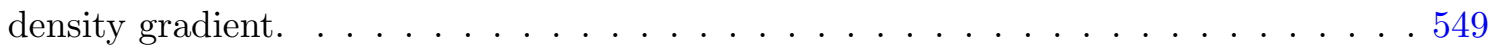


6.22 Trajectory of a zirconium grain with $a=0.05 \mu \mathrm{m}$, started at the dust thermal speed in the $x$-direction at $(x, y)=(0.1,0) \mathrm{m}$ for the profile shown in figure $6.17 \mathrm{using}$ the OML model with no drag forces are present. The solid red circle indicates the boundary of the vacuum vessel, the smaller, solid magenta circle indicates the uniform magnetic field region, and the black line shows the grain trajectory. The grain charges non-instantaneously for the plasma conditions specified in figure 6.17 and the trajectory includes the effects of an electric field that is consistent with the density gradient. . . . . . . . . . . . . . . . . . . . . 550

6.23 Radial excursion of the $a=0.05 \mu \mathrm{m}$ grain and grain charge corresponding to the trajectory in figure 6.22 when the grain is started with the in-situ equilibrium grain charge, using the OML model, and no drag forces are present. The grain charges non-instantaneously for the plasma conditions specified in figure 6.17 and the radial excursion includes the effects of an electric field that is consistent with the density

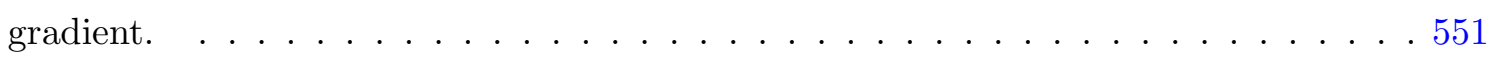

6.24 Trajectory of a zirconium grain with $a=0.05 \mu \mathrm{m}$, started at the dust thermal speed in the $x$-direction at $(x, y)=(0.1,0) \mathrm{m}$ for the profile shown in figure 6.17 using the OML model. The solid red circle indicates the boundary of the vacuum vessel, the smaller, solid magenta circle indicates the uniform magnetic field region, and the black line shows the grain trajectory. The grain charges non-instantaneously for the plasma conditions specified in figure 6.17 and the trajectory includes the effects of an electric field that is consistent with the density gradient. Neutral drag is assumed, but ion drag is not. . . . . . . . . . . . . . . . . . . . . . . . . . . . .

6.25 Radial excursion of the grain and grain charge corresponding to the trajectory in figure 6.24 when the $a=0.05 \mu \mathrm{m}$ grain is started with the in-situ equilibrium grain charge and using the OML model. The grain charges non-instantaneously for the plasma conditions specified in figure 6.17 and the radial excursion includes the effects of an electric field that is consistent with the density gradient. Neutral drag is assumed, but ion drag is not. . . . . . . . . . . . . . . . . . 553 
6.26 Radial excursion of the grain and grain charge corresponding to the trajectory in figure 6.24 when the $a=0.05 \mu \mathrm{m}$ grain is started with $q(t=0)=0$ and using the OML model. The grain charges non-instantaneously for the plasma conditions specified in figure 6.17 and the radial excursion includes the effects of an electric field that is consistent with the density gradient. Neutral drag is assumed, but ion drag

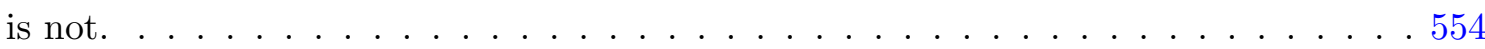

6.27 Radial excursion of the $a=0.05 \mu \mathrm{m}$ grain and grain charge is compared for instantaneous and non-instantaneous charging, using the OML model. The grain charges non-instantaneously for the plasma conditions specified in figure 6.17 and the radial excursion includes the effects of an electric field that is consistent with the density gradient. Neutral and ion drag are considered for this trajectory. . . . . . . . . . 555

6.28 Trajectory of a zirconium grain with $a=0.05 \mu \mathrm{m}$, started at the dust thermal speed in the $x$-direction at $(x, y)=(0.1,0) \mathrm{m}$ for the profile shown in figure 6.17 using the Gatti-Kortshagen model. The solid red circle indicates the boundary of the vacuum vessel, the smaller, solid magenta circle indicates the uniform magnetic field region, and the black line shows the grain trajectory. The grain charges non-instantaneously for the plasma conditions specified in figure 6.17 and the grain trajectory includes the effects of an electric field that is consistent with the density gradient. Neutral drag is assumed, but ion drag is not. . . . . . . . . . . . . . . 556

6.29 Radial excursion of the grain and grain charge corresponding to the trajectory in figure 6.28 when the grain is started with the in-situ equilibrium grain charge and using the Gatti-Kortshagen model. The grain charges non-instantaneously for the plasma conditions specified in figure 6.17 and the radial excursion includes the effects of an electric field that is consistent with the density gradient. Neutral drag is assumed, but ion drag is not. . . . . . . . . . . . . . . . . . 557

6.30 A profile of the Saturnian system to a radial distance of $10 R_{\text {sat }} . \ldots \ldots 58$

6.31 Trajectory of an $a=0.015 \mu \mathrm{m}$ grain launched from Enceladus in the co-rotating frame of Saturn when no UV is considered. In the leftmost panel, the trajectory is depicted by the solid black line, while the outer cyan circle shows the geosynchronous orbit and inner red circle shows the radial extent of Saturn's surface. . . . . . . . 559 
6.32 Trajectory of an $a=0.015 \mu \mathrm{m}$ grain launched from Enceladus in the co-rotating frame of Saturn when UV is considered. In the leftmost panel, the trajectory is depicted by the solid black line, while the outer cyan circle shows the geosynchronous orbit and inner red circle shows the radial extent of Saturn's surface. . . . . . . . . . 559

6.33 Comparison between the radial excursion and grain charge as a function of time for the trajectories from figures 6.31 and 6.32. The solid line represents the grain trajectory when UV is not considered and the dashed line represents the grain trajectory when UV is considered. . . . . . . . . . . . . . . . . . . 560

6.34 Trajectory of an $a=0.015 \mu \mathrm{m}$ grain launched from Enceladus in the co-rotating frame of Saturn when UV is considered and there is an electron temperature gradient. In the leftmost panel, the trajectory is depicted by the solid black line, while the outer cyan circle shows the geosynchronous orbit and inner red circle shows the radial extent of Saturn's surface. The rightmost panel shows the radial excursion of the dust grain. Neutral and ion drag forces are not considered for this grain trajectory.

6.35 Trajectory of an $a=0.025 \mu \mathrm{m}$ grain launched from Enceladus in the co-rotating frame of Saturn when UV is considered and there is an electron and ion temperature gradient. Neutral drag force is considered for this grain trajectory. In the leftmost panel, the trajectory is depicted by the solid black line, while the outer cyan circle shows the geosynchronous orbit and inner red circle shows the radial extent of Saturn's surface. The rightmost panel shows the radial excursion of the dust grain. 561

6.36 Trajectory of an $a=0.025 \mu \mathrm{m}$ grain launched from Enceladus in the co-rotating frame of Saturn when UV is considered and there is an electron and ion temperature gradient. Neutral and ion drag forces are considered for this grain trajectory. In the leftmost panel, the trajectory is depicted by the solid black line, while the outer cyan circle shows the geosynchronous orbit and inner red circle shows the radial extent of Saturn's surface. The rightmost panel shows the radial excursion of the dust grain. This figure is not noticeably different from 6.35 , which shows that the effect of ion drag on this grain trajectory is negligible. . . . . . . . . . . . . 5 562 


\section{List of Symbols}

$\begin{array}{ll}a & =\text { Dust grain radius } \\ C_{d} & =4 \pi \epsilon_{0}\left(1+a / \lambda_{D}\right)=\text { Dust grain capacitance } \\ C_{n} & =\text { Dust-neutral collision frequency } \\ C_{s} & =\sqrt{8 k_{b} T_{n} /\left(\pi m_{n}\right)}=\text { Mean speed of plasma species } s \\ e & =\text { Elementary charge } \\ f_{u v} & =\text { UV photoelectron flux } \\ g & =\text { Local acceleration due to gravity } \\ I_{s} & =\text { Collected current of species } s \\ k_{b} & =\text { Boltzmann constant } \\ \mathrm{Kn}_{a} & =\lambda_{i} / a=\text { Knudsen number }\end{array}$

$\mathrm{Kn}_{R 0}=\lambda_{i} /\left[2(1.22) R_{0}\right]=$ Knudsen capture radius number

$M_{t h s}=v_{s} / v_{t h s}=$ Thermal Mach number of plasma species $s$

$M_{s} \quad=v_{s} / v_{b}=$ Mach number of plasma species $s$

$m_{s} \quad=$ Mass of species $s$

$N \quad=$ Number of charges on a dust grain

$N_{D e} \quad=4 / 3 \pi n_{e} \lambda_{D}^{3}=$ Number of electrons per Debye sphere

$n_{0} \quad=n_{e}=n_{i}=$ Density of quasineutral plasma

$n_{s} \quad=$ Density of species $s$

$P_{0} \quad=$ Probability for an ion to have no collisions with neutral gas atoms in the dust grain sheath

$P_{1} \quad=$ Probability for an ion to have approximately one collision with a neutral gas atom in the dust grain sheath

$P_{>1} \quad=$ Probability for an ion to have many collisions with neutral gas atoms in the dust grain sheath

$q_{1} \quad=$ The in-situ equilibrium grain charge in abrupt inhomogeneity corresponding to the minimum (more negative) charge state 
$q_{2} \quad=$ The $i n$-situ equilibrium grain charge in abrupt inhomogeneity corresponding to the maximum (less negative) charge state

$q_{a} \quad=$ Amplitude of charge state modulation during a gyro-orbit

$q_{e q} \quad=$ In-situ equilibrium grain charge

$R_{0} \quad=$ Capture radius

$R_{L s} \quad=$ Gyro-radius of species $s$

$T_{s} \quad=$ Temperature of species $s$

$V_{d} \quad=V_{\text {surf }}-V_{s}=q_{d} / C_{d}=$ Dust grain surface potential

$V_{s} \quad=$ Space potential

$V_{\text {surf }}=$ Surface potential

$v_{b} \quad=\sqrt{\frac{k_{b} T_{e}}{m_{i}}}=$ Bohm speed, ion acoustic speed

$v_{s} \quad=$ Fluid drift speed of plasma species $s$

$v_{t h s}=\left(\frac{2 k_{b} T_{s}}{m_{s}}\right)^{2}=$ Thermal speed of plasma species $s$

$w_{s} \quad=\left|v-v_{s}\right|=$ Relative drift speed between a dust grain and plasma species $s$ 


\section{Greek Symbols}

$\alpha \quad=$ Adjustable charging rate parameter

$\delta \quad=$ Coefficient for dust-neutral collisions

$\delta q \quad=$ RMS grain charge fluctuation

$\epsilon_{0} \quad=$ Permittivity of free space

$\lambda_{D} \quad=$ Debye length

$\zeta=C_{d}\left(\phi_{1}\right) / C_{d}\left(\phi_{\max }\right)=$ Amplitude of dust grain capacitance modulation during gyro-orbit

$\theta \quad=\theta_{0}+\int_{\tau_{g}}^{0} \omega_{c d}\left(t^{\prime}\right) \mathrm{d} t^{\prime}=$ Integral of dust gyro-frequency

$\lambda_{i} \quad=$ Mean free path for ion-neutral collisions

$\nu_{i n} \quad=\frac{32 \sigma_{e x} n_{n} \sqrt{\frac{k_{b} T_{i}}{m_{i}}}}{3 \sqrt{\pi}}=$ Ion-neutral charge exchange frequency

$\nu_{d n} \quad=\delta \frac{4 \pi}{3} a^{2} n_{n} \frac{m_{n}}{m_{d}}=$ Dust-neutral collision frequency

$\rho_{d} \quad=$ Dust grain mass density

$\sigma_{e x}=$ Ion-neutral charge exchange cross-sectional area

$\tau_{c} \quad=$ Charging time

$\tau_{g} \quad=\frac{2 \pi}{\omega_{c d}}=$ Dust grain gyro-period

$\tau_{p e} \quad=\frac{2 \pi}{\omega_{p e}}=$ Electron plasma period

$v \quad=f_{u v} /\left(n_{e} v_{t h e}\right)=$ Normalized UV photo-electron flux

$\phi \quad=$ Gyro-phase angle

$\phi_{1} \quad=$ Gyro-phase angle where dust grain is most negatively charged

$\phi_{\max }=$ Gyro-phase angle where dust grain is least negatively charged

$\chi_{e} \quad=e V_{d} /\left(k_{b} T_{e}\right)=$ Normalized dust grain surface potential

$v \quad=f_{u v} /\left(n_{e} v_{\text {the }}\right)$ Normalized UV photoelectron flux

$\omega_{c s} \quad=$ Angular gyro-frequency of species $s$

$\omega_{p s} \quad=$ Angular plasma frequency of species $s$

$\Omega \quad=$ Angular frequency of co-rotating reference frame 


\section{List of Acronyms and Initialisms}

$\begin{array}{ll}\text { GK } & - \text { Gatti-Kortshagen charging model } \\ \text { MDPX } & - \text { Magnetized Dusty Plasma Experiment } \\ \text { MPE } & - \text { Max Planck Institute for Extraterrestrial Physics } \\ \text { OML } & - \text { Orbit Motion Limited } \\ \text { PH } & - \text { Patacchini-Hutchinson charging model } \\ \text { PIV } & - \text { Particle Imaging Velocimetry } \\ \text { PTV } & - \text { Particle Tracking Velocimetry } \\ \text { RMS } & \text { - Root Mean Squared } \\ \text { SCEPTIC } & - \text { Specialized-Coordinate Electrostatic Particle and Thermals in Cell } \\ \text { UV } & \end{array}$




\section{Chapter I}

\section{Introduction}

Multi-phase systems involving small particulate matter, neutral atoms and molecules, and ionized gas exist in many space, laboratory, and industrial regimes. This small particulate matter is called dust. The presence of dust in ionized gas modifies the collective behavior of the ionized gas. In this chapter, the multi-phase case of solid particulates in partially-ionized gas is presented and the utility for studying the non-instantaneous charging of macroscopic particles in these types of systems is discussed.

\section{A Multi-Phase Case of Solid Particulates in Ionized Gas}

Plasma, or ionized gas, is ubiquitous in nature and comprises nearly $99 \%$ of the visible matter in the universe (Gurnett and Bhattacharjee, 2005). The terms plasma and ionized gas both refer to a medium where free electrons and ions are present with approximately equal charge densities, with the possible presence of neutral gas atoms. The degree of ionization is represented by the ionization fraction $n_{n} / n_{0}$, where $n_{n}$ is the neutral gas atom number density and $n_{0}$ is the number density of electrons. Small values of $n_{n} / n_{0}$ indicate weakly ionized plasma, while large values of $n_{n} / n_{0}$ indicate strongly ionized plasma. When the temperature of a gas is raised high enough, neutral gas atoms become sufficiently energetic that collisions between gas atoms strip off electrons, forming a mixture of unbound electrons and ions in addition to the presence of the neutral gas atoms. Because the process of ionization takes place above a well-defined temperature, plasma is often referred to as the fourth state of matter (Gurnett and Bhattacharjee, 2005; Chen, 2006). Additionally, when an 
electric field is present, the electric field accelerates free electrons to ionize neutral gas atoms upon impact, producing plasma. Photons with sufficient incident energy can also ionize neutral gas atoms to produce plasma. Electrons are always singly charged but ions can have multiple ionization states. With increasingly energetic photons incident on neutral gas, ions can lose additional electrons. The process of recombination in partially ionized plasma can also occur during electron-ion collisions, leading to newly formed neutral gas atoms. In order for the plasma to exist in a steady state, the rate at which gas atoms are ionized must be equal to or greater than the rate of recombination.

Dust grains are small pieces of particulate matter. In the context of this dissertation, particulate matter with radii smaller than $100 \mu \mathrm{m}$ are considered dust grains. As discussed by Goertz (1989), dust is quite common throughout the universe. This natural dust exists in supernova remnants, planetary nebulae, molecular clouds, and it is also scattered throughout galaxies in the interstellar medium. Dust comprises $1 \%$ of the interstellar medium by mass (Boulanger et al., 2000). The presence of dust in molecular clouds and the interstellar medium blocks visible light from the Milky Way. Dust does not radiate in the visible spectrum because the black body temperature of dust is too small, approximately $10 \mathrm{~K}$, which corresponds to radiation in the infared spectrum. The presence of dust can significantly affect the behavior of the plasma or other systems. As an example, Draine and Sutin (1987) discuss how charged dust grains become the dominant coupling agent between the neutral gas and the background magnetic field in molecular clouds when the ratio of ion density to neutral gas atom density is small. The motion of charged dust grains is thus of great importance for understanding the behavior of dense molecular clouds throughout the interstellar medium. Dust can also affect equilibrium plasma conditions. For example, water ice near Enceladus causes electron density to decrease significantly near this Saturnian moon (Farrell et al., 2009). Dust grains are also formed in the manufacturing process of silicon wafers for the semiconductor industry. The formation of dust grains from the reactive gases used in etching plasmas is an unavoidable part of semiconductor manufacture. The study of charged dust formation, growth, and transport is necessary to mitigate the damaging effects of dust on semiconductors.

There have been many dust experiments and observations done to understand multi-phase dusty plasma systems in the presence of magnetic fields. These experiments can be subdivided into regimes where the dust grains do not have magnetized orbits and those experiments where magnetized orbit dust grains should exist or have been observed. For dust grains to have magnetized 


\section{CHAPTER I. INTRODUCTION}

orbits, their gyro-radii must be small enough to fit inside some observational scale length and the gyro-frequency is greater than the frequency of collisions with neutral gas atoms or ions. The dust-electron collision frequency is not relevant because the electron mass is small enough that collisions with plasma electrons do not change the dust grain momentum appreciably. Table 1.1 gives an overview of the plasma parameters present in selected dusty plasma experiments and regimes. Table 1.2 puts some of the parameters from table 1.1 into the context of the dimensionless parameters discussed throughout this dissertation. Some examples of experiments where dust grains are not magnetized inside the observational volume include the experiments performed by Nunomura et al. (1997), Thompson et al. (1997), and Trottenberg et al. (2006). Nunomura et al. (1997) used a relatively small magnetic field of $0.0875 \mathrm{~T}$ to observe the effects of ion flow on dust grain trajectories in simulation and experiment. In this experiment, the magnetic field indirectly affected the trajectories of dust grains via the motion of plasma ions. Thompson et al. (1997) investigated dust acoustic waves, and the presence of a magnetic field of $0.09 \mathrm{~T}$ assisted with plasma production in the DC glow discharge plasma. The magnetic field was not large enough throughout the volume of the experiment to affect grain motion. Likewise, the experiments performed by Trottenberg et al. (2006) used a magnetic field of 0.02 for the purpose of plasma production, rather than modification to grain trajectories.

Examples of experiments where magnetized orbit dust grains should be possible or have been observed include the experiments of Sato et al. (2001), Amatucci et al. (2004), Schwabe et al. (2011), Carstensen et al. (2012), and Thomas et al. (2012). Sato et al. (2001) had an apparatus that was capable of producing magnetic fields of up to $4 \mathrm{~T}$, which should be sufficient to allow for magnetized orbit dust grains in the experiment. However, stable discharges were not possible for field strengths greater than $1 \mathrm{~T}$, and the neutral gas pressures were too large to permit magnetized orbit dust grains. The global rotation of the dusty plasma cloud was solely due to the presence of the magnetized orbit plasma ions and the effects of magnetized orbit dust grains were too small to measure. Amatucci et al. (2004) claim to have observed magnetized orbit dust grains in the DUPLEX experimental device at the Naval Research Laboratory. Measurements with a CCD camera unambiguously show a horizontal oscillation for a few distinctly different dust grains which could be consistent with gyro-motion. However, for the neutral gas pressures in these experiments, the dust-neutral collision frequency is much greater than the gyro-frequency as predicted using 
the Epstein drag formula. As shown in table 1.2, the ratio of dust gyro-frequency to dust-neutral collision frequency should be quite small for the experiments performed by Amatucci et al. (2004), $\omega_{c d} / \nu_{d n}=10^{-4}$. If their intrepretation of the horizontal oscillation as evidence of gyro-motion is correct, then the Epstein drag model may be incorrect and gyro-motion should be easily detected in MDPX. Schwabe et al. (2011) used magnetic field strengths of up to $2 \mathrm{~T}$ in the Magnetized Dusty Plasma Experiment (MDPX) at the Max Planck Institute for Extraterrestrial Physics. In these experiments, gyration is nominally possible $\left(\omega_{c d} / \nu_{d n} \approx 1\right)$, but there were no direct observations or evidence of dust grain gyration. The Suleiman Device at Kiel University is capable of fields up to 4 T, and Carstensen et al. (2012) looked at the effect of strong magnetic fields on the ion wakes on dust grains. Ion wakes are formed around dust grains or other solid objects when plasma ions have a fluid drift speed, and this situation occurs whenever dust grains levitate in a planar sheath. Carstensen et al. (2012) aligned the magnetic field along the direction of the ion flow. Even though Carstensen et al. (2012) used a large magnetic field of $4 \mathrm{~T}$, the dust grains and neutral gas pressures were too large to permit gyration $\left(\omega_{c d} / \nu_{d n} \ll 1\right)$. Future experiments with the Suleiman device might be used to see if ion rotation couples to the neutral gas, and how this affects the motion of charged dust grains. Similar to this line of inquiry, Kählert et al. (2012) successfully magnetized a dusty plasma without a magnetic field. By rotating an electrode, Kählert et al. (2012) produced a Coriolis force using the neutral drag force, coupling the neutral gas atom motion to the dust grains. Experiments like Carstensen et al. (2012) and Kählert et al. (2012) suggest the possiblity of studying how dust grain motion can couple with the neutral gas motion, or to quantify how charged dust grains couple the neutral gas to the background magnetic field, which is a situation that can arise in dense molecular clouds (Draine and Sutin, 1987).

The MDPX at Auburn should be the first experiment to deliver a strong enough magnetic field, low enough neutral gas pressures, and small enough dust grains to convincingly demonstrate grain gyration (Thomas et al., 2012). For careful choice of experiment parameters, grain gyration should be possible in MDPX, as shown in table 1.2. Additionally, MDPX has the advantage of a larger diameter and experimental volume than other, similar high magnetic field devices. This larger diameter in the plane perpendicular to the magnetic field attempts to accommodate the gyro-radii of charged dust grains, which are much greater than the gyro-radii of plasma electrons or ions. Despite these all of these favorable conditions, Bonitz et al. (2013) claim that it is practically 
impossible to magnetize a strongly coupled dusty plasma, because the ratio of dusty gyro-frequency to the dust plasma frequency is too large for imagible grains. By imagible grains, we refer to grains that are sufficiently large enough to permit standard imaging techniques like Particle Tracing Velocimetry (PTV) and Particle Imaging Velocimetry (PIV). These concerns might be addressed by using a lower dust grain density so that the intergrain spacing is large enough to prevent a strongly coupled system. Ultimately, experiments in the Auburn MDPX will reveal whether these criticisms are valid.

\section{B Motivation}

Because dust-in-plasma or dusty-plasma multi-phase systems are frequently encountered in space, laboratory, and industrial settings, it is of interest to build intuitive models of how grains modify the plasmas they inhabit and how charging affects the dynamics of single grains. Just as single-particle analysis leads to important intuition about collective plasma effects in confinement in toroidal fusion devices, single-particle analysis is a precursor to developments in the collective behavior of many grains. Analysis of the behavior of a single dust grain can also provide knowledge about its in-situ plasma environment. The effect of non-stationary charging in the charging processes of grains that affect grain dynamics in multi-phase systems can be used to study properties of complex plasmas (Nunomura et al., 1999), and as a sensitive diagnostic for sheath mechanisms or other plasma processes.

\section{Applications of Dusty Plasma}

Grains act as in-situ probes in plasmas (Samarian and James, 2005; Wang and Ticos, 2008; Basner et al., 2009), diagnostics for plasma surface interaction (Kersten et al., 2003), and point scatterers of starlight. Direct analysis of dust trajectories in particular has proven to be a valuable diagnostic tool for high temperature fusion plasma (Wang et al., 2007; Wang and Ticos, 2008). Injected Aerosols have also been shown to suppress Edge Localized Mode disruptions in Tokamaks (Mansfield et al., 2010). 


\section{SECTION B MOTIVATION}

\section{State of Knowledge of Grain Charging}

The origin of charging models for dust grains is Langmuir probe theory, which described electron and ion current collection to conducting objects immersed in plasma. Mott-Smith and Langmuir (1926) developed the Orbit Motion Limited (OML) charging model, valid for spheres or cylinders, that is still used unmodified in dusty plasma literature today. Allen et al. (1957) developed a radial motion theory for collisionless plasmas for situations in which the electron temperature is much greater than the ion temperature. The radial motion theory is equivalent to presuming that ions start nearly from rest far away from the probe surface while electron motion is thermal and electron density obeys the Boltzmann relation.

The OML approach separates the plasma around an object into distinct sheath and plasma regions. Bernstein and Rabinowitz (1959) were able to employ mono-energetic ions to numerically calculate the space potential around spherical or cylindrical probes without having to distinguish the sheath boundary. This approach was used to produce numerical predictions for arbitrary shielding length by Laframboise (1966), who included thermally-distributed ions. Other refinements to OML theory include flowing plasma for spheres and cylinders (Kanal, 1962, 1964). The problem of arbitrary probe shape in this framework was treated by Laframboise and Parker (1973). Northrop and Birmingham (1996) found the equilibrium surface potential and equilibrium surface charge for spheres and cylinders in flowing plasmas. The spherical case is readily applicable to grains in flowing plasma. Concurrently with the development of the OML approach, Boyd (1951) presented a theory for a high plasma density discharge that was refined by Su and Lam (1963) and Kiel (1969) to produce the hydrodynamic model for grain charging in collisional plasma. The ion current is limited by mobility in the hydrodynamic framework for the continuum (fluid) limit. Su and Kiel (1966) considered ellipsoidal probe shapes for mobility-limited ion current, permitting deviation from spherical and cylindrical probes. Chang and Laframboise (1976) offered further refinements in this model for the limit of large shielding length and for non-flowing plasma.

Recent developments in grain charging have concerned the certain peculiarities of ion-neutral collisions within grain sheaths, the effect of arbitrary magnetic field magnitude on the grain, and the dust grain shape. Inherent shortcomings with the OML model pertaining to ion-neutral collisions and probes were first theorized by Talbot and Chou (1969). The OML model is designed for 


\section{CHAPTER I. INTRODUCTION}

situations where there are no collisions between ions and neutrals in the grain sheath, and the hydrodynamic model is designed for situations where there are many collisions between ions and neutrals in the grain sheath. The opposite case of few collisions between ions and neutrals in the sheath is predicted to have very different results than the predictions from the OML and hydrodynamic models. Zakrzewski and Kopiczynski (1974) showed experimental evidence for the case of relatively few ion-neutral collisions in a DC glow discharge plasma. Ion-neutral charge exchange generates a population of trapped ions around spherical grains in plasma (Goree, 1992), and these trapped ions lead to a marked increase in ion current (Lampe et al., 2001). This feature of trapped ions was ultimately exploited by Lampe et al. (2003) to produce an analytical model of collision-enhanced ion current for the regime of weak collisionality in the grain sheath. The molecular dynamics simulations of Zobnin et al. (2000) demonstrated the profound effect that even a few ion-neutral collisions can have on surface charge. Gatti and Kortshagen (2008) developed a particularly useful analytical model for grain charging that describes charging along a continuum of ion collisional mean free path, reducing to OML for collisionless plasma and to the hydrodynamic approach for collisional plasma. At present, the Gatti-Kortshagen charge model provides the most comprehensive description for ion current over all collisionality regimes.

Magnetized-orbit electrons and ions are a problem within the OML framework because the magnetic field complicates the theory of collection and renders analytical solutions intractible. Rubinstein and Laframboise (1982) found well-defined upper and lower limits of electron collection in a collisionless, magnetized plasma. Patacchini et al. (2007) developed an analytical theory, based empircally on simulations, for electron current with arbitrary magnetic fields and arbitrary shielding length. The Patacchini-Hutchinson model offers a complete description for electron collection in the presence of magnetic fields, including the motions of electrons in the grain sheath. As of this writing, a similar comprehensive description for magnetized ions does not exist.

In addition to the advancements that have been made in understanding the basic charging processes present in magnetized plasmas, there has also been an incorporation of charging processes to account for Ultra-Violet illumination, secondary emission, and thermionic emission. These processes have long been known to laboratory scientists in such forms as the photo-electric effect and Richardson's law (Edison effect) for hot surfaces. Secondary electron emission has long been exploited for plasma generation and for use in photomultiplier tubes. An early application of UV 


\section{SECTION B MOTIVATION}

charging came in the form of spacecraft charging, discussed by Guernsey and Fu (1970); Feuerbacher and Fitton (1972); Whipple (1981). Sternglass (1957) developed a theory for secondary electron emission that closely matched experimental observations. Secondary electron emission and thermionic electron emission are important to understanding dust in Tokamaks (Pigarov et al., 2005; Smirnov et al., 2007; Bacharis et al., 2010; Gunn, 2012; Vaverka et al., 2014).

While tribo-electric charging was known to the ancient Greeks, many questions about these charging process are not completely resolved (Castle, 1997). Desch and Cuzzi developed a computational model for contact charging in order to simulate lightning in the solar nebula. Farrell et al. (2006) used contact-charging principles to develop a successful proof of concept for predicting Ultra Low Frequency waves from dust devils on Mars, supported by the earlier experimental and observational work of Farrell et al. (2004).

\section{$3 \quad$ Utility of examining nonstationary charging effects}

Dust grain motion is influenced by the grain charge, which is a variable parameter. When immersed in plasma, grains easily attain a net charge through the collection and emission of ions and electrons. Unlike electrons, which cannot change charge state, or ions, which can only change charge state through absorption of energy above ionization thresholds, grains change charge state as a result of changes in the local plasma conditions that affect the stochastic as well as coherent collection and emission of electrons and ions. Like uncharged grains that are introduced into a plasma, all macroscopic particles require a finite time to reach an equilibrium charge (Choi and Kushner, 1994; Goree, 1994), with both the equilibrium charge and the relaxation time predicted by a charging model that takes into account the surrounding plasma conditions. Charging models describe how the local plasma parameters determine the collection currents and the equilibrium grain charge. Inhomogeneous plasma is characterized by spatial variation of the plasma conditions, such as density, magnetic field, electron or ion temperature, electron or ion flow speed, the collisional mean free path of plasma species, ionization state of ions, ion species composition, or UV illumination, and we expect that grain charge changes if the grain were to move through the plasma. The in-situ-equilibrium grain charge is the charge state associated with a stationary grain at a given spatial location. A grain reaches the in-situ-equilibrium grain charge if stationary at a given spatial location for a long time. The charging time is the time it takes for an uncharged grain 


\section{CHAPTER I. INTRODUCTION}

to reach some fraction of the in-situ-equilbrium grain charge, where this fraction is generally given by $q\left(t=\tau_{c}\right)=(1-1 / e) q_{0}, q$ is the dust charge as a function of time, $\tau_{c}$ is the charging time, and $q_{0}$ is the in-situ-equilibrium grain charge. Even under equilibrium conditions in homogeneous plasma, grain charge fluctuates imperceivably due to the stochastic nature of electron and ion bombardment (Cui and Goree, 1994). Charge-state fluctuations can be modelled having a characteristic timescale based on the dust and plasma parameters. Gyro-synchronous modulation of grain charge will lead to a gyro-phase-averaged diamagnetic drift of the grain's guiding center.

Northrop and Hill (1983) discussed variable grain charge in the context of producing gyrophase drift, an adiabatic guiding center drift motion for magnetized-orbit dust grains. Northrop et al. (1989) then used gyro-phase drift induced by non-stationary charging to explain fundamental structuring of dust in Jupiter's Gossamer ring. Nunomura et al. (1999) later used the concept of non-stationary charging to explain unstable vertical oscillations of grains in a laboratory planar sheath. Nunomura discussed the mechanism by which dust grains can gain energy as a result of phase-lagged charge modulation in inhomogeneous plasma. Nunomura convincingly showed that delayed charging that occurs when the grain does not immediately reach the in-situ-equilibrium grain charge is responsible for dynamic instability. Walker et al. (2014) used gyro-synchronously modulated grain charge to describe guiding center drift for the case of an abrupt inhomegeneity, while Koepke et al. (2013) and Walker et al. (2014), as a part of this dissertation work, suggested that non-stationary charging might be used as a diagnostic for planar and dust sheath mechanisms and, potentially as a testbed for comparing and validating charge models. One of the goals of this dissertation is to show how the non-stationary charging feature of dust grains manifests itself in guiding center drift for different charging models and sheath mechanisms over a broad range of plasma and grain parameters. Another goal is to reassert that charging dynamics matter when it comes to grain trajectories, and to show examples where grain dynamics for the case of nonstationary charging significantly deviate from grain dynamics for the case of instantaneous charging.

\section{Scope of Dissertation}

In this dissertation, a semi-analytical treatment for the guiding center motion of a magnetized-orbit dust grain in an abrupt inhomogeneity in the presence of neutral drag is developed to study how 
charging-model and sheath mechanisms affect gyro-phase drift over a range of dust and plasma parameters. Comparisons are made model-to-model, and within each model. Also, a single-particle trajectory tracking code is developed that permits corroboration of the semi-analytic treatment of the abrupt inhomogeneity and, very importantly, it allows the study of grain trajectories in gradual inhomogeneity where analytical results are not possible. Guiding center drift is analyzed for some un-realistic conditions to illustrate important features, but is also analyzed for the specific applications of evaluating charge models in Auburn's MDPX. A major point of this dissertation is that how a grain's charge evolution affects its trajectory can reveal difficult-to-measure aspects of mechanisms that affect grain charging and electric field profiles in electrostatic plasma sheaths.

Chapter II describes basic parameters in homogeneous and inhomogeneous plasma that are important in the context of grain charging. Chapter III discusses the grain-charging process, the charging models that describe these processes, the forces that act on dust grains, and how these forces result in guiding center drift for magnetized-orbit dust grains. Chapter IV discusses the simulation and analysis codes developed for this dissertation, while chapter $\mathrm{V}$ presents the semianalytical and simulation results. Applications of these results to the Auburn Magnetized Dusty Plasma Experiment (MDPX) and to the Saturnian environment are discussed in chapter VI, with further discussion on the quantitative error bars of these results given in chapter VII. Conclusions are outlined in chapter VIII. 


\section{Chapter II}

\section{Description of Plasma}

A plasma is an ensemble of partially ionized gas that can be described by several parameters. Properties of quasi-neutral homogeneous plasma include the shielding length, relaxation of charges due to charge separation, collisional mean free path, and charged particle gyro-radius. All of these parameters are important for characterizing plasma in general and further, inhomogeneity in these parameters have implications for grain charging. This chapter reviews the homogeneous and inhomogeneous plasma parameters that are important in the context of grain charging.

\section{A Parameters of Homogeneous Plasma}

In homogeneous plasmas, the plasma is quasi-neutral, meaning that the number density of the ions, including all species, is nearly equal to the number density of electrons, to the extent consistent with thermal motion of the particles. For singly ionized single ion species plasma, this is mathematically expressed by the relation $n_{i} \approx n_{e}$. Non-neutral plasma is found where electric fields expel one charged species and attract the other. In many plasmas, including astrophysical settings, more than one ion species can be present. In all cases investigated here, the following relationship will hold:

$$
\sum_{s} Z_{s}(\boldsymbol{r}, t) n_{s}(\boldsymbol{r}, t) \approx n_{e}(\boldsymbol{r}, t),
$$


where $s$ denotes the ion species, and $Z_{s}(\boldsymbol{r}, t)$ represents the spatially-dependent charge state of ion species. In this dissertation, assume that the ion charge state is homogenenous and unity, expressed as $Z_{s}(\boldsymbol{r}, t)=Z_{s}=1$. Additionally, species $s$ generally refers to Argon in this dissertation, with a few exceptions. Dust grains are often considered as a separate plasma species, but the finite spectrum of dust grain charge state $Z_{d}$ and the orders of magnitude disparity of scales between plasma particles and dust grains suggests that dust needs to be treated differently than electrons, which have one value of $Z_{e}$, and ions which have very few values of $Z_{i}$ (Shukla, 2001). When negatively charged single-charge-state dust is present in a singly-ionized plasma, equation 2.1 is modified to

$$
n_{i}(\boldsymbol{r}, t) \approx n_{e}(\boldsymbol{r}, t)+Z_{d}(\boldsymbol{r}, t) n_{d}(\boldsymbol{r}, t)
$$

where $Z_{d}(\boldsymbol{r}, t)$ is the number of charges on each grain, and $n_{d}(\boldsymbol{r}, t)$ is the number density of dust grains. The regimes $\frac{n_{e}}{n_{n}} \ll 1$ and $\frac{n_{e}}{n_{n}} \gg 1$ correspond to weakly ionized and strongly ionized plasmas, respectively, and $n_{n}$ is the neutral gas atom density. Glow discharges (Nunomura et al., 1999; Amatucci et al., 2004) and radio-frequency (RF) plasmas (Schwabe et al., 2011; Thomas et al., 2012) generally produce weakly ionized plasmas $\left(n_{e} / n_{n} \approx 10^{-6}\right.$ or smaller), while Q-machines (196) produce strongly ionized plasmas $\left(n_{e} / n_{n} \approx 0.99\right)$.

An unbounded, quasi-neutral plasma has an approximately uniform potential everywhere in space, in the absence of plasma waves. This can be demonstrated through Poisson's equation:

$$
\nabla^{2} V_{s}(\mathbf{r})=-\frac{\rho}{\epsilon_{0}}=-\frac{e}{\epsilon_{0}}\left(n_{i}-n_{e}\right)
$$

where $V_{s}$ is the space potential, $\rho$ is the charge density, which is approximately zero due to quasineutrality, $e=1.602 \times 10^{-19} \mathrm{C}$ is the elementary charge, and $\epsilon_{0}=8.854 \times 10^{12} \mathrm{Farad} / \mathrm{meter}$ is the permittivity of free space. Because $n_{i} \approx n_{e}$, the Laplacian of $V_{s}$ is approximately equal to zero, and since the plasma is unbounded, the only solution is for $V_{s} \approx$ constant everywhere. Because the space potential is approximately constant everywhere, it necessarily follows that the electric field is approximately zero everywhere, by $\mathbf{E}(\mathbf{r})=-\nabla V_{s}(\mathbf{r})$. The principle of quasi-neutrality will be exploited in the following sub-sections to determine the important scale lengths and relaxation 
times that characterize homogeneous plasmas. Inhomogeneous plasma is treated in section B of this chapter.

\section{Debye Length}

Conducting or non-conducting spheres (such as spherical dust grains) charge negatively when placed within an unbounded, homogeneous plasma because free electrons with non-zero temperature have a higher mobility than free ions. This is true as long as $T_{i} \geq T_{e} \frac{m_{i}}{m_{e}}$, which is the case for most situations of interest. When given enough time to relax to equilibrium, the sphere attains a surface potential that is negative relative to the local space potential, or in other words, $V_{\text {surf }}(t)-V_{s}<0$ when $t \rightarrow \infty$. This difference, $V_{f l}=V_{\text {surf }}(t \rightarrow \infty)-V_{s}$, is called the floating potential (MottSmith and Langmuir, 1926). The symbol $V_{d}(t)=V_{\text {surf }}(t)-V_{s}$ will be used to describe the, in general, time-dependent potential difference between a dust grain's surface and the unperturbed space potential. The criterion $V_{d}(t \rightarrow \infty)=V_{f l}=V_{\text {surf }}(t \rightarrow \infty)-V_{s}$ holds for stationary grains allowed to reach equilibrium conditions in the plasma.

Quasi-neutrality dictates that far away from the conducting sphere, the equilibrium potential is given by $V_{s}$. The region of space where the potential deviates from $V_{s}$ in proximity of the grain surface is called the sheath. The sheath's electrostatic potential profile $V_{s h}(r)$ is resolved through Poisson's equation and appropriate boundary conditions and varies a function of radial distance from the sphere, according to Poisson's equation,

$$
\nabla^{2} V_{s h}(r)=\frac{1}{r^{2}} \frac{\partial}{\partial r}\left(r^{2} \frac{\partial V_{s h}}{\partial r}\right)=-\frac{e}{\epsilon_{0}}\left[n_{i}(r)-n_{e}(r)\right]
$$

The electron and ion densities as function of space are given by $n_{e}(r)=n_{e 0} \exp \left(\frac{e V_{s h}(r)}{k_{b} T_{e}}\right)$ and $n_{i}(r)=n_{i 0} \exp \left(-\frac{e V_{s h}(r)}{k_{b} T_{i}}\right)$ respectively, assuming Maxwell-Boltzmann distributed electrons and ions. Electron and ion densities far from the sphere are given by $n_{e 0}$ and $n_{i 0}$ respectively. When the electron and ion temperatures are not equal, the two species are not thermalized with respect to each other and the plasma is referred to as non-equilibrium (Bonitz et al., 2010).

The assumption of Maxwell-Boltzmann distributed electrons and ions in equation 2.4 leads to 
the differential equation

$$
\frac{2}{r} \frac{\partial}{\partial r} V_{s h}(r)+\frac{\partial^{2}}{\partial r^{2}} V_{s h}(r)=\frac{e}{\epsilon_{0}} n_{e} \exp \left(\frac{e V_{s h}(r)}{k_{b} T_{e}}\right)-\frac{e}{\epsilon_{0}} n_{i} \exp \left(-\frac{e V_{s h}(r)}{k_{b} T_{i}}\right) \approx 0 .
$$

This non-linear differential equation can be solved by assuming that the magnitude of potential variation in the sheath is small compared to the temperature of electrons or ions. Quasi-neutrality implies that $n_{e 0} \approx n_{i 0}$, and this simplifies the linear differential equation to

$$
\frac{2}{r} \frac{\partial}{\partial r} V_{s h}(r)+\frac{\partial^{2}}{\partial r^{2}} V_{s h}(r)-\frac{e^{2}}{\epsilon_{0} k_{b}}\left(\frac{n_{e 0}}{T_{e}}+\frac{n_{i 0}}{T_{i}}\right) V_{s h}(r)=0
$$

The factor $\frac{e^{2}}{\epsilon_{0} k_{b}}\left(\frac{n_{e 0}}{T_{e}}+\frac{n_{i 0}}{T_{i}}\right)$ has units $\mathrm{m}^{-2}$, and the association of this quantity with $1 / \lambda_{D}^{2}$, where $\lambda_{D}$ is the Debye length is expressed

$$
\lambda_{D}=\sqrt{\frac{\epsilon_{0} k_{b}}{e^{2}}\left(\frac{n_{e 0}}{T_{e}}+\frac{n_{i 0}}{T_{i}}\right)^{-1}}=\sqrt{\frac{\epsilon_{0} k_{b} T_{e} T_{i}}{e^{2}\left(n_{e 0} T_{i}+n_{i 0} T_{e}\right)}} .
$$

In the literature, $\lambda_{D}$ is often referred to as the linearized Debye length, since this length scale results from linearizing equation 2.5. The Debye length is equivalent to the reciprocal sum of electron and ion Debye lengths, or

$$
\frac{1}{\lambda_{D}^{2}}=\frac{1}{\lambda_{D e}^{2}}+\frac{1}{\lambda_{D i}^{2}}
$$

where $\lambda_{D e}=\sqrt{\frac{\epsilon_{0} k_{b} T_{e}}{e^{2} n_{e 0}}}$ is the electron Debye length, and $\lambda_{D i}=\sqrt{\frac{\epsilon_{0} k_{b} T_{i}}{e^{2} n_{i 0}}}$ is the ion Debye length. The smaller of the two length scales dominates the size of the linearized Debye length. Because $n_{e 0} \approx n_{i 0}$, the species with the smaller temperature dominates the effective value of Debye length, for $e V_{s h} /\left(k_{b} T_{s}\right) \ll 1$.

The solution for the differential equation in equation 2.6, using the appropriate boundary conditions $V_{\text {sh }}(r=a)=V_{\text {surf }}$ and $V_{\text {sh }}(r \rightarrow \infty)=V_{s}$, is given by (Debye and Huckel, 1923)

$$
V_{s h}(r)=V_{d} \frac{a}{r} \exp \left(-\frac{r-a}{\lambda_{D}}\right)+V_{s}
$$

Equation 2.9 satisfies the differential equation and the boundary conditions, and is called the 
Debye-Hückel, or Yukawa, potential, which decreases more rapidly than for a point charge, or Coloumbic, potential (Daugherty et al., 1992). Figure 2.1 shows a comparison between Coloumbic and Debye-Hückel sheath potential models when $a / \lambda_{D} \ll 1$.

There are some limitations for using $\lambda_{D}$ to describe the characteristic shielding length of a small sphere or dust grain. In order to achieve this characteristic length scale and the sheath potential variation in equation 2.9, it is necessary to assume $e V_{s h}(r) /\left(k_{b} T_{e}\right) \ll 1$ and $e V_{s h}(r) /\left(k_{b} T_{i}\right) \ll 1$, and Maxwell-Boltzmann ion and electron distribution functions. Both electrons and ions participate in the shielding when assuming $n_{e}=n_{0} \exp \left(\frac{e V_{s h}(r)}{k_{b} T_{e}}\right)$ and $n_{i}=n_{0} \exp \left(-\frac{e V_{s h}(r)}{k_{b} T_{i}}\right)$. Many introductory texts on Debye shielding (Gurnett and Bhattacharjee, 2005; Chen, 2006) assume constant ion density in their derivations, which modifies equation 2.9 , replacing $\lambda_{D}$ with $\lambda_{D e}$. In other words, only the electrons contribute to the shielding and ions are not stratified. In a low temperature or weakly ionized plasma, $\lambda_{D} \ll \lambda_{D e}$, which means we need to be careful to assign the correct parameter for the shielding length. The results of Daugherty et al. (1992) suggest that using $n_{i}=n_{0} \exp \left(-\frac{e V_{s h}}{k_{b} T_{i}}\right)$ is perfectly acceptable, and the shielding length is given by $\lambda_{D}$, when $a / \lambda_{D} \ll 1$. As the ratio $a / \lambda_{D}$ increases, the shielding length in the exponential factor of equation 2.9 increases and $\lambda_{D}$ becomes a poor description for the shielding length. When $a / \lambda_{D} \approx 1$, Daugherty et al. (1992) found that the shielding length is accurately described by $\lambda_{D e}$ instead of $\lambda_{D}$. A planar sheath begins to form when $a / \lambda_{D} \gg 1$, or when the dust grain radius is much larger than the linearized Debye length (Daugherty et al., 1992). The separate but related topic of planar sheaths is discussed further in section B. Numerically solving the coupled Poisson and Vlasov equations, Daugherty et al. (1992) found that the Debye-Hückel solution in equation 2.9 does not accurately predict the potential variation in the grain sheath when $a / \lambda_{D} \gg 1$. Because of these problems, the condition $a \lambda_{D}<1$ is generally imposed for dust grains, unless stated otherwise. Another concern regarding the derivation of equation 2.9 is when the ions are mono-energetic, or have some flow speed, instead of being purely a Maxwell-Boltzmann distribution. For mono-energetic ions, equation 2.9 changes so that the Debye length is given by $\lambda_{D}=\sqrt{\frac{\epsilon_{0}\left(2 E_{0} T_{e}\right)}{e\left(2 E_{0} n_{e 0}+T_{e} n_{i 0}\right)}}$ (Bernstein and Rabinowitz, 1959; Daugherty et al., 1992), where $E_{0}$ is the energy of the mono-energetic ions. The shielding length has also been discussed for other non-Maxwell-Boltzmann distributions, such as the Kappa distribution often encountered in space physics (Bryant, 1996). More specific considerations of the sheath will follow in chapter III, section B. 


\section{Number of Electrons in a Debye Sphere}

In homogeneous plasma, with a characteristic shielding length $\lambda_{D}$ described in section 1 , the number of electrons in a Debye sphere is given by

$$
N_{D e}=n_{e 0}\left(\frac{4}{3} \pi\right) \lambda_{D}^{3}
$$

Collective effects dominate over binary collisions for $N_{D e} \gg 1$ (Chen, 2006). Binary collisions dominate over collective effects when $N_{D e} \ll 1$. The criterion $N_{D e}>1$ is imposed as a requirement for plasma, and the parameter $N_{D e}$ will show up later in this dissertation when important quantities are placed into dimensionless form. The criterion $N_{D e}>1$ will not be violated in this dissertation.

The effect of many successive, small angle collisions is described by the Coloumb logarithm

$$
\ln \left(\frac{b_{m}}{b_{0}}\right)=\ln \left(\frac{\lambda_{D}}{b_{0}}\right)=\ln \left(9 N_{D e}\right)
$$

where $b_{m}$ is the maximum impact parameter, generally taken to be the Debye length because

electric fields are screened outside of this distance, and $b_{0}=\frac{q_{s}}{q_{s^{\prime}}} 4 \pi \epsilon_{0} \mu_{s s^{\prime}}\left|\boldsymbol{v}-\boldsymbol{v}^{\prime}\right|$ is the distance of closest approach or Coloumb impact parameter, where $\mu_{s s^{\prime}}=m_{s} m_{s^{\prime}} /\left(m_{s}+m_{s^{\prime}}\right), q$ is particle charge, $s$ and $s^{\prime}$ represent the two different species. The plasma parameter is given by $\Lambda=9 N_{D e}$, and $\ln \Lambda$ ranges from 10-40 for nearly all plasmas of interest (Gurnett and Bhattacharjee, 2005).

\section{Electron Plasma frequency}

For a cold $\left(T_{s} \approx 0\right)$, quasi-neutral, bounded, homogeneous plasma, a separation of charges caused by displacing the positions of electrons from equilibrium relative to a fixed background of ions produces an electric field, which in turn acts as a restoring force to bring the electrons back to their equilibrium position in the plasma. As the electrons return to equilibrium, they gain energy from the electric field and overshoot their equilibrium position. Describe the oscillating perturbed position of the electrons as $x=x_{e q}+\Delta x$, where $x_{e q}$ is the equilibrium position of the cold electron slab. The equation of motion for the cold slab of electrons is given by (Gurnett and Bhattacharjee, 


$$
\frac{\mathrm{d} \Delta x}{\mathrm{~d} t}=-\frac{e^{2} n_{e 0}}{\epsilon_{0} m_{e}} \Delta x
$$

where the electric field $E_{x}=\frac{e n_{0} \Delta x}{\epsilon_{0}}$ is along the direction of perturbation, $\Delta x$.

In equation 2.12 , the harmonic perturbation $\Delta x$ has angular frequency

$$
\omega_{p e}=\sqrt{\frac{e^{2} n_{e 0}}{\epsilon_{0} m_{e}}} .
$$

The analysis can be repeated with mobile ions to obtain

$$
\omega_{p}^{2}=\omega_{p e}^{2}+\omega_{p i}^{2}
$$

where $\omega_{p}$ is the angular frequency of the plasma oscillations and $\omega_{p i}=\sqrt{\frac{e^{2} n_{i 0}}{\epsilon_{0} m_{i}}}$ is the ion plasma frequency. Equation 2.14 shows that the larger of the two quantities $\omega_{p e}$ or $\omega_{p i}$ dominates the general plasma oscillation. Because electrons are much less massive than ions, $\omega_{p} \approx \omega_{p e}$.

For the plasma relevant to this dissertation, $\frac{2 \pi}{\omega_{p e}}$ will always correspond to the shortest time scale in the system. Because the plasma period corresponds to the shortest dynamical time scale in the dust in plasma system, normalizing by $\omega_{p e}$ represents the best choice for accurately resolving the time-dependent grain charge and producing dimensionless charging equations for all charging models in this investigation. Note that the electron plasma frequency, electron Debye length, and electron thermal speed are related by $\omega_{p e} \lambda_{D e}=v_{t h e} / \sqrt{2}$.

\section{Mean Free Path and Knudsen Number}

The collisional mean free path is the average distance traveled by a particle in a given species between collisions with another particle. The Knudsen number $\mathrm{Kn}$ is defined as the ratio of a collisional mean-free-path length scale $\lambda_{m f p}$ and the grain radius $a$. Specifically,

$$
\mathrm{Kn}_{a}=\frac{\lambda_{m f p}}{a}
$$


In this dissertation, the length scale $a$ is the dust grain radius, and $\lambda_{m f p}$ is the average collisional length for the more collisional species pair. For low temperature or weakly ionized plasmas, the most collisional species pair is the ion-neutral collisional pair, specifically the ion-neutral chargeexchange mean free path, so $\lambda_{m f p}=\lambda_{i}$ refers to the ion-neutral charge-exchange mean free path in this dissertation. The grain radius $a$ is a sensitive parameter in the charging models (Patacchini et al., 2007; Gatti and Kortshagen, 2008) being assessed for gyro-phase drift in gradual and abrupt inhomogeneity. It is also meaningful to discuss whether or not a plasma source is collisional over the length scale of the plasma size $L$. Collisional processes cause plasma species to assume Maxwell-Boltzmann distributions, therefore plasma constituents should be expected to be Maxwell-Boltzmann distributions if $\lambda_{i}<L$. Since plasma sources are much larger than dust grains,

the Knudsen number of a dust grain $\left(\lambda_{i} / a\right)$ is much larger than the Knudsen number characterizing the size of the plasma itself $\left(\lambda_{i} / L\right)$. Goree (1994) and, later, Lampe et al. (2001) showed that the Knudsen parameter is not the best way to distinguish collisionless and collisional regimes for grain charging because the trapped charged particles travel far enough in their many orbits around a spherical conductor or grain to make a charge-exchange collision with a neutral gas atom. The details of this problem is further discussed in chapter III, section C.

\section{Electron, Ion Gyro-frequency and gyro-radius}

Magnetized-orbit charged particles within homogeneous plasma gyrate around the direction of the magnetic field. The Lorentz force equation describes the motion of a charged particle in a magnetic field in the absence of an electric field, given by

$$
\boldsymbol{F}_{\text {Lorentz }}=m_{s} \frac{\mathrm{d} \boldsymbol{v}}{\mathrm{d} t}=q_{s} \boldsymbol{v} \times \boldsymbol{B}(\boldsymbol{r})
$$

where the $q_{s}$ is the charge of the particle species. The magnetic field is along the $\hat{z}$ direction, and has a magnitude $B$.

The equation of motion for a magnetized-orbit particle's $x$-position in a uniform magnetic field is

$$
\ddot{x}+\left(\frac{e B}{m_{s}}\right)^{2} x=\left(\frac{e B}{m_{s}}\right)\left(\left(\frac{e B}{m_{s}}\right) x_{0} \pm v_{0 y}\right)
$$


and the $y$-component is

$$
\ddot{y}+\left(\frac{e B}{m_{s}}\right)^{2} y=\left(\frac{e B}{m_{s}}\right)\left[\left(\frac{e B}{m_{s}}\right) y_{0} \mp v_{0 x}\right],
$$

where the zero subscript denotes initial values. The resulting equations 2.17 and 2.18 are identical to the harmonic oscillator equation, with terms on the right hand side that depend on the initial conditions. These terms ensure that circular motion or gyration will result, with the characteristic gyro-frequency of the gyration given by

$$
\omega_{c s}=\frac{e B}{m_{s}}
$$

The gyro-radius of each species $R_{L s}$ is given by $R_{L s}=\frac{v_{\perp}}{\omega_{c s}}$, where $v_{\perp}=\sqrt{v_{x}^{2}+v_{y}^{2}}$. The mean gyro-radius is $\sqrt{\pi} v_{t h s} /\left(2 \omega_{c s}\right)$, where the thermal speed is given by $v_{t h s}=\sqrt{\frac{2 k_{b} T_{s}}{m_{s}}}$. The dimensionless magnetization number for each species is $\beta_{s}=\frac{a}{R_{L s}}$ (Patacchini et al., 2007), where $a$ is the dust grain radius, or some other scale size of interest. A plasma species $s$ is not magnetized on the length scale $a$ if $\beta_{s} \ll 1$. By contrast, when $\frac{a}{R_{L s}}$ is much larger than unity, then that plasma species $s$ is magnetized on the length scale $a$. It is also important to note that as an additional criterion for magnetization, the gyro-frequency of a species must be larger than the collision frequency, or $\omega_{c s}>\nu$. When judging magnetization, the effective collision frequency $\nu$ is the largest collision frequency for a given species in the plasma. In this dissertation, $\omega_{c s}>\nu$ will be assumed for electrons and ions, because this condition is satisfied in relevant laboratory experiments (Konopka et al., 2000; Schwabe, 2006; Schwabe et al., 2011; Thomas et al., 2012) and space conditions (Khurana et al., 2008).

\section{Mach numbers}

The thermal Mach number of a plasma species $s$ is expressed (Horányi, 1996) as

$$
M_{t h s}=\frac{v_{s}}{v_{t h s}}
$$

where $v_{s}$ is the species fluid speed and $v_{t h s}=\sqrt{\frac{2 k_{b} T_{s}}{m_{s}}}$ is the thermal speed of the same species. A suitable thermal speed can be established for non-Maxwellian distributions, defined as a measure 
of the average energy $T_{s}=(2 / 3)\left\langle\varepsilon_{s}\right\rangle$ (Godyak and Demidov, 2011). The thermal Mach number is a natural parameter for describing fluid drifts for a continuous distribution of particle energies, but it is insufficient for describing cold, mono-energetic particles. Instead, use the parameter $M_{s}=v_{s} / v_{B}$, where $v_{s}$ still describes the fluid drift of the mono-energetic particles, but it is now scaled to $v_{B}=\sqrt{\left(e Z_{i} T_{e}+\gamma_{i} T_{i}\right) / m_{i}}$, called the ion acoustic speed, ion sound speed, or Bohm speed, where $\gamma_{i}=1$ for isothermal ions, and $\gamma_{i}=3$ for ions with one degree of freedom. In this dissertation, $T_{e} \gg T_{i}$ is assumed unless otherwise noted, which means $v_{B}=\sqrt{\left(e Z_{i} T_{e}\right) / m_{i}}$. This Mach number $M_{s}$ is analagous to the Mach number from fluid mechanics.

\section{B Inhomogeneous Plasmas}

This section discusses some sources of inhomogeneous plasma, and some of the properties that might potentially be measured by gyro-phase drift in space plasma and in sheaths. As discussed by Wang and Ticos (2008), grains can be effectively used as probes for inhomogeneous high temperature fusion plasmas, while Samarian and James (2001); Basner et al. (2009) used grains to measure properties of sheaths. Homogeneous-plasma parameters will be modified, if needed, for use with inhomogeneous plasma.

\section{Planar Sheaths}

Real plasma is necessarily confined to a finite-sized region of space, such as the extent of the vacuum chamber. Electrons are more mobile than plasma ions and leave the plasma, reaching the chamber walls faster than the ions. The chamber walls of an experimental device charge negatively with respect to the plasma until electron and ion net currents to the walls are balanced and the plasma electrically floats with respect to the boundary. The sheath separates the boundary and the electrically floating plasma. The surface floats below the potential in the center of the plasma, and is given by (Gurnett and Bhattacharjee, 2005)

$$
V_{f l}=-\frac{k_{b} T_{e}}{2 e}\left[\ln \left(\frac{m_{i}}{m_{e}}\right)+\ln \left(\frac{T_{e}}{T_{i}}\right)\right] .
$$


The potential variation in the plasma at a distance $z$ from the surface in contact with the plasma becomes

$$
V_{s h}(z)=V_{f l} \exp \left(-\frac{z}{\lambda_{D e}}\right)
$$

as discussed many standard introductory texts (Chen, 2006; Chabert and Braithwaite, 2011) when $\left|e V_{s h}\right| /\left(k_{b} T_{e}\right)<1, L / \lambda_{D e}>1$, and constant ion density are assumed. Note that $\left|e V_{s h}\right| /\left(k_{b} T_{e}\right)<1$ does not actually hold very close to the planar surface, because $\left(e V_{f l}\right) /\left(k_{b} T_{e}\right)=-\frac{1}{2}\left[\ln \left(\frac{m_{i}}{m_{e}}\right)+\ln \left(\frac{T_{e}}{T_{i}}\right)\right]$, and $m_{i} / m_{e} \gg 1$. Equation 2.22 becomes more accurate as $z$ increases. This is a similar solution to equation 2.9, but a slab geometry has been assumed for the planar sheath and the shielding length is characterized by the electron Debye length $\lambda_{D e}$ rather than the Debye length $\lambda_{D}$. The potential profile in equation 2.22 indicates that plasmas are inhomogeneous near boundaries.

Biasing the planar surface negatively with respect to the plasma results in a Child-Langmuir sheath (Child, 1911), specifically when $\left|e V_{0}\right| /\left(k_{b} T_{e}\right) \gg 1$ and $V_{0}<V_{f l}<0$, where $V_{0}$ is the bias voltage. The sheath size increases as a function of the applied bias voltage $V_{0}$. The formation of a planar sheath produces inhomogeneous space potential profiles near the plasma boundaries and it also produces inhomogeneous space potential in the plasma bulk. The electric field produced by the inhomogeneous space potential is important for dusty laboratory plasma, since this field permits the levitation of dust grains (Nunomura et al., 1999; Arnas et al., 1999; Fortov et al., 2001; Sickafoose et al., 2002; Robertson et al., 2003; Thomas et al., 2012, 2013). This electric field also accelerates ions in the plasma bulk so that they obtain the Bohm speed at the sheath edge. Properties of the sheath, such as ion-neutral collisionallity, modify the ion energy distribution function. Controlling the ion energy distribution is important for industrial concerns, specifically in plasma etching reactors. Much work has been done to produce anisotropic ion beams for this purpose. One of the goals of the Department of Energy's Low Temperature Plasma Science Center was to measure and control distribution functions in laboratory and industrial plasma, listed as priority 1 in (Graves and Kushner, 2008). Samarian and James (2001); Basner et al. (2009) established that dust can be used as a plasma diagnostic by measuring planar sheath properties through the levitation height. Following in this spirit, dust grain guiding center motion, specifically gyro-phase drift, might be used to measure other properties in planar sheaths such as ion energy 
distribution function.

There are many models in the literature to describe planar sheath properties for glow discharges, radio frequency plasmas, collisionallity regimes, negative ions, and other plasma processes. Tomme et al. (2000) provides a brief, graphical overview of the potential profiles for many different sheath models. In this dissertation, the focus on planar sheaths will be restricted to the collisionless and weakly collisional Child-Langmuir sheaths, producing mono-energetic and drifting Maxwell-Boltzmann ions, respectively. The mechanism of ion-neutral charge exchange determines this difference between these energy distribution functions. Radovanov et al. (1995) convincingly showed that the neutral gas pressure in an Argon-Hydrogen gas mixture plasma controls the shape of the ion energy distribution function through collisionality. In the absence of collisions, the cold ions are mono-energetic and have a fluid drift equal to the Bohm speed. Ion-neutral collisions in the sheath cause the ion distribution function, initially mono-energetic in the plasma bulk, to assume a drifting Maxwell-Boltzmann distribution with the Bohm speed as the drift speed. In reality, the distribution functions are more complicated for collisionless ions, which have a cusp-like shape with a peak in the energy distribution function near $\varepsilon=k_{b} T_{e}$ (Tonks and Langmuir, 1929; Sheridan, 2001), although the simplistic model of unidirectional mono-energetic ions has been used in some studies of dust charging (Nunomura et al., 1999). Likewise, collisions produce ion distribution functions with an increased number of ions at lower energies as compared to the collisionless ion case (Chabert and Braithwaite, 2011), and are not necessarily a simple drifting Maxwell-Boltzmann distribution. The simplistic generalization of mono-energetic ions for collisionless conditions and drifting Maxwell-Boltzmann ions for collisional conditions is imposed to show the sensitivity of gyro-phase drift to ion distribution function shape and hence as a useful testbed for measuring sheath properties. As shown later in chapter V, when test grains levitate in a planar sheath, gyrophase drift acts as a sensitive indicator for the planar sheath mechanisms of ion flow, flow-shifted Maxwellian ions versus mono-energetic ions, ion-neutral charge-exchange collisions [via the charging model developed by Gatti and Kortshagen (2008)], and plasma species magnetization [via the charging model developed by Patacchini et al. (2007)]. All of these mechanisms have relevance to dust charging in experimental devices (Konopka et al., 2005a; Thomas et al., 2012).

To obtain the collisionless Child-Langmuir solution for flat, planar sheaths in plasma, assume that $n_{e}=n_{i}$ at the boundary $z=z_{s h}$ between the sheath and the plasma bulk, the potential in the 
sheath satisfies $\left|e V_{s h}\right| /\left(k_{b} T_{e}\right) \gg 1$ and $V_{s h}<0$, the Maxwell-Boltzman electrons are nearly absent in the sheath $\left(n_{e} \ll n_{i}\right)$, ions are cold $\left(T_{i} \ll T_{e}\right)$, and all ions are accelerated by the potential profile in the sheath because ion flux and energy are conserved. Conservation of ion flux means that the density of ions decreases because they are accelerated in the sheath. The following solutions for collisionless, weakly collisional, and fully collisional sheaths are one-dimensional models that are valid for partially or fully ionized plasma. Two-dimensional sheath models do exist, but Luginsland et al. (1996) showed that when the sheath inhomogeneity direction aligns with the magnetic field direction, the sheath properties along this direction are not appreciably altered by magnetic field strength.

The potential profile of a collisionless Child-Langmuir sheath is given by (Chabert and Braithwaite, 2011)

$$
V_{s h}(z)=-\left[\left(-\frac{3}{2} \sqrt{\frac{e n_{i}\left(z=z_{s h}\right) v_{i}\left(z=z_{s h}\right)}{\epsilon_{0}} \sqrt{\frac{m_{i}}{2 e}}}\right) z+\left(-V_{0}\right)^{3 / 4}\right]^{4 / 3}
$$

where $z$ corresponds to the distance above the planar surface, $z_{s h}>0$ is the location of the plasma sheath boundary, while $n_{i}\left(z=z_{s h}\right)$ and $v_{i}\left(z=z_{s h}\right)$ correspond to the ion density and ion flow speed at the plasma sheath boundary, respectively. The solution given in equation 2.23 provides $V_{s h}\left(z=z_{s h}\right)=0$. The sheath potential $V_{s h}(z)<0$ for $z<z_{s h}$, and accelerates ions from the sheath edge. The ion flow speed as a function of space is easily predicted from the potential profile in equation 2.23 using conservation of energy, and is given by $v_{i}(z)=\sqrt{v_{i}\left(z=z_{s h}\right)^{2}-\frac{2 e}{m_{i}} V_{s h}(z)}$. In the collsionless Child-Langmuir model of the planar sheath, the ions enter the sheath with a mono-energetic distribution, and they remain mono-energetic when they strike the surface. The upper panel of figure 2.2 shows the resulting profile along the sheath direction for a collisionless Child-Langmuir sheath.

When the ion-neutral mean free path is not large with respect to the size of the sheath, a collisional model is more valid than the description given by equation 2.23. Collisions act as a drag term in the ion momentum equation, and the ion flow becomes limited by mobility. Ion flux is still conserved, and the ion drift speed is given by $\mu_{i} E(z)$, where $E(z)$ is the electric field in the sheath and the ion mobility is given by $\mu_{i}=\frac{e}{m_{i} \nu_{i n}}$, where $\nu_{i n}$ is the ion-neutral charge exchange 
collision frequency. The resulting space profile for the collisional Child-Langmuir sheath is given by (Chabert and Braithwaite, 2011)

$$
V_{s h}(z)=-\sqrt{\frac{2 e n_{i}\left(z=z_{s h}\right) v_{i}\left(z=z_{s h}\right)}{\epsilon_{0} \mu_{i}}}\left(\left(z_{s h}-z\right)\right)^{3 / 2}+V_{0} .
$$

While the ions still enter the sheath at the sound speed $v_{i}\left(z=z_{s h}\right)=v_{B}$, the presence of ionneutral collisions imply that the ion energy distribution function must be a flow-shifted or drifting Maxwellian (Meige et al., 2007).

As discussed by Chabert and Braithwaite (2011), the ion fluid speed always exceeds the ion thermal speed, which means that the above description is inaccurate for high pressure sheaths.

The solution to this problem is to correct the ion mobility by using $\mu_{i}=\frac{2 e \lambda_{i}}{\pi m_{i}\left|v_{i}\right|}$, where $\lambda_{i}$ is the ion-neutral mean free path. The resulting space profile for the weakly collisional Child-Langmuir sheath is given by (Chabert and Braithwaite, 2011)

$$
V_{s h}(z)=-\frac{3}{2}\left(\frac{2 e n_{i}\left(z=z_{s h}\right)}{3 \epsilon_{0}} \sqrt{\frac{\pi T_{e}}{2 \lambda_{i n}}}\right)^{2 / 3}\left(z_{s h}-z\right)^{5 / 3}+V_{0}
$$

Again, the ions enter the sheath at the sound speed but collisions produce a flow-shifted or drifting Maxwellian ion energy distribution function. The lower panel of figure 2.2 shows the resulting profile along the sheath direction for a weakly collisional, partially-ionized Child-Langmuir sheath.

To summarize this section briefly, ion-neutral charge exchange collisions shape the ion energy distribution function in planar sheaths. Mono-energetic and drifting Maxwell-Boltzmann ions are adopted in this dissertation to measure the effect of different ion distribution functions on guiding center drift magnitude and direction. The presence of neutral gas atoms also manifests in the effect of ion-neutral charge exchange in the grain sheath, enhancing the ion current to dust grains, lowering grain charge. This change in grain charge and the presence of neutral drag should affect grain trajectories.

\section{Space Plasma}

Northrop and Hill (1983) established the analytical foundations for the adiabatic drifts in the co-rotating frame of a planet and the gyro-phase drift for dust grains orbiting a planet within 
a magnetic field. Further, Northrop and Hill (1983) explained how grain gyration in a plasma co-rotating with the planet leads to inhomogeneous ion flow relative to the dust grain during a gyro-orbit. In other words, for ions in the co-rotating plasma with temperature $T_{i}, v_{i}(r) / v_{t h i}=M_{i}$ is spatially inhomogeneous because $\left|v_{i}(r)\right|=\Omega r$, where $\Omega$ corresponds to the angular speed of the planet. An analytical solution for gyro-phase drift is possible for the condition $\left|v_{d}-v_{i}\right| \gg v_{t h i}$, where $v_{d}$ is the dust grain speed perpendicular to the magnetic field direction. As a side note, in homogeneous slab geometry, gyro-phase drift can still occur even if the ion flow is homogeneous, provided $\left|v_{d}-v_{i}\right| \approx v_{t h i}$ or $\left|v_{d}-v_{i}\right|>v_{t h i}$, because the gyration of the grain means that the relative ion flow will not be constant during a gyro-orbit, leading to charge-state modulation (Bliokh et al., 1994). Figure 2.3 shows an example trajectory of a grain around Saturn with no other inhomogeneities except for the relative velocity between the grain and the co-rotating plasma. For simplicity, this situation will also be referred to as inhomogeneous. Possible inhomogeneities present in space plasmas include ion mass composition, magnetic field, plasma density, neutral gas atom density, and inhomogeneous UV illumination. These inhomogeneities are summarized below.

Northrop and Morfill (1984) established that sub-micron dust grains can be radially transported by inhomogeneous temperature or ion mass composition. A temperature gradient exists in the Io plasma torus in Jupiter's plasmasphere, as discussed by Northrop et al. (1989), which can plausibly transport grains radially. The magnetic field of a planet can be approximately characterized as a dipole. Because a dipole field is spatially inhomogeneous, the magnetic field surrounding a planet

in space is spatially inhomogeneous. Surrounding a planet, the co-rotating plasma can also have a density gradient. Because the density of neutral gas can also have a gradient, the ion-neutral meanfree path can change during a grain gyro-orbit. In space it is also possible to have inhomogeneous UV illumination. As an example, grains can transit into and out of UV illumination when orbiting a celestial body such as a planet or moon, which is best modelled as an abrupt inhomogeneity.

\section{Basic parameters of Inhomogeneous Plasma}

The basic parameters outlined in section A were for homogeneous plasmas. These parameters can also be extended for inhomogeneous plasmas with some caution. Because plasma inhomogeneity is necessary for gyro-phase drift to occur (Northrop and Hill, 1983), it is imperative to correctly modify these basic parameters. Debye length, number of electrons in a Debye sphere, electron 
plasma frequency, mean free path, and gyro-frequency are extended to inhomogeneous plasma.

The plasma conditions far from the sheath surrounding a small spherical conductor or dust grain determine the Debye length in an inhomogeneous plasma. Small here means that $L \gg a$, or that the plasma is much larger than the grain size. With the assumption of electron, ion densities and temperatures $n_{i}, n_{e}$, and $T_{e}, T_{i}$ respectively, the definition in equation 2.7 will be relatively unchanged and is given by

$$
\lambda_{D}=\sqrt{\frac{\epsilon_{0} k_{b}}{e^{2}}\left(\frac{n_{e}}{T_{e}}+\frac{n_{i}}{T_{i}}\right)^{-1}}=\sqrt{\frac{\epsilon_{0} k_{b} T_{e} T_{i}}{e^{2}\left(n_{e} T_{i}+n_{i} T_{e}\right)}}
$$

Even though $n_{e}$ and $n_{i}$ are not required to be equal, this solution is still valid if we assume Maxwell-Boltzman distributed electrons and ions and that $e V(r) /\left(k_{b} T_{s}\right) \ll 1$. When performing the expansion on the right hand side of equation 2.5, we obtain the result to first order

$$
\frac{2}{r} \frac{\partial}{\partial r} V_{s h}(r)+\frac{\partial^{2}}{\partial r^{2}} V_{s h}(r)-\frac{e}{\epsilon_{0}}\left(\frac{n_{e 0}}{T_{e}}+\frac{n_{i 0}}{T_{i}}\right) V_{s h}(r)+\frac{e}{\epsilon_{0}}\left(n_{e}-n_{i}\right) .
$$

Notice that the only difference from equation 2.6 is that we have an offset $\frac{e}{\epsilon_{0}}\left(n_{e}-n_{i}\right)$, which means that the solution is essentially the same as the solution given in equation 2.9, except that there is an offset:

$$
V_{s h}(r)=V_{d} \frac{a}{r} \exp \left(-\frac{r-a}{\lambda_{D}}\right)+V_{s}-\lambda_{D}^{2} \frac{e}{\epsilon_{0}}\left(n_{e}-n_{i}\right)
$$

By inspection, the solution in equation 2.28 satisfies the differential equation in equation 2.27. Note also that the electron or ion Debye length can be written:

$$
\lambda_{D e}=\lambda_{D} \sqrt{1+\frac{n_{i}}{n_{e}} \frac{T_{e}}{T_{i}}}
$$

and

$$
\lambda_{D i}=\lambda_{D} \sqrt{1+\frac{n_{e}}{n_{i}} \frac{T_{i}}{T_{e}}} .
$$


When ions are best described by a drifting Maxwellian with flow speed $v_{i} \gg v_{t h i}$, then

$$
\lambda_{D}=\lambda_{D e} \sqrt{1+\frac{\frac{T_{e}}{T_{i}}}{2 \frac{n_{e}}{n_{i}} M_{t h i}^{2}}},
$$

where $M_{t h i}=v_{i} / v_{t h i}$ is the ion thermal Mach number. When ions are best described as a monoenergetic, then

$$
\lambda_{D}=\lambda_{D e} \sqrt{1+\frac{1}{\frac{n_{e}}{n_{i}} M_{i}^{2}}},
$$

where $M_{i}=v_{i} / \sqrt{\frac{k_{b} T_{e}}{m_{i}}}$ now corresponds to the regular Mach number, i.e., the ratio of the ion flow speed to the acoustic or Bohm speed (Daugherty et al., 1992). The definition for the number of electrons in a Debye sphere in equation 2.10 will remain unchanged for inhomogeneous plasma, except that this number need not be constant everywhere in the plasma.

The electron plasma frequency varies spatially when the electron density varies. The local electron plasma frequency is a proxy for electron number density. Despite the possibility for electron density variation, the electron plasma frequency generally remains the shortest time scale for inhomogeneous plasmas, which is true for all cases presented in this dissertation.

The mean free path can also vary spatially. For ion-neutral charge exchange collisions, the primary mean free path length scale in this dissertation, this quantity is dependent on the neutral gas atom number density. The neutral gas atom number density will be considered constant in the surrounding plasma throughout this dissertation.

The gyro-frequency of a plasma species varies spatially when an inhomogeneous magnetic field is present. The simplest, lowest order description of this change is given by $\omega_{c s}(\mathbf{r})=\frac{e B(\mathbf{r})}{m_{s}}$, where the magnetic field varies spatially. However, as first demonstrated by Parker (1958), the plasma particles move in a more complicated fashion in the plane perpendicular to the magnetic field direction than described by equations 2.17 and 2.18 when the magnetic field is homogeneous and the electric field is inhomogeneous. The original derivation assumes that there is shear in the plasma flow, but because the flow speed $\boldsymbol{u}$, the electric field $\boldsymbol{E}$, and the magnetic field $\boldsymbol{B}$ are related by $\boldsymbol{E}=-\boldsymbol{u} \times \boldsymbol{B}$, the treatment can also be done when the electric field is inhomogeneous along the electric field direction. 
To summarize the effects of inhomogeneous electric field on charged particle motion, the equations of motion for a charged particle in the plane perpendicular to the magnetic field direction become

$$
\ddot{x}= \pm \omega_{c s} \dot{y}
$$

and

$$
\ddot{y}= \pm \omega_{c s} \frac{E_{y}(y, t)}{B_{0}} \mp \omega_{c s} \dot{x}
$$

assuming $B_{0}$ is constant, the perpendicular electric field $E_{y}$ is inhomogeneous in the field coordinate $y$, perpendicular to the magnetic field direction $\hat{z}$, and the upper signs indicate positively charged plasma particles while the lower signs indicate negatively charged plasma particles. Integrate equation 2.33 to get $\dot{x}= \pm \omega_{c s}\left(y-y_{0}\right)$, where $y_{0}=y(t=0)$ is the initial position of the plasma particle in the $y$-direction. Substitute the integration of equation 2.33 into equation 2.34 to yield

$$
\ddot{y}= \pm \omega_{c s}\left(\frac{E_{y}(y, t)}{B_{0}} \mp \omega_{c s} y \pm \omega_{c s} y_{0}\right)
$$

which can be solved using the WKB approximation to produce elliptical equations of motion. The frequency for oscillation $\omega$ in the $\boldsymbol{E} \times \boldsymbol{B}$-direction is given by

$$
\omega=\omega_{c s}\left(1-\frac{\xi(y, t)}{\omega_{c s}}\right)^{1 / 2}
$$

where $\xi=\left.\frac{\partial E_{y}(y, t)}{\partial y}\right|_{y=y_{0}} / B_{0}$, evaluated at the particle's initial position in the $y$-direction, is called the shear parameter (Parker, 1958; Ganguli, 1995; Gavrishchaka, 1996). Whenever the electric field is inhomogeneous, the gyro-frequency is no longer equal to $\omega_{c s}$, and the particle travels in an ellipse. Likewise, the gyro-radius is no longer given by the simple relation $v_{\perp} / B_{0}$, and instead the semi-major axes of the ellipse are given by $v_{\perp} /\left(\omega_{c s}\left(1-\xi(t) / \omega_{c s}\right)^{3 / 4}\right)$ for the $x$-direction and $v_{\perp} /\left(\omega_{c s}\left(1-\xi(t) / \omega_{c s}\right)^{1 / 4}\right)$ for the $y$-direction. These effects were important for the experiments and simulations of Reynolds et al. (2006).

Lastly, the standard and thermal Mach numbers $\left(M_{s}\right.$ and $\left.M_{t h s}\right)$ are inhomogeneous in the 
presence of inhomogeneous electron or ion flow. This situation of inhomogeneous Mach number in also encountered whenever the difference between the grain velocity and plasma flow changes during a gyro-orbit. Inhomogeneous Mach numbers permit charge state modulation of gyrating dust grains, elaborated in subsequent chapters. 


\section{Chapter III}

\section{Description of Dust in Plasma}

Particulate matter in ionized gas becomes charged, so electromagnetic forces become relevant. In this chapter, the basics of the charging process for dust grains and the forces on dust grains are introduced.

\section{A Distinction between Dust in Plasma and Dusty Plasma}

Dust in plasma means the density of dust grains is small enough that dust grains are, on average, sufficiently distant from one another that their Debye sheaths do not overlap. This average inter-

grain spacing $d$ is predicted by $d=\left[3 / 4\left(\pi n_{d}\right)\right]^{1 / 3}$, where $n_{d}$ is the dust number density. Shukla and Mamun (2002) provide a robust definition to distinguish two distinct possibilities, noting that the situation $a \ll \lambda_{D}<d$ (in which charged dust particles are considered as a collection of isolated screened grains) corresponds to the dust in a plasma condition, while the situation $a \ll d<\lambda_{D}$ (in which charged dust particles participate in the collective behavior) corresponds to the dusty plasma condition.

When considering the charging of grains in a dusty plasma, the background plasma of electrons and ions cannot be treated as being dust-absent. The coupling parameter $\Gamma$, the ratio of Coloumb energy to thermal energy of dust grains, further characterizes the regime of dusty plasma, and is given by

$$
\Gamma=\frac{q_{d}^{2}}{4 \pi \epsilon_{0} d k_{b} T_{d}}
$$


where $q_{d}$ is the average charge on the grains and $T_{d}$ is the kinetic temperature of dust (Merlino, 2006). When $\Gamma$ exceeds the critical value $\Gamma \approx 170$, the dust grains are arranged in an approximate lattice and is called a plasma crystal or Coloumb crystal (Ikezi, 1986). This dissertation will focus exclusively on dust in plasma, which means the criterion $a \ll \lambda_{D}<d$ is used, rather than dusty or coupled plasma. The assumption of dust in plasma simplifies the calculation of grain charge for test particle trajectories in abrupt and gradual inhomogeneity.

\section{Unmagnetized-orbit and Magnetized-orbit Dust grains}

As discussed earlier in chapter II, section 5, a charged particle species $s$ is considered magnetized with respect to the grain radius $a$ if $R_{L s}<a$ or with respect to the plasma volume $L$ if $R_{L s}<L$ and the collision frequency of the species is smaller than the gyro-frequency. For a weakly ionized plasma, the highest collisional timescale for dust is the dust-neutral collision frequency (Thomas et al., 2012). In a fully ionized plasma, neutral gas atoms are not present, so the highest collisional timescale is the dust-ion collision frequency. Dust collisions with electrons produce negligible changes to dust grain motion, and are not considered. The dust-neutral collision frequency will be discussed at greater length in section 4. In order for the gyro-motion of grains to exist, magnetized-orbit grains are required. In other words, the gyro-frequency of dust must be larger than the dust-neutral collision frequency $\left(\omega_{c d}>\nu_{d n}\right)$ and the dust-ion collision frequency $\left(\omega_{c d}>\nu_{d i}\right)$. Because neither grad-q nor gyro-phase drift occurs in the absence of dust gyration, this dissertation will focus on magnetized-orbit grain trajectories. Some example trajectories are shown later in chapter $\mathrm{V}$ for when the dust-neutral collision frequency is greater than the dust gyro-frequency to illustrate that gyration does not occur.

\section{B Physics of the Grain Sheath in Dusty Plasma}

\section{Debye-Huckel Grain Sheath Potential Profile}

As discussed earlier in chapter II, section 1, and given by equation 2.9, the sheath potential profile

around a spherical conductor is given by $V_{s h}(r)=V_{d} \frac{a}{r} \exp \left(-\frac{r-a}{\lambda_{D}}\right)+V_{s}$, where $V_{s}$ is the space potential far away from the grain, or outside of the grain's Debye sphere. The spatial variation of $V_{s}$ outside of the grain's Debye sphere does not pose any problems, provided the inhomogeneity 
scale length of $V_{s}$ is much larger than the grain radius a. Importantly, Daugherty et al. (1992) showed that the Debye-Huckel solution is no longer valid when the grain size is comparable to or greater than any of the Debye lengths. It becomes impossible to fit a Debye length to the solution in 2.9 when the grain size is larger than the electron Debye length. As $a$ is increased beyond $\lambda_{D e}$, the grain sheath becomes planar-like. Equation 2.9 and $a \lesssim \lambda_{D}$ will be assumed for negatively charged grains and will pervade this dissertation. Here, the profile for a spherical conductor will hold for dust grains.

\section{Non-monotonic Grain Sheath Potential Profiles}

For emitting grains, which includes photo-emitting, thermionic emitting, and secondary electron emitting grains, the potential profile surrounding spherical surface can be non-monotonic. That is to say, the sheath may have a local minimum (or maximum) in the potential profile. This is also refered to as a virtual cathode or space charge-limited sheath (Intrator et al., 1988; Guernsey and Fu, 1970; Poppe and Horányi, 2010). Guernsey and Fu (1970) first predicted the formation of a non-monotonic planar sheath. Poppe and Horányi (2010) simulated a photo-electric planar sheath, relevant to the lunar surface, which produced a non-monotonic profile. For grain sheaths, Delzanno et al. (2004) demonstrated that non-monotonic sheaths can form around a thermionically emitting grain. The presence of a non-monotonic grain sheath should affect current collection for the charging models of Patacchini et al. (2007) and Gatti and Kortshagen (2008), because these models consider the profile of the sheath when determining the collected currents. The orbit motion limited charge model is not prepared to take this into account, although Delzanno et al. (2005) provide an extension to OML for emitting surfaces. The emitted photo-electron currents in this dissertation are generally small, so the effect on the grain sheath is expected to be negligible.

\section{Ion-Neutral Charge-Exchange Collisions in the Dust Grain Sheath}

Goree (1992) explained that ion-neutral charge-exchange collisions alter the sheath potential profile through the process of creating trapped ion orbits. Lampe et al. (2001) was the first to provide a self-consistent model for the dust grain sheath potential, and showed how this differed from a pure Debye-Hückel solution. The sheath potential profile is still monotonic, although $V_{s h}$ deviates significantly from the Debye-Hückel solution after 5 Debye lengths away from the grain. Lampe 
et al. (2003); Gatti and Kortshagen (2008) incorporated trapped ions to predict the enhanced ion current to the dust grain, but they assumed a Debye-Hückel solution when calculating the capture radius $R_{0}$. Hence, in this dissertation, this strategy is used and deviations to the Debye-Hückel solution, although present, are neglected.

\section{Grain Capacitance and Dimensionless Surface Potential}

The capacitance of a spherical grain immersed in a plasma is given by (Whipple et al., 1985; Barnes et al., 1992)

$$
C_{d}=4 \pi \epsilon_{0} a\left(1+\frac{a}{\lambda_{D}}\right)
$$

This is easily derived using Gauss' law and evaulating the electric field at the surface, starting with the equation

$$
E_{r}(r=a)=\frac{q_{d}}{4 \pi \epsilon_{0} a^{2}}
$$

where $q_{d}$ is the net charge on the grain. Proceed by equating the electric field with the negative gradient of the Debye-Hückel solution to the sheath potential profile given in equation 2.9 to obtain

$$
E_{r}(r=a)=-\left.\frac{\partial V_{s h}}{\partial r}\right|_{r=a}=\left.V_{d}\left(\frac{a}{r^{2}}+\frac{a}{r \lambda_{D}}\right) \exp \left(-\frac{r-a}{\lambda_{D}}\right)\right|_{r=a}=V_{d}\left(\frac{\lambda_{D}+a}{\lambda_{D} a}\right)=\frac{q_{d}}{4 \pi \epsilon_{0} a^{2}}
$$

Capacitance is defined by $C \equiv q / \Delta V$, and here, $\Delta V=V_{d}=V_{\text {surf }}-V_{s}$ is the potential difference between the surface of the sphere and the potential outside of the Debye sheath. Applying the definition of capacitance to equation 3.4 produces the result given in equation 3.2. A dimensionless surface potential $\chi_{e}$ can then be defined

$$
\chi_{e}=\frac{e V_{d}}{k_{b} T_{e}}=\frac{e q_{d}}{C_{d} k_{b} T_{e}} .
$$

Whenever the quantity $e V_{d} /\left(k_{b} T_{i}\right)$ arises in a charging model, this reduces to $\frac{T_{e}}{T_{i}} \chi_{e}$. 


\section{Charging of Dust Grains in the Plasma}

The relative flux of electrons and ions to the grain surface determines grain charge. Secondary electron emission occurs for electron temperatures as small as $T_{e} \approx 2$ to $5 \mathrm{eV}$. Secondary electron emission from the dust grain surface yields a positive current contribution. Thermionic emission from sufficiently hot dust grain surfaces and photo-electron emission due to ultra-violet light also modifies the in-situ equilibrium dust charge to a more positive value. Photo-emission of dust grains is of particular importance in the background of a tenuous quasi-neutral plasma (Colwell et al., 2005). A background magnetic field alters the electron collection of a grain (Patacchini et al., 2007). The presence of ion-neutral charge exchange collisions in the dust sheath (Lampe et al., 2001, 2003) is an example of a mechanism where ion current is enhanced and the dust charges to a more positive value.

Non-equilibrium conditions such as plasma inhomogeneity are responsible for additional charging processes. The distribution function determines the flux of electrons or ions to the dust grain surface, and the distribution functions are not necessary Maxwell-Boltzmann, as indicated earlier in section II. When the plasma inhomogeneity scale length is much smaller than the electron energy relaxation length, the electron distribution function is non-local (Tsendin, 1995; Demidov et al., 2002). Filippov et al. (2003) has developed a dust charging model for non-local electrons. As shown

in chapter II, section 1, ions can be modelled as mono-energetic or a drifting Maxwell-Boltzmann at the planar sheath edge, which implies a deviation from Maxwell-Boltzmann distribution functions. Additionally, grains with large grain speed relative to ion thermal speed collect a modified current (Northrop and Hill, 1983).

In this section, the charging processes relevant for dust grains in space and laboratory are discussed. Emphasis will be placed on the OML model (Mott-Smith and Langmuir, 1926) including both mono-energtic and drifting Maxwell-Boltzmann ion flow, the charging model of Patacchini et al. (2007), the charging model of Gatti and Kortshagen (2008), and UV photo-electron emission because these are the processes investigated in this dissertation. Secondary electron emission and thermionic electron emission are also important grain charging processes, but these will not be considered for the work done in this dissertation because these effects should be negligible in MDPX. 


\section{Charging Time}

The charging time of dust grains is a crucial parameter in this dissertation. In Cui and Goree (1994) and Goree (1994), the characteristic charging time $\left(\tau_{\mathrm{ch}}\right)$ of a dust grain is given by

$$
\tau_{\text {charge }}=K_{\tau} \frac{\left(k_{b} T_{e}\right)^{1 / 2}}{a n_{e}}
$$

is used, where $k_{b}$ is the Boltzmann constant, $n_{e}$ corresponds to the background plasma density, $T_{e}$ is the electron temperature in $\mathrm{eV}$, and $K_{\tau}$ is a function of both $T_{i} / T_{e}$ and $m_{i} / m_{e}$. As is clear by inspection of equation $3.6, \tau_{\mathrm{ch}}$ decreases with increasing dust grain radius or plasma density. Larger dust grains charge faster, with the collection area scaling as $\propto a^{2}$. Higher density implies a greater flux of incident charge carriers to the dust grain, and it follows intuitively that this must bring the dust grain to its equilibrium value at a greater rate (smaller $\tau_{\mathrm{ch}}$.) For OML theory in a homogeneous plasma, the background density will not affect the total charge; density only affects the charging rate. Electron temperature also increases the charging time, since the equilibrium charge for a dust grain will be a larger value for a hotter plasma. The charging time can be specified for other charging models and can include other charging processes such as photo-electron emission, etc.

A different, but related concept to the charging time is the time for a grain to gain or lose one electron. This is given by

$$
t_{1 e}=\frac{1}{3} \frac{\frac{\lambda_{D}}{a} \sqrt{1+\frac{T_{e}}{T_{i}} \frac{n_{i}}{n_{e}}} N_{D e}}{\frac{2 \pi}{\omega_{p e}} \frac{\mathrm{d}\left|\chi_{e}\right|}{\mathrm{d} t}}
$$

where $\chi_{e}=e V_{d} /\left(k_{b} T_{e}\right)$ is the dimensionless surface potential. When the charging model currents are made dimensionless, the resulting differential equation is given by $\frac{\mathrm{d} \chi_{e}}{\mathrm{~d} t}=I_{t o t}\left(\chi_{e}\right) /\left(2 \sqrt{\pi} e n_{e} v_{t h e} a^{2}\right)$. The charging equation can be solved numerically at each timestep by computing the currents, evaluating the time to gain or lose an electron $t_{1 e}$, and adding the change to the dimensionless surface potential by one electron using $\chi_{e}^{n+1}=\chi_{e}^{n}+\frac{\mathrm{d} \chi_{e}}{\mathrm{~d} t} t_{1 e}$, where $\chi^{n}$ is the present value of the dimensionless surface potential. For the next time step, the process is repeated. Note that $t_{1 e}$ changes because the current changes as a function of $\chi_{e}$, so this is an adaptive timestep that cares only about the time necessary to gain or lose an electron based on grain and plasma conditions. Once the grain flips between neighboring charge states, or when $\chi_{e}^{n+2}=\chi_{e}^{n}$, the grain has reached the 
in-situ-equilibrium charge.

Using molecular dynamics simulations, Cui and Goree (1994) found that grain charge varies stochastically, especially when the grain charge is close to the in-situ equilibrium charge. Larger grains have smaller fractional charge fluctuations, as compared to smaller grains. Because of these charge fluctuations, small enough grains can even charge positively if the in-situ equilibrium grain

charge is negative. For this dissertation, the larger fractional charge fluctuations of smaller grains implies that the uncertainty in grain charge due to stochastic variation and hence the resulting grain trajectories and drifts is more important for smaller grains. Cui and Goree (1994) explain that while the stochastic collection of charges is related to counting statistics (Morfill et al., 1980), it is not straightforward to apply Poisson stastics because the probabilities for electron or ion collection depends in part on the dust grain surface potential $V_{d}$. From simulations, Cui and Goree (1994) determined that the fractional root-mean-square square fluctuation level is given by

$$
\frac{\Delta q}{q_{e q}}=\frac{\sqrt{\left[q_{e q}-q(t)\right]^{2}}}{q_{e q}}=\frac{1}{2}|N|^{-1 / 2},
$$

where $q_{e q}$ is the in-situ-equilibrium charge, $q(t)$ is the charge on the grain as a function of time, and $N$ is the number of charges on the grain. The timescale for charge fluctuations is an important timescale to consider. Cui and Goree (1994) stress that the coefficient $\frac{1}{2}$ cannot necessarily be predicted using Poisson statistics, but this coefficient describes the fluctations for the specific parameters chosen in their simulations.

\section{Specifics of OML Model}

The Orbit Motion Limited (OML) charging model (Mott-Smith and Langmuir, 1926) is the simplest form of the more general Orbit Motion (OM) theory; the full OM theory involves simultaneously solving the non-linear Possion equation and the particle trajectories (Delzanno et al., 2005). The Orbit Motion Limited (OML) charging model is valid for negligible collisionality, in other words, for high Knudsen number $\mathrm{Kn}_{a}, \lambda_{m f p} / a \gg 1$, where $\lambda_{m f p}$ is some characteristic collisional length scale. and $a$ is the characteristic length scale of the probe or object. In this dissertation, the mean free path is determined by the ion-neutral charge-exchange mean free path and $\lambda_{m f p}=\lambda_{i}$. In the OML model, the plasma is treated as being collisionless on a lengthscale $a$. Also in OML theory, 
it is assumed that $\lambda_{D} \gg a$. Specifically, OML is valid for $a / \lambda_{D} \leq 3$ (Zakrzewski and Kopiczynski, 1974).

In OML theory the collection of unmagnetized ions and electrons is treated as a central force problem. An impact parameter, $h$, determines the maximum approaching distance at which an ion will be collected by the dust grain, and this is determined solely by the conservation of energy and angular momentum, and whether incident ions or electrons experience an attractive or retarding potential. Collection here means that the ion or electron will at least graze the surface of the probe or dust grain. The table 2.1 clarifies attractive and retarding potentials.

For the attracted species, the collection is limited by the angular momentum of the species about the probe or dust grain; large velocity components transverse to the sphere's surface normal vector will have too much angular momentum to be collected by the dust grain.

For the species being repelled by the probe, the collection is still limited by angular momentum considerations but it is also limited by the energy of the species. The particles (electrons for a negatively charged sphere, ions for a positively charged sphere) must have enough energy to make it to the probe surface.

\section{i Unmagnetized Stationary grain}

The derivation is briefly summarized in the appendix A, section 1, with the main assumptions and results quoted here. The grain is assumed to be stationary with respect to the plasma. The electron and ion distribution functions do not have to be Maxwellian, but this will be assumed for simplicity and provides a baseline description of charging currents in plasma. A non-Maxwellian distribution function may entail a more difficult integration in the energy and angular momentum space. Also, assume that the grain is negatively charged, so that ions are the attracted species and

electrons are the repelled species. In all the dissertation results, this will be the case. The sphere is a spherically symmetric collector.

Far from the grain surface, where the plasma is unperturbed by the dust sheath, the energy of the ions with respect to the grain surface is given by

$$
E_{\infty}=\frac{1}{2} m_{i} \boldsymbol{v}_{\infty}^{2}+e V_{s}
$$


where $V_{s}$ is the space potential of the plasma several Debye lengths from the grain where the sheath has no effect to attract or retard particles. The energy of an ion that just grazes the surface of the grain of radius $a$ is given by

$$
E_{a}=\frac{1}{2} m_{i}\left[\boldsymbol{v}_{\boldsymbol{t}}(r=a)\right]^{2}+e V_{\text {surf }}
$$

where $m_{i}$ is the ion mass, $\boldsymbol{v}_{\infty}=\boldsymbol{v}_{\boldsymbol{t}}+\boldsymbol{v}_{\boldsymbol{r}}$ is the velocity very far away from the grain, $\boldsymbol{v}_{\boldsymbol{t}}(\boldsymbol{a})$ is the velocity of the ion that grazes the grain surface, $\boldsymbol{v}_{\boldsymbol{t}}$ is the velocity component transverse or perpendicular to the grain's radial direction and $\boldsymbol{v}_{\boldsymbol{r}}$ is the velocity component parallel to the grain's radial direction. The velocity far from the grain is specified by the distribution function in the plasma bulk, which does not necessarily have to be Maxwellian. Because OML assumes no collisions occur in the sheath, conservation of energy tells us that equations 3.9 and 3.10 are equal to each other. Also, the angular momentum of the ions with respect to the grain must be conserved. Because the angular momentum is given by $\boldsymbol{J}=\boldsymbol{r}_{\boldsymbol{i}} \times m_{i} \boldsymbol{v}$, only the transverse velocity component contributes to the angular momentum in this coordinate system, where $\boldsymbol{r}_{\boldsymbol{i}}$ is the position of ions and $\boldsymbol{v}$ is the velocity of ions. This results in an expression for the current density collected in terms of the distribution function in the plasma bulk,

$$
J_{\infty}=m_{i} v_{t}(r \rightarrow \infty) h
$$

and

$$
J_{a}=m_{i} v_{t}(a) a,
$$

where $h$ is a yet-to-be determined impact parameter. The values of $J_{\infty}$ and $J_{a}$ are equivalent, and when the two expressions in equations 3.11 and 3.12 are combined with the conservation of energy, the impact parameter is given by (Allen, 1992)

$$
h=a \sqrt{1+\frac{2\left(V_{s}-V_{\text {surf }}\right)}{m_{i} v_{\infty}^{2}}} .
$$

The parameter $V_{d} \equiv V_{s}-V_{\text {surf }}$ was defined earlier in chapter II, section 1 . 
The complete derivation, including the integration over energy and momentum space is carried out in the appendix A, section 1. Assuming the ion density far from the grain is $n_{i}$, the ion current is given by (Mott-Smith and Langmuir, 1926; Laframboise, 1966; Allen, 1992)

$$
I_{i}=e 4 \pi a^{2} n_{i} \frac{v_{t h i}}{2 \sqrt{\pi}}\left(1-\frac{e V_{d}}{k_{b} T_{i}}\right)
$$

Non-Maxwellian ion distribution functions will modify the result given by equation 3.14. As is evident in equation 3.14, increases in ion temperature correspond to decreases in the (negative) quantity $\frac{e V_{d}}{k_{b} T_{i}}$, which results in reduced ion current, even though $v_{t h i}$ increases. This is entirely a consequence of angular momentum conservation; higher energy ions have a smaller value of maximum impact parameter for collection. This is demonstrated in figure 3.2, where Merlino (2006) shows that raising the ion temperature relative to electron temperature raises the dimensionless surface potential. However, the effect is not monotonic, and near $T_{e} / T_{i} \approx 0.1$ the dimensionless surface potential becomes less negative because the increasing ion temperature results in a larger thermal flux of ions, even though the angular momentum corresponding to the thermal speed increases.

Electrons are repelled from the (negatively-charged) spherical grain, and the description in equation 3.10 is modifed by:

$$
E_{a}=\frac{1}{2} m_{e} v_{t}(a)^{2}+e V_{\text {surf }}=\frac{1}{2} m_{e} \boldsymbol{v}_{\infty}^{2}+e V_{s}=E_{\infty}
$$

We note also that for the repelled electrons, only particles with energy $E>e V(r=a)$ can be collected. Like the ions, the electrons are assumed to have a Maxwellian distribution far from the probe, and electron energy and momentum are conserved. Using the procedures described in the appendix A and elsewhere (Allen, 1992; Laframboise, 1966; Mott-Smith and Langmuir, 1926), the electron current to the probe is given by

$$
I_{e}=-e 4 \pi a^{2} n_{e} \frac{v_{t h e}}{2 \sqrt{\pi}} \exp \left(\frac{e V_{d}}{k_{b} T_{e}}\right)
$$

Equilibrium charge scales linearly with electron temperature in the stationary OML model, which reinforces the utility of the dimensionless surface potential, $\chi_{e}=e q_{d} /\left(C_{d} k_{b} T_{e}\right)$. The results from 
equations 3.14 and 3.16 correspond to currents for a negatively charged grain. The currents are switched when the grain is positively charged, that is to say, equation 3.16 describes ion current collection for $V_{d}>0$ if we change all the subscripts from $e$ to $i$ and there is a minus sign in the argument of the exponential but no minus sign in front of the prefactor $e 4 \pi a^{2} n_{i} \frac{v_{t h i}}{2 \sqrt{\pi}}$, and equation 3.14 describes electron current collection for $V_{d}>0$ if we change all the subscripts from $i$ to $e$

and there is no minus sign in front of the $\frac{e V_{d}}{k_{b} T_{e}}$ term but there is a minus sign in the prefactor $-e 4 \pi a^{2} n_{e} \frac{v_{t h e}}{2 \sqrt{\pi}}$. All of these possibilities are addressed in table 2.1. The dimensionless charging equation for the stationary OML model is given by

$$
\frac{2 \pi}{\omega_{p e}} \frac{\mathrm{d} \chi_{e}}{\mathrm{~d} t}=-\frac{\sqrt{2 \pi}}{\frac{\lambda_{d}}{a} \sqrt{1+\frac{n_{i} T_{e}}{n_{e} T_{i}}}\left(1+\frac{a}{\lambda_{d}}\right)} \exp \left(\chi_{e}\right)+\frac{\sqrt{2 \pi} \frac{n_{i}}{n_{e}}}{\frac{\lambda_{d}}{a} \sqrt{1+\frac{n_{i} T_{e}}{n_{e} T_{i}}}\left(1+\frac{a}{\lambda_{d}}\right)} \sqrt{\frac{m_{e} T_{i}}{m_{i} T_{e}}}\left(1-\frac{T_{e}}{T_{i}} \chi_{e}\right)
$$

where $\chi_{e} \equiv e V_{d} /\left(k_{b} T_{e}\right)=q_{d} /\left(C_{d} k_{b} T_{e}\right)$ is the dimensionless surface potential.

\section{ii Unmagnetized Non-stationary Granule}

The results in the previous section i can be extended to the case of a moving grain, or whenever there is a relative velocity between attracted/repelled plasma constituents and a dust grain. It is straightforward to obtain a solution for the special case of mono-energetic, unidirectional ion flow using the techniques discussed in section i, without the need for integration. The result for this simple case is given by (Nunomura et al., 1999),

$$
I_{i}=e \pi a^{2} n_{i} v_{i}\left(1-\frac{2 e V_{d}}{m_{i} v_{i}^{2}}\right)
$$

Because the ions are mono-energetic and unidirectional, the collection area is $\pi a^{2}$. For planar sheaths in low pressure discharges, mono-energetic ion flow to the planar sheath surface can provide a satisfactory model of ion current collection (Nunomura et al., 1999). If the ion flow speeds are to be replaced with ion thermal Mach numbers in equation 3.18, the most sensible scaling is the Bohm speed or ion-acoustic speed which is $v_{b}=\sqrt{\frac{k_{b} T_{e}}{m_{i}}}$.

The solution for drifting Maxwellian populations is more involved. Kanal (1962) first obtained the result for attractive and retarded currents to a spherical object in warm flowing plasma, later extended to cylindrical objects by Kanal (1964) and summarized by Whipple (1981), while Northrop 
and Birmingham (1996) discussed at length the equilibrium solutions for spheres and cylinders. Nagy et al. (1963) used these results to make measurements in the ionosphere. Patacchini (2007) provides a very clear derivation in an appendix. This result will not be derived here, but the main results for Maxwellian ion and electron distributions will be quoted.

Just as in the stationary case, we are not limited to drifting Maxwellian-Boltzmann ions; as long as a suitable distribution function in energy and angular momentum $f(E, J, \varphi)$ can be constructed from the velocity distribution function $f(\boldsymbol{v})$, an expression for the current from attracted and repelled particles can be derived. The resulting integrals may not necessarily be analytically tractable, but it is possible to numerically integrate them using gaussian quadrature or other numerical methods.

The ion current to a negatively-charged dust grain in warm flowing plasma is given by (Northrop and Birmingham, 1996; Horányi, 1996)

$$
I_{i}=e \sqrt{\pi} a^{2} n_{i} v_{t h i}\left[\left(M_{t h i}^{2}+\frac{1}{2}-\frac{e V_{d}}{k_{b} T_{i}}\right) \frac{\sqrt{\pi}}{M_{t h i}} \operatorname{erf}\left(M_{t h i}\right)+\exp \left(-M_{t h i}^{2}\right)\right]
$$

while the electron current to a negatively-charged dust grain in flowing plasma is given by (Hinteregger, 1961; Kanal, 1962)

$$
\begin{aligned}
I_{e}= & -e \frac{\sqrt{\pi}}{2} a^{2} n_{e} \frac{v_{\text {the }}}{M_{\text {the }}}\left\{\sqrt{\pi}\left(M_{\text {the }}^{2}+\frac{1}{2}+\frac{e V_{d}}{k_{b} T_{e}}\right)\left[\operatorname{erf}\left(M_{\text {the }}+\sqrt{-\frac{e V_{d}}{k_{b} T_{e}}}\right)+\operatorname{erf}\left(M_{\text {the }}-\sqrt{-\frac{e V_{d}}{k_{b} T_{e}}}\right)\right]\right. \\
& +\left(\sqrt{-M_{\text {the }} \frac{e V_{d}}{k_{b} T_{e}}}+M_{\text {the }}\right) \exp \left(-\left(M_{\text {the }}-\sqrt{-\frac{e V_{d}}{k_{b} T_{e}}}\right)^{2}\right) \\
& \left.-\left(\sqrt{-M_{\text {the }} \frac{e V_{d}}{k_{b} T_{e}}}-M_{\text {the }}\right) \exp \left(-\left(M_{\text {the }}+\sqrt{-\frac{e V_{d}}{k_{b} T_{e}}}\right)^{2}\right)\right\},
\end{aligned}
$$

where erf is the error function, $M_{t h s}=w_{s} / v_{t h s}=\left|\boldsymbol{v}_{\boldsymbol{s}}-\boldsymbol{v}\right| / v_{t h s}$ corresponds to the normalized relative velocity between grain and plasma. As discussed by Northrop and Hill (1983), the electron thermal speed $v_{\text {the }}$ is typically much larger than any relative plasma flow, or $v_{\text {the }} \gg w_{s}$, where $w_{s}=\left|\boldsymbol{v}_{\boldsymbol{s}}-\boldsymbol{v}\right|$ is the relative fluid drift speed between a plasma species and the dust grain. This condition $v_{\text {the }} \gg w_{s}$ means that equation 3.20 reduces to the form given in equation 3.16 , and so 
this form is often used even in the case of a relative electron drift (Horányi, 1996). Equation 3.20 describes ion current if the sphere is positively charged, all of the subscripts are changed from $i$ to

$e$, and there is no minus sign in front of the ratio $\frac{e V_{d}}{k_{b} T_{e}}$ or the prefactor $e \frac{\sqrt{\pi}}{2} a^{2} n_{e} \frac{v_{t h e}}{M_{t h e}}$. Equation 3.19 describes electron current if all of the subscripts are changed from $i$ to $e$ and there is no minus sign

in front of the ratio $\frac{e V_{d}}{k_{b} T_{e}}$, but there is a minus sign in front of the prefactor $-e \sqrt{\pi} a^{2} n_{e} v_{t h e}$. Drifting Maxwell-Boltzmann ions are used in throughout this work to characterize ion flow in collisional planar sheaths, and to describe collection current when there is a relative drift motion between dust grains and Maxwell-Boltzmann ions. Meige et al. (2007) also suggest that drifting Maxwellian ion populations can exist even in lower pressure plasmas. Grains might be used as a diagnostic tool to discriminate between mono-energetic and drifting Maxwellian ions.

\section{iii Magnetized Grain}

The OML model cannot readily describe arbitrary electron or ion magnetization, but it is possible to express results for the $B \rightarrow \infty$ limit. The helical orbits of electrons and ions as they approach a probe imply an upper and lower limit to current collection, as shown by Rubinstein and Laframboise (1982). Arbitrary electron magnetization is in the purview of the charging model developed by Patacchini et al. (2007), which is discussed later in section 4 For the $B \rightarrow \infty$ limit, the equation 3.16 is modified by the introduction of a prefactor $\frac{1}{2}$. Infinite magnetic field means that the gyroradii of electrons is zero, so only the electrons directly above or below the grain along the field line can contribute to the current, so that the effective collecting area is $\pi a^{2}$. The exponential $\frac{e V_{d}}{k_{b} T_{e}}$ is retained because a negatively charged sphere will repel any incoming electrons along the field line unless the kinetic energy is greater than $V_{d}$. Also in this same limit, only ions above or below the grain along the field line can contribute to the current, and the ion current is described by

$$
I_{i}=e \frac{\pi}{2} n_{i} a^{2} v_{t h i}
$$

Magnetized electron or ion currents will be assumed if the gyro-radius of the species is less than the radius of the dust grain. 


\section{Specifics of Gatti-Kortshagen Model}

In the context of grain charging, the Kortshagen charging model (Gatti and Kortshagen, 2008) is valid for negatively charged grains, but it is applicable to the entire range of collisionality. The effects of electron or ion magnetization on current collection were not originally treated by this model because $R_{L s} / a>1$, so they will be neglected here. For this dissertation, the magnetized OML electron and ion currents will be assumed if $R_{L s} / a<1$. The electron current used is the same as for the case of electron collection in a retarding potential, i.e., $I_{e}=4 \pi a^{2} n_{e} e v_{t h e} \exp \left(e V_{d} / k_{b} T_{e}\right)$, where $V_{d}$ is the difference between the grain surface potential and the ambient plasma potential. The collected ion current however is split into three parts, with contributions from three separate charging models. These are represented by $I_{i}^{\mathrm{OML}}$ (collected ion current from the OML model), $I_{i}^{\mathrm{CEC}}$ (enhanced ion current due to ion-neutral collisions in the grain sheath), and $I_{i}^{\text {Hyd }}$ (ion current from the Hydrodynamic model.) Characteristic of the enhanced current due to ion-neutral chargeexchange collisions is a capture radius $R_{0}$ which was developed by Zobnin et al. (2000); Lampe et al. (2003); Khrapak et al. (2005), following the ideas of Zakrzewski and Kopiczynski (1974). If ions with kinetic energy $\frac{3}{2} k_{b} T_{i}$ pass within this length, it will likely be collected by the dust grain with probability $P_{1}$. Assuming that $\lambda_{D} \gg a$, the capture radius (Gatti and Kortshagen, 2008) is defined as:

$$
R_{0}=\frac{e|\Delta V| a\left(1+\frac{a}{\lambda_{D}}\right)}{\frac{3}{2} k_{b} T_{i}+e|\Delta V| \frac{a}{\lambda_{D}}} .
$$

In this charging model, all of the currents are added together, and each one is weighted with the probability of an ion to undergo no collisions inside the capture radius sphere $\left(P_{0}\right.$ corresponding to the OML term), exactly one collision inside the capture radius sphere $\left(P_{1}\right.$ corresponding to the collision-enhanced current term), and more than one collision inside the capture radius $\operatorname{sphere}\left(P_{>1}\right.$ corresponding to the Hydrodynamic term.) This can be written succinctly as:

$$
I_{i}=P_{0} I_{i}^{\mathrm{OML}}+P_{1} I_{i}^{\mathrm{CEC}}+P_{>1} I_{i}^{\mathrm{Hyd}}
$$

where $I_{i}^{\mathrm{OML}}$ is given equation $3.14,3.18$, or 3.19 , depending on whether the ions are stationary, 
mono-energetic, or drifting Maxwell-Boltzmann ions, respectively,

$$
I_{i}^{\mathrm{CEC}}=e 4 \pi\left(\alpha R_{0}\right)^{2} n_{i} \frac{v_{t h i}}{2 \sqrt{\pi}}
$$

and

$$
I_{i}^{\mathrm{Hyd}}=e \frac{3}{2} \pi a n_{i} \frac{v_{t h i}}{\sqrt{\pi}} \lambda_{i}\left|\frac{e V_{d}}{k_{b} T_{i}}\right| .
$$

Khrapak et al. (2005); Gatti and Kortshagen (2008) define a Knudsen capture radius number, $\mathrm{Kn}_{R}=\lambda_{i} /\left(2 \alpha R_{0}\right)$, where $\lambda_{i}$ is the mean free path for ions, and $\alpha \approx 1.22$ is the constant obtained by averaging equation 3.22 over a Maxwell-Boltzmann energy distribution:

$$
\alpha R_{0}=\int_{0}^{\infty} R_{0}(E) f(E) \mathrm{d} E=1.22 R_{0}\left(\frac{3}{2} e T_{i}\right) .
$$

This allows the probabilities to be written:

$$
\begin{aligned}
& P_{0}=\exp \left(-\frac{1}{\mathrm{Kn}_{R}}\right) \\
& P_{1}=\frac{1}{\mathrm{Kn}_{R}} \exp \left(-\frac{1}{\mathrm{Kn}_{R}}\right) \\
& P_{>1}=1-\left(P_{0}+P_{1}\right) .
\end{aligned}
$$

The Gatti-Kortshagen charging model is thus an extension of the Collision-Enhanced current charge models developed by Zakrzewski and Kopiczynski (1974); Zobnin et al. (2000); Lampe et al. (2003); Khrapak et al. (2005), because it allows a smooth transition between the three major ion regimes of collisionless, weakly collisional, and collisional. It should be noted that for the $I_{i}^{\mathrm{Hyd}}$ term, the estimate for $R_{\text {capture }}$ relies on using a Yukawa potential with Debye length $\lambda_{D}$ (Gatti and Kortshagen, 2008). This model is therefore valid only when the dust grain radius is smaller than the shielding length, or $a<\lambda_{D}$. In principle, a non-linearized Yukawa potential can be

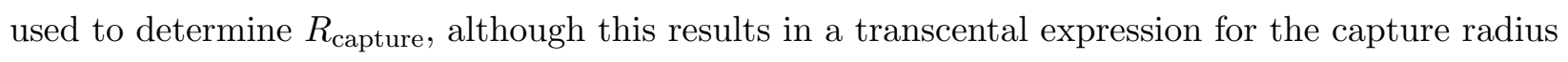


(Khrapak et al., 2005) and so an analytical expression for $R_{0}$ would be unavailable. Because the capture radius was derived using a Yukawa potential, the Kortshagen charging model has an implicit dependence on this sheath profile, in contrast to the OML model where the sheath profile was not considered. The normalized surface potential $\chi_{e}=e q_{d} /\left(C_{d} k_{b} T_{e}\right)$ for the charging model of Gatti and Kortshagen (2008) is shown in figure 3.3.

Note that equation 3.23 is valid only in the limit of small grain velocity with respect to neutral thermal velocity, or $\left|\boldsymbol{v}_{\boldsymbol{n}}-\boldsymbol{v}_{\boldsymbol{d}}\right| / v_{t h n} \ll 1$. The capture radius given by equation 3.22 assumes a Maxwell-Boltzmann neutral gas atom distribution, which needs to be recalculated for other distribution functions. Maxwell-Boltzmann neutral gas atom distributions are assumed throughout this dissertation because this is a good approximation for laboratory experiments, but the procedure to obtain the capture radius is instructive. An ion resulting from charge-exchange with a thermal neutron is captured when its kineitc energy is equal to its potential energy in the grain sheath, expressed by

$$
e V_{s h}\left(r=R_{0}\right)=E \text {. }
$$

When the grain sheath is not the given by the Debye-Hückel solution, such as in a non-monotonic sheath which may arise from photo-emission (Poppe and Horányi, 2010), equation 3.22 is no longer valid. Equation 3.30 needs to be inverted in order to determine the capture radius as a function of energy, which may not lead to a closed form solution when the grain sheath potential profile deviates from the Debye-Hückel solution. Throughout this dissertation, the deviation from the Debye-Hückel solution is considered small because the photo-electric current is considered small. To determine the capture radius, integrate over the neutral gas distribution function to obtain

$$
R_{0} \equiv\left\langle R_{0}\right\rangle=\int_{0}^{\infty} R_{0}(E) f_{n}(E) \mathrm{d} E,
$$

and the Knudsen capture radius number becomes $\mathrm{Kn}_{R}=\lambda_{i} /\left(2 R_{0}\right)$. As an additional caveat, equation 3.23 is only valid for the case of unmagnetized ions, i.e., the ion gyro-radius is larger than the size of the dust grain. 


\section{Specifics of Patacchini and Hutchinson Model}

An analytical model for electron collection to a spherical probe was developed by Patacchini et al. (2007) for arbitrary $a / R_{L e}$ ratios. The model described by Patacchini et al. (2007) does not consider arbitrary $a / R_{L i}$ ratios, and the OML ion current is assumed. The model is briefly described by Patacchini et al. (2007), while Patacchini (2007) provides a description of this theory in even greater depth. Below are quoted the major results with some of the necessary background. Patacchini et al. (2007) fitted empirical polynomial fits to output from the SCEPTIC code (Hutchinson, 2002).

In section $\mathrm{i}$, the unmagnetized current for $B=0$ is described by equation 3.16 as

$$
I_{e}=-e 4 \pi a^{2} n_{e} \frac{v_{t h e}}{2 \sqrt{\pi}} \exp \left(\frac{e V_{d}}{k_{b} T_{e}}\right)
$$

while in section iii the magnetized current for $B \rightarrow \infty$ is half of this quantity, or

$$
I_{e}=-e 2 \pi a^{2} n_{e} \frac{v_{t h e}}{2 \sqrt{\pi}} \exp \left(\frac{e V_{d}}{k_{b} T_{e}}\right)
$$

The upper and lower bounds for electron curent collection are given by Rubinstein and Laframboise (1982) if the magnetic field is not considered zero or infinite. If the grain is at the local space potential, or $V_{d}=0$, Patacchini et al. (2007) provide an empirical estimate for $\iota^{*}$ to within $0.3 \%$ for the electron current collection, which is given by

$$
\iota^{*}=-e 4 \pi a^{2} n_{e} \frac{v_{\text {the }}}{2 \sqrt{\pi}}\left(1.000-0.0946 z-0.305 z^{2}+0.950 z^{3}-2.200 z^{4}+1.150 z^{5}\right)
$$

where $z=\frac{a}{R_{L e}} /\left(1+\frac{a}{R_{L e}}\right)$. Sonmor and Laframboise (1991) calculated the exact numerical solution for the collected electron current for large shielding radius compared to the grain radius or $\lambda_{D} \gg a$, corroborated by Patacchini et al. (2007). Through dimensional analysis, Patacchini et al. (2007) determined that the only relevant parameter for the electron current when $\lambda_{D} \gg a$ is given by the dimensionless number

$$
\eta=-\frac{e V_{d}}{k_{b} T_{e}} \frac{R_{L e}}{a} .
$$


Once again, a fit can be made to results of the SCEPTIC simulations over this parameter to yield

$$
I_{e}=-e 4 \pi a^{2} n_{e} \frac{v_{t h e}}{2 \sqrt{\pi}}\left[A^{*}(w)+\left(1-A^{*}(w)\right) \iota^{*}(z)\right] \exp \left(\frac{e V_{d}}{k_{b} T_{e}}\right)
$$

where

$$
A^{*}(w)=0.678 w+1.543 w^{2}-1.212 w^{3}
$$

and $w=\eta /(1+\eta)$.

Patacchini et al. (2007) extend the result in equation 3.36 even further, to the case of arbitrary shielding length. The requirement that $\lambda_{D} \gg a$ is lifted by adjusting the parameter $\eta$ in equation 3.35 , so that it is described by

$$
\eta=-\frac{e V_{d}}{k_{b} T_{e}} \frac{R_{L e}}{a}\left[1+\frac{1}{4} \frac{R_{L e}}{a}-\exp \left(-4 \frac{a}{R_{L e}} \frac{a}{\lambda_{D}}\right)\right]
$$

In this dissertation, all references to the Patacchini-Hutchinson charging model will refer to OML ions and the electron current given by equation 3.36 , where $A^{*}(w)$ is given by 3.37 , using the definition of $\eta$ given by equation 3.38 .

\section{Photoemission and Grain Work Function}

The photo-emission of electrons has been reviewed extensively by Horányi (1996); Shukla (2001), but a brief summary is presented here. UV radiation performs the role of abrupt inhomogeneity which is analyzed in chapters V and VI. Because the grains are in a quasi-neutral plasma, a nonneutral photo-electric sheath (Sickafoose et al., 2000; Poppe and Horányi, 2010) will not be assumed. Photo-electrons charge the grain less negatively, but regimes where $V_{d}>0$ are not considered and the grain charge will always be negative. For the case of single-sided UV illumination when $V_{d}>0$,

$I_{U V}=e \pi a^{2} f_{U V} \exp \left(\frac{e V_{d}}{k_{b} T_{p e}}\right)$, where $f_{U V}$ is the flux of photo-electrons from the surface and $T_{p e}$ is the temperature of the Maxwell-Boltzmann distribution of photo-electrons.

If $V_{d}<0$, the current due to single-sided UV illumination is given by

$$
I_{U V}=e \pi a^{2} f_{U V}
$$


which is a positive current because electrons leave the surface of the grain, charging it less negatively. If the UV illumination is double sided, then a prefactor of 2 must be included in equation 3.39, and if the UV illumination is isotropic, a prefactor of 4 is required. The flux $f_{U V}$ is dependent on properties of the source spectrum and the material properties of the emitting surface. More precisely, this flux is determined by

$$
f_{U V}=\int_{0}^{\lambda_{1}} F(\lambda) Y(\lambda) \mathrm{d} \lambda
$$

where $F(\lambda)$ is the flux of UV photons as a function of wavelength $\lambda$ for a UV source, $Y(\lambda)$ is the quantum efficiency of photoemission for the grain material, and $\lambda_{1}$ is the wavelength cutoff, representing the longest wavelength capable of producing a photo-electron for a given work function (Colwell et al., 2005).

As an example, lunar regolith in the presence of solar UV radiation produces a photo-electron flux of

$$
f_{U V}=2.5 \times 10^{14} \frac{\kappa}{d^{2}}
$$

where $d$ is the distance of an object from the sun in astronomical units, $\kappa$ is the efficiency, close to 0.1 for dielectrics, nearly unity for conductors, and $f_{U V}$ is given in units of $\mathrm{m}^{-2} \mathrm{~s}^{-1}$ (Colwell et al., 2005; Whipple, 1981; Horányi, 1996).

This flux can take on an infinitude of values for different properties of UV source and material composition (work function), so the approach for this dissertation is to consider the UV current as some fraction of the thermal electron current, or

$$
I_{U V}=\left(e 4 \pi a^{2} n_{e} \frac{v_{\text {the }}}{2 \sqrt{\pi}}\right)\left(\frac{\sqrt{\pi} f_{U V}}{2 n_{e} v_{\text {the }}}\right)=\left(e 4 \pi a^{2} n_{e} \frac{v_{\text {the }}}{2 \sqrt{\pi}}\right) \frac{\sqrt{\pi}}{2} v
$$

where $v=I_{U V} /\left(n_{e} v_{\text {the }}\right)$ is the dimensionless quantity of photo-electron current normalized by the thermal electron flux. For stationary plasma, the grain attains a negative $i n$-situ equilibrium grain charge when $v<2 / \sqrt{\pi}$, and a positive charge when $v>2 / \sqrt{\pi}$. The work in this dissertation is restricted to the $v<2 / \sqrt{\pi}$ regime, and furthermore no considerations for UV photons with energies greater than $12 \mathrm{eV}$ are made, to ensure that the UV illumination ionization of neutral gas atoms 
can be neglected (Rosenberg and Mendis, 1995).

\section{Forces acting on a Solid-State particle in Plasma}

A solid state particle, or dust grain, is subject to numerous forces when it is in a plasma. In a homogeneous plasma, dust grains acquire a net negative charge due to the higher mobility of electrons, as discussed earlier in section 1 Because grains have much larger mass than ions or electrons, the gravitational force can not be ignored. In the case of larger grains it can be the dominant force. However, the small size of dust grains means that grains are typically light enough that electromagnetic forces are also important, and so these forces must be included when describing the dynamics of grains in plasma. Additionally, due to the much larger collision cross-sections of grains, drag forces are important for the dust dynamics, especially in gas discharges. There are also other forces, such as the thermophoretic force and others which are treated as distinct forces in their own right which are absent for particles such as electrons and ions. In this section, we will review the important forces acting on a dust grain. Not all of these forces will be in the scope of this dissertation, but they are included for completeness. I will assess the applicability of each force to the work provided in this dissertation. Many of these forces can be neglected under certain conditions.

\section{Gravity}

For dust grains that are of micron or submicron size, the gravitational force is an important force that must be included in order to obtain grain trajectories (Nunomura et al., 1997). Because grains have larger mass than ions or electrons, dust grains are subject to the $\boldsymbol{g} \times \boldsymbol{B}$ drift, which is described later in appendix A, section 4. It is for this reason that magnetized dusty plasma experiments (Konopka et al., 2005a; Schwabe et al., 2011; Thomas et al., 2012) are typically designed to allow for rotation of the vacuum vessel relative to the direction of the gravitational field in order to produce or eliminate the $\boldsymbol{g} \times \boldsymbol{B}$ drift. The levitation height of dust grains in a planar sheath is in part determined by the gravitational force. The gravitational force on a dust grain is given by

$$
\boldsymbol{F}_{\boldsymbol{g}}=m_{d} \boldsymbol{g}(\boldsymbol{r}),
$$


where the $\boldsymbol{g}(\boldsymbol{r})$ is the local gravitational acceleration. For laboratory experiements on the surface of earth, $g \approx 9.8 \mathrm{~m} \mathrm{~s}^{-2}$. In general, the local gravitational acceleration will be given by

$$
\boldsymbol{g}(\boldsymbol{r})=\frac{G M}{\left|\boldsymbol{r}_{\boldsymbol{d}}-\boldsymbol{r}_{\boldsymbol{b}}\right|^{3}}\left(\boldsymbol{r}_{\boldsymbol{g}}-\boldsymbol{r}_{\boldsymbol{b}}\right)
$$

where $M$ is the mass of a celestial body, such as a star, planet, moon, comet, or asteroid that is sufficiently large enough to produce a gravitational field, $\boldsymbol{r}_{\boldsymbol{b}}$ is the position of this celestial body in a chosen coordinate system, and $\boldsymbol{r}_{\boldsymbol{d}}$ is the position of the dust grain in this coordinate system.

When dealing with dust grains in orbit around a celestial body, it is often easier to choose a coordinate system that is co-located with the celestial body's center of mass (Northrop and Hill, 1983). In this case, $\boldsymbol{r}_{\boldsymbol{b}}=\mathbf{0}$, and we are free to choose $\boldsymbol{r}=\boldsymbol{r}_{\boldsymbol{d}}$, and equation 3.44 reduces to $\boldsymbol{g}(\boldsymbol{r})=\frac{G M_{b}}{r^{2}} \hat{r}$.

\section{Electric Force}

The force on a charged grain in a plasma due to the electric force is computed in the same way as for electrons in the plasma (Bliokh et al., 1994),

$$
\boldsymbol{F}_{\boldsymbol{E}}=q_{d} \boldsymbol{E}\left(\boldsymbol{r}_{\boldsymbol{d}}, t\right)
$$

where $q_{d}$ is the charge on the dust grain, and $\boldsymbol{E}\left(\boldsymbol{r}_{\boldsymbol{d}}, t\right)$ is the electric field measured at the grain position $\boldsymbol{r}_{\boldsymbol{d}}$. Quasi-neutral plasmas tend to shield electric fields from penetrating the volume of the plasma, generally preventing the charge separation of electrons and ions. For many dusty plasma experiments (Nunomura et al., 1999), the ion-rich planar sheath provides a vertical electric field that levitates grains against gravity. In the co-axial plasma described by Zimmermann et al. (2010), sheaths develop on the inner and outer concentric cylinders, and there is a radial electric field in the quasi-neutral pre-sheath or plasma bulk. 


\section{Magnetic Force}

The force on a charged dust grain in a static, uniform magnetic field is the same as for ions and electrons, and is given by (Bliokh et al., 1994; Gurnett and Bhattacharjee, 2005)

$$
\boldsymbol{F}_{\boldsymbol{B}}=q_{d}\left[\boldsymbol{v}_{\boldsymbol{d}} \times \boldsymbol{B}(\boldsymbol{r}, t)\right]
$$

Evident from equation 3.46 is that the magnetic force is zero if the grain has no velocity. In the absence of other forces and for constant grain charge $q_{d}$, the dust grain gyrates in a circle in the plane perpendicular to the magnetic field direction. The radius of this circle is (Gurnett and Bhattacharjee, 2005)

$$
R_{L d}=\frac{m_{d} v_{\perp}}{\left|q_{d} B\right|}
$$

where $v_{\perp}$ is the constant component of the dust grain velocity perpendicular to the magnetic field direction. This is the same description as for more elementary plasma constituents, such as ions and electrons.

The definition given by equation 3.47 is an important scale length for electrons, ions, and dust grains. This parameter was discussed for electrons and ions in chapter II, section 5, and in the same way we use the term magnetized-orbit dust to describe dust grains that have $L>R_{L d}$, where $R_{L d}$ is the gyro-radius of dust grains and $L$ is the size of the plasma or region of space in question. Because dust grains are more massive than electrons or ions, it is necessary to employ larger fields to achieve the magnetized-orbit dust condition compared to the magnetized plasma condition. The larger collision cross-section of dust grains also means that the frequency of momentum-changing binary collisions with neutral gas atoms is much higher, which also poses a problem for maintaining the magnetized-orbit dust condition; the details of this are explored in greater depth in section E.

\section{Neutral Drag Force}

The neutral drag force is of particular importance for laboratory dust experiments and in space regimes where there are significant populations of neutrals present (Northrop and Birmingham, 1996). There are three important regimes for characterizing the neutral drag force on grains: the 
hydrodynamic regime, the kinetic regime with relative dust-neutral velocity much less than the neutral thermal speed, also known as Epstein drag (Epstein, 1924), and the kinetic regime with the relative dust-neutral velocity much greater than the neutral thermal speed. All three regimes can be explored by the test particle code supplied by this dissertation, but the Epstein drag regime is the focus for this dissertation. I will briefly review the three regimes.

For small Knudsen numbers $\mathrm{Kn}_{a}=\frac{\lambda_{i}}{a}$ the dust grain is in the collisional or hydrodynamic regime. For small Reynolds numbers $a v / \nu$, where $v$ is the speed of the grain relative to the fluid and $\nu$ is the kinematic viscosity of the fluid, viscous forces are much greater than inertial forces. When both the Knudsen and Reynolds numbers are small, the neutral drag force is given by Stokes' law

$$
\boldsymbol{F}_{\boldsymbol{n}}=-6 \pi \eta a\left(\boldsymbol{v}_{\boldsymbol{d}}-\boldsymbol{v}_{\boldsymbol{n}}\right)
$$

where $\eta$ is the dynamic viscosity of the gas, and the quantity $\left(\boldsymbol{v}_{\boldsymbol{d}}-\boldsymbol{v}_{\boldsymbol{n}}\right)$ is the relative velocity between the dust grain $\boldsymbol{v}_{\boldsymbol{d}}$ and the neutral fluid speed $\boldsymbol{v}_{\boldsymbol{n}}$ (Paeva, 2005). For large Reynolds numbers, equation 3.48 is no longer valid. Because the Knudsen numbers considered in this dissertation exceed unity, the hydrodynamic drag will not be considered. The neutral drag force on dust grains will be evaluated using a kinetic treatment, which determines the momentum transferred from a statistical ensemble of neutral gas atoms. Because of this kinetic treatment, the Reynolds number is irrelevant for grains in this dissertation because the viscosity is not defined.

For large Knudsen numbers, the dust grain is in the collisionless or kinetic regime. The kinetic neutral drag force must further be divided into categories of large relative velocity of dust grains to neutrals compared with the neutral thermal speed, or small relative velocity of dust grains to neutrals compared with the neutral thermal speed. For the former case, $\frac{\left|\boldsymbol{v}_{d}-\boldsymbol{v}_{n}\right|}{v_{t h n}} \gg 1$, and the neutral drag force is given by

$$
\boldsymbol{F}_{\boldsymbol{n}}=-\pi a^{2} m_{n} n_{n} v_{t h n}\left(\boldsymbol{v}_{\boldsymbol{d}}-\boldsymbol{v}_{\boldsymbol{n}}\right)\left|\boldsymbol{v}_{\boldsymbol{d}}-\boldsymbol{v}_{\boldsymbol{n}}\right|
$$

where $m_{n}$ is the mass of neutrals, $n_{n}$ is the neutral atom density, and $v_{t h n}=\sqrt{\frac{2 k_{b} T_{n}}{m_{n}}}($ Paeva, 2005). In this case, the drag force is proportional to the square of relative velocity of dust grains to 
neutrals. Grains do not generally satisfy $\frac{\left|\boldsymbol{v}_{d}-\boldsymbol{v}_{n}\right|}{v_{t h n}} \gg 1$ under laboratory conditions, but it is possible for this situation in space (Northrop et al., 1989; Northrop, 1992; Northrop and Birmingham, 1996). Because the drag force is quadratic in the relative velocity of dust grains to neutrals, it is not possible to incorporate it into a Boris computational scheme. However, it can be treated using the iterative leapfrog method provided in this dissertation. Additionally, quadratic drag does permit analytic solutions for grain trajectories in some cases.

For the last case, or small relative velocity of dust grains to neutrals compared with the neutral thermal speed, the Epstein drag force (Epstein, 1924) can be used. Epstein drag is given by

$$
\boldsymbol{F}_{\boldsymbol{n}}=-\delta \frac{4}{3} \pi a^{2} m_{n} n_{n} C_{n}\left(\boldsymbol{v}_{\boldsymbol{d}}-\boldsymbol{v}_{\boldsymbol{n}}\right)
$$

where $\delta \approx 1$ is a coefficient that accounts for the collision between gas atoms and the dust grain. For melamine grains, $\delta=1.6 \pm .13$ (Liu et al., 2003). It should be noted that the average thermal

speed of neutrals, $C_{n}=\sqrt{\frac{8 k_{b} T_{n}}{\pi m_{n}}}$, is used, rather than the thermal speed $v_{t h n}$. In this case, the drag force is linear in the relative velocity of dust grains to neutrals, so it is straightforward to incorporate into the Boris computational algorithm. The Epstein drag readily permits analytic solutions for grain trajectories.

\section{$5 \quad$ Ion Drag Force}

Just as the neutral drag force is the force on a dust grain due to collisions with neutral gas atoms, the ion drag force is the force on a dust grain due to collisions with ions. Because electrons are much less massive than ions, dust-electron collisions do not appreciably alter the dust grain's momentum. There are two components to the ion drag, ion collection force and ion orbit force. When ions strike the dust surface, they are collected by the grain and the ions impart their momentum to the grain, which corresponds to the ion collection force. If ions pass close enough for the central field of the dust grain to deflect the ion but it is not collected, a force is exerted on the grain which corresponds to the ion orbit force (Merlino, 2006). Like the neutral drag, in the kinetic limit there are expressions for the ion drag force for large relative dust ion velocity $\left|\boldsymbol{v}_{\boldsymbol{d}}-\boldsymbol{v}_{\boldsymbol{i}}\right| / v_{t h i} \gg 1$, and small relative dust ion velocity $\left|\boldsymbol{v}_{\boldsymbol{d}}-\boldsymbol{v}_{\boldsymbol{i}}\right| / v_{t h i} \ll 1$.

In the high velocity limit $\left|\boldsymbol{v}_{\boldsymbol{d}}-\boldsymbol{v}_{\boldsymbol{i}}\right| / v_{t h i} \gg 1$, the ion collection force is given by (Barnes et al., 
1992; Merlino, 2006)

$$
\boldsymbol{F}_{\boldsymbol{i c}}=-n_{i} m_{i}\left(\boldsymbol{v}_{\boldsymbol{d}}-\boldsymbol{v}_{\boldsymbol{i}}\right) v_{s} \pi b_{c}^{2},
$$

where $\boldsymbol{v}_{\boldsymbol{i}}$ is the directed or fluid ion flow, $v_{s}=\sqrt{\frac{8 k_{b} T_{i}}{\pi m_{i}}+\left(\boldsymbol{v}_{\boldsymbol{d}}-\boldsymbol{v}_{\boldsymbol{i}}\right)^{2}}$, and $b_{c}=a \sqrt{1-\frac{2 e V_{d}}{m_{i} v_{s}^{2}}}$ is the impact parameter for ion collection. Recall that a similar impact parameter was encountered earlier in section 2 This impact parameter is determined by charge model, so the ion collection drag force is charge model dependent. The ion orbit force is given by (Barnes et al., 1992)

$$
\boldsymbol{F}_{\boldsymbol{i o}}=-n_{i} m_{i}\left(\boldsymbol{v}_{\boldsymbol{d}}-\boldsymbol{v}_{\boldsymbol{i}}\right) v_{s}(4 \pi) b_{90}^{2} \Gamma
$$

where $b_{90}=\frac{e q_{d}}{4 \pi \epsilon_{0} m_{i} v_{s}^{2}}$ is the impact parameter for $90^{\circ}$ collisions, and $\Gamma=\frac{1}{2} \frac{\lambda_{D}^{2}+b_{90}^{2}}{b_{c}^{2}+b_{90}^{2}}$ is the Coloumb logarithm integrated from $b_{90}$ to $\lambda_{D}$. These integration limits are used because $b_{90}$ is the closest distance ions can approach without being collected, and the central field of the dust grain does not affect ions at distances greater than $\lambda_{D}$. This Coloumb logarithm is essentially the same as the one discussed in section 2, except that $b_{c}$ and $b_{90}$ are different for dust when compared to electrons and ions. The impact parameter $b_{90}$ is not charge model dependent, unlike $b_{c}$. The total ion drag force is the sum of the two components in equations 3.51 and 3.52 , or $\boldsymbol{F}_{\boldsymbol{i}}=\boldsymbol{F}_{\boldsymbol{i c}}+\boldsymbol{F}_{\boldsymbol{i o}}$.

Note that in the high velocity limit, both the collection and orbit forces vary non-trivially on the dust velocity relative to ion flow. It is only possible to achieve analytical results for magnetizedorbit grain trajectories if the grain charge does not change with time. The high velocity limit of the ion drag force cannot be included into a Boris scheme, but the iterative leapfrog solver provided in this dissertation can handle these complications.

Barnes et al. (1992); Fortov et al. (2005) provide a description for the ion drag in the low velocity limit $\left|\boldsymbol{v}_{\boldsymbol{d}}-\boldsymbol{v}_{\boldsymbol{i}}\right| / v_{t h i} \ll 1$. The ion collection force in this limit is given by (Bacharis et al., 2010)

$$
\boldsymbol{F}_{\boldsymbol{i c}}=\pi a^{2} m_{i} n_{i} \sqrt{\frac{8 k_{b} T_{i}}{\pi m_{i}}}\left(\boldsymbol{v}_{\boldsymbol{d}}-\boldsymbol{v}_{\boldsymbol{i}}\right)\left(1-\frac{e V_{d}}{k_{b} T_{i}}\right)
$$


and the ion orbit force is given by (Fortov et al., 2005; Bacharis et al., 2010)

$$
\boldsymbol{F}_{\boldsymbol{i o}}=\frac{\sqrt{32 \pi}}{3} \frac{\left(\boldsymbol{v}_{\boldsymbol{d}}-\boldsymbol{v}_{\boldsymbol{i}}\right)}{v_{t h n}} \epsilon_{0} T_{i}^{2} \Lambda \beta_{T}^{2}
$$

where the Coloumb logarithm is approximated by $\Lambda \approx \exp \left(\beta_{T} / 2\right) \operatorname{Ei}\left(-\beta_{T} / 2\right)$, Ei is the exponential

integral, $\beta_{T}=a\left|e V_{d}\right| /\left(\lambda_{D} k_{b} T_{i}\right)$ is the thermal scattering parameter. As discussed by Bacharis et al. (2010) and demonstrated by Hutchinson (2006) through simulations, the low velocity version of the ion orbit drag force described by Fortov et al. (2005) is valid for thermal Mach numbers up to $\left|\boldsymbol{v}_{\boldsymbol{d}}-\boldsymbol{v}_{\boldsymbol{i}}\right| / v_{t h i}=1.5$.

Unlike in the high velocity limit, the low velocity limit drag force is linear rather than quadratic in $\left(\boldsymbol{v}_{\boldsymbol{d}}-\boldsymbol{v}_{\boldsymbol{i}}\right)$, and it can be included into a Boris scheme. Analytic solutions to magnetized-orbit grain trajectories are permitted if grain charge is constant, but it may be possible to find approximate analytical solutions if the grain charge changes slowly enough during a gyro-orbit, and the dependence on grain charge is weak.

For dust experiments using planar sheaths, ion flow into the sheath provides a force on the grain that points from the bulk plasma to the biased planar surface (Nunomura et al., 1999). The ion drag force affects the levitation height, though it can often be neglected in the vertical (sheath) direction when compared to gravity and the electric force in laboratory experiments (Nunomura et al., 1999). If a vertical magnetic field is present along with a radial electric field, an azimuthal flow of ions is present, which alters dust dynamics because the ion drag is now in the plane rather than a simple change in the levitation height. For fusion relevant plasmas, the ion drag can be the dominant force present (Smirnov et al., 2007).

\section{Thermophoretic Force}

The thermophoretic force arises from a neutral gas temperature gradient. This force is a result of the higher random thermal flux of neutral gas atoms from the hotter side of a temperature gradient when compared to the flux of neutral gas atoms on the colder side of the temperature gradient. The thermophoretic force is given by (Rohatschek, 1985, 1995; Rosenberg et al., 1999; Merlino, 2006)

$$
\boldsymbol{F}_{\boldsymbol{t h}}=\frac{16 \sqrt{\pi}}{15} \frac{a^{2} \kappa_{T}}{v_{t h n}} \nabla T_{n}
$$


where $\kappa_{T}$ is the translational component of the thermal conductivity. As discussed by Merlino (2006), the thermophoretic force can be induced by heating one of the electrodes in a planar discharge. In this dissertation, there will be no gradients in the neutral gas background, so this force will not be considered.

\section{Photo-electron Recoil and Radiation Pressure Forces}

Electrons can leave the surface of the grain if UV photons incident on a grain surface have sufficient energy. As an example, if photons with frequency $\nu_{U V}$ and energy $h \nu_{U V}$ illuminate a grain with

work function $W$, then electrons leave the surface with energy $W-h \nu_{U V}$. When an electron leaves the surface, conservation of momentum implies that the grain recoils in the opposite direction from where photo-electron leaves the surface. An upper limit to this photo-electron recoil force for single-sided illumination is given by (Rosenberg and Mendis, 1995)

$$
F_{r c} \approx \frac{1}{2} \kappa J_{U V} m_{e} v_{p e} \pi a^{2}
$$

where $\kappa$ is the efficiency of photo-electrons, close to 0.1 for dielectrics, nearly unity for conductors as mentioned earlier in section $5, J_{U V}$ is the flux of UV photons, and $v_{p e}$ is an ensemble averaged velocity of photo-electrons. Alternatively, the product $\kappa J_{U V} v_{p e}$ can be replaced by integrating

$$
\kappa J_{U V} v_{p e}=\int_{0}^{\lambda_{1}} F(\lambda) \chi(\lambda) \sqrt{\frac{2}{m_{e}}\left(\frac{h c}{\lambda}-W\right)} \mathrm{d} \lambda
$$

to obtain the ensemble average of all quantities. When UV illumination is along the magnetic field or planar sheath direction, the photo-electron recoil force only changes the levitaiton height. When UV illumination is perpendicular to the magnetic field direction, an $\boldsymbol{F}_{\boldsymbol{r} \boldsymbol{c}} \times \boldsymbol{B}$ drift results, where $\boldsymbol{F}_{\boldsymbol{r} \boldsymbol{c}}$ is the photo-electron recoil force. There is also a radiation pressure force, but because UV illumination is assumed to be along the magnetic field direction and the radiation pressure force is two orders of magnitude smaller than the photo-electron recoil force (Rosenberg and Mendis, 1995), it is not considered. 


\section{E Gyration and Charge Model Parameter Space}

In order to characterize gyro-phase drift in the context of the charge models in section C, specifically the subsections 2, 3, and 4, some perspective is necessary. These charge models all lie along a spectrum of collisionallity and ion or electron magnetization number. While collisionality is interesting from a charge model perspective, for gyro-phase drift it brings with it the inevitable complication of dust grain collisions with neutral atoms. For laboratory experiments with $\mid \boldsymbol{v}_{\boldsymbol{n}}-$ $\boldsymbol{v}_{\boldsymbol{d}} \mid / v_{t h n} \ll 1$, the Epstein drag force (Epstein, 1924) can destroy gyro-motion if the collision frequency of a gyrating dust grain is greater than the dust gyro-frequency (Thomas et al., 2012).

Because the collisionality of the plasma with respect to the dust grain is best parameterized by the Knudsen capture radius number $\mathrm{Kn}_{R}$, it is useful to determine the capture radius number dependence on the ratio of dust grain gyro-frequency to dust-neutral collision frequency. Starting with equation 3.22 , it is possible to reduce factors within this expression to some fundamental scaling parameters. The Knudsen capture radius number is given by (Gatti and Kortshagen, 2008)

$$
\mathrm{Kn}_{R}=\frac{\mathrm{Kn}_{a}}{(2)(1.22)}\left(\frac{\frac{3}{2} \frac{C_{d} k_{b} T_{i}}{e\left|q_{d}\right|} \frac{\lambda_{D}}{a}+1}{\frac{\lambda_{D}}{a}+1}\right)=\frac{\operatorname{Kn}_{a}}{(2)(1.22)}\left(\frac{\frac{3}{2}\left|\chi_{e}\right|^{-1} \frac{T_{i}}{T_{e}} \frac{\lambda_{D}}{a}+1}{\frac{\lambda_{D}}{a}+1}\right)
$$

where $T_{i}=\frac{1}{2} m_{i} v_{t h i}^{2} / k_{b}$ is the ion temperature and $q_{d}<0$ is the charge on the dust grain. If the dust-neutral collision frequency described in section 4 is given by (Epstein, 1924; Thomas et al., 2012)

$$
\nu_{d n}=\delta \frac{4 \pi}{3} a^{2} n_{n} \frac{m_{n}}{m_{d}} C_{n},
$$

where $C_{n}=\sqrt{8 k_{b} T_{n} /\left(\pi m_{n}\right)}$ is the mean speed of neutrals if $T_{n}$ is the temperature of neutrals, $m_{n} \approx m_{i}$ is the mass of neutrals, and the dust gyro-frequency is given by $\left|q_{d}\right| B / m_{d}$, then

$$
\frac{\omega_{c d}}{\nu_{d n}}=\frac{3\left|q_{d}\right| B}{4 \pi a^{2} n_{n} C_{n} m_{n}}
$$

where $n_{n}$ is the neutral atom density in the plasma. Rearranging equation 3.60 in terms of $q_{d}$ allows substitution of equation 3.60 into equation 3.59 and if the relationships $T_{e} \gg T_{i}$ and $n_{e} \approx n_{i}=n_{0}$ 
hold, this yields

$\mathrm{Kn}_{R}=\frac{9 \pi}{(1.22)(8)} \frac{\left(1+\frac{n_{e} T_{i}}{n_{i} T_{e}}\right)\left(1+\frac{a}{\lambda_{D}}\right) \frac{a}{R_{L i}} \frac{n_{i}}{n_{n}} \sqrt{\frac{m_{e}}{m_{n}}} \sqrt{\frac{T_{e}}{T_{n}}} \mathrm{Kn}_{a}\left(\frac{\lambda_{D}}{a}\right)^{3}}{\left(\frac{\lambda_{D}}{a}+1\right)}\left(\frac{\omega_{c d}}{\nu_{d n}}\right)^{-1}+\frac{\mathrm{Kn}_{a}}{(2)(1.22)\left(\frac{\lambda_{D}}{a}+1\right)}$,

where $\lambda_{i}$ is the mean free path for ion-neutral charge exchange collisions, $\frac{n_{i}}{n_{n}}$ is the ionization fraction of the plasma, and $\beta_{i}=\frac{a}{R_{L i}}$ is the ion magnetization number. The ion magnetization number is related to the electron magnetization number, $\beta_{e}=\frac{a}{R_{L e}}$. This relation is given by $\beta_{i}=\beta_{e} \sqrt{\frac{m_{e} T_{e}}{m_{i} T_{i}}}$. Note that equation 3.61 is truly only applicable for stationary grains in homogeneous plasmas, but it serves as an approximation for the grain when considering its gyro-averaged plasma parameters during one gyro-orbit. The parameter space diagram is shown in figure 3.1. Of course, a grain can transition through different parameter space regimes during a gyro-orbit, which is an issue we will explore later in this dissertation.

At first sight, it is unclear what has been gained by writing $\mathrm{Kn}_{R}$ in this new and different way, especially since so many free parameters appear in equation 3.61. However, many of these dimensionless fractions are constrained. The dimensionless number $\frac{\lambda_{D}}{a}$ is allowed to vary, but it can only take on values much greater than unity, meaning $\frac{\lambda_{D}}{a}+1 \approx \frac{\lambda_{D}}{a}$. For most dusty plasma laboratory experiments, $\frac{T_{i}}{T_{n}} \approx 1$, and since we take the square root of this quantity this ratio is nearly unity. It is possible to approximate equation 3.61 by

$$
\mathrm{Kn}_{R} \approx \frac{9 \pi}{(1.22)(8)}\left(\frac{a}{R_{L i}}\right)\left(\frac{n_{i}}{n_{n}}\right) \mathrm{Kn}_{a}^{3}\left(1+\frac{n_{e} T_{i}}{n_{i} T_{e}}\right)^{-1}\left(\frac{\lambda_{i}}{\lambda_{D}}\right)^{-2}\left(\frac{\omega_{c d}}{\nu_{d n}}\right)^{-1}+\frac{1}{(2)(1.22)}\left(\frac{\lambda_{i}}{\lambda_{D}}\right)
$$

For quasineutral plasma, $n_{e} \approx n_{i}$.

We are left to discuss the free parameters $\frac{n_{i}}{n_{n}}, \frac{a}{R_{L i}}, \mathrm{Kn}_{a}$, and $\frac{\lambda_{i}}{\lambda_{D}}$. In general, all of these parameters can be controlled in some part in experiments, although it is clear that some of these parameters are inter-related. For example, $\frac{n_{i}}{n_{n}}$ and $\lambda_{i}$ are related through the neutral gas pressure. For weakly ionized plasmas, especially glow discharge plasmas, the ionization fraction $\frac{n_{i}}{n_{n}}$ can be 0.01 or lower while in Q-machines the ionization fraction is near unity. The ionization fraction represents how much quasi-neutral plasma exists compared to the background gas. For many glow- 
discharge and RF plasma laboratory experiments, the ionization fraction can be changed by the appropriate adjustment of input power. For the purposes of these parameter space diagrams, treat the ionization fraction as a free parameter. The parameter $\frac{a}{R_{L i}}\left(\right.$ or $\left.\frac{a}{R_{L i}}=\sqrt{\frac{m_{e} T_{e}}{m_{i} T_{i}}} \frac{a}{R_{L e}}\right)$ represents the magnetization of ions (or electrons), which is an important parameter in all charging models. Arbitrary electron magnetization $\frac{a}{R_{L e}}$ is only fully treated by the charging model of Patacchini et al. (2007). The Knudsen number $\mathrm{Kn}_{a}$ will still be used as a parameter to characterize the collisionality of the plasma. The final free parameter, $\frac{\lambda_{i}}{\lambda_{D}}=\mathrm{Kn}_{a} / \frac{\lambda_{D}}{a}$, describes the ratio between the ion-neutral collision scale length and the Debye length. If this ratio is near unity, and for $a \ll \lambda_{D}$, there is, on average, approximately one ion-neutral charge exchange collision in the dust sheath. This actually corresponds to having slightly more than ion-neutral charge exchange collision, because the size of the entire dust sheath $s$ might be $s \approx 5 \lambda_{D}$. The collision enhanced ion current is at a near maximum when this is the case, and the left hand side of equation 3.62 trends toward $K n_{R}=10^{0}$ for large values of $\left(\frac{\omega_{c d}}{\nu_{d n}}\right)^{-1}$, which is shown in figure 3.1. This parameter is notable in that it also strongly affects the behavior of equation 3.62 for smaller values of $\left(\frac{\omega_{c d}}{\nu_{d n}}\right)^{-1}$. By increasing $\frac{\lambda_{i}}{\lambda_{D}}$ in an effort to obtain higher $\operatorname{Kn}_{R}$ values, the slope of the resulting $\operatorname{Kn}_{R}\left(\left(\frac{\omega_{c d}}{\nu_{d n}}\right)^{-1}\right)$ curves is flattened at the smaller values of $\left(\frac{\omega_{c d}}{\nu_{d n}}\right)^{-1}$. This is demonstrated in figure 3.1.

The product of $\frac{n_{i}}{n_{n}}, \frac{a}{R_{L i}}$, and $\mathrm{Kn}_{a}^{2}$ can be used to adjust the behavior of the $\operatorname{Kn}_{R}\left(\left(\frac{\omega_{c d}}{\nu_{d n}}\right)^{-1}\right)$ curves without affecting the behavior as the gyration parameter gets very large, or in other words, $\left(\frac{\omega_{c d}}{\nu_{d n}}\right)^{-1} \rightarrow \infty$. Specifically, this number product is given by

$$
\text { Number Product }=\frac{9 \sqrt{\pi}}{8(1.22)} \sqrt{\frac{m_{i} T_{i}}{m_{e} T_{e}}} \beta_{e} \frac{n_{i}}{n_{n}} \operatorname{Kn}_{a}^{3}\left(1+\frac{n_{e} T_{i}}{n_{i} T_{e}}\right)
$$

This is evident in figure 3.1, where the different values of this number product do not affect the Knudsen capture parameter at large values of the gyration parameter. However, these different values of the number product yield very different parameter curves at smaller values of the gyration parameter. Note that all connection to the particle charge has been eliminated from the description given in equation 3.62; the particle charge must be eliminated in order to get an expression in terms of the gyration ratio, $\frac{\omega_{c d}}{\nu_{d n}}$. The expression in equation 3.62 is not necessarily fundamental, but it allows us to more readily understand the relevant parameter space for a specific charge model.

The figure 3.1 shows where the OML and hydrodynamic models are relevant. For $\mathrm{Kn}_{R}>10^{2}$, 
the grain is effectively in a collisionless regime and the OML theory is sufficient to describe the charging process. For $\mathrm{Kn}_{R}=1$, the grain is in the regime where there is approximately one ionneutral collision in the grain sheath, which results in an enhanced ion current to the grain. For $\mathrm{Kn}_{R}<10^{-2}$, there are many ion-neutral charge-exchange collisions, and the ion current is limited by ion mobility, which means that the ion current decreases with decreasing Knudsen capture parameter and the charge on the grain becomes more negative. Figure 1 of Gatti and Kortshagen (2008) shows this specific relationship quite clearly. It should be noted that the charging model of Gatti and Kortshagen (2008) yields the asymptotic results of the hydrodynamic, OML, and collision-enhanced current charging models, but distinguishes itself from all of these in that it offers predictive power over all Knudsen capture parameter values. Hence, the charging model of Gatti and Kortshagen (2008) claims to predict in-situ equilibrium grain charge in the range of $10^{2}>\mathrm{Kn}_{R}>10^{-2}$. Also in this range of the Knudsen capture parameter number, the charging rates of these models may be different. It is of interest therefore to see if gyro-phase drift, which is charging-rate dependent, can distinguish between the Gatti-Kortshagen charging model and another charge model, such as OML, in a region where the effects of orbit-motion-limited ion current, collisionally-enhanced ion current, and hyrodynamic current are all non-negligible, such as $\mathrm{Kn}_{R} \approx 10^{1}$.

As mentioned earlier, gyro-motion exists if $\frac{\omega_{c d}}{\nu_{d n}}>1$, but this does not guarantee that the charging-rate feature of dust grains gives rise to gyro-phase drift. A grain can exist anywhere in the parameter space but if there is no charge modulation, then there can be no gyro-phase drift. Additionally, the charge state modulation must be sufficiently large to measure gyro-phase drift. Since the ratio of gyro-averaged gyro-period to gyro-averaged charging time $\left(\tau_{g} / \tau_{c}\right) \approx 1$ produces the maximum amplitude of the gyro-phase drift, it is necessary to determine $\tau_{g} / \tau_{c}$ ratios near unity using the free parameters. 


\section{Chapter IV}

\section{Methodology for Test Particle}

\section{Investigation}

Test particle simulations elucidate the motions of charged particles and particulate matter in the presence of electric, magnetic, and other force fields. In order to obtain trajectories and drift motions for grains in arbitrary plasma profiles, and to provide independent corroboration of guiding center drift predicted by Northrop's adiabatic approach in a gradual inhomogeneity and later for the new approach for an abrupt inhomogeneity in chapter $\mathrm{V}$, we require a computational method for solving the equations of motion and the charge evolution of the dust grain. Predicting the guiding center drift motions using Northrop's adiabatic approach a priori is not possible for the most general descriptions of grain charging because the charge modulation $q_{1}$ is not known and the relationship to the DC offset $q_{0}$ may not be known due to the non-stationary charging effects. Guiding center drifts can be completely predicted using Northrop's simplified charging model, where grain velocity relative to ions is much larger than the ion thermal speed, limiting the scope of the theory sufficiently that a more general approach is needed. Numerical simulations provide a tool to solve grain motion for analytically intractible situations. Simulation codes such as the Dust in TOKamakS (DTOKS) developed at Imperial College London (Martin et al., 2008), the DUST Transport (DUSTT) developed at University of San Diego (Pigarov et al., 2005), and the DEMON

code developed at Auburn (Jefferson et al., 2010) provide sophisticated treatment of forces and charging mechanisms, but these codes treat the charging process instantaneously. Treating grain 
charging as instantaneous leads to inaccuracies in determining grain motion when the charging time is small compared to gyro-period, or other grain dynamical time scales. The code described in this chapter provides an energy conserving leapfrog integrator and incorporates drag forces that are non-linear in velocity, a feature not seen in codes mentioned earlier. An adaptive time step handles the grain charging process, allowing the ramifications of non-stationary charging on grain motion and guiding center drifts, specifically gyro-phase drift, to be investigated. This code is also the first to incorporate the Gatti-Kortshagen and Patacchini-Hutchinson charging models, including the effects of a drifting Maxwellian and mono-energetic ion flow. In this chapter, the simulation code is described, and analytically tractable examples are compared to simulation results to provide confidence in the approach.

\section{A Numerical Treatment of Forces}

The treatment of forces is synonomous here with the evolution of the grain trajectory in phase space, or solving the equations of motion resulting from the sum of all forces on the grain. All of the applicable forces, including electric, magnetic, gravitational, neutral drag, and ion drag were described in chapter III, so this will not be repeated here. There are many numerical options available to solve the resulting equations of motion, such as explicit or implicit Runge-Kutta, velocity Verlet, and leapfrog schemes. Implicit solvers have an advantage over explicit solvers in that they are resistant to numerical instabilities. Velocity Verlet and leapfrog methods inherently conserve energy, a useful feature. For this reason, and because of the straightforward implementation, a leapfrog method based on the Boris scheme (Boris, 1970) was chosen, and an iterative leapfrog was also developed. Both of these numerical methods used a fixed timestep at the beginning of the simulation, and had a separate, adaptive timestep for grain charging. Examples of different numerical methods used in other dust simulation codes include the DEMON code, which uses the explicit Runge-Kutta scheme RK4 (Jefferson et al., 2010), and the DTOKS code, which uses leapfrog integration (Martin et al., 2008; Bacharis et al., 2010). The Boris method, as applied to dust grains, will be discussed in greater detail in section 1, but the basic scheme is outlined below.

The leapfrog method for updating velocity in one spatial dimension can be illustrated with the 
scheme

$$
\frac{\mathrm{d} v_{x}}{\mathrm{~d} t}=\frac{F_{x}(x)}{m_{d}} \rightarrow v_{x}^{n+1 / 2}=v_{x}^{n-1 / 2}+\frac{F_{x}\left(x^{n}, y^{n}\right)}{m_{d}} \Delta t_{\mathrm{Nwt}},
$$

where $F_{x}\left(x^{n}\right)$ is the force on the particle evaluated at the full time step $n, m$ is the mass of the particle, and $\Delta t_{\mathrm{Nwt}}$ is the Newton time step. The position in one dimension is updated according to

$$
\frac{\mathrm{d} x}{\mathrm{~d} t}=v_{x} \rightarrow x^{n+1}=x^{n}+v_{x}^{n+1 / 2} \Delta t_{\mathrm{Nwt}} .
$$

To make the first computation of the velocity at $v_{x}^{1 / 2}$ requires that the term $v_{x}^{-1 / 2}$ is known. Because the initial conditions are specified at $n=0$, equation 4.1 is modified according to

$$
v_{x}^{-1 / 2}=v_{x}^{0}-\frac{F_{x}\left(x^{0}, y^{0}\right)}{m_{d}}\left(\frac{1}{2} \Delta t_{\mathrm{Nwt}}\right),
$$

so that the simulation can be started with a known value for $v_{x}^{-1 / 2}$. The procedure for computing equation 4.3 is equivalent to running the simulation backwards by a half time step. An important technique used in the Boris method is to add half the electric impulse before applying the rotation from the magnetic field, and adding the other half of the electric impulse afterward. For more details on the Boris method, see Boris (1970); Birdsall and Langdon (2005). The next subsection will describe how the Boris method was modifed in this dissertation to find trajectories of dust grains in the presence of Epstein drag.

\section{Modification to Boris Algorithm}

For the case of dust grains, the presence of the neutral drag force requires slight modification to the Boris method. The code in this dissertation is restricted to grain motion in two dimensions, since we are exclusively interested in the grain motion in the plane perpendicular to the magnetic field direction. This adaptation to the Boris method uses a fixed timestep for the grain motion, and is not adaptive. Updating the position is the same as in the Boris algorithm, but the drag force term 
is included in the two-dimensional, finite difference equation for velocity,

$$
\begin{aligned}
\frac{\boldsymbol{v}^{\boldsymbol{n}+\mathbf{1} / \mathbf{2}}-\boldsymbol{v}^{\boldsymbol{n}-\mathbf{1} / \mathbf{2}}}{\Delta t_{\mathrm{Nwt}}}= & \frac{q^{n}}{m_{d}}\left(\boldsymbol{E}\left(x^{n}, y^{n}, t^{n}\right)+\frac{\boldsymbol{v}^{\boldsymbol{n}+\mathbf{1} / \mathbf{2}}+\boldsymbol{v}^{\boldsymbol{n}-\mathbf{1} / \mathbf{2}}}{2} \times \boldsymbol{B}\left(x^{n}, y^{n}, t^{n}\right)\right)+\boldsymbol{g}\left(x^{n}, y^{n}\right) \\
& -\nu_{d n}\left(x^{n}, y^{n}, t^{n}\right) \frac{\boldsymbol{v}^{\boldsymbol{n}+\mathbf{1} / \mathbf{2}}+\boldsymbol{v}^{\boldsymbol{n}-\mathbf{1} / \mathbf{2}}}{2}+\nu_{d n}\left(x^{n}, y^{n}, t^{n}\right) \boldsymbol{v}_{\boldsymbol{n}}\left(x^{n}, y^{n}, t^{n}\right),
\end{aligned}
$$

where $q^{n}$ is the grain charge at the $n$th timestep, $m_{d}$ is the grain mass, $\boldsymbol{E}\left(x^{n}, y^{n}, t^{n}\right)$ is the temporally and spatially dependent electric field, $\boldsymbol{B}\left(x^{n}, y^{n}, t^{n}\right)$ is the temporally and spatially dependent magnetic field which is taken to be parallel to the $\hat{z}$-direction, $\boldsymbol{g}\left(x^{n}, y^{n}\right)$ corresponds to the spatially-dependent gravitational force term, $\nu_{d n}\left(x^{n}, y^{n}, t^{n}\right)$ is the dust-neutral collision frequency, and $\boldsymbol{v}_{\boldsymbol{n}}\left(x^{n}, y^{n}, t^{n}\right)$ is the temporally and spatially dependent neutral flow velocity. The last two terms in equation 4.4 arise from the neutral drag force, $\boldsymbol{F}_{\boldsymbol{n}}=m_{d} \nu_{d n}\left(x^{n}, y^{n}, t^{n}\right)\left(\boldsymbol{v}_{\boldsymbol{n}}\left(x^{n}, y^{n}, t^{n}\right)-\boldsymbol{v}\right)$. In equation 4.4, velocity-dependent forces use the average velocity of the new and old time steps, given by $\left(v^{n+1 / 2}+v^{n-1 / 2}\right) / 2$. This average value is used instead of $\boldsymbol{v}^{\boldsymbol{n}-\mathbf{1} / \mathbf{2}}$ in order to improve the accuracy of the solver. Following the Boris scheme, a velocity vector $\boldsymbol{v}^{-}$is defined by

$$
\begin{aligned}
\boldsymbol{v}^{-}= & \boldsymbol{v}^{\boldsymbol{n}-\mathbf{1 / 2}}+\frac{\Delta t_{\mathrm{Nwt}}}{2} \frac{q^{n}}{m_{d}}\left(\boldsymbol{E}\left(x^{n}, y^{n}, t^{n}\right)+\frac{\Delta t_{\mathrm{Nwt}}}{2} \boldsymbol{g}\left(x^{n}, y^{n}\right)\right. \\
& \left.+\frac{\Delta t_{\mathrm{Nwt}}}{2} \nu_{d n}\left(x^{n}, y^{n}, t^{n}\right) \boldsymbol{v}_{\boldsymbol{n}}\left(x^{n}, y^{n}, t^{n}\right)\right) .
\end{aligned}
$$

The point of this definition is to add half the impulse due to all force terms except the magnetic and grain-velocity-dependent drag forces because these are velocity dependent. The velocity vector $\boldsymbol{v}^{+}$is defined by

$$
\begin{aligned}
\boldsymbol{v}^{+}= & \boldsymbol{v}^{\boldsymbol{n}+\mathbf{1} / \mathbf{2}}-\frac{\Delta t_{\mathrm{Nwt}}}{2} \frac{q^{n}}{m_{d}}\left(\boldsymbol{E}\left(x^{n}, y^{n}, t^{n}\right)-\frac{\Delta t_{\mathrm{Nwt}}}{2} \boldsymbol{g}\left(x^{n}, y^{n}\right)\right. \\
& \left.-\frac{\Delta t_{\mathrm{Nwt}}}{2} \nu_{d n}\left(x^{n}, y^{n}, t^{n}\right) \boldsymbol{v}_{\boldsymbol{n}}\left(x^{n}, y^{n}, t^{n}\right)\right)
\end{aligned}
$$

Equation 4.4 can then be rewritten with linear combinations of $\boldsymbol{v}^{-}$and $\boldsymbol{v}^{+}$, resulting in the equation

$$
\frac{\boldsymbol{v}^{+}-\boldsymbol{v}^{-}}{\Delta t_{\mathrm{Nwt}}}=\frac{q^{n}}{2 m_{d}}\left(\boldsymbol{v}^{+}+\boldsymbol{v}^{-}\right) \times \boldsymbol{B}\left(x^{n}, y^{n}, t^{n}\right)-\left(\boldsymbol{v}^{+}+\boldsymbol{v}^{-}\right) \frac{\nu_{d n}\left(x^{n}, y^{n}, t^{n}\right)}{2} .
$$


Inserting the definitions from equations 4.5 and 4.6 into equation 4.7 produces the original finite difference representation of $\frac{\mathrm{d} \boldsymbol{v}}{\mathrm{d} t}$ in equation 4.4 .

The procedure continues by solving equation 4.7 for $\boldsymbol{v}^{+}$. Once $\boldsymbol{v}^{+}$is known, then $\boldsymbol{v}^{\boldsymbol{n}+\mathbf{1 / 2}}$ can be computed using equation 4.6, which is equivalent to adding the remaining half impulse from all force terms except the magnetic and grain-velocity-dependent drag forces. Because the magnetic field is assumed to be along the $\hat{z}$ direction, equation 4.7 can be written in matrix form

$$
\begin{aligned}
{\left[\begin{array}{c}
v_{x}^{+} \\
v_{y}^{+}
\end{array}\right]=} & {\left[\begin{array}{c}
v_{x}^{-} \\
v_{y}^{-}
\end{array}\right]+\left(\Delta t_{\mathrm{Nwt}} \frac{q^{n} B\left(x^{n}, y^{n}, t^{n}\right)}{2 m_{d}}\right)\left[\begin{array}{c}
v_{y}^{+} \\
-v_{x}^{+}
\end{array}\right] } \\
& +\left(\Delta t_{\mathrm{Nwt}} \frac{q^{n} B\left(x^{n}, y^{n}, t^{n}\right)}{2 m_{d}}\right)\left[\begin{array}{c}
v_{y}^{-} \\
-v_{x}^{-}
\end{array}\right] \\
& -\left(\Delta t_{\mathrm{Nwt}} \frac{\nu_{d n}\left(x^{n}, y^{n}, t^{n}\right)}{2}\right)\left[\begin{array}{c}
v_{x}^{+}+v_{x}^{-} \\
v_{y}^{+}+v_{y}^{-}
\end{array}\right] .
\end{aligned}
$$

Defining $A=\Delta t_{\mathrm{Nwt}} q_{d}\left(t^{n}\right) B\left(x^{n}, y^{n}, t^{n}\right) /\left(2 m_{d}\right)$ and some algebraic manipulation results in the solutions for $\boldsymbol{v}^{+}$

$$
v_{x}^{+}=\frac{\left(1-A^{2}-\left(\frac{\Delta t_{\mathrm{Nwt}} \nu_{d n}\left(x^{n}, y^{n}, t^{n}\right)}{2}\right)^{2}\right) v_{x}^{-}+2 A v_{y}^{-}}{\left(1+\frac{\Delta t_{\mathrm{Nwt}} \nu_{d n}\left(x^{n}, y^{n}, t^{n}\right)}{2}\right)^{2}+A^{2}}
$$

and

$$
v_{y}^{+}=\frac{\left(1-A^{2}-\left(\frac{\Delta t_{\mathrm{Nwt}} \nu_{d n}\left(x^{n}, y^{n}, t^{n}\right)}{2}\right)^{2}\right) v_{x}^{-}-2 A v_{x}^{-}}{\left(1+\frac{\Delta t_{\mathrm{Nwt}} \nu_{d n}\left(x^{n}, y^{n}, t^{n}\right)}{2}\right)^{2}+A^{2}} .
$$

Now that the solutions for $v_{x}^{+}$and $v_{y}^{-}$are known, equation 4.6 can be used to solve for $v_{x}^{n+1 / 2}$ and $v_{y}^{n+1 / 2}$. In this process, $v_{x}^{n+1 / 2}$ and $v_{y}^{n+1 / 2}$ effectively only depend on $v_{x}^{n-1 / 2}$ and $v_{y}^{n-1 / 2}$, making this an explicit method.

The user must specify the value for the Newton timestep $\Delta t_{\mathrm{Nwt}}$ The default option is to use the in-situ-equilibrium dust gyro-period at $t=0$ with $\Delta t_{\mathrm{Nwt}}=2 \pi /\left(\omega_{c d}(t=0) N\right)$, where $N$ is the user input number of points per gyro-cycle at the in-situ-equilibrium plasma conditions. This 
Newton timestep, unlike the charging timestep, is not adaptive, and remains fixed throughout the simulation. A smaller time step is recommended if the conditions or the dust grain charge change dramatically over the course of a simulation.

\section{Iterative Velocity Solver}

The iterative method developed in this dissertation is capable of solving force terms that are nonlinear in velocity, such as ion drag, or neutral drag for large relative dust-neutral drift speeds compared to the neutral thermal speed. This iterative method uses a fixed timestep for the grain motion, and is not adaptive. Computation of position, grain charge, and position-dependent forces is no different than in the Boris method, but the non-linear equation for velocity must be solved and, here, the half-impulse is not added before and after velocity is calculated as it is done in the Boris scheme. The finite difference scheme for the velocity is illustrated with the equation

$$
\begin{aligned}
\frac{\boldsymbol{v}^{\boldsymbol{n + 1 / 2}}-\boldsymbol{v}^{\boldsymbol{n - 1 / 2}}}{\Delta t_{\mathrm{Nwt}}}= & \frac{q^{n}}{m_{d}}\left(\boldsymbol{E}\left(x^{n}, y^{n}, t^{n}\right)+\frac{\boldsymbol{v}^{\boldsymbol{n + 1 / 2}}+\boldsymbol{v}^{\boldsymbol{n - 1 / 2}}}{2} \times \boldsymbol{B}\left(x^{n}, y^{n}, t^{n}\right)\right) \\
& +\boldsymbol{g}\left(x^{n}, y^{n}\right)+\beta_{n}\left(x^{n}, y^{n},\left(\frac{v_{x}^{n-1 / 2}+v_{x}^{n+1 / 2}}{2}\right),\left(\frac{v_{y}^{n-1 / 2}+v_{y}^{n+1 / 2}}{2}\right), t^{n}\right) \\
& +\beta_{i}\left(q^{n}, x^{n}, y^{n},\left(\frac{v_{x}^{n-1 / 2}+v_{x}^{n+1 / 2}}{2}\right),\left(\frac{v_{y}^{n-1 / 2}+v_{y}^{n+1 / 2}}{2}\right), t^{n}\right),
\end{aligned}
$$

where $\beta_{n}(\boldsymbol{r}, \boldsymbol{v}, t)=\boldsymbol{F}_{\boldsymbol{n}} / m_{d}$, when $\boldsymbol{F}_{\boldsymbol{n}}$ can be defined by either equation 3.50 or 3.49 depending on whether dust-neutral relative velocity compared to the neutral thermal speed is small or large, respectively, and $\beta_{i}(q, \boldsymbol{r}, \boldsymbol{v}, t)=\left(\boldsymbol{F}_{\boldsymbol{i c}}+\boldsymbol{F}_{\boldsymbol{i o}}\right) / m_{d}$, when $\boldsymbol{F}_{\boldsymbol{i c}}$ and $\boldsymbol{F}_{\boldsymbol{i o}}$ are defined by equations 3.51 and 3.52 respectively.

All velocity-dependent quantities in equation 4.11 use the average velocity of the $n-1 / 2$ and $n+1 / 2$ time steps to improve accuracy and stability of the solver. Because drag terms depend on both $\boldsymbol{v}^{\boldsymbol{n}-\mathbf{1 / 2}}$ and $\boldsymbol{v}^{\boldsymbol{n}+\mathbf{1 / 2}}$ in equation 4.11, this is an implicit method for velocity. This results in a set of coupled, non-linear equations for $\boldsymbol{v}^{\mathbf{n + 1 / 2}}$. In matrix form, we encounter an equation for the 
velocity vector that looks like

$$
\left[\begin{array}{c}
v_{x}^{n+1 / 2} \\
v_{y}^{n+1 / 2}
\end{array}\right]=\left[\begin{array}{c}
v_{x}^{n-1 / 2}+\frac{\Delta t_{\mathrm{Nwt}}}{m_{d}} F_{x}\left(q^{n}, x^{n}, y^{n},\left(\frac{v_{x}^{n-1 / 2}+v_{x}^{n+1 / 2}}{2}\right),\left(\frac{v_{y}^{n-1 / 2}+v_{y}^{n+1 / 2}}{2}\right)\right) \\
v_{y}^{n-1 / 2}+\frac{\Delta t_{\mathrm{Nwt}}}{m_{d}} F_{y}\left(q^{n}, x^{n}, y^{n},\left(\frac{v_{x}^{n-1 / 2}+v_{x}^{n+1 / 2}}{2}\right),\left(\frac{v_{y}^{n-1 / 2}+v_{y}^{n+1 / 2}}{2}\right)\right)
\end{array}\right],
$$

where $F_{x}$ corresponds to all force terms in the $x$-direction and $F_{y}$ corresponds to all force terms in the $y$-direction.

To solve equation 4.12, note the velocity components on the left hand side can be subtracted from the right hand side to equal zero, producing

$$
f_{1}=-u_{x}+u_{0 x}+\frac{\Delta t_{\mathrm{Nwt}}}{m_{d}} F_{x}\left(q^{n}, x^{n}, y^{n},\left(\frac{u_{0 x}+u_{x}}{2}\right),\left(\frac{u_{0 y}+u_{y}}{2}\right)\right)=0
$$

and

$$
f_{2}=-u_{y}+u_{0 y}+\frac{\Delta t_{\mathrm{Nwt}}}{m_{d}} F_{y}\left(q^{n}, x^{n}, y^{n},\left(\frac{u_{0 x}+u_{x}}{2}\right),\left(\frac{u_{0 y}+u_{y}}{2}\right)\right)=0,
$$

where we have replaced $\boldsymbol{v}^{\boldsymbol{n}+\mathbf{1} / \mathbf{2}}$ with $\boldsymbol{u}$ and $\boldsymbol{v}^{\boldsymbol{n}-\mathbf{1} / \mathbf{2}}$ with $\boldsymbol{u}_{\mathbf{0}}$ for clarity when applying the iterative method to this system of equations. If we can find the values of $u_{x}$ and $u_{y}$ that make $f_{1}=0$ and $f_{2}=0$, then we have solved the equations of motion for the dust grain. The values $u_{0 x}$ and $u_{0 y}$ are from the previous timestep and are treated like constants. Because $f_{1}$ and $f_{2}$ constitute a system of equations in variables $u_{x}$ and $u_{y}$, solving the coupled equations 4.13 and 4.14 corresponds to the Newton method for root-finding as applied to matrices. In operator notation, the procedure can be expressed by

$$
\hat{\boldsymbol{J}} \boldsymbol{\delta}=-\boldsymbol{f}
$$

where $\hat{\boldsymbol{J}}$ is the Jacobian matrix, $\boldsymbol{f}$ is a vector function of $\boldsymbol{u}, \boldsymbol{\delta}=\boldsymbol{u}^{(k+1)}-\boldsymbol{u}^{(k)}$ is the vector describing the error, and $(k)$ denotes the $k^{\text {th }}$ iteration. The non-linear vector function of velocity $\boldsymbol{f}$ approaches zero with successive iterations to an error that is within a user-defined tolerance. The velocity calculated at the $k^{\text {th }}$ iteration is simply $\boldsymbol{u}^{(k)}+\boldsymbol{\delta}$. In matrix notation, solve an equation of 
the form

$$
\left[\begin{array}{cc}
\frac{\partial f_{1}}{\partial u_{x}^{(k)}} & \frac{\partial f_{1}}{\partial u_{y}^{(k)}} \\
\frac{\partial f_{2}}{\partial u_{x}^{(k)}} & \frac{\partial f_{2}}{\partial u_{y}^{(k)}}
\end{array}\right]\left[\begin{array}{c}
\delta_{1}=u_{x}^{(k+1)}-u_{x}^{(k)} \\
\delta_{2}=u_{y}^{(k+1)}-u_{y}^{(k)}
\end{array}\right]=\left[\begin{array}{c}
f_{1}\left(u_{x}^{(k)}, u_{y}^{(k)}\right) \\
f_{2}\left(u_{x}^{(k)}, u_{y}^{(k)}\right)
\end{array}\right] .
$$

The matrix on the left hand side of equation 4.16 is technically a tri-diagonal matrix, so the fast and efficient Thomas algorithm readily finds the $\boldsymbol{\delta}$ vector. This nice tri-diagonal property does not hold for three dimensions, because the non-linear drag terms couple all three velocity components together. Because this method requires an initial guess for $\boldsymbol{u}^{(0)}$, this is set to the velocity vector from the last time step, $\boldsymbol{u}^{(0)}=\boldsymbol{u}_{\mathbf{0}}=\boldsymbol{v}^{\boldsymbol{n}-\mathbf{1} / \mathbf{2}}$. The method generally converges very quickly with this choice of $\boldsymbol{u}^{(0)}$, because the velocity vector changes slowly when $\Delta t_{\mathrm{N}}$ is based on the gyro-period. In other words, while $\boldsymbol{v}^{\boldsymbol{n + 1 / 2}}$ and $\boldsymbol{v}^{\boldsymbol{n}-\mathbf{1 / 2}}$ are different, the velocities at successive time levels are not substantially different. This method can be used to obtain arbitrary precision in velocity during a timestep, provided the maximum number of iterations is not exceeded. The new positions are computed by $\boldsymbol{r}^{\boldsymbol{n + 1}}=\boldsymbol{r}^{\boldsymbol{n}}+\boldsymbol{v}^{\boldsymbol{n + 1 / 2}} \Delta t_{\mathrm{Nwt}}$, which is the same procedure used for the Boris method. For the results quoted in this dissertation that use the iterative leapfrog method, a maximum of 1000 iterations was used, a limit that can be adjusted. The code for the iterative leapfrog method is included in appendix B, section iv.

\section{Adjustments for Non-Inertial Reference Frames}

Some of the results in this dissertation and in the context of space applications are best studied in a co-rotating reference frame. Co-rotating reference frames require the Boris and iterative leapfrog schemes to be rewritten slightly. In the co-rotating reference frame, $\boldsymbol{E}=\boldsymbol{B} \times(\boldsymbol{\Omega} \times \boldsymbol{r})$, where $\boldsymbol{\Omega}$ corresponds to the angular velocity vector of the rotating system, $\boldsymbol{g}$ and $\boldsymbol{B}$ are no longer functions of time, and the relative motion between grain and plasma is determined solely by grain motion in this frame (we do not need to account for $\boldsymbol{v}_{\boldsymbol{i}}$ terms). This results in an equation of motion (Northrop and Hill, 1983)

$$
\left(\frac{\mathrm{d} \boldsymbol{v}}{\mathrm{d} t}\right)_{c}=\boldsymbol{v}_{c} \times\left(\frac{q(t)}{m_{d}} \boldsymbol{B}(\boldsymbol{r})+2 \boldsymbol{\Omega}\right)-\boldsymbol{\Omega} \times(\boldsymbol{\Omega} \times \boldsymbol{r})+\boldsymbol{g}(\boldsymbol{r})
$$


where the $c$ subscripts now indicate quantities calculated in the co-rotating frame. No restrictions need to be placed on the magnetic field; it can have arbitrary spatial dependence. The penultimate term of equation $4.17, \boldsymbol{\Omega} \times(\boldsymbol{\Omega} \times \boldsymbol{r})$, reduces to $-(x \hat{x}+y \hat{y}) \Omega^{2}$ because analysis occurs in the corotating frame. For treatment using the Boris scheme, add the half impulse for all of the spatiallydependent psuedo-forces in equation 4.17 before applying velocity dependent forces, and add the other half impulse afterward. These fictitious forces produce an $\boldsymbol{E} \times \boldsymbol{B}$-like drift in the co-rotating frame.

\section{Adjustments for Ion Flow along Magnetic Field Direction}

As a final note, the Boris and iterative leapfrog schemes are also configured for the possibility of ion flow in the planar sheath of a laboratory experiment, assumed to be parallel or anti-parallel to the magnetic field direction. In the simulation code, the grain charge $q^{n+1}$ is computed after $\boldsymbol{r}^{\boldsymbol{n}+\boldsymbol{1}}$ and $\boldsymbol{v}^{\boldsymbol{n + 1 / 2}}$ have been calculated, using the plasma conditions at $\boldsymbol{r}^{\boldsymbol{n + 1}}$ and the relative drift between the dust grain and the electrons and ions. The relative drift between dust grain and electrons or ions is the flow speed $w_{e, i}$ and it is given by

$$
w_{e, i}\left(x^{n+1}, y^{n+1}, t^{n+1}\right)=\left|\boldsymbol{v}^{n+1 / 2}-\boldsymbol{v}_{e, i}\left(x^{n}, y^{n}, t^{n}\right)\right|,
$$

where $v_{e, i}$ is the electron or ion drift speed. Even when grains are constrained to two dimensions, electron and ion flow can have components in three dimensions. In order for the flow to be correct, it is necessary to account for both the electron or ion flow relative to the grain in the plane perpendicular to the magnetic field direction and the ion flow along the magnetic field. Because the code is two dimensional, there is no specified third component of ion flow, so the sheath particle-moving schemes use the electron flow in the $x$-direction to store this third component, and the electron flow in the $y$-direction is associated with a flow in the $\varphi$-direction, where $\varphi$ is the azimuthal angle in cylindrical geometry. In a planar sheath there is little or no electron flow along the magnetic field, so we do not need to worry about treating this component. Nothing changes with respect to the calculation of forces; the sheath particle-moving schemes reconfigure the flow output so that it is correct when this information enters the charging algorithm. 


\section{B Numerical Treatment of Charging}

When a magnetized dust grain samples different plasma conditions (temperature, space potential, ultraviolet photons, species, etc.) along its gyro-orbit, the dust grain's floating potential will adjust to the new in-situ parameters. Because dust grains have capacitance, they do not respond instantaneously to each new set of plasma conditions at each location if $\left(\tau_{\mathrm{c}} / \tau_{\mathrm{g}}\right) \not \approx 1$. Just as a capacitor has a characteristic charging time, dust grains have characteristic charging times based on their size and the local plasma conditions (Goree, 1994), so if the dust grain moves fast enough through these varying conditions it never reaches the $i n$-situ equilibrium charge. In the literature, this situation is referred to as delayed charging (Nunomura et al., 1999). Currently, no major dust simulation codes treat non-instantaneous charging, instead relying on the in-situ equilibrium value for their equations of motion. Failure to account for non-instantaneous charging produces erroneous grain trajectories under certain conditions, as shown in chapters V and VI. The option to use instantaneous charging is included with the charging code, described in appendix B.

The integration schemes described in section A solve the equations of motion, and the charging algorithm described in this section updates grain charge. During each charging timestep $\Delta t_{c}$, the analytical currents for the selected charging model are applied to the dust grain using $\Delta q_{n}=I_{t o t} \Delta t_{c}$. This is an adaptive timestep $\Delta t_{c}$ to ensure that the non-linear differential equation for the grain charge is solved correctly in accordance with the specified model simultaneously with the coupled differential equations for the grain motion from the Lorentz force.

At the beginning of the simulation, and at the beginning of each Newton timestep, $\Delta t_{c}$ is calculated according to $\Delta t_{c}=e /\left|I_{t o t}\right|$, which is the time for the grain to gain or lose 1 electron. If $\Delta t_{c}>\Delta t_{\mathrm{Nwt}}$, then $q(t)$ is held fixed at its current value and a timer is started with $t_{a c c}=\Delta t_{\mathrm{Nwt}}$. This timer $t_{a c c}$ keeps track of the accumulated time that has passed since the last charge update. During subsequent timesteps, if $t_{a c c}<\Delta t_{\mathrm{Nwt}}$, then another Newton timestep is added to $t_{a c c}$. In other words, if $t_{a c c}<\Delta t_{c}$, then $t_{a c c}=t_{a c c}+\Delta t_{\mathrm{Nwt}}$. When the condition $t_{a c c} \geq \Delta t_{\mathrm{Nwt}}$ is satisfied, then 1 electron is added or subtracted to $q(t)$, depending on the sign of $I_{t o t}$, and the accumulated time is set to $t_{a c c}=\Delta t_{\mathrm{Nwt}}-\operatorname{Remainder}\left(\Delta t_{c} / \Delta t_{\mathrm{Nwt}}\right)$. This way, when $\Delta t_{c}$ is calculated from the new value of $I_{\text {tot }}$ at the next Newton timestep, the exact value since the last charge update is known. For this numerical scheme, $q(t)$ is only recorded at the end of every Newton timestep. 
The total current becomes very small when the grain surface is near the in-situ equilibrium grain charge, and $\Delta t_{c}$ becomes large. The grain fluctuates between neighboring charge states because in general, $C_{d} V_{d}$ is not an exact integer multiple of the elementary charge $e$. As a precaution to prevent wasted computation, if the grain charge equals the value it had two iterations previously $\left(q^{(n)}=q^{(n-2)}\right)$, the charging procedure stops because it will only flip back and forth between these charge states. This implies that the equilibrium grain charge has been reached, within \pm 1 electron.

The charging rate can also be arbitrarily controlled in this model by introducing an adjustable charging parameter $\alpha$ used in the following way: $q_{n+1}=q_{n}+\Delta q_{n}^{\prime}$, where $\Delta q_{n}^{\prime}=\alpha \Delta q$ as is done in Koepke et al. (2013). This is equivalent to calculating $\Delta t_{c}=\frac{e}{\alpha I_{t o t}}$. For $\alpha=1$, the dust grain charges without restriction, while $\alpha<1$ implies that the grain charges more slowly. This adjustable charging rate parameter is discussed here only insofar as $\alpha$ can be used as a possible way of changing the value of $\tau_{c h}$ in simulations when other plasma parameters, such as the background plasma density $n_{0}$ are fixed. Examining a particular charging model in terms of non-stationary charging by introducting parameter $\alpha$ permits differences in the prediction sensitivity to be assessed within the model's own validity regime.

\section{Gyro-averaged Quantities}

When discussing guiding center drifts, we need a good way of discussing how grain charge, position, velocity, gyro-radius, etc. change over successive gyro-cycles. An analysis code was developed for the simulation program described in the previous sections that finds instantaneous guiding center positions and calculates gyro-averaged quantities. The code's main features are highlighted in this section. The entire code can be found in appendix B.

Gyro-phase averaging poses some computational challenges. The relationship $\frac{\mathrm{d} \phi}{\mathrm{d} t}=\frac{q(t) B(t)}{m}=$ $\omega_{c d}(t)$ holds only for trivial of cases of magnetized-orbit motion in homogeneous plasma, where $\phi$ is the gyro-phase, and does not even hold for regular $\boldsymbol{E} \times \boldsymbol{B}$ motion. It is not possible to use the gyro-frequency alone to specify the gyro-phase for many cases of interest. Additionally, the

expression $R_{L d}=\frac{v_{\perp} m}{q B}$ is invalid for many cases of interest, so more consistently valid working definitions are needed for use in discussing instantaneous guiding center position and gyro-motion. Describing the gyrating particle about its guiding center starts by finding the angle of the grain in 
velocity space, or the arctangent of the ratio of two velocity vectors in the plane perpendicular to the magnetic field. The arctangent function needs to be extended beyond the domain of $[-1,1]$ and treated correctly so that a full $360^{\circ}$ is swept out in configuration space during a gyro-cycle. The details of this geometric treatment are handled in the code descriptions in B. Examples are given in the paragraphs below, where the gyro-radius can then be defined by

$$
R_{L d}=\frac{v_{\perp}}{\frac{\mathrm{d} \phi}{\mathrm{d} t}},
$$

which is effectively a consequence of the Frenet-Serret formulas. This expression holds for every case of interest, including the case of a net force $\boldsymbol{F}$ acting on the grain at time $t$, and it reduces to non-drifting gyro-motion in the trivial case of $\frac{\mathrm{d} \phi}{\mathrm{d} t}=\omega_{c d}(t)$. Computing gyro-phase allows us to look at grain quantities as functions of gyro-phase instead of just as functions of time, and this ultimately permits accurate calculation of gyro-period and guiding center drifts from simulation data.

In slab geoemetry, the gyro-phase angle is best defined by

$$
\phi=\arctan \left(\frac{v_{y}}{v_{x}}\right)
$$

where $\phi$ is the gyro-phase angle of the grain in velocity space and the $x$ and $y$ components are defined after the choice of coordinate system, but not necessarily the guiding center, is made. Whereas the gyro-phase angle is determined by the ratio of velocity components, the gyro-radius magnitude is determined by how these components are changing with respect to each other. To illustrate how the result of this calculation maps correctly in configuration space, or in the $x, y$ plane, consider as an example when $v_{x}=0$ and $v_{y}>0$. Even though $\arctan \infty=\pi$, this velocity pair corresponds to $\phi=0$, for clockwise or counter-clockwise gyrating grains, because the tangent line to the trajectory (the velocity vector) would correspond to an angle of 0 on the unit circle. See figure 4.1 for clarity. The direction of gyration does not affect the calculation, it merely produces positive values for $\frac{\mathrm{d} \phi}{\mathrm{d} t}$ for counter-clockwise rotation and negative values for clockwise rotation. 
This leads to the expression for the gyro-radius

$$
R_{L d}(t)=\frac{\left(v_{\perp}(t)\right)^{3}}{v_{x} \dot{v_{y}}-v_{y} \dot{v}_{x}} .
$$

Instantaneous guiding center positions are now readily calculated with

$$
x_{c}(\phi)=x(\phi)-R_{L d}(\phi) \cos \phi
$$

and

$$
y_{c}(\phi)=y(\phi)-R_{L d}(\phi) \sin \phi \text {. }
$$

Ultimately, these relations are a consequence of $\boldsymbol{r}_{\boldsymbol{c}}=\boldsymbol{r}-\boldsymbol{\rho}_{\boldsymbol{g}}$ described earlier in chapter III, where

$\boldsymbol{\rho}_{\boldsymbol{g}}$ plays the role of the vector that points from the grain's position in configuration space to the grain's guiding center.

\section{Benchmarking Simulations to Analytical Examples}

Analytical results can be compared to simulation results for simple cases, such as $\boldsymbol{E} \times \boldsymbol{B}$ drift with constant grain charge, that serve both to benchmark or validate the simulations to build intuition on the way to more complex cases. Where an analytical solution is tractable, it will be compared to simulation results. It should be noted that because the particle motion simulations can be done using either the Boris method or the iterative leapfrog method developed in this dissertation, the results for both will be presented and compared simultaneously in cases where both methods are valid. This section starts with the familiar, simplistic models for gyro-motion and increasing complexity is added incrementally to illustrate features of the simulation code. Some minor, but important details will be shown along the way that have important implications for grain trajectories and confinement. 


\section{$1 E \times B$ Drift in slab Geometry}

The general solution for the position $x_{d}$ and $y_{d}$ of a grain having fixed charge state and in the presence of electric and magnetic fields is given by (Chen, 2006)

$$
\begin{aligned}
x_{d}(t)= & \left(\frac{v_{0 x d}}{\omega_{c d}}-\frac{E_{0 y}}{\omega_{c d} B_{0}}\right) \sin \left(\omega_{c d} t\right) \mp\left(\frac{v_{0 y d}}{\omega_{c d}}+\frac{E_{0 x}}{\omega_{c d} B_{0}}\right) \cos \left(\omega_{c d} t\right)+ \\
& \frac{E_{0 y}}{B_{0}} t \pm\left(\frac{v_{0 y d}}{\omega_{c d}}+\frac{E_{0 x}}{\omega_{c d} B_{0}}\right)+x_{0 d}
\end{aligned}
$$

and

$$
\begin{aligned}
y_{d}(t)= & \left(\frac{v_{0 y d}}{\omega_{c d}}+\frac{E_{0 x}}{\omega_{c d} B_{0}}\right) \sin \left(\omega_{c d} t\right) \pm\left(\frac{v_{0 x d}}{\omega_{c d}}-\frac{E_{0 y}}{\omega_{c d} B_{0}}\right) \cos \left(\omega_{c d} t\right)- \\
& \frac{E_{0 x}}{B_{0}} t \mp\left(\frac{v_{0 x d}}{\omega_{c d}}-\frac{E_{0 y}}{\omega_{c d} B_{0}}\right)+y_{0 d} .
\end{aligned}
$$

Figure 4.2 shows a comparison between the analytical prediction and the solution from the Boris time evolution and iterative leapfrog time evolution for a $1 \mu$ m diameter grain with crossed $E$ and $B$ fields. We see close agreement among all solutions, providing confidence in the computational methods. The $\boldsymbol{E} \times \boldsymbol{B}$ drift is $0^{t h}$ order in the parameter $m_{d} / q_{d}$, so drift motion will always be the same. However, as $m_{d} / q_{d}$ increases, the effective gyro-radius becomes very large. For a grain that starts at the origin with a velocity in the $\hat{x}$ direction, $\boldsymbol{E}_{\mathbf{0}}$ along $-\hat{x}$ and $\boldsymbol{B}$ along $\hat{z}$, the maximum

distance from the line $x=0$ is given by $\frac{2 m_{d} E_{0}}{q_{d} B^{2}}$. More generally, using the definition for gyro-radius from equation 4.19 , we get the gyro-radius of the grain as a function of time

$$
R_{L d}=\frac{m_{d}}{q_{d} B} \frac{v_{\perp}^{3}}{v_{\perp}^{2}+\frac{E_{0}}{B} v_{y}} .
$$

Bear in mind that even for constant fields and grain charge, equation 4.26 involves the time dependent quantities $v_{y}(t)$ and $v_{\perp}(t)$. Figure 4.3 shows how the ambient electric field enlarges the effective gyro-radius of dust grains.

\section{$2 \quad \boldsymbol{F} \times \boldsymbol{B}$ Drift in slab Geometry with Epstein Drag}

The addition of drag into the situation provides some more complexity, and an additional test of the computational approach. The analytical solution of $x_{d}$ and $y_{d}$ for $E, B$ fields and neutral drag 
(including flows) for fixed charge state are given by

$$
\begin{aligned}
x_{d}(t)= & \left(v_{0 x d}-\frac{C_{1}}{\nu_{d n}^{2}+\omega_{c d}^{2}}\right) \exp \left(-\nu_{d n} t\right) \frac{\omega_{c d} \sin \left(\omega_{c d} t\right)-\nu_{d n} \cos \left(\omega_{c d} t\right)}{\nu_{d n}^{2}+\omega_{c d}^{2}} \mp \\
& \left(v_{0 y d}-\frac{C_{2}}{\nu_{d n}^{2}+\omega_{c d}^{2}}\right) \exp \left(-\nu_{d n} t\right) \frac{\nu_{d n} \sin \left(\omega_{c d} t\right)+\omega_{c d} \cos \left(\omega_{c d} t\right)}{\nu_{d n}^{2}+\omega_{c d}^{2}}+\frac{C_{1}}{\nu_{d n}^{2}+\omega_{c d}^{2}} t+ \\
& \frac{\nu_{d n}}{\nu_{d n}^{2}+\omega_{c d}^{2}}\left(v_{0 x d}-\frac{C_{1}}{\nu_{d n}^{2}+\omega_{c d}^{2}}\right) \pm \frac{\omega_{c d}}{\nu_{d n}^{2}+\omega_{c d}^{2}}\left(v_{0 y d}-\frac{C_{2}}{\nu_{d n}^{2}+\omega_{c d}^{2}}\right)+x_{0 d}
\end{aligned}
$$

and

$$
\begin{aligned}
y_{d}(t)= & \left(v_{0 y d}-\frac{C_{2}}{\nu_{d n}^{2}+\omega_{c d}^{2}}\right) \exp \left(-\nu_{d n} t\right) \frac{\omega_{c d} \sin \left(\omega_{c d} t\right)-\nu_{d n} \cos \left(\omega_{c d} t\right)}{\nu_{d n}^{2}+\omega_{c d}^{2}} \pm \\
& \left(v_{0 x d}-\frac{C_{1}}{\nu_{d n}^{2}+\omega_{c d}^{2}}\right) \exp \left(-\nu_{d n} t\right) \frac{\nu_{d n} \sin \left(\omega_{c d} t\right)+\omega_{c d} \cos \left(\omega_{c d} t\right)}{\nu_{d n}^{2}+\omega_{c d}^{2}}+\frac{C_{2}}{\nu_{d n}^{2}+\omega_{c d}^{2}} t+ \\
& \frac{\nu_{d n}}{\nu_{d n}^{2}+\omega_{c d}^{2}}\left(v_{0 y d}-\frac{C_{2}}{\nu_{d n}^{2}+\omega_{c d}^{2}}\right) \mp \frac{\omega_{c d}}{\nu_{d n}^{2}+\omega_{c d}^{2}}\left(v_{0 x d}-\frac{C_{1}}{\nu_{d n}^{2}+\omega_{c d}^{2}}\right)+y_{0 d},
\end{aligned}
$$

where

$$
\begin{aligned}
& C_{1}=\nu_{d n}^{2} v_{x n} \pm \frac{\nu_{d n} \omega_{c d} E_{0 x}}{B_{0}} \pm \nu_{d n} \omega_{c d} v_{y n}+\frac{\omega_{c d}^{2} E_{0 y}}{B_{0}}, \\
& C_{2}=\nu_{d n}^{2} v_{y n} \pm \frac{\nu_{d n} \omega_{c d} E_{0 y}}{B_{0}} \mp \nu_{d n} \omega_{c d} v_{x n}-\frac{\omega_{c d}^{2} E_{0 x}}{B_{0}},
\end{aligned}
$$

$\nu_{d n}$ is the dust-neutral collision rate, $\boldsymbol{v}_{\boldsymbol{n}}$ corresponds to the velocity of neutrals as measured in a stationary reference frame, and the top signs correspond to positively charged grains while the bottom signs correspond to negatively charged grains.

Figure 4.4 demonstrates that flowing neutrals produce an $\boldsymbol{F} \times \boldsymbol{B}$ guiding center drift by introducing a neutral flow along the $-\hat{x}$ direction. The neutral gas pressure is very small, about $0.01 \mathrm{~m}$ Torr so that this grain can perform multiple gyrations. Using $\frac{1}{q_{d}} \frac{\boldsymbol{F} \times \boldsymbol{B}}{B^{2}}$, produces a value of $v_{y g c}=-1.0068 \mathrm{~m} / \mathrm{s}$, while analysis from simulation yields the close agreement of $v_{y g c}=-1.0064 \mathrm{~m} / \mathrm{s}$. The two values do not agree completely, because in addition to the $\boldsymbol{F} \times \boldsymbol{B}$ drift from the flowing neutrals, there is an inherent drift from the gyro-synchronously modified gyro-radius; as the grain moves through the plasma the gyro-motion damps so that $v_{\perp}$ decreases. It is noted that gyro-phase drift results from the gyro-radius being modulated gyro-synchronously with a phase offset, and that 
gyro-synchronously modulated gyro-radius results in a guiding center drift

Figure 4.5 shows a trajectory where neutral gas flow is present, but the neutral gas pressure is much higher, about 1 mTorr. The grain does not even perform a visible gyration because the neutral drag force dominates. This is in stark contrast to the results of Amatucci et al. (2004), $\omega_{c d} / \nu_{d n}=10^{-4}$. If their intrepretation of the horizontal oscillation as evidence of gyro-motion is correct then the Epstein drag model may be incorrect, which means that the trajectory shown in figure 4.5 would not be overdamped.

Figure 4.6 shows an example trajectory for $1 \mu \mathrm{m}$ diameter grain in crossed $E=100 \mathrm{~V} / \mathrm{m}, B=4 \mathrm{~T}$ fields, flowing neutrals, and a small enough pressure so that grain gyration is not completely damped, like in figure 4.4. For this case, the electric field is along $-\hat{x}$ and is strong enough to overcome the $\boldsymbol{F} \times \boldsymbol{B}$ drift from the neutral drag force so that the grain drifts along $\hat{y}$. Calculating the $\boldsymbol{E} \times \boldsymbol{B}$ and $\boldsymbol{F} \times \boldsymbol{B}$ drifts separately yields $v_{y g c}=25 \mathrm{~m} / \mathrm{s}$ and $v_{y g c}=-1.0068 \mathrm{~m} / \mathrm{s}$ for the electric and drag forces respectively, which add together to produce a combined $\boldsymbol{F} \times \boldsymbol{B}$ drift of $v_{y g c}=23.99 \mathrm{~m} / \mathrm{s}$. The simulation analysis produces a result of $v_{y g c}=23.860 \mathrm{~m} / \mathrm{s}$.

\section{$3 \quad F \times B$ Drift with Flowing Ions}

Just as neutral drag produces $\boldsymbol{F} \times \boldsymbol{B}$ drift, flowing ions produce an $\boldsymbol{F} \times \boldsymbol{B}$ drift from the ion drag force. However, the Boris scheme cannot treat non-linear drag forces, so as an alternative the iterative leapfrog method can be used to simulate grain trajectories. Exact, analytical predictions for the grain trajectory cannot be made without assuming constant grain charge, $\boldsymbol{v}_{\boldsymbol{i}} \gg \boldsymbol{v}$, and $\left|\boldsymbol{v}_{\boldsymbol{i}}-\boldsymbol{v}\right| / v_{t h i} \ll 1$. This restriction results in a limited range of applicability, and suggests the necessity of using simulations for most situations. As discussed in chapter III, the work done by Barnes et al. (1992); Fortov et al. (2005); Hutchinson (2006) paved the way for using linear ion drag in simulations (Pigarov et al., 2005; Smirnov et al., 2007; Martin et al., 2008; Bacharis et al., 2010), valid for the conditions listed above. The iterative leapfrog scheme in this dissertation extends the computational determination of ion drag beyond these the linear regime of ion drag.

Figure 4.7 shows an example trajectory using the iterative leapfrog method when the ion flow is $v_{x i}=-200 \mathrm{~m} / \mathrm{s}$, the ion density is $10^{12} \mathrm{~m}^{-3}$, the magnetic field is $B=4 \mathrm{~T}$, and no other forces are present. The ion drag force causes the grain to drift along $-\hat{y}$, because this is the direction

of $\boldsymbol{F} \times \boldsymbol{B}$ drift. Computing $\frac{1}{q_{d}} \frac{\boldsymbol{F} \times \boldsymbol{B}}{B^{2}}$ predicts a drift $v_{y g c}=-0.0420 \mathrm{~m} / \mathrm{s}$, while simulation analysis 
yields $v_{y g c}=-0.044 \mathrm{~m} / \mathrm{s}$. The iterative leapfrog method provides a valid approach to compute ion drag forces. 


\section{Chapter V}

\section{Analytical and Simulation Results}

Gyro-synchronous dust grain charge modulation from a spatially-dependent plasma parameter leads to a dynamic grain gyro-center. The grain's gyro-center drifts over the course of a gyro-cycle, with a velocity predicted using the adiabatic theory for charged-particle motion. Analysis of charged particle motion has been used to infer field quantities, and in the case of variable grain charge, monitoring the gyro-phase drift can be used to measure plasma non-uniformities because the gyrophase drift component is sensitive to grain charging rate, with the added benefit that the gyro-phase drift component is exclusively perpendicular to diamagnetic drifts such as $\boldsymbol{E} \times \boldsymbol{B}$, grad-B, magnetic curvature drift, and grad-q drift. Gyro-phase drift, therefore, serves as a sensitive, untainted indicator for phenomena that depend on charging rate. In this chapter, the semi-analytical and simulation results for an abrupt inhomogeneity quantify how gyro-phase drift is used to discriminate among charge models for planar sheath mechanisms, and both the semi-analytical results for abrupt inhomogeneity and simulation results for gradual inhomogeneity reveal that the maximal gyro-phase drift amplitude occurs when the charging time is comparable to the gyro-period. Prior to this research, there has been no attempt to find the gyro-phase drift in abrupt inhomogeneity, and the dependence of gyro-phase drift magnitude and direction has not been demonstrated over a wide range of gyro-period to charging time ratios. Furthermore, gyro-phase drift has not been characterized for a wide range of parameters and sheath mechanisms, and its ability to measure plasma sheath properties has not been demonstrated. The semi-analytical results for abrupt inhomogeneity show that, under certain regimes, gyro-phase drift discriminates charge model and planar sheath mechanisms. 


\section{A General Solutions}

This section will expand upon the derivation of Walker et al. (2014), with discussions regarding the presence of an electric field and the Epstein drag. Ion drag in the plane perpendicular to the magnetic field direction is considered negligible compared to electric, magnetic, and neutral drag forces, so it is not included. The grain trajectory and gyro-phase drift can be computed once an analytical expression for the dust grain velocity vector is in hand. Start with the Lorentz force equation, including the Epstein drag force (Epstein, 1924) to obtain the grain trajectory, and permit the grain charge to be time-dependent,

$$
\boldsymbol{F}=q(t) \boldsymbol{E}+q(t) \boldsymbol{v} \times \boldsymbol{B}+-m_{d} \nu_{d n}\left(\boldsymbol{v}-\boldsymbol{v}_{\boldsymbol{n}}\right),
$$

where $q(t)$ is the time-dependent grain charge, $m_{d}$ is the dust grain mass, $\boldsymbol{v}_{\boldsymbol{n}}$ is the neutral-gas fluid flow velocity, $\boldsymbol{v}$ is the dust grain velocity, and $\nu_{d n}$ is the dust-neutral collision frequency. It is assumed that the fields $\boldsymbol{E}$ and $\boldsymbol{B}$ are uniform in space, $\boldsymbol{B}$ is along the $\hat{z}$-axis with magnitude $B$, so that the time-evolution of the grain charge can be conveniently described. The equation of motion can be obtained exactly for some conditions when the grain charge is an arbitrary but known function of time, but not for all conditions. The value of $q(t)$ must be inserted at each time step in order to obtain grain trajectories, and this procedure will be explained after general solutions for the grain velocity vector are obtained. The prediction for $q(t)$ for the case of abrupt inhomogeneity is a new result of this dissertation.

Using the Lorentz force equation, differential equations for the $x$ and $y$ velocities of the grain can be found in the plane perpendicular to the magnetic field,

$$
\begin{aligned}
& \frac{\mathrm{d} v_{x}}{\mathrm{~d} t}= \pm \frac{q E_{0 x}}{m_{d}} \pm \frac{q B}{m_{d}} v_{y}-\nu_{d n}\left(v_{x}-v_{n x}\right) \\
& \frac{\mathrm{d} v_{y}}{\mathrm{~d} t}= \pm \frac{q E_{0 y}}{m_{d}} \mp \frac{q B}{m_{d}} v_{x}-\nu_{d n}\left(v_{y}-v_{n y}\right),
\end{aligned}
$$

where $E_{0 x}$ is the electric field along the $\hat{x}$-axis, $E_{0 y}$ is the electric field along the $\hat{y}$-axis, $v_{n x}$ is the neutral gas flow along the $\hat{x}$-axis, $v_{n y}$ is the neutral-gas flow along the $\hat{y}$-axis, and the top signs correspond to the motion for a positively charged grain while the bottom signs correspond to the motion for a negatively charged grain. These two differential equations for $v_{x}$ and $v_{y}$ are coupled, 
but they can be decoupled using the substitution (Taylor, 2005)

$$
\psi=v_{x}+\mathrm{i} v_{y}
$$

where $\psi$ describes the grain velocity in the complex plane. It is also possible to make the substitution $\omega_{c d}(t)=\frac{q(t) B}{m_{d}}$, noting that $\omega_{c d}(t)$ is a function of time, which results in the differential equation

$$
\dot{\psi}= \pm \frac{\omega_{c d}(t)}{B}\left(E_{0 x}+\mathrm{i} E_{0 y}\right)+\nu_{d n}\left(v_{n x}+\mathrm{i} v_{n y}\right)+\left(\mp \mathrm{i} \omega_{c d}(t)-\nu_{d n}\right) \psi
$$

The differential equation 5.5 cannot be solved in general, but it can be solved exactly for several cases. The difficulty is that the parameter $\omega_{c d}$ is time-dependent. The general problem can be treated using the simulation codes included in this dissertation. For the exact case where $E_{0 x} / B=v_{n y}$ and $E_{0 y} / B=-v_{n x}$, the single-grain solution is given by

$$
\psi=A \exp \left(\mp \mathrm{i}\left(\theta(t)-\theta_{0}\right)-\nu_{d n} t\right)+\left(-\mathrm{i} \frac{E_{0 x}}{B}+\frac{E_{0 y}}{B}\right)
$$

where the constant $A$ is determined using the initial conditions, $\theta$ is defined by $\omega_{c d} \equiv \frac{\mathrm{d} \theta}{\mathrm{d} t}$, and $\theta_{0}$ is a constant. The constant $\theta_{0}$ can be associated with the initial gyro-phase of the grain, and $\theta(t)$ can be associated with the gyro-phase angle only in the absence of an electric field. The dependence of guiding center drift on the initial gyro-phase angle is explored in the subsequent sections B and C. This distinction between $\theta$ and the real-time gyro-phase angle $\phi$ will be important in later discussions. Because the case where $E_{0 x} / B=-v_{n y}$ and $E_{0 y} / B=-v_{n x}$ is contrived, unique, and of limited utility, it will not be discussed further.

In the presence of an electric field in the plane to which the magnetic field direction is normal and in the absence of neutral drag $\left(\omega_{c d} \gg \nu_{d n}\right)$, the solution for the differential equation 5.5 is

$$
\psi=A \exp \left(\mp \mathrm{i}\left(\theta(t)-\theta_{0}\right)\right)+\left(-\mathrm{i} \frac{E_{0 x}}{B}+\frac{E_{0 y}}{B}\right)
$$

which is very similar to the solution in equation 5.6 except that there is no assumed time-dependence on the dust-neutral collision frequency. The constant $A$ is determined by the initial conditions, 
assuming $\theta_{0}=\theta(t=0), \psi(t=0)=v_{x}(0)+i v_{y}(0)$, and we have the solution

$$
\begin{aligned}
A & =\left(v_{x 0}-\frac{E_{0 y}}{B}\right)+\mathrm{i}\left(v_{0 y}+\frac{E_{0 x}}{B}\right) \\
v_{x}(t) & =\operatorname{Re}[\psi(t)]=\frac{E_{0 y}}{B}+\left(v_{0 x}-\frac{E_{0 y}}{B}\right) \cos \left(\theta-\theta_{0}\right) \pm\left(v_{0 y}+\frac{E_{0 x}}{B}\right) \sin \left(\theta-\theta_{0}\right) \\
v_{y}(t) & =\operatorname{Im}[\psi(t)]=-\frac{E_{0 x}}{B}+\left(v_{0 y}+\frac{E_{0 x}}{B}\right) \cos \left(\theta-\theta_{0}\right) \mp\left(v_{0 x}-\frac{E_{0 y}}{B}\right) \sin \left(\theta-\theta_{0}\right),
\end{aligned}
$$

where $v_{0 x} \equiv v_{x}(t=0)$, and $v_{0 y} \equiv v_{y}(t=0)$. It is interesting to note that for this solution, retracing gyro-orbits will not occur unless $\left(\sqrt{E_{0 x}^{2}+E_{0 y}^{2}}\right) / B \approx \sqrt{v_{0 x}^{2}+v_{0 y}^{2}}$. Here, retracing means that the grain spends some of its time moving in the opposite direction of the diamagnetic drift vector during a gyro-orbit. The solutions in equations 5.9 and 5.10 may be applicable for grains in some space situations where neutral drag is absent $\left(\omega_{c d} \gg \nu_{d n}\right)$ and the slab geometry is satisfied. These solutions are applicable for a fully ionized plasma, assuming that the ion drag force is also considered negligible. Figure 5.1 shows an example of a retracing trajectory in an abrupt inhomogeneity where drag is absent. In this example, $E_{0 x} /\left(B v_{\perp}\right)=-1$, to ensure a retracing gyro-orbit and to provide an $\boldsymbol{E} \times \boldsymbol{B}$ drift in the $+\hat{y}$ direction. The instantaneous guiding center is correctly represented in figure 5.1 by using $v_{\perp}(t) / \frac{\mathrm{d} \phi}{\mathrm{d} t}$.

The most elucidating application for the results of this dissertation, is the absence of perpendicular electric field and neutral gas flow, but neutral gas is present. Zero perpendicular electric field might be achieved in the laboratory through boundary conditions provided by the use of a multi-disk electrode that can compensate the natural tendency for a non-zero radial potential profile to form in a plasma column (Carroll et al., 1994; Koepke et al., 2008). With these restrictions, it is straightforward to show that the general solution to the differential equation 5.5 becomes

$$
\begin{aligned}
& \operatorname{Re}(\psi)=v_{x}(t)=v_{0 x} \cos \left(\theta-\theta_{0}\right) \exp \left(-\nu_{d n} t\right) \pm v_{0 y} \sin \left(\theta-\theta_{0}\right) \exp \left(-\nu_{d n} t\right) \\
& \operatorname{Im}(\psi)=v_{y}(t)=v_{0 y} \cos \left(\theta-\theta_{0}\right) \exp \left(-\nu_{d n} t\right) \mp v_{0 x} \sin \left(\theta-\theta_{0}\right) \exp \left(-\nu_{d n} t\right) .
\end{aligned}
$$

The specific case where $\nu_{d n}=0$ is shown in figure 5.2. An example where $\nu_{d n} \neq 0$ is shown in figure 5.3 , and this is compared to a $\nu_{d n}=0$ trajectory. The decreasing gyro-radius, due to decreasing $v_{\perp}$ over the course of the gyro-orbit, results in a trajectory with a smaller guiding center drift along 
the $\hat{x}$ direction compared to the $\nu_{d n}=0$ trajectory. In principle, the grain continues to gyrate but the gyro-radius decreases exponentially so that there are practically no magnetized-orbit effects.

In summary, closed form solutions are possible for the conditions $E \neq 0, \omega_{c d} \gg \nu_{d n}$, or for the separate case $E=0$ and $v_{n}=0$. If the grain charge $q(t)$ can be specified, then $\theta(t)$ can be specified and analytical results are possible for the guiding center drift. This is problematic for the use of gyro-phase drift monitoring to diagnose sensitively the sheath mechanisms or other plasma processes in that $q(t)$ depends on the grain motion through inhomogeneous plasma, so $\theta(t)$ is not necessarily known and may change over the course of several gyro-cycles. Northrop and Hill (1983) assumed a sinusoidal charge modulation around some DC offset, but it is clear that $q(t)$ must change over successive gyro-orbits and so the gyro-phase drift must change over time. Furthermore, Northrop's theory only predicts behavior $m_{d} / q_{d}>1 \mathrm{~kg} / \mathrm{C}$. By using the direct solutions to the equation of motion, gyro-phase drift can be established for arbitrary $m_{d} / q_{d}$ ratios.

\section{B Abrupt Inhomogeneity}

In this section, both theoretical and simulation results for an abrupt inhomogeneity will be presented. The semi-analytical method developed here for a dust grain in an abrupt inhomogeneity allows the prediction of the gyro-phase drift vector over many orders of magnitude of plasma conditions, which offers a significant advantage over simulations. The result from Walker et al. (2014) is extended from the drag-absent limit to include Epstein drag (low relative velocity between dust and neutral gas atoms), with the stipulation that gyration occurs, or $\omega_{c d} / \nu_{d n}>1$ (Thomas et al., 2012). Trajectories are assessed for $\omega_{c d} / \nu_{d n}<1$, but they are not particularly interesting because gyration does not properly exist for $\omega_{c d} / \nu_{d n}<1$. An abrupt inhomogeneity is characterized by a plasma parameter having a very small, compared to the size of the dust gyro-radius, transition region between two parameter values. This analysis is possible for two neighboring regions of plasma that are individually homogeneous, with each region having different plasma parameters and hence different in-situ equilibrium charge states (Walker et al., 2014) for a given dust grain. Associate $q_{1}$ with the more negative in-situ charge state and $q_{2}$ with the less negative in-situ charge state. For simplicity and without loss of generality, assume that $x=0$ in the plane perpendicular to the magnetic field direction divides these two regions, so that for $x<0, q=q_{1}$, and for $x>0$, 
$q=q_{2}$. The $i n$-situ equilibrium grain charge values $q_{1}$ and $q_{2}$ can also be associated with in-situ equilibrium dimensionless dust surface potential values $\chi_{e}^{(1)}$ and $\chi_{e}^{(2)}$. The quantities grain charge and dimensionless grain surface potential are equivalently useful for describing the non-stationary state of the dust grain. From a theoretical standpoint, working with dimensionless grain surface potential is easier, but thinking in terms of grain charge is more intuitive. In order to obtain closed-form solutions for the grain's velocity components, it is necessary to know the charge state as a function of time. When $I_{t o t}$ includes all of the relevant charging currents for a given charge model and relevant mechanisms, $\frac{\mathrm{d} q}{\mathrm{~d} t}=I_{t o t}$ yields this function.

In general, this differential equation is non-linear and cannot be solved analytically, largely because the electron current contains a term proportional to $\exp (q / C)$ in most charging models. However, it is possible to approximate the grain charge relaxation with the familiar equations for a discharging/charging capacitor. When the grain discharges or loses electrons to go from $q_{1}$ to $q_{2}$,

$$
q(t)=\left(q_{1}-q_{2}\right) \exp \left(-t / \tau_{2}\right)+q_{2}
$$

where $\tau_{2}$ is the discharging time. Similar to the definition of charging time used by Goree (1994) for the case of a grain going from $q=0$ to the $i n$-situ equilibrium charge state, $\tau_{2}$ is defined as the time elapsed between $q(t=0)=q_{1}$ and $q\left(t=\tau_{2}\right)=q_{1}+\frac{1}{\mathrm{e}}\left(q_{1}-q_{2}\right)$ for a given charge model. To clarify, $\tau_{2}$ is obtained by numerically solving the non-linear differential equation for $q(t)$ for a given charge model, and $q(t)$ is approximated as a discharging capacitor with $\tau_{2}$ as the time constant. Figure 5.4 shows this process. The analytical model shows a reasonably close agreement with the numerical solution to the non-linear charging equation, although the discharging capacitor approximation overestimates the grain charge when $t<\tau_{2}$, and underestimates the grain charge when $t>\tau_{2}$. The characteristic time for the grain to discharge $\tau_{2}$ is different among charging models, which has implications on the resulting gyro-phase drift. Using the definition $\frac{\mathrm{d} \theta}{\mathrm{d} t}=\omega_{c d}$, the quantity $\theta(t)$ can be calculated according to

$$
\theta(t)-\theta_{0}=\frac{B}{m_{d}} \int_{t^{\prime}=0}^{t^{\prime}=t}|q(t)| \mathrm{d} t
$$

Likewise, if the grain starts with $q(t=0)=q_{2}$ and moves into the region where the in-situ- 
equilibrium charge is $q_{1}$, the grain charges more negatively with a characteristic charging time $\tau_{1}$. In general, $\tau_{1} \neq \tau_{2}$. The theoretical approach is valid for arbitrary starting angle $\theta_{0}$ and initial charge $q_{1}$ or $q_{2}$, provided the grain undergoes a sudden transition between the two neighboring homogeneous regions. In the absence of an electric field or ion drag, the quantity $\theta$ from equation 5.14 is the same as the gyro-phase angle $\phi$. The method is illustrated for one gyro-cycle.

Assume that the grain starts at the origin with grain charge $q(t=0)=q_{1}$ and with gyro-phase angle $\theta_{0}=\frac{3 \pi}{2}$ (this starting angle means that the grain's initial velocity is only in the $\hat{x}$-direction). Assuming the grain is negatively charged and the magnetic field is along the $\hat{z}$-direction or out of the page, the grain gyrates counterclockwise and enters the $x>0$ region, where the in-situ equilbrium grain charge is $q_{2}$. As it gyrates, the grain continuously adusts to the new in-situ equilibrium charge $q_{2}$. The quantity $\theta(t)$ can be obtained by integrating $|q(t)|$,

$$
\begin{aligned}
& \theta(t)=\int_{t^{\prime}=0}^{t^{\prime}=t} \frac{\mathrm{d} \theta\left(t^{\prime}\right)}{\mathrm{d} t^{\prime}} \mathrm{dt}^{\prime}+\theta(t=0)=\int_{t^{\prime}=0}^{t^{\prime}=t}\left(\frac{\left|q\left(t^{\prime}\right)\right| B}{m_{d}}\right) \mathrm{d} t^{\prime}+\theta_{0} \\
& \theta(t)=\omega_{2}\left[\tau_{2}\left(\frac{q_{2}-q_{1}}{q_{2}}\right)\left(\exp \left(-\frac{t}{\tau_{2}}\right)-1\right)+t\right]+\theta_{0},
\end{aligned}
$$

where $\omega_{2}=\left|q_{2}\right| B / m_{d}$. The equation for $\theta(t)$ given by equation 5.16 can be inserted into the analytic expressions for the grain motion in the presence of electric field without drag (equations 5.9, 5.10), or for grain motion in the absence of electric field but including drag (equations 5.11 and 5.12).

Ideally, the next step would be to integrate the grain velocity components to obtain the grain trajectory. Unfortunately, the expressions for $v_{x}$ and $v_{y}$ cannot be analytically integrated due to terms involving sinusoidal functions of exponentials, so it is necessary to integrate these expressions numerically. The grain has a guiding center drift in the positive $\hat{x}$-direction due to the presence of neutral drag force, and the grad-q and gyro-phase drifts cease when the ratio $\omega_{c d} / \nu_{d n} \approx 1$. For this scenario, the grain never transitions back to the $q_{1}$ region, i.e., $x(t)$ is positive for all times $t$, and an example trajectory is shown in figure 5.5.

A new $\theta(t)$ must be prescribed if the grain does make a transition to the $q_{1}$ region. Call $t_{2 f}$ the time at which the grain leaves the $q_{2}$ region. The charge state $q(t)$ from equation 5.13 gives the result $q\left(t=t_{2 f}\right)=q_{2 f}$, and so $q_{2 f}$ is the initial charge for the next part of the gyro-cycle. This distinction is necessary because $q_{2 f}$ may not necessarily equal $q_{2}$ due to the time-dependent charge modification to the grain trajectory. For instantaneous charging, $q_{2 f}=q_{2}$, but we are interested in 
the more general case. Likewise, equation 5.18 says that the gyro-phase angle of the grain is given by $\theta\left(t=t_{2 f}\right)=\theta_{2 f}$, which becomes the initial angle for the next part of the gyro-cycle. The charge as a function of time is given by

$$
q(t)=\left(q_{2 f}-q_{1}\right) \exp \left(-t / \tau_{1}\right)+q_{1},
$$

where $\tau_{1}$ characterizes the amount of time needed to attain the charge $q_{2 f}+\frac{1}{\mathrm{e}}\left(q_{2 f}-q_{1}\right)$ for a given charge model. The gyro-phase angle as a function of time for this next part of the gyro-cycle is then given by

$$
\theta(t)=\omega_{1}\left[\tau_{1}\left(\frac{q_{1}-q_{2 f}}{q_{1}}\right)\left(\exp \left(-\frac{t}{\tau_{1}}\right)-1\right)+t\right]+\theta_{0}
$$

where $\omega_{1}$ is the in-situ-equilibrium gyro-frequency in the $q_{1}$ region and $\theta_{0}=\theta_{2 f}$ as explained earlier. Because $\theta_{0}$ can be an arbitrary value, it is important to note that the initial velocity for this next part of the gyro-cycle can have $x$ and $y$ components. Equation 5.18 can be inserted into the analytic expressions for the grain motion in the presence of electric field without drag (equations 5.9, 5.9), or for grain motion in the absence of electric field but including drag (equations 5.11 and 5.12). Again, numerically integrate the resulting grain velocity components to obtain grain trajectories. It is important to note that the grain does not transition back to the $x>0$ half-plane when $\tau_{g} \approx \tau_{c}$, and an example trajectory is shown in figure 5.6. The gyro-phase drift is sufficiently large enough that the grain leaves the region of inhomogeneity, and the gyro-center becomes stationary.

In the absence of drag, the grain will not transition from the $q_{1}$ region to the $q_{2}$ region before $\theta=\frac{7 \pi}{2}$. In other words, the grain only goes from $x<0$ to $x>0$ once during one gyro-orbit. When Epstein drag is present, this is not necessarily true and the grain can go into the $q_{2}$, i.e., $x>0$, region again. In this case, repeat the general procedure by using $t_{1 f}$ to denote the time at which the grain leaves the $q_{1}$ region, use equation 5.17 to obtain the charge on the grain as it leaves the $q_{1}$ region $q\left(t=t_{1 f}\right)=q_{1 f}$, and use the equation 5.18 to obtain the gyro-phase angle as it leaves the $q_{1}$ region $\theta\left(t=t_{1 f}\right)=\theta_{1 f}$. The grain discharges when it reaches the $q_{2}$ region, so $q(t)$ is given by

$$
q(t)=\left(q_{1 f}-q_{2}\right) \exp \left(-t / \tau_{2}^{(\operatorname{Re}-\text { entry })}\right)+q_{2},
$$


where $\tau_{2}^{(\text {Re-entry) }}$ characterizes the time necessary for the grain to attain the charge $q_{1 f}+\frac{1}{\mathrm{e}}\left(q_{1 f}-q_{2}\right)$ using a given charge model. Insert equation 5.16 into the analytic expressions for the grain motion in the absence of electric field but including drag (equations 5.11 and 5.12) after subsituting $q_{1}=$ $q_{1 f}$. This produces the last possible section of the gyro-orbit. The time it takes for the grain to go from $x=0$ to $\theta=\frac{7 \pi}{2}$ is given by $t_{2 f}^{(\operatorname{Re}-\text { entry })}$. Hence, the entire gyro-period is given by $\tau_{g}=t_{2 f}+t_{1 f}+t_{2 f}^{(\operatorname{Re}-\text { entry })}$ because this is the time required for the grain to go from $\theta=\frac{3 \pi}{2}$ to $\theta=\frac{7 \pi}{2}$, or $2 \pi$ in gyro-phase. Figure 5.7 shows an example of this re-entry phenomenon. A code that fulfils the entire procedure outlined in the last few paragraphs is included appendix B.

The dependence on individual trajectories on the gyration parameter $\omega_{c d} / \nu_{d n}$ has been demonstrated for a few specific cases (figures 5.5 5.6, 5.7), but it is instructive to witness the behavior for a wide range of this parameter. Figure 5.8 shows the behavior of guiding center drift magnitude and direction dependence on the gyration parameter $\omega_{c d} / \nu_{d n}$. The gyro-phase drift magnitude increases as $\omega_{c d} / \nu_{d n}$ decreases.

The guiding center drift depends on the initial gyro-phase, even when the initial starting position is held constant. Changing the initial gyro-phase angle indirectly alters the amount of time the grain spends on either side of the UV inhomogeneity. Figure 5.9 shows the dependence of the guiding center drift magnitude and direction on the initial gyro-phase angle $\theta_{0}$ with the maximum amplitude of the gyro-phase drift occurring near $\theta_{0}=270^{\circ}$.

It is now a natural question to ask: how do grain trajectories and guiding center drifts for this abrupt inhomogeneity depend on the charging time? When do the components of the guiding center drifts reach a maximum value, and how does this min-max interval depend on the charging time and gyro-period? The guiding center drift in the $\hat{x}$ and $\hat{y}$ directions are given by

$$
v_{x g c}=\frac{x\left(t=\tau_{g}\right)-x(t=0)}{\tau_{g}}-\left[\frac{v_{\perp}\left(t=\tau_{g}\right)}{\frac{\mathrm{d} \phi\left(t=\tau_{g}\right)}{\mathrm{d} t} \tau_{g}} \cos \left(\phi\left(t=\tau_{g}\right)\right)-\frac{v_{\perp}(t=0)}{\frac{\mathrm{d} \phi(t=0)}{\mathrm{d} t} \tau_{g}} \cos (\phi(t=0))\right],
$$

and

$$
v_{y g c}=\frac{y\left(t=\tau_{g}\right)-y(t=0)}{\tau_{g}}-\left[\frac{v_{\perp}\left(t=\tau_{g}\right)}{\frac{\mathrm{d} \phi\left(t=\tau_{g}\right)}{\mathrm{d} t} \tau_{g}} \sin \left(\phi\left(t=\tau_{g}\right)\right)-\frac{v_{\perp}(t=0)}{\frac{\mathrm{d} \phi(t=0)}{\mathrm{d} t} \tau_{g}} \sin (\phi(t=0))\right],
$$

where $v_{\perp}(t)=\sqrt{v_{x}^{2}(t)+v_{y}^{2}(t)}$, which is not necessarily constant throughout a gyro-orbit, and $\phi(t)$ 
is the actual gyro-phase angle in configuration space. In the absence of an electric field, $\phi(t)$ and $\theta(t)$ are equivalent. In the presence of an electric field, equations 5.16, 5.18, 5.19, and the grain velocity equations are still valid, but $\theta(t)$ no longer describes the gyro-phase angle. In order to get the correct value of the instantaneous gyro-radius in the presence of an electric field, the relationship $\phi(t)=\arctan \left(\frac{v_{y}}{v_{x}}\right)$ must be used, and $R_{L d}=v_{\perp}(t) /\left(\frac{\mathrm{d} \phi}{\mathrm{d} t}\right)$ instead of $v_{\perp} /\left(\frac{\mathrm{d} \theta}{\mathrm{d} t}\right)$. This description is always correct, whether an electric field, drag, or any other forces are present, and this method is used exclusively when analyzing simulation data.

For the entire gyro-period, characterize the gyro-averaged charging time using

$$
\tau_{c}=\tau_{2 f} \frac{t_{2 f}}{\tau_{g}}+\tau_{1 f} \frac{t_{1 f}}{\tau_{g}}+\tau_{2 f}^{(\mathrm{Re}-\text { entry })} \frac{t_{2 f}^{(\mathrm{Re}-\text { entry })}}{\tau_{g}} .
$$

The most natural parameter to probe here is $\tau_{g} / \tau_{c}$, which is shown in figure 5.10, in the absence of drag or an electric field. It is assumed that single-sided UV illumination provides the $\left|q_{2}\right|<\left|q_{1}\right|$ region, although the details will be left to the following sections. For now, we only assume that UV illumination produces a $q_{2} / q_{1}<1$ ratio, although if another plasma parameter can be made suitably abrupt over the course of a grain's gyro-orbit, this approach would also be valid. We see in figure 5.10 that, in the absence of neutral drag, the $x$-component of the guiding center drift is at a maximum when $\tau_{g} / \tau_{c} \approx 1$. The $x$-component of the guiding center drift becomes zero as $\tau_{g} / \tau_{c} \rightarrow \infty$, or when the charging is instantaneous. It also becomes zero as $\tau_{g} / \tau_{c} \rightarrow 0$, because as $\tau_{c}$ increases, the grain charge hardly changes over the course of a gyro-period. We unequivocally see that the $x$-component of the guiding center drift velocity depends on charging rate and we associate this guiding center drift with gyro-phase drift.

When charging is instantaneous, the $y$-component of the guiding center drift is non-zero. We Associate this guiding center drift with the grad-q drift because it arises from charge-state modulation even when charging is instantaneous. The grad-q drift is certainly affected by the charging rate, but in the limit of instantaneous charging, in the absence of drag, and with $\theta_{0}=3 \pi / 2$, it is described by

$$
\boldsymbol{v}_{\nabla q}=2 \frac{v_{\perp}}{\pi}\left(\frac{\left|q_{1}\right|-\left|q_{2}\right|}{\left|q_{1}\right|+\left|q_{2}\right|}\right) \hat{y} .
$$


The figure 5.2 compares the instantaneous charging grain $\left(\tau_{g} / \tau_{c} \rightarrow \infty\right)$ to a non-instantaneous charging grain with $\tau_{g} / \tau_{c} \approx 20$. For the case of the abrupt UV inhomogeneity and instantaneous charging $\left(\tau_{g} / \tau_{c} \rightarrow \infty\right)$, the grad-q drift is given by equation 5.23. Because the grain instantaneously reaches the in-situ equilibrium grain charge, in the absence of drag the grain velocity as a function of time for the first gyro-cycle can be written as

$$
\boldsymbol{v}=\left\{\begin{array}{lll}
v_{x}=v_{\perp} \cos \left(\omega_{2} t\right) & v_{y}=v_{\perp} \sin \left(\omega_{2} t\right) & 0<t<\frac{\pi}{\omega_{2}} \\
v_{x}=v_{\perp} \cos \left(\omega_{1} t-\frac{\omega_{1}+\omega_{2}}{\omega_{2}} \pi\right) & v_{y}=v_{\perp} \sin \left(\omega_{1} t-\frac{\omega_{1}+\omega_{2}}{\omega_{2}} \pi\right) & \frac{\pi}{\omega_{2}}<t<\frac{\pi}{\omega_{1}}+\frac{\pi}{\omega_{2}}
\end{array}\right.
$$

The gyro-radius as a function of gyro-phase angle for instantaneous and non-instantaneous charging is shown in figure 5.12 .

When the charging is not instantaneous, the gyro-period $\tau_{g}$ can be controlled by ratio of the dust grain mass to electron mass $m_{d} / m_{e}$. This is evident when looking at the dimensionless form of the dust gyro-period in electron plasma periods,

$$
\tau_{g}=\frac{2}{3 \sqrt{\pi} \frac{a}{R_{L e}}\left|\chi_{e}\right|\left(1+\frac{a}{\lambda_{D}}\right)\left(1+\frac{T_{e}}{T_{i}} \frac{n_{i}}{n_{e}}\right)^{3 / 2} N_{D e}}\left(\frac{m_{d}}{m_{e}}\right) .
$$

The gyro-period described by equation 5.25 does not include initial gyro-phase or other effects but shows to lowest order how the gyro-period depends on the dimensionless parameters. This affirms that the approximate gyro-period of dust grains is proportial to dust mass, and inversely proportional to the electron magnetization $a / R_{L e}$, dimensionless surface potential $\chi_{e}=e V_{d} /\left(k_{b} T_{e}\right)$, and number of electrons in a Debye sphere or the plasma parameter $N_{D e}$. Also note that $n_{e} / n_{i}$ and $T_{e} / T_{i}$ provide non-trivial dependence. The only one of these parameters which does not enter into every charging model is the parameter $m_{d} / m_{e}$. Because the dimensionless gyro-period is linear with the ratio $m_{d} / m_{e}$, this ratio is a proxy for the gyro-period, and the $m_{d} / m_{e}$ parameter can be swept without directly affecting charging processes from a model perspective. In summary, less massive dust grains gyrate faster, and only the parameter $m_{d} / m_{e}$ allows control of the gyro-period without affecting charging processes, and it is for this reason that $\tau_{g} / \tau_{c}$ sweeps will generally be produced by sweeping this parameter in the range $10^{8} \leq m_{d} / m_{e} \leq 10^{22}$. The semi-analytic method readily permits solutions for this large range, which would be prohibitive with simulations. 
In inhomogeneous plasma, the dimensionless dust grain surface potential varies over the course of a gyro-period, and the quantity $\tau_{g}$ in equation 5.25 is time-dependent. The gyro-period is not actually known until the grain travels $2 \pi$ in gyro-phase. To plot the semi-analytical trajectory, a gyro-period must be chosen before numerically integrating $v_{x}$ and $v_{y}$. The in-situ-equilibrium dimensionless surface potential corresponding to the UV-present condition is chosen to ensure that the grain reaches either the $x=0$ or $\phi=\pi / 2$ condition; using $q(t=0)=q_{2}$ produces the smallest gyro-frequency possible and hence gives the grain ample time to reach either the $x=0$ or $\phi=\pi / 2$ condition. As an example for why this is necessary, consider the case $\tau_{c} \gg \tau_{g}$ and $q_{1} / q_{2}=10$. Using the dimensionless surface potential corresponding to the UV-absent condition, i.e., $q(t=0)=q_{1}$ for the gyro-period prevents the condition $\phi=\pi / 2$ from being attained because the grain only moves until $t=\tau_{1} / 2$, when it needs to at least move until $t=\tau_{2} / 2$, and $\tau_{2}=10 \tau_{1}$. Despite uncertainties, equation 5.25 establishes that the parameter $m_{d} / m_{e}$ can be swept to produce a range of $\tau_{g}$ values without directly affecting $\tau_{c}$. While $\tau_{c}$ does depend on grain radius $a$, and $m_{d}$ depends on grain radius $a$ through $m_{d}=4 / 3 \pi \rho_{d} a^{3}$, using the parameter $m_{d} / m_{e}$ alone to change $\tau_{g}$ without affecting $\tau_{c}$ is permissible because it is possible to use a wide range of material densities $\rho_{d}$. A range of materials and grain sizes should be considered in order to best determine the range of $m_{d} / m_{e}$. A reasonable lower cutoff might be $a=5 \mathrm{~nm}$, while the least dense material possible for grains would be aerogel $\left(\rho_{d}=1 \mathrm{~kg} \mathrm{~m}^{-3}\right)$. A reasonable upper cutoff might be $a=50 \mu \mathrm{m}$ using the element Osmium, the densest known material with $\rho_{d}=22 \times 10^{3} \mathrm{~kg} \mathrm{~m}^{-3}$. This choice of bounds produces $6 \times 10^{8} \leq m_{d} / m_{e} \leq 10^{22}$, slightly more than 13 orders of magnitude. The upper limit is not as relevant to gyro-phase drift, because, as $m_{d} / m_{e}$ gets larger, the charging of a grain crossing an abrupt inhomogeneity is more instantaneous because the grain surface area increases, and this trend does not change as $\tau_{g}$ continues to increase. The lower limit is more relevant to gyro-phase drift and for any set of other parameters chosen, $m_{d} / m_{e}=6 \times 10^{8}$ should be seen as a practical lower limit.

To produce the plot shown in figure 5.10, the ratio $m_{d} / m_{e}$ was swept from $10^{15}$ to $10^{20}$ and the surface properties were assumed to remain constant. Keeping the surface properties constant assumes that the interaction between UV illumination and surface material produces the same photo-electric current and hence the same in-situ equilibrium grain charge. This requirement is imposed so that $q_{1} / q_{2}$ ratios do not change as $m_{d} / m_{e}$ changes, which would otherwise obscure the 
dependence of gyro-phase drift on the $\tau_{g} / \tau_{c}$ ratio. One could imagine coating spherical grains of disparate materials with the same functional material, so that for a given UV flux, the photo-current should be the same. It is also possible to imagine that the UV flux could be changed in tandem with the photo-electric yield and work function of the grain surface so that the photo-electric current remains constant across the range of materials, fixing the $q_{1} / q_{2}$ ratio. For plots featuring variable $\tau_{g} / \tau_{c}$, it will be assumed that the grain surface and $q_{1} / q_{2}$ ratio are considered constant for the abrupt inhomogeneity unless otherwise noted.

Also included in figure 5.10 is the prediction for guiding center drift using Northrop's method. As demonstrated in figure 5.11 for a few different values of $\tau_{g} / \tau_{c}$, the grain charge as a function of time is not sinuisoidally varying in an abrupt inhomogeneity. Undeterred, we can model the grain charge using Northrop's adiabatic theory for dust grains to see the comparison with the semianalytic method. As discussed in appendix A, section 4, the gyro-phase drift vector for grains in slab geometry is given by

$$
\dot{\boldsymbol{R}_{\perp}}=\frac{q_{a} v_{\perp}}{q_{e q}}\left(\hat{x} \sin \phi_{1}-\hat{y} \cos \phi_{1}\right)
$$

where $q_{a}$ corresponds to the amplitude of the sinuisoidal charge modulation during a gyro-orbit around some gyro-averaged value $q_{e q}$, and $\phi_{1}$ corresponds to the angle at which the grain is most negatively charged. In principle, the charge as a function of time can be Fourier decomposed, so that that the capacitor-like charge dependence can be expressed exactly as a function of cosines, $q(t)=q_{0}+q_{a} \cos \left(\phi-\phi_{1}\right)+q_{2} \cos \left(\phi-\phi_{2}\right)+\ldots$ The amplitude for each Fourier component could be obtained and the guiding center averaging technique for adiabatic grain motion from appendix A, section 4 can be used to find the resulting guiding center drift. However, such a procedure is tedious and impractical compared to the method outlined in this section, and it does not immediately lend itself to producing a charging time, an important result of the method of this dissertation. When $q(t)$, calculated using the procedure outlined in this chapter, is fitted with a sinuisoidal approximation, the Northrop prediction in figure 5.10 is obtained. The Northrop prediction offers a reasonable approximation to the more accurate method of directly integrating the analyticallyderived velocity vector. However, in order to make a prediction based on Northrop's method, the grain charge $q(t)$ must be known, which was only possible through the use of the abrupt theory 
developed in this dissertation.

Figure 5.13 shows the dependence of the gyro-phase drift magnitude and direction when an electric field is present and drag is absent. In this figure, $m_{d} / m_{e}$ is swept from $10^{10}$ to $10^{20}$, and $E_{0 x} /\left(B v_{\perp}(t=0)\right)=-1$, where $v_{\perp}(t=0)$ signifies the perpendicular velocity of the grain at the start of the gyro-orbit, which is $v_{\perp}=\sqrt{v_{0 x}^{2}+v_{0 y}^{2}}=v_{0 x}$. The electric field is chosen along $-\hat{x}$ so that the $\boldsymbol{E} \times \boldsymbol{B}$ drift is in the $\hat{y}$-direction, and the $E_{0 x} /\left(B v_{0 x}\right)$ ratio is kept relatively small so that the retracing gyro-orbit is evident, but neither of these conditions are required. In the presence of an electric field, the grain trajectory is still modified by the time-dependent charging of the grain, and we see again that for high $\tau_{g} / \tau_{c}$ ratios that there is no charging-rate modification to the trajectory. The grain drifts with only the grad-q and $\boldsymbol{E} \times \boldsymbol{B}$ drift when $\tau_{g} / \tau_{c} \rightarrow \infty$. Also apparent in the bottom panel of figure 5.13 is that the guiding center angle does not deviate far from $90^{\circ}$, or from the $\hat{y}$ direction. The addition of an electric field enhances the drift along the $\hat{y}$-direction and exaggerates the $\hat{x}$-direction excursion during the gyro-orbit.

The ratio of in-situ equilbrium grain charges on either side of the inhomogeneity $q_{1} / q_{2}$ has a strong effect on both grad-q and gyro-phase drift, as demonstrated in figure 5.14 in the absence of an electric field and neutral drag. For small values of $q_{1} / q_{2}$, the grad-q and gyro-phase drifts are small. When $q_{1} / q_{2}$ increases, grad-q and gyro-phase drifts increase. For large enough values of $q_{1} / q_{2}$, the grad-q drift behaves non-monotonically in that it does not achieve a maximum amplitude when $\tau_{g} / \tau_{c} \rightarrow \infty$. The dependence of the guiding center drift on the ratio $q_{1} / q_{2}$ is shown in figure 5.14 for four different values of $q_{1} / q_{2}$. As the $q_{1} / q_{2}$ ratio increases, the $x$-component of the guiding center drift, i.e., the charging rate component of guiding center drift, increases and the peak of $v_{x g c}$ shifts toward smaller values of $\tau_{g} / \tau_{c}$. This happens because as $q_{1} / q_{2}$ increases, the gyro-averaged quantity of dimensionless surface potential $\chi_{e}$ increases, and the gyro-period is inversely proportional to $\chi_{e}$, shown clearly in equation 5.25. The dimensionless surface potential also determines the charging rate, and while the charging time increases as $\chi_{e}$ increases, this dependence is weak. Hence, the decreasing values of $\tau_{g}$ win out, and the ratio $\tau_{g} / \tau_{c}$ decreases, shifting the entire graph to lower values of this ratio as $q_{1} / q_{2}$ increases.

The ratio $m_{e} / m_{i}$ is another important parameter, and we will determine how gyro-phase drift depends on this quantity. The ratio $m_{e} / m_{i}$ helps to determine charging rate and in-situ equilibrium charge, but makes no contribution to the gyro-period. Figure 5.15 shows how the gyro-phase drift 
magnitudes change when the $m_{e} / m_{i}$ ratio is varied, and $q_{1} / q_{2}=2$ is kept constant. It is apparent from figure 5.15 that $m_{e} / m_{i}$ makes no difference to the guiding center drift magnitude dependence, making it is clear that lower values of $m_{e} / m_{i}$ allow access to smaller values of $\tau_{g} / \tau_{c}$.

Figure 5.16 shows guiding center drift magnitudes for varying $m_{e} / m_{i}$ ratios, but the UV photocurrent is kept constant. Again, a lower $m_{e} / m_{i}$ ratio allows access to lower values of $\tau_{g} / \tau_{c}$, but the drift magnitudes are also larger. This is because lower $m_{e} / m_{i}$ ratio reduces the ion current, meaning the charge variation over the course of a gyro-orbit is larger, and the $q_{1} / q_{2}$ ratio is larger.

The ratio of $T_{e} / T_{i}$ determines both grain charge and to an extent, charging time. Unlike the ratio $m_{e} / m_{i}$, the parameter $T_{e} / T_{i}$ does make a direct contribution to the gyro-period, and it is also important for grain charging. Figure 5.17 shows how the gyro-phase drift magnitudes change when the $T_{e} / T_{i}$ varies, and $q_{1} / q_{2}=2$ is kept constant. Like $m_{e} / m_{i}$, the parameter $T_{e} / T_{i}$ does not affect the guiding center drift dependence, but it does allow access to different regions of $\tau_{g} / \tau_{c}$. Higher values of $T_{e} / T_{i}$ allow access to lower values of $\tau_{g} / \tau_{c}$ in a much more dramatic fashion than seen due to lower $m_{e} / m_{i}$ ratios. According to equations 5.25 and 3.7, higher values of $T_{e} / T_{i}$ decrease the gyro-period and decrease the time for the grain to gain or lose one electron. However, in equation 5.25 the dependence on the temperature ratio goes like $\left(1+\frac{T_{e}}{T_{i}} \frac{n_{i}}{n_{e}}\right)^{-3 / 2}$, while in equation 3.7 , the time interval needed to gain or lose one electron has a dependence that goes like $\left(1+\frac{T_{e}}{T_{i}} \frac{n_{i}}{n_{e}}\right)^{-1}$. Hence, the gyro-period wins out over the charging time which leads to smaller values of $\tau_{g} / \tau_{c}$.

Figure 5.18 shows the affect of $T_{e} / T_{i}$ ratio on guiding center drift magnitudes with a constant photo-current for each temperature ratio. Notice that smaller $T_{e} / T_{i}$ ratios produce greater gyrophase drift magnitude, but at the cost of reducing the range of accessible $\tau_{g} / \tau_{c}$ values. Lower $T_{e} / T_{i}$ ratios lead to more negative grain charge, and hence greater charge modulation over a gyro-orbit. So while a lower $T_{e} / T_{i}$ ratio produces a greater gyro-phase drift magnitude, larger values of $T_{e} / T_{i}$ permit lower $\tau_{g} / \tau_{c}$ values when all other plasma parameters are kept constant. Ultimately, we want as much access to these lower values to ensure that gyro-phase drift exists. I will generally use $40<T_{e} / T_{i}<200$ in the ensuing plots to ensure access to these lower values, which is a perfectly valid range for glow discharge and RF discharge plasmas.

The plasma parameter $N_{D e}$ affects charging time and gyro-period. Figure 5.19 shows the dependence of guiding center drift on the plasma parameter or number of electrons per Debye sphere. Higher values of $N_{D e}$ allow access to lower values of $\tau_{g} / \tau_{c}$, as is evident in this figure. 
Figure 5.20 demonstrates that guiding center drift magnitude and direction is the same for all values of $N_{D e}$. Note that $N_{D e}$ only affects $\tau_{g} / \tau_{c}$ ratios but not grain charge. Along with the mass ratio $m_{d} / m_{e}, N_{D e}$ helps determine $\tau_{g} / \tau_{c}$.

Figure 5.21 shows the resulting guiding center drifts when the $a / R_{L e}$ parameter is varied for constant UV photocurrent. Higher values of this parameter allow access to lower $\tau_{g} / \tau_{c}$ values, which is evident in equation 5.25 because the gyro-period is inversely proportional to the electron magnetization parameter. Additionally, the gyro-phase drift magnitude increases with this parameter; this is because the current collection regime goes from unmagnetized electron and ion current collection at very low values of $a / R_{L e}<1$ (the first two plots), to the magnetized electron and unmagnetized ion current collection regime (the next two plots), and finally to the magnetized electron and ion current collection regime. The general trend is that as $a / R_{L e}$ increases, the gyrophase drift magnitude and direction change because the charge modulation becomes greater. For the $a / R_{L e}>1$ but $a / R_{L i}<1$ regime, the electron current decreases because the collection area of the sphere for electrons becomes $2 \pi a^{2}$. When nearing $a / R_{L i}>1$, the impact parameter for ions no longer depends on the grain surface potential and the ion current is simply the thermal flux along the magnetic field lines. This drastic reduction in ion current produces the greatest charge modulation during a gyro-orbit. Electrons generally become magnetized before ions as the magnetic field is increased, unless $T_{i}>>T_{e}$. For the OML model, shown in figure 5.25, the change among these regimes is abrupt, or not generally considered. The model of Patacchini et al. (2007) allows the $a / R_{L e}$ parameter to vary gradually, although $a / R_{L i}$ is not considered.

Figure 5.22 shows the resulting guiding center drifts for $\omega_{c d} / \nu_{d n}=10^{4}, 10^{3}, 10^{2}$, and 10 . Figure 5.8 already showed the guiding center drifts for variable $\omega_{c d} / \nu_{d n}$ ratio at a fixed value of $m_{d} / m_{e}$. For large values, the drag force is absent and has no effect on grain trajectories. There is very little difference between the $\omega_{c d} / \nu_{d n}=10^{3}$ and $\omega_{c d} / \nu_{d n}=10^{4}$ trajectories, and larger ratios will produce the same guiding center drift magnitudes. As this parameter decreases to 100 or less, changes to the guiding center drift magnitudes in both directions are evident. The $x$-component of the guiding center drift begins to have a positive component for large values of $\tau_{g} / \tau_{c}$. Hence, there is an inherent drift in the positive $\hat{x}$-direction solely due to the drag force decreasing the gyro-radius of the grain. This inherent drift can be overcome by gyro-phase drift in the negative $\hat{x}$-direction for values $\tau_{g} / \tau_{c} \approx 1$. At some value of $\tau_{g} / \tau_{c}$, the gyro-phase drift and the inherent drift due to neutral 
drag will match, which is seen near $\tau_{g} / \tau_{c}=10$ in figure 5.22. In terms of relevant parameters, the gyration ratio $\omega_{c d} / \nu_{d n}$ from equation 3.60 can be rewritten

$$
\frac{\omega_{c d}}{\nu_{d n}}=\frac{3}{4}\left(\frac{\lambda_{D}}{a}\right)^{2}\left(1+\frac{n_{i}}{n_{e}} \frac{T_{e}}{T_{i}}\right)\left(1+\frac{a}{\lambda_{D}}\right) \sqrt{\frac{m_{e}}{m_{i}}} \sqrt{\frac{T_{e}}{T_{n}}} \frac{a}{R_{L e}} \frac{n_{e}}{n_{n}}\left|\chi_{e}\right|
$$

Because $\chi_{e}=\chi_{e}(t)$, the gyration ratio can vary over a gyro-orbit. This ratio is dependent on a few other parameters, such as the mass ratio of electrons to ions, the electron magnetization parameter, the ratios of electron to ion and neutral temperatures, the size of the Debye length relative to the grain radius $\lambda_{D} / a$, and the electron to neutral gas density. These last two parameters are important since they correspond to dust grain size and ionization fraction respectively; for large enough values of $\lambda_{D} / a$ and $n_{e} / n_{n}$, the drag force becomes negligible. It should also be noted that the $\omega_{c d} / \nu_{d n}$ values in figure 5.22 should be seen as approximate values, because the grain charge changes during a gyro-orbit and so the ratio $\omega_{c d} / \nu_{d n}$ also changes.

\section{Charging Model Comparisons with Sheath Mechanisms}

In this section, the OML charging model, the Patacchini et al. (2007) charging model, and the Gatti and Kortshagen (2008) charging model will be assessed for a wide range of plasma parameters and for the sheath mechanisms of ion flow including both mono-energetic ions and drifting Maxwellian ions, ion-neutral charge exchange, dust-neutral drag, and electron magnetization. In order to study the effect of the Gatti-Kortshagen charging model on grain trajectory for the abrupt inhomogeneity, the neutral gas pressure must be non-zero. In order to compare all three models for regimes where ion-neutral charge exchange effects might be important, neutral drag will be present, and it is for this reason, and also the presence of neutral drag in the MDPX (Thomas et al., 2012, 2013), that it is necessary to include drag in the analysis. Assume that $\omega_{c d}>\nu_{d n}$ as before, since the trajectories where gyro-motion occurs comprise the interesting cases. In order to restrict the analysis to the analytically tractable regime, it is necessary to enforce the condition that $E=0$, which might be achieved in a laboratory plasma through the use of a multi-disk electrode (Carroll et al., 1994; Koepke et al., 2008). The ion-neutral charge exchange in the dust sheath mechanism can be analyzed for low drag situations, but such an analysis is not very realistic or relevant to experiment because grain gyration ratio $\omega_{c d} / \nu_{d n}$ is marginal for experimentally-achievable values of $\mathrm{Kn}_{R 0}$. 
Figure 5.23 shows the dependence of guiding center drift upon the Knudsen number $\lambda_{i} / a$. We see that while the Patacchini-Hutchinson and OML models are in complete agreement throughout this large range of Knudsen numbers, the Gatti-Kortshagen charging model differs from both of these greatly, with a minimum drift seen near $\lambda_{i} / a \approx 10^{3}$. This is no coincidence; as stated earlier, the Knudsen capture radius parameter is near unity when $\lambda_{i} \approx \lambda_{D}$, and this corresponds to the region where the collision-enhanced current is largest. In figure $5.23, \lambda_{D} / a=10^{3}$. The enhanced ion current reduces charge state modulation during a gyro-orbit, and so the gyro-phase drift decreases. Also note that the Gatti-Kortshagen charging model is different from the OML and Patacchini-Hutchinson models for all of the Knudsen numbers shown; for $\lambda_{i} / \lambda_{D} \gg 1$ or $\lambda_{i} / \lambda_{D} \ll 1$ the Gatti-Kortshagen charging model should give the same results as the other two models. In a laboratory experiment where gyro-motion occurs, $\lambda_{i} / \lambda_{D} \geq 10^{2}$ is a reasonable expectation. The high value of this ratio is a consequence from the need to have a small enough neutral gas pressure so that the neutral drag force does not dominate the grain dynamics. Even when $\lambda_{i} \neq \lambda_{D}$, figure 5.23 unequivocally displays that the enhanced current due to ion-neutral charge exchange changes the character of the gyro-phase drift dramatically. Indeed, even when the ion mean free path $\lambda_{i} / a=10^{4}$, the plasma is collisionless yet the effect of ion-neutral charge exchange on guiding center trajectory is quite pronounced. Because $\lambda_{i} / a$ does not exist as a parameter in the OML model or Patacchini-Hutchinson models, we see no change in the gyro-phase or grad-q drift for these models over a broad range of $\tau_{g} / \tau_{c}$ values. Figure 5.24 shows a similar style of plot, but here $a / R_{L e}=1$ and we see pronounced differences among the three charging models throughout the range of collisionality.

Figure 5.25 shows how the guiding center drifts depend on the electron magnetization number $a / R_{L e}$ for $\lambda_{i} / \lambda_{D}=10^{2}$. Note that Gatti-Kortshagen generally never agrees with either the OML or Patacchini-Hutchinson models because the ion-neutral charge exchange mean free path and the Debye length are dissimilar, i.e., $\lambda_{i} / \lambda_{D}=10^{2}$ for figure 5.25. As mentioned earlier, if $\lambda_{i} / \lambda_{D} \gg 1$ or $\lambda_{i} / \lambda_{D} \ll 1$ then we do not expect significant enhanced ion current due to ion-neutral charge exchange and, in these limits, the Gatti-Kortshagen charge model will produce the same results as OML. Large and small values of the $a / R_{L e}$ parameter produce nearly identical plots for OML and Patacchini-Hutchinson, but for $1<a / R_{L e}<10$, there is some deviation between them. The OML and Gatti-Kortshagen charging models are described by the magnetized electron charging regime 
at $a / R_{L e}=1$, which reduces electron current on both sides of the abrupt inhomogeneity. So, while electron magnetization produces a less negative in-situ equilibrium grain charge on both sides of the inhomogeneity, the presence of UV illumination has not changed so that the grain charges much less negatively when it is in the UV-absent region. This means that the $q_{1} / q_{2}$ ratio has increased.

We are left to discuss the role of the $\lambda_{D} / a$ parameter as it concerns gyro-phase drift for all three models. Figures 5.26 and 5.27 show the dependence of the guiding center drift magnitudes on $\lambda_{D} / a$ for $a / R_{L e}=10^{-1}$ and $a / R_{L e}=1.1$ respectively.

There is more to the story for the $\lambda_{D} / a$ parameter, however. All three of the charging models have a different response to this parameter as shown in figure 5.28 since this parameter is important for determining charging time. Sweeping the $\lambda_{D} / a$ parameter results in a $\tau_{g} / \tau_{c}$ sweep, even though the ratio of $m_{d} / m_{e}$ is kept constant. Fluctuations seen at small values of $\tau_{g} / \tau_{c}$ happen because the charging time is so much smaller than the gyro-period, and the fact that grain charge can be off by up to one electron causes this problem in the graph. Figure 5.28 demonstrates, with all other parameters unchanged, that gyro-phase drift discriminates the three charge models. Gyro-phase drift may permit a direct measurement of $\lambda_{D} / a$ for a given charge model, which is discussed later in chapters VI and VII.

Figure 5.28 shows what happens when $\lambda_{D} / a$ is varied, assuming all other important parameters such as $\lambda_{i} / a, a / R_{L e}$, and $m_{d} / m_{e}$ are kept constant. The only way to vary the $\lambda_{D} / a$ parameter is to change the size of the dust grain $a$ if the plasma conditions are kept constant, such as electron/ion temperature or electron density. This can be done self-consistently by noting that if $\lambda_{D} / a$ changes by some factor $r_{\text {fact }}$, defined as $\lambda_{D} / a=r_{\text {fact }}\left(\lambda_{D} / a\right)_{0}$, where $\left(\lambda_{D} / a\right)$ is pre-specified. In this case, $\left(\lambda_{D} / a\right)_{0}=100$. Likewise, $\lambda_{i} / a=r_{\text {fact }}\left(\lambda_{i} / a\right)_{0}, R_{L e} / a=r_{\text {fact }}\left(\lambda_{D} / a\right)_{0}\left(R_{L e} / \lambda_{D}\right)$, and $m_{d} / m_{e}=$ $\left(m_{d 0} / m_{e}\right) / r_{\text {fact }}^{3}$. By adopting idealized assumptions for grain uniformity and shape, we gain the convenience of linking parameter scans to the quantity $r_{\text {fact }}$.

Figure 5.29 shows the results when $\lambda_{D} / a, \lambda_{i} / a, a / R_{L e}$, and $m_{d} / m_{e}$ all vary self consistently, assuming that $\omega_{c d} / \nu_{d n} \approx 10$ still holds. The sharp discontinuity in the OML and Gatti-Kortshagen models immediately jumps out in these plots, and this occurs near $\lambda_{D} / a=10^{3}\left(r_{\text {fact }}=1000\right)$ in figure 5.29. This discontinuity is simply due to each of these two models treating electrons as being either unmagnetized in one regime and magnetized in another regime with no smooth variation between the two regimes. Because the Patacchini-Hutchinson model has a smooth transition between 
electron magnetization regimes, this discontinuity is absent. For sufficiently large $\lambda_{D} / a\left(>10^{5}\right)$ values, there is little difference between the models, but smaller values of $\lambda_{D} / a(<100)$ show a difference between all charge models. The $\lambda_{D} / a$ parameter still discriminates one charge model from each another.

An important point that should be made here is that because the guiding center drift is sensitive to the $\lambda_{D} / a$ parameter, gyro-phase drift permits measurement of the shielding length $\lambda_{D}$, assuming the dust grain size $a$ is readily adjustable. This allows the possibility to test different models of the grain sheath. For example, Daugherty et al. (1992), in the presence of ion flow, the Debye length is given by

$$
\lambda_{D}=\sqrt{\frac{\epsilon_{0}}{e^{2} n_{0}}\left(\frac{1}{k_{b} T_{e}}+\frac{1}{2 E_{i}}\right)^{-1}}
$$

where $E_{i}$ is the ion energy. For grains levitating near the sheath edge, ions flow at the Bohm speed so therefore $E_{i}=\frac{1}{2} k_{b} T_{e}$. The ion term in equation 5.28 depends on electron temperature instead of ion temperature, which indicates that the shielding length is much larger than if we assumed $E_{i}=\frac{1}{2} k_{b} T_{i}$

In a laboratory experiment, dust grains levitate at or near a planar sheath boundary, which implies that flowing ions are present. The ion population is either mono-energetic if the DC glow discharge plasma is collisionless on the length scale of the planar sheath size, or a drifting MaxwellBoltzmann distribution if the DC glow discharge plasma is collisional on the length scale of the planar sheath size. The presence of flowing ions modifies ion current, and modifies the grain sheath size because the ion kinetic energy is no longer accurately represented by the random thermal energy $T_{i}$ in the plasma. Instead, $T_{i} \approx T_{e}$ better represents ion kinetic energy near the planar sheath edge. In the ensuing paragraphs, we show that the inherent planar sheath mechanism of flowing ions also affects the gyro-phase drift magnitude and direction for dust grains. The flowing ions can be treated as mono-energetic, which is consistent with dust suspended in a collisionless Child-Langmuir sheath, or the flowing ions can be treated as a drifting Maxwellian population, which should be consistent with a weakly-collisional Child-Langmuir sheath. Gyro-phase drift distinguishes these models based on this planar sheath mechanism.

Figures 5.30 and 5.31 show how the guiding center drifts depend on the thermal Mach number 
for drifting Maxwellian ion populations in the case of electron magnetization parameters of $a / R_{L e}=$ $10^{-1}$ and $a / R_{L e}=1.1$, respectively. In these figures, the ion flow speed has been normalized to the Bohm speed; this differs by a factor of $\sqrt{\frac{T_{e}}{2 T_{i}}}$ from the thermal Mach number. The Bohm speed is a natural parameter to use, since the ion flow speed is equal to the Bohm speed at the sheath edge where dust grains levitate. Also for both of these figures, $\lambda_{i} / \lambda_{D}=10^{2}$ so that the effect of ion-neutral charge exchange collisions is non-negligible, but not at its largest value. In figure 5.30, OML and Patacchini-Hutchinson are indistinguishable as far as ion flow speed is concerned, but Gatti-Kortshagen is quite different for most values. For high enough values of $v_{i} / v_{t h i}$, the Gatti-Kortshagen charge model will produce the same results as the other two models, because the high flux of ions is sufficient to dominate all other charging processes. The OML and PatacchiniHutchinson models have the highest increased charge-state modulation, hence largest gyro-phase drift magnitudes for thermal Mach numbers $<10^{2}$.

When considering magnetized electrons or $a / R_{L e}=1.1$ as in figure 5.31 , the picture is different. None of the models consistently agree with each other throughout the full range of ion thermal Mach number. The OML and Patacchini-Hutchinson models never agree, while OML and GattiKortshagen models agree for very large thermal Mach numbers.

Figures 5.32 and 5.33 show how the guiding center drifts depend on the thermal Mach number with mono-energetic ions for the electron magnetization parameters of $a / R_{L e}=10^{-1}$ and $a / R_{L e}=$ 1.1, respectively. Again, the ion flow speed has been normalized to the Bohm speed. The qualitative differences between mono-energetic and drifting Maxwellian ions are substantial when all other parameters are equal. The peak in the $x$-component of the guiding center drift velocity occurs at much lower values than for the drifting Maxwellian ion case. In figure 5.32, the Gatti-Kortshagen charging model shows almost no charging-rate modifications to guiding center motion. In figure 5.32, the OML and Patacchini-Hutchinson models cannot be distinguished, but they are clearly quite different when $a / R_{L e}=1.1$, as shown in figure 5.33 .

Figure 5.34 offers a comparison among the three charge models for drifting Maxwellian ions and mono-energetic ions. We see that the guiding center drift magnitudes differ within a charge model for mono-energetic or drifting Maxwellian ion flow. Gyro-phase drift thus discriminates between mono-energetic or drifting Maxwellian ion flow within a charge model.

Figure 5.35 shows a comparison between simulation and the semi-analytical theory, using drift- 
ing Maxwell-Boltzmann ions and other parameters similar to the conditions present in MDPX. Both simulation and theory suggest that the OML and Patacchini-Hutchinson charge models are indistinguishable for these conditions, but the Gatti-Kortshagen charge model is different. In this plot, the reasonable agreement between theory and simulation validates the semi-analytical approach.

\section{Gradual Inhomogeneity}

The analytical description for guiding center velocity components shown in section A are still valid for a gradual inhomogeneity, although specifying $q(t)$ a priori and hence $\theta(t)$ can be difficult. The solutions in equations $5.9,5.10,5.11,5.12$ from section A demonstrate that the grain velocity components $v_{x}$ and $v_{y}$ only vary linearly with $v_{0 x}$ or $v_{0 y}$; guiding center drift is proportional to the perpendicular velocity components. This is still true for the gradual inhomogeneity. However, for a gradual inhomogeneity, grain charge modulation depends on gyro-radius, which in turn does depend on the velocity perpendicular to the magnetic field direction.

In the case of sinusoidal variation of grain charge, in a gradual inhomogeneity the approach of (Northrop and Hill, 1983) suffices to describe the gyro-phase drift as long as $m_{d} / q \ll 1 \mathrm{~kg} / \mathrm{C}$ and the amplitude of charge modulation during a gyro-orbit is much less than the in-situ equilibrium grain charge evaluated at the guiding center. With simulations, it is possible to explore regimes where $m_{d} / q \ll 1 \mathrm{~kg} / \mathrm{C}$ and $m_{d} / q \gg 1 \mathrm{~kg} / \mathrm{C}$ and compare these to the Northrop prediction, shown in equation A.80. Many types of gradual inhomogeneities can be postulated, such as inhomogeneous electron or ion temperature, magnetic field, or ion mass composition, but the focus here will be on the inhomogeneous $n_{e} / n_{i}$ ratio. An example of a radial electric field and varying $n_{e} / n_{i}$ ratio in a magnetized plasma column is described analytically by Zimmermann et al. (2010), although it is apparent that $n_{e} / n_{i}$ only changes significantly inside the sheath, and the plasma is quasineutral $\left(n_{e} / n_{i} \approx 1\right)$ outside the sheath. Inhomogeneous $n_{e} / n_{i}$ ratio produces an electric field, but the electric field is ignored in this section to focus on the properties of guiding center drift that are dependent on charging rate.

The results from section B provide a source of intuition for the gradual inhomogeneity case. The idea of the guiding center drift sensitivity to the $\tau_{g} / \tau_{c}$ ratio, because the grain does not immediately reach the in-situ equilibrium grain charge, can also be applied to a gradual inhomogeneity. Here, 
$q(t)$ is no longer described by an exponential function as in the abrupt inhomogeneity case. As a means to artificially delay charging, and force the charging to not be instantaneous regardless of the plasma conditions, an adjustable charging parameter $\alpha$ can be used like in the abrupt inhomogeneity case $q_{n+1}=q_{n}+\Delta q_{n}^{\prime}$, where $\Delta q_{n}^{\prime}=\alpha \Delta q_{n}$. For $\alpha=1$, the dust grain charges and discharges without artificial restriction to what the model otherwise would predict. A problem with this parameter, is that fractional elementary charge is introduced, which is a small nuisance.

\section{Linear Profile}

The single-grain trajectory in a dust-absent plasma using a linear profile of electron and ion densities is computed, assuming an inertial lab frame. We examine the case of a gradual change in the ratio between ion and electron densities, as shown in figure 5.36, with each species characterized by a Maxwellian velocity distribution having a temperature $T_{i}$ and $T_{e}$, respectively. It is evident that figure 5.36 shows an unrealistic profile for a quasi-neutral plasma, but this profile serves to demonstrate how a gradual inhomogeneity in the $n_{e} / n_{i}$ ratio can lead to charge state modulation and hence gyro-phase drift. We also ignore the complicating factor of electric field in this profile to focus on the gyro-synchronous charging/de-charging modulation required for gyro-phase drift.

The parameter $\alpha$ is used to adjust the rate of charge evolution much like how density can be used to adjust the rate of charge evolution. Unlike in Northrop's case (Northrop and Hill, 1983; Northrop et al., 1989), we examine a laboratory relevant scenario where the dust thermal speed is much smaller than the ion thermal speed. For this specific case, the electric field in the plane of the gyro-motion is taken to be zero. The ion drag force, neutral drag force, gravitational force, and other typical forces on a dust grain are ignored for simplicity in this section. The magnetic field is 4T. We assume room-temperature ions $\left(T\left[A r^{+}\right]=0.0025 \mathrm{eV}\right)$ and atoms and $1.6 \mathrm{eV}$ temperature electrons for the sake of modeling the Auburn Magnetized Dusty Plasma Experiment (MDPX) (Thomas et al., 2012). The initial grain speed is oriented in the inhomogeneity direction. We also use $n_{0}=10^{16} \mathrm{~m}^{-3}$ as the background density for $a=0.015 \mu \mathrm{m}$ radius dust grains.

The simulation code is described in chapter IV and appendix B. It is a symplectic, leapfrog integrator which solves the equations of motion resulting from the Lorentz force. Within the larger Newton timestep, an adaptive charging step was used to ensure that only an integer number of charges were collected at a time. It is necessary to include this timestep within the larger Newton 
timestep because, for our choice of parameters, the dust grain will have undergone many charging timesteps during each Newton timestep. Within the charging timestep, the analytical currents for a given charging model are applied to the dust grain. We will focus on OML first before investigating other charging models.

The grain is fully charged in a matter of microseconds for our set of parameters, while the gyro-period is two to three times larger. The grain charges to the in-situ-equilibrium charge at each spatial location during a gyro-orbit for charging time much less than gyro-period. The grad-q drift takes place, but no gyro-phase drift occurs for $a=0.015 \mu \mathrm{m}, n_{0}=10^{16} \mathrm{~m}^{-3}$, and $\alpha=1$. The dust grain charge effectively never changes if $\alpha=0$, no matter how large the current may be to the dust grain. It should be noted that the numerical method does not assume any particle drifts or charge modulation of the dust grain a priori; the integrator solves the equation of motion of the dust grain resulting from the Lorentz force during the Newtonian time step while it computes the ion and electron currents, which are both functions of the dust grain charge, i.e., dust grain surface potential, at each charging timestep. For the Newtonian time step, 2000 points/gyrocycle was used.

Figure 5.37 shows a comparison of different grain trajectories for the gradual $n_{e} / n_{i}$ inhomogeneity (figure 5.36) using the OML model with instantaneous $(\alpha=1)$ and non-instantaneous ( $\alpha=0.0105$ ) grain charging. All trajectories start at $x=0, y=0$, with an initial velocity of $v_{x}=-11, v_{y}=0$ (in units of meters/second). Also at $t=0$, the particle charge and gyroradius for this dust grain is $-43 \mathrm{e}$ and $5.7 \mathrm{~mm}$, respectively. The grain was initialized with the in-situ-equilibrium charge in order to avoid complications involved with the unwanted and irrelevant transient effects. The trajectory of the instantaneous gyro-centers are also plotted, which are represented by the helical lines having smaller radial excursions than the actual grain trajectories. The gyro-averaged guiding centers are along these lines, depicted as squares for the $\alpha=1$ grain trajectory, and diamonds for the $\alpha=0.0105$ trajectory. A density of $1000 \mathrm{~kg} \mathrm{~m}^{-3}$ was chosen for the dust grain, which means that $m_{d} / q_{d} \approx 2 \times 10^{-3} \mathrm{~kg} / \mathrm{C}$, meaning that we can expect the adiabatic approximation for guiding center drift to be valid.

To accurately predict guiding center drift components by using Northrop's adiabatic drift approximation, it is necessary to fit $q(t)$ to a function of the form $q_{0}+q_{1} \cos \left(\phi-\phi_{1}\right)$, where $\phi_{1}$ is the gyro-phase angle at which the grain is most negatively charged. Performing this fit allows us 
to use equation A.80 to determine guiding center drifts for one gyro-cycle, but we should keep in mind that $q_{0}$ and $q_{1}$ are not generally known a priori based on the plasma conditions, and hence predictions offered by Northrop's theory can only be made a posteri. Figure 5.39 demonstrates this process for the grain with $\alpha=0.0105$, although this process can be applied to any grain where the charge modulation is strictly sinusoidal, or nearly so, during a gyro-orbit. The sinuisoidal fit does not perfectly match $q(t)$ for this $a=0.015 \times 10^{-6} \mathrm{~m}, \alpha=0.0105$ grain. For the grain with $\alpha=1$ with $v_{\perp}=11 \mathrm{~m} / \mathrm{s}$, this results in a predicted grad-q drift of $0.775 \mathrm{~m} / \mathrm{s}$, while the value obtained from gyro-averaging the trajectories from the simulation is $0.726 \mathrm{~m} / \mathrm{s}$. For the $\alpha=0.0105$ grain with $v_{\perp}=11 \mathrm{~m} / \mathrm{s}$, the sinusoidal fit or Northrop prediction produces guiding center drift components $v_{x g c}=-0.278 \mathrm{~m} / \mathrm{s}, v_{y g c}=0.58 \mathrm{~m} / \mathrm{s}$, while the simulation produces guiding center drift components $v_{x g c}=-0.21 \mathrm{~m} / \mathrm{s}, v_{y g c}=0.49 \mathrm{~m} / \mathrm{s}$. It is important to note that only the terms from equation A.80 were used, while the grad-q drift mentioned in equation A.71 was not used, and that including the grad-q drift term from A.71 causes erroneous results. Equation A.80 makes predictions consistent with the simulation results for the guiding center drift vector even in the limit of instantaneous grain charging.

The grain charge as a function of gyro-phase for the two different trajectories in figure 5.37 is shown in figure 5.40. For both trajectories, the grain charge at each gyro-phase angle is normalized by the in-situ, equilibrium grain charge that the same grain would attain at the instantaneous guiding-center corresponding to this gyro-phase angle, which can be expressed as $q(\theta) / q_{e q}(x=$ $\left.x_{g c}(t)\right)$. A grain having a fixed, gyro-phase independent, grain charge would be displayed as a circle with radius of 1 in this kind of plot. The evolution of particle charge is tracked for approximately 5 gyro-periods. For the $\alpha=1$ trajectory, the resulting plot in gyro-phase angle is nearly circular in shape, although its center is offset from the origin, as expected for the inhomogeneous-plasma case. During a gyro-orbit, the grain has the fewest number of electrons at $\phi=0$, and the grain is maximally charged negatively at $\phi=\pi$. The discrete steps apparent in this plot, which yields a circular sawblade-like appearance, are representative of a net loss or a gain of 1 electron from the previous, discrete, circular-arc step. Additionally, between these sudden transitions, the grain continuously gains and loses an electron, causing its charge state to fluctuate rapidly between each new pair of neighboring charge states. This fluctuation increases the line thickness $\delta q=\left|q_{n+1}-q_{n}\right|$ to be $e$. During each gyro-cycle, the same pattern is retraced in the $q$ versus gyro-phase angle plot 
for a dust grain having $\alpha=1$. The choice of initial gyro-phase does change the in-situ-equilibrium charge at the guiding center $q_{0}$, but it does not appreciably alter the plots in figure 5.40 .

In contrast, for the $\alpha=0.0105$ polar plot in figure 5.40, the same pattern is not retraced for each gyro-cycle. If both $\alpha=1$ and $\alpha=0.0105$ grains start at $\theta=\pi / 2$ in gyro-phase, the $\alpha=0.0105$ grain will subsequently become undercharged with respect to $\alpha=1$ as both grains follow trajectories in physical space that go into increasingly non-neutral (negative) plasma. As $\theta$ increases past $\theta=\pi$, both grains are leaving the region where the $n_{e} / n_{i}$ ratio is highest. However, because the $\alpha=0.0105$ grain does not immediately reach the in-situ-equilibrium, it stays undercharged with respect to $\alpha=1$ past $\theta=\pi$, reaching a maximum value at $\theta=200^{\circ}$ with standard deviation of $9^{\circ}$ in figure 5.40. This maximum charge state for the $\alpha=0.0105$ grain is between the $\theta=\pi$ charge states of the two grains. The $\alpha=0.0105$ grain is now overcharged with respect to $\alpha=1$ after $\theta=200^{\circ}$. For $\theta>\frac{3}{2} \pi$, both grains are entering the region where the $n_{e} / n_{i}$ ratio is decreasing, and are becoming less negatively charged. The $\alpha=0.0105$ grain does not immediately reach the lower in-situ-equilibrium charge state, achieving its smallest charge state when the two grains again match, at $\theta \approx 20^{\circ}$ with a standard deviation of $1^{\circ}$. The capacitive effects of grain charging that are inherently present in the OML model ensure that the dust grain never reaches the insitu-equilibrium charge during a gyro-orbit if the charging rate is low enough, as is qualitatively demonstrated in figure 5.41 .

The magnitude of the gyrophase drift velocity and the direction with respect to the inhomogeneity direction varies with the value of the charge delay parameter $\alpha$, reaching a peak near $10^{-2}$ (figure 5.41). For the chosen electron density, the no-delay case of $\alpha=1$, the grain charges to the in-situ equilibrium charge much faster than it completes a gyro-orbit, so there is no modification to the particle trajectory beyond the effect of the grad-q drift (which is an effect arising only from gyro-synchronous charge variation). As $\alpha$ is lowered, the gyro-phase drift reaches a peak when the charging timescale is comparable to the gyro-period timescale (Walker et al., 2014). As $\alpha$ is further lowered, the grain changes charge state sufficiently slowly during a gyro-orbit that it does not change charge state enough during a gyro-orbit that negligible modification of the $\alpha=1$ gyromotion occurs and both gyro-phase and grad-q drift become negligible. This is reflected in figure 5.41 , in that the gyro-phase drift magnitude steadily decreases and the direction of the guiding center drift approaches $\theta_{\text {drift }}=\pi / 2$ (using the same polar coordinates) as $\alpha$ is lowered beyond 
$\alpha=10^{-2}$, consistent with the grad-q drift also steadily decreasing.

The gyro-phase drift magnitude and direction sensitivities can be recast in terms of the $\tau_{g} / \tau_{c}$ parameter instead of $\alpha$. Figure 5.42 shows this dependence for the same data as figure 5.41, but for each data point $\tau_{c}$ is calculated in the following way: $\tau_{c}$ is the amount of time for a grain with starting no charge to reach $[1-\exp (-1)] q_{e q}\left(x_{g c}\right)$ for the plasma conditions at the grain's gyro-averaged guiding center, where $q_{e q}$ is the in-situ equilibrium grain charge. In figure 5.42 , this method produces a peak for $\tau_{g} / \tau_{c}$ values between 1 and 10 . This behavior is very similar to the $\tau_{g} / \tau_{c}$ plots seen earlier for the abrupt inhomogeneity. It may seem that $\tau_{c}$ is an unsatisfactory parameter for grain charging time in a gradual inhomogeneity since the charge varies gyro-synchronously for a gradual inhomogeneity and the charging process does not involve a monotonic incrementation of charge. Nevertheless, figure 5.42 demonstrates that casting the sensitivities in terms of $\tau_{g} / \tau_{c}$ produces results that are consistent with the intuition developed for the abrupt inhomogeneity.

It is also possible to modulate certain plasma parameters, such as number density, temperature, and also grain size to provide a range of the $\tau_{g} / \tau_{c}$ parameter. For the figures $5.43,5.44,5.45$, and 5.46, corresponding to grain sizes $a=0.0105, a=0.5, a=5$, and $a=10$ microns, the plasma density $n_{0}$ varies to produce a range of $\tau_{g} / \tau_{c}$. These figures show the trend of both the $x$ and $y$ components of guiding center drift go to zero for very small values of $\tau_{g} / \tau_{c}$ because the charging time is so long that the grain does not appreciably change charge during a gyro-orbit. Figure 5.43 has a rough appearance since charge modulation is a significant proportion of the grain's in-situ equilibrium charge at the gyro-averaged guiding center. The Northrop fit for the $q_{0}$ or $q_{1}$ value may be off by 1 electron, which causes the uncertainty.

Also in all of these figures, both the $x$ and $y$ components of guiding center drift reach zero or start to aproach zero for very large values of $\tau_{g} / \tau_{c}$. The $x$ and $y$ components of guiding center drift approach zero together because the charge state modulation approaches zero, as shown in figure 5.47. There is a very large range of $\tau_{g} / \tau_{c}$ over which the ratio of charge state modulation to in-situ equilibrium charge at the gyro-averaged guiding center $q_{1} / q_{0}$ is flat. Koepke et al. (2013) refers to this regime as instantaneous charging, but for very large values of $\tau_{g} / \tau_{c}$ (even more-instantaneous charging), the $q_{1} / q_{0}$ ratio decreases to zero. This happens because the grain capacitance is described by $C=4 \pi \epsilon_{0} a\left(1+a / \lambda_{D}\right)$, where $\lambda_{D}$ was described earlier in equation 2.7 and depends on electron and ion temperature and electron and ion density. This decrease in $q_{1} / q_{0}$ is impossible to avoid 
for a gradual inhomogeneity in density or temperature, because plasma density and temperature parameters affect the Debye length and hence the grain capacitance, so the qualitative appearance for figures 5.43, 5.44, 5.45, and 5.46 will remain unchanged. An inhomogeneity in ion species composition should not cause this kind of effect because the Debye length has no dependence on ion mass. The change in the $q_{1} / q_{0}$ ratio can be expressed

$$
\frac{q_{1}}{q_{0}}=\frac{q_{\max }-q_{\min }}{q_{\max }+q_{\min }}=\frac{\chi_{e 0}+\chi_{e 1}-\frac{C_{d}\left(\phi_{\max }\right)}{C_{d}\left(\phi_{1}\right)}\left(\chi_{e 0}-\chi_{e 0}\right)}{\chi_{e 0}+\chi_{e 1}+\frac{C_{d}\left(\phi_{\max }\right)}{C_{d}\left(\phi_{1}\right)}\left(\chi_{e 0}-\chi_{e 1}\right)}
$$

where $C_{d}$ corresponds to the gyro-phase-dependent capacitance of the grain, we assume changes to the sheath thickness are nearly instantaneous compared to the grain gyro-frequency, $\phi_{\max }$ corresponds to the angle where the grain is least negatively charged $\left(q=q_{0}+q_{1}\right), \phi_{1}$ corresponds to the angle where the grain is most negatively charged $\left(q=q_{0}-q_{1}\right), \chi_{e 0}$ corresponds to the DC-offset of the dimensionless surface potential variation, and $\chi_{e 1}$ is the amplitude of the dimensionless surface potential variation. Equation 5.29 also determines the $q_{1} / q_{0}$ ratio if only the dimensionless surface potential is known. While the dimensionless surface potential variation, or the ratio $\chi_{1 e} / \chi_{0 e}$ does not change appreciably as the density increases further, the $q_{1} / q_{0}$ ratio changes because the capacitance is gyro-phase dependent due to the spatial variation in $n_{e}$ and $n_{i}$. An important consequence here is that charge modulation can occur if only the quasi-neutral plasma density is inhomogeneous, because this will change the grain capacitance and hence change the grain charge during a gyro-orbit. In other words, the grain capacitance is gyro-phase dependent, and if we define $\zeta=C_{d}\left(\phi_{1}\right) / C_{d}\left(\phi_{\max }\right)$, then

$$
\frac{q_{1}}{q_{0}}=\frac{1-\zeta}{1+\zeta}
$$

A caveat here is that the change in plasma density over the course of a gyro-orbit must be large to appreciably alter the grain charge, and microscopic charging models assume $a / \lambda_{D} \ll 1$. Additionally, as explained by Daugherty et al. (1992), the regime where $a / \lambda_{D} \gg 1$ imply that the grain sheath becomes a Child-Langmuir sheath, so the OML model is not necessarily accurate for these regions of high $a / \lambda_{D}$ or very high $\tau_{g} / \tau_{c}$. 


\section{Cylindrical Geometry}

The addition of cylindrical geometry presents some complications, but it is important to explore the consequences of this geometry since all magnetized-orbit dusty plasma experiments are done in cylindrical machines (Konopka et al., 2005b; Schwabe et al., 2011; Thomas et al., 2012), and because magnetized-orbit dusty plasmas in space will generally exist in a dipole-like magnetic field surrounding a celestial body. The two salient classes of cylindrical geometries are cylindrical devices with inhomogeneities in the laboratory frame, and co-rotating reference frames of a celestial body. Producing radial gradients in plasma parameters with gradient-scale-length comparable to the gyro-radius of grains to produce charge-state modulation in a laboratory plasma is difficult, as will be discussed. It is much easier to find comparable gradient-scale-length and gyro-radius in space (Northrop et al., 1989). Northrop and Hill (1983) have already explored the case of gyrating grains in plasma gradients within the co-rotating frame of a planet, so the focus is on grain trajectories in the laboratory frame of a magnetized cylindrical discharge.

To demonstrate how charging rate effects might affect trajectories in cylindrical geometry, the model cases of constant charge, non-instantaneous charging, and non-instantaneous charging when drag is present are examined. To produce the charge modulation, the linear profile discussed earlier is adapted to cylindrical geometry. Figure 5.48 shows the grain trajectory when grain charge is constant and drag is absent. Figure 5.49 shows the trajectory for the same grain when charging is non-instantaneous, and the introduction of non-instantaneous charging leads to radial transport and $v_{\perp}$ increases over successive gyro-orbits. When drag is introduced in figure 5.50, the radial excursion steadily decreases over many gyro-orbits as $v_{\perp}$ steadily decreases. Grain trajectories in self-consistent electric field and electron density profiles are discussed further in chapter VI.

\section{Gradual versus Step-Function Inhomogeneity}

Having explored the ramifications of gradual and abrupt inhomogeneity in terms of gyro-phase drift, we can draw some comparisons. Both the abrupt and gradual inhomogeneities show a similar peak in the gyro-phase drift when $\tau_{g} \approx \tau_{c}$, even when using $\tau_{c}$ to characterize the time needed to reach in-situ equilibrium charge state for the gradual inhomogeneity. The peak at $\tau_{g} \approx \tau_{c}$ in figures 5.41, 5.42, 5.43,5.44, 5.45, and 5.46 validate this use of $\tau_{c}$. All of these figures used 
gradual density to make the plots, so we can immediately compare this to the results of the abrupt inhomogeneity for the $\lambda_{D} / a$ parameter that was shown earlier in figure 5.28. The results for the gradual inhomogeneity compared to the abrupt inhomogeneity are shown in figure 5.51.

The gradual inhomogeneity has a much smaller guiding center drift amplitude, and this is largely due to the gradual inhomogeneity producing less charge state modulation during a gyroorbit. The best way to compare the gradual and abrupt inhomogeneities is to compare the values of $\chi_{1 e} / \chi_{2 e}$ from the abrupt inhomogeneity to the same values of $\left(q_{0}-q_{1}\right) /\left(q_{0}+q_{1}\right)$ from the gradual inhomogeneity. In this way, there is the same amplitude of charge state modulation for both. It is difficult to produce the same amplitude of charge state modulation and consequently the gyrophase drift magnitude in a gradual inhomogeneity as compared to the abrupt inhomogeneity. More discussions about abrupt and gradual inhomogeneity as applied to MDPX follow in chapter VI. 


\section{Chapter VI}

\section{Applications}

Measuring dust grain motion provides information about the background plasma. Predictions are most useful when a relevant case can be observed or replicated in a laboratory or space setting, so that classes of models might be discarded or improved so that the models are more consistent with observations. The Auburn Magnetized Dusty Plasma Experiment and the dusty environment in the Saturn-Enceladus system provide two regimes of application where dust grain motion can provide clues to plasma processes and grain charging. To solidify the utility of gyro-phase drift for discriminating among charge models and sheath mechanisms, specific behavior must be predicted in space and laboratory plasmas where evidence of the behavior can be documented by measurement. In this chapter, we argue the predicted gyro-phase drift in an abrupt inhomogeneity discriminates among charge models in the Auburn Magnetized Dusty Plasma experiment (MDPX) for ideal conditions. The predicted gyro-phase drift, while present in a gradual inhomogeneity, does not discriminate among charge models in MDPX. The resulting experimental evidence for gyro-phase drift of a gradual inhomogeneity would be outside the limits of detection, but it should reduce dust grain transport of very small grains $(a<0.1 \mu \mathrm{m})$ out of MDPX. Previous to this research, the viability of gyro-phase drift experiments in the laboratory has not been assessed.

\section{A Application to Auburn Magnetized Dusty Plasma Experiment}

The Auburn Magnetized Dusty Plasma Experiment (MDPX) is described in Thomas et al. (2012, 2013), and the relevant parameters are shown in table 1.1. It is important to consider how measure- 
ments can be or will be made in order to determine or document guiding center drift magnitudes and to match up with theory and simulation in some way. Grains can be directly imaged using Particle Tracking Velocimetry (PTV) for a sparsely seeded grain population (dust in plasma condition), or using Particle Imaging Velocimetry (PIV) for a densely seeded grain population (dusty plasma condition) (Boessé et al., 2004). Imaging grain trajectories provides the most information and would be the ideal candidate to study dust dynamics, and this method can provide an immediate, direct comparison between different charging models and planar sheath mechanisms. However, there are certain limitations to the imaging method that must be considered. The imaging that takes place in PTV and PIV uses visible light to image dust grains, so $a \approx 200 \mathrm{~nm}$ is a reasonable lower limit on dust grain size that can be imaged unless shorter wavelength light is used, which has practical inconveniences. Friedel and Greulich-Weber (2006) were able to reliably produce pyrolized carbon spheres as small as $100 \mathrm{~nm}$ in diameter, so it is possible to produce mono-disperse spherical grains with $a \approx 200 \mathrm{~nm}$ for experiments.

Extremely small grains $(a<100 \mathrm{~nm})$ possess a much higher value of $\omega_{c d} / \nu_{d n}$, as discussed later in section A, subsection 1 and they also have a much slower charging rate. The slower charging rate makes sub-micron grains ideally suited for studying charging rate modification to grain motion, but there is a penalty - these small grains cannot be imaged using the standard PTV or PIV techniques or any other established method. Experiments that use grains too small to image with PIV require probe techniques (Barkan et al., 1994).

For a collection of dust grains for a dust-in-plasma situation, the guiding center drift will vary as a function of grain size, so details of the size distribution is important. As far as making predictions for gyro-phase drift consistent with experimental conditions, it is necessary to account for the grain size distribution. It would be easiest to work with mono-disperse grains, so that the dust parameters are the same for all grains in a dust in plasma situation. The synthesis results of Friedel and Greulich-Weber (2006) suggests that a mono-disperse dust population is feasible for dust experiments.

To restrict grains to move only in the plane perpendicular to $\boldsymbol{B}$, an electric field is necessary to levitate the dust grains, which is provided by a planar sheath. The planar sheath results in a flow of ions to the planar electrode; charging models must include ion flow to be accurate. Additionally, predictions should be made for both mono-energetic and drifting Maxwellian ion flow, with the 
expectation that gyro-phase drift may distinguish between them.

\section{Accessible Parameter-Space Regimes in MDPX}

An important consideration, discussed by Kählert et al. (2012); Thomas et al. (2012) is that gyromotion does not formally exist when $\omega_{c d} / \nu_{d n}<1$. This was also demonstrated earlier in this dissertation with figures 4.5 and 5.8. Another important result is that the dust-dust collision frequency must be much smaller than the dust gyro-frequency. The limit where $\omega_{c d} / \nu_{d n}>1$ is a valid regime for tracking the grain over many successive gyro-periods. The gyration ratio was

discussed earlier in chapter $\mathrm{V}$, but is rewritten here to rely on parameters that are more useful from an experimenter's perspective, resulting in the expression,

$$
\frac{\omega_{c d}}{\nu_{d n}}=\frac{3 \pi \epsilon_{0}\left(1+\frac{a}{\lambda_{D}}\right) B \sqrt{\frac{8 e T_{n}}{\pi m_{n}}} T_{e}\left|\chi_{e}\right|}{8 a P_{n}}
$$

where $P_{n}$ is the neutral gas pressure. Note that the gyration ratio depends on dust grain size, and not explicitly on the dust grain's mass. From an experimenter's perspective of DC glow and RF Discharges, only $P_{n}$ is easily controlled, $a$ can reasonably be controlled, $B$ can be controlled but is limited to a practical DC maximum of $4 \mathrm{~T}$ (Thomas et al., 2012), and only weak variation over a small range of $T_{e}$ or $T_{n}$ is possible. The absorbed $\mathrm{RF}$ power can be adjusted to change $T_{e}$, and also $n_{e}$ and $n_{i}$, for an RF discharge plasma. Beyond the gyration ratio, the plasma densities $n_{e}$ and $n_{i}$ are important parameters because they determine charging rate. These $n_{e}$ and $n_{i}$ densities, in combination with dust grain size, alter a trajectory when the densities are small enough to limit charging rate enough for gyro-phase drift to exist.

The dimensionless surface potential, $\chi_{e}=e V_{d} /\left(k_{b} T_{e}\right)$, cannot be easily controlled and, due to the presence of planar and grain sheath mechanisms, is not typically known to great precision. Also, due to non-stationary charging, the dimensionless surface potential felt by the grain can change with time or during a gyro-orbit, which means that the gyration ratio can change during a gyro-orbit. We can use the result from OML theory, using an equlibrium in-situ charge, to get an estimate for the dimensionless surface potential.

For analyzing magnetized-orbit dust in MDPX, the DC magnetic field must be as large as the device allows $(4 \mathrm{~T})$, while the dust grain size and neutral gas pressure must be as small the 
diagnostics allow $(a>0.2 \mu \mathrm{m})$. For the evaluation of charge models, this poses a problem. The requirement for small neutral gas pressures means that there is access to a limited region of ionneutral collisionallity for gyro-phase drift experiments.

A good compromise of parameters can be made for $B=4 \mathrm{~T}: a \approx 0.2 \mu \mathrm{m}, P_{n} \approx 1$ mTorr, $T_{e}=5 \mathrm{eV}, T_{n}=0.025 \mathrm{eV}$ (room temperature neutrals), and $n_{e}=10^{14} \mathrm{~m}^{-3}$. This ensures noninstantaneous charging $\left(\tau_{g} / \tau_{c h}<1\right), \omega_{c d} / \nu_{d n}>1, a / R_{L e} \approx 0.1$, and the effects of ion-neutral charge exchange in the dust sheath are still important. Variation in these parameters is considered, both to allow for variation in these plasma parameters and to predict a range of guiding center drift. A ratio of $\omega_{c d} / \nu_{d n}=4$ is obtained if $\left|\chi_{e}\right| \approx 2$ is assumed in equation 6.1. Access to $a / R_{L e}=1$ and larger is desirable for comparing the Patacchini-Hutchinson model to the OML and Gatti-Kortshagen models, but this is not feasible with MDPX at this time. For the parameters

discussed, $R_{L e}=1.67 \times 10^{-6} \mathrm{~m}$, so $a / R_{L e}=0.12$. Larger grains will easily satisfy the magnetizedelectron-current condition, which is an interesting regime to examine as seen earlier in chapter V, but gyration will not occur because $\omega_{c d} / \nu_{d n}<1$. To satisfy $a / R_{L e} \approx 1$ for $a \approx 0.2 \mu \mathrm{m}$ grains, a magnetic field of nearly $40 \mathrm{~T}$ is required.

A consequence of all these issues is that the gyro-phase drift for a wide range of parameters shown in chapter $\mathrm{V}$ is not possible in Auburn MDPX. Certain parameters are effectively restricted, and these restrictions will be discussed in the following sections.

\section{Abrupt Inhomogeneities in Auburn MDPX}

For the abrupt inhomogeneity, some plasma condition must be discontinuous across a boundary. While an inhomogeneity in $n_{e}, T_{e}$, or other parameters is possible or can possibly be constructed in the lab with an electron cathode or by other means, we will again rely on an abrupt inhomogeneity in UV illumination. An abrupt inhomogeneity in UV illumination has an advantage in that important parameters, such as grain shielding length, mean free path, and gyro-radius will be independent of the inhomogeneity, simplifying the problem. It is important that the UV spectrum does not exceed the ionization energy of the neutral gas species, otherwise this can increase electron and ion density, and the semi-analytical and simulation results will be erroneous. Additionally, we will assume that the UV source is aligned with the magnetic field direction, so that the photo-recoil force is along the magnetic axis and does not perturb the motion of the grain in the plane perpendicular to $\mathbf{B}$. 
The grain will experience an additional $\boldsymbol{F} \times \boldsymbol{B}$ drift due to the photo-electron recoil force when the UV source is not aligned with the magnetic axis, which can be a significant effect.

For laboratory experiments, the UV source specifics need to be assumed. Electromagnetic sources are described by their spectral irradiance as a function of wavelength, so that integration over the entire spectrum produces the intensity of the radiation. In this dissertation, a spectrum comparable to the UV lamps described by Dove et al. (2012) is assumed, because it is readily obtainable and has been used successfully to produce photo-emitting grains. More about their results will be discussed shortly. The Osram Xeradex 20 lamp is so named because it consumes 20 W of electrical power. The Osram Xeradex 20 lamp emits $8 \mathrm{~W}$ of power in the ultraviolet, centered around $\lambda_{c}=172 \mathrm{~nm}$, corresponding to a photon energy of $7.21 \mathrm{eV}$. The resulting spectrum has a FWHM of $\lambda_{\text {FWHM }}=14 \mathrm{~nm}$, and this is shown in figure 6.1. Although this lamp provides insufficient power for charge state modulation, it serves as a known baseline for the ensuing predictions. The radiation intensity at the lamp surface is $45 \mathrm{~mW} \mathrm{~cm}^{-2}$, or $450 \mathrm{Wm}^{-2}$. The integral described by Colwell et al. (2005), discussed earlier and provided in equation 3.40, can be performed by approximating the spectrum as a Gaussian centered at $172 \mathrm{~nm}$. Using the Gaussian fit, and declaring $\lambda_{\mathrm{FWHM}}=2 \sqrt{2 \ln 2} \Delta \lambda$, where $\Delta \lambda$ is taken as the standard deviation, the intensity of light as a function of wavelength is given by

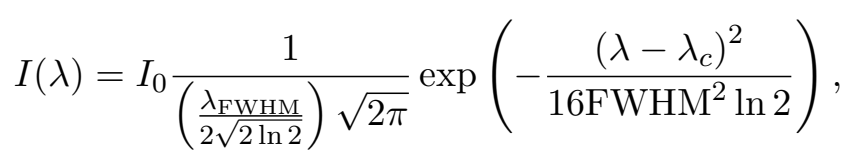

where $\lambda_{c}=172 \mathrm{~nm}$ is the peak wavelength, and $I_{0}=P /\left(4 \pi r^{2}\right)$ is UV flux intensity, assuming the UV lamp is a point source, $P$ is the power of the source, and $r$ is the distance of grains away from the source. Alternatively, $I_{0}$ can be the intensity measured at some distance from the UV source. Integration of equation 6.2 over $\lambda$ results in the value $I_{0}$, as expected. The careful reader may notice that equation 6.2 gives an expression for the spectral irradiance $I(\lambda)$, when the flux of photons as a function of wavelength $F(\lambda)$ is sought. Replace $P$ with $P=\lambda /(h c)$, where $h$ is Planck's constant, and $c$ is the speed of light, which transforms equation 6.2 into the photon flux $F(\lambda)$ needed to evaluate equation 3.40 .

The lower integration cutoff in equation equation 3.40 is given by $\lambda=0$. To determine the upper cutoff $\lambda_{1}$, consider the material being irradiated with UV photons. Only a few different materials 
are considered for this section, with reasons given for their inclusion. These materials are Carbon $\left(W=4.81 \mathrm{eV}, \lambda_{1}=258 \mathrm{~nm}, \rho_{d} \approx 2000 \mathrm{~kg} \mathrm{~m}^{-3}\right)$, Zirconium $\left(W=4.05 \mathrm{eV}, \lambda_{1}=306 \mathrm{~nm}, \rho_{d} \approx 4600\right.$ $\left.\mathrm{kg} \mathrm{m}{ }^{-3}\right)$, and Platinum $\left(W=5.65 \mathrm{eV}, \lambda_{1}=219 \mathrm{~nm}, \rho_{d} \approx 21.45 \times 10^{3} \mathrm{~kg} \mathrm{~m}^{-3}\right)$. As discussed in chapter III, a dimensionless photo-electron current fraction is used to represent the magnitude of the photo-current normalized to the electron saturation current, or $v=f_{u v} /\left(n_{e} v_{t h e}\right)$ because the assignment of this number determines the in-situ-equilibrium grain charge in the UV-present region of plasma. Because simply quoting the photo-electron current fraction $v$ in this way hides information about the UV spectrum and the grain material properties, photo-electron flux $f_{u v}$ will also be quoted. For the results in chapter V, the coefficient of UV illumination was held constant while the ratio $\tau_{g} / \tau_{c h}$ varied but, if UV flux is held constant, then $v$ must change throughout the range of electron plasma density $n_{e}$ values, and hence $\tau_{c h}$ values change. As a consequence, the dependence of $v$ on density will be shown for each case examined.

The pyrolyzed melamine spheres (Friedel and Greulich-Weber, 2006) are included here because the resulting grains are smooth spheres of pure carbon and the grains are monodisperse. The smooth nature of the surface and the spherical shape of these grains makes them a perfect testbed for the guiding center drift study in this dissertation. These spheres provide the idealized spherical shape assumed in the main charging and grain sheath models. The photo-electron yield must be characterized to determine the outward photo-electron flux. Feuerbacher and Fitton (1972) performed experiments to determine the photo-yield for graphite, vitreous (glassy) carbon, and Aquadag, a colloidal dispersion of graphite in water. These measurements, shown in figure 6.2, form the basis for an analytical expression for the yield as a function of wavelength. We assume that the yield curve for graphite approximates the work function for pyrolized melamine spheres, because the grains are allowed to cool slowly, allowing crystallization to occur. The yield curve for graphite serves as graphite's work function lower limit for photo-electrons even if the pyrolyzed spheres do not exactly have the same properties as graphite.

Zirconium and Platinum are also included. Eastman (1971) measured the photo-electron yield of Zirconium, shown in figure 6.3, while Lin et al. (1971) measured the photo-electron yield of Platinum, shown in figure 6.4. Zirconium easily forms into spheres. Platinum also forms easily into spheres of arbitrary size even as small as $a=2.5 \mathrm{~nm}$. Platinum spheres can be made with a narrow size distribution, which is another useful property for studies of grain charging. Although the size 
distribution for Platinum spheres may be narrow, there is still a size distribution so this must be considered in the analysis. Dove et al. (2012) measured a photo-electron flux of $F_{U V}=1.57 \times 10^{16}$ $e \mathrm{~m}^{-2} \mathrm{~s}^{-1}$ for Zirconium, and $F_{U V}=1.87 \times 10^{16} \mathrm{em}^{-2} \mathrm{~s}^{-1}$ for Platinum at a distance of $17 \mathrm{~cm}$ from the UV lamp. Even though Zirconium has a lower work function, Platinum has a much higher photo-electron yield which results in a higher photo-electron flux. Earlier, we assumed that $2 \leq T_{e} \leq 5 \mathrm{eV}$ and $10^{13} \leq n_{e} \leq 10^{16} \mathrm{~m}^{-3}$ as practical limits for Auburn, which means we have $1.41 \times 10^{-6} \leq v \leq 2.23 \times 10^{-3}$ for Platinum grains, and $1.18 \times 10^{-6} \leq v \leq 1.87 \times 10^{-3}$ for Zirconium grains. Earlier in chapter $\mathrm{V}$ fraction values as large as $v=0.25$ were used for plots, though we see now that this is very large compared to the measurements provided by Dove et al. (2011, 2012).

Grain velocity perpendicular to the magnetic field direction is another important consideration, because this implicitly determines the size of the gyro-orbit. The abrupt inhomogeneity approach is not valid if the UV transition region cannot be made small with respect to the size of a grain's gyro-orbit. Small gyro-orbits also pose a problem for PIV and PTV measurements because the error bars on grain velocity and position may become larger than the gyro-orbits themselves. Note also that the shapes of the gyro-orbits depend on the ratio $v_{\perp} /\left(E_{0} / B\right)$ when an electric field is present, as discussed in chapter $\mathrm{V}$ and demonstrated in figure 5.1. An assumption of small or large must be made for the grain velocity component that is perpendicular to the magnetic field direction compared to the neutral-atom thermal velocity. The most straightforward approach is to assume that the population of dust in plasma is in thermal equilibrium with the neutral gas atoms. In a DC glow or RF discharge, the ions and neutral gas atoms are in thermal equilibrium with each other, and both species are near room temperature. Even though low dust density invokes the dust-in-plasma rather than dusty-plasma condition, a statistical ensemble of grain velocities still exists, with a thermal speed characterized by $v_{t h d}$.

Assume that for the population of grains used in an experiment, little control can be placed over their initial position and gyro-phase; the grains may be dropped into the plasma (Thomas, 1999, 2001), after which PIV measurements begin. Because the neutral drag force for $a=400$ $\mathrm{nm}$ grains quickly reduces the grain's perpendicular speed, the size of gyro-orbits decays noticably after only a few gyro-cycles. Therefore, it is of paramount importance that PIV measurements are started quickly after the grains are introduced into the plasma, near the UV illumination region. UV illumination should be turned on when the grains have reached the in-situ-equilibrium charge 
(after several charging periods $\tau_{1}$ ). With enough grains and with a large enough gyro-radius, there will be grains near the boundary between UV-present and UV-absent regions of plasma. Unlike in chapter V, grains will in general have a random assortment of initial gyro-phase, meaning that they do not necessarily encounter the boundary between UV absent and present plasma at $270^{\circ}$. Grains will charge negatively in the plasma, so the grains will gyrate in a counter-clockwise direction when viewed from above, and the grain trajectories will look like those described in chapter V. It is necessary to consider the range of initial gyro-phase angles $-180 \leq \theta_{0} \leq 0$ in order to allow grains starting with positions $x<0$ to reach the transition region before analyzing their trajectories using the PIV data from the hypothetical experiment. Also, because the UV-present region will be of finite size, there will be grains "on the other side of the inhomogeneity" that start in the UV-present region and transition to UV-absent plasma during a gyro-orbit. As long as the boundaries of the UV region are known, the position, velocity and hence gyro-phase information for grains in an experiment can be determined through PIV analysis.

Acknowledging that dust grains with size $a=200 \mathrm{~nm}$ are the smallest grains that can be imaged and that either mono-energetic or drifting Maxwellian ion flow exists, it is possible to make some predictions for guiding center drift, addressing the issues of grain material and varying strengths of UV source, type of ion flow for a range of electron temperatures and densities. The results are discussed one at a time for each grain material for the range of experimental parameters considered earlier, $2 \leq T_{e} \leq 5 \mathrm{eV}, 10^{13} \leq n_{e} \leq 10^{16} \mathrm{~m}^{-3}$, and mono-energetic or drifting Maxwell-Boltzmann ions, along with some discussion about the confidence in the results. The three charge models (OML, Patacchini-Hutchinson, and Gatti-Kortshagen) are considered.

Figure 6.5 shows the guiding center drift for the three different charging models for a Platinum grain and a range of electron plasma densities $n_{e}=n_{0}$ and drifting Maxwell-Boltzmann ions. The coefficient of UV illumination $v=f_{u v} /\left(n_{e} v_{t h e}\right)$ used for figure 6.5 is shown in figure 6.6, which corresponds to ten times the amount produced by a Xeradex UV lamp $17 \mathrm{~cm}$ away from a grain. The smaller dotted lines indicate the upper and lower bounds of the guiding center drift, assuming that $T_{e}=5 \mathrm{eV}$, with an uncertainty of $\pm 0.5 \mathrm{eV}$. The experimental parameters are insufficient to discriminate between OML and Patacchini-Hutchinson charge models, but these two models are quite different from the predictions offered by the Gatti-Kortshagen charge model, especially at lower densities. Grains that charge according to the Gatti-Kortshagen model travel in the $-y$ - 
direction, another notable difference distinguishing it from the OML and Pattacchini-Hutchinson models. The ratio of the $i n$-situ-equilibrium grain surface potentials $\chi_{e}^{(1)} / \chi_{e}^{(2)}$ is shown in figure 6.7 .

Figure 6.8 shows the guiding center drift for the three different charging models for a Platinum grain and a range of electron plasma densities $n_{e}=n_{0}$, assuming mono-energetic ions and using the same coefficient of UV illumination shown in figure 6.6, which also corresponds to ten times the amount produced by a Xeradex UV lamp $17 \mathrm{~cm}$ away from a grain. Again, OML and PatacchiniHutchinson models are indistinguishable. The mono-energetic results show different values for the guiding center drift magnitudes as compared to the flow-shifted Maxwellian ions, especially for the Gatti-Kortshagen charge model. The other two models can be compared for the planar sheath mechanism of mono-energetic versus flow-shifted Maxwellian ions using their $y$-component of guiding center drift. The ratio of the in-situ-equilibrium grain surface potentials $\chi_{e}^{(1)} / \chi_{e}^{(2)}$ is shown in figure 6.9.

Zirconium is not as efficient at producing photo-electrons as Platinum, resulting in a photocurrent that is $84 \%$ that of Platinum (Dove et al., 2012). This is still sufficient to produce charge state modulation. Zirconium is also much less dense than Platinum, so the important ratio $m_{d} / m_{e}$ will be smaller. Figure 6.10 shows the guiding center drift for drifting Maxwellian ions, with the coefficient for UV illumination over the range of densities shown in figure 6.11. At $n_{0}=10^{13} \mathrm{~m}^{-3}$, zirconium grains have a small enough $m_{d} / m_{e}$ ratio that they have $\tau_{g} / \tau_{c h} \approx 1$ and they drift in the negative $x$-direction. As before, the Patacchini-Hutchinson and OML models agree closely, but these two both disagree with the predictions for the Gatti-Kortshagen model. The ratio $\chi_{e}^{(1)} / \chi_{e}^{(2)}$ shown in figure 6.12 is slightly smaller for the platinum grain throughout the range of densities, owing to the lower photo-electric yield of zirconium.

Figure 6.13 shows the guiding center drift for the three charging models for the Zirconium grain through the range of plasma densities, and the ratio $\chi_{e}^{(1)} / \chi_{e}^{(2)}$ is shown in figure 6.14. There is a considerable difference between the guiding center drifts for the flowing Maxwellian ions of figure 6.10 as compared to the mono-energetic ions of figure 6.13 , revealing that this sheath mechanism can be compared within a given model.

The initial gyro-phase of grains was held at $\theta_{0}=3 \pi / 2$ and the initial position was chosen as $x_{0}=x_{b}$ for all of the trajectories shown in the preceding figures. This restriction is not strictly 
necessary, and it is possible to consider any set of initial conditions. The preceding figures provide the most direct representation for testing guiding center drifts, but the analysis can easily be extended to any initial starting angle with no difficulty.

The intensity of UV illumination is a large factor in producing guiding center drift, but it has no effect unless there is sufficient photo-electric yield. Thus, the photo-electric yield function and the UV spectrum determine in large part the resulting guiding center drift in a quasi-neutral plasma. Both of these properties affect the UV photo-electron flux and hence determine in-situ-equilibrium grain charge. As shown earlier in figure 5.14, larger $q_{1} / q_{2}$ ratios produce greater gyro-phase and grad-q drift magnitude. As long as the photo-electron flux is not so large to make the grain charge positively, guiding center drift will increase as the ratio $q_{1} / q_{2}$ increases. The analysis above shows that in order to maximize the photo-electron flux, a grain material with a high photo-electric yield

function is desirable. Cesium or Cesium-coated surfaces, for example, have very large photo-electric yields. Having a UV spectrum that is peaked at higher photon energies is also beneficial for all materials, because the yield increases with UV photon-energy, up to some maximum. The work function is not so important for producing gyro-phase drift; as long as the energy of UV photons is greater than the work function, the yield curve determines the photo-electric flux. Carbon grains have a much lower work function than platinum, but drift less than platinum grains because the photo-electron yield is so much smaller for Carbon. It should be noted however that for materials with high yields and high work functions (such as Platinum), having a peak in the UV spectrum near the work function produces photo-electrons that leave the surface with very little kinetic energy, and these electrons might readily form a Space-Charge-Limited sheath.

In MDPX, the prediction made by the Gatti-Kortshagen charging model should produce the correct results. The electron current is effectively the same in all three models, but only GattiKortshagen accounts for ion-neutral charge exchange collisions in the grain sheath. An experiment set up in accordance with this section should provide a crucial test for the validity of the GattiKortshagen charging model.

\section{Gradual Inhomogeneities in MDPX}

There are a few possibilities for gradual inhomogeneities in MDPX that we might use as candidates for observing gyro-phase drift. These possibilities are summarized, with an assessment for 
each of these in MDPX. An immediate consequence of a gradual inhomogeneity is that an electric field is generally present, which enlarges grain gyro-orbits (chapters IV and V) and leads to problems with dust confinement for larger size grains $(a>200 \mathrm{~nm})$. Furthermore, the grain-size dependent drag forces damp the gyro-motion, which gives even further reason to use small grains $(a<200 \mathrm{~nm})$. The use of small grains prevents observation of any differences between the OML and Patacchini-Hutchinson models because the magnetization parameter $a / R_{L e}$ will be extremely small. A complete list of gradual inhomogeneities of which we can conceive for the Auburn MDPX is inhomogeneous $n_{e} / n_{i}$, inhomogeneous $T_{e}$ or $T_{i}$, inhomogeneous ion mass composition, inhomogeneous ion charge state, inhomogeneous quasi-neutral plasma density, and inhomogeneous electron or ion flow during a gyro-orbit. Inhomogeneous ion mass composition is discarded due to the technical challenges required to produce an ion mass composition gradient in an experiment. Inhomogeneous ion charge state may be present with a meaningful spatial dependence in Tokamaks or other high temperature plasma, but this is ignorable in MDPX and singly-ionized ions are a justifiable assumption. Inhomogeneous electron or ion temperature profiles are present in Tokamaks and Stellerators, but they do not arise in any meaningful way in a magnetized RF discharge such as MDPX. This leaves three sources of gradual inhomogeneity that may give rise to charge modulation during a gyro-orbit and possibly grad-q and gyro-phase drift, which are inhomogeneous quasi-neutral plasma density, inhomogeneous $n_{e} / n_{i}$ ratio, and inhomogeneous electron or ion flow. These inhomogeneities merit further discussion.

As discussed in chapter $\mathrm{V}$, a guiding center drift is present when only the quasi-neutral plasma density is inhomogeneous, which is a common feature of many magnetized plasma experiments. However, in order for grain charge to change appreciably during gyro-motion due to plasma density, the ratio $\lambda_{D} / a$ must change substantially during gyro-motion, which generally requires either very dense plasma or very large grains. Very dense plasma increases charging time, limiting the magnitude of the gyro-phase drift, and very large grains will experience very large neutral drag forces that spoil gyration. Hence, inhomogeneous quasi-neutral plasma density has insignificant effect on charge modulation during gyro-motion in MDPX, on the basis that the $\lambda_{D} / a$ parameter is a very large number for any conceivable dust experiment.

Typical laboratory plasma, a few Debye lengths from foreign material objects, is quasineutral. Some of the electrons are lost due to the walls due to the higher thermal speeds of electrons as 
compared to ions. Although no deviation from quasi-neutrality is to be expected in MDPX, find an electric field self-consistent with ion and electron densities in cylindrical geometry relevant to MDPX by assuming a plasma with a radial and vertical electric field, which arises naturally in many laboratory experiments. For azimuthal symmetry, Gauss' law becomes

$$
\frac{1}{r} \frac{\partial\left[r E_{r}\right)}{\partial r}+\frac{\partial E_{z}}{\partial z}=\frac{e}{\epsilon_{0}}\left(n_{i}(r, z)-n_{e}(r, z)\right]
$$

Only the values of these quantities when they are evaluated at $z=z_{s h}$ are pertinent, because this corresponds to the vertical distance above the electrode where the grains levitate. According to Child-Langmuir theory of planar sheaths, the plasma potential varies slowly or is constant with respect to $z$ at $z=z_{s h}$, so the $\partial E_{z} / \partial z=\partial^{2} V_{s} / \partial z^{2}$ term is dropped from equation 6.3. As long as two of the three quantities $n_{e}, n_{i}$, or $\boldsymbol{E}(\boldsymbol{r})$ are known, the missing quantity can be determined using equation 6.3. The assumption of Maxwell-Boltzmann electrons translates into the ability to obtain ion and electron densities if $E_{r}$ is known. Consider the radial profiles for an ECR plasma with a biasing ring shown in figure 6.15 (Nunomura et al., 1997). Approximate the radial electric field profile from figure 6.15 as a gaussian function centered at $r_{0}=4 \mathrm{~cm}$ with a FWHM of 3 $\mathrm{cm}$. The plots shown in figure 6.16 show the plasma profile when assuming Maxwell-Boltzmann electrons, which provides a reasonable approximation to figure 6.15. Note that the ratio $n_{e} / n_{i}$ does vary spatially, although it is nearly unity throughout the plasma. As the plasma density $n_{0}$ increases, according to equation 6.3 , the $n_{e} / n_{i}$ variation must decrease unless the relative strength of the electric field increases. An assessment of gyro-phase drift for the profiles like those shown in figures 6.15 and 6.16 will be provided.

The last inhomogeneity to discuss is that of inhomogeneous ion flow during the course of a gyroorbit. Inhomogeneous ion flow happens naturally when a dust grain has very high speed relative to the plasma, or in other words $\left|\boldsymbol{v}_{\boldsymbol{i}}-\boldsymbol{v}_{\boldsymbol{d}}\right| / v_{t h i}$, where $\boldsymbol{v}_{\boldsymbol{i}}$ is the ion drift velocity in the lab frame, and $\boldsymbol{v}_{\boldsymbol{d}}$ is the dust grain velocity in the lab frame. This can also arise in the presence of a magnetic field gradient, because the $\operatorname{grad}-B$ drifts for electrons and ions are different, and a diamagnetic current is carried through the plasma. The background uniform-plasma $\boldsymbol{E} \times \boldsymbol{B}$ drift does not carry a current, so it cannot cause charge state modulation, but the drifting ions do produce a drag force which must be considered when determining grain trajectories. The root-mean square 
or thermal speed of dust grains is proportional to $a^{-3 / 2}$, so small grains offer an advantage here for producing charge modulation because the ratio $v_{g} / v_{t h i}$ is larger for smaller grains. The dust thermal speed is $v_{\text {thd }}=0.54 \mathrm{~m} / \mathrm{s}$ for zirconium grains with $a=0.01 \mu \mathrm{m}$ in thermal equilibrium with room temperature neutral gas atoms, which hardly approaches the thermal speed of neutrals $v_{t h n}=346 \mathrm{~m} / \mathrm{s}$. One might consider grains with velocities much greater than the dust thermal speed because such grains will be present in a statistical ensemble. Additionally, the presence of a radial electric field increases the maximum perpendicular speed experienced by a dust grain during its $\boldsymbol{E} \times \boldsymbol{B}$ drifting gyro-orbit, so modulated ion flow during a dust grain gyro-orbit is unavoidable.

Figure 6.17 shows a possible profile of self-consistent plasma parameters, using a gaussian electric field centered at $r_{0}=0.1 \mathrm{~m}$ with $\mathrm{FWHM}=0.03 \mathrm{~m}$ and Boltzmann electrons. The equilibrium charge is shown for all three charge models. Such a large deviation from quasi-neutrality is not very realistic, and this exact electric field profile might not be possible for the plasma density on axis $n_{0}=10^{14} \mathrm{~m}^{-3}$. Bear in mind, however, that this profile serves as an upper limit for the detection of gyro-phase drift in MDPX. The OML and Patacchini-Hutchinson models give the same answer for equilibrium grain charge, as expected for small grains and small $a / R_{L e}$ ratios. These equilibrium charge profiles include the effects of the drifting Maxwellian ions in the $z$-direction that enter the planar sheath at the Bohm speed, and also the azimuthal electron and ion flow due to the $\boldsymbol{E} \times \boldsymbol{B}$ flow. The ions that enter the sheath in the $z$-direction dominate the ion flow because $\sqrt{\frac{k_{b} T_{e}}{m_{i}}} \gg E / B$ for this profile. There is a smaller quasi-neutral plasma density than in figure $6.15, n_{0}=10^{14} \mathrm{~m}^{-3}$. The plasma density is not exactly $10^{14} \mathrm{~m}^{-3}$ at $x=0$ in this figure because grains will levitate at the sheath edge in the $z$-direction, where $n_{e}(r=0)=n_{i}(r=0)=n_{0} \exp (-1 / 2)$. The profile in figure 6.17 is notable in that $n_{e} / n_{i}$ varies considerably near $r=7.5 \mathrm{~cm}$, so this profile is a good candidate for charge state modulation. The gaussian, hence inhomogeneous, shape of the electric field means that there is an additional drift in the $\varphi$-direction associated with this non-uniformity. Grain charge also varies considerably near $r=7.5 \mathrm{~cm}$ in this kind of profile, so there is a possibility for gyro-phase drift.

Before including ion drag and neutral drag, it is instructive to see some example grain trajectories for the gaussian electric field profile. Constant grain charge constitues the simplest example. The grain charge $q=-695 e$ is chosen because this corresponds to the in-situ equilbrium grain charge for the OML model at $r=0.1 \mathrm{~m}$. Figure 6.18 shows the grain trajectory for 80 gyro-cycles. 
This trajectory closely matches figure 5.17a on page 289 of Gavrishchaka (1996), suggesting that the axis-encircling trajectory of this grain is consistent with regular $\boldsymbol{E} \times \boldsymbol{B}$ drift in cylindrical geometry. Figure 6.19 shows the radial excursion of the grain. The grain does not drift inward or outward; the radial excursion is bounded as expected for pure $\boldsymbol{E} \times \boldsymbol{B}$ drift.

We add more complexity to the situation by allowing the grain to change charge state instantaneously. Figure 6.20 shows approximately 10 gyro-cycles of the grain trajectory for a grain that charges instantaneously to the in-situ-equilibrium grain charge. Figure 6.20 is very similar in appearance to figure 6.18. The radial excursion shown in figure 6.21 is similar to figure 6.19 , but there is now a grad-q drift term that changes the pattern of radial excursion slightly, although the upper bound of $r=0.1 \mathrm{~m}$ (the initial position of the grain) remains the same.

As the final step before adding drag terms, we examine what happens when the grain does not charge instantaneously. Figure 6.22 presents a different picture for the grain trajectory when non-instantaneous charging is permitted. The radial excursion in figure 6.23 shows that noninstantaneous charging significantly alters the grain trajectory and, ultimately, the grain drifts out of the plasma in this gaussian electric field profile, in contrast to the bounded radial excursion seen in figure 6.19. In figure 6.23, both the lower and upper bound of the radial excursion are increasing, even though it is difficult to see the small increase for the lower bound in this figure. Armed with this intuition from the drag-absent scenarios, it is now time to add drag forces and analyze the resulting grain trajectories.

Figure 6.24 shows an example trajectory for non-instantaneous charging for the profile shown earlier in figure 6.17 when only the neutral drag is included from the drag force terms. The many gyro-orbits present in figure 6.24 make it difficult to see anything quantitatively and a more illuminating visualization is needed to diagnose the grain motion. Figure 6.25 shows the radial excursion of the grain and the charge-state as a function of time. The charge state modulation and the radial excursion of the grain decreases in time. The grain in figure 6.25 starts with the in-situ-equilibrium charge. Next consider what happens when the grain starts with no grain charge, a possibility when grains are first introduced to the plasma. The transient charging case is shown in figure 6.26. Except for the beginning of the trajectory, where the grain starts charging negatively, the radial excursion exhibits similar behavior and their radial positions are nearly identical after the same amount of time. 
However, when both the ion and neutral drag are included as drag force terms, a different picture for the grain position and charge evolution emerges. As implied by figure 6.17, the ion flow causes an ion drag force in the $\varphi$-direction, meaning that there is a $\boldsymbol{F} \times \boldsymbol{B}$ force, and negatively charged grains drift radially outward. The concurrent presence of neutral drag may ameliorate this effect somewhat, because this force acts to decrease the gyro-radius of grains. Figure 6.27 suggests that the radial transport of grains is different for instantaneous charging and non-instantaneous charging, and non-instantaneous charging leads to grain confinement in MDPX for the inhomogeneity profile discussed in this chapter.

An obvious question to ask is whether the charging model choice can make a difference to grain trajectories. Figure 6.17 shows that the Gatti-Kortshagen charge model produces very different values for the in-situ-equilibrium grain charge as compared to Patacchini-Hutchinson and OML models, so one might expect some difference in the grain trajectories. Figure 6.28 shows an example trajectory for the Gatti-Kortshagen charging model, which seems promising since it visually looks different. The trajectory shown includes neutral drag, but not ion drag. Figure 6.29 shows the radial excursion and charge state for the grain for the trajectory shown in figure 6.28. A very different pattern of charge evolution emerges when the Gatti-Kortshagen charge model is used in figure 6.28 as compared to figures 6.25 and 6.26. Unfortunately, the grain reaches the same final radial position for both models at about the same time, so it is too difficult to distinguish these models in a real experiment.

\section{B Application to Enceladus}

Saturn and its rings and moons form a dusty plasma system. Given the presence of dust grains of all sizes throughout Saturn's plasmasphere, it is natural to ask whether non-stationary charging processes can lead to stratification of dust. Northrop et al. (1989) answered this question for the case of Jupiter and explaining the formation and structure of Jupiter's gossamer ring, although no treatment was offered for UV illumination or ion-neutral charge exchange processes. Khurana et al. (2008) provide a useful collection of parameters for the Saturnian moons, shown in table 6.1. Note that the increase in electron and ion temperatures strongly suggests the presence of temperature gradients, and an attempt to model this profile of the Saturn system is shown in figure 6.30. The 
electron temperature is modelled as exponentially increasing with increasing radius from Saturn's center in a similar fashion as Northrop et al. (1989) and Northrop (1992). The magnetic field of Saturn is treated as a dipole field and the magnetic field axis is closely aligned with its rotation axis. As discussed by Farrell et al. (2009), near Enceladus in the E-ring in Saturn's gas torus, neutral gas atoms and molecules also exist, with concentrations as large as $n_{n} \approx 10^{11} \mathrm{~m}^{-3}$. Naïvely, one might suspect that these neutral gas atoms lead to ion-neutral charge exchange collisions in the grain sheath and affect grain dynamics. However, $\lambda_{i} / \lambda_{D} \gg 1$ in the Saturn-Enceladus system, which suggests that ion-neutral charge exchange collisions do not play a role in grain charging. The presence of neutral gas does mean dust-neutral collisions occur, damping gyro-motion which affects grain dynamics.

Figure 2.3 gives an example trajectory for conditions present near Enceladus in Saturn's magnetosphere for a grain with $a=0.025 \mu \mathrm{m}$, but with no inhomogeneities except for ion flow modulation during grain gyrorbit. Given the conditions near Enceladus including the weak magnetic field of $B \approx 370 \mathrm{nT}$, only very small grains are magnetized with respect to Saturn's magnetosphere, and the grain gyro-orbits are generally larger than Enceladus' diameter. Nonetheless, it is possible to observe the difference that the presence of UV can make on grain trajectories, specifically the change in UV illumination that occurs when a grain transits from a UV-present region to a UVabsent region. Figure 6.31 shows an example trajectory of a water ice grain $(a=0.025 \mu \mathrm{m})$ that has sufficient energy to escape the gravity of Enceladus, and so travels at the Kepler speed around the equatorial plane of Saturn. The grain has an inward radial drift. No UV illumination is considered in figure 6.31. Figure 6.32 shows the same grain, but this time considering UV illumination, with the UV flux being modelled by assuming the Solar UV spectrum and regolith as the grain material (Colwell et al., 2005). The astute observer may notice that the trajectory is slightly different from figure 6.31. Because the grain may transit behind Saturn, a UV-absent region or cylindrical shadow sweeps around Saturn like a "darkhouse" in the co-rotating frame. Grains are close enough to Saturn that the region of shadow should be the diameter of Saturn, and no penumbral effects are considered. This constitutes an abrupt inhomogeneity in UV illumination. Figure 6.33 shows the direct comparison between the UV and no-UV grain trajectories. The differences in grain charge modulation lead to differences in radial excursion in figure 6.33. The abrupt inhomogeneity enlarges the radial excursion of the grain. 
It is also possible to examine grain trajectories for a temperature gradient. Figure 6.34 shows a temperature gradient and the UV "darkhouse" function is also included, but no drag forces are considered. There is an obvious drift toward larger radial excursions from Saturn; the grain gets transported towards Rhea and the outer moons of the Saturnian system. The gyro-radius increases as the grain drifts radially outward because the magnetic field is decreasing. Figure 6.35 refines this picture by including neutral drag. The grain still drifts in the radial direction, but the gyro-radius is decreasing due to the neutral drag. Figure 6.36 introduces ion drag, but the results are the same as found in figure 6.35 because the ion drag force on this $a=0.025 \mu \mathrm{m}$ grain is so small in Saturn's plasmasphere. 


\section{Chapter VII}

\section{Discussion}

The main issue in this chapter is to discuss whether or not gyro-phase drift is observable in laboratory experiments and to evaluate the limits on its detection based on predictions of observables from the model for abrupt and gradual inhomogeneities.

\section{A Optimizing the Detection of Gyro-phase Drift in MDPX}

The following considerations can be made regarding uncertainty quantification of guiding-center drift for the abrupt inhomogeneity. Discrepancy between theory and experimental observation of guiding center drift caused by over-simplification or inaccuracy of the theoretical models can be called model error. Measurement uncertainty concerning dust grain trajectories and measurements concerning plasma dimensionless parameters will be discussed here.

The grain position and grain velocity sampling rate serve as important parameters when discussing possible error in grain trajectories, represented by the capability of laser strobing and CCD frame capture rate. Thomas (1999) discussed a PIV system where $0.0005 \mathrm{~ms}<\Delta t_{\text {laser }}<30 \mathrm{~ms}$. For the $a=2 \times 10^{-7} \mathrm{~m}$ grains discussed in chapter VI, the gyro-period corresponds to $\approx 0.1-10 \mathrm{~s}$. A sampling rate of $1 \mathrm{kHz}$ yields a minimum of $\approx 30$ data points per gyro-orbit, which should be sufficient for documenting the velocity of the grain. As an example of the spatial resolution of the CCD camera used in typical PIV studies, Thomas and Watson (1999) provide a reasonable bound on grain position by using a pixel size of $25 \mu \mathrm{m}$.

In order to measure single grain trajectories in a way that is consistent with the predicted grain 
trajectories in this dissertation, particle tracking velocimetry is needed because PIV measures the dust population as an ensemble rather than individual particles. PTV allows tracking of individual grains for many successive frames (Feng et al., 2011). With PTV, a sparsely seeded field is necessary to maintain a relatively large interparticle spacing, otherwise PTV algorithms are inefficient (Boessé et al., 2004). In the context of this dissertation, a sparsely seeded field refers to the dust-in-plasma condition, where the Debye sheaths of individual dust grains do not overlap. A sparsely seeded field has another advantage in that the dust-dust collision frequency is reduced. The velocity at a time halfway between two video frames can be calculated using

$$
\boldsymbol{v}_{n+1 / 2}=\frac{\boldsymbol{r}_{n+1}-r_{n}}{\Delta t}
$$

where $\Delta t$ refers to the time between video frames and the subscripts represent time steps. Using the propogation of errors, Feng et al. (2011) showed that the error in velocity is given by

$$
(\delta \boldsymbol{v})^{2}=2\left(\frac{\delta \boldsymbol{r}}{\Delta t}\right)^{2}
$$

where $\delta \boldsymbol{r}$ is the inherent error in grain position and $\delta \boldsymbol{v}$ is the error in velocity.

A consequence of equation 7.2 is that faster frame rates increase the error in velocity. However, Feng et al. (2011) showed that by skipping some image frames for the velocity calculation, $\Delta t$ is increased, resulting in smaller errors in velocity but lowering the sampling rate for velocity measurements. To further discuss the possibilities for measurement error using PTV, it is instructive to look at some PTV observations in dust experiments in the literature. Feng et al. (2011) obtained a pixel width of $31 \mu \mathrm{m}$ at a capture rate of 250 frames per second, successfully imaging $a=4 \mu \mathrm{m}$ grains having a thermal speed of $1 \mathrm{~mm} / \mathrm{s}$, which is comparable to the perpendicular velocities assumed in chapter VI. The gyro-period for the dust grains in chapter VI is approximately $1 \mathrm{~s}$, so a $250 \mathrm{hz}$ sampling rate results in 250 images per gyro-period. Feng et al. (2007) used a setup where the pixel width was $7.4 \mu \mathrm{m}$ for imaging $a=4 \mu \mathrm{m}$ grains. Feng et al. (2007) point out that the image size is not necessarily given by the pixel size due to diffraction by the camera aperature and imperfect focusing. The $8 \mu \mathrm{m}$ dust grains used by Feng et al. (2007) produced images that were larger than one pixel, even though the pixel size was $7.4 \mu \mathrm{m}$. With their grain and optical 
parameters, Feng et al. (2007) obtained accuracy in grain position to within 0.017 pixel using their technique. Setting the uncertainy in grain position $\delta r=(0.017) 7.4 \mu \mathrm{m}=0.126 \mu \mathrm{m}$ with a representative sampling rate of 250 frames per second results in a velocity error of $\delta v=4.5 \mu \mathrm{m} / \mathrm{s}$, which corresponds to $4.5 \%$ of the grain velocity perpendicular to the magnetic field. In figures $6.10,6.9,6.12$, and 6.14 , the largest difference between models is comparable to this number, which suggests that a higher UV flux is needed in order to directly observe gyro-phase drift.

However, if a similar algorithm to the one developed in appendix B, section 3 of this dissertation is used to find quantities such as the gyro-period, gyro-phase angles, and guiding center drift magnitudes from the PTV data that relies only on the grain position, rather than using the velocity, this error might be reduced by using relationships of the form $x_{g c}=\left[x(t=0)-x\left(t=\tau_{g}\right)\right] / \tau_{g}$. Assuming the gyro-period $\tau_{g}$ can be determined to within $\pm 1 /$ SamplingRate, dividing the initial and final positions by the longer timescale $\tau_{g}$, rather than the time between successive images, has the effect of decreasing the error in $v_{x g c}$. In other words, $\delta v_{x g c} \approx \sqrt{2} \delta x / \tau_{g}$. For the best case scenario, this results in $\delta v_{x g c} / v_{\perp} \approx 0.1 \%$ for $\tau_{g} \approx 1 \mathrm{~s}$, which should be sufficient to distinguish charging models and ion distribution functions for the UV fluxes used in figures 6.7, 6.9, 6.12, and 6.14 .

To address the issue of the possible photo-electron currents that might be produced for the abrupt inhomogeneity, Dove et al. (2012) measured UV photo-currents of $F_{U V}=(1.87 \pm 0.3) \times$ $10^{16} \mathrm{em}^{-2} \mathrm{~s}^{-1}$ for Platinum and $F_{U V}=(1.57 \pm 0.3) \times 10^{16} \mathrm{em}^{-2} \mathrm{~s}^{-1}$ for Zirconium using the Xeradex lamp. These photo-current amplitudes are not enough to produce a measurable gyro-phase drift for the abrupt inhomogeneity in UV illumination for typical plasma parameters in MDPX. Gyro-phase drift is predicted to be visible when the luminosity is increased by a factor of 10 for the same UV spectrum, which might be achieved through the use of multiple UV lamps or a more powerful UV lamp. Higher UV output is advantageous for observing gyro-phase drift.

Measuring electron temperature using Langmuir probes poses some difficulty, and in chapter VI an uncertainty of $\pm 0.5 \mathrm{eV}$ was assumed to correspond with the inherent uncertainty of electron temperature for Maxwell-Boltzmann electrons. For electron distributions that have heavier tails than a Maxwell-Boltzmann distribution, such as a Kappa distribution, the in-situ-equilibrium grain charge increases, the charging time increases, and the gyro-phase drift magnitude increases as compared to the Maxwell-Boltzmann case with all other parameters being identical. The ion temperature is 
assumed to be room temperature $\left(T_{i}=0.025 \mathrm{eV}\right)$, and because grains are at the sheath edge, the flow velocity, perpendicular to both gyro-phase and grad-q drifts, should be considered to be at the Bohm speed. The speed of ions does depend on the electron temperature here, uncertain by $\pm 0.5 \mathrm{eV}$, with this feature already factored into the plots shown in chapter VI. Plasma instabilities, such as streaming instabilities, might increase the ion temperature or modify the flow velocity near the sheath edge. In general, higher electron temperatures and ion temperatures increase the magnitude of gyro-phase drift.

A challenge in implementing the abrupt inhomogeneity experimentally is maintaining sufficiently sharp isolation of the two inhomogeneity regions. To ensure that the transition between UV absent and present regimes is abrupt, a cylindrical tube surrounding the UV light source and leading into the plasma would be recommended, maintaining collimated light and producing a well-defined UV-illuminated region. As mentioned many times throughout this dissertation, the collection of electrons and ions is an inherently stochastic process, and hence charge fluctuations (Jana et al., 1993; Cui and Goree, 1994) can be expected during charge-state evolution as grains move between regions of different plasma conditions. Addressing these concerns requires a random component in the charge state evolution on top of a spatial profile, instead of the exponential approximation used in the theory. Vaulina et al. (1999) note that charging flucutations lead to changes in dust ensemble temperature, causing uncertainty in $T_{d}$. This should not be a practical problem for experiment because $v_{\perp}$ is typically decoupled from the dust population thermal speed and because velocities will be determined from the PIV analysis.

In summary, to optimize the detection of gyro-phase drift in MDPX for abrupt and gradual inhomogeneity, some general guidelines can be prescribed. Lower neutral gas atom densities decrease the neutral drag force on dust grains, which increases the signature of gyro-phase drift. The presence of more grains is helpful for PIV analysis, but the resolution of individual grain motion is not possible and the dust-in-plasma criterion is not applicable when the inter-particle spacing is smaller than the Debye length. Zirconium and Carbon both have smaller work functions than Platinum but, as was demonstrated in chapter VI, the combination of photo-electric yield function and UV source spectrum is the most important factor for achieving higher $q_{1} / q_{2}=\chi_{1 e} / \chi_{2 e}$ ratio in the abrupt inhomogeneity, which in turn increases the observed gyro-phase drift magnitude. Platinum and Zirconium make better choices than Carbon for a gyro-phase drift experiment with 
abrupt inhomogeneity in UV luminosity. Zirconium is an ideal choice for dust grain material, since the photo-electric yield function is high enough that the grain's new in-situ-equilibrium grain charge is significantly lowered by commercially-available UV-illumination $(\approx 10 \%$ less electrons on the grain surface for the conditions discussed in this dissertation), and because grains have mass density sufficiently low enough that the ratio $\tau_{g} / \tau_{c h} \approx 1$ is attainable. A UV lamp with a similar spectrum, but with higher radiation intensity than the Xeradex lamp used by Dove et al. (2012) should be sufficient to produce a measurable gyro-phase drift.

\section{B Model Uncertainty}

To make predictions for gyro-phase drift in MDPX, reliance on models for the dust grain sheath, the planar sheath, and charging currents are necessary. For the abrupt inhomogeneity, gyro-phase drift was predicted for an uncertainty in the electron temperature of $\pm 0.5 \mathrm{eV}$ and for a density range of $10^{13}<n_{0}<10^{15} \mathrm{~m}^{-3}$. The very small grain sizes required, so that grain orbits fit inside MDPX, implies that charge fluctuations will be even more dominant in MDPX when considering a gradual inhomogeneity. Charge fluctuations should lead to an increase in gyro-phase drift. At every timestep, the iterative leapfrog solver has a maximum error of $10^{-10} \mathrm{~m} / \mathrm{s}$; this can easily be adjusted in the simulation code for additional accuracy. Moving the particle in simulations should not be a significant source of error; rather, it is the uncertainty in the grain charge and other transient plasma conditions that would cause deviation in grain trajectories.

To accurately model the Debye length of the dust grain, the temperature or average kinetic energy of each species must be known, and the species with the smallest temperature controls the shielding length because the temperatures are added reciprocally. For grains suspended in a planar sheath, electrons have a well-defined temperature, but ions flow toward the biasing electrode so that they have higher kinetic energy than is represented by their thermal energy. When a magnetic field is introduced into the plasma with the field lying along the planar sheath direction, there is a pronounced anisotropy between the parallel and perpendicular ion temperature, with $T_{i, \|} \gg T_{i, \perp}$. In this dissertation, the parallel ion temperature was used in computations for the Debye length of the dust grain, and the possibility of an elipsoidal grain sheath was not accounted for. Additional complications come from the fact that Gatti and Kortshagen (2008) assume a 
Debye-Huckel potential profile, when we know from the work of Goree (1992) and Lampe et al. (2001) that the trapped ions produce changes to the potential profile of the grain sheath. For the plots using the Gatti-Kortshagen charge model, these deviations are assumed to be small, so that the effect on the capture radius is small.

The discrete and stochastic nature of charge collection by a dust grain implies that the grain charge is not a continuous function of time. The semi-analytical method used for the abrupt inhomogeneity in this dissertation does not take into account either the discrete or stochastic nature of charge collection, while the simulations only treat the discrete nature of charge collection. In the simulations, the grain charge fluctuates around the in-situ-equilibrium grain charge by one electron, but this does not entirely represent the stochastic nature of charge collection. Despite the fact that the semi-analytical method does not use discrete charging, the semi-analytical method agrees very closely with the simulation results, which is evident in figure 5.12. Cui and Goree (1994) found in their stochastic grain charging simulations that for the OML model, the RMS grain charge fluctuation normalized to the in-situ-equilibrium charge is given by

$$
\frac{\Delta q}{q_{e q}}=\frac{1}{2} \frac{1}{|\langle N\rangle|^{1 / 2}}=\frac{1}{2} \frac{1}{\left|3 N_{D e} \frac{a}{\lambda_{D}}\left(1+\frac{a}{\lambda_{D}}\right)\left\langle\chi_{e}\right\rangle\right|^{1 / 2}},
$$

where $\Delta q$ is the RMS grain charge fluctuation, $q_{e q}$ is the in-situ equilibrium charge, $\left\langle\chi_{e}\right\rangle$ is the in-situ equilibrium dimensionless dust grain surface potential, and $|\langle N\rangle|$ corresponds to number of charges on the dust grain at in-situ equilibrium conditions. Equation 7.3 is the same as 3.8, but has been included here again, recast in terms of the in-situ-equilibrium dimensionless dust grain surface potential. This allows an estimation for the RMS grain charge fluctuation when dimensionless parameters are used, such as in the semi-analytical method. For smaller grains, $|\langle N\rangle|$ is a smaller number and so the grain experiences greater charge fluctuations around the in-situ-equilibrium charge.

The stochastic model of Cui and Goree (1994) does not deviate greatly from a continuous model for $0<t<\tau_{c}$, where $\tau_{c}$ is the charging time, which is shown in figure 4 of (Cui and Goree, 1994). It is when the grain charge is close to the in-situ-equilibrium grain charge that the deviations from the continuous model become significant. This is a problem for the gradual inhomogeneity, since the grain charge does not deviate greatly from the in-situ equilibrium grain charge during a gyro-orbit. 
As an example, the very small grains $a=0.05 \mu \mathrm{m}$ used for the gradual inhomogeneity in MDPX in figures $6.23,6.25,6.27,|\langle N\rangle| \approx 700$, which implies $\frac{\Delta q}{q_{e q}} \leq 0.02$. This corresponds to an RMS fluctuation of 14 electrons, which is larger than the amplitude of charge state modulation during a gyro-orbit $\left(q_{a} \approx 3 e\right)$.

For the dust grains used in the abrupt inhomogeneity, much larger grains were considered. For these grains, the in-situ-equilibrium grain charge exceeds 2000 electrons, so that $\frac{\Delta q}{q_{e q}} \leq 0.01$, or $\Delta q \leq 20$ when $t>\tau_{c}$. When $n_{0}=10^{13} \mathrm{~m}^{-3}$, figures $6.10,6.9,6.12$, and 6.14 show a charge state modulation of $\approx 10 \%$ for the Patacchini-Hutchinson and OML models. This charge state modulation due to the abrupt inhomogeneity should be sufficient to distinguish it from the GattiKortshagen model in a possible experiment in MDPX. The ratio of stochastic charging to the charge state modulation during a gyro-orbit is best described by the relations $\frac{\Delta q}{q_{a}}>1$ for the gradual inhomogeneity investigated, and $\frac{\Delta q}{q_{a}}>1$ for the abrupt inhomogeneity, which implies that gyrophase drift is easier to detect in an abrupt inhomogeneity. According to Northrop and Hill (1983), stochastic charging introduces a randomness or diffusive behavior superposed on the unidirectional radial drift. Hence, the stochastic nature of grain charging does not eliminate gyro-phase drift, it just reduces the gyro-phase drift magnitude and manifests statistically among participating dust grains.

\section{Confinement in MDPX}

Just as the vertically-aligned sheath electric field (along the direction of the magnetic field) provides vertical confinement for dust grains, a radial electric field controls dust confinement properties. In unmagnetized dusty plasma, radial confinement is achieved through a combination of the radial electric field, ion drag force, and thermophoretic forces resulting in formation of dusty disk in a plasma discharge. In magnetized dusty plasma, a radial electric field enlarges the gyro-radius and the radial excursion of a dust grain, which was demonstrated in figure 4.3. This puts limits on the grain size that can be successfully confined in a gradual inhomogneity in MDPX, with a reasonable upper limit given by $a=0.1 \mu \mathrm{m}$ for $E_{r}=100 \mathrm{~V} / \mathrm{m}$.

In MDPX, there is a greater radial excursion for negatively charged grains in a radially-inward electric field as compared to a radially-outward electric field. This can be explained as follows: 
the grain trajectory has a higher radius of curvature in regions of smaller or more negative space potential whenever the ratio of electric and magnetic fields is much greater than the initial grain velocity, which is satisfied in MDPX for non-zero electric field. The "small" loop of the gyro-orbit lies on the high-potential side, while the "large" loop of the gyro-orbit lies on the low-potential side. Electric fields that are radially-outward offer better confinement properties because the grain trajectory's radius of curvature enlarges as it travels radially inward, leading to confined, spirograph trajectories like in figures 6.19 and 6.20 . Contrast these plots with grain trajectories with radiallyinward electric fields in figures 5.48, 5.49, and 5.50 to see why outwardly directed electric fields are advantageous to grain confinement. Because neutral drag damps gyro-motion, the presence of neutrals allows the possibility of confinement although the neutral drag ultimately destroys the grain gyration that we are interested in observing. Ion drag poses a confinement problem, because the resulting $\boldsymbol{E} \times \boldsymbol{B}$ drift for ions from an outwardly-directed electric field produce an azimuthal ion drag, which in turn produces an $\boldsymbol{F} \times \boldsymbol{B}$ drift that is outwardly-directed.

The ratio of $\tau_{g} / \tau_{c h}$ is grain-size dependent. Therefore, when all other plasma parameters are kept constant, a non-monodisperse dust population has a spread in normalized gyro-phase drift magnitude owing to the inherent spread in $\tau_{g} / \tau_{c h}$ values. Irregular grain shape is another concern worth consideration for gyro-phase drift. Irregular grain shape can lead to increased capacitance, especially in the case of fractal or cauliflower-shaped grains. Increased capacitance leads to an increase in the in-situ-equilibrium grain charge $q_{0}$ and charging time $\tau_{c h}$. Because $\tau_{g} \propto q_{0}^{-1}$ and $\tau_{c h}$ increases with increasing grain charge, $\tau_{g} / \tau_{c h}$ ratios decrease. This may either increase or decrease the gyro-phase drift magnitude of irregularly-shaped dust as compared to spherical dust with the plasma parameters kept constant, depending on whether $\tau_{g} / \tau_{c h}>1$ or $\tau_{g} / \tau_{c h}<1$. For the $a=100 \mathrm{~nm}$ Zirconium and Platinum grains and plasma conditions $\left(n_{e} \approx 10^{14}, T_{e} \approx 5 \mathrm{eV}\right.$, $T_{i} \approx 0.025 \mathrm{eV}$ ) discussed in the context of abrupt inhomogeneity in chapter VI, the gyro-phase drift magnitude should increase compared to the spherical case. In addition to the increased capacitance, irregularly-shaped grains also rotate and have magnetic moments. Tsytovich et al. (2003) were the first to explain that dust shape asymetry leads to grain rotation from charging processes. Because spinning dust grains have magnetic moments, there is a magnetic interaction force between grains in a dusty plasma, but it is orders of magnitude smaller than the Coloumb interaction force between grains. For a single irregularly-shaped grain in dust-absent conditions, the dust grain magnetic 
moment aligns with the magnetic field. Apart from this alignment, there should be no noticable effect on gyro-phase drift due to the dust grain's inherent magnetic moment.

\section{Gyro-phase Drift in Space}

A common theme throughout this dissertation is that gyro-phase drift causes dust grains to leave regions of inhomogeneity. Gas giants such as Jupiter and Saturn are known to have a radial temperature gradient where both electron and ion temperatures increase with radial distance. For example, the tabulated temperature measurements in table 6.1 clearly indicate that the electron and ion temperatures increase with radial distance from Saturn. When both electron and ion temperatures increase with radial distance, the in-situ-equilibrium grain charge also increases with radial distance. The presence of these temperature gradients around gas giants implies that gyrophase drift transports magnetized-orbit dust grains radially outward. Plasma drag decreases the radial excursion of sub-micron grains over time. The combined effect of gyro-phase drift and plasma

drag causes gyro-centers to accumulate in a radial band that is directed radially outward from their place of origin. 


\section{Chapter VIII}

\section{Conclusions}

In this dissertation, a semi-analytical solution was presented for guiding center drift resulting from an abrupt inhomogeneity for a broad range of parameters, and predictions were made for grain trajectories in both abrupt and gradual inhomogeneities in the Auburn Magnetized Dusty Plasma Experiment. The gyro-phase drift resulting from the adiabatic theory of Northrop and Hill (1983) has been extended to include abrupt inhomogeneties, and a simulation code has been developed to study non-stationary charging effects when grain charge state modulation is not known a priori. The inclusion of non-stationary charging and non-linear drag force terms distinguish this code from other simulation schemes (Pigarov et al., 2005; Martin et al., 2008; Jefferson et al., 2010). The semi-analytical method developed in this dissertation permits modelling over a large range of parameter space, which is an advantage over simulations. In this dissertation, gyro-phase drift, when detectable, is predicted to be a sensitive indicator of sheath mechanisms. The noninstantaneous charging feature of dust grains also allows discrimination of charging models based on the resulting gyro-phase drift. This dissertation also provides the first quantitative prediction that gyro-phase drift is at a maximum when the ratio of gyro-period to charging timescale is near unity for both abrupt and gradual plasma inhomogeneities.

For the abrupt inhomogeneity, the dependence of guiding center drift on ion-neutral collisions, the planar sheath mechanisms of flowing ions (drifting Maxwellian or mono-energetic) and ionneutral charge exchange were demonstrated semi-analytically in the presence of Epstein or linear

neutral drag for a broad range of parameters. Simulations corroborate these semi-analytical results and demonstrate the utility of analyzing grain motion to discriminate among charge models. The 
exhaustive treatment using all relevant parameters that affect grain charging in standard charging models provides an intuitive qualitative picture for how different charging mechanisms alter charge state modulation, the ratio of gyro-period to charging time, and consequently guiding center drift. A range of predictions are offered for the case of the abrupt inhomogeneity in the Auburn Magnetized Dusty Plasma Experiment including uncertainty in the electron temperature of $\pm 0.5 \mathrm{eV}$ and several orders of magnitude in the electron density. Disparate results are obtained when comparing the guiding center drift magnitudes of dust grains for mono-energetic ions to flow-shifted Maxwellian ions for all charge models tested in this dissertation. This suggests that experiments can use dust grains to assess the validity of either sheath mechanism, but the constraints on realistic dusty plasma measurments make this unlikely in the near future. The consistent differences in guiding center drift between the Gatti-Kortshagen charging model and OML/Patacchini-Hutchinson models, even for the low gas pressures expected in MDPX, suggest that MDPX-like experiments should be capable of assessing the validity of the Gatti-Kortshagen charging model if large photo-electron fluxes are used $\left(f_{u v} /\left(n_{e} v_{t h e}\right)>0.01\right)$. The electron current is not greatly affected by the magnetic field and other parameters for imagible grains in MDPX, which cause the OML and Patacchini-Hutchinson charge models to be indistinguishable. Because neither OML and Patacchini-Hutchinson charge models includes the ion-neutral charge exchange grain sheath mechanism, the Gatti-Kortshagen charging model, adjusted for ion flow, should make the most accurate prediction for grain trajectories. The abrupt inhomogeneity was also shown to have particular relevance when investigating sub-micron grains around Saturn, maximizing the chance of a significant role in the resulting single-grain dynamics in the absence of other plasma gradients.

The gradual inhomogeneity has also been analyzed in the context of planar sheath mechanisms and charge model choice and compared to the features of the abrupt inhomogeneity. It was shown that grain capacitance modulation, as a result of changes in the Debye length during a gyro-orbit can lead to charge state modulation and gyro-phase drift, even when the dimensionless surface potential $\chi_{e}=e V_{d} /\left(k_{b} T_{e}\right)$ is constant during the gyro-orbit. Predictions were made for grain trajectories assuming dust-in-plasma conditions in plausible plasma profiles for MDPX, including the full effects of neutral drag and non-linear ion drag, made possible by the development of an iterative leapfrog method in this dissertation. Sub-micron grains are predicted to not drift out of the plasma when non-instantaneous charging is assumed in a gaussian electric field in MDPX, 
whereas these grains will drift radially outward with almost negligible magnitude for radial electric fields that point inward when instantaneous charging is assumed. For the profiles from chapter VI, the ion drag force produces a drift that is in the opposite direction to the gyro-phase drift, meaning grains will not drift out of the plasma.

Analysis of the guiding center drifts for gradual and abrupt inhomogeneities demonstrates the utility of considering non-stationary charging. Even when these dust grains cannot directly be measured, their motions are affected by non-stationary charging. Non-stationary charging mechanisms, first discussed by Nunomura et al. (1999) for grain oscillation in a planar sheath, can be used under certain conditions to measure plasma parameters and validate charge models. 
Appendices 



\section{Appendix A}

\section{Derivations}

\section{Orbit-motion Limited Charge Model derivation}

As the de facto charge model used extensively for collisionless plasmas, the straightforward derivation of Orbit-Motion-Limited charge model is summarized here. The derivation was originally for the application of probes in plasma (Mott-Smith and Langmuir, 1926; Laframboise, 1966), but it is readily applicable to a dust grain under certain assumptions (Shukla, 2001). For a stationary grain, assume a spherically symmetric collector and consider an attracted species with charge $Z e$ and mass $m$. The sphere is unmagnetized with respect to the attracted particle species, meaning that the gyro-radius $R_{L}$ is larger than the grain radius $a$. The sphere is immersed in a plasma, and the attracted particles move isotropically toward the small grain. Knowing that the energy and angular momentum of an attracted particle approaching the spherical grain from far away must be conserved, we seek to find the largest angular momentum of an attracted particle that can be collected. Because the particles are attracted to the spherical grain, particles of all energies can be collected. The energy of the attracted particle far away from the spherical grain is given by

$$
E_{\infty}=\frac{1}{2} m \boldsymbol{v}_{\infty}^{2}+Z e V_{s}
$$

where $V_{s}$ is the space potential of the plasma several Debye lengths from the grain, $m$ is the mass of the attracted species, $\boldsymbol{v}_{\infty}=\boldsymbol{v}_{\boldsymbol{t}}+\boldsymbol{v}_{\boldsymbol{r}}$ is the velocity very far away from the grain, $\boldsymbol{v}_{\boldsymbol{t}}$ is the velocity component transverse the grain's radial direction, and $\boldsymbol{v}_{\boldsymbol{r}}$ is the velocity component parallel to the 
grain's radial direction. The energy of the attracted particle as it just grazes the grain surface must be given by:

$$
E_{a}=\frac{1}{2} m v_{t}(a)^{2}+Z e V_{\text {surf }}(a)
$$

where $r=a$ is the radius of the spherical grain and $V_{\text {surf }}$ is the potential on the surface of the grain. Note that because we are considering an attracted particle whose trajectory just grazes the grain surface, there is no radial velocity component. If we set $E_{\infty}$ from equation A.1 to $E_{a}$ in equation A.2, we can derive an expression for the transverse velocity $\boldsymbol{v}_{\boldsymbol{t}}(a)$ for this attracted particle:

$$
\boldsymbol{v}_{\boldsymbol{t}}(a)=\sqrt{\frac{2}{m}\left(\frac{1}{2} m v_{\infty}^{2}-Z e V_{d}\right)}
$$

where $V_{d}=V_{s}-V_{\text {surf }}$, and $\frac{1}{2} m v^{2}$ equates to the kinetic energy $E$ of the attracted species far from the grain, described by the distribution function. The critical magnitude of the angular momentum $J_{c}$ for this attracted particle that just grazes the spherical grain surface is given by

$$
J_{c}=m\left|\boldsymbol{v}_{\boldsymbol{t}}(a)\right| a=a \sqrt{2 m\left(E-Z e V_{d}\right)}
$$

Only those attracted particles whose angular momentum satifies $0<J<J_{c}$ will be collected by the spherical grain.

The current to the grain from the attracted species $I_{a}$, through current closure, is given by

$$
I_{a}=e \int_{S} r^{2} \sin \phi \mathrm{d} \varphi \mathrm{d} \phi \iiint \boldsymbol{v} \cdot(-\hat{r}) f(\boldsymbol{r}, \boldsymbol{v}) \mathrm{d}^{3} v
$$

where the surface integral is carried out at some radius $r \gg a, f(\boldsymbol{r}, \boldsymbol{v})$ is the velocity distribution function, and the minus sign in the product $\boldsymbol{v} \cdot(-\hat{r})$ indicates that we are interested in attracted particles that are travelling radially inward to the spherical grain. We will associate $u(r)$, the inward radial velocity of attracted particles at some radial distance $r>a$ from the spherical grain, with $\boldsymbol{v} \cdot(\hat{r})$. Additionally, because we have $\boldsymbol{v}_{\infty}=\boldsymbol{v}_{\boldsymbol{t}}+\boldsymbol{v}_{\boldsymbol{r}}$, the appropriate velocity volume integration 
element is given by

$$
\mathrm{d}^{3} v=v_{t} \mathrm{~d} v_{t} \mathrm{~d} v_{r} \mathrm{~d} \varphi
$$

We can therefore rewrite equation A.5 as

$$
I_{a}=-e \int_{\varphi=0}^{\varphi=2 \pi} r^{2} \mathrm{~d} \varphi \int_{\phi=0}^{\phi=\pi} \sin \phi \mathrm{d} \phi \int_{\varphi=0}^{\varphi=2 \pi} \mathrm{d} \varphi \int_{v_{t}=0}^{v_{t}=\infty} v_{t} \mathrm{~d} v_{t} \int_{v_{r}=-\infty}^{v_{r}=\infty} u(r) f\left(\boldsymbol{r}, v_{r}, v_{t}, \varphi\right)
$$

Because the limit on energy and angular momentum has been defined earlier intuitively, we will transform from $v_{t}, v_{r}$ velocity space into energy $E$ and angular momentum $J$ space. The integral and velocity volume integration elements in A.7 will be transformed into a dependence on energy and momentum through the use of the Jacobian (Kennedy and Allen, 2003). Before making this transformation, define $U=v_{t}^{2}$, so that $\mathrm{d} U=2 v_{t} \mathrm{~d} v_{t}$. Hence, $\mathrm{d}^{3} v=\frac{1}{2} \mathrm{~d} v_{r} \mathrm{~d} U \mathrm{~d} \varphi$. Because the angular momentum is given by

$$
J=m\left|v_{t}(r)\right| r
$$

our subsitution $U=v_{t}^{2}$ means that

$$
U=\frac{J^{2}}{m^{2} r^{2}}
$$

It should also be noted that

$$
u(r)=v_{r}=\sqrt{\frac{2}{m}[E-e V(\boldsymbol{r})]-\frac{J^{2}}{m^{2} r^{2}}}
$$

where $V(\boldsymbol{r})$ is the potential variation anywhere in space, contrasted with $V_{s}$, the space potential several Debye lengths from the spherical grain. As shown in the subsequent steps, the dependence on potential variation disappears when the integral of equation A.7 is transformed into an integral over kinetic energy and angular momentum space.

The velocity volume integration elements are related to the energy and angular momentum 
volume elements by

$$
\mathrm{d}^{3} v=\frac{1}{2} \mathrm{~d} v_{r} \mathrm{~d} U \mathrm{~d} \varphi=\frac{1}{2} \hat{J} \mathrm{~d} E \mathrm{~d} J \mathrm{~d} \varphi
$$

where $\hat{J}$ is the Jacobian. The Jacobian is given by the absolute value of the determinant:

$$
\hat{J}=\left|\begin{array}{cc}
\frac{\partial v_{r}}{\partial E} & \frac{\partial v_{r}}{\partial J} \\
\frac{\partial U}{\partial E} & \frac{\partial U}{\partial J}
\end{array}\right|=\left|\begin{array}{cc}
\frac{1}{m u(r)} & \frac{J}{m^{2} r^{2} u(r)} \\
0 & \frac{2 J}{m^{2} r^{2}}
\end{array}\right|=\frac{2 J}{m^{3} r^{2} u(r)}
$$

We can now integrate over the angles $\varphi$ and $\phi$ and rewrite equation A.7 in terms of energy and momentum with appropriate limits, which yields

$$
I_{a}=-e 4 \pi r^{2} \int_{\varphi=0}^{\varphi=2 \pi} \int_{E=0}^{E=\infty} \int_{J=0}^{J=J_{c}} u(r) f(\boldsymbol{r}, E, J, \varphi) \frac{J}{m^{3} r^{2} u(r)} \mathrm{d} J \mathrm{~d} E \mathrm{~d} \varphi .
$$

Notice that the sphere of attracted plasma constituents characterized by radius $r \rightarrow \infty$ cancels, as does $u(r)$; we have removed all dependence on the radial position and the radial velocity. The distribution function no longer has dependence on $\boldsymbol{r}$ because we have taken $r \rightarrow \infty$, meaning that we are using the distribution function far outside the dust sphere sheath, corresponding to the conditions of the attracted species within the plasma. We will now make some restrictions on the energy and momentum distribution function. We assume spherical symmetry, so that the distribution function has no dependence on $\varphi$ and integrate over $\varphi$, which produces a factor of $2 \pi$. The current to the grain from the attracted particles is given by:

$$
I_{a}=-e 4 \pi \int_{E=0}^{E=\infty} \int_{J=0}^{J=J_{c}} f(E, J) \frac{2 \pi J}{m^{3}} \mathrm{~d} E \mathrm{~d} J
$$

For simplicity, we will assume that the attracted particles are in a Maxwellian distribution far away from the dust grain, and a suitable energy and momentum distribution function is given by

$$
f(\boldsymbol{v})=n_{a}\left(\frac{m}{2 \pi k_{b} T_{a}}\right)^{3 / 2} \exp \left(-\frac{m \boldsymbol{v}^{2}}{2 k_{b} T_{a}}\right)=n_{a}\left(\frac{m}{2 \pi k_{b} T_{a}}\right)^{3 / 2} \exp \left(-\frac{E}{k_{b} T_{a}}\right)
$$

where $\boldsymbol{v}$ is the velocity of attracted particles far away from the sphere, $n_{a}$ is the number density of particles far away from the sphere, and $T_{a}$ is the temperature of the particles. Note that this 
simple Maxwell-Boltzmann distribution has no dependence on the angular momentum $J$. In the OML approach, we are not limited to Maxwell-Boltzmann distributions. Any suitable distribution function can be chosen, but here we assume a Maxwell-Boltzmann distribution to illustrate how to reach the common result given by Allen (1992).

Using the energy distribution defined in equation A.15, we can put this into equation A.14 and integrate over $J$ to obtain

$$
I_{a}=-e 4 \pi n_{a} \int_{E=0}^{E=\infty}\left(\frac{m}{2 \pi k_{b} T_{a}}\right)^{3 / 2} \exp \left(-\frac{E}{k_{b} T_{a}}\right) \frac{2 \pi J_{c}^{2}}{m^{3}} \mathrm{~d} E .
$$

When we use the critical value for the angular momentum of an attracted particle that just grazes the probe that was given in equation A.4, we get

$$
I_{a}=-e 4 \pi a^{2} n_{a} \frac{\sqrt{2}}{2 \sqrt{\pi m}}\left(\frac{1}{k_{b} T_{a}}\right)^{3 / 2} \int_{E=0}^{E=\infty}\left(E-e V_{d}\right) \exp \left(-\frac{E}{k_{b} T_{a}}\right) \mathrm{d} E .
$$

Integration of equation ?? yields

$$
I_{a}=e 4 \pi a^{2} n_{a} \sqrt{\frac{2 e T_{a}}{m}} \frac{1}{2 \sqrt{\pi}}\left(1-\frac{e V_{d}}{k_{b} T_{a}}\right) .
$$

If we associate the thermal speed of the attracted particles with $v_{t h, a}=\sqrt{\frac{2 k_{b} T_{a}}{m}}$, then we can rewrite

$$
I_{a}=e 4 \pi a^{2} n_{a} \frac{v_{t h a}}{2 \sqrt{\pi}}\left(1-\frac{e V_{d}}{k_{b} T_{a}}\right)
$$

which gives the familiar result for an attracted species to a spherical probe (Mott-Smith and Langmuir, 1926; Laframboise, 1966; Allen, 1992).

For particles that are repelled from the spherical grain, the description in equation A.2 is modifed by:

$$
E_{r}=\frac{1}{2} m \boldsymbol{v}_{\boldsymbol{t}}(\boldsymbol{a})_{\infty}^{2}+e V_{\text {surf }}
$$

We note also that for the repelled species, only particles with energy $E>e V_{d}$ can be collected. The closure of current to the grain has a similar form to that of the attracted species, and is given 
by

$$
I_{r}=-e 4 \pi \int_{\varphi=0}^{\varphi=2 \pi} \int_{E=e V_{d}}^{E=\infty} \int_{J=0}^{J=J_{c}} f(\boldsymbol{r}, E, J, \varphi) \frac{J}{m^{3}} \mathrm{~d} \varphi \mathrm{d} E \mathrm{~d} J .
$$

The current due to the repelled particles is in terms of an arbitrary distribution function in energy, angular momentum, and azimuthal velocity about the grain. As before, we will assume spherical symmetry and use a Maxwellian distribution of energies to obtain

$$
I_{r}=-e 4 \pi a^{2} n_{r} \frac{\sqrt{2}}{2 \sqrt{\pi m}}\left(\frac{1}{e T_{r}}\right)^{3 / 2} \int_{E=e V_{d}}^{E=\infty}\left(E+e V_{d}\right) \exp \left(-\frac{E}{e T_{a}}\right) \mathrm{d} E .
$$

When equation A.22 is integrated, we obtain

$$
I_{r}=-e 4 \pi a^{2} n_{r} \sqrt{\frac{2 e T_{a}}{m}} \frac{1}{2 \sqrt{\pi}} \exp \left(\frac{V_{d}}{T_{r}}\right)
$$

and using $v_{t h, r}=\sqrt{\frac{2 e T_{r}}{m}}$, then we can rewrite

$$
I_{r}=-e 4 \pi a^{2} n_{r} \frac{v_{t h, r}}{2 \sqrt{\pi}} \exp \left(\frac{V_{p}}{T_{r}}\right)
$$

which gives the familiar result for a repelled species to a spherical probe (Laframboise, 1966; Allen, 1992).

\section{$2 E \times B$ Drift in Slab Geometry}

In this section, the simple case of $\boldsymbol{E} \times \boldsymbol{B}$ drift with a constant grain charge is investigated, and sample trajectories are computed for the analytical theory, Boris scheme, and iterative scheme. These trajectories are qualitatively the same as for the $\boldsymbol{E} \times \boldsymbol{B}$ drift for particles such as ions and electrons, although the much lower charge to mass ratio of dust grains necessitates that the gyroradius will be greatly enlarged, as is implied by $R_{L d}=\frac{m_{d} v_{\perp}}{q_{d} B}$. Also, as demonstrated in figure 4.3, the much larger mass of dust grains (compared to ions or electrons) means that in the presence of an electric field, the dust grain will have an effective gyro-radius that is much larger than in the $\boldsymbol{E}=0$ case. 
The motion for a dust grain in electric and magnetic fields and no other forces can be solved analytically for constant grain charge. This amounts to finding the equations of motion that satisfy the Lorentz force equation:

$$
\boldsymbol{F}=q_{d} \boldsymbol{E}+q_{d} \boldsymbol{v}_{\boldsymbol{d}} \times \boldsymbol{B} .
$$

Without loss of generality, we can specify that the magnetic field is solely along the $\hat{z}$-axis. Any electric field that is parallel or anti-parallel to the magnetic field will not affect the grain's trajectory in the plane of gyration. The effect of an electric field along the magnetic field is to accelerate the particle along magnetic field lines. For this derivation, we consider only the motion in this plane of gyration, and not the grain motion along the magnetic field. This leaves us with

$$
m_{d} \frac{\mathrm{d} \boldsymbol{v}_{\boldsymbol{d}}}{\mathrm{d} t}=q_{d} E_{x} \hat{x}+q_{d} E_{y} \hat{y}+q_{d} B v_{y d} \hat{x}-q_{d} B v_{x d} \hat{y}
$$

where $E_{x}$ and $E_{y}$ are the $x$ and $y$ components of the electric field, respectively, which can be positive or negative and $B$ is the magnitude of the magnetic field at a given spatial location. If we restrict the motion to uniform fields $\boldsymbol{E}=E_{0 x} \hat{x}+E_{0 y} \hat{y}$ and $\boldsymbol{B}=B_{0} \hat{z}$, we are left with the coupled set of differential equations

$$
v_{x d}^{\cdot}=\frac{q_{d} E_{0 x}}{m_{d}}+\frac{q_{d} B_{0} v_{y d}}{m_{d}}
$$

and

$$
v_{y d}^{\cdot}=\frac{q_{d} E_{0 y}}{m_{d}}-\frac{q_{d} B_{0} v_{x d}}{m_{d}}
$$

To solve this coupled set of ordinary differential equations, use the substitution

$$
\psi=v_{x d}+\mathrm{i} v_{y d}
$$

Taking one time derivative, recognizing that $\omega_{c d}=\frac{|q| B_{0}}{m_{d}}$ and $B_{0}$ is defined as being always positive, 
because $+\hat{z}$ has been chosen as the magnetic field direction yields the result

$$
\dot{\psi}=v_{x d}^{\dot{i}}+\dot{i} \dot{y}_{y d}= \pm \frac{\omega_{c d}}{B_{0}}\left(E_{0 x}+\mathrm{i} E_{0 y}\right) \pm \omega_{c d} v_{y d} \mp \mathrm{i} \omega_{c d} v_{x d}= \pm \frac{\mathrm{i} \omega_{c d}}{B_{0}}\left(E_{0 y}-\mathrm{i} E_{0 x}\right) \mp \mathrm{i} \omega_{c d} \psi,
$$

where the upper sign corresponds to a positively charged grain, and the lower sign corresponds to a negatively charged grain. Hence, we have a simple (uncoupled) differential equation for $\psi$,

$$
\dot{\psi} \pm \mathrm{i} \omega_{c d} \psi= \pm \frac{\mathrm{i} \omega_{c d}}{B_{0}}\left(E_{0 y}-\mathrm{i} E_{0 x}\right)
$$

We can assume a solution of the general form:

$$
\psi=A \exp \left(\mp \mathrm{i} \omega_{c d} t\right)+\frac{1}{B_{0}}\left(E_{0 y}-\mathrm{i} E_{0 x}\right)
$$

where $A$ is a constant determined by the initial conditions of the dust grain, the upper sign again signifies positive grain charge, and the lower sign signifies negative grain charge. By inspection, the definition of $\psi$ given in equation A.32 is the solution to the differential equation in A.31. Evident in equation A.32 is that the sign of the grain charge affects only the direction of gyration, not the direction of the $\boldsymbol{E} \times \boldsymbol{B}$ drift.

It now remains to determine $A$, which is achieved by setting

$$
\psi(t=0)=v_{x d}(t=0)+\mathrm{i} v_{y d}(t=0) .
$$

Represent $v_{x d}(t=0)$ with $v_{0 x d}$ and $v_{y d}(t=0)$ with $v_{0 y d}$. The constant $A$ is then given by:

$$
A=v_{0 x d}-\frac{E_{0 y}}{B_{0}}+\mathrm{i}\left(v_{0 y d}+\frac{E_{0 x}}{B_{0}}\right)
$$

where again the lower sign signifies positive grain charge, and the upper sign signifies negative grain charge.

The $x$-component of the dust grain velocity must be given by $\operatorname{Re}(\psi)$, while the $y$-component of 
the dust grain velocity must be given by $\operatorname{Im}(\psi)$. This results in

$$
v_{x d}(t)=\operatorname{Re}(\psi)=\left(v_{0 x d}-\frac{E_{0 y}}{B_{0}}\right) \cos \left(\omega_{c d} t\right) \pm\left(v_{0 y d}+\frac{E_{0 x}}{B_{0}}\right) \sin \left(\omega_{c d} t\right)+\frac{E_{0 y}}{B_{0}}
$$

and

$$
v_{y d}(t)=\operatorname{Im}(\psi)=\left(v_{0 y d}+\frac{E_{0 x}}{B_{0}}\right) \cos \left(\omega_{c d} t\right) \mp\left(v_{0 x d}-\frac{E_{0 y}}{B_{0}}\right) \sin \left(\omega_{c d} t\right)-\frac{E_{0 x}}{B_{0}} .
$$

The expression for the $x$-component of the dust grain trajectory is obtained by integrating equation A.35. If we represent $x_{d}(t=0)$ with $x_{0 d}$ and $y_{d}(t=0)$ with $y_{0 d}, x_{d}(t)$ can be written

$$
\begin{aligned}
x_{d}(t)= & \left(\frac{v_{0 x d}}{\omega_{c d}}-\frac{E_{0 y}}{\omega_{c d} B_{0}}\right) \sin \left(\omega_{c d} t\right) \mp\left(\frac{v_{0 y d}}{\omega_{c d}}+\frac{E_{0 x}}{\omega_{c d} B_{0}}\right) \cos \left(\omega_{c d} t\right)+ \\
& \frac{E_{0 y}}{B_{0}} t \pm\left(\frac{v_{0 y d}}{\omega_{c d}}+\frac{E_{0 x}}{\omega_{c d} B_{0}}\right)+x_{0 d}
\end{aligned}
$$

and $y_{d}(t)$ can be written

$$
\begin{aligned}
y_{d}(t)= & \left(\frac{v_{0 y d}}{\omega_{c d}}+\frac{E_{0 x}}{\omega_{c d} B_{0}}\right) \sin \left(\omega_{c d} t\right) \pm\left(\frac{v_{0 x d}}{\omega_{c d}}-\frac{E_{0 y}}{\omega_{c d} B_{0}}\right) \cos \left(\omega_{c d} t\right)- \\
& \frac{E_{0 x}}{B_{0}} t \mp\left(\frac{v_{0 x d}}{\omega_{c d}}-\frac{E_{0 y}}{\omega_{c d} B_{0}}\right)+y_{0 d} .
\end{aligned}
$$

An example trajectory for $E_{0 x}=-100 \mathrm{~V} / \mathrm{m}, E_{0 y}=-100 \mathrm{~V} / \mathrm{m}$, and $B=4 \mathrm{~T}$ is shown in figure 4.2. Evident in this figure is the close agreement between the simulated (Boris and iterative leapfrog) and analytically derived trajectories of a $1 \mu-\mathrm{m}$ dust grain with $q_{d} \approx 1400$ electrons. The gyro-radius for $\boldsymbol{E}=0$ is $\approx 0.6 \mathrm{~mm}$. It is clear that the presence of an electric field has greatly enlarged the gyro-radius of the dust grain several orders of magnitude larger than $R_{L d}$.

Although the gyro-radius is naïvely given by $R_{L d}=\frac{m_{d} v_{\perp}}{q_{d} B}$, the presence of an electric field enlarges the excursion of a dust grain during a gyro-orbit. Even when assuming that $v_{0 x d}=0$, $v_{0 y d}=0$, which guarantees that the dust grain's gyro-radius is as small as possible, a grain that starts at the origin will be at a significantly greater distance from the origin than $R_{L d}$ when the grain attains an angle of $\phi=\frac{\pi}{4}$ in gyro-phase. In the slab geometry, the direction of the electric field can be chosen as one of the basis vectors so that only one vector component is needed. So for simplicity and to correspond with A.37, if we assume $E_{0 x}=-100 \mathrm{~V} / \mathrm{m}, B=4 \mathrm{~T}, v_{0 x d}=0$, 
$v_{0 y d}=0$, and a dust grain that starts at the origin, at $\phi=\frac{\pi}{2} x_{d}(t)$ is given by

$$
x_{d}\left(t=\frac{\pi}{2 \omega_{c d}}\right)=-\frac{2 E_{0 x}}{\omega_{c d} B_{0}}=\frac{2 m_{d}\left|E_{0 x}\right|}{q_{d} B_{0}^{2}}=R_{e f f} .
$$

Figure 4.3 shows how the ambient radial electric field enlarges the effective gyro-radius of dust grains for a variety of grain sizes and electric fields.

\section{$3 E \times B$ Drift with Linear Drag in Slab Geometry}

In this section, the case of $\boldsymbol{E} \times \boldsymbol{B}$ drift along with a linear (Epstein) drag is investigated. The analytical result is similar to the results from the previous section, although now there is a damping term that causes the amplitude of the gyro-radius of the dust grain to decay exponentially at the dust-neutral collision frequency.

As discussed by Thomas et al. (2012), the requirement $\omega_{c d}>\nu_{d n}$ is necessary for dust grain gyration, where $\omega_{c d}$ is the dust-cylotron frequency and $\nu_{d n}$ is the dust-neutral collision frequency. The dust-neutral collision frequency is given by

$$
\nu_{d n}=\delta \frac{4 \pi}{3} a^{2} n_{n} \frac{m_{n}}{m_{d}} C_{n}
$$

which is achieved by setting the Epstein drag force 3.50 equal to

$$
\boldsymbol{F}_{n}=-m_{d} \nu_{d n}\left(\boldsymbol{v}_{d}-\boldsymbol{v}_{n}\right)
$$

To obtain the equation of motion for a dust grain with neutral drag in addition to electric and magnetic forces, we start with the Lorentz force equation, which results in the following coupled differential equations for $v_{x d}$ and $v_{y d}$

$$
v_{x d}^{\cdot}= \pm \frac{\omega_{c d} E_{0 x}}{B_{0}} \pm \omega_{c d} v_{y d}-\nu_{d n}\left(v_{x d}-v_{x n}\right)
$$

and

$$
v_{y d}^{\dot{y}}= \pm \frac{\omega_{c d} E_{0 y}}{B_{0}} \mp \omega_{c d} v_{x d}-\nu_{d n}\left(v_{y d}-v_{y n}\right)
$$


where the upper sign corresponds to positive dust grain charge, and the lower sign corresponds to negative dust grain charge. The terms $v_{x n}$ and $v_{y n}$, which are the velocity of neutrals in the $x$ and $y$ direction respectively in the lab frame, are retained to include the effect of arbitrary neutral flow.

If we use the definition of $\psi$ given by equation A.29, then we ultimately arrive at a new differential equation for $\psi$ :

$$
\dot{\psi}=\left(\nu_{d n} v_{x n} \pm \frac{\omega_{c d} E_{0 x}}{B_{0}}\right)+\mathrm{i}\left(\nu_{d n} v_{y n} \pm \frac{\omega_{c d} E_{0 y}}{B_{0}}\right)+\left(-\nu_{d n} \mp \mathrm{i} \omega_{c d}\right) \psi
$$

It is readily apparent from equation A.44 that the solution is damped oscillatory motion due to the presence of $\nu_{d n} \psi$ in this differential equation. Velocities will be damped at the dust-neutral collision frequency, while the dust grain will gyrate at the dust gyro-frequency.

The general solution for the differential equation A.44 is

$$
\psi=A \exp \left(-\nu_{d n} t \mp \mathrm{i} \omega_{c d} t\right)+\frac{C_{1}+\mathrm{i} C_{2}}{\nu_{d n}^{2}+\omega_{c d}^{2}}
$$

where

$$
C_{1}=\nu_{d n}^{2} v_{x n} \pm \frac{\nu_{d n} \omega_{c d} E_{0 x}}{B_{0}} \pm \nu_{d n} \omega_{c d} v_{y n}+\frac{\omega_{c d}^{2} E_{0 y}}{B_{0}}
$$

and

$$
C_{2}=\nu_{d n}^{2} v_{y n} \pm \frac{\nu_{d n} \omega_{c d} E_{0 y}}{B_{0}} \mp \nu_{d n} \omega_{c d} v_{x n}-\frac{\omega_{c d}^{2} E_{0 x}}{B_{0}}
$$

We determine the constant $A$ by the same method shown in equation A.33 and we obtain

$$
A=v_{0 x d}-\frac{C_{1}}{\nu_{d n}^{2}+\omega_{c d}^{2}}+\mathrm{i}\left(v_{0 y d}-\frac{C_{2}}{\nu_{d n}^{2}+\omega_{c d}^{2}}\right)
$$

The $x$ and $y$ components of the dust grain velocity are then given by

$$
\begin{aligned}
v_{x}=\operatorname{Re}(\psi)= & \left(v_{0 x d}-\frac{C_{1}}{\nu_{d n}^{2}+\omega_{c d}^{2}}\right) \exp \left(-\nu_{d n} t\right) \cos \left(\omega_{c d} t\right) \pm \\
& \left(v_{0 y d}-\frac{C_{2}}{\nu_{d n}^{2}+\omega_{c d}^{2}}\right) \exp \left(-\nu_{d n} t\right) \sin \left(\omega_{c d} t\right)+\frac{C_{1}}{\nu_{d n}^{2}+\omega_{c d}^{2}}
\end{aligned}
$$


and

$$
\begin{aligned}
v_{y}=\operatorname{Im}(\psi)= & \left(v_{0 y d}-\frac{C_{2}}{\nu_{d n}^{2}+\omega_{c d}^{2}}\right) \exp \left(-\nu_{d n} t\right) \cos \left(\omega_{c d} t\right) \mp \\
& \left(v_{0 x d}-\frac{C_{1}}{\nu_{d n}^{2}+\omega_{c d}^{2}}\right) \exp \left(-\nu_{d n} t\right) \sin \left(\omega_{c d} t\right)+\frac{C_{2}}{\nu_{d n}^{2}+\omega_{c d}^{2}} .
\end{aligned}
$$

Integrating equations A.49 and A.50 with repsect to time gives us the $x$ and $y$ components of the dust grain trajectory:

$$
\begin{aligned}
x_{d}(t)= & \left(v_{0 x d}-\frac{C_{1}}{\nu_{d n}^{2}+\omega_{c d}^{2}}\right) \exp \left(-\nu_{d n} t\right) \frac{\omega_{c d} \sin \left(\omega_{c d} t\right)-\nu_{d n} \cos \left(\omega_{c d} t\right)}{\nu_{d n}^{2}+\omega_{c d}^{2}} \mp \\
& \left(v_{0 y d}-\frac{C_{2}}{\nu_{d n}^{2}+\omega_{c d}^{2}}\right) \exp \left(-\nu_{d n} t\right) \frac{\nu_{d n} \sin \left(\omega_{c d} t\right)+\omega_{c d} \cos \left(\omega_{c d} t\right)}{\nu_{d n}^{2}+\omega_{c d}^{2}}+\frac{C_{1}}{\nu_{d n}^{2}+\omega_{c d}^{2}} t+ \\
& \frac{\nu_{d n}}{\nu_{d n}^{2}+\omega_{c d}^{2}}\left(v_{0 x d}-\frac{C_{1}}{\nu_{d n}^{2}+\omega_{c d}^{2}}\right) \pm \frac{\omega_{c d}}{\nu_{d n}^{2}+\omega_{c d}^{2}}\left(v_{0 y d}-\frac{C_{2}}{\nu_{d n}^{2}+\omega_{c d}^{2}}\right)+x_{0 d}
\end{aligned}
$$

and

$$
\begin{aligned}
y_{d}(t)= & \left(v_{0 y d}-\frac{C_{2}}{\nu_{d n}^{2}+\omega_{c d}^{2}}\right) \exp \left(-\nu_{d n} t\right) \frac{\omega_{c d} \sin \left(\omega_{c d} t\right)-\nu_{d n} \cos \left(\omega_{c d} t\right)}{\nu_{d n}^{2}+\omega_{c d}^{2}} \pm \\
& \left(v_{0 x d}-\frac{C_{1}}{\nu_{d n}^{2}+\omega_{c d}^{2}}\right) \exp \left(-\nu_{d n} t\right) \frac{\nu_{d n} \sin \left(\omega_{c d} t\right)+\omega_{c d} \cos \left(\omega_{c d} t\right)}{\nu_{d n}^{2}+\omega_{c d}^{2}}+\frac{C_{2}}{\nu_{d n}^{2}+\omega_{c d}^{2}} t+ \\
& \frac{\nu_{d n}}{\nu_{d n}^{2}+\omega_{c d}^{2}}\left(v_{0 y d}-\frac{C_{2}}{\nu_{d n}^{2}+\omega_{c d}^{2}}\right) \mp \frac{\omega_{c d}}{\nu_{d n}^{2}+\omega_{c d}^{2}}\left(v_{0 x d}-\frac{C_{1}}{\nu_{d n}^{2}+\omega_{c d}^{2}}\right)+y_{0 d} .
\end{aligned}
$$

As discussed b efore, the upper sign corresponds to positive grain charge, and the lower sign corresponds to negative grain charge. The descriptions in equations A.51 and A.52 give the most general solution for dust grain motion in the plane perpendicular to a magnetic field in slab geometry for arbitrary electric field, particle charge, magnetic field strength, and relative flow between the dust grain and neutrals. The relative flow in a direction parallel to the magnetic field acts to slow the dust grain only in the $\hat{z}$ direction, while the velocity components perpendicular to the magnetic field remain unchanged from equations A.49 and A.50.

\section{Northrop's Adiabatic Approximation to Guiding Center Motion}

The adiabatic approach developed by Kruskal (1959); Berkowitz and Gardner (1959); Northrop (1961, 1963); Northrop and Rome (1978); Northrop and Hill (1983) to describe guiding center drifts 
in gradual inhomogeneity is summarized here. The new semi-analytical approach to the guiding center motion for grains in abrupt inhomogeneity is shown in chapter V. The basic approach used by Northrop (1961) yields solutions for the guiding center drift of an elementary particle (an ion or electron) in a plane perpendicular to the background magnetic field, culminating with the results of Northrop and Hill (1983), which yield gyro-phase and grad-q drifts for dust grains with large charge to mass ratios. Drag forces have been included in this treatment. Because the case of dust grains immersed in the co-rotating frame of a planet has been treated previously and completely, (Northrop and Hill, 1983; Northrop et al., 1989; Northrop, 1992), it will only be summarized briefly here. The drift motions discussed in this section, with the exception of drag, grad-q, and gyro-phase drifts, are applicable to ions and electrons, so these constituents will not be discussed separately.

\section{i Adiabatic Approximation to Guiding Center Motion in Arbitrary Geometry}

A complete description for dust grain gyro-motion including drag forces comes from solutions to the Lorentz force equation,

$$
\boldsymbol{F}=q_{d}\left(\boldsymbol{r}_{\boldsymbol{d}}\right) \boldsymbol{E}\left(\boldsymbol{r}_{\boldsymbol{d}}\right)+q_{d}\left(\boldsymbol{r}_{\boldsymbol{d}}\right) \boldsymbol{v}_{\boldsymbol{d}} \times \boldsymbol{B}\left(\boldsymbol{r}_{\boldsymbol{d}}\right)+m_{d} \boldsymbol{g}\left(\boldsymbol{r}_{\boldsymbol{d}}\right)+\boldsymbol{F}_{\boldsymbol{d n}}\left(\boldsymbol{r}_{\boldsymbol{d}},\left(\boldsymbol{v}_{\boldsymbol{n}}-\dot{\boldsymbol{r}_{\boldsymbol{d}}}\right)\right)+\boldsymbol{F}_{\boldsymbol{d i}}\left(\boldsymbol{r}_{\boldsymbol{d}},\left(\boldsymbol{v}_{\boldsymbol{i}}-\dot{\boldsymbol{r}_{\boldsymbol{d}}}\right), q_{d}\right)
$$

where $\boldsymbol{r}_{\boldsymbol{d}}$ is the position of the dust grain, $F_{d n}$ is the drag force on the dust grain due to collisions with neutral atoms, dependent on grain position and the velocity of neutrals $\boldsymbol{v}_{\boldsymbol{n}}$ relative to the dust grain velocity $\dot{\boldsymbol{r}}_{\boldsymbol{d}}$ as measured in some reference frame $\left(\boldsymbol{v}_{\boldsymbol{n}}-\dot{\boldsymbol{r}}_{\boldsymbol{d}}\right), F_{d i}$ is the drag force on the dust grain due to collisions with ions, including both ion collection and ion-orbit collisions, which is dependent on grain position, the velocity of ions $\boldsymbol{v}_{\boldsymbol{i}}$ relative to the dust grain velocity $\dot{\boldsymbol{r}}_{\boldsymbol{d}}$ as measured in some reference frame $\left(\boldsymbol{v}_{\boldsymbol{i}}-\dot{\boldsymbol{r}_{\boldsymbol{d}}}\right)$, and grain charge, and the gravitational acceleration $\boldsymbol{g}\left(\boldsymbol{r}_{\boldsymbol{d}}\right)$ has been retained because gravitational forces can be non-negligible for dust grains. The equation for the dust grain motion is then given by

$\ddot{\boldsymbol{r}_{\boldsymbol{d}}}=\frac{q_{d}\left(\boldsymbol{r}_{\boldsymbol{d}}\right)}{m_{d}} \boldsymbol{E}\left(\boldsymbol{r}_{\boldsymbol{d}}\right)+\frac{q_{d}\left(\boldsymbol{r}_{\boldsymbol{d}}\right)}{m_{d}} \dot{\boldsymbol{r}_{\boldsymbol{d}}} \times \boldsymbol{B}\left(\boldsymbol{r}_{\boldsymbol{d}}\right)+\boldsymbol{g}\left(\boldsymbol{r}_{\boldsymbol{d}}\right)+\frac{1}{m_{d}} \boldsymbol{F}_{\boldsymbol{d} \boldsymbol{n}}\left(\boldsymbol{r}_{\boldsymbol{d}},\left(\boldsymbol{v}_{\boldsymbol{n}}-\dot{\boldsymbol{r}_{\boldsymbol{d}}}\right)\right)+\frac{1}{m_{d}} \boldsymbol{F}_{\boldsymbol{d} \boldsymbol{i}}\left(\boldsymbol{r}_{\boldsymbol{d}},\left(\boldsymbol{v}_{\boldsymbol{i}}-\dot{\boldsymbol{r}_{\boldsymbol{d}}}\right), q_{d}\right)$. 
For now, assume instantaneous charging, so that the grain charge $q_{d}\left(\boldsymbol{r}_{\boldsymbol{d}}\right)$ can be a function of the grain position only, because it is always at the local in-situ equilibrium charge state. If the dust grain charge varies spatially, it follows necessarily that it must be changing with respect to time, which seems to contradict the assumption that grain charge $q_{d}$ has no time dependence. This issue of grain charge time dependence will be explored in the next section.

If the magnetic field $\boldsymbol{B}$ is chosen as a preferred direction, with $\hat{b}=\boldsymbol{B} /|\boldsymbol{B}|$, tangent to the local magnetic field direction, the grain gyrates in a plane perpendicular to this direction. It is also convenient to define a unit vector, perpendicular to the local magnetic field direction and which is directed away from the local center of curvature of the magnetic field line $\hat{c}$. Define also an azimuthal unit vector, $\hat{a}$. These symbols are chosen for the unit vectors due to their association with their repsective directions, such as $\hat{c}$ for the direction away from the center of the magnetic field curvature, $\hat{a}$ is in an azimuthal direction, while $\hat{b}$ is a unit vector along $\boldsymbol{B}$, and these three unit vectors satisfy the relationships

$$
\begin{aligned}
& \hat{a} \times \hat{b}=\hat{c} \\
& \hat{c} \times \hat{a}=\hat{b} \\
& \hat{b} \times \hat{c}=\hat{a},
\end{aligned}
$$

which are easy to remember. Note that Northrop (1961) choose a coordinate system with a unit vector pointing toward the center of magnetic field curvature, so this is in a direction opposite to $\hat{c}$ as defined in A.55. Northrop (1961) also chooses the third unit vector so that if $\hat{e}_{1}=\hat{b}$ and $\hat{e}_{2}=-\hat{c}, \hat{e}_{1} \times \hat{e}_{2}=\hat{e}_{3}$, which means that the unit vector $\hat{a}$ from A.55 is commensurate with the unit vector $-\hat{e}_{3}$ given by Northrop (1961). In slab geometry, with $\hat{z}$ chosen along $\boldsymbol{B}, \hat{c}=\hat{x}, \hat{a}=\hat{y}$, $\hat{b}=\hat{z}$. In such a geometry (strictly parallel magnetic field lines,) no center of curvature exists but this association is still valid. In cylindrical geometry, where $\hat{z}$ is again along $\boldsymbol{B}, \hat{c}=\hat{r}, \hat{a}=\hat{\varphi}$, $\hat{b}=\hat{z}$. As with slab geometry, if all magnetic field lines are considered to be strictly parallel, there will again be no center of curvature, but the association holds. In arbitrary geometry, these unit vectors might in general be functions of field coordinates, i.e., $\hat{b}=\hat{b}\left(\boldsymbol{r}_{\boldsymbol{d}}\right)$.

It is possible to separate the motion of the grain into motion parallel to the magnetic field, and perpendicular to the magnetic field. The postion vector $\boldsymbol{r}_{\boldsymbol{d}}$ can be separated into a gyro- 
averaged guiding center position $\boldsymbol{R}$, and a phase-dependent gyro-vector from this gyro-averaged guiding center position $\boldsymbol{\rho}$. This vector has the same length as the time-dependent gyro-radius, so $\rho=v_{\perp}(t) / \dot{\phi}$. Assuming the magnetic field is along $+\hat{b}$, as mentioned earlier, the gyro-vector can be entirely described by

$$
\boldsymbol{\rho}=\hat{c} \rho \cos \phi+\hat{a} \rho \sin \phi
$$

where $\phi$ is the gyro-phase angle. Equation A.58 should apply even in cases where the gyro-orbit is not circular; in such cases the gyro-radius of the dust grain becomes gyro-phase dependent, or $\rho=\rho(\phi)$. Rosenbluth and Longmire (1957) established that $\rho$ is first order in the parameter $m_{d} / q_{d}$. The relationship between these three vectors is described by

$$
r_{d}=R+\rho
$$

Substituting equation A.59 for $\boldsymbol{r}_{\boldsymbol{d}}$ into equation A.54 yields

$$
\begin{aligned}
\ddot{\boldsymbol{R}}+\ddot{\boldsymbol{\rho}}= & \frac{q_{d}(\boldsymbol{\rho}+\boldsymbol{R})}{m_{d}} \boldsymbol{E}(\boldsymbol{\rho}+\boldsymbol{R})+\frac{q_{d}(\boldsymbol{\rho}+\boldsymbol{R})}{m_{d}}(\dot{\boldsymbol{R}}+\dot{\boldsymbol{\rho}}) \times \boldsymbol{B}(\boldsymbol{\rho}+\boldsymbol{R})+\boldsymbol{g}(\boldsymbol{\rho}+\boldsymbol{R})+ \\
& \frac{1}{m_{d}} \boldsymbol{F}_{\boldsymbol{d n}}\left(\boldsymbol{\rho}+\boldsymbol{R},\left(\boldsymbol{v}_{\boldsymbol{n}}-\dot{\boldsymbol{R}}-\dot{\boldsymbol{\rho}}\right)\right)+\frac{1}{m_{d}} \boldsymbol{F}_{\boldsymbol{d i}}\left(\boldsymbol{\rho}+\boldsymbol{R},\left(\boldsymbol{v}_{\boldsymbol{i}}-\dot{\boldsymbol{R}}-\dot{\boldsymbol{\rho}}\right), q_{d}\right) .
\end{aligned}
$$

It is possible to completely recast equation A.54 from an equation in the dust grain position $\boldsymbol{r}_{\boldsymbol{d}}$ into an equation of motion for the dust grain guiding center $\boldsymbol{R}$ if equation A.60 is averaged over gyro-phase $\phi$ and the fields $\boldsymbol{E}, \boldsymbol{B}$, and $\boldsymbol{g}$ are Taylor expanded about the guiding center $\boldsymbol{R}$. The result of doing the Taylor expansion yields

$$
\begin{aligned}
\ddot{\boldsymbol{R}}+\ddot{\boldsymbol{\rho}}= & \left(\frac{q_{d}(\boldsymbol{R})}{m_{d}}+\left(\boldsymbol{\rho} \cdot \nabla_{\boldsymbol{R}}\right) \frac{q_{d}(\boldsymbol{R})}{m_{d}}+\ldots\right)\left[\boldsymbol{E}(\boldsymbol{R})+\left(\boldsymbol{\rho} \cdot \boldsymbol{\nabla}_{\boldsymbol{R}}\right) \boldsymbol{E}(\boldsymbol{R})+\ldots\right]+ \\
& \left(\frac{q_{d}(\boldsymbol{R})}{m_{d}}+\left(\boldsymbol{\rho} \cdot \nabla_{\boldsymbol{R}}\right) \frac{q_{d}(\boldsymbol{R})}{m_{d}}+\ldots\right)\left[(\dot{\boldsymbol{R}}+\dot{\boldsymbol{\rho}}) \times \boldsymbol{B}(\boldsymbol{R})+(\dot{\boldsymbol{R}}+\dot{\boldsymbol{\rho}}) \times\left(\boldsymbol{\rho} \cdot \boldsymbol{\nabla}_{\boldsymbol{R}}\right) \boldsymbol{B}(\boldsymbol{R})+\ldots\right]+ \\
& {\left[\boldsymbol{g}(\boldsymbol{R})+\left(\boldsymbol{\rho} \cdot \boldsymbol{\nabla}_{\boldsymbol{R}}\right) \boldsymbol{g}(\boldsymbol{R})+\ldots\right]+} \\
& \frac{1}{m_{d}} \boldsymbol{F}_{\boldsymbol{d n}}\left(\boldsymbol{R},\left(\boldsymbol{v}_{\boldsymbol{n}}-\dot{\boldsymbol{R}}\right)\right)+\frac{1}{m_{d}}\left(\boldsymbol{\rho} \cdot \boldsymbol{\nabla}_{\boldsymbol{R}}-\dot{\boldsymbol{\rho}} \cdot \nabla_{\dot{\boldsymbol{R}}}\right) \boldsymbol{F}_{\boldsymbol{d} \boldsymbol{n}}\left(\boldsymbol{R},\left(\boldsymbol{v}_{\boldsymbol{n}}-\dot{\boldsymbol{R}}\right)\right)+ \\
& \frac{1}{m_{d}} \boldsymbol{F}_{\boldsymbol{d i}}\left(\boldsymbol{R},\left(\boldsymbol{v}_{\boldsymbol{i}}-\dot{\boldsymbol{R}}\right), q_{d}\right)+\frac{1}{m_{d}}\left(\boldsymbol{\rho} \cdot \boldsymbol{\nabla}_{\boldsymbol{R}}-\dot{\boldsymbol{\rho}} \cdot \nabla_{\dot{\boldsymbol{R}}}\right) \boldsymbol{F}_{\boldsymbol{d i}}\left(\boldsymbol{R},\left(\boldsymbol{v}_{\boldsymbol{i}}-\dot{\boldsymbol{R}}\right), q_{d}\right)
\end{aligned}
$$


where $\nabla_{\boldsymbol{R}}$ has components that correspond to the coordinate-component gradient in the guiding center averaged cooridinate system, and $\nabla_{\dot{\boldsymbol{R}}}$ has components that correspond to the velocitycomponent gradients in the guiding center averaged coordinate system. The $\boldsymbol{R}$ subscript will be dropped from the gradient operator in the ensuing discussion and it will be assumed that gradients will be with respect to the guiding center averaged coordinate system. The drag terms with the $\nabla_{\dot{\boldsymbol{R}}}$ operator will be dropped, demonstrated later.

After doing this Taylor expansion, average both sides of the equation over a period in gyrophase $\phi$, i.e., $\langle f(\phi)\rangle=\frac{1}{2 \pi} \int_{0}^{2 \pi} f(\phi) \mathrm{d} \phi$. The terms $\dot{\boldsymbol{R}}$ and $\ddot{\boldsymbol{R}}$ refer to the velocity and the acceleration of the gyro-averaged guiding center, respectively, which means that these quantities are not gyrophase dependent. Likewise, the field quantities are evaluated at the gyro-averaged guiding center position. Although the field quantities are, in general, gyro-phase dependent, the expansion in equation A.61 evaluates field quantities at the gyro-averaged guiding center position, so these quantities do not depend on the gyro-phase angle. Keep terms up to first in the parameter $m_{d} / q_{d}$ or $\rho$ from equation A.61. This is a valid adiabatic expansion parameter, provided $m_{d} / q_{d} \ll 1$ $\mathrm{Kg} / \mathrm{C}$, which may not hold for all dust grain sizes of interest. Knowing that $\rho \propto m_{d} / q_{d}$, gyroaveraging $(\boldsymbol{\rho} \cdot \boldsymbol{\nabla}) \boldsymbol{E}(\boldsymbol{R}), \dot{\boldsymbol{R}} \times(\boldsymbol{\rho} \cdot \boldsymbol{\nabla}) \boldsymbol{B}(\boldsymbol{R}),(\boldsymbol{\rho} \cdot \boldsymbol{\nabla}) \boldsymbol{g}(\boldsymbol{R}), \frac{1}{m_{d}}\left(\boldsymbol{\rho} \cdot \nabla_{\boldsymbol{R}}\right) \boldsymbol{F}_{\boldsymbol{d n}}\left(\boldsymbol{R},\left(\boldsymbol{v}_{\boldsymbol{n}}-\dot{\boldsymbol{R}}\right)\right)$, and $\frac{1}{m_{d}}\left(\boldsymbol{\rho} \cdot \nabla_{\boldsymbol{R}}\right) \boldsymbol{F}_{\boldsymbol{d i}}\left(\boldsymbol{R},\left(\boldsymbol{v}_{\boldsymbol{i}}-\dot{\boldsymbol{R}}\right), q_{d}\right)$ yields zero for each term.

To handle the terms with $\dot{\boldsymbol{\rho}}$ or $\ddot{\boldsymbol{\rho}}$ in equation A.61, it is necessary to compute the time derivatives of $\boldsymbol{\rho}$ in order to determine the order of $m_{d} / q_{d}$ within each term. The first time derivative of the gyro-vector $\rho$ is given by

$$
\dot{\boldsymbol{\rho}}=\left(\dot{\rho} \cos \phi-v_{\perp} \sin \phi\right) \hat{c}+\left(\dot{\rho} \sin \phi+v_{\perp} \cos \phi\right) \hat{a}+\cos \phi \frac{\mathrm{d}(\hat{c} \rho)}{\mathrm{d} t}+\sin \phi \frac{\mathrm{d}(\hat{a} \rho)}{\mathrm{d} t}
$$

Terms involving $\dot{\rho}, \frac{\mathrm{d} \hat{c} \rho}{\mathrm{d} t}$, or $\frac{\mathrm{d} \hat{a} \rho}{\mathrm{d} t}$ are first order in the parameter $m_{d} / q_{d}$, so these terms are dropped if they are not multiplied by another quantity that is at least first order in $q_{d} / m_{d}$ anywhere within equation A.61. Additionally, the presence of drag in equation A.61 implies that $v_{\perp}=v_{\perp}(t)=v_{\perp}(\phi)$, because the drag forces will cause the grain to gain or lose energy over a gyro-orbit depending on the orientation of the drag forces with respect to the background magnetic field. This fact must be acknowledged when including drag forces in the analysis, and the following steps in this section are taken with the assumption that $\mathrm{d} / \mathrm{d} t\left(v_{\perp}\right) \approx 0$, which will have to be re-assessed later. 
Discard the terms $\frac{1}{m_{d}}\left(\dot{\boldsymbol{\rho}} \cdot \nabla_{\dot{\boldsymbol{R}}}\right) \boldsymbol{F}_{\boldsymbol{d n}}\left(\boldsymbol{R},\left(\boldsymbol{v}_{\boldsymbol{n}}-\dot{\boldsymbol{R}}\right)\right)$ and $\frac{1}{m_{d}}\left(\dot{\boldsymbol{\rho}} \cdot \nabla_{\dot{\boldsymbol{R}}}\right) \boldsymbol{F}_{\boldsymbol{d i}}\left(\boldsymbol{R},\left(\boldsymbol{v}_{\boldsymbol{i}}-\dot{\boldsymbol{R}}\right), q_{d}\right)$ from equation A.62 because the highest order terms are only $0^{\text {th }}$ order in $m_{d} / q_{d}$, which are $\approx v_{\perp} \sin \phi$ and $\approx v_{\perp} \cos \phi$, and these terms integrate to zero. The factor of $1 / m_{d}$ beside the drag terms does not affect anything, because the neutral and ion drag must be linear in $m_{d}$, as discussed earlier in chapter III, sections 4 and 5 , so the relative "smallness" of $m_{d}$ is unimportant here when determining the order of terms.

The second time derivative of the gyro-radius vector $\boldsymbol{\rho}$ is given by

$$
\begin{aligned}
\ddot{\boldsymbol{\rho}}= & -\frac{v_{\perp}^{2}}{\rho} \cos \phi \hat{c}-\sin \phi \frac{\mathrm{d}\left(\hat{c} v_{\perp}\right)}{\mathrm{d} t}-\frac{v_{\perp}^{2}}{\rho} \sin \phi \hat{a}+\cos \phi \frac{\mathrm{d}\left(\hat{a} v_{\perp}\right)}{\mathrm{d} t} \\
& -\frac{v_{\perp}}{\rho} \sin \phi \frac{\mathrm{d}(\hat{c} \rho)}{\mathrm{d} t}+\cos \frac{\mathrm{d}^{2}(\hat{c} \rho)}{\mathrm{d} t^{2}}+\frac{v_{\perp}}{\rho} \cos \phi \frac{\mathrm{d}(\hat{a} \rho)}{\mathrm{d} t}+\sin \frac{\mathrm{d}^{2}(\hat{c} \rho)}{\mathrm{d} t^{2}} .
\end{aligned}
$$

This allows the elimination of $\ddot{\boldsymbol{\rho}}$ from equation A. 61 because all the terms up to $0^{\text {th }}$ order in $m_{d} / q_{d}$ gyro-average to zero.

The term $\dot{\boldsymbol{\rho}} \times(\boldsymbol{\rho} \cdot \boldsymbol{\nabla}) \boldsymbol{B}(\boldsymbol{R})$, related to the grad-B drift, can be simplified by

$$
\langle\dot{\boldsymbol{\rho}} \times(\boldsymbol{\rho} \cdot \boldsymbol{\nabla}) \boldsymbol{B}(\boldsymbol{R})\rangle=\frac{v_{\perp} \rho}{2}(\hat{a} \times \hat{b}(\hat{c} \cdot \nabla) \boldsymbol{B}-\hat{c} \times \hat{b}(\hat{a} \cdot \nabla) \boldsymbol{B})=-\frac{v_{\perp} \rho}{2} \nabla B(\boldsymbol{R}),
$$

which is the result from Northrop (1961). The term $\frac{1}{m_{d}} \boldsymbol{\rho} \cdot \boldsymbol{\nabla} q_{d}(\dot{\boldsymbol{\rho}} \times \boldsymbol{B})$, related to the grad-q drift, can be simplified to

$$
\frac{1}{m_{d}}\left\langle\boldsymbol{\rho} \cdot \nabla q_{d}(\dot{\boldsymbol{\rho}} \times \boldsymbol{B})\right\rangle=\frac{v_{\perp}^{2}}{2 q_{d}} \nabla q_{d}
$$

Some of the steps have been left out for these results, see the references. The expansion in A.61 can now be written as

$$
\begin{aligned}
\ddot{\boldsymbol{R}}= & \frac{q_{d}}{m_{d}} \boldsymbol{E}(\boldsymbol{R})+\frac{q_{d}}{m_{d}} \dot{\boldsymbol{R}} \times \boldsymbol{B}(\boldsymbol{R})-\frac{v_{\perp}^{2}}{2 B} \nabla B(\boldsymbol{R})+\frac{v_{\perp}^{2}}{2 q_{d}} \nabla q_{d}(\boldsymbol{R})+\boldsymbol{g}(\boldsymbol{R})+ \\
& \frac{1}{m_{d}} \boldsymbol{F}_{\boldsymbol{d n}}\left(\boldsymbol{R},\left(\boldsymbol{v}_{\boldsymbol{n}}-\dot{\boldsymbol{R}}\right)\right)+\frac{1}{m_{d}} \boldsymbol{F}_{\boldsymbol{d i}}\left(\boldsymbol{R},\left(\boldsymbol{v}_{\boldsymbol{i}}-\dot{\boldsymbol{R}}\right), q_{d}\right) .
\end{aligned}
$$

The result given by equation A.66 gives a differential equation for the guiding center equation of motion. We are only interested in the guiding center velocity, and we will not attempt to solve this differential equation. Rather, to achieve our goal of obtaining all drift motions for the dust grain, 
we need to isolate an expression for the guiding center velocity $\dot{\boldsymbol{R}}$. Henceforth, the dependence of grain charge and field quantities on $\boldsymbol{R}$ will be assumed, e.g., $\boldsymbol{B}(\boldsymbol{R})=\boldsymbol{B}$, etc. We can take the vector product of $\boldsymbol{B}$ with both sides of A.66, using the definition for the guiding center drift velocity in the plane perpendicular to $\boldsymbol{B}$ which is $\dot{\boldsymbol{R}_{\perp}}=\dot{\boldsymbol{R}}-\boldsymbol{B}(\dot{\boldsymbol{R}} \cdot \boldsymbol{B}) / B^{2}$, divide by $B^{2}$ and obtain

$$
\begin{aligned}
\dot{\boldsymbol{R}_{\perp}}= & \frac{\boldsymbol{E} \times \boldsymbol{B}}{B^{2}}+\frac{m_{d} v_{\perp}^{2}}{2 q_{d} B} \frac{\boldsymbol{B} \times \nabla B}{B^{2}}+\frac{m_{d} v_{\perp}^{2}}{2 q_{d}^{2}} \frac{\nabla q_{d} \times \boldsymbol{B}}{B^{2}}+\frac{m_{d}}{q_{d}} \frac{\boldsymbol{g} \times \boldsymbol{B}}{B^{2}}+\frac{1}{q_{d}} \frac{\boldsymbol{F}_{\boldsymbol{d} \boldsymbol{n}} \times \boldsymbol{B}}{B^{2}}+\frac{1}{q_{d}} \frac{\boldsymbol{F}_{\boldsymbol{d} i} \times \boldsymbol{B}}{B^{2}} \\
& -\frac{m_{d}}{q_{d}} \frac{\ddot{\boldsymbol{R}} \times \boldsymbol{B}}{B^{2}} .
\end{aligned}
$$

We need to remove the $\ddot{\boldsymbol{R}}$ term. To do this, we drop all terms that are above $0^{\text {th }}$ order in $m_{d} / q_{d}$. Recall from chapter III, sections 4 and 5 that the drag terms are linear in $m_{d}$, meaning that the drag terms are of order $m_{d} / q_{d}$. So even though the drag forces are functions of $\dot{\boldsymbol{R}}$, they are multiplied by the factor $m_{d} / q_{d}$, so we can ignore their $\dot{\boldsymbol{R}}$ dependence and these terms are dropped. We are left with only

$$
\dot{\boldsymbol{R}_{\perp}}=\frac{\boldsymbol{E} \times \boldsymbol{B}}{B^{2}}
$$

The full vector $\dot{\boldsymbol{R}}$, not just the perpendicular component, is given by

$$
\dot{\boldsymbol{R}}=v_{\|} \hat{b}+\frac{\boldsymbol{E} \times \boldsymbol{B}}{B^{2}}
$$

Take one time derivative of A.69 and we have

$$
\begin{aligned}
\ddot{\boldsymbol{R}}= & \frac{\mathrm{d} v_{\|}}{\mathrm{d} t} \hat{b}+v_{\|} \frac{\partial \hat{b}}{\partial t}+v_{\|}^{2} \frac{\partial \hat{b}}{\partial s_{\|}}+v_{\|}\left(\frac{\boldsymbol{E} \times \boldsymbol{B}}{B^{2}} \cdot \boldsymbol{\nabla}\right) \hat{b} \\
& +\frac{\partial}{\partial t}\left(\frac{\boldsymbol{E} \times \boldsymbol{B}}{B^{2}}\right)+v_{\|} \frac{\partial}{\partial s_{\|}}\left(\frac{\boldsymbol{E} \times \boldsymbol{B}}{B^{2}}\right)+\left(\frac{\boldsymbol{E} \times \boldsymbol{B}}{B^{2}} \cdot \boldsymbol{\nabla}\right) \frac{\boldsymbol{E} \times \boldsymbol{B}}{B^{2}},
\end{aligned}
$$

where $s_{\|}$is the distance along the magnetic field. See Northrop (1961) for the details on how to go from A.69 to A.70, but to summarize the procedure briefly, the process is achieved by taking the total time derivative of all quantities in A.69. We put the result for $\ddot{\boldsymbol{R}}$ from A.70 into A.67 and 
obtain the final result (Northrop, 1961)

$$
\begin{aligned}
\dot{\boldsymbol{R}}_{\perp}= & \frac{\boldsymbol{E} \times \boldsymbol{B}}{B^{2}}+\frac{m_{d} v_{\perp}^{2}}{2 q_{d} B} \frac{\boldsymbol{B} \times \boldsymbol{\nabla} B}{B^{2}}+\frac{m_{d} v_{\perp}^{2}}{2 q_{d}^{2}} \frac{\boldsymbol{\nabla} q_{d} \times \boldsymbol{B}}{B^{2}} \\
& +\frac{m_{d}}{q_{d}} \frac{\boldsymbol{g} \times \boldsymbol{B}}{B^{2}}+\frac{1}{q_{d}} \frac{\boldsymbol{F}_{\boldsymbol{d} \boldsymbol{n}} \times \boldsymbol{B}}{B^{2}}+\frac{1}{q_{d}} \frac{\boldsymbol{F}_{\boldsymbol{d} \boldsymbol{i}} \times \boldsymbol{B}}{B^{2}} \\
& +\frac{m_{d}}{q_{d} B} v_{\|}^{2} \hat{b} \times \frac{\partial \hat{b}}{\partial s_{\|}}+\frac{m_{d}}{q_{d} B} v_{\|} \hat{b} \times \frac{\partial \hat{b}}{\partial t}+\frac{m_{d}}{q_{d} B} v_{\|} \hat{b} \times\left(\frac{\boldsymbol{E} \times \boldsymbol{B}}{B^{2}} \cdot \boldsymbol{\nabla}\right) \hat{b} \\
& +\frac{m_{d}}{q_{d} B} \hat{b} \times \frac{\partial}{\partial t}\left(\frac{\boldsymbol{E} \times \boldsymbol{B}}{B^{2}}\right)+\frac{m_{d}}{q_{d} B} v_{\|} \hat{b} \times \frac{\partial}{\partial s_{\|}}\left(\frac{\boldsymbol{E} \times \boldsymbol{B}}{B^{2}}\right)+\frac{m_{d}}{q_{d} B} \hat{b} \times\left(\frac{\boldsymbol{E} \times \boldsymbol{B}}{B^{2}} \cdot \boldsymbol{\nabla}\right) \frac{\boldsymbol{E} \times \boldsymbol{B}}{B^{2}} .
\end{aligned}
$$

The first term in A.71 is the familiar $\boldsymbol{E} \times \boldsymbol{B}$ drift, the second term is the grad-B drift, the third term is the grad-q drift, the fourth term is the $\boldsymbol{g} \times \boldsymbol{B}$ drift, while the neutral and ion drag forces give rise to generic $\boldsymbol{F} \times \boldsymbol{B}$ drifts, which are the fifth and sixth terms respectively. The general $\boldsymbol{F} \times \boldsymbol{B}$ drift is described by Chen (2006). The seventh term of A.71, the curvature drift, combined with the rest of the third line of A.71 comprise the total inertial drift. The terms in the fourth line of A.71 are explained by Northrop (1961).

In the scope of this dissertation, the fields $\boldsymbol{E}$ and $\boldsymbol{B}$ will be assumed to be constant in time. Additionally, $v_{\|}$will be taken as zero, because we are interested in grains levitated in a planar sheath or launched along the equitorial plane of a planet. This means that the curvature drift and other inertial drift terms can be ignored, which greatly simplifies the expression in A.71. The third line in the expression can be discarded, as can the final line of the expression except for the very last term, $\frac{m_{d}}{q_{d} B} \hat{b} \times\left(\frac{\boldsymbol{E} \times \boldsymbol{B}}{B^{2}} \cdot \nabla\right) \frac{\boldsymbol{E} \times \boldsymbol{B}}{B^{2}}$. For most of the applications in this thesis, this term will be zero, however.

\section{ii Adiabatic Approximation to Guiding Center Motion for Time-dependent Grain Charge}

In section i, we obtained all of the familiar guiding center drifts for dust grains, including a few obscure terms such as the grad-q drift and the $\boldsymbol{F} \times \boldsymbol{B}$ drift due to drag forces. We will now admit time-dependence of the dust grain, allowing the dust grain to vary gyro-synchronously. Following Northrop and Hill (1983); Northrop et al. (1989); Northrop (1992), we will assume that the grain 
charge oscillates sinusoidally around some constant value $q_{0}<0$ during its gyro-orbit and write the grain charge $q_{d}(\boldsymbol{R}, t)$ as a Fourier decomposition

$$
q_{d}\left(\boldsymbol{r}_{\boldsymbol{d}}, t\right)=q_{d}\left(\boldsymbol{r}_{\boldsymbol{d}}, \phi\right)=q_{0}\left(\boldsymbol{r}_{\boldsymbol{d}}\right)+q_{1} \cos \left(\phi-\phi_{1}\right)=q_{0}\left(\boldsymbol{r}_{\boldsymbol{d}}\right)+q_{1}\left(\cos \phi_{1} \cos \phi+\sin \phi_{1} \sin \phi\right),
$$

where $q_{1}<0$ is the coefficient of the charge state modulation, $q_{1} / q_{0} \ll 1, \phi_{1}$ is the gyro-phase angle where the grain is most negatively charged, and we have used gyro-phase dependence as a proxy for time-dependence. A time-dependent grain charge is equivalent to a gyro-phase-dependent grain charge.

The description of $q_{d}$ given by equation A.72, as discussed by Northrop and Hill (1983), is somewhat simplistic, because the grain charge can be any arbitrary function of gyro-phase. As mentioned by Northrop and Hill (1983), higher harmonics $\left(q_{2} \cos 2\left(\phi-\phi_{2}\right)+\ldots\right)$ of grain charge modulation are possible, but are neglected by this truncated expression. Additionally, we are specifying the grain's gyro-phase dependence and the angle at which it is most negatively charged a priori, when in actuality these pieces of information might not be available unless there is some manner of predicting $\phi_{1}$ based on the charging rate and gyro-period of the dust grain. The angle during its gyro-orbit when it is most negatively charged $\phi_{1}$ can also in general be time-dependent, changing with each successive gyro-cycle; we will ignore this complication for now. However, the gyro-phase dependence of A.72 is simple and intuitive enough to illustrate some qualitative features of gyro-phase drift, and we will proceed with this definition for this section.

We start with equation A.61, but this time we include the gyro-phase dependent term $q_{1} \cos \left(\phi-\phi_{1}\right)$. For the sake of brevity, we will discard all of the same terms that we did in section i when we assumed $q_{d}=q_{0}\left(\boldsymbol{r}_{\boldsymbol{d}}\right)$ and performed gyro-averages and ordering of terms in $m_{d} / q_{d}$, and only show the new terms. This leaves us with

$$
\begin{aligned}
\ddot{\boldsymbol{R}}= & \frac{q_{0}}{m_{d}} \boldsymbol{E}+\frac{q_{1} \cos \left(\phi-\phi_{1}\right)}{m_{d}}(\boldsymbol{E}+(\boldsymbol{\rho} \cdot \nabla) \boldsymbol{E}) \\
& +\frac{q_{0}}{m_{d}}(\dot{\boldsymbol{R}}+\dot{\boldsymbol{\rho}}) \times \boldsymbol{B}+\frac{q_{1}}{m_{d}} \cos \left(\phi-\phi_{1}\right)(\dot{\boldsymbol{R}}+\dot{\boldsymbol{\rho}}) \times \boldsymbol{B}+\left(\frac{\boldsymbol{\rho} \cdot \nabla q_{0}}{m_{d}}\right)(\dot{\boldsymbol{R}}+\dot{\boldsymbol{\rho}}) \times \boldsymbol{B} \\
& +\frac{q_{0}}{m_{d}}(\dot{\boldsymbol{R}}+\dot{\boldsymbol{\rho}}) \times(\boldsymbol{\rho} \cdot \nabla) \boldsymbol{B}+\frac{q_{1}}{m_{d}} \cos \left(\phi-\phi_{1}\right)(\dot{\boldsymbol{R}}+\dot{\boldsymbol{\rho}}) \times(\boldsymbol{\rho} \cdot \boldsymbol{\nabla}) \boldsymbol{B}+\left(\frac{\boldsymbol{\rho} \cdot \nabla q_{0}}{m_{d}}\right)(\dot{\boldsymbol{R}}+\dot{\boldsymbol{\rho}}) \times(\boldsymbol{\rho} \cdot \boldsymbol{\nabla}) \boldsymbol{B} \\
& +\boldsymbol{g}+\frac{1}{m_{d}} \boldsymbol{F}_{\boldsymbol{d n}}+\frac{1}{m_{d}} \boldsymbol{F}_{\boldsymbol{d i}} .
\end{aligned}
$$


We can tidy up equation A.73 quickly by using the properties of $\boldsymbol{\rho}$ and $\dot{\boldsymbol{\rho}}$ that were used in section i, because the definition in equation A.58 allows arbitrary phase dependence of the gyro-phase vector. Note, however, that now

$$
\rho(\phi)=\frac{v_{\perp} m_{d}}{\left[q_{0}+q_{1} \cos \left(\phi-\phi_{1}\right)\right] B} \approx \frac{v_{\perp} m_{d}}{q_{0} B} .
$$

Performing the gyro-average and discarding terms of order $m_{d} / q_{d}$ and higher thus leaves us with the terms:

$$
\begin{aligned}
\ddot{\boldsymbol{R}}= & \frac{q_{0}}{m_{d}} \boldsymbol{E}+\left\langle\frac{q_{1} \cos \left(\phi-\phi_{1}\right)}{m_{d}}(\boldsymbol{\rho} \cdot \boldsymbol{\nabla}) \boldsymbol{E}\right\rangle \\
& +\frac{q_{0}}{m_{d}} \dot{\boldsymbol{R}} \times \boldsymbol{B}+\left\langle\frac{q_{1}}{m_{d}} \cos \left(\phi-\phi_{1}\right) \dot{\boldsymbol{\rho}} \times \boldsymbol{B}\right\rangle+\left\langle\frac{\boldsymbol{\rho} \cdot \nabla q_{0}}{m_{d}}(\dot{\boldsymbol{\rho}} \times \boldsymbol{B})\right\rangle \\
& +\left\langle\frac{q_{0}}{m_{d}} \dot{\boldsymbol{\rho}} \times(\boldsymbol{\rho} \cdot \boldsymbol{\nabla}) \boldsymbol{B}\right\rangle+\left\langle\frac{q_{1}}{m_{d}} \cos \left(\phi-\phi_{1}\right)\left(\dot{\boldsymbol{r}}_{\boldsymbol{d}}+\dot{\boldsymbol{\rho}}\right) \times(\boldsymbol{\rho} \cdot \boldsymbol{\nabla}) \boldsymbol{B}\right\rangle \\
& +\boldsymbol{g}+\frac{1}{m_{d}} \boldsymbol{F}_{\boldsymbol{d} \boldsymbol{n}}+\frac{1}{m_{d}} \boldsymbol{F}_{\boldsymbol{d i}} .
\end{aligned}
$$

Two of these gyro-averaged terms were described in section i, i.e., grad-B and grad-q drift. We seek to handle the three new terms which do not obviously disappear when gyro-averaging or keeping terms up to $0^{\text {th }}$ order in $m_{d} / q_{d}$ or $q_{1} / q_{0}$, namely

$$
\begin{aligned}
& \frac{q_{1}}{m_{d}}\left\langle\cos \left(\phi-\phi_{1}\right) \dot{\boldsymbol{\rho}} \times \boldsymbol{B}\right\rangle, \\
& \frac{q_{1}}{m_{d}}\left\langle\cos \left(\phi-\phi_{1}\right) \dot{\boldsymbol{r}_{\boldsymbol{d}}} \times(\boldsymbol{\rho} \cdot \nabla) \boldsymbol{B}\right\rangle,
\end{aligned}
$$

and

$$
\frac{q_{1}}{m_{d}}\left\langle\cos \left(\phi-\phi_{1}\right) \dot{\boldsymbol{\rho}} \times(\boldsymbol{\rho} \cdot \boldsymbol{\nabla}) \boldsymbol{B}\right\rangle
$$

The terms given by A.77 and A.78 can be discarded because they are actually of order $q_{1} / q_{0}$. 
In the adiabatic limit, we only need to deal with A.76. We get:

$$
\begin{aligned}
& \frac{q_{1}}{m_{d}}\left\langle\cos \left(\phi-\phi_{1}\right) \dot{\boldsymbol{\rho}} \times \boldsymbol{B}\right\rangle=\frac{q_{1}}{m_{d}}\left\langle\left(\cos \phi_{1} \cos \phi+\sin \phi_{1} \sin \phi\right) \times\right. \\
& \left.\left(-v_{\perp} \sin \phi \hat{c}+v_{\perp} \cos \phi \hat{a}+\cos \phi \frac{\mathrm{d}(\hat{c} \rho)}{\mathrm{d} t}+\sin \phi \frac{\mathrm{d}(\hat{a} \rho)}{\mathrm{d} t}\right) \times B \hat{b}\right\rangle \\
& =\frac{q_{1} B v_{\perp}}{m_{d}}\left\langle\left(\cos \phi_{1} \cos \phi+\sin \phi_{1} \sin \phi\right)(\sin \phi \hat{a}+\cos \phi \hat{c})\right\rangle \\
& =\frac{q_{1} B v_{\perp}}{m_{d}}\left\langle\left(\frac{1}{2} \cos \phi_{1} \sin 2 \phi+\sin \phi_{1} \sin ^{2} \phi\right) \hat{a}+\left(\frac{1}{2} \sin \phi_{1} \sin 2 \phi+\cos \phi_{1} \cos ^{2} \phi\right) \hat{c}\right\rangle \\
& =\frac{q_{1} B v_{\perp}}{2 m_{d}}\left(\sin \phi_{1} \hat{a}+\cos \phi_{1} \hat{c}\right) \text {. }
\end{aligned}
$$

The $\frac{\mathrm{d}(\hat{c} \rho)}{\mathrm{d} t}$ and $\frac{\mathrm{d}(\hat{a} \rho)}{\mathrm{d} t}$ terms seen in the first line of A.79 are discarded because they are first order in $q_{1} / q_{0}$ while being first order in $m_{d} / q_{d}$. Because we are left with one new extra term from A.79, we append this term to A.71 and obtain

$$
\dot{\boldsymbol{R}}_{\perp}=\frac{q_{1} v_{\perp}}{2 q_{0}}\left(\hat{c} \sin \phi_{1}-\hat{a} \cos \phi_{1}\right)+\ldots \text { other terms }
$$

One can also consider the vector $\hat{a} \sin \phi_{1}+\hat{c} \cos \phi_{1}=\hat{n}$, as done by Northrop and Hill (1983); Northrop (1992). If $\phi_{1}=0, n \pi$, where $n$ is any integer number, then there is only a drift component along $\hat{a}$. Likewise, if $\phi_{1}=n \frac{\pi}{2}$, where $n$ is any integer number, then there is only a drift component along $\hat{c}$. The $\hat{c}$ component of A.80 gives the exact same expression for the radial drift as Northrop (1992), in the limit where $\mathscr{B}=B$, i.e., a non-rotating reference frame. 


\section{Appendix B}

\section{Computer codes}

To analyze the effect of charging rate on the trajectory of a single dust grain, a code, dust_trajectory.m has been written and developed using MATLAB. The single particle code contains the main program and moves the dust grain in configuration space, but it also requires several other subroutines, which will be explained. The main program uses either the Boris algorithm or an iterative method to update the particle position and velocity, which is a good compromise of speed, accuracy, and stability. This code only supports trajectories in two dimensions, which is all that is needed to model a magnetized orbit dust grain. Motion of the dust grain is given with reference to a coordinate system where the z-axis is defined as the magnetic field direction. The main program is capable of modeling particle trajectories in inhomogeneous plasma, where the inhomogeneity is described analytically. An Inhomogeneous $n_{e} / n_{i}$ ratio can, for example, lead to an in-situ grain charge that varies as a function of space. This changing in-situ grain charge can lead to an alteration of the grain trajectory, which we call the grad-q drift. The free parameter $\alpha$ is used as an input to the program to artificially delay the charging of the dust grain. When $\alpha<1$, the dust grain is made to charge more slowly than the natural charging rate. When the charging rate of the dust grain is significant when compared to the gyro-period, this leads to an alteration of the grain trajectory which we refer to as the gyro-phase drift. In this appendix, examples of MATLAB code used for the simulations is reproduced.

The dust trajectory code relies on a suite of other sub-functions that have been written specifi-

cally to support dust_trajectory.m in the purpose of tracking the dust grains charge and trajectory. Also, some helpful scripts have been written that take advantage of the sub-functions to plot 
information regarding in-situ grain charge, $n_{e} / n_{i}$ profiles, etc. Starting with dust_trajectory.m, each of these components will be described in turn, starting with the larger scope functions and working down to the functions which are smaller in scope. These sub-functions can and may need to be modified if additional situations are to be modeled. The shortcomings of any sub-function will be addressed in the summary of each one. Codes used for analysis of the simulation data, such as gyrophaser.m will also be included.

\section{Grain Motion}

This section contains brief descriptions along with the MATLAB codes for the necessary routines and subroutines needed to obtain grain trajectories. The subroutines from section 4 are also needed for grain trajectories.

\section{i Main Simulation Routine}

The program dust_trajectory.m is the main simulation routine, which uses several parameters as inputs, allows the user to specify the number of gyro-cycles and number of Newton timesteps per gyro-cycle. There are various error-checking procedures to make sure that negative temperatures and other spurious quantities cannot be entered.

This routine uses the inputs: ion species (a positive integer), a charge model selection (which must be entered as a string, e.g., "oml"), a profile type (which must be entered as a string, e.g., 'uniform_E'), the plasma density $n_{0}$ which is given in units of $\mathrm{m}^{-3}$, a representative electron temperature $T_{e 0}$ which is given in units of Volts, a representative ion temperature $T_{i 0}$ which is given in units of Volts, the ionization number of the ion species Z (which is a positive integer), the neutral gas pressure $\mathrm{P}$ which must be input in units of Pascals, and $\alpha_{m}$, which is the charging rate control parameter (a number, generally between 0 and 1 which delays or slows down the charging rate of dust grains.) The main program outputs the following data arrays when a simulation is run:

1.) $\mathrm{t}$, which is an array for the time data where $t_{n+1}=t_{n}+\Delta t$ where $n$ is the Newton timestep number

2.) $\mathrm{q}$, which is an array containing the discretized representation of $q(t)$

3.) $\mathrm{x}$ is an array containing the discretized particle position $\mathrm{x}(\mathrm{t})$ for the $\mathrm{x}$-direction of the grain at 
integer multiples of $\Delta t$

4.) $\mathrm{y}$ is an array containing the discretized particle position $\mathrm{y}(\mathrm{t})$ for the $\mathrm{y}$-direction of the grain at integer multiples of $\Delta t$

5.) $\mathrm{vx}$ is an array containing the discretized particle velocity of the grain in the $\mathrm{x}$-direction at half integer multiples of $\Delta t$. It should be noted that $\operatorname{vx}(1)$ is the velocity at $t=-\Delta t / 2$, due to the nature of the Boris or leapfrog method that is used

6.) vy is an array containing the discretized particle velocity of the grain in the $\mathrm{x}$-direction at half integer multiples of $\Delta t$. It should be noted that $\mathrm{vy}(1)$ is the velocity at $t=-\Delta t / 2$, due to the nature of the Boris or leapfrog method that is used

7.) RLd is an array containing the discretized particle

8.) $\mathrm{s}$

9.) ne_time

10.) ni_time

11.) ne_s

12.) ni_s

13.) E_xt

14.) $\mathrm{E}_{-} \mathrm{yt}$

15.) E_sx

16.) E_sy

As an additional feature, dust_trajectory.m saves nearly all the data arrays and constants used in the main body of the program into a .mat file; this is coded in the last line. To save the file into a different format, such as ASCII, the last line of the program should be changed accordingly.

The flow of the main program is as follows: the initial values and local plasma parameters are calculated based on the block of user input at the beginning of the program and from the output of profiles.m (assuming a valid profile has been chosen). Based on the local plasma parameters, the equilibrium dust charge is found using charging_models.m, and $q(t=0)$ is given this value. Choosing $q(t=0)=q_{e q}$ is the preferred option since it starts the dust grain out at the in-situ equilibrium grain charge, but other choices can be made which will result in transient behavior of $q(t)$. Such a choice will need to be input manually into the program, by setting $q(1)$ equal to the 
desired initial dust grain charge. Arrays for $\mathrm{t}, \mathrm{q}(\mathrm{t}), \mathrm{x}(\mathrm{t}), \mathrm{y}(\mathrm{t}), \mathrm{vx}(\mathrm{t}), \mathrm{vy}(\mathrm{t}), \mathrm{RL}(\mathrm{t}), \operatorname{ne}(\mathrm{t}), \operatorname{ni}(\mathrm{t})$, $\operatorname{Ex}(t), \operatorname{Ey}(t), f x(t), f y(t)$, and lambda_d(t) are allocated and initialized. The last step before the main time loop begins is to run the Boris algorithm backwards to obtain vx(- $\Delta t / 2)$ and vy(- $\Delta t / 2)$.

In the main loop, the particle position $\mathrm{x}$ and $\mathrm{y}$ are calculated at the full timestep, meaning for example that in the first time through this loop, $\mathrm{x}(t=\Delta t)$ and $\mathrm{y}(t=\Delta t)$ are calculated. Any field or parameter that is a function of $\mathrm{x}$ or $\mathrm{y}$ is computed at this time as well. Velocities are calculated at half timesteps, meaning that in the first time through the main loop, $\mathrm{vx}(t=\Delta t)$ and $\mathrm{vy}(t=\Delta t)$ are computed. It is possible to configure the code so that velocities and positions are calculated at the same timestep, which means that the velocities must be given a half rotation backward due to the magnetic field, and a half backward acceleration must be applied. If this is done, then there will be one less element in the velocity arrays than in the position arrays. Currently, velocities are offset by half a timestep from the position and field quantities. The positions, field quantities, profile quantities, grain charge, and velocities are computed using the Boris algorithm, which is explained below:

1.) Velocities are calculated according to:

2.) Positions are calculated according to:

3.) Profile quantities, such as electric field, densities as function of space, and other quantities are calculated using profiles.m

4.) With the local plasma parameters now specified via profiles.m, the grain charge is updated in time using accumulate_charge.m.

5.) The time array is updated according to $t_{\text {new }}=t_{\text {old }}+d t$.

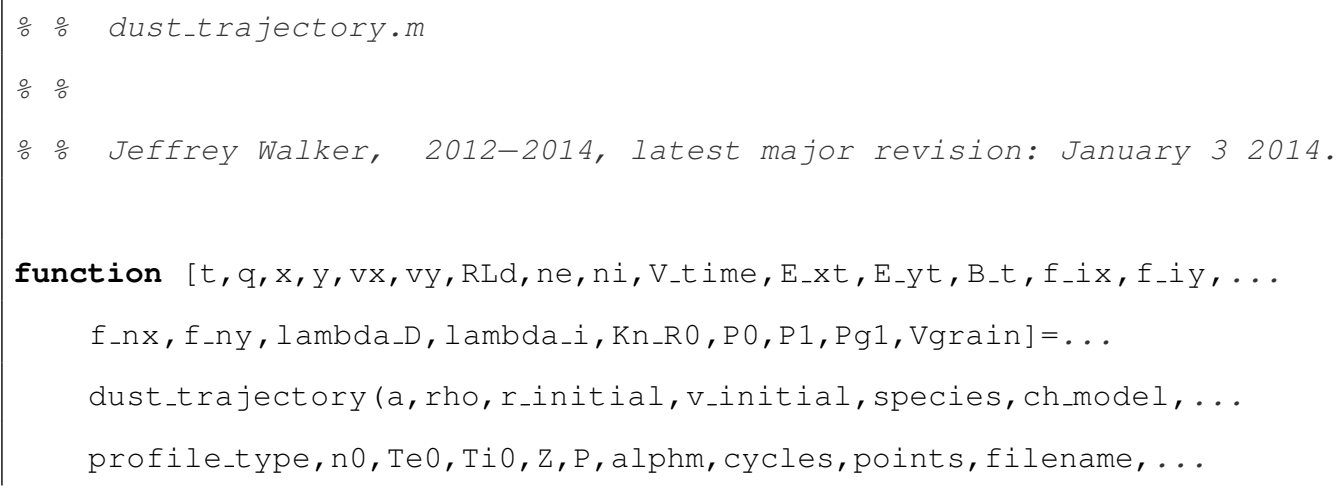


particle_pusher)

oxplanation of inputs:

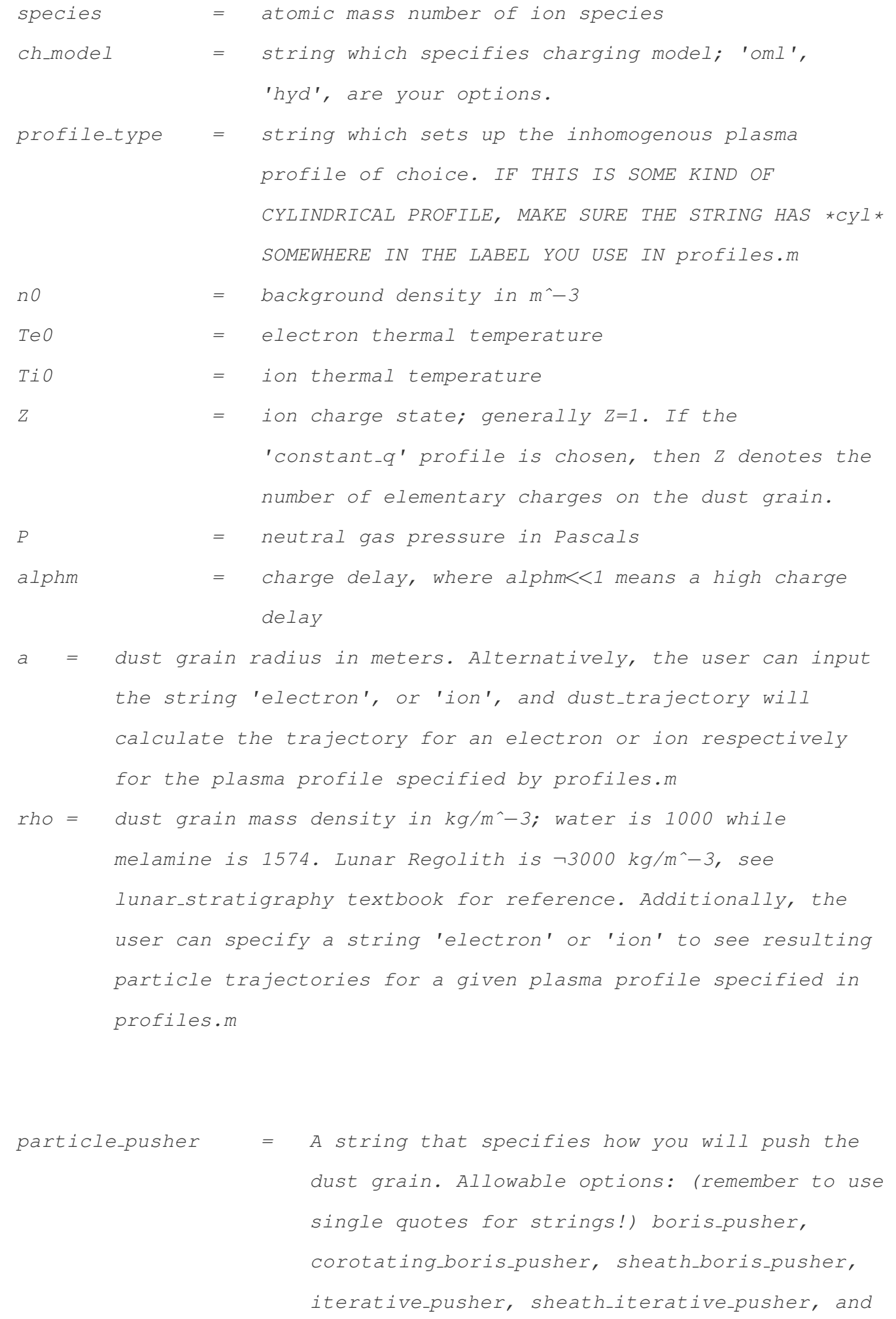




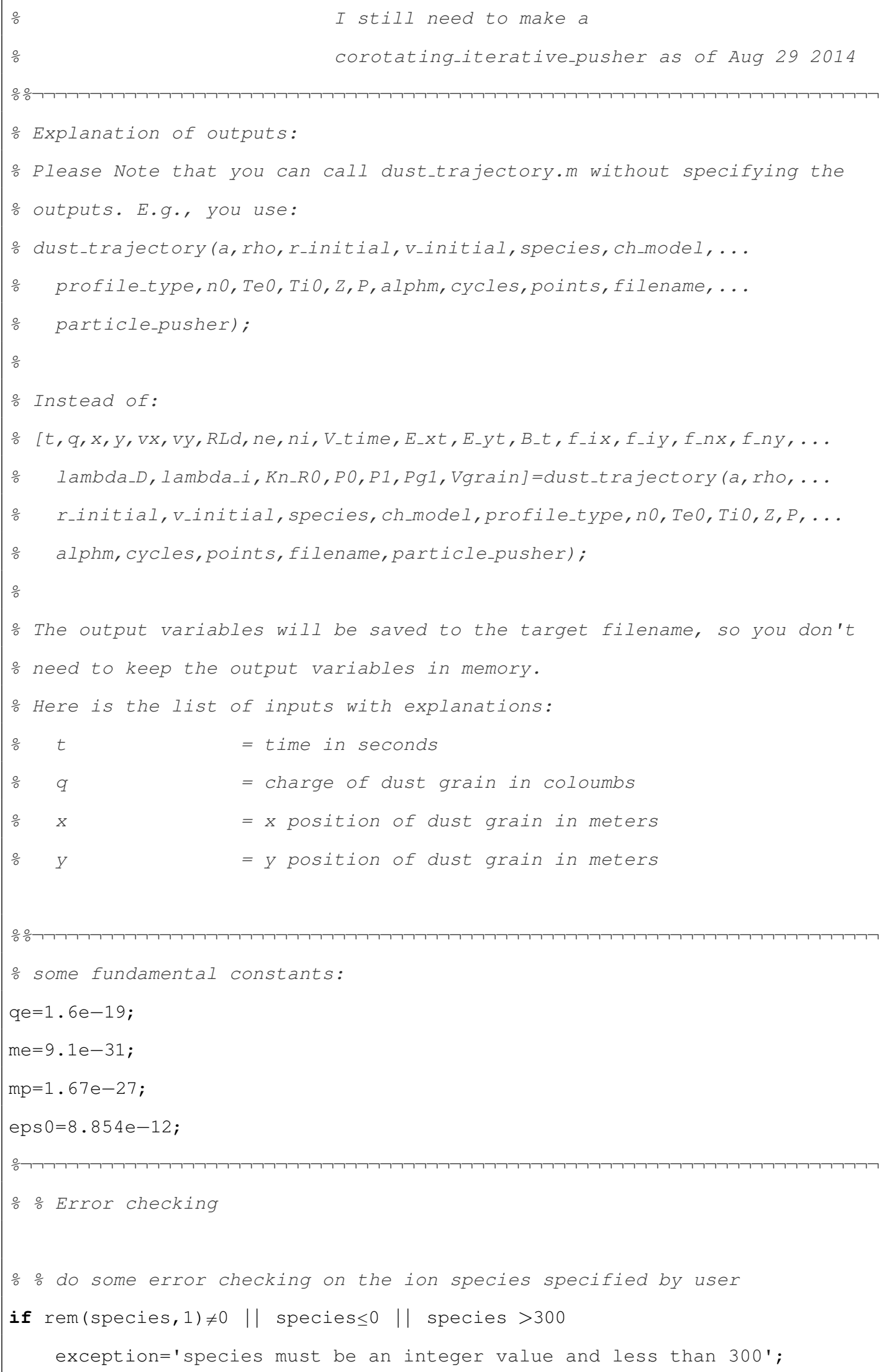




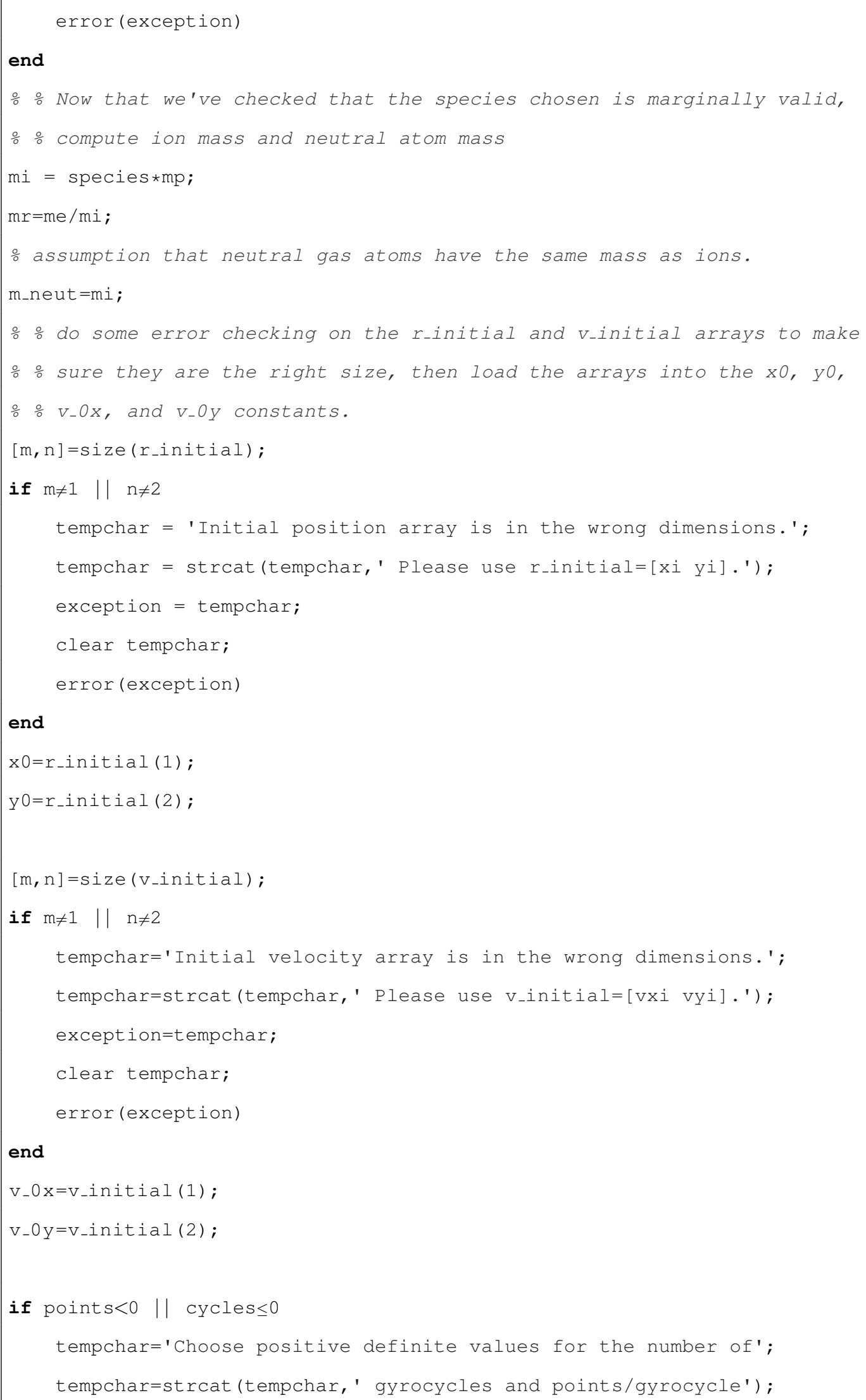


exception=tempchar;

clear tempchar;

error (exception)

\section{end}

o check to make sure background plasma density, electron temperature, ion

- temperature, and adjustable charge rate parameter are all greater than

응 zero.

if $\mathrm{n} 0 \leq 0 \quad|| \operatorname{Te} 0 \leq 0 \quad|| \mathrm{Ti} 0 \leq 0 \quad|| \mathrm{al} h \mathrm{ph} \leq 0$

tempchar='Choose positive definite values for plasma density,';

tempchar=strcat (tempchar,' ion/electron temperatures (in eV), and');

tempchar=strcat (tempchar,' adjustable charge rate parameter alpha,m.');

exception=tempchar:

clear tempchar;

error (exception)

\section{end}

$\div$ now that we now TiO is okay, set Tn=Tio.

May 2014: Maybe this should be set as an input??

$\operatorname{Tn}=\mathrm{Ti} 0$

o determine if a is input as a number (the usual state of affairs) or as a

o string. If 'electron' or 'ion' are input for a, the code will run using

음 the electron or ion mass as the dust grain mass. Hence, it can be used to

o produce electron/ion trajectories for a given profile.

if ischar $(a)==0$

if $a \leq 0 \quad|| a>0.01$

tempchar='Choose positive definite values for the dust grain';

tempchar=strcat (tempchar,' radius. Also, choose $a<0.01 \mathrm{~m}$ ');

exception=tempchar;

clear tempchar;

error(exception)

end

․ if the user has chosen a floating value for a, or a numerical value

응 $a$, and has not specified 'constant_q' as the charge model, then

o the ion charge number $z$ must be restricted to positive definite

- values, and probably less than 118, since that is the atomic number

응 the heaviest known element.

$\div \quad$ if $(Z \leq 0|| Z>118||$ floor $(Z) \neq Z) \quad \& \& \ldots$ 


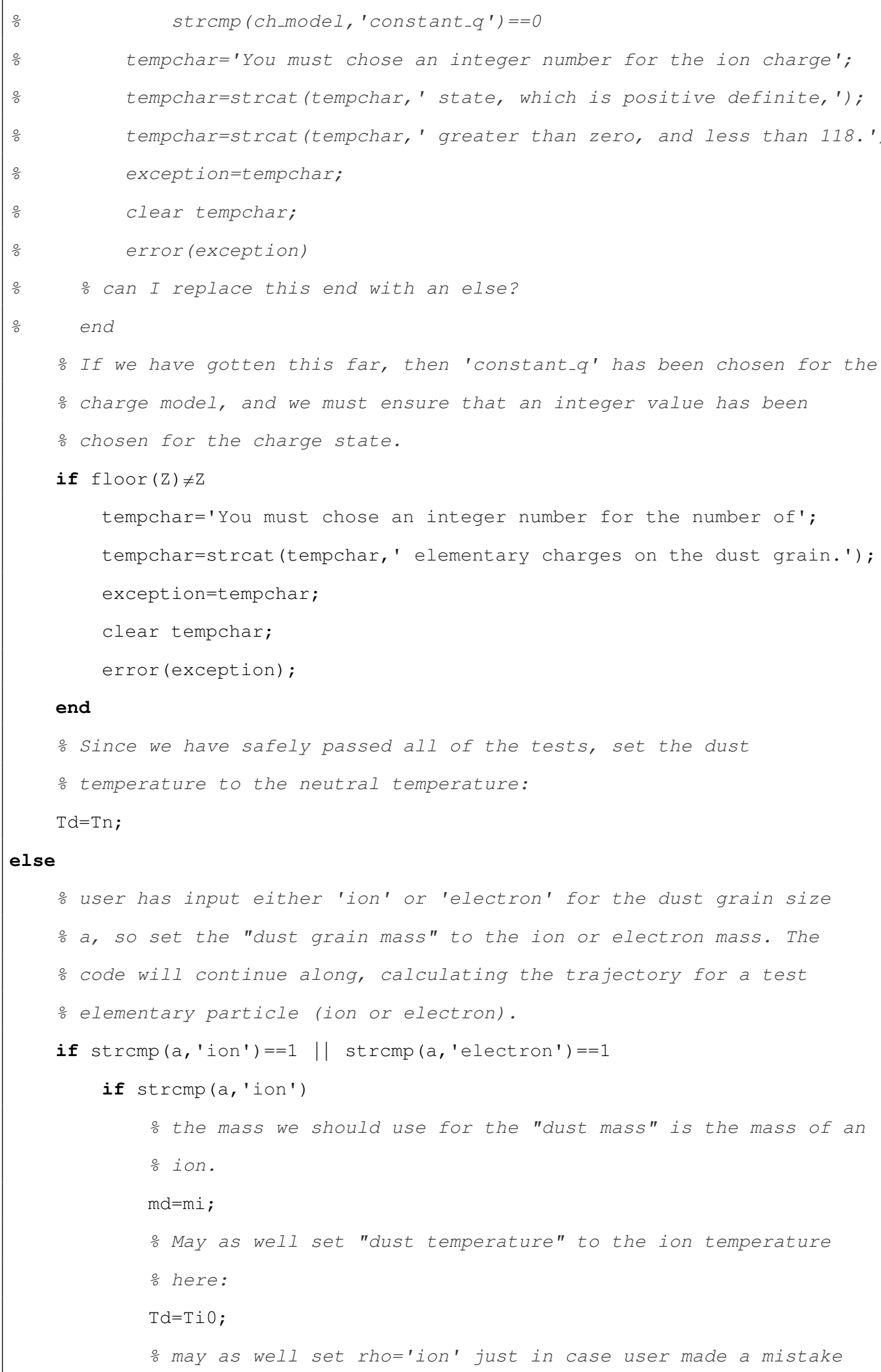




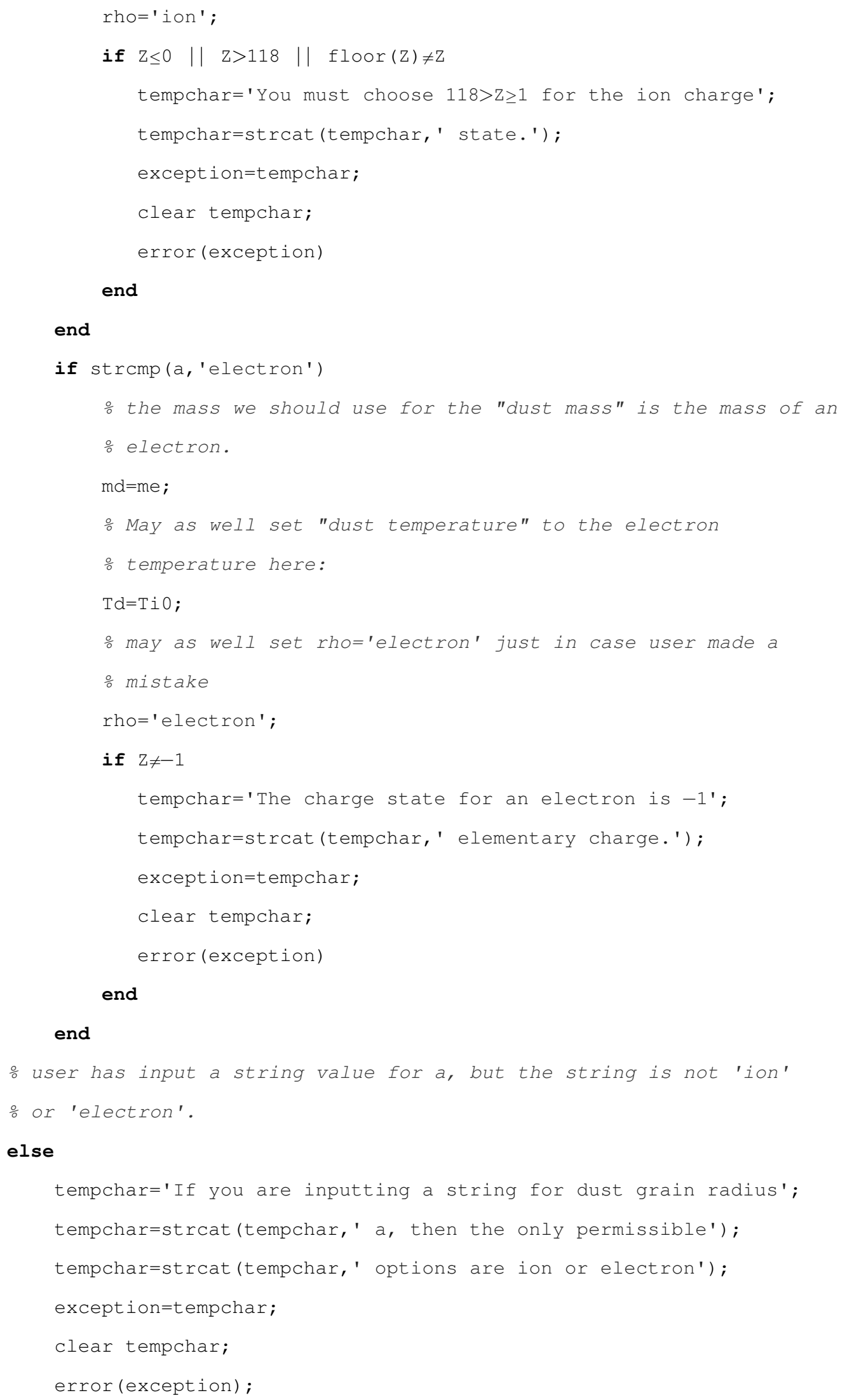




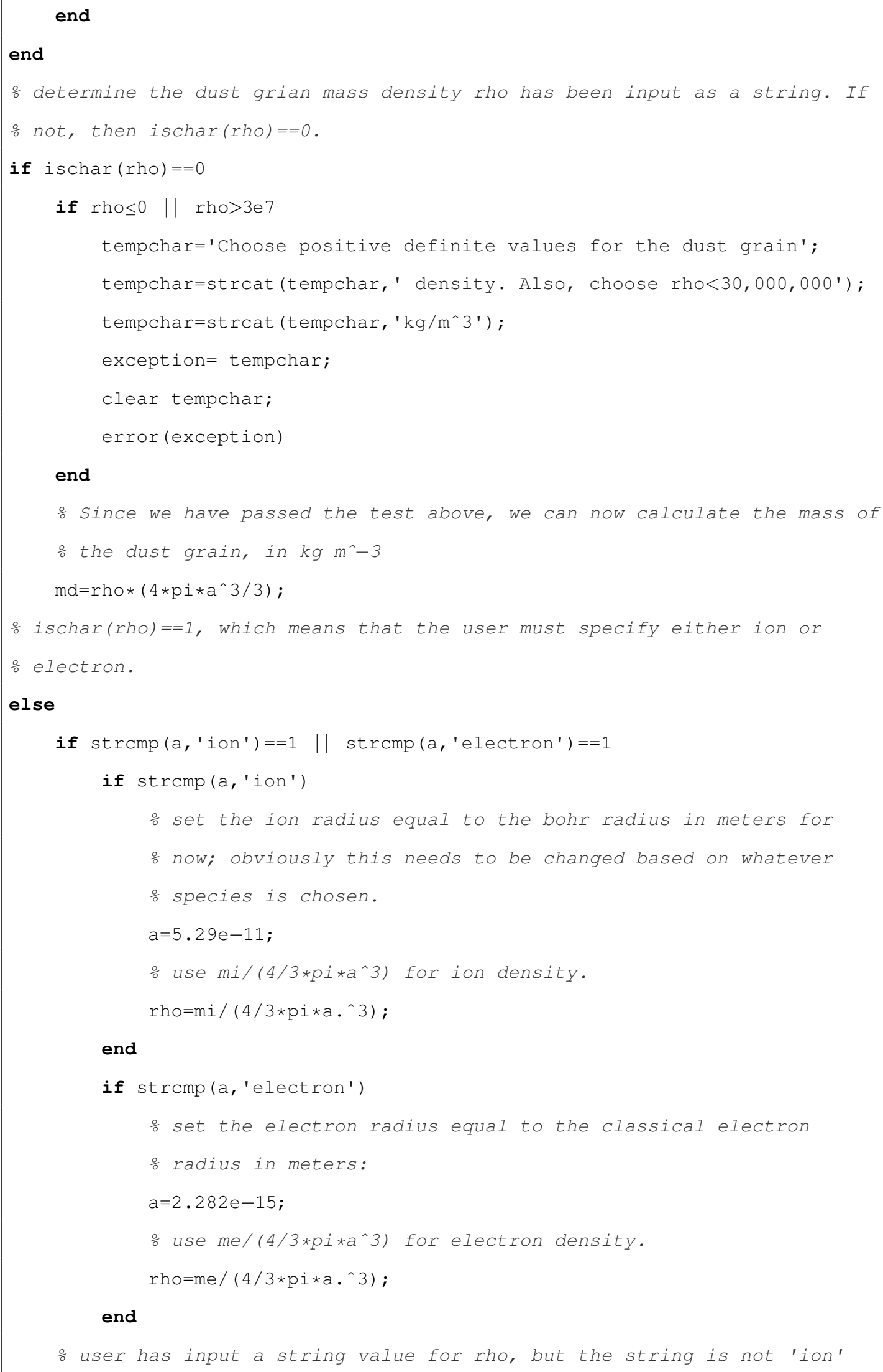


or 'electron'.

else

tempchar='If you are inputting a string for dust grain density';

tempchar=strcat (tempchar,' rho, then the only permissible');

tempchar=strcat (tempchar,' options are ion or electron');

exception=tempchar;

clear tempchar;

error(exception);

end

end

․ Make sure the user does not input a negative value for the neutral gas

응 pressure!

if $\mathrm{P}<0$

tempchar='Choose positive definite values for the neutral gas';

tempchar=strcat (tempchar,' pressure $(\mathrm{P} \geq 0)$. ');

exception=tempchar;

clear tempchar;

error (exception)

\section{end}

능 shis segment of code may no longer be necessary.

if $z \leq 0 \quad|| z>200$

․ tempchar='Choose a positive definite value for the ion charge state.';

\% tempchar=strcat (tempchar, 'Also, Z is restricted to <200.');

exception=tempchar;

olear tempchar;

error(exception)

$\therefore$ end

음 if a filename is not specified, then the output will be written to

$\div$ test.mat

if length (filename)==0 || ischar (filename) $==0$

filename=' test' ;

tempchar='A valid filename was not chosen, so data will be written';

tempchar=strcat (tempchar,' to test.mat') ;

disp (tempchar);

clear tempchar;

end 


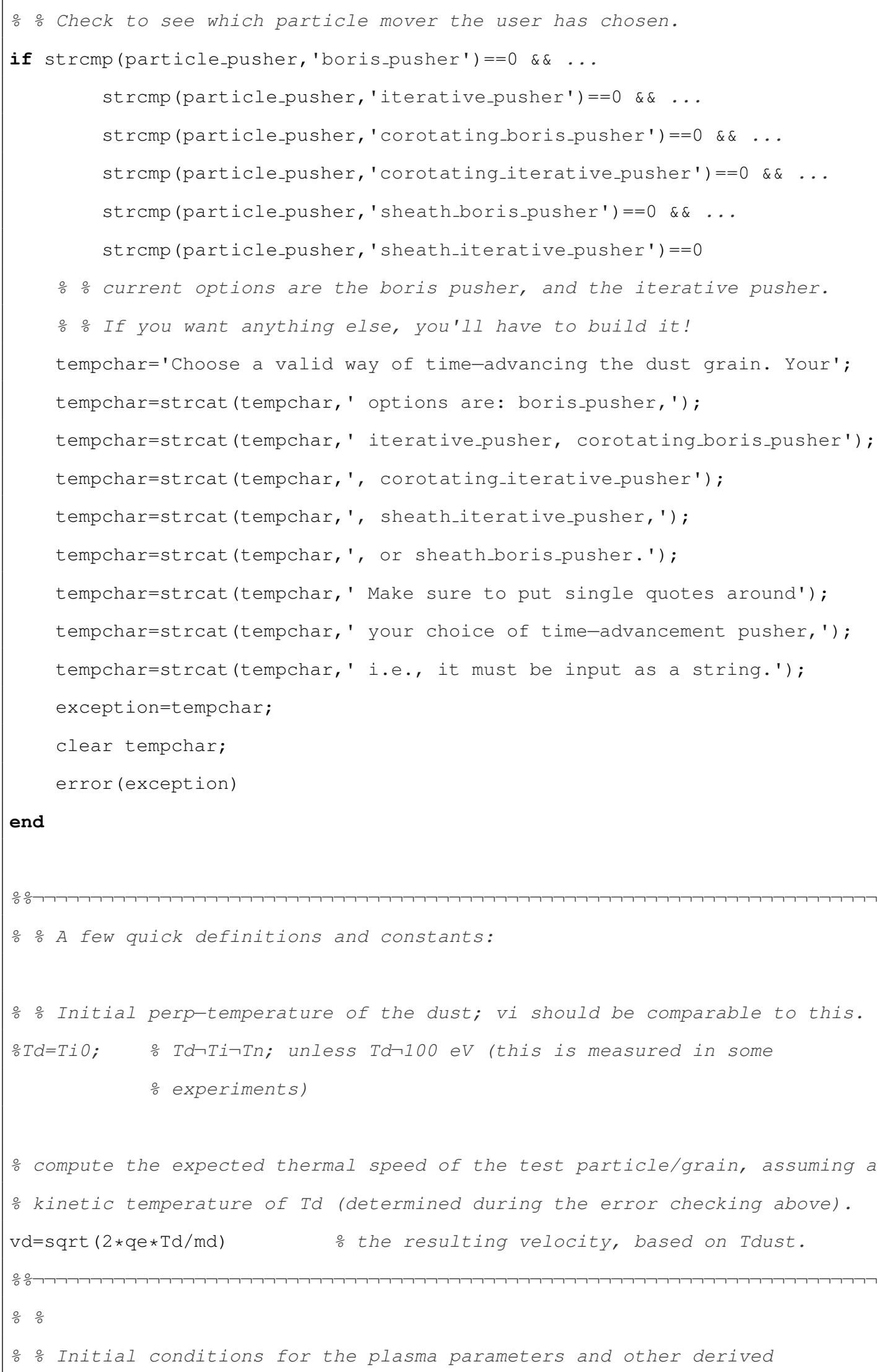




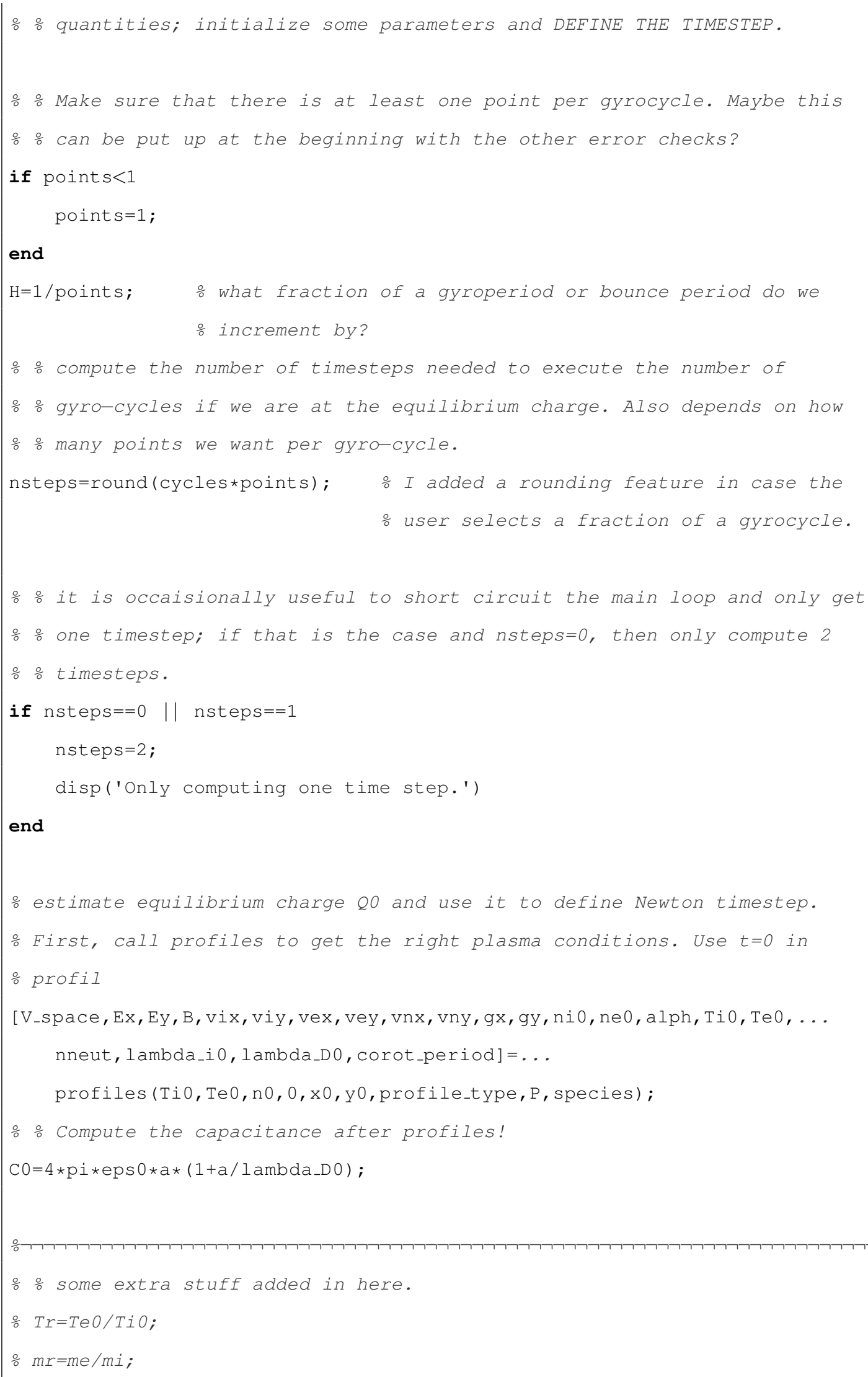




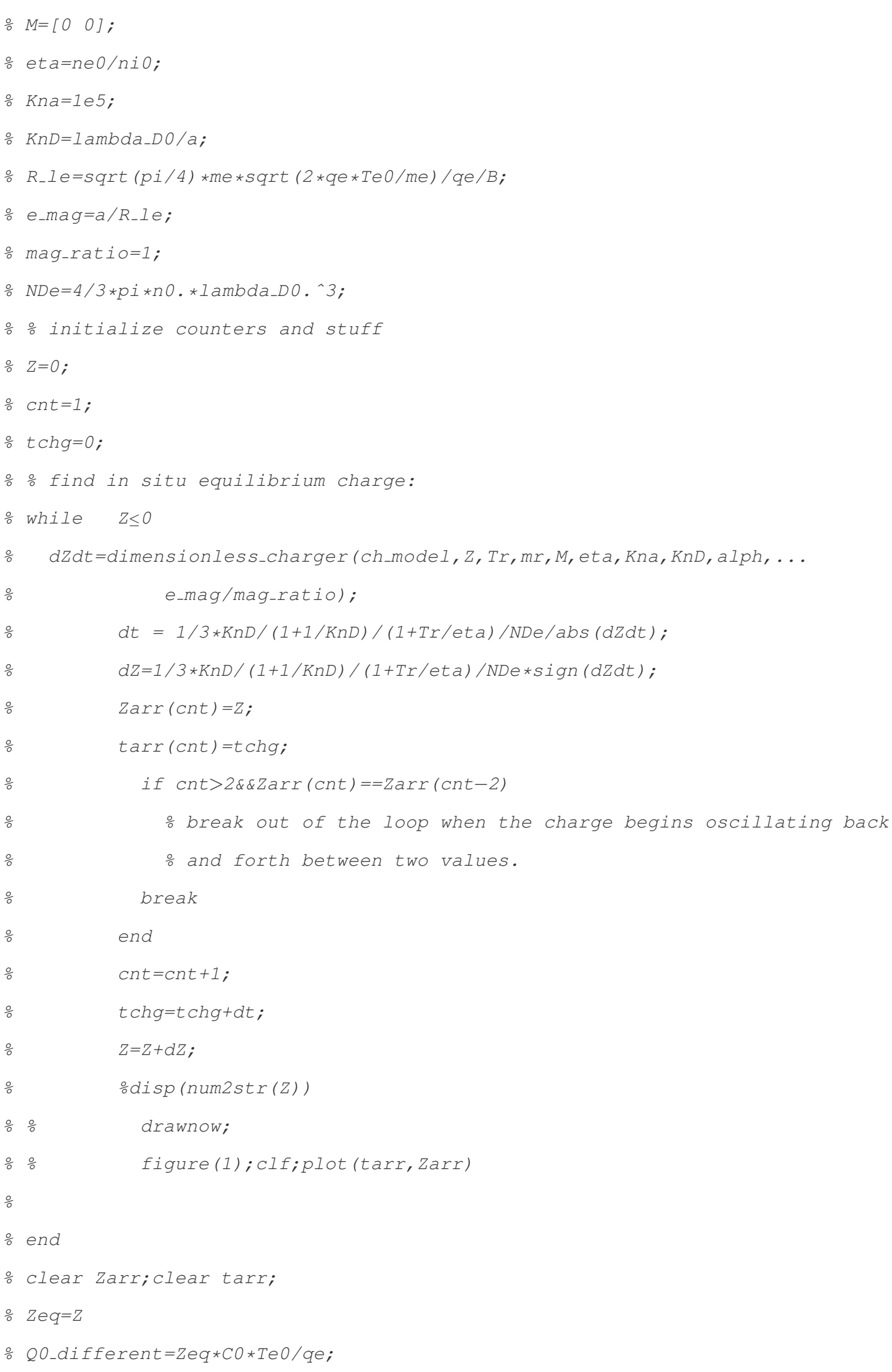




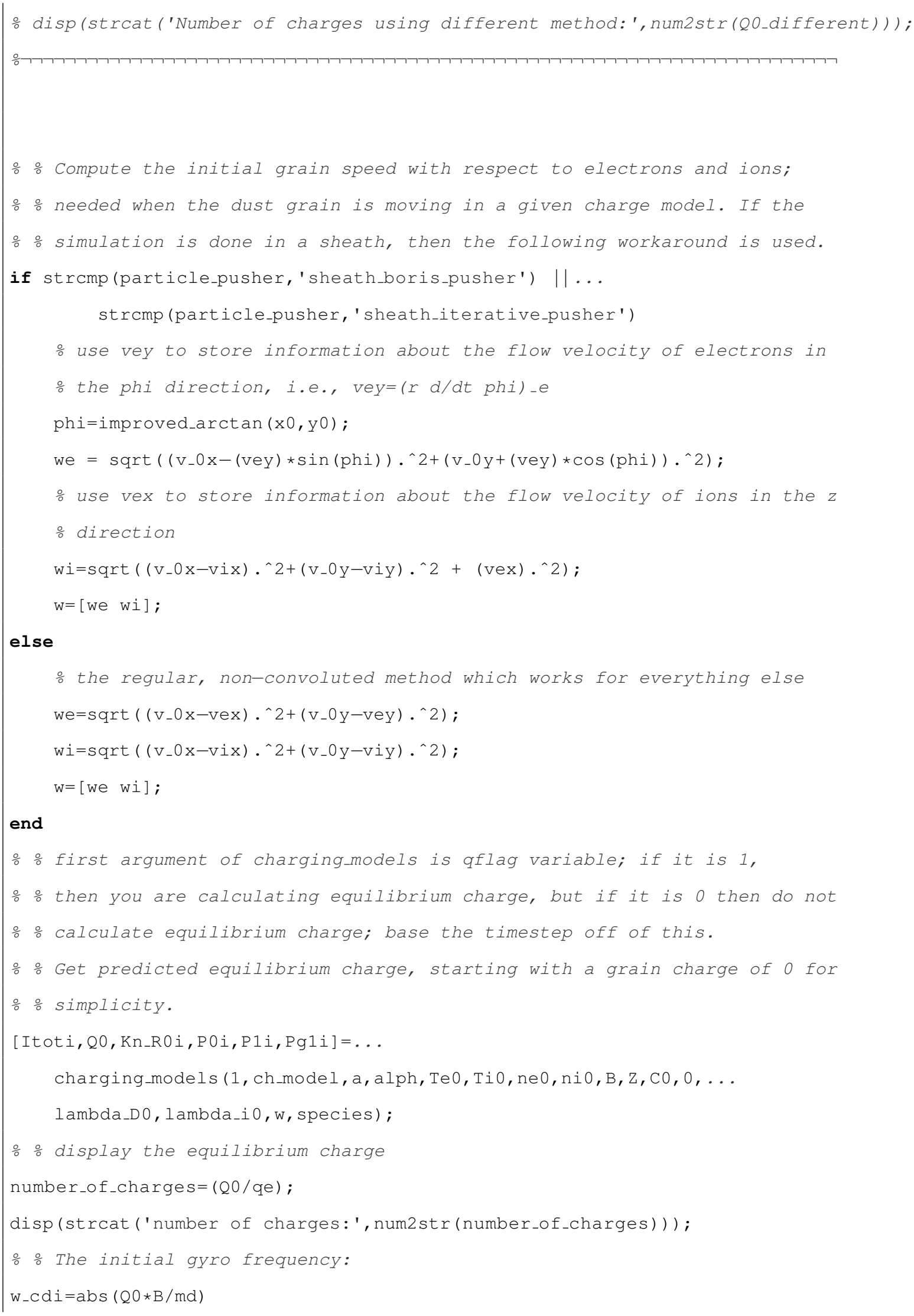




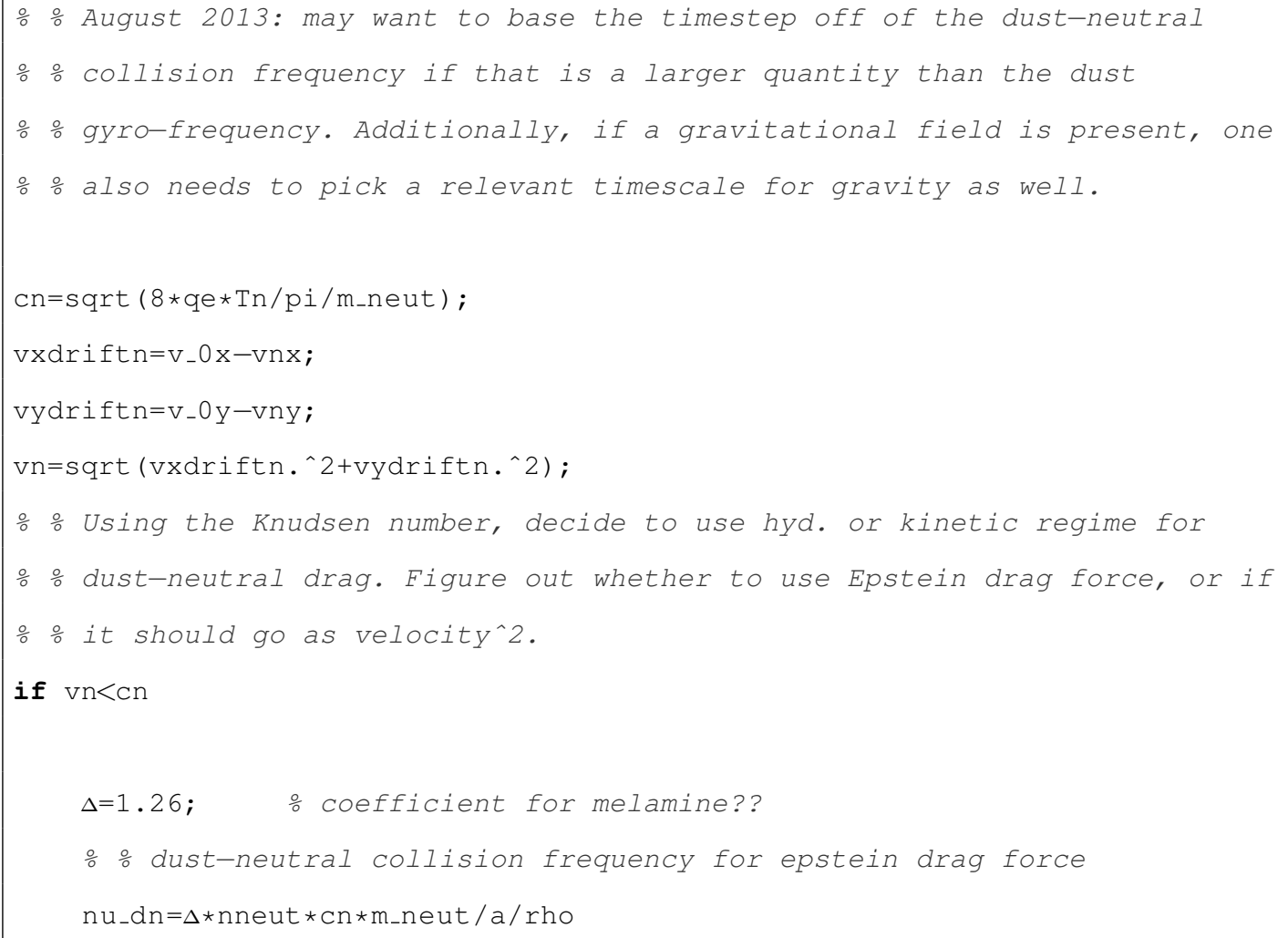


음 right below this comment is where the electric field should be

응 checked. it may be possible to define some frequency which relies

$\div$ 응 $|E|$.

\% $\frac{\circ}{0}$ attempt to find a bounce period in order to specify a time step.

dtNwt $=0.001 ; \quad \therefore$ Just a guess right now

\section{end}

응 Now check which is the largest freqency, and choose the smallest

응 timescale for the timestep. If all quantities are zero, you will bypass

응 this loop and dtNwt will be fixed according to the if statement above.

if $W_{-} c d i>n u_{-} d n$

if corot_period $==0$

$\mathrm{dtNwt}=\mathrm{H} * 2 * \mathrm{pi} / \mathrm{w}_{-} \mathrm{Cdi}$

else

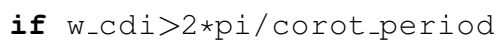

$\mathrm{d} t \mathrm{Nw} t=\mathrm{H} \star 2 * \mathrm{pi} / \mathrm{w}_{-} \mathrm{Cdi}$

else

$d t N w t=H *$ corot_period

end

end

else

if corot_period $==0$

응 this case, check to see if $B=0$; this next set of statements

응 is used for small oscillations in a sheath. This requires that

ion flow must be non-zero. Dust charge must also be non-zero. See

응 notebook \#7, page 53.

if $B==0 \quad \& \& \quad W(2) \neq 0 \quad \& \& \quad Q 0 \neq 0$

tempcharl='Using small oscillation frequency.';

tempchar2=' Make sure grain starts slightly below the sheath';

tempchar3=' boundary.' ;

disp (strcat (tempchar1, tempchar2, tempchar3));

$\therefore d t N w t=H * 2 * p i / n u_{-} d n / 50$

use profiles again to find out what is the potential

응 difence between the plasma and the lower electrode:

$[\mathrm{V} 0, \neg, \neg, \neg, \neg, \neg, \neg, \neg, \neg, \neg, \neg, \neg, \neg, \neg, \neg, \neg, \neg, \neg, \neg, \neg, \neg]=\ldots$

profiles (Ti0, Te0, n0, 0,0,0,profile_type,P, species); 


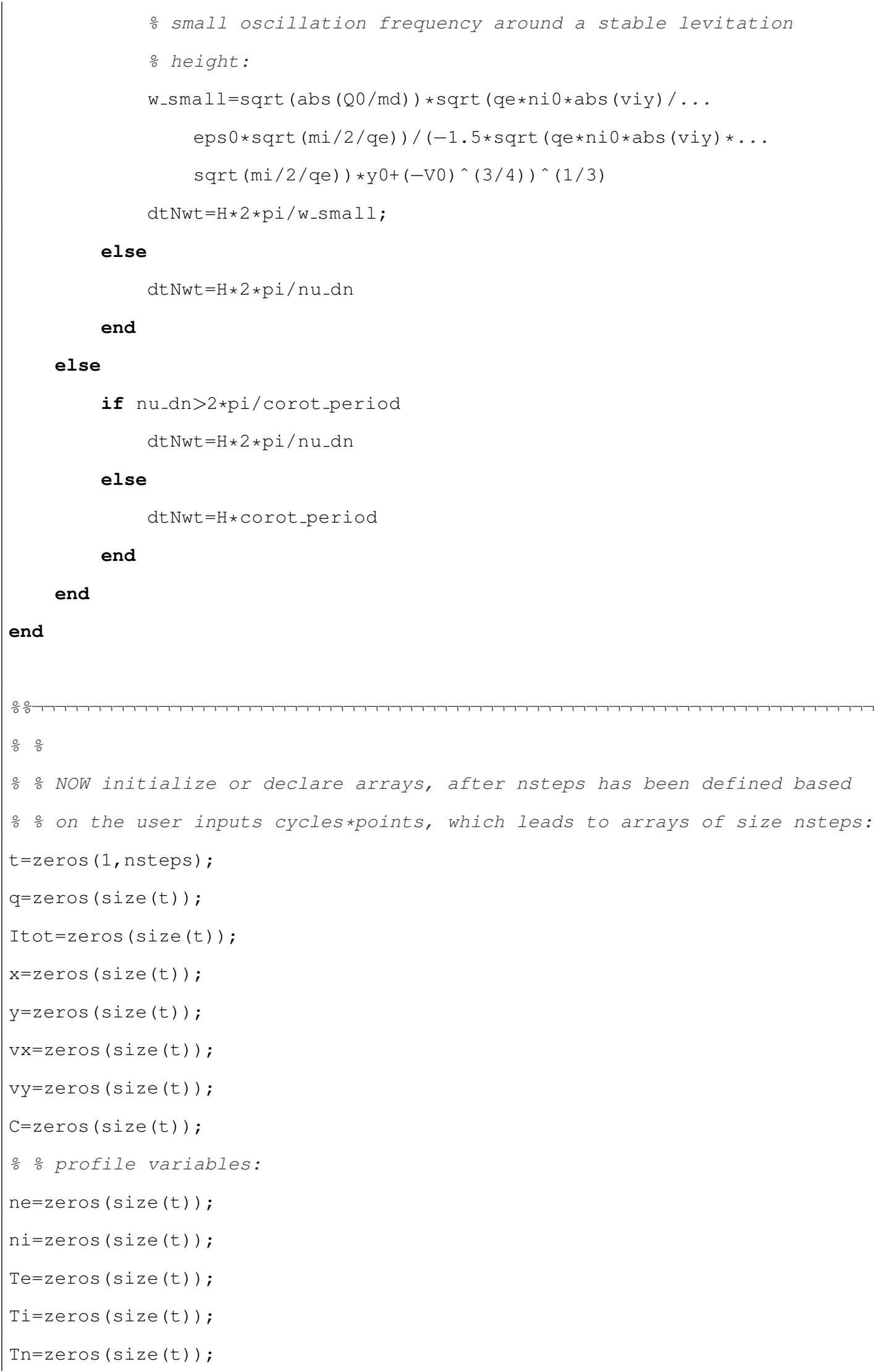




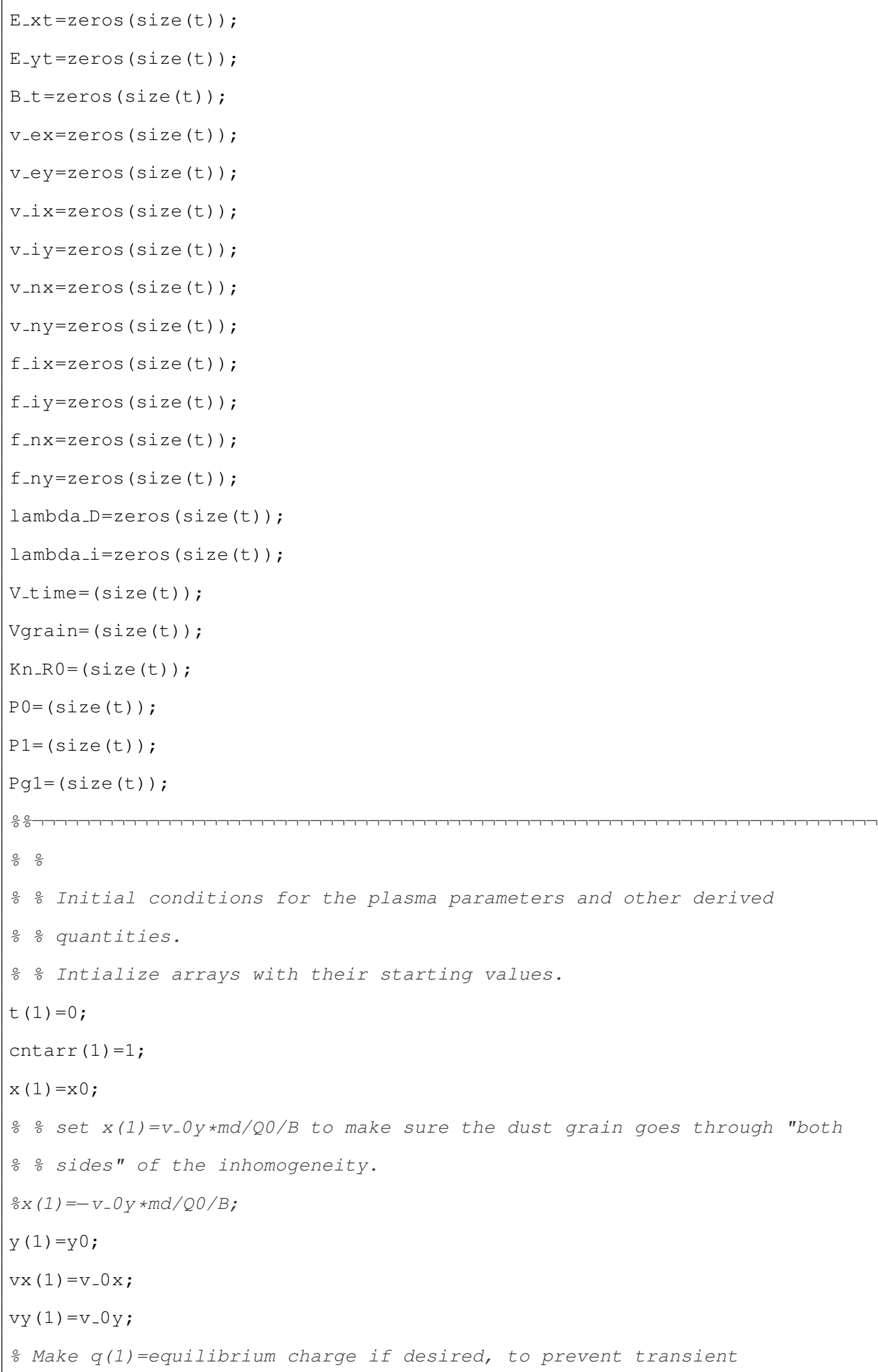




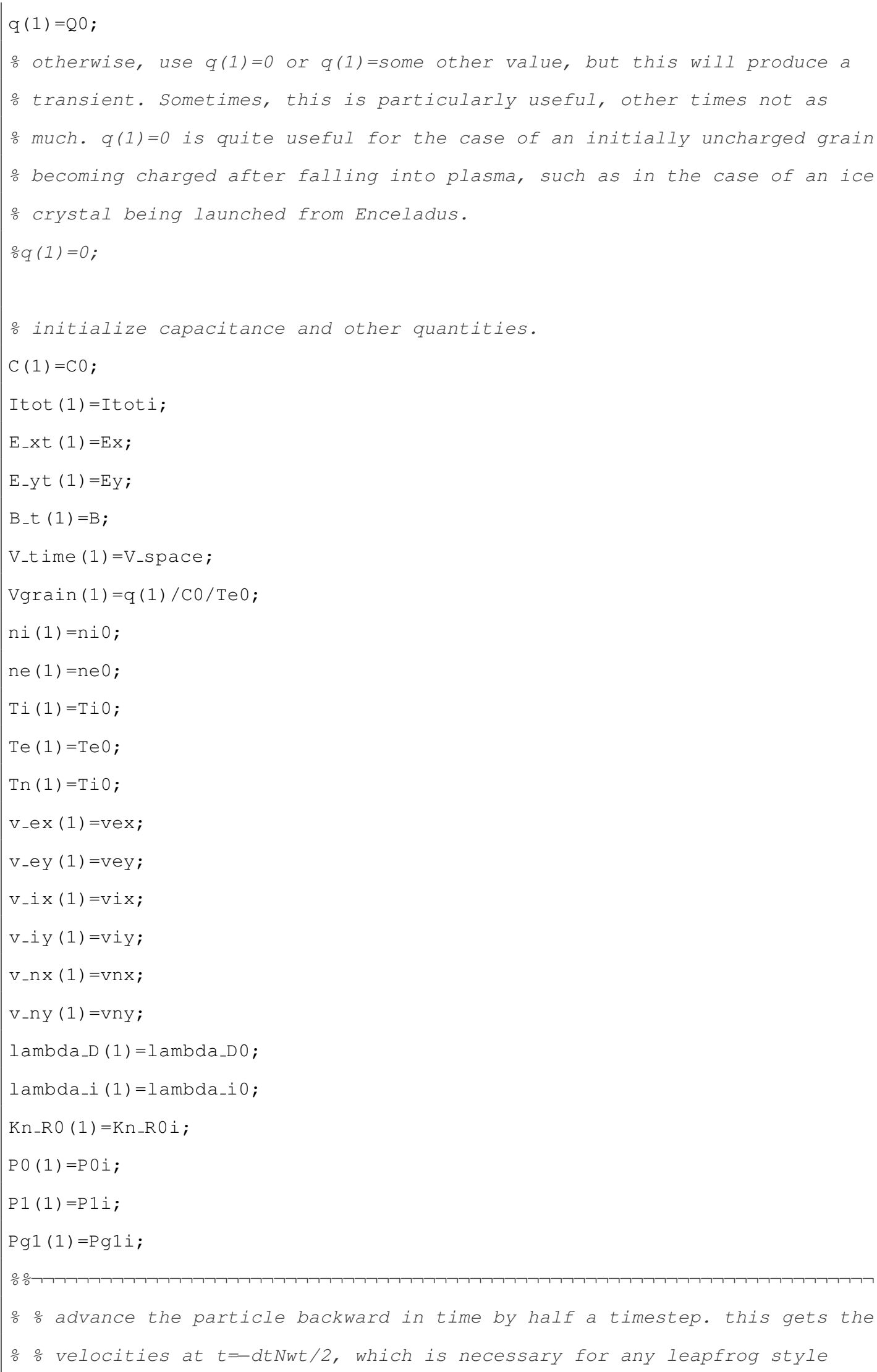




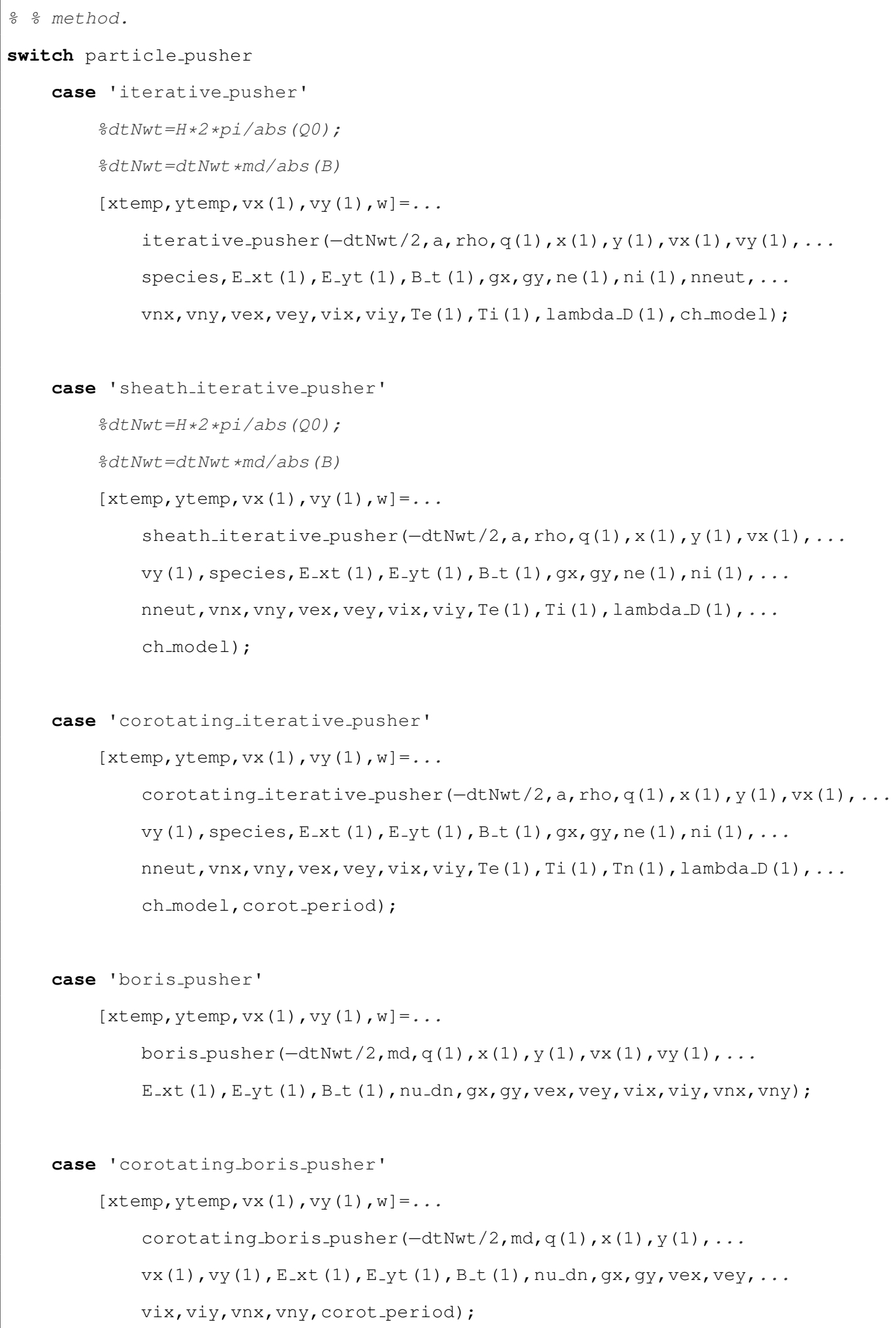


case 'sheath_boris_pusher'

$[\mathrm{xtemp}, \mathrm{ytemp}, \mathrm{vx}(1), \mathrm{vy}(1), \mathrm{w}]=\ldots$

sheath_boris_pusher (-dtNwt/2, md, $q(1), x(1), y(1), \ldots$

$\mathrm{vx}(1), \mathrm{vy}(1), \mathrm{E}_{-} \mathrm{xt}(1), \mathrm{E}_{-} \mathrm{yt}(1), \mathrm{B}_{-} \mathrm{t}(1), \mathrm{nu} \mathrm{d}_{-} \mathrm{n}, \ldots$

gx, gy, vex, vey, vix, viy, vnx, vny);

\section{end}

응 xtemp and ytemp are not needed when we go back just a half step.

음 you will get the wrong answer if you try to get $x(1)$ and $y(1)$ from the

응 command written above.

clear xtemp; clear ytemp;

응 Now, all the initial values have been assigned, time to start the main

$\div$ 응

응 응

응 The Main Ioop.

t_acc=0; $\quad$ o the accumulated time since a charge update starts at zero.

for $k=2$ :nsteps

을

음 display progress.

if $\neg \bmod (\mathrm{k}-2$, nsteps/20)

disp ( [num2str ( (k-2)/nsteps*100, '\%.2f') '\%'])

end

ㅇagnostic stuff

\% rsat $=60268000$;

ㅇaㅁ draw;

o subplot $(1,2,1)$;

응 plot $\left(x, y,{ }^{\prime} . b^{\prime}\right)$; hold on;

ㅁ phi_sat $=$ Iinspace $(0,2 * p i, 1 e 2)$;

응 $\quad x_{\text {sat }}=r_{\text {ssat }} * \cos \left(p h i_{\text {_sat }}\right)$;

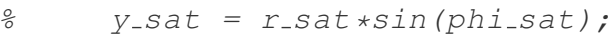

잉 $x \lim \left(\left[-10 * r_{-}\right.\right.$sat $10 * r_{-}$sat $\left.]\right)$;

yㅣㅁ $\quad y \lim \left(-10 * r_{-}\right.$sat $10 * r_{-}$sat $\left.]\right)$;

음 plot (x_sat, y_sat, '--r'); 


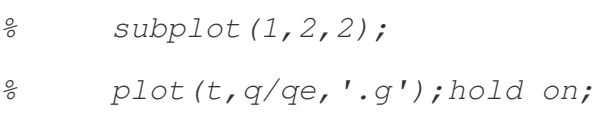




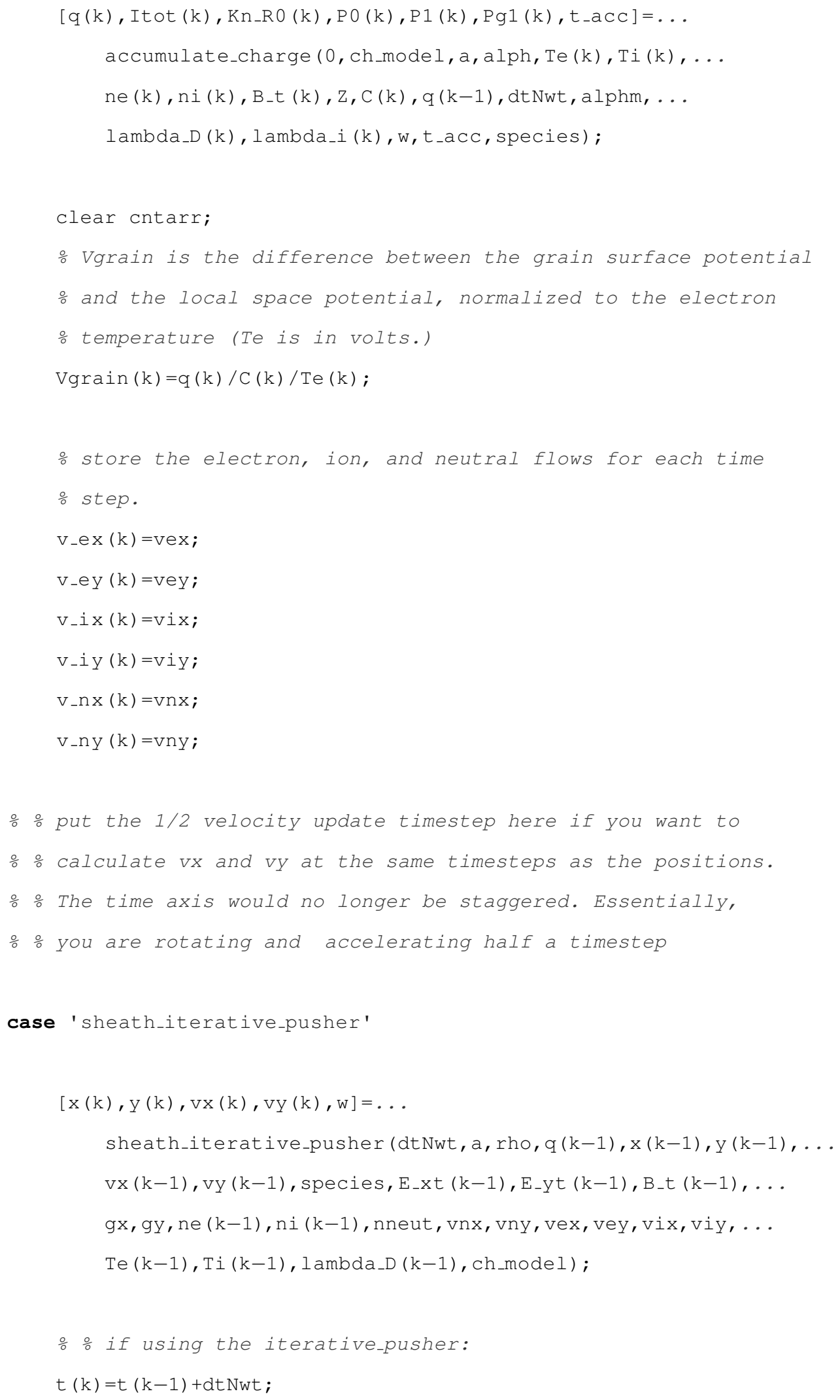




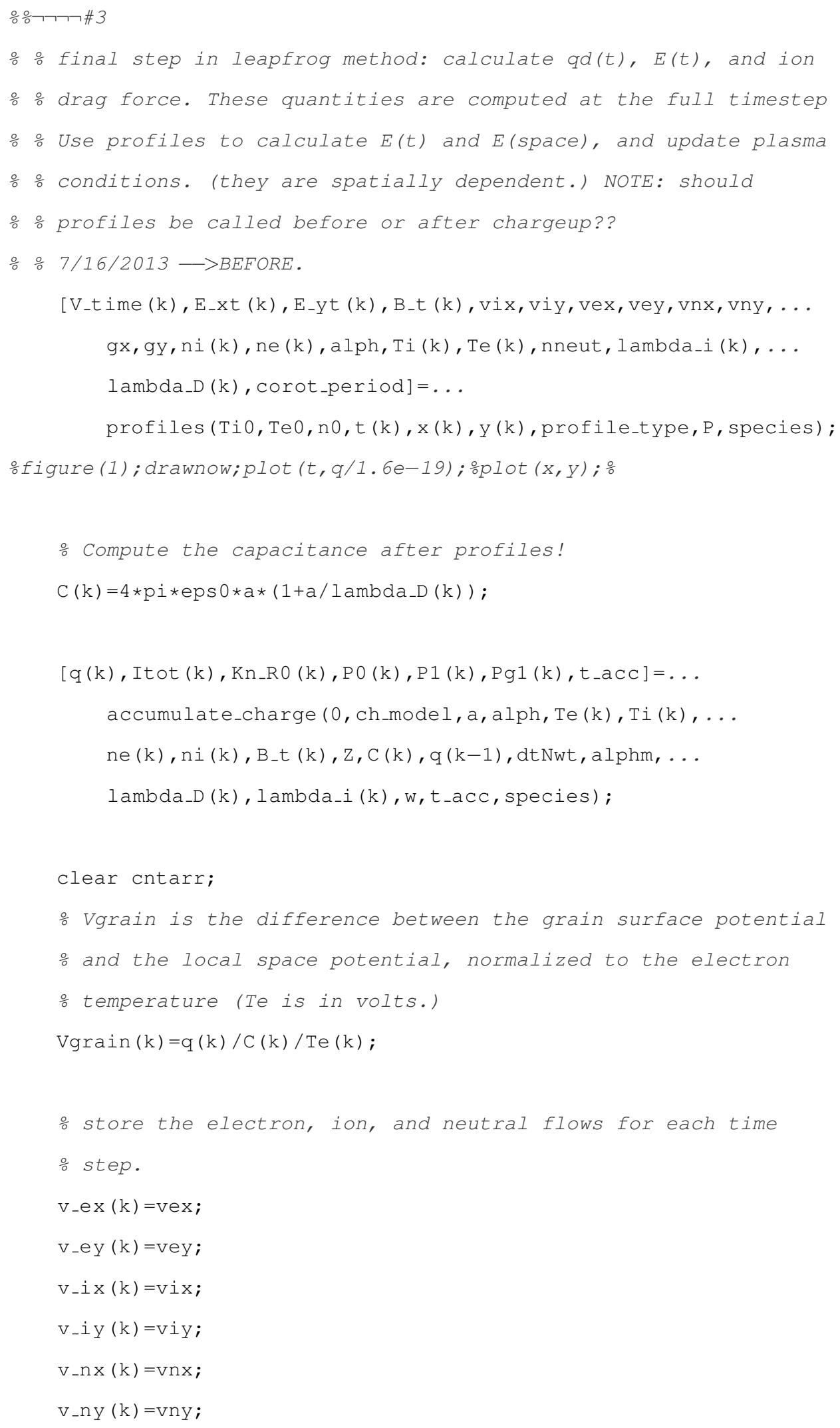


응 put the $1 / 2$ velocity update timestep here if you want to

응 calculate $v x$ and $v y$ at the same timesteps as the positions.

응 The time axis would no longer be staggered. Essentially,

응 you are rotating and accelerating half a timestep

case 'corotating_iterative_pusher'

$[\mathrm{x}(\mathrm{k}), \mathrm{y}(\mathrm{k}), \mathrm{vx}(\mathrm{k}), \mathrm{vy}(\mathrm{k}), \mathrm{w}]=\ldots$ corotating_iterative_pusher (dtNwt, a, rho, $q(k-1), x(k-1), \ldots$ $y(k-1), v x(k-1), V y(k-1)$, species, E_xt $(k-1), E_{-} y t(k-1), \ldots$ B_t $(k-1), g x, g y, n e(k-1), n i(k-1)$, nneut, vnx, vny, vex, vey, ... vix, viy, Te $(k-1)$, Ti $(k-1)$, Tn $(k-1)$, lambda_D $(k-1)$, ch_model, . . corot_period);

응 if using the iterative_pusher:

$t(k)=t(k-1)+d t N w t ;$

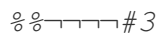

응 final step in leapfrog method: calculate qd(t), $E(t)$, and ion

$\div$ orag force. These quantities are computed at the full timestep

응 Use profiles to calculate $E(t)$ and E(space), and update plasma

응 conditions. (they are spatially dependent.) NOTE: should

음 profiles be called before or after chargeup??

응 7/16/2013 - $>B E F O R E$.

[V_time (k), E_xt (k), E_yt (k), B_t (k), vix, viy, vex, vey, vnx, vny, ...

gx, gy, ni (k), ne (k), alph, Ti (k), Te (k), nneut, lambda_i (k), ..

lambda_D (k), corot_period] $=$. . .

profiles(Ti0, Te0, no, t (k), x(k), y (k), profile_type, P, species);

ofigure (1); drawnow;plot (t, $q / 1.6 e-19)$; ำlot $(x, y)$; 응

ㅇ Compute the capacitance after profiles!

$C(k)=4 * p i * e p s 0 * a *\left(1+a / l a m b d a \_D(k)\right)$;

$\left[\mathrm{q}(\mathrm{k}), \operatorname{Itot}(\mathrm{k}), \mathrm{Kn}_{-} \mathrm{R} 0(\mathrm{k}), \mathrm{PO}(\mathrm{k}), \mathrm{P} 1(\mathrm{k}), \mathrm{Pg} 1(\mathrm{k}), \mathrm{t}_{-} \mathrm{acC}\right]=\ldots$ accumulate_charge (0, ch_model, a, alph, Te (k), Ti (k), . . $n \in(k), n i(k), B_{-} t(k), z, C(k), q(k-1), d t N w t, a l p h m, \ldots$ lambda_D (k), lambda_i (k), w,t_acc, species) ; 


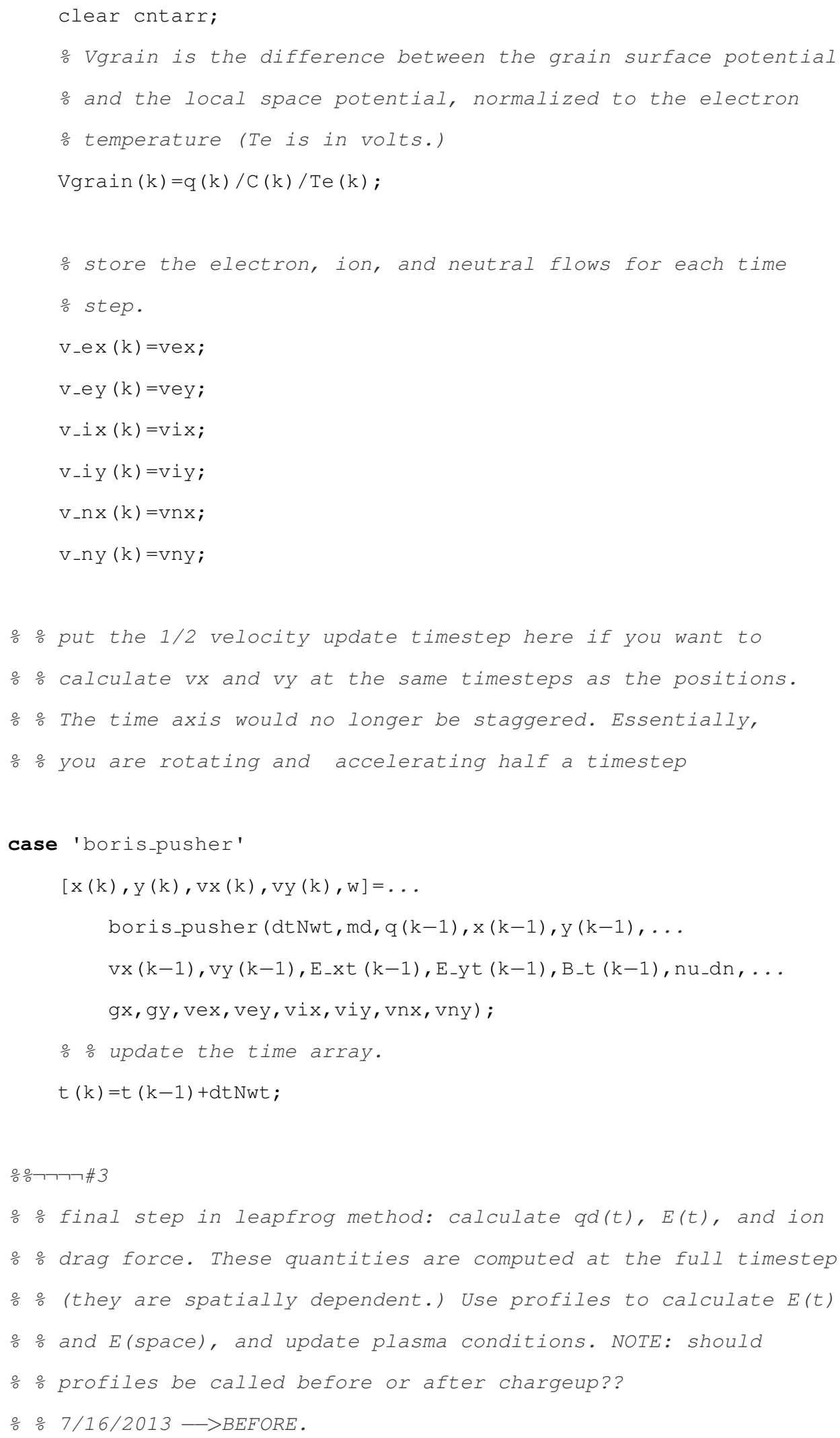


[V_time (k), E_xt (k), E_yt (k), B_t (k), vix, viy, vex, vey, vnx, vny,... gx, gy, ni (k), ne (k), alph, Ti (k), Te (k), nneut, lambda_i (k),...

lambda_D (k), corot_period] $=$. .

profiles(Ti0, Te0, n0,t(k), x(k),y(k), profile_type,P, species) ;

- Compute the capacitance after profiles!

$\mathrm{C}(\mathrm{k})=4 * \mathrm{pi} * \operatorname{eps} 0 * \mathrm{a} *\left(1+\mathrm{a} / \mathrm{lambda}_{-} \mathrm{D}(\mathrm{k})\right)$;

$\left[q(k), \operatorname{Itot}(k), K_{\_} R 0(k), P 0(k), P 1(k), P g 1(k), t_{-} a c c\right]=\ldots$ accumulate_charge (0, ch_model, a, alph, Te (k), Ti (k),... ne (k), ni (k), B_t (k), z, C (k), $q(k-1), d t N w t, a l p h m, \ldots$ lambda_D (k), lambda_i (k), w, t_acc, species) ;

clear cntarr;

V Vrain is the difference between the grain surface potential

o and the local space potential, normalized to the electron

을 temperature ( $T e$ is in volts.)

$\operatorname{Vgrain}(\mathrm{k})=\mathrm{q}(\mathrm{k}) / \mathrm{C}(\mathrm{k}) / \mathrm{Te}(\mathrm{k})$;

응 store the electron, ion, and neutral flows for each time

step.

V_ex $(k)=$ vex;

V_ey $(k)=$ vey;

$\mathrm{v}_{-} \mathrm{ix}(\mathrm{k})=\mathrm{vix}$;

$\mathrm{v}_{-} \mathrm{iy}(\mathrm{k})=\mathrm{viy}$;

$\mathrm{V} \_\mathrm{nx}(\mathrm{k})=\mathrm{vnx}$;

V_ny $(k)=$ vny ;

응 For ion and neutral drag, you have to assume vions-vdust>>vthi, $\frac{\circ}{0}$ and V_neut-vdust> >thn in order for Boris method to work.

$\frac{\circ}{0}$ If you want to compute the drag forces as functions of time, 응 put them here.

응 I think the ion drag should actually use $q(k)$ instead of

$\div$ 응 $(k-1)$, because $q$ should be evaluated at a spatial location - 
$\frac{0}{0}\left[f_{-} i x(k), f_{-} i y(k)\right]=i o n_{-} d r a g\left(q(k), a_{1}, T e(k), T i(k), n i(k), C, l a m b d a \_D(k), l a m b d a-i\right.$

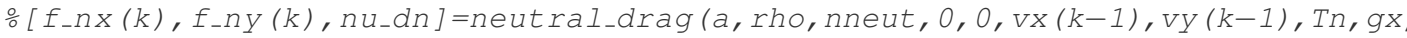

응 put the 1/2 velocity update timestep here if you want to calculate

응 VX and Vy at the same timesteps as the positions. The time axis

\% would no longer be staggered. Essentially, you are rotating and

응 accelerating half a timestep

ㅇ use corotating boris pusher when you are dealing with dust and a o planet or moon, or other celestial body.

case 'corotating_boris_pusher'

$[x(k), y(k), V x(k), V y(k), w]=\ldots$ corotating_boris_pusher (dtNwt, $m d, q(k-1), x(k-1), y(k-1), \ldots$ $v x(k-1), v y(k-1), E_{-} x t(k-1), E_{-} y t(k-1), B_{-} t(k-1), n u \_d n, \ldots$ gx, gy, vex, vey, vix, viy, vnx, vny, corot_period) ;

응 update the time array.

$t(k)=t(k-1)+d t N w t ;$

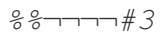

응 final step in leapfrog method: calculate $q d(t), E(t)$, and ion

응 drag force. These quantities are computed at the full timestep

응 (they are spatially dependent.) Use profiles to calculate $E(t)$

응 and E(space), and update plasma conditions. NOTE: should

응 profiles be called before or after chargeup??

$\therefore \div 7 / 16 / 2013 \longrightarrow$ 응

[V_time (k), E_xt (k), E_yt (k), B_t (k), vix, viy, vex, vey, vnx, vny,...

gx, gy, ni (k), ne (k), alph, Ti(k), Te (k), nneut, lambda_i (k),...

lambda_D (k), corot_period] $=$. . .

profiles (Ti0, Te0, no, t (k), x(k), y (k), profile_type, P, species);

- Compute the capacitance after profiles!

$\mathrm{C}(\mathrm{k})=4 * \mathrm{p} i * \operatorname{eps} 0 * \mathrm{a} *(1+\mathrm{a} / \mathrm{lambda} \mathrm{D}(\mathrm{k}))$; 
$\left[q(k), \operatorname{Itot}(k), K_{-} R 0(k), P 0(k), P 1(k), P g 1(k), t_{-} a c c\right]=\ldots$ accumulate_charge (0, ch_model, a, alph, Te (k), Ti (k),... $n e(k), n i(k), B_{-} t(k), z, C(k), q(k-1)$, dt Nwt, alphm, .. lambda_D (k), lambda_i (k),w,t_acc, species) ;

clear cntarr;

-Vgrain is the difference between the grain surface potential

응 the local space potential, normalized to the electron

temperature (Te is in volts.)

$\operatorname{Vgrain}(\mathrm{k})=\mathrm{q}(\mathrm{k}) / \mathrm{C}(\mathrm{k}) / \mathrm{Te}(\mathrm{k})$;

o store the electron, ion, and neutral flows for each time

$\therefore$ step.

$\mathrm{v}_{-} \mathrm{ex}(\mathrm{k})=\mathrm{vex}$;

v_ey $(k)=$ vey ;

$\mathrm{v}_{-} \mathrm{ix}(\mathrm{k})=\mathrm{vix}$;

$v_{-i y}(k)=v i y$;

$\mathrm{V} \_\mathrm{nx}(\mathrm{k})=\mathrm{vnx}$;

v_ny $(\mathrm{k})=\mathrm{vny}$;

응 For ion and neutral drag, you have to assume vions-vdust>>vthi,

응 and V_neut-vdust> >thn in order for Boris method to work.

응 If you want to compute the drag forces as functions of time,

응 put them here.

음 I think the ion drag should actually use $q(k)$ instead of

$\div$ q(k-1), because $q$ should be evaluated at a spatial location -

$\therefore 5 / 23 / 2013$

을 $\left.f_{-} x(k), f_{-} i y(k)\right]=i o n_{-} d r a g\left(q(k), a, T e(k), T i(k), n i(k), C, I a m b d a \_D(k), I a m b d a_{-} i\right.$ $\frac{0}{0}\left[f_{-} n x(k), f_{-} n y(k), n u_{-} d n\right]=n e u t r a I_{-} d r a g(a, r h o, n n e u t, 0,0, v x(k-1), v y(k-1), T n, g x$,

(k), vix, viy, o gy, species);

$\div \frac{0}{0}$ put the $1 / 2$ velocity update timestep here if you want to 
응 calculate $v x$ and $v y$ at the same timesteps as the positions.

음 The time axis would no longer be staggered. Essentially, you

$\div$ 응 rotating and accelerating half a timestep

\% January 2014: use sheath_boris_pusher if you want work with dust

o grains levitated in a planar sheath.

case 'sheath_boris_pusher'

$[\mathrm{x}(\mathrm{k}), \mathrm{y}(\mathrm{k}), \mathrm{vx}(\mathrm{k}), \mathrm{vy}(\mathrm{k}), \mathrm{w}]=\ldots$

sheath_boris_pusher (dtNwt, $m d, q(k-1), x(k-1), y(k-1), \ldots$

$v x(k-1), v y(k-1), E_{-} x t(k-1), E_{-} y t(k-1), B_{-} t(k-1), n u \_d n, \ldots$

gx, gy, vex, vey, vix, viy, vnx, vny) ;

응 update the time array.

$t(k)=t(k-1)+d t N w t ;$

응그าフ\#3

응 final step in leapfrog method: calculate $q d(t), E(t)$, and ion

응 drag force. These quantities are computed at the full timestep

응 (they are spatially dependent.) Use profiles to calculate $E(t)$

응 and E(space), and update plasma conditions. NOTE: should

응 profiles be called before or after chargeup??

응 $7 / 16 / 2013->B E F O R E$.

[V_time (k), E_xt (k), E_yt (k), B_t (k), vix, viy, vex, vey, vnx, vny, .. .

gx, gy, ni (k), ne (k), alph, Ti (k), Te (k), nneut, lambda_i (k),...

lambda_D (k), corot_period] $=\ldots$

profiles(Ti0, Te0, no, t(k), x(k),y(k), profile_type, P, species);

응 Compute the capacitance after profiles!

$\mathrm{C}(\mathrm{k})=4 * \mathrm{pi} * \operatorname{eps} 0 * \mathrm{a} *(1+\mathrm{a} / \mathrm{lambda} \mathrm{D}(\mathrm{k}))$;

$\left[q(k), \operatorname{Itot}(k), K_{\_} R 0(k), P 0(k), P 1(k), P g l(k), t \_a c c\right]=\ldots$

accumulate_charge $(0, \mathrm{ch}$ model, a, alph, Te $(\mathrm{k}), \mathrm{Ti}(\mathrm{k}), \ldots$

$n e(k), n i(k), B_{-} t(k), z, C(k), q(k-1), d t N w t, a l p h m, \ldots$

lambda_D (k), lambda_i (k), w,t_acc, species) ;

clear cntarr; 


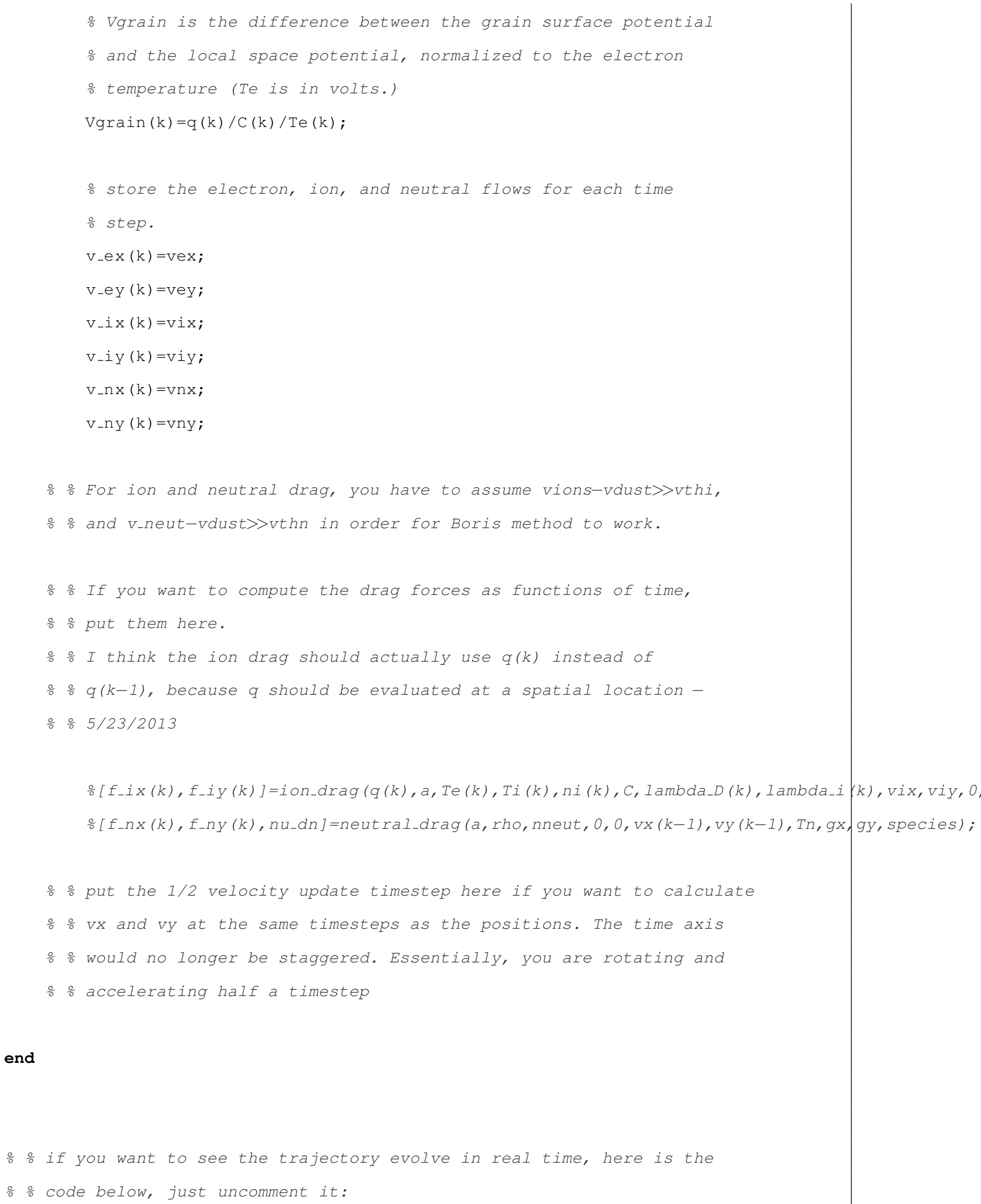




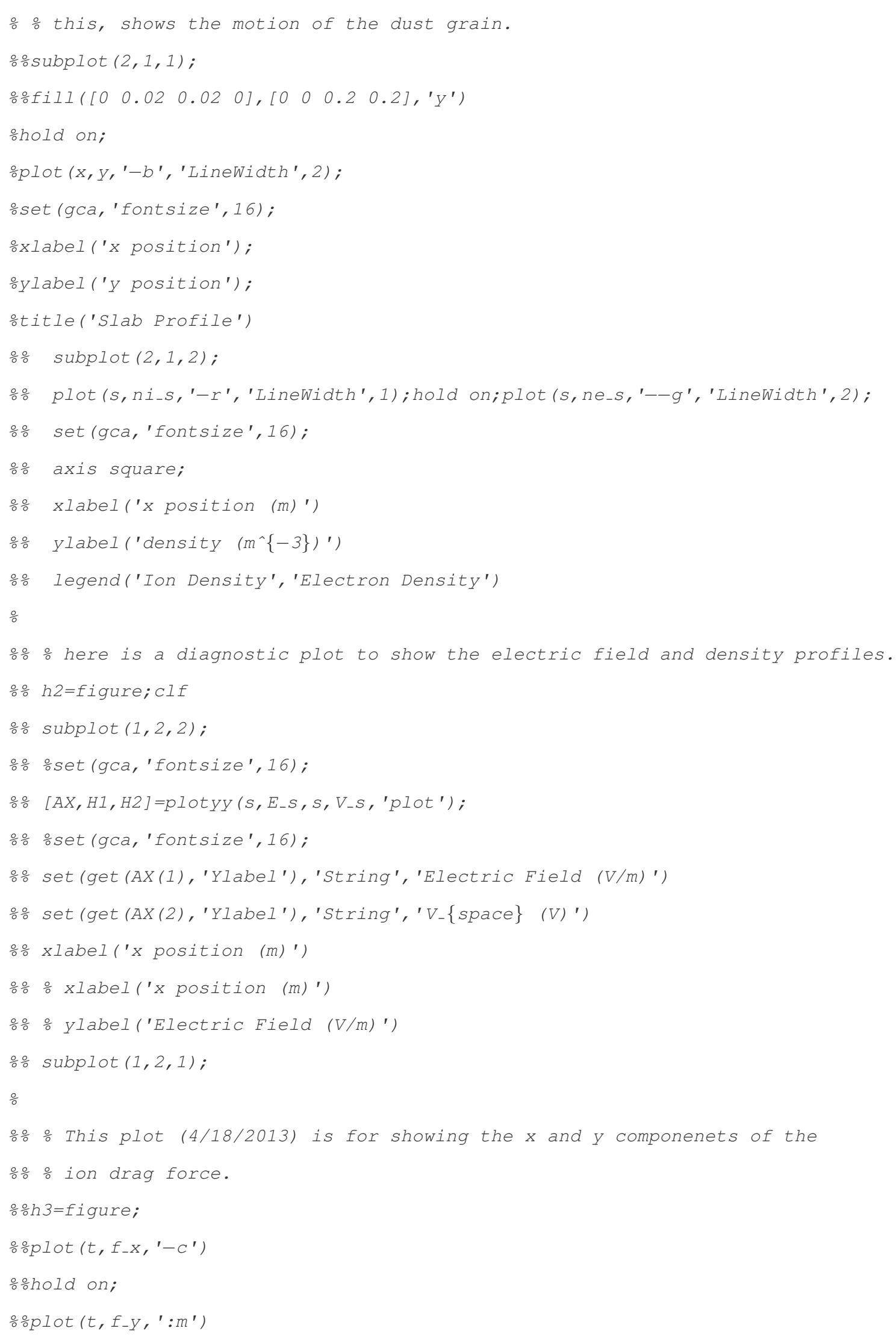




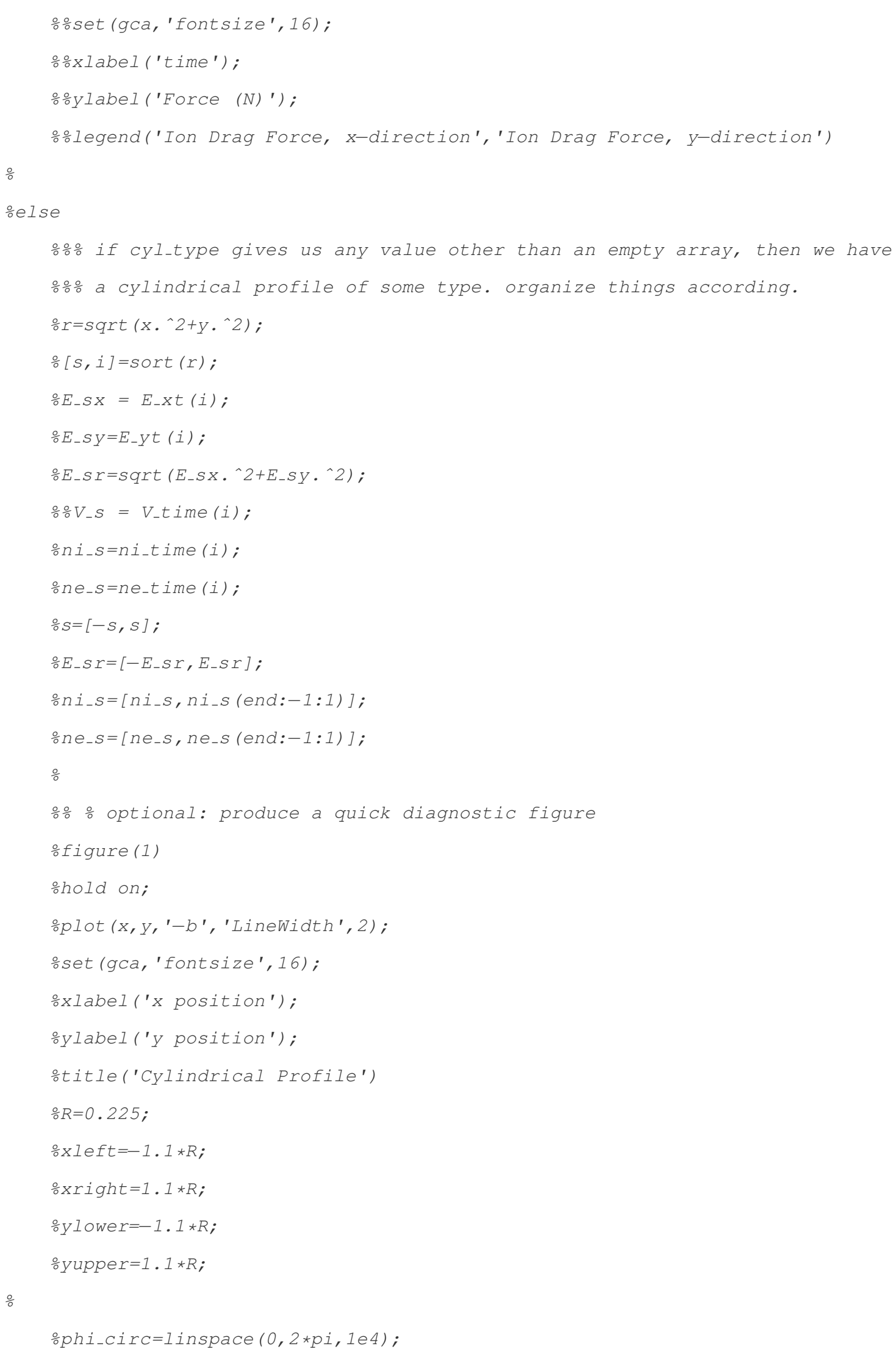




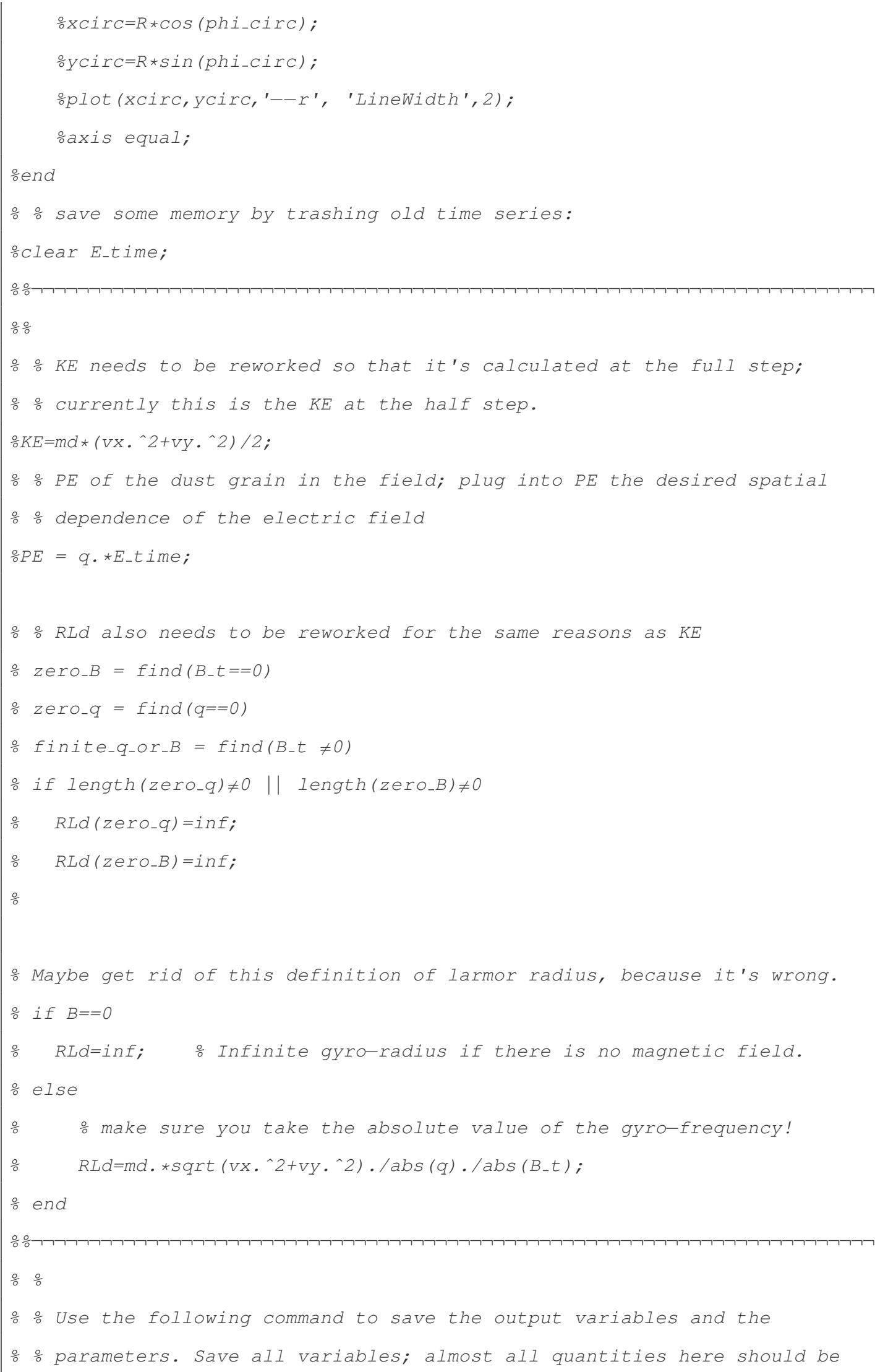




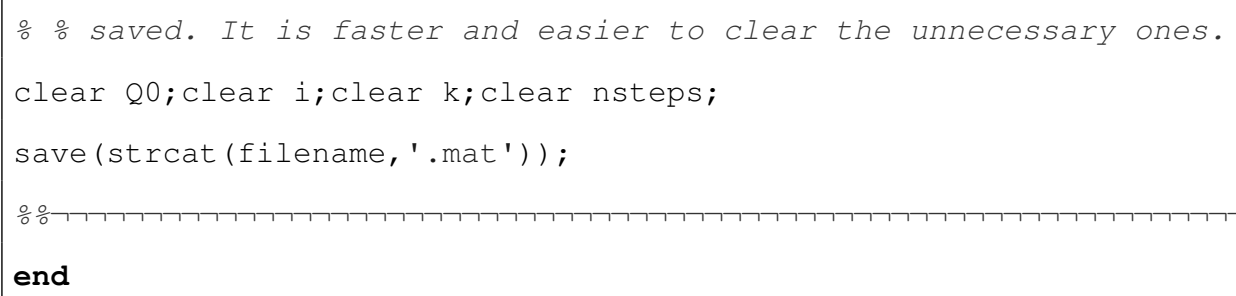

\section{i Example Input Script}

It is generally easiest to run the main program by setting up a script .m file. Input the necessary parameters, as shown in the code below. The script file example_dust_trajectory_script.m names an output file based on input parameters and saves the data in .mat format. By default, gyrophaser.m is enabled, but this command can be commented out if desired.

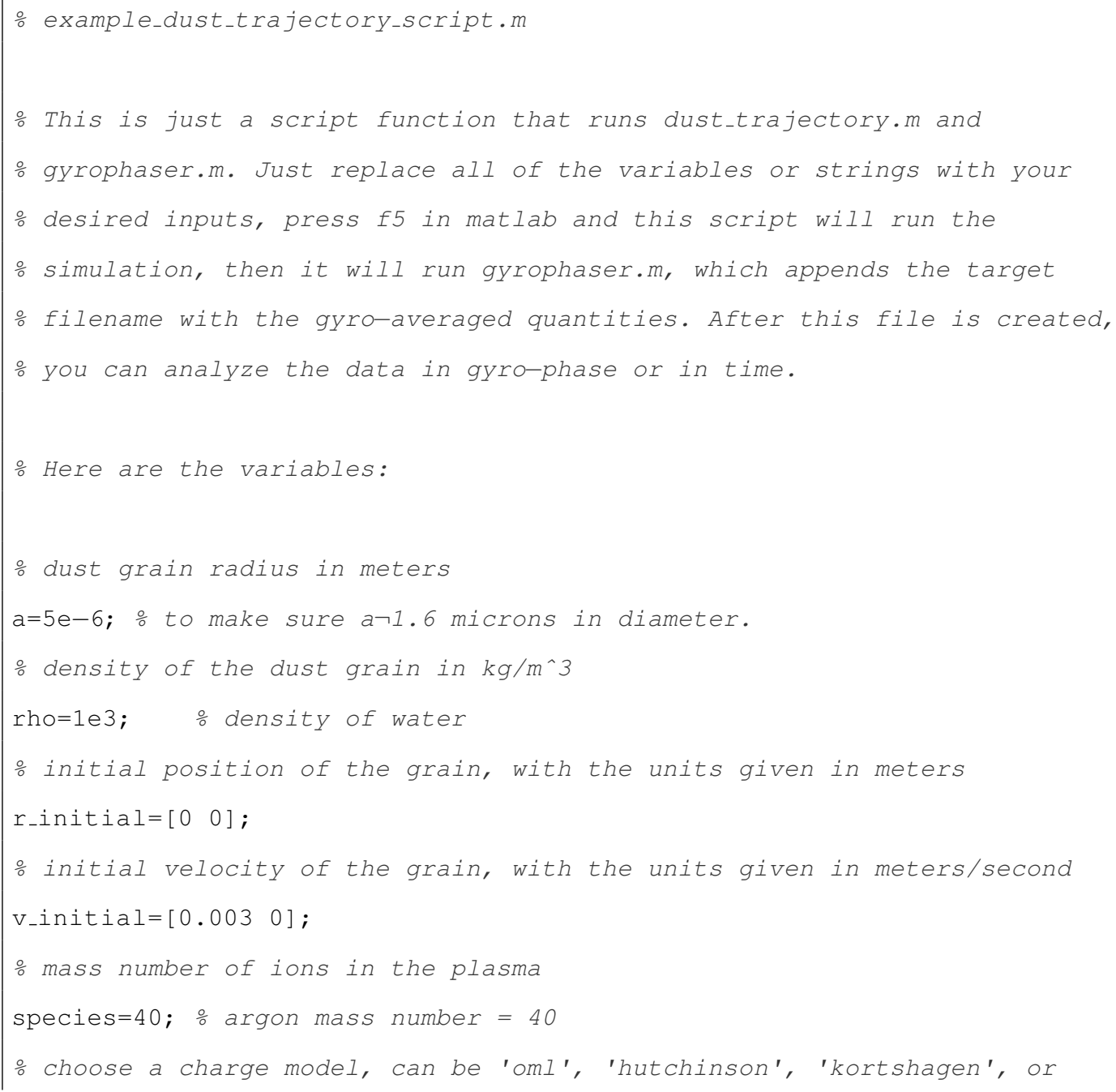




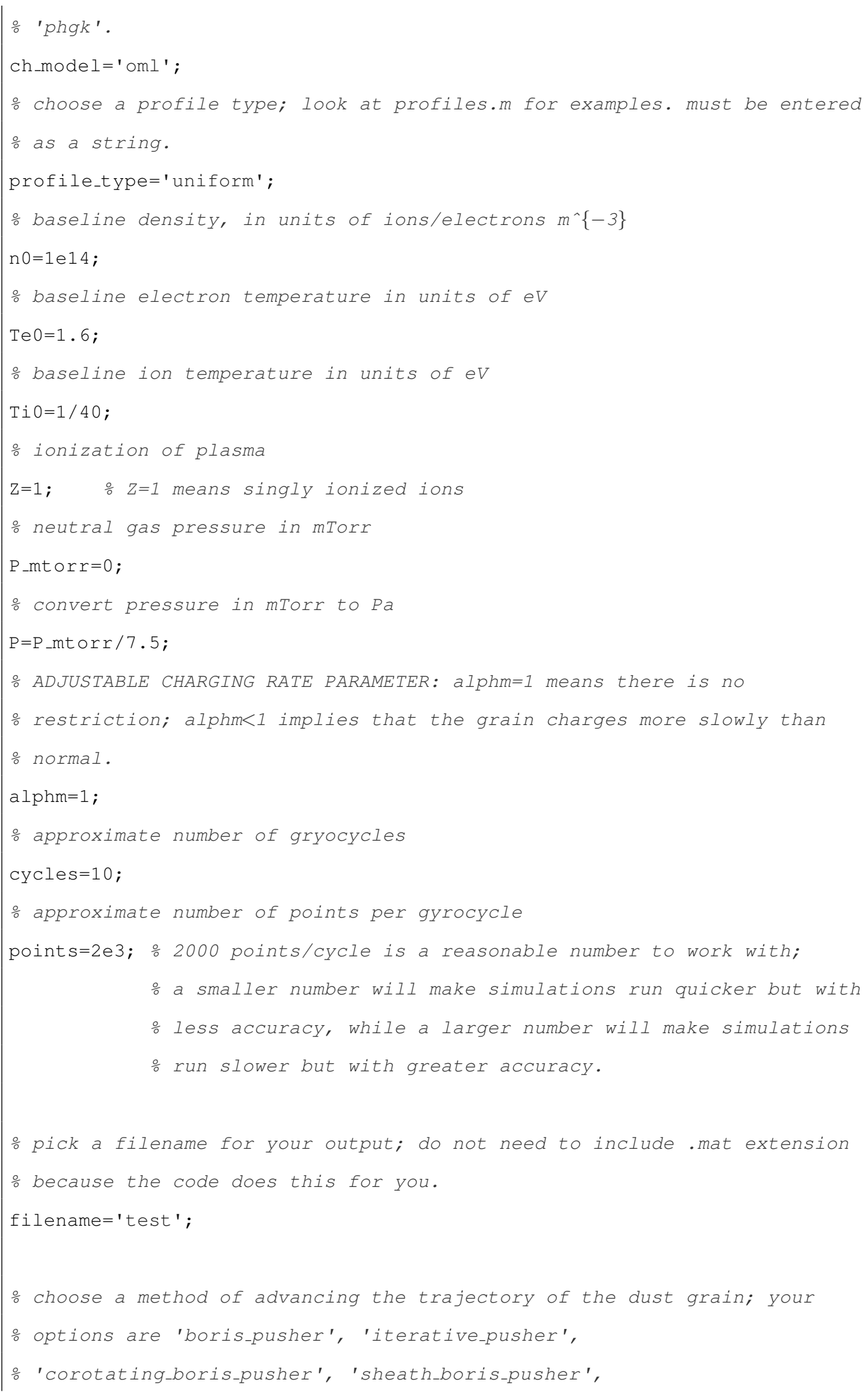




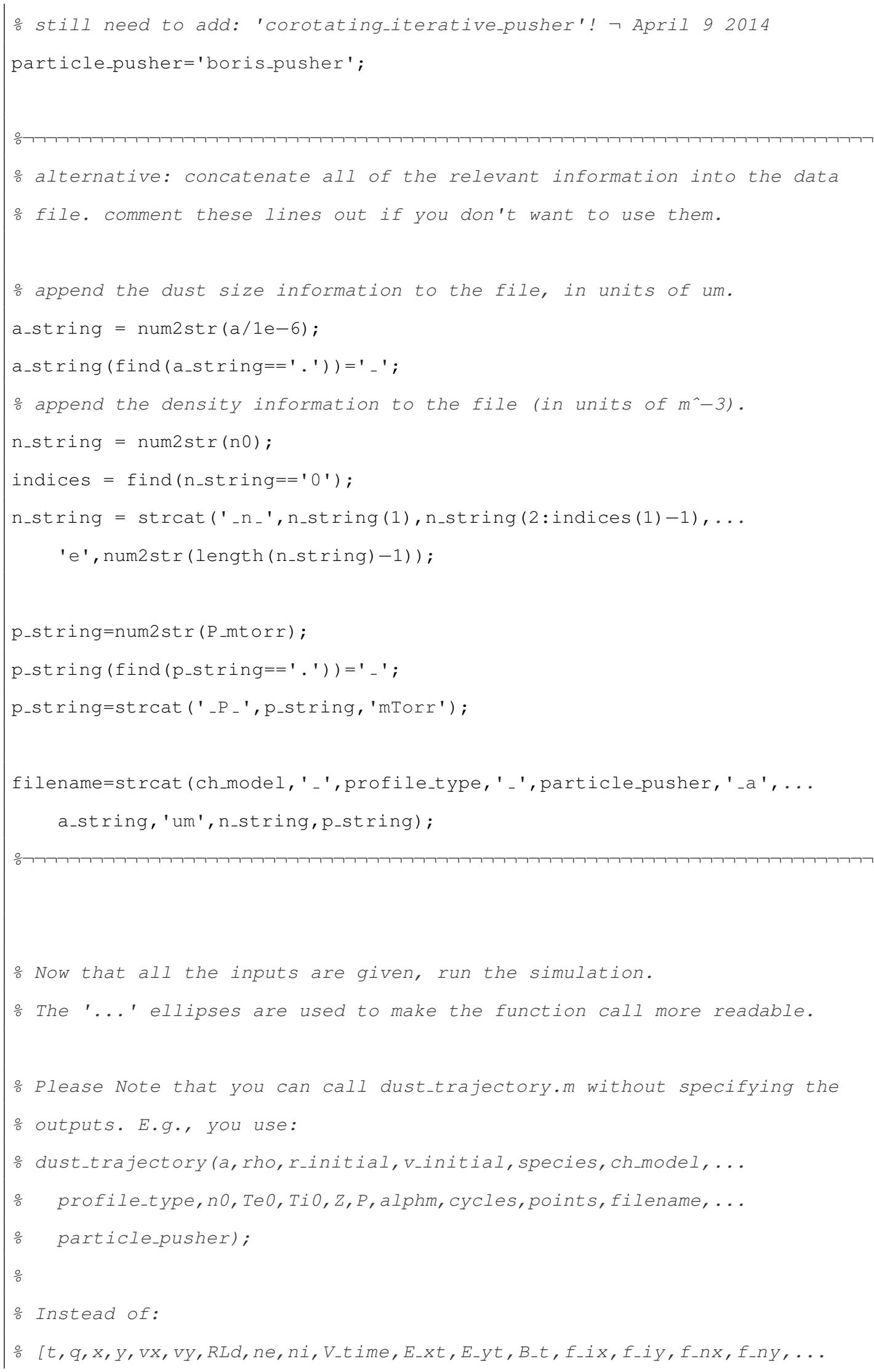




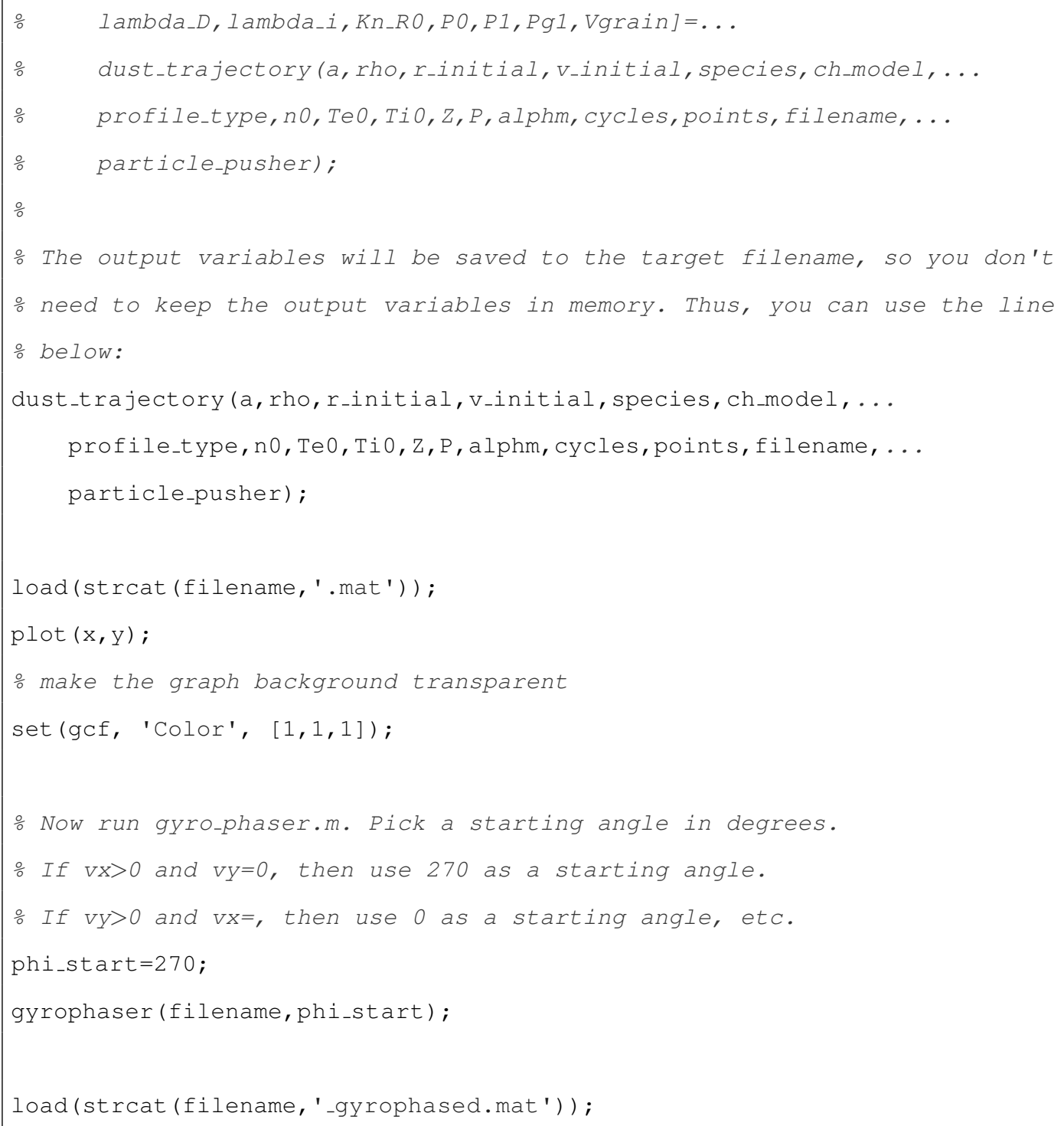

\section{ii Miscellaneous Subroutines}

The subroutine improved_arctan. $\mathrm{m}$ is included here, since it is required for computation of electric fields or other quantities in cylindrical geometry. This is a simple function that expands the range of the function so that it can produce the correct values between 0 and $360^{\circ}$, instead of being limited to $-90^{\circ}$ and $90^{\circ}$.

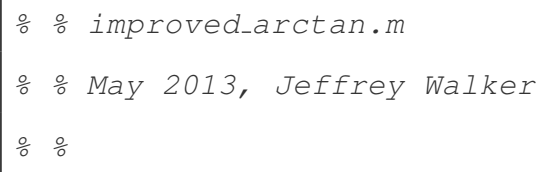




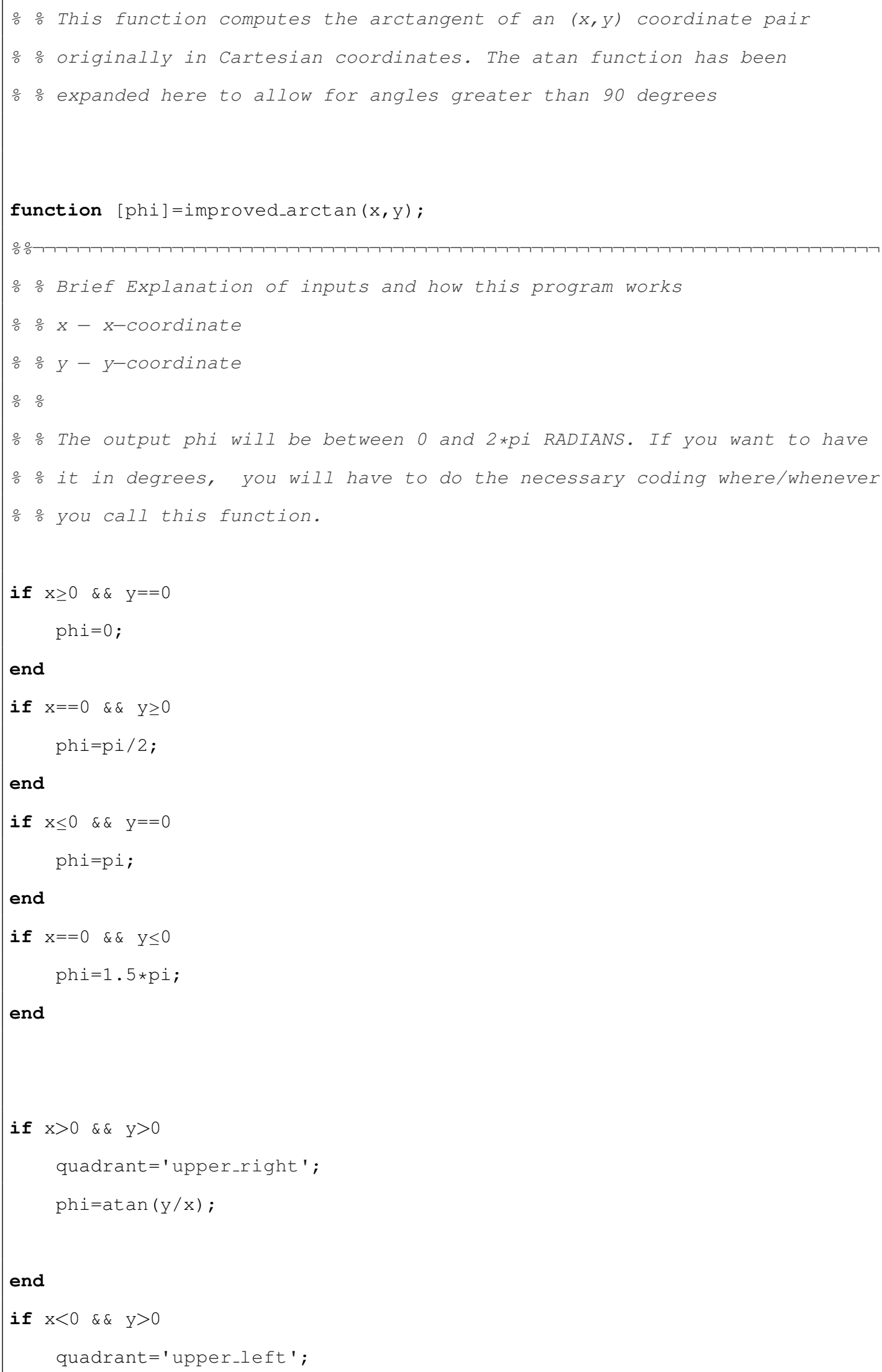




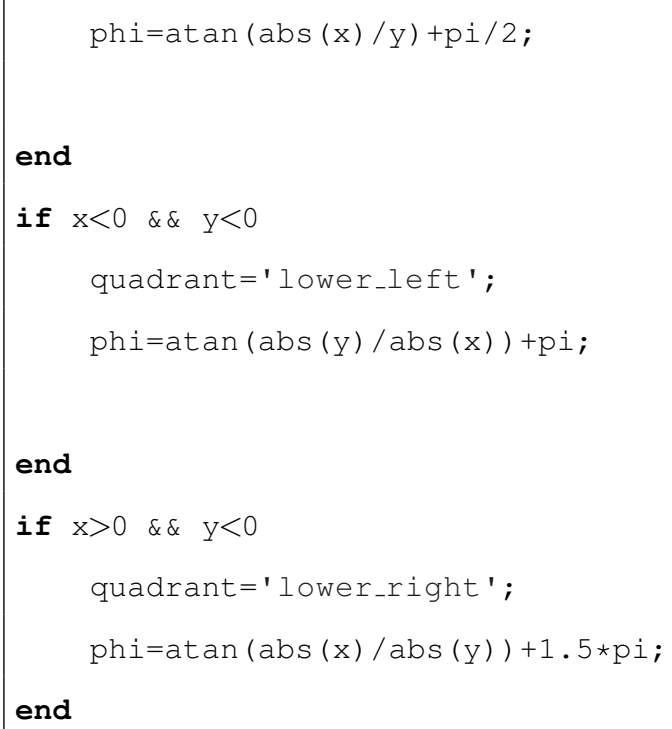

\section{ii Position and Velocity Advancement}

The dust_trajectory.m code allows for the use of several different ways of time-stepping the grain, finding the resulting position and velocity based on the forces on the grain. All of these methods use a leapfrog integration scheme, but each has certain unique features that are discussed below. As a caveat, these algorithms were written to determine the grain motion in a two-dimensional plane perpendicular to the magnetic field direction.

\section{i Boris Solver}

The function boris_pusher.m uses the standard Boris method (Boris, 1970) to advance the grain position and velocity. The Boris algorithm has been slightly modified to include the velocitydependent, linear, Epstein drag force. Gravitational forces are also included, because this force can be significant for dust grains.

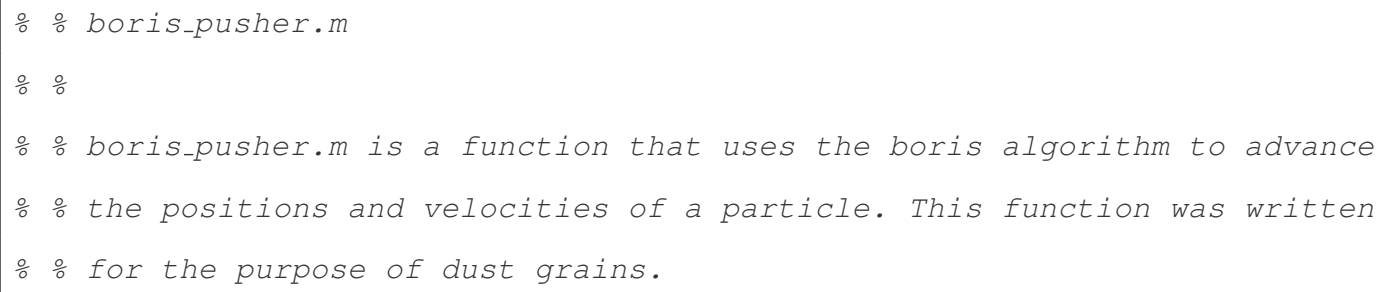




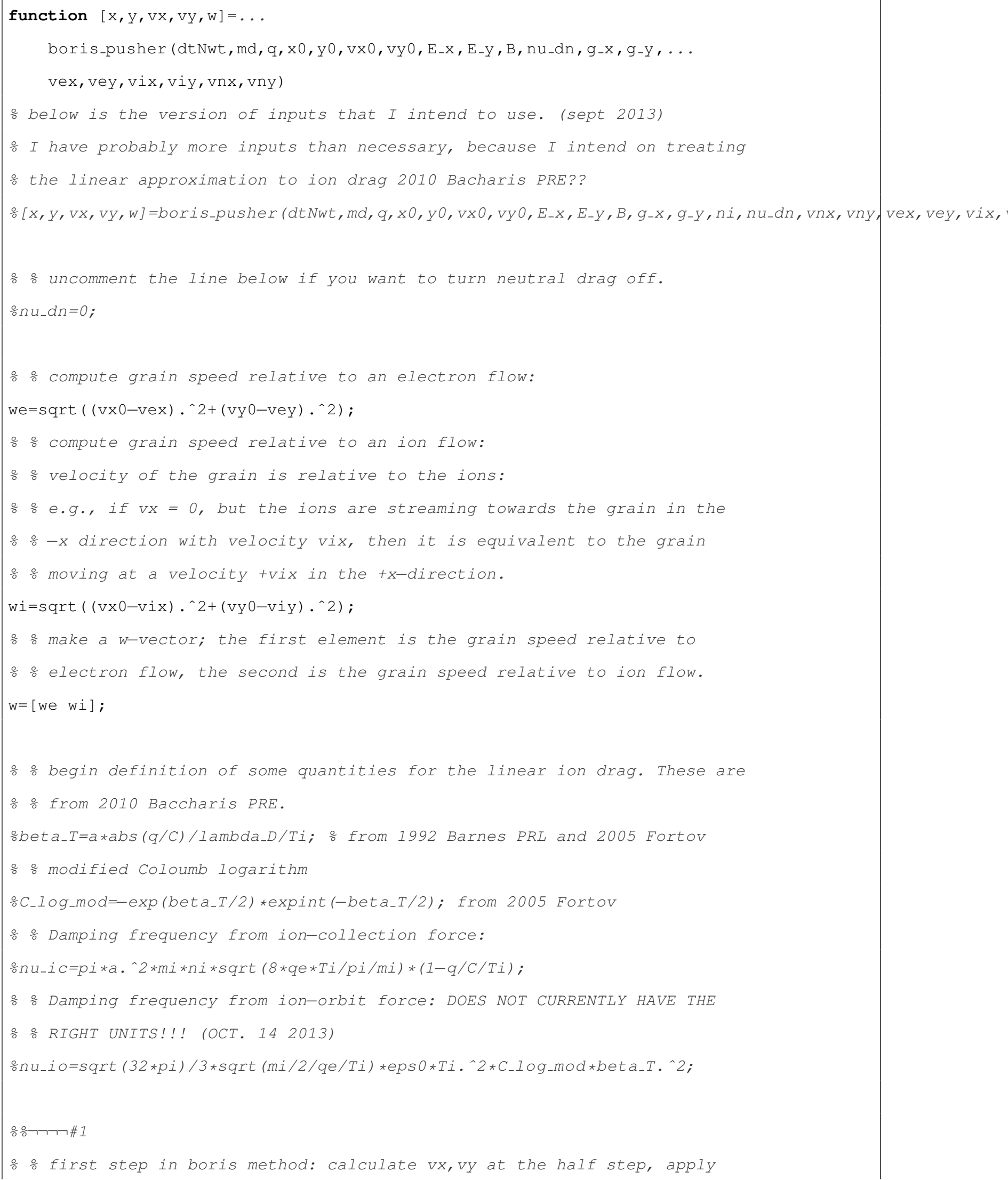




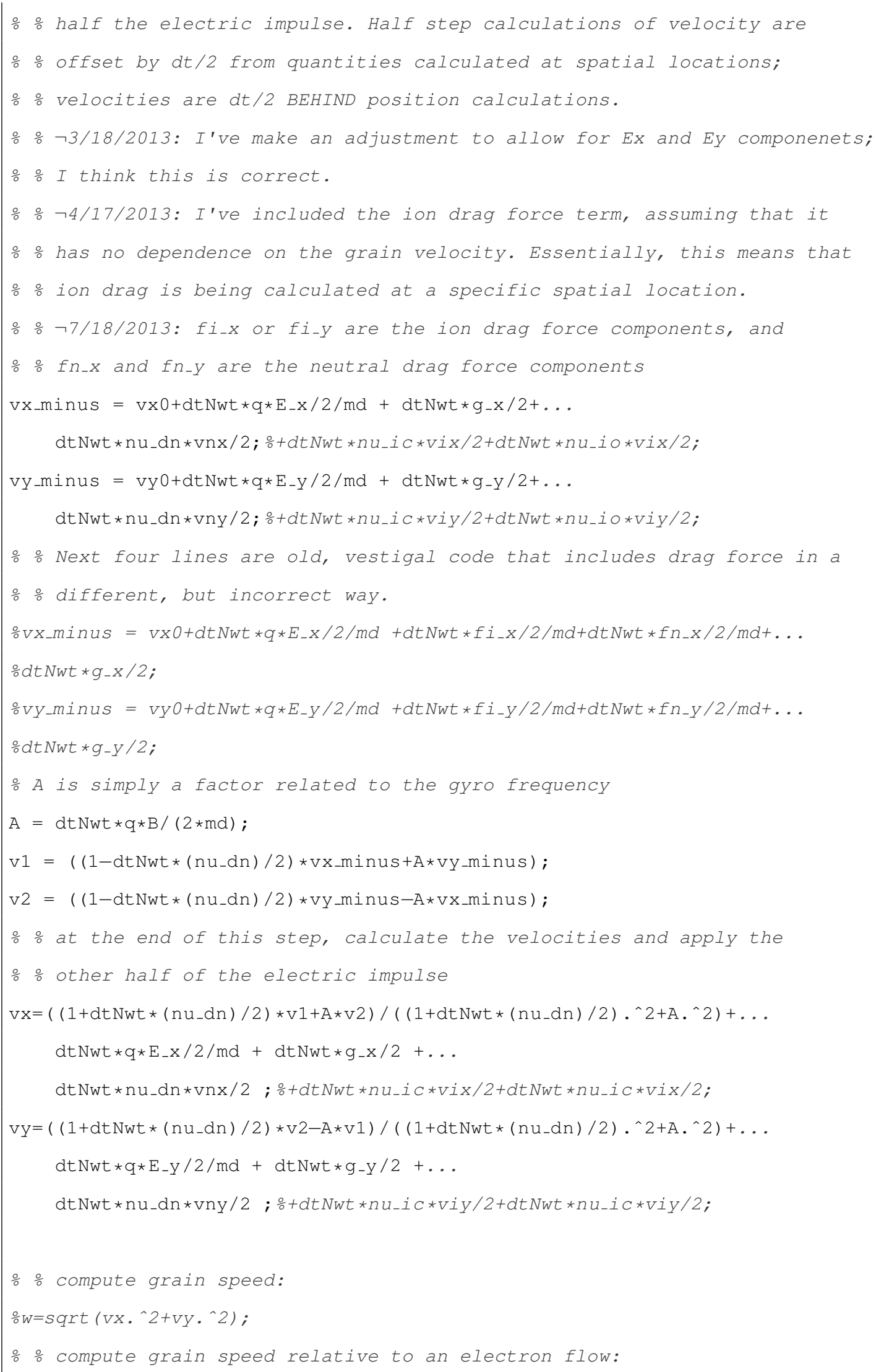




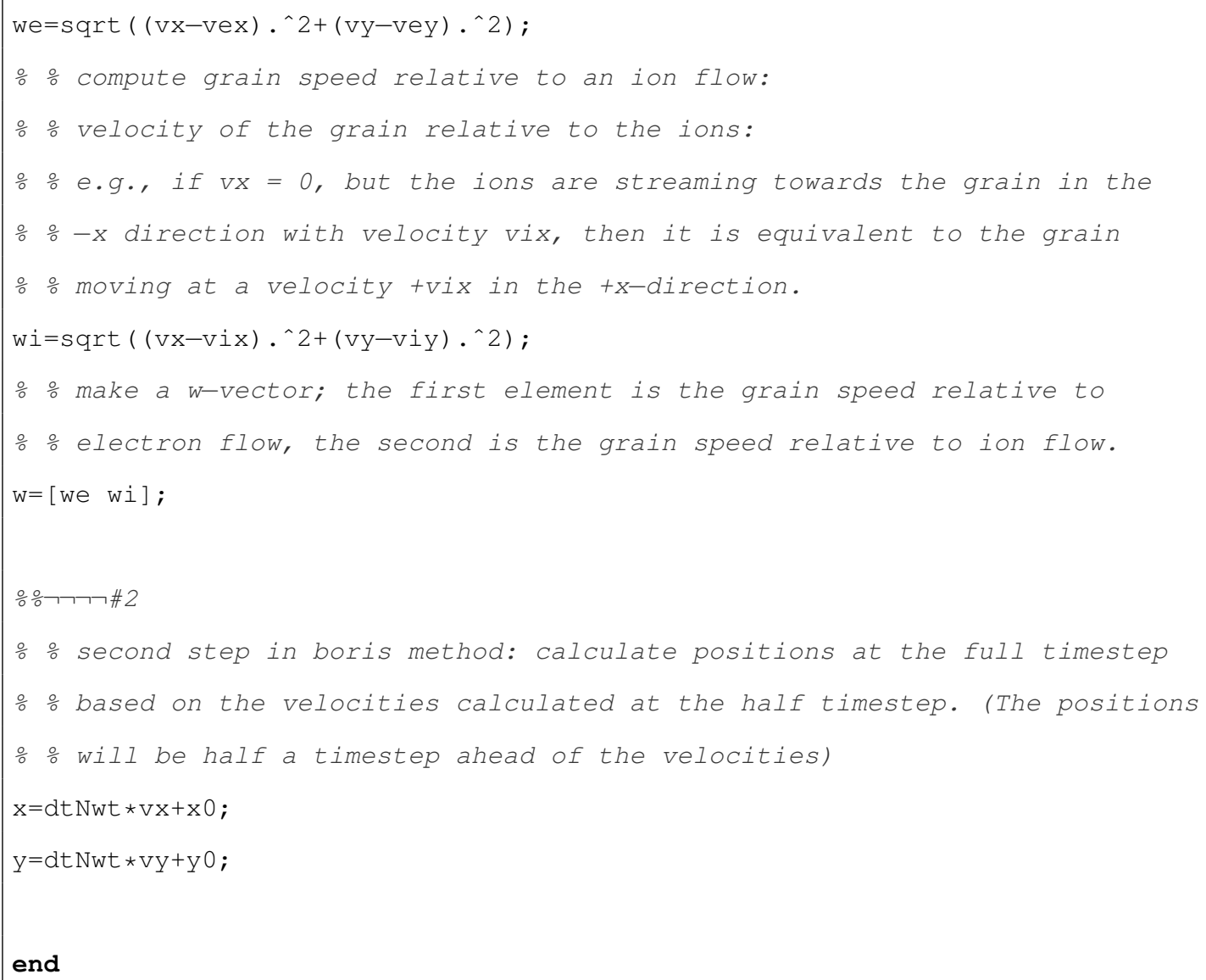

\section{ii Boris Solver for Grains in Sheaths}

The function sheath_boris_pusher.m is nearly identical to boris_pusher.m, except that it assumes that the variable $v_{e x}$ is used to store the information about the ion flow along the $z$-direction. This is necessary because the code is only 2-dimensional. When grains levitate in a plasma sheath, there can be an ion flow in the plane perpendicular to the magnetic field, but there can also be ion flow along the magnetic field. Because $v_{e x}$ is used for $v_{i z}$, it cannot be used to specify electron flow along the $x$-direction. If electron flow is azimuthally symmetric, then all of the flow information can be specified by $v_{e y}$, which is a feature used for cylindrical profiles. In the sheaths encountered in laboratory situations, the flow of electrons along the $z$-direction is generally negligible.

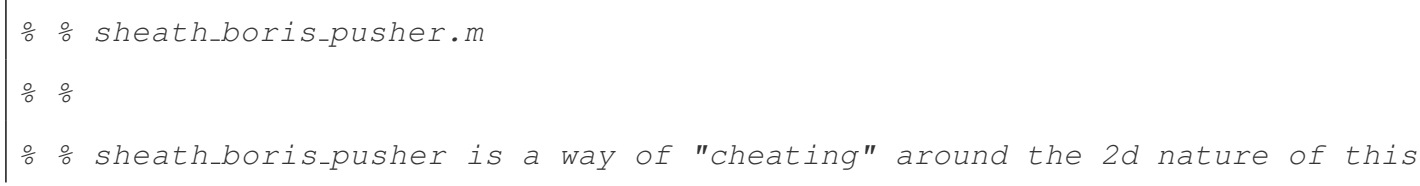




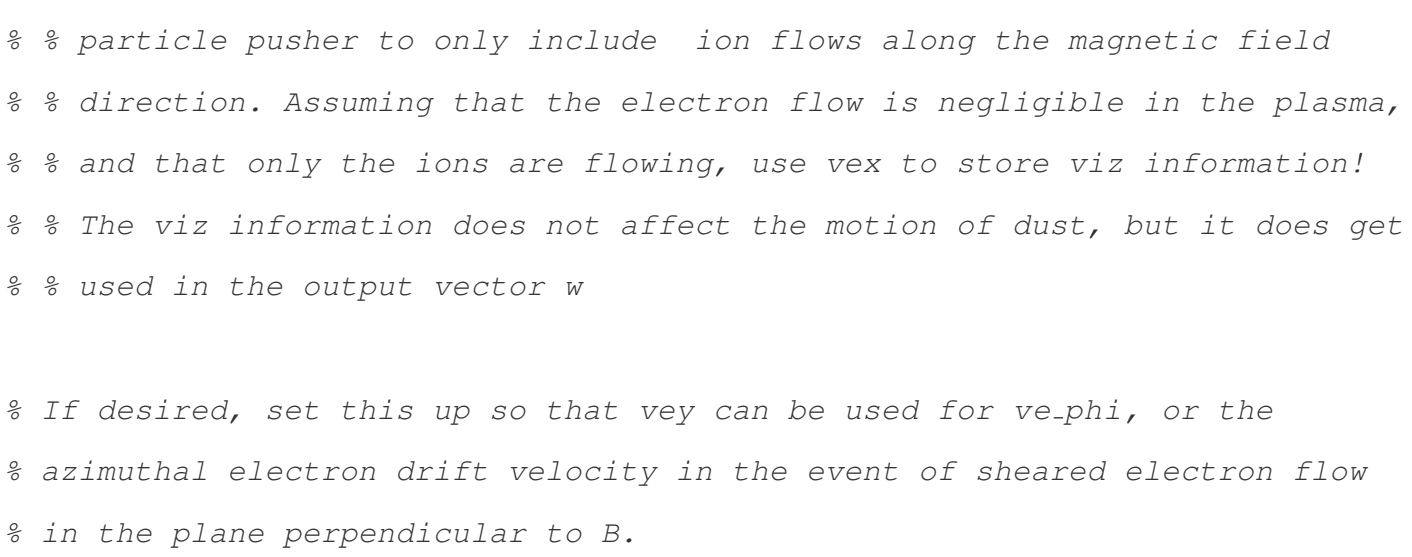




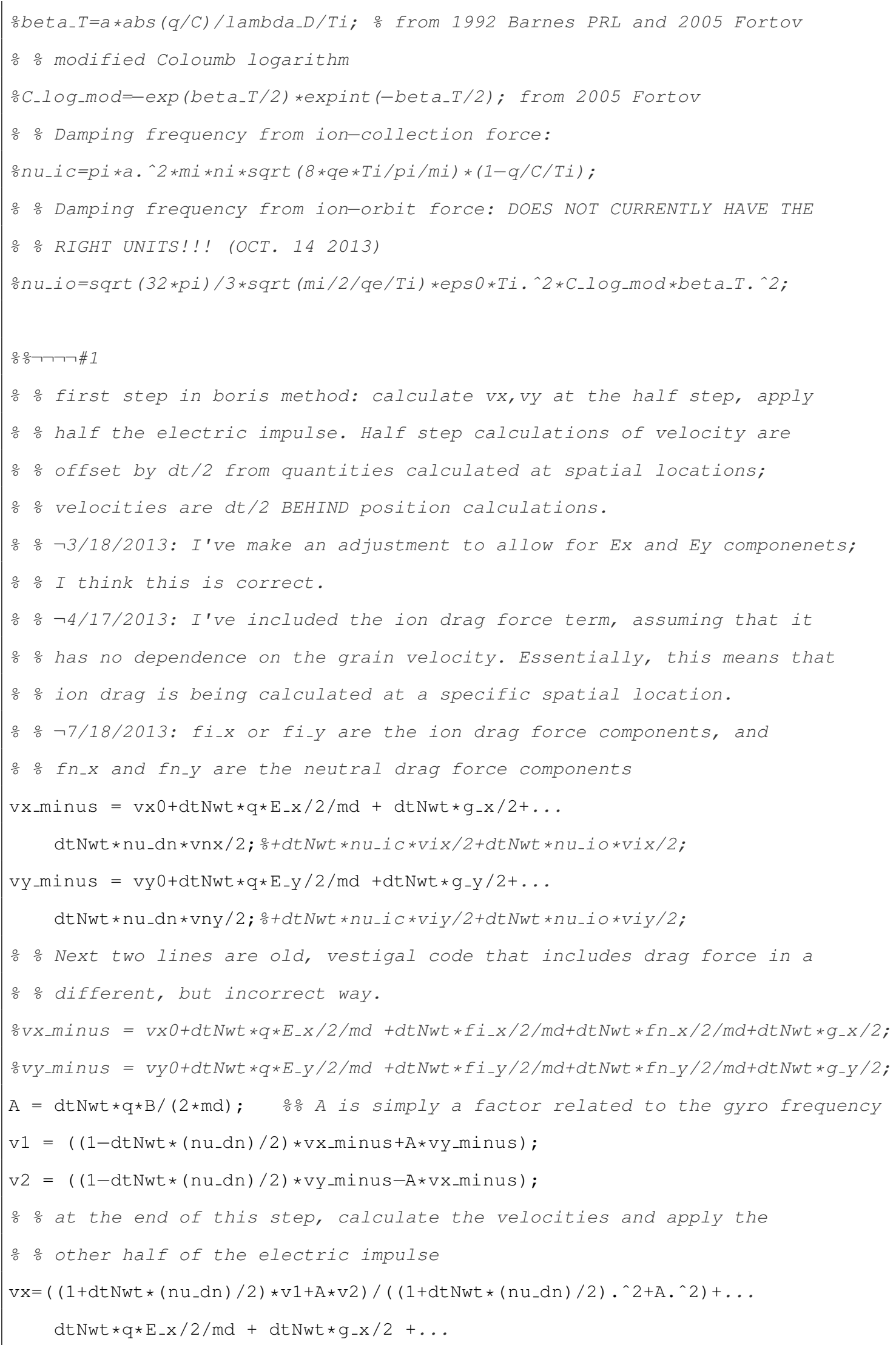




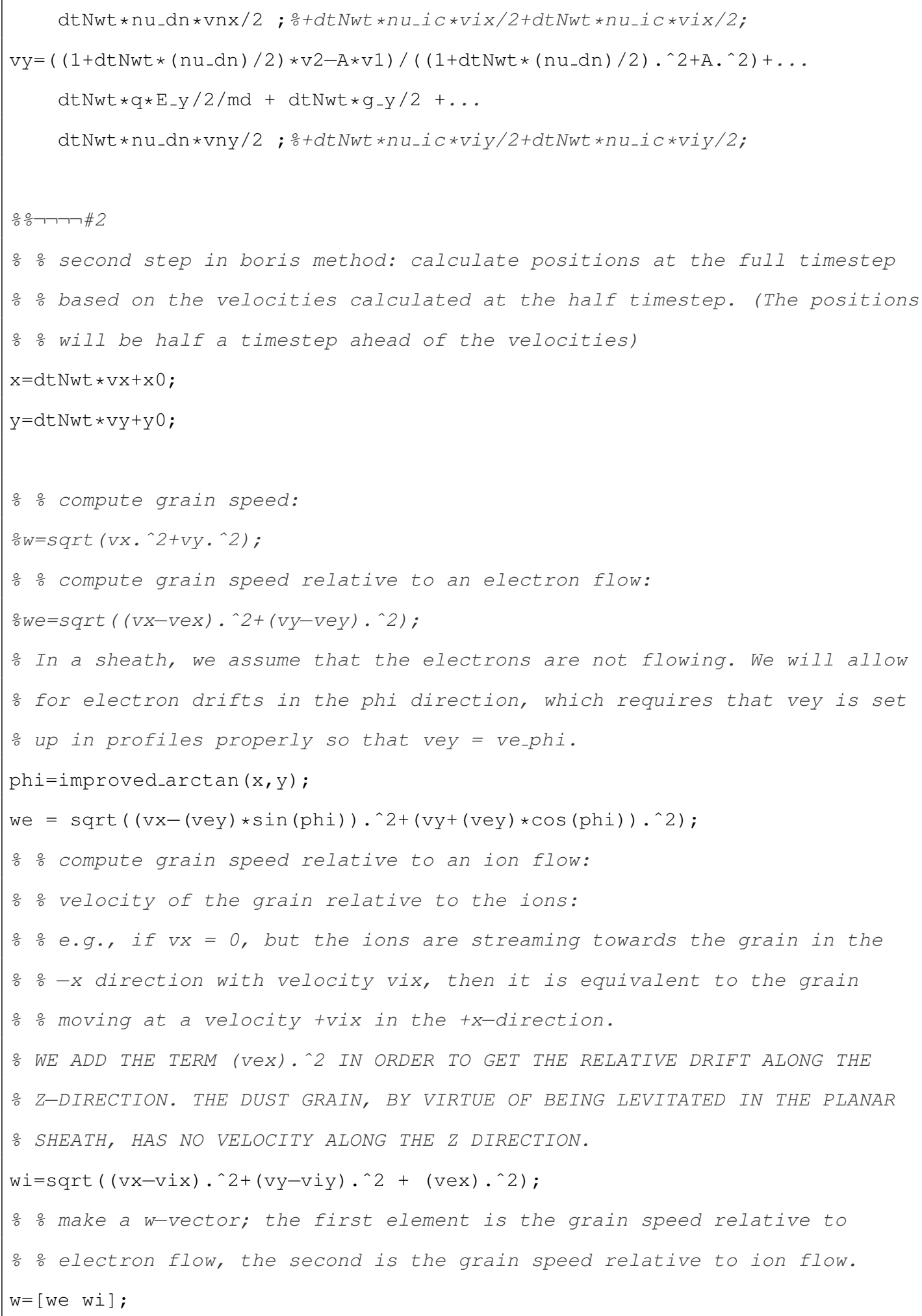




\section{iii Boris Solver for Grains in Co-rotating Reference frames}

The function corotating_boris_pusher.m provides the time advancement of the grain in the frame that co-rotates with a planet, or other rotating system. This is particularly useful for determining grain motion relative to an observer on Saturn or Jupiter, for example.

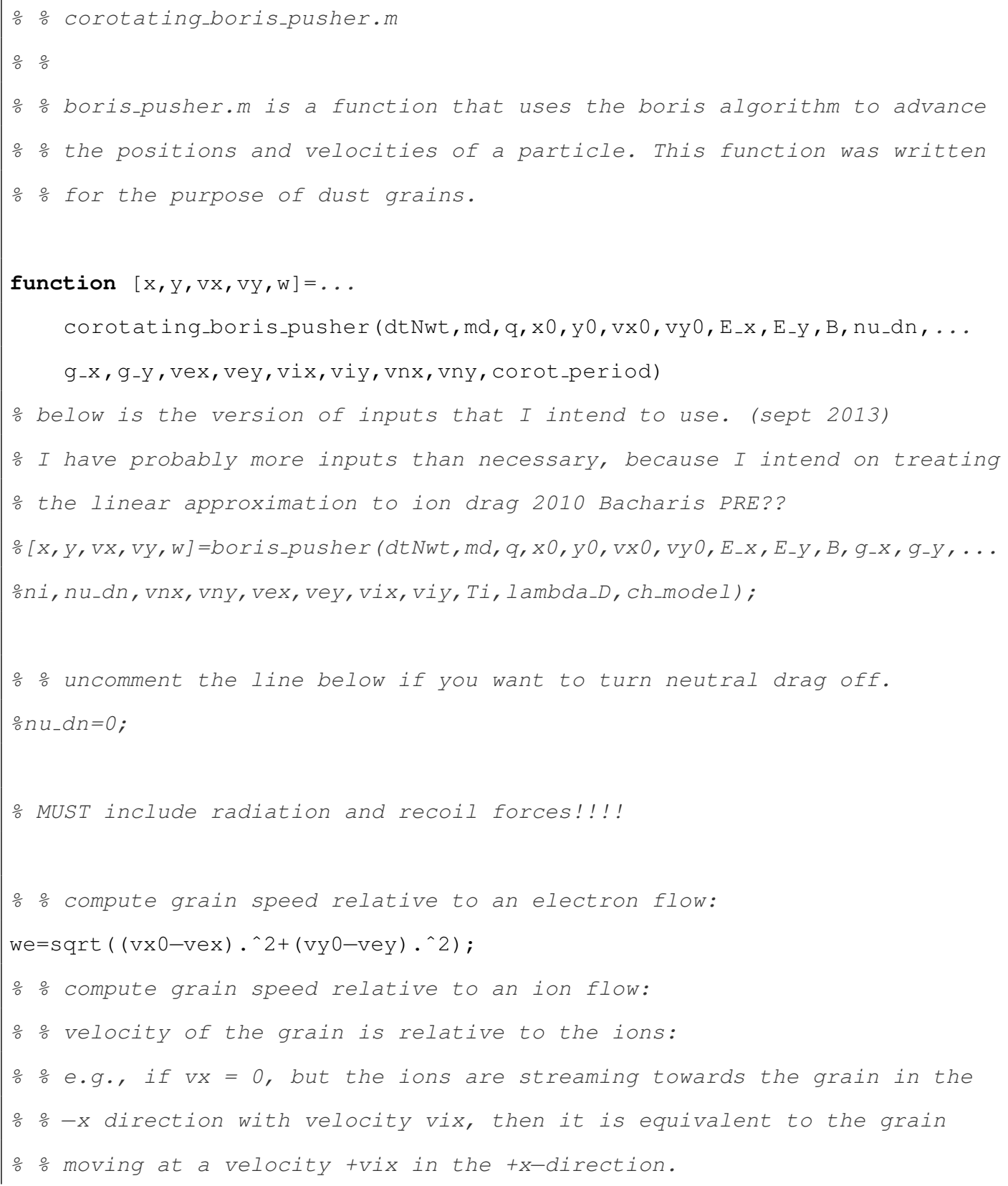




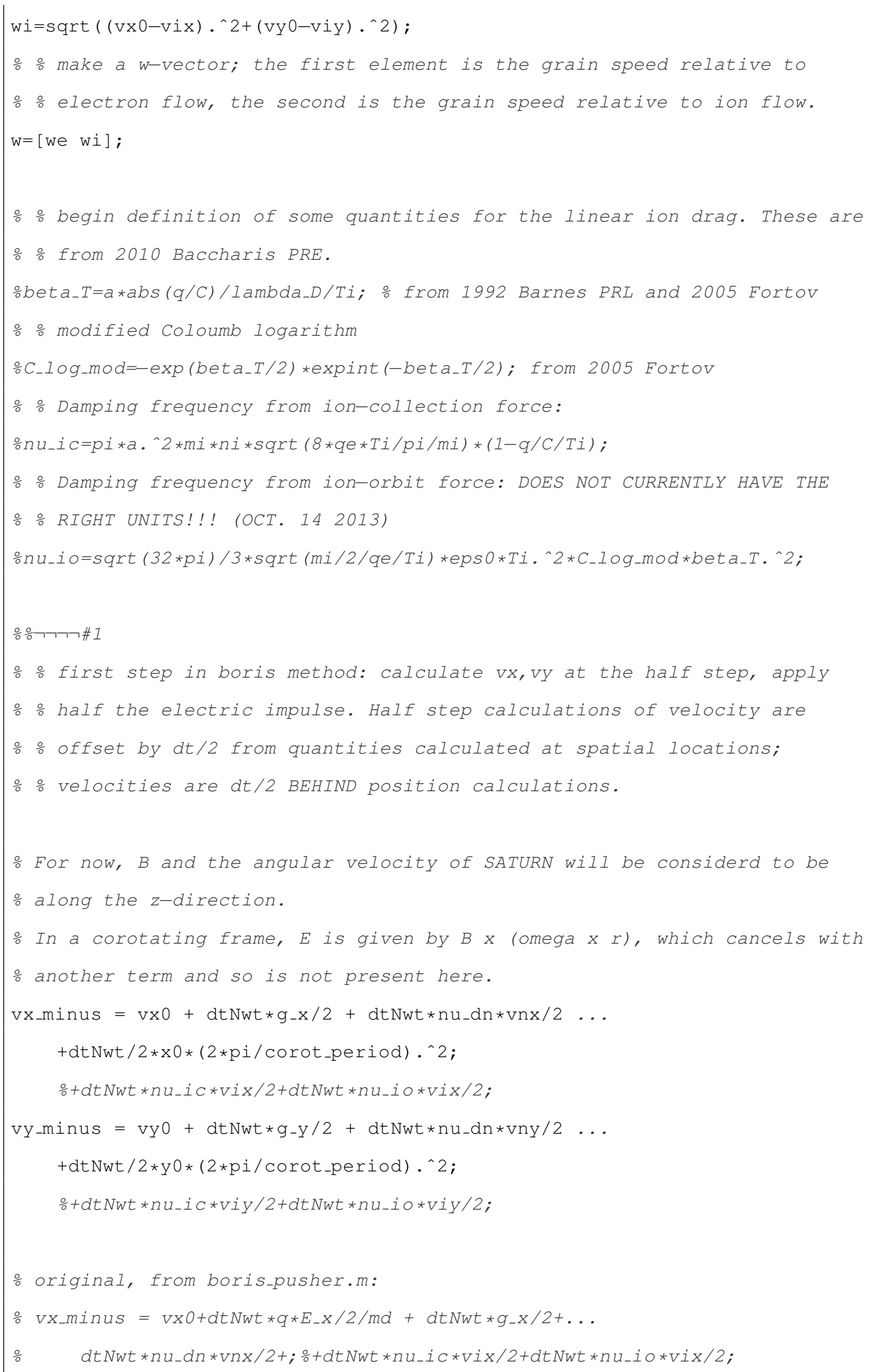




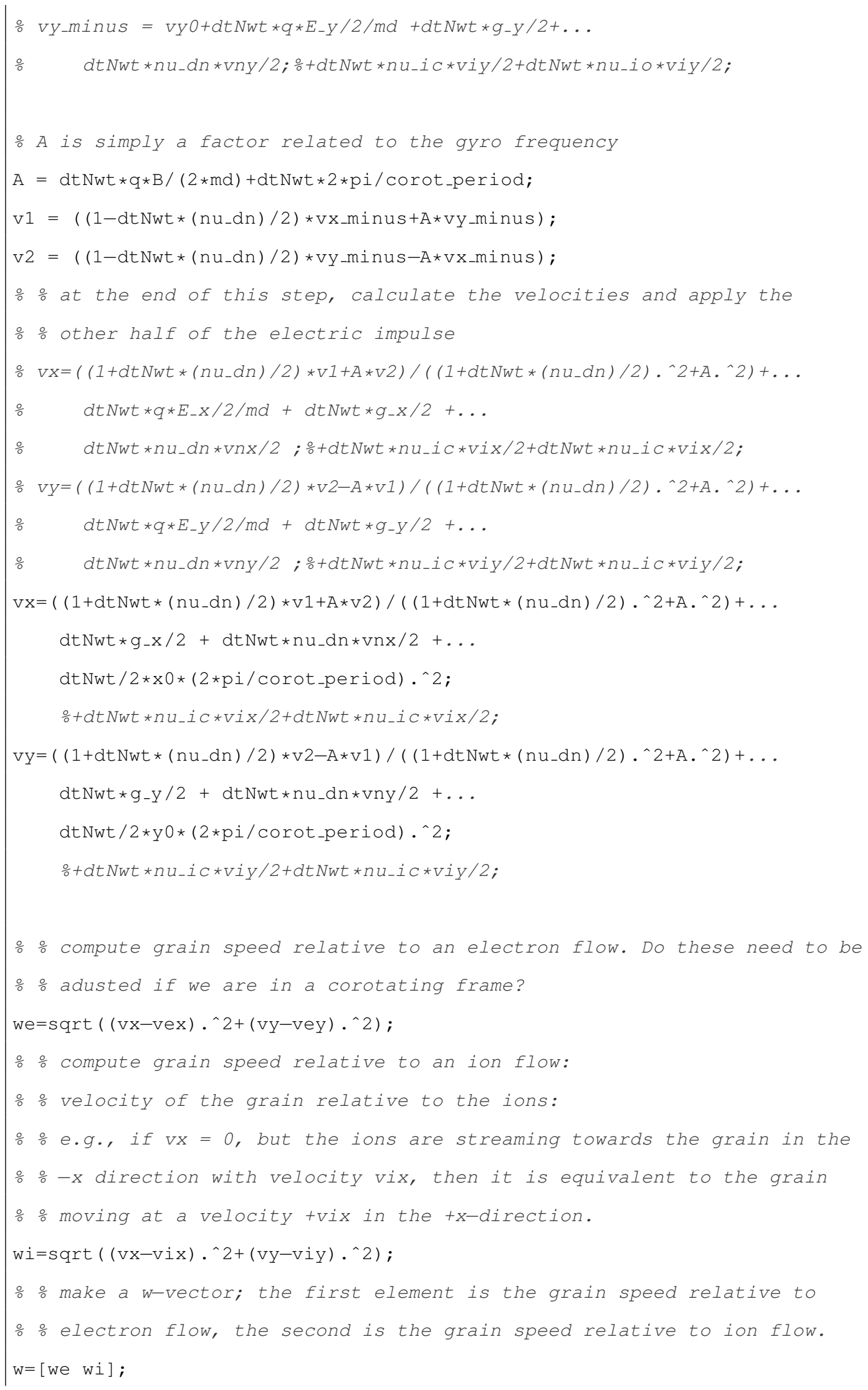




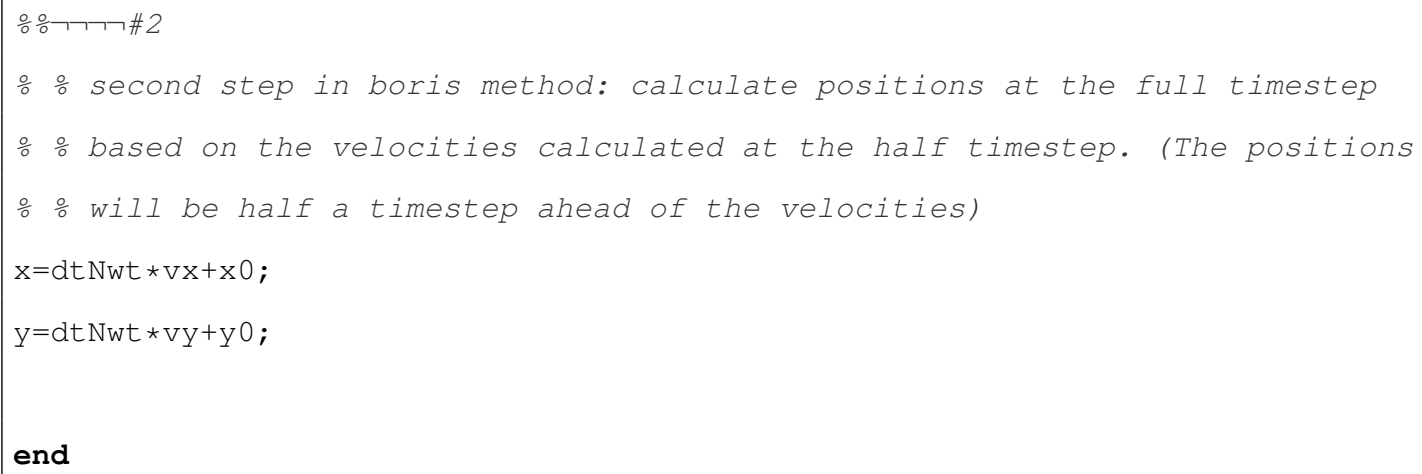

\section{iv Iterative Leapfrog Solver}

The function iterative_pusher.m provides the time advancement of the grain for the case of non-linear, velocity dependent drag forces. An iterative, Newton method is used to determine the resulting grain position and velocity. The maximum number of iterations and the error tolerance can be specified by the user within the subroutine; these values are currently set at 1000 iterations and $10^{-10} \mathrm{~m} / \mathrm{s}$, respectively, which provide a reasonable degree of accuracy without compromising performance. Note that this method is an implicit solver.

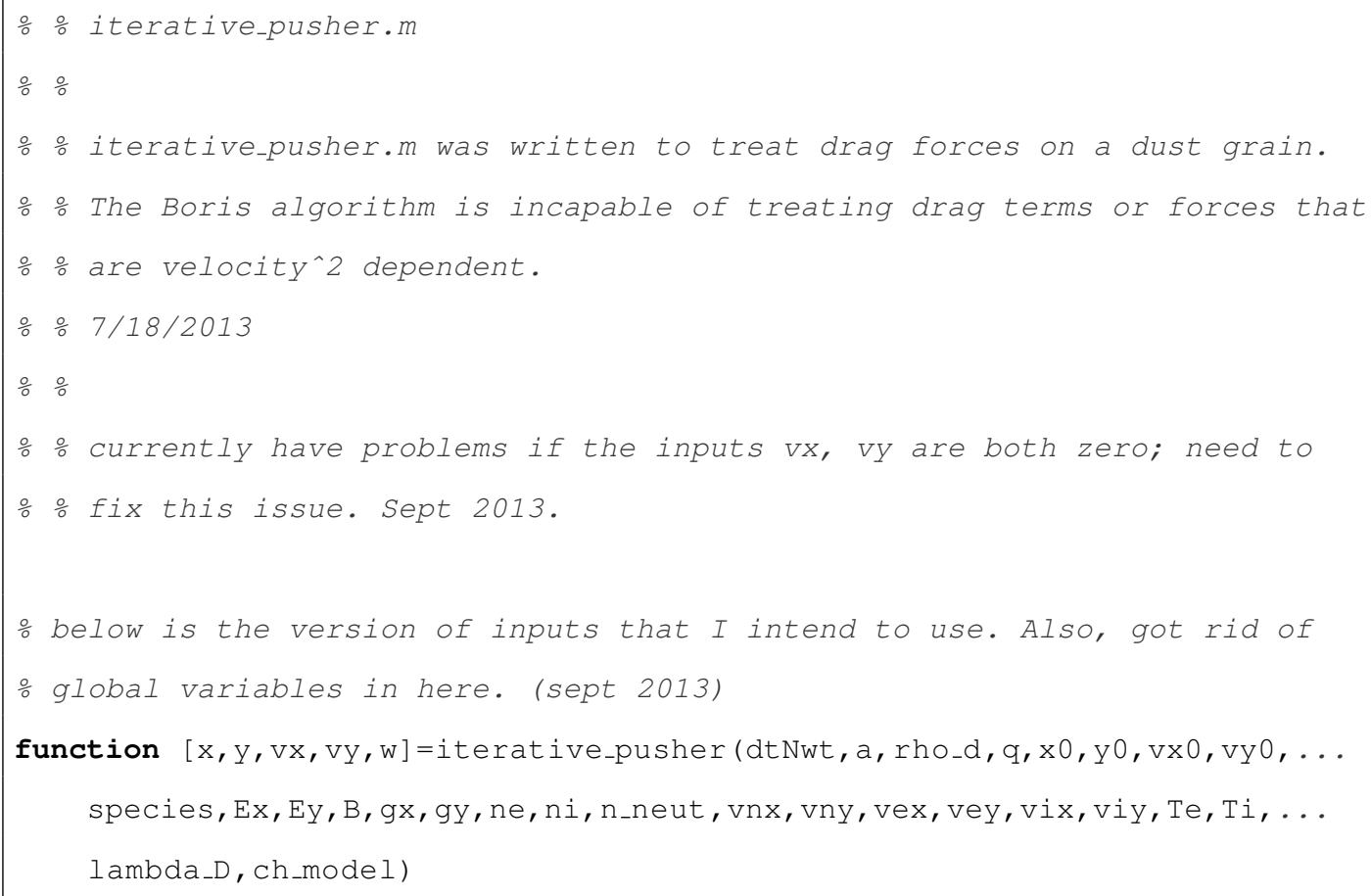




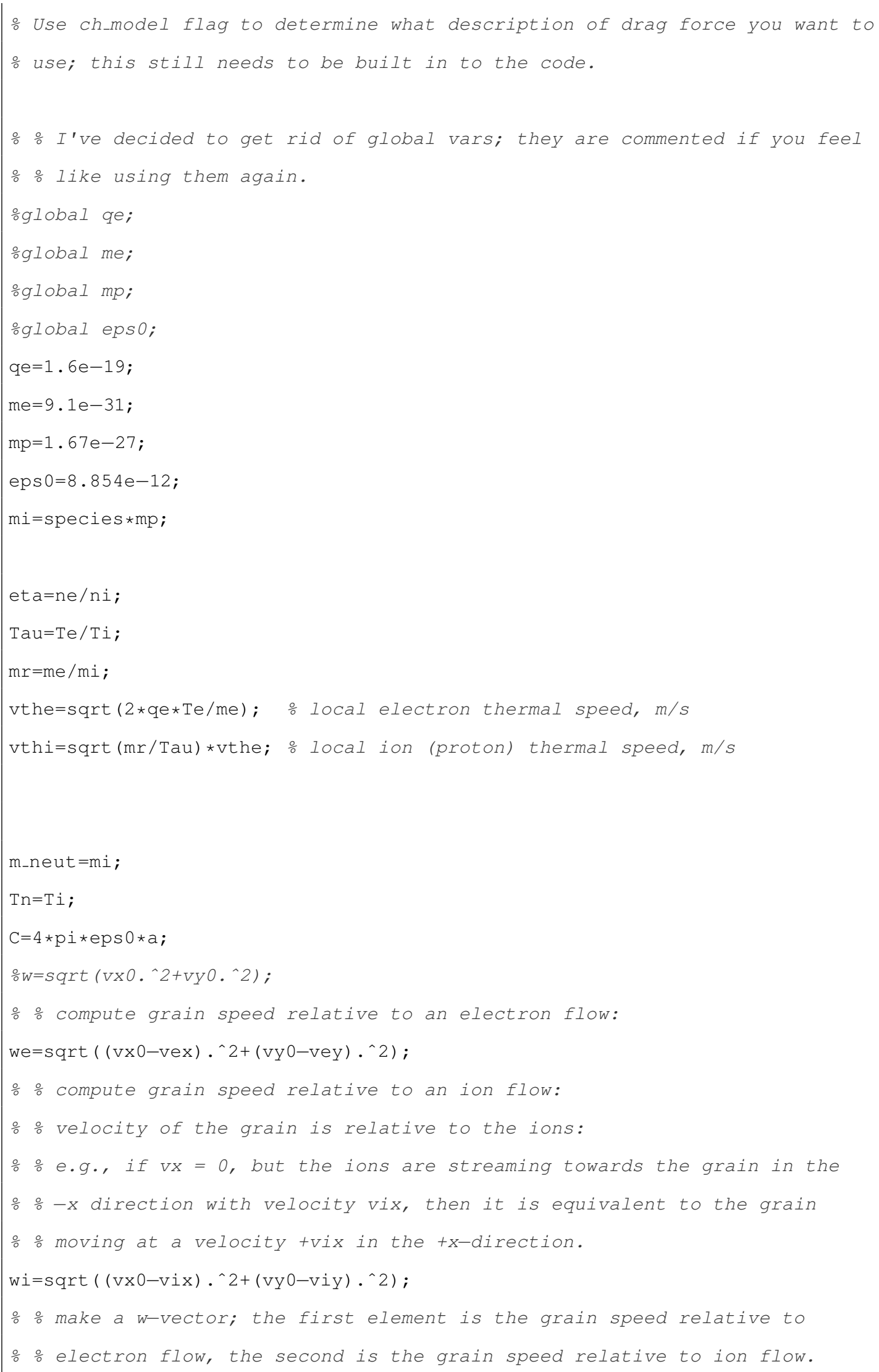




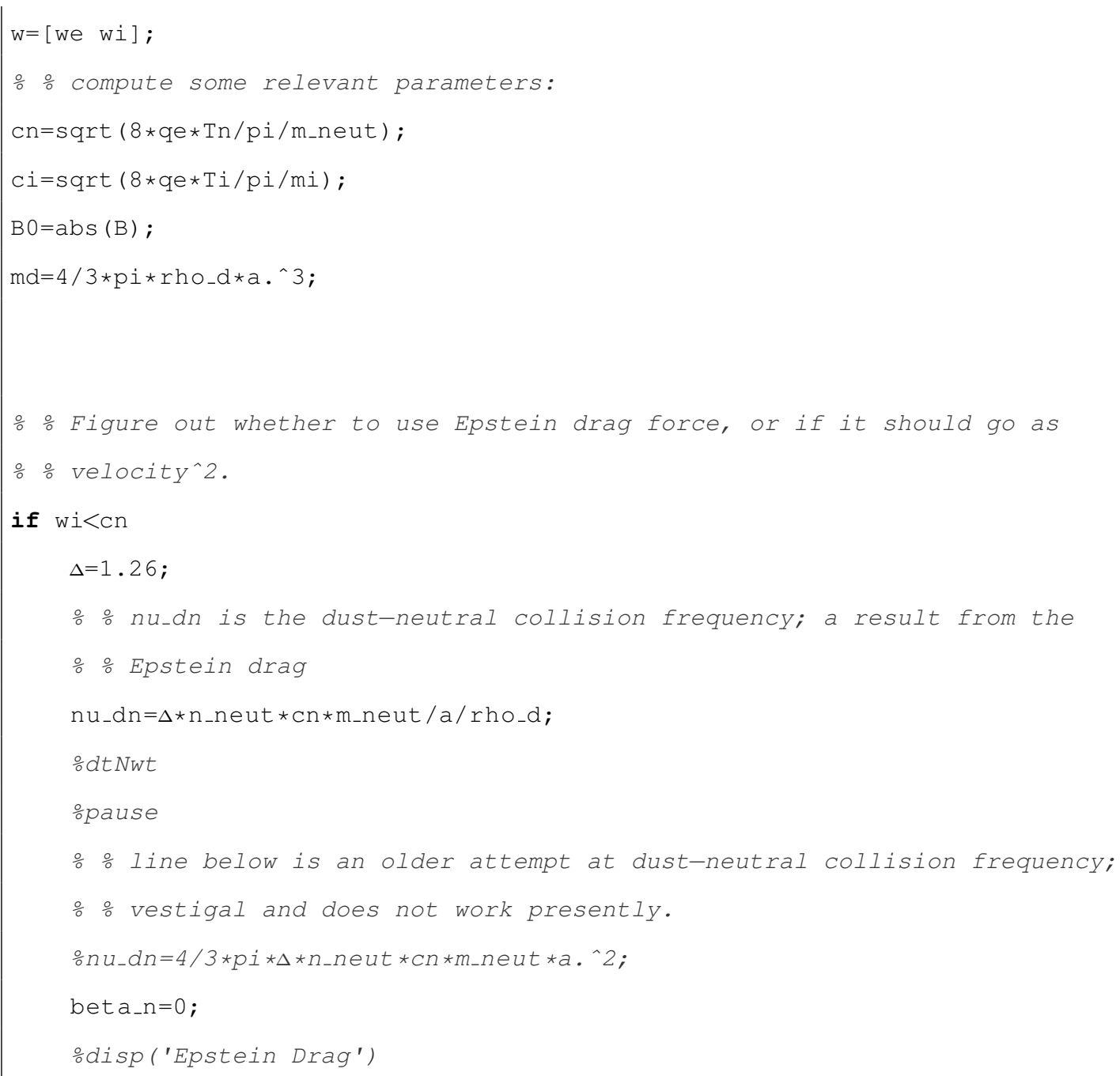




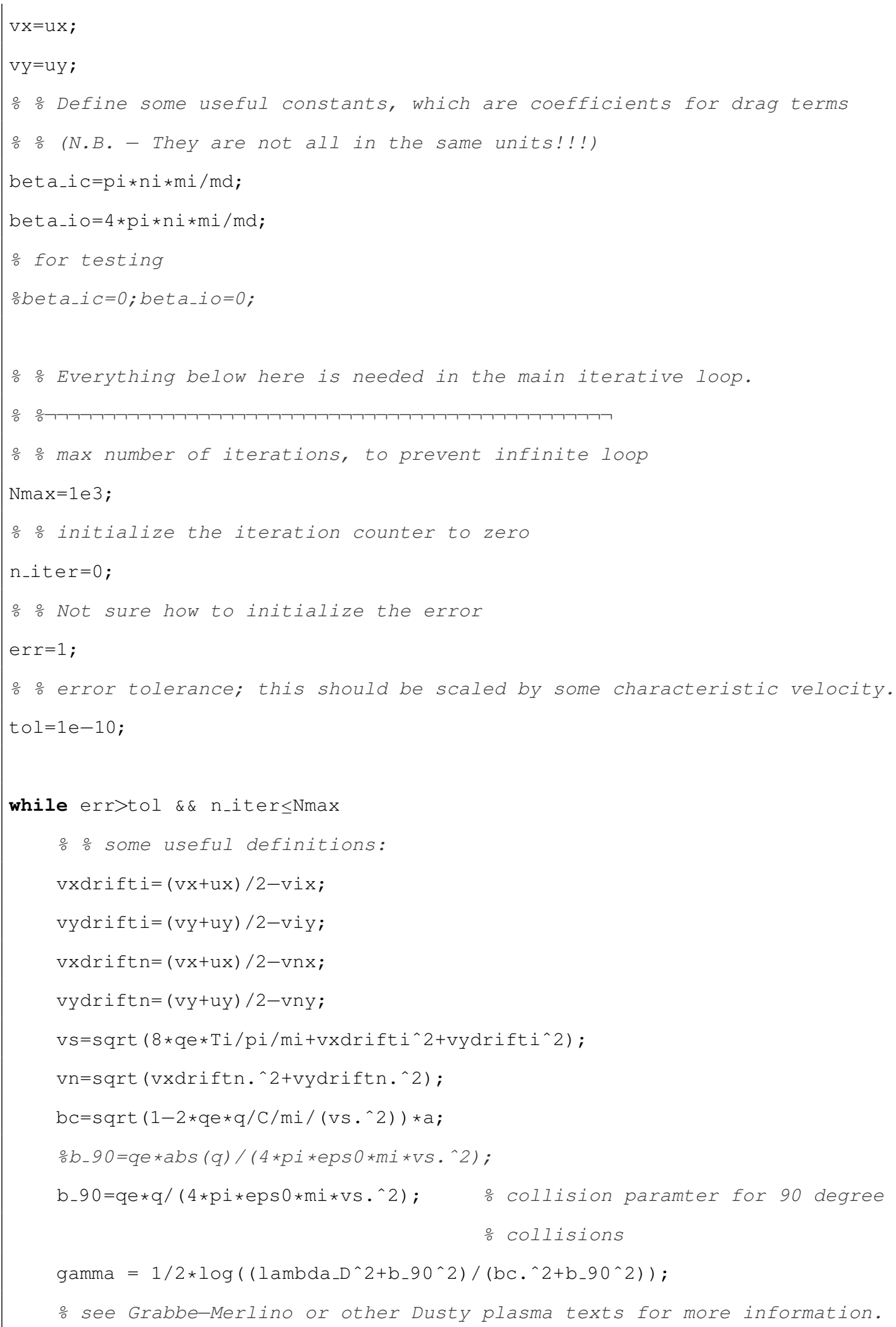




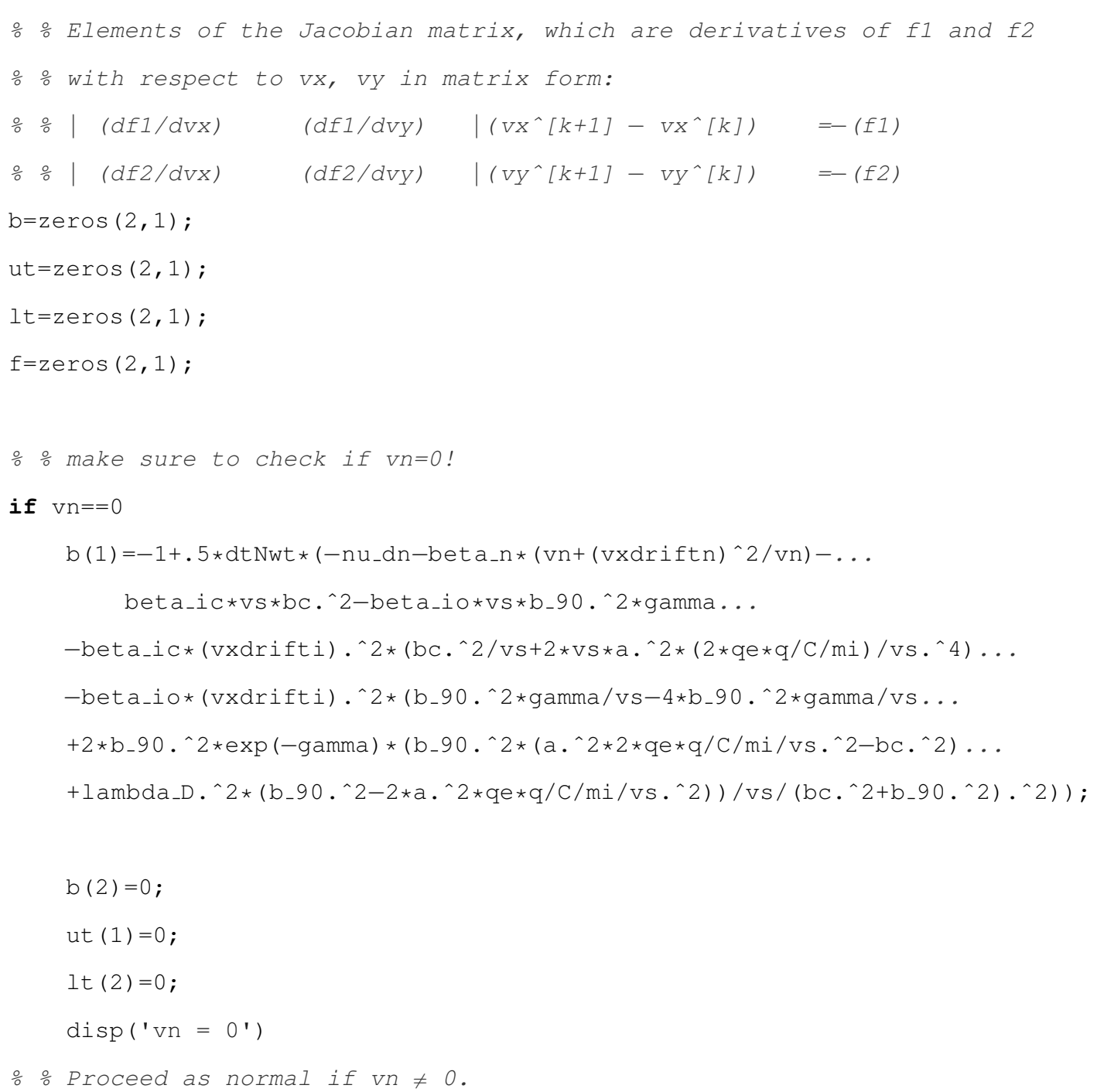




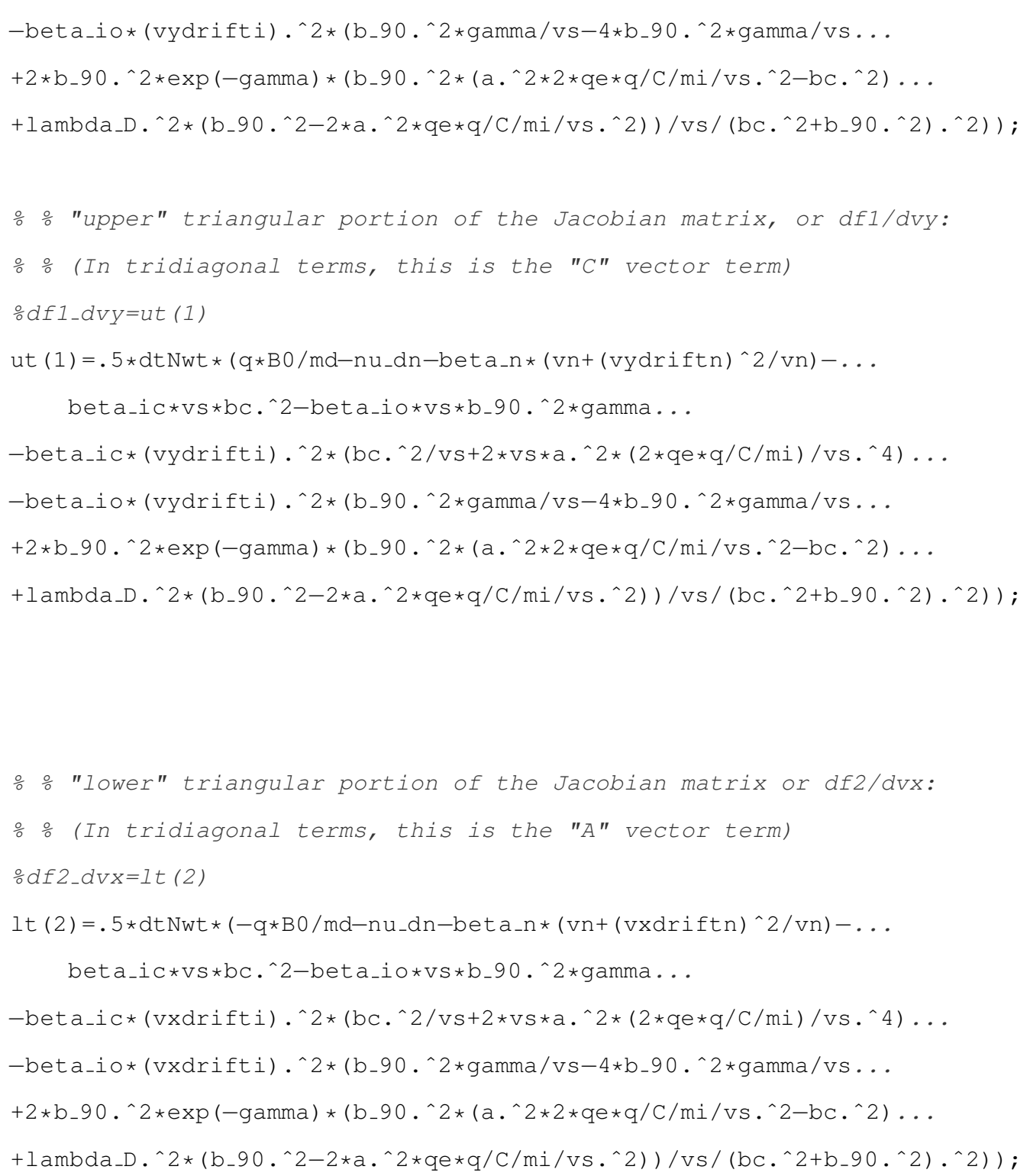




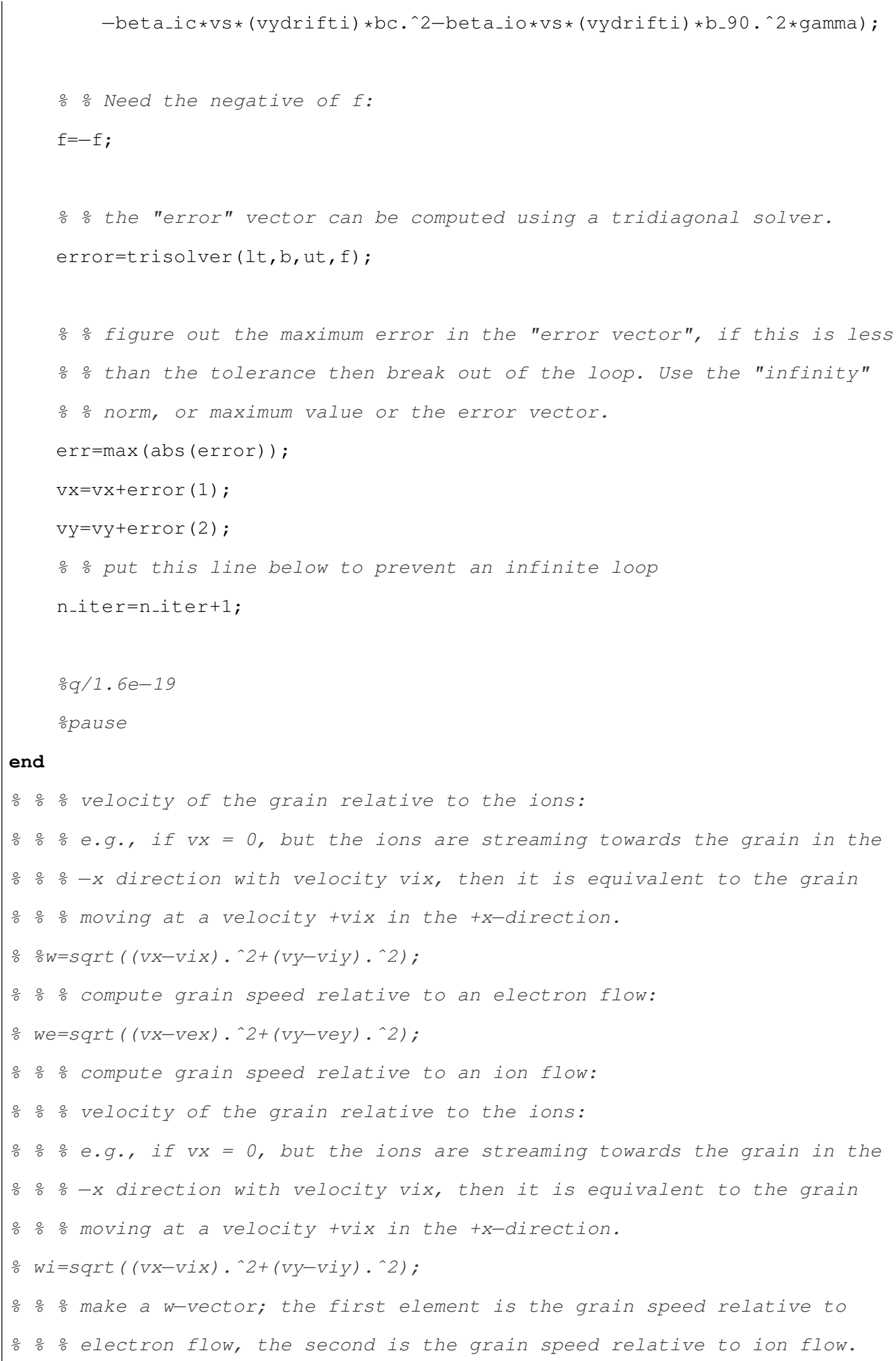




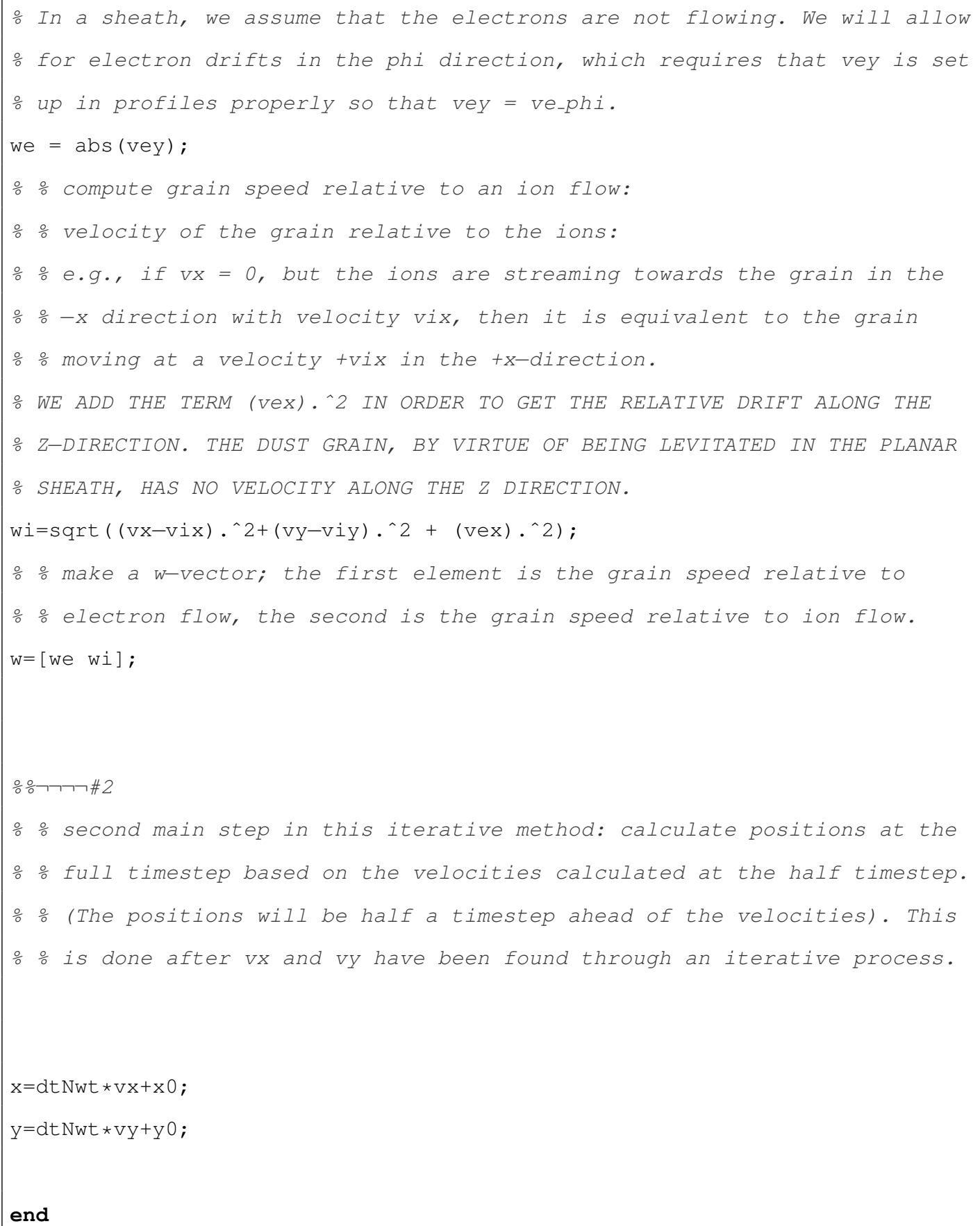

\section{v Iterative Leapfrog Solver for Grains in Sheaths}

The function sheath_iterative_pusher.m is nearly identical to iterative_pusher.m, except that it assumes that the variable $v_{e x}$ is used to store the information about the ion flow along the $z$-direction, instead of storing information about the electron flow in the $x$-direction. 


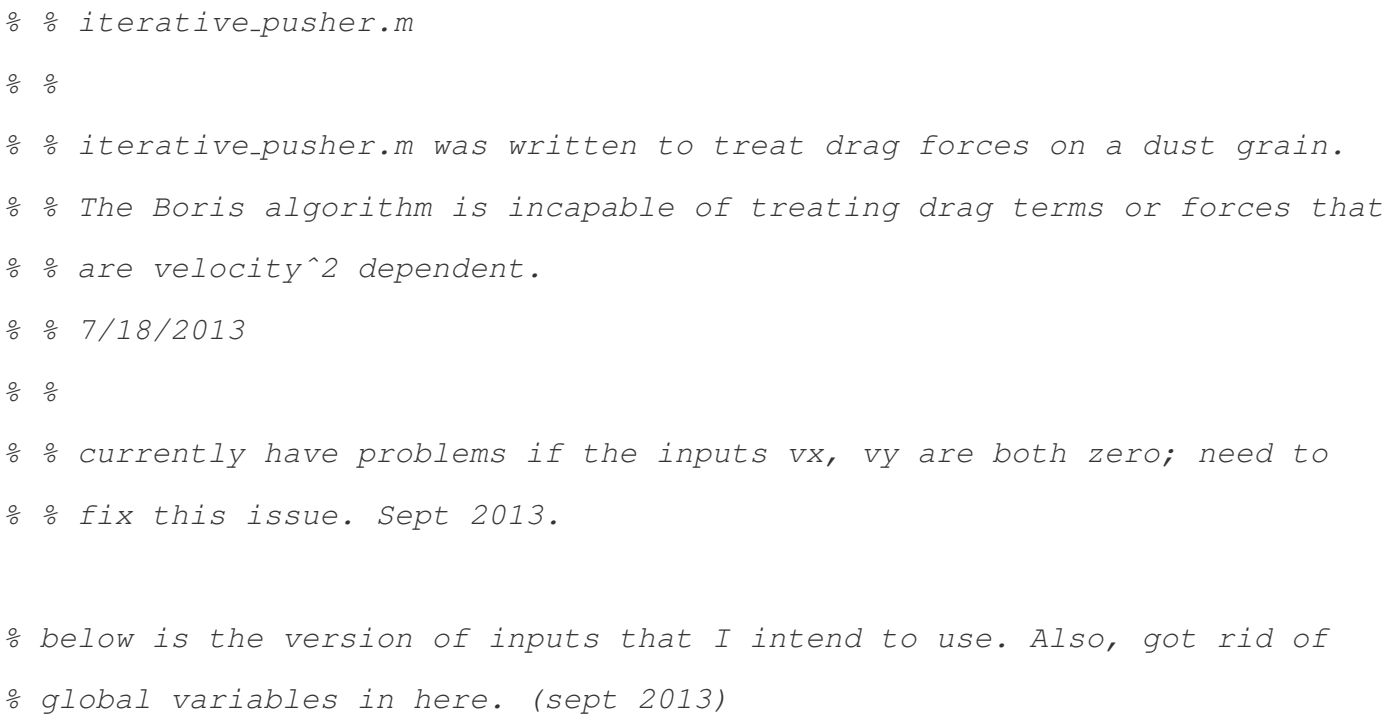




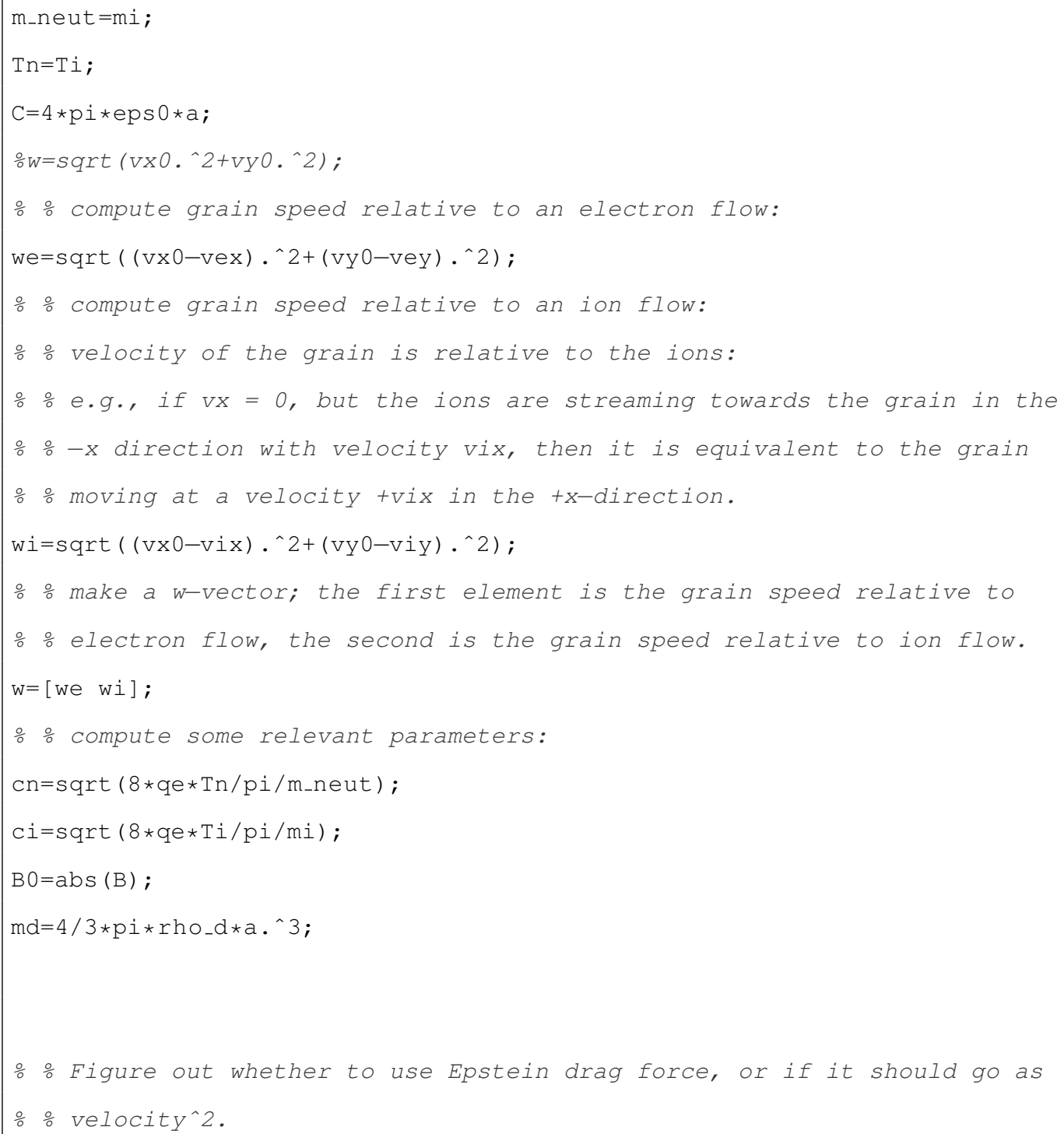


odisp ('Epstein Drag')

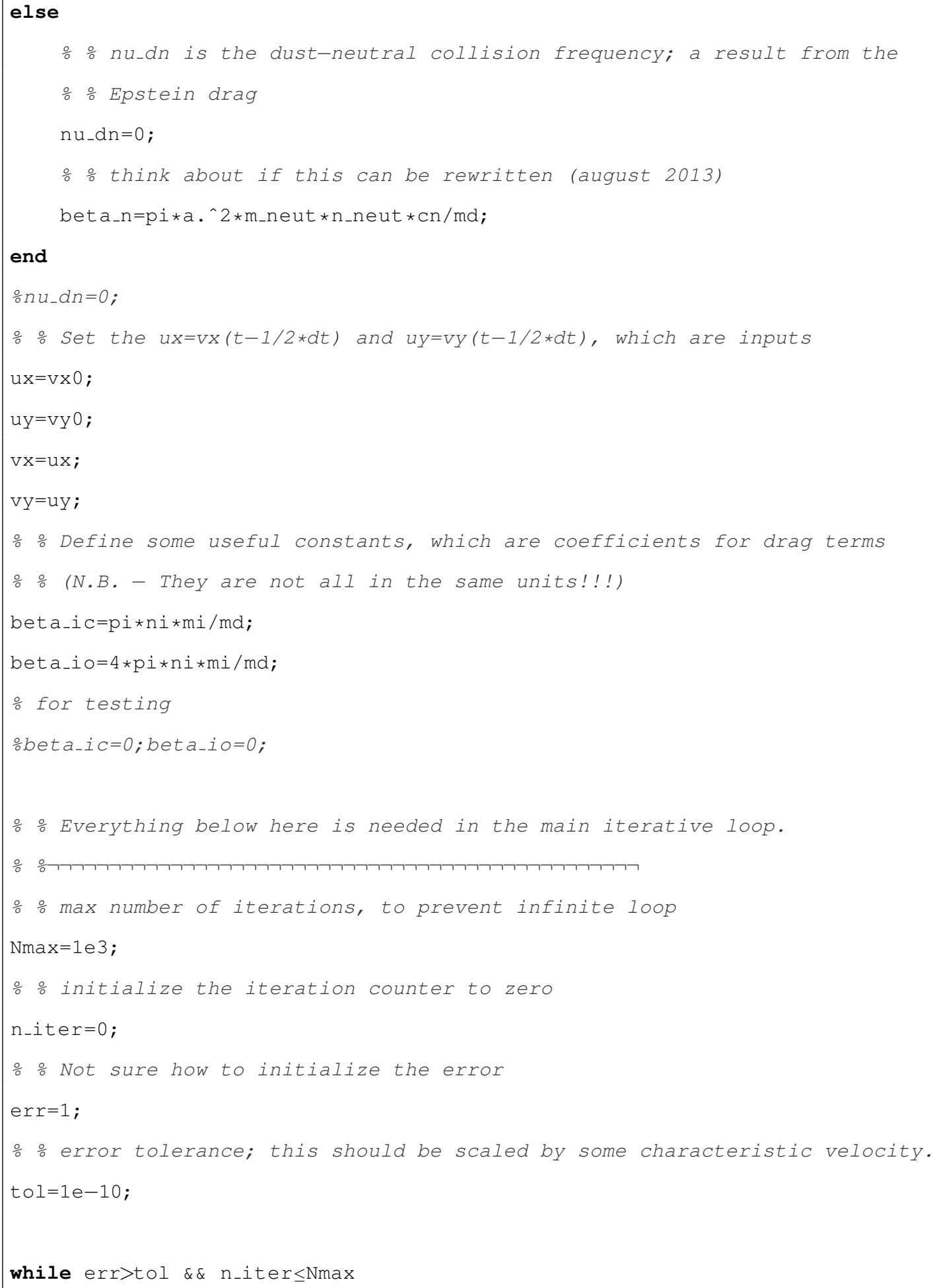




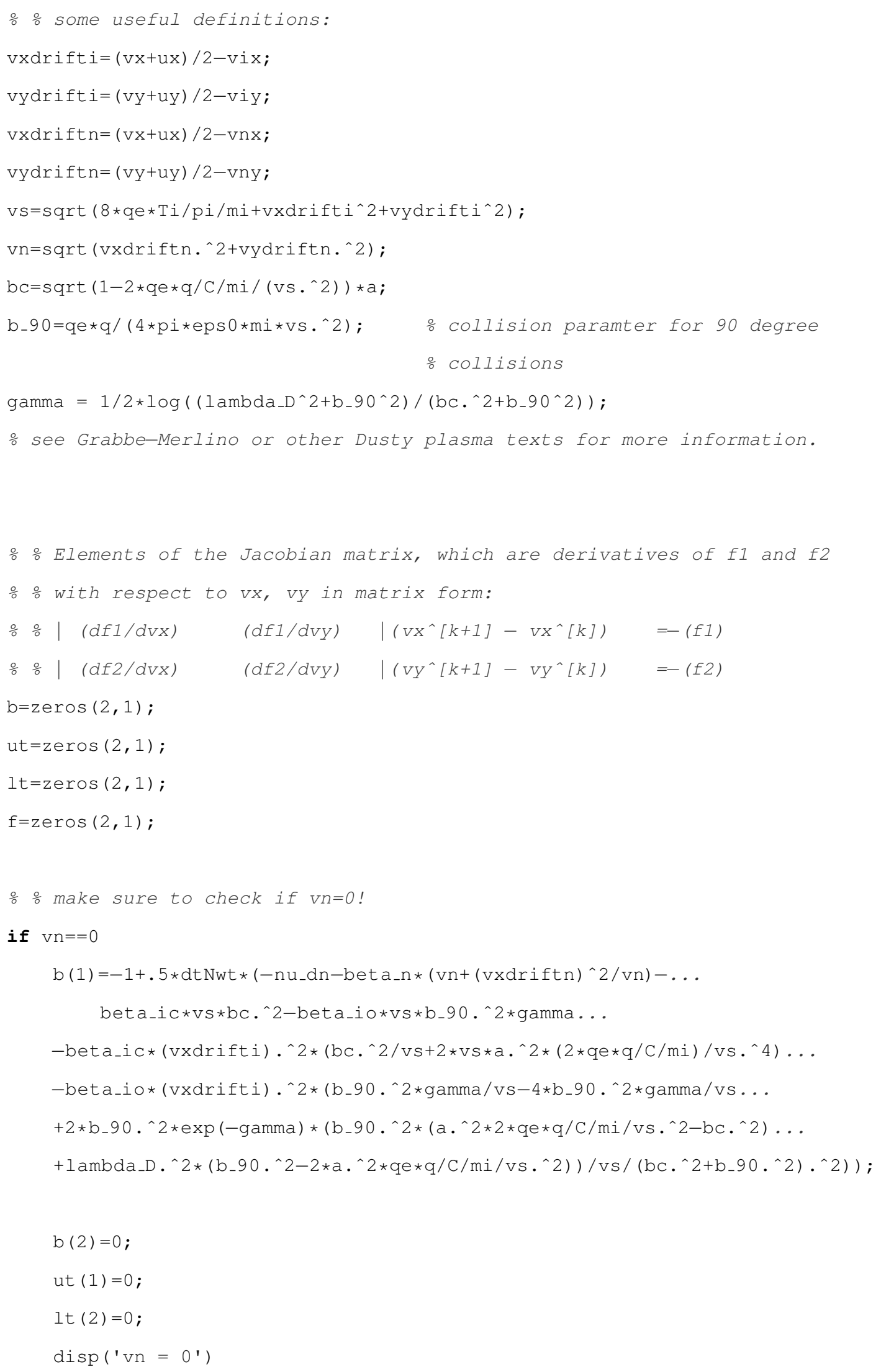


응 Proceed as normal if $v n \neq 0$.

else

$\div \quad d f 1 / d v x:$

$\mathrm{b}(1)=-1+.5 * d t N w t *\left(-n u_{-} d n-\right.$ beta_n $*\left(v n+(\text { vxdriftn })^{\wedge} 2 / v n\right)-\ldots$

beta_ic*vs*bc.^2-beta_io*vs*b_90 . ^2*gamma...

-beta_ic*(vxdrifti) .`2 $2 *\left(b c \cdot{ }^{\wedge} 2 / v s+2 * v s * a \cdot{ }^{\wedge} 2 *(2 * q e * q / C / m i) / v s \cdot \wedge 4\right) \ldots$

-beta_io*(vxdrifti) .^2*(b_90.^2*gamma/vs-4*b_90.^2*gamma/vs...

$+2 * b_{-} 90 \cdot{ }^{\wedge} 2 * \exp (-g a m m a) *\left(b_{-} 90 \cdot{ }^{\wedge} 2 *\left(a \cdot{ }^{\wedge} 2 * 2 * q e * q / C / m i / v s \cdot{ }^{\wedge} 2-b c \cdot{ }^{\wedge} 2\right) \ldots\right.$

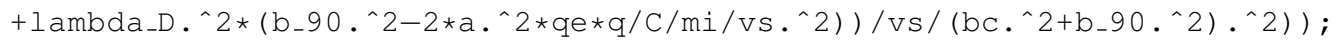

$\div \quad d f 2 / d v y:$

$\mathrm{b}(2)=-1+.5 * d t N w t *\left(-n u_{-} d n-\right.$ beta_n $*(v n+($ vydriftn $) \wedge 2 / v n)-\ldots$

beta_ic*Vs*bc.`2-beta_io*vs*b_90.^2*gamma...

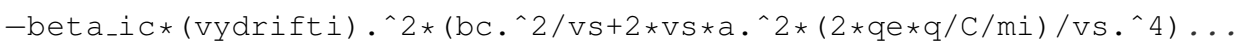

-beta_io*(vydrifti) .^2*(b_90.^2*gamma/vs-4*b_90.^2*gamma/vs...

$+2 \star b_{-} 90 \cdot \wedge 2 \star \exp (-g a m m a) *\left(b_{-} 90 \cdot \wedge 2 *\left(a \cdot{ }^{\wedge} 2 * 2 \star q e \star q / C / m i / v s \cdot \wedge 2-b c \cdot \wedge 2\right) \ldots\right.$

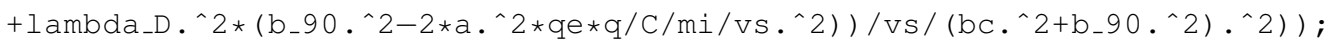

응 "upper" triangular portion of the Jacobian matrix, or dfl/dvy:

응 (In tridiagonal terms, this is the "C" vector term)

$\circ d f 1_{-} d v y=u t(1)$

ut $(1)=.5 * \operatorname{dtNwt} *\left(q * B 0 / m d-n u_{-} d n-\right.$ beta_n $*(v n+(\operatorname{vydriftn}) \wedge 2 / v n)-\ldots$

beta_ic*vs*bc.^2-beta_io*vs*b_90 • ^2*gamma...

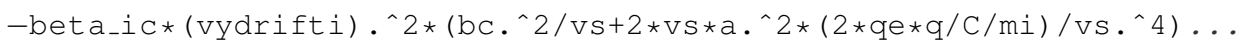

-beta_io*(vydrifti).^2*(b_90.^2*gamma/vs $-4 * b_{-} 90$.^2*gamma/vs...

$+2 * b_{-} 90 \cdot{ }^{\wedge} 2 * \exp (-g a m m a) *\left(b_{-} 90 \cdot{ }^{\wedge} 2 *\left(a \cdot{ }^{\wedge} 2 * 2 * q e * q / C / m i / v s \cdot{ }^{\wedge} 2-b c \cdot{ }^{\wedge} 2\right) \ldots\right.$

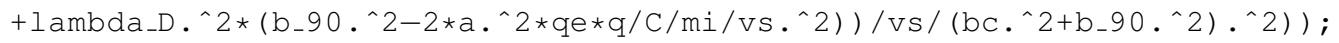

응 "lower" triangular portion of the Jacobian matrix or df2/dvx:

응 (In tridiagonal terms, this is the "A" vector term)

$\circ d f 2 \_d v x=1 t(2)$

lt $(2)=.5 * d t \operatorname{Nwt} *\left(-q * B 0 / m d-n u_{-} d n-\right.$ beta_n $*\left(v n+(\operatorname{vxdriftn})^{\wedge} 2 / v n\right)-\ldots$

beta_ic*vs*bc.^2-beta_io*vs*b_90.^2*gamma...

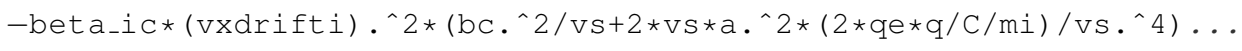


-beta_io*(vxdrifti) .^2*(b_90.^2*gamma/vs-4*b_90.^2*gamma/vs ...

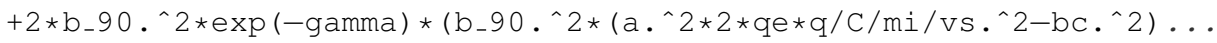

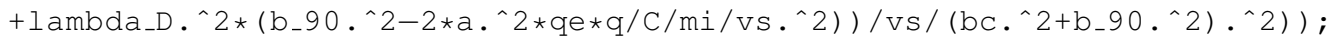

end

$\div$ 능 the functions of $v x$, Vy:

$f(1)=-v x+u x+d t N w t *\left(g x+q * E x / m d+q * B 0 *(v y+u y) / 2 / m d-n u \_d n * \ldots\right.$

(vxdriftn) - beta_n*(vxdriftn $) *$ vn $\ldots$

-beta_ic*vs*(vxdrifti)*bc.^2-beta_io*vs*(vxdrifti)*b_90.^2*gamma)；

$f(2)=-v y+u y+d t N w t *\left(g y+q * E y / m d-q * B 0 *(v x+u x) / 2 / m d-n u \_d n *(v y d r i f t n)-\ldots\right.$

beta_n*(vydriftn $) * v n \ldots$

-beta_ic*vs*(vydrifti)*bc.^2-beta_io*vs*(vydrifti)*b_90.^2*gamma)；

$\div$ 응 Need the negative of $f$ :

$\mathrm{f}=-\mathrm{f}$;

응 the "error" vector can be computed using a tridiagonal solver.

error=trisolver $(l t, b, u t, f)$;

응 figure out the maximum error in the "error vector", if this is less

$\div$ ․ than the tolerance then break out of the loop. Use the "infinity"

응 norm, or maximum value or the error vector.

$\operatorname{err}=\max (\operatorname{abs}($ error $))$;

$\mathrm{vx}=\mathrm{vx}+\operatorname{error}(1)$;

$\mathrm{vy}=\mathrm{vy}+\operatorname{error}(2)$;

응 put this line below to prevent an infinite loop

n_iter=n_iter+1;

$\circ q / 1.6 e-19$

opause

end

응금 $\neg \neg \neg \# 2$ 


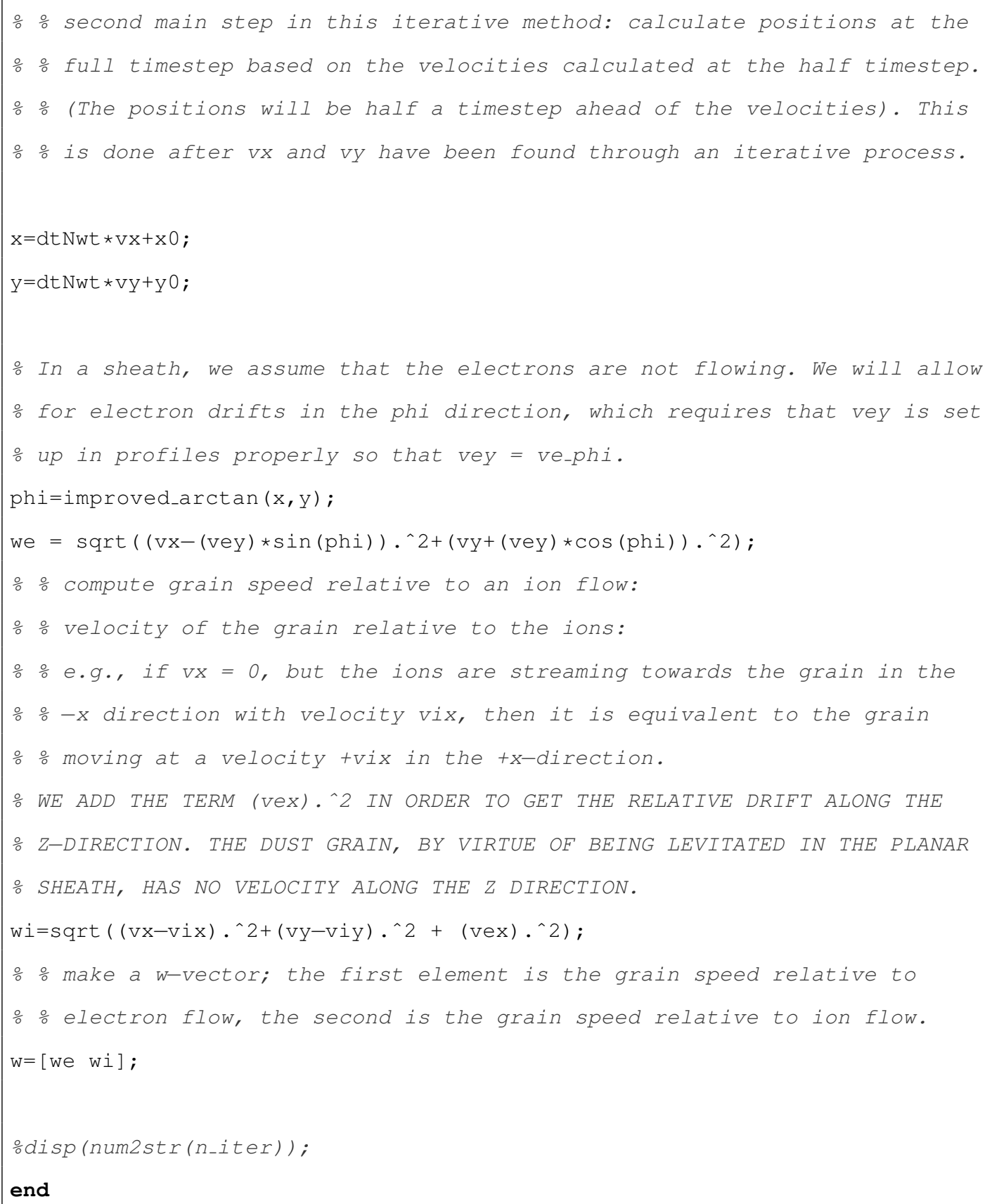

\section{vi Iterative Leapfrog Solver for Grains in Co-rotating Reference frames}

The function corotating_iterative_pusher.m incorporates non-linear ion drag for grains in the reference frame that co-rotates with a planet or moon.

응 corotating_iterative_pusher.m 


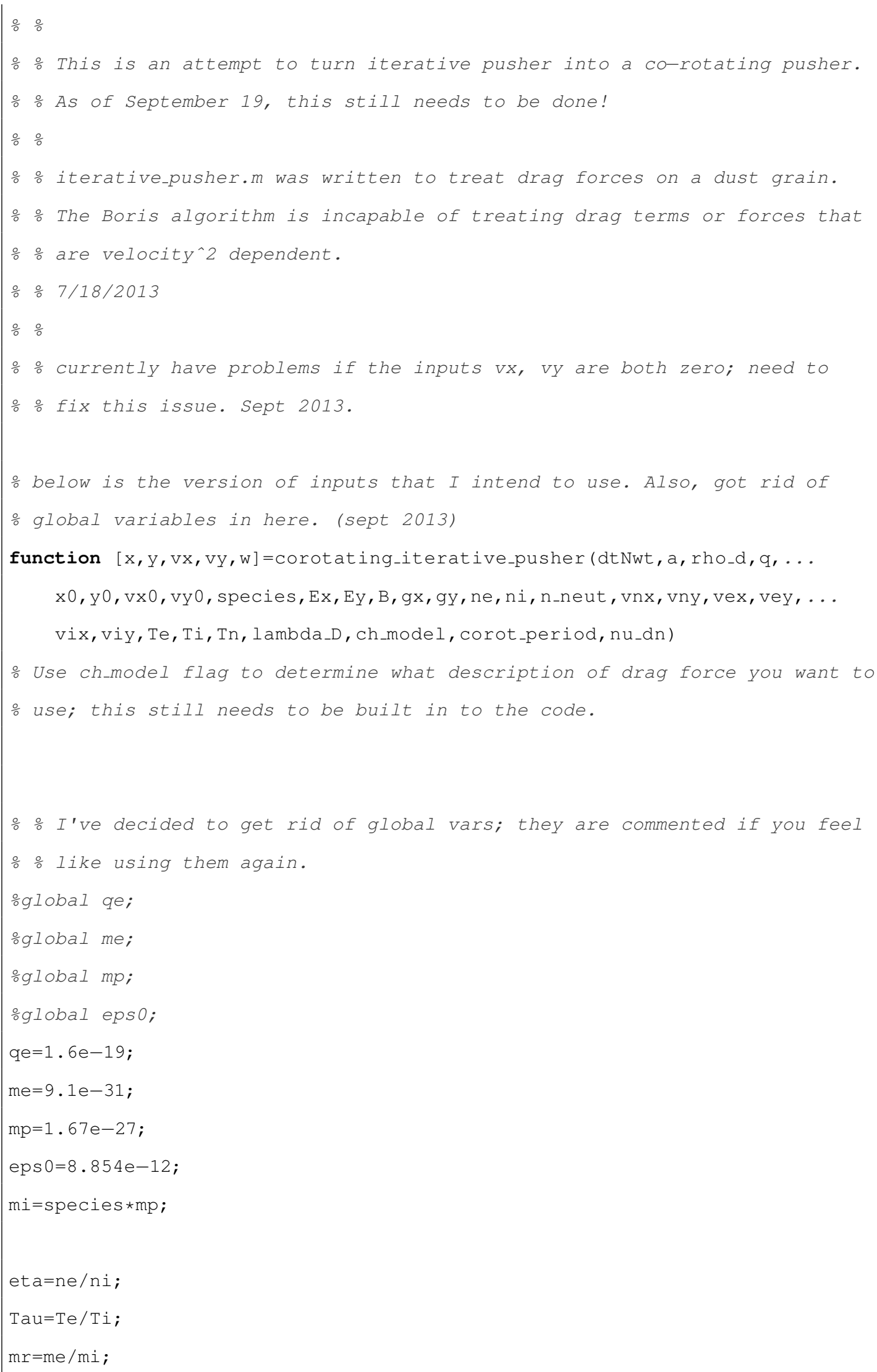




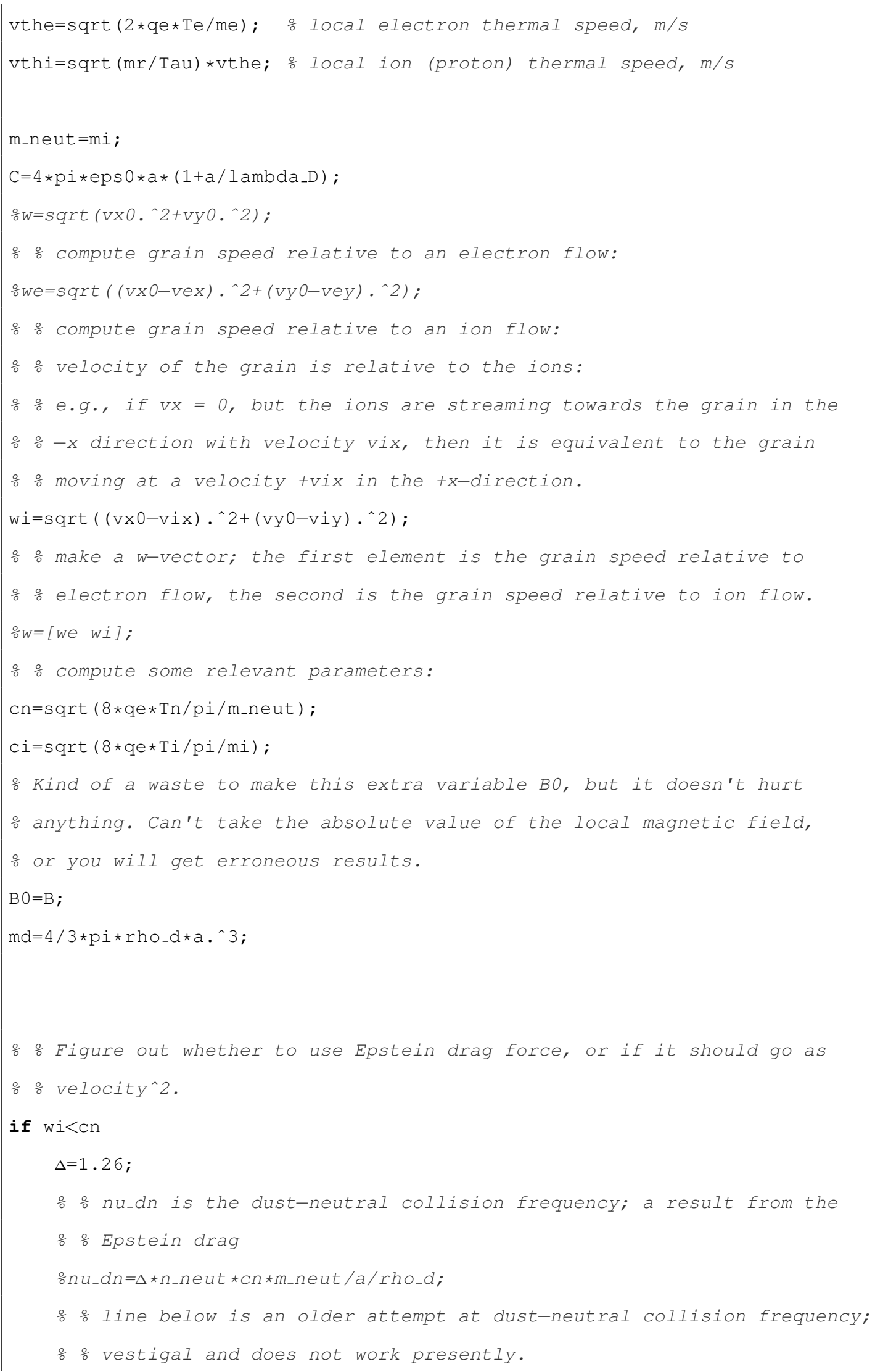




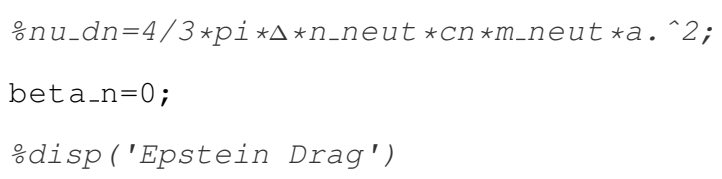

else

응 nu_dn is the dust-neutral collision frequency; a result from the

$\div$ Epstein drag

$\mathrm{nu} \mathrm{u}_{-} \mathrm{dn}=0$;

응 think about if this can be rewritten (august 2013)

beta_n $=\mathrm{pi} * \mathrm{a} \cdot{ }^{\wedge} 2 * \mathrm{~m} \_$neut $* \mathrm{n}_{-}$neut $* \mathrm{cn} / \mathrm{md}$;

\section{end}

$\frac{\circ}{\circ} n u_{-} d n=0$;

$\div \frac{\circ}{0}$ Set the ux=vx $(t-1 / 2 * d t)$ and uy=vy $(t-1 / 2 * d t)$, which are inputs

$\mathrm{ux}=\mathrm{vx} 0$;

uy $=v y 0$;

$\mathrm{vx}=\mathrm{ux}$;

$\mathrm{vy}=\mathrm{uy}$;

음 Define some useful constants, which are coefficients for drag terms

응 (N.B. - They are not all in the same units!!!)

beta_ic $=\mathrm{pi} \star \mathrm{ni} * \mathrm{mi} / \mathrm{md}$;

beta_io $=4 * \mathrm{p} i * \mathrm{ni} * \mathrm{mi} / \mathrm{md}$;

응 testing

obeta_ic=0; beta_io=0;

$\therefore n u_{-} d n=0 ;$ beta $n=0$;

응 Everything below here is needed in the main iterative loop.

응 응

$\div$ o max number of iterations, to prevent infinite loop

$\operatorname{Nmax}=1 e 3$

응 initialize the iteration counter to zero

n_iter $=0$;

$\therefore$ Not sure how to initialize the error

err $=1$;

응 error tolerance; this should be scaled by some characteristic velocity. 


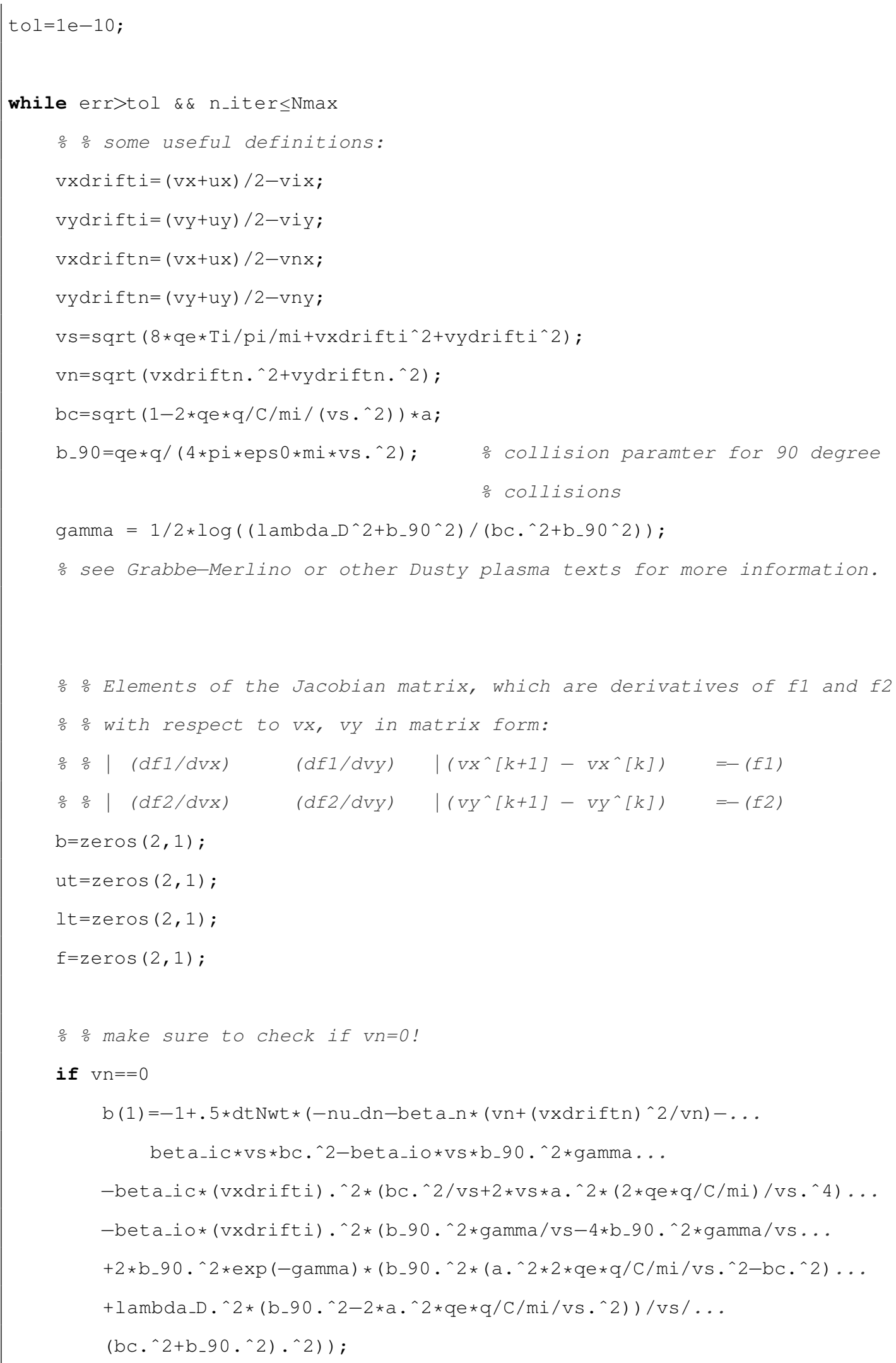




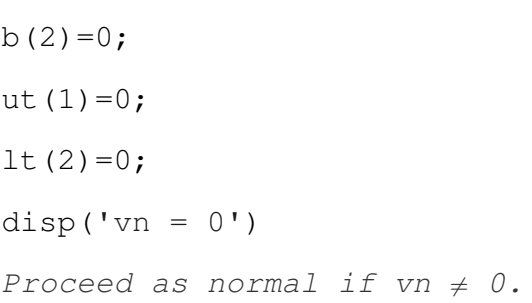

else

$\div \frac{\circ}{0} d f 1 / d v x$ :

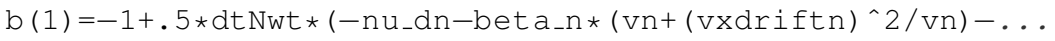
beta_ic*vs*bc.^2-beta_io*vs*b_90 .^2*gamma...

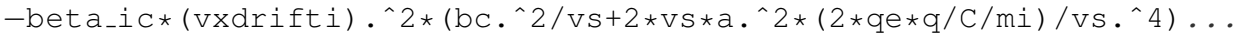
-beta_io*(vxdrifti) .^2*(b_90.^2*gamma/vs $-4 * b \_90 . \wedge 2 * g a m m a / v s . .$. $+2 * b_{-} 90 \cdot{ }^{\wedge} 2 * \exp (-\mathrm{gamma}) *\left(\mathrm{~b}_{-} 90 \cdot{ }^{\wedge} 2 *\left(\mathrm{a} \cdot{ }^{\wedge} 2 * 2 * \mathrm{qe} * \mathrm{q} / \mathrm{c} / \mathrm{mi} / \mathrm{vs} \cdot{ }^{\wedge} 2-\mathrm{bc} \cdot{ }^{\wedge} 2\right) \ldots\right.$ +1 ambda_D . $\left.{ }^{\wedge} 2 *\left(b_{-} 90 \cdot{ }^{\wedge} 2-2 * a \cdot{ }^{\wedge} 2 * q e * q / C / m i / v s \cdot{ }^{\wedge} 2\right)\right) / v s / \ldots$ $\left.\left.\left(\mathrm{bc} \cdot{ }^{\wedge} 2+b_{-} 90 \cdot{ }^{\wedge} 2\right) \cdot \wedge 2\right)\right) ;$

$\div \frac{\circ}{0} d f 2 / d v y:$

$\mathrm{b}(2)=-1+.5 * d t \operatorname{Nwt} *\left(-\mathrm{nu} \mathbf{d}_{-} \mathrm{n}-\right.$ beta_n $*\left(\mathrm{vn}+(\text { vydriftn })^{\wedge} 2 / \mathrm{vn}\right)-\ldots$ beta_ic*vs*bc.^2-beta_io*vs*b_90 . ^2*gamma...

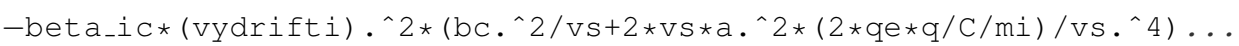
-beta_io*(vydrifti) .`2*(b_90.^2*gamma/vs $-4 * b \_90 \cdot{ }^{\wedge} 2 * g a m m a / v s . .$. $+2 * b_{-} 90 \cdot{ }^{\wedge} 2 * \exp (-g a m m a) *\left(b_{-} 90 \cdot{ }^{\wedge} 2 *\left(a \cdot{ }^{\wedge} 2 * 2 * q e * q / C / m i / v s \cdot{ }^{\wedge} 2-b c \cdot{ }^{\wedge} 2\right) \ldots\right.$

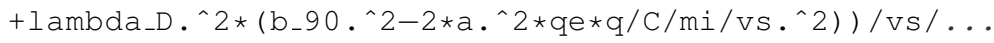
$\left.\left.\left(\mathrm{bcc} \cdot{ }^{\wedge} 2+\mathrm{b}_{-} 90 \cdot{ }^{\wedge} 2\right) \cdot{ }^{\wedge} 2\right)\right)$;

응 "upper" triangular portion of the Jacobian matrix, or df1/dvy: 응 (In tridiagonal terms, this is the "C" vector term) $\therefore d f 1 \_d v y=u t(1)$ ut $(1)=.5 * d t N w t *(q * B 0 / m d+4 * p i /$ corot_period $-\ldots$ beta_n*(vn+(vydriftn $\left.)^{\wedge} 2 / v n\right)-\ldots$ beta_ic*vs*bc.^2-beta_io*vs*b_90 . ^2*gamma...

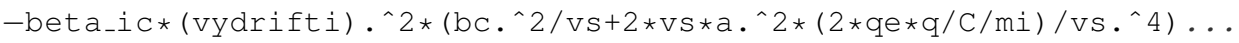
-beta_io*(vydrifti) .^2*(b_90.^2*gamma/vs-4*b_90 .^2*gamma/vs... $+2 * b_{-} 90 \cdot{ }^{\wedge} 2 * \exp (-g a m m a) *\left(b_{-} 90 \cdot{ }^{\wedge} 2 *\left(a \cdot{ }^{\wedge} 2 * 2 * q e * q / C / m i / v s \cdot{ }^{\wedge} 2-b c \cdot{ }^{\wedge} 2\right) \ldots\right.$

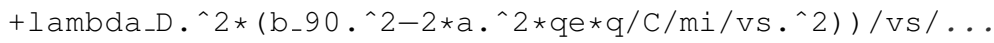
$\left.\left.\left(\mathrm{bc} \cdot{ }^{\wedge} 2+b_{-} 90 \cdot \wedge 2\right) \cdot \wedge 2\right)\right) ;$ 


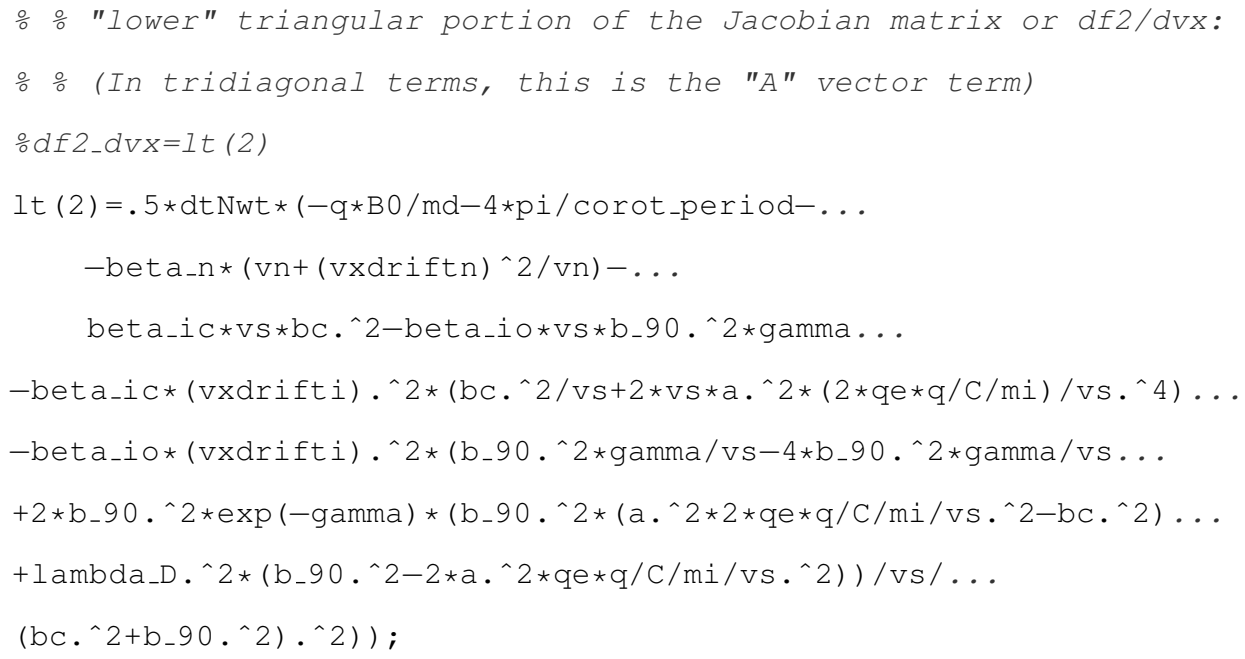


응 norm, or maximum value or the error vector.

err $=\max ($ abs (error)) ;

$\mathrm{vx}=\mathrm{vx}+\operatorname{error}(1)$;

$\mathrm{vy}=\mathrm{vy}+\operatorname{error}(2)$;

응 put this line below to prevent an infinite loop

n_iter=n_iter+1;

$\div 9 / 1.6 e-19$

opause

\section{end}

$\div \frac{\circ}{0}$ velocity of the grain relative to the ions:

$\therefore \frac{\circ}{0} \mathrm{o} .9 .$, if $V X=0$, but the ions are streaming towards the grain in the

$\div \frac{\circ}{0}-x$ direction with velocity vix, then it is equivalent to the grain

응 응 moving at a velocity +vix in the +x-direction.

$\frac{\circ}{0} \mathrm{w}=\operatorname{sqrt}\left((\mathrm{vx}-\mathrm{vix}) \cdot{ }^{\wedge} 2+(v y-v i y) \cdot{ }^{\wedge} 2\right) ;$

응 ompute grain speed relative to an electron flow:

응 we=sqrt $\left((\right.$ vx-vex $\left.) \cdot{ }^{\wedge} 2+(v y-v e y) \cdot{ }^{\wedge} 2\right)$;

응 ompute grain speed relative to an ion flow:

응 $\frac{0}{0}$ vecity of the grain relative to the ions:

$\div \frac{\circ}{0} \mathrm{o} .9 .$, if $V X=0$, but the ions are streaming towards the grain in the

$\div \frac{\circ}{0}-x$ direction with velocity vix, then it is equivalent to the grain

$\therefore \frac{\circ}{0}$ moving at a velocity +vix in the +x-direction.

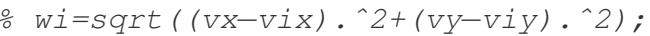

$\div \frac{\circ}{0}$ make a w-vector; the first element is the grain speed relative to

$\therefore \div \frac{\circ}{0}$ electron flow, the second is the grain speed relative to ion flow.

$\therefore w=\left[\begin{array}{ll}\text { we } & \text { wi }]\end{array}\right.$;

음 compute grain speed relative to an electron flow. Do these need to be

$\div$ 응 adusted if we are in a corotating frame?

we=sqrt $(($ vx-vex $) \cdot \wedge 2+($ vy-vey $) \cdot \wedge 2) ;$

응 compute grain speed relative to an ion flow:

응 velocity of the grain relative to the ions:

$\frac{\circ}{\circ}$ e.g., if $V x=0$, but the ions are streaming towards the grain in the

응 $-x$ direction with velocity vix, then it is equivalent to the grain

응 moving at a velocity +vix in the +x-direction.

wi=sqrt $\left((v x-v i x) \cdot{ }^{\wedge} 2+(v y-v i y) \cdot \wedge 2\right)$; 


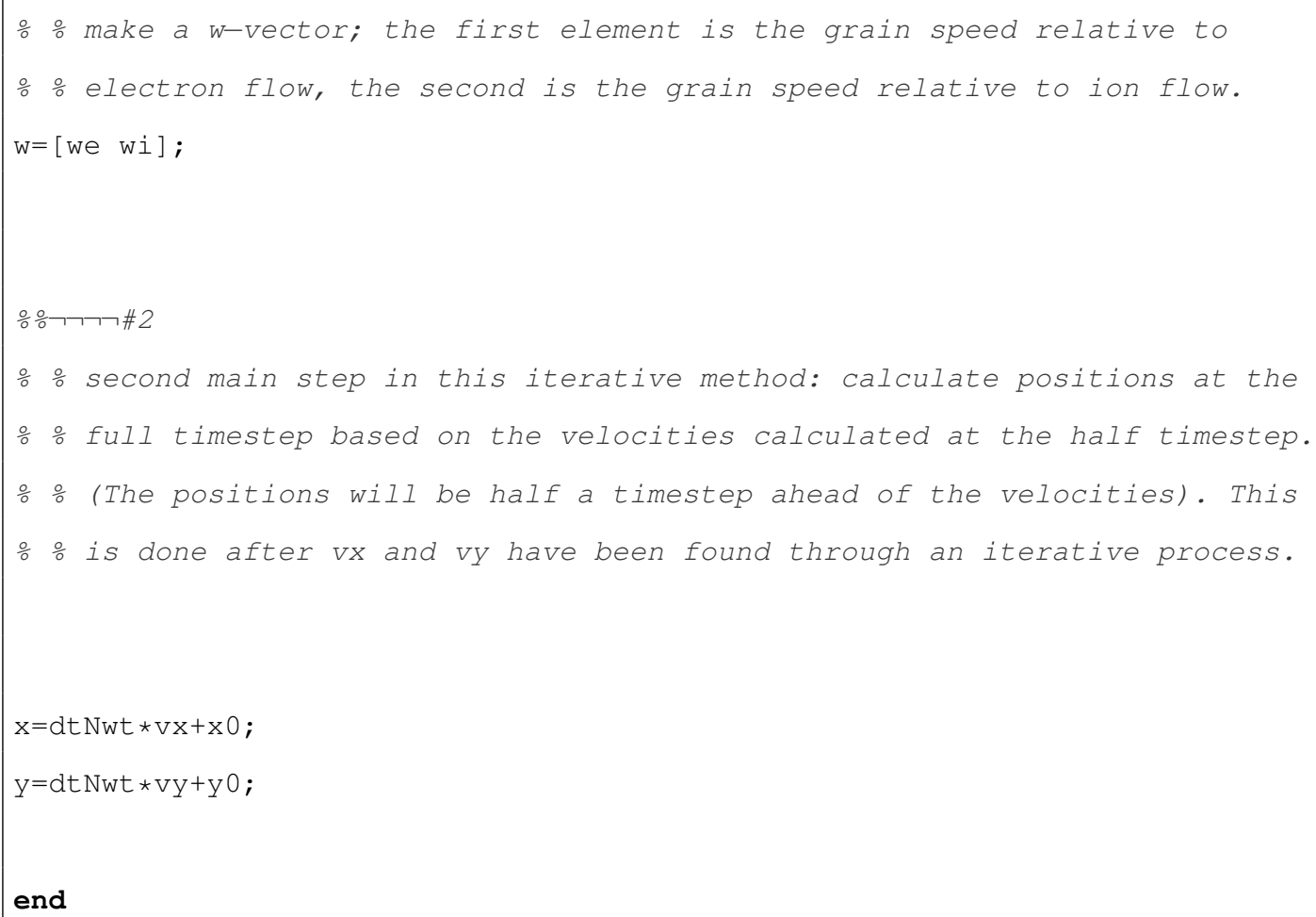

\section{iii Abrupt Inhomogeneity Theory Code}

The theory code for producing one gyro-orbit in an abrupt inhomogeneity, abrupt_omega_theory.m is included here. For a description of the basic theory behind this code, see sections A and B. The process is described there. The final position of the grain in $x, y$ coordinate space can be used as inputs to the abrupt_omega_theory.m function in order to produce multiple gyro-orbits. This function can be used to find the guiding center drifts over a wide range of plasma parameters and conditions for the abrupt inhomogeneity. This routine requires dimensionless_charger.m, which is described later in 4 .

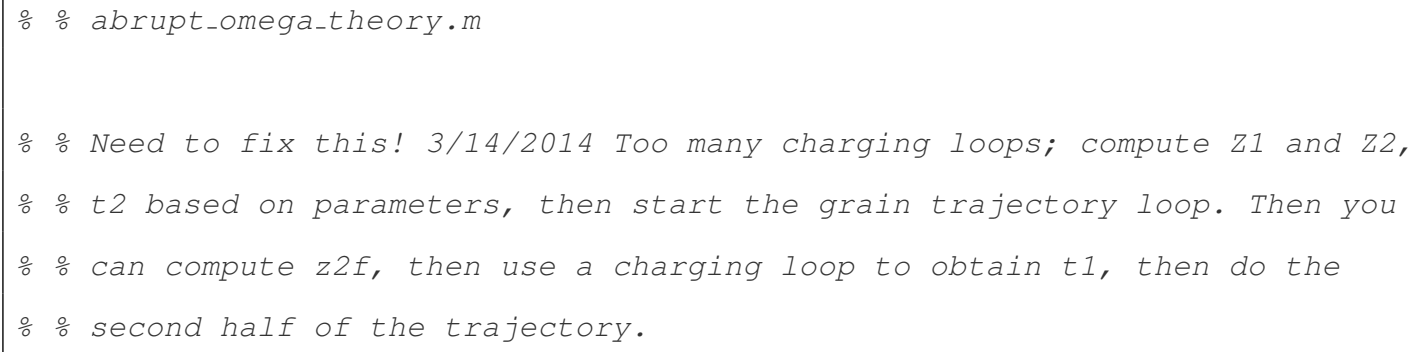




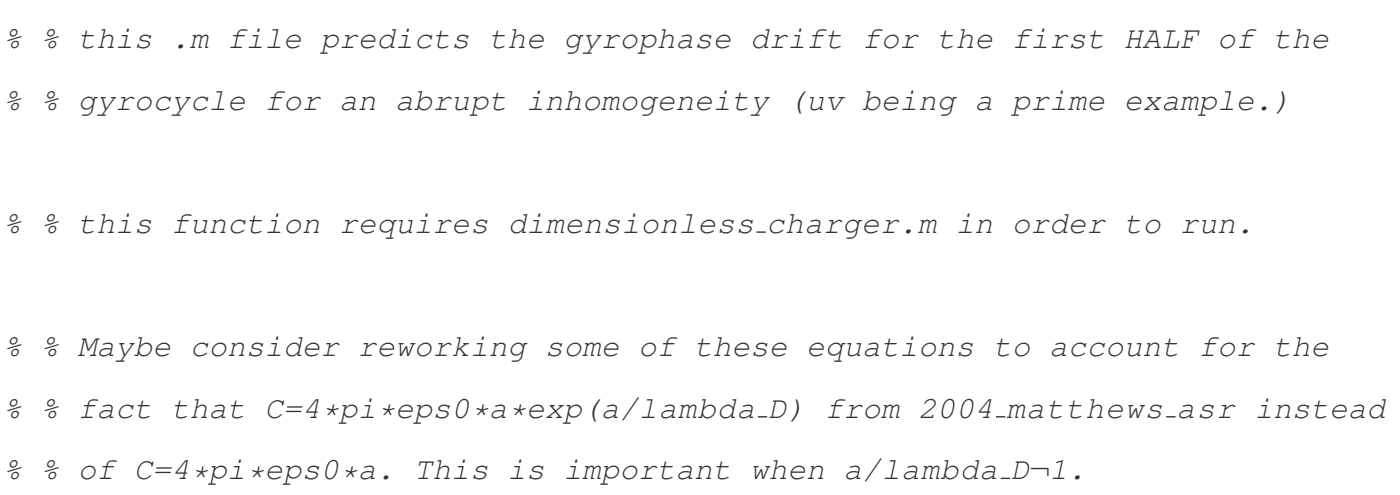




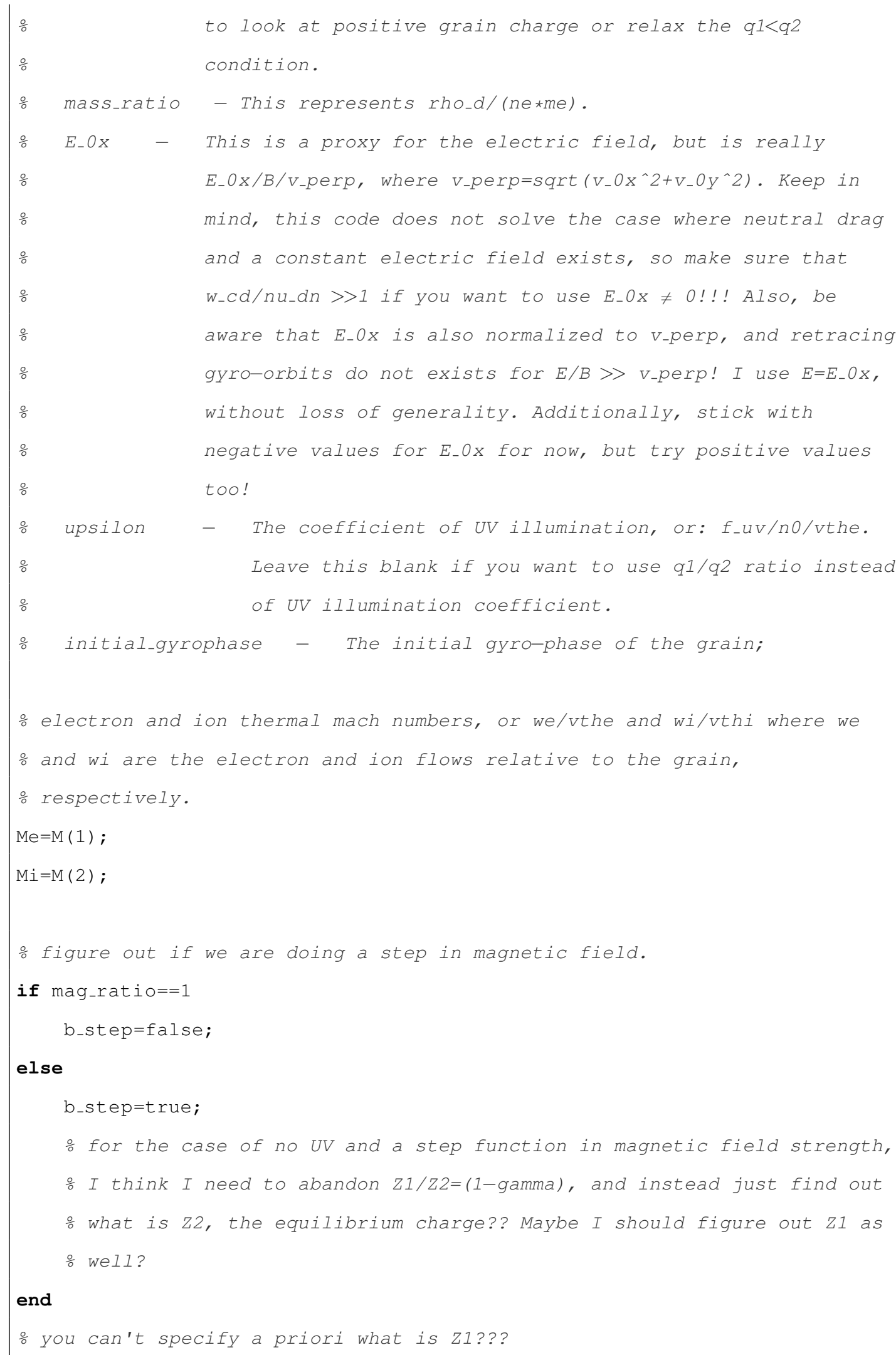




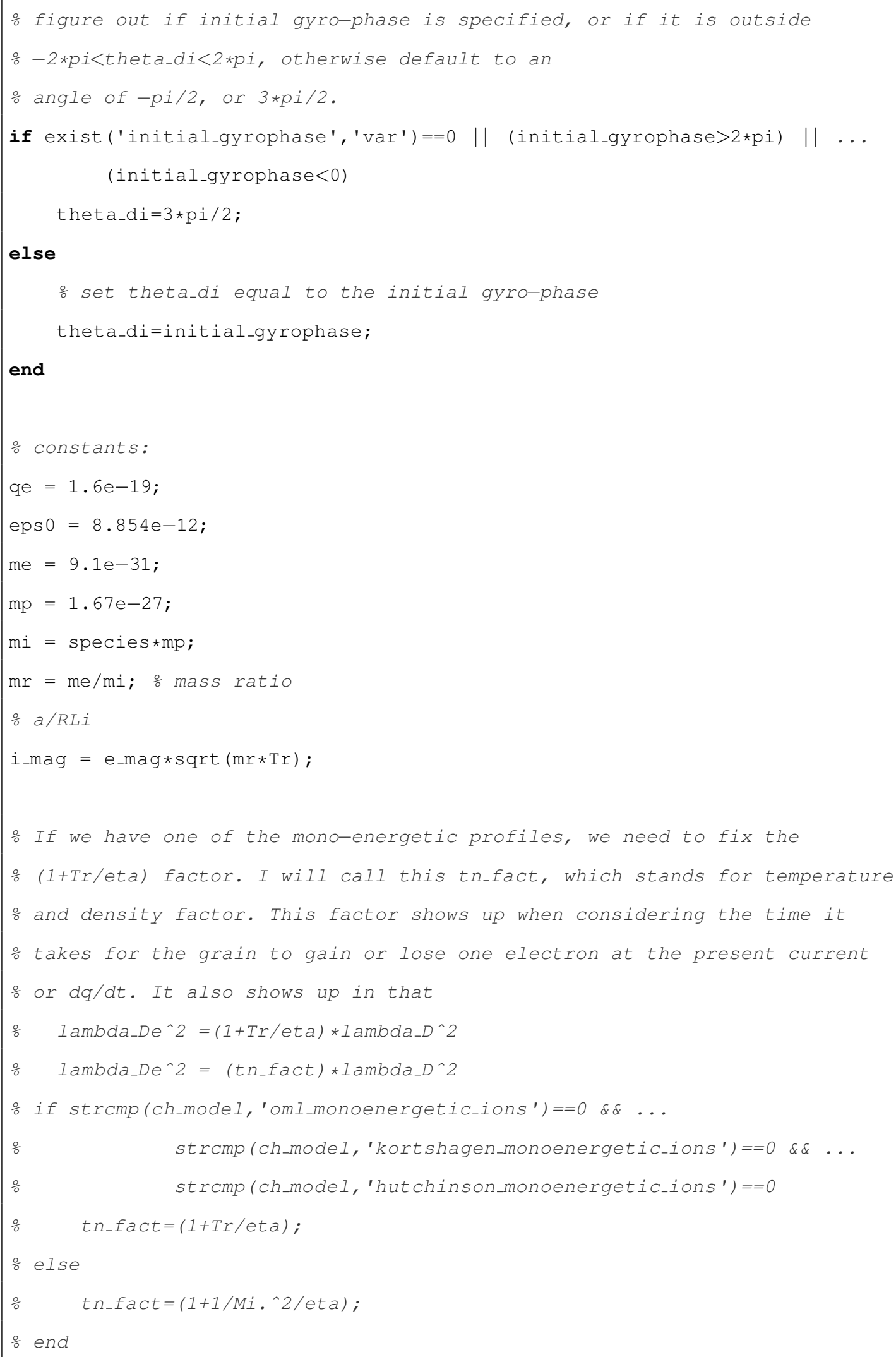




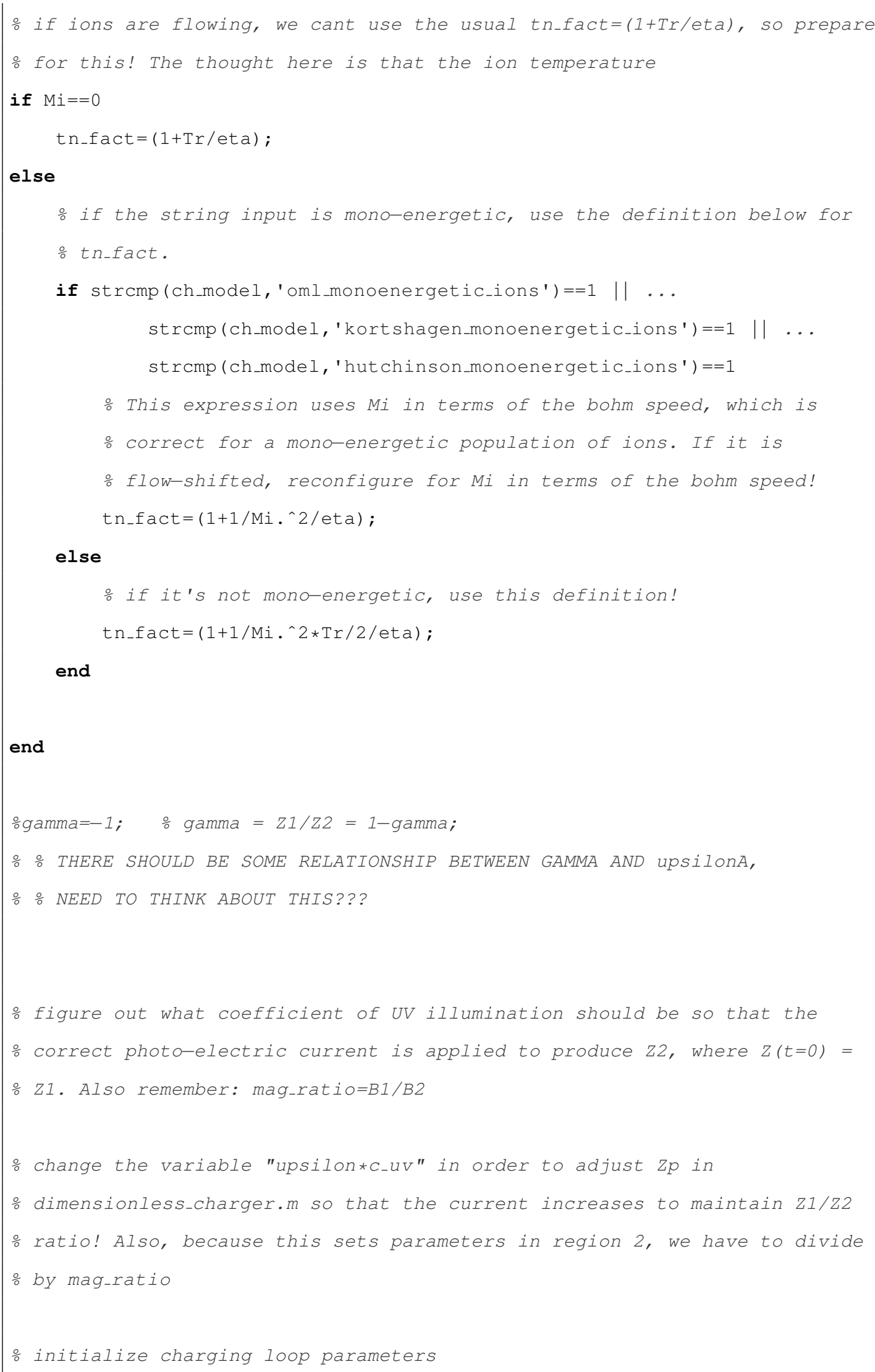




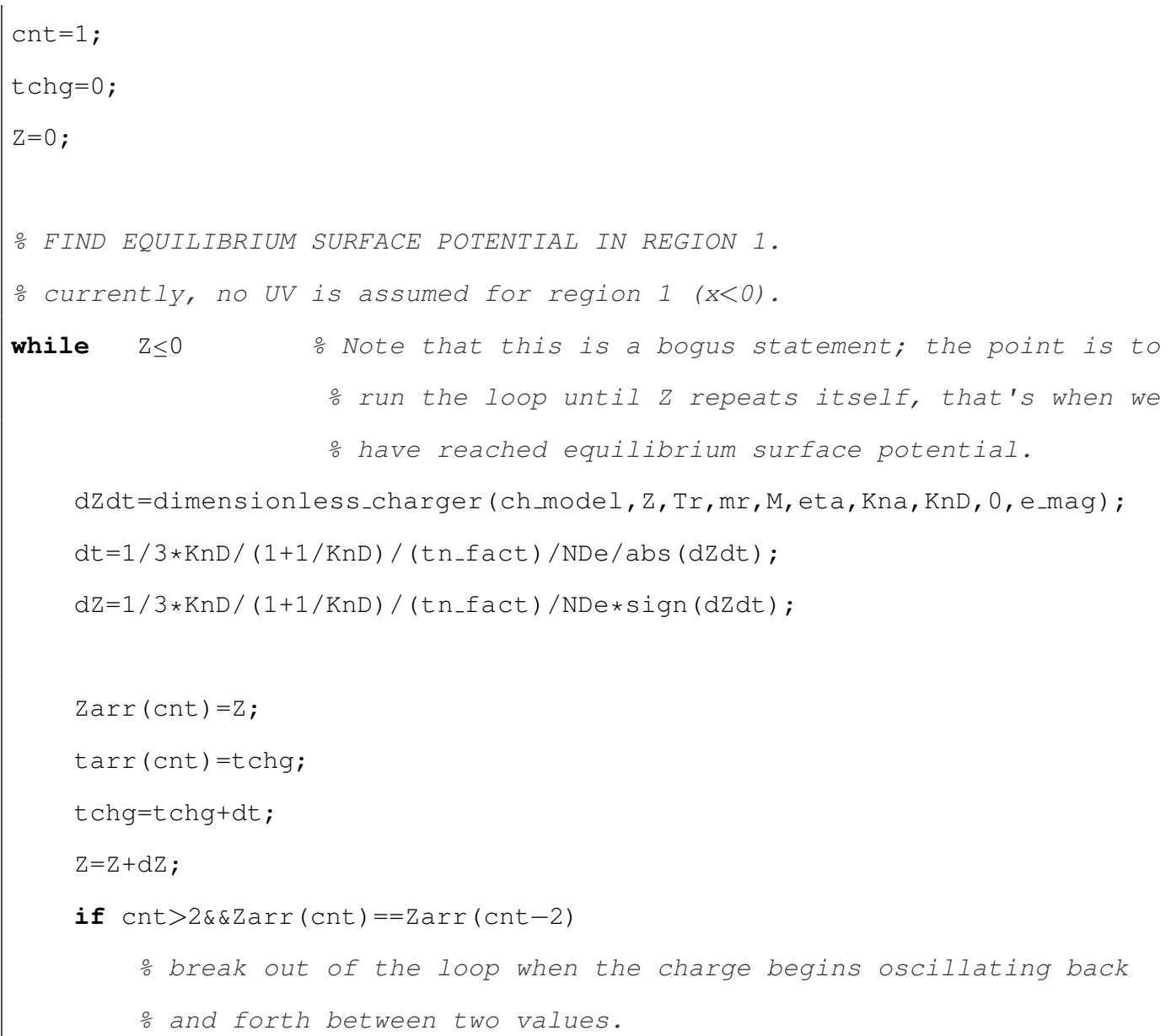




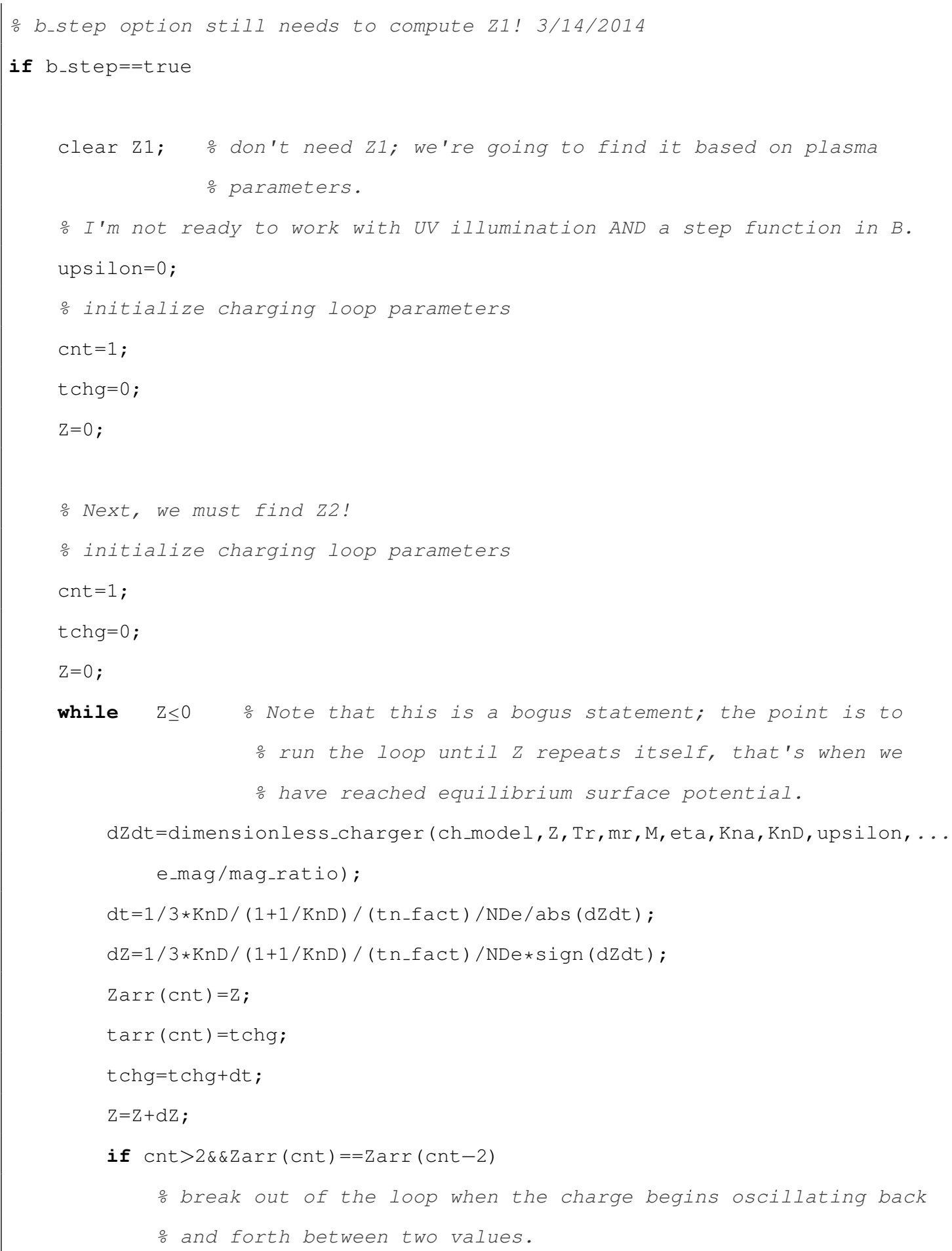




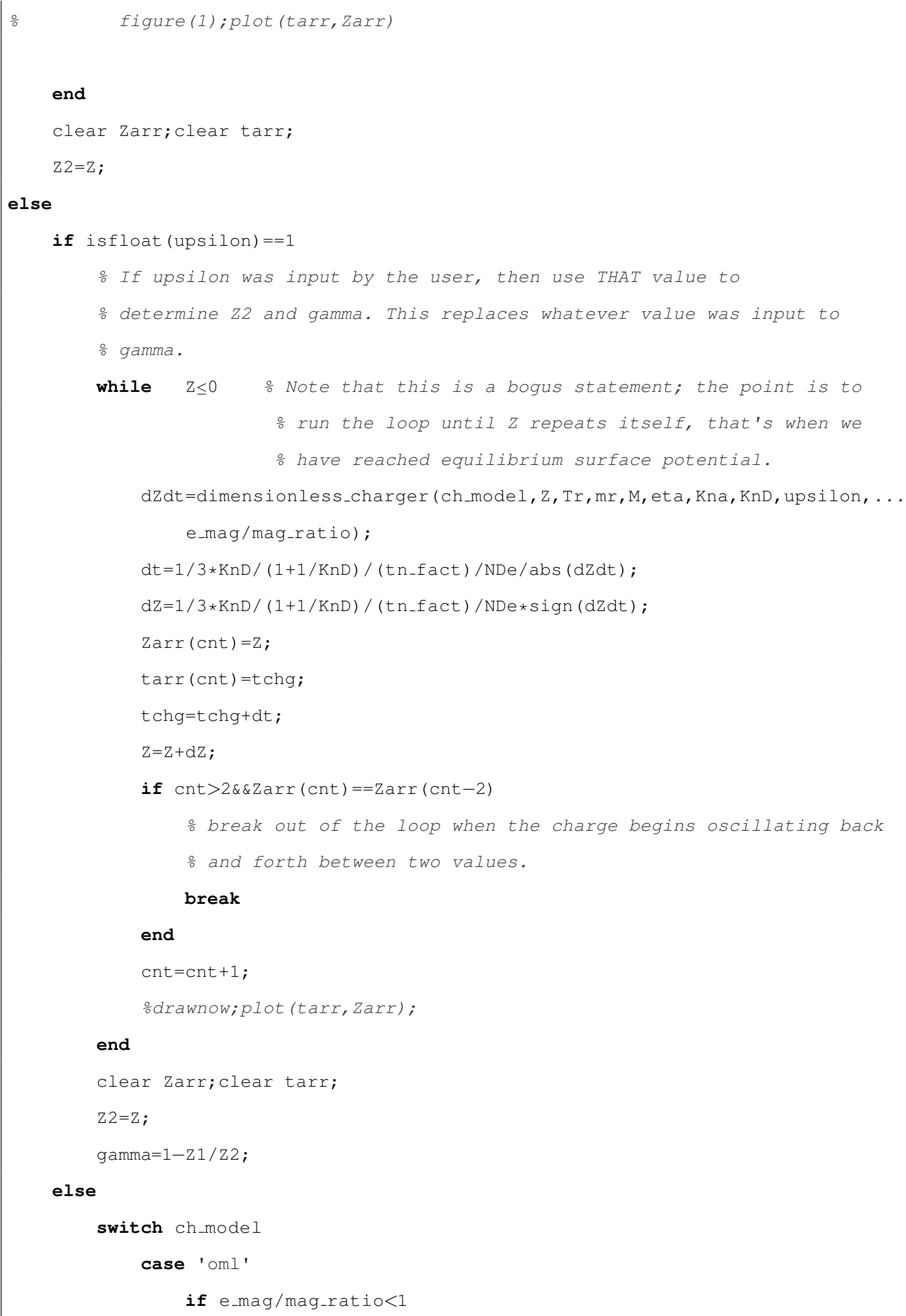


if i_mag/mag_ratio<1

응 for model ONLY!!!

$\therefore C_{-} U V=1$

upsilon=2/sqrt (pi) *exp ( 22$)-2 / \operatorname{sqrt}(p i) * \operatorname{sqrt}(\operatorname{mr} / \operatorname{Tr}) \ldots$

leta*(1-Tr*Z2);

ouv_coeff = upsilona*C_uv!!! so it includes the

illumination factor

ouv_coeff $=4 * \exp (Z 2)-4 * \operatorname{sqrt}(\operatorname{mr} / \operatorname{Tr}) * e t a *(1-\operatorname{Tr} * Z 2)$;

else

upsilon $=2 / \operatorname{sqrt}(\mathrm{pi}) * \exp (\mathrm{z} 2)-1 / \operatorname{sqrt}(\mathrm{pi}) * \operatorname{sqrt}(\mathrm{mr} / \mathrm{Tr}) /$ eta;

end

else

if i_mag/mag_ratio<1

o for omI model ONLY!!!

Factor of $1 / 2$ in front of ion current term.

$\therefore \quad C_{-} U V=1$

upsilon $=1 / \operatorname{sqrt}(p i) * \exp (z 2)-2 / \operatorname{sqrt}(p i) * \operatorname{sqrt}(\operatorname{mr} / \operatorname{Tr}) \ldots$

/eta* $(1-\operatorname{Tr} * \mathrm{Z} 2)$;

uv_coeff = upsilona*C_uv!!! so it includes the illumination

ㅇaㅇ

ouv_coeff $=4 * \exp (Z 2)-4 * \operatorname{sqrt}(\operatorname{mr} / \operatorname{Tr}) * \operatorname{eta} *(1-\operatorname{Tr} * Z 2)$;

else

upsilon $=1 / \operatorname{sqrt}(\mathrm{pi}) * \exp (\mathrm{z} 2)-1 / \operatorname{sqrt}(\mathrm{pi}) * \operatorname{sqrt}(\mathrm{mr} / \mathrm{Tr}) /$ eta; end

end

$\therefore C_{-} U V=2$

oupsilona $=2 \star \exp (Z 2)-2 * \operatorname{sqrt}(\mathrm{mr} / \operatorname{Tr}) * \operatorname{eta} a(1-\operatorname{Tr} * Z 2)$

case 'oml_monoenergetic_ions'

응 fill this out!!!

case 'kortshagen'

- must compute Knudsen capture radius parameter based on the

o dimensionless grain potential, $z 2$ (so that we can derive the

\% necessary photo-current to produce equilbrium grain potential

$\div \quad 22)$ 


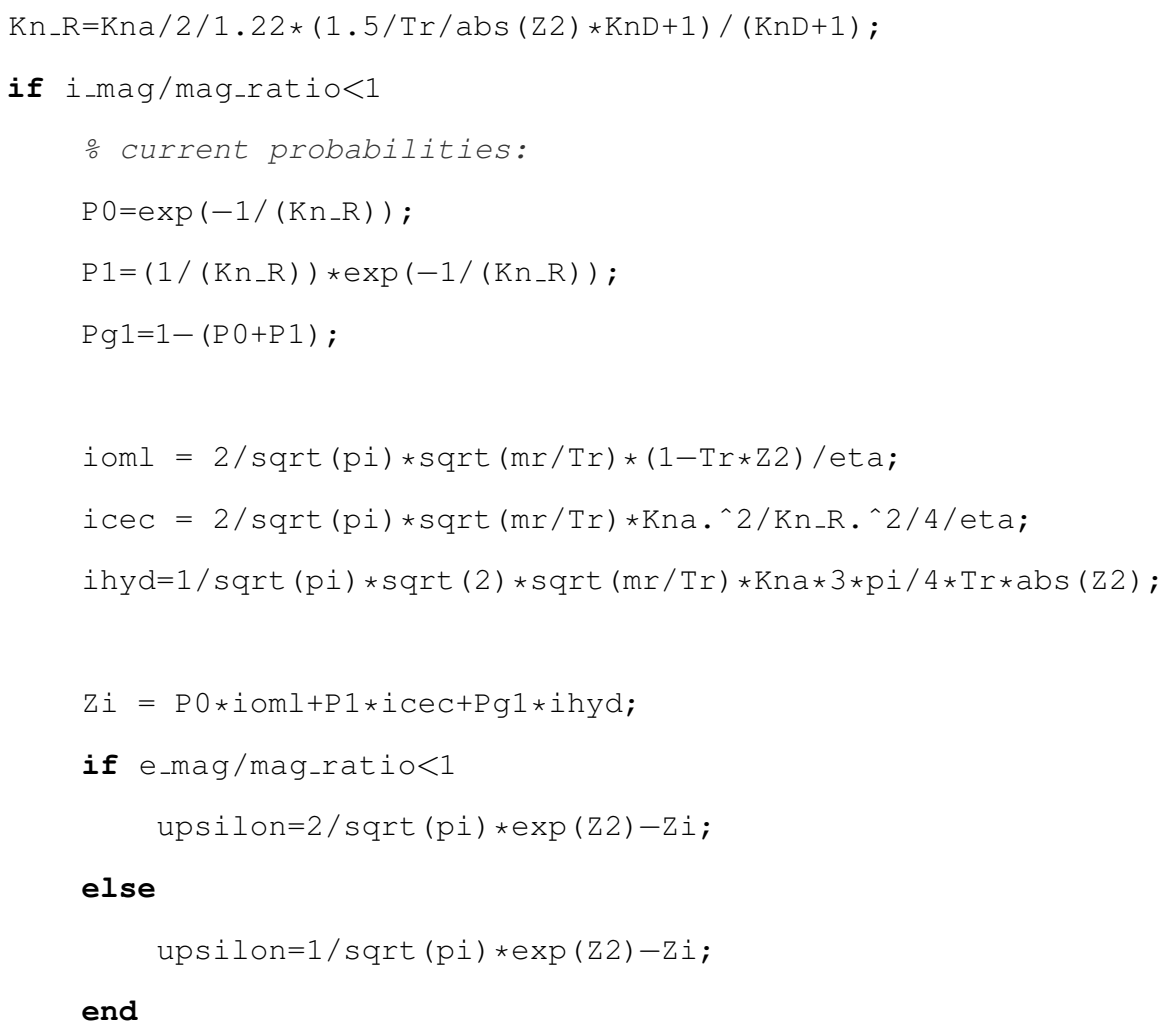

else

if e_mag/mag_ratio $<1$

$\mathrm{Zi}=1 / \operatorname{sqrt}(\mathrm{pi}) * \operatorname{sqrt}(\mathrm{mr} / \mathrm{Tr}) *(1-\mathrm{Tr} * \mathrm{Z} 2) /$ eta;

upsilon=2/sqrt (pi) *exp ( 22$)-z i$;

else

$\mathrm{Zi}=1 / \operatorname{sqrt}(\mathrm{pi}) * \operatorname{sqrt}(\mathrm{mr} / \mathrm{Tr}) *(1-\mathrm{Tr} * \mathrm{Z} 2) / \mathrm{eta} ;$

upsilon=1/sqrt (pi) *exp (Z2)-Zi;

end

end

case 'kortshagen_monoenergetic_ions'

일 this out!

case 'hutchinson'

- put hutchinson case in here.

z=e_mag/mag_ratio/(1+e_mag/mag_ratio) ; 
o iota* in Patacchini and Hutchinson:

iot $a=1-0.0946 * z-0.305 * z \cdot{ }^{\wedge} 2+0.95 * z \cdot{ }^{\wedge} 3-2.2 * z \cdot{ }^{\wedge} 4+1.15 * z \cdot{ }^{\wedge} 5$;

응 FOR LAMBDA_D = FINITE, AND DEBYE-HUCKEL POTENTIAL:

o eta, which is now dependent on grain sheath size:

eta_mag=-Z2/e_mag/mag_ratio*...

$(1+$ e_mag $/ 4 *(1-\exp (-4 / \mathrm{KnD} /$ e_mag/mag_ratio $)))$;

응 which is eta/(1+eta):

w_mag=eta_mag/(1+eta_mag) ;

$\therefore A$, the fitting polynomial, a function of $\mathrm{w}$ :

A_fit $=0.678 * w \_m a g+1.543 * w \_m a g \cdot{ }^{\wedge} 2-1.212 * w \_m a g \cdot ` 3 ;$

응 Ie*, which is the empirical formula for electron current as a

응 function of magnetization

$\div Z e=-\operatorname{sqrt}(2 * p i) / K n D * \exp (Z) *\left(A_{-} f i t+\left(1-A_{-} f i t\right) * i o t a\right) ;$

: Patacchini-Hutchinson model can include the spatial

\% dependence of electron flux to the sphere. I have chosen not

to put this in at the current time, since it is not necessary

o for determining the total grain charge.

if i_mag/mag_ratio $<1$

upsilon=2/sqrt $(p i) * \exp (Z 2) *\left(A_{-} f i t+\left(1-A_{-} f i t\right) * i o t a\right)-\ldots$

$2 / \operatorname{sqrt}(p i) * \operatorname{sqrt}(\mathrm{mr} / \mathrm{Tr}) / e t a *(1-\operatorname{Tr} * \mathrm{z} 2)$;

else

upsilon=1/sqrt (pi) $* \exp (\mathrm{Z} 2) *\left(\mathrm{~A}_{-} \mathrm{fit}+\left(1-\mathrm{A}_{-} \mathrm{fit}\right) *\right.$ iota $)-\ldots$

$2 * \operatorname{sqrt}(\mathrm{mr} / \operatorname{Tr}) / \operatorname{eta} *(1-\operatorname{Tr} * \mathrm{Z} 2)$;

end

case 'hutchinson_monoenergetic_ions'

응 fil this out!

end

o determine 22, for redundancy purposes.

o initialize charging loop parameters

$\mathrm{cnt}=1$;

tchg $=0$;

$\mathrm{Z}=0$ ； 


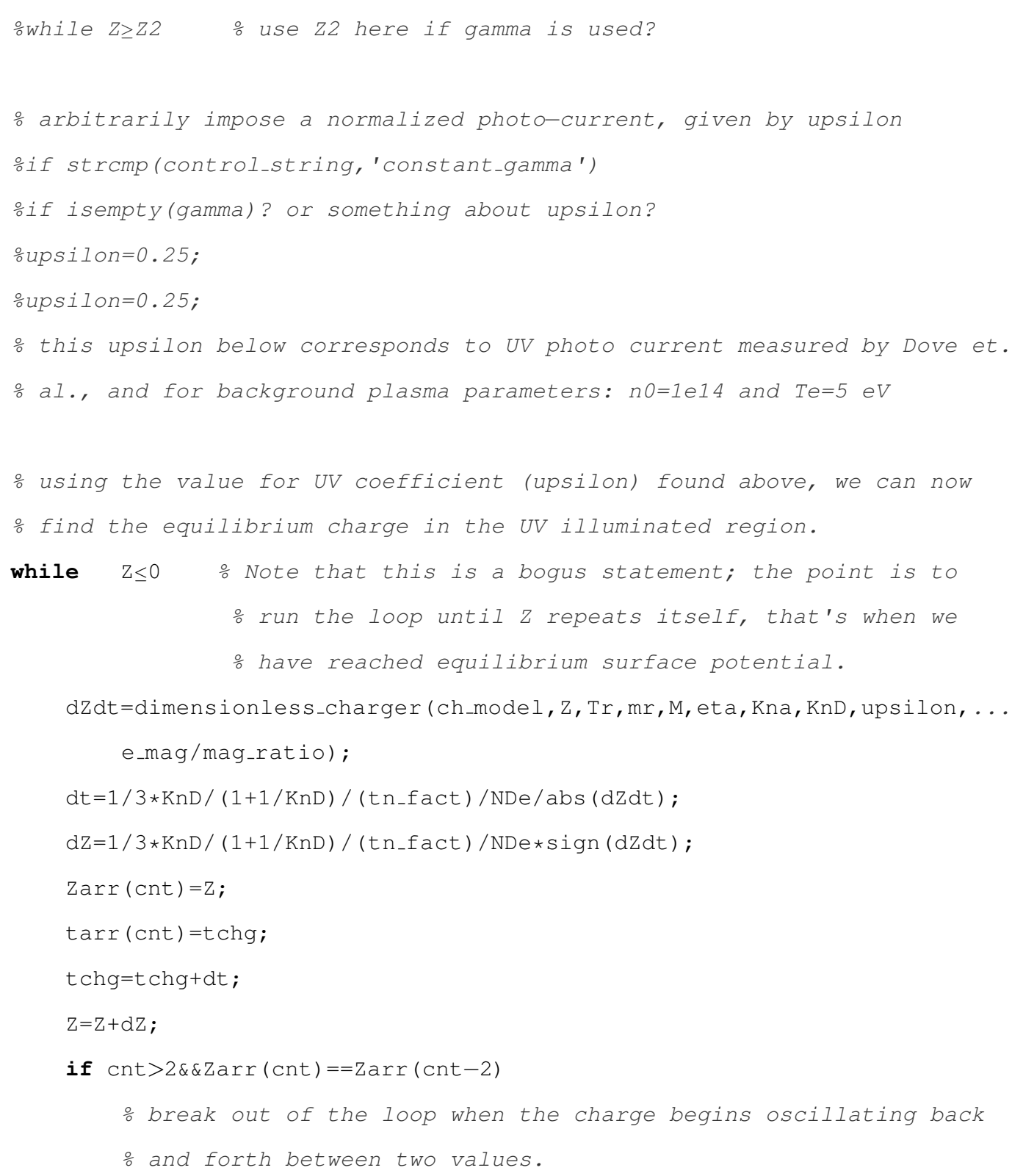

\section{break}

end

$\mathrm{cnt}=\mathrm{cnt}+1$

odrawnow;plot (tarr, Zarr);

end

clear Zarr;clear tarr;

$\mathrm{Z} 2=\mathrm{Z} ;$

gamma=1-Z1/Z2 ;

end

end 


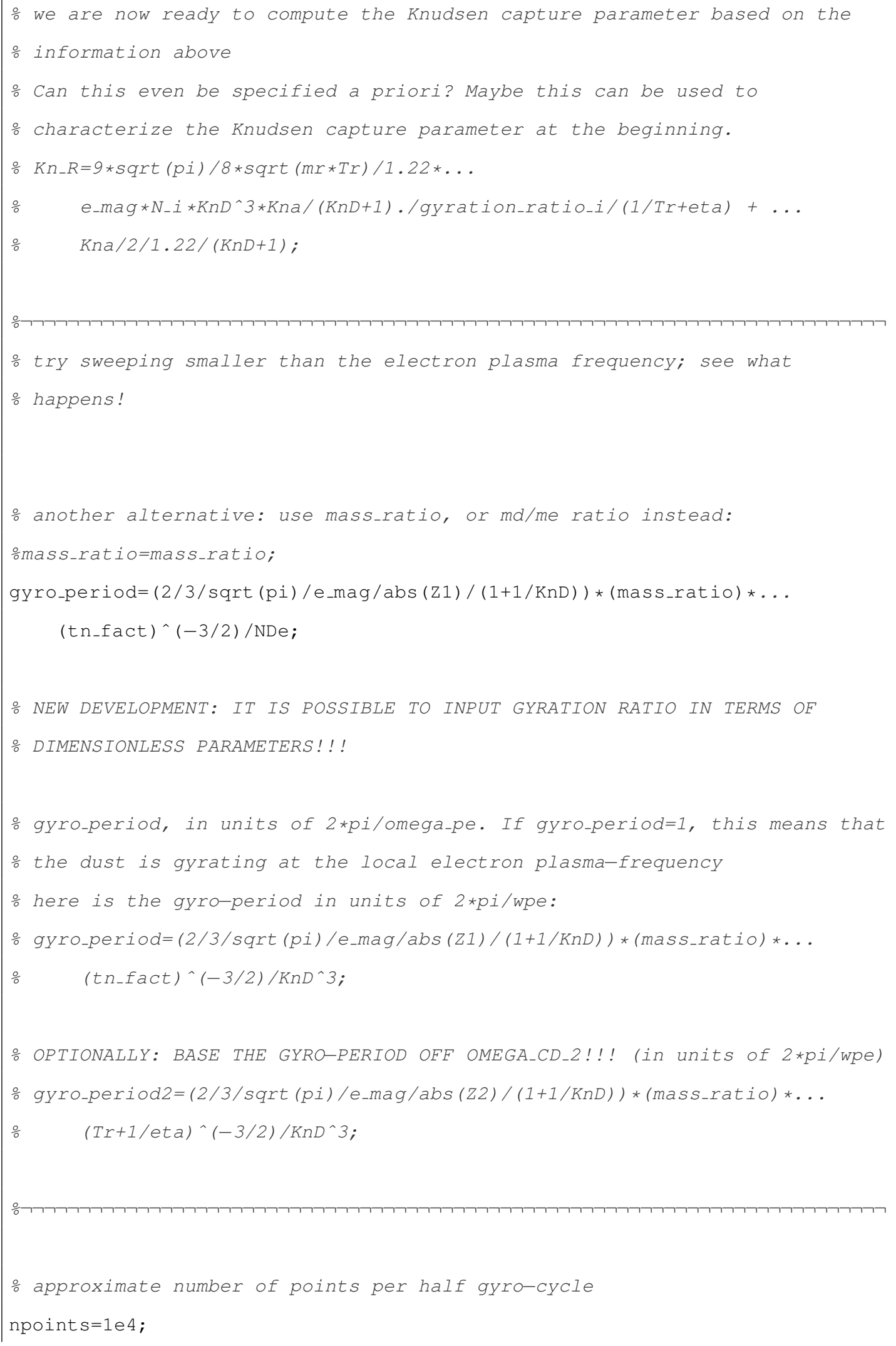




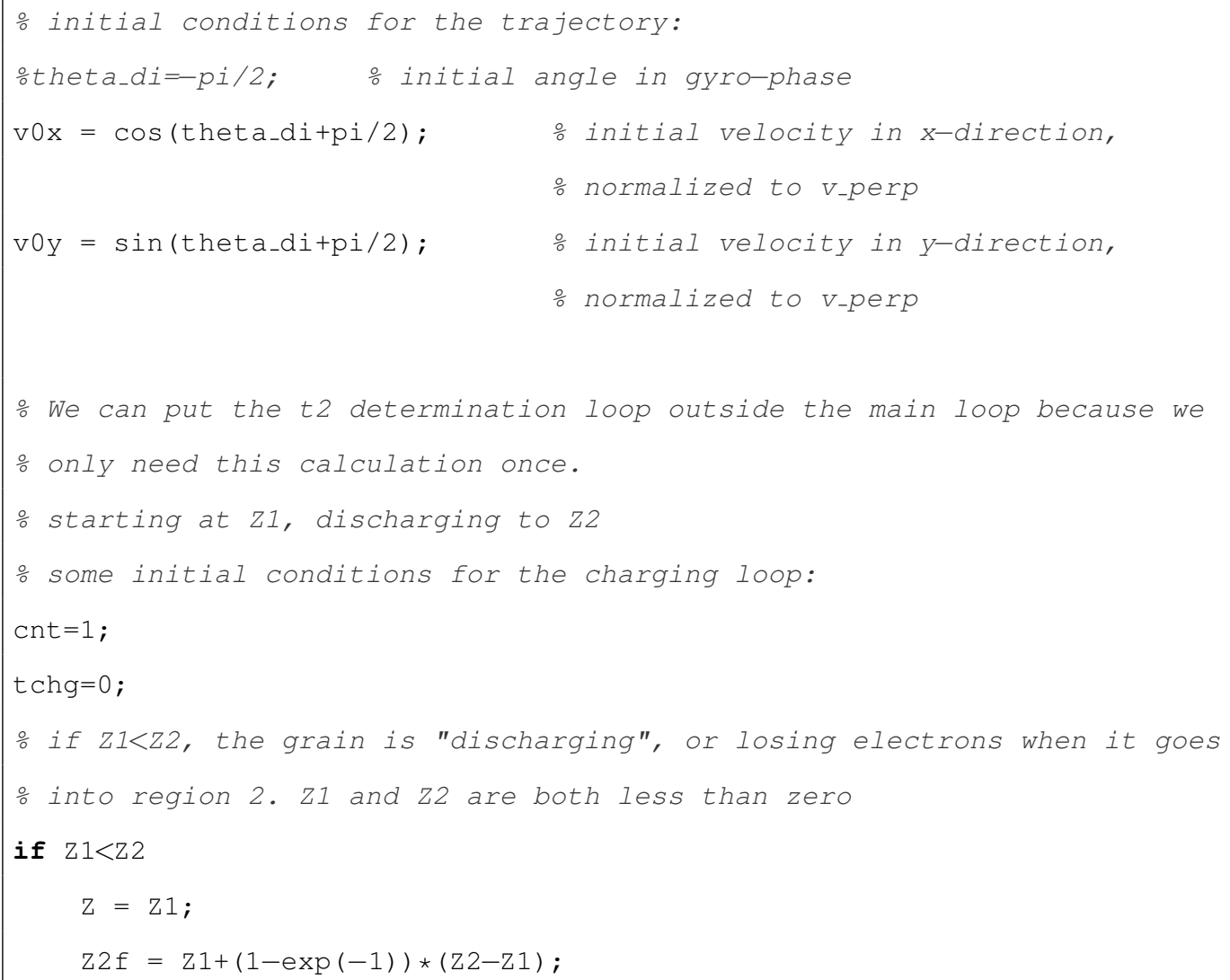




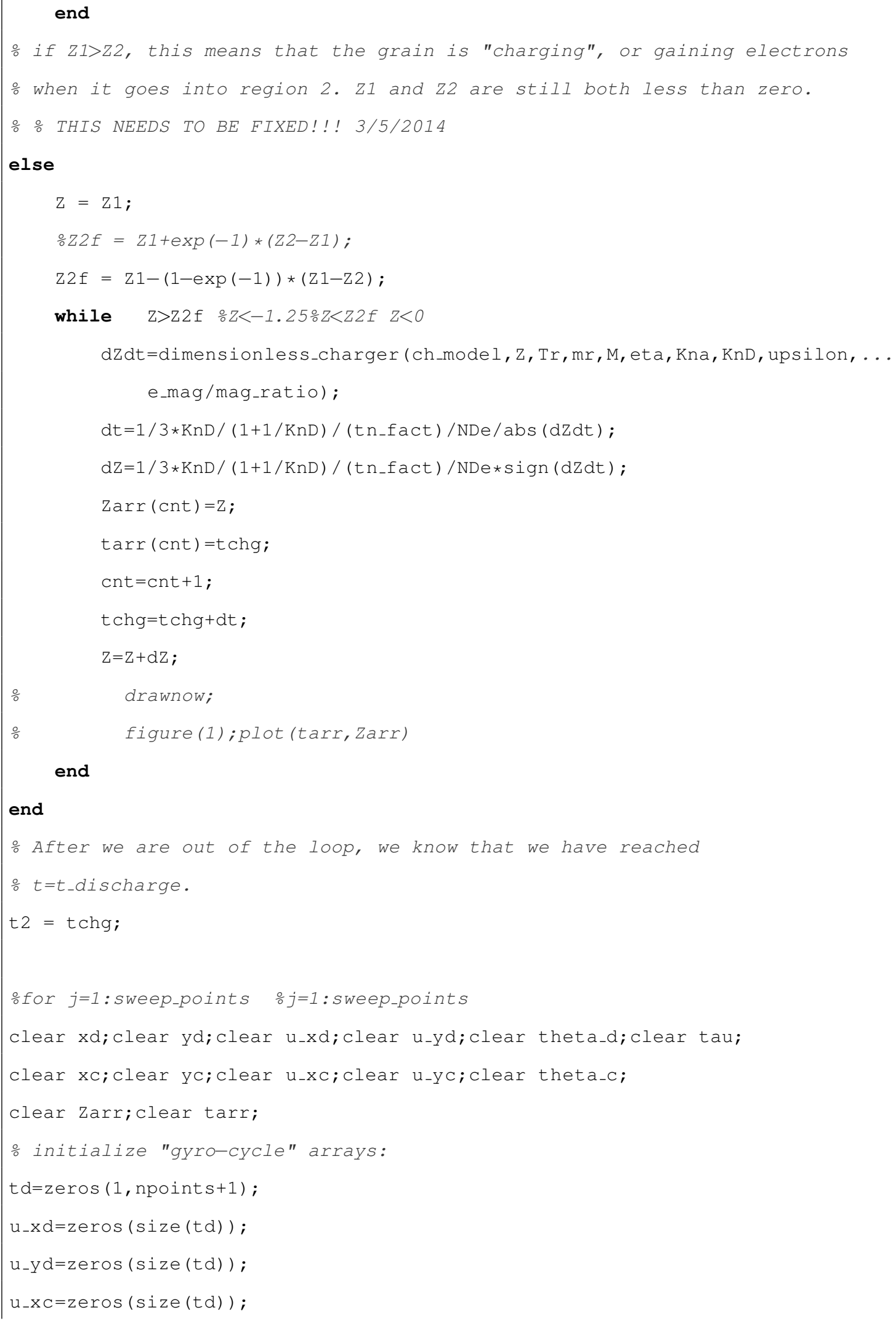




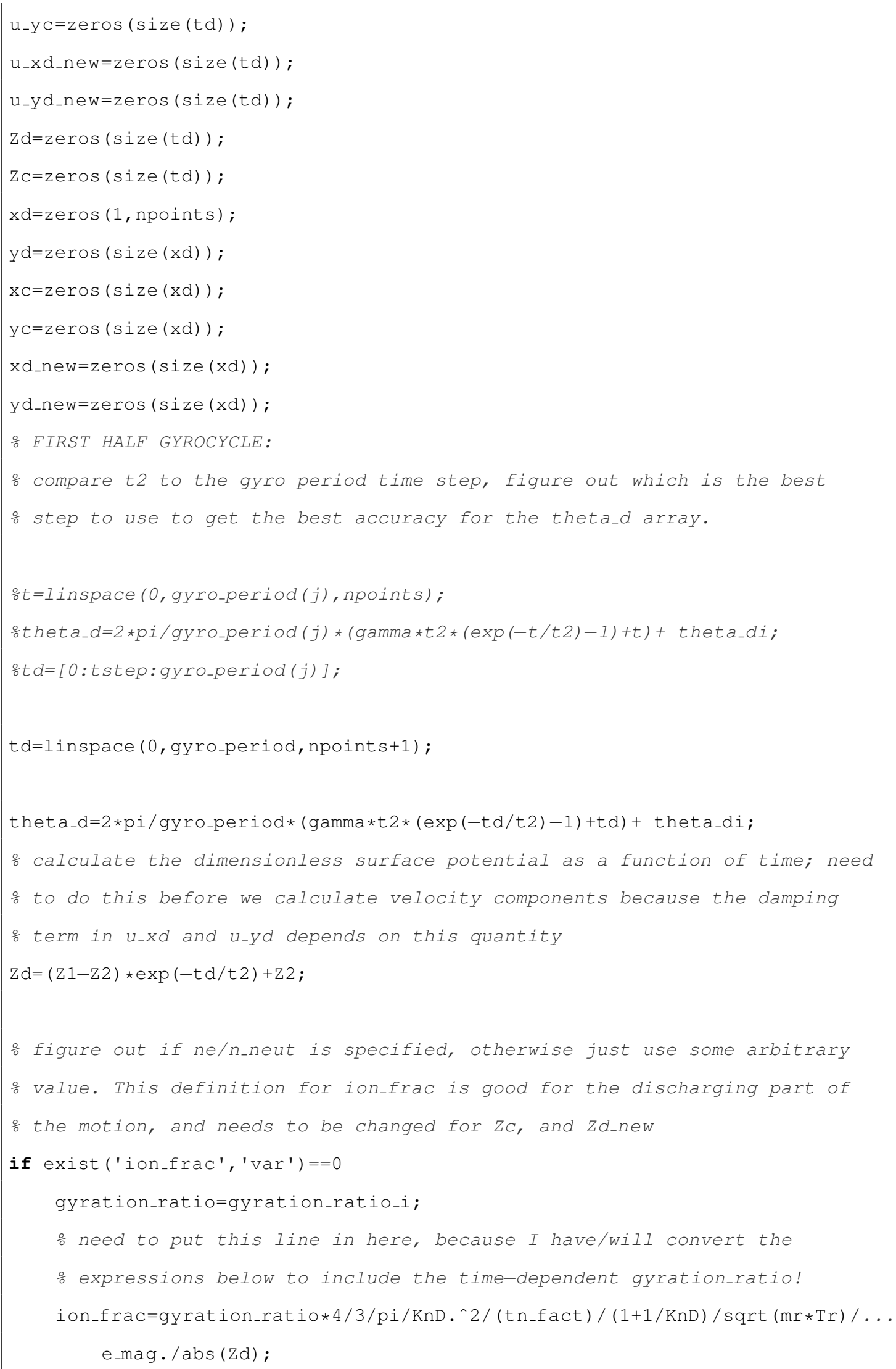


const_gyration_ratio=true;

\section{else}

const_gyration_ratio=false;

\section{end}

- Compute dimensionless (normalized by v-perp) velocities. These equations

o look a little complicated right now because the damping terms depend on

응 dimensionless surface potential

$u_{-} x d=v 0 x * \cos ($ theta_d-theta_di) .*...

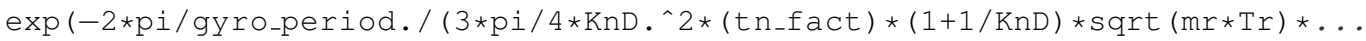
e_mag $\left.\left.\star a b s(Z d) . * i o n \_f r a c\right) . * t d\right)-\ldots$

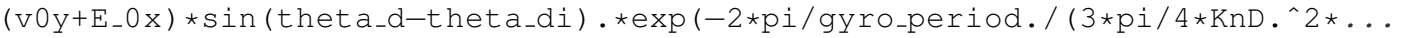
$\left.\left.\left(t n_{-} f a c t\right) *(1+1 / K n D) * \operatorname{sqrt}(m r * T r) * e \_m a g * a b s(Z d) . * i o n_{-} f r a c\right) . * t d\right)$;

$u_{-} y d=v 0 x * \sin ($ theta_d-theta_di).*..

$\exp (-2 * \mathrm{pi} /$ gyro_period./(3*pi/4*KnD.^ $2 *($ tn_fact $) *(1+1 / \mathrm{KnD}) * \operatorname{sqrt}(\operatorname{mr} * \operatorname{Tr}) * \ldots$ $e_{-} \operatorname{mag} * a b s(Z d)$. *ion_frac) . *td) $-E_{-} 0 x+\ldots$

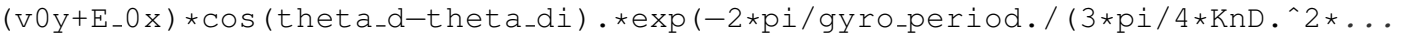
$\left.\left.\left(t n_{-} f a c t\right) *(1+1 / K n D) * \operatorname{sqrt}(m r * T r) * e_{-} m a g * a b s(Z d) . * i o n_{-} f r a c\right) . * t d\right)$;

음 initial conditions for position; just start at $x=0$ and $y=0$, most

응 sensible way to start.

$\mathrm{xd}(1)=0$;

$\operatorname{yd}(1)=0$;

$\div x$ and $y$ are in units of v-perp*gyro_period/2/pi

yd $(1)=-2$ *gyro_period $(j) / 2 / p i$;

$\therefore$ olot (td, theta_d)

o use while loop instead??? This gaurantees that the last element of $x d$

is at $x=0$ for the "non-drag" case, and also permits high drag

coefficients!

을 need to initialize counter

$i=1$

$\circ i=$ length $(t d)-2$;

oclear $x d$; clear $y d$;

\% does not appear to be breaking out of the loop correctly. 3/5/2014

- This problem appears to have been fixed.

while $x d(i) \geq 0 \quad \& \& \quad i \leq l$ ength $(t d)-1$ 


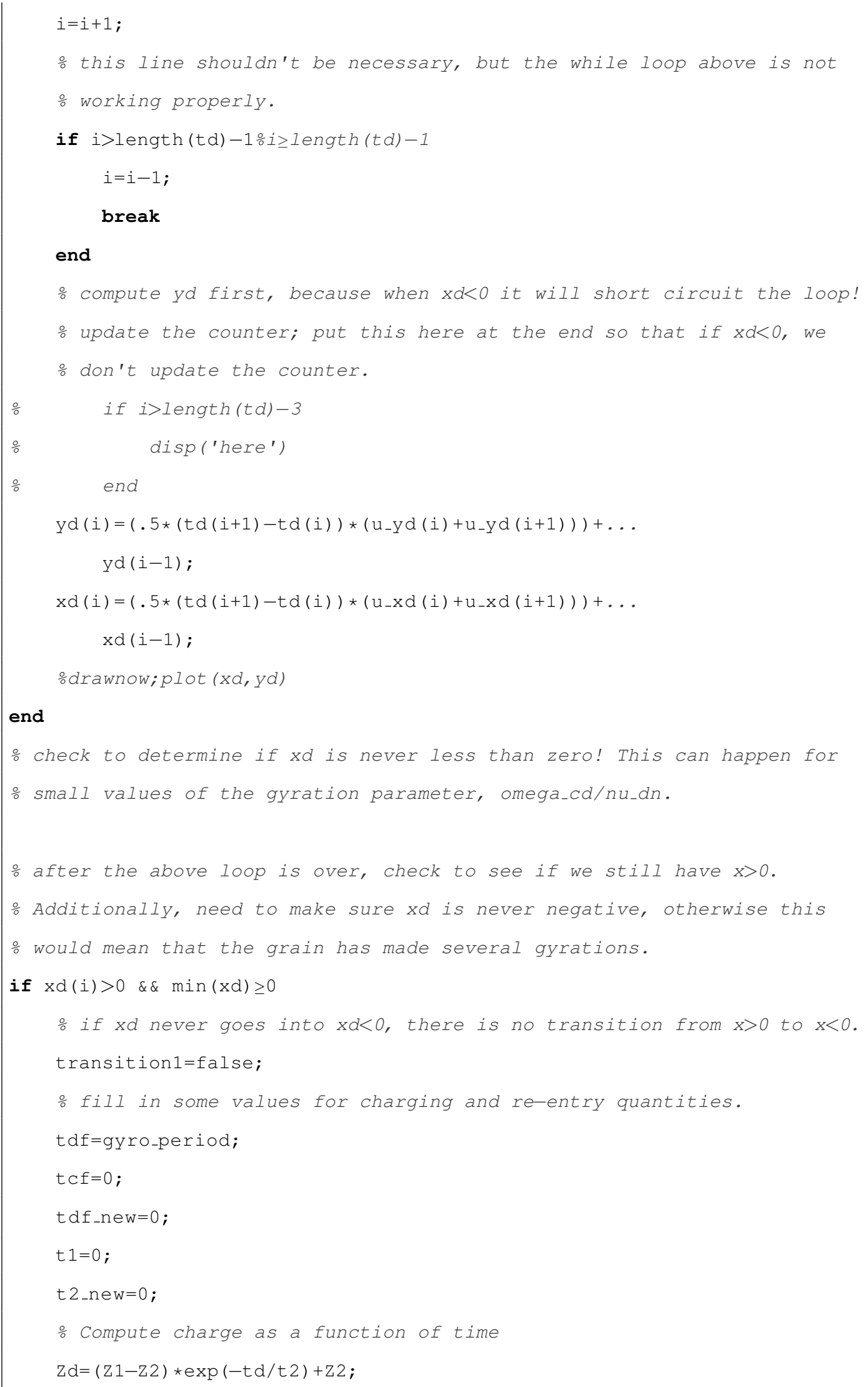




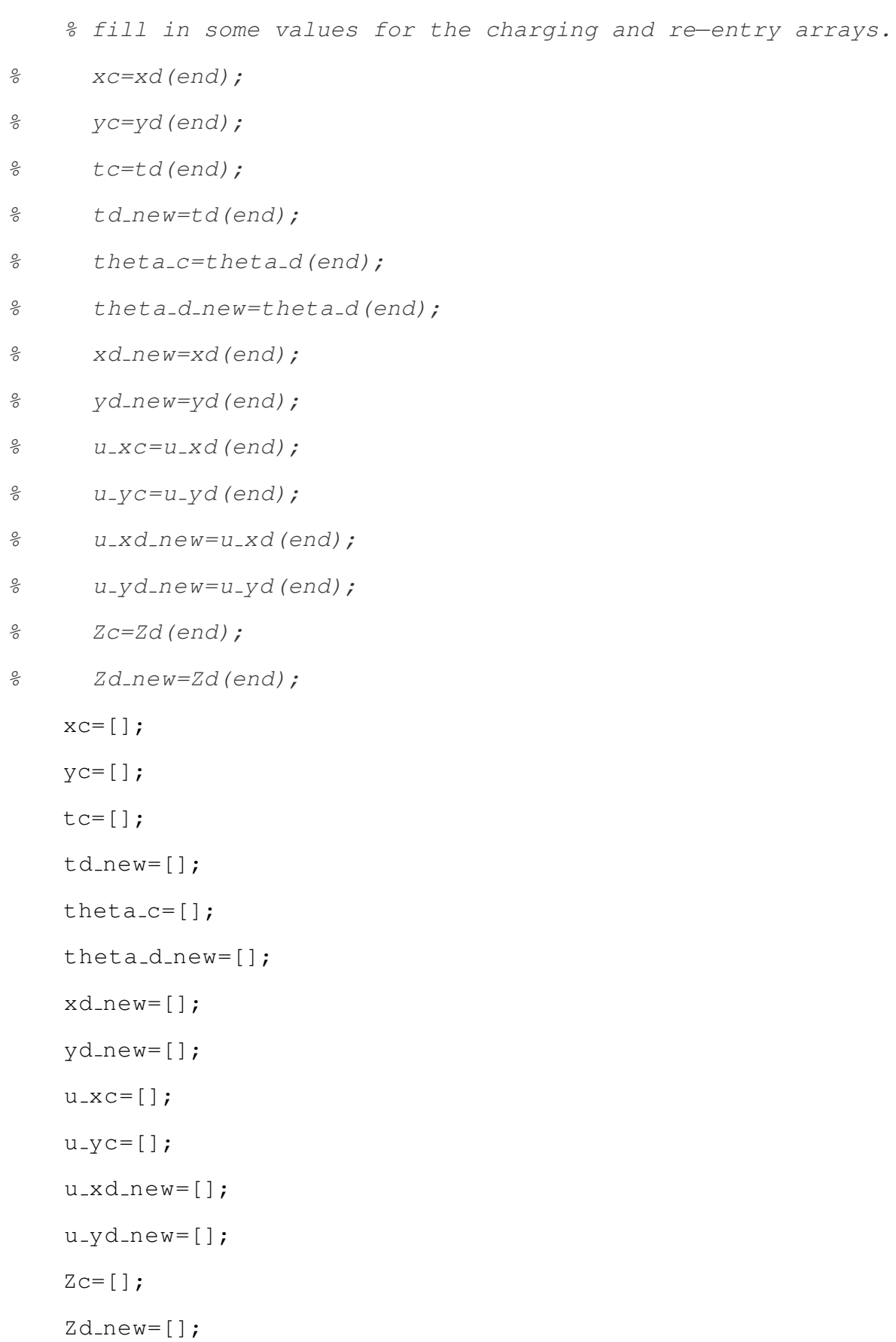




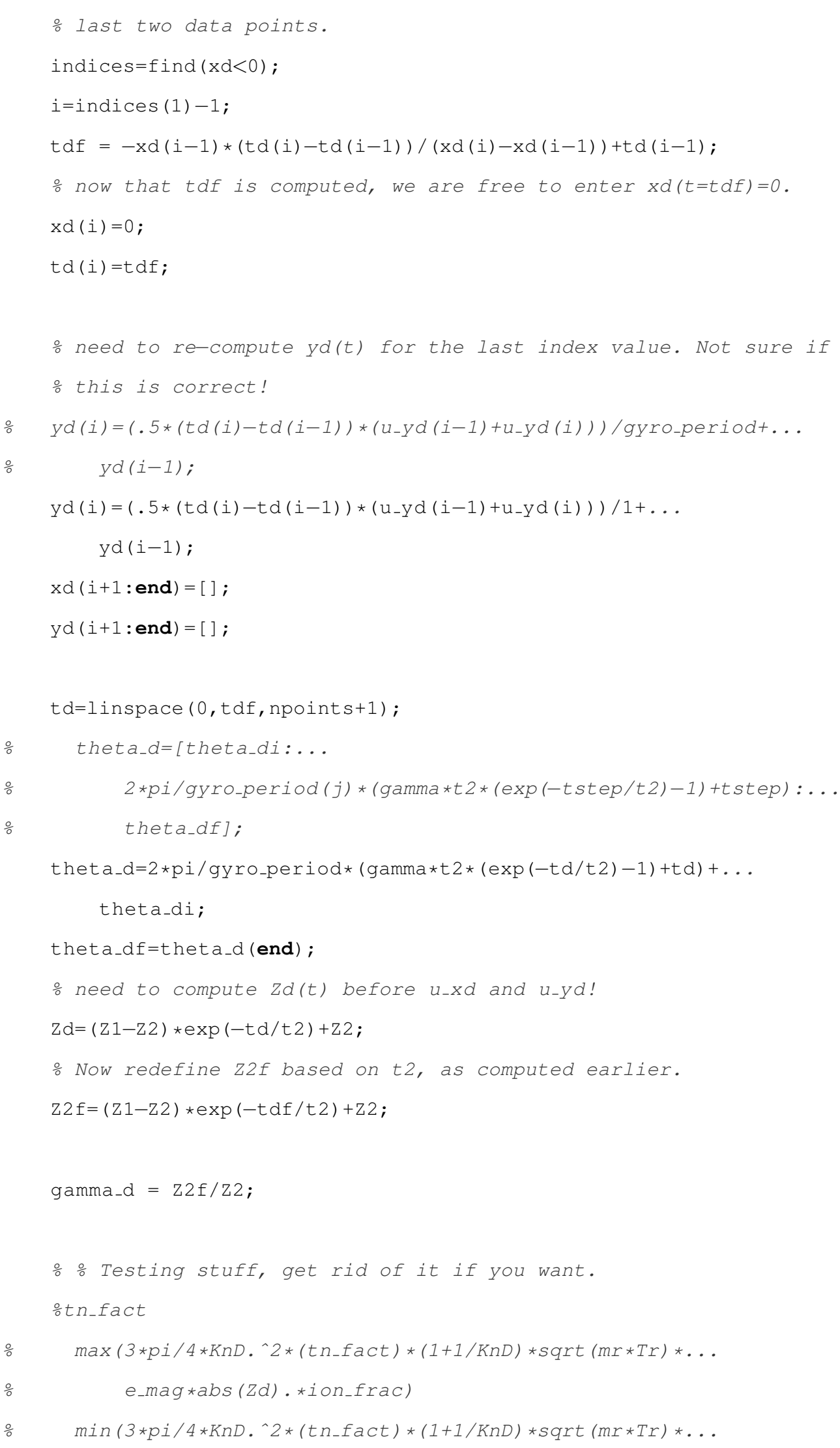




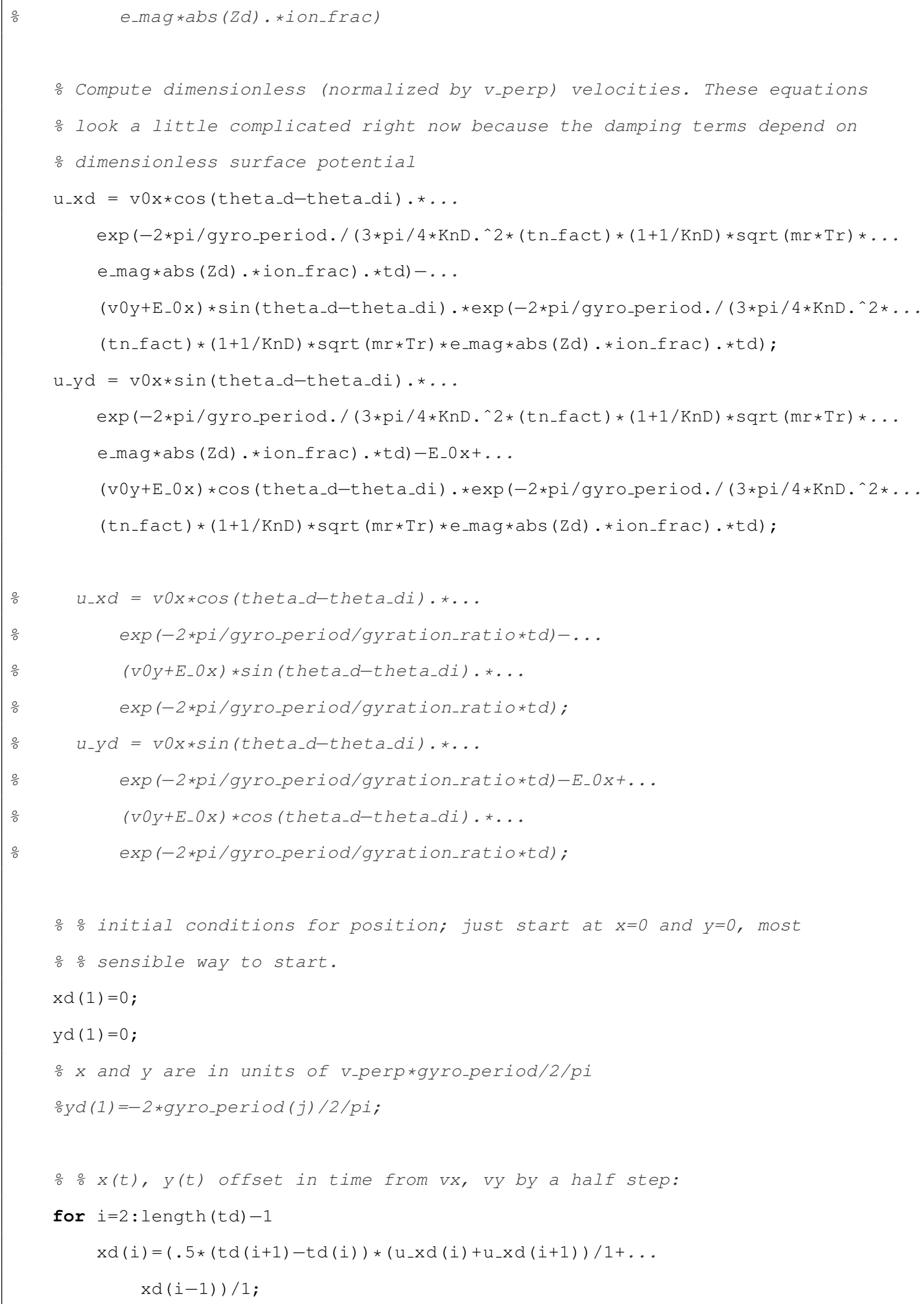




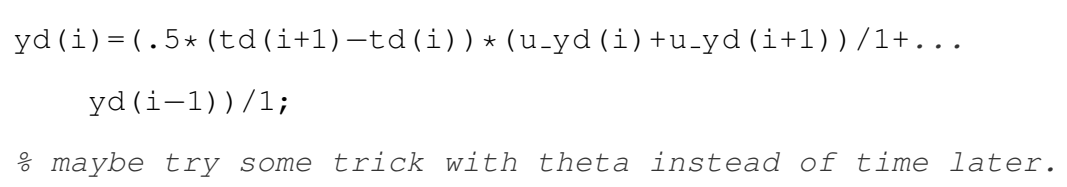




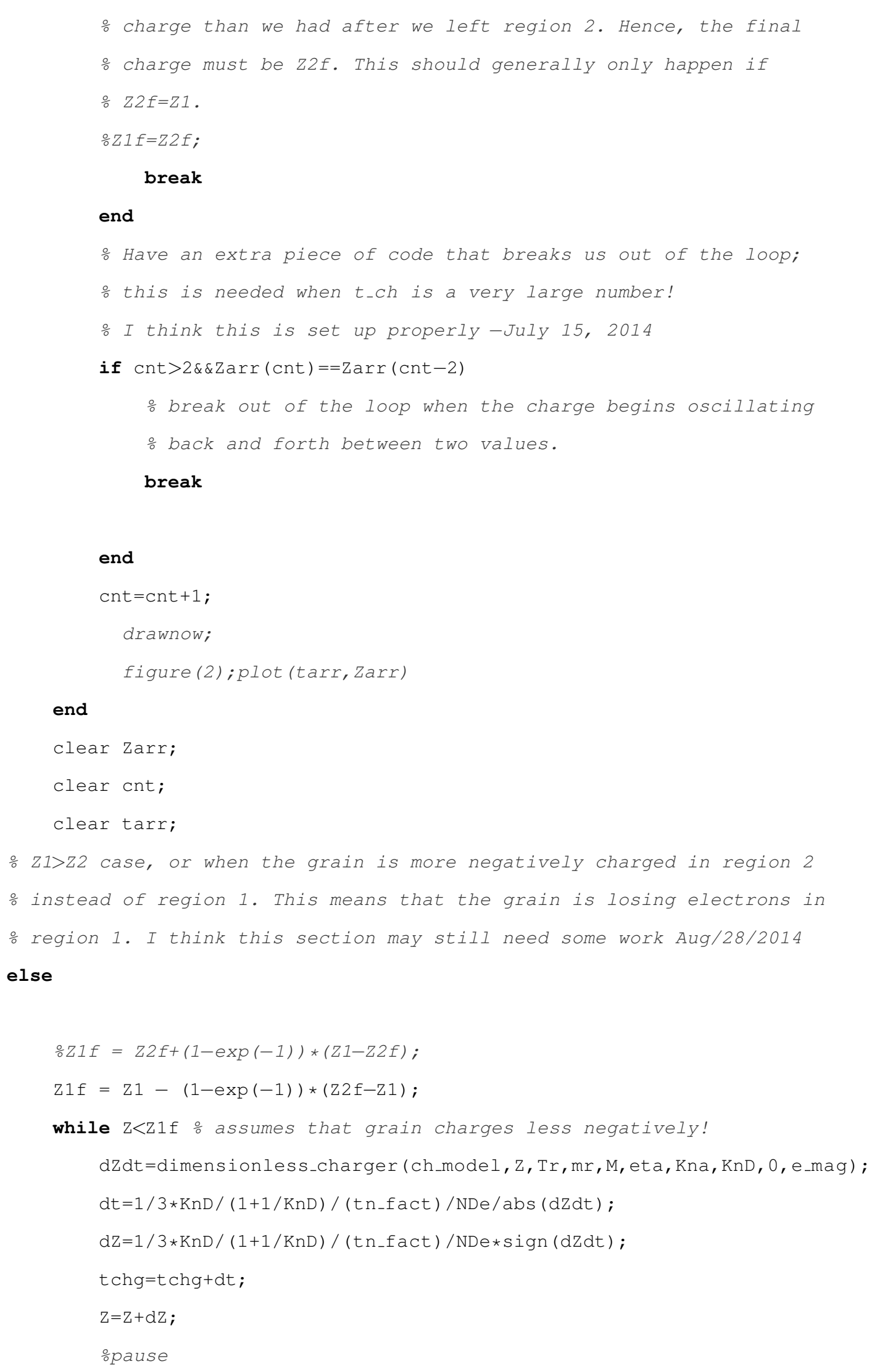




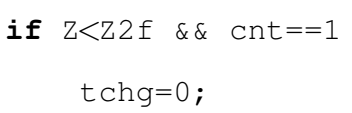

break

end

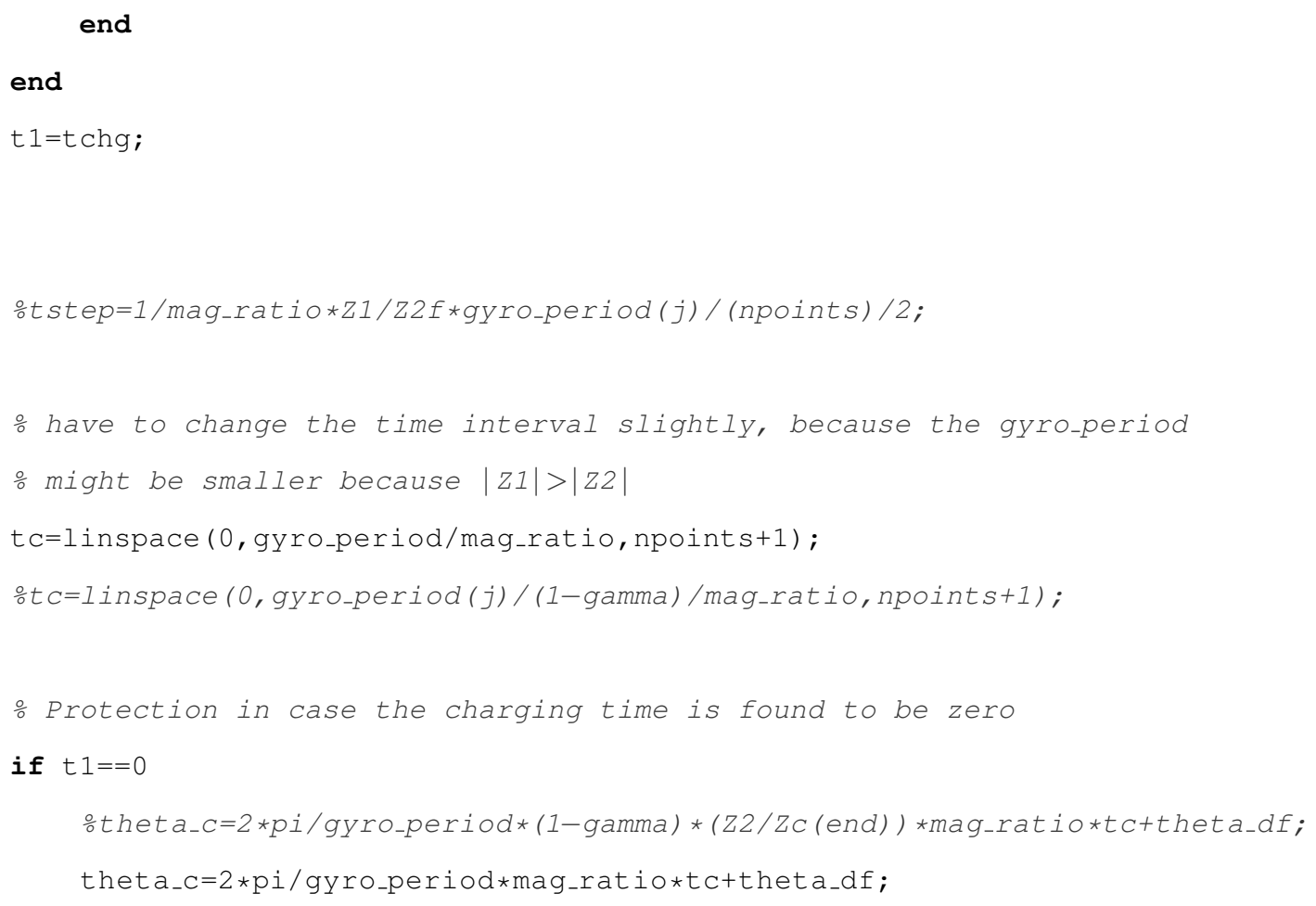




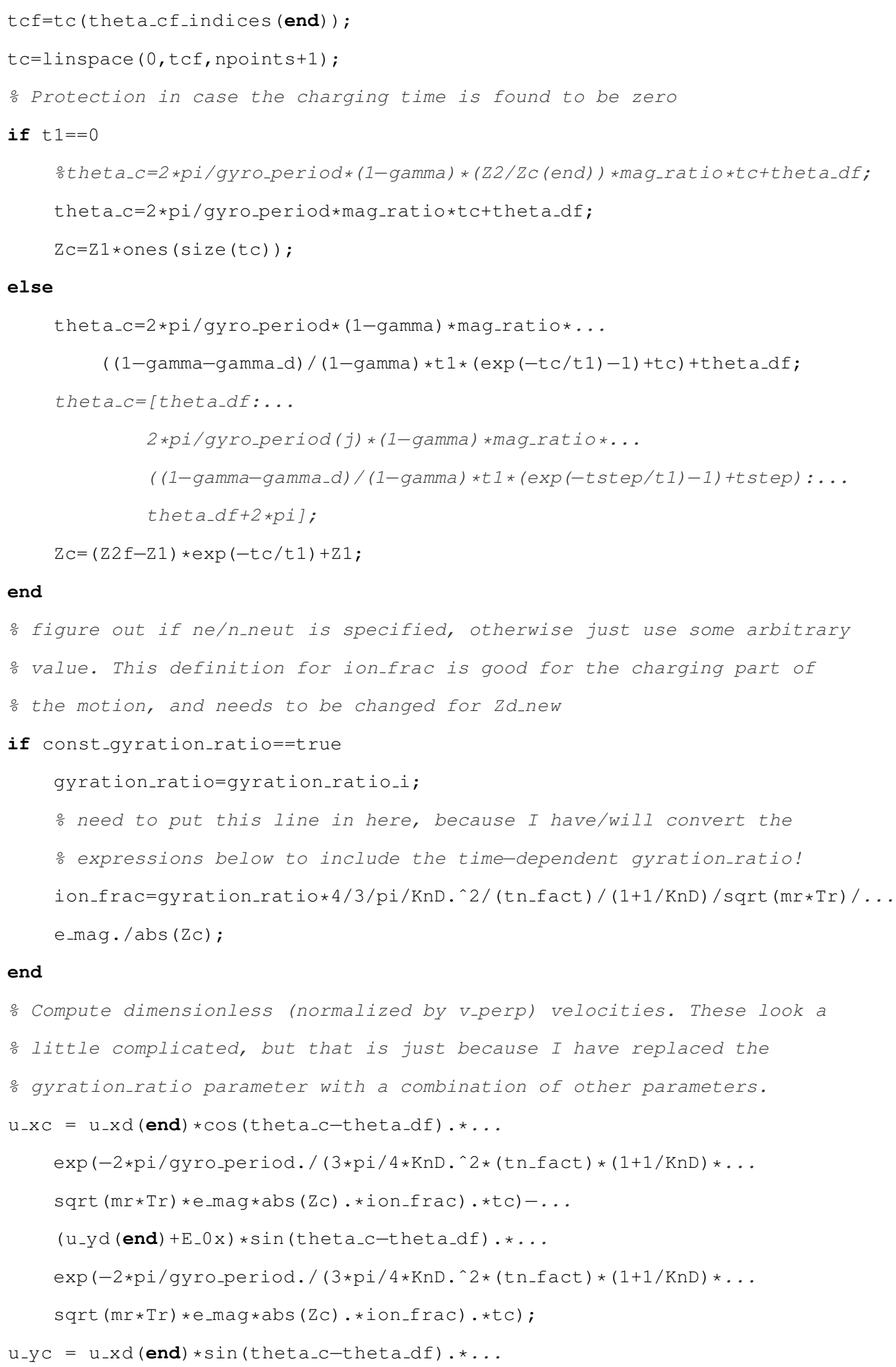




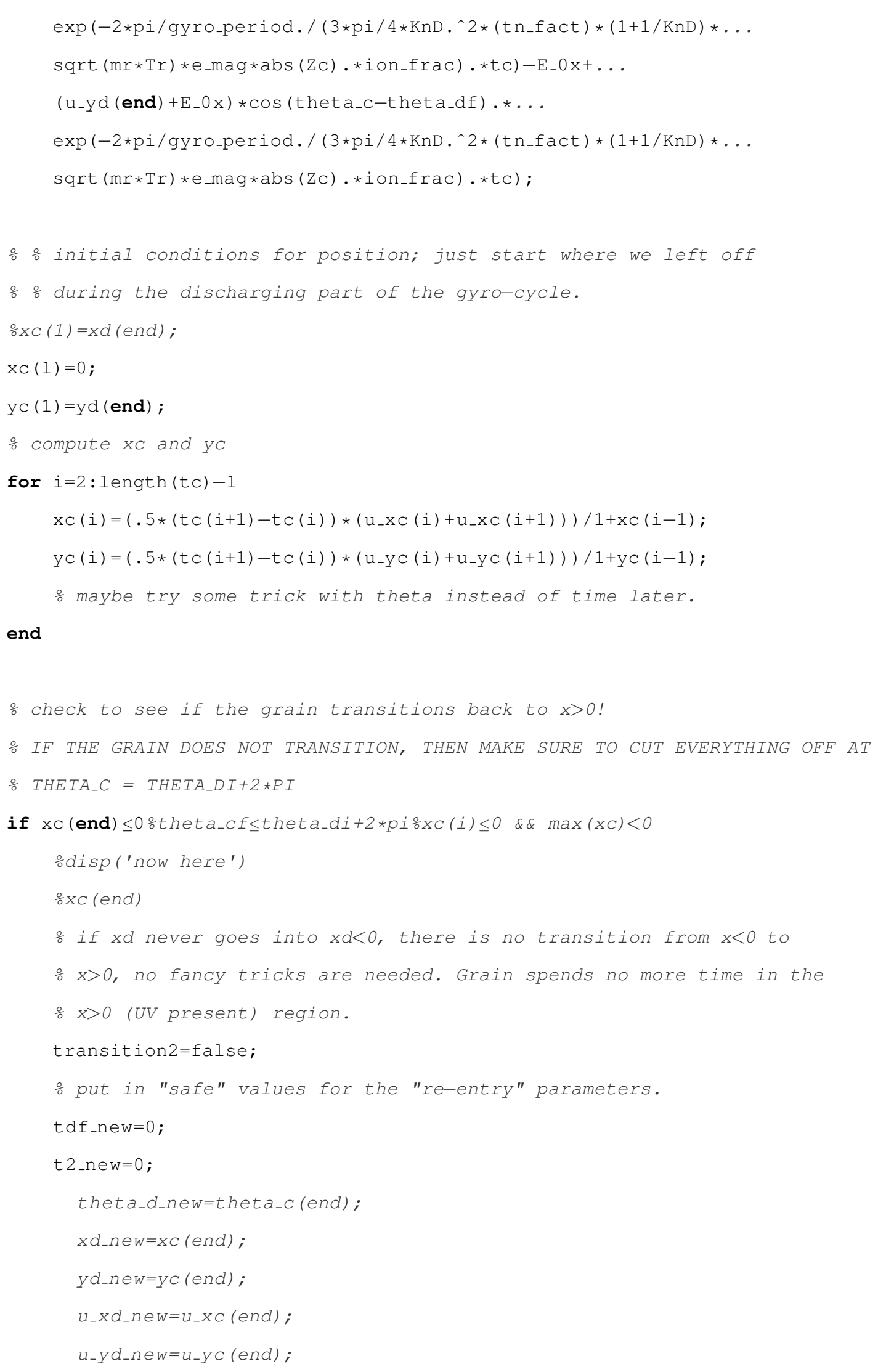




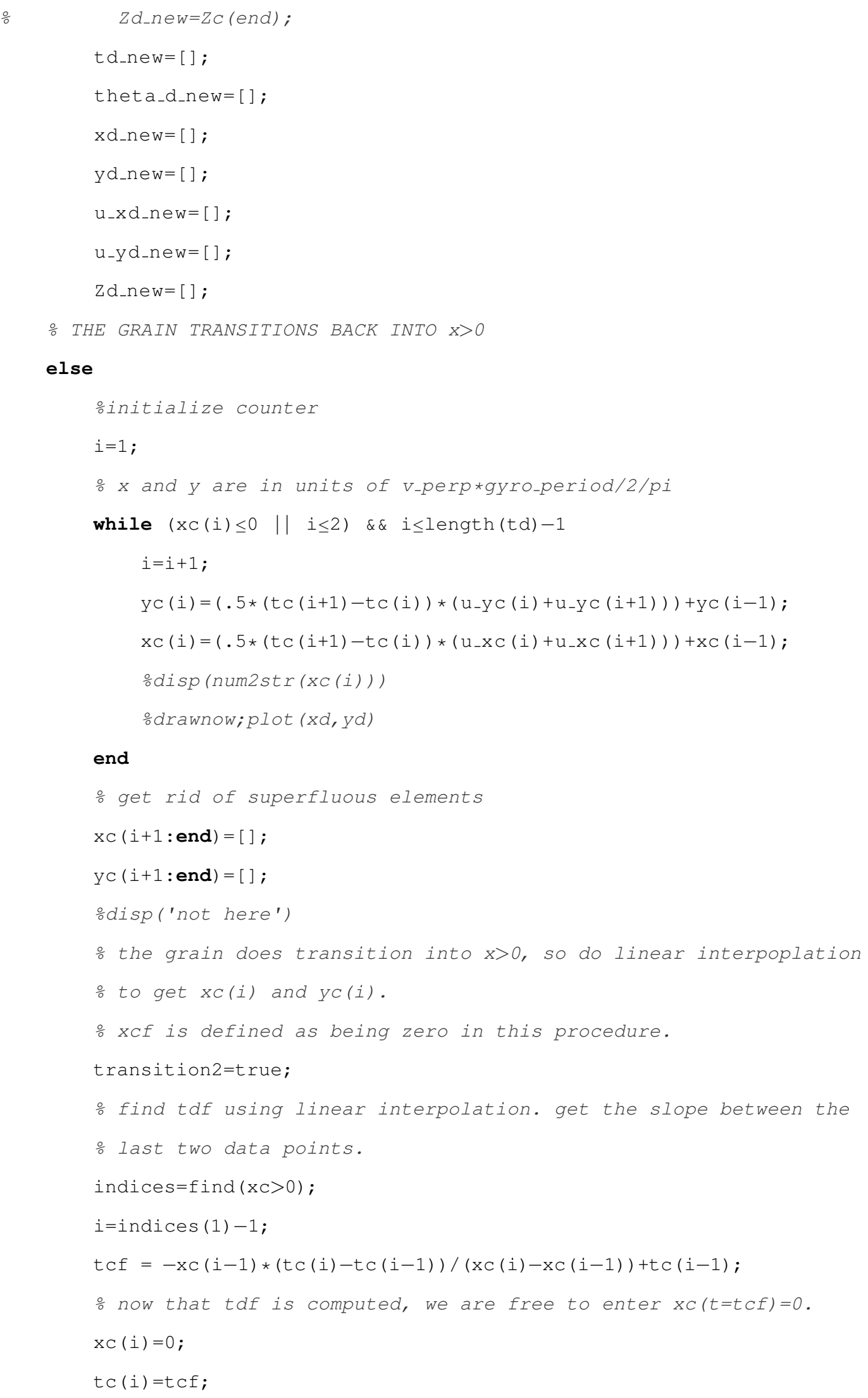




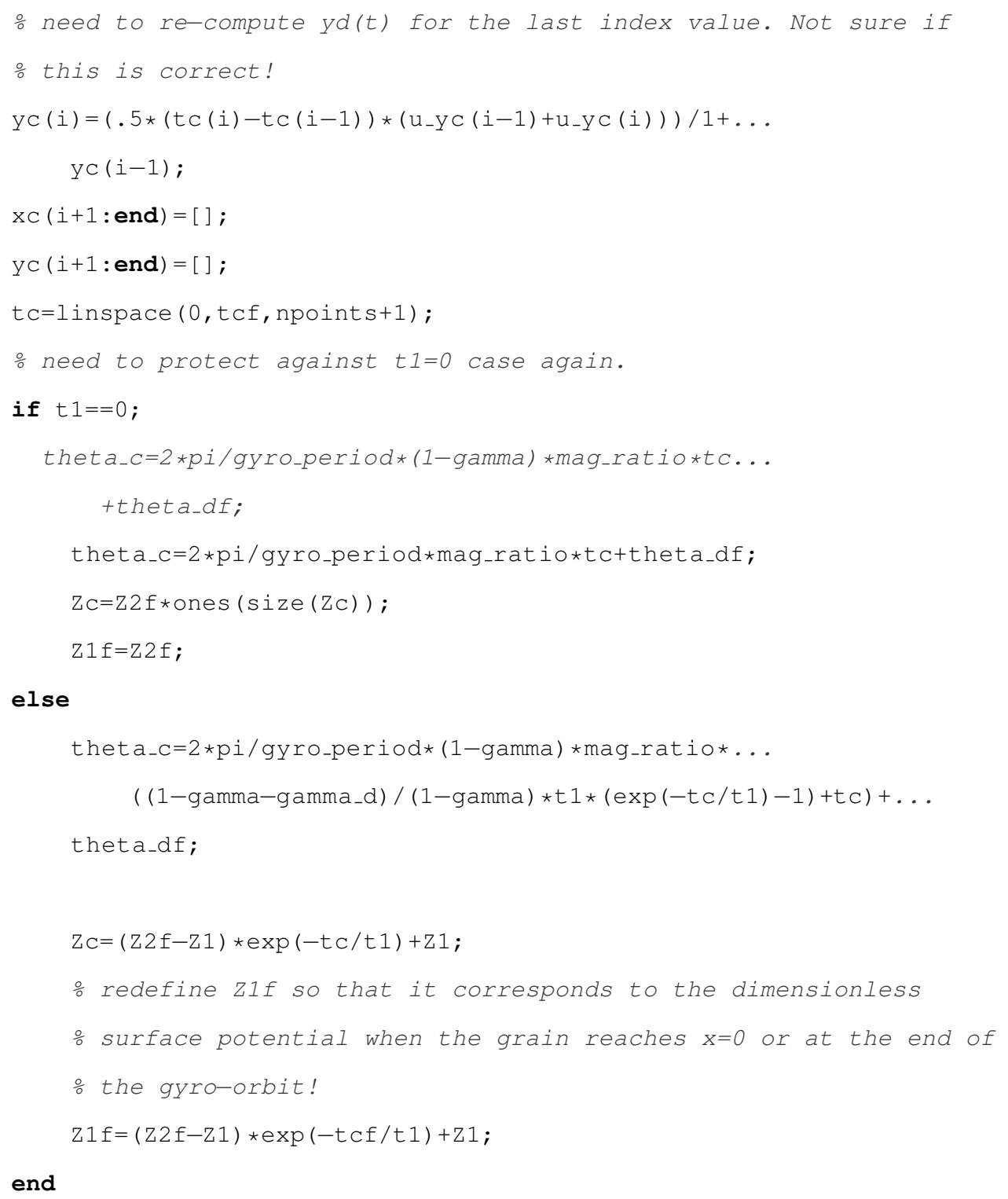




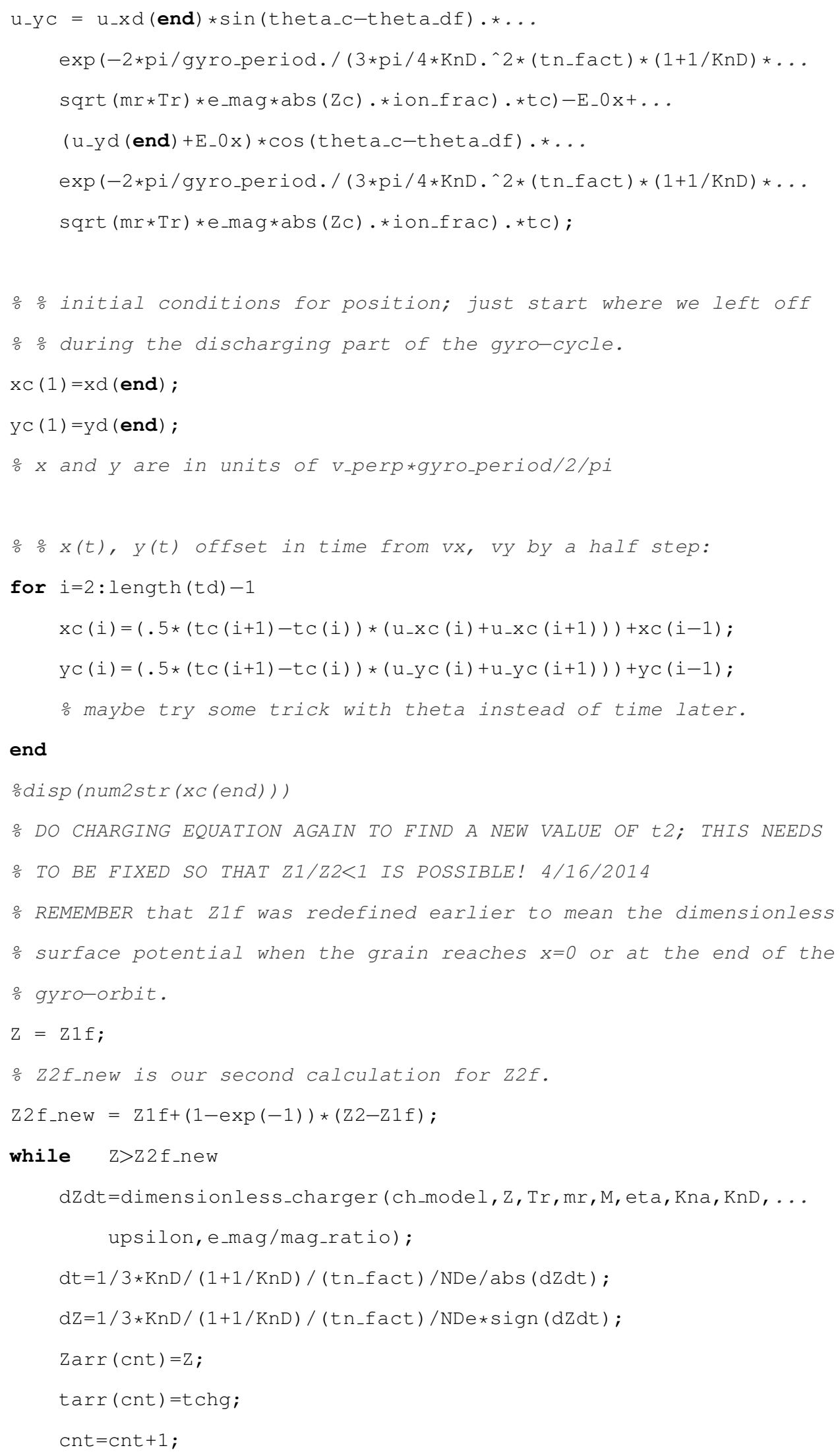




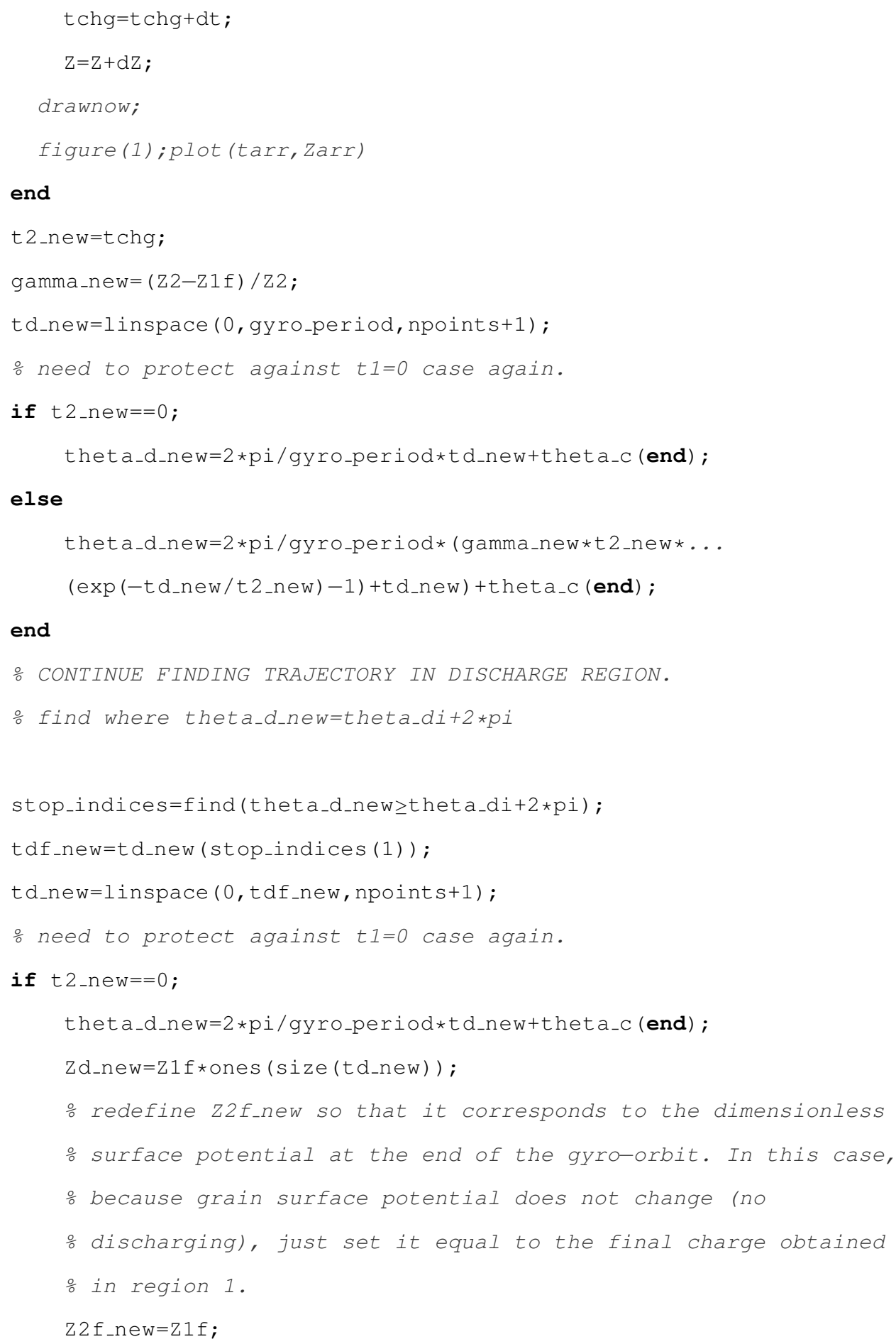


o surface potential at the end of the gyro-orbit!

Z2f_new $=(\mathrm{Z} 1 \mathrm{f}-\mathrm{Z} 2) * \exp \left(-\mathrm{tdf} \_n e w / t 2 \_n e w\right)+Z 2$;

\section{end}

o figure out if ne/n_neut is specified, otherwise just use some

\% arbitrary value. This definition for ion_frac is good for the 2nd

o discharging part of the motion, and needs to be changed for

으을

if const_gyration_ratio==true

gyration_ratio=gyration_ratio_i;

\% need to put this line in here, because I have/will convert the

o expressions below to include the time-dependent gyration_ratio!

ion_frac=gyration_ratio*4/3/pi/KnD.^2/(tn_fact) / $(1+1 / \mathrm{KnD}) \ldots$

/sqrt (mr*Tr) / e_mag./abs (Zd_new);

\section{end}

$u_{-} x d_{-}$new $=u_{-} x c($ end $) \star \cos ($ theta_d_new-theta_c $($ end $)) . \star \ldots$

$\exp \left(-2 *\right.$ pi /gyro_period./(3*pi/4*KnD. ${ }^{\wedge} 2 *\left(t n_{-}\right.$fact $) *(1+1 / \mathrm{KnD}) * \ldots$

$\operatorname{sqrt}(\operatorname{mr} * T r) * e_{\_} m a g * a b s\left(Z d_{-} n e w\right)$. *ion_frac $\left.) . * t d \_n e w\right)-\ldots$

$\left(u_{-} y c(\right.$ end $\left.)+E_{-} 0 x\right) * \sin ($ theta_d_new-theta_c (end) ) ...

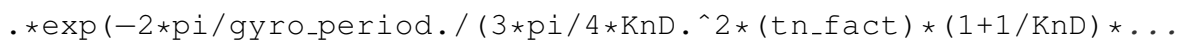

$\operatorname{sqrt}(m r * T r) * e \_m a g * a b s\left(Z d \_n e w\right)$ * *ion_frac) . *td_new) ;

$u_{-y} d_{-}$new $=u_{-} x c($ end $) * \sin ($ theta_d_new-theta_c $($ end $)) . \star .$.

$\exp \left(-2 * \mathrm{pi} /\right.$ gyro_period./(3*pi/4*KnD. ${ }^{\wedge} 2 *($ t n_fact $) *(1+1 / \mathrm{KnD}) * \ldots$

$\operatorname{sqrt}(m r * T r) \star e_{\_} m a g * a b s\left(Z d_{-} n e w\right)$. *ion_frac $\left.) . \star t d \_n e w\right)-E \_0 x+\ldots$

$\left(u_{-} y c(\right.$ end $\left.)+E_{-} 0 x\right) \star c o s\left(t h e t a_{-} d_{-} n e w-t h e t a_{-} c(\right.$ end $\left.)\right) \ldots$

. $\star \exp \left(-2 * \mathrm{pi} / \mathrm{gyro}\right.$ period. / ( $3 * \mathrm{pi} / 4 * \mathrm{KnD} .{ }^{\wedge} 2 *($ tn_fact $) \star(1+1 / \mathrm{KnD}) \star \ldots$

$\left.\operatorname{sqrt}(\operatorname{mr} * \mathrm{Tr}) * e \_m a g * a b s\left(Z d \_n e w\right) . * i o n \_f r a c\right)$ *td_new) ;

응 initial conditions for position; just start at $x=x c$ (end) and

응 $y=y c(e n d)$, most sensible way to start.

xd_new $(1)=x c($ end $)$;

yd_new $(1)=y c($ end $)$;

$\therefore \circ x(t), y(t)$ offset in time from $v x$, vy by a half step:

for $i=2:$ length $(t d)-1$

xd_new $(i)=(.5 *($ td_new $(i+1)-$ td_new $(i)) \ldots$

*(u_xd_new (i) +u_xd_new $\left.(i+1)) / 1+x d \_n e w(i-1)\right) / 1$;

yd_new $(i)=\left(.5 *\left(t d \_n e w(i+1)-t d \_n e w(i)\right) \ldots\right.$ 


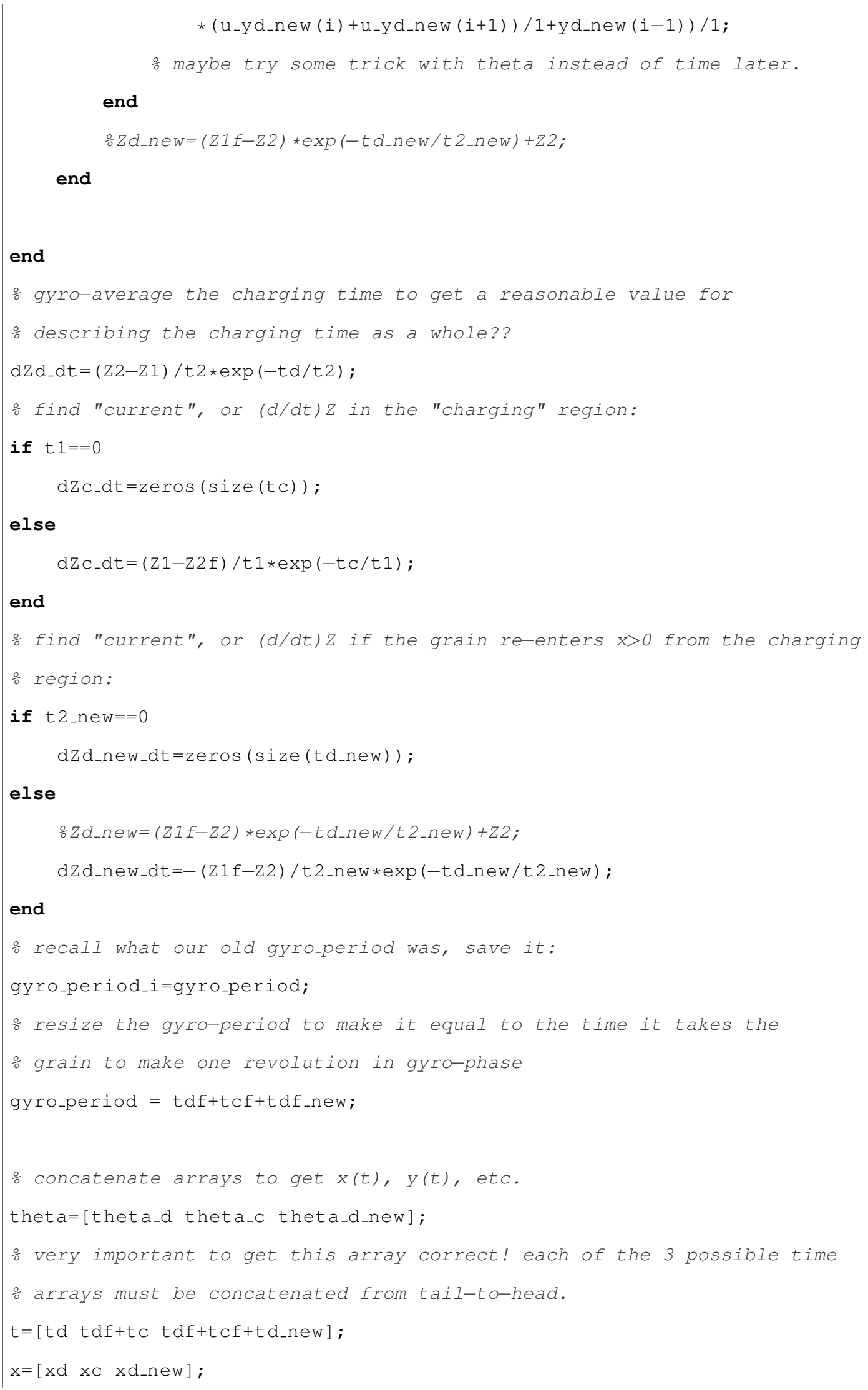




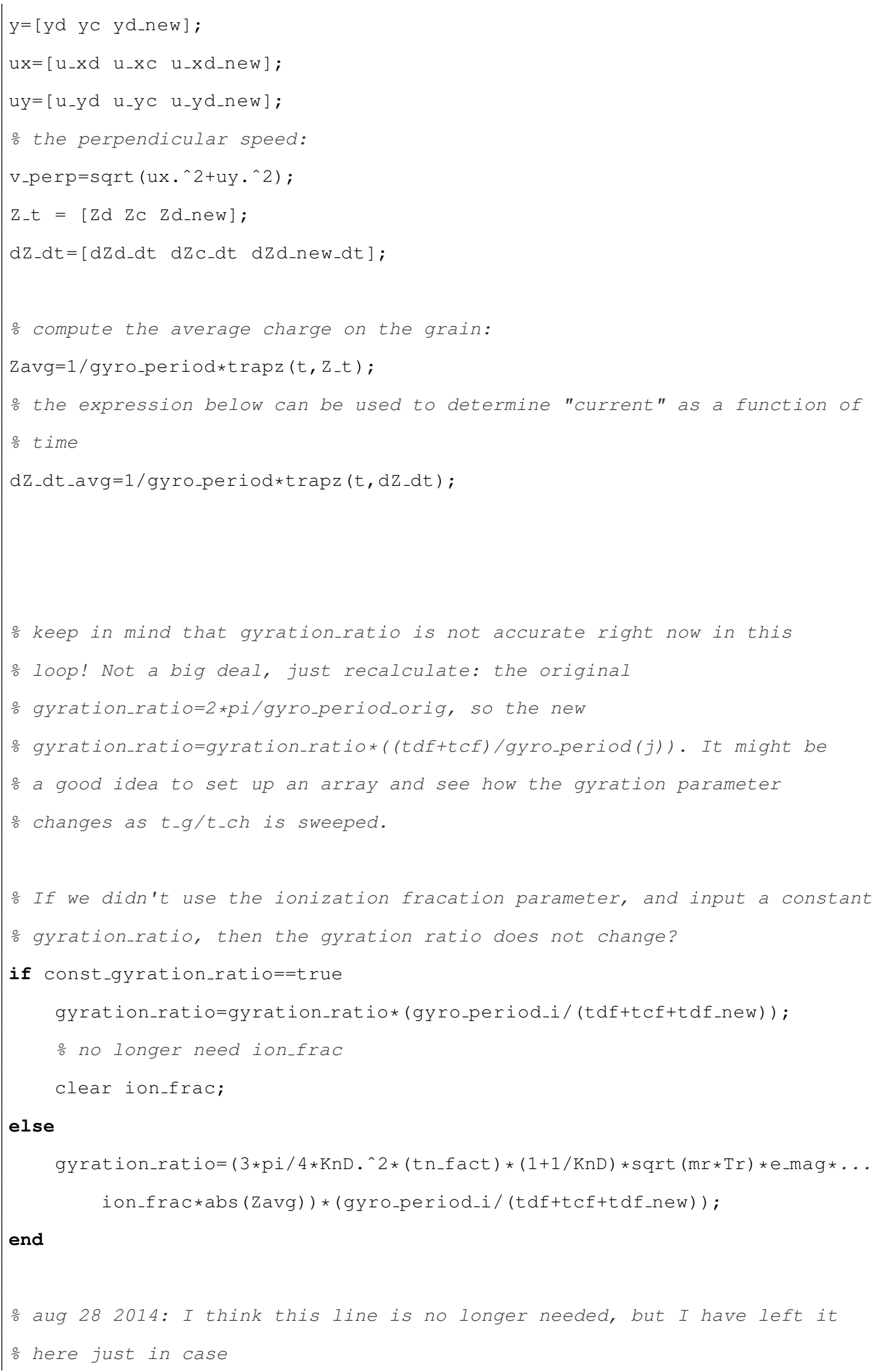




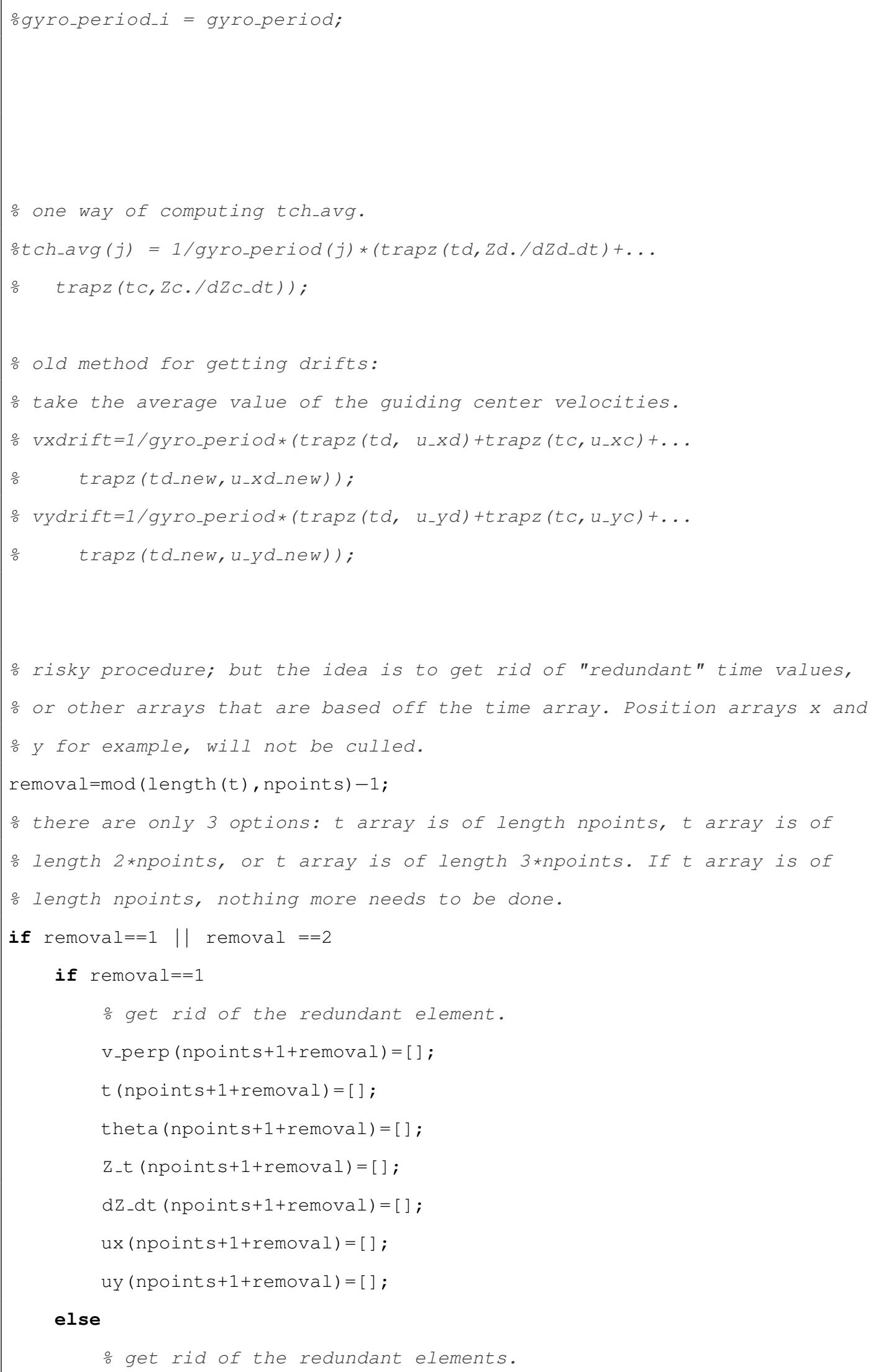




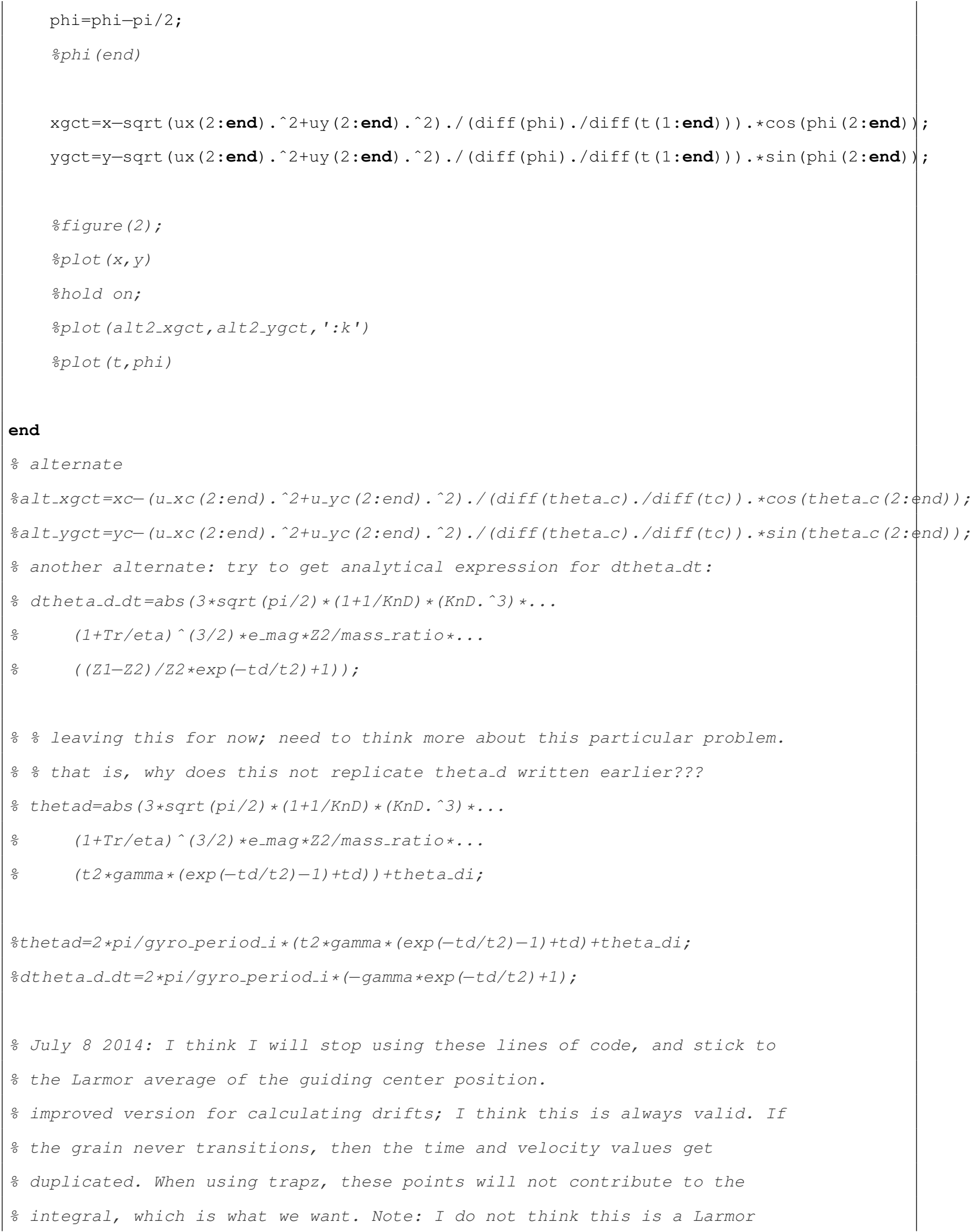




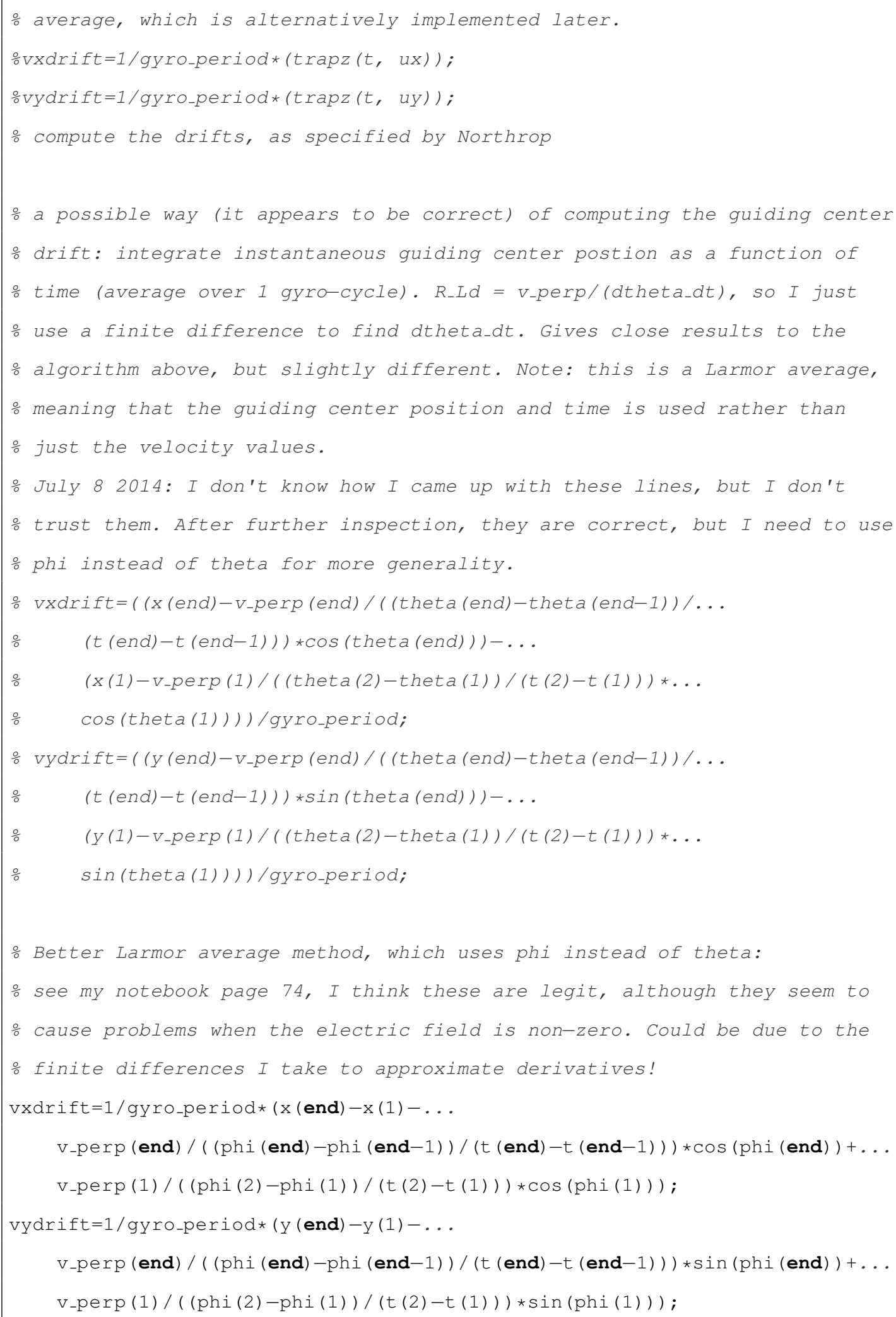




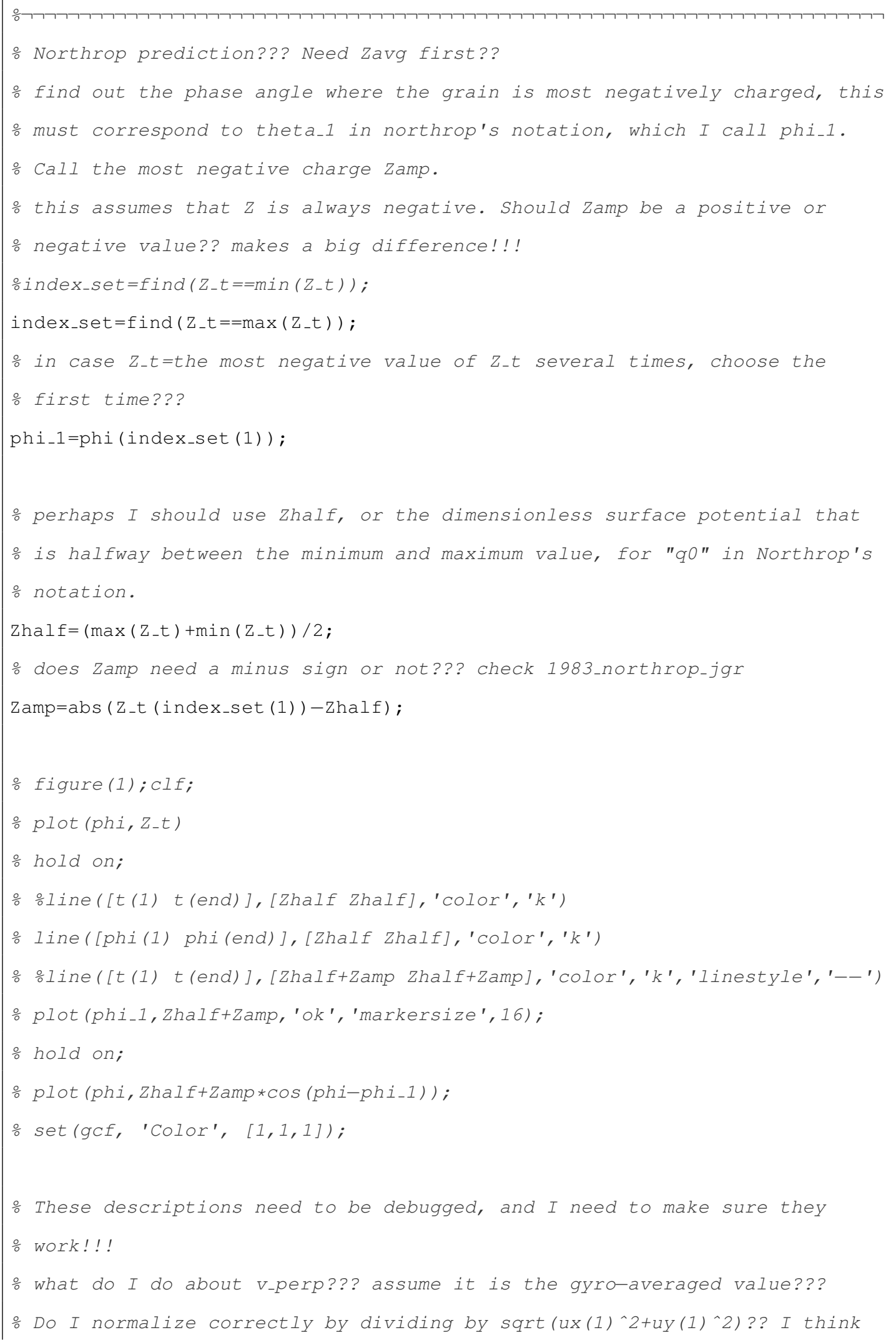




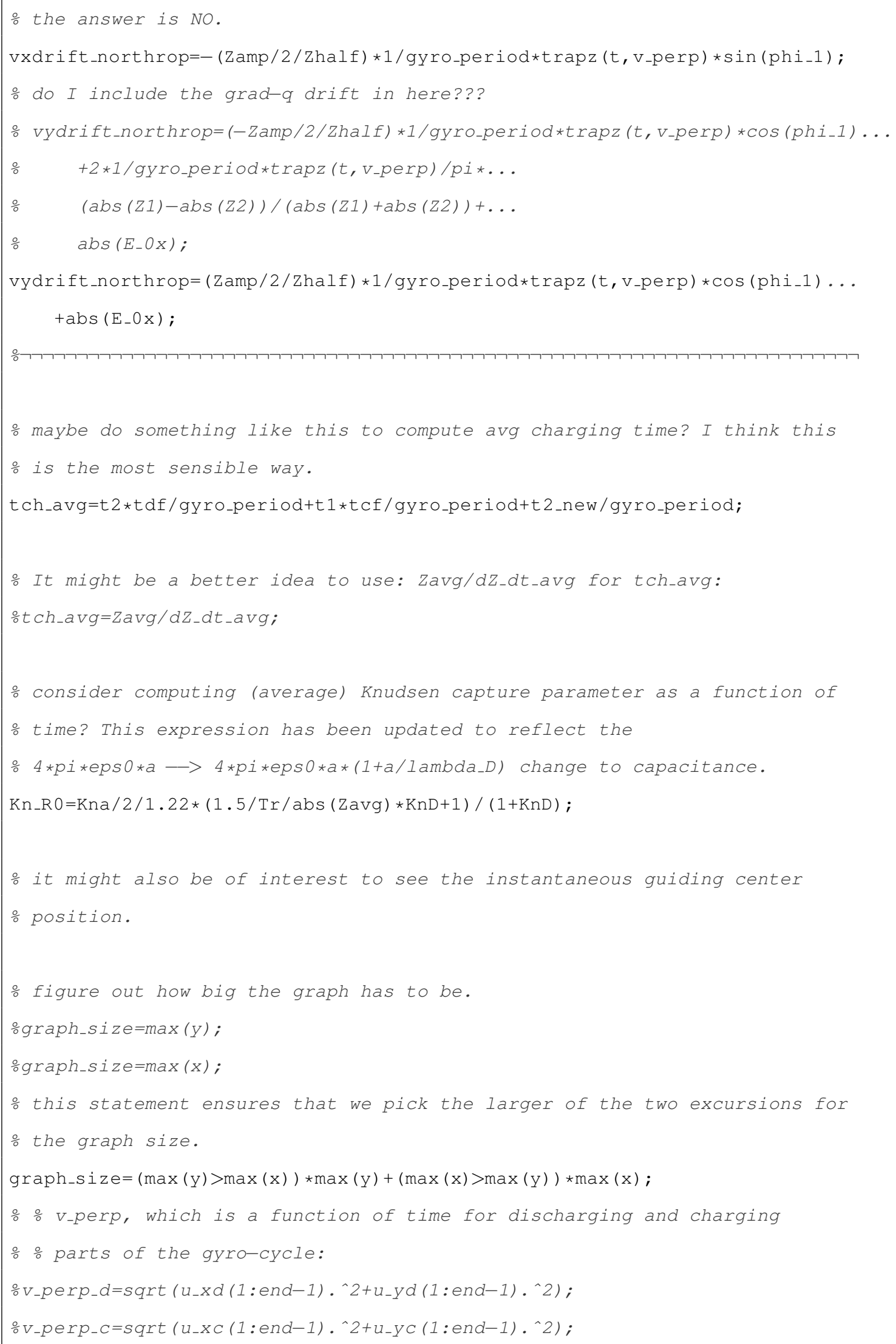




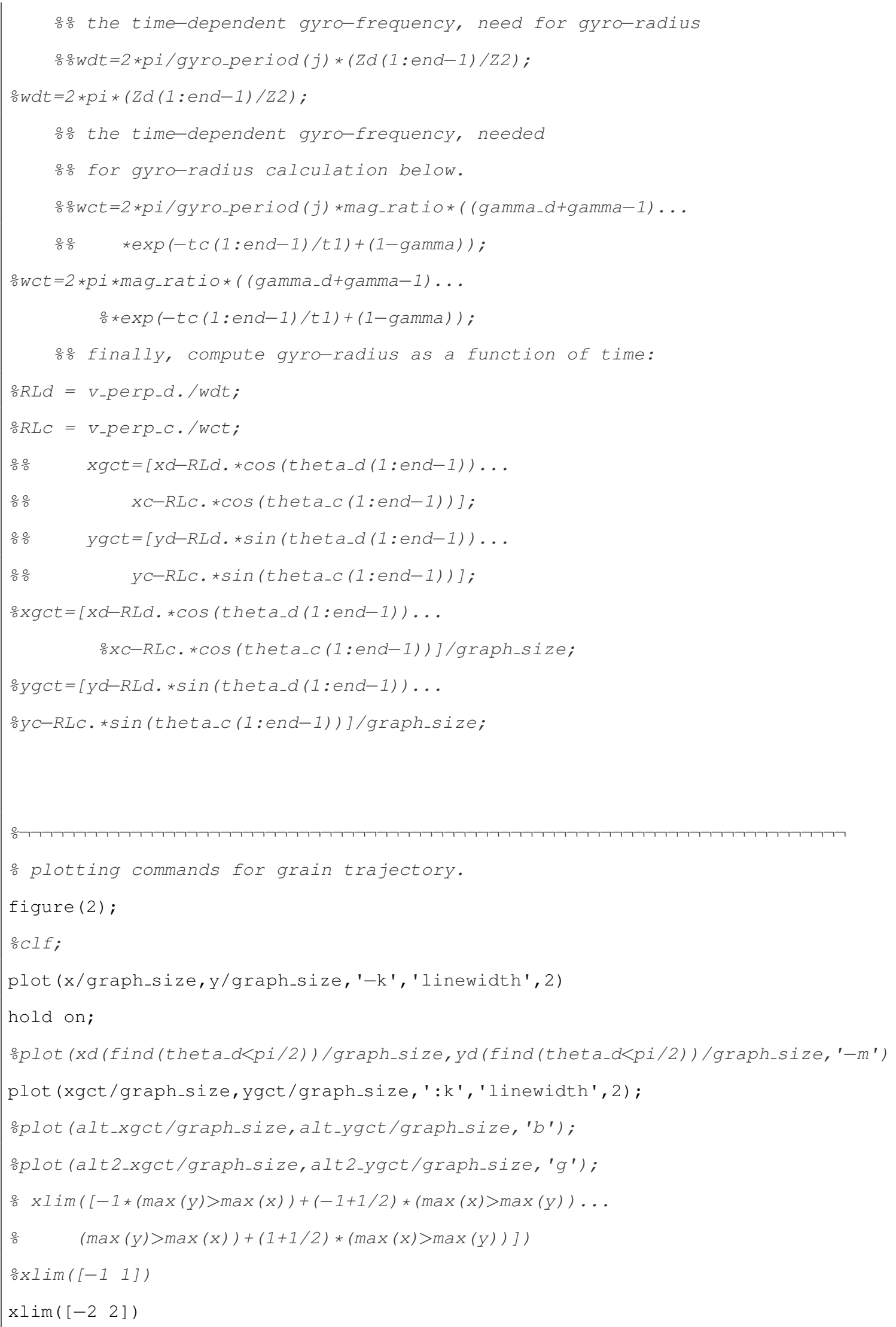




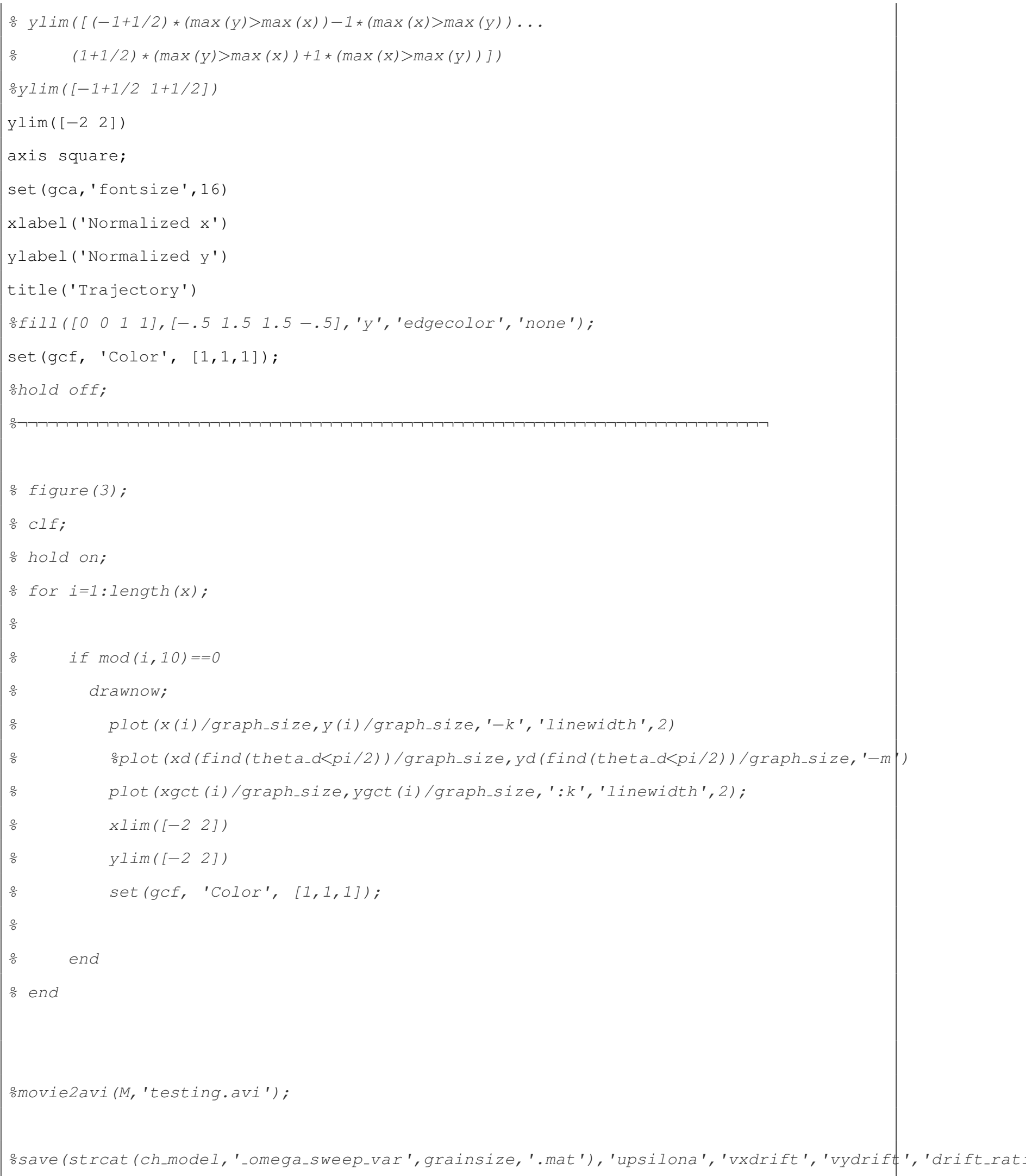




\section{Specifying the Space or Laboratory Plasma Profile}

The purpose of profiles.m is to provide an analytical spatial description for the plasma or system of interest. The output of profiles, such as electron and ion density and electric fields etc., will ultimately be applied to the equations of motion for the dust grain and for determining the current to the dust grain. This program can also be called to make spatial plots of a specific profile. Many profiles have been constructed so far, and examples of these will be discussed in later sections of this report, but it will generally be up to the user to create new profiles, or modify existing profiles when running simulations.

Profiles is used by dust_trajectory.m, but can be used as a subroutine or function in its own right.

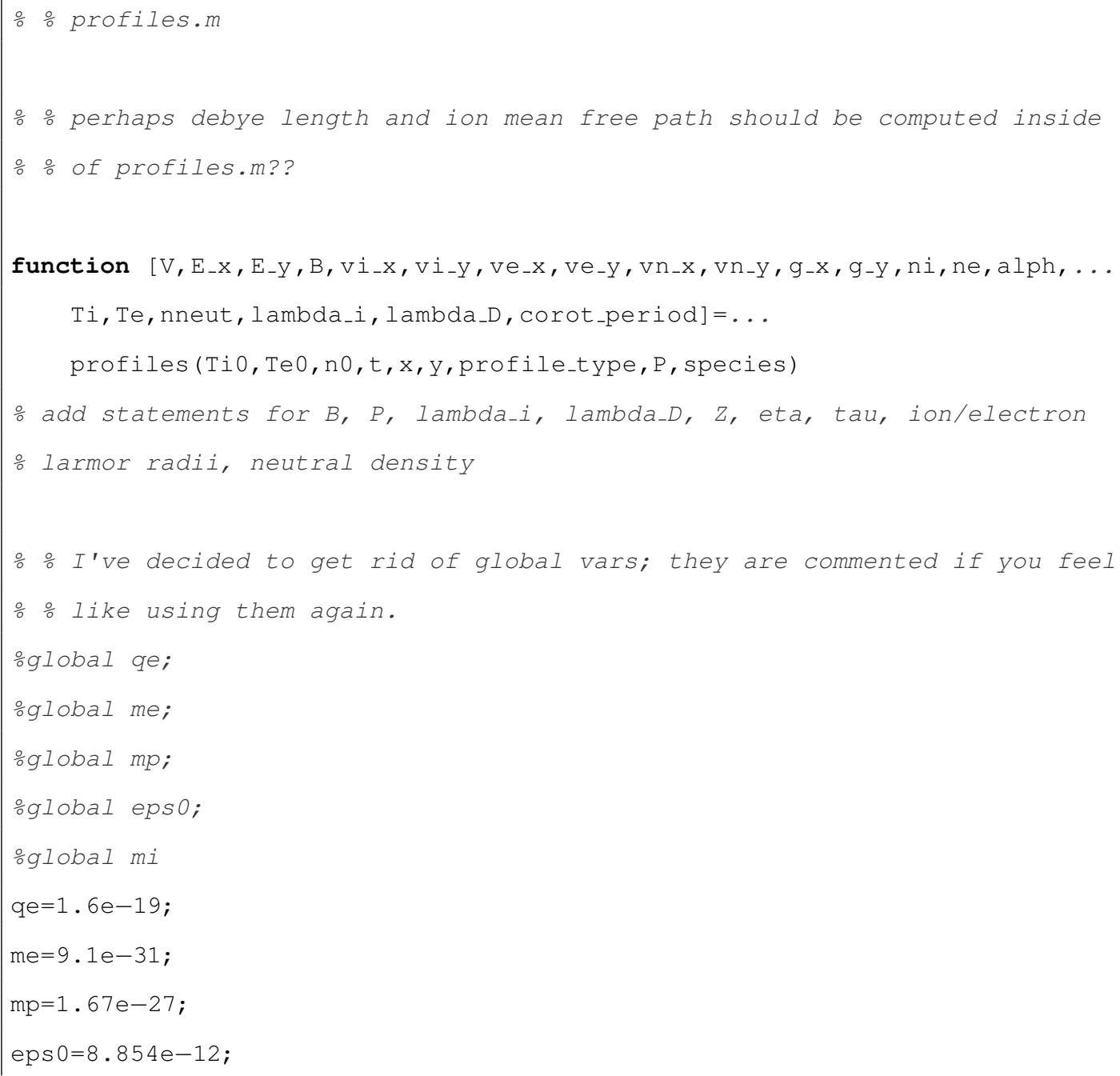




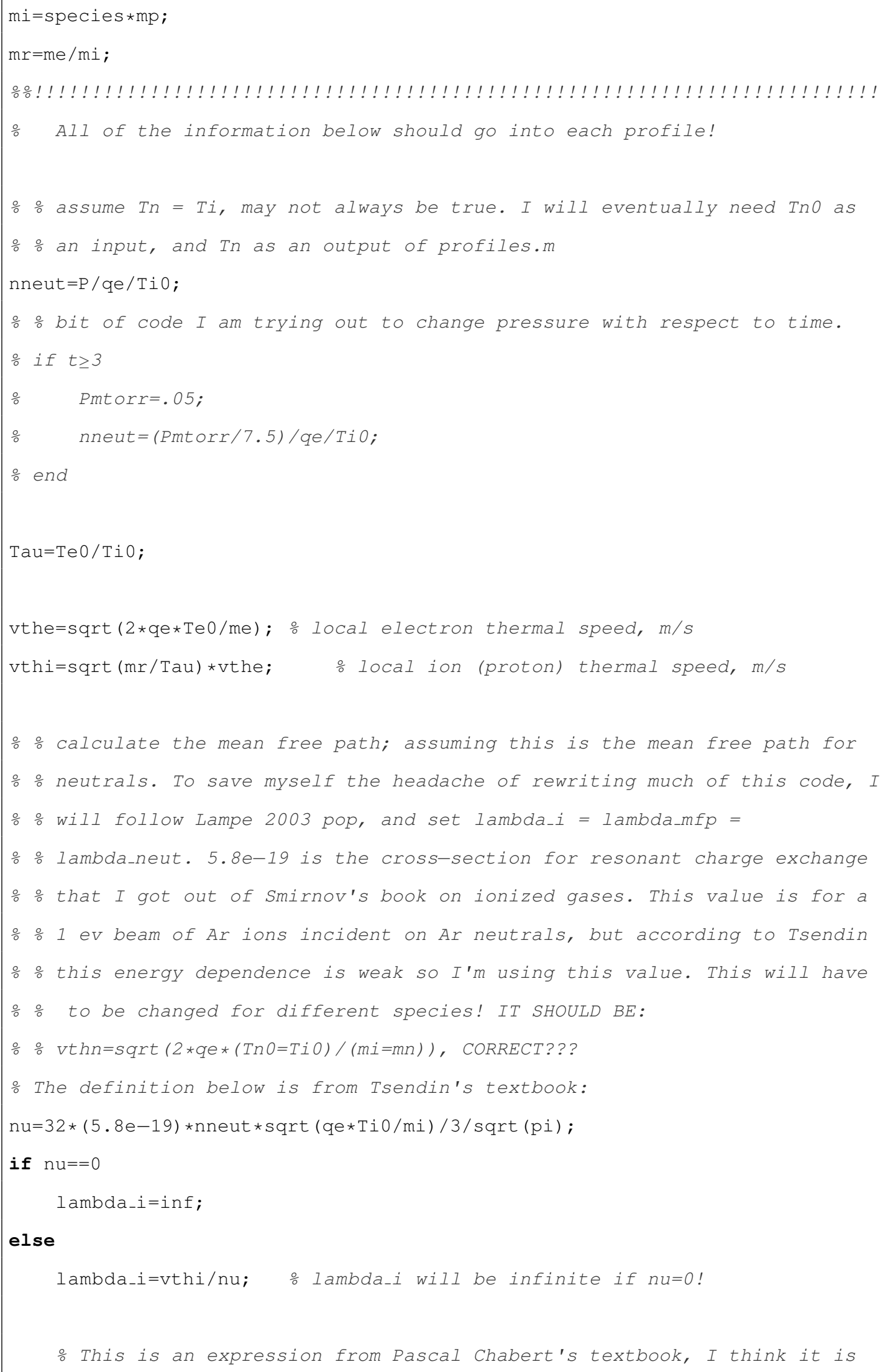


- not as correct as the Tsendin expression above.

ㄴ Iambda_i=1/5.8e-19/nneut;

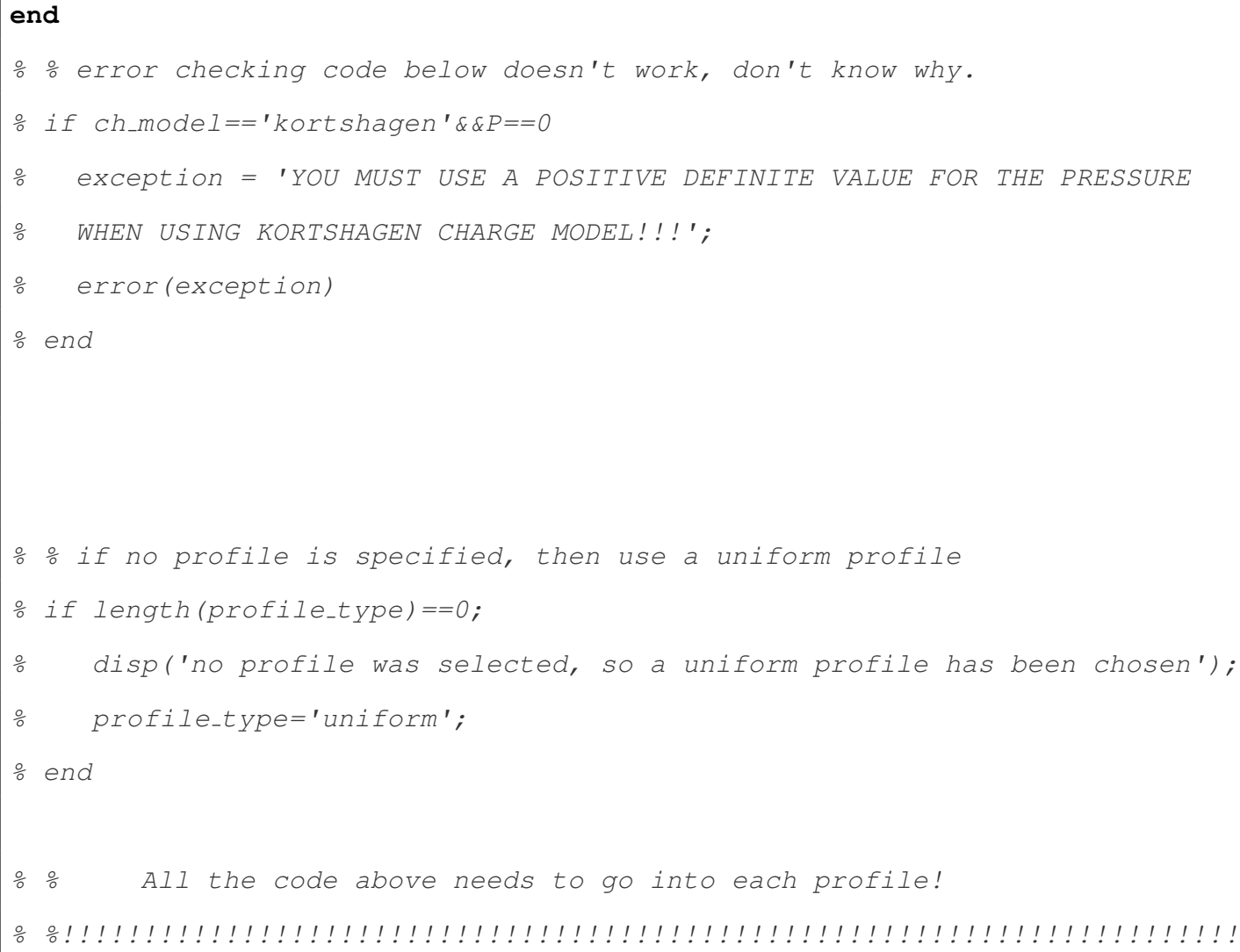




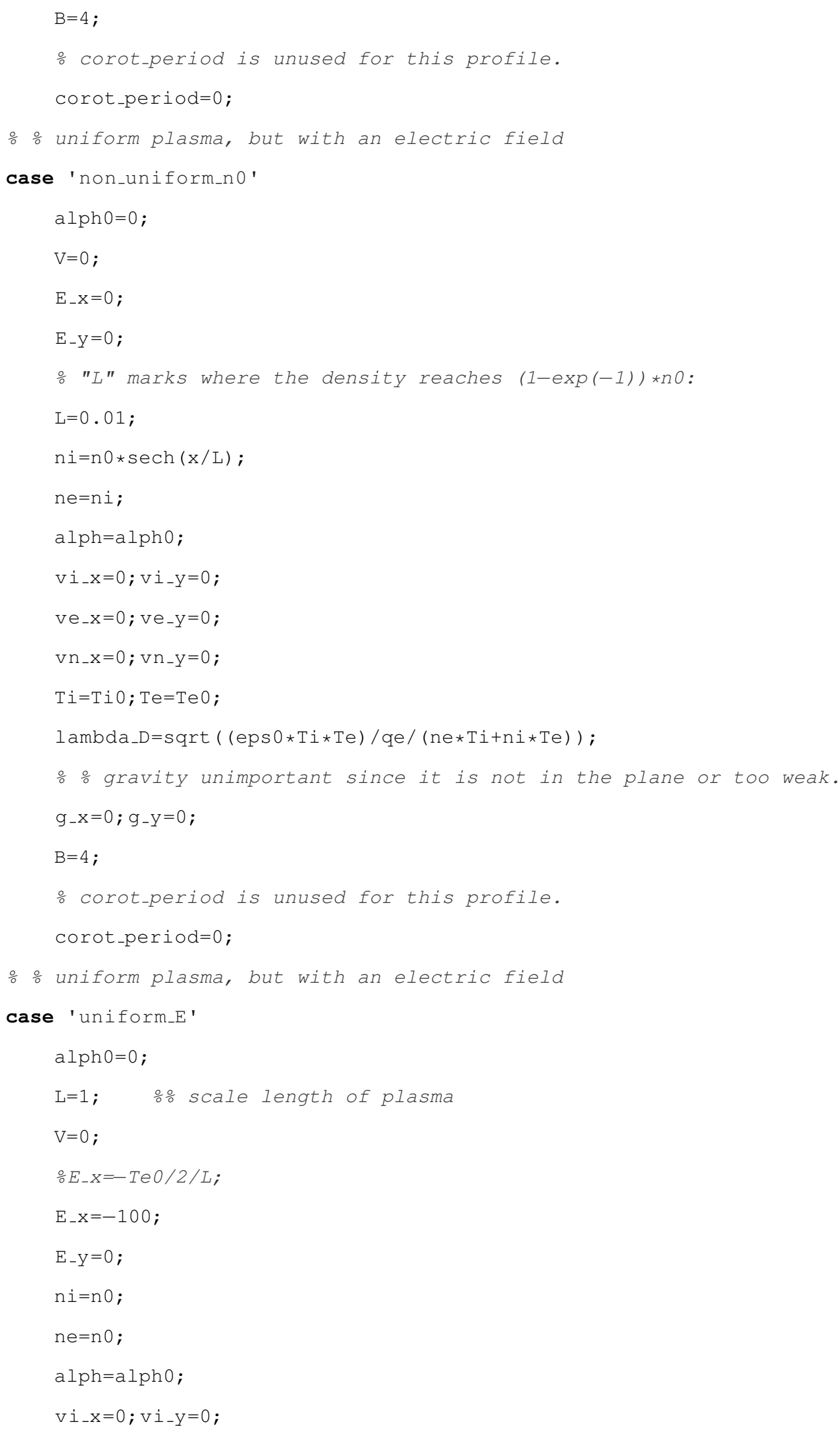




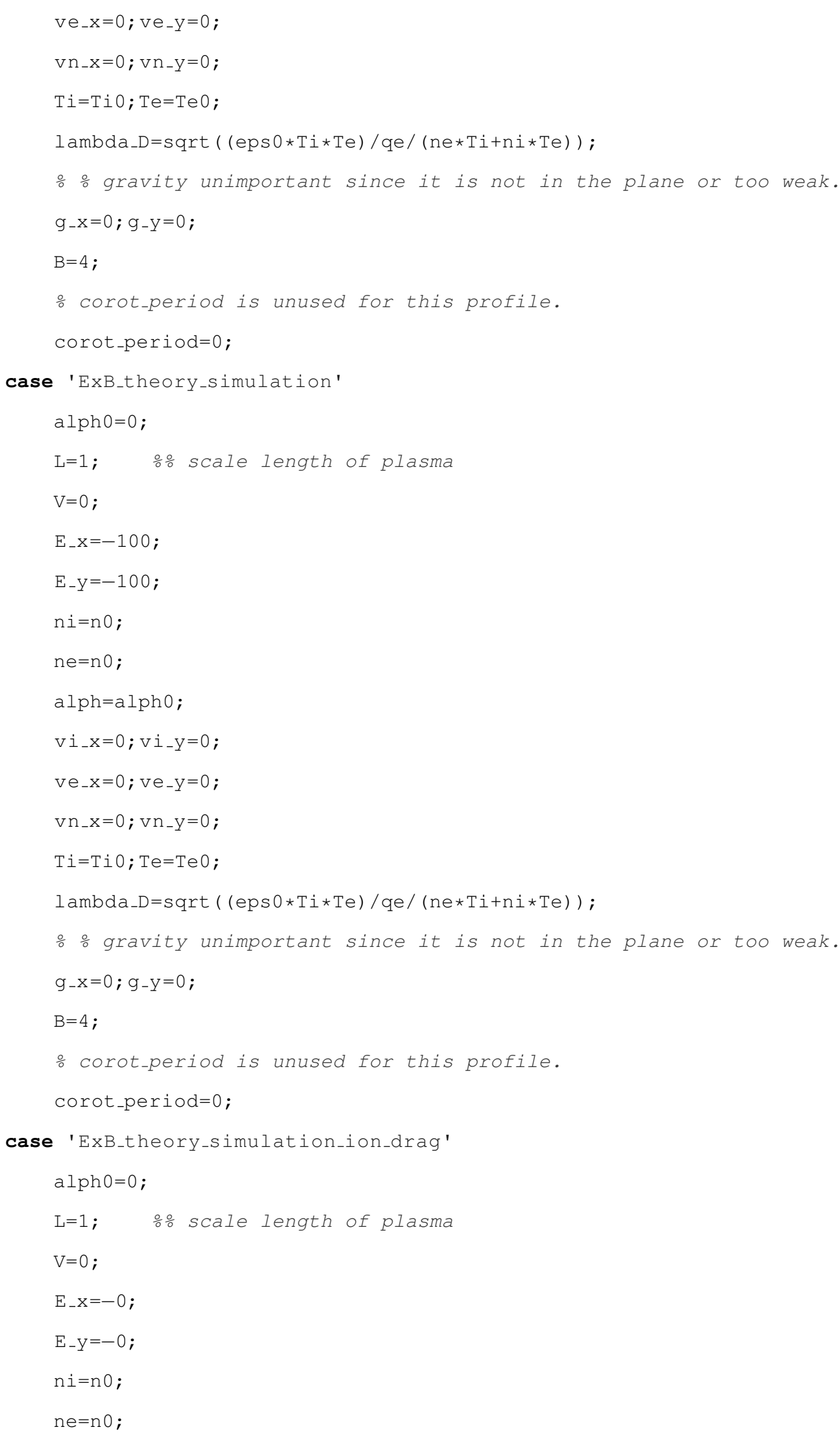




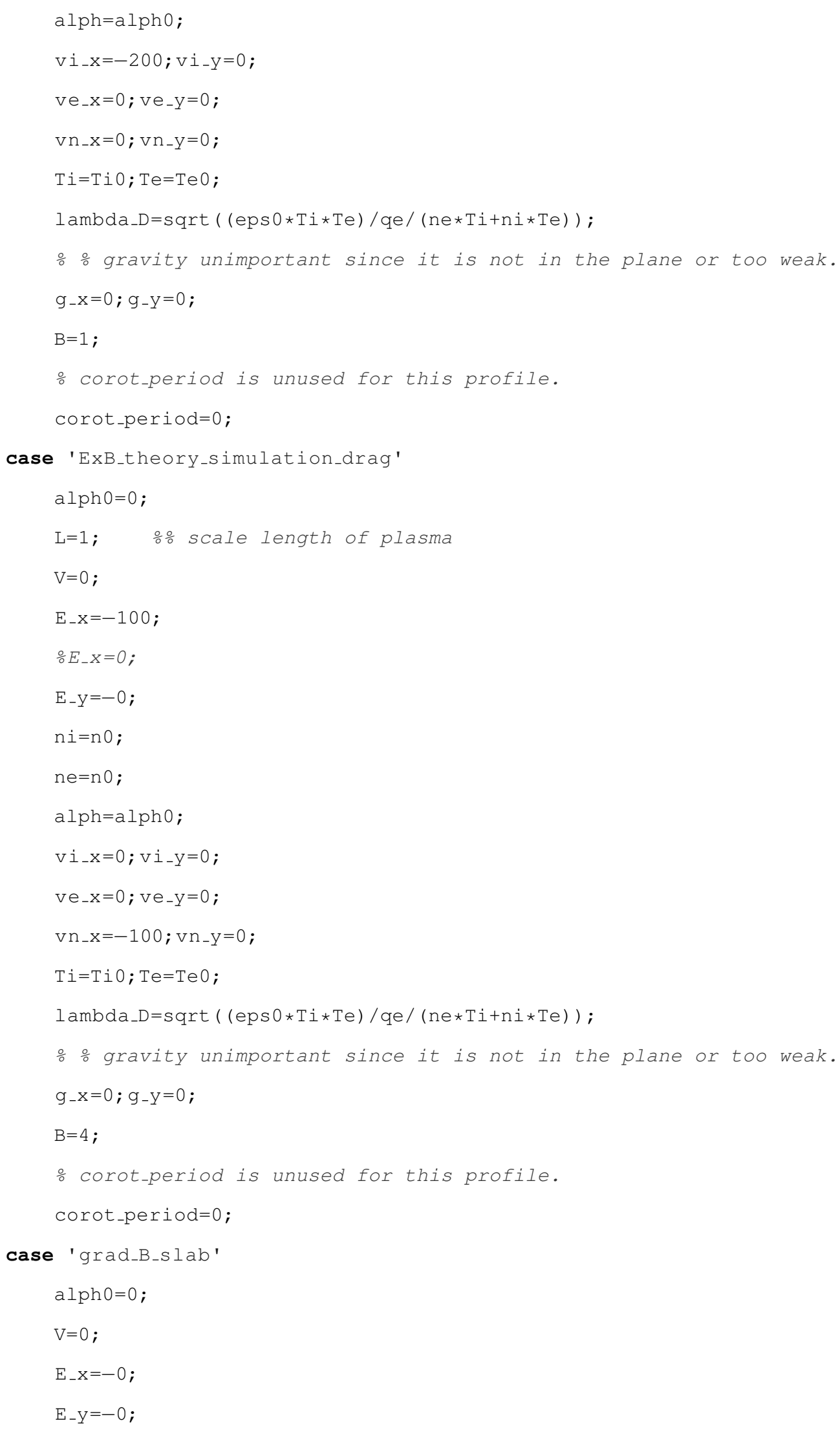




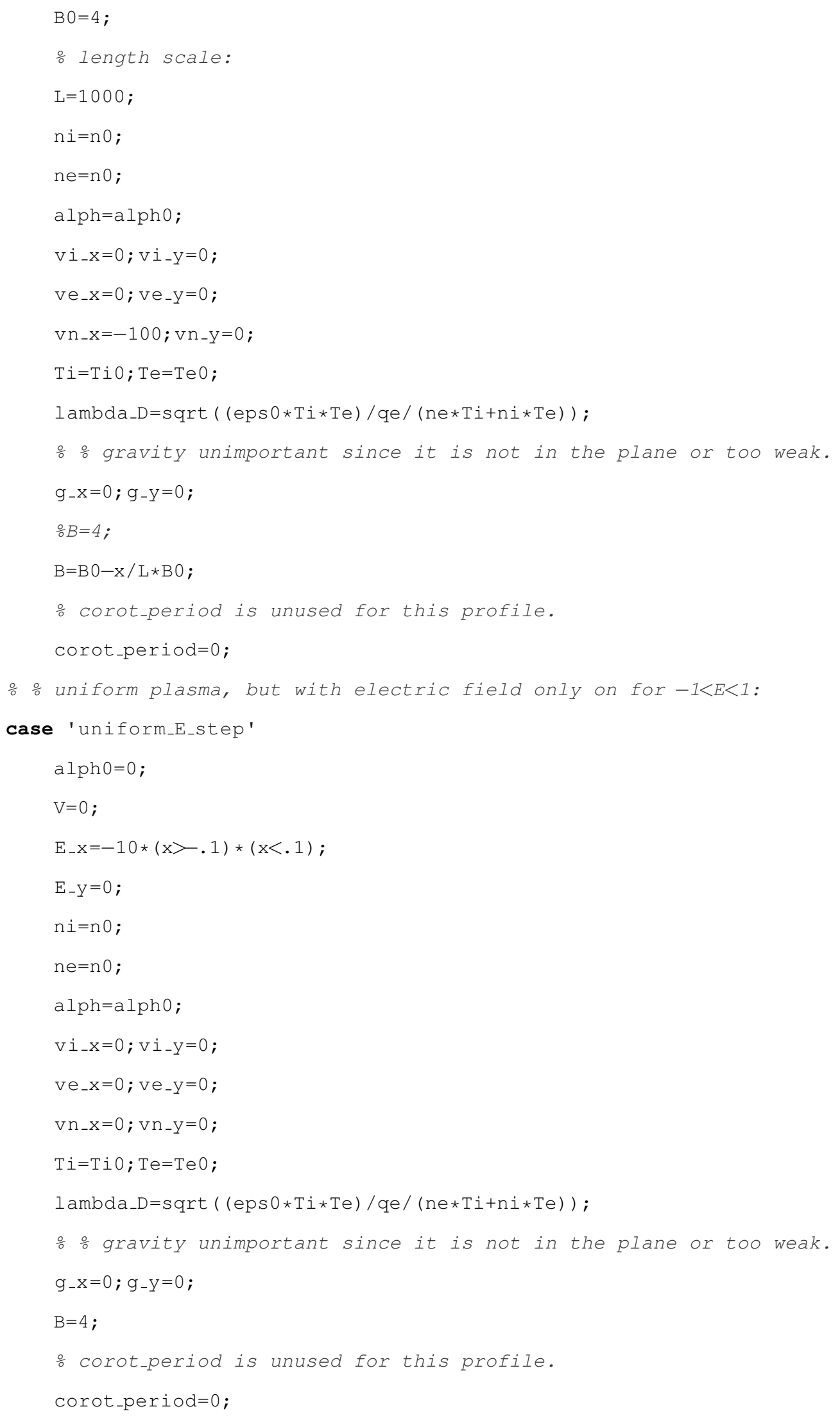




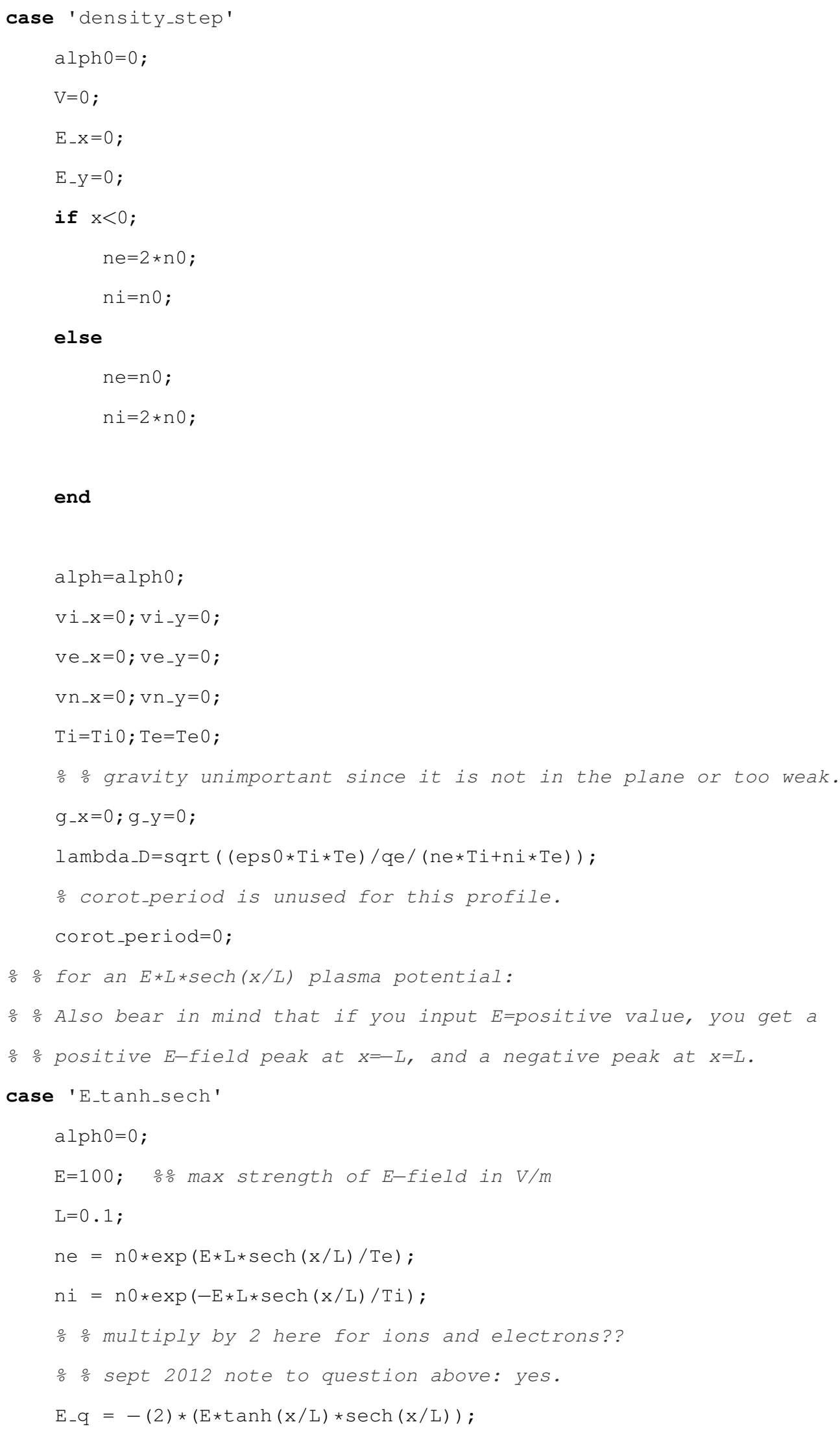




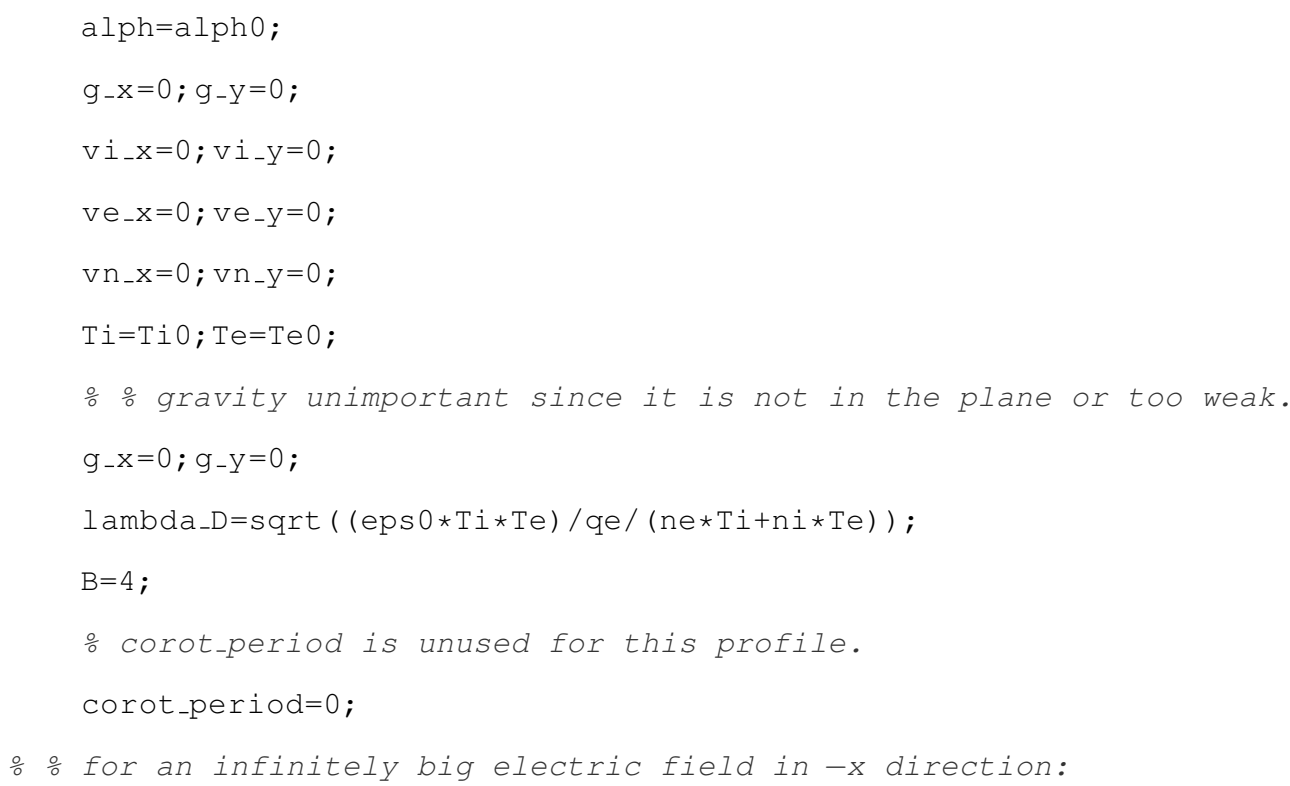




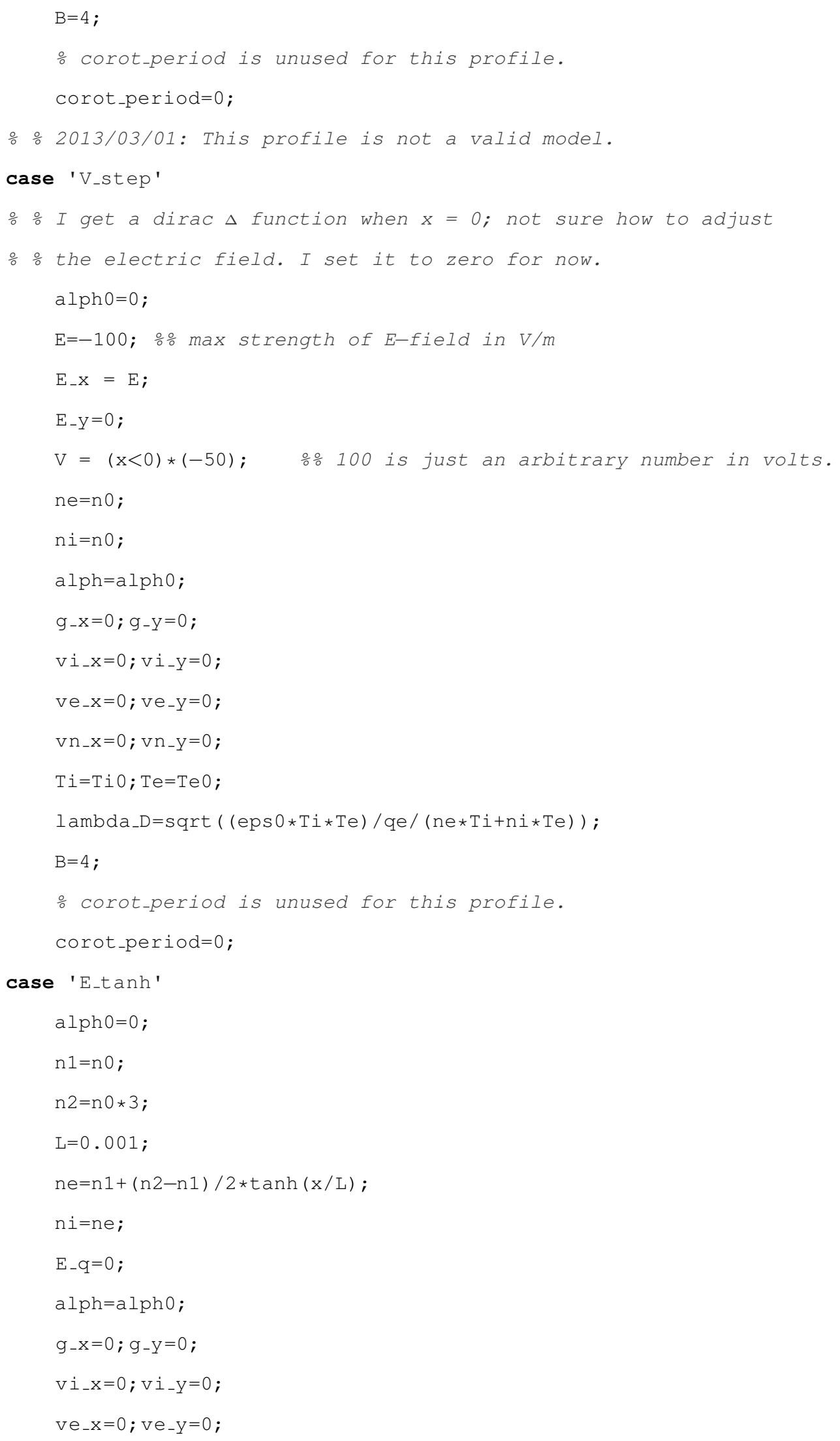




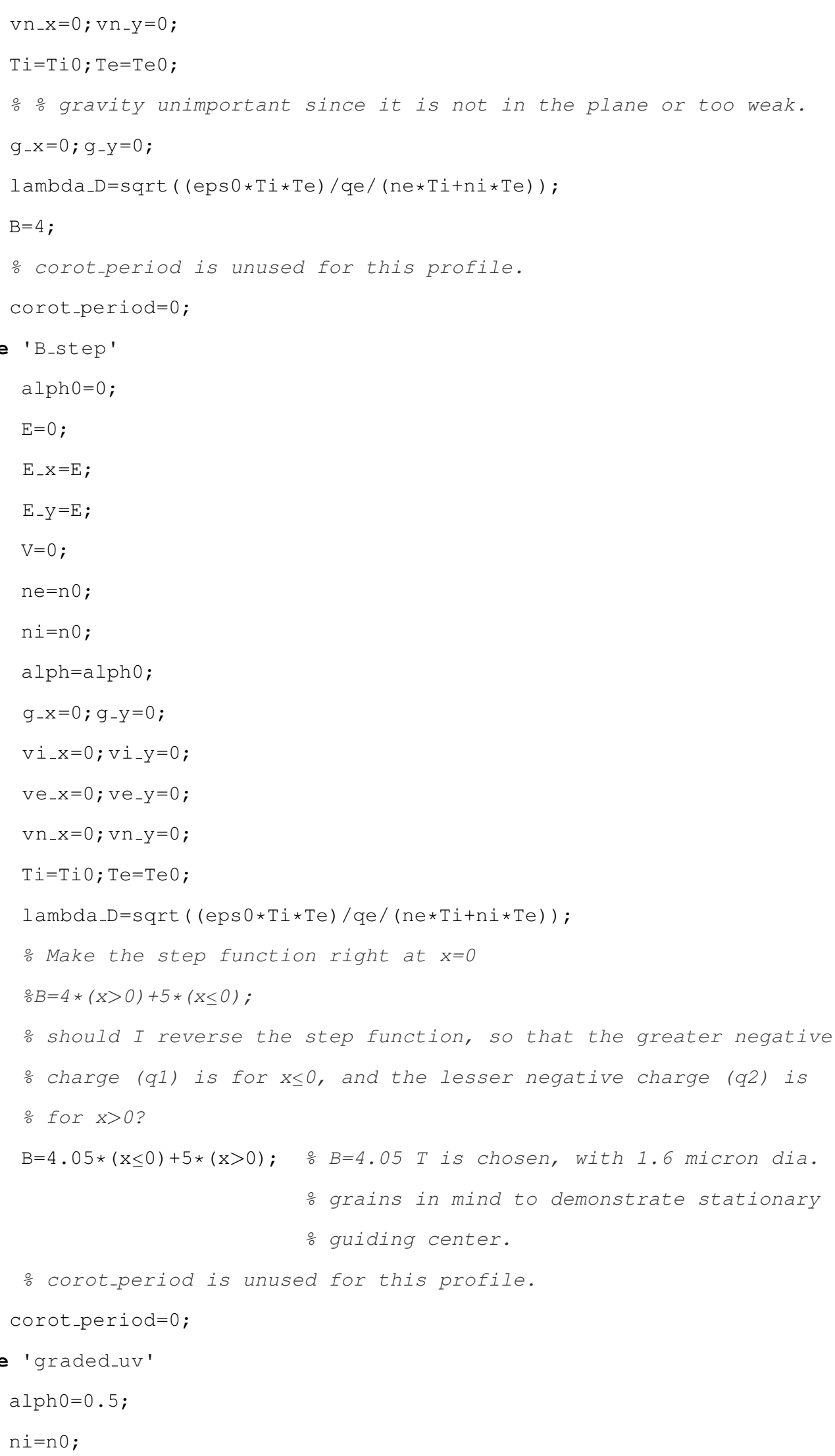




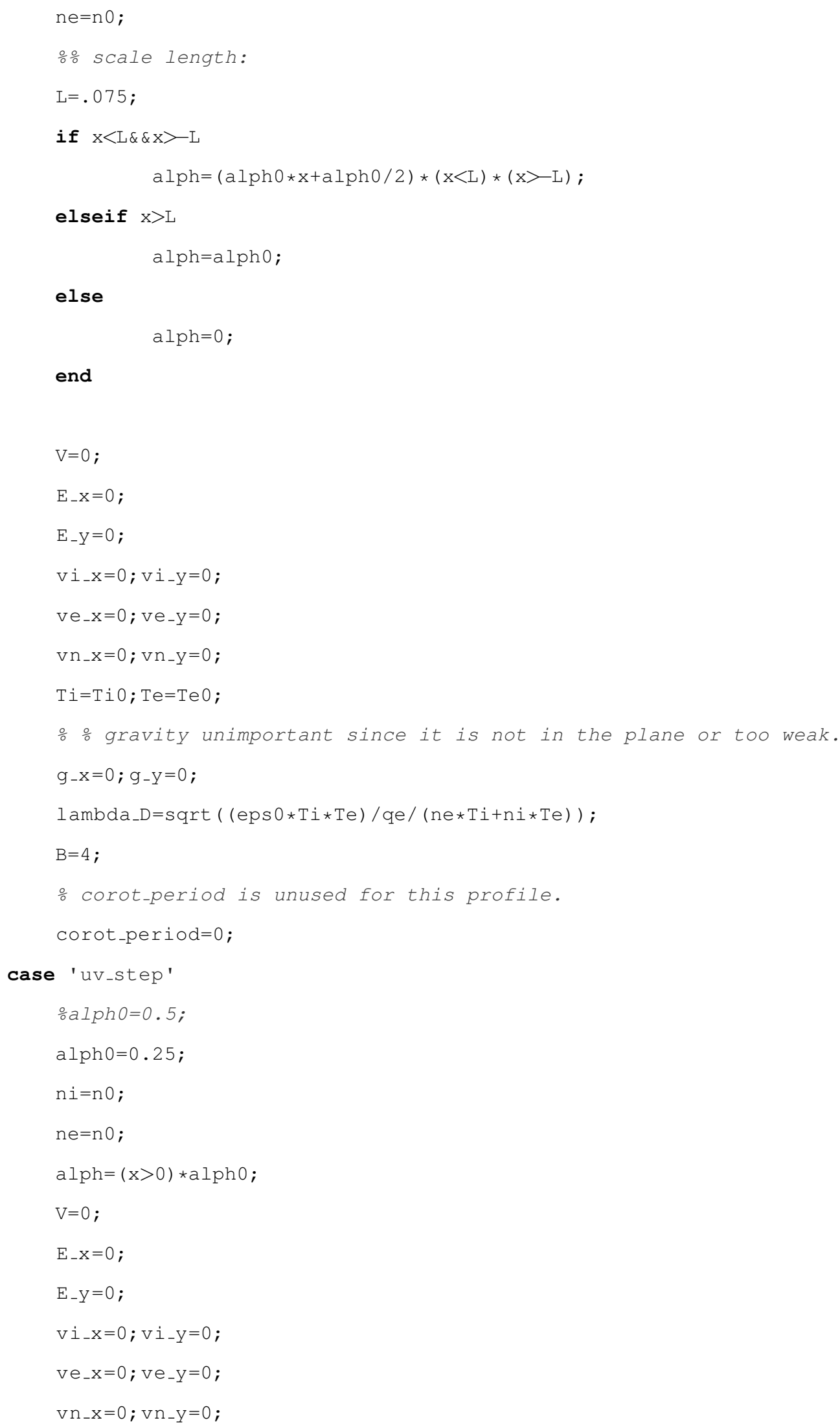




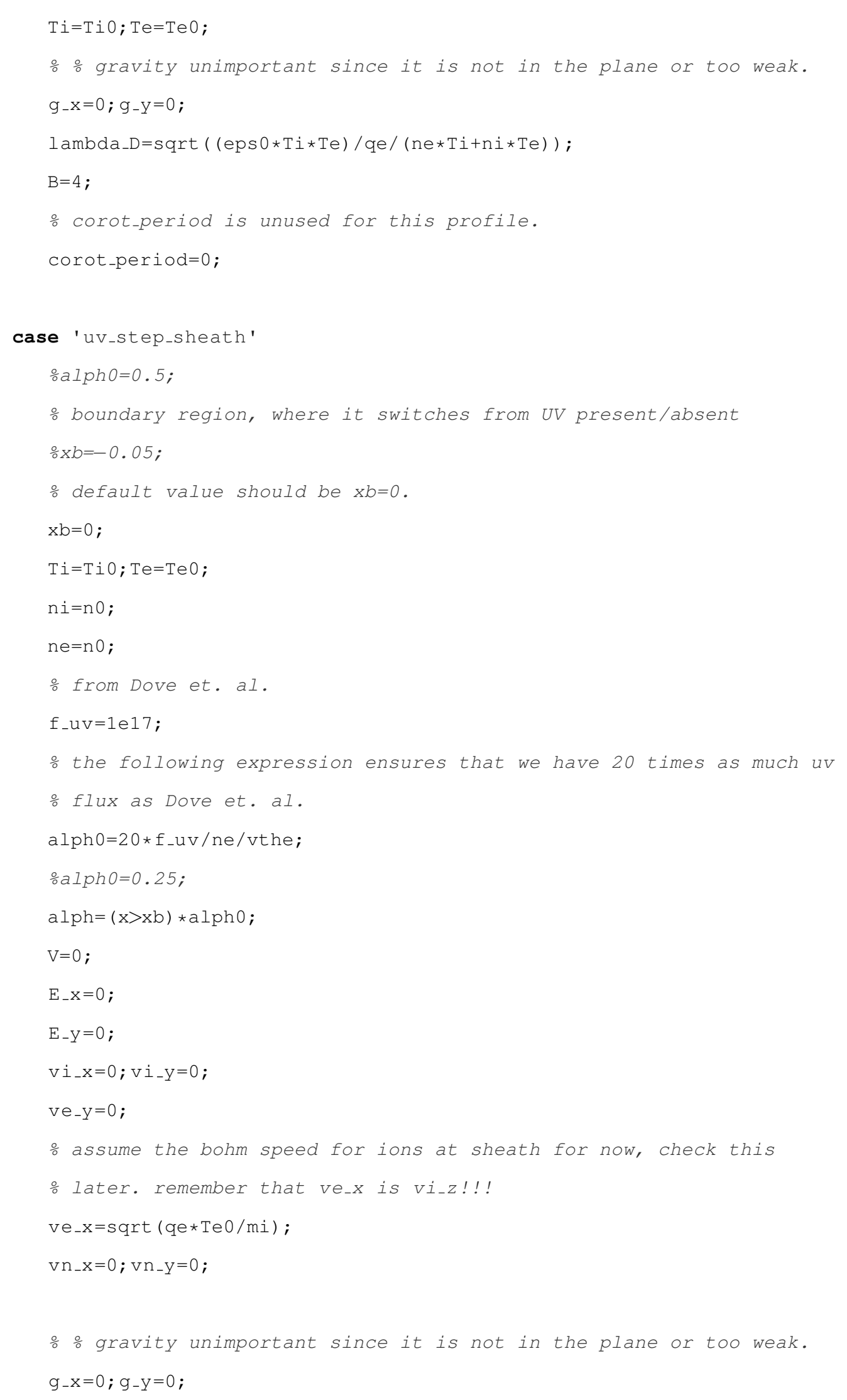




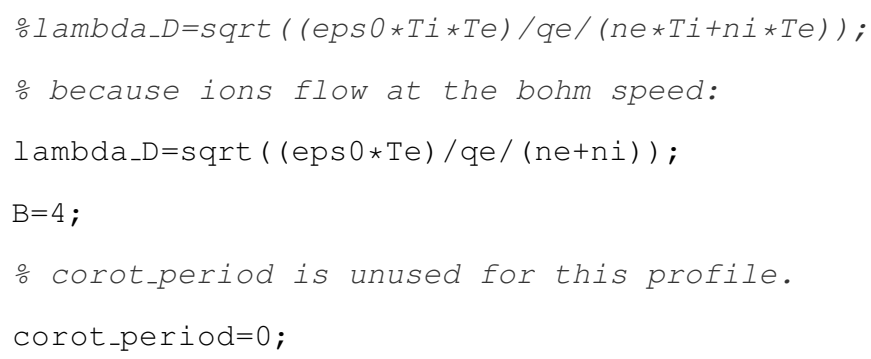




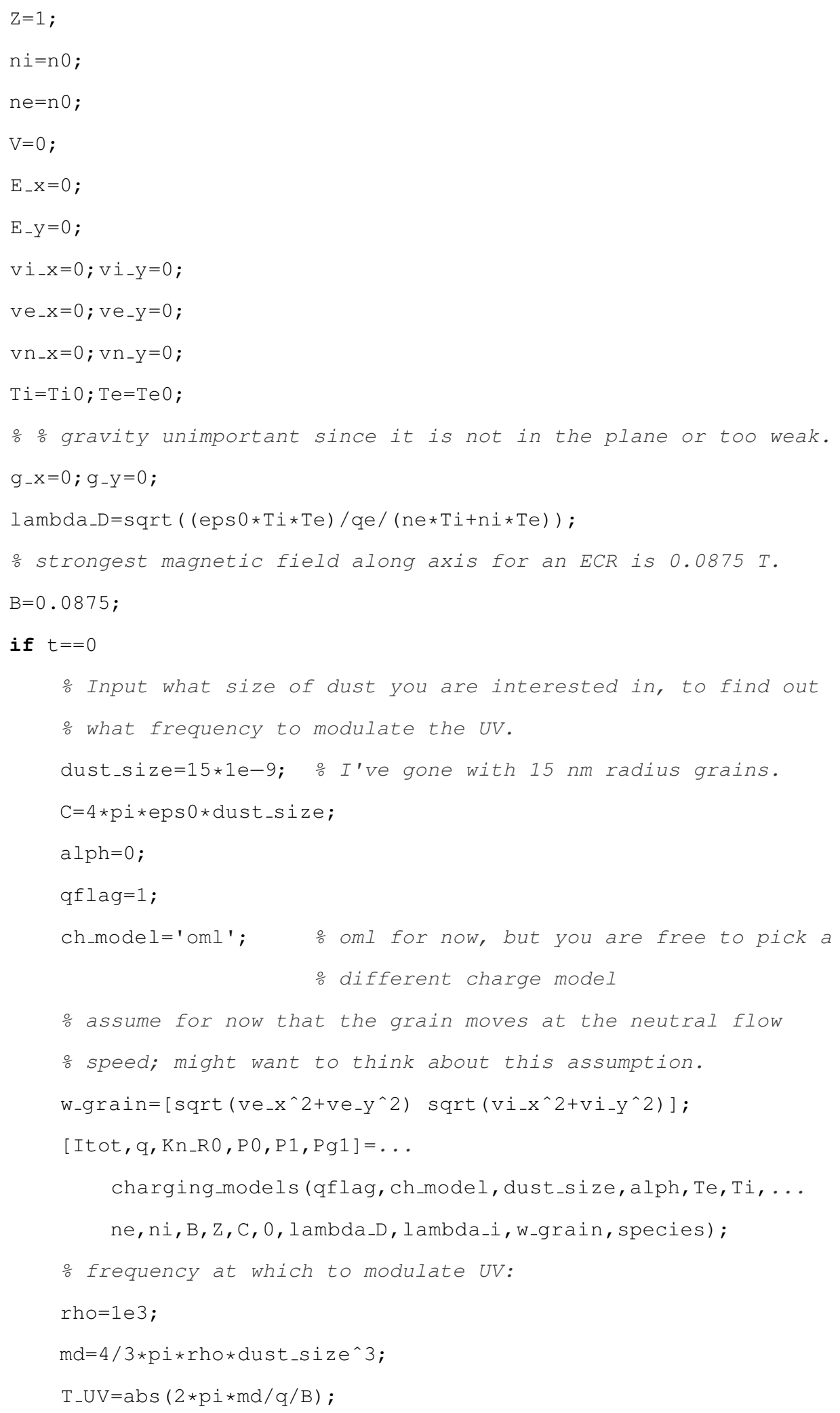


응 CIRCLE!

$\mathrm{T} 1=0.1476 ;$

$\mathrm{T} 2=.3095 ;$

$\therefore T_{-} U V=0.1476$;

$\mathrm{T}_{-} \mathrm{UV}=\mathrm{T} 1 / 2+\mathrm{T} 2 / 2$

o integer multiples of $T_{-} U V$ :

n_whole $=$ floor $\left(t / T_{-} U V\right)$;

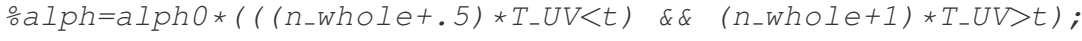

ㅇ I think this bottom line works:

alph=alpho* ( (n_whole *T_UV+T1/2<t) \&\& (n_whole+1)*T_UV>t $)$;

o corot_period is unused for this profile.

corot_period=0;

case 'uv_gradient'

alph0=0.25;

$\mathrm{ni}=\mathrm{n} 0$;

$\mathrm{ne}=\mathrm{n} 0$;

$\mathrm{alph}=(\mathrm{x}>0) * \mathrm{alph} 0$;

$\therefore$ 능

$\frac{\circ}{\circ} L=.0015$;

응 alternatively, use a smaller gradient:

$\mathrm{L}=0.00015$

alph increases from 0 at $x=0$ to a max value at $x=L$, and stays at

․ this max value for $x>L$.

alph=alph $0 *(x \geq L)+a l p h 0 * x / L *(x \geq 0) *(x \leq L) ;$

$\mathrm{V}=0$;

$E_{-} \mathrm{x}=0$;

$E_{-} \mathrm{Y}=0$;

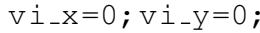

ve_x=0; ve $-\mathrm{y}=0$;

$\mathrm{vn} \_\mathrm{x}=0 ; \mathrm{vn}-\mathrm{y}=0$;

$\mathrm{Ti}=\mathrm{Ti} 0 ; \mathrm{Te}=\mathrm{Te} 0$;

응 gravity unimportant since it is not in the plane or too weak.

$g-\mathrm{x}=0 ; \mathrm{g}-\mathrm{y}=0$;

lambda_D $=\operatorname{sqrt}((\operatorname{eps} 0 * \mathrm{Ti} * \mathrm{Te}) / \mathrm{qe} /($ ne $* \mathrm{Ti}+\mathrm{ni} * \mathrm{Te}))$;

o corot_period is unused for this profile. 
corot_period=0;

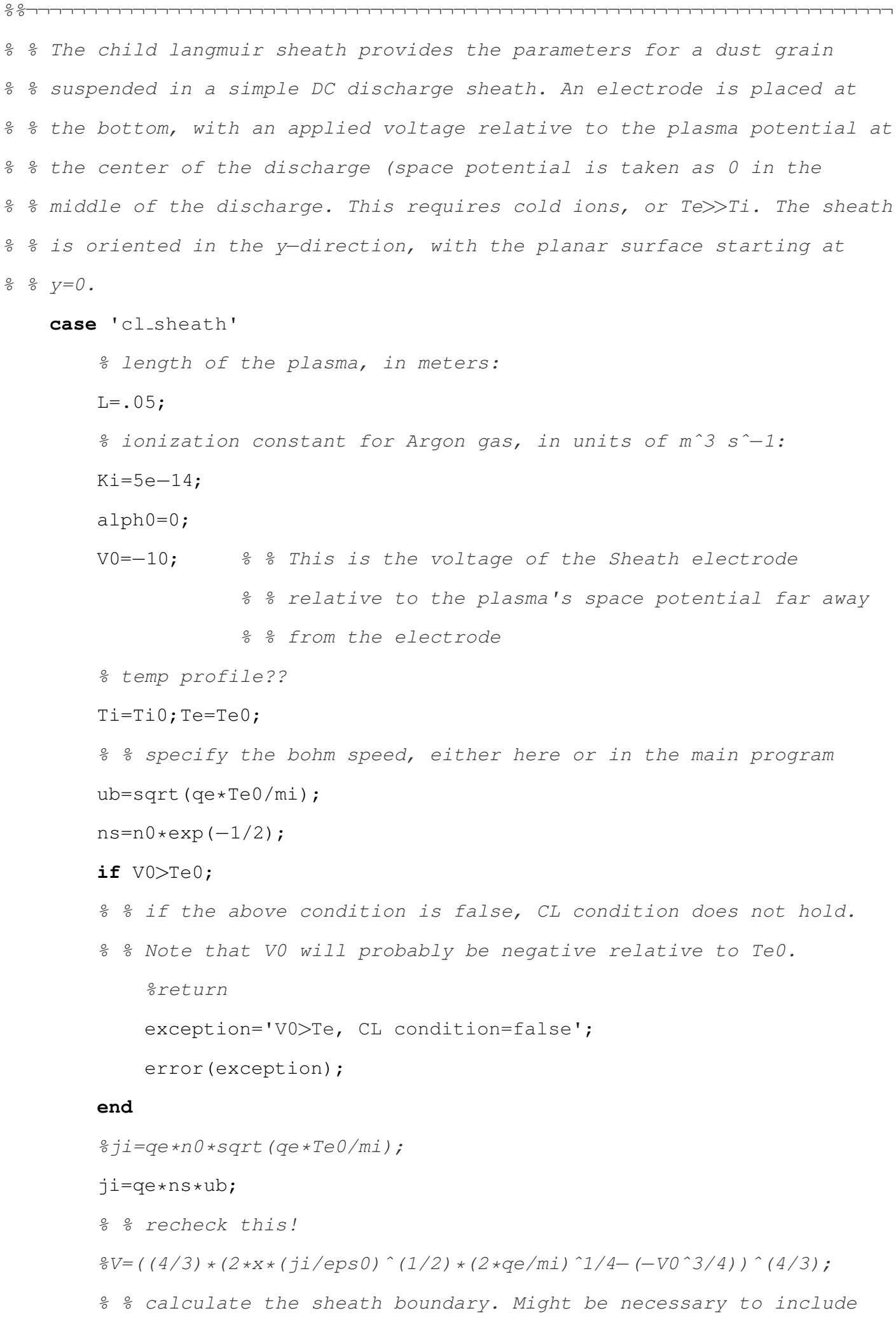




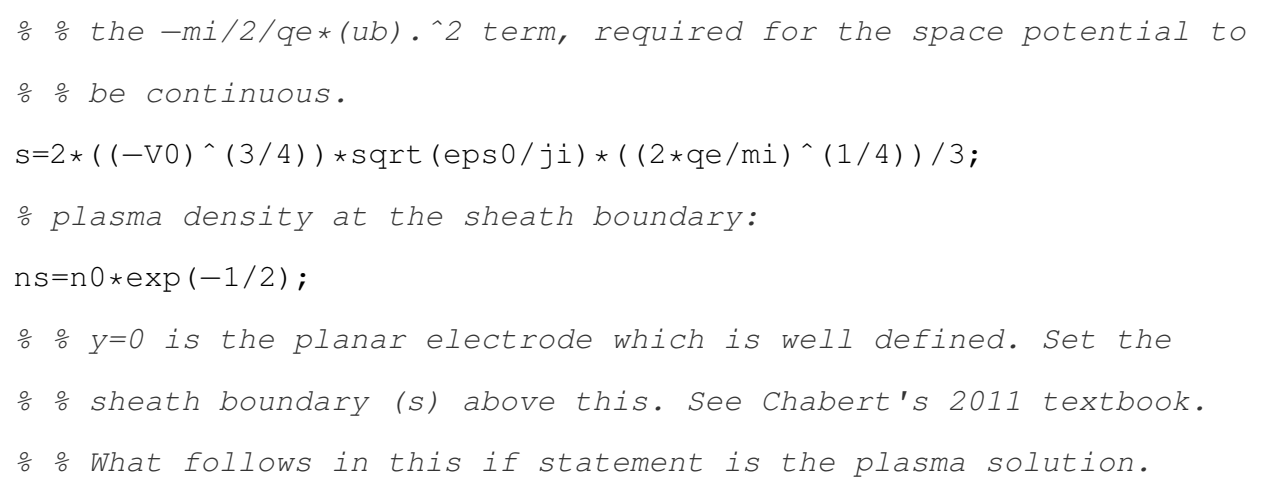




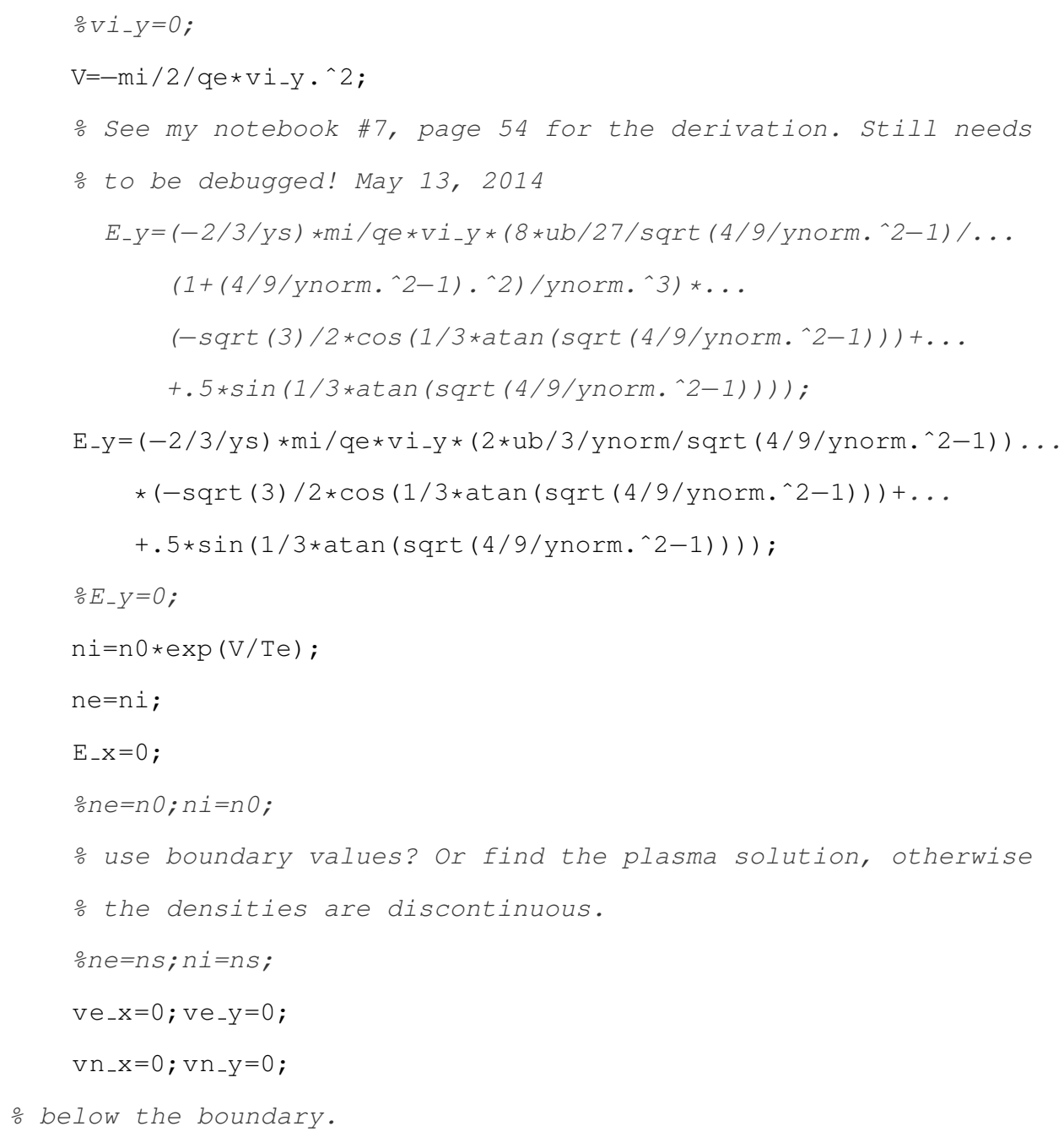




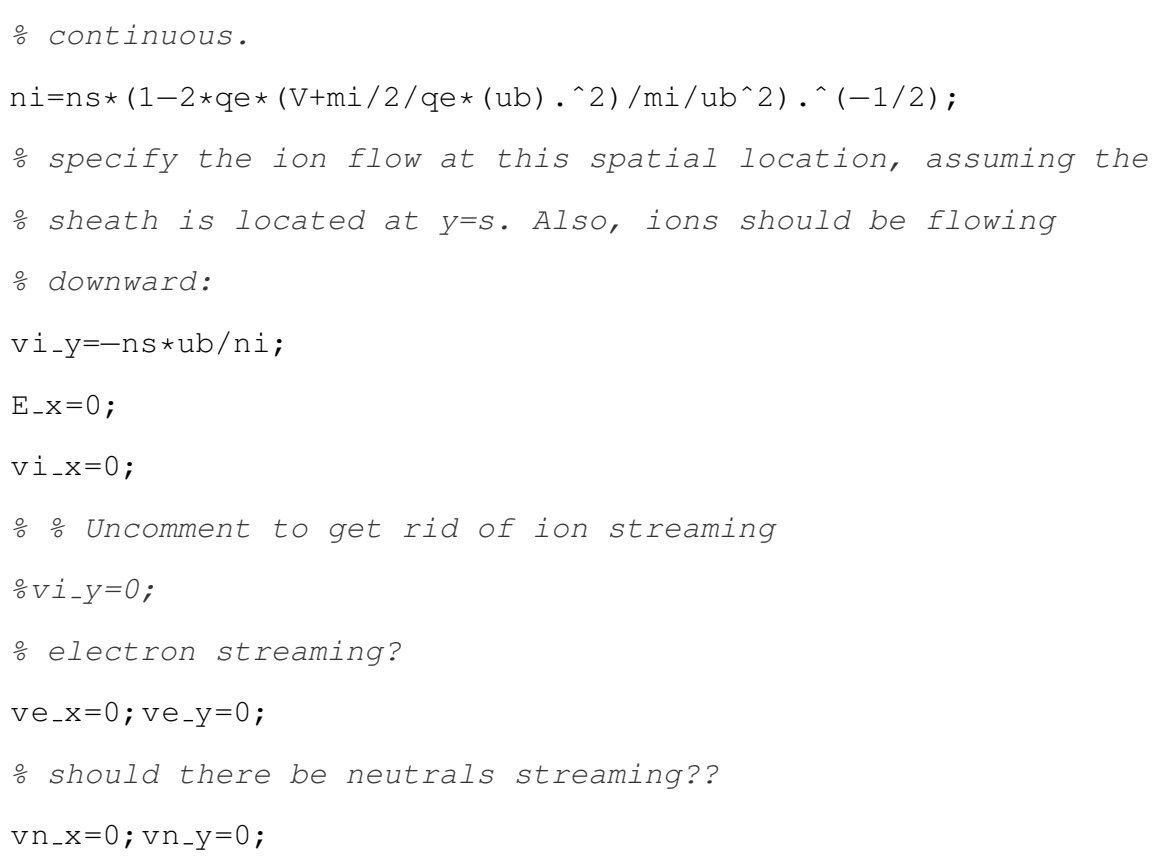




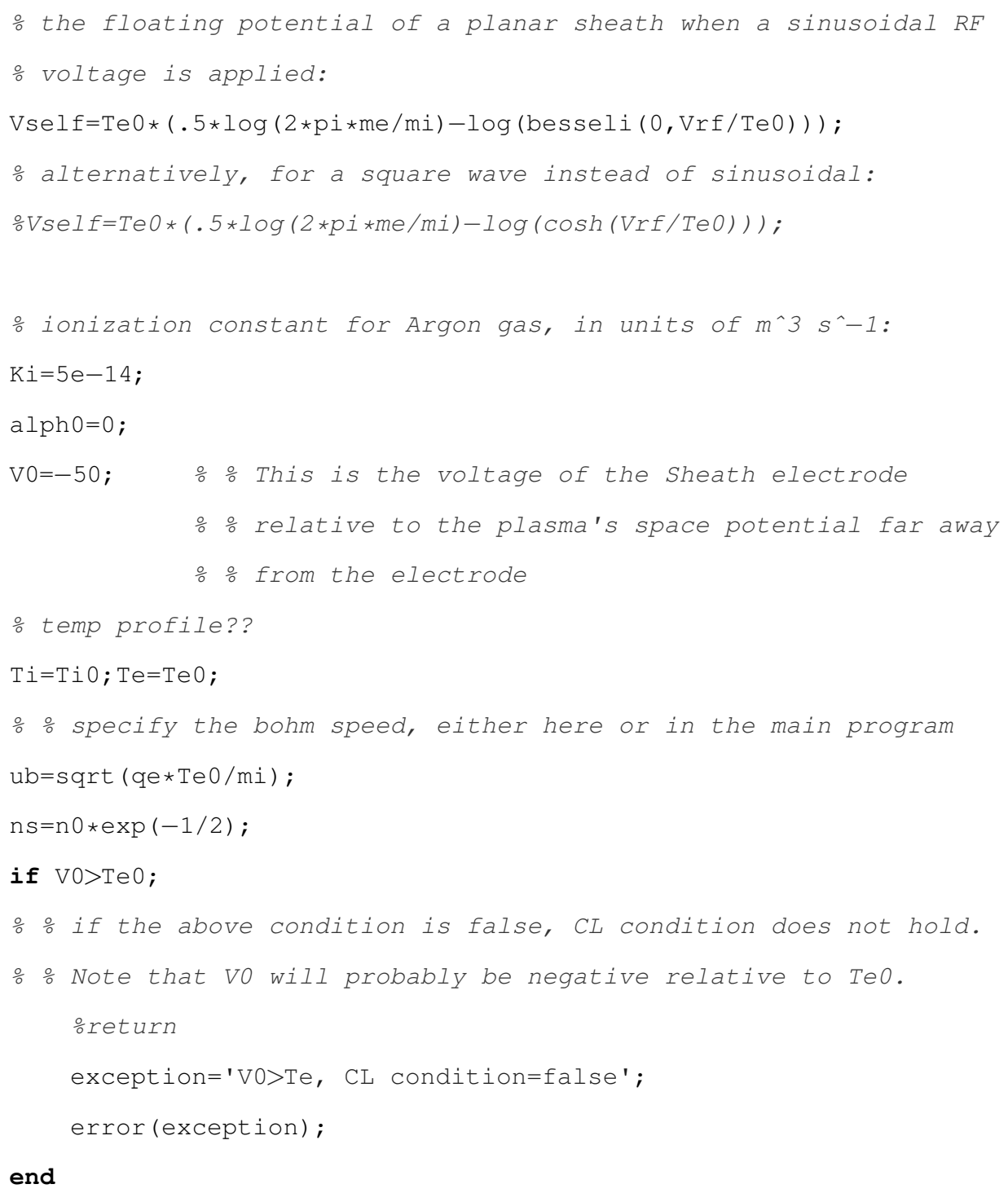




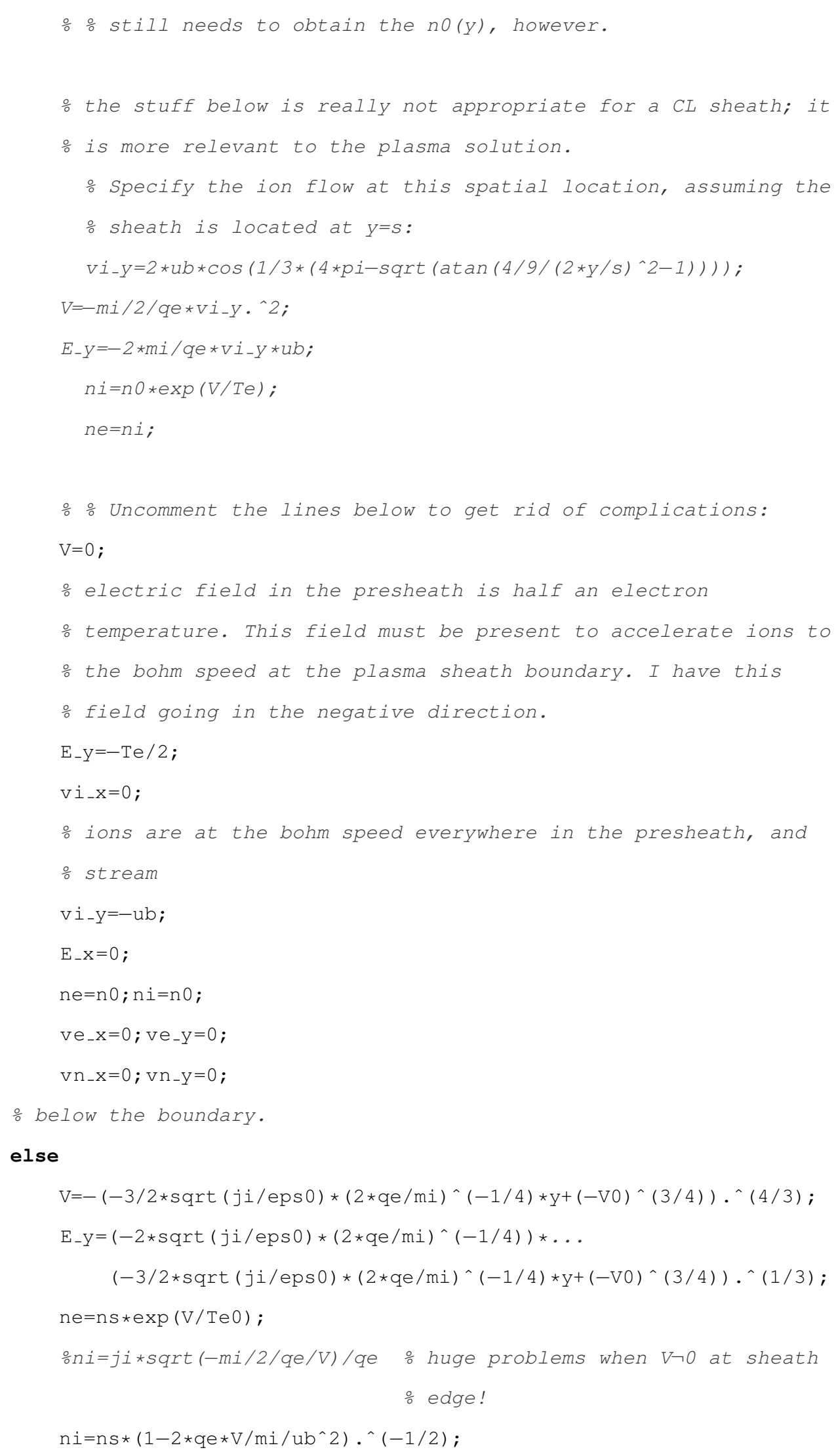




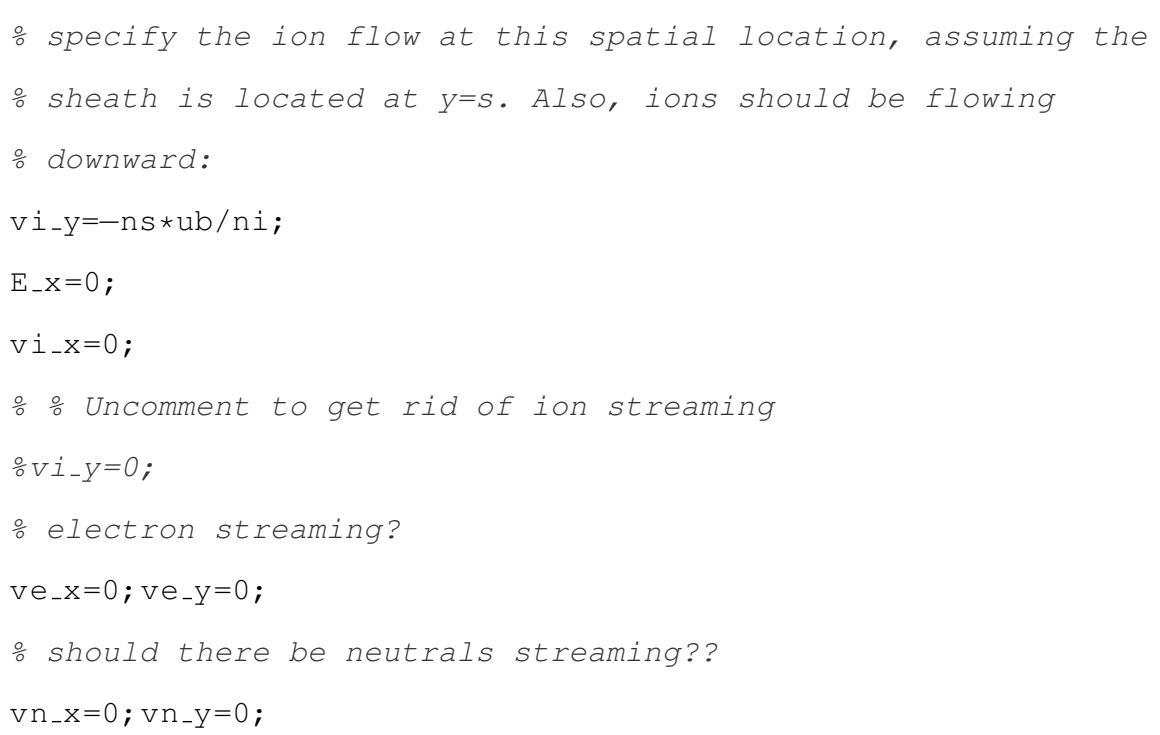




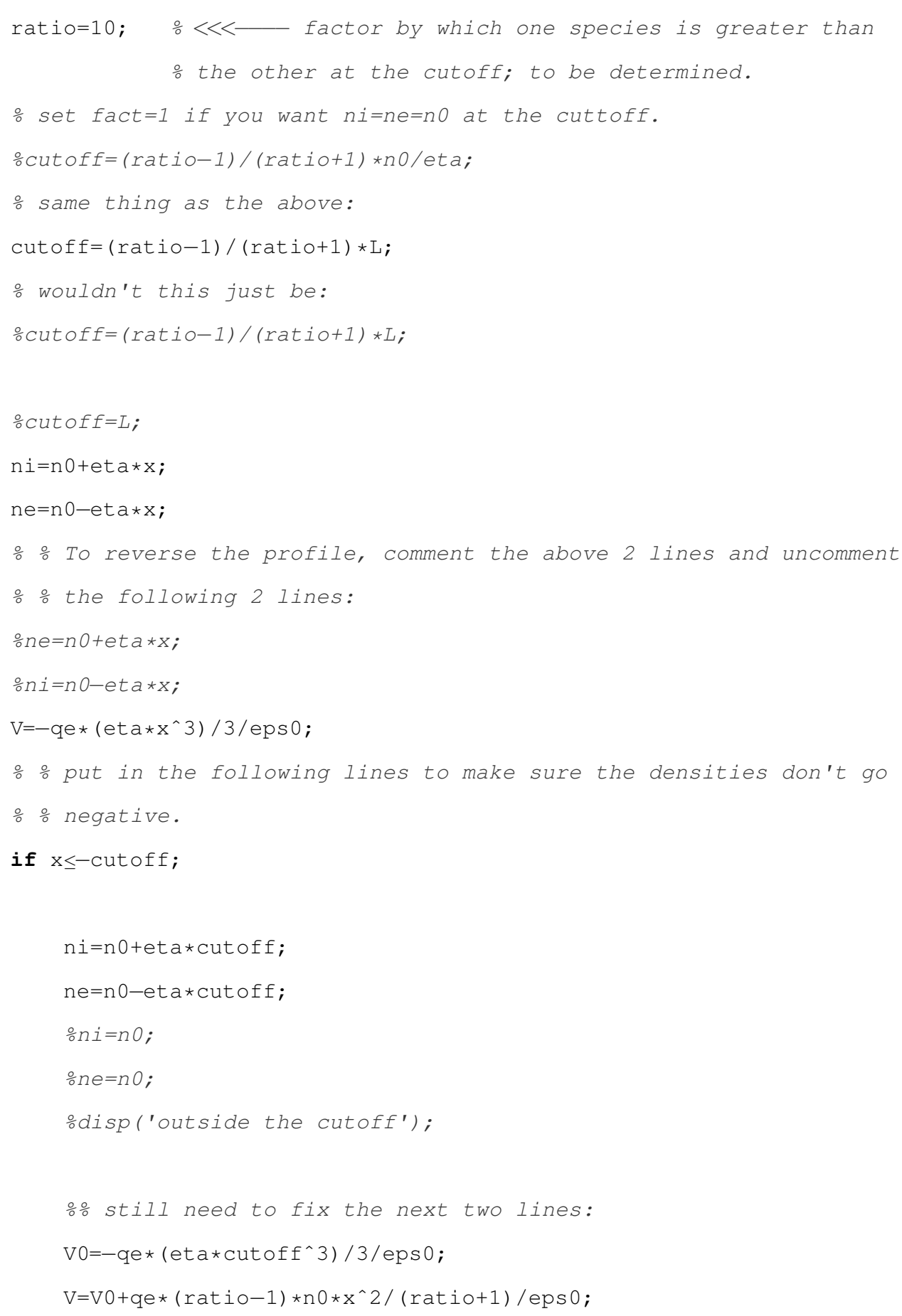




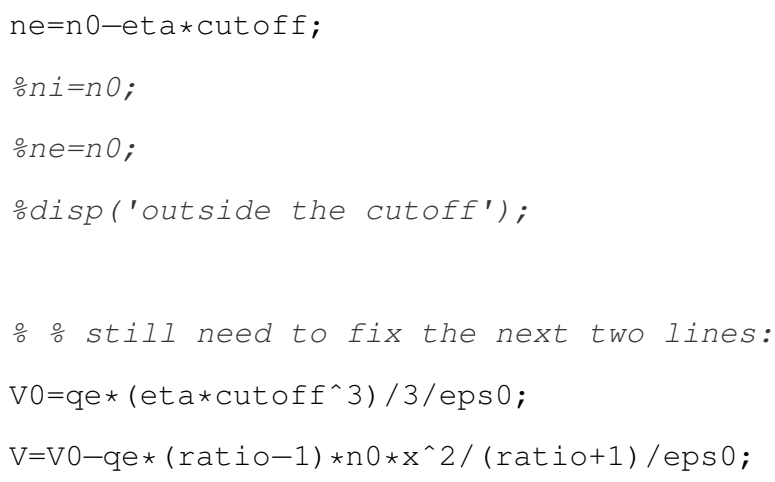




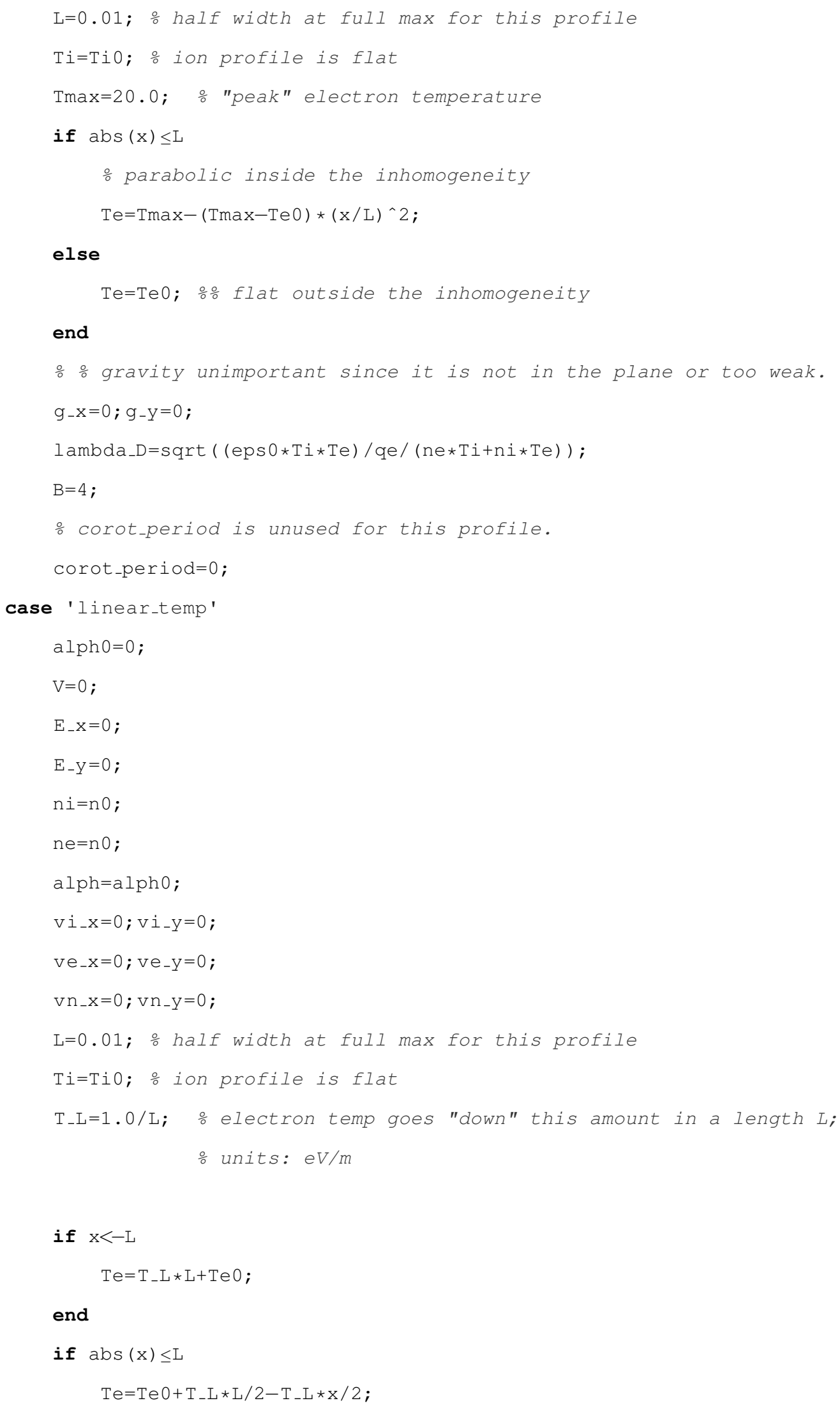




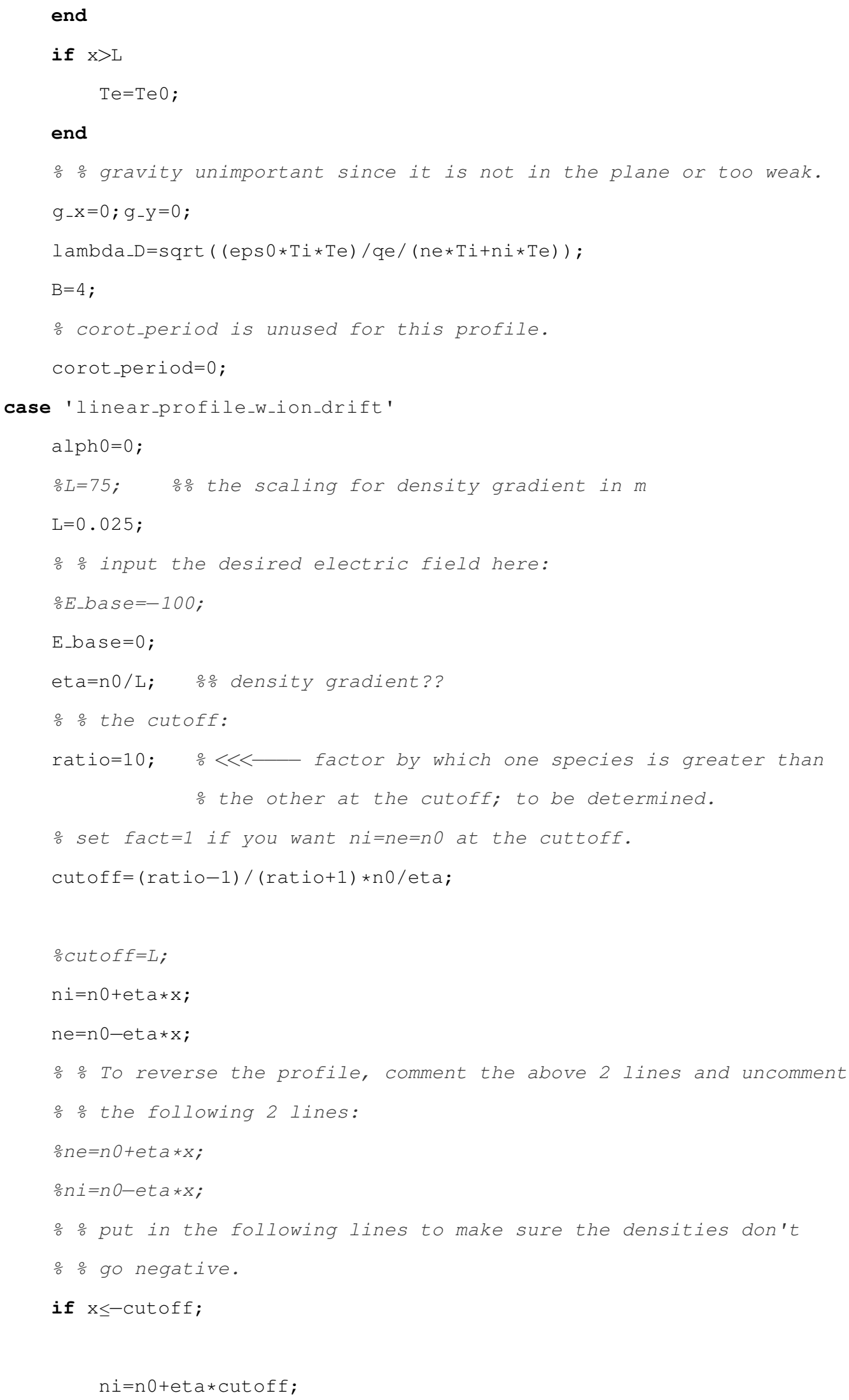


ne $=$ no-eta $*$ cut off

은 $n O$;

을 $=$ i

odisp('outside the cutoff');

end

if $x \geq$ cutoff;

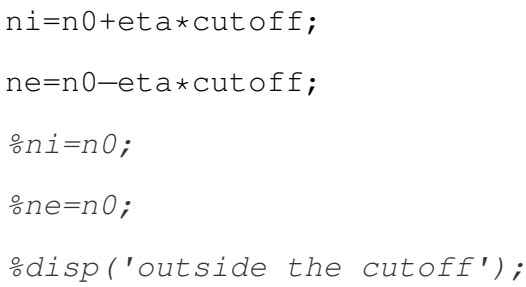


\% radius of the experimental volume, in meters

$\mathrm{R}=0.225$;

This corresponds exactly with the "central experimental volume"

on the Auburn machine; the smaller, "uniform region" radius is

$\div 0.1$

Er $=-100 ;$

$\mathrm{ni}=\mathrm{n} 0$;

$\mathrm{ne}=\mathrm{n} 0 ;$

radius $=\operatorname{sqrt}\left(\mathrm{x}^{\wedge} 2+\mathrm{y}^{\wedge} 2\right)$;

$\mathrm{alph}=0 ;$

$\mathrm{V}=0$;

$\mathrm{E}_{-} \mathrm{x}=\mathrm{E} r * \cos (\mathrm{phi})$;

$E_{-} \mathrm{Y}=\mathrm{E} r * \sin (\mathrm{phi})$;

$\mathrm{vi}-\mathrm{x}=0 ; \mathrm{vi}-\mathrm{y}=0$;

$v e_{-} \mathrm{x}=0 ; \mathrm{ve}-\mathrm{y}=0$;

응 If you want to use a sheath:

assume the bohm speed for ions at sheath for now, check this

o later. remember that ve-x is $v i_{-} z ! ! !$

ve_x $=\operatorname{sqrt}(q e * \mathrm{Te} 0 / \mathrm{mi})$;

$\mathrm{vn} \_\mathrm{x}=0 ; \mathrm{vn}-\mathrm{y}=0$;

$\mathrm{Ti}=\mathrm{Ti} 0 ; \mathrm{Te}=\mathrm{Te} 0$;

$\mathrm{Ti}=\mathrm{Ti} 0 ; \mathrm{Te}=\mathrm{Te} 0$

lambda_D $=\operatorname{sqrt}((\operatorname{eps} 0 * \mathrm{Ti} * \mathrm{Te}) / \mathrm{qe} /(\mathrm{ne} * \mathrm{Ti}+\mathrm{ni} * \mathrm{Te}))$

응 gravity unimportant since it is not in the plane or too weak.

$g_{-} \mathrm{x}=0 ; \mathrm{g}_{-} \mathrm{y}=0$;

$\mathrm{B}=4$;

o corot-period is unused for this profile.

corot_period=0;

case 'cylindrical_profile'

$\mathrm{B}=4$;

$\therefore$ 응 rius of the experimental volume, in meters

$\mathrm{R}=0.225$;

This corresponds exactly with the "central experimental volume"

음 on the Auburn machine; the smaller, "uniform region" radius is

$\div 0.1$ 


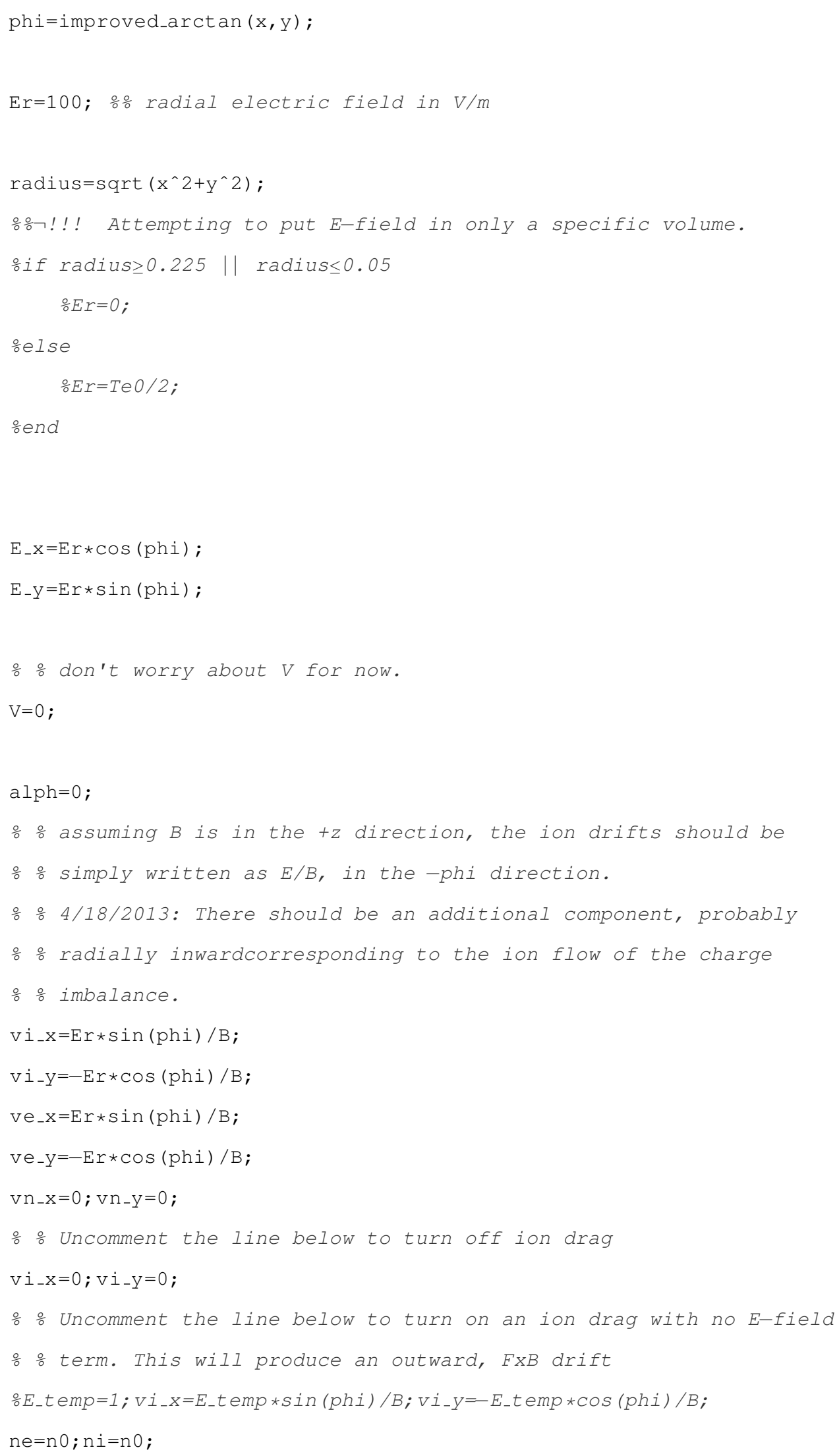


$\mathrm{Ti}=\mathrm{Ti} 0 ; \mathrm{Te}=\mathrm{Te} 0$;

응 gravity unimportant since it is not in the plane or too weak.

$g_{-} \mathrm{x}=0 ; \mathrm{g}_{-} \mathrm{Y}=0$

lambda_D $=\operatorname{sqrt}((\operatorname{eps} 0 * \mathrm{~T} i \star \mathrm{Te}) / \mathrm{qe} /(\mathrm{ne} * \mathrm{~T} i+\mathrm{n} i \star \mathrm{Te}))$;

o corot period is unused for this profile.

corot_period=0;

case 'linear_cylindrical_profile'

응 This is like the linear-profile, but instead this is in

응 cylindrical geometry

$\div$ ㅇaius of the experimental volume, in meters

$\mathrm{R}=0.225$

응 This corresponds exactly with the "central experimental volume"

o on the Auburn machine; the smaller, "uniform region" radius is

응 0.1

phi=improved_arctan $(x, y)$;

radius $=\operatorname{sqrt}\left(\mathrm{x}^{\wedge} 2+\mathrm{y}^{\wedge} 2\right)$

$\mathrm{R} 1=0.15 ;$

$\mathrm{R} 2=0.175 ;$

$\mathrm{dR}=\mathrm{R} 2-\mathrm{R} 1 ; \quad \div \mathrm{R} 1$ and $\mathrm{R} 2$ have been chosen such that $\mathrm{dR}=0.025$ meters.

o keep in mind that the center of the gradient, where

$\therefore$ ne=ni is at $R 0=0.1625$; use this as an initial

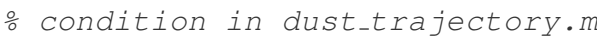

$\mathrm{R} 0=\mathrm{R} 1+\mathrm{dR} / 2$; $\frac{\circ}{0}$ center of the density gradient

ratio=10; 는 ratio signifies by how many times larger is the

ㅇ electron density than the ion density at the lower

o cutoff, and by what factor the ion density is larger

o than the electron density at the upper cutoff.

o lower radius cutoff:

r_lower $=(\mathrm{R} 1+(1-$ ratio $) * \mathrm{dR}+$ ratio $* \mathrm{R} 2) /(1+$ ratio $)$;

o upper radius cutoff;

r_upper=2*R0-r_lower; 


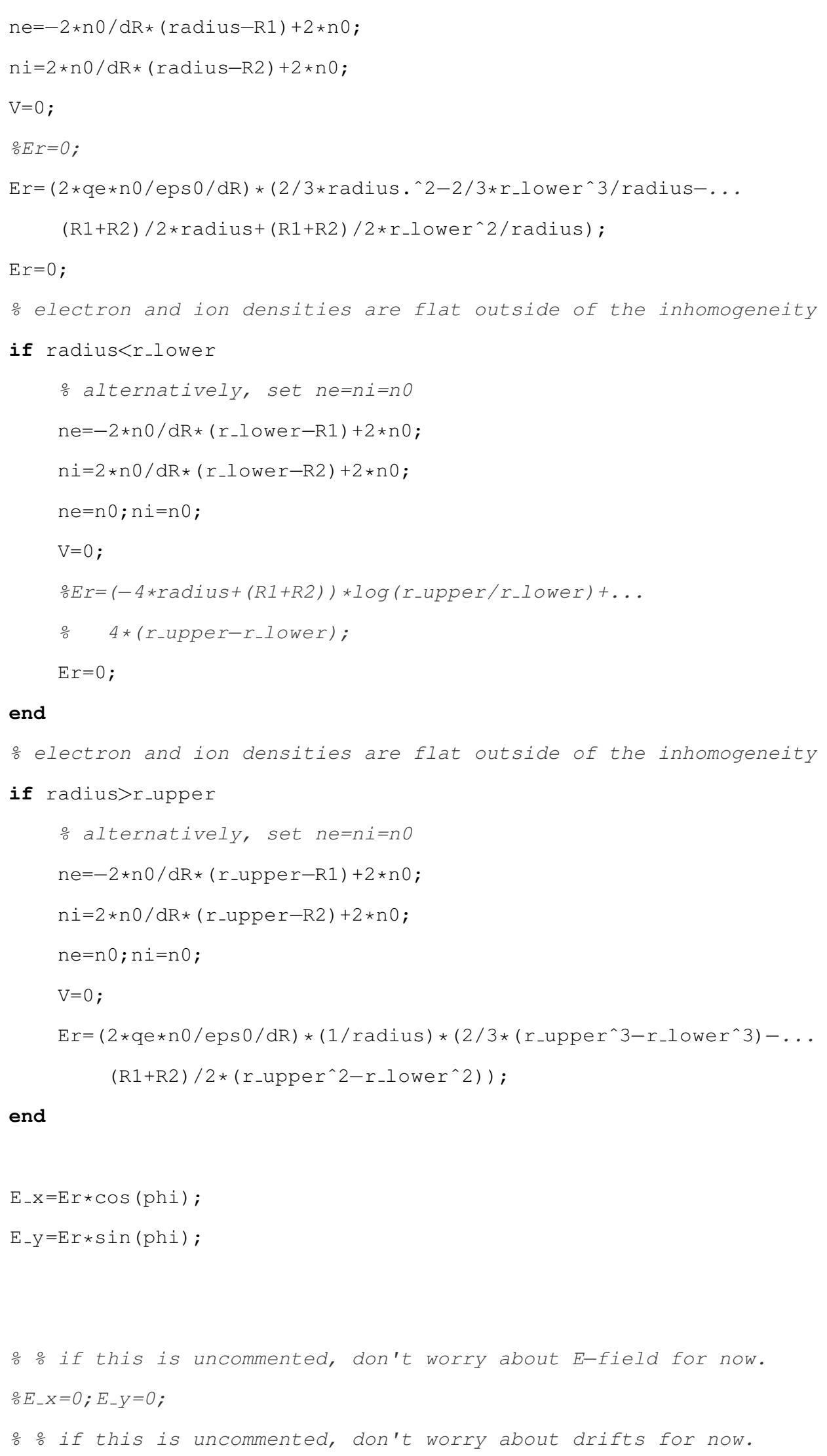




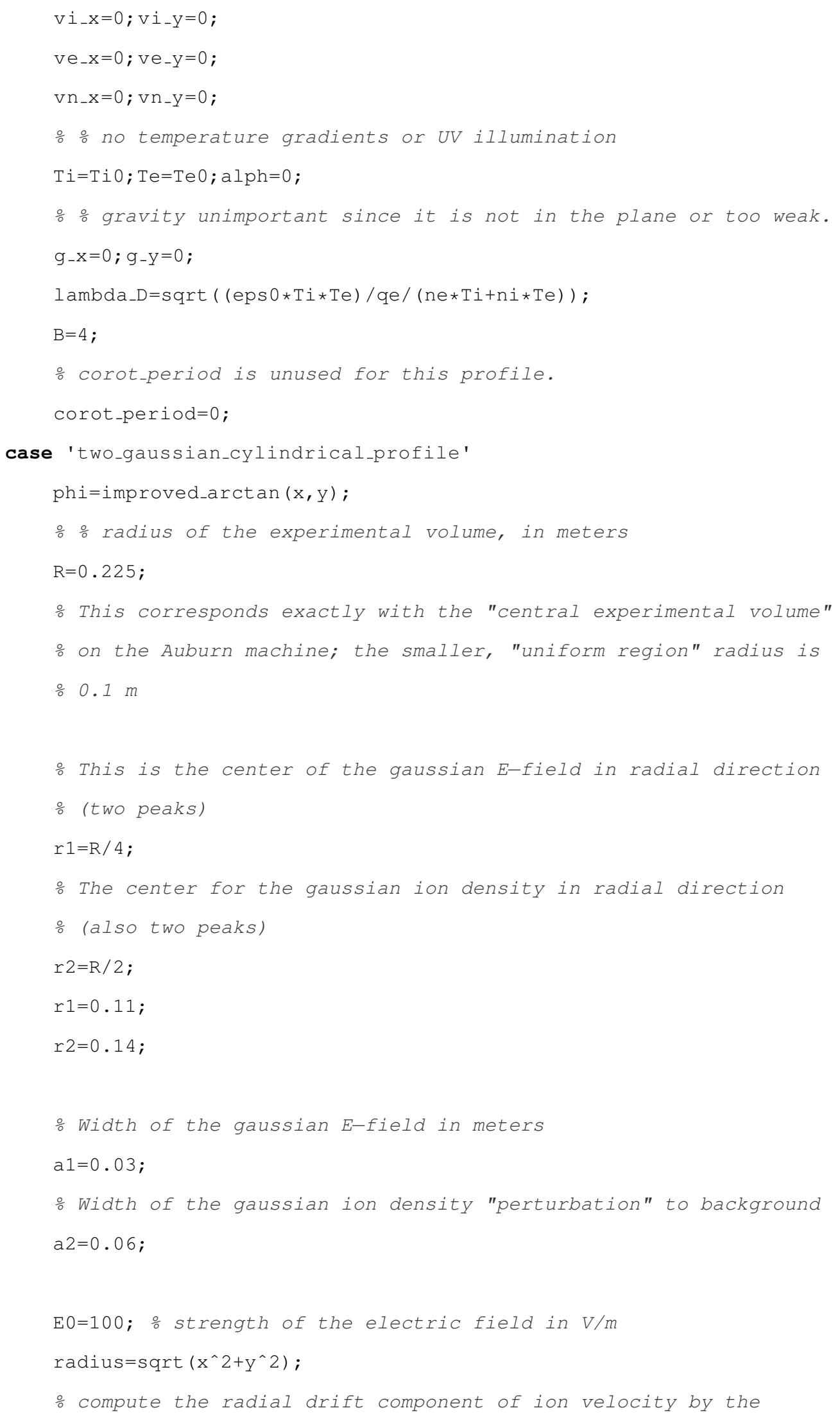




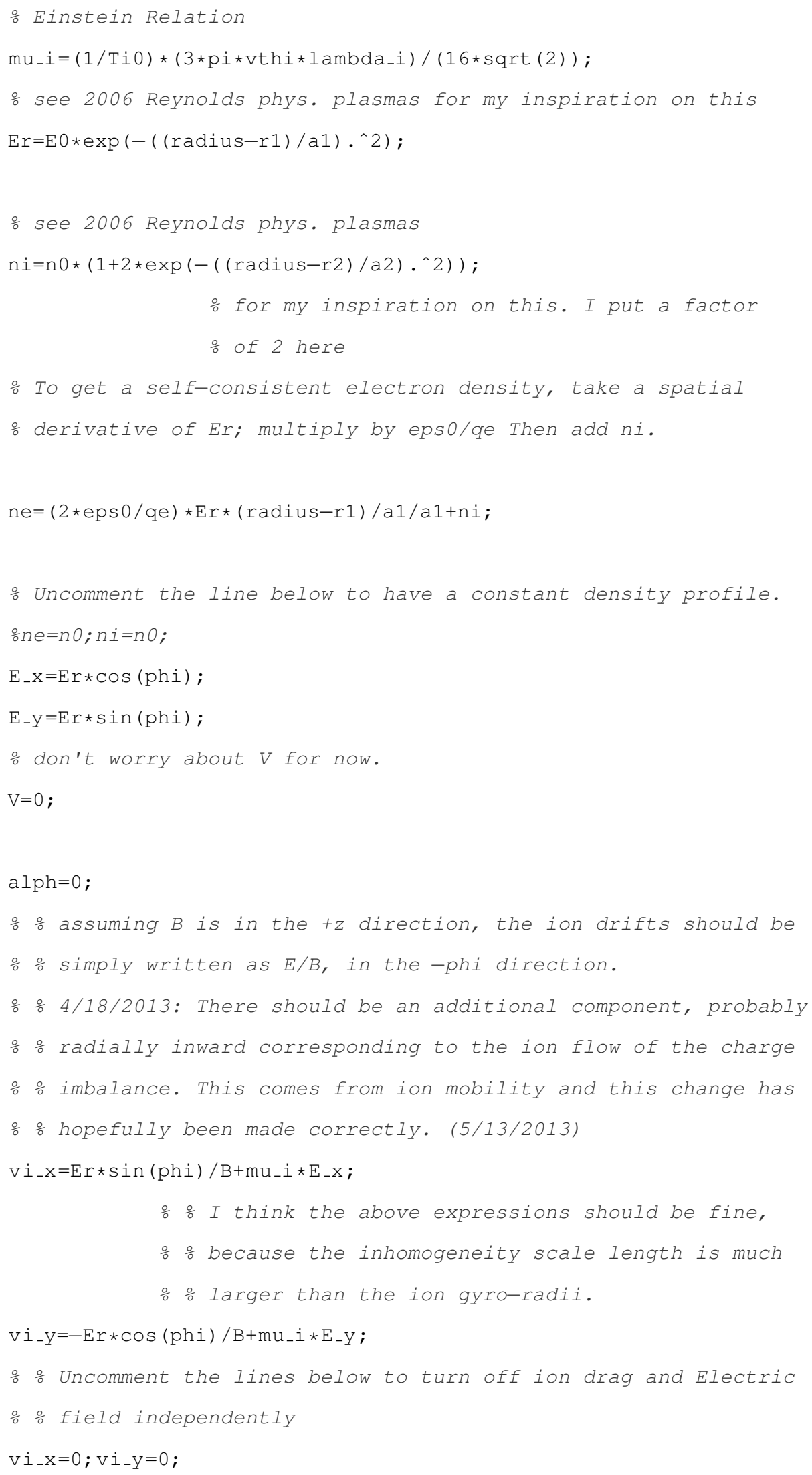


응 Not sure what to do with electron terms; temporarily set to

$\frac{\circ}{0}$ zero.

$\mathrm{ve} \mathrm{x}_{\mathrm{x}}=0 ; \mathrm{ve} \mathrm{e}_{-} \mathrm{y}=0$;

$\mathrm{vn} \_\mathrm{x}=0 ; \mathrm{vn}-\mathrm{y}=0$;

$\therefore E_{-} X=0 ; E_{-} y=0$;

$\mathrm{Ti}=\mathrm{Ti} 0 ; \mathrm{Te}=\mathrm{Te} 0$;

응 gravity unimportant since it is not in the plane or too weak.

$g_{-} \mathrm{x}=0 ; \mathrm{g}_{-} \mathrm{Y}=0$;

lambda_D $=\operatorname{sqrt}((\operatorname{eps} 0 * \mathrm{Ti} * \mathrm{Te}) / \mathrm{qe} /(\mathrm{ne} * \mathrm{~T} i+\mathrm{n} i * \mathrm{Te}))$;

$\mathrm{B}=4$ ；

o corot_period is unused for this profile.

corot_period=0;

case 'parabolic_cylindrical_profile'

phi=improved_arctan $(\mathrm{x}, \mathrm{y})$;

응 radius of the experimental volume, in meters

응 This corresponds exactly with the "central experimental volume"

$\mathrm{R}=0.225$;

응 on the Auburn machine; the smaller, "uniform region" radius is

$\div 0.1$

$\div \frac{\circ}{\circ}$ The radius at which $E \neg 0$. If $r>r_{-}$field, $E=0$, space potential is

$\frac{\circ}{0}$ constant.

r_field=0.075;

응 the space potential at the edge of the parabolic profile.

V_edge $=2.5$;

$\div$ 응 potential when $r=0$.

V_max $=25$;

$\frac{\circ}{0} 0=1 e 2 ; \quad \frac{\circ}{0}$ strength of the electric field in $\mathrm{V} / \mathrm{m}$

radius $=\operatorname{sqrt}\left(\mathrm{x}^{\wedge} 2+\mathrm{y}^{\wedge} 2\right)$;

if radius $\leq r_{-}$field

Er $=-2 *\left(V_{-}\right.$edge-V_max $) *$ radius/r_field/r_field;

$\mathrm{ne}=\mathrm{n} 0$;

$\mathrm{ni}=\mathrm{n} 0$;

$\div \frac{0}{0}$ assuming $B$ is in the $+z$ direction, the ion drifts should be 
응 simply written as $E / B$, in the -phi direction.

응 4/18/2013: There should be an additional component,

응 probably radially inward corresponding to the ion flow of

응 the charge imbalance.

vi_x=Er*sin(phi)/B; $\frac{\circ}{0}$ I think these expressions should be

응 fine, because the inhomogeneity scale

응 length is much larger than the ion

응 gyro-radii.

vi-y=-Er*Cos $(p h i) / B$;

응 Uncomment the line below to turn off ion drag

$\frac{\circ}{\partial} i_{-} x=0 ; v i_{-} y=0$;

else

Er=0；

응 4/18/2013: There should be an additional component,

응 probably radially inward corresponding to the ion flow of

응 the charge imbalance.

$\mathrm{vi}$ - $_{-}=0$

$\mathrm{vi}-\mathrm{y}=0$;

$\mathrm{ne}=\mathrm{n} 0$;

$\mathrm{ni}=\mathrm{n} 0$;

end

응 Uncomment the line below to have a constant density profile.

$\therefore n e=n O ; n i=n O ;$

$\mathrm{E} \_\mathrm{X}=\mathrm{E} r * \operatorname{Cos}(\mathrm{phi})$;

$\mathrm{E}_{-} \mathrm{Y}=\mathrm{Er} * \sin (\mathrm{phi})$;

$\div$ on't worry about $V$ for now.

$\mathrm{V}=0$;

alph=0;

$\mathrm{Ti}=\mathrm{Ti} 0 ; \mathrm{Te}=\mathrm{Te} 0$;

응 gravity unimportant since it is not in the plane or too weak.

$g_{-} \mathrm{x}=0 ; g_{-} \mathrm{y}=0$;

lambda_D $=\operatorname{sqrt}((\operatorname{eps} 0 * \mathrm{Ti} * \mathrm{Te}) / \mathrm{qe} /(\mathrm{ne} * \mathrm{~T} i+\mathrm{n} i * \mathrm{Te}))$;

응 not sure what to do with electron terms; temporarily set to

$\therefore$ zero. 


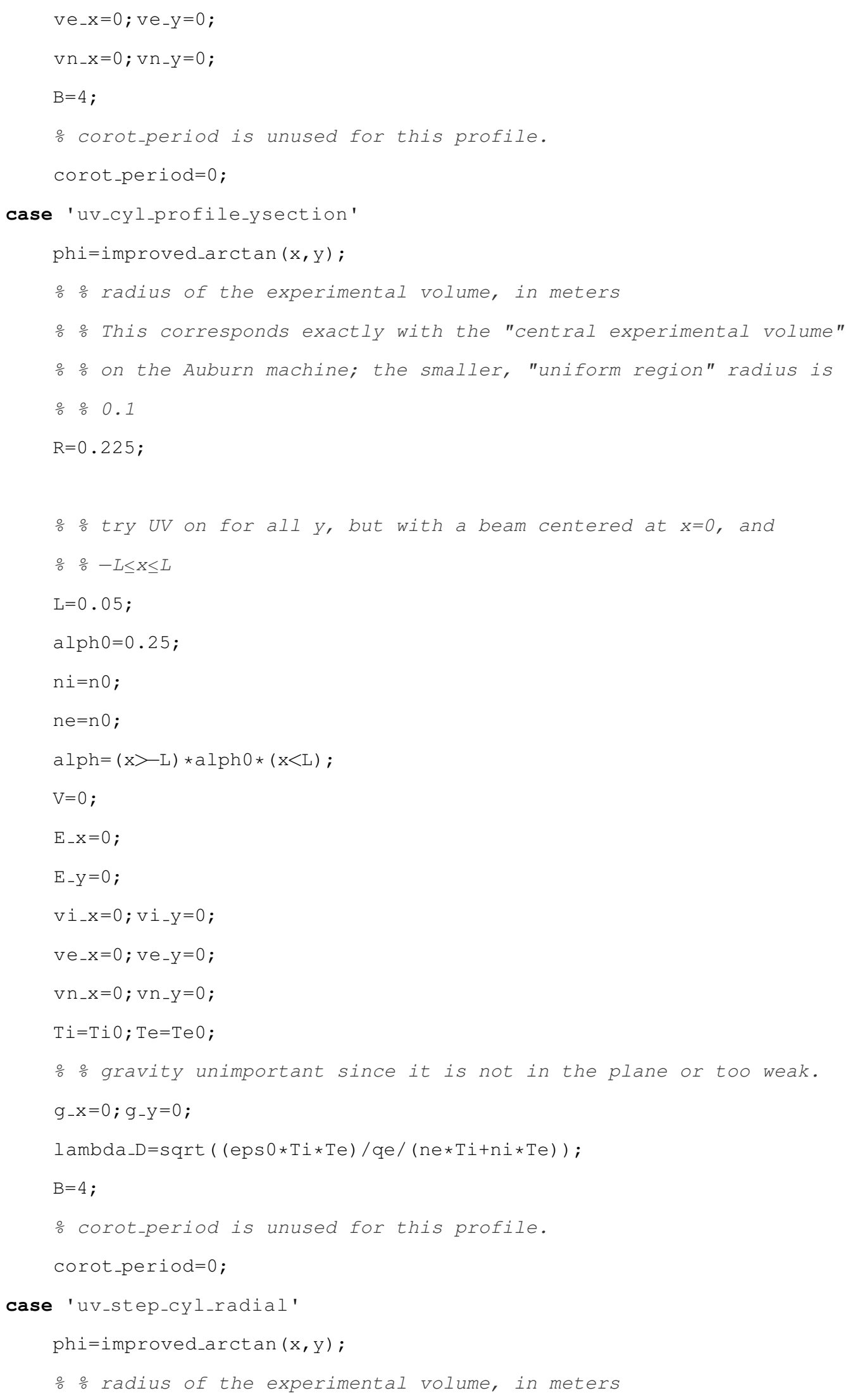




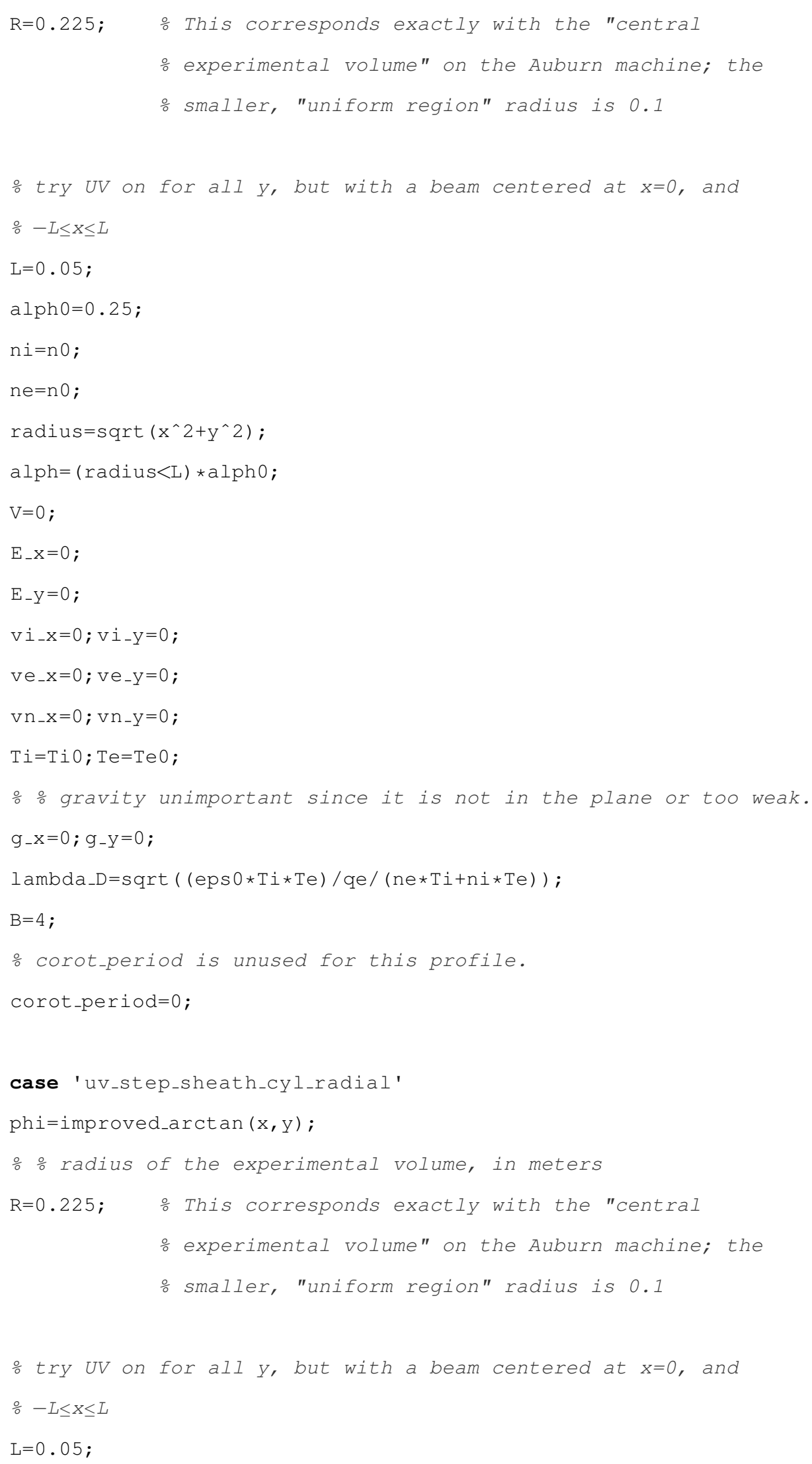




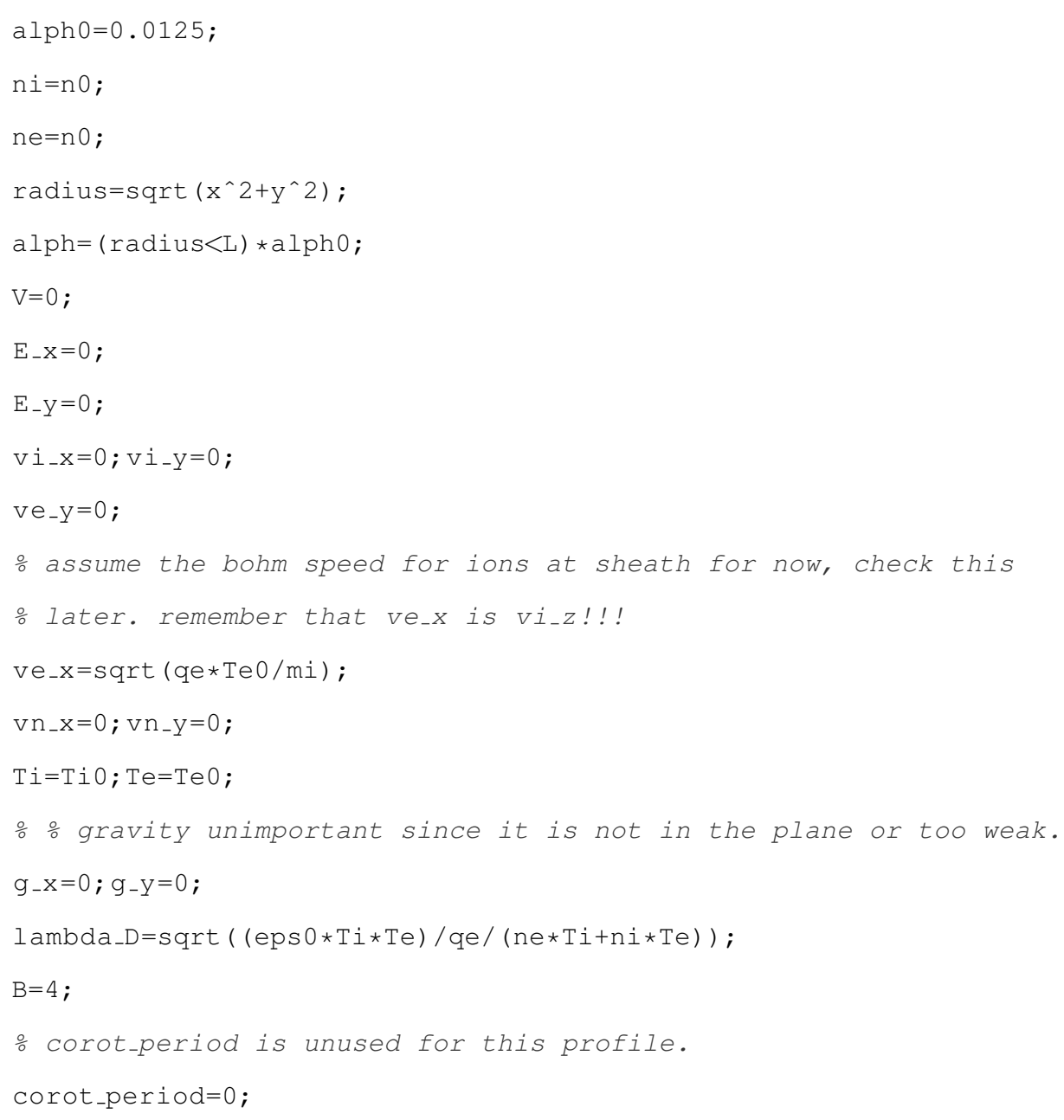


- Using a parabola for now, try gaussian or sech later.

$\mathrm{Te}=\mathrm{Te} \_\mathrm{max}-\left(\mathrm{Te} \_\mathrm{max}-\mathrm{T} \_\right.$edge $) *(\operatorname{radius} / \mathrm{R}) \cdot{ }^{\wedge} 2$;

$\mathrm{Ti}=\mathrm{T} i \_m a x-\left(\mathrm{Ti}\right.$ max $-\mathrm{T} \_$edge $) *(\operatorname{radius} / \mathrm{R}) \cdot{ }^{\wedge} 2$;

if radius $>R$

$\mathrm{Te}=\mathrm{T}_{-}$edge $;$

$\mathrm{Ti}=\mathrm{T}_{-}$edge ;

end

능 the density profiles are nearly flat, provided $r \leq R$.

$\mathrm{ni}=\mathrm{n} 0$

$\mathrm{ne}=\mathrm{n} 0$;

\% fill in electric field and ion drift information later.

$\mathrm{V}=0$;

$E_{-} \mathrm{X}=0$;

$\mathrm{E}_{-} \mathrm{Y}=0$;

$\mathrm{vi}-\mathrm{x}=0 ; \mathrm{vi}-\mathrm{y}=0$;

ve_x $=0 ; \mathrm{ve}-\mathrm{y}=0$;

$\mathrm{vn} \mathrm{x}_{-}=0 ; \mathrm{v} \mathrm{n}_{-} \mathrm{y}=0$;

$\mathrm{alph}=0$

응 gravity unimportant since it is not in the plane or too weak.

$g_{-} \mathrm{X}=0 ; \mathrm{g}_{-} \mathrm{y}=0$;

lambda_D $=\operatorname{sqrt}((\operatorname{eps} 0 * \mathrm{Ti} * \mathrm{Te}) / \mathrm{qe} /(\mathrm{ne} * \mathrm{~T} i+\mathrm{n} i * \mathrm{Te})) ;$

$\mathrm{B}=4$;

o corot_period is unused for this profile.

corot_period=0

case 'enceladus_cyl'

ocase 'enceladus'

o please note: this profile is set up to be used with

"Corotating_boris_pusher.m" exclusively! Things will be different

․․․ if you work in a non-corotating frame.

phi=improved_arctan (x,y);

radius $=\operatorname{sqrt}\left(\mathrm{x}^{\wedge} 2+\mathrm{y}^{\wedge} 2\right)$;

o radius of saturn, in meters, $+/-4000 \mathrm{~m}$ :

r_sat $=60268 \mathrm{e} 3$;

- space potential is set to zero, for now

$\mathrm{V}=0$; 


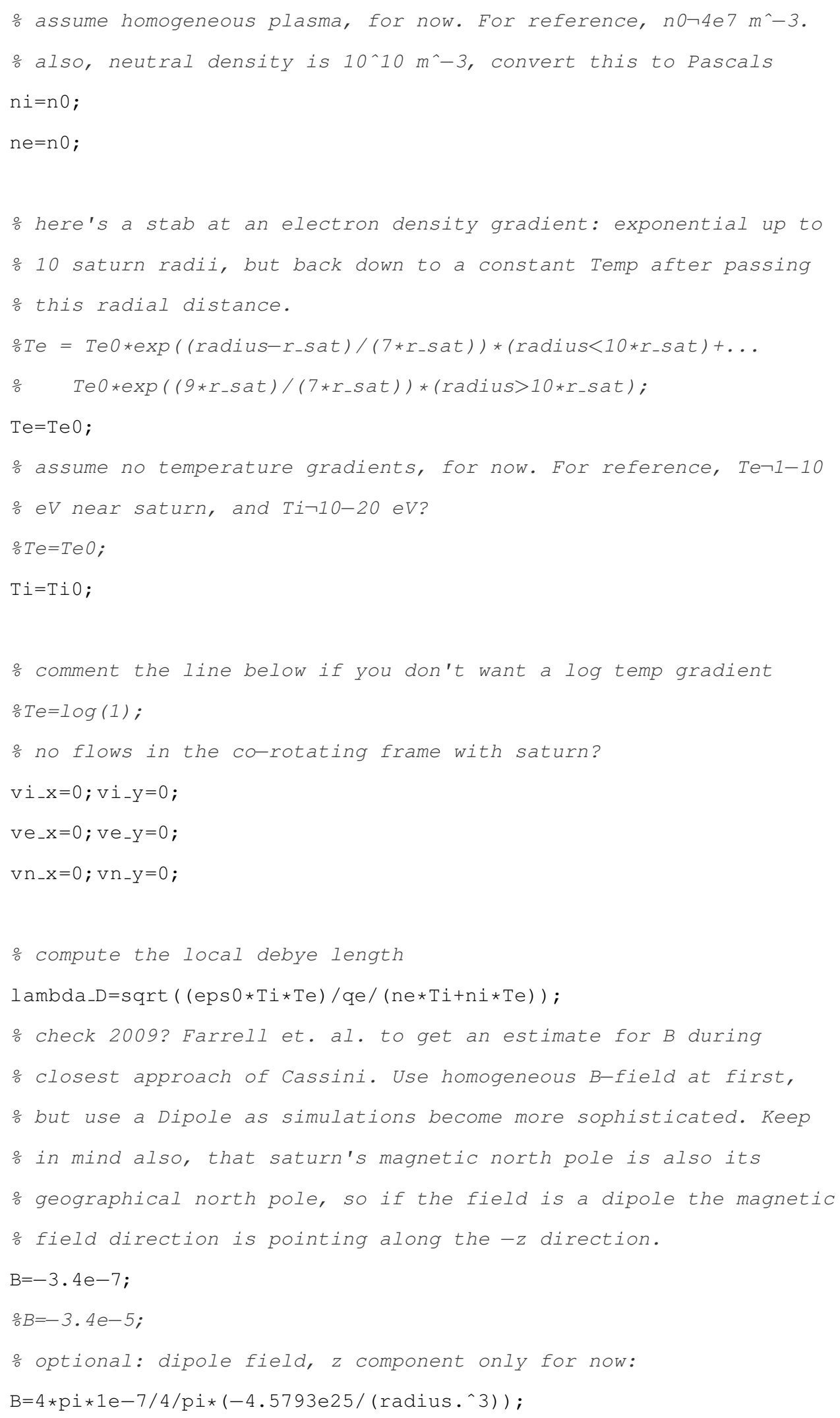




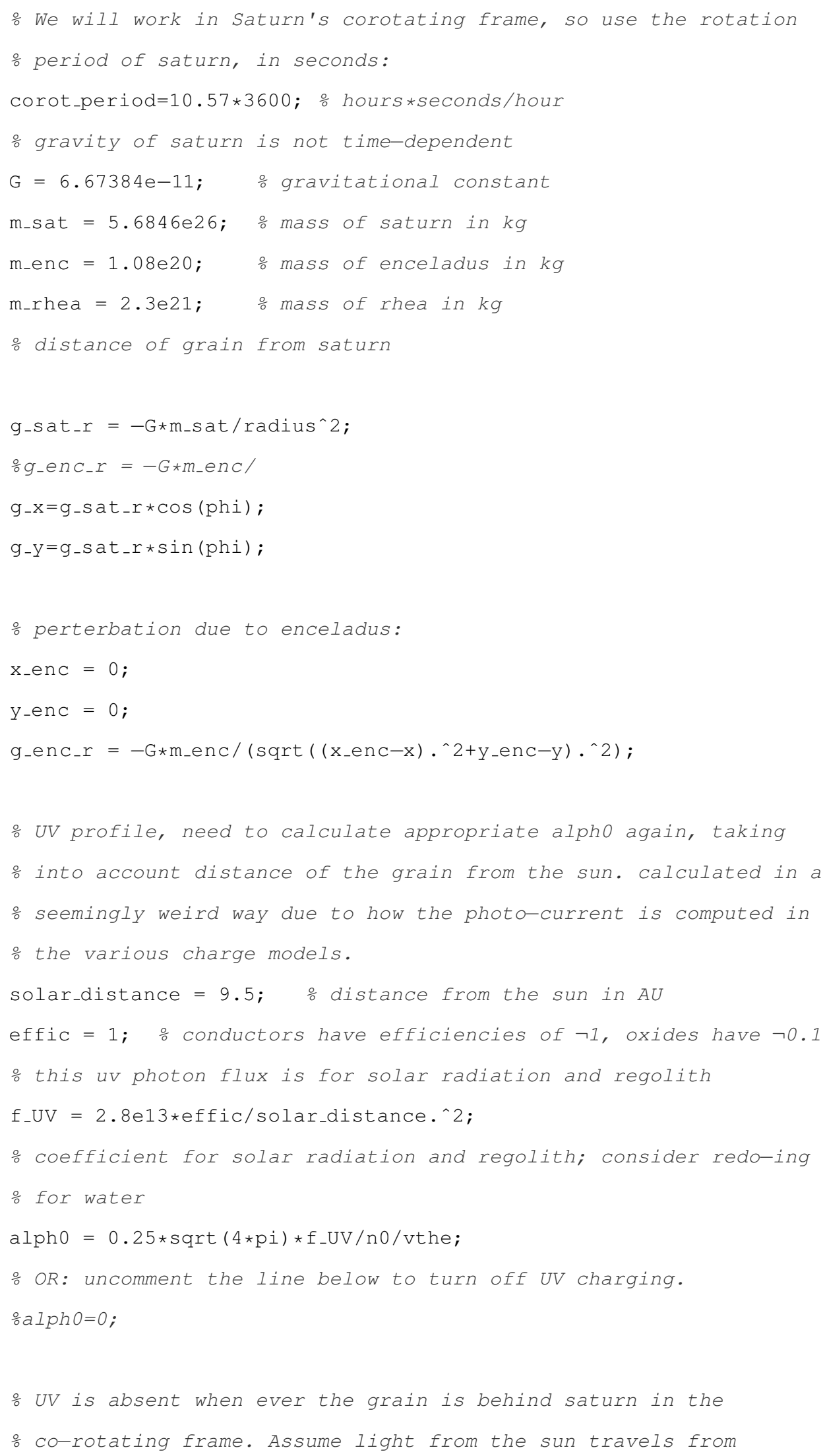




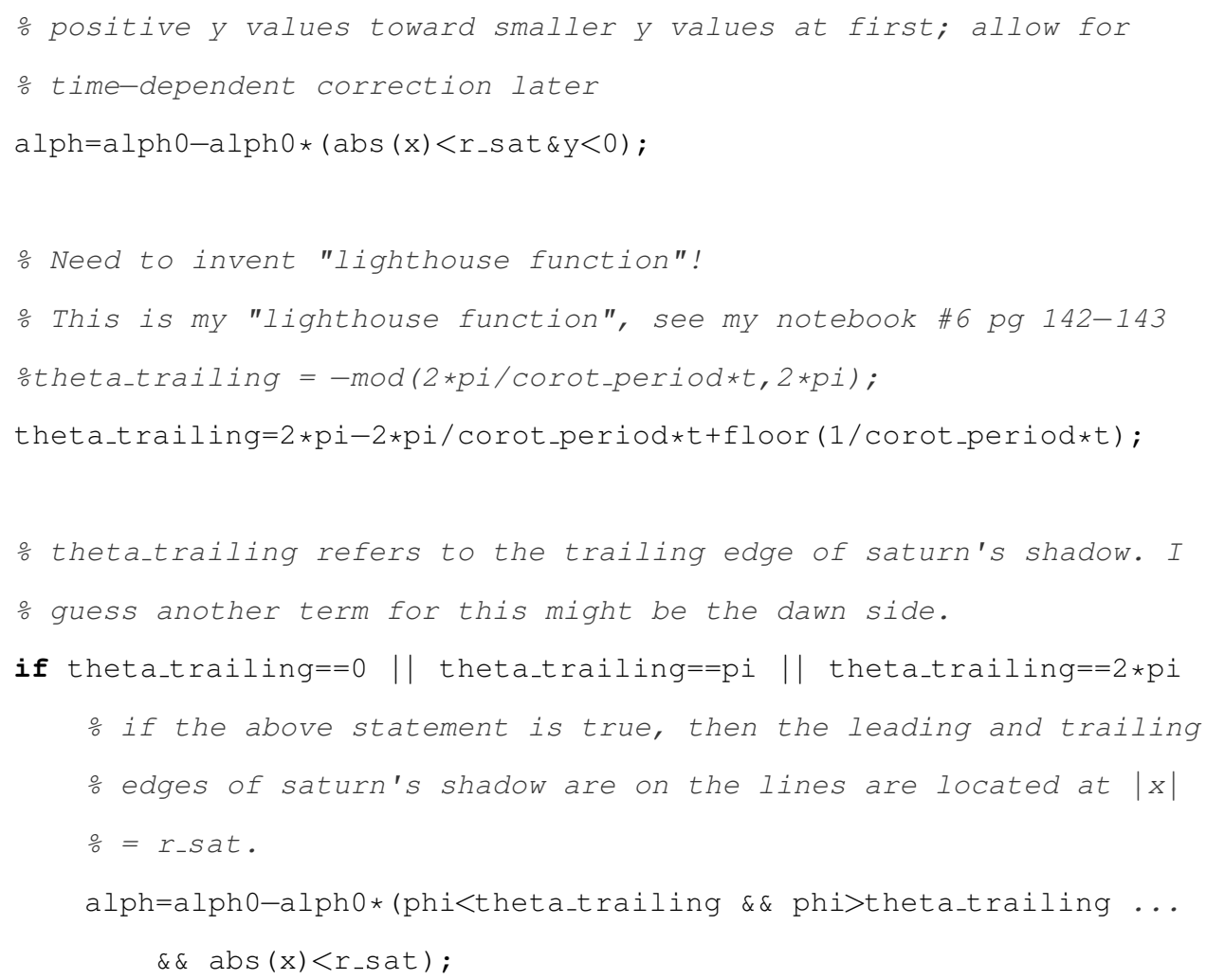




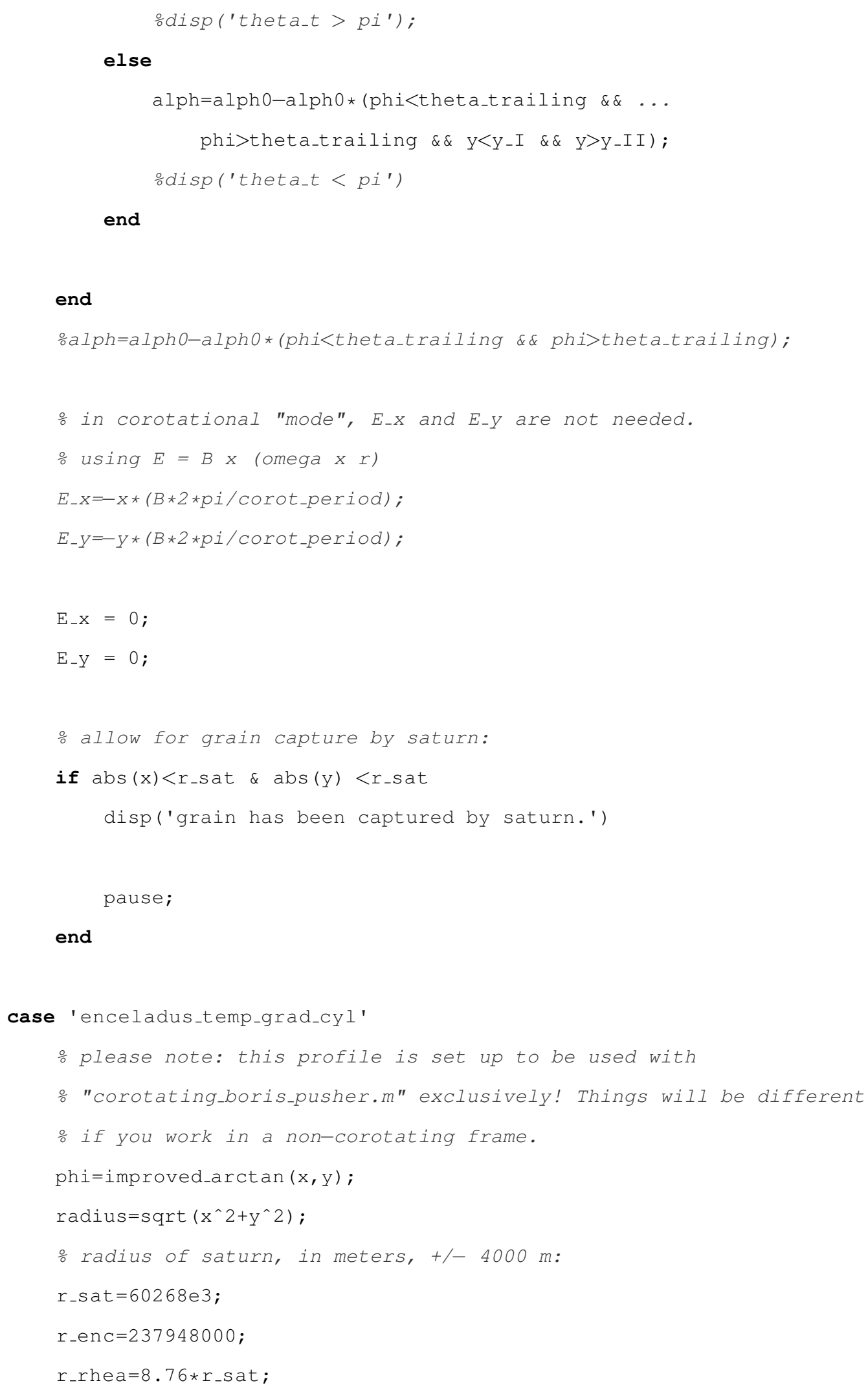




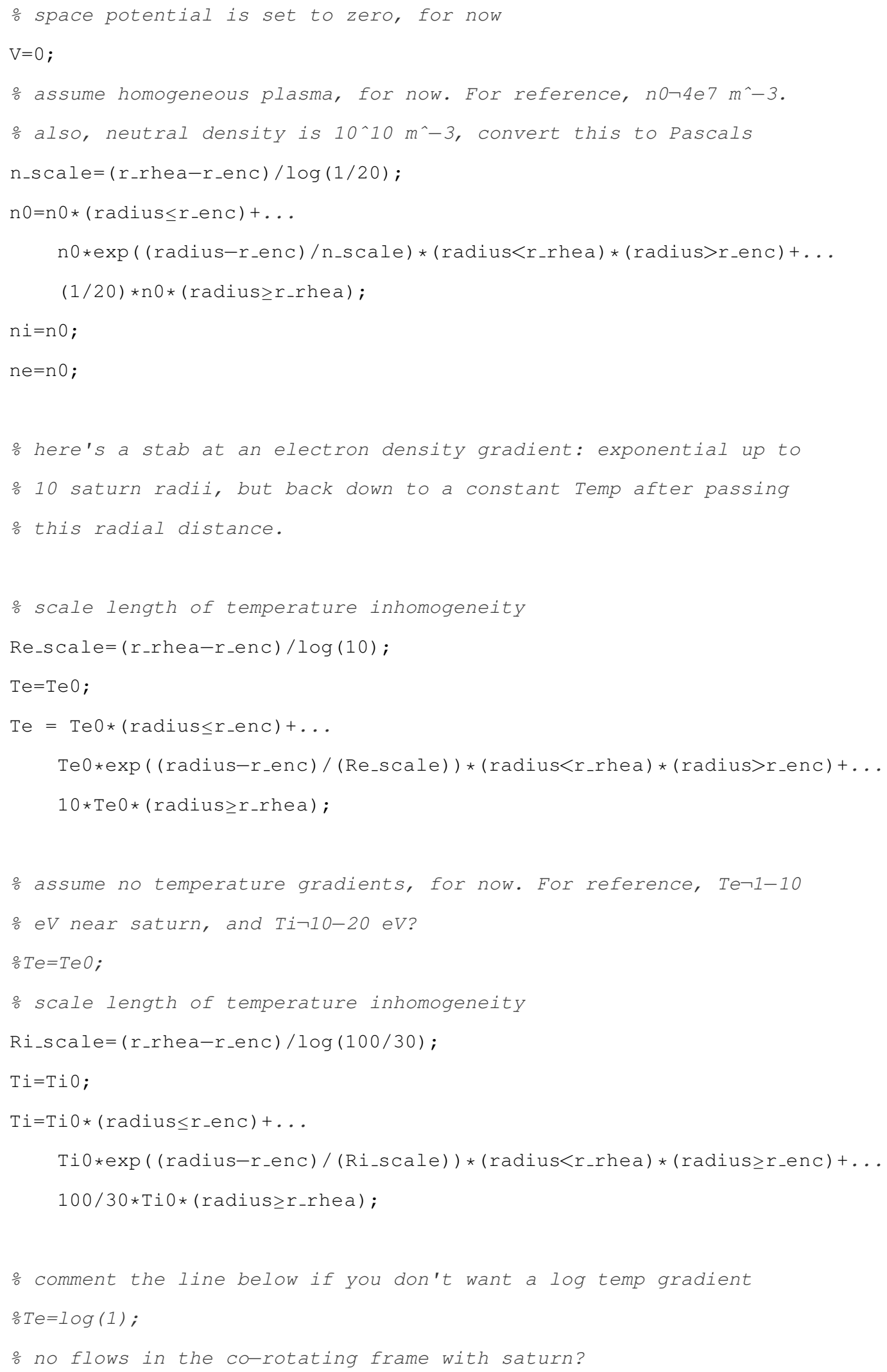




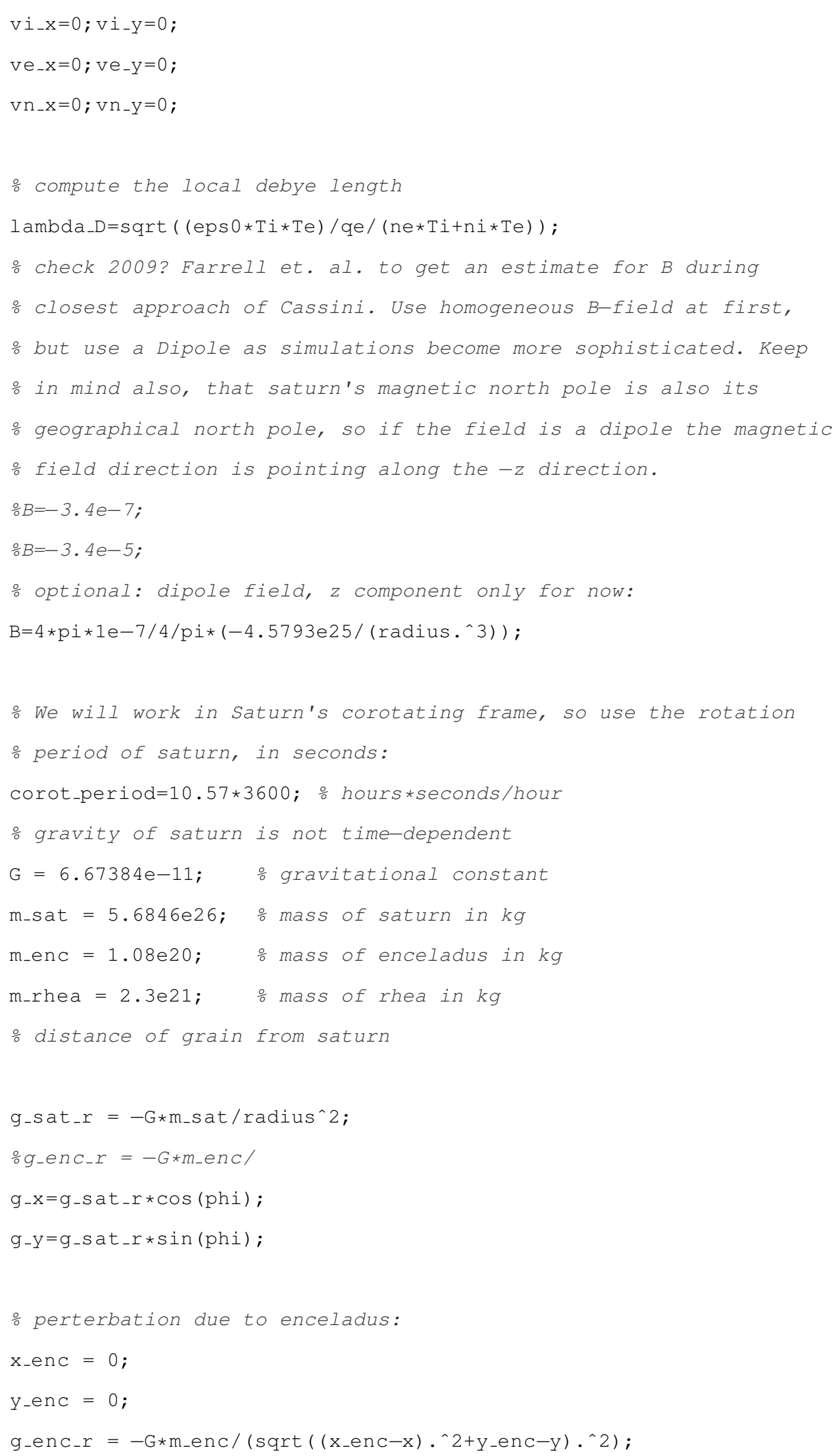


- UV profile, need to calculate appropriate alpho again, taking

o into account distance of the grain from the sun. calculated in a

응 seemingly weird way due to how the photo-current is computed in

․ the various charge models.

solar_distance $=9.5 ; \quad \circ$ distance from the sun in $A U$

effic $=1 ; \quad$ o conductors have efficiencies of $\neg 1$, oxides have $\neg 0.1$

․ㅡㄴ this uv photon flux is for solar radiation and regolith

$f_{-U V}=2.8 e 13 *$ effic/solar_distance. ${ }^{` 2}$;

o coefficient for solar radiation and regolith; consider redo-ing

응 water

alpho $=0.25 * \operatorname{sqrt}(4 * \mathrm{pi}) * \mathrm{f}_{-} \mathrm{UV} / \mathrm{n} 0 / \mathrm{vthe}$;

ㅇ OR: uncomment the line below to turn off UV charging.

$\div$ alphO=0;

UV is absent when ever the grain is behind saturn in the

o co-rotating frame. Assume light from the sun travels from

o positive y values toward smaller y values at first; allow for

을 time-dependent correction later

alph=alph0-alph0*(abs $(x)<r_{-}$sat \& $\left.y<0\right)$;

Need to invent "lighthouse function"!

․ㅡㄴ is is my "Iighthouse function", see my notebook \#6 pg 142-143

otheta_trailing $=-\bmod (2 *$ pi/corot_period*t, $2 * p i)$;

theta_trailing=2*pi-2*pi/corot_period*t+floor(1/corot_period*t);

o theta-trailing refers to the trailing edge of saturn's shadow. I

ㅁ guess another term for this might be the dawn side.

if theta_trailing==0 || theta_trailing==pi || theta_trailing==2 $\star p i$

의 the above statement is true, then the leading and trailing

o edges of saturn's shadow are on the lines are located at $|x|$

$\therefore=r_{-}$sat.

alph=alph0-alpho* (phi<theta_trailing \&\& phi>theta_trailing ... $\& \& \operatorname{abs}(\mathrm{x})<r_{-}$sat $)$;

else 


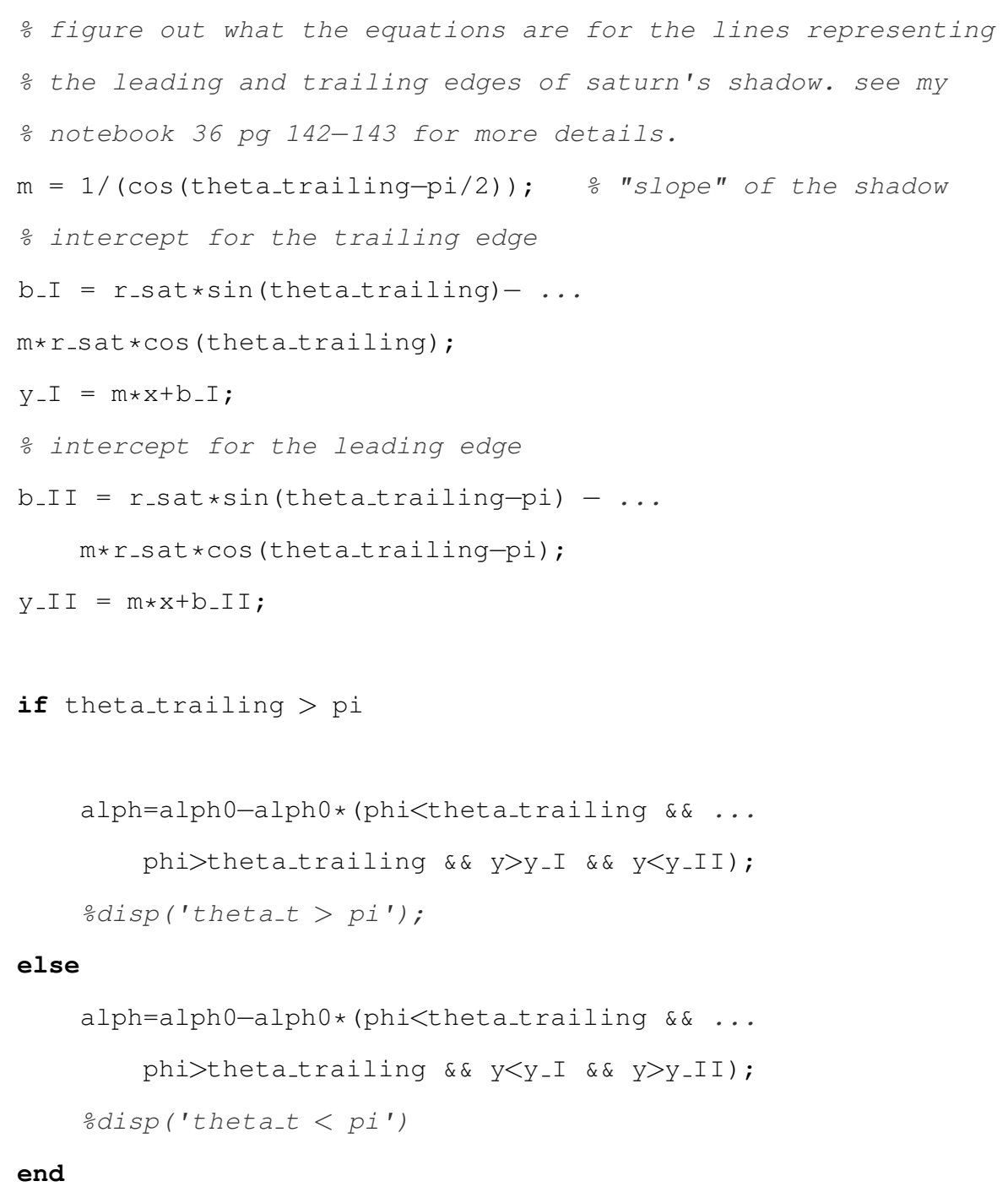




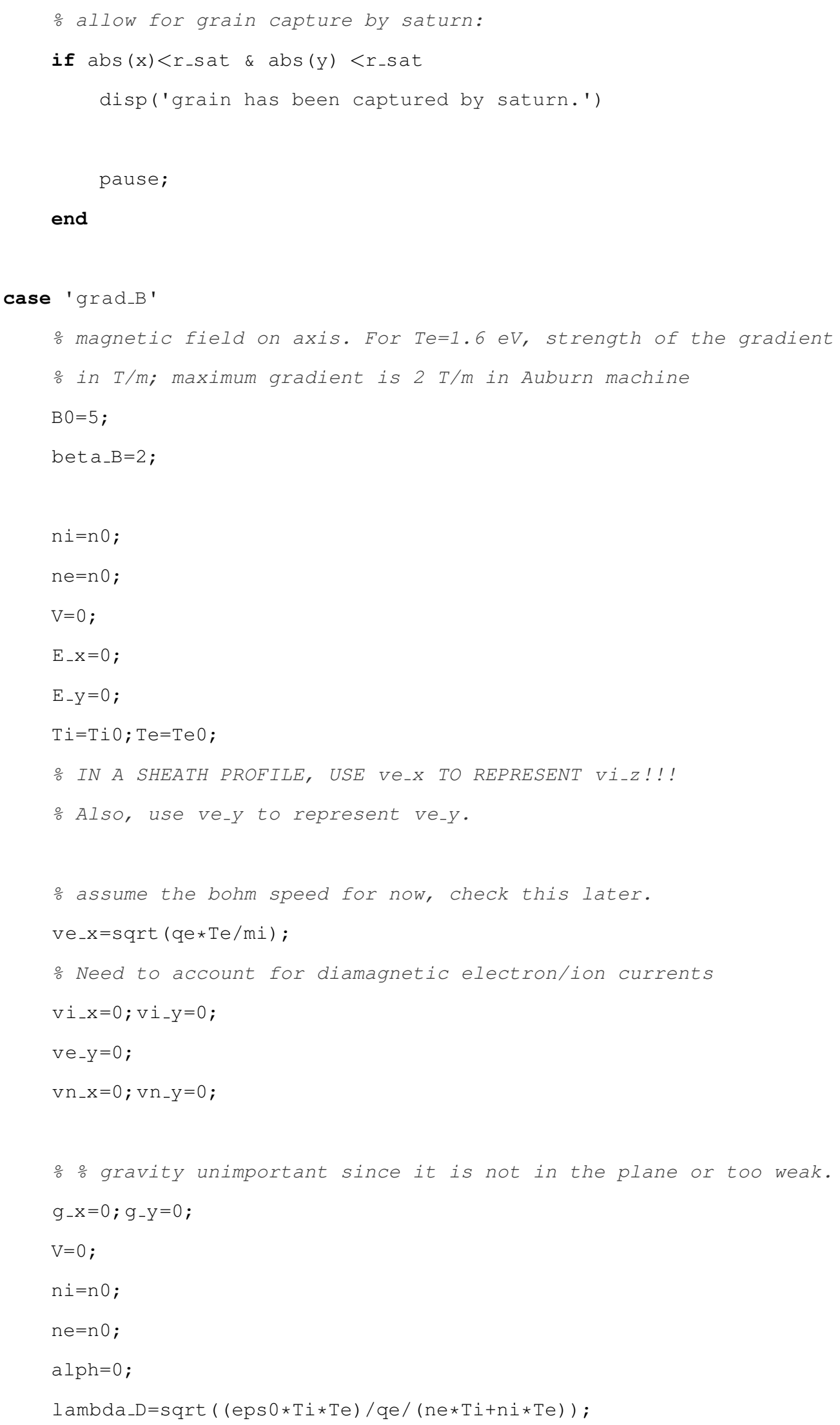




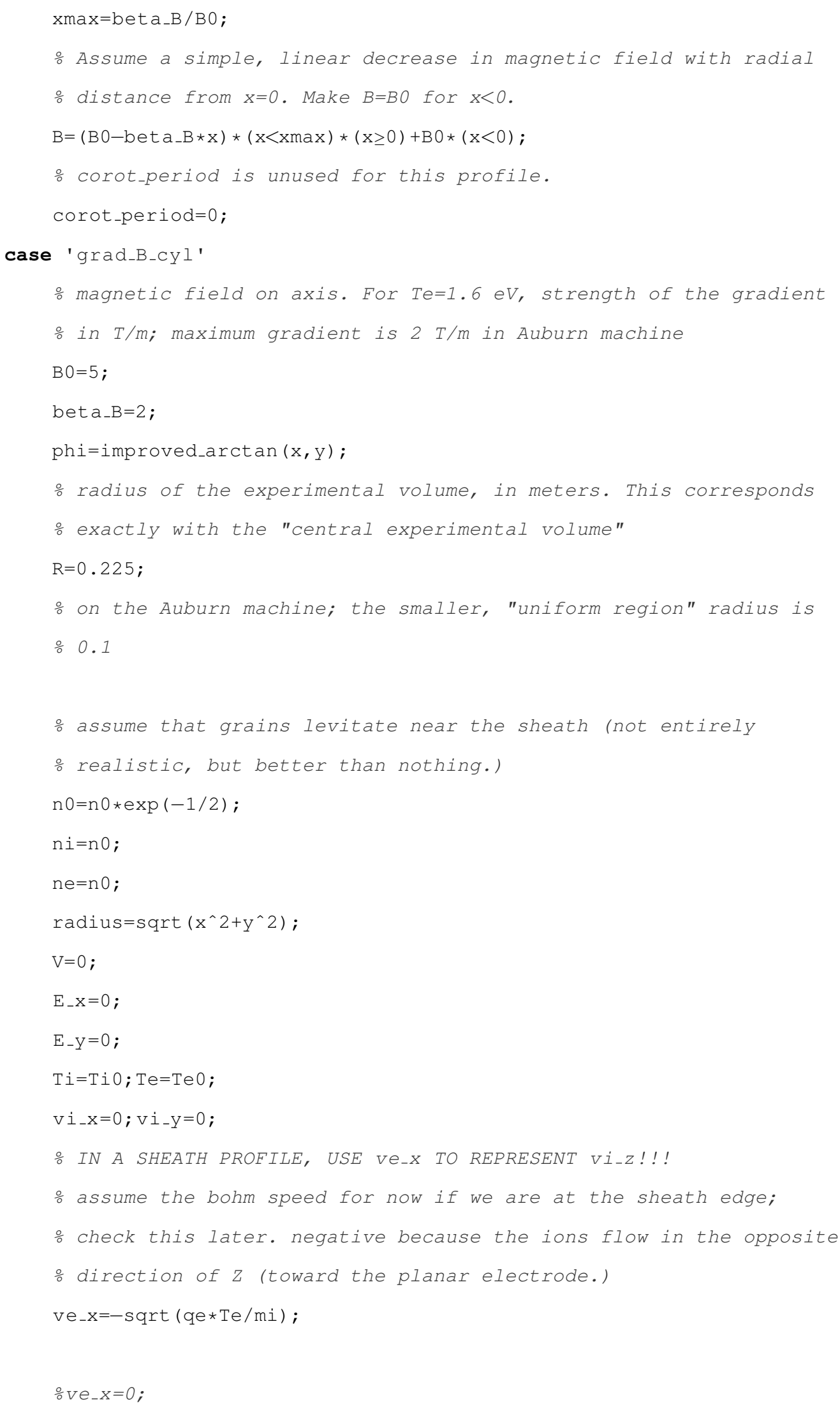


- Need to account for diamagnetic electron/ion currents

ve $-\mathrm{y}=0$;

$\mathrm{vn} \_\mathrm{x}=0 ; \mathrm{vn}-\mathrm{Y}=0$;

응 gravity unimportant since it is not in the plane or too weak.

$g_{-} \mathrm{x}=0 ; \mathrm{g}_{-} \mathrm{y}=0$;

$\mathrm{V}=0$;

$\mathrm{ni}=\mathrm{n} 0$;

$\mathrm{ne}=\mathrm{n} 0$;

$\mathrm{alph}=0$;

lambda_D $=\operatorname{sqrt}((\operatorname{eps} 0 * \mathrm{Ti} * \mathrm{Te}) / \mathrm{qe} /(\mathrm{ne} * \mathrm{Ti}+\mathrm{ni} * \mathrm{Te}))$;

N NEED TO CHECK THIS LINE BELOW! I AM BASING IT OFF OF

ㅇ 1992_DAUGHERTY_JAP. THE IDEA HERE IS THAT NEAR-MONO-ENERGETIC

․ IONS THAT FALL OUT OF THE SHEATH HAVE AN ENERGY COMPARABLE TO Te,

응 SO THIS ENLARGES THE SIZE OF THE SHEATH.

I $\operatorname{ambda} D=\operatorname{sqrt}((\operatorname{eps} 0 * T e * T e) / q e /(n e * T e+n i * T e))$;

․ Assume a simple, linear decrease in magnetic field with radial

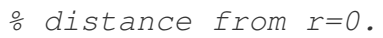

$\mathrm{B}=\mathrm{B} 0-$ beta_B*radius;

o corot-period is unused for this profile.

corot_period=0;

case 'auburn_cyl'

ocase 'auburn'

․ magnetic field on axis. For $\mathrm{Te}=1.6 \mathrm{eV}$, strength of the gradient

․ in $\mathrm{T} / \mathrm{m}$; maximum gradient is $2 \mathrm{~T} / \mathrm{m}$ in Auburn machine

$\therefore B O=5$;

$\mathrm{B} 0=4 ;$

을et $a_{-} B=2$;

phi=improved_arctan $(\mathrm{x}, \mathrm{y})$;

radius of the experimental volume, in meters. This corresponds

을 exactly with the "central experimental volume"

$\mathrm{R}=0.225$;

ㅇ on the Auburn machine; the smaller, "uniform region" radius is

$\div 0.1$ 


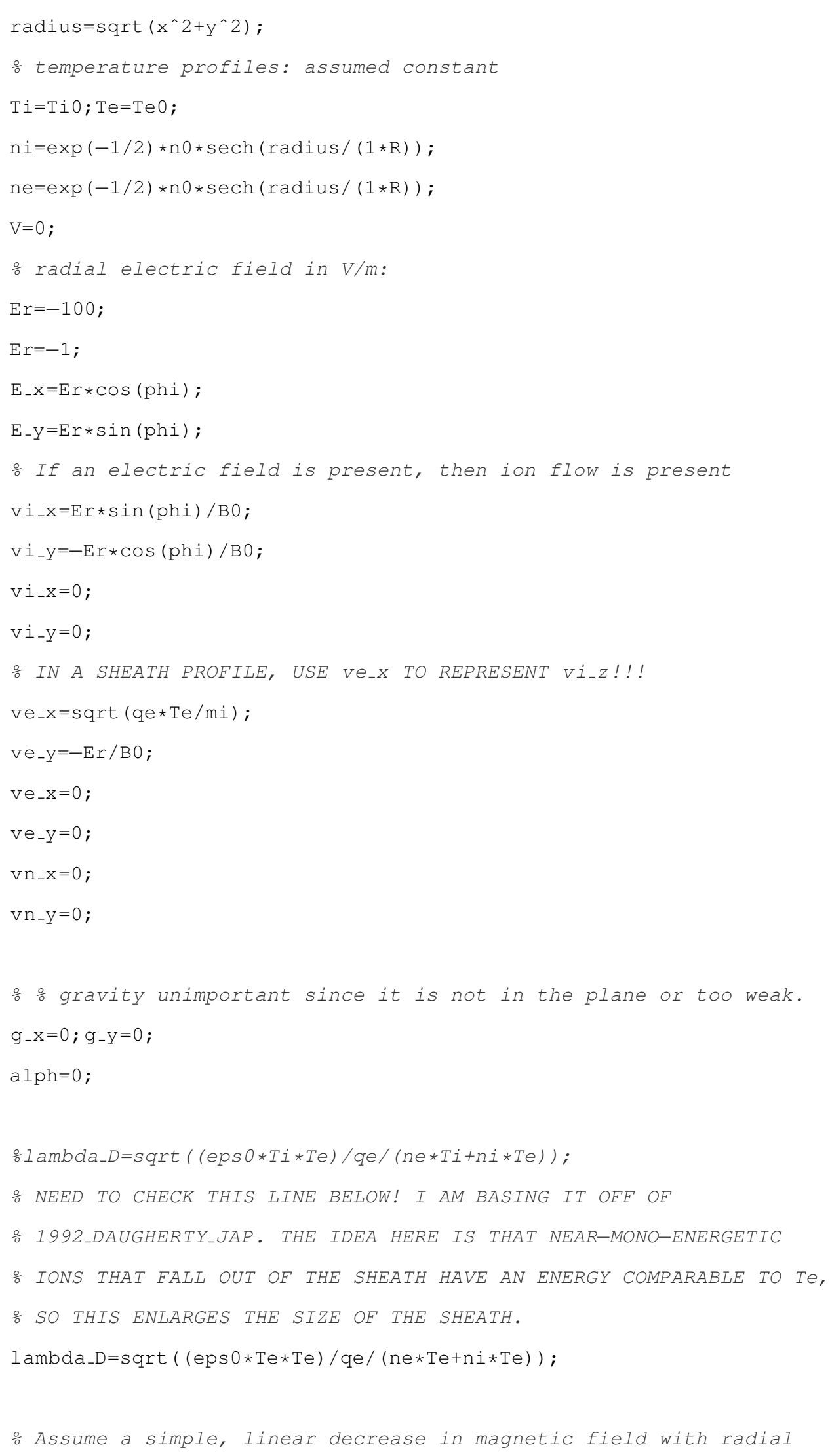




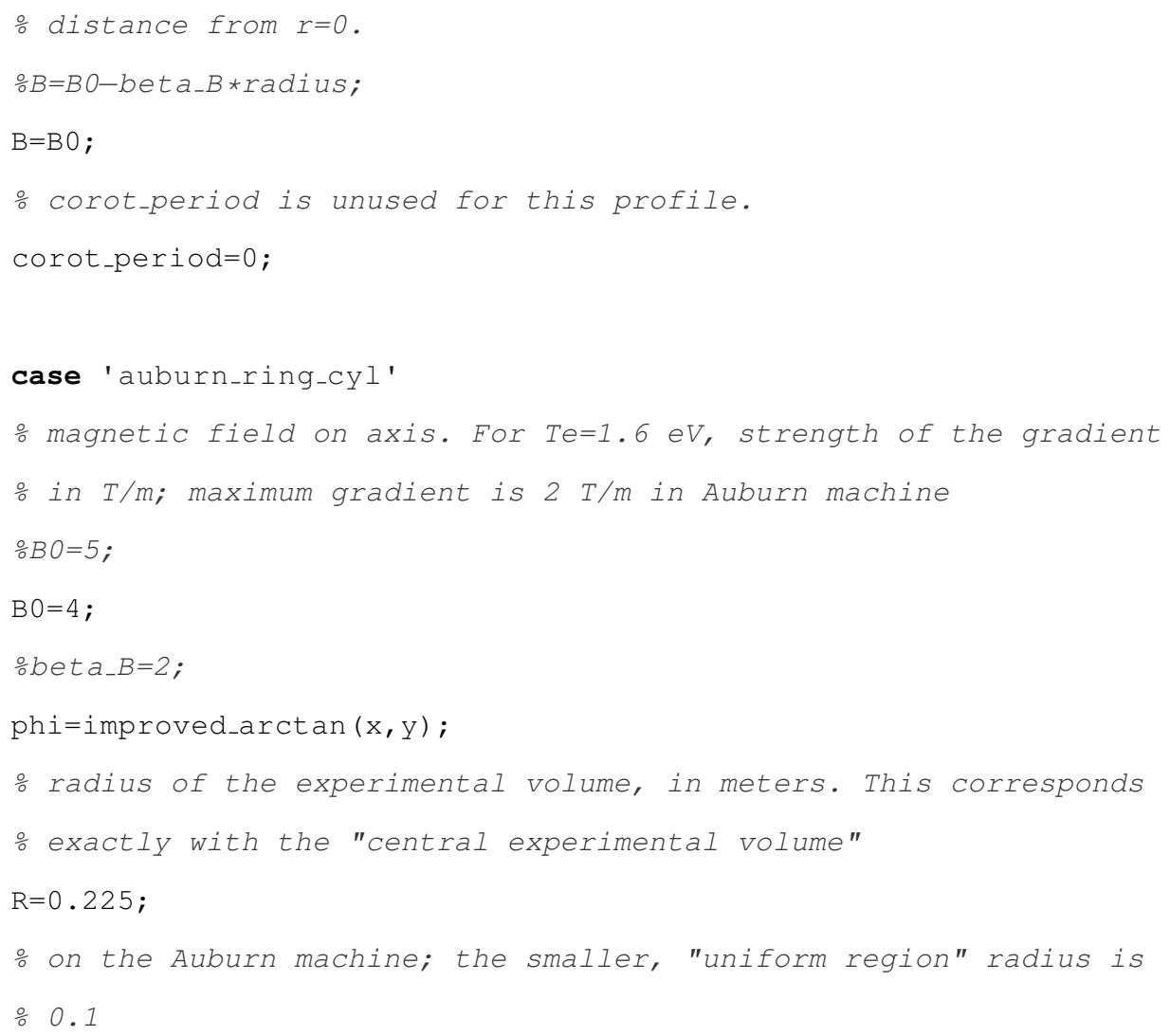




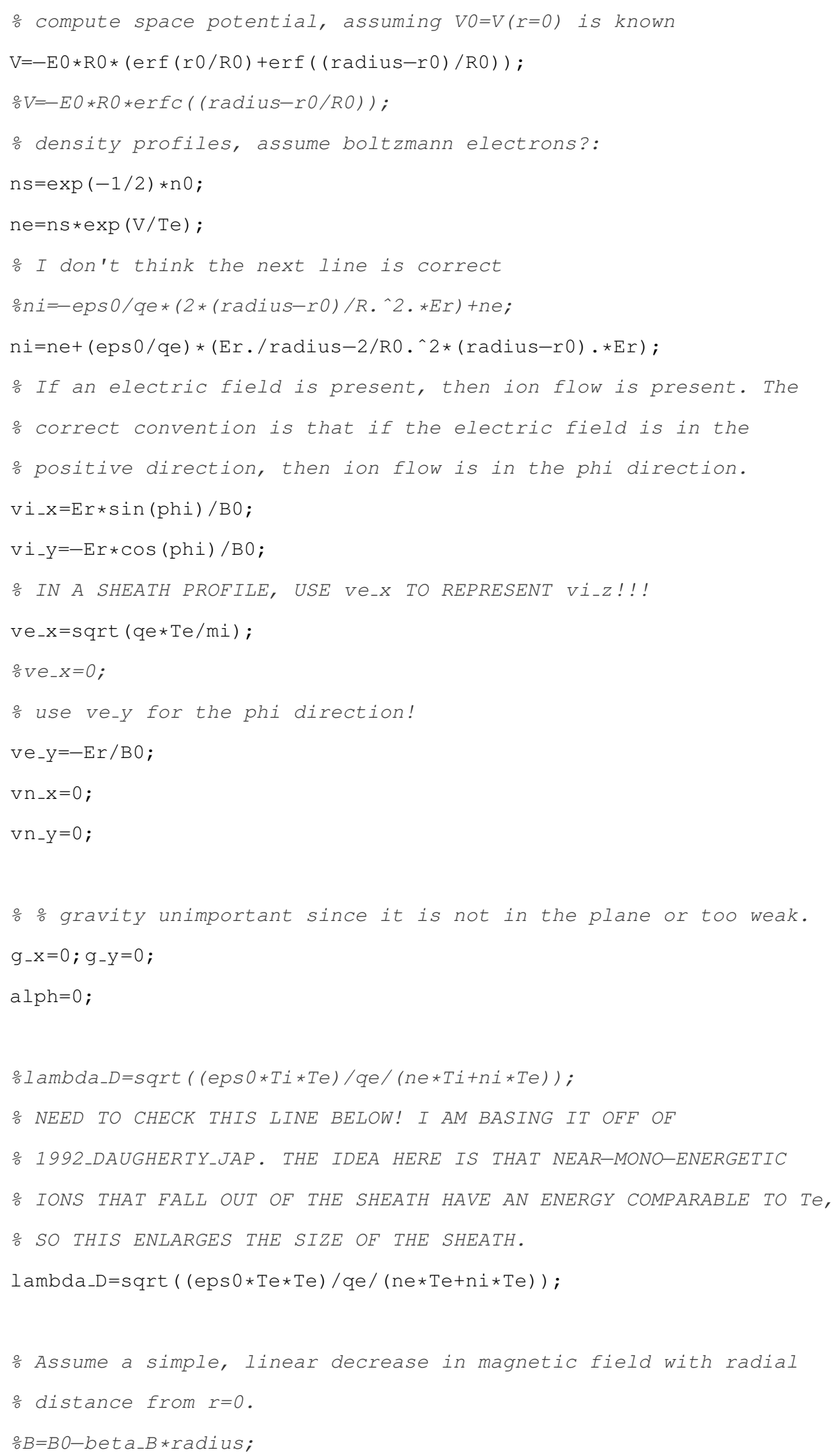


$\mathrm{B}=\mathrm{BO}$ ；

o corot-period is unused for this profile.

corot_period=0;

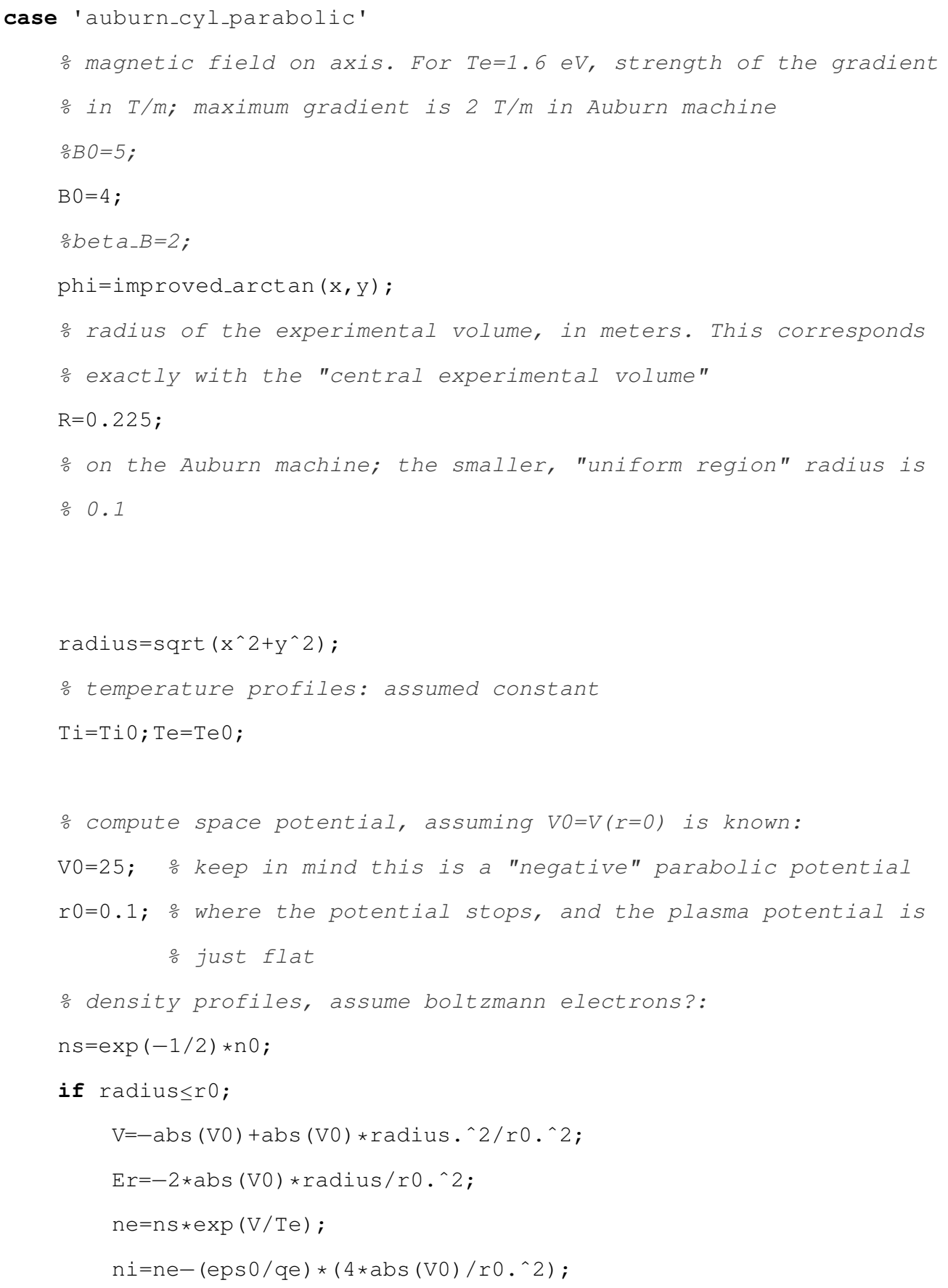




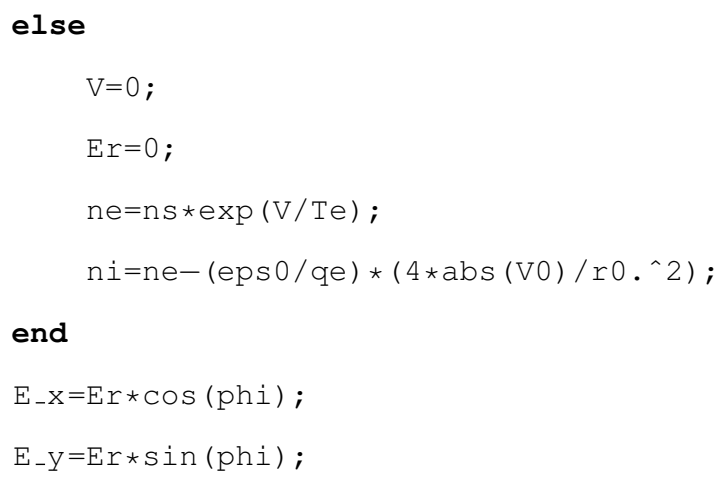




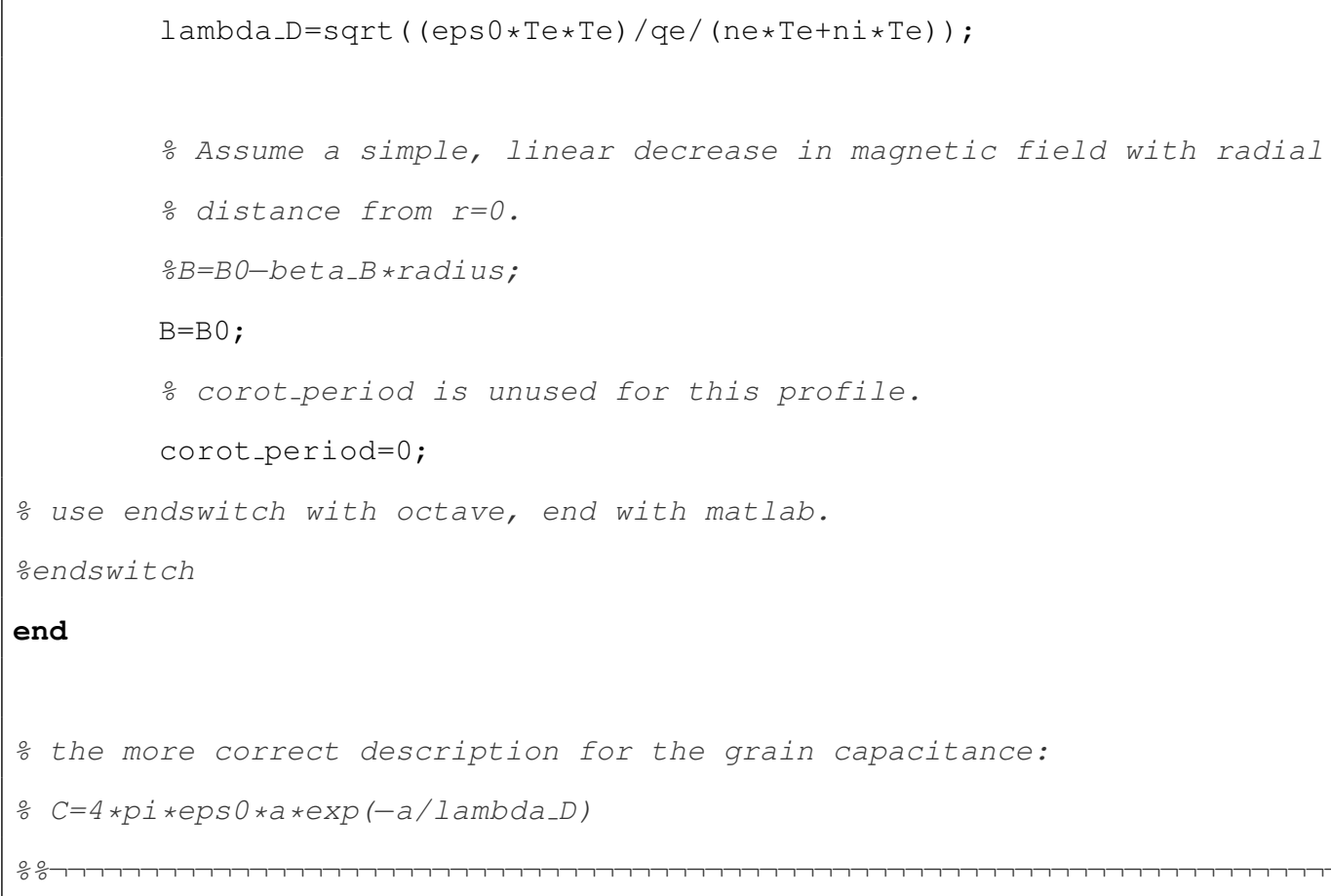

\section{Gyro-phase Analysis Code}

The main routine dust_trajectory.m produces trajectories in $x, y$ configuration space. In order to put quantities into gyro-phase space, or to determine gyro-phase angle, and dependence of quantities on gyro-phase, an analysis code is needed. This is done using the routine gyrophaser.m.

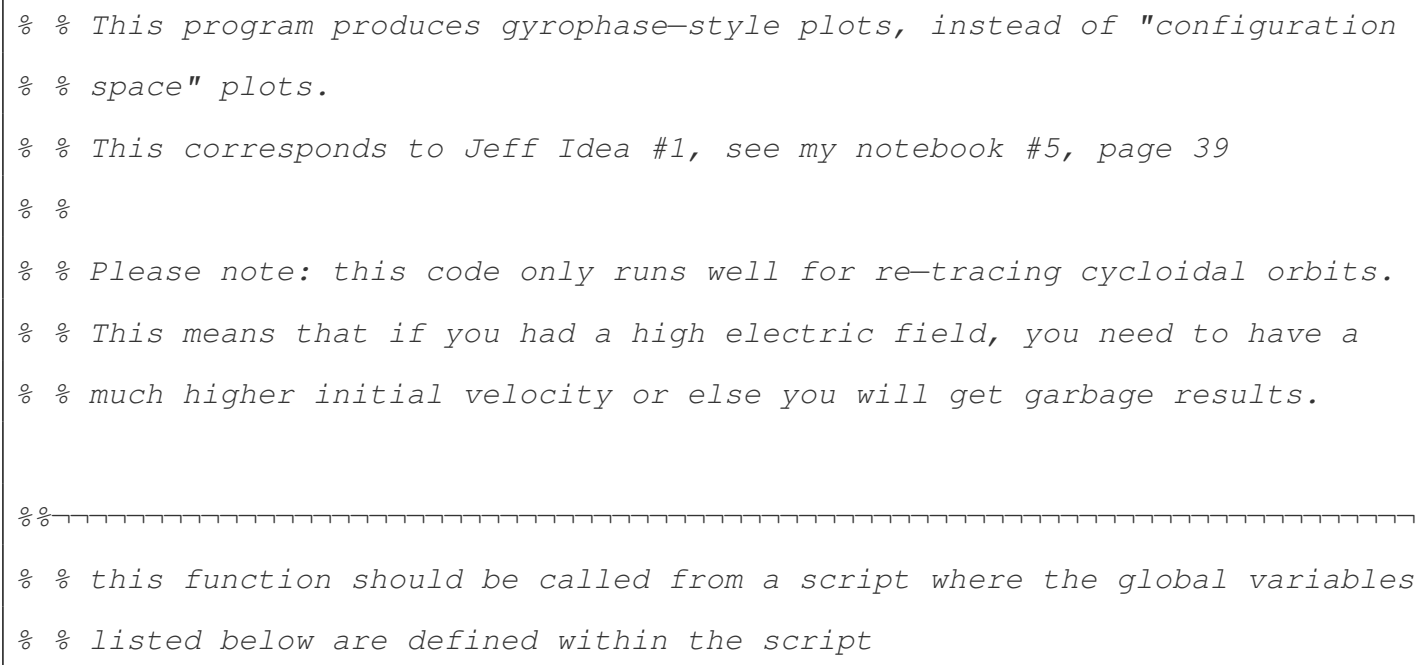




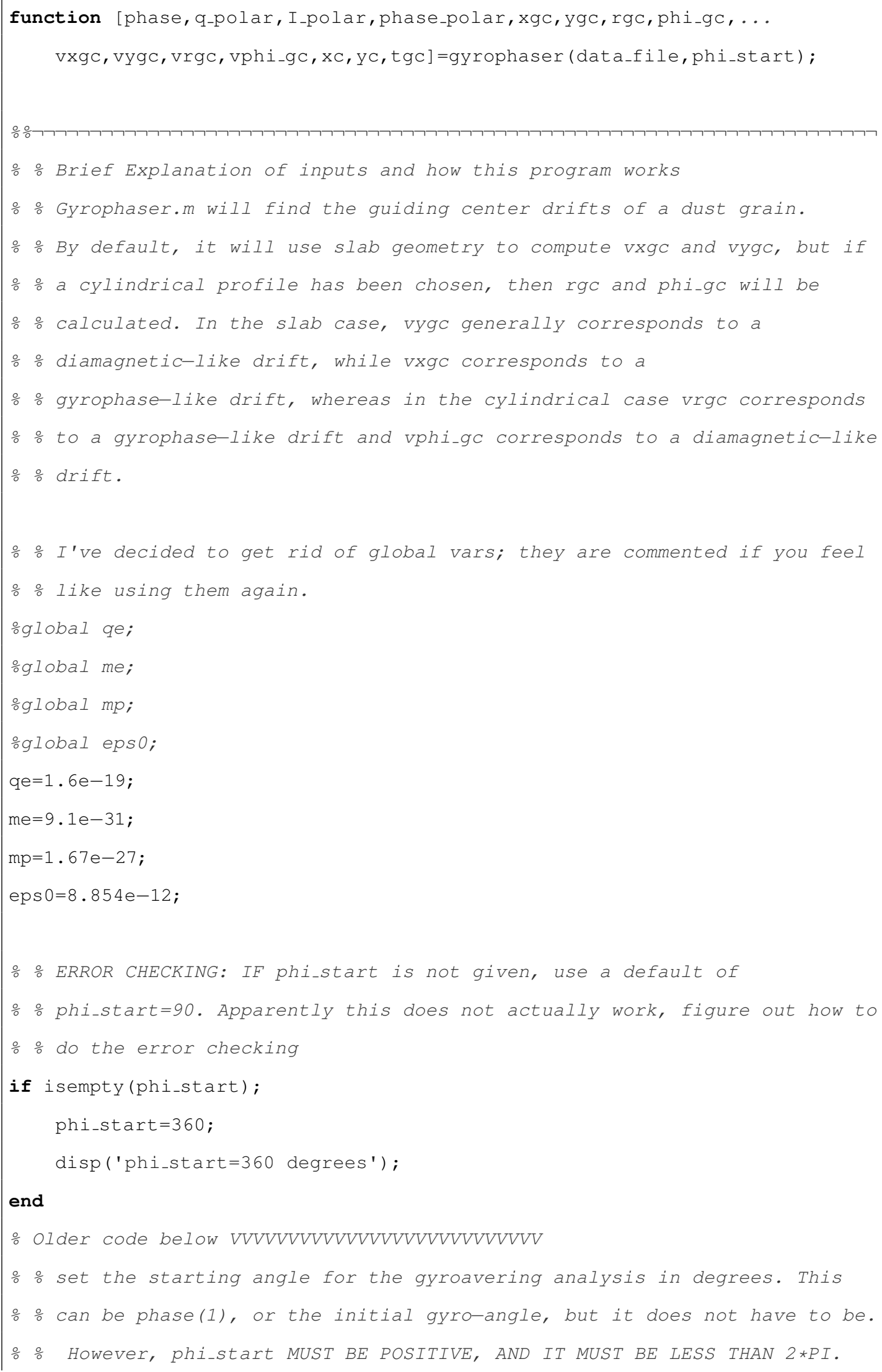




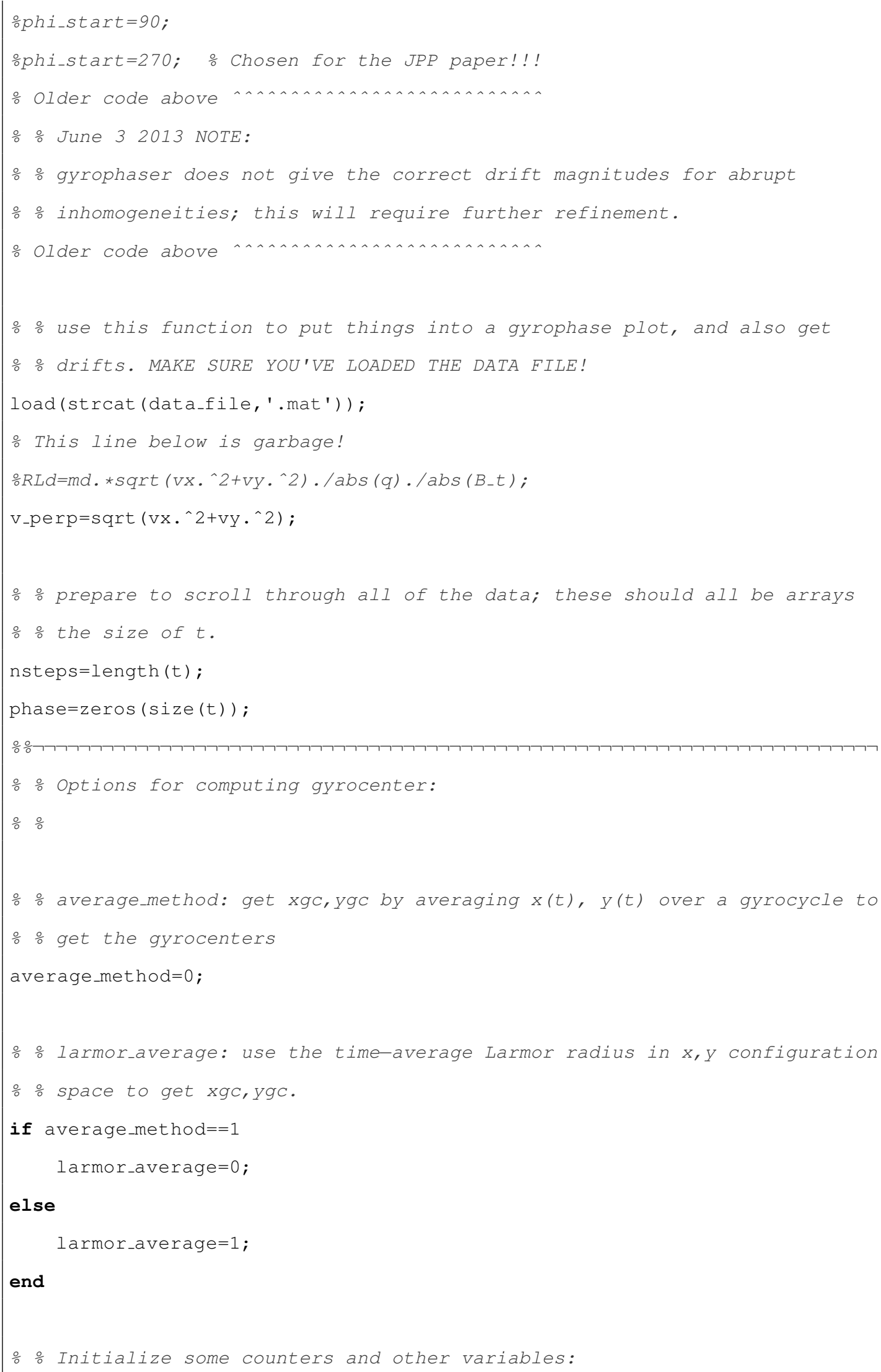




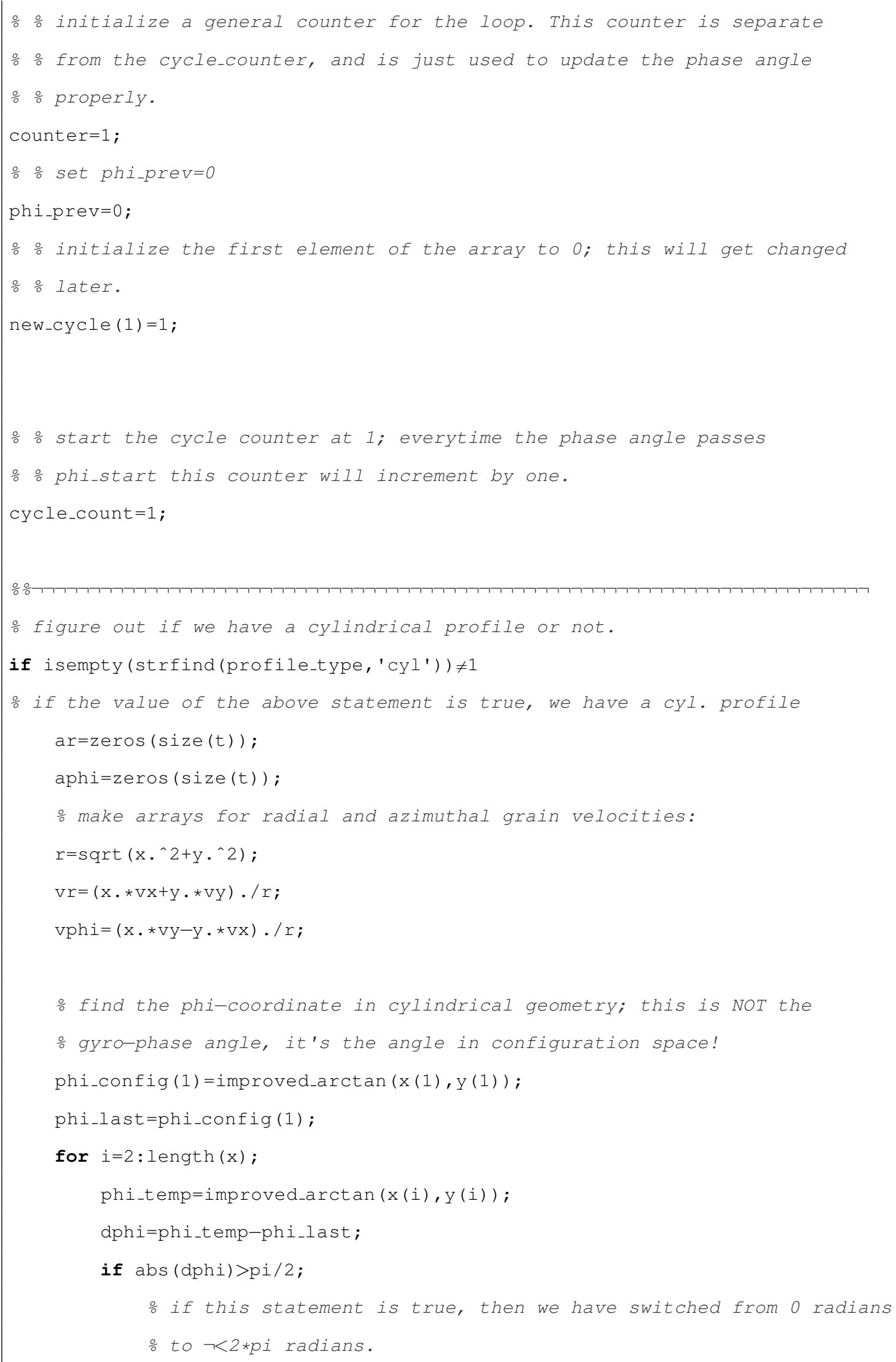




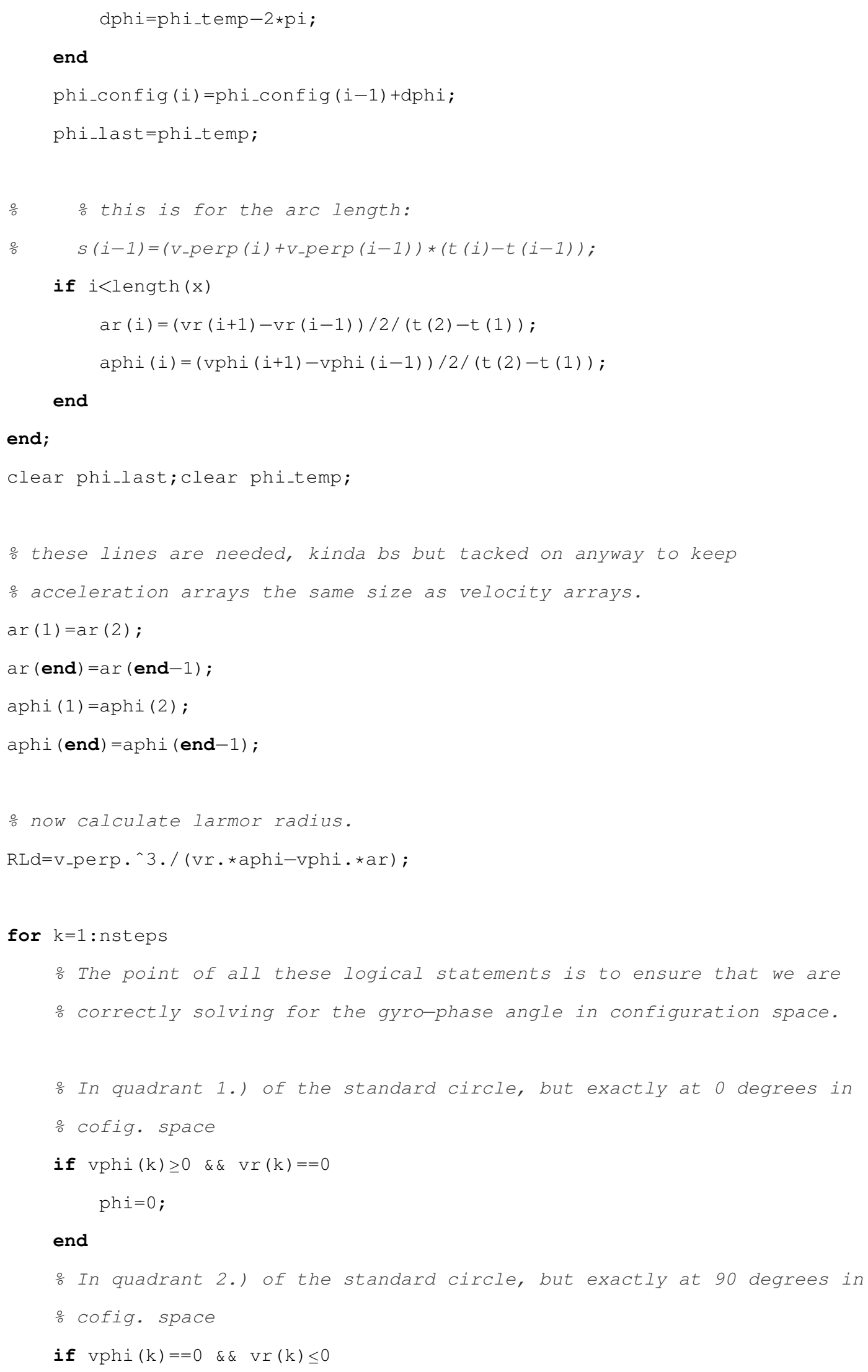


phi=pi/2;

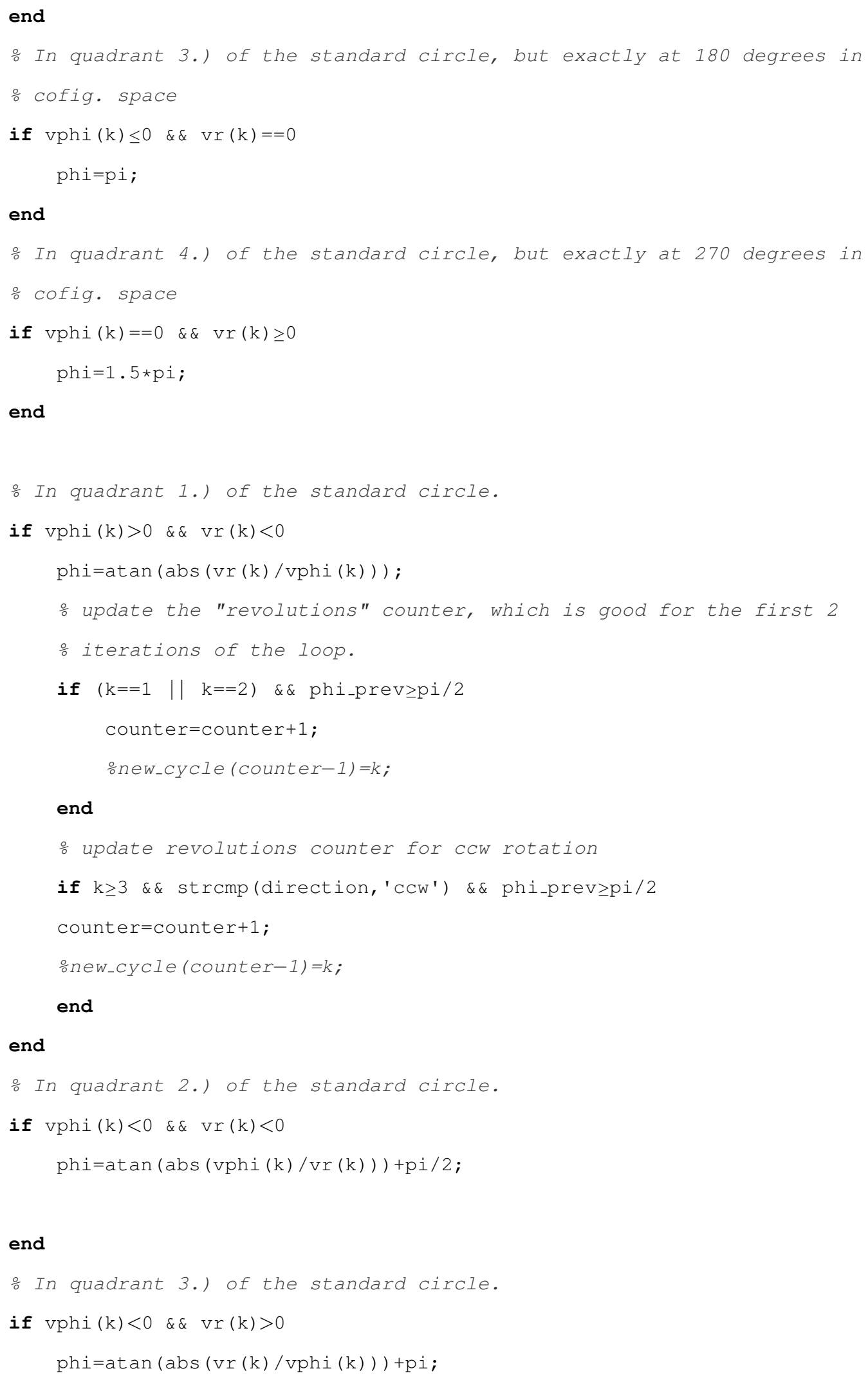




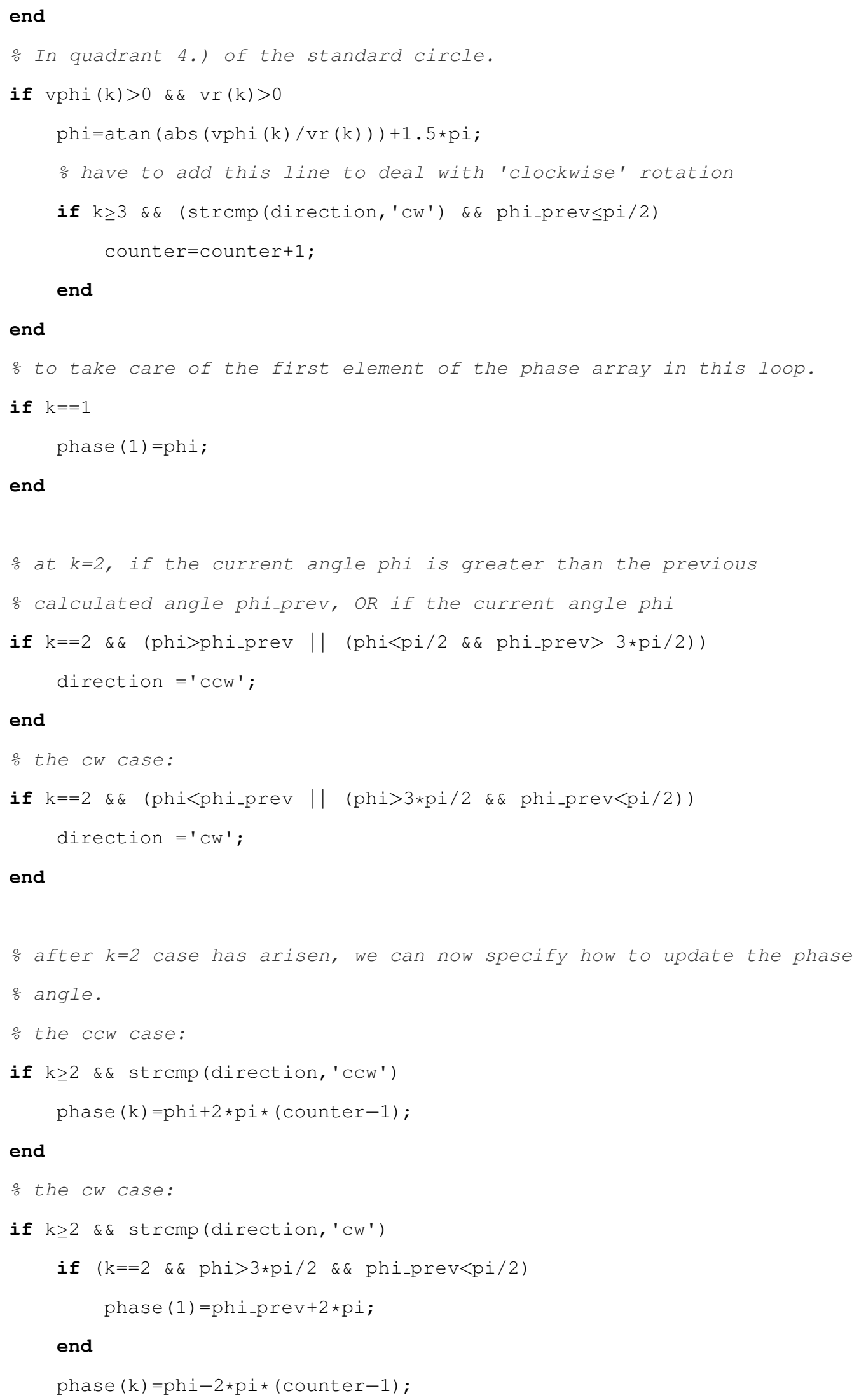




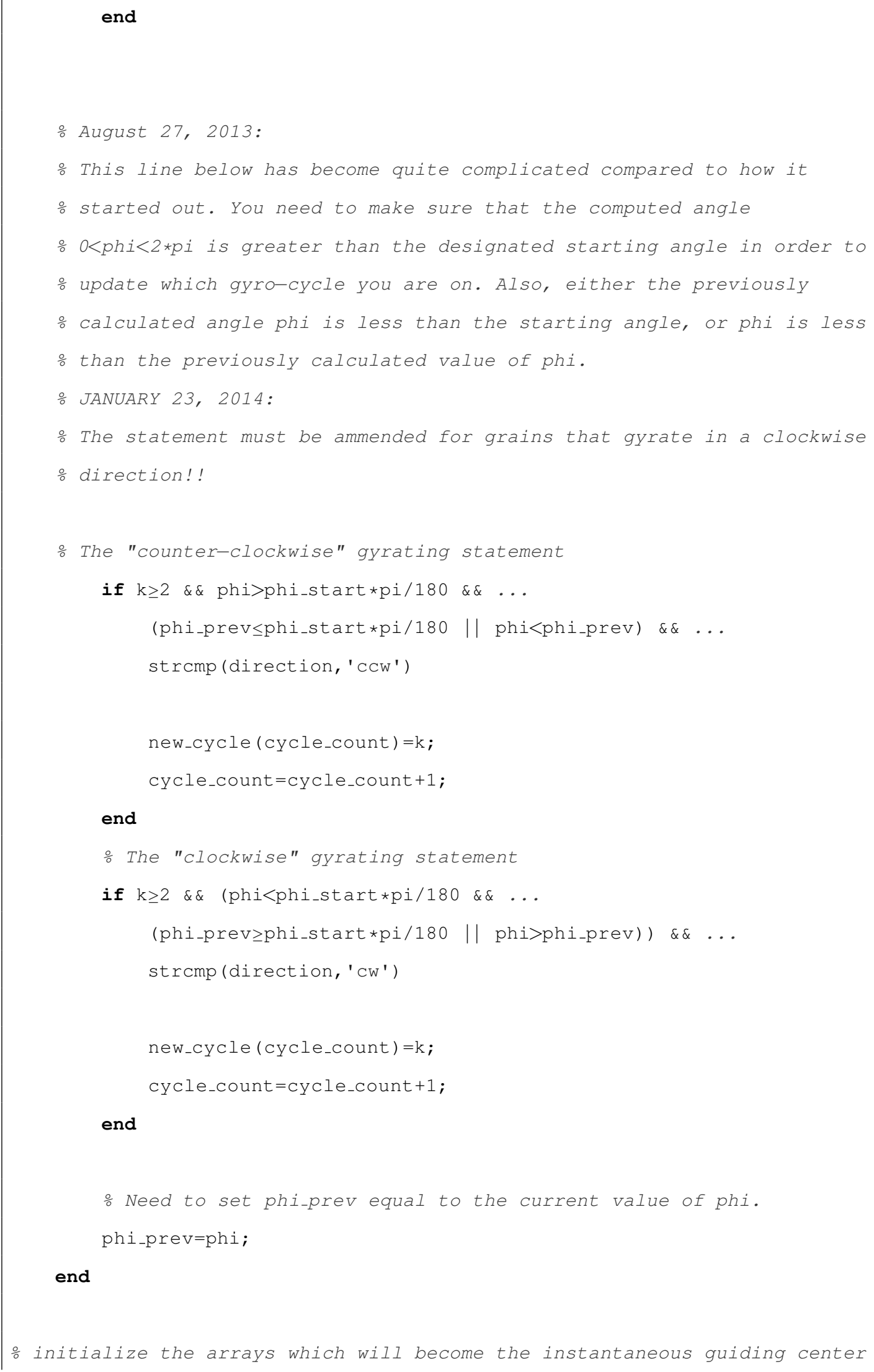




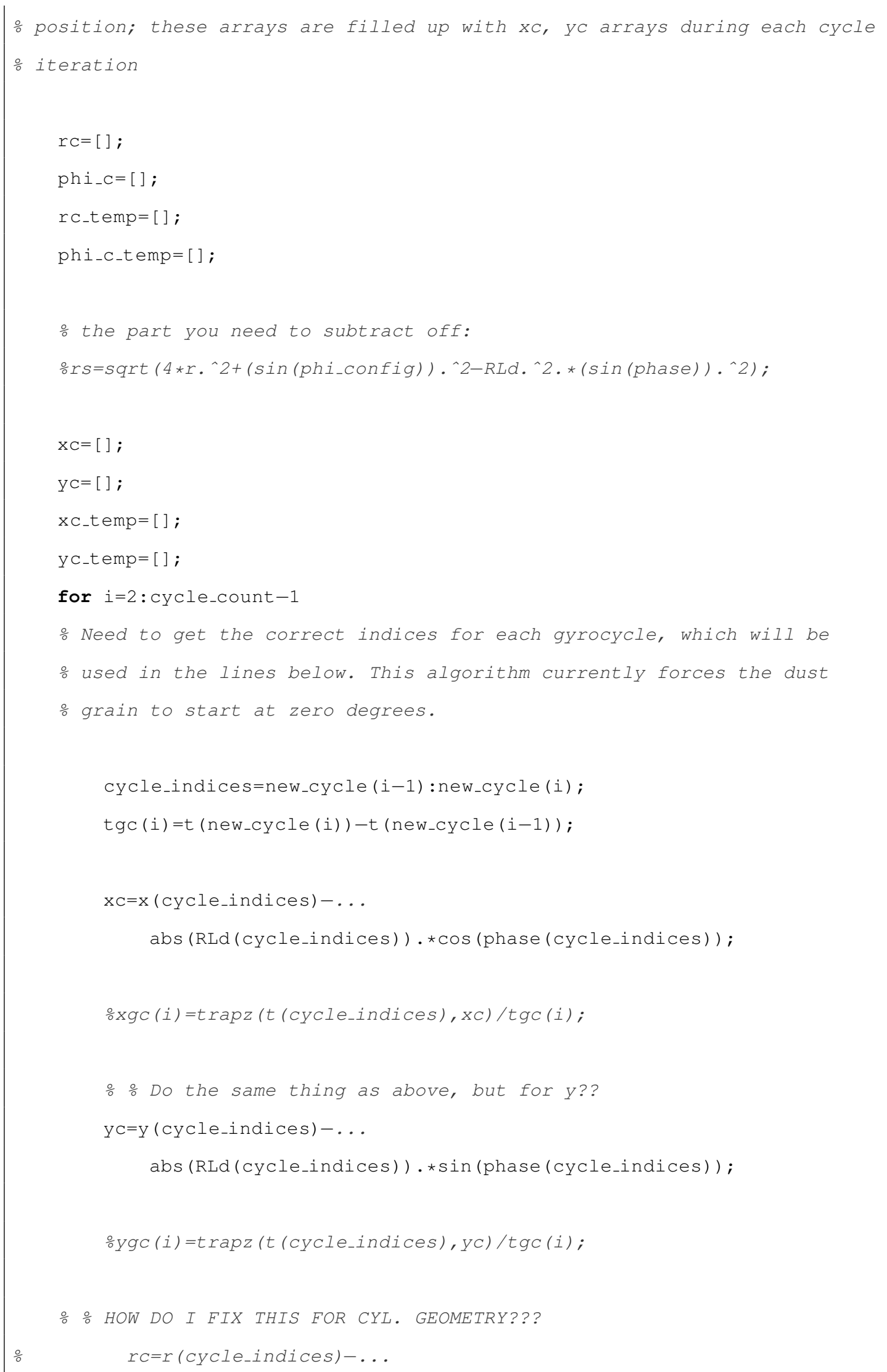




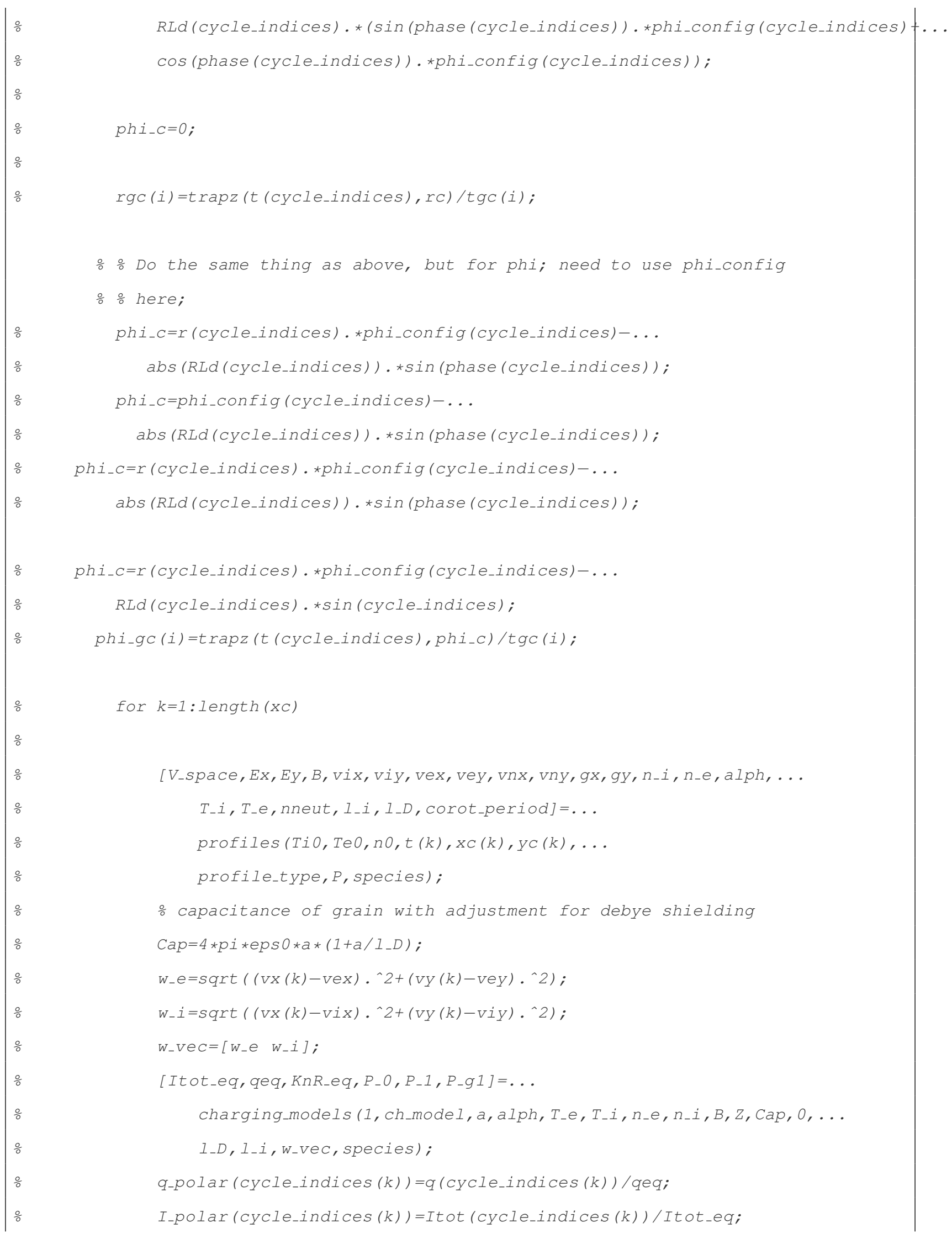

RLd(cycle_indices).*(sin(phase (cycle_indices)) . *phi_config(cycle_indices) f... cos (phase (cycle_indices)) . *phi_config(cycle_indices));$$
p h i_{-}=0 \text {; }
$$

$\operatorname{rgc}(i)=\operatorname{trapz}\left(t\left(c y c l e_{-} i n d i c e s\right), r c\right) / \operatorname{tgc}(i) ;$

음 Do the same thing as above, but for phi; need to use phi_config

응 here;

phi_c=r(cycle_indices) . *phi_config(cycle_indices)-...

abs (RLd(cycle_indices)) . *sin (phase (cycle_indices));

phi_c=phi_config (cycle_indices)-...

abs (RLd (cycle_indices)) . *sin(phase (cycle_indices));

phi_c $=r\left(c y c l e_{-} i n d i c e s\right) . \star p h i_{-}$config (cycle_indices)-...

abs (RLd(cycle_indices)) . *sin (phase (cycle_indices));

phi_c=r(cycle_indices) . *phi_config(cycle_indices)-...

RLd(cycle_indices).*sin (cycle_indices);

phi_gc $(i)=\operatorname{trapz}\left(t\left(c y c l e_{-} i n d i c e s\right), p h i_{-} c\right) / \operatorname{tgc}(i)$;

for $k=1$ : length $(x C)$

[V-space, Ex, Ey, B, vix, viy, vex, vey, vnx, vny, gx, gy, n-i, n-e, alph, . .

$T_{-} i, T_{-} e$, nneut, $I_{-} i, I_{-} D$, corot_period] $=\ldots$

profiles(TiO, TeO, no, t $(k), x C(k), y c(k), \ldots$

profile_type, $P$, species);

o capacitance of grain with adjustment for debye shielding

$C a p=4 * p i * e p s 0 * a *\left(1+a / I_{-} D\right) ;$

$w_{-} e=\operatorname{sqrt}\left((\operatorname{vx}(k)-v e x) \cdot{ }^{\wedge} 2+(v y(k)-v e y) \cdot{ }^{\wedge} 2\right) ;$

$w_{-} i=\operatorname{sqrt}\left((\operatorname{vx}(k)-v i x) \cdot{ }^{\wedge} 2+(v y(k)-v i y) \cdot{ }^{\wedge} 2\right) ;$

$w_{-}$vec $=\left[\begin{array}{ll}w_{-} e & w_{-} i\end{array}\right]$;

[Itot_eq, qeq, KnR_eq, $\left.P_{-} 0, P_{-} 1, P_{-} g 1\right]=\ldots$

charging_models (1, ch_model, a, alph, $T_{-} e, T_{-} i, n_{-} e, n_{-} i, B, z, C a p, 0, \ldots$

I_D, I_i, w_vec, species);

q-polar (cycle_indices (k)) =q(cycle_indices (k)) /qeq;

I_polar (cycle_indices (k)) =Itot (cycle_indices (k))/Itot_eq; 


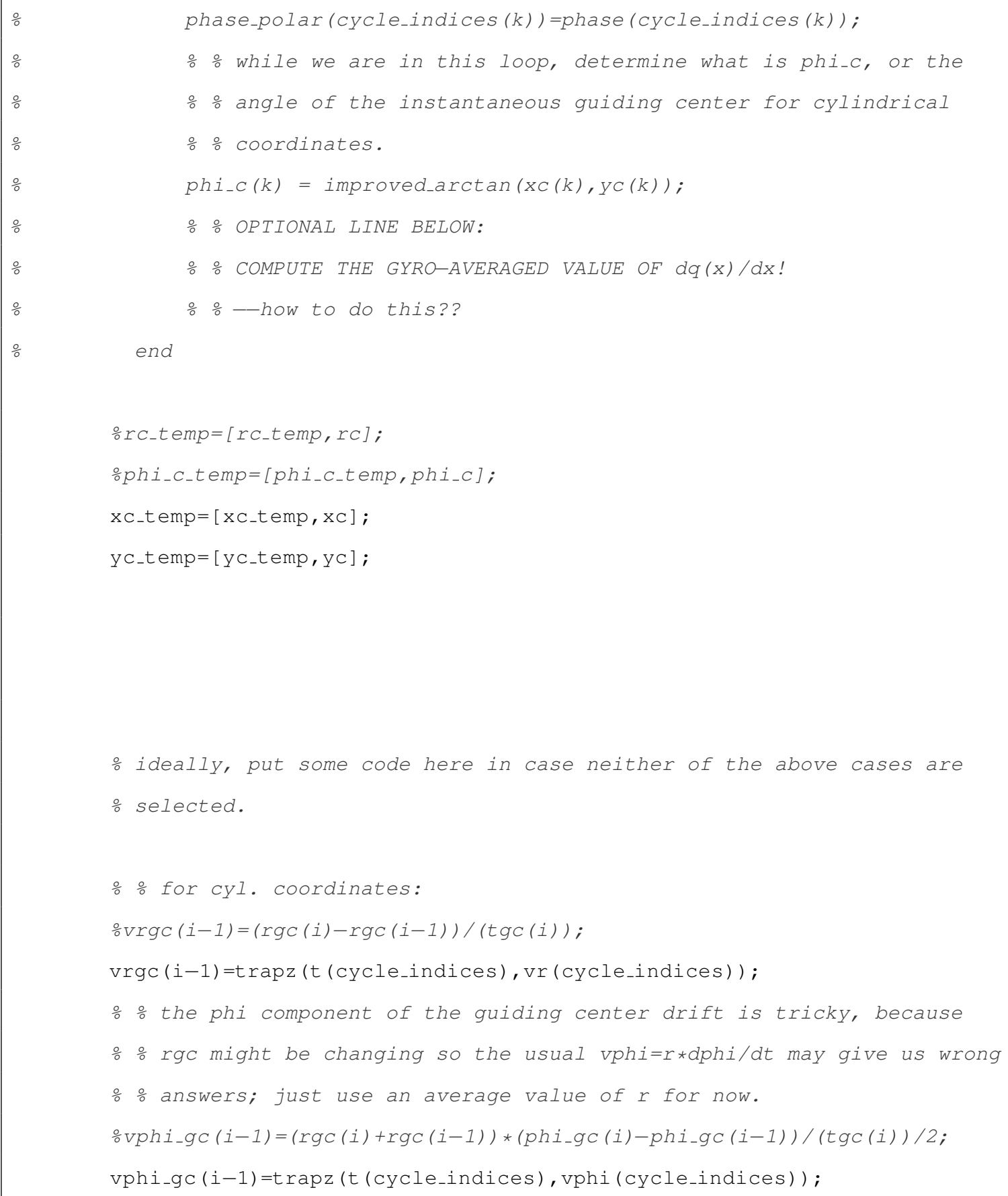




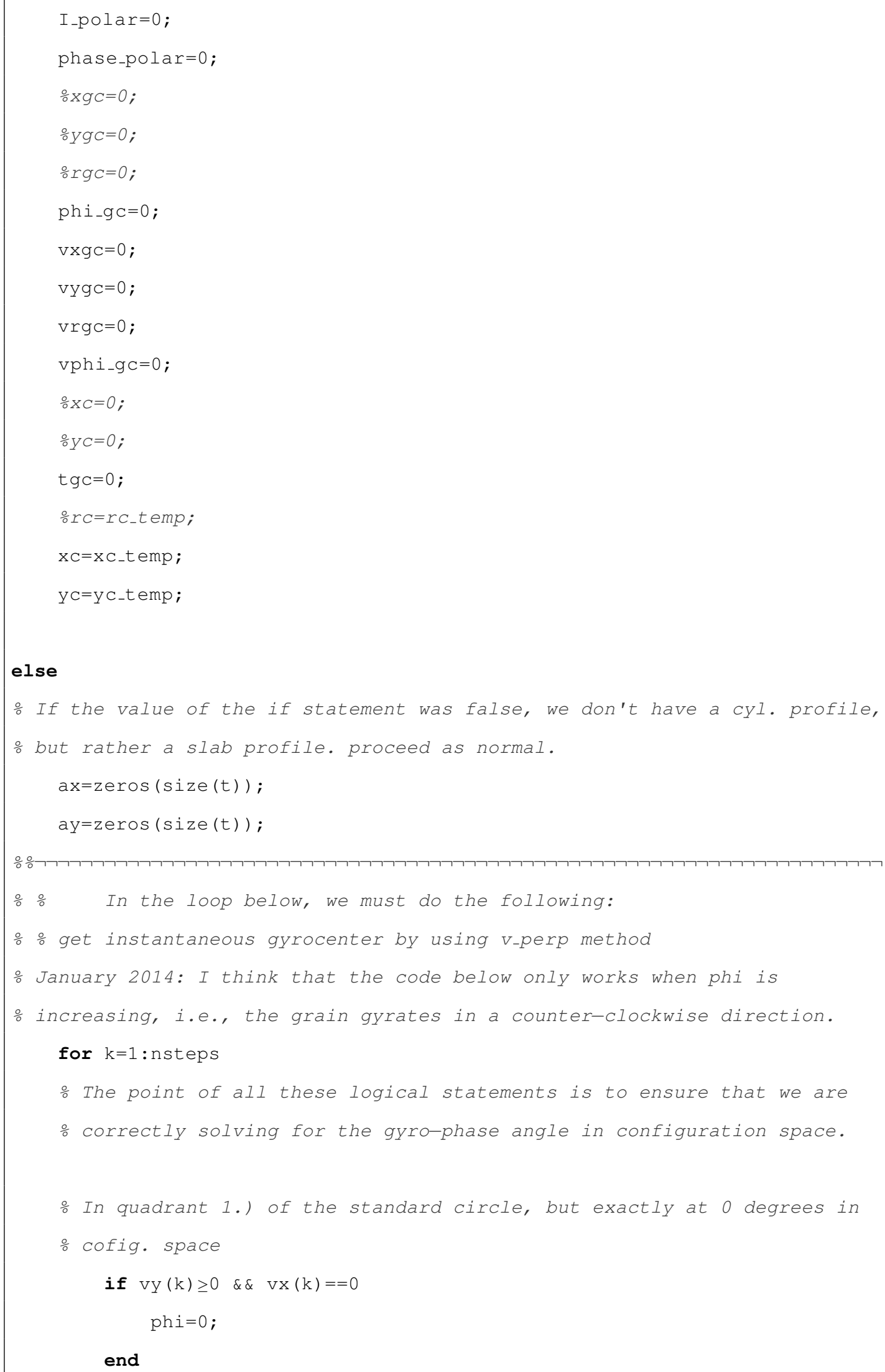


- In quadrant 2.) of the standard circle, but exactly at 90 degrees in

응 cofig. space

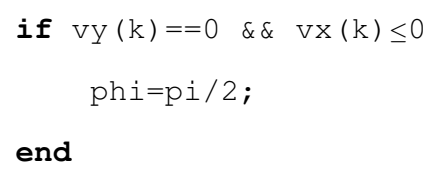

․ In quadrant 3.) of the standard circle, but exactly at 180 degrees in 응 cofig. space

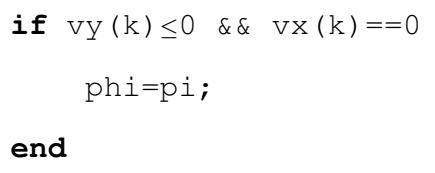


- In quadrant 3.) of the standard circle.

if $\operatorname{Vy}(k)<0$ \&\& $\operatorname{Vx}(k)>0$

phi=atan (abs (vx (k)/vy (k))) +pi;

end

응 quadrant 4.) of the standard circle.

if $\operatorname{Vy}(k)>0 \& \& \quad \mathrm{Vx}(\mathrm{k})>0$

phi=atan $(\operatorname{abs}(\mathrm{vy}(\mathrm{k}) / \mathrm{vx}(\mathrm{k})))+1.5 * \mathrm{pi}$;

o have to add this line to deal with 'clockwise' rotation

if $\mathrm{k} \geq 3$ \&\& (strcmp (direction, ' $\mathrm{cW}$ ') \&\& phi_prev $\leq \mathrm{pi} / 2$ )

counter=counter+1;

end

end

을 $(k)=p h i+2 * p i *($ counter -1$) ;$

- to take care of the first element of the phase array in this loop.

if $\mathrm{k}==1$

phase (1)=phi;

end

응 $k=2$, if the current angle phi is greater than the previous

o calculated angle phi-prev, OR if the current angle phi

if $k==2$ \&\& (phi>phi_prev ||$(p h i<p i / 2$ \&\& phi_prev> 3*pi/2))

direction $={ }^{\prime} \mathrm{CCW}$ ';

end

ㄴ the cw case:

if $k==2$ \&\& (phi<phi_prev ||$\quad(p h i>3 * p i / 2 \& \&$ phi_prev<pi/2))

direction $={ }^{\prime} \mathrm{CW}^{\prime}$;

end

after $k=2$ case has arisen, we can now specify how to update the

ㅇ phase angle.

응 the CCW case:

if $\mathrm{k} \geq 2$ \&\& $\operatorname{strcmp}($ direction, ' $\mathrm{ccw}$ ')

phase $(\mathrm{k})=\mathrm{phi}+2 \star \mathrm{p} i *($ counter -1$)$;

end 
o the cw case:

if $\mathrm{k} \geq 2$ \&\& stremp (direction, ' $\mathrm{CW}$ ')

if $(\mathrm{k}==2$ \&\& phi> $3 * \mathrm{pi} / 2$ \&\& phi_prev<pi/2)

phase (1) =phi_prev+2 $\star$ pi ;

end

phase $(\mathrm{k})=\mathrm{phi}-2 \star \mathrm{p} i *($ counter -1$)$;

end

응 August 27, 2013:

o This line below has become quite complicated compared to how it

\% started out. You need to make sure that the computed angle

응 $0<$ phi<2 $\mathrm{p} i$ is greater than the designated starting angle in order to

update which gyro-cycle you are on. Also, either the previously

o calculated angle phi is less than the starting angle, or phi is less

o than the previously calculated value of phi.

JANUARY 23, 2014:

The statement must be ammended for grains that gyrate in a clockwise

ㅇirection!!

응 "counter-clockwise" gyrating statement

if $\mathrm{k} \geq 2 \& \&$ phi>phi_start $\star \mathrm{pi} / 180 \& \& \ldots$

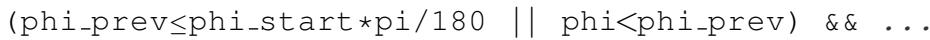

strcmp (direction, 'ccw')

new_cycle (cycle_count) $=\mathrm{k}$;

cycle_count $=$ cycle_count +1 ;

\section{end}

음 "clockwise" gyrating statement

if $\mathrm{k} \geq 2 \& \&($ phi<phi_start $* \mathrm{pi} / 180$ \&\& $\ldots$

(phi_prev $\geq$ phi_start*pi/180 || phi>phi_prev)) \&\& ...

strcmp (direction, ' $\mathrm{cw}$ ')

new_cycle (cycle_count) $=\mathrm{k}$;

cycle_count $=$ cycle_count +1 ;

end 
№ed to set phi-prev equal to the current value of phi.

phi_prev=phi;

last little part here is needed for calculating RLd.

if $\mathrm{k} \geq 2 \quad \& \& \quad \mathrm{k} \leq \mathrm{nsteps}-1$

$\mathrm{ax}(\mathrm{k})=(\mathrm{vx}(\mathrm{k}+1)-\mathrm{vx}(\mathrm{k}-1)) / 2 /(\mathrm{t}(2)-\mathrm{t}(1)) ;$

$\operatorname{ay}(k)=(v y(k+1)-v y(k-1)) / 2 /(t(2)-t(1))$;

end

end

\% for the clockwise direction, I need to subtract off 180 degrees to

을 the correct phase.

if strcmp (direction, ' $\mathrm{cW}$ ')

phase=phase-pi;

end

: these lines are needed, kinda bs but tacked on anyway to keep

acceleration arrays the same size as velocity arrays.

$\operatorname{ax}(1)=\operatorname{ax}(2)$

$\operatorname{ax}($ end $)=\operatorname{ax}($ end -1$)$

$\operatorname{ay}(1)=\operatorname{ay}(2) ;$

ay $($ end $)=a y($ end -1$)$;

- now calculate larmor radius.

RLd=v_perp.^3./ (vx.*ay-vy •*ax);

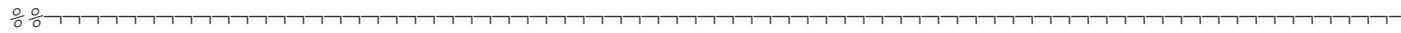

응 make a plot to check on the phase, if desired.

ㄷigure (1); clf;

oplot (phase, '. ')

$\div$ Not sure if the following can go into the loop above, but the idea

응 here is to calculate the average guiding center over a gyroperiod.

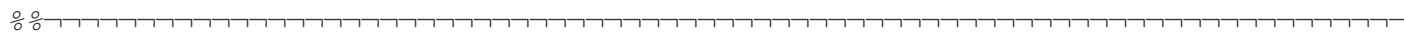

응 set the initial xgc position to the initial $x$-position

\% below must be fixed for arbitrary starting gyro-average angle

$\therefore \operatorname{xgc}(1)=x($ new_cycle (1)) - RLd (new_cycle (1)) *cos (new_cycle (1));

응ㅅ Is that right??? 


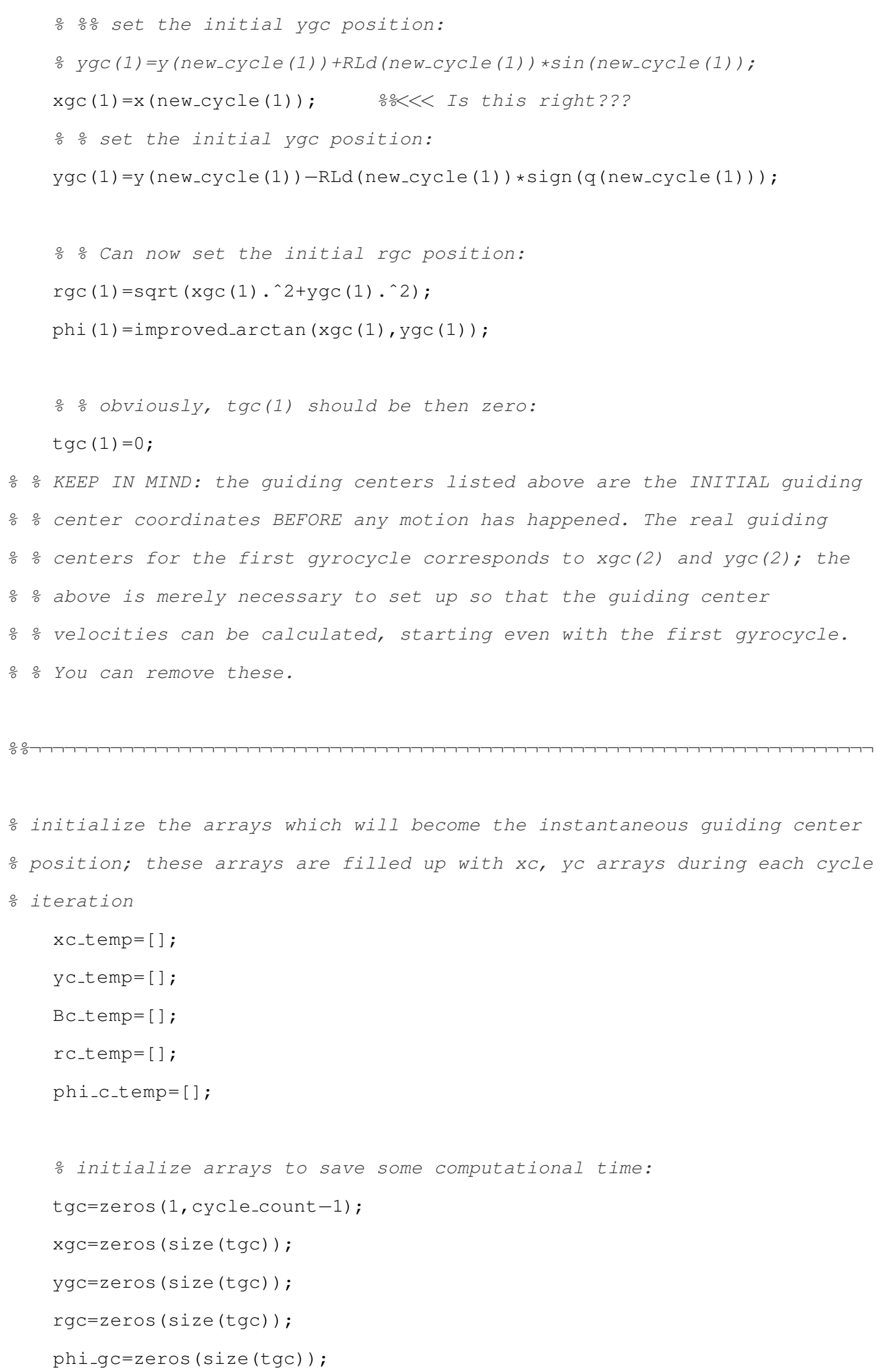




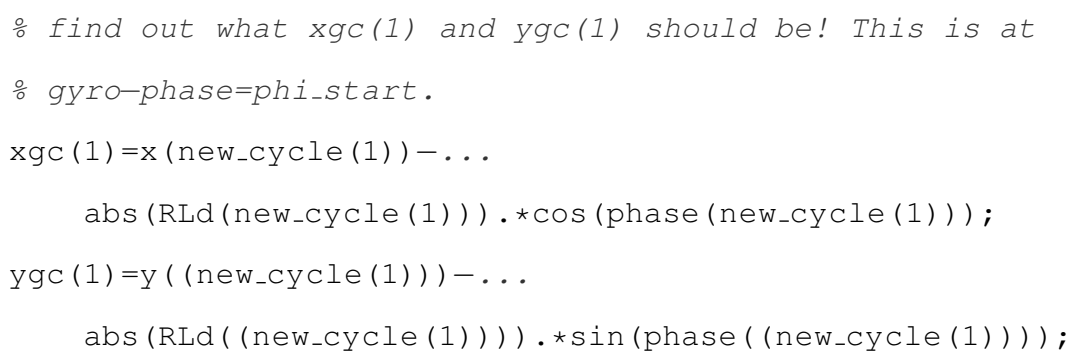




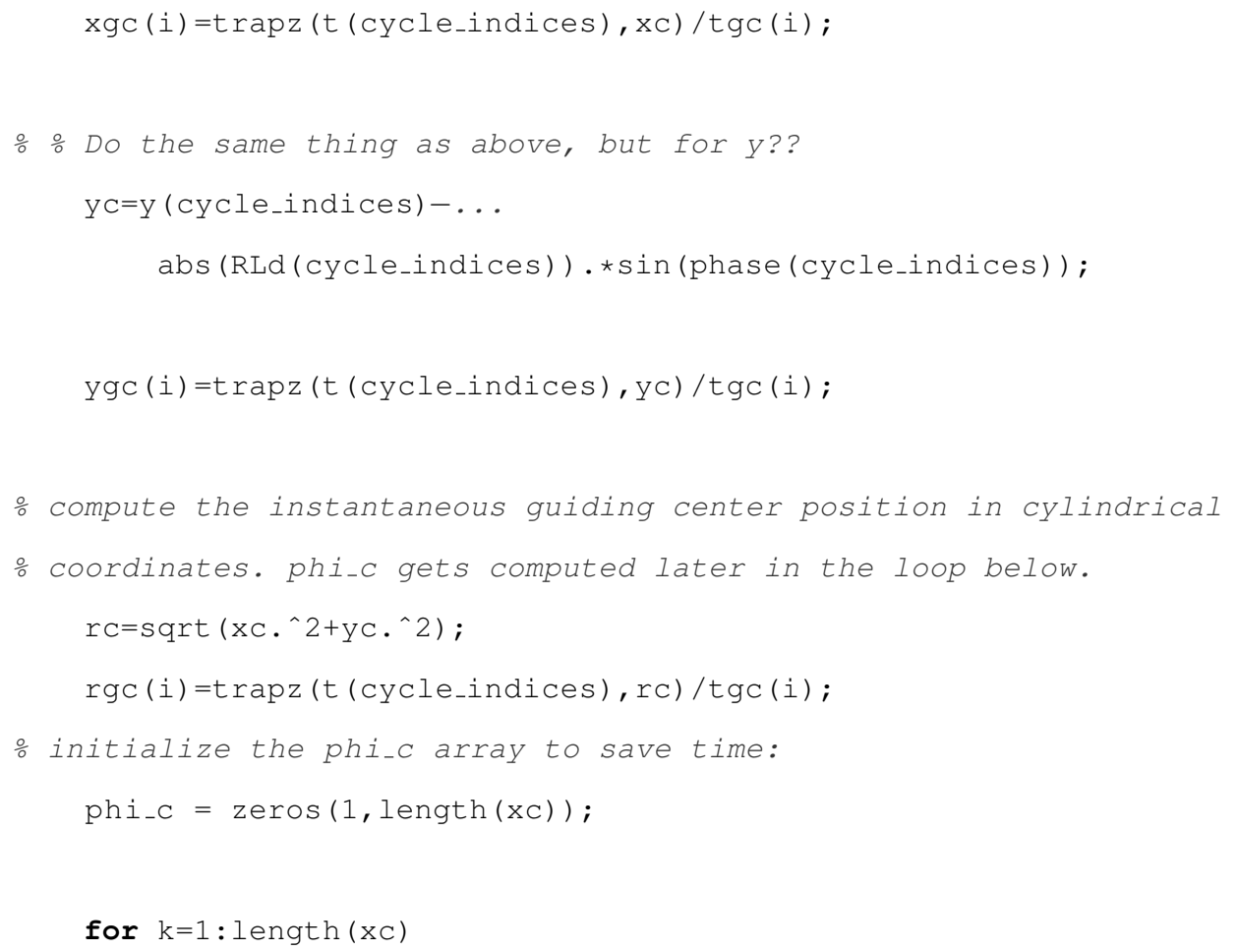




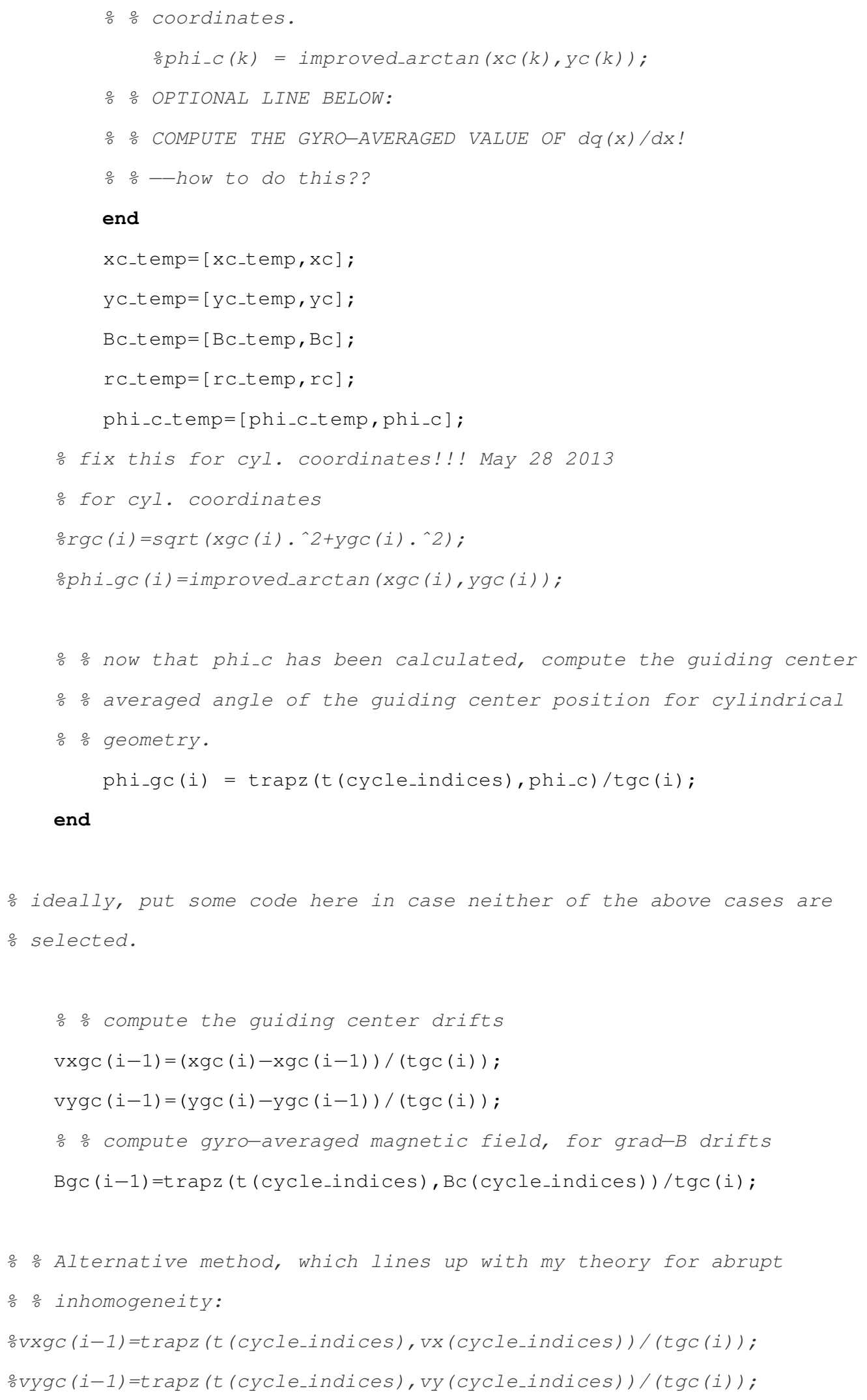




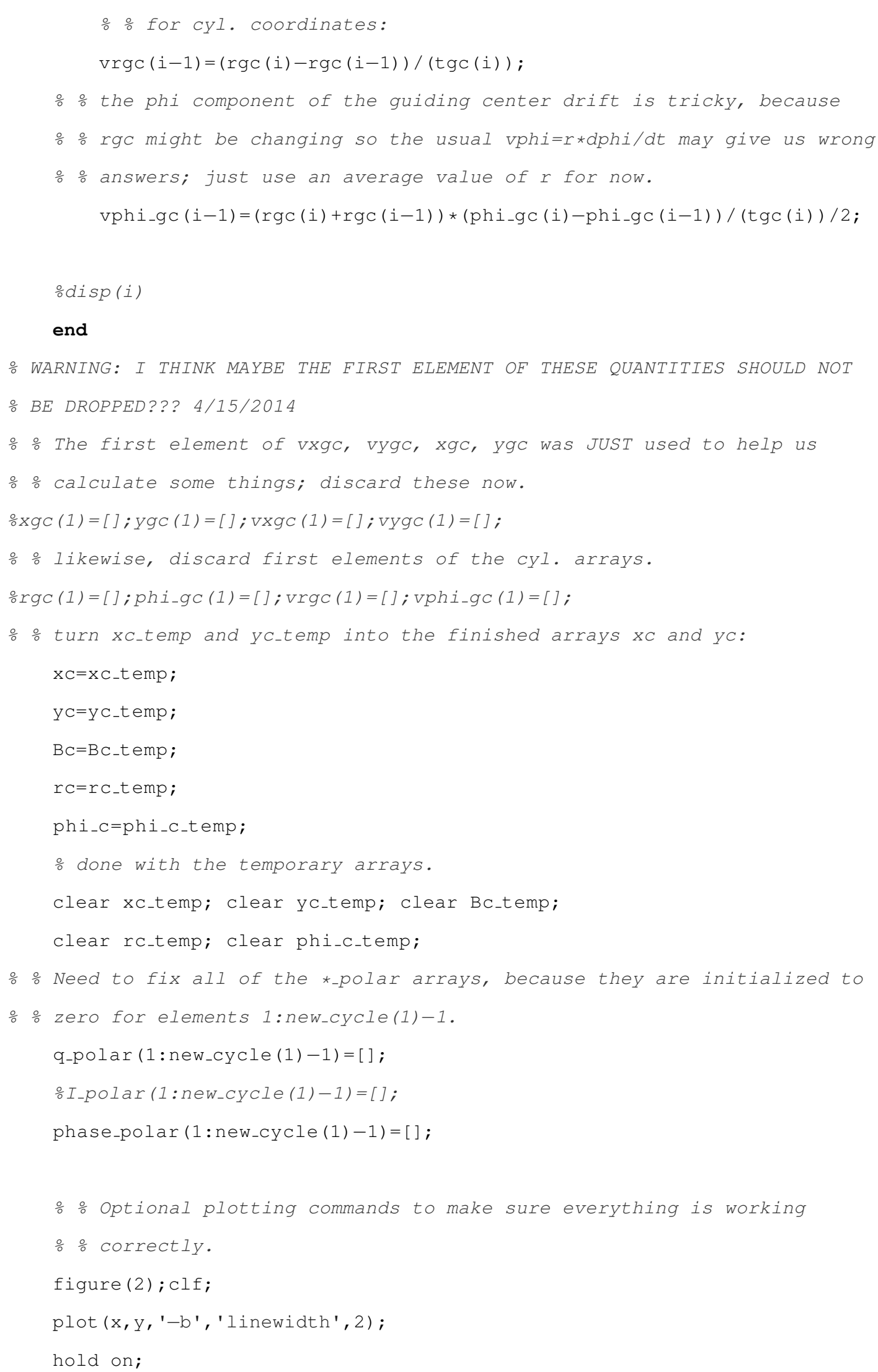




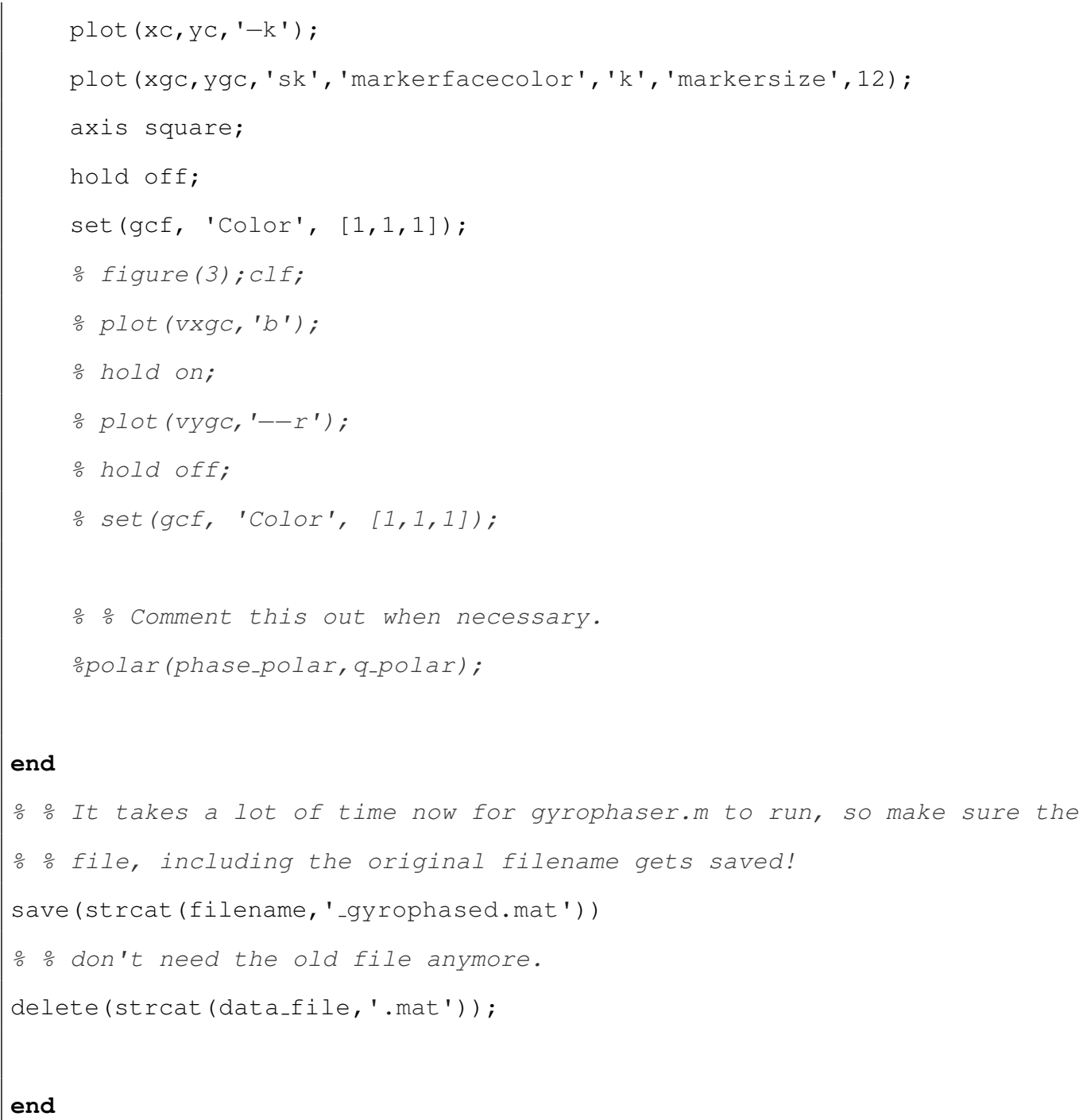

\section{Charging Algorithm Codes}

The subroutine accumulate_charge.m is used to update charge on the grain. For a more detailed description of the charging scheme, consult chapter IV.

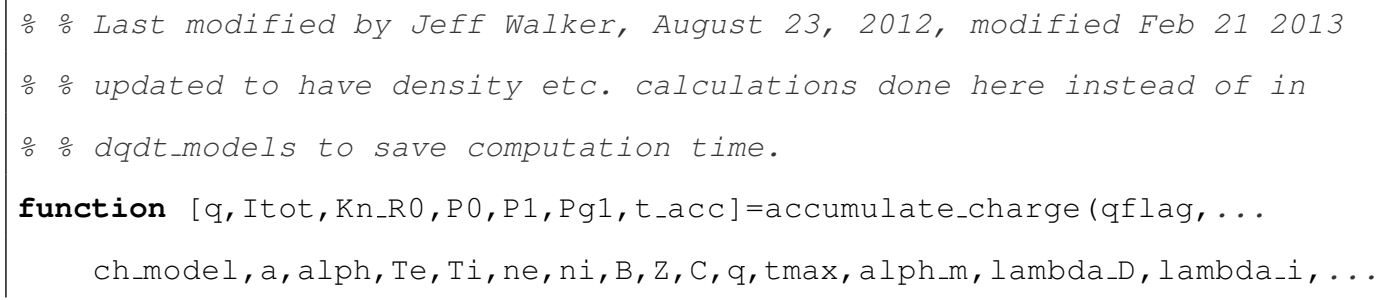




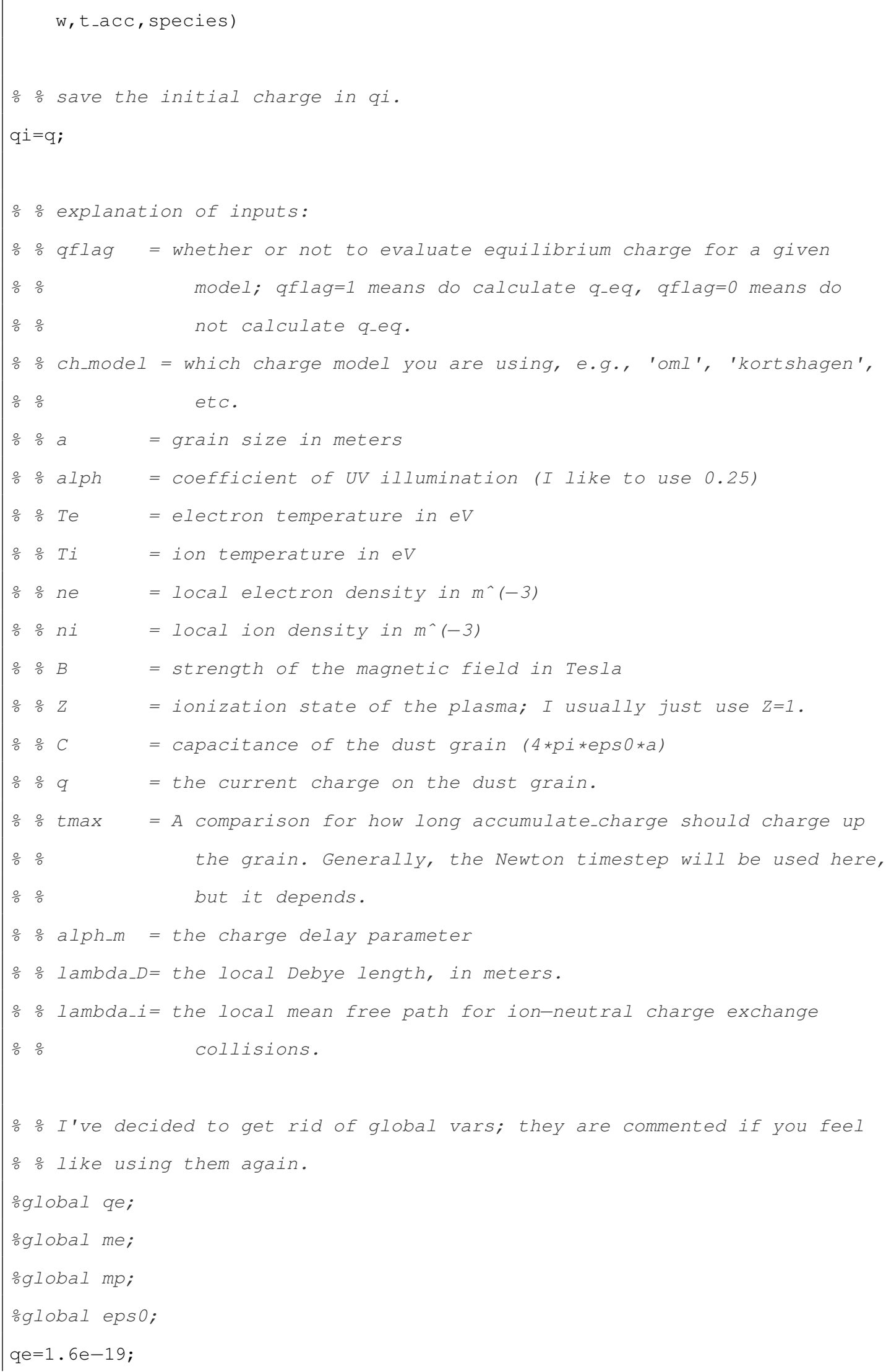




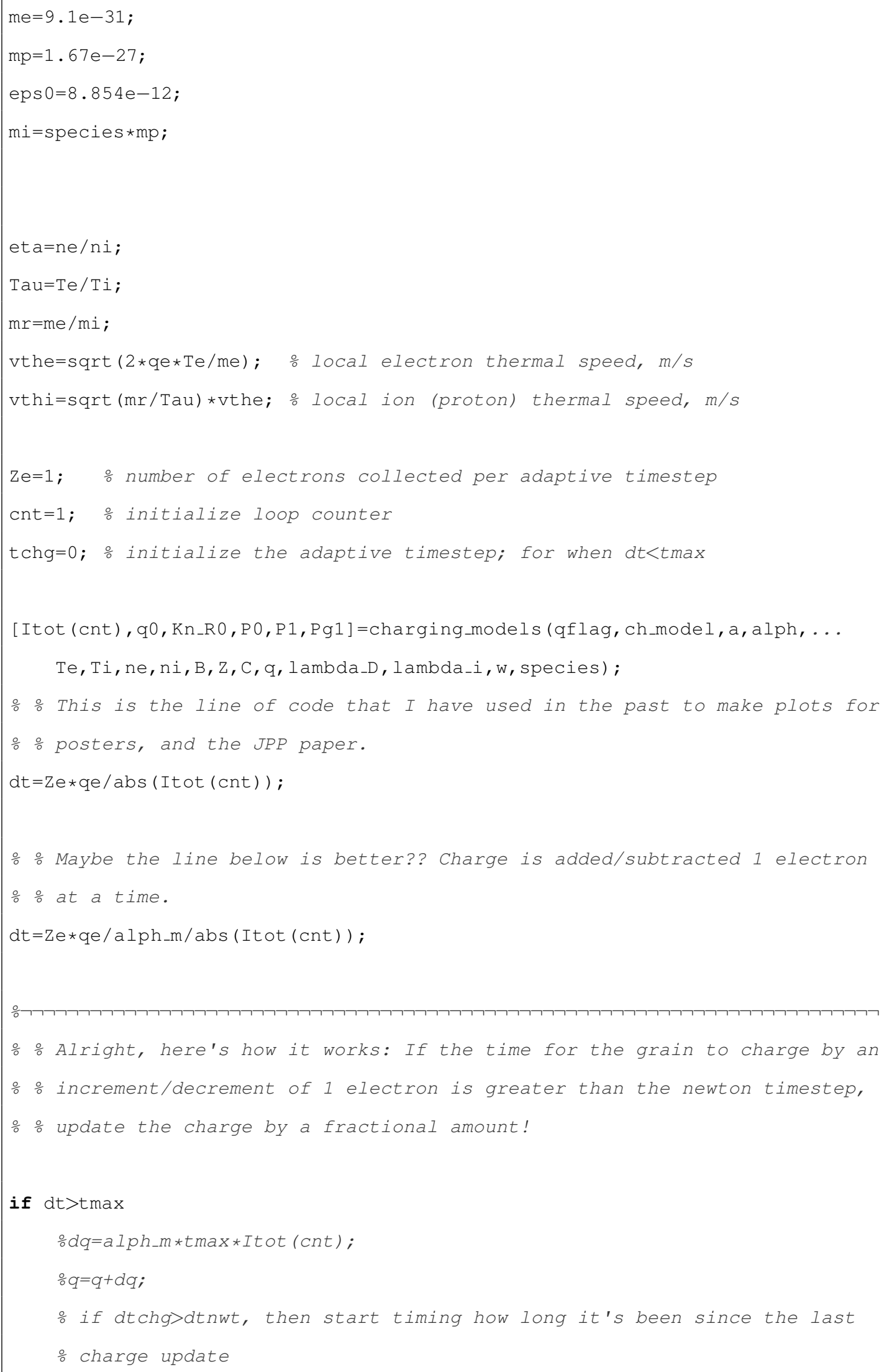




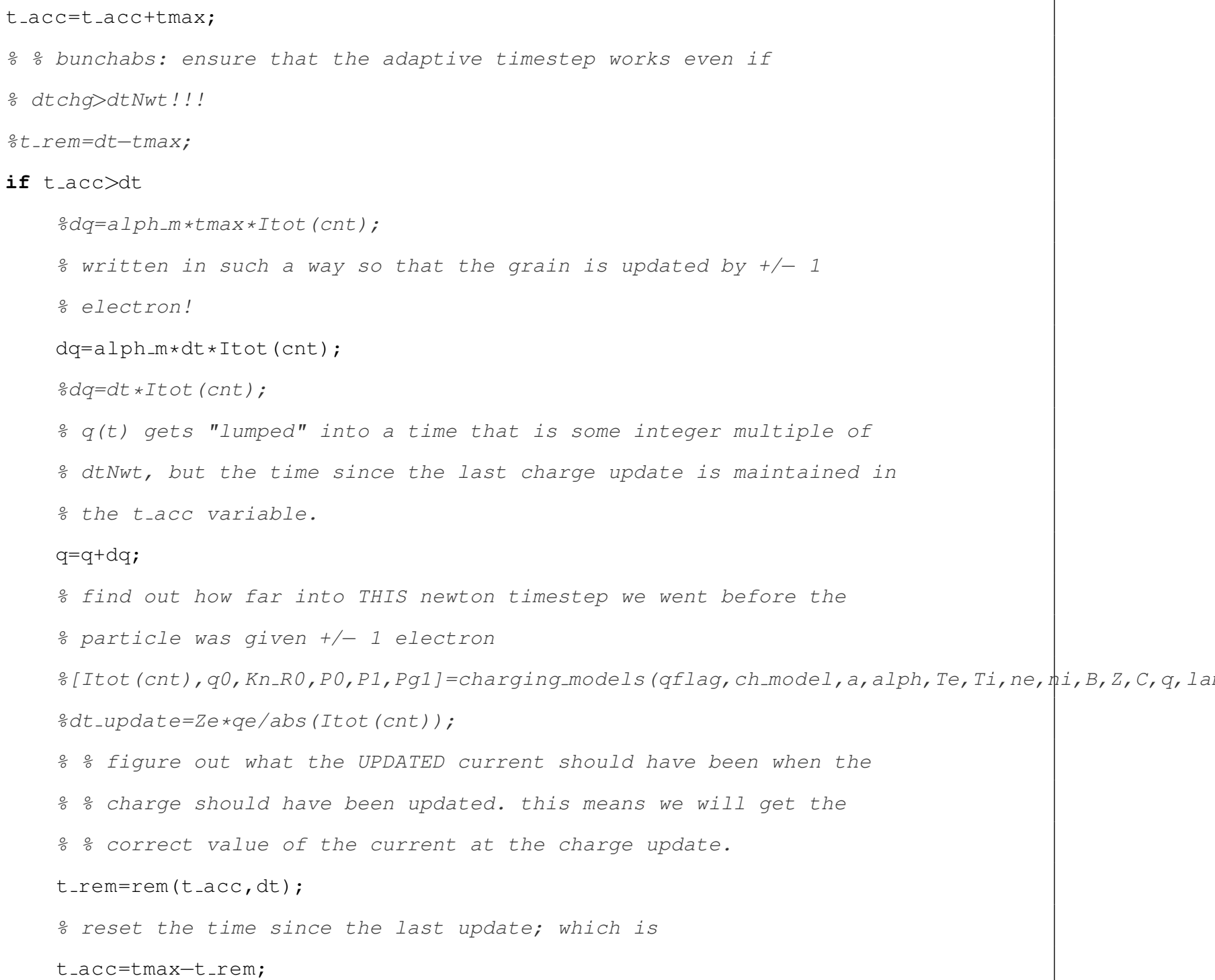

else

while tchg<tmax

o iterate to find equilibrium charge;

ㄴ $\max$ is the newton timestep

$\therefore$ disp ('tchg<dtNwt')

응 $q 0$ is an output of charging models; maybe it is not needed if 
응 using accumulate_charge function

[ Itot (cnt) , q0, Kn_R0, P0, P1, Pg1] =charging_models (qflag, ch_model,a, ... alph, Te, Ti, ne, ni, B, z, C, q, lambda_D , lambda_i, w, species) ;

음 if there is no current to the dust grain, this will be dividing

응 by zero!

$d t=Z e * q e / a b s($ Itot $(c n t))$;

응 Maybe the line below is better??

$\frac{\circ}{o} d t=Z e * q e / a I p h \_m / a b s($ Itot (cnt)) ;

$\div$ 응 the next line is the charge delay

$d q=a l p h \_m * d t * I t o t(c n t) ;$

응 if not using an alpha:

$\therefore d q=d t * I t o t(c n t)$;

if $a b s(d q / q e)<1$

if $d t>t \max$

! !

else

$d t=q e / a b s\left(a l p h \_m * d q d t(c n t)\right) ;$

$d q=q e ;$

end

end

$q=q+d q ;$

tchg=tchg+dt ;

응 How do I fix things to include t_acc? August 2013

$\frac{\circ}{0} t c h g=t \_a c c+t c h g+d t$;

qarr $(\operatorname{cnt})=q$;

o optional: make a time array

$\mathrm{t}(\mathrm{cnt})=\mathrm{tchg}$;

if $\operatorname{cnt}>2 \& \& q \operatorname{arr}(\mathrm{cnt})==\operatorname{qarr}(\mathrm{cnt}-2)$

응 because we have broken out of the loop early, we need to

$\div$ 응 out how much time was left in the newton timestep.

ot_rem=tmax-tchg;

ot_acc=t_rem; $\quad$ o the grain reached its final charge value 


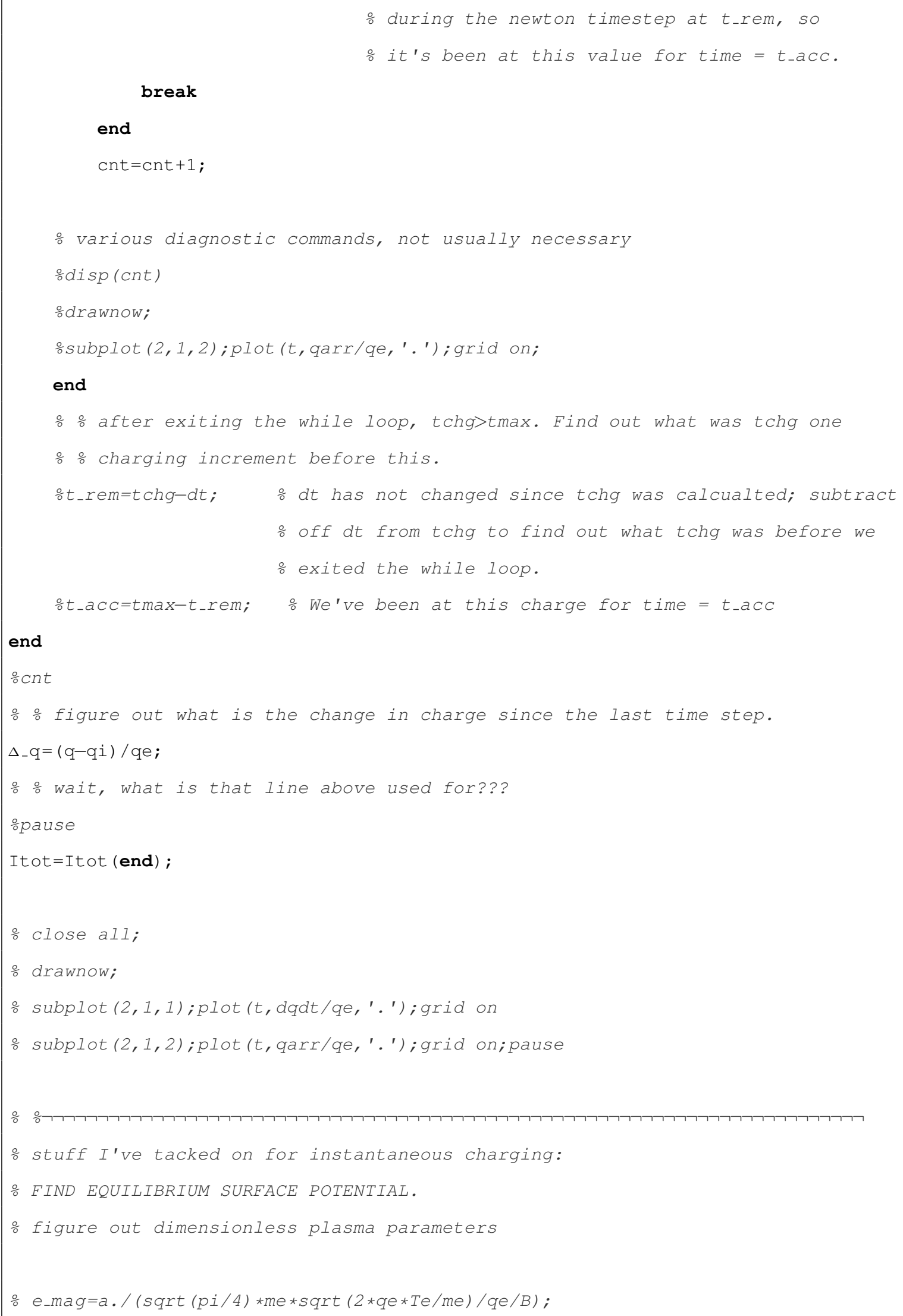




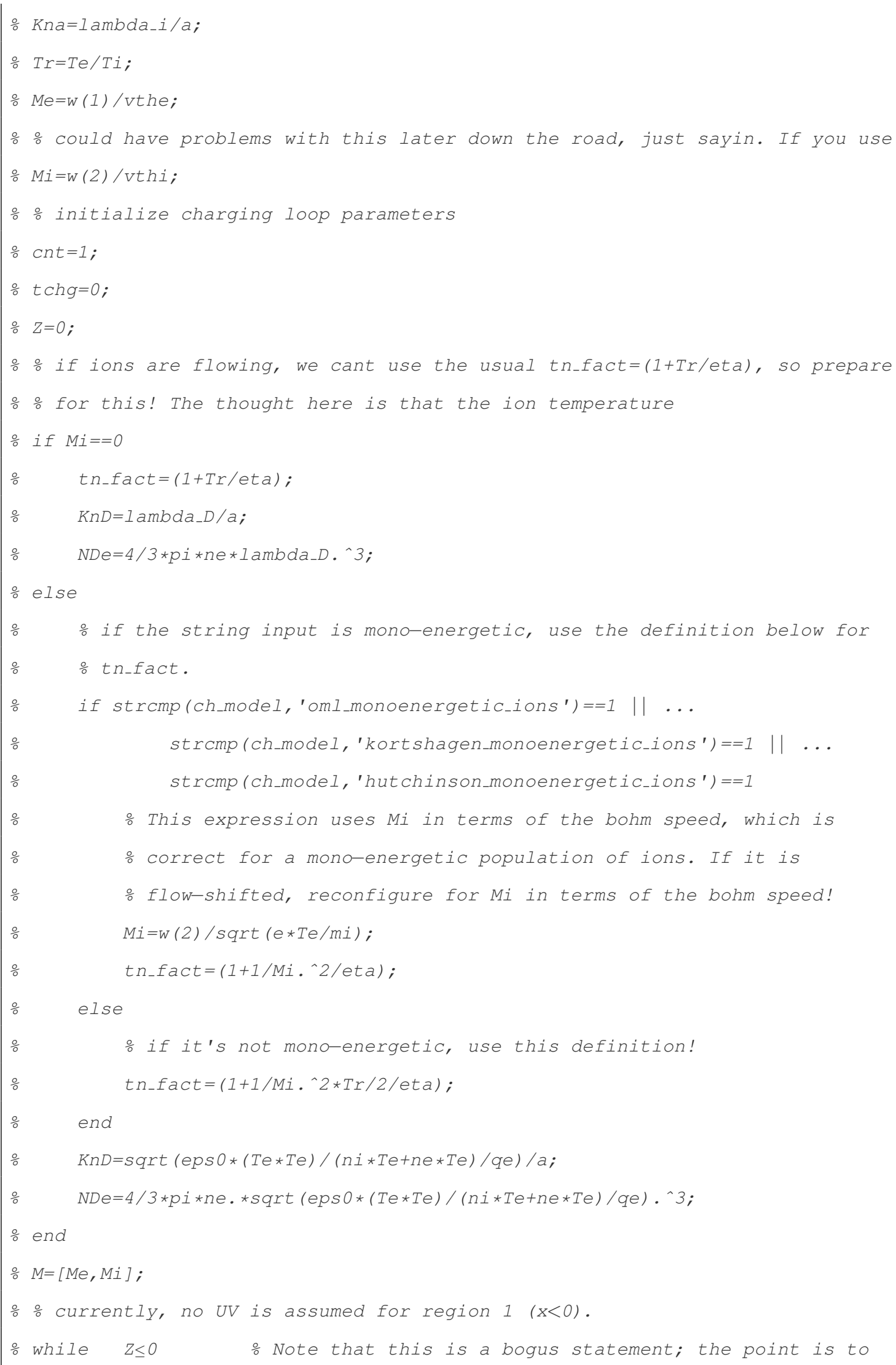




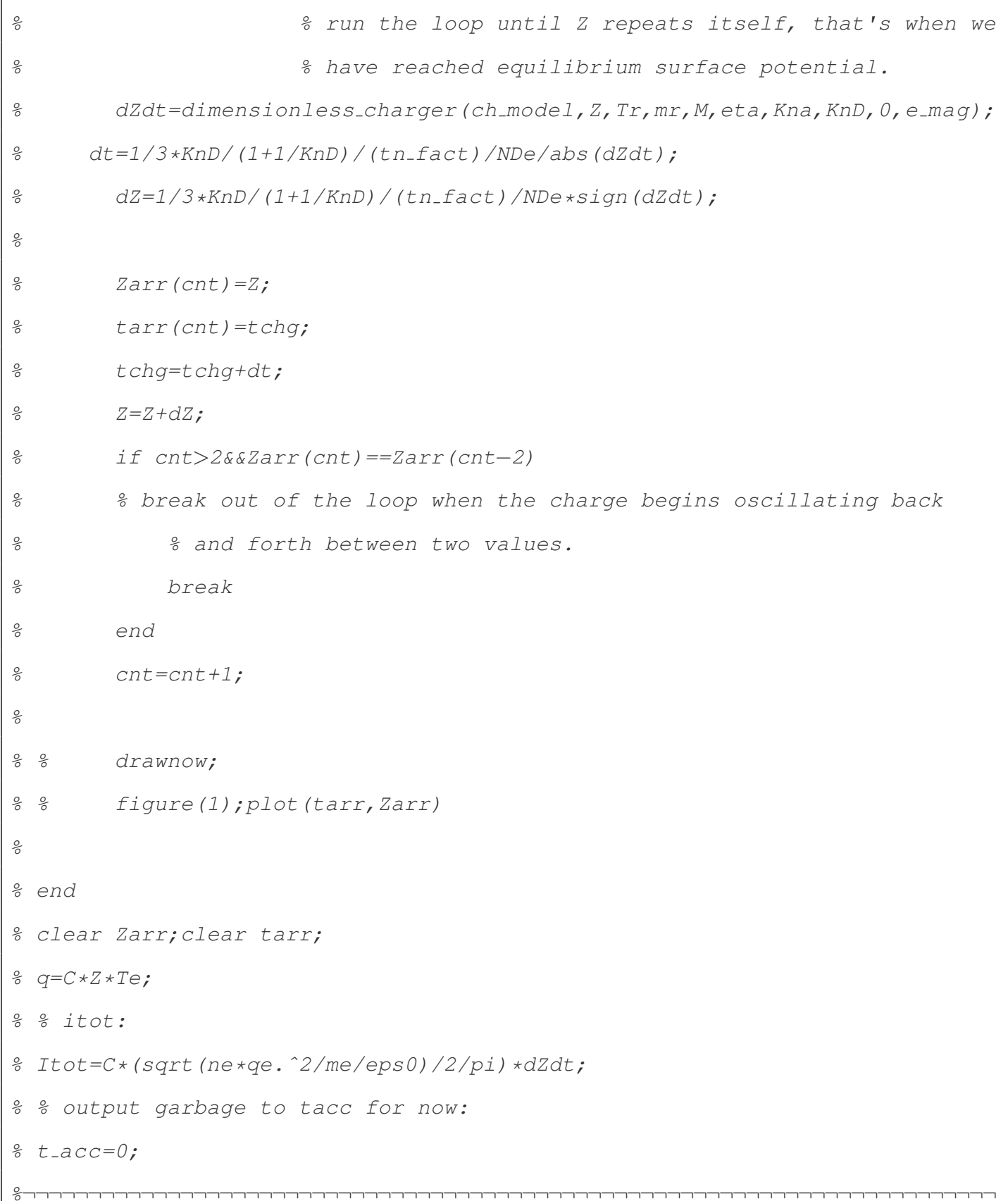

\section{i Specifying Charging Model}

The subroutine charging_models.m allows the user to calculate the current to the dust grain for the local plasma conditions based on the dust grain's current charge state. The user can specify whether to compute the in-situ, equilibrium grain charge, which uses a bisection method. The bisection method codes are listed at the end of section ??. Bear in mind that computing the 
in-situ equilibrium grain charge is not generally recommended when running dust_trajectory.m, since this greatly slows down the program. Instead, use the instantaneous charging feature option in accumulate_charge.m if you want to find trajectories for instantaneous grain charging. The program charging models.m is a subroutine in dust_trajectory.m, but it can also be called independently to find in-situ equilibrium grain charge, charging currents, Knudsen capture radius, and also the probabilities for zero, one, and many ion-neutral charge exchange collisions in the grain sheath. Charging_models.m current supports several charge models, included after this subsection.

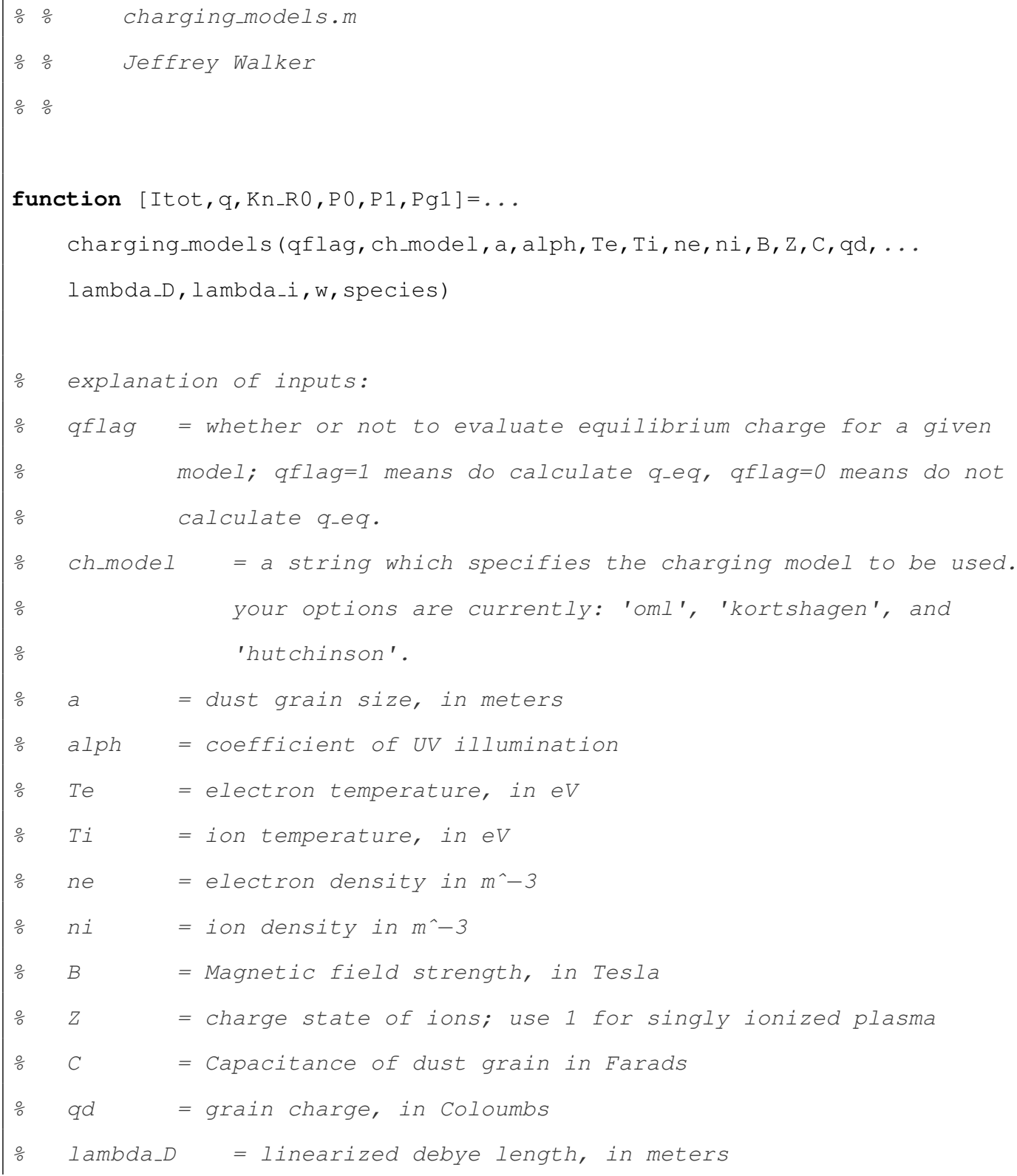




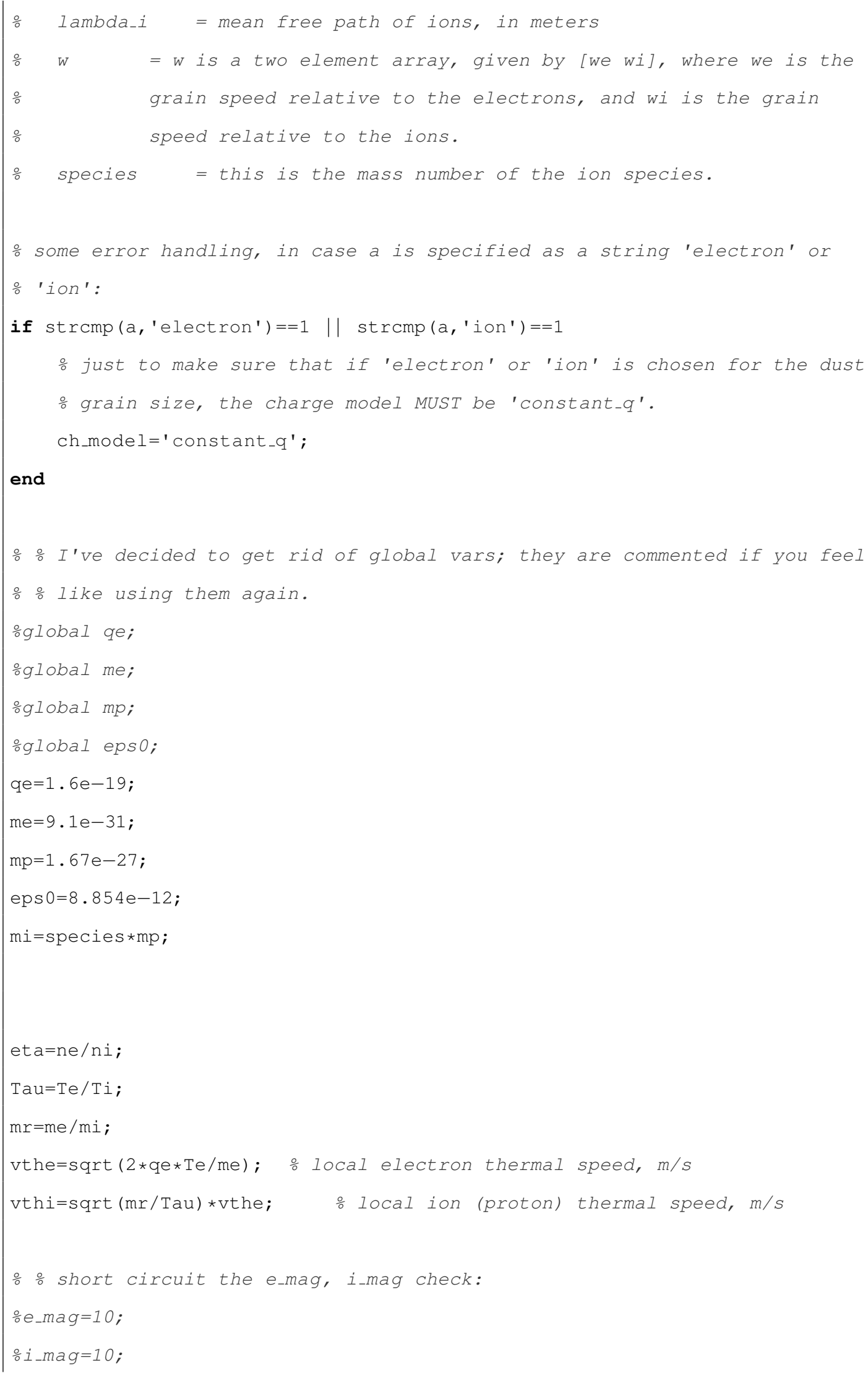


switch ch_model

ㅇ I still have not written the "northrop" charging model function, but

응 is the limiting case of high dust grain velocity relative ions

․ when compared to the ion thermal speed. Electrons are treated as

ㅇ being much faster than the dust grain velocity relative to electrons.

case 'northrop'

[ Itot, $q]=$ northrop $(\mathrm{a}, \mathrm{alph}$, species, mi, . .

Ti, vthi,Rli, ni, Te, vthe, Rle, ne, B, z, C, qd, er, vx, vy, V_space) ;

case 'oml'

[ Itot, q, Kn_R0, P0, P1, Pgl] =oml (qflag, a, alph, Ti, ne, ni, Te, B, z, c, qd, . . lambda_D, lambda_i, w, species) ;

case 'simple'

[Itot, q] = simple(qflag, alph, ne, ni, eta, vthe, vthi,a, qd, c) ;

case 'kortshagen'

[ Itot, $q$, Kn_R0, P0, P1, Pgl] =kortshagen (qflag, a, alph, Ti, ni, Te, ne, . .

B, Z, C, qd, lambda_D, lambda_i, w, species) ;

case 'hutchinson'

[ Itot, q, Kn_R0, P0, P1,Pg1] =hutchinson (qflag, a, alph, species,...

Ti, ne, ni, Te, B, Z, C, qd, lambda_D, lambda_i, w) ;

case 'oml_monoenergetic_ions'

[ Itot, q, Kn_R0, P0, P1,Pgl] =oml_monoenergetic_ions (qflag, a, alph, Ti, ne, ni, Te, B, Z, C, qd, . . . lambda_D , lambda_i,w, species);

constant_q charge model fixes the grain charge to a given number.

\% This can be used to show particle trajectories for ions or electrons

․ in a profile specified by profiles.m.

case 'constant_q'

\% not using Knudsen capture parameters for this profile.

$\mathrm{Kn} \_\mathrm{RO}=0 ; \mathrm{PO}=0 ; \mathrm{P} 1=0 ; \mathrm{Pg} 1=0$;

․ no current, because the charge is specified to be constant.

Itot $=0$ 


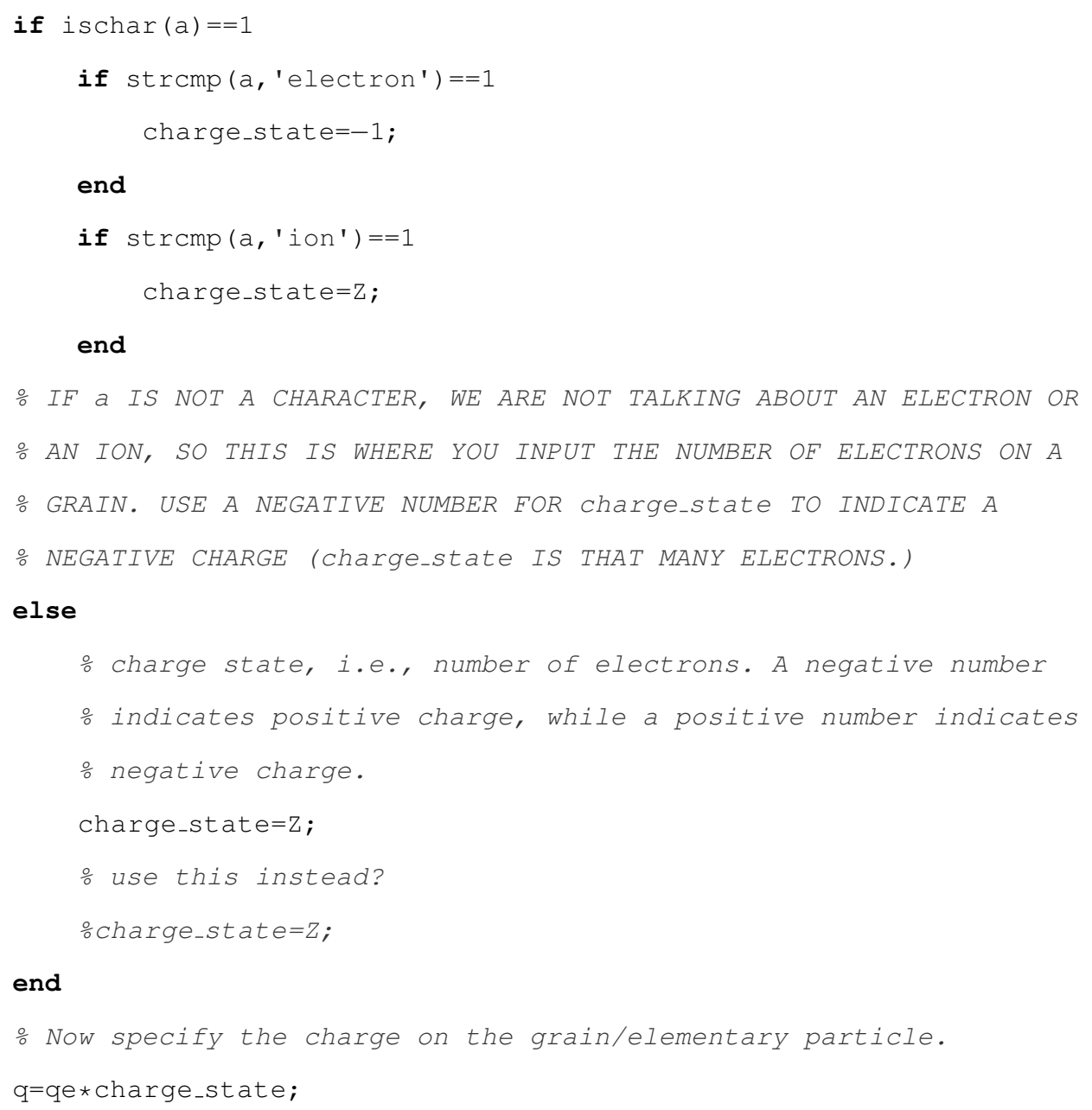

\section{ii Charging Model Selection}

This subsection includes all of the MATLAB code for the different charging models investigated in this dissertation.

\section{i OML Model Codes}

Included below is the MATLAB code for oml.m, which is the OML model for grain charging and includes drifting Maxwellian electron and drifting Maxwellian ion populations. 


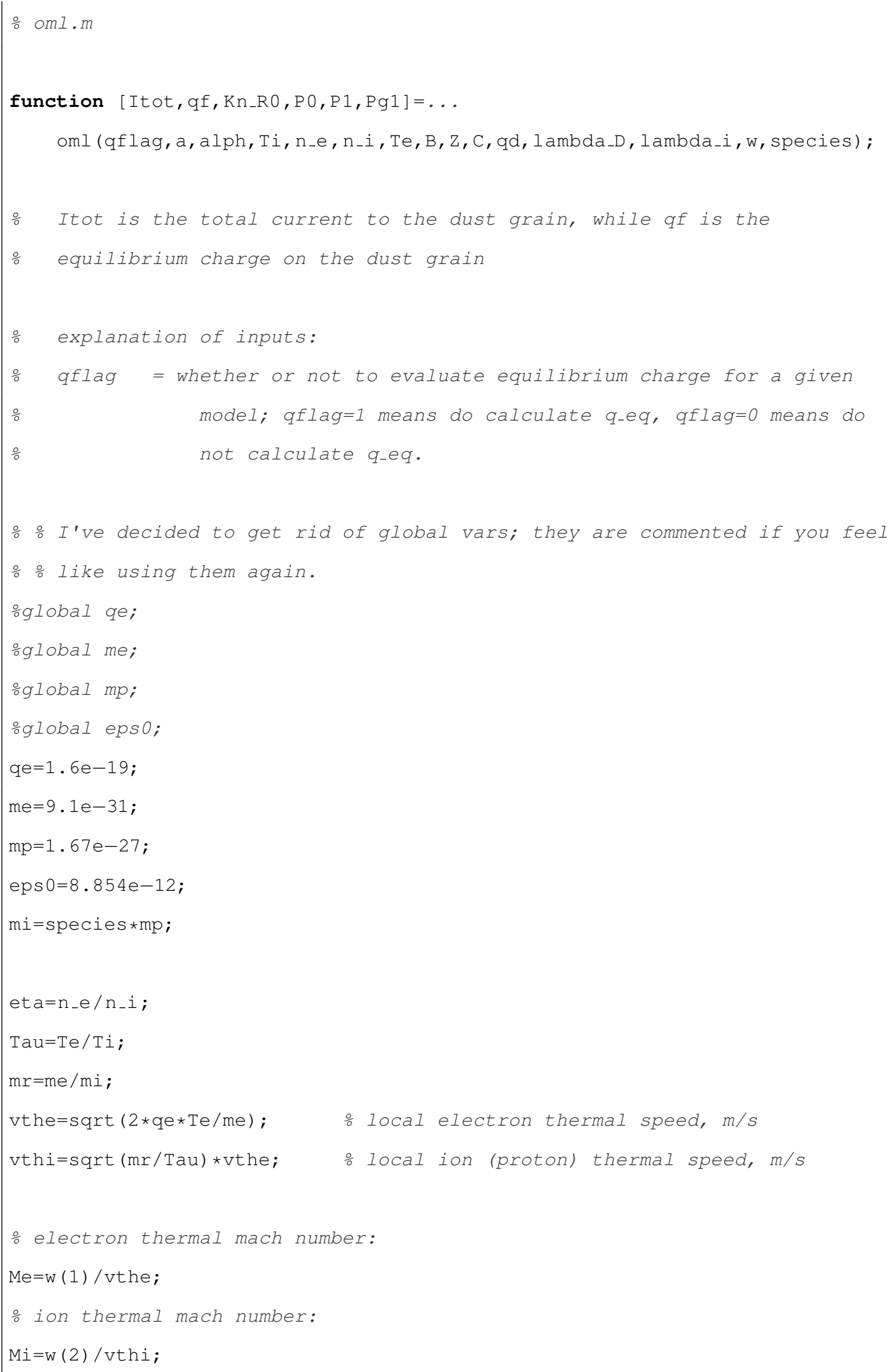




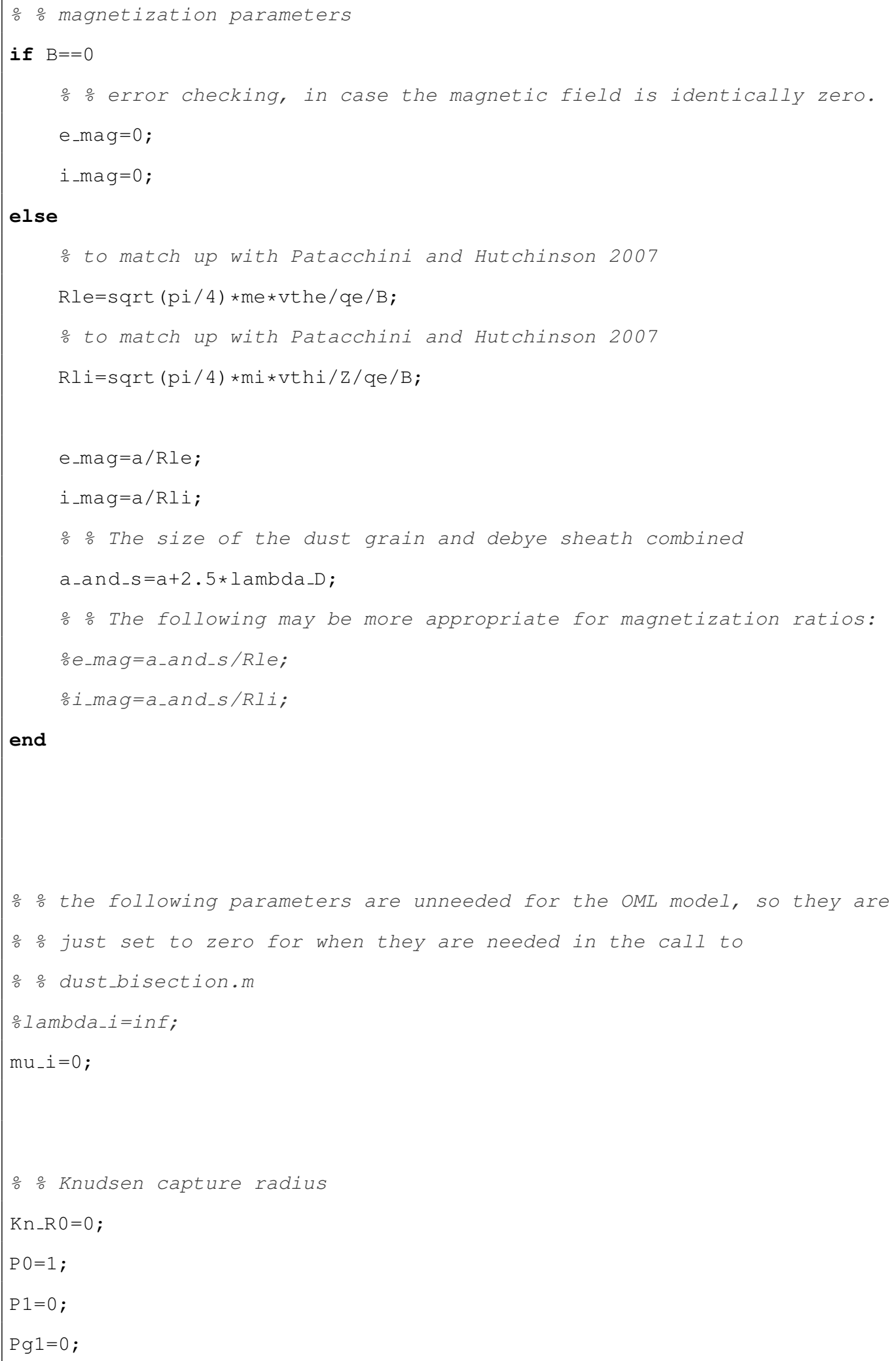


- Calculate plasma currents to particle

if $q d \leq 0$ 을 negative dust potential (phi=qd/C)

if e_mag<1 응 UNMAGNETIZED ELECTRONS

if $\mathrm{Me}=0$

음 derived via integration of Maxwellian from vmin=sqrt (2*qe*phi/me)

응 to infinity, given $4 \star p i \star a^{\wedge} 2$ collection area

$\therefore I e=-n e * q e *\left(4 * p i * a^{\wedge} 2\right) * v t h e * \exp (q d / C / T e)$;

$\mathrm{Ie}=-\mathrm{n}_{-} \mathrm{e} * \mathrm{qe} *\left(4 * \mathrm{pi} * \mathrm{a}^{\wedge} 2\right) / \operatorname{sqrt}(4 * \mathrm{pi}) * \mathrm{vthe} * \exp (\mathrm{qd} / \mathrm{C} / \mathrm{Te})$;

o expression above matches Patacchini and Hutchinson, 2007

else

$\frac{\circ}{\circ} e=-n_{-} e * q e *\left(4 * p i * a^{\wedge} 2\right) / \operatorname{sqrt}(4 * p i) * v t h e * \exp (q d / C / T e) ;$

Ie $=-.5 * \operatorname{sqrt}(p i) * a \cdot{ }^{\wedge} 2 * n_{-} e * q e * v t h e / \operatorname{Me} *(\ldots$

$\left(\mathrm{Me} \cdot{ }^{\wedge} 2+.5+q d / C / \mathrm{Te}\right) * \operatorname{sqrt}(\mathrm{pi}) *(\operatorname{erf}($ Me $+\operatorname{sqrt}(-\mathrm{qd} / \mathrm{C} / \mathrm{Te}))+\ldots$

$\operatorname{erf}(\mathrm{Me}-\operatorname{sqrt}(-\mathrm{qd} / \mathrm{C} / \mathrm{Te})))+\ldots$

$(\operatorname{sqrt}(-q d / C / T e)+M e) \star \exp \left(-(M e-\operatorname{sqrt}(-q d / C / T e)) \cdot{ }^{\wedge} 2\right)-\ldots$

$\left.(\operatorname{sqrt}(-\mathrm{qd} / \mathrm{C} / \mathrm{Te})-\mathrm{Me}) * \exp \left(-(\mathrm{Me}+\operatorname{sqrt}(-\mathrm{qd} / \mathrm{C} / \mathrm{Te})) \cdot{ }^{\wedge} 2\right)\right)$;

end

else 응 MAGNETIZED ELECTRONS; this

음 same as unmagnetized case, except collection area reduced due

응 to magnetization; may be off by some constant factor due to

$\div$ 응-dependence of incidence angle

if $\mathrm{Me}==0$

을 derived via integration of Maxwellian from vmin=sqrt (2*qe*phi/me)

응 to infinity, given $4 * p i * a^{\wedge} 2$ collection area

$\frac{\circ}{\circ} I e=-n e * q e *\left(4 * p i * a^{\wedge} 2\right) * v t h e * \exp (q d / C / T e)$;

$\mathrm{Ie}=-.5 * \mathrm{n}_{-} \mathrm{e} * \mathrm{qe} *\left(4 * \mathrm{pi} * \mathrm{a}^{\wedge} 2\right) / \operatorname{sqrt}(4 * \mathrm{pi}) * v \operatorname{the} * \exp (\mathrm{qd} / \mathrm{C} / \mathrm{Te})$;

expression above matches Patacchini and Hutchinson, 2007

else

$\therefore I e=-n_{-} e * q e *\left(4 * p i * a^{\wedge} 2\right) / \operatorname{sqrt}(4 * p i) * v t h e * \exp (q d / C / T e) ;$

$\mathrm{Ie}=-.25 * \operatorname{sqrt}(\mathrm{pi}) * \mathrm{a} .{ }^{\wedge} 2 * \mathrm{n}_{-} \mathrm{e} * \mathrm{qe} * \mathrm{vthe} / \operatorname{Me} *(\ldots$

$\left(\mathrm{Me} \cdot{ }^{\wedge} 2+.5+\mathrm{qd} / \mathrm{C} / \mathrm{Te}\right) * \operatorname{sqrt}(\mathrm{pi}) *(\operatorname{erf}($ Me $+\operatorname{sqrt}(-\mathrm{qd} / \mathrm{C} / \mathrm{Te}))+\ldots$

$\operatorname{erf}(\mathrm{Me}-\operatorname{sqrt}(-\mathrm{qd} / \mathrm{C} / \mathrm{Te})))+\ldots$

$(\operatorname{sqrt}(-\mathrm{Me} \star \mathrm{qd} / \mathrm{C} / \mathrm{Te})+\mathrm{Me}) \star \exp \left(-(\mathrm{Me}-\operatorname{sqrt}(-\mathrm{qd} / \mathrm{C} / \mathrm{Te})) \cdot{ }^{\wedge} 2\right)-\ldots$ 
end

end

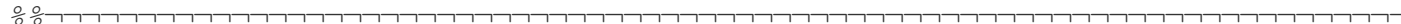

if i_mag $<1$ 응 UNMAGNETIZED IONS

응 OML current, cf. Allen, Phys. Scr. 45 (1992), eq. 51

ㅇ $I i=n i * q e *\left(4 * p i * a^{\wedge} 2\right) * \operatorname{sqrt}(-2 * q e * q d / \mathrm{C} / \mathrm{mi})$;

응 I'm replacing the above line with the current I see more

응 often in the literature (Shukla 2001 pop, for example)

- Include a statement for $\mathrm{w}==0$ so that there is no division by

ㄱero.

if $\mathrm{Mi}==0$

$\mathrm{Ii}=\mathrm{n}_{-} i * \mathrm{qe} *\left(4 * \mathrm{pi} * \mathrm{a}^{\wedge} 2\right) / \operatorname{sqrt}(4 * \mathrm{pi}) * \mathrm{vthi} *(1-\mathrm{qd} / \mathrm{C} / \mathrm{Ti})$;

응 expression above matches Patacchini and Hutchinson, 2007

else

Ii $=\operatorname{sqrt}(p i) * a \cdot{ }^{\wedge} 2 * n_{-} i * Z * q e * \operatorname{vth} i *(\ldots$

(Mi.^2+0.5-qd/C/Ti) *sqrt (pi)/Mi*erf (Mi) +exp (-Mi .^2))；

응 see 1992_northrop_ps or 1981_whipple_repprogphys for the

응 above. Also, 1996 Horanyi and 1996 Northrop.

end

$\div$ Call the Newton-Raphson method to find the equilibrium charge FOR

$\div$ THE INPUT CONDITIONS.

if $\mathrm{qflag}==1$

응 a charge between 0 and leg elementary charges is a good

$\frac{\circ}{0}$ search interval.

[output]=dust_bisection (1e9, 'oml' , (qd/qe), eta, alph, Ti, Te,...

e_mag, i_mag, C, lambda_D, lambda_i,a,w, species) ;

$q f=$ round (output);

$q f=q f * q e$;

else

응 return 0 if you do not wish to calculate equilibrium charge

$\div \quad(q f l a g=0$.

$q f=0$; 
\% just return zero, value does not matter we just want to

응 skip the charge calculation step.

end

o now that charge, potential profile, and ion mean free path are

․ known, the Capture Radius can be calculated at the mean ion

응 thermal kinetic energy.

$\mathrm{RO}=\left(\mathrm{abs}(\mathrm{qd} / \mathrm{C}) * \mathrm{a} *\left(1+\mathrm{a} / \mathrm{l} \mathrm{ambda}_{-} \mathrm{D}\right)\right) /\left(1.5 * \mathrm{~T} i+\mathrm{abs}(\mathrm{qd} / \mathrm{C}) * \mathrm{a} / \mathrm{l} \mathrm{ambda}_{-} \mathrm{D}\right) ;$

if $\mathrm{R} 0==0 \quad||$ lambda_i==inf

$\therefore$ If $R O=0$, use OML currents??

$\mathrm{P} 0=1$;

$\mathrm{P} 1=0 ;$

$\operatorname{Pg} 1=0$;

$\mathrm{Kn}_{-} \mathrm{RO}=0$;

else

- compute the Knudsen Capture radius; see 2008 Gatti PRE for

응 detils

Kn_R0 = lambda_i $/(2 * 1.22 * R 0) ;$

$\mathrm{P} 0=\exp \left(-1 /\left(\mathrm{Kn}_{-} \mathrm{R} 0\right)\right)$;

$\mathrm{P} 1=\left(1 /\left(\mathrm{Kn}_{-} \mathrm{R} 0\right)\right) * \exp \left(-1 /\left(\mathrm{Kn}_{-} \mathrm{R} 0\right)\right)$;

$\mathrm{Pg} 1=1-(\mathrm{P} 0+\mathrm{P} 1) ;$

end

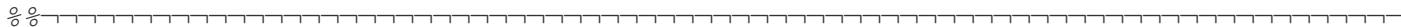

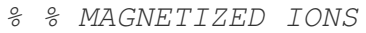

else

응 simple thermal flux of ions, assuming ballistic trajectories

응 along field lines w/ reduced collection area due to

응 magnetization

$\mathrm{I} i=\mathrm{n}_{-} i \star \mathrm{qe} *\left(2 \star \mathrm{p} i \star \mathrm{a}^{\wedge} 2\right) * \mathrm{vthi}$

\% reference for the above?? Maybe 1982 Rubenstein phys. fluids

응 for magnetized ions and electrons, a simple charge model is

응 sufficient.

if $q f \operatorname{lag}==1$

$q f=0.5 * C * \operatorname{Te} * \log (\mathrm{mr} / \mathrm{Tau} /$ eta/eta $) ;$ 


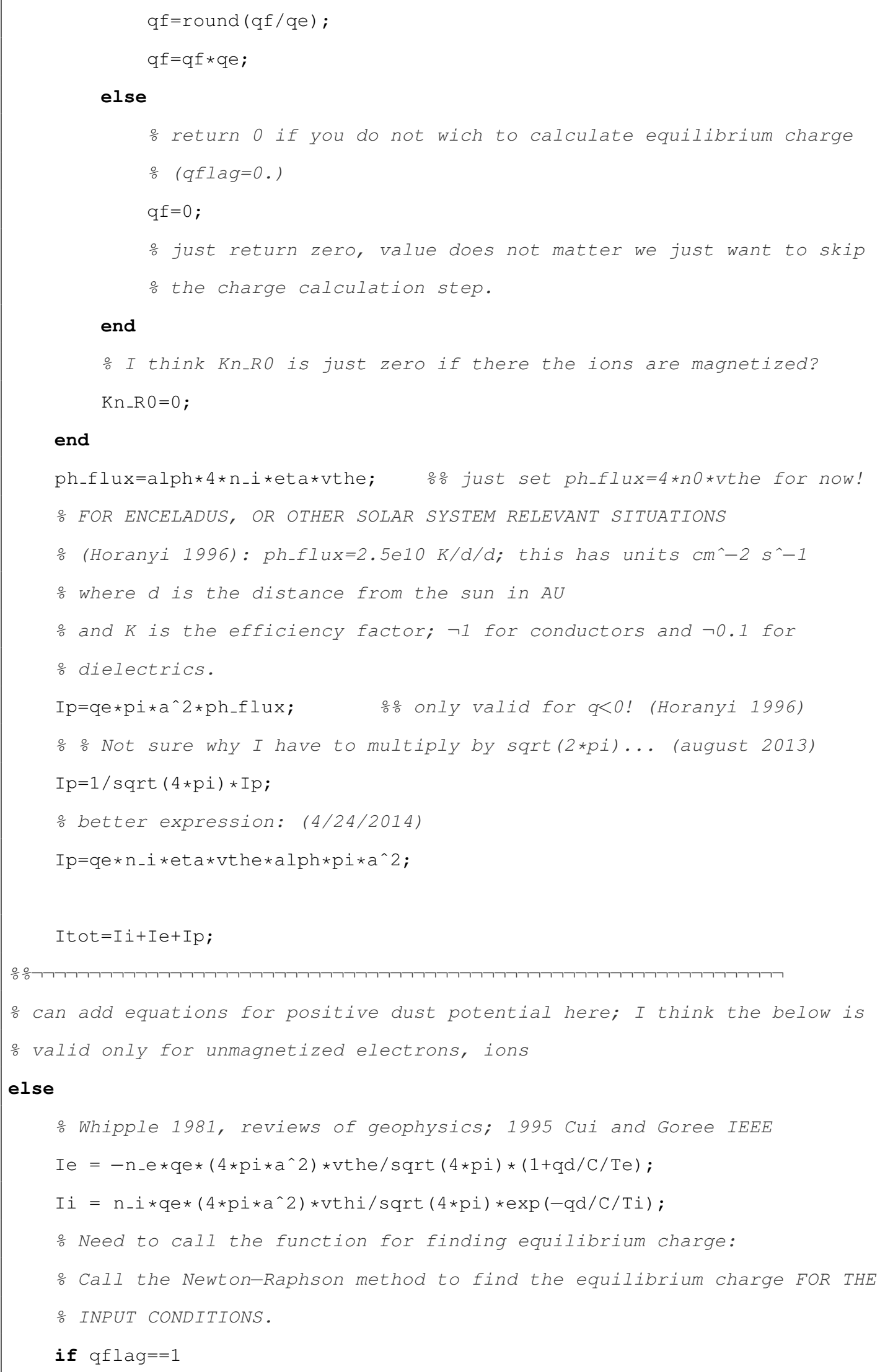


- a charge between 0 and leg elementary charges is a good search

interval.

[output]= dust_bisection (le9, ' oml', (qd/qe), eta, alph, Ti, Te, . .

e_mag, i_mag, C, lambda_D, lambda_i,mu_i,a,w, species) ;

qferound (output);

$q f=q f \star q e ;$

else

\% return 0 if you do not wich to calculate equilibrium charge

$\therefore \quad(q f l a g=0$.

$q f=0$

\% just return zero, value does not matter we just want to skip

응 the charge calculation step.

end

응응응응응 응응 FOLLOWING NEEDS TO BE CHANGED FOR POSITIVE DUST POTENTIAL!!

ph_flux=alph*4*n_i*eta*vthe; 응 just set ph_flux=4*no*vthe for now!

FOR ENCELADUS, OR OTHER SOLAR SYSTEM RELEVANT SITUATIONS

응 (Horanyi 1996): ph-flux=2.5e10 $\mathrm{K} / \mathrm{d} / \mathrm{d}$; this has units $\mathrm{cm}^{\wedge}-2 \mathrm{~s}^{\wedge}-1$

응 where $d$ is the distance from the sun in $A U$

$\therefore$ and $K$ is the efficiency factor; $\neg 1$ for conductors and $\neg 0.1$ for

응 diectrics.

Ip $=q e * p i * a^{\wedge} 2 * p_{-f l u x} \quad \quad \frac{\circ}{0}$ only valid for $q<0 !$ (Horanyi 1996)

응 better expression: (4/24/2014)

$I p=q e * n_{-} i * e t a * v t h e * a l p h * p i * a^{\wedge} 2 ;$

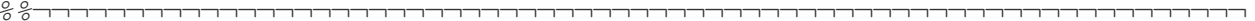

Ip=qe $* p i * a^{\wedge} 2 * p h_{\text {fflux }}$ ～응 only valid for $q<0 !$ (Horanyi 1996)

Qabs=1; $\quad$ 응 absorption efficiency is $\neg 1$ for $2 * p i * a / l a m b d a \_u v>1$.

능 the yield of photoelectrons; perhaps use $Y=1$ ? so we get 1

- photoelectron for every uv photon?

$\therefore Y=1$;

$\frac{\circ}{0} u v=a I p h * 1 e 15 ;$ 응 UV photon flux, in units of $m^{\wedge}-2 s^{\wedge}-1$ ?

Thermal energy of photo electrons; for now I'm assuming $T p h=1$ eV for

응 convenience.

$\therefore \mathrm{Tph}=1$ ；

응 only valid for $q>0$ ! (Shukla 2001)

I $p=q e * p i * a^{\wedge} 2 * Q a b s * Y * j u v * \exp (-q e * q d / C / T p h)$;

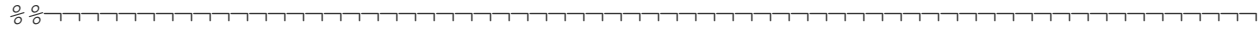


Itot $=I i+I e+I p ;$

o Knudsen capture radius does not exist.

$\mathrm{Kn} \_\mathrm{RO}=0$ ；

end

Included below is the matlab code for oml_monoenergetic_ions.m, which includes Maxwellian electrons and mono-energetic ion populations. This model differs from OML because the ions are assumed to be cold, having only some relative drift velocity.

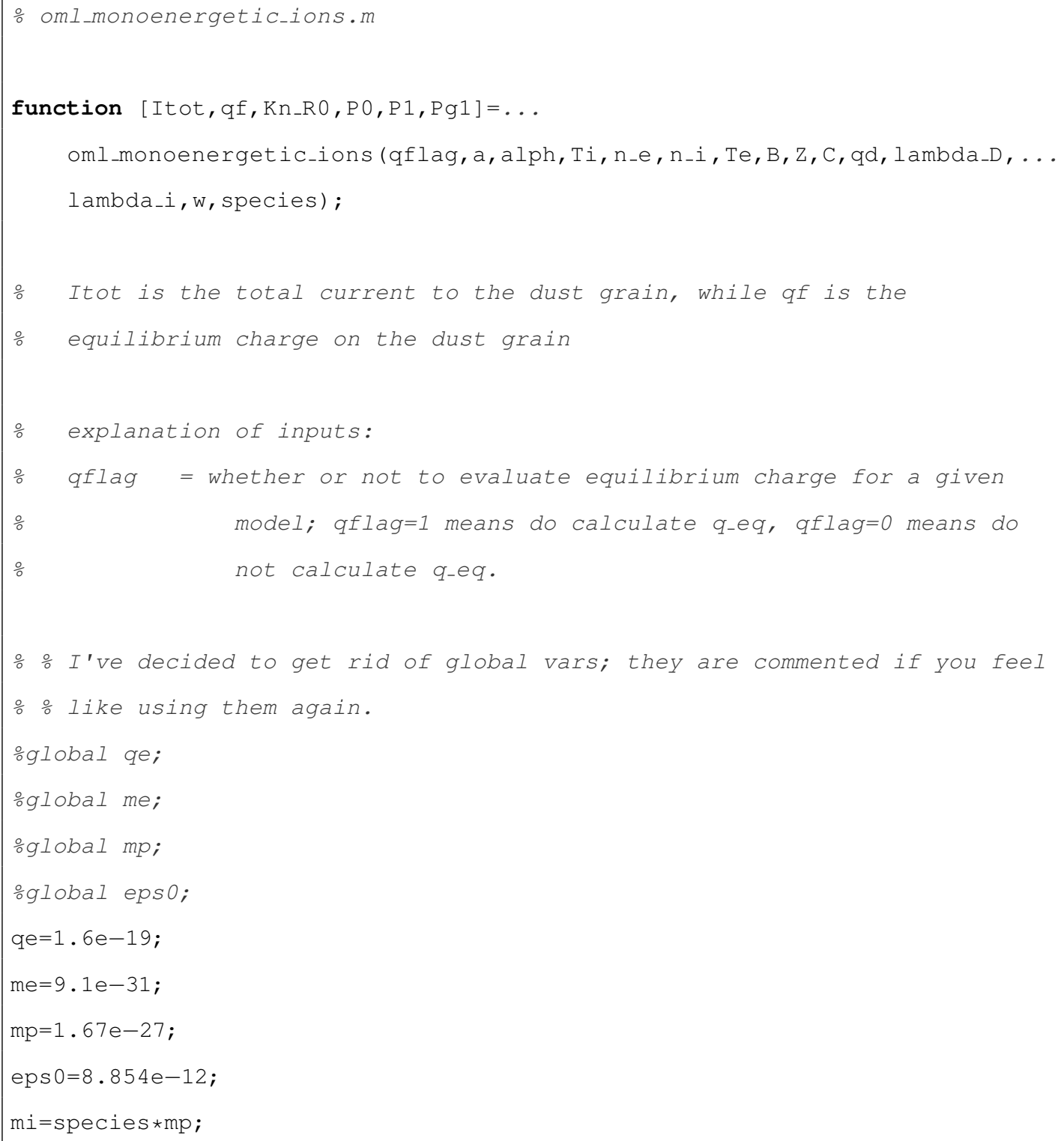




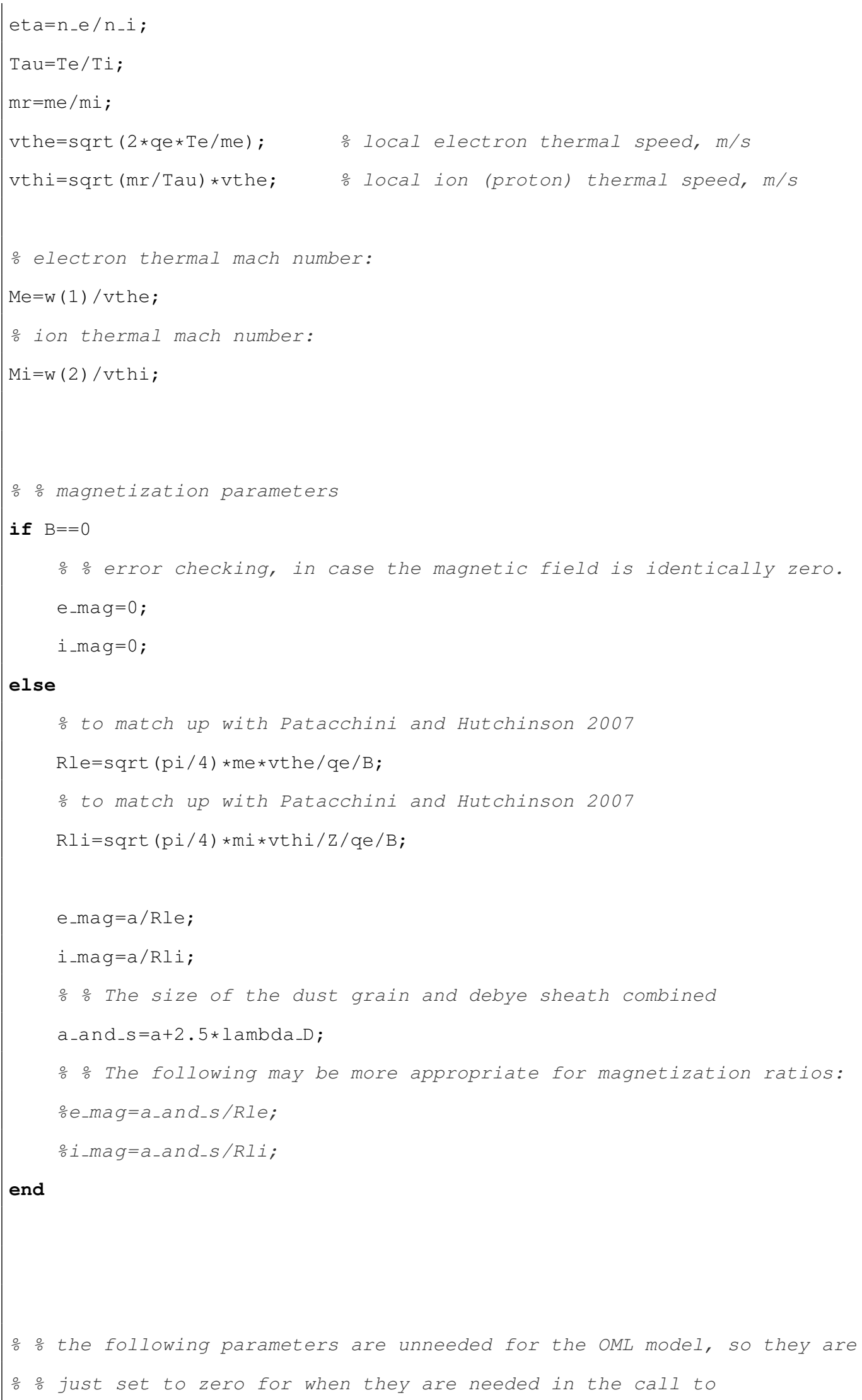




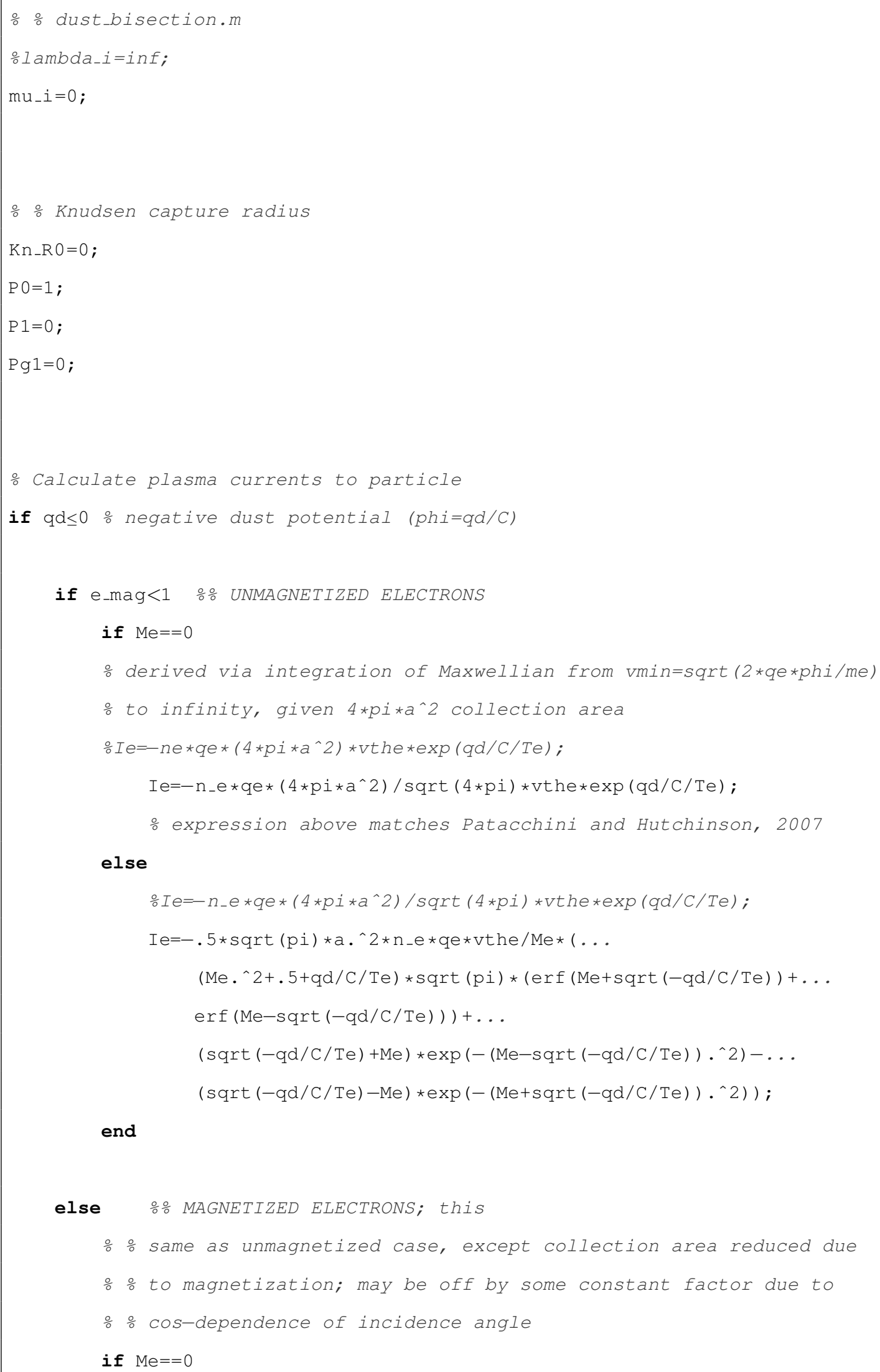


o derived via integration of Maxwellian from vmin=sqrt ( 2 *qe*phi/me)

응 to infinity, given $4 * p i * a^{\wedge} 2$ collection area

$\frac{\circ}{\circ} \mathrm{e}=-n e * q e *\left(4 * p i * a^{\wedge} 2\right) * v t h e * \exp (q d / C / T e)$;

$\mathrm{Ie}=-.5 * \mathrm{n}_{-} \mathrm{e} * \mathrm{qe} *\left(4 * \mathrm{pi} * \mathrm{a}^{\wedge} 2\right) / \operatorname{sqrt}(4 * \mathrm{pi}) * \mathrm{vthe} * \exp (\mathrm{qd} / \mathrm{C} / \mathrm{Te})$;

응 expression above matches Patacchini and Hutchinson, 2007

else

을 $=-n_{-} e * q e *\left(4 * p i * a^{\wedge} 2\right) / \operatorname{sqrt}(4 * p i) * v t h e * \exp (q d / C / T e)$;

$I e=-.25 * \operatorname{sqrt}(p i) * a . \wedge 2 * n_{-} e * q e * v t h e / M e *(\ldots$

$\left(\mathrm{Me} \cdot{ }^{\wedge} 2+.5+q d / C / T e\right) * \operatorname{sqrt}(p i) *(\operatorname{erf}($ Me $+\operatorname{sqrt}(-q d / C / T e))+\ldots$

$\operatorname{erf}(\mathrm{Me}-\operatorname{sqrt}(-\mathrm{qd} / \mathrm{C} / \mathrm{Te})))+\ldots$

$(\operatorname{sqrt}(-\mathrm{qd} / \mathrm{C} / \mathrm{Te})+\mathrm{Me}) * \exp \left(-(\mathrm{Me}-\operatorname{sqrt}(-\mathrm{qd} / \mathrm{C} / \mathrm{Te})) \cdot{ }^{\wedge} 2\right)-\ldots$

$\left.(\operatorname{sqrt}(-\mathrm{qd} / \mathrm{C} / \mathrm{Te})-\mathrm{Me}) * \exp \left(-(\mathrm{Me}+\operatorname{sqrt}(-\mathrm{qd} / \mathrm{C} / \mathrm{Te})) \cdot{ }^{\wedge} 2\right)\right)$;

end

end

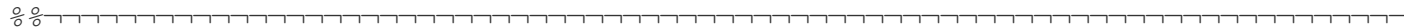

if i_mag<1 응 UNMAGNETIZED IONS

응 OML current, cf. Allen, Phys. Scr. 45 (1992), eq. 51

ㅇ $I i=n i * q e *\left(4 * p i * a^{\wedge} 2\right) * \operatorname{sqrt}(-2 * q e * q d / \mathrm{C} / \mathrm{mi})$;

응 I'm replacing the above line with the current I see more

응 often in the literature (Shukla 2001 pop, for example)

을 Iude a statement for $w==0$ so that there is no division by

$\therefore$ zero.

if $\mathrm{Mi}==0$

this is OML theory for mono-energetic ions, so if there is no

ion flow, there can be no ion current.

$\mathrm{Ii}=0$;

$\frac{\circ}{\circ} i=n_{-} i * q e *\left(4 * p i * a^{\wedge} 2\right) / \operatorname{sqrt}(4 * p i) * v t h i *(1-q d / C / T i)$;

\% expression above matches Patacchini and Hutchinson, 2007

else

$\mathrm{Ii}=\mathrm{p} i * \mathrm{n}_{-} i * \mathrm{qe} * \mathrm{a}^{\wedge} 2 * \mathrm{w}(2) *\left(1-\mathrm{qe} * \mathrm{qd} / \mathrm{C} /\left(\mathrm{mi} * \mathrm{w}(2) \cdot{ }^{2}\right)\right) ;$

Ii $=\operatorname{sqrt}(p i) * a \cdot{ }^{\wedge} 2 * n_{-} i * z * q e * \operatorname{vth} i *(\ldots$

$\left.\left(M i \cdot{ }^{\wedge}+0.5-q d / C / T i\right) \star \operatorname{sqrt}(p i) / M i \star \operatorname{erf}(M i)+\exp \left(-M i \cdot{ }^{\wedge} 2\right)\right)$;

\% 응 see 1992_northrop_ps or 1981_whipple_repprogphys for the

응 above. Also, 1996 Horanyi and 1996 Northrop. 


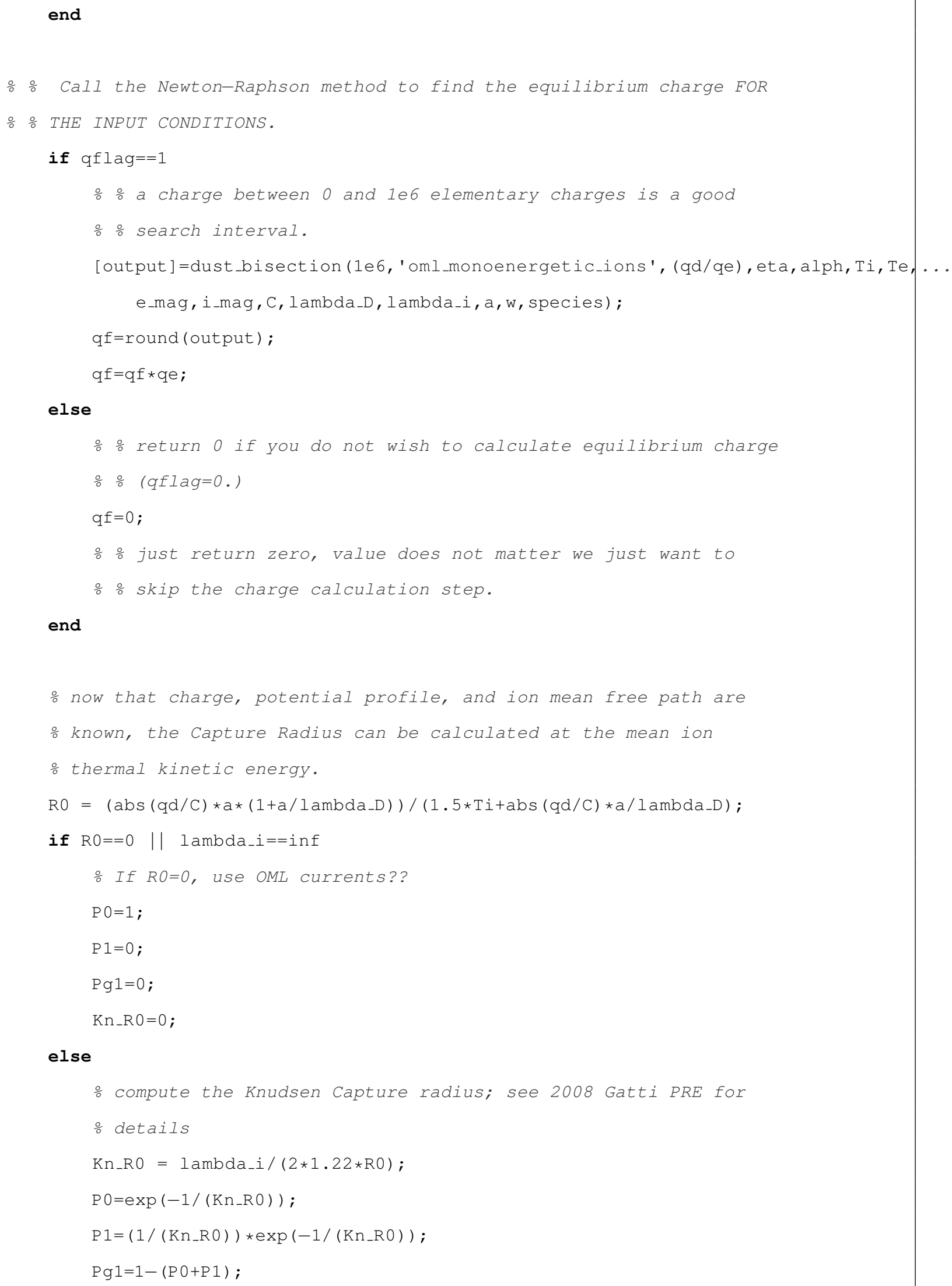




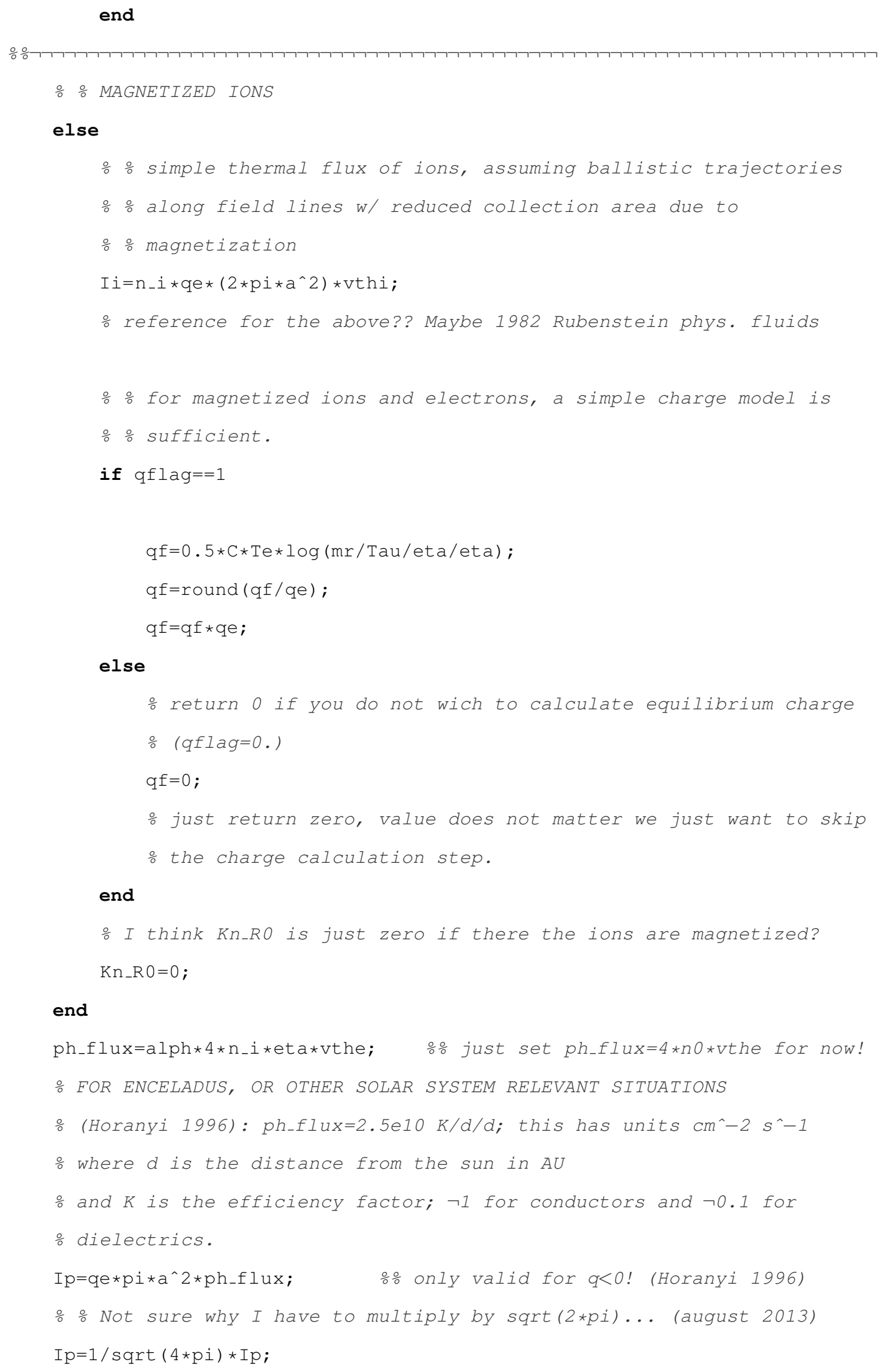


응 better expression: (4/24/2014)

I $p=q e * n_{-} i * e t a * v t h e * a l p h * p i * a^{\wedge} 2 ;$

Itot $=\mathrm{Ii}+\mathrm{Ie}+\mathrm{Ip}$

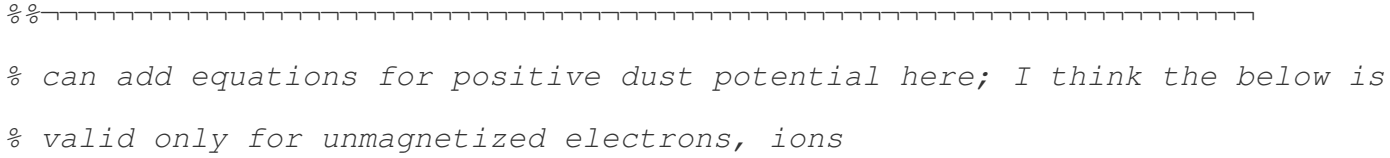




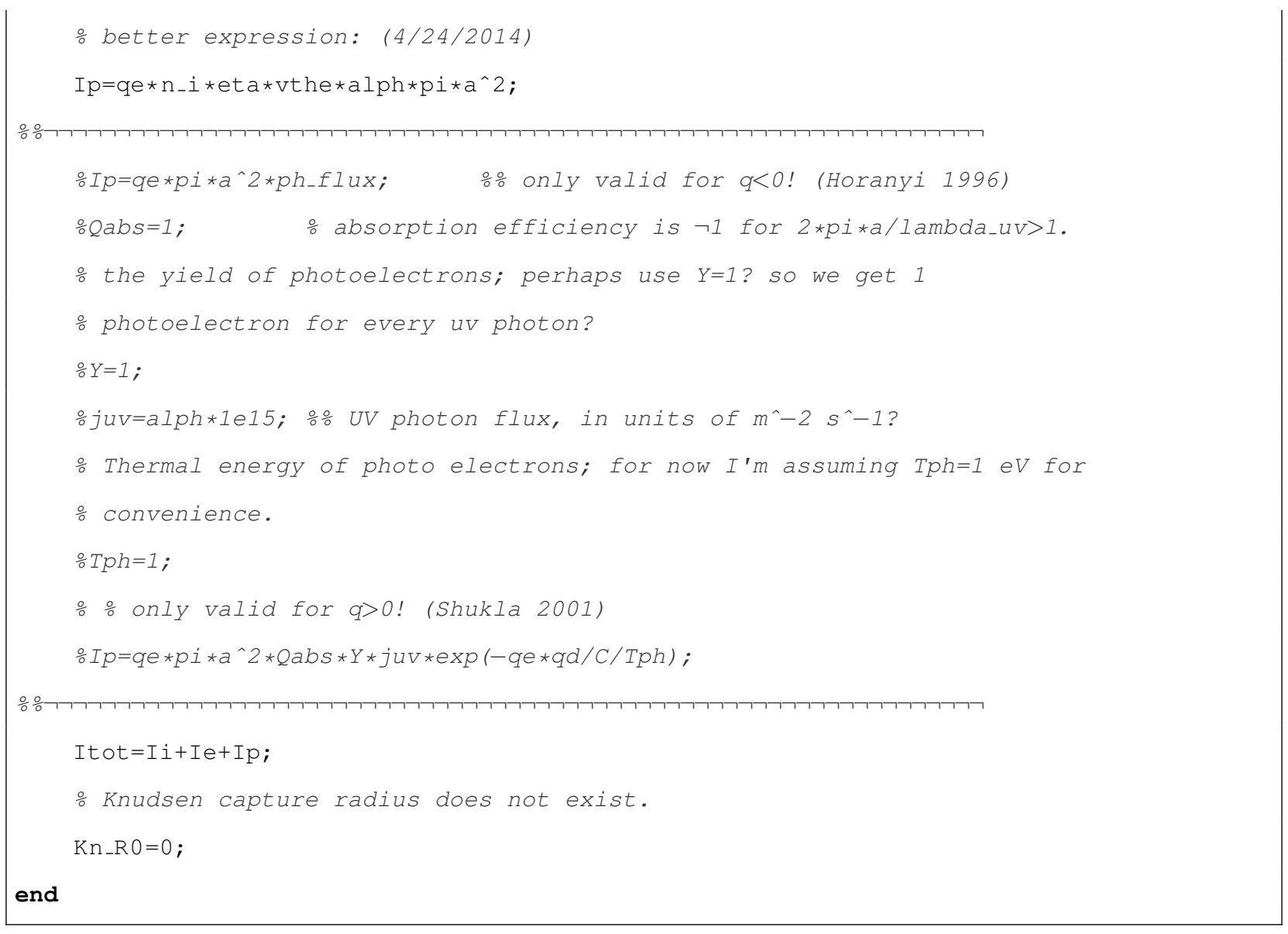

\section{ii Patacchini-Hutchinson Model Code}

Included below is the matlab code for hutchinson.m, which is the Patacchini-Hutchinson model for grain charging and includes Maxwellian electron and drifting Maxwellian ion populations. This model differs from OML because it includes finite-Larmor radius effects of the electrons.

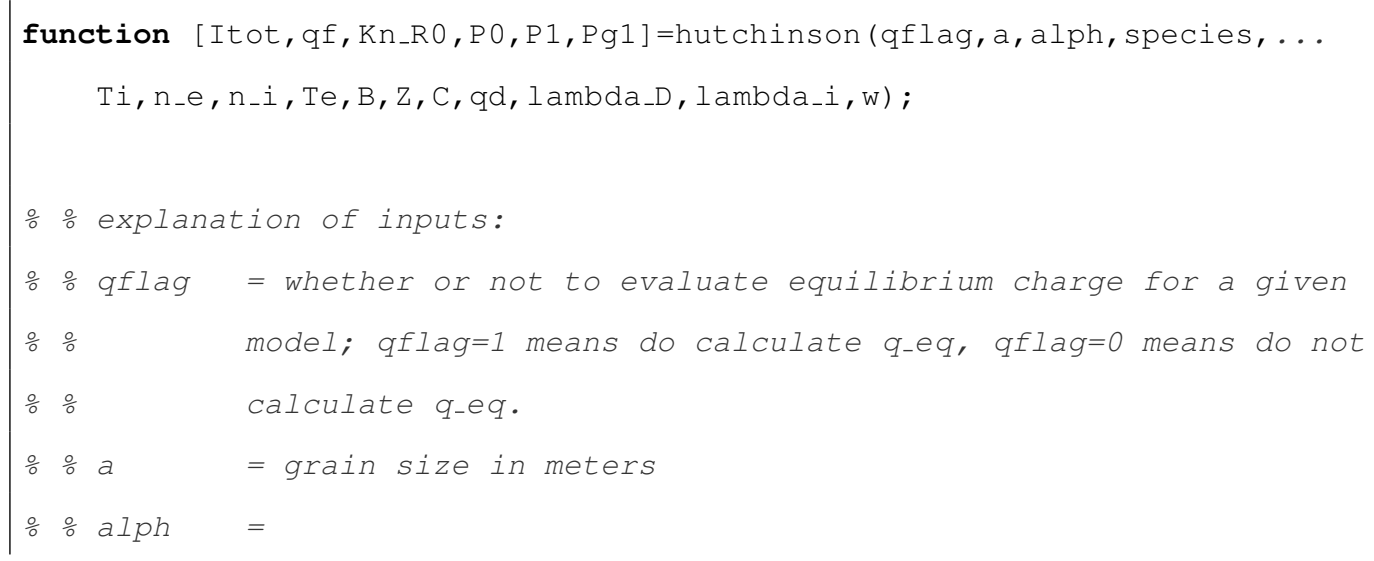




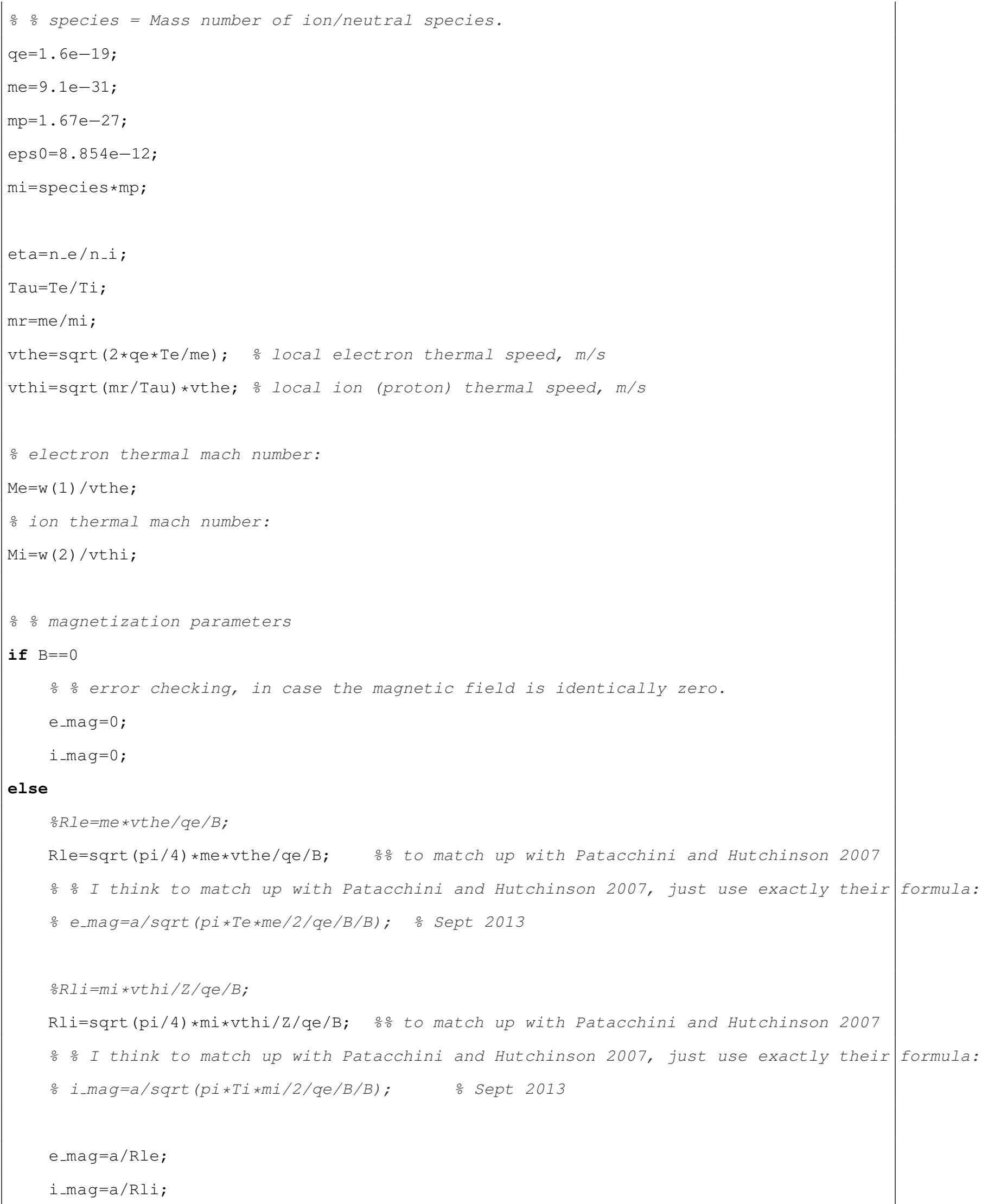




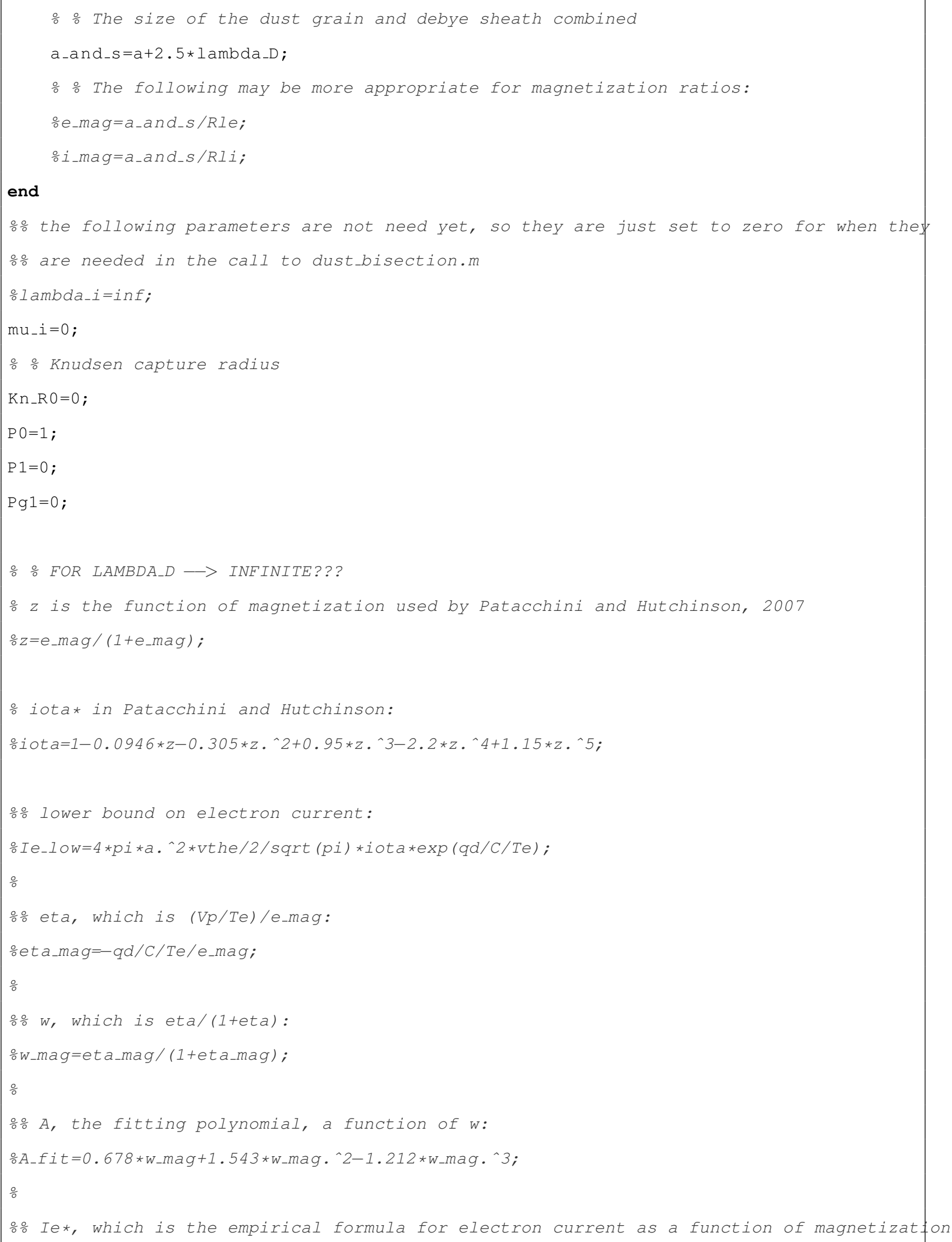




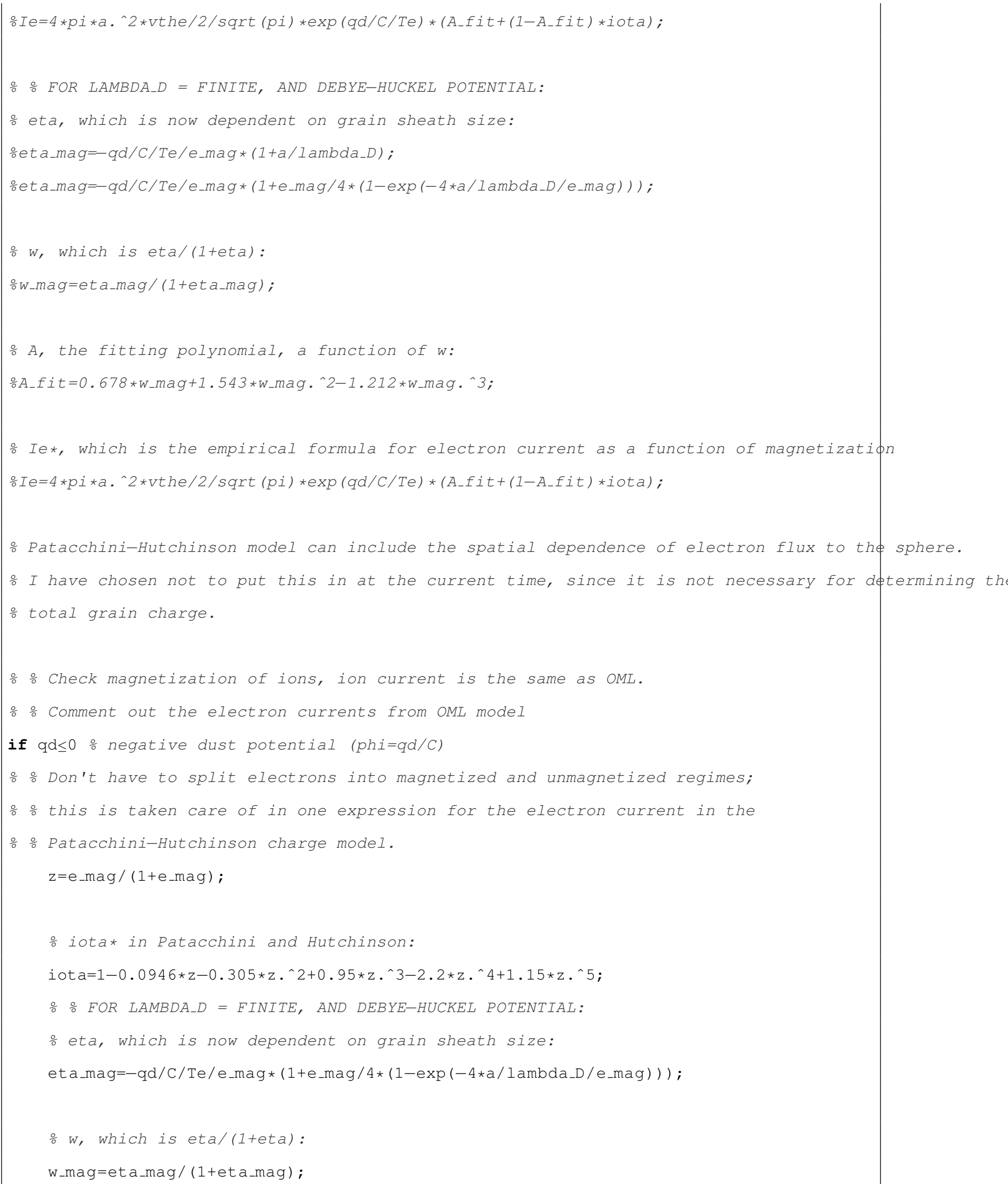




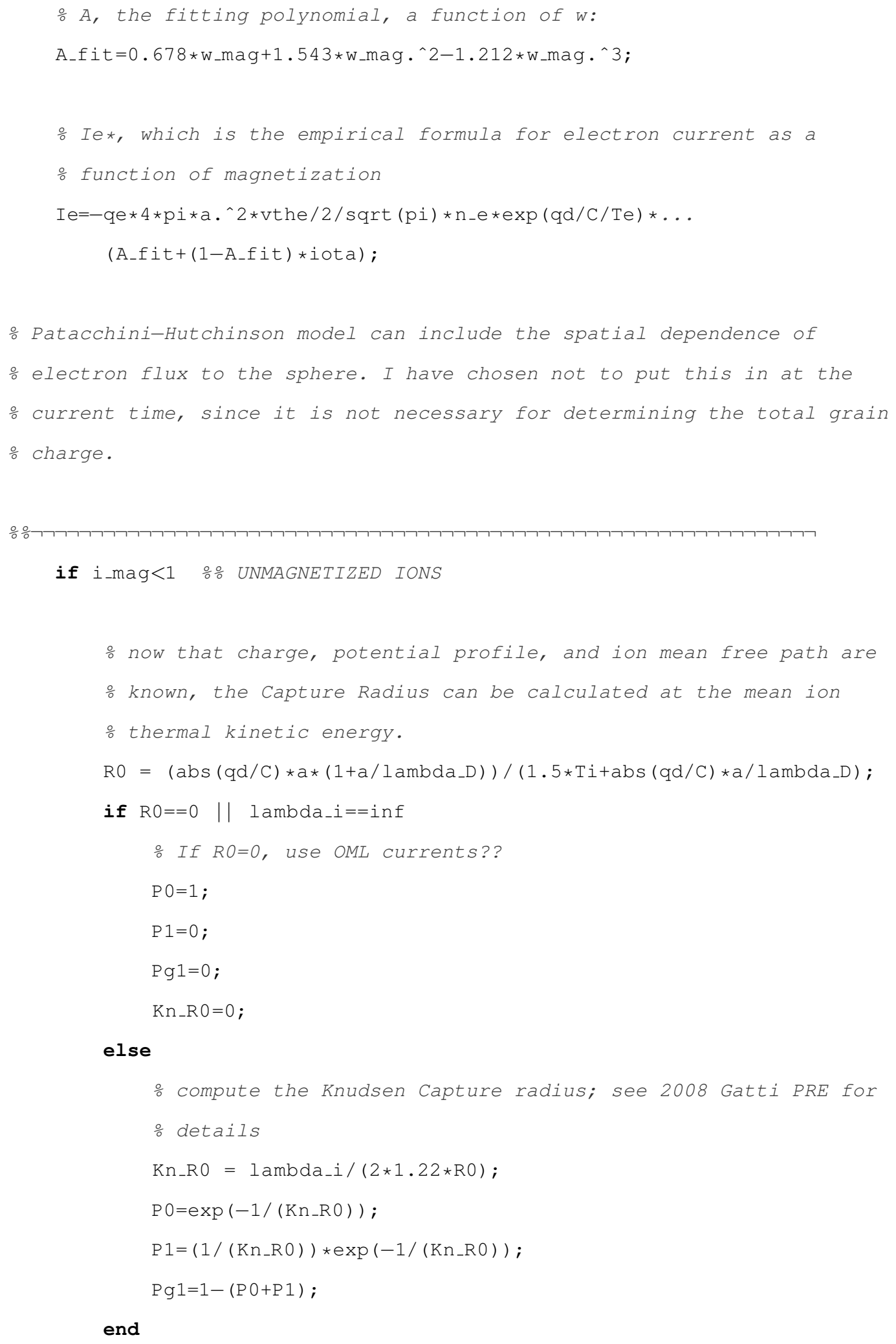


응 current, for all thermal mach numbers.

if $\mathrm{Mi}==0$

$\mathrm{Ii}=\mathrm{n}_{-} i \star \mathrm{qe} *\left(4 \star \mathrm{p} i \star \mathrm{a}^{\wedge} 2\right) / \operatorname{sqrt}(4 \star \mathrm{p} i) \star \mathrm{vthi} *(1-\mathrm{qd} / \mathrm{C} / \mathrm{Ti})$;

oxpression above matches Patacchini and Hutchinson, 2007

else

$\mathrm{Ii}=\operatorname{sqrt}(\mathrm{pi}) * \mathrm{a} \cdot{ }^{\wedge} 2 * \mathrm{n}_{-} i * \mathrm{Z} * \mathrm{qe} * \operatorname{vth} i *\left(\left(\mathrm{Mi} \cdot{ }^{\wedge} 2+0.5-\mathrm{qd} / \mathrm{C} / \mathrm{Ti}\right) * \ldots\right.$

$\left.\operatorname{sqrt}(\mathrm{pi}) / \mathrm{Mi} * \operatorname{erf}(\mathrm{Mi})+\exp \left(-\mathrm{Mi} \cdot{ }^{\wedge} 2\right)\right)$;

\% 응 see 1992_northrop_ps or 1981_whipple_repprogphys for the

응 above. Also, 1996 Horanyi and 1996 Northrop.

end

응 Call the Newton-Raphson method to find the equilibrium charge FOR

응 THE INPUT CONDITIONS.

if $q f \operatorname{lag}==1$

응 a charge between 0 and $1 e 4$ elementary charges is a good

$\frac{\circ}{0}$ search interval.

[output] = dust_bisection(1e6, 'hutchinson', (qd/qe), eta,alph,...

Ti, Te, e_mag, i_mag, C, lambda_D, lambda_i,a,w, species) ;

qf=round (output) ;

$q f=q f \star q e$;

else

$\frac{\circ}{0}$ return 0 if you do not wish to calculate equilibrium charge

$\therefore \quad(q f l a g=0$.

$\mathrm{qf}=0 ; \quad \therefore$ just return zero, value does not matter we just

응 want to skip the charge calculation step.

end

else 응 MAGNETIZED IONS

응 simple thermal flux of ions, assuming ballistic trajectories

응 along field lines $\mathrm{w} /$ reduced collection area due to magnetization

$\mathrm{Ii}=\mathrm{n}_{-} \mathrm{i} * \mathrm{qe} *\left(2 * \mathrm{p} i \star \mathrm{a}^{\wedge} 2\right) \star \mathrm{vthi} ; \quad \frac{\circ}{0}$ reference for this?? Maybe 1982 Rubenstein phys. Fluids

응 for magnetized ions and electrons, a simple charge model is sufficient.

if $\mathrm{qflag}==1$ 
$\mathrm{qf}=0.5 * \mathrm{C} * \mathrm{Te} * \log (\mathrm{mr} / \mathrm{Tau} / \mathrm{eta} /$ eta $) ;$

$q f=r o u n d(q f / q e)$;

$q f=q f \star q e ;$

else

o응 return 0 if you do not wich to calculate equilibrium charge (qflag=0.) qf=0; 응 just return zero, value does not matter we just want to skip 응 the charge calculation step.

end

end

ph_flux=alph*4*n_i*eta*vthe; 응 just set ph_flux=4*no*vthe for now!

음 FOR ENCELADUS, OR OTHER SOLAR SYSTEM RELEVANT SITUATIONS (HOranYi 1996):

응믄 phux $=2.5 e 10 \mathrm{~K} / \mathrm{d} / \mathrm{d}$; this has units $\mathrm{cm}^{\wedge}-2 \mathrm{~s}^{\wedge}-1$

응 where $d$ is the distance from the sun in $A U$

$\frac{\circ}{0}$ and $K$ is the efficiency factor; $\neg 1$ for conductors and $\neg 0.1$ for dielectrics.

Ip $=q e * p i * a^{\wedge} 2 * p h \_f l u x ; \quad \quad \frac{0}{0}$ only valid for $q<0 !$ (Horanyi 1996)

응 Not sure why I have to multiply by sqrt(2*pi)... (august 2013)

Ip $=1 / \operatorname{sqrt}(4 * \mathrm{pi}) \star I p ;$

obeter expression: (4/24/2014)

$\mathrm{Ip}=\mathrm{qe} * \mathrm{n}_{-} i * e t \mathrm{a} * \mathrm{vthe} * \mathrm{al} \mathrm{ph} * \mathrm{p} i * \mathrm{a}^{\wedge} 2$;

Itot=Ii+Ie+Ip;

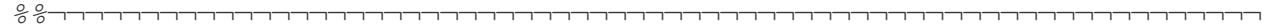

else

o can add equations for positive dust potential here; I think the below is valid onfy for unmagn 응 electrons, ions

$\mathrm{Ie}=-\mathrm{n}_{-} \mathrm{e} * \mathrm{qe} *\left(4 * \mathrm{pi} * \mathrm{a}^{\wedge} 2\right) * \mathrm{vthe} / \operatorname{sqrt}(4 * \mathrm{pi}) *(1+\mathrm{qd} / \mathrm{C} / \mathrm{Te}) ; \quad$ 응 Whipple 1981, reviews of geophysics;

$\mathrm{Ii}=\mathrm{n}_{-} i * \mathrm{qe} *\left(4 * \mathrm{p} i * \mathrm{a}^{\wedge} 2\right) * \operatorname{vthi} / \operatorname{sqrt}(4 * \mathrm{pi}) * \exp (-\mathrm{qd} / \mathrm{C} / \mathrm{Ti})$;

응 Need to call the function for finding equilibrium charge:

응 Call the Newton-Raphson method to find the equilibrium charge FOR THE INPUT CONDITIONS.

if $q f \operatorname{lag}==1$

응 a charge between 0 and le4 elementary charges is a good search interval.

[output] = dust_bisection (1e6, 'oml', (qd/qe), eta, alph, Ti, Te, e_mag, i_mag, C, lambda_D, lambda_i, mu

음output]=dust_newt_meth (Ie4, 'oml', (qd/qe), eta, alph, Ti, Te, e_mag, i_mag, C) ;

$q f=r o u n d$ (output) ;

$q f=q f \star q e$; 


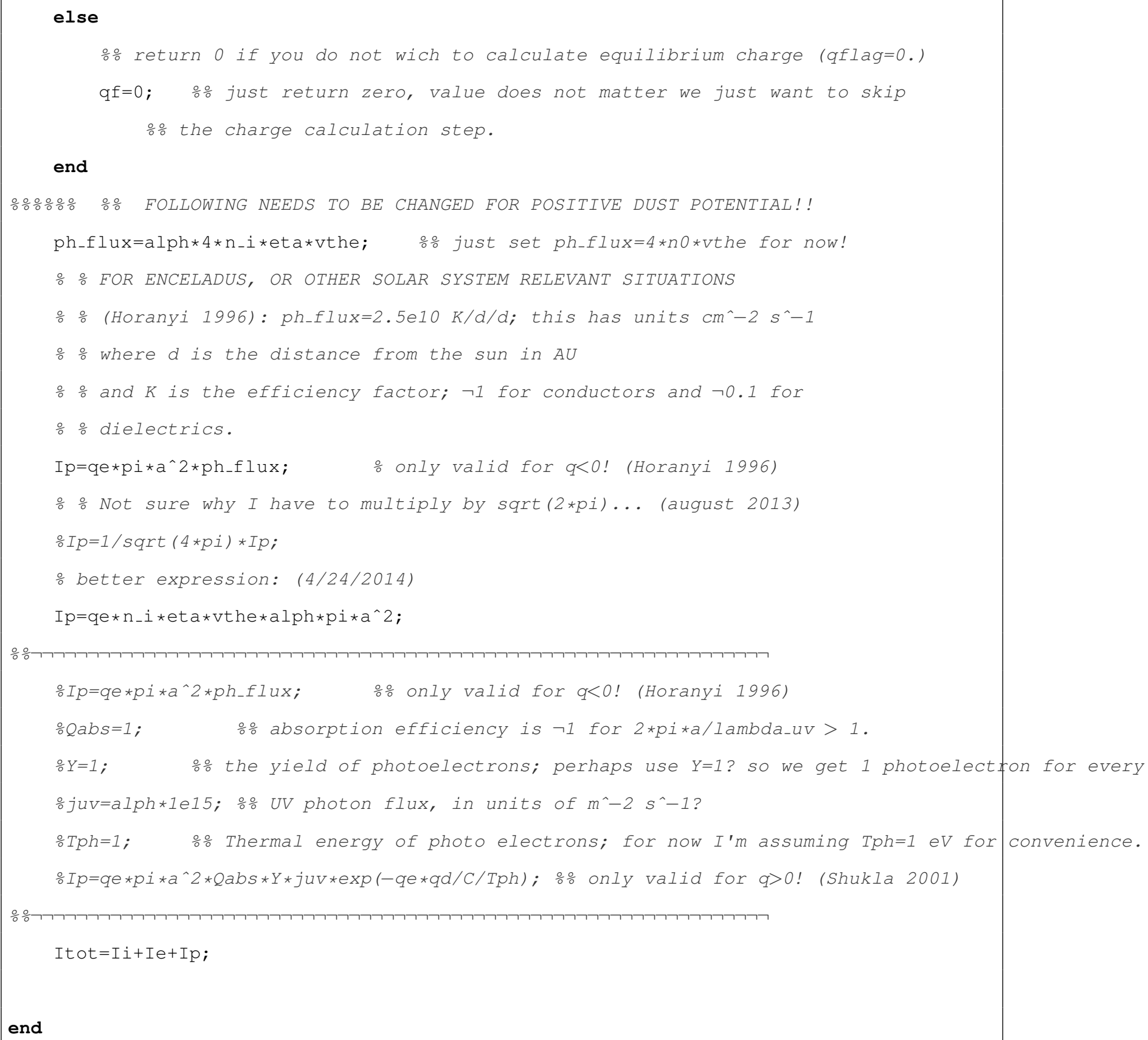

\section{iii Gatti-Kortshagen Model Code}

Included below is the matlab code for kortshagen.m, which is the Gatti-Kortshagen model for grain charging and includes drifting Maxwellian electron and drifting Maxwellian ion populations. This charging model differs from OML because it includes the effects of ion-neutral charge exchange collisions in the grain sheath, and is valid over a wide range of collisionality. 


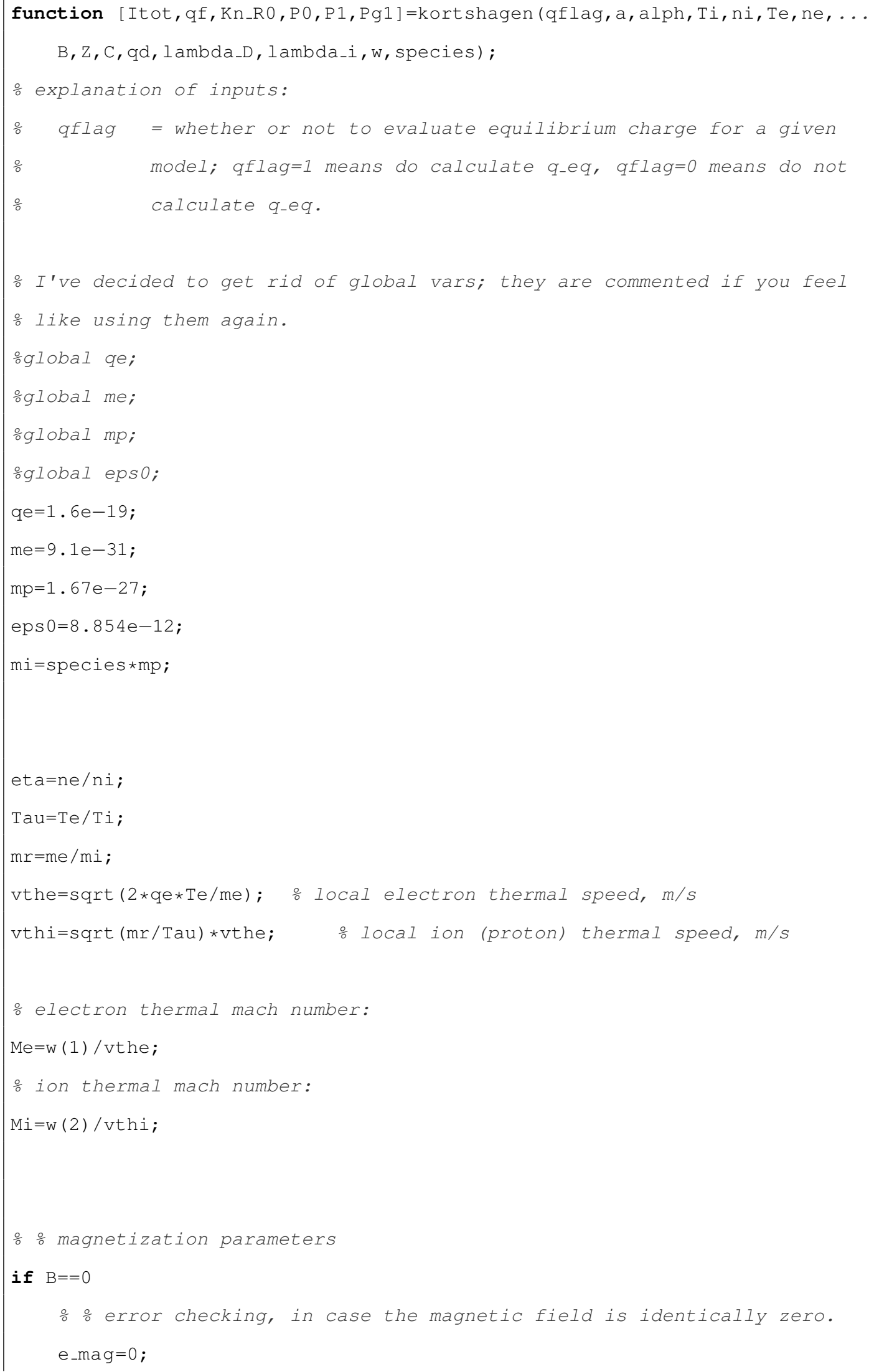


i_mag=0;

else

이 $\mathrm{e}=\mathrm{me} * \mathrm{vthe} / \mathrm{qe} / \mathrm{B}$;

Use below to match up with Patacchini and Hutchinson 2007

Rle=sqrt (pi/4) *me*vthe/qe/B;

$\circ R I i=m i * v t h i / z / q e / B$;

․ Use below to match up with Patacchini and Hutchinson 2007

Rli=sqrt $(\mathrm{pi} / 4) \star \mathrm{mi} * \mathrm{vthi} / \mathrm{z} / \mathrm{qe} / \mathrm{B}$;

e_mag=a/Rle;

$i_{\_} \operatorname{mag}=a / R l i$;

end

음 The size of the dust grain and debye sheath combined

a_and_s $=a+2.5 * 1$ ambda_D;

응 The following may be more appropriate for magnetization ratios:

$\div e_{-} m a g=a_{-} a n d_{-} s / R I e$;

은 mag=a_and_s/RIi;

o now that charge, potential profile, and ion mean free path are known,

- the Capture Radius can be calculated at the mean ion thermal

응 kinetic energy.

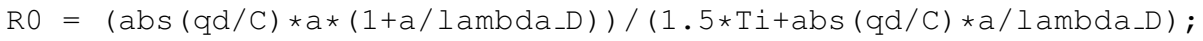

응 calculate the ion mobility. confusingly, DO NOT MULTIPLY BY qe!!!

$m u_{-} i=(1 / T i) *\left(3 * p i * v t h i * 1 a_{m b d a} i\right) /(16 * \operatorname{sqrt}(2))$;

if $m u_{-} i==i n f$;

obviously, if mu-i is infinite, or the plasma is essentially

collisionless, we are in the OML regime so the hydrodynamic current

o is basically zero. To enforce this, just set mu_i=0 if lambda_i=inf $m u_{-} i=0$;

end

- Calculate plasma currents to particle

if $q d \leq 0$ 응 negative dust potential (phi=qd/C) 


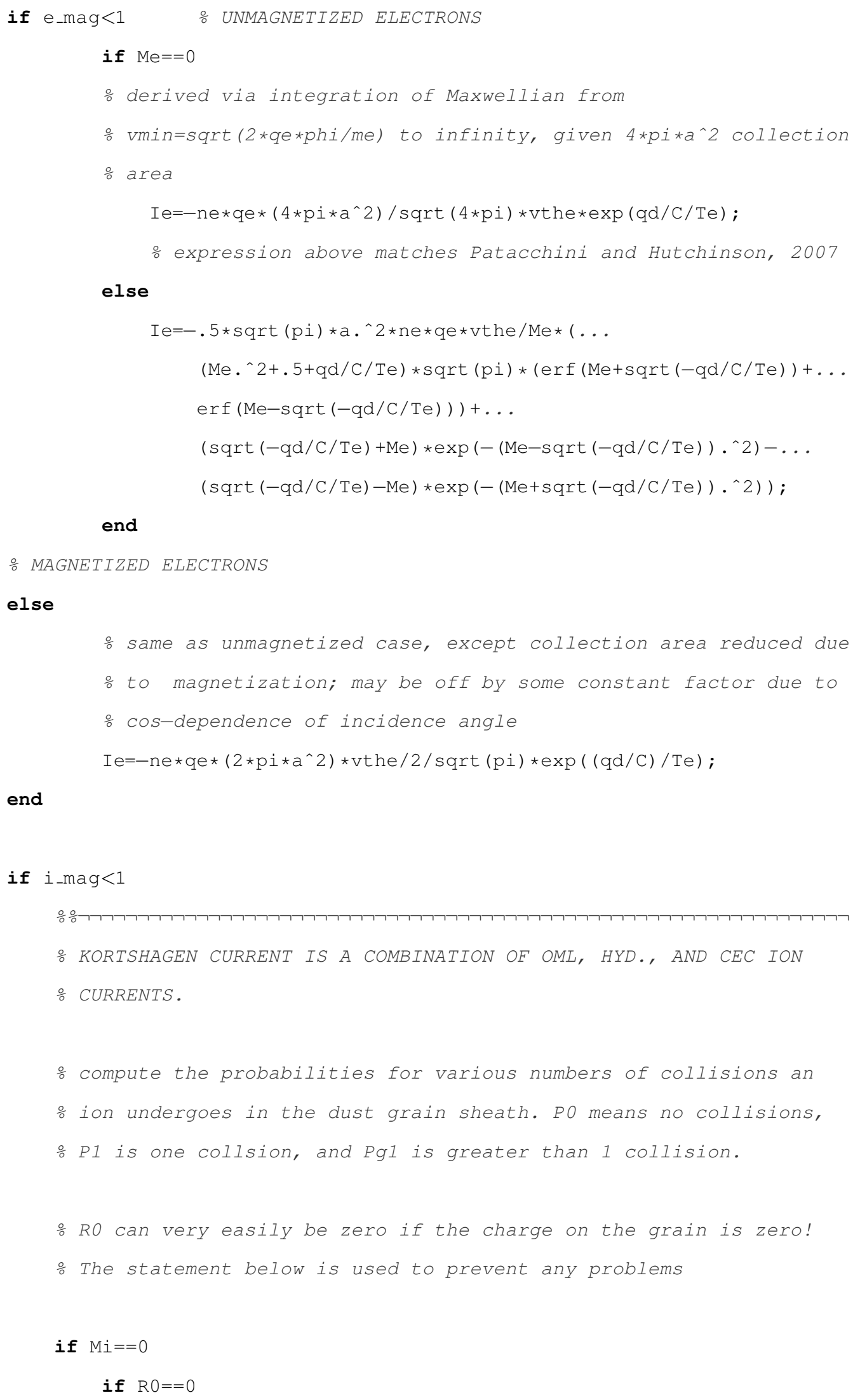


응 $R O=0$, use OML currents??

$\mathrm{PO}=1$;

$\mathrm{P} 1=0$;

$\operatorname{Pg} 1=0 ;$

$\mathrm{Kn} \_\mathrm{RO}=0$ ；

ioml=ni $* \mathrm{qe} *\left(4 * \mathrm{pi} * \mathrm{a}^{\wedge} 2\right) * \mathrm{vthi} / 2 / \operatorname{sqrt}(\mathrm{pi}) *(1-\mathrm{qd} / \mathrm{C} / \mathrm{Ti})$;

icec $=0$;

ihyd=0;

\section{else}

compute the Knudsen Capture radius; see 2008 Gatti PRE

for details

Kn_R0 $=1$ ambda_i $/(2 * 1.22 * R 0) ;$

$\mathrm{P} 0=\exp \left(-1 /\left(\mathrm{K} \mathrm{n}_{-} \mathrm{R} 0\right)\right) ;$

$\mathrm{P} 1=\left(1 /\left(\mathrm{Kn}_{-} \mathrm{R} 0\right)\right) * \exp \left(-1 /\left(\mathrm{Kn}_{-} \mathrm{R} 0\right)\right) ;$

$\mathrm{Pg} 1=1-(\mathrm{P} 0+\mathrm{P} 1)$

ioml=ni $*$ qe $*\left(4 * p i * a^{\wedge} 2\right) * v t h i / 2 / \operatorname{sqrt}(p i) *(1-q d / C / T i)$;

icec $=\mathrm{ni} * \mathrm{qe} *\left(4 * \mathrm{pi} *(1.22 * \mathrm{R} 0)^{\wedge} 2\right) * \mathrm{vthi} / 2 / \operatorname{sqrt}(\mathrm{pi})$;

- calculate the ion current due to hyd. charge model, from

\% Gotti et. al. Phys Rev E 2008, might want to find other

$\div$ sources too

ihyd=16*pi*a*qe*ni*mu_i*abs ( (qd)/C)/2/sqrt (pi);

end

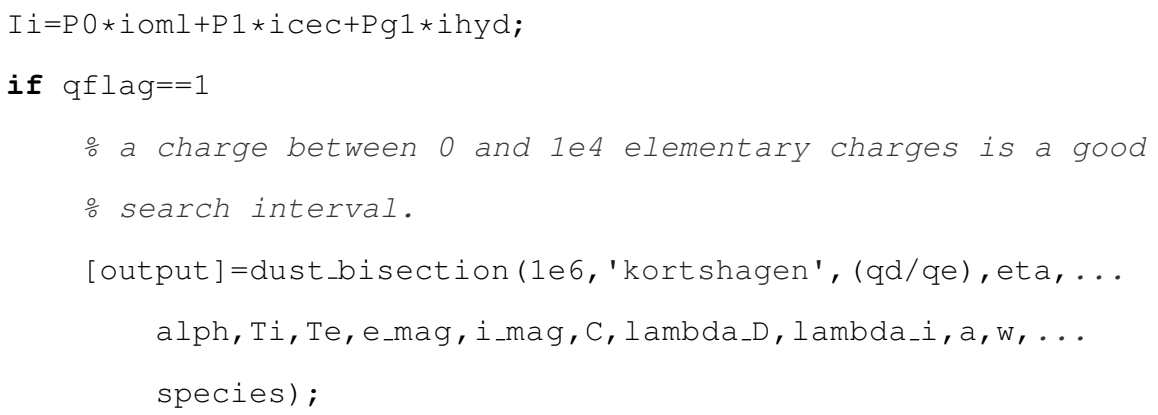




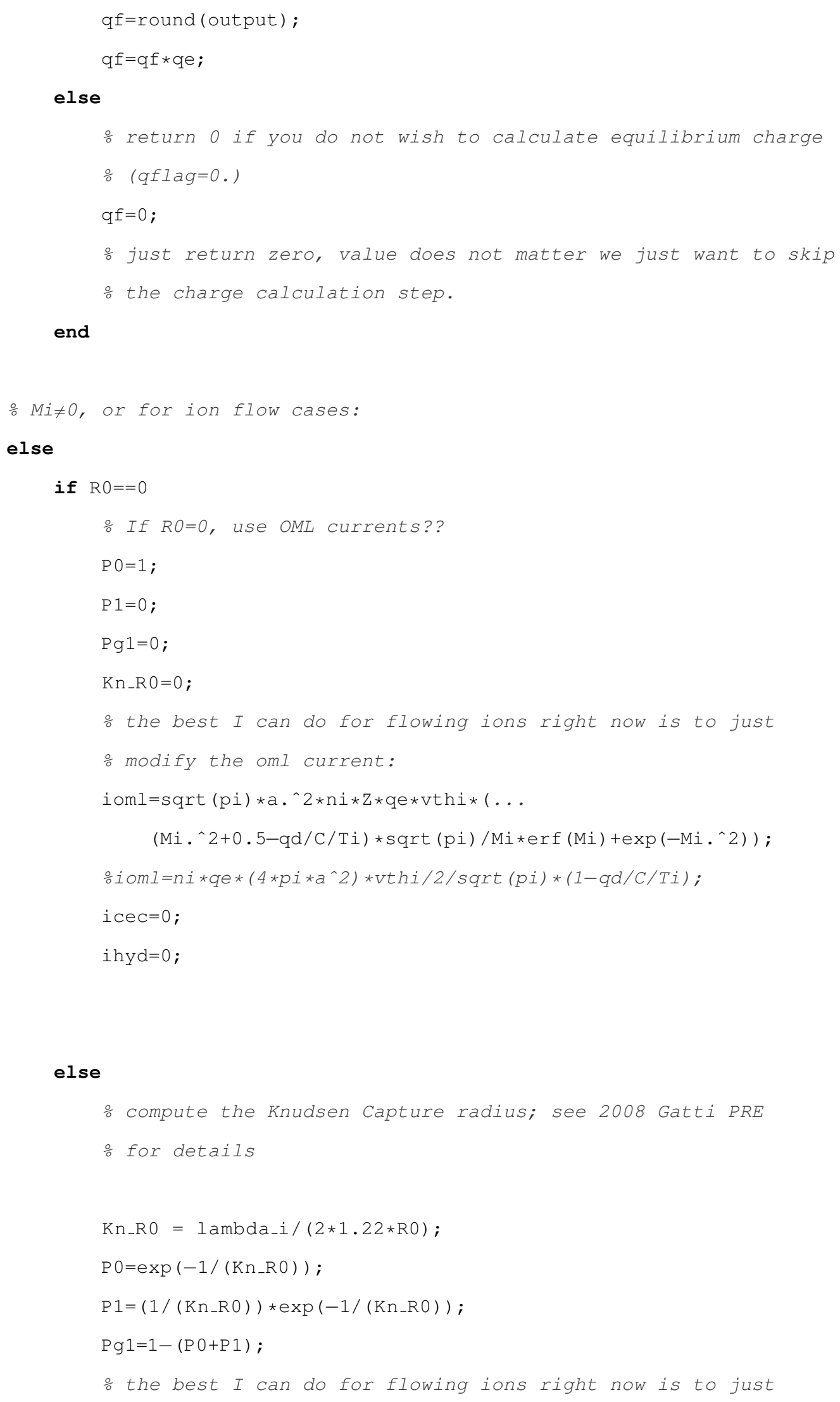




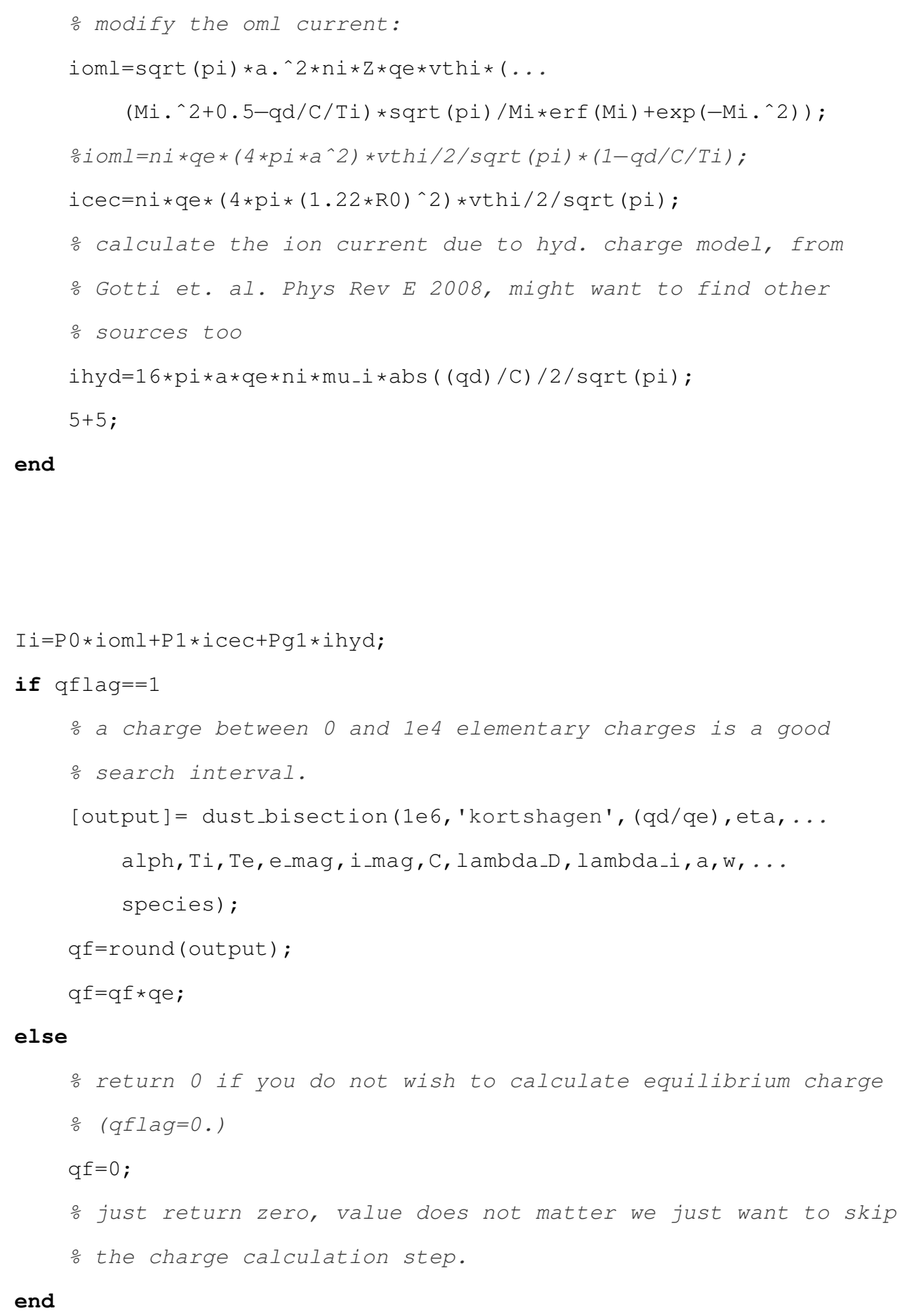




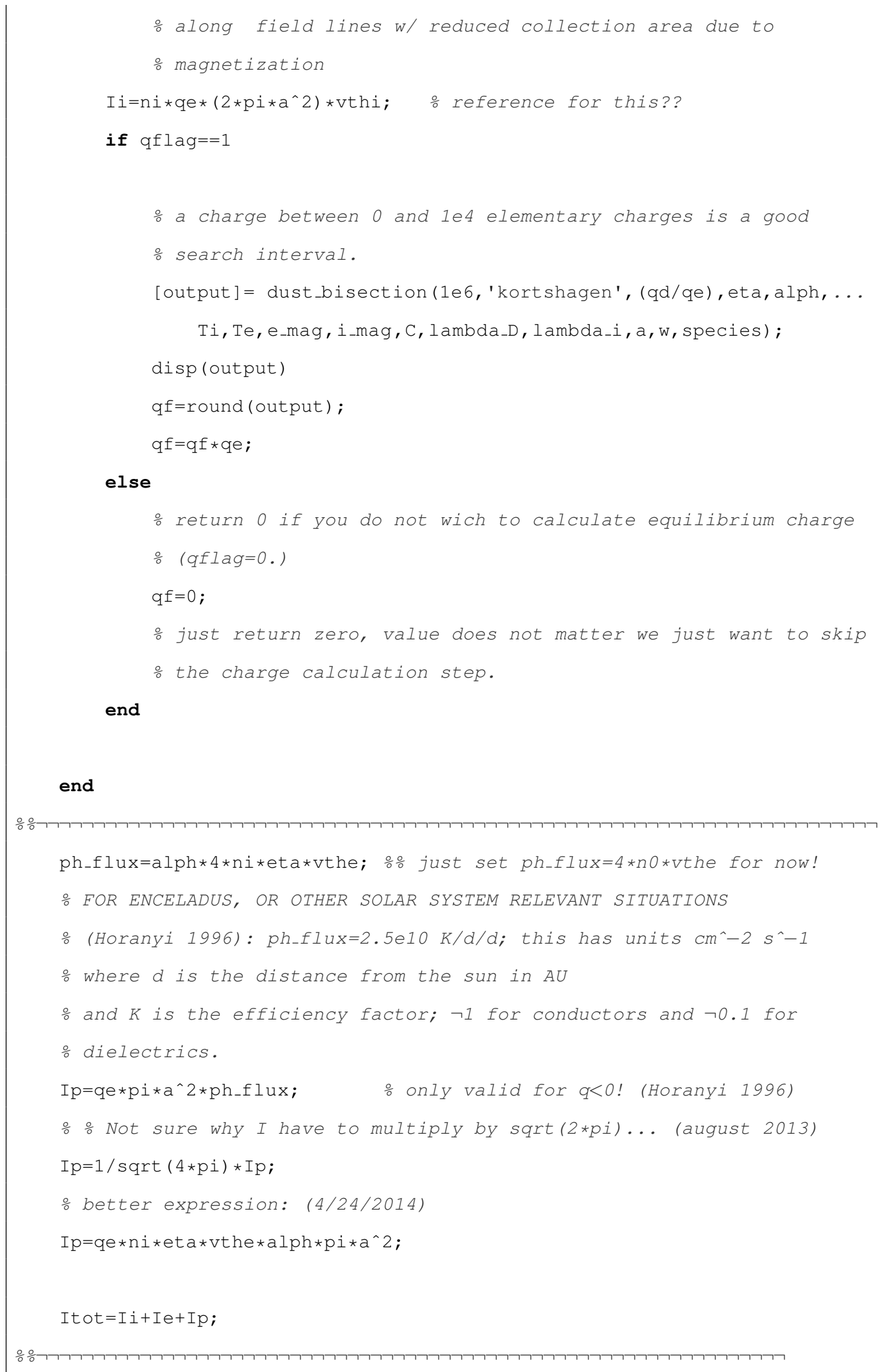




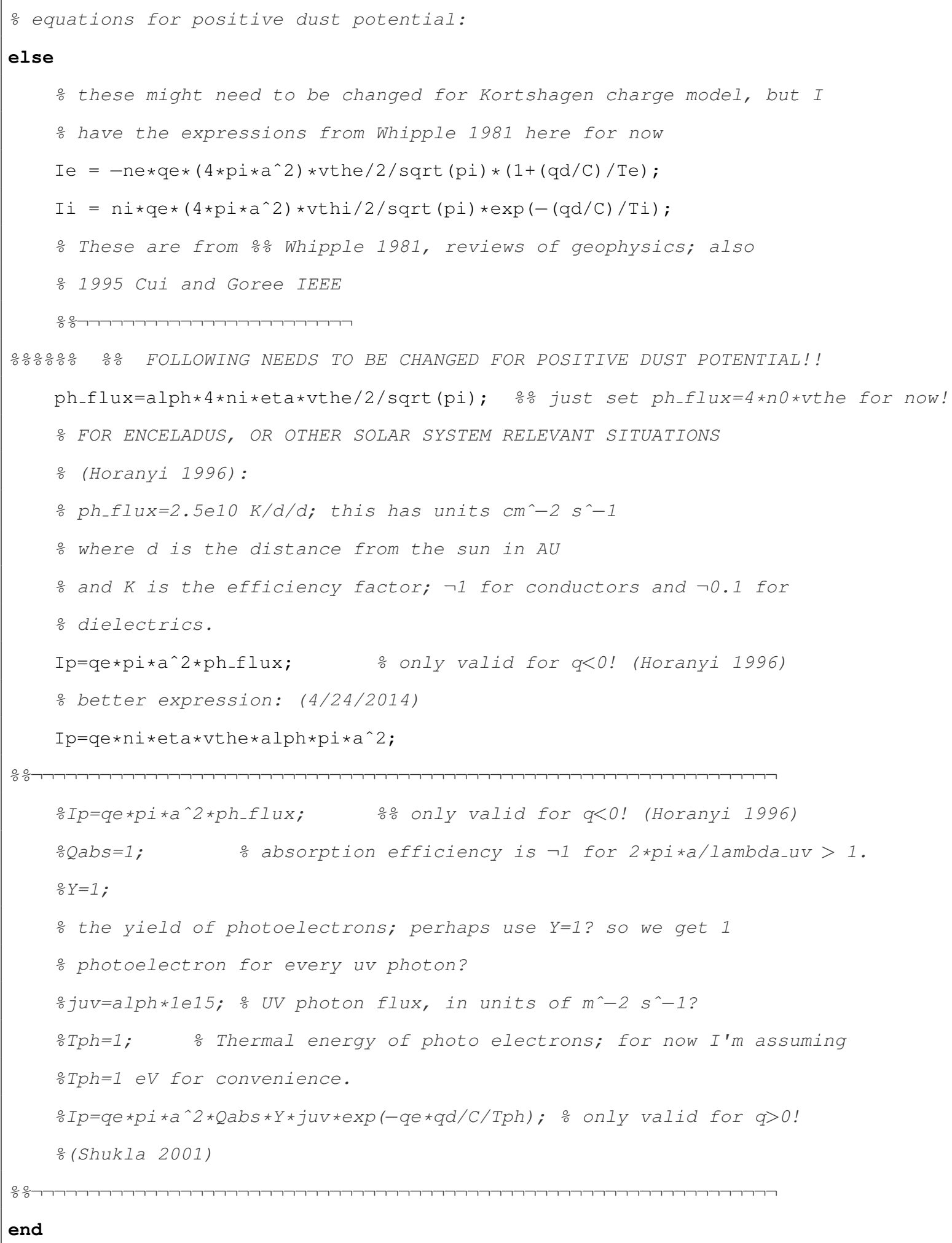




\section{iii Bisection Method}

The bisection method is used to solve the transcendental equation that results when trying to find equilibrium charge on the grain. The bisection method is capable of solving any polynomial or transcendental algebraic equation, making it a particularly useful tool. This particular bisection function was set up to include plasma parameters as inputs, and can be called by any of the charging model subroutines to find equilibrium grain charge. This code, dust_bisection.m, is described below.

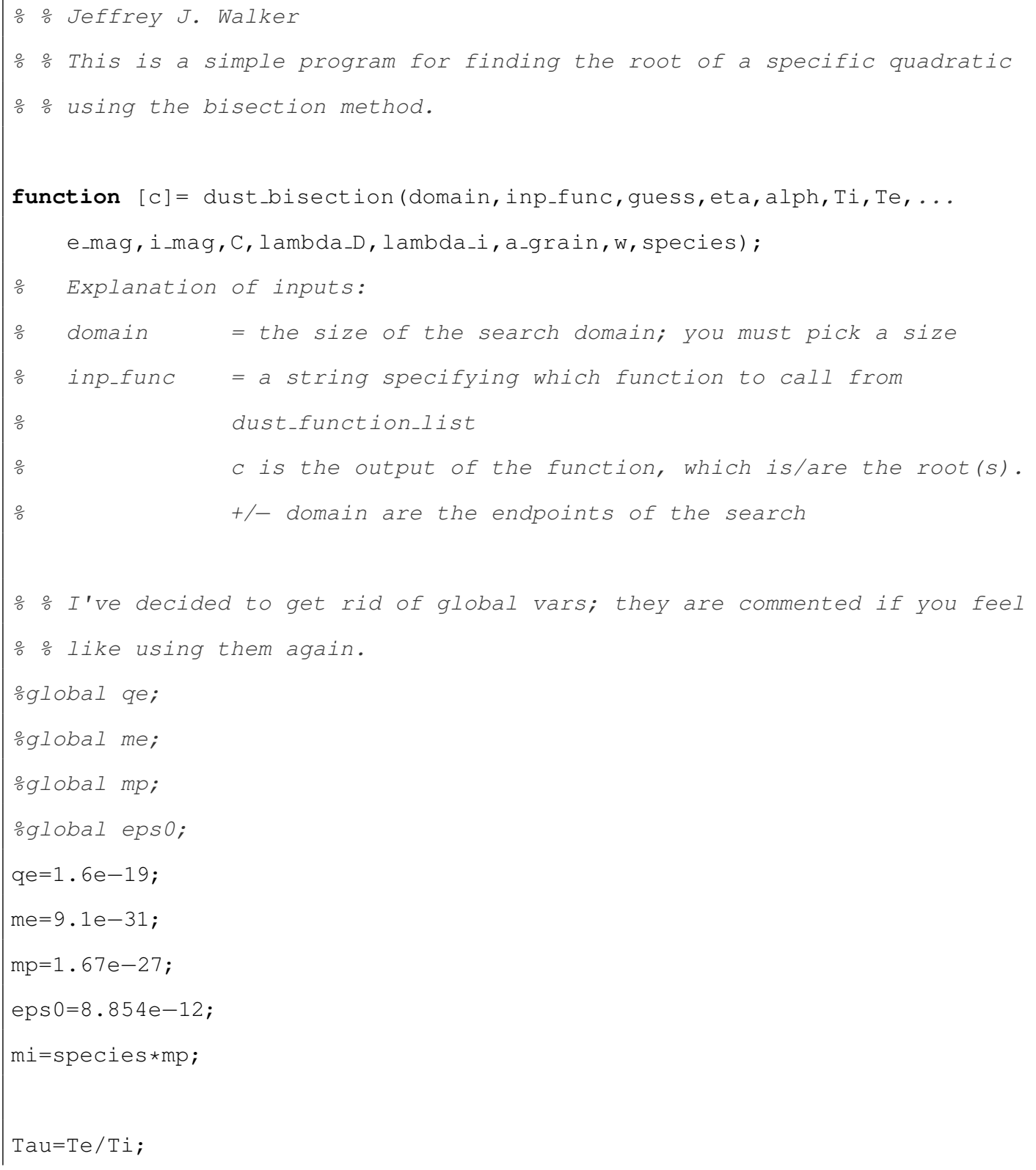




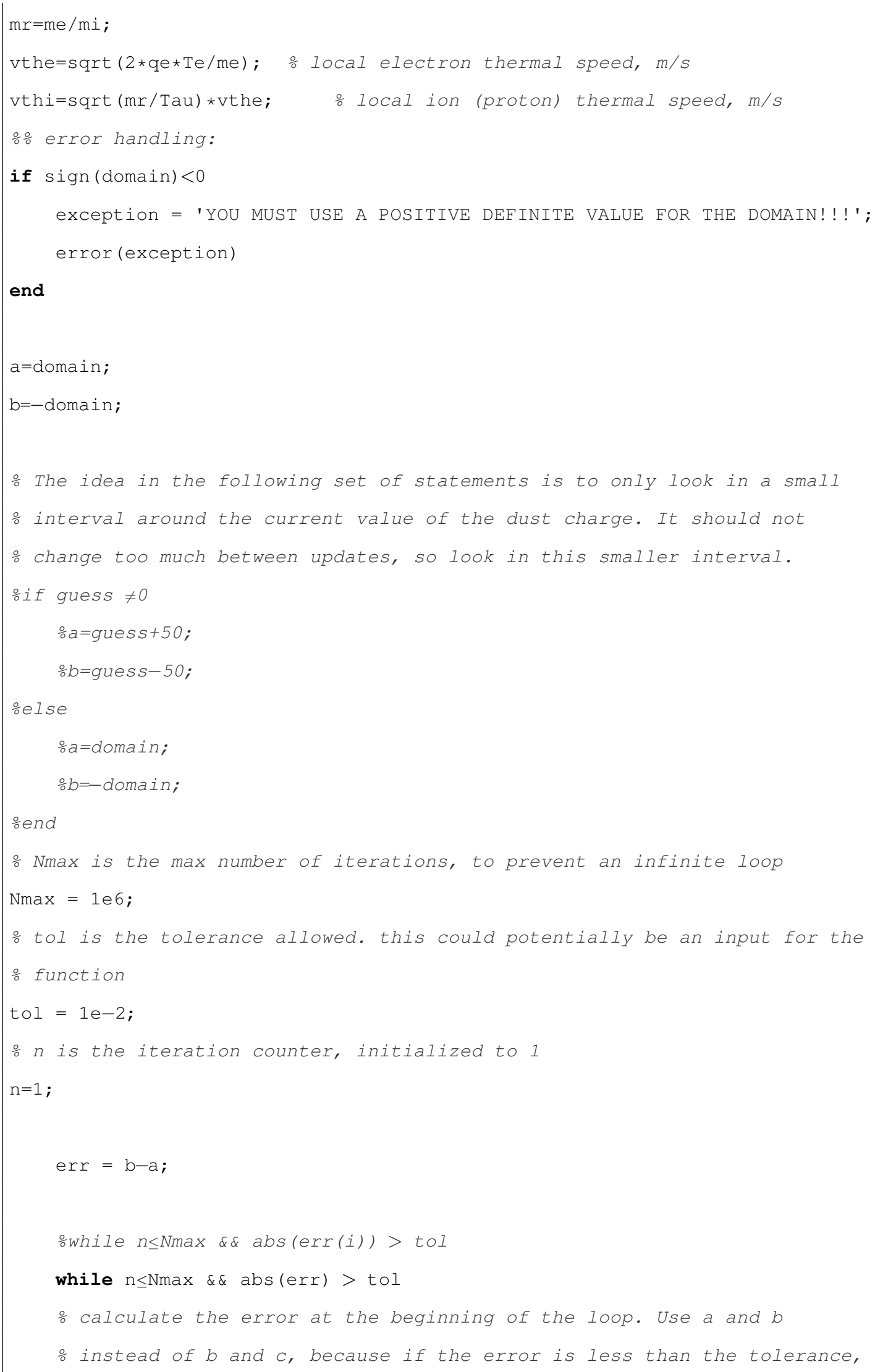




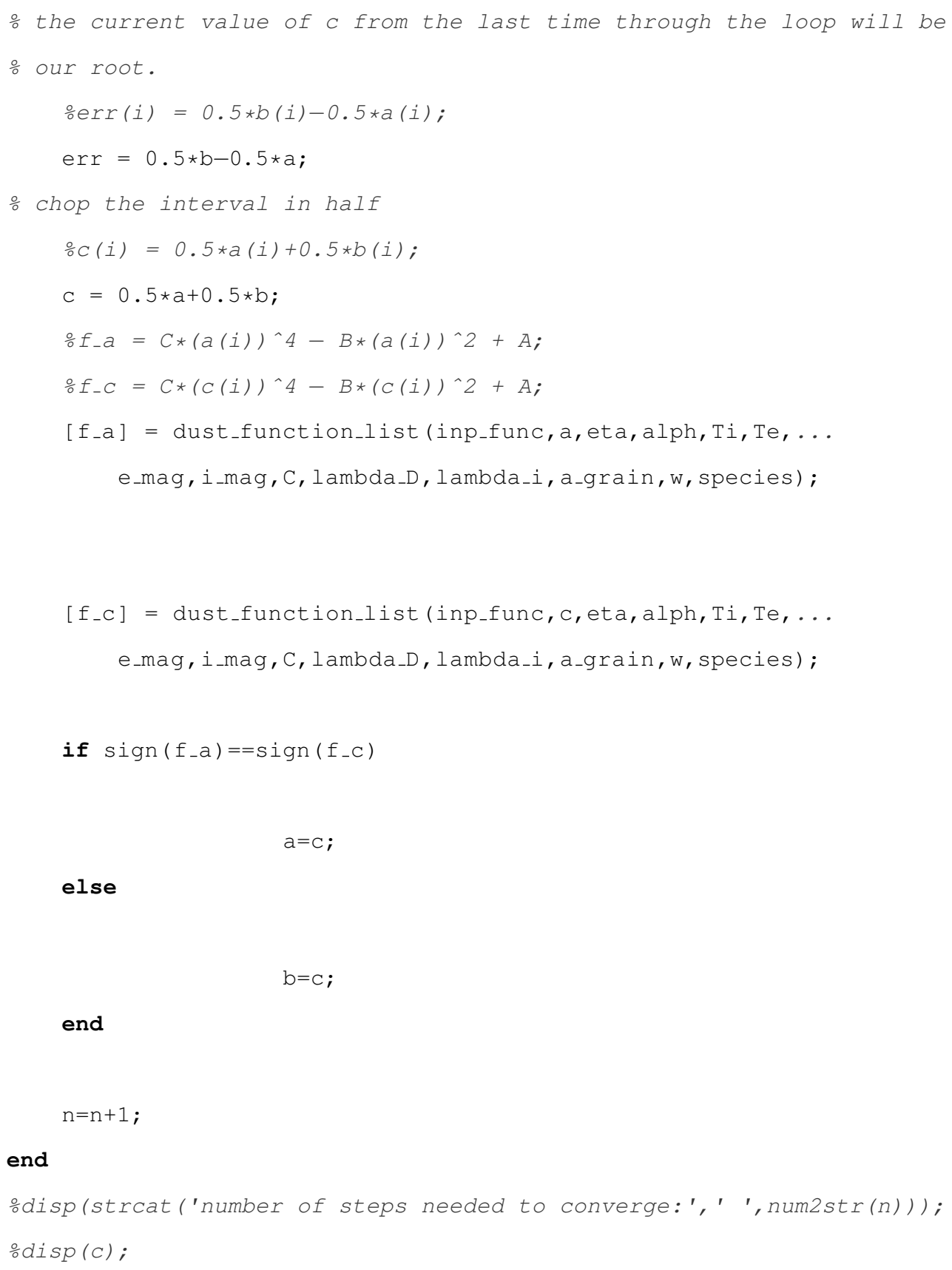

The program dust_bisection.m requires a function list. The program dust_function_list.m provides a list of dimensionless charging equations, and it is shown below.

function $\left[f_{-} x, f_{-} d e r i v\right]=$ dust_function_list (case_label, x, eta, alph, Ti, Te, ..

e_mag, i_mag, C, lambda_D, lambda_i,a,w, species)

응 3/11/13 note: NEED to add alph to the omI cases!!! 


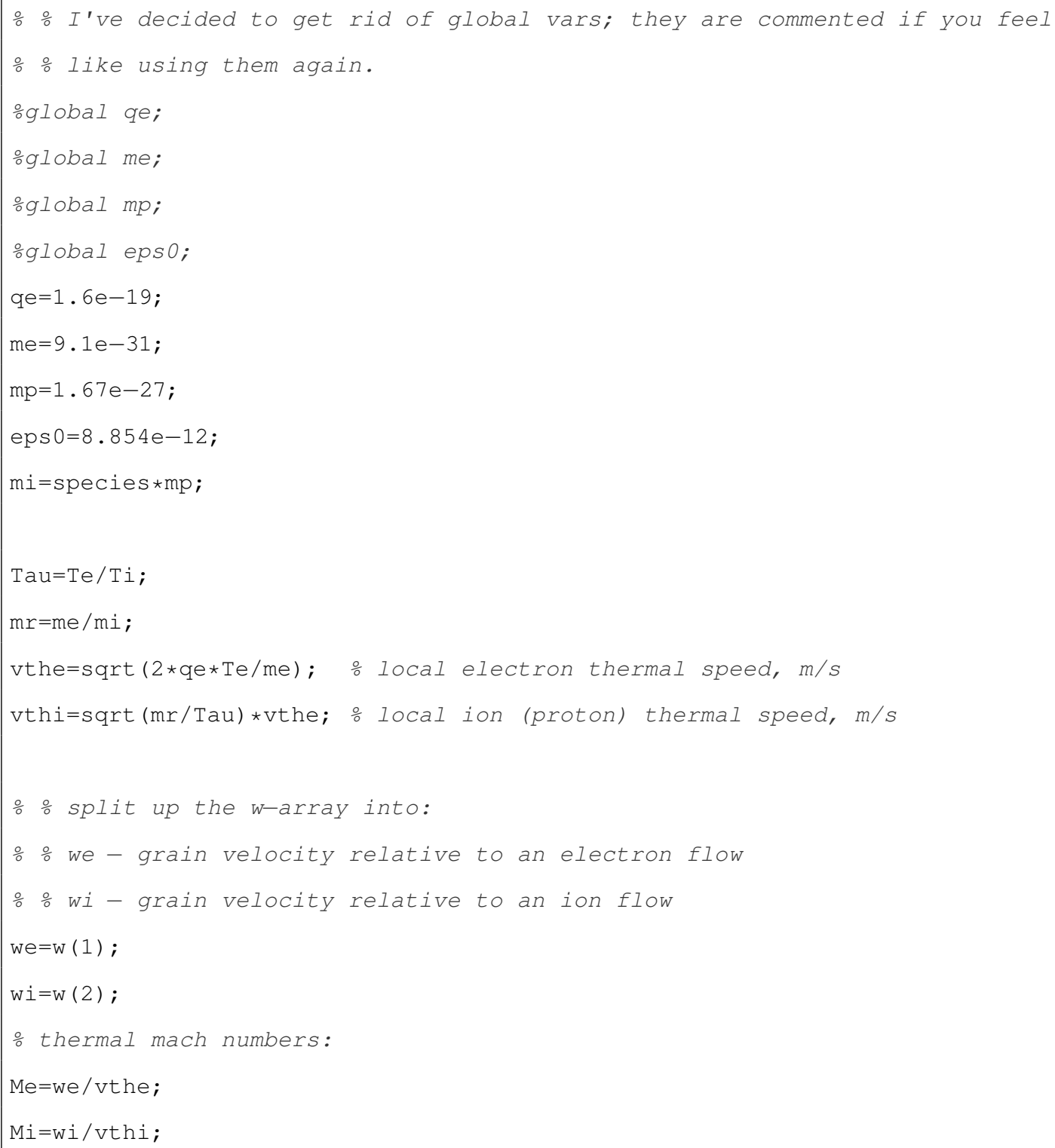




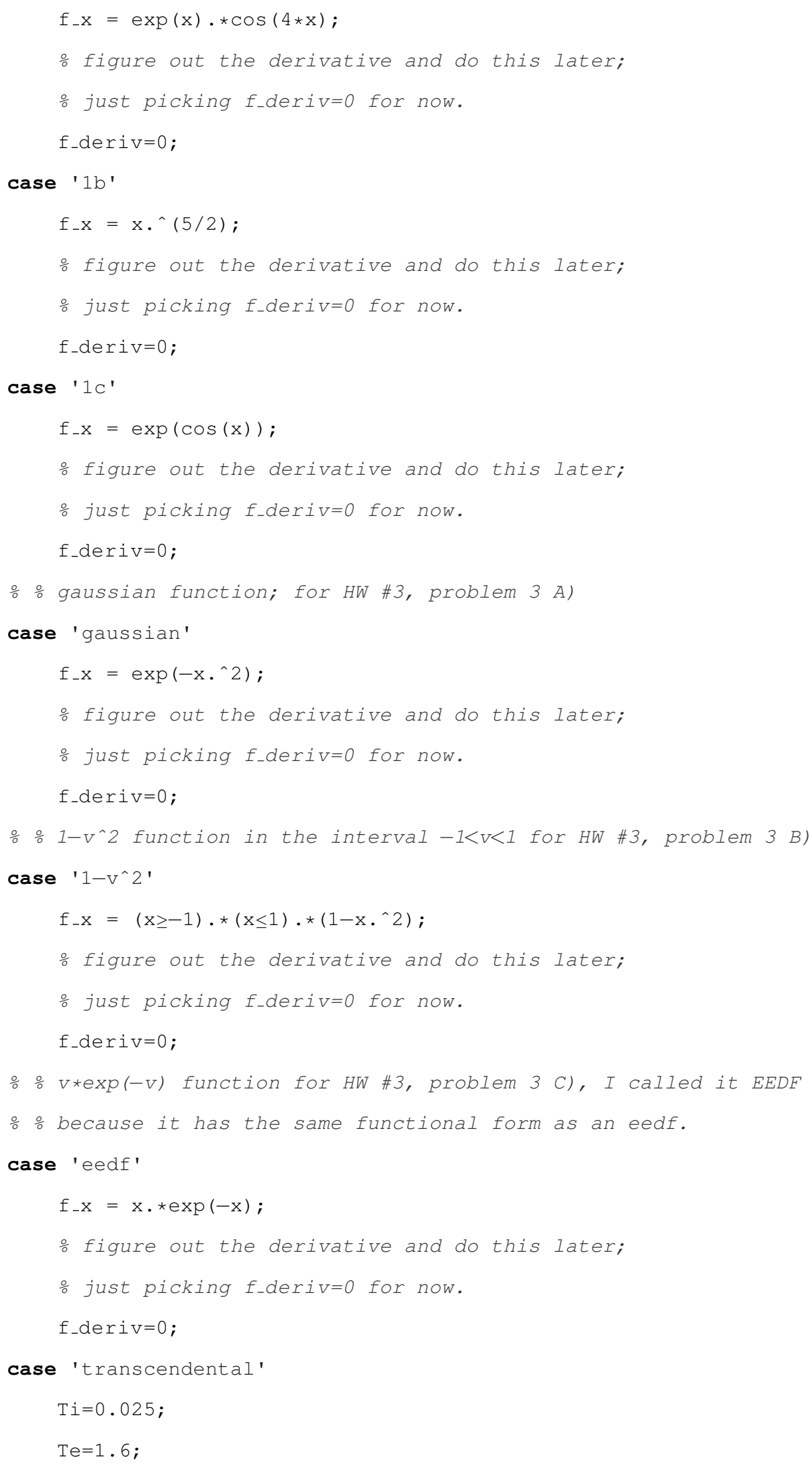


$\mathrm{E} 0=100$

species $=40 ;$ Argon

$\mathrm{me}=9.1 \mathrm{e}-31$

$\mathrm{mi}=1.67 e-27 \star$ species;

․ x_coord tells you where you are in space in order to calculate

․ the space potential. (in $m$ )

$\mathrm{x}_{-} \operatorname{coord}=-.1$;

- calculate Vspace in the prescribed way for a linear E-field

V_space $=100 *\left(x_{-}\right.$coord $) ;$

$\frac{\circ}{0} \mathrm{x}$ here represents surface potential.

$f_{-} x=\operatorname{sqrt}(\mathrm{Ti} / \mathrm{mi}) *\left(1-\left(\mathrm{x} / \mathrm{Ti}-\mathrm{V}_{-}\right.\right.$space $\left.\left./ \mathrm{Ti}\right)\right)-\ldots$

$\operatorname{sqrt}(\mathrm{Te} / \mathrm{me}) \star \exp \left(\left(\mathrm{x}-\mathrm{V}_{-}\right.\right.$space $\left.) / \mathrm{Te}\right)$;

case 'oml_monoenergetic_ions'

if $x \geq 0$

$f_{-} x=\operatorname{sqrt}(p i) *(w i / v t h e) / 2 *(1-q e * q e * x / C /(m i * w i . \wedge 2))-\ldots$ et $a * \exp (q e \star x / C / T e)$;

f_deriv=0;

else

$f_{-x}=\operatorname{sqrt}(p i) *(w i / v t h e) / 2 * \exp \left(-q e * q e * x / C /\left(m i * w i{ }^{\wedge} 2\right)\right)-\ldots$ et $a *(1+q e * x / C / T e)$;

f_deriv=0;

end

case 'oml' $\frac{\circ}{0} \circ<-\ldots$

$\div$ Note: $x=e l e m e n t a r y$ charges on dust grain.

$\therefore$ both ions and electrons are unmagnetized if temp==1.

temp=e_mag $<1 \quad \& \& \quad$ i_mag $<1$;

응 $x \leq 0$, then the grain is negatively charged.

if temp==1;

oml_case='unmag' ;

end

$\div \frac{\circ}{0}$ electrons = magnetized, ions = unmagnetized.

temp=e_mag $>1$ \&\& i_mag $<1$;

if temp $==1$;

oml_case='mag_e' ;

end

$\div \frac{\circ}{0}$ electrons $=$ magnetized, ions = magnetized . 


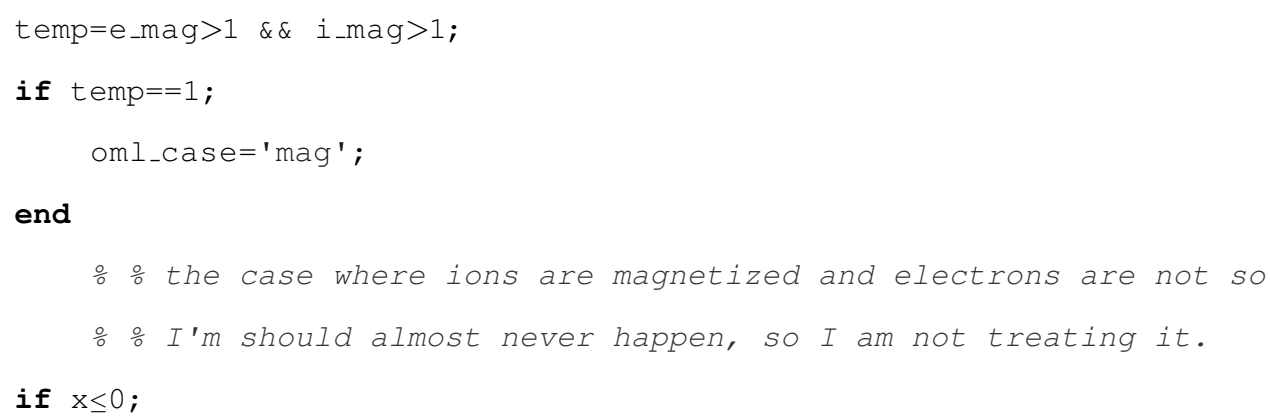


f_deriv=0;

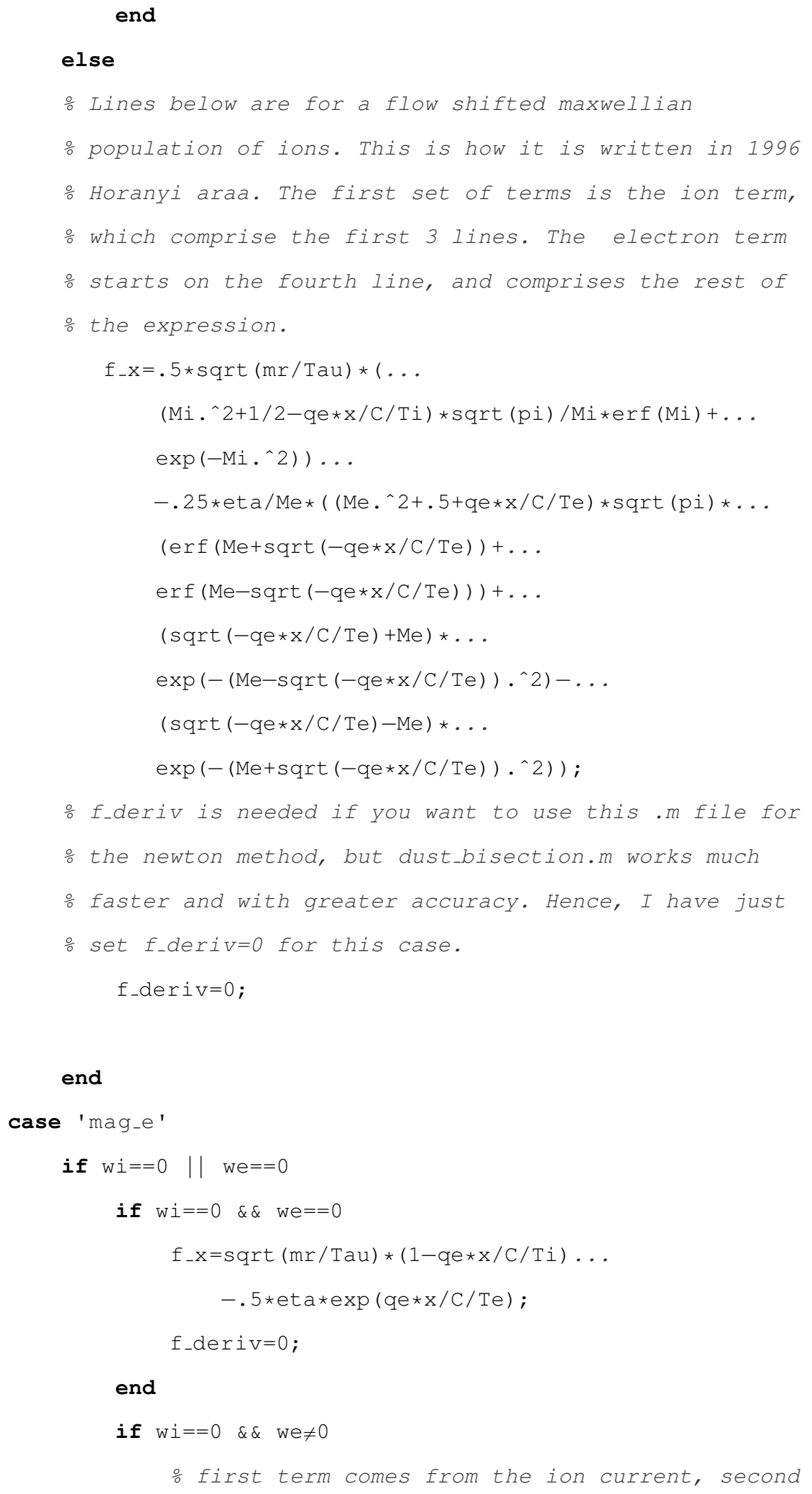




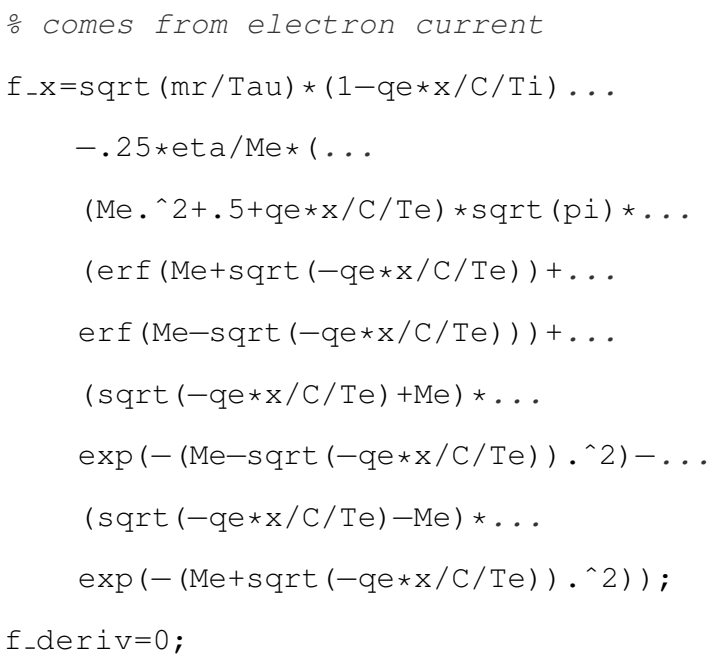




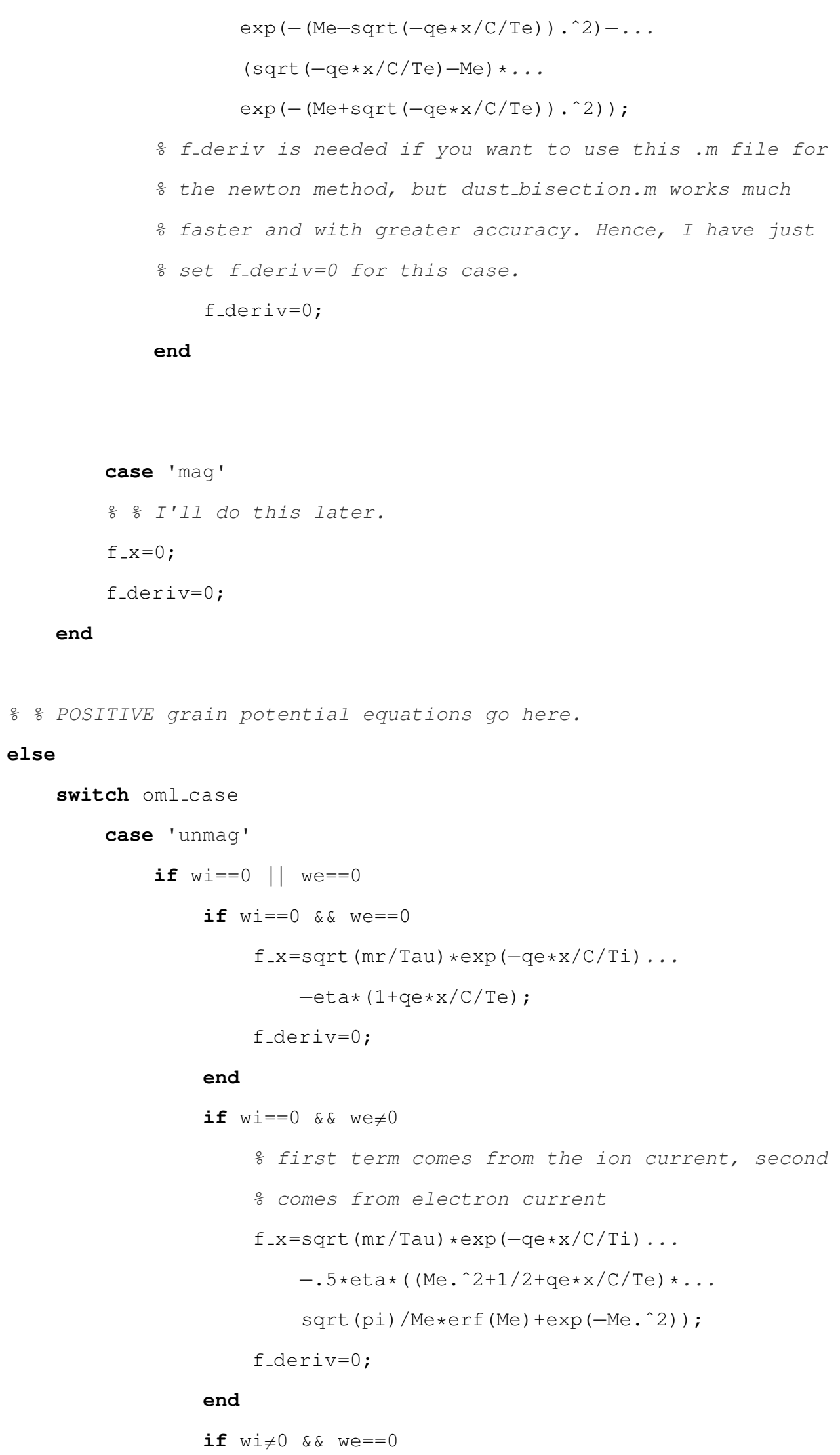




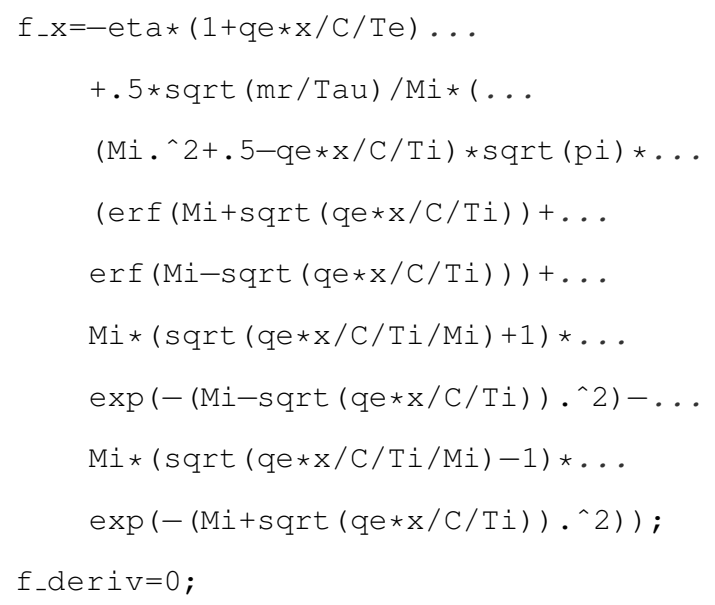




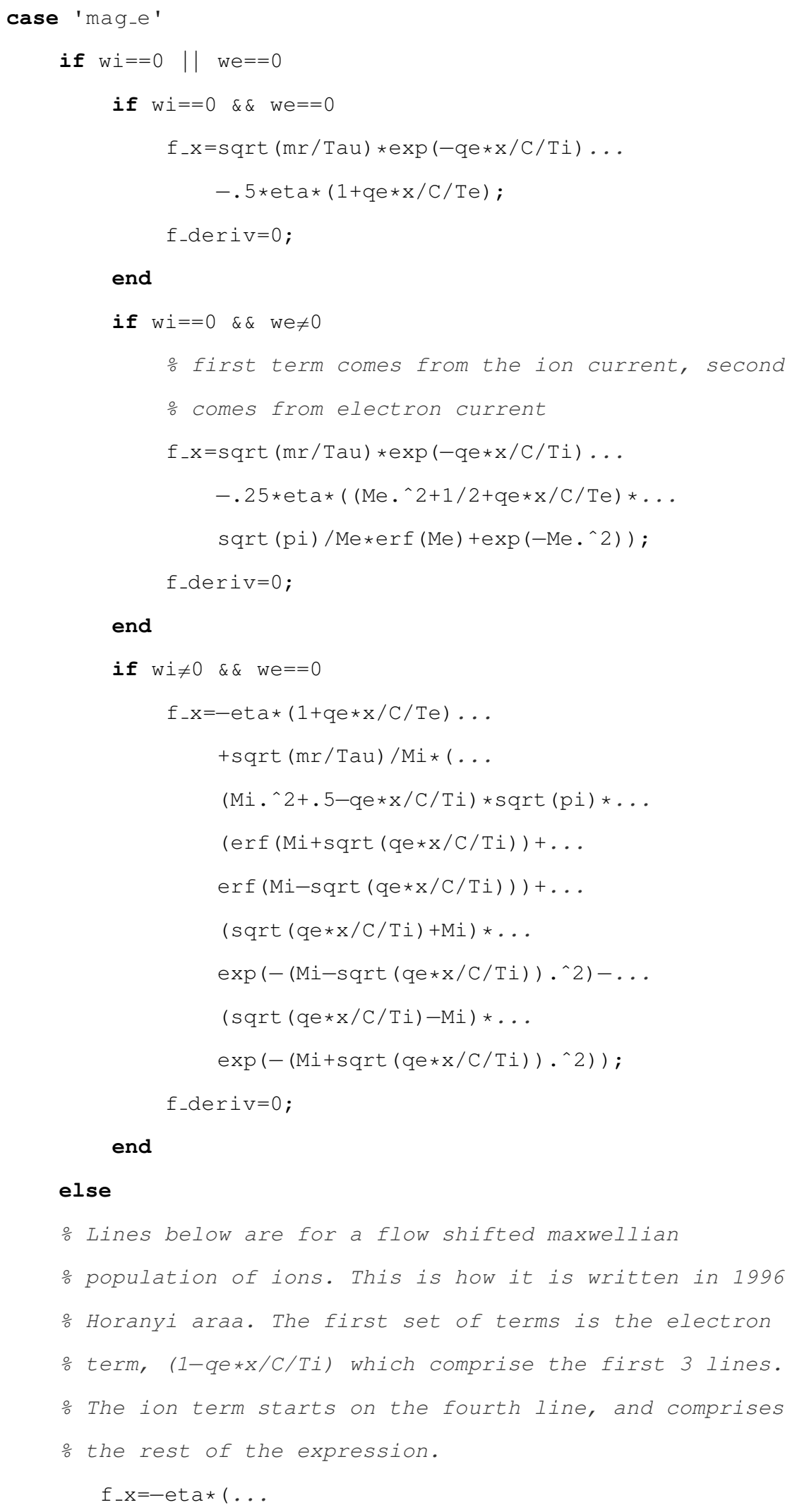




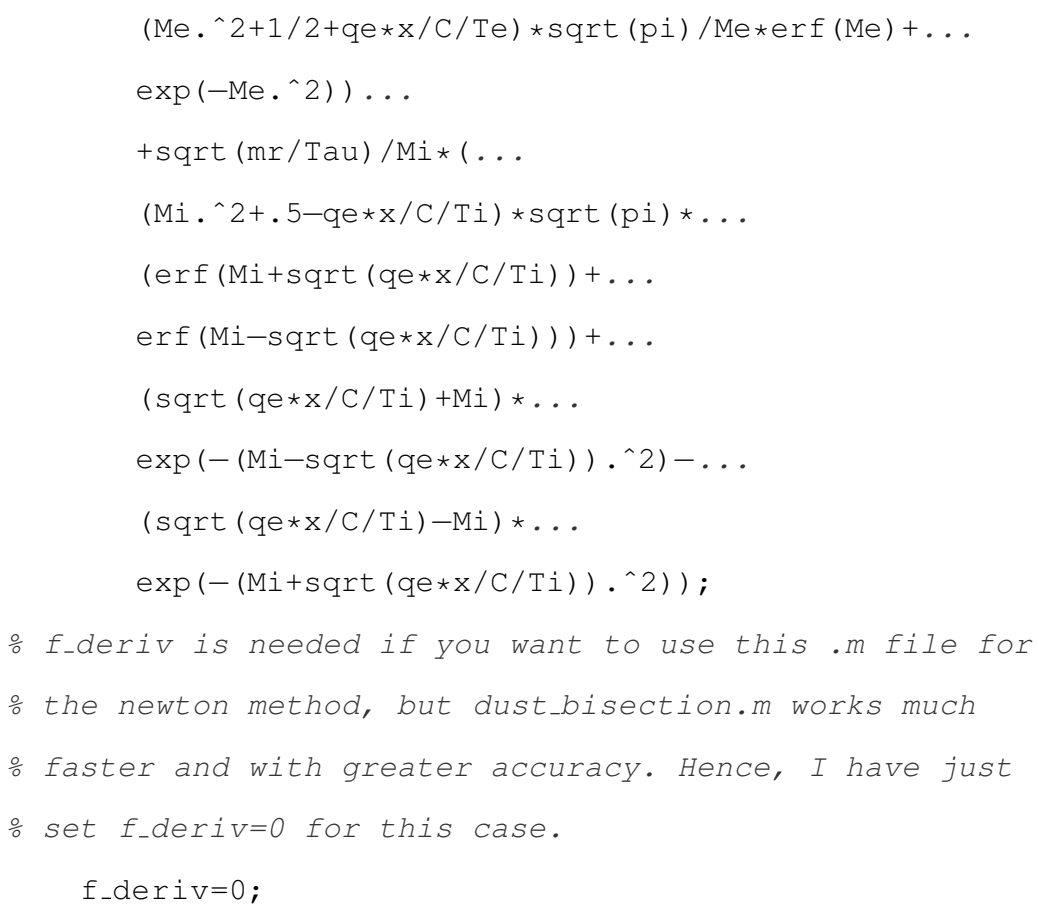


$k_{-}$case $=$'mag' ;

end

음 the case where ions are magnetized and electrons are not

응 should almost never happen, so I'm not treating it.

switch k_case

case 'unmag'

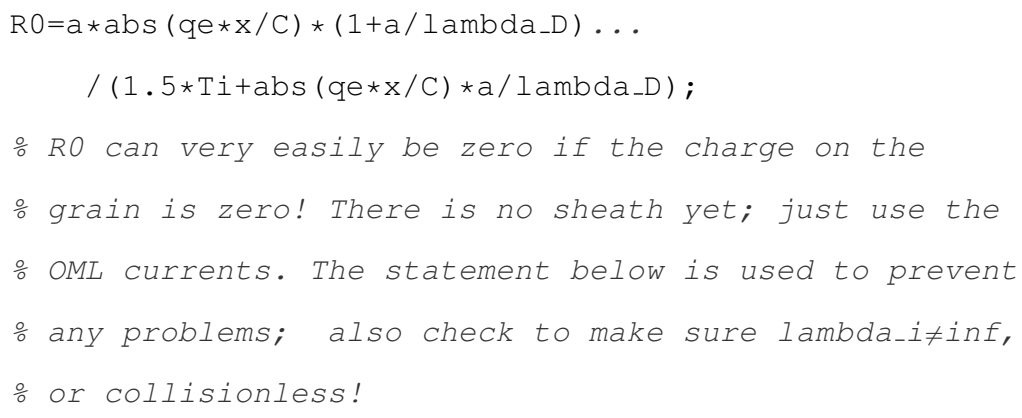




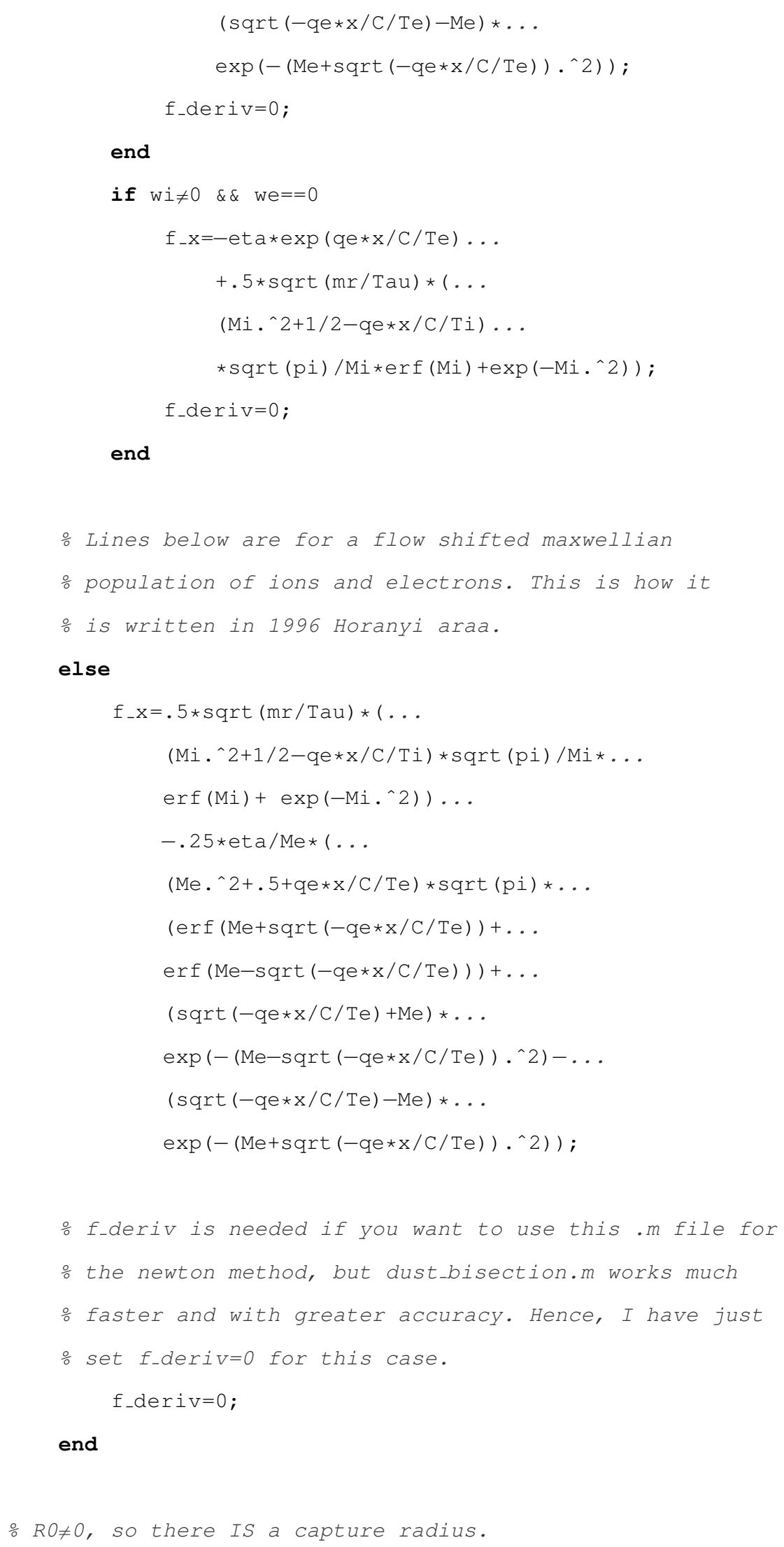




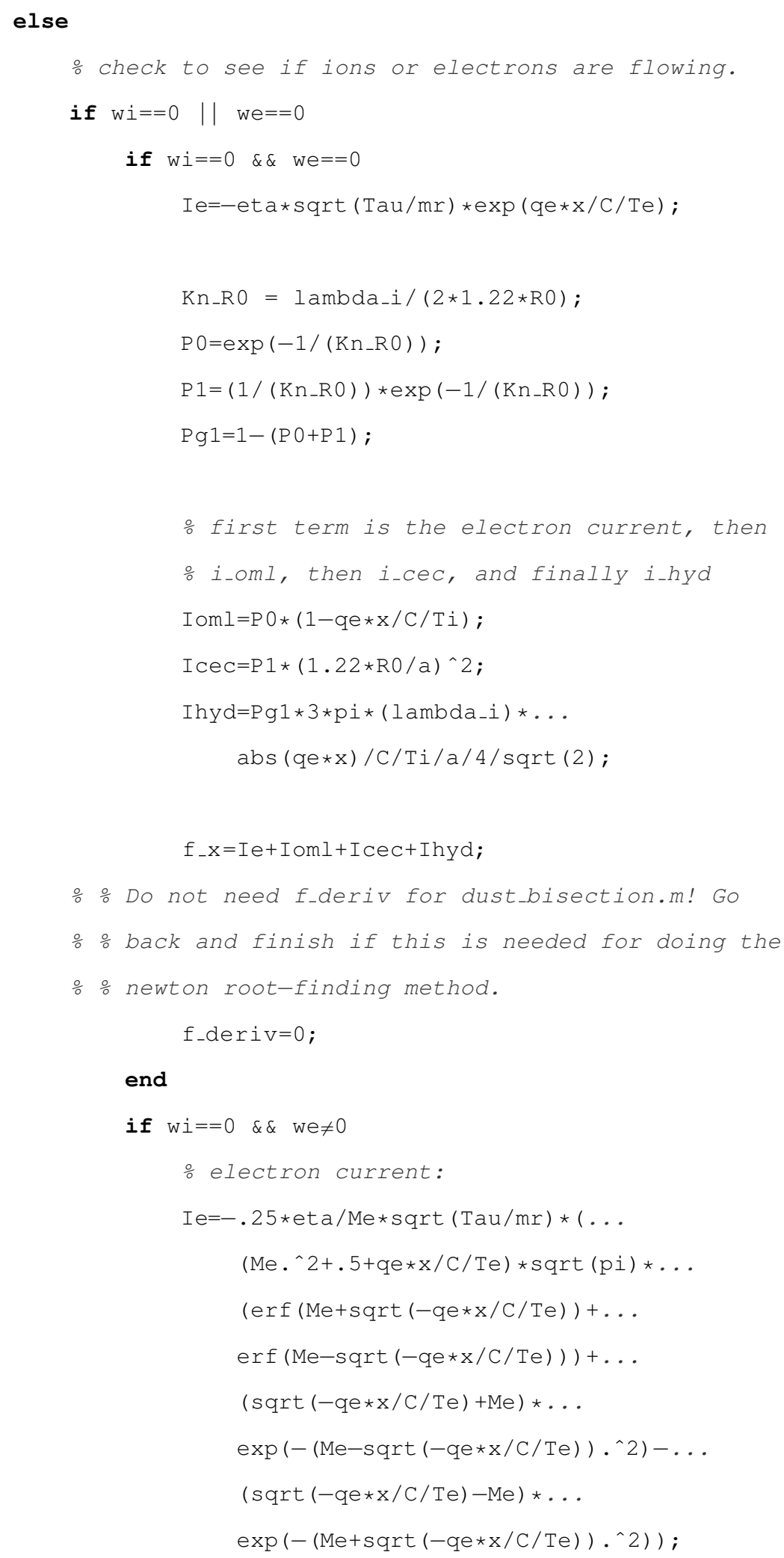




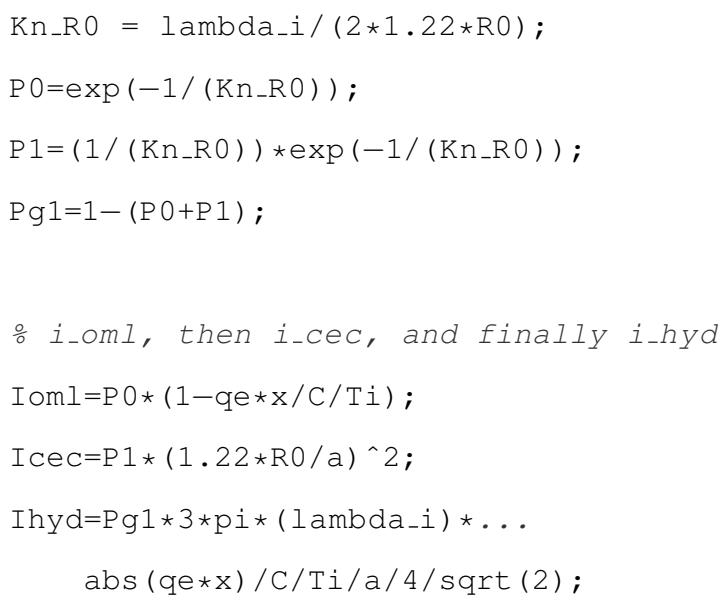


\% Do not need f_deriv for dust_bisection.m! Go

응 back and finish if this is needed for doing the

응 newton root-finding method.

$$
\text { f_deriv=0; }
$$

end

응 this case, the capture radius exists and both

ions and electrons are flowing.

\section{else}

Ie $=-.25 *$ eta $/$ Me $* \operatorname{sqrt}(\mathrm{Tau} / \mathrm{mr}) *(\ldots$

$\left(\mathrm{Me} \cdot{ }^{\wedge} 2+.5+q e * x / C / T e\right) * \operatorname{sqrt}(p i) * \ldots$

$(\operatorname{erf}(\mathrm{Me}+\operatorname{sqrt}(-\mathrm{ge} * \mathrm{x} / \mathrm{C} / \mathrm{Te}))+\ldots$

$\operatorname{erf}(\mathrm{Me}-\operatorname{sqrt}(-\mathrm{ge} * \mathrm{x} / \mathrm{C} / \mathrm{Te})))+\ldots$

$(\operatorname{sqrt}(-q e * x / C / T e)+M e) * \ldots$

$\exp \left(-(\right.$ Me-sqrt $\left.(-q e * x / C / T e)) \cdot{ }^{\wedge} 2\right)-\ldots$

$(\operatorname{sqrt}(-q e \star x / C / T e)-M e) \star \ldots$

$\left.\exp \left(-(\operatorname{Me}+\operatorname{sqrt}(-q e * x / C / \mathrm{Te})) \cdot{ }^{\wedge} 2\right)\right) ;$

$\mathrm{Kn}_{-} \mathrm{R} 0=1 \mathrm{ambda}_{-} \mathrm{i} /(2 * 1.22 * \mathrm{R} 0) ;$

$\mathrm{P} 0=\exp \left(-1 /\left(\mathrm{Kn}_{-} \mathrm{R} 0\right)\right)$;

$\mathrm{P} 1=\left(1 /\left(\mathrm{Kn}_{-} \mathrm{R} 0\right)\right) * \exp \left(-1 /\left(\mathrm{Kn}_{-} \mathrm{R} 0\right)\right) ;$

$\mathrm{Pg} 1=1-(\mathrm{P} 0+\mathrm{P} 1) ;$

o i-oml, then i_cec, and finally i_hyd

$\operatorname{Ioml}=.5 * \mathrm{P} 0 *\left(\left(\mathrm{Mi} \cdot{ }^{\wedge} 2+1 / 2-\mathrm{qe} * \mathrm{x} / \mathrm{C} / \mathrm{Ti}\right) * \ldots\right.$

$\left.\operatorname{sqrt}(\mathrm{pi}) / \mathrm{Mi} * \operatorname{erf}(\mathrm{Mi})+\exp \left(-\mathrm{Mi} \cdot{ }^{\wedge} 2\right)\right) ;$

든 $=P O *(1-q e * x / C / T i)$;

$\mathrm{Icec}=\mathrm{P} 1 *(1.22 * \mathrm{R} 0 / \mathrm{a}){ }^{\wedge} 2$;

Ihyd $=\mathrm{Pg} 1 * 3 * \mathrm{p} i *\left(1 \mathrm{ambda}_{-} i\right) * \ldots$

abs (qe*x)/C/Ti/a/4/sqrt (2);

$\mathrm{f}_{-\mathrm{x}}=\mathrm{Ie}+\mathrm{Ioml}+\mathrm{Icec}_{\text {Ihyd }}$

end

end 


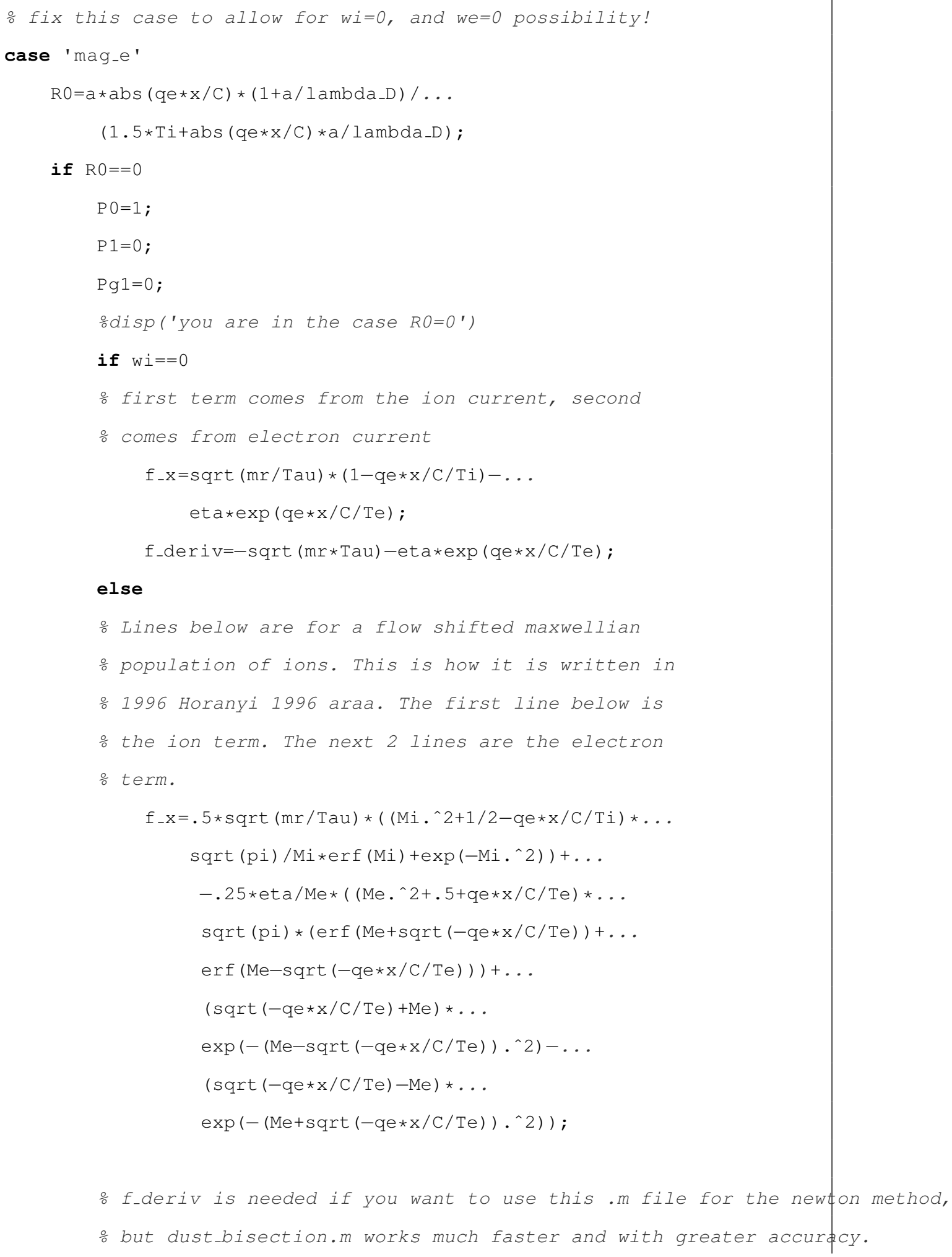


\% Hence, I have just set $f_{-d e r i v}=0$ for this case.

$$
\text { f_deriv }=0 \text {; }
$$

end

else

은 $n_{-} a=1 a m b d a_{-} i / a$;

Kn_RO = lambda_i/ $(2 * 1.22 * R 0)$;

$\mathrm{P} 0=\exp \left(-1 /\left(\mathrm{Kn} \_\mathrm{RO}\right)\right)$;

$\mathrm{P} 1=\left(1 /\left(\mathrm{Kn}_{-} \mathrm{R} 0\right)\right) \star \exp \left(-1 /\left(\mathrm{Kn}_{-} \mathrm{R} 0\right)\right)$;

$\mathrm{Pg} 1=1-(\mathrm{P} 0+\mathrm{P} 1)$;

odisp('You are in the case $R O !=0$ ')

음 first term is the electron current, then i_oml,

응 then i_cec, and finally i_hyd

응 is reduced by factor of $1 / 2$ for magnetization

$\mathrm{Ie}=-.5 * e t a * \operatorname{sqrt}(\mathrm{Tau} / \mathrm{mr}) * \exp (\mathrm{qe} * \mathrm{x} / \mathrm{C} / \mathrm{Te})$;

$\operatorname{Ioml}=\mathrm{P} 0 *(1-\mathrm{qe} * \mathrm{x} / \mathrm{C} / \mathrm{Ti})$;

$\operatorname{Icec}=\mathrm{P} 1 *(1.22 * \mathrm{R} 0 / \mathrm{a})^{\wedge} 2$;

Ihyd $=\mathrm{Pg} 1 * 3 * \mathrm{pi} *($ lambda_i $) * \mathrm{abs}(\mathrm{qe} * \mathrm{x}) / \mathrm{C} / \mathrm{Ti} / \mathrm{a} / 4 / \operatorname{sqrt}(2)$;

$\mathrm{f}_{-\mathrm{x}}=\mathrm{Ie}+\mathrm{Ioml}+\mathrm{Icec}+\mathrm{Ihyd}$;

f_deriv=0;

end

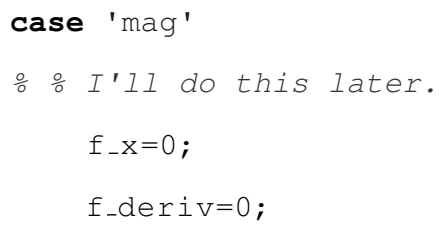


응 THIS WILL NEED TO BE REDONE FOR KORTSHAGEN MODEL.

else

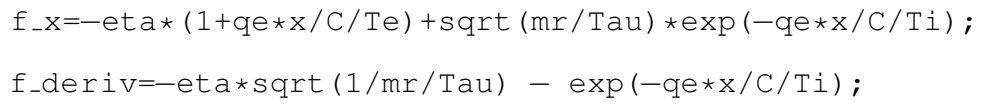

end

case 'hutchinson'

음 fill this out for OML charge model to find equilibrium charge. x=elementary chatges on dust

응 both ions and electrons are unmagnetized if temp==1.

temp=e_mag $<1 \&$ \& i_mag $<1$;

if $x \leq 0$

응 negative grain potential.

$z=e \_m a g /\left(1+e \_m a g\right)$;

o iota* in Patacchini and Hutchinson:

iot $a=1-0.0946 * z-0.305 * z \cdot{ }^{\wedge} 2+0.95 * z \cdot \wedge 3-2.2 * z \cdot \wedge 4+1.15 * z \cdot{ }^{\wedge} 5$;

$\therefore$ ㄷR LAMBDA_D = FINITE, AND DEBYE-HUCKEL POTENTIAL:

o eta, which is now dependent on grain sheath size:

eta_mag $=-q e * x / C / T e / e \_m a g *\left(1+e \_m a g / 4 *(1-\exp (-4 * a / 1\right.$ ambda_D / e_mag $\left.))\right)$;

음 which is eta/(1+eta):

w_mag=eta_mag/(1+eta_mag) ;

$\therefore$, the fitting polynomial, a function of $\mathrm{w}$ :

A_fit $=0.678 * w \_m a g+1.543 * w \_m a g . ` 2-1.212 * w \_m a g \cdot ` 3$;

if i_mag $_{<} 1$

응 unmagnetized ions:

if $\mathrm{Mi}==0$

oxpression above Patacchini and Hutchinson, 2007

$f_{-x}=\operatorname{sqrt}(\mathrm{mr} / \mathrm{Tau}) *(1-q e * x / C / T i)-e t a *\left(A_{-} f i t+\left(1-A_{-} f i t\right) * i o t a\right) * \exp (q e * x / \phi / T e) ;$

f_deriv=0;

else

$f_{-x}=.5 * \operatorname{sqrt}(\mathrm{mr} / \mathrm{Tau}) *\left(\left(\mathrm{Mi} \cdot{ }^{\wedge} 2+1 / 2-\mathrm{qe} * \mathrm{x} / \mathrm{C} / \mathrm{Ti}\right) * \operatorname{sqrt}(\mathrm{pi}) / \operatorname{Mi} * \operatorname{erf}(\mathrm{Mi})+\exp \left(-\mathrm{Mi} \cdot{ }^{\wedge} 2\right)\right)+\ldots$

$-\operatorname{eta}\left(A_{-} f i t+\left(1-A_{-} f i t\right) * i o t a\right) * \exp (q e * x / C / T e) ;$

응 see 1992_northrop_ps or 1981_whipple_repprogphys for the above. 
응 Also, 1996 Horanyi and 1996 Northrop.

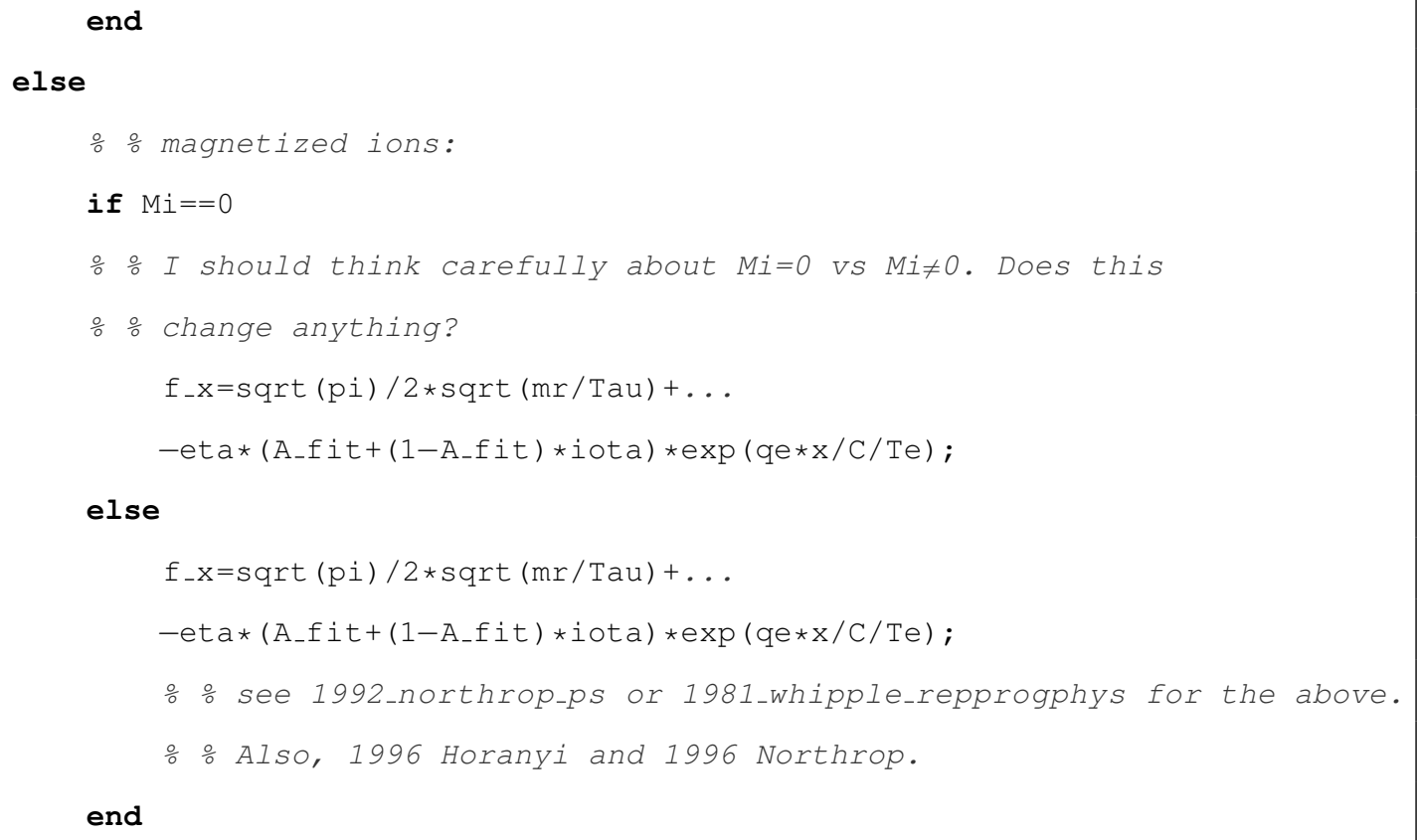




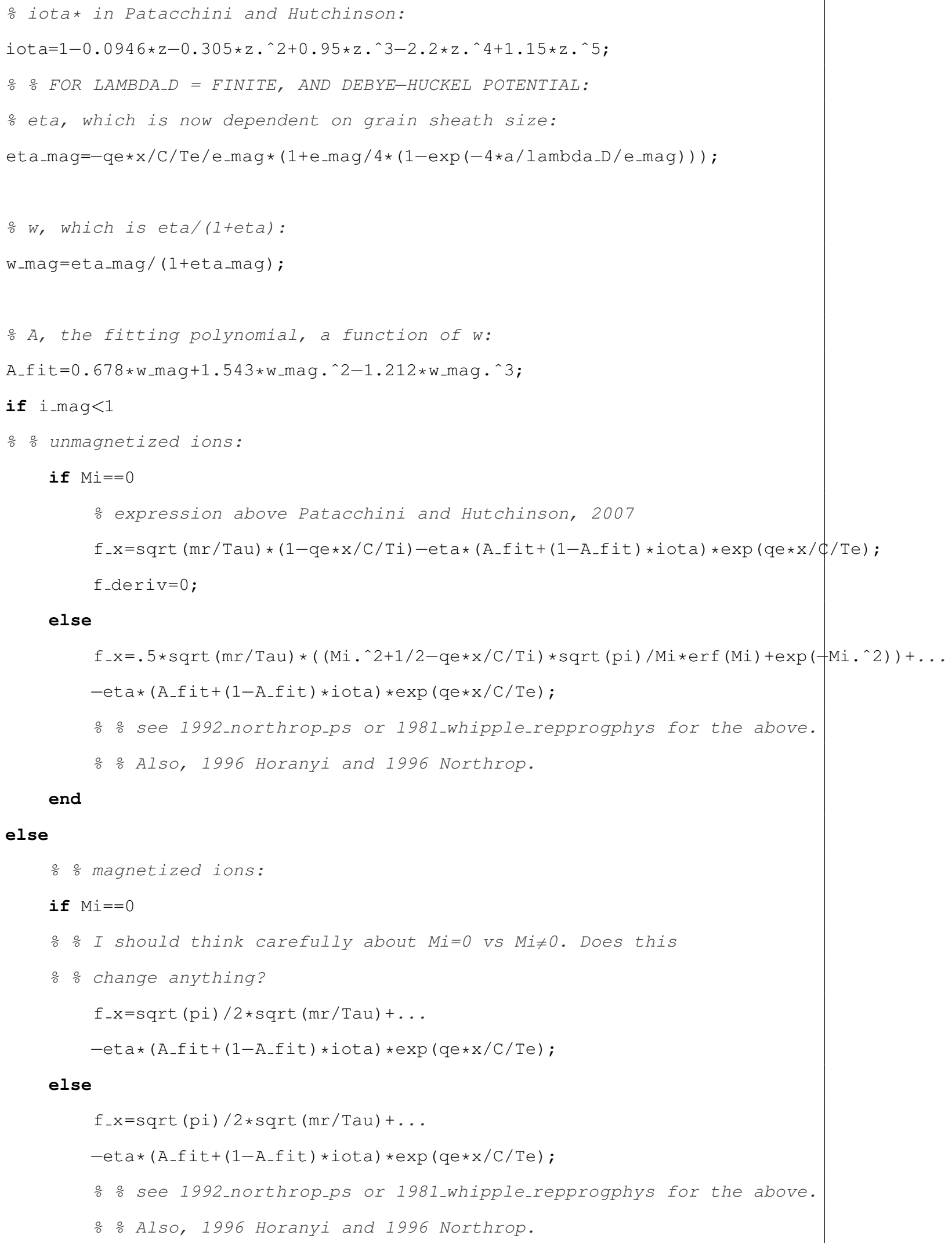




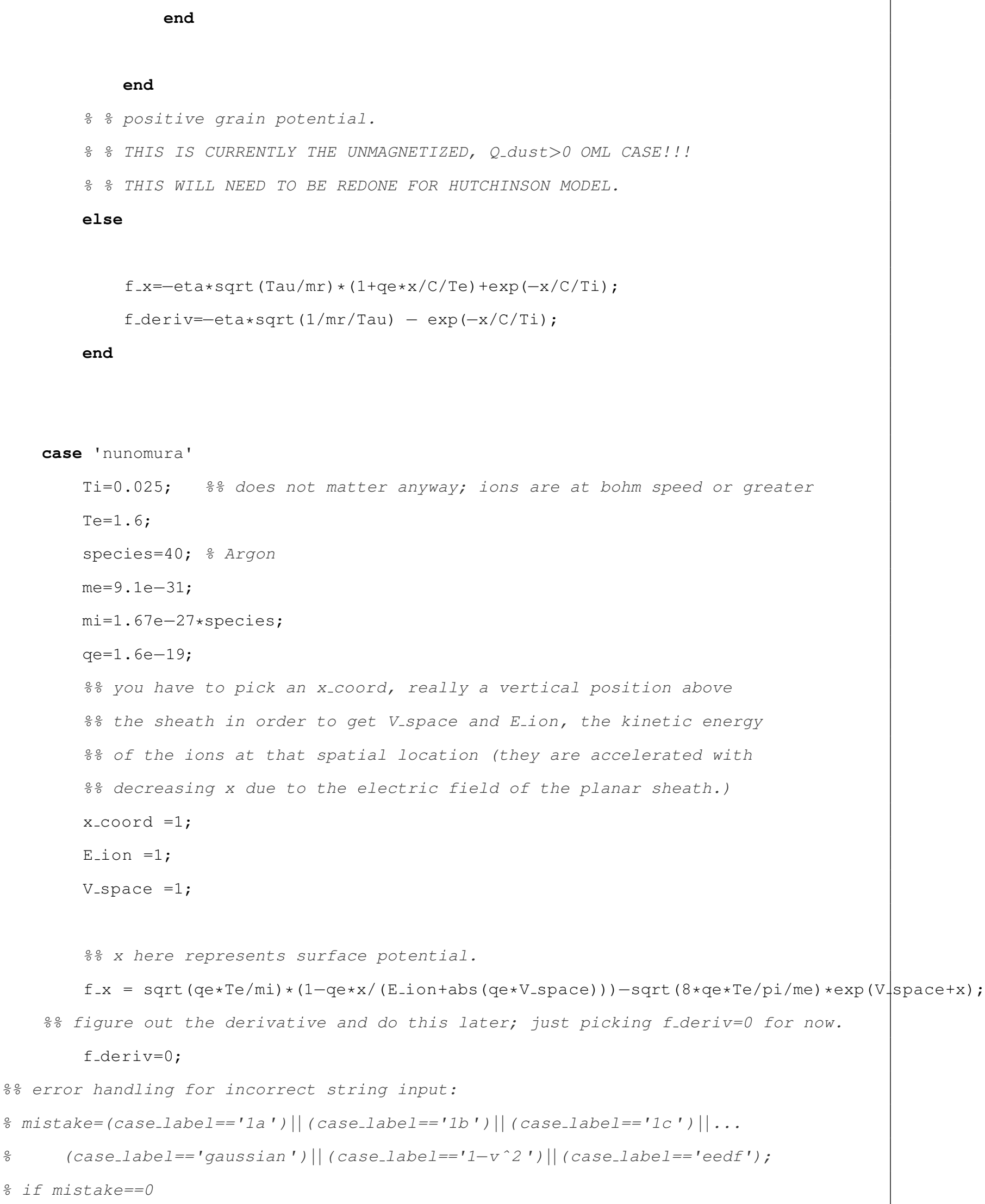




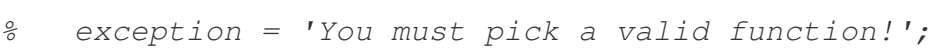

$\therefore \operatorname{error}($ exception)

$\therefore$ end

end

\section{iv Dimensionless Charging Equation}

The function dimensionless_charger.m is called by the theory code, abrupt_omega_theory.m. This charging code can also be used to find equilibrium dimensionless surface potential faster than the bisection algorithm discussed earlier in subsection iii.

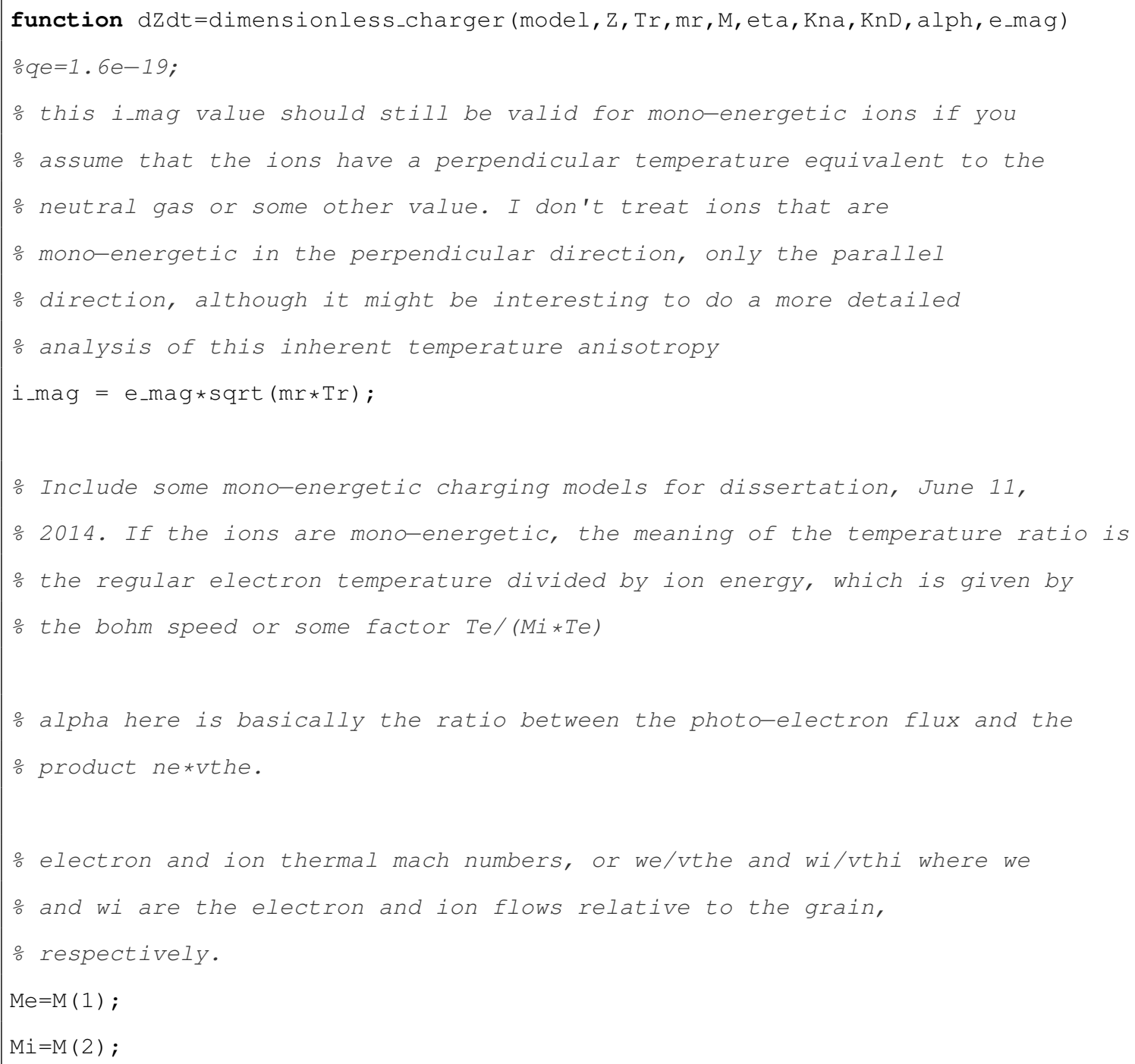




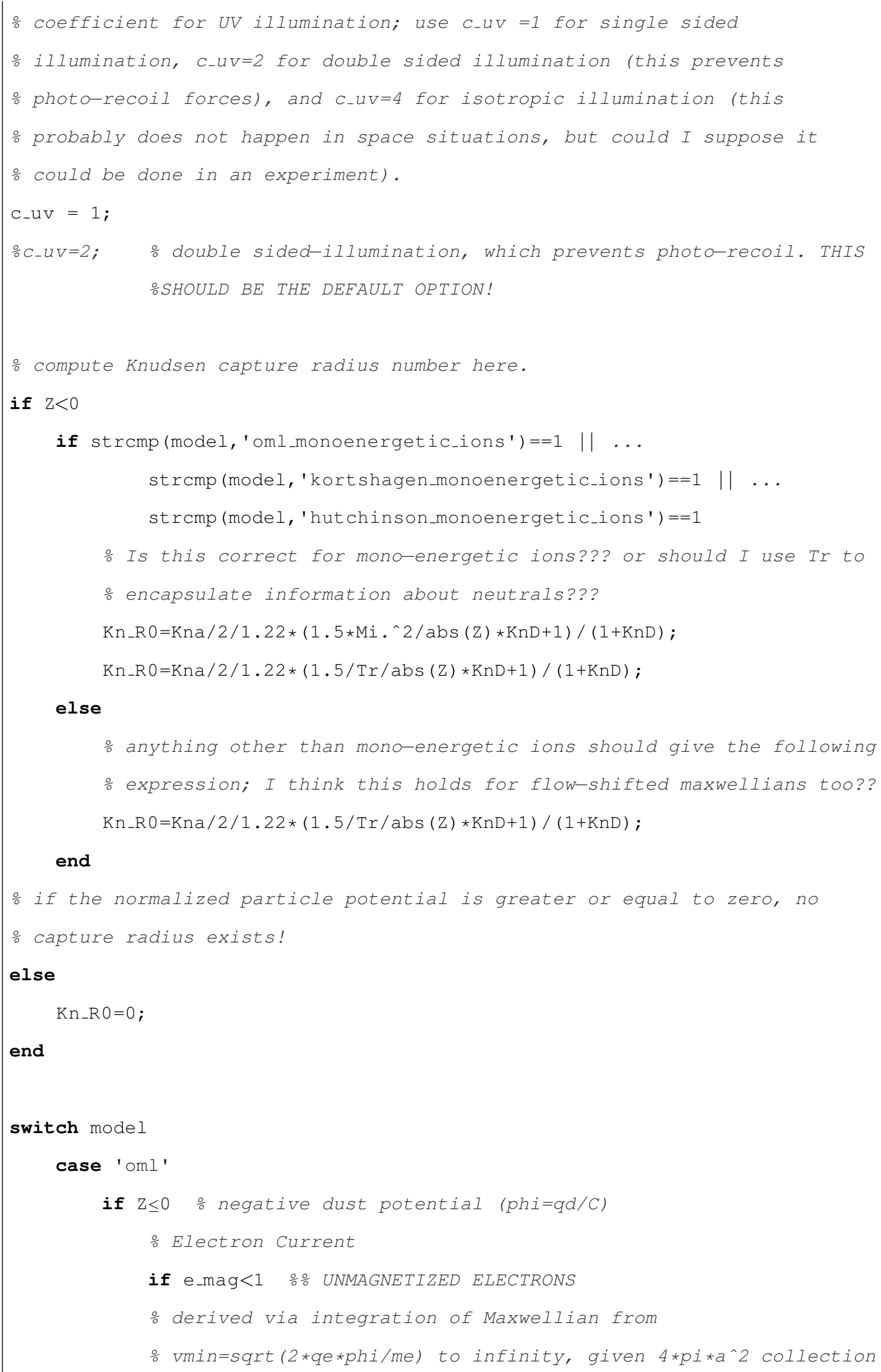


area

if $\mathrm{Me}==0$

$\mathrm{Ze}=-\operatorname{sqrt}(2 \star \mathrm{pi}) / \mathrm{KnD} / \operatorname{sqrt}(1+\mathrm{Tr} / \mathrm{eta}) /(1+1 / \mathrm{KnD}) \star \exp (\mathrm{Z})$;

- check to make sure the photocurrent is written

응 correcty!

$\therefore Z p=\operatorname{sqrt}(p i) / \operatorname{sqrt}(2) / 2 * a I p h / K n D / \operatorname{sqrt}(1+\operatorname{Tr} /$ eta $) / \ldots$

$\circ(1+1 / K n D) * C_{-} U V$;

o seems like it should actually be:

$\mathrm{Zp}=\mathrm{pi} / \operatorname{sqrt}(2) \star \mathrm{al} \mathrm{ph} / \mathrm{KnD} / \operatorname{sqrt}(1+\operatorname{Tr} /$ eta $) / \ldots$

$(1+1 / \mathrm{KnD}) * \mathrm{C}_{-} \mathrm{uv}$;

If there is an electron flow, need to use this

응 description from 1981 Whipple rev. geophys.

else

$\mathrm{Ze}=-.5 * \operatorname{sqrt}(2 * \mathrm{pi}) / \mathrm{KnD} / \operatorname{sqrt}(1+\mathrm{Tr} / \mathrm{eta}) /(1+1 / \mathrm{KnD}) / \mathrm{Me} *(\ldots$

$($ Me.`2+.5+Z)*sqrt $(p i) *(\operatorname{erf}($ Me $+\operatorname{sqrt}(-Z))+\ldots$

$\operatorname{erf}(\operatorname{Me}-\operatorname{sqrt}(-\mathrm{Z})))+\ldots$

$(\operatorname{sqrt}(-\mathrm{Me} * \mathrm{Z})+\mathrm{Me}) * \exp \left(-(\mathrm{Me}-\operatorname{sqrt}(-\mathrm{Z})) \cdot{ }^{\wedge} 2\right)-\ldots$

$\left.(\operatorname{sqrt}(-\mathrm{Me} \star \mathrm{Z})-\mathrm{Me}) \star \exp \left(-(\mathrm{Me}+\operatorname{sqrt}(-\mathrm{Z})) \cdot{ }^{\wedge} 2\right)\right)$;

$\therefore Z p=\operatorname{sqrt}(p i) / \operatorname{sqrt}(2) / 2 * a I p h / K n D / \operatorname{sqrt}(1+\operatorname{Tr} /$ eta $) / \ldots$

$\therefore \quad(1+1 / K n D) * C_{-} U V$;

응 seems like it should actually be:

$\mathrm{Zp}=\mathrm{pi} / \operatorname{sqrt}(2) * \mathrm{alph} / \mathrm{KnD} / \operatorname{sqrt}(1+\mathrm{Tr} /$ eta $) / \ldots$

$(1+1 / \mathrm{KnD}) * \mathrm{C}-\mathrm{uv}$;

end

else $\quad$ MAGNETIZED ELECTRONS

- same as unmagnetized case, except collection area reduced due

to magnetization; may be off by some constant factor due to

o cos-dependence of incidence angle

if $\mathrm{Me}==0$

$\mathrm{Ze}=-.5 * \operatorname{sqrt}(2 * \mathrm{pi}) / \mathrm{KnD} / \operatorname{sqrt}(1+\mathrm{Tr} / \mathrm{eta}) /(1+1 / \mathrm{KnD}) * \exp (\mathrm{Z})$;

UV is incident along magnetic field direction.

$\therefore Z p=\operatorname{sqrt}(p i) / \operatorname{sqrt}(2) / 2 * a I p h / K n D / \operatorname{sqrt}(1+\operatorname{Tr} /$ eta $) / \ldots$ 


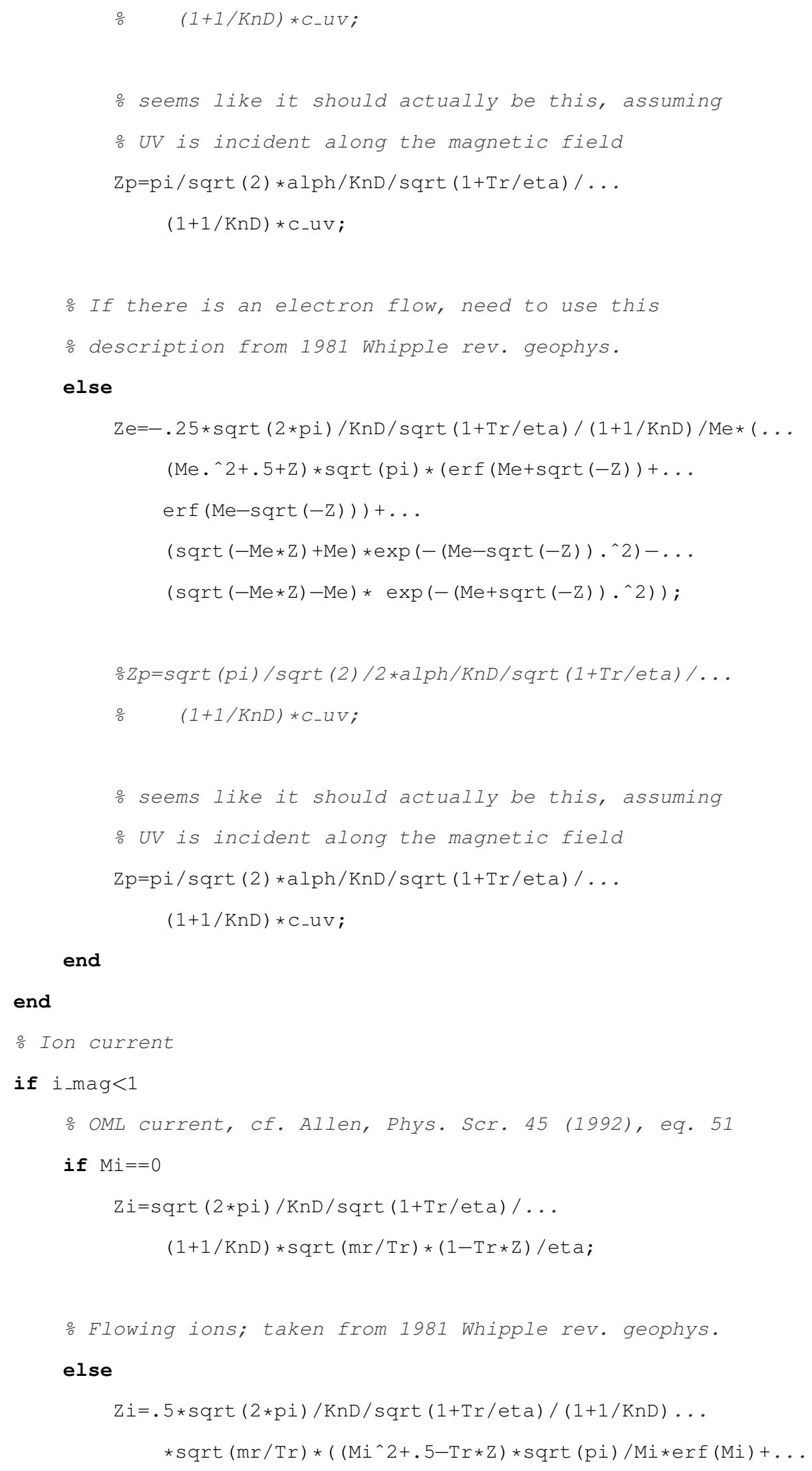




$$
\left.\exp \left(-M i^{\wedge} 2\right)\right) / \text { eta; }
$$

end

\section{else}

o simple thermal flux of ions, assuming ballistic trajectories

o along field lines w/ reduced collection area due to

․ognetization

$\mathrm{Zi}=.5 * \operatorname{sqrt}(2 * \mathrm{pi}) / \mathrm{KnD} / \operatorname{sqrt}(1+\mathrm{Tr} / \mathrm{eta}) / \ldots$

$(1+1 / \mathrm{KnD}) * \operatorname{sqrt}(\mathrm{mr} / \mathrm{Tr}) /$ eta;

o reference for this?

end

can add equations for positive dust potential here.

응 4/4/2014: THIS STILL NEEDS TO BE DONE PROPERLY!!!

else

응 Electron Current

if e_mag $<1$ 응 UNMAGNETIZED ELECTRONS

$\mathrm{Ze}=-\operatorname{sqrt}(2 * \mathrm{pi}) / \mathrm{KnD} / \operatorname{sqrt}(1+\mathrm{Tr} /$ eta $) /(1+1 / \mathrm{KnD}) *(1+\mathrm{Z})$;

else

$\mathrm{Ze}=-.5 * \operatorname{sqrt}(2 * \mathrm{pi}) / \mathrm{KnD} / \operatorname{sqrt}(1+\mathrm{Tr} / \mathrm{eta}) /(1+1 / \mathrm{KnD}) ;$

end

Ion current

if i_mag $<1$ ㄴ Unmagnetized ions

$\mathrm{Zi}=\operatorname{sqrt}(2 * \mathrm{pi}) / \mathrm{KnD} / \operatorname{sqrt}(1+\mathrm{Tr} / \mathrm{eta}) / \ldots$

$(1+1 / \mathrm{KnD}) * \operatorname{sqrt}(\mathrm{mr} / \mathrm{Tr}) * \exp (-\mathrm{Tr} * \mathrm{Z}) /$ eta

else

$\mathrm{Zi}=.5 * \operatorname{sqrt}(2 * \mathrm{pi}) / \mathrm{KnD} / \operatorname{sqrt}(1+\mathrm{Tr} / \mathrm{eta}) / \ldots$

$(1+1 / \mathrm{KnD}) * \operatorname{sqrt}(\mathrm{mr} / \mathrm{Tr}) * \exp (-\operatorname{Tr} * \mathrm{Z}) /$ eta $;$

end

o check to make sure the photocurrent is written

응 correctly!

$\mathrm{Zp}=\mathrm{pi} / \operatorname{sqrt}(2) * \mathrm{alph} / \mathrm{KnD} / \operatorname{sqrt}(1+\mathrm{Tr} /$ eta $) /(1+1 / \mathrm{KnD}) * \mathrm{C}_{-} u v$;

․ Whipple 1981, reviews of geophysics; 1995 Cui and Goree IEEE

end

$d z d t=Z i+Z e+Z p ;$

case 'oml_monoenergetic_ions'

stop everything if $M i=0$, because $M i \neq 0$ is necessary for 
응 monenergetic ions.

if $\mathrm{Mi}==0$

exception='Mi cannot be zero!' ;

error(exception);

end

․ If we get through the statement above, we can continue

if $\mathrm{Z} \leq 0$ 응 negative dust potential (phi=qd/C)

․ Electron Current

if e_mag $<1$ 응 UNMAGNETIZED ELECTRONS

o derived via integration of Maxwellian from

․ vmin=sqrt $(2 * q e * p h i / m e)$ to infinity, given $4 * p i * a^{\wedge} 2$ collection

area

if $M e==0$

$\mathrm{Ze}=-\operatorname{sqrt}(2 \star \mathrm{pi}) / \mathrm{KnD} / \operatorname{sqrt}\left(1+1 / \mathrm{Mi} \cdot{ }^{\wedge} 2 / \mathrm{eta}\right) / \ldots$

$(1+1 / \mathrm{KnD}) * \exp (Z) ;$

o check to make sure the photocurrent is written

응 correctly!

$\therefore Z p=\operatorname{sqrt}(p i) / \operatorname{sqrt}(2) / 2 * a I p h / K n D / \operatorname{sqrt}(1+\operatorname{Tr} /$ eta $) / \ldots$

$\therefore(1+1 / K n D) * C_{-} U V$;

o seems like it should actually be:

$\mathrm{Zp}=\mathrm{pi} / \operatorname{sqrt}(2) * \mathrm{al} \mathrm{ph} / \mathrm{KnD} / \operatorname{sqrt}(1+1 / \mathrm{Mi} \cdot \wedge 2 / \mathrm{eta}) / \ldots$

$(1+1 / \mathrm{KnD}) * \mathrm{C}-\mathrm{uv}$;

․ If there is an electron flow, need to use this

응 description from 1981 Whipple rev. geophys.

else

$\mathrm{Ze}=-.5 * \operatorname{sqrt}(2 * \mathrm{pi}) / \mathrm{KnD} / \operatorname{sqrt}\left(1+1 / \mathrm{Mi} \cdot{ }^{\wedge} 2 / \mathrm{eta}\right) /(1+1 / \mathrm{KnD}) / \mathrm{Me} *(\ldots$

$\left(\right.$ Me $\left.\cdot{ }^{\wedge} 2+.5+Z\right) * \operatorname{sqrt}(p i) *(\operatorname{erf}(\operatorname{Me}+\operatorname{sqrt}(-\mathrm{Z}))+\ldots$

$\operatorname{erf}($ Me-sqrt $(-Z)))+\ldots$

$(\operatorname{sqrt}(-Z)+\mathrm{Me}) * \exp \left(-(\mathrm{Me}-\operatorname{sqrt}(-\mathrm{Z})) \cdot{ }^{\wedge} 2\right)-\ldots$

$\left.(\operatorname{sqrt}(-Z)-M e) * \exp \left(-(\operatorname{Me}+\operatorname{sqrt}(-Z)) \cdot{ }^{\wedge} 2\right)\right)$;

$\div Z p=\operatorname{sqrt}(p i) / \operatorname{sqrt}(2) / 2 * a I p h / K n D / \operatorname{sqrt}(1+\operatorname{Tr} /$ eta $) / \ldots$

$\therefore \quad(1+1 / K n D) * C_{-} U V ;$ 


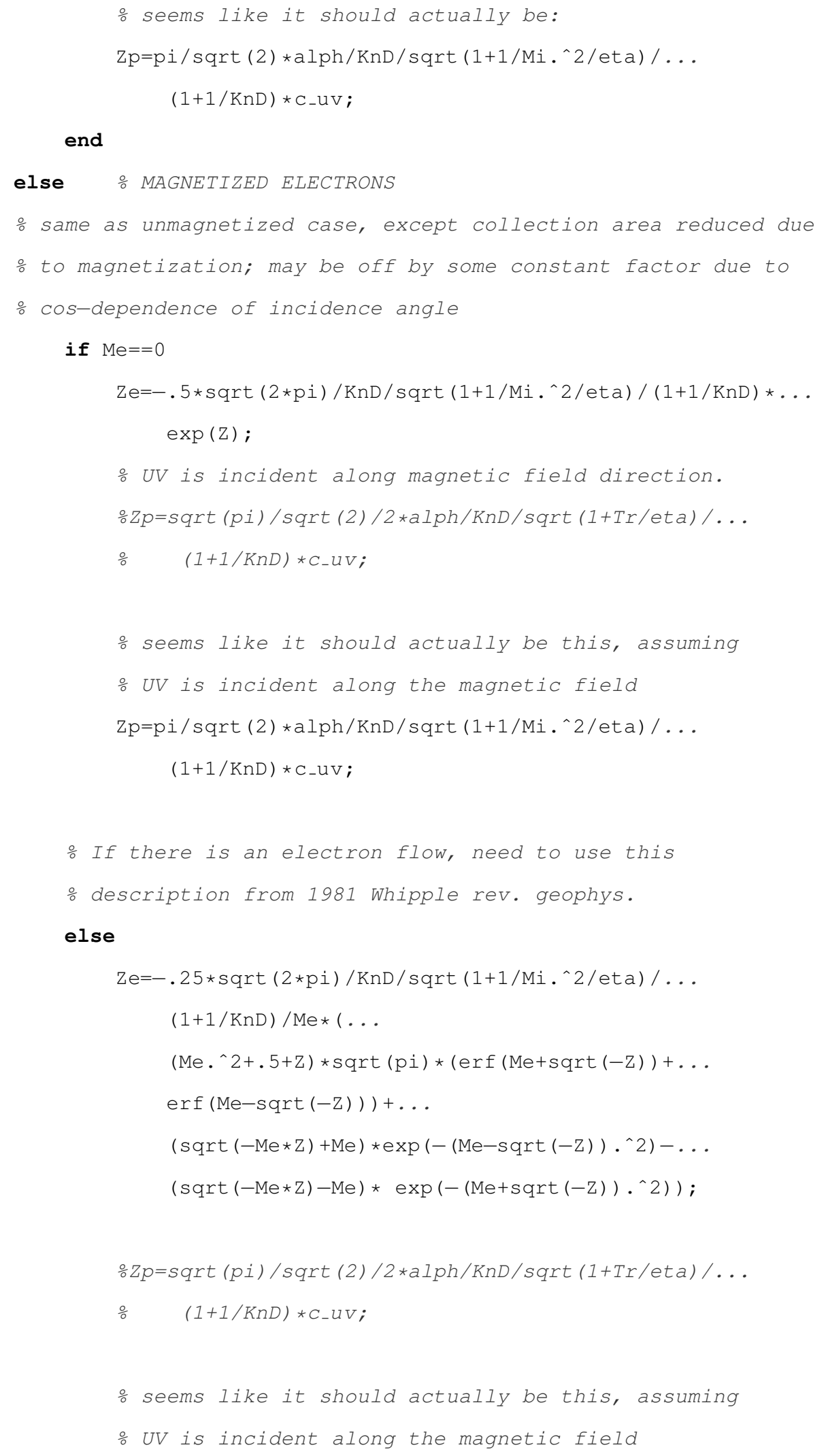


$\mathrm{Zp}=\mathrm{pi} / \operatorname{sqrt}(2) * \mathrm{alph} / \mathrm{KnD} / \operatorname{sqrt}(1+1 / \mathrm{Mi}$ •^2/eta) / . .

$$
(1+1 / \mathrm{KnD}) * \mathrm{C}-\mathrm{uv} \text {; }
$$

end

end

ㅇon current

if i_mag $_{<1}$

응 Because we have mono-energetic ions, cannot have $\mathrm{Mi}==0$

case!!

$\mathrm{Zi}=\mathrm{pi} / 2 / \mathrm{KnD} / \mathrm{sqrt}\left(1+1 / \mathrm{Mi} \cdot{ }^{\wedge} 2 /\right.$ eta $) /(1+1 / \mathrm{KnD}) / \ldots$

eta*sqrt $(m r) * M i *(1-m r * Z /(m r / 2 * M i ` 2))$;

\section{else}

o simple thermal flux of ions, assuming ballistic trajectories

along field lines w/ reduced collection area due to

o magnetization

else

Electron Current

if e_mag $<1$ 응 UNMAGNETIZED ELECTRONS $\mathrm{Ze}=-\operatorname{sqrt}(2 * \mathrm{pi}) / \mathrm{KnD} / \operatorname{sqrt}\left(1+1 / \mathrm{Mi} \cdot{ }^{\wedge} 2 / \mathrm{eta}\right) /(1+1 / \mathrm{KnD}) *(1+\mathrm{Z}) ;$

else

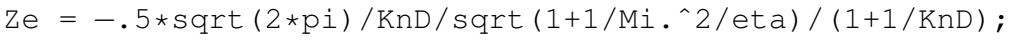

end

응 current 


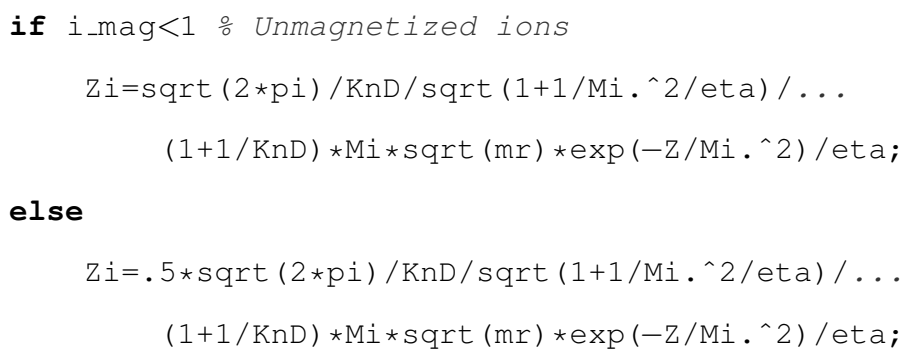




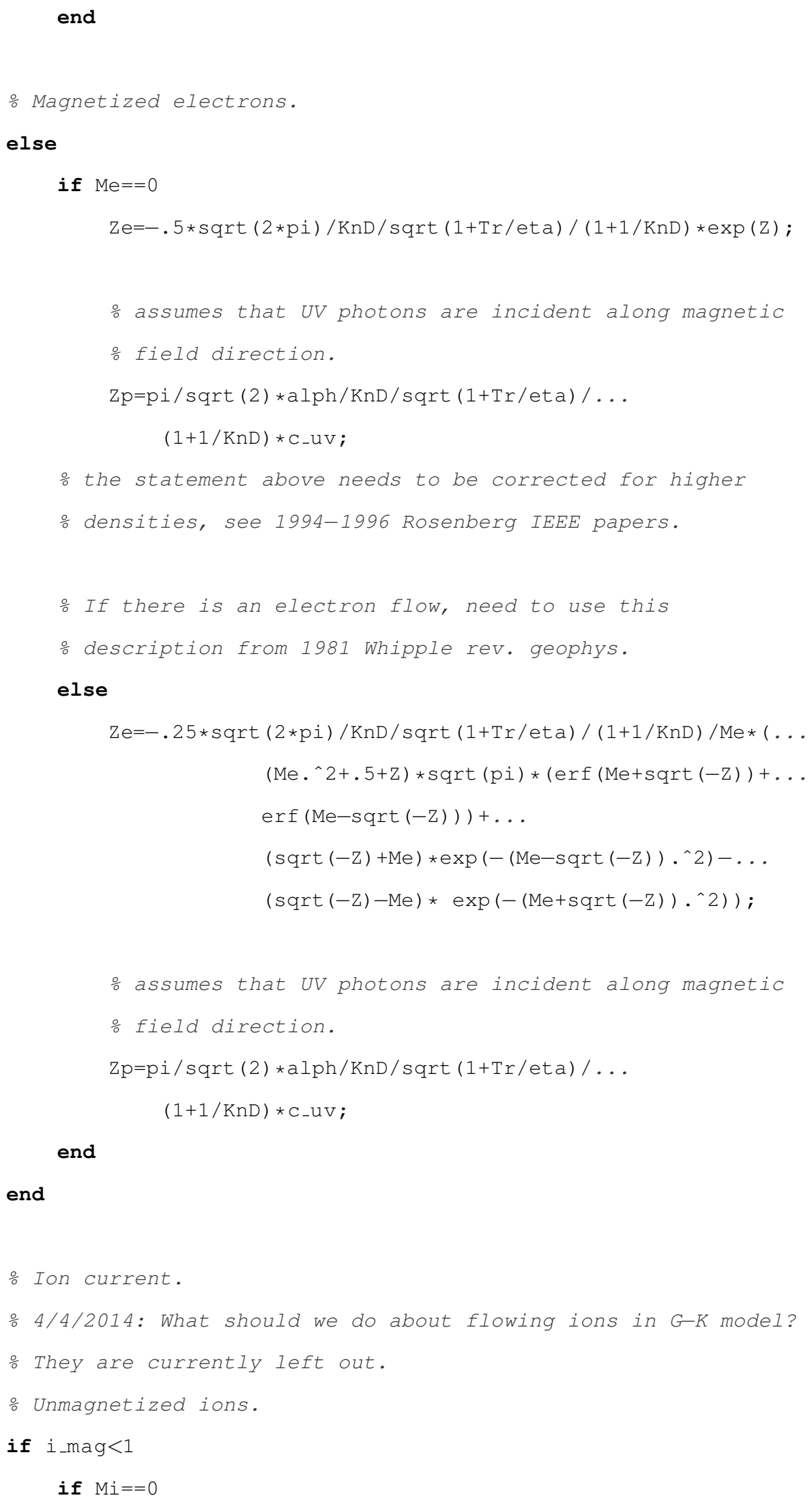


if $\mathrm{Kn}_{-} \mathrm{RO}==0$

$\mathrm{PO}=1$;

$\mathrm{P} 1=0$;

$\operatorname{Pg} 1=0$;

ioml $=\operatorname{sqrt}(2 \star \mathrm{pi}) / \mathrm{KnD} / \operatorname{sqrt}(1+\mathrm{Tr} /$ eta $) / \ldots$

$(1+1 / \mathrm{KnD}) * \operatorname{sqrt}(\mathrm{mr} / \mathrm{Tr}) *(1-\mathrm{Tr} * \mathrm{Z}) /$ eta $;$

icec=0;

ihyd=0;

else

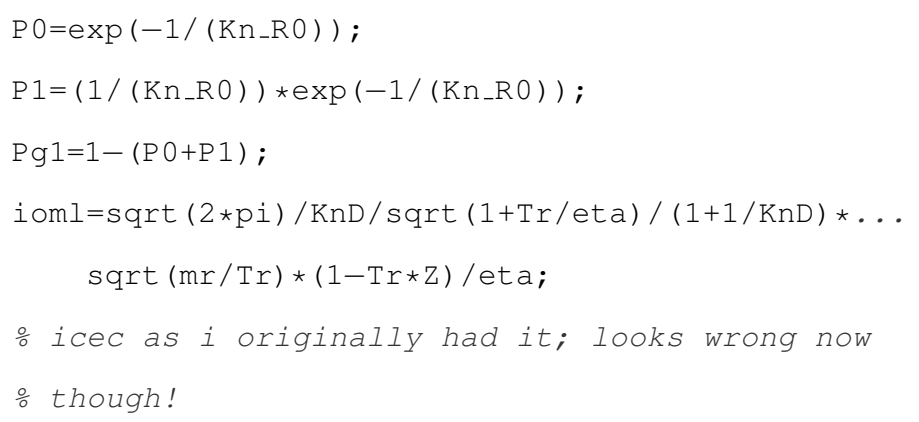


else

$$
(1+1 / \mathrm{KnD}) * \operatorname{sqrt}(\mathrm{mr} / \mathrm{Tr}) *(1-\mathrm{Tr} * \mathrm{Z}) / \text { eta; }
$$

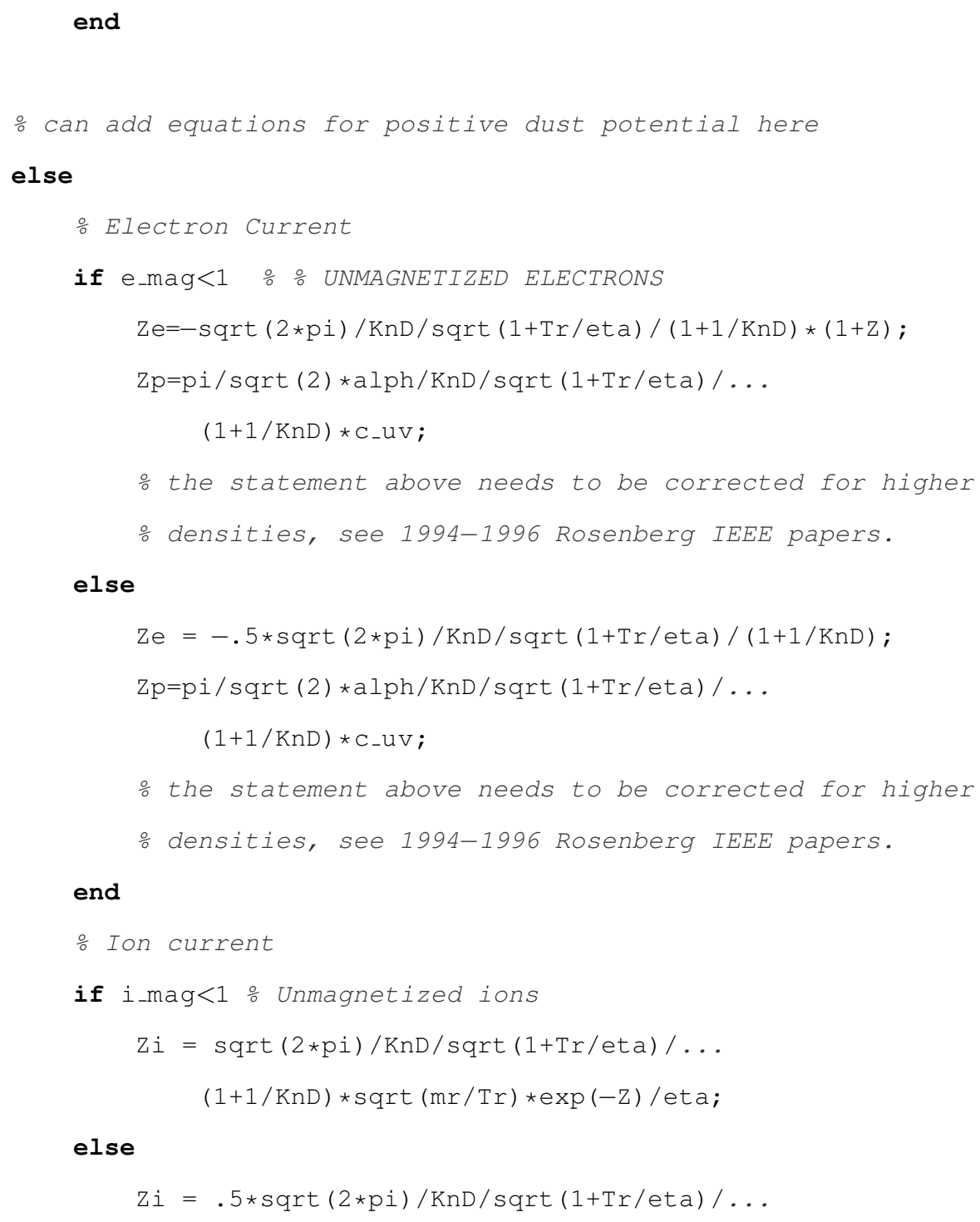




$$
(1+1 / \mathrm{KnD}) * \operatorname{sqrt}(\mathrm{mr} / \mathrm{Tr}) * \exp (-\mathrm{Z}) / \text { eta }
$$

\section{end}

o check to make sure the photocurrent is written

응 correctly!

end

$d z d t=Z i+Z e+Z p ;$

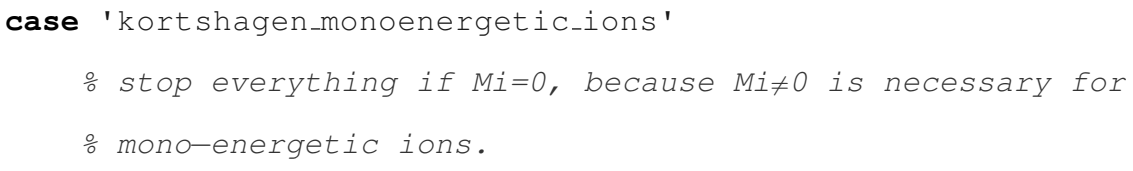


$\mathrm{Zp}=\mathrm{pi} / \operatorname{sqrt}(2) \star \mathrm{alph} / \mathrm{KnD} / \operatorname{sqrt}(1+1 / \mathrm{Mi}$ •`2/eta) / . .

$(1+1 / \mathrm{KnD}) * \mathrm{C}_{-} \mathrm{uv}$;

end

․ Magnetized electrons.

else

if $\mathrm{Me}==0$

$\mathrm{Ze}=-.5 * \operatorname{sqrt}(2 * \mathrm{pi}) / \mathrm{KnD} / \operatorname{sqrt}\left(1+1 / \mathrm{Mi} \cdot{ }^{\wedge} 2 / \mathrm{eta}\right) /(1+1 / \mathrm{KnD}) * \ldots$ $\exp (Z) ;$

- assumes that UV photons are incident along magnetic

으이 direction.

$\mathrm{Zp}=\mathrm{pi} / \operatorname{sqrt}(2) \star \mathrm{alph} / \mathrm{KnD} / \operatorname{sqrt}\left(1+1 / \mathrm{Mi} \cdot{ }^{\wedge} 2 / \mathrm{eta}\right) / \ldots$

$(1+1 / \mathrm{KnD}) * \mathrm{C}_{-} \mathrm{uv}$;

\% the statement above needs to be corrected for higher

응 densities, see 1994-1996 Rosenberg IEEE papers.

․ If there is an electron flow, need to use this

응 description from 1981 Whipple rev. geophys.

else

$\mathrm{Ze}=-.25 * \operatorname{sqrt}(2 * \mathrm{pi}) / \mathrm{KnD} / \operatorname{sqrt}(1+1 / \mathrm{Mi} \cdot \wedge 2 / \mathrm{eta}) /(1+1 / \mathrm{KnD}) \ldots$

$/ \operatorname{Me} \star\left(\left(\operatorname{Me} \cdot{ }^{\wedge} 2+.5+Z\right) * \operatorname{sqrt}(p i) \star(\operatorname{erf}(\operatorname{Me}+\operatorname{sqrt}(-Z))+\ldots\right.$

$\operatorname{erf}(\operatorname{Me}-\operatorname{sqrt}(-\mathrm{Z})))+\ldots$

$(\operatorname{sqrt}(-Z)+M e) * \exp \left(-(\operatorname{Me}-\operatorname{sqrt}(-Z)) \cdot{ }^{\wedge} 2\right)-\ldots$

$\left.(\operatorname{sqrt}(-Z)-M e) * \exp \left(-(\operatorname{Me}+\operatorname{sqrt}(-Z)) \cdot{ }^{\wedge} 2\right)\right)$;

- assumes that UV photons are incident along magnetic

드이 direction.

$\mathrm{Zp}=\mathrm{pi} / \operatorname{sqrt}(2) * \mathrm{al} \mathrm{ph} / \mathrm{KnD} / \operatorname{sqrt}\left(1+1 / \mathrm{Mi} \cdot{ }^{\wedge} 2 / \mathrm{eta}\right) / \ldots$

$(1+1 / \mathrm{KnD}) * \mathrm{C}_{-} \mathrm{uv}$;

end

end

응 current.

\% 4/4/2014: What should we do about flowing ions in G-K model? 


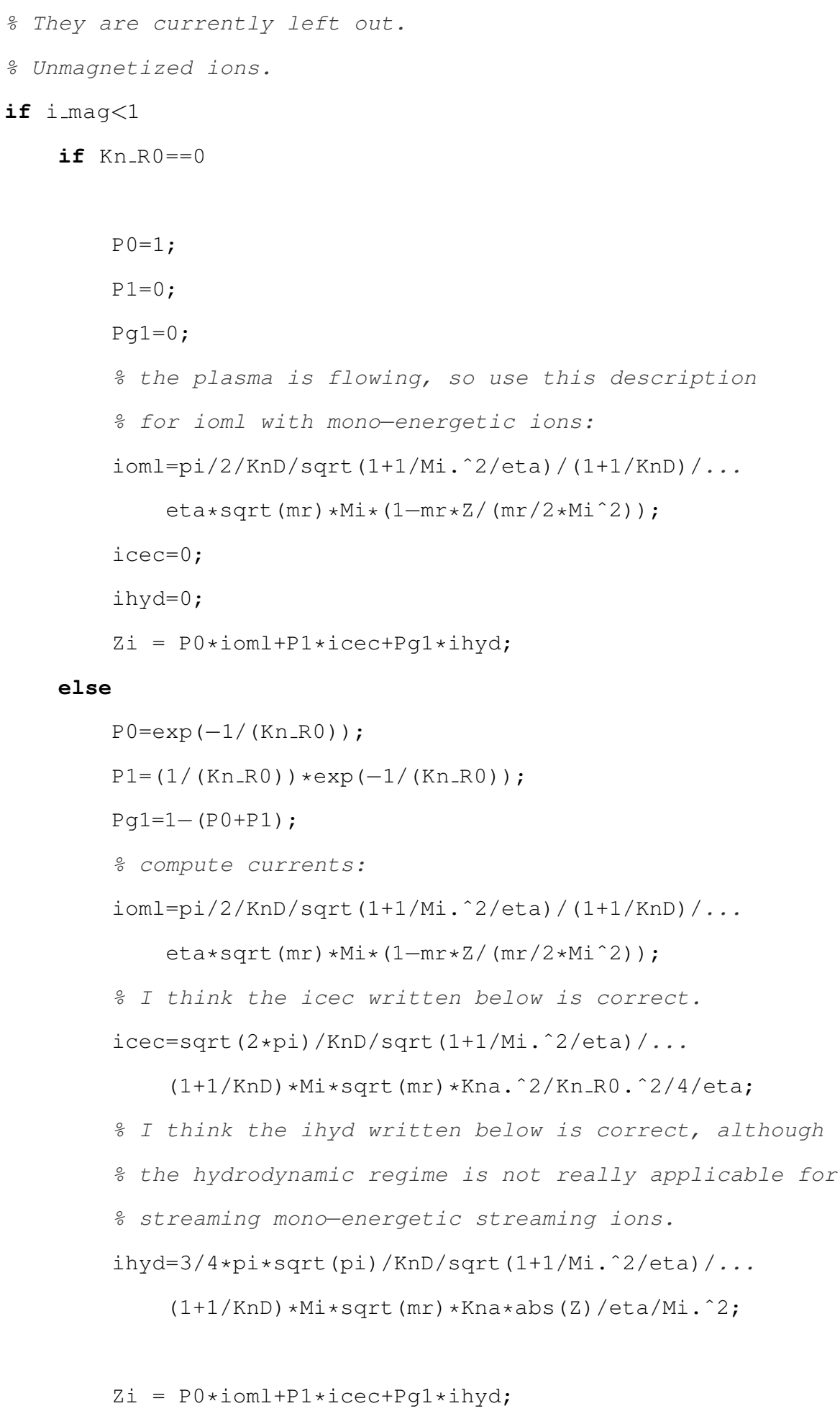


o "magnetized". Maybe make this the same as the lines above,

응 now, or try working these out.

else

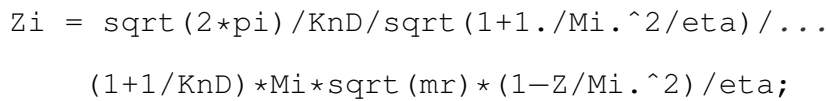

end

o can add equations for positive dust potential here

else

․ㅡㄹ Electron Current

if e_mag<1 응 UNMAGNETIZED ELECTRONS

$\mathrm{Ze}=-\operatorname{sqrt}(2 * \mathrm{pi}) / \mathrm{KnD} / \operatorname{sqrt}(1+1 / \mathrm{Mi} \cdot \hat{2} 2 / \mathrm{eta}) /(1+1 / \mathrm{KnD}) \star(1+\mathrm{Z}) ;$

$\mathrm{Zp}=\mathrm{pi} / \operatorname{sqrt}(2) \star \mathrm{alph} / \mathrm{KnD} / \operatorname{sqrt}(1+1 / \mathrm{Mi} \cdot \wedge 2 / \mathrm{eta}) / \ldots$

$(1+1 / \mathrm{KnD}) * \mathrm{C}_{-} \mathrm{uv}$;

․ the statement above needs to be corrected for higher

응 densities, see 1994-1996 Rosenberg IEEE papers.

else

$\mathrm{Ze}=-.5 * \operatorname{sqrt}(2 * \mathrm{pi}) / \mathrm{KnD} / \operatorname{sqrt}(1+1 / \mathrm{Mi} \cdot \hat{2} 2 / \mathrm{eta}) /(1+1 / \mathrm{KnD})$;

$\mathrm{Zp}=\mathrm{pi} / \operatorname{sqrt}(2) \star \mathrm{alph} / \mathrm{KnD} / \operatorname{sqrt}(1+1 / \mathrm{Mi} \cdot \wedge 2 /$ eta $) / \ldots$

$(1+1 / \mathrm{KnD}) * \mathrm{C}_{-} \mathrm{uv}$;

․ㅡㄹ the statement above needs to be corrected for higher

응 densities, see 1994-1996 Rosenberg IEEE papers.

end

응 current

if i_mag<1 을 Unagetized ions

$\mathrm{Zi}=\operatorname{sqrt}(2 \star \mathrm{pi}) / \mathrm{KnD} / \operatorname{sqrt}(1+1 / \mathrm{Mi} \cdot \wedge 2 /$ eta $) / \ldots$

$(1+1 / \mathrm{KnD}) \star \mathrm{Mi} * \operatorname{sqrt}(\operatorname{mr}) \star \exp (-\mathrm{Z}) / \mathrm{eta}$

else

$\mathrm{Zi}=.5 * \operatorname{sqrt}(2 * \mathrm{pi}) / \mathrm{KnD} / \operatorname{sqrt}(1+1 / \mathrm{Mi} . \wedge 2 / \mathrm{eta}) / \ldots$

$(1+1 / \mathrm{KnD}) \star \mathrm{Mi} * \operatorname{sqrt}(\mathrm{mr}) \star \exp (-\mathrm{z}) /$ eta;

end

- check to make sure the photocurrent is written

응 correctly!

end 
$d z d t=Z i+Z e+Z p ;$

case 'hutchinson'

if $\mathrm{Z} \leq 0$ 응 negative dust potential (phi=qd/C)

Electron Current

$z=e \_m a g /\left(1+e \_m a g\right) ;$

․ iota* in Patacchini and Hutchinson:

iota $=1-0.0946 * z-0.305 * z \cdot{ }^{\wedge} 2+0.95 * z \cdot{ }^{\wedge} 3-2.2 * z \cdot{ }^{\wedge} 4+1.15 * z \cdot{ }^{\wedge} 5$;

$\therefore$ FOR LAMBDA_D = FINITE, AND DEBYE-HUCKEL POTENTIAL:

o eta, which is now dependent on grain sheath size:

eta_mag $=-Z /$ _e_mag* $(1+$ e_mag $/ 4 *(1-\exp (-4 / \mathrm{KnD} /$ e_mag $)))$;

음 which is eta/(1+eta):

w_mag=eta_mag/(1+eta_mag) ;

$\therefore$ A, the fitting polynomial, a function of $w$ :

A_fit $=0.678 * w \_m a g+1.543 * w \_m a g \cdot{ }^{\wedge} 2-1.212 * w \_m a g \cdot{ }^{\wedge} 3$;

o Ie*, which is the empirical formula for electron current as a

․ function of magnetization

$\mathrm{Ze}=-\operatorname{sqrt}(2 \star \mathrm{pi}) / \mathrm{KnD} / \operatorname{sqrt}(1+\mathrm{Tr} / \mathrm{eta}) / \ldots$

$(1+1 / \mathrm{KnD}) * \exp (\mathrm{Z}) *\left(\mathrm{~A}_{-} \mathrm{f} i t+\left(1-\mathrm{A}_{-} \mathrm{f} i t\right) * i o t a\right) ;$

o Patacchini-Hutchinson model can include the spatial

dependence of electron flux to the sphere. I have chosen not

o to put this in at the current time, since it is not necessary

응 determining thetotal grain charge.

음 Ion current; still "binary", so it is either magnetized or

o unmagnetized; not sure if/how this has been treated by

- Patacchini and Hutchinson.

if $i_{-} \operatorname{mag}<1$

if $\mathrm{Mi}==0$

응 current, cf. Allen, Phys. Scr. 45 (1992), eq. 51

$\mathrm{Zi}=\operatorname{sqrt}(2 \star \mathrm{pi}) / \mathrm{KnD} / \operatorname{sqrt}(1+\mathrm{Tr} /$ eta $) / \ldots$

$(1+1 / \mathrm{KnD}) * \operatorname{sqrt}(\mathrm{mr} / \mathrm{Tr}) *(1-\operatorname{Tr} * \mathrm{Z}) /$ eta $;$

o Flowing ions; taken from 1981 Whipple rev. geophys. 
else

$\mathrm{Zi}=.5 * \operatorname{sqrt}(2 \star \mathrm{pi}) / \mathrm{KnD} / \operatorname{sqrt}(1+\mathrm{Tr} / \mathrm{eta}) /(1+1 / \mathrm{KnD}) \ldots$

$\star \operatorname{sqrt}(\mathrm{mr} / \mathrm{Tr}) *\left(\left(\mathrm{Mi}^{\wedge} 2+.5-\mathrm{Tr} * \mathrm{Z}\right) * \operatorname{sqrt}(\mathrm{pi}) / \mathrm{Mi} * \operatorname{erf}(\mathrm{Mi})+\ldots\right.$

$\left.\exp \left(-\mathrm{Mi}^{\wedge} 2\right)\right) /$ eta;

end

else

ㅇ simple thermal flux of ions, assuming ballistic trajectories

\% along field lines w/ reduced collection area due to

agnetization

$\mathrm{Zi}=.5 * \operatorname{sqrt}(2 * \mathrm{pi}) / \mathrm{KnD} / \operatorname{sqrt}(1+\mathrm{Tr} /$ eta $) / \ldots$

$(1+1 / \mathrm{KnD}) * \operatorname{sqrt}(\mathrm{mr} / \mathrm{Tr}) /$ eta;

응 refence for this?

end

- put photo-electron current here?

$\mathrm{Zp}=\mathrm{pi} / \operatorname{sqrt}(2) * \mathrm{al} \mathrm{ph} / \mathrm{KnD} / \operatorname{sqrt}(1+\mathrm{Tr} /$ eta $) / \ldots$

$(1+1 / \mathrm{KnD}) * \mathrm{C} \_\mathrm{Uv}$;

can add equations for positive dust potential here. How is this

o done in the Hutchinson model??

\section{else}

․ Electron Current

if e_mag $<1$ 음 UNMAGNETIZED ELECTRONS

$\mathrm{Ze}=-\operatorname{sqrt}(2 \star \mathrm{pi}) / \mathrm{KnD} / \operatorname{sqrt}(1+\mathrm{Tr} / \mathrm{eta}) /(1+1 / \mathrm{KnD}) *(1+\mathrm{Z}) ;$

else

$\mathrm{Ze}=-.5 * \operatorname{sqrt}(2 * \mathrm{pi}) / \mathrm{KnD} / \operatorname{sqrt}(1+\mathrm{Tr} / \mathrm{eta}) /(1+1 / \mathrm{KnD}) ;$

end

Ion current

if i_mag $<1$ 는 Unagnetized ions

$\mathrm{Zi}=\operatorname{sqrt}(2 \star \mathrm{pi}) / \mathrm{KnD} / \operatorname{sqrt}(1+\mathrm{Tr} /$ eta $) / \ldots$

$(1+1 / \mathrm{KnD}) * \operatorname{sqrt}(\mathrm{mr} / \mathrm{Tr}) * \exp (-\operatorname{Tr} * \mathrm{Z}) /$ eta $;$

else

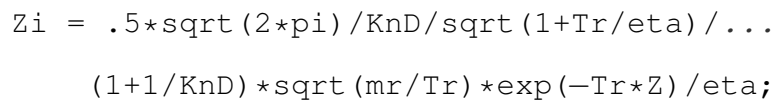

end

- check to make sure the photocurrent is written 
correctly! Might be different in $P-H$ model.
$\mathrm{Zp}=\mathrm{pi} / \mathrm{sqrt}(2) \star \mathrm{alph} / \mathrm{KnD} / \mathrm{sqrt}(1+\mathrm{Tr} / \mathrm{eta}) /(1+1 / \mathrm{KnD}) \star \mathrm{C}-\mathrm{uv} ;$
$\therefore$ Whipple 1981, reviews of geophysics; $1995 \mathrm{Cui}$ and Goree IEEE

end

$d z d t=z i+Z e+Z p ;$

case 'hutchinson_monoenergetic_ions'

stop everything if $M i=0$, because $M i \neq 0$ is necessary for

․ mono-energetic ions.

if $\mathrm{Mi}==0$

exception='Mi cannot be zero!';

error(exception);

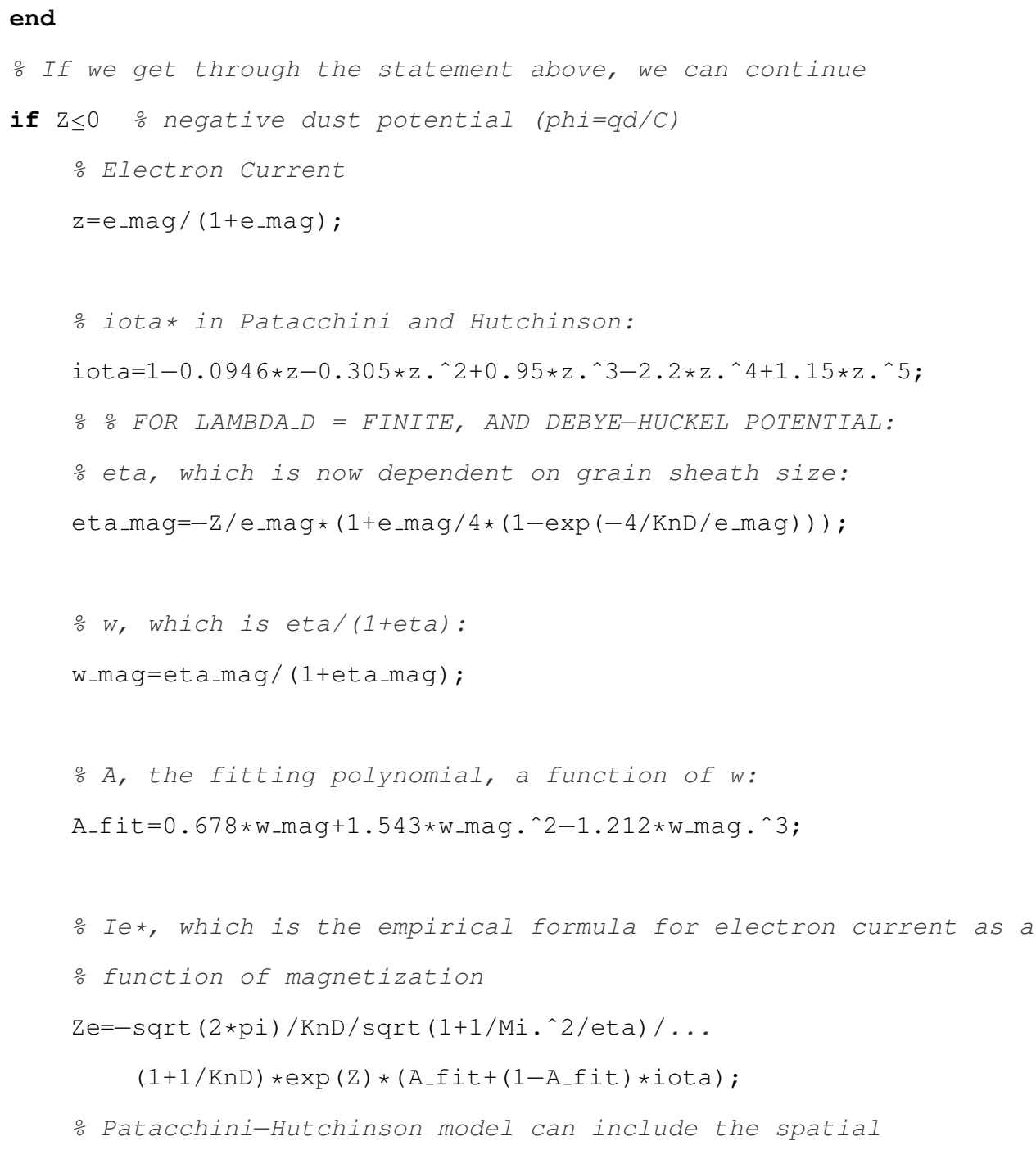


dependence of electron flux to the sphere. I have chosen not

- to put this in at the current time, since it is not necessary

응 determining thetotal grain charge.

o Ion current; still "binary", so it is either magnetized or

․ unmagnetized; not sure if/how this has been treated by

․ Patacchini and Hutchinson.

if i_mag $<1$

$\mathrm{Zi}=\mathrm{pi} / 2 / \mathrm{KnD} / \mathrm{sqrt}\left(1+1 / \mathrm{Mi} \cdot{ }^{\wedge} 2 / \mathrm{eta}\right) /(1+1 / \mathrm{KnD}) / \ldots$

eta $* \operatorname{sqrt}(m r) \star M i *(1-m r * Z /(m r / 2 * M i \wedge 2))$;

else

o simple thermal flux of ions, assuming ballistic trajectories

along field lines w/ reduced collection area due to

․ magnetization

reference for this?

$\mathrm{Zi}=\mathrm{pi} / 2 / \mathrm{KnD} / \operatorname{sqrt}(1+1 / \mathrm{Mi}$ •^2/eta)/(1+1/KnD)/eta*sqrt $(\mathrm{mr}) \star \mathrm{Mi} ;$

end

o put photo-electron current here?

$\mathrm{Zp}=\mathrm{pi} / \operatorname{sqrt}(2) \star \mathrm{alph} / \mathrm{KnD} / \operatorname{sqrt}\left(1+1 / \mathrm{Mi} \cdot{ }^{\wedge} 2 /\right.$ eta $) / \ldots$

$(1+1 / \mathrm{KnD}) * \mathrm{C}_{-} \mathrm{uv}$;

- can add equations for positive dust potential here. How is this

o done in the Hutchinson model??

else

․ Electron Current

if e_mag $<1$ 응 UNMAGNETIZED ELECTRONS

$\mathrm{Ze}=-\operatorname{sqrt}(2 * \mathrm{pi}) / \mathrm{KnD} / \operatorname{sqrt}\left(1+1 / \mathrm{Mi} \cdot{ }^{\wedge} 2 / \mathrm{eta}\right) /(1+1 / \mathrm{KnD}) *(1+\mathrm{Z}) ;$

else

$\mathrm{Ze}=-.5 * \operatorname{sqrt}(2 * \mathrm{pi}) / \mathrm{KnD} / \operatorname{sqrt}\left(1+1 / \mathrm{Mi} \cdot{ }^{\wedge} 2 / \mathrm{eta}\right) /(1+1 / \mathrm{KnD}) ;$

end

응 Ion current

if i_mag $<1$ 을 Unagnetized ions

$\mathrm{Zi}=\operatorname{sqrt}(2 \star \mathrm{pi}) / \mathrm{KnD} / \operatorname{sqrt}\left(1+1 / \mathrm{Mi} \cdot{ }^{\wedge} 2 / \mathrm{eta}\right) / \ldots$ 


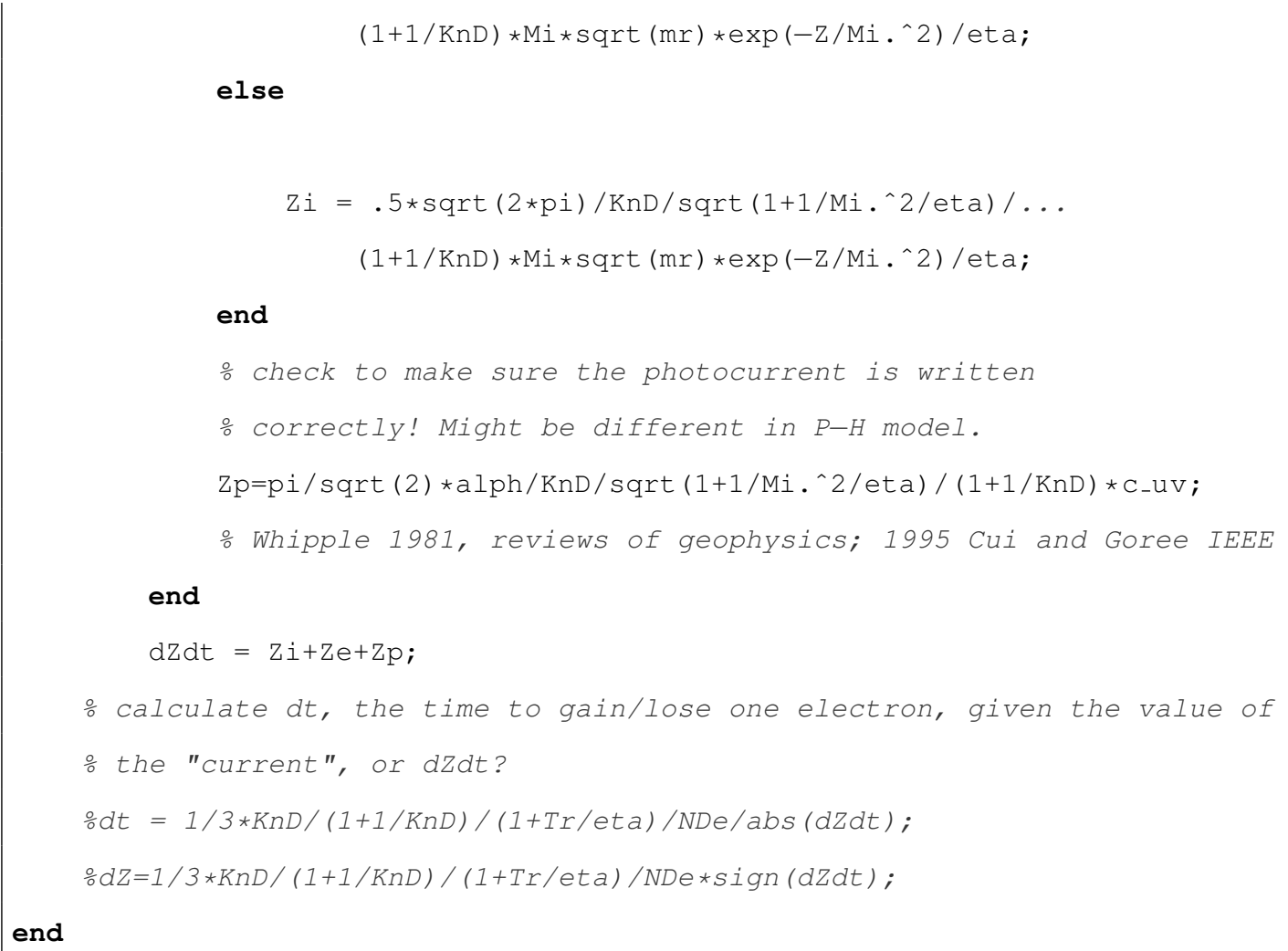




\section{References}

() .

Allen, J. E. (1992), Probe theory - the orbital motion approach, Physica Scripta, 45(5), 497.

Allen, J. E., R. L. F. Boyd, and P. Reynolds (1957), The collection of positive ions by a probe immersed in a plasma, Proceedings of the Physical Society. Section B, 70(3), 297.

Amatucci, W. E., D. N. Walker, G. Gatling, and E. E. Scime (2004), Direct observation of microparticle gyromotion in a magnetized direct current glow discharge dusty plasma, Physics of Plasmas, $11(5)$, 2097-2105, doi:10.1063/1.1669396.

Arnas, C., M. Mikikian, and F. Doveil (1999), High negative charge of a dust particle in a hot cathode discharge, Phys. Rev. E, 60, 7420-7425, doi:10.1103/PhysRevE.60.7420.

Bacharis, M., M. Coppins, and J. E. Allen (2010), Critical issues for modeling dust transport in tokamaks, Phys. Rev. E, 82, 026,403, doi:10.1103/PhysRevE.82.026403.

Barkan, A., N. D'Angelo, and R. L. Merlino (1994), Charging of dust grains in a plasma, Phys. Rev. Lett., 73, 3093-3096, doi:10.1103/PhysRevLett.73.3093.

Barnes, M. S., J. H. Keller, J. C. Forster, J. A. O’Neill, and D. K. Coultas (1992), Transport of dust particles in glow-discharge plasmas, Phys. Rev. Lett., 68, 313-316, doi:10.1103/PhysRevLett.68. 313.

Basner, R., F. Sigeneger, D. Loffhagen, G. Schubert, H. Fehske, and H. Kersten (2009), Particles as probes for complex plasmas in front of biased surfaces, New Journal of Physics, 11 (1), 013,041. 


\section{REFERENCES}

Berkowitz, J., and C. S. Gardner (1959), On the asymptotic series expansion of the motion of a charged particle in slowly varying fields, Communications on Pure and Applied Mathematics, 12(3), 501-512, doi:10.1002/cpa.3160120307.

Bernstein, I. B., and I. N. Rabinowitz (1959), Theory of electrostatic probes in a low-density plasma, Physics of Fluids, 2(2), 112-121, doi:10.1063/1.1705900.

Birdsall, C. K., and A. B. Langdon (2005), Plasma physics via computer simulation, Taylor and Francis, New York.

Bliokh, P., V. Sinitsin, and V. Yaroshenko (1994), Dusty and Self-Gravitational Plasmas in Space, Kluwer Academic Publishers, Dordrecht, The Netherlands.

Boessé, C., M. Henry, T. Hyde, and L. Matthews (2004), Digital imaging and analysis of dusty plasmas, Advances in Space Research, 34(11), 2374 - 2378, doi:http://dx.doi.org/10.1016/j.asr.2003. 02.069, scientific Exploration, Planetary Protection, Active Experiments and Dusty Plasmas.

Bonitz, M., A. Filinov, J. Böning, and J. W. Dufty (2010), Introduction to quantum plasmas, Springer.

Bonitz, M., H. Khlert, T. Ott, and H. Lwen (2013), Magnetized strongly coupled plasmas and how to realize them in a dusty plasma setup, Plasma Sources Science and Technology, 22(1), 015,007.

Boris, J. P. (1970), Relativistic plasma simulation-optimization of a hybrid code, Proceeding of Fourth Conference on Numerical Simulations of Plasmas.

Boulanger, F., P. Cox, and A. Jones (2000), Dust in the interstellar medium, in Astronomie spatiale infrarouge, aujourdhui et demain Infrared space astronomy, today and tomorrow, Les Houches Ecole dEte de Physique Theorique, vol. 70, edited by F. Casoli, J. Lequeux, and F. David, pp. 251-335, Springer Berlin Heidelberg, doi:10.1007/3-540-45573-6_7.

Boyd, R. L. F. (1951), The mechanism of positive ion collection by a spherical probe in a dense gas, Proceedings of the Physical Society. Section B, 64(9), 795.

Bryant, D. A. (1996), Debye length in a kappa-distribution plasma, Journal of Plasma Physics, 56, 87-93, doi:10.1017/S0022377800019115. 


\section{REFERENCES}

Carroll, I., J. J., M. Koepke, W. Amatucci, T. Sheridan, and M. Alport (1994), A segmented disk electrode to produce and control parallel and transverse particle drifts in a cylindrical plasma, Review of Scientific Instruments, 65(9), 2991-2995, doi:10.1063/1.1144590.

Carstensen, J., F. Greiner, and A. Piel (2012), Ion-wake-mediated particle interaction in a magnetized-plasma flow, Phys. Rev. Lett., 109, 135,001, doi:10.1103/PhysRevLett.109.135001.

Castle, G. (1997), Contact charging between insulators, Journal of Electrostatics, 4041(0), 13 20, doi:http://dx.doi.org/10.1016/S0304-3886(97)00009-0, proceedings of the 8th International Conference on Electrostatics.

Chabert, P., and N. Braithwaite (2011), Physics of Radio-Frequency Plasmas, 1st ed., Cambridge University Press, The Edinburgh Building, Cambridge CB2 8RU, UK.

Chang, J., and J. G. Laframboise (1976), Probe theory for arbitrary shape in a large debye length, stationary plasma, Physics of Fluids (1958-1988), 19(1).

Chen, F. (2006), Introduction to Plasma Physics and Controlled Fusion, 2nd ed., Springer, 233 Spring Street, New York, NY 10013, USA.

Child, C. D. (1911), Discharge from hot cao, Phys. Rev. (Series I), 32, 492-511, doi:10.1103/ PhysRevSeriesI.32.492.

Choi, S. J., and M. Kushner (1994), A particle-in-cell simulation of dust charging and shielding in low pressure glow discharges, Plasma Science, IEEE Transactions on, 22(2), 138-150, doi: 10.1109/27.279017.

Colwell, J. E., A. A. Gulbis, M. Hornyi, and S. Robertson (2005), Dust transport in photoelectron layers and the formation of dust ponds on eros, Icarus, 175(1), 159 - 169, doi:http://dx.doi.org/ 10.1016/j.icarus.2004.11.001.

Cui, C., and J. Goree (1994), Fluctuations of the charge on a dust grain in a plasma, Plasma Science, IEEE Transactions on, 22(2), 151 -158, doi:10.1109/27.279018.

Daugherty, J. E., R. K. Porteous, M. D. Kilgore, and D. B. Graves (1992), Sheath structure 


\section{REFERENCES}

around particles in low-pressure discharges, Journal of Applied Physics, 72(9), 3934-3942, doi: $10.1063 / 1.352245$.

Debye, P., and E. Huckel (1923), The interionic attraction theory of deviations from ideal behavior in solution, Z. Phys, 24, 185.

Delzanno, G. L., G. Lapenta, and M. Rosenberg (2004), Attractive potential around a thermionically emitting microparticle, Phys. Rev. Lett., 92, 035,002, doi:10.1103/PhysRevLett.92.035002.

Delzanno, G. L., A. Bruno, G. Sorasio, and G. Lapenta (2005), Exact orbital motion theory of the shielding potential around an emitting, spherical body, Physics of Plasmas, 12(6), 062102, doi:10.1063/1.1914546.

Demidov, V. I., S. V. Ratynskaia, and K. Rypdal (2002), Electric probes for plasmas: The link between theory and instrument, Review of Scientific Instruments, 73(10), 3409-3439, doi:10. $1063 / 1.1505099$.

Desch, S. J., and J. N. Cuzzi (), The Generation of Lightning in the Solar Nebula.

Dove, A., S. Robertson, M. Horanyi, A. Poppe, and X. Wang (2011), Operation of a langmuir probe in a photoelectron plasma, AIP Conference Proceedings, 1397(1), 387-388, doi:10.1063/1. 3659856.

Dove, A., M. Horanyi, X. Wang, M. Piquette, A. R. Poppe, and S. Robertson (2012), Experimental study of a photoelectron sheath, Physics of Plasmas, 19(4), 043502, doi:10.1063/1.3700170.

Draine, B., and B. Sutin (1987), Collisional charging of interstellar grains, The Astrophysical Journal, 320, 803-817.

Eastman, D. (1971), Photoemission studies of scandium, titanium, and zirconium, Electronic Density of States, p. 205.

Epstein, P. S. (1924), On the resistance experienced by spheres in their motion through gases, Physical Review, 23(6), 710. 


\section{REFERENCES}

Farrell, W. M., J. R. Marshall, S. A. Cummer, G. T. Delory, and M. D. Desch (2006), A model of the ulf magnetic and electric field generated from a dust devil, Journal of Geophysical Research: Planets, 111 (E11), n/a-n/a, doi:10.1029/2006JE002689.

Farrell, W. M., W. S. Kurth, D. A. Gurnett, R. E. Johnson, M. L. Kaiser, J.-E. Wahlund, and J. H. Waite (2009), Electron density dropout near enceladus in the context of water-vapor and water-ice, Geophysical Research Letters, 36(10), n/a-n/a, doi:10.1029/2008GL037108.

Farrell, W. M., et al. (2004), Electric and magnetic signatures of dust devils from the 20002001 matador desert tests, Journal of Geophysical Research: Planets, 109 (E3), n/a-n/a, doi:10.1029/ 2003JE002088.

Feng, Y., J. Goree, and B. Liu (2007), Accurate particle position measurement from images, Review of Scientific Instruments, 78(5), 053704, doi:http://dx.doi.org/10.1063/1.2735920.

Feng, Y., J. Goree, and B. Liu (2011), Errors in particle tracking velocimetry with high-speed cameras, Review of Scientific Instruments, 82(5), 053707, doi:http://dx.doi.org/10.1063/1.3589267.

Ferrière, K. M. (2001), The interstellar environment of our galaxy, Rev. Mod. Phys., 73, 1031-1066, doi:10.1103/RevModPhys.73.1031.

Feuerbacher, B., and B. Fitton (1972), Experimental investigation of photoemission from satellite surface materials, Journal of Applied Physics, 43(4).

Filippov, A., N. Dyatko, A. Pal, and A. Starostin (2003), Development of a self-consistent model of dust grain charging at elevated pressures using the method of moments, Plasma Physics Reports, 29(3), 190-202, doi:10.1134/1.1561112.

Fortov, V., A. Ivlev, S. Khrapak, A. Khrapak, and G. Morfill (2005), Complex (dusty) plasmas: Current status, open issues, perspectives, Physics Reports, 421(12), 1 - 103, doi:http://dx.doi. org/10.1016/j.physrep.2005.08.007.

Fortov, V. E., A. P. Nefedov, V. I. Molotkov, M. Y. Poustylnik, and V. M. Torchinsky (2001), Dependence of the dust-particle charge on its size in a glow-discharge plasma, Phys. Rev. Lett., 87, 205,002, doi:10.1103/PhysRevLett.87.205002. 


\section{REFERENCES}

Friedel, B., and S. Greulich-Weber (2006), Preparation of monodisperse, submicrometer carbon spheres by pyrolysis of melamineformaldehyde resin, Small, 2(7), 859-863, doi:10.1002/smll. 200500516.

Ganguli, G. (1995), Interrelationship of Local and Global Physics in the Low Altitude Ionosphere, pp. 23-36, American Geophysical Union, doi:10.1029/GM093p0023.

Gatti, M., and U. Kortshagen (2008), Analytical model of particle charging in plasmas over a wide range of collisionality, Phys. Rev. E, 78, 046,402, doi:10.1103/PhysRevE.78.046402.

Gavrishchaka, V. (1996), Collective phenomena in a magnetized plasma with a field-aligned drift and inhomogeneous transverse flow, Ph.D. thesis, West Virginia University.

Godyak, V. A., and V. I. Demidov (2011), Probe measurements of electron-energy distributions in plasmas: what can we measure and how can we achieve reliable results?, Journal of Physics D: Applied Physics, $44(23), 233,001$.

Goertz, C. K. (1989), Dusty plasmas in the solar system, Reviews of Geophysics, 27(2), 271-292, doi:10.1029/RG027i002p00271.

Goree, J. (1992), Ion trapping by a charged dust grain in a plasma, Phys. Rev. Lett., 69, 277-280, doi:10.1103/PhysRevLett.69.277.

Goree, J. (1994), Charging of particles in a plasma, Plasma Sources Science and Technology, 3(3), 400.

Graves, D., and M. Kushner (2008), Low temperature plasma science: not only the fourth state of matter but all of them, in Report of the Department of Energy Office of Fusion Sciences Workshop Low Temperature Plasmas.

Guernsey, R. L., and J. H. M. Fu (1970), Potential distribution surrounding a photo-emitting, plate in a dilute plasma, Journal of Geophysical Research, 75(16), 3193-3199, doi:10.1029/ JA075i016p03193.

Gunn, J. P. (2012), Evidence for strong secondary electron emission in the tokamak scrape-off layer, Plasma Physics and Controlled Fusion, 54(8), 085,007. 


\section{REFERENCES}

Gurnett, D. A., and A. Bhattacharjee (2005), Introduction to Plasma Physics, 1st ed., Cambridge University Press, The Edinburgh Building, Cambridge, CB2 2RU, UK.

Hinteregger, H. E. (1961), Unpulished results, 1961.

Horányi, M. (1996), Charged dust dynamics in the solar system, Annual Review of Astronomy and Astrophysics, 34(1), 383-418, doi:10.1146/annurev.astro.34.1.383.

Hutchinson, I. H. (2002), Ion collection by a sphere in a flowing plasma: I. quasineutral, Plasma Physics and Controlled Fusion, 44(9), 1953.

Hutchinson, I. H. (2006), Collisionless ion drag force on a spherical grain, Plasma Physics and Controlled Fusion, 48(2), 185.

Ikezi, H. (1986), Coulomb solid of small particles in plasmas, Physics of Fluids (1958-1988), 29(6).

Intrator, T., M. H. Cho, E. Y. Wang, N. Hershkowitz, D. Diebold, and J. DeKock (1988), The virtual cathode as a transient double sheath, Journal of Applied Physics, 64(6).

Jana, M. R., A. Sen, and P. K. Kaw (1993), Collective effects due to charge-fluctuation dynamics in a dusty plasma, Phys. Rev. E, 48, 3930-3933, doi:10.1103/PhysRevE.48.3930.

Jefferson, R. A., M. Cianciosa, and E. Thomas (2010), Simulations of one- and two-dimensional complex plasmas using a modular, object-oriented code, Physics of Plasmas (1994-present), 17(11), 113704, doi:http://dx.doi.org/10.1063/1.3501025.

Kählert, H., J. Carstensen, M. Bonitz, H. Löwen, F. Greiner, and A. Piel (2012), Magnetizing a complex plasma without a magnetic field, Phys. Rev. Lett., 109, 155,003, doi:10.1103/PhysRevLett. 109.155003

Kanal, M. (1962), Theory of current collection of moving spherical probe, 1962.

Kanal, M. (1964), Theory of current collection of moving cylindrical probes, Journal of Applied Physics, 35(6), 1697-1703, doi:10.1063/1.1713722.

Kennedy, R. V., and J. E. Allen (2003), The floating potential of spherical probes and dust grains. ii: Orbital motion theory, Journal of Plasma Physics, 69(06), 485-506, doi:10.1017/ S0022377803002265. 


\section{REFERENCES}

Kersten, H., et al. (2003), Examples for application and diagnostics in plasmapowder interaction, New Journal of Physics, 5(1), 93.

Khrapak, S. A., et al. (2005), Particle charge in the bulk of gas discharges, Phys. Rev. E, 72, 016,406, doi:10.1103/PhysRevE.72.016406.

Khurana, K. K., C. Russell, and M. Dougherty (2008), Magnetic portraits of tethys and rhea, Icarus, 193(2), 465-474.

Kiel, R. E. (1969), Continuum electrostatic probe theory for large sheaths on spheres and cylinders, Journal of Applied Physics, 40(9).

Knist, S., F. Greiner, F. Biss, and A. Piel (2011), Influence of negative ions on drift waves in a low-density ar/o2-plasma, Contributions to Plasma Physics, 51(8), 769-784, doi:10.1002/ctpp. 201000109.

Koepke, M. E., S. M. Finnegan, S. Vincena, D. J. Knudsen, and C. Chaston (2008), Integrated campaign to study the stationary inertial alfvn wave in the laboratory and space regimes, Plasma Physics and Controlled Fusion, 50(7), 074,004.

Koepke, M. E., J. J. Walker, M. I. Zimmerman, W. M. Farrell, and V. I. Demidov (2013), Signature of gyro-phase drift, Journal of Plasma Physics, 79, 1099-1105, doi:10.1017/S0022377813001128.

Konopka, U., D. Samsonov, A. V. Ivlev, J. Goree, V. Steinberg, and G. E. Morfill (2000), Rigid and differential plasma crystal rotation induced by magnetic fields, Phys. Rev. E, 61, 1890-1898, doi:10.1103/PhysRevE.61.1890.

Konopka, U., M. Schwabe, C. Knapek, M. Kretschmer, and G. E. Morfill (2005a), Complex plasmas in strong magnetic field environments, AIP Conference Proceedings, 799(1), 181-184, doi:10. $1063 / 1.2134595$.

Konopka, U., et al. (2005b), Charge-induced gelation of microparticles, New Journal of Physics, $7(1), 227$.

Kruskal, M. (1959), The Gyration of a Charged Particle, Tech. rep., Princeton University. 


\section{REFERENCES}

Laframboise, J. G. (1966), Theory of spherical and cylindrical langmuir probes in a collisionless, maxwellian plasma at rest, Ph.D. thesis, University of Toronto Institute for Aerospace Studies.

Laframboise, J. G., and L. W. Parker (1973), Probe design for orbitlimited current collection, Physics of Fluids (1958-1988), 16(5).

Lampe, M., V. Gavrishchaka, G. Ganguli, and G. Joyce (2001), Effect of trapped ions on shielding of a charged spherical object in a plasma, Phys. Rev. Lett., 86, 5278-5281, doi: 10.1103/PhysRevLett.86.5278.

Lampe, M., R. Goswami, Z. Sternovsky, S. Robertson, V. Gavrishchaka, G. Ganguli, and G. Joyce (2003), Trapped ion effect on shielding, current flow, and charging of a small object in a plasma, Physics of Plasmas, 10(5), 1500-1513, doi:10.1063/1.1562163.

Lin, S. F., D. T. Pierce, and W. E. Spicer (1971), Photoemission studies of platinum, Phys. Rev. B, 4, 326-329, doi:10.1103/PhysRevB.4.326.

Liu, B., J. Goree, V. Nosenko, and L. Boufendi (2003), Radiation pressure and gas drag forces on a melamine-formaldehyde microsphere in a dusty plasma, Physics of Plasmas (1994-present), $10(1)$

Luginsland, J. W., Y. Y. Lau, and R. M. Gilgenbach (1996), Two-dimensional child-langmuir law, Phys. Rev. Lett., 77, 4668-4670, doi:10.1103/PhysRevLett.77.4668.

Mansfield, D., A. Roquemore, H. Schneider, J. Timberlake, H. Kugel, and M. Bell (2010), A simple apparatus for the injection of lithium aerosol into the scrape-off layer of fusion research devices, Fusion Engineering and Design, 85(6), 890 - 895, doi:http://dx.doi.org/10.1016/j.fusengdes. 2010.08.033, proceedings of the 1st International Workshop on Lithium Applications for the Boundary Control in Fusion Devices.

Martin, J. D., M. Bacharis, M. Coppins, G. F. Counsell, and J. E. Allen (2008), Modelling dust transport in tokamaks, EPL (Europhysics Letters), 83(6), 65,001.

Meige, A., O. Sutherland, H. B. Smith, and R. W. Boswell (2007), Ion heating in the presheath, Physics of Plasmas (1994-present), 14(3), 032104, doi:http://dx.doi.org/10.1063/1.2709648. 


\section{REFERENCES}

Merlino, R. L. (2006), Dusty plasmas and applications in space and industry, Plasma Physics Applied, 81, 73-110.

Morfill, G., E. Grün, and T. Johnson (1980), Dust in jupiter's magnetosphere: Physical processes, Planetary and Space Science, 28(12), 1087 - 1100, doi:http://dx.doi.org/10.1016/0032-0633(80) 90067-7.

Mott-Smith, H. M., and I. Langmuir (1926), The theory of collectors in gaseous discharges, Phys. Rev., 28, 727-763, doi:10.1103/PhysRev.28.727.

Nagy, A. F., L. H. Brace, G. R. Carignan, and M. Kanal (1963), Direct measurements bearing on the extent of thermal nonequilibrium in the ionosphere, Journal of Geophysical Research, 68(24), 6401-6412, doi:10.1029/JZ068i024p06401.

Northrop, T., and G. Morfill (1984), Dust in magnetised plasmas: Basic theory and some applications, Advances in Space Research, 4(9), 63 - 73, doi:http://dx.doi.org/10.1016/0273-1177(84) 90009-7.

Northrop, T., D. Mendis, and L. Schaffer (1989), Gyrophase drifts and the orbital evolution of dust at jupiter's gossamer ring, Icarus, 79(1), 101 - 115, doi:http://dx.doi.org/10.1016/0019-1035(89) 90110-3.

Northrop, T. G. (1961), The guiding center approximation to charged particle motion, Annals of Physics, 15(1), 79 - 101, doi:http://dx.doi.org/10.1016/0003-4916(61)90167-1.

Northrop, T. G. (1963), Adiabatic charged-particle motion, Reviews of Geophysics, 1(3), 283-304.

Northrop, T. G. (1992), Dusty plasmas, Physica Scripta, 45(5), 475.

Northrop, T. G., and T. J. Birmingham (1996), Equilibrium electric potential of spherical, cylindrical, and planar dust grains moving through a plasma, Journal of Geophysical Research: Space Physics, 101 (A5), 10,793-10,796, doi:10.1029/96JA00459.

Northrop, T. G., and J. R. Hill (1983), The adiabatic motion of charged dust grains in rotating magnetospheres, Journal of Geophysical Research: Space Physics, 88(A1), 1-11, doi:10.1029/ JA088iA01p00001. 


\section{REFERENCES}

Northrop, T. G., and J. A. Rome (1978), Extensions of guiding center motion to higher order, Physics of Fluids (1958-1988), $21(3)$.

Nunomura, S., N. Ohno, and S. Takamura (1997), Effects of ion flow by $\mathbf{E} \times \mathbf{B}$ drift on dust particle behavior in magnetized cylindrical electron cyclotron resonance plasmas, Japanese Journal of Applied Physics, 36(Part 1, No. 2), 877-883, doi:10.1143/JJAP.36.877.

Nunomura, S., T. Misawa, N. Ohno, and S. Takamura (1999), Instability of dust particles in a coulomb crystal due to delayed charging, Phys. Rev. Lett., 83, 1970-1973, doi:10.1103/ PhysRevLett.83.1970.

Paeva, G. V. (2005), Sheath phenomena in dusty plasmas, Ph.D. thesis, Eindhoven University of Technology, The Netherlands.

Parker, E. N. (1958), Interaction of the solar wind with the geomagnetic field, Physics of Fluids (1958-1988), 1(3).

Patacchini, L. (2007), Collisionless ion collection by a sphere in a weakly magnetized plasma, Ph.D. thesis, Massachusetts Institute of Technology Department. of Nuclear Science and Engineering.

Patacchini, L., I. H. Hutchinson, and G. Lapenta (2007), Electron collection by a negatively charged sphere in a collisionless magnetoplasma, Physics of Plasmas (1994-present), 14(6), 062111, doi: http://dx.doi.org/10.1063/1.2741249.

Pigarov, A. Y., S. I. Krasheninnikov, T. K. Soboleva, and T. D. Rognlien (2005), Dust-particle transport in tokamak edge plasmas, Physics of Plasmas (1994-present), 12(12), 122508, doi: http://dx.doi.org/10.1063/1.2145157.

Poppe, A., and M. Horányi (2010), Simulations of the photoelectron sheath and dust levitation on the lunar surface, Journal of Geophysical Research: Space Physics (1978-2012), 115(A8).

Radovanov, S. B., J. K. Olthoff, R. J. Van Brunt, and S. Djurovi (1995), Ion kineticenergy distributions and balmeralpha (h) excitation in arh2 radiofrequency discharges, Journal of Applied Physics, 78(2). 


\section{REFERENCES}

Reynolds, E. W., M. E. Koepke, J. J. Carroll, and S. Shinohara (2006), Inhomogeneity scale lengths in a magnetized, low-temperature, collisionless, q-machine plasma column containing perpendicular-velocity shear, Physics of Plasmas (1994-present), 13(9), 092106, doi:http://dx. doi.org/10.1063/1.2338293.

Richardson, J. D. (1998), Thermal plasma and neutral gas in saturn's magnetosphere, Reviews of Geophysics, 36(4), 501-524, doi:10.1029/98RG01691.

Robertson, S., A. Gulbis, J. Colwell, and M. Hornyi (2003), Dust grain charging and levitation in a weakly collisional sheath., Physics of Plasmas, $10(10), 3874$.

Rohatschek, H. (1985), Direction, magnitude and causes of photophoretic forces, Journal of Aerosol Science, 16(1), 29 - 42, doi:http://dx.doi.org/10.1016/0021-8502(85)90018-7.

Rohatschek, H. (1995), Semi-empirical model of photophoretic forces for the entire range of pressures, Journal of Aerosol Science, 26(5), 717 - 734, doi:http://dx.doi.org/10.1016/0021-8502(95) 00011-Z.

Rosenberg, M., and D. A. Mendis (1995), Uv-induced coulomb crystallization in a dusty gas, Plasma Science, IEEE Transactions on, 23(2), 177-179, doi:10.1109/27.376584.

Rosenberg, M., D. A. Mendis, and D. P. Sheehan (1999), Positively charged dust crystals induced by radiative heating, Plasma Science, IEEE Transactions on, 27(1), 239-242, doi:10.1109/27. 763125 .

Rosenbluth, M., and C. Longmire (1957), Stability of plasmas confined by magnetic fields, Annals of Physics, 1 (2), 120 - 140, doi:http://dx.doi.org/10.1016/0003-4916(57)90055-6.

Rubinstein, J., and J. G. Laframboise (1982), Theory of a spherical probe in a collisionless magnetoplasma, Physics of Fluids (1958-1988), 25(7).

Samarian, A., and B. James (2001), Sheath measurement in rf-discharge plasma with dust grains, Physics Letters A, 287(12), 125 - 130, doi:http://dx.doi.org/10.1016/S0375-9601(01)00470-4.

Samarian, A. A., and B. W. James (2005), Dust as fine electrostatic probes for plasma diagnostic, Plasma Physics and Controlled Fusion, 47(12B), B629. 


\section{REFERENCES}

Sato, N., G. Uchida, T. Kaneko, S. Shimizu, and S. Iizuka (2001), Dynamics of fine particles in magnetized plasmas, Physics of Plasmas, 8(5), 1786-1790, doi:10.1063/1.1342229.

Schwabe, M. (2006), Microparticles as probes in a highly magnetized plasma, Ph.D. thesis, Max Planck Institute for Physics, Munich.

Schwabe, M., U. Konopka, P. Bandyopadhyay, and G. E. Morfill (2011), Pattern formation in a complex plasma in high magnetic fields, Phys. Rev. Lett., 106, 215,004, doi:10.1103/PhysRevLett. 106.215004 .

Sheridan, T. E. (2001), Solution of the plasma-sheath equation with a cool maxwellian ion source, Physics of Plasmas (1994-present), 8(9).

Shukla, P., and A. Mamun (2002), Introduction to dusty plasma physics, Plasma Physics and Controlled Fusion, $44(3), 395$.

Shukla, P. K. (2001), A survey of dusty plasma physics, Physics of Plasmas, 8(5), 1791-1803, doi:10.1063/1.1343087.

Sickafoose, A. A., J. E. Colwell, M. Horányi, and S. Robertson (2000), Photoelectric charging of dust particles in vacuum, Phys. Rev. Lett., 84, 6034-6037, doi:10.1103/PhysRevLett.84.6034.

Sickafoose, A. A., J. E. Colwell, M. Hornyi, and S. Robertson (2002), Experimental levitation of dust grains in a plasma sheath, Journal of Geophysical Research: Space Physics, 107(A11), SMP 37-1-SMP 37-11, doi:10.1029/2002JA009347.

Smirnov, R. D., A. Y. Pigarov, M. Rosenberg, S. I. Krasheninnikov, and D. A. Mendis (2007), Modelling of dynamics and transport of carbon dust particles in tokamaks, Plasma Physics and Controlled Fusion, 49(4), 347.

Sonmor, L. J., and J. G. Laframboise (1991), Exact current to a spherical electrode in a collisionless, largedebyelength magnetoplasma, Physics of Fluids B: Plasma Physics (1989-1993), 3(9).

Sternglass, E. J. (1957), Theory of secondary electron emission by high-speed ions, Phys. Rev., 108, 1-12, doi:10.1103/PhysRev.108.1. 


\section{REFERENCES}

Su, C. H., and R. E. Kiel (1966), Continuum theory of electrostatic probes, Journal of Applied Physics, 37(13).

Su, C. H., and S. H. Lam (1963), Continuum theory of spherical electrostatic probes, Physics of Fluids (1958-1988), 6(10).

Talbot, L., and Y. Chou (1969), Langmuir probe response in the transition regime, Rarefied Gas Dynamics, 2(Suppl 5), 1723-1737.

Taylor, J. R. (2005), Classical Mechanics, 1st ed., University Science Books, Sausalito, California, USA.

Thomas, E. (1999), Direct measurements of two-dimensional velocity profiles in direct current glow discharge dusty plasmas, Physics of Plasmas (1994-present), 6(7).

Thomas, E. (2001), Observations of high speed particle streams in dc glow discharge dusty plasmas, Physics of Plasmas (1994-present), 8(1).

Thomas, E., and M. Watson (1999), First experiments in the dusty plasma experiment device, Physics of Plasmas (1994-present), 6(10).

Thomas, E., R. L. Merlino, and M. Rosenberg (2012), Magnetized dusty plasmas: the next frontier for complex plasma research, Plasma Physics and Controlled Fusion, 54(12), 124,034.

Thomas, E., R. Merlino, and M. Rosenberg (2013), Design criteria for the magnetized dusty plasma experiment, Plasma Science, IEEE Transactions on, 41(4), 811-815, doi:10.1109/TPS.2012. 2236360.

Thompson, C., A. Barkan, N. DAngelo, and R. L. Merlino (1997), Dust acoustic waves in a direct current glow discharge, Physics of Plasmas (1994-present), 4(7).

Tomme, E., D. Law, B. Annaratone, and J. Allen (2000), Parabolic plasma sheath potentials and their implications for the charge on levitated dust particles, Physical review letters, 85(12), $2518-2521$.

Tonks, L., and I. Langmuir (1929), A general theory of the plasma of an arc, Physical Review, $34(6), 876$. 


\section{REFERENCES}

Trottenberg, T., D. Block, and A. Piel (2006), Dust confinement and dust-acoustic waves in weakly magnetized anodic plasmas, Physics of Plasmas (1994-present), 13(4), 042105, doi:http://dx. doi.org/10.1063/1.2196347.

Tsendin, L. D. (1995), Electron kinetics in non-uniform glow discharge plasmas, Plasma Sources Science and Technology, 4(2), 200.

Tsytovich, V. N., N. Sato, and G. E. Morfill (2003), Note on the charging and spinning of dust particles in complex plasmas in a strong magnetic field, New Journal of Physics, 5(1), 43.

Vaulina, O. S., S. A. Khrapak, A. P. Nefedov, and O. F. Petrov (1999), Charge-fluctuation-induced heating of dust particles in a plasma, Phys. Rev. E, 60, 5959-5964, doi:10.1103/PhysRevE.60. 5959.

Vaverka, J., I. Richterov, M. Vyinka, J. Pavl, J. afrnkov, and Z. Nmeek (2014), The influence of secondary electron emission on the floating potential of tokamak-born dust, Plasma Physics and Controlled Fusion, 56(2), 025,001.

Walker, J. J., M. E. Koepke, M. I. Zimmerman, W. M. Farrell, and V. I. Demidov (2014), Analytical model for gyro-phase drift arising from abrupt inhomogeneity, Journal of Plasma Physics, 80, 395-404, doi:10.1017/S0022377813001359.

Wang, Z., and C. M. Ticos (2008), Dust as a versatile matter for high-temperature plasma diagnostica), Review of Scientific Instruments, 79(10), 10.

Wang, Z., C. M. Ticoş, and G. A. Wurden (2007), Dust trajectories and diagnostic applications beyond strongly coupled dusty plasmas, Physics of Plasmas (1994-present), 14(10), 103701, doi:http://dx.doi.org/10.1063/1.2778416.

Whipple, E. C. (1981), Potentials of surfaces in space, Reports on Progress in Physics, 44(11), 1197.

Whipple, E. C., T. G. Northrop, and D. A. Mendis (1985), The electrostatics of a dusty plasma, Journal of Geophysical Research: Space Physics, 90(A8), 7405-7413, doi:10.1029/ JA090iA08p07405. 
Zakrzewski, Z., and T. Kopiczynski (1974), Effect of collisions on positive ion collection by a cylindrical langmuir probe, Plasma Physics, 16(12), 1195.

Zimmermann, T. M. G., M. Coppins, and J. E. Allen (2010), Coaxial discharge with axial magnetic field: Demonstration that the boltzmann relation for electrons generally does not hold in magnetized plasmas, Physics of Plasmas (1994-present), 17(2), 022301, doi:http://dx.doi.org/ 10.1063/1.3299390.

Zobnin, A., A. Nefedov, V. Sinelshchikov, and V. Fortov (2000), On the charge of dust particles in a low-pressure gas discharge plasma, Journal of Experimental and Theoretical Physics, 91 (3), 483-487, doi:10.1134/1.1320081. 


\section{Tables}




\begin{tabular}{|l|l|l|l|l|l|l|l|l|}
\hline $\begin{array}{l}\text { Dust Experiment } \\
\text { (Units) }\end{array}$ & $\begin{array}{l}a \\
(\mu \mathrm{m})\end{array}$ & $\begin{array}{l}B \\
(\mathrm{~T})\end{array}$ & $m_{i} / m_{p}$ & $\begin{array}{l}n_{i} \\
\left(\mathrm{~m}^{-3}\right)\end{array}$ & $\begin{array}{l}\mathrm{P} \\
(\mathrm{mTorr})\end{array}$ & $\begin{array}{l}T_{d} \\
(\mathrm{eV})\end{array}$ & $\begin{array}{l}T_{e} \\
(\mathrm{eV})\end{array}$ & $\begin{array}{l}T_{i} \\
(\mathrm{eV})\end{array}$ \\
\hline $\begin{array}{l}\text { DPD } \\
\text { (Thompson et al., 1997) }\end{array}$ & 0.8 & 0.09 & 14 & $10^{15}$ & 100 & 2.5 & $0.03-0.1$ \\
\hline $\begin{array}{l}\text { DUPLEX } \\
\text { (Amatucci et al., 2004) }\end{array}$ & 0.6 & 0.25 & 40 & $10^{15}$ & 250 & 0.025 & 1.6 & 0.025 \\
\hline $\begin{array}{l}\text { DUSTWHEEL } \\
\text { (Knist et al., 2011) }\end{array}$ & 0.5 & 40 & $10^{14}$ & $1-10$ & & 2.5 & 0.03 \\
\hline $\begin{array}{l}\text { Matilda II } \\
\text { (Trottenberg et al., 2006) }\end{array}$ & 0.47 & 0.02 & 40 & $10^{15}$ & 15 & 0.025 & 2.7 & 0.1 \\
\hline $\begin{array}{l}\text { MDPX } \\
\text { (Thomas et al., 2012) }\end{array}$ & $0.3-0.5$ & 4 & $10,14,40$ & $10^{14}-10^{16}$ & 1 & 0.025 & $3-4$ & 0.025 \\
\hline $\begin{array}{l}\text { MDPX at MPE } \\
\text { (Schwabe et al., 2011) }\end{array}$ & $1.3-2.2$ & 2 & $10,14,40$ & $10^{14}-10^{16}$ & 2.5 & & $3-4$ & 0.025 \\
\hline $\begin{array}{l}\text { Molecular Clouds } \\
\text { (Ferrière, 2001) }\end{array}$ & $10^{-4}-0.1$ & $10^{-9}$ & 1 & $10-10^{10}$ & $<10^{-9}$ & $10^{-3}$ & 1 & 1 \\
\hline (Nunomura et al., 1997) & 30 & 0.09 & 4 & $10^{16}$ & 0.34 & & 5 & 0.5 \\
\hline (Sato et al., 2001) & 10 & $0.04-4$ & 40 & $10^{14}$ & 100 & & 2 & 0.025 \\
\hline $\begin{array}{l}\text { Saturn's E ring } \\
\text { (Khurana et al., 2008) }\end{array}$ & $0.01-10$ & $10^{-7}$ & 18 & $10^{6}$ & $10^{-10}$ & & 10 & 30 \\
\hline $\begin{array}{l}\text { Suleiman Device } \\
\text { (Carstensen et al., 2012) }\end{array}$ & 5.8 & 4 & 40 & $10^{14}$ & 60 & & 3 & 0.025 \\
\hline
\end{tabular}

Table 1.1: Parameters for laboratory dust experiments and other regimes with magnetic fields. 


\begin{tabular}{|l|l|l|l|l|l|l|l|l|l|}
\hline Dust Experiment & $a / R_{L e}$ & $a / R_{L i}$ & $\mathrm{Kn}_{R 0}$ & $N_{D e}$ & $R_{L d}(m)$ & $T_{e} / T_{i}$ & $\lambda_{D} / a$ & $\lambda_{i} / a$ & $\omega_{c d} / \nu_{d n}$ \\
\hline $\begin{array}{l}\text { DPD } \\
\text { (Thompson et al., 1997) }\end{array}$ & $10^{-2}$ & $10^{-3}$ & 1 & $10^{5}$ & $10^{-1}$ & 50 & 500 & $10^{2}$ & $10^{-4}$ \\
\hline $\begin{array}{l}\text { DUPLEX } \\
\text { (Amatucci et al., 2004) }\end{array}$ & $10^{-2}$ & $10^{-3}$ & 1 & $10^{4}$ & $10^{-2}$ & 64 & 500 & $10^{2}$ & $10^{-4}$ \\
\hline $\begin{array}{l}\text { Matilda II } \\
\text { (Trottenberg et al., 2006) }\end{array}$ & $10^{-3}$ & $10^{-5}$ & $10^{2}$ & $10^{5}$ & $10^{-1}$ & 27 & 830 & $>10^{4}$ & $10^{-2}$ \\
\hline $\begin{array}{l}\text { MDPX } \\
\text { (Thomas et al., 2012) }\end{array}$ & $0.2-0.4$ & $10^{-2}$ & 20 & $10^{4}$ & $10^{-3}$ & 140 & $>10^{3}$ & $>10^{4}$ & 10 \\
\hline $\begin{array}{l}\text { MDPX at MPE } \\
\text { (Schwabe et al., 2011) }\end{array}$ & 1 & $10^{-2}$ & $10^{-1}$ & $10^{4}$ & $10^{-3}$ & 140 & $10^{3}-10^{4}$ & $10^{2}$ & 1 \\
\hline $\begin{array}{l}\text { Molecular Clouds } \\
\text { (Ferrière, 2001) }\end{array}$ & $10^{-10}$ & $10^{-12}$ & $>10^{4}$ & $>10^{6}$ & $>10^{5}$ & 1 & $>10^{6}$ & $>10^{14}$ & $1-10^{4}$ \\
\hline $\begin{array}{l}\text { (Nunomura et al., 1997) } \\
\text { Nunom's E ring }\end{array}$ & 0.4 & $10^{-1}$ & 2 & $10^{4}$ & $10^{-1}$ & 200 & 6 & $10^{3}$ & $10^{-2}$ \\
\hline $\begin{array}{l}\text { Saturn's } \\
\text { (Khurana et al., 2008) }\end{array}$ & $10^{-7}$ & $10^{-9}$ & $10^{9}$ & $10^{9}$ & $>10^{5}$ & 0.1 & $>10^{8}$ & $>10^{15}$ & $10-10^{4}$ \\
\hline $\begin{array}{l}\text { Suleiman Device } \\
\text { (Carstensen et al., 2012) }\end{array}$ & 4.5 & $10^{-1}$ & $10^{-1}$ & $10^{5}$ & $10^{-3}$ & 120 & 220 & 34 & $10^{-3}$ \\
\hline
\end{tabular}

Table 1.2: Dimensionless Parameters for laboratory dust experiments with magnetic fields. Even experiments with large magnetic fields $(B>1 \mathrm{~T})$ do not necessarily produce magnetized orbit dust grains due to the presence of neutral drag, which is represented in this table by the $\omega_{c d} / \nu_{d n}$ parameter. Parameters that depend on the gyro-radii quoted here correspond to the mean gyroradius $R_{L s}=\sqrt{\frac{\pi k_{b} T_{s}}{2 m_{e}}} / \omega_{c s}$. The dust gyro-radius $R_{L d}$ quoted in this table does not include the enlarging effect of an applied electric field that is perpendicular to the magnetic field. Dust grains are levitated in a planar sheath where the ions have a fluid drift $v_{i}=\sqrt{\frac{k_{b} T_{e}}{m_{i}}}$ directed along the sheath dimension, which means that the Debye length is given by $\lambda_{D}=\sqrt{\frac{\epsilon_{0} k_{b} T_{e}}{e^{2} n_{i}}}$ (Carstensen et al., 2012). The dust-neutral collision frequency $\nu_{d n}$ is calculated assuming Epstein drag and is given by equation 3.59 .

\begin{tabular}{|l|l|l|}
\hline & Species & Potential Type \\
\hline$V_{d}=V_{\text {surf }}-V_{s}>0$ & Ions & Retarding Potential \\
\hline$V_{d}=V_{\text {surf }}-V_{s}>0$ & Electrons & Attractive Potential \\
\hline$V_{d}=V_{\text {surf }}-V_{s}<0$ & Ions & Attractive Potential \\
\hline$V_{d}=V_{\text {surf }}-V_{s}<0$ & Electrons & Retarding Potential \\
\hline
\end{tabular}

Table 2.1: Summary of retarding and attracting conditions for electrons and ions. 


\begin{tabular}{|l|l|l|l|l|}
\hline Moon & Enceladus & Tethys & Dione & Rhea \\
\hline Radial distance $\left(R_{\text {Sat }}\right)$ & 3.95 & 4.89 & 6.26 & 8.74 \\
$|\boldsymbol{B}|(\mathrm{nT})$ & 370 & 167 & 75 & 25 \\
$n_{e}\left(\mathrm{~m}^{-3} / 10^{6}\right)$ & 40 & 30 & 13 & 2 \\
$n_{n}\left(\mathrm{~m}^{-3} / 10^{6}\right)$ & 400 & 400 & 100 & \\
$m_{i}(\mathrm{AMU})$ & 18 & 17 & 17 & 17 \\
$T_{e}(\mathrm{eV})$ & 10 & 20 & 60 & 100 \\
$T_{i}(\mathrm{eV})$ & 30 & 50 & 90 & 100 \\
$C_{s}(\mathrm{~km} / \mathrm{s})$ & 19 & 26 & 37 & 43 \\
\hline
\end{tabular}

Table 6.1: Selected plasma parameters throughout the Saturnian system, from Khurana et al. (2008); Richardson (1998). 


\section{Figures}



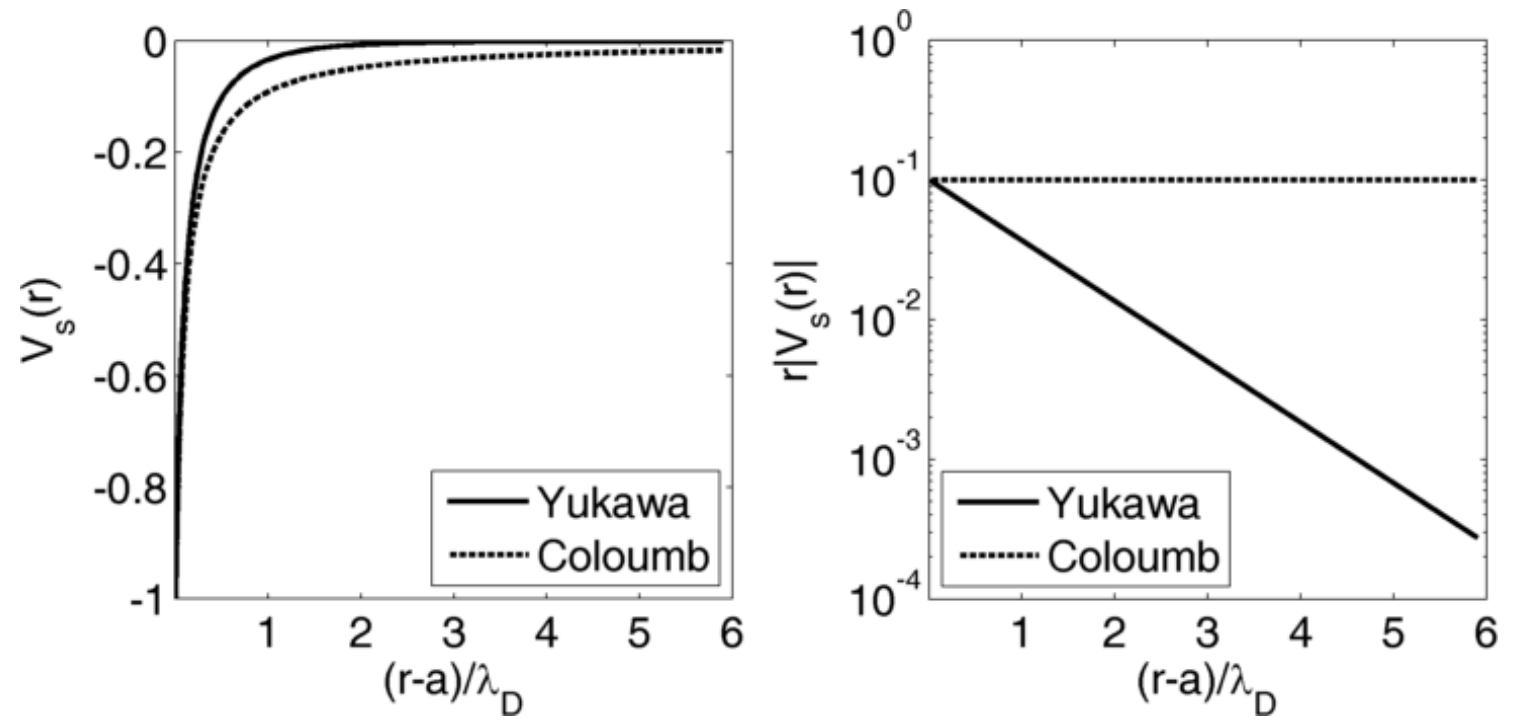

Figure 2.1: For a small spherical conductor $a \ll \lambda_{D}$, the Yukawa, or Debye-Huckel potential is compared to a Coloumb potential. 


\section{Collisionless Child-Langmuir Sheath}

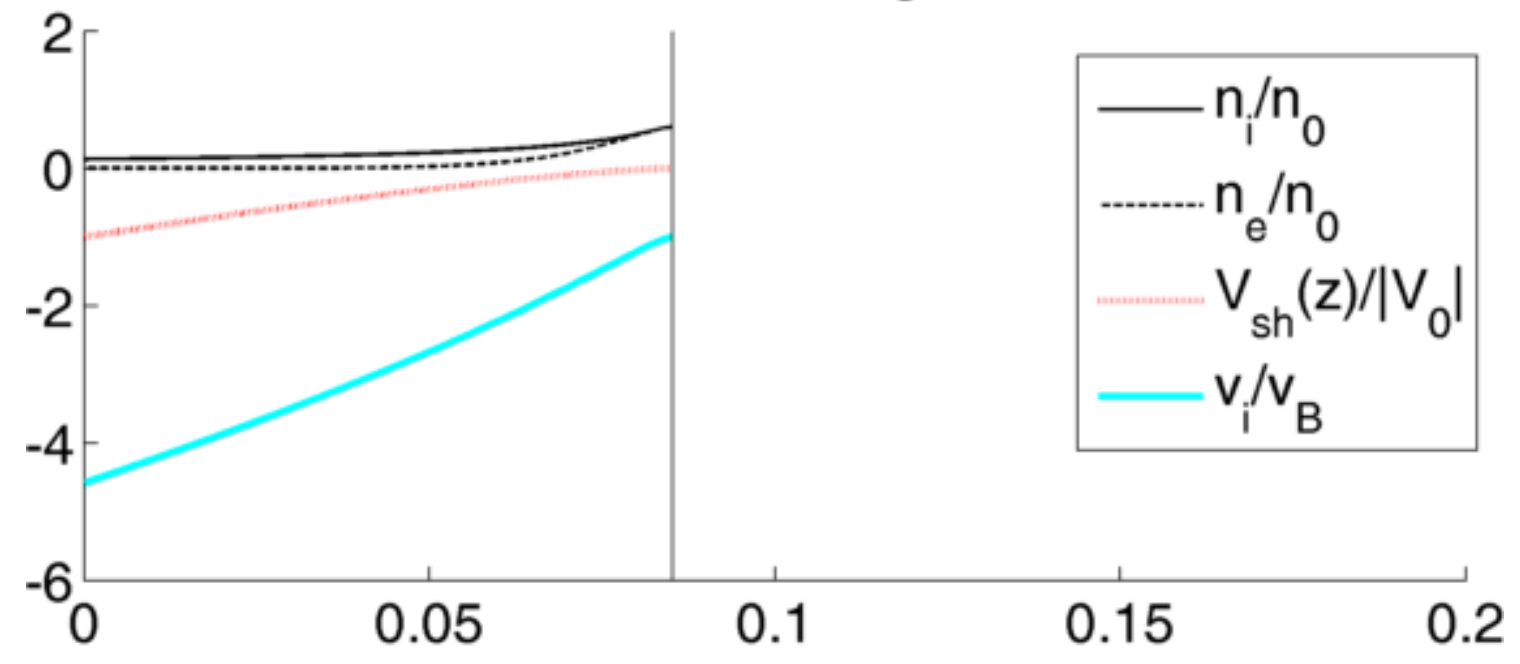

Collisional Child-Langmuir Sheath

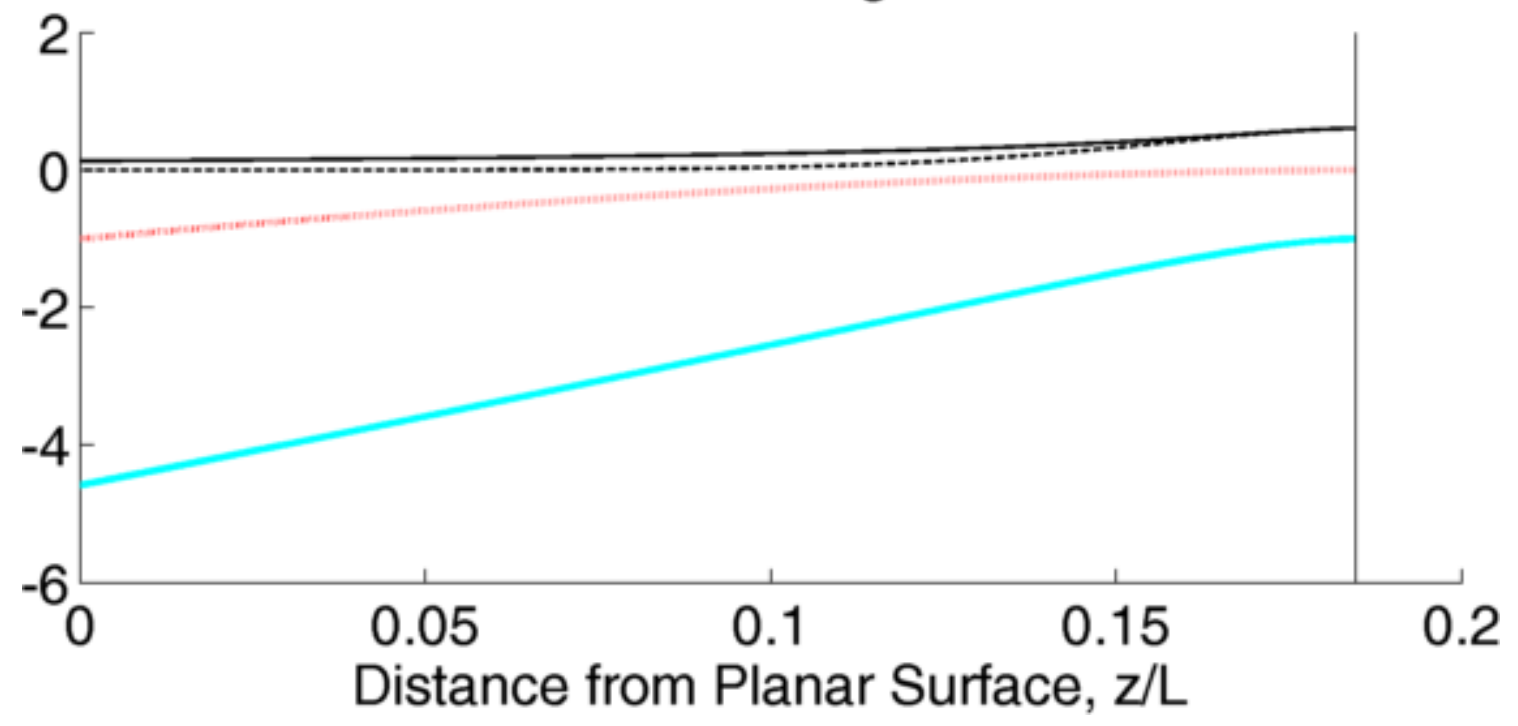

Figure 2.2: Space potential, electron and ion densities, and ion fluid velocity along the sheath direction for a collisionless and weakly collisional Child-Langmuir sheath, represented by the top and bottom panels, respectively. For the same plasma parameters, the sheath is larger in the weakly collisional case. 

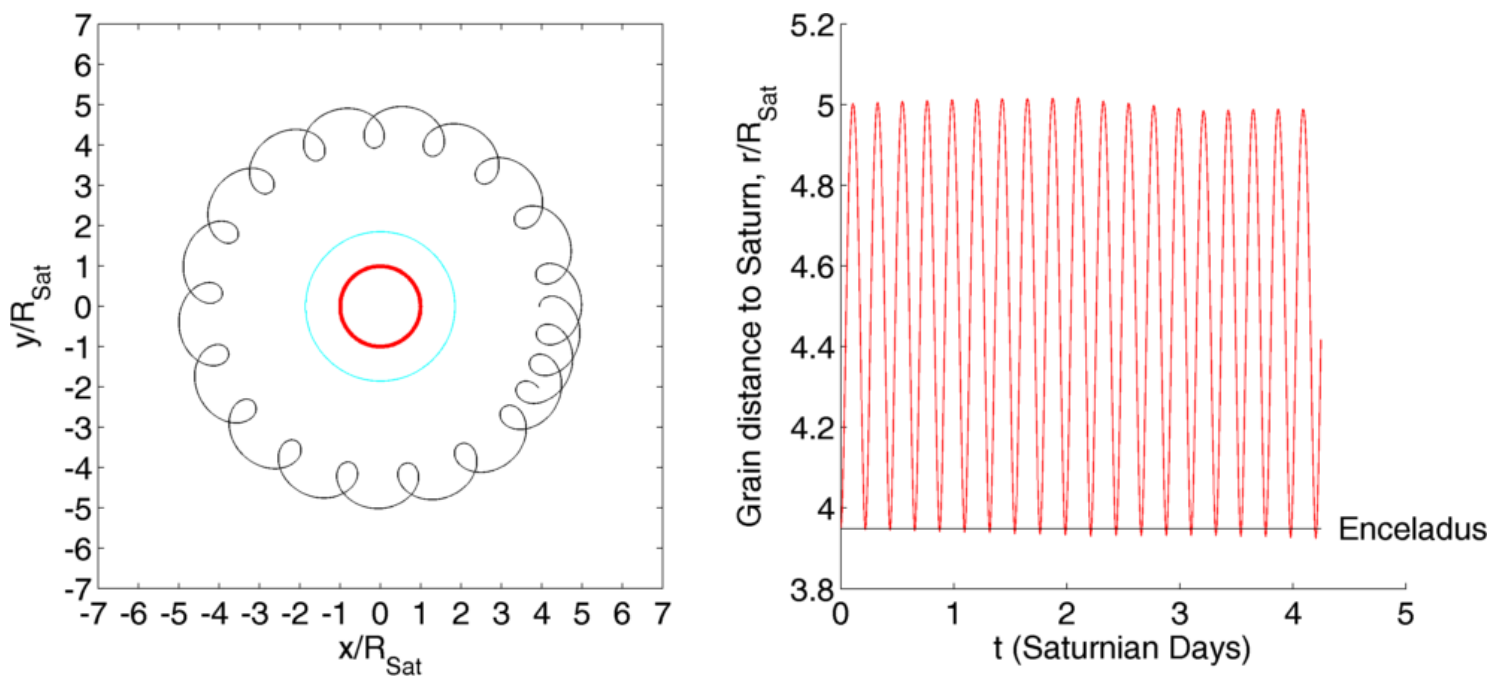

Figure 2.3: Example grain trajectory around Saturn in the corotating reference frame. The $a=$ $0.025 \mu \mathrm{m}$ grain is launched from Enceladus at the Kepler velocity. On the left side of the figure, the thick red line in the center shows the extent of Saturn, the larger, concentric cyan circle shows the radius for geosynchronous orbit, and the thin black line corresponds to the grain trajectory. A dipole magnetic field is assumed, and the parameters in the corotating plasma are $n_{0}=4 \times 10^{7}$ $\mathrm{m}^{-3}, T_{e}=5 \mathrm{eV}, T_{i}=10 \mathrm{eV}$, with ions having $20 \mathrm{amu}$. On the right side of the figure, the radial excursion of the grain as a function of time is plotted. The grain drifts radially inward. 


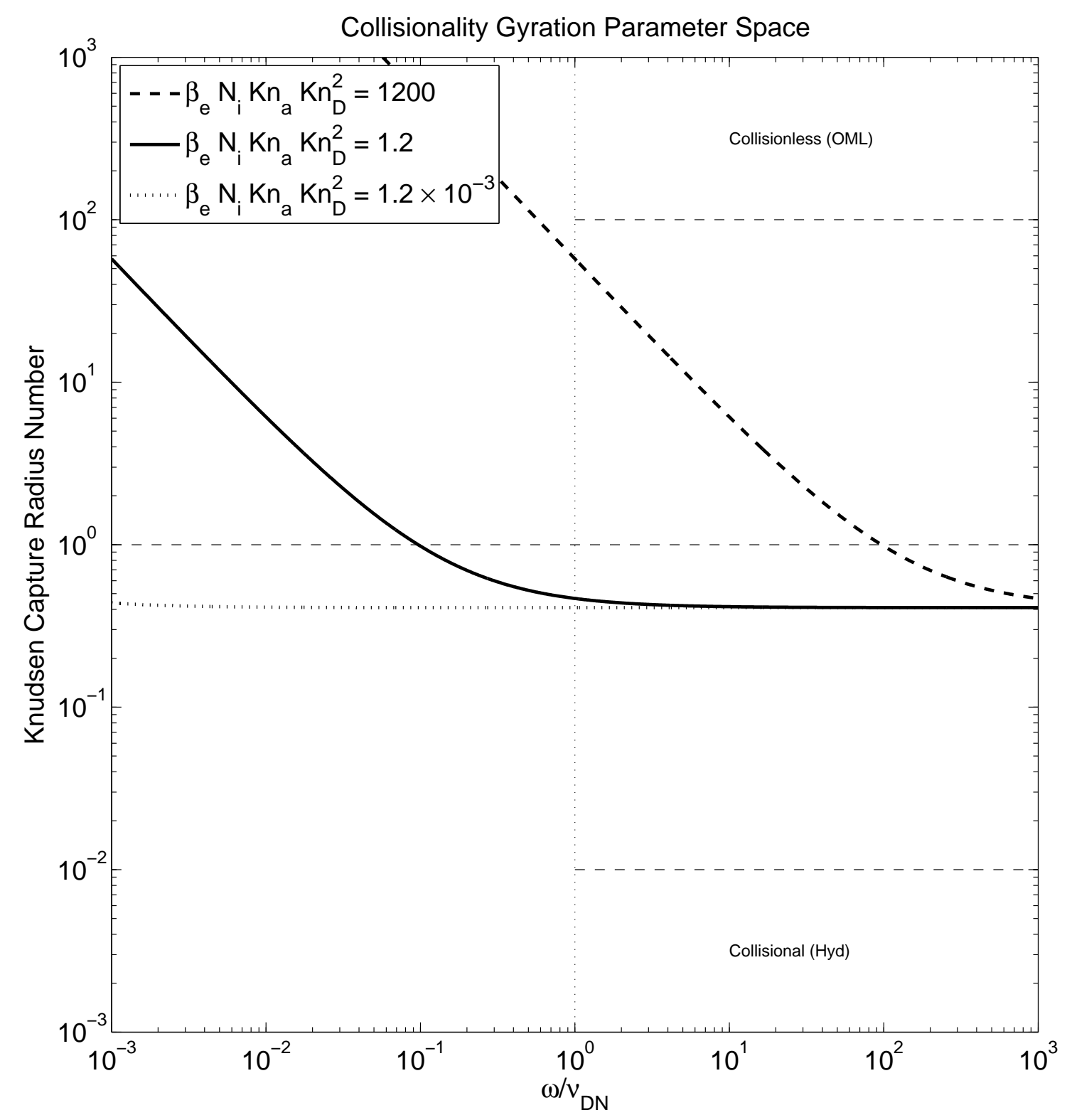

Figure 3.1: Knudsen capture parameter $K n_{R}$ as a function of the gyration parameter, $\omega_{c d} / \nu_{d n}$. The number product is expressed as $\frac{9 \sqrt{\pi}}{8(1.22)} \sqrt{\frac{m_{i} T_{i}}{m_{e} T_{e}}} \beta_{e} \frac{n_{i}}{n_{n}} K n_{a}^{3}\left(\frac{T_{i}}{T_{e}}+\frac{n_{e}}{n_{i}}\right)^{-1}$. 


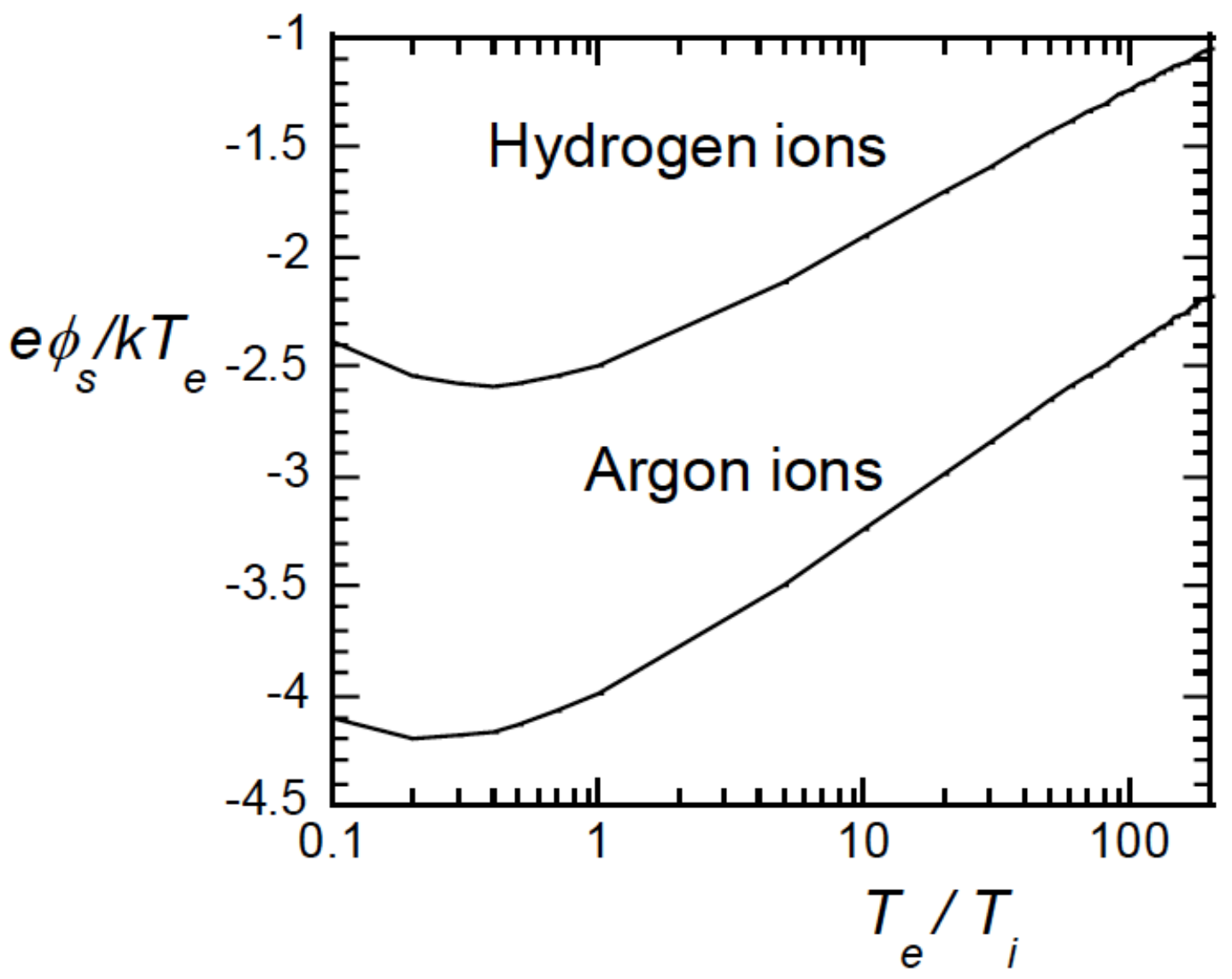

Figure 3.2: Figure from Merlino (2006) which shows how dimensionless surface potential $\chi_{e}=$ $e V_{d} /\left(k_{b} T_{e}\right)$ depends on the ratio of electron to ion temperature. 


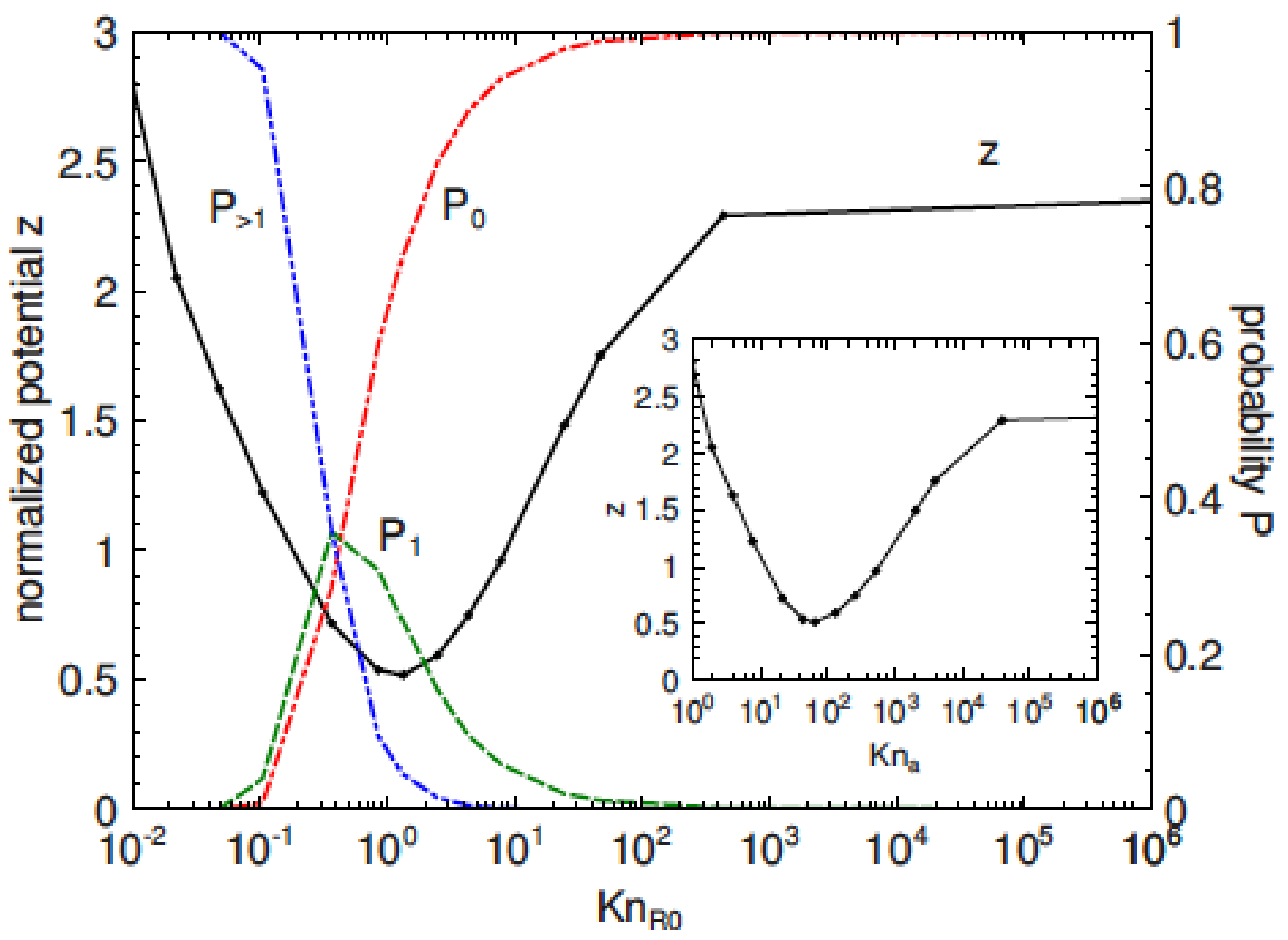

Figure 3.3: Figure from Gatti and Kortshagen (2008). This shows the normalized particle potential $\chi_{e}=q_{d} /\left(C_{d} T_{e}\right)$ as a function of the capture radius Knudsen number $K n_{R 0}$ for a particle with a diameter of $500 \mathrm{~nm}, n_{i 0}=10^{10} \mathrm{~cm}^{3}, T_{e}=2.5 \mathrm{eV}, T_{i}=0.025 \mathrm{eV}$, and $n_{d} / n_{i 0} \approx 0$, meaning the plasma is treated as dust in plasma rather than dusty-plasma. Also plotted are the probabilities of performing zero $\left(P_{0}\right)$, one $\left(P_{1}\right)$, and more than one collision $P_{>1}$ inside the capture radius sphere. 


\section{Gyro-phase Diagram}

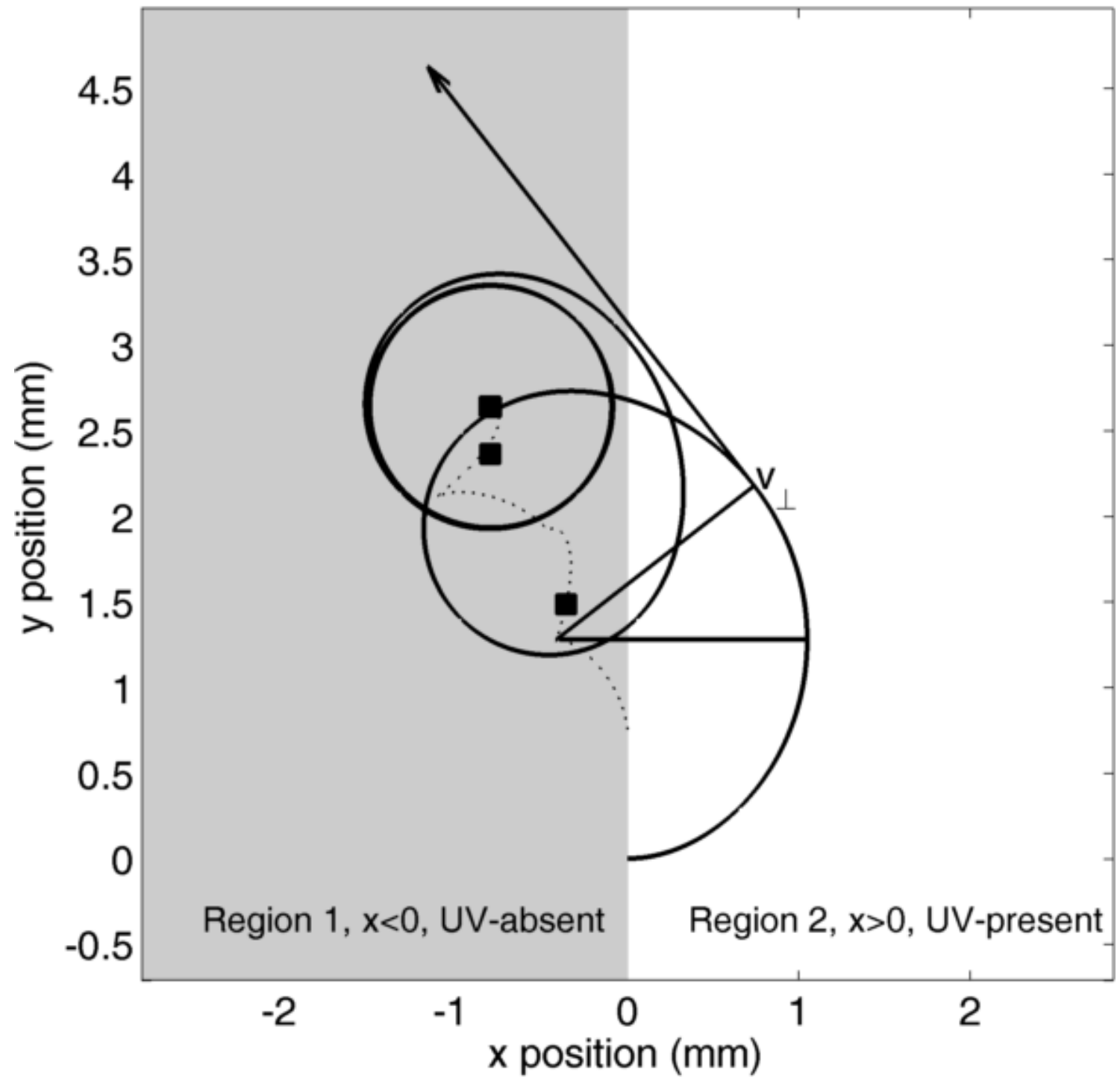

Figure 4.1: This diagram shows the relationship between the velocity vector and the gyro-phase angle $\phi$ for an example grain trajectory. 

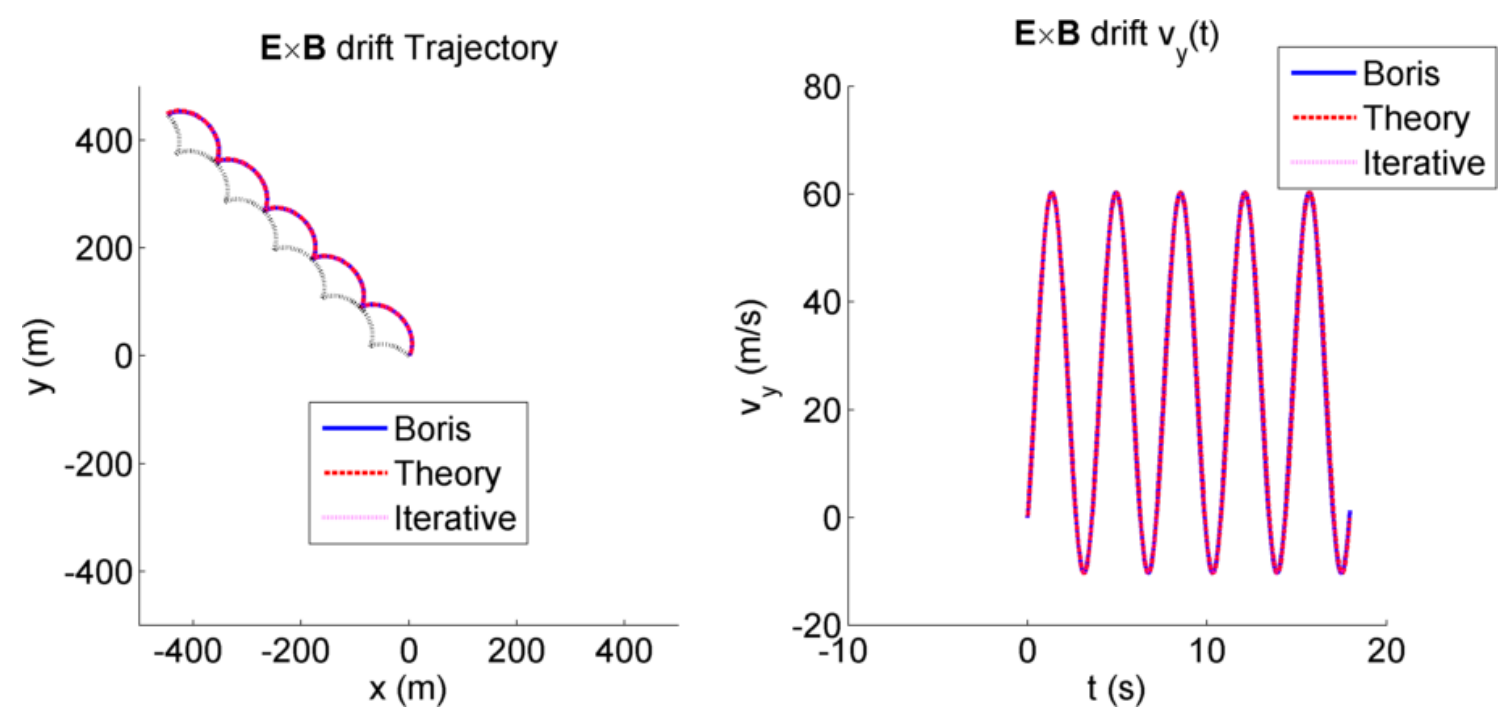

Figure 4.2: A $1 \mu \mathrm{m}$ diameter grain starts at the origin, gyrates, and drifts in a magnetic field $B=4$ $\mathrm{T}$ and an electric field $E_{x}=100 \mathrm{~V} / \mathrm{m}, E_{y}=100 \mathrm{~V} / \mathrm{m}$. The grain has $\approx 1400$ electrons on its surface.
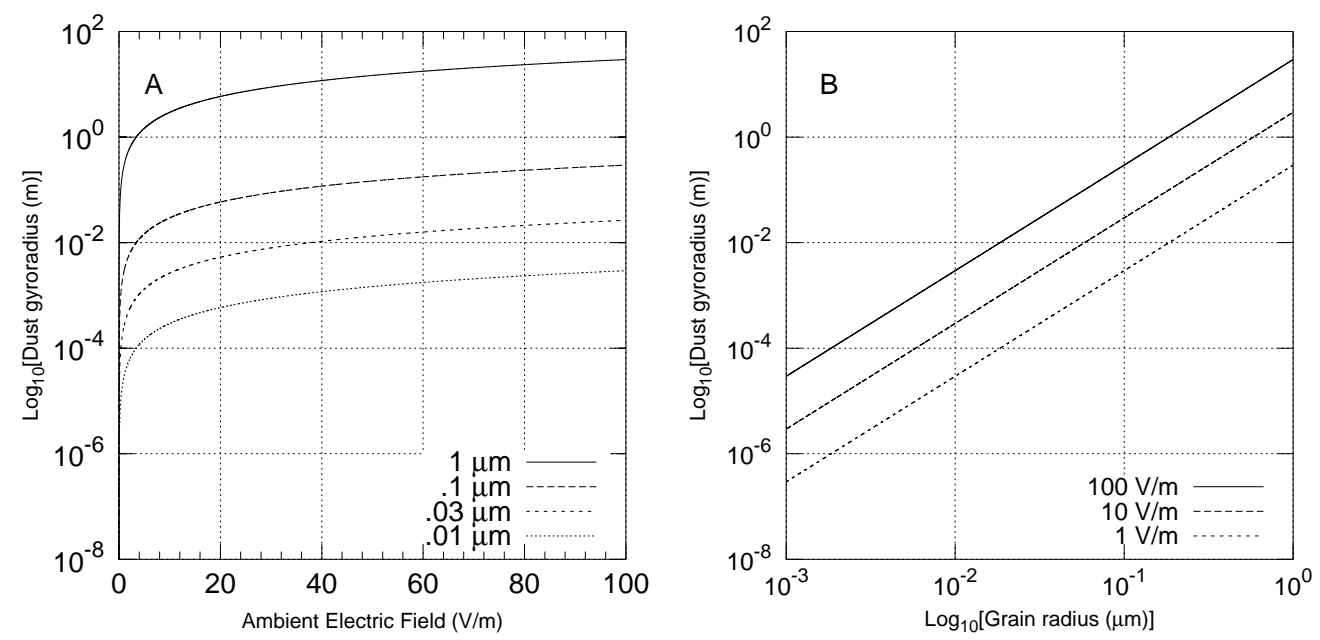

Figure 4.3: The effective gyro-radius can be accomodated in a laboratory vacuum vessel only for small magnitude of radial electric field and small grain radius. A: Effective dust grain gyro-radius as a function of a constant, ambient electric field is plotted for grain diameters $a=1 \mu \mathrm{m}, a=0.1 \mu \mathrm{m}$, $a=.015 \mu \mathrm{m}$, and $a=0.01 \mu \mathrm{m}$. B: Effective gyro-radius as a function of grain radius is plotted for constant, ambient electric field values of $100 \mathrm{~V} / \mathrm{m}, 10 \mathrm{~V} / \mathrm{m}$, and $1 \mathrm{~V} / \mathrm{m}$. 

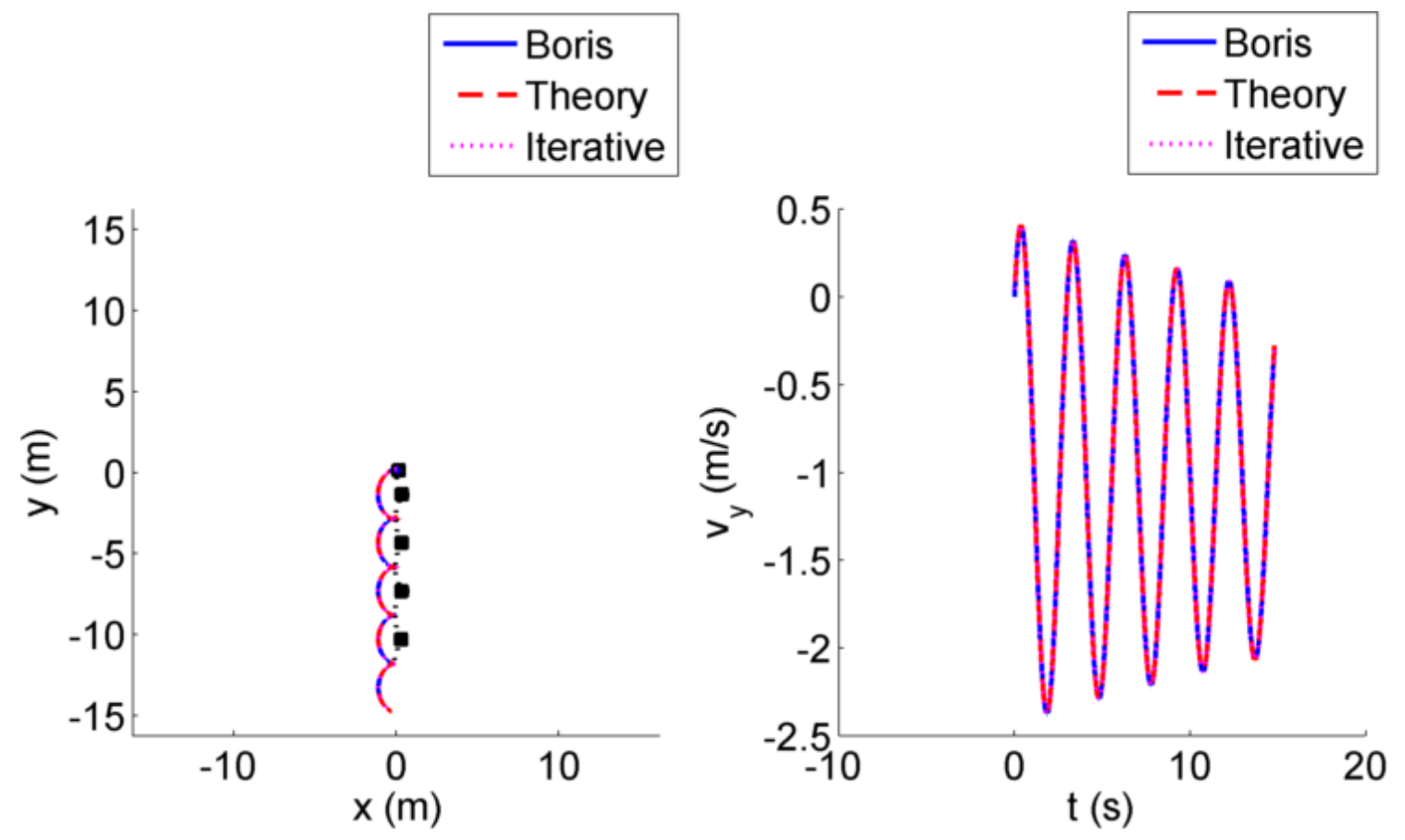

Figure 4.4: A $1 \mu \mathrm{m}$ diameter grain starts at the origin and moves in a magnetic field $B=4 \mathrm{~T}$, an electric field $E_{x}=100 \mathrm{~V} / \mathrm{m}, E_{y}=100 \mathrm{~V} / \mathrm{m}$, a neutral gas pressure of $1 \mathrm{mTorr}$, with the neutrals flowing at $v_{x n}=-100 \mathrm{~m} / \mathrm{s}$, which produces a drag force of magnitude $\approx 10^{-15} \mathrm{~N}$. By comparison, the magnitude of the magnetic force on this grain $\approx 10^{-18} \mathrm{~N}$. The grain has $\approx 1400$ electrons on its surface, $\omega_{c d} \approx 2 \mathrm{~s}^{-1}, \nu_{d n} \approx 2 \times 10^{-2} \mathrm{~s}^{-1}$ so that $\omega_{c d} / \nu_{d n} \gg 1$. 

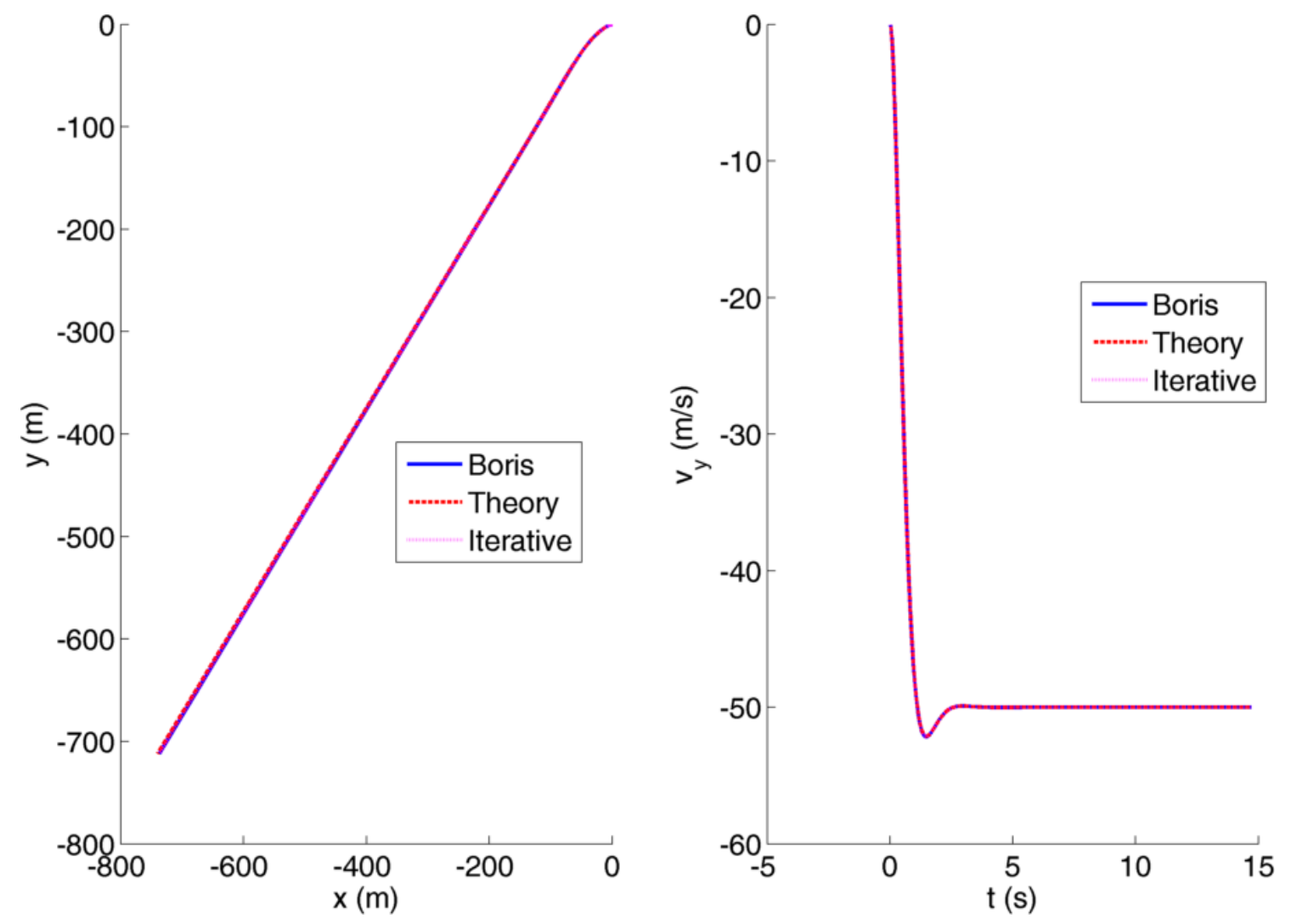

Figure 4.5: A $1 \mu \mathrm{m}$ diameter grain starts at the origin and moves in a magnetic field $B=4 \mathrm{~T}$, an electric field $E_{x}=100 \mathrm{~V} / \mathrm{m}, E_{y}=100 \mathrm{~V} / \mathrm{m}$, a neutral gas pressure of $1 \mathrm{mTorr}$, with the neutrals flowing at $v_{x n}=-100 \mathrm{~m} / \mathrm{s}$ which produces a drag force of magnitude $\approx 10^{-15} \mathrm{~N}$. By comparison, the magnitude of the magnetic force on this grain $\approx 10^{-18} \mathrm{~N}$. The grain has $\approx 1400$ electrons on its surface, $\omega_{c d} \approx 2 \mathrm{~s}^{-1}, \nu_{d n} \approx 2$ so that $\omega_{c d} / \nu_{d n} \approx 1$ and the grain does not make a complete gyration. 

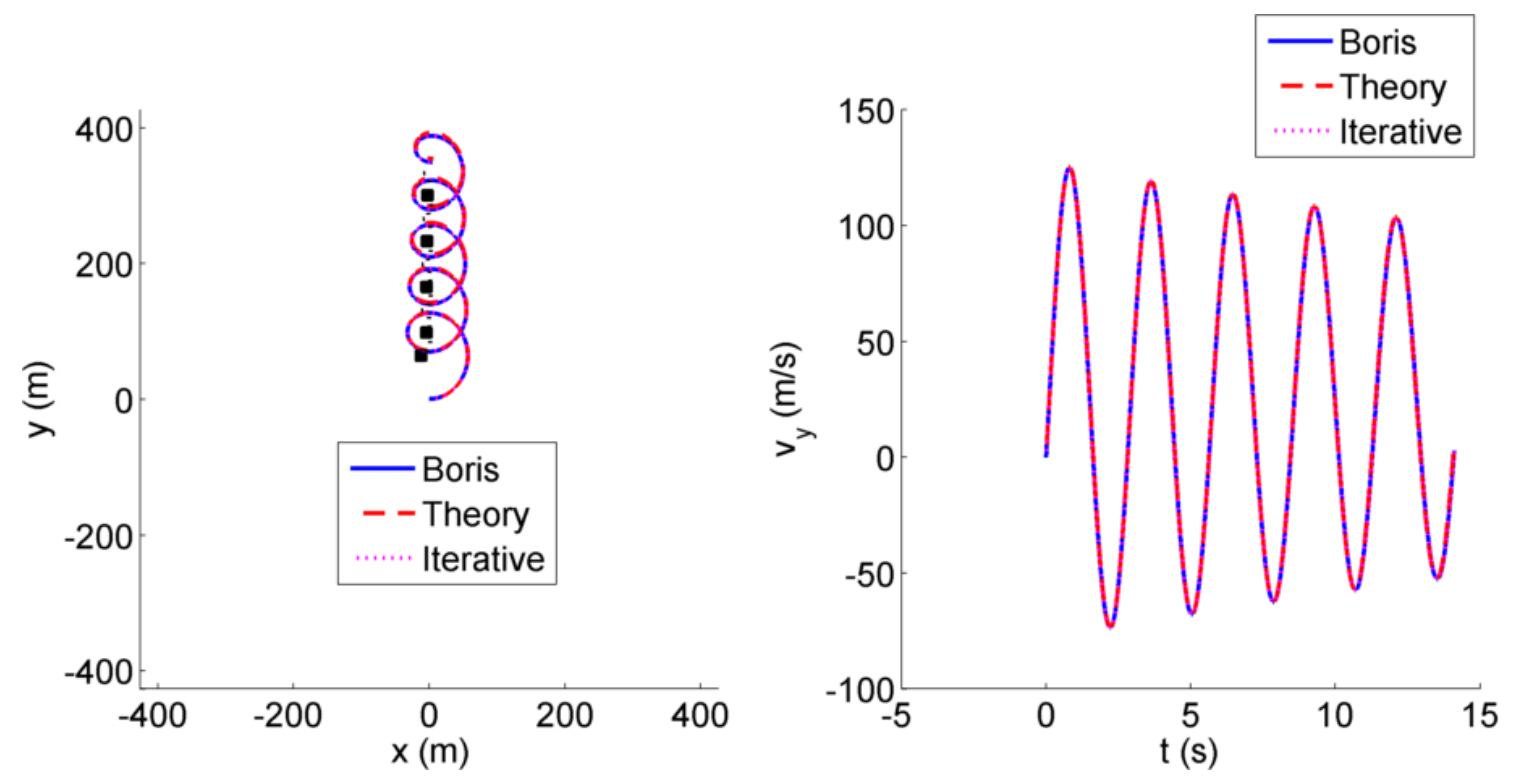

Figure 4.6: A $1 \mu \mathrm{m}$ diameter grain starts at the origin and moves in a magnetic field $B=4 \mathrm{~T}$, an electric field $E_{x}=-100 \mathrm{~V} / \mathrm{m}$, a neutral gas pressure of $0.01 \mathrm{mTorr}$, with the neutrals flowing at $v_{x n}=-100 \mathrm{~m} / \mathrm{s}$, which produces a drag force of magnitude $\approx 10^{-15} \mathrm{~N}$. By comparison, the magnitude of the magnetic force on this grain $\approx 10^{-18} \mathrm{~N}$. The grain has $\approx 1400$ electrons on its surface. 


\section{Ion Drag}

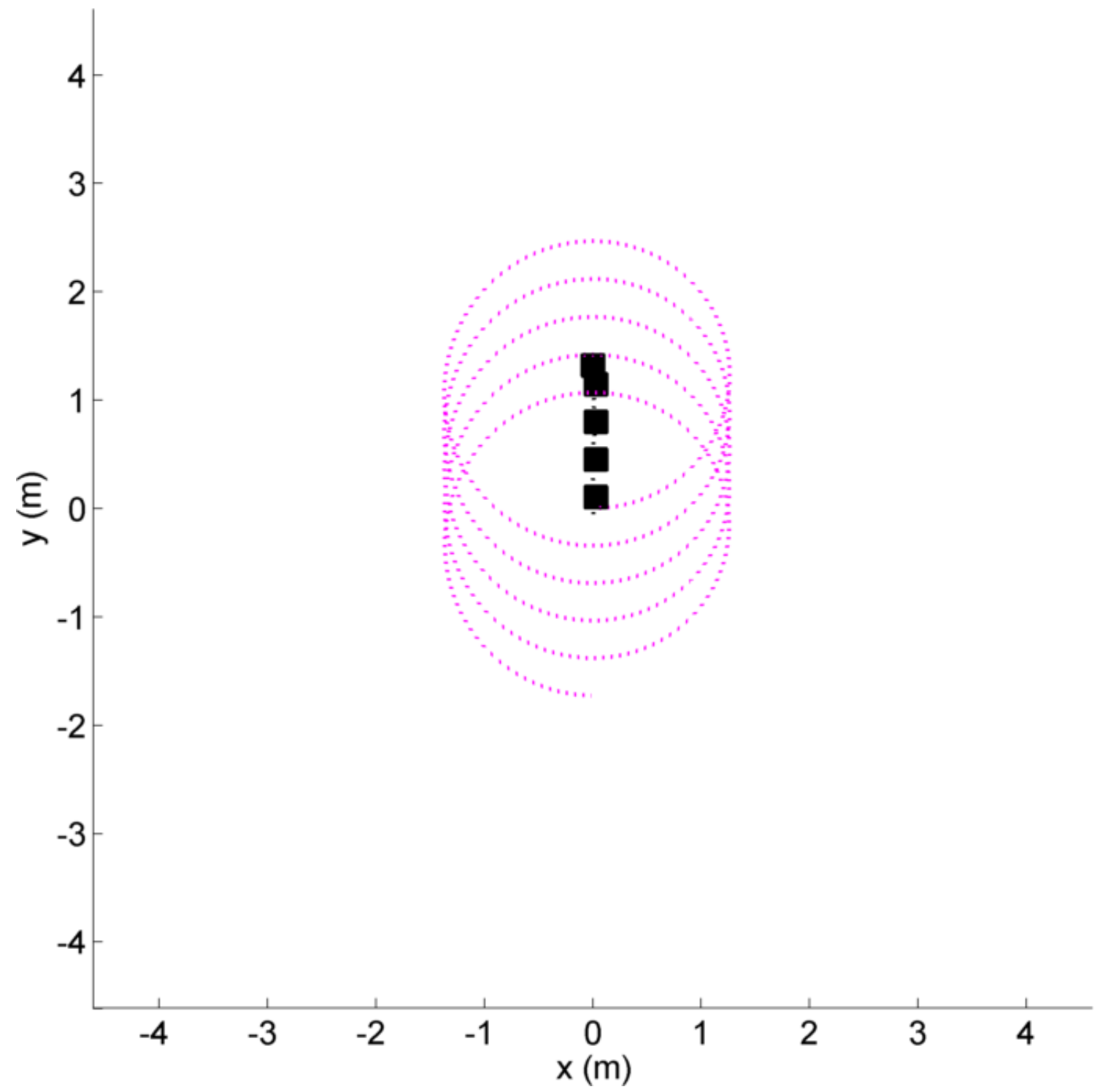

Figure 4.7: A $1 \mu \mathrm{m}$ diameter grain starts at the origin and moves in a magnetic field $B=4 \mathrm{~T}$, an ion density of $10^{12} \mathrm{~m}^{-3}$, with the ions flowing at $v_{x i}=-200 \mathrm{~m} / \mathrm{s}$, which produces a drag force of magnitude $\approx 10^{-17} \mathrm{~N}$. By comparison, the magnitude of the magnetic force on this grain $\approx 10^{-18} \mathrm{~N}$. The grain has $\approx 1400$ electrons on its surface. 


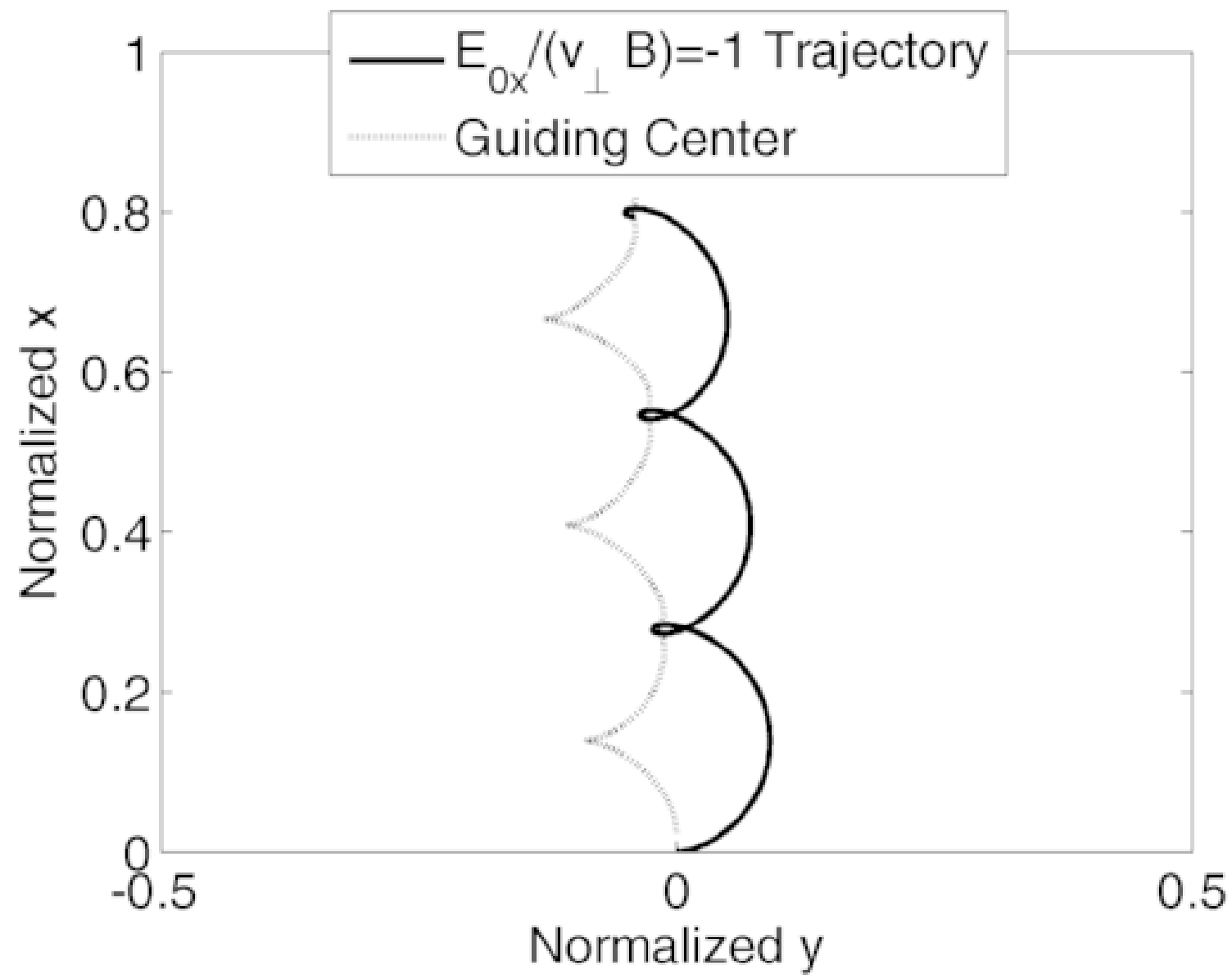

Figure 5.1: Grain trajectory for $E_{0 y}=0, E_{0 x} /\left(B v_{\perp}\right)=-1, \lambda_{D} / a=10^{3}, \lambda_{i} / a=10^{5}, m_{d} / m_{e}=$ $10^{11}, q_{1} / q_{2}=1.74, \omega_{c d} / \nu_{d n}=10^{4}$, and $\tau_{g} / \tau_{c}=5$. In this case, the instantaneous gyro-radius must be specified by the more general $v_{\perp}(t) / \frac{\mathrm{d} \phi}{\mathrm{d} t}$, rather than $v_{\perp}(t) / \omega_{c d}(t)$, where $\phi$ is the gyro-phase angle. The dotted line represents the guiding center position. 


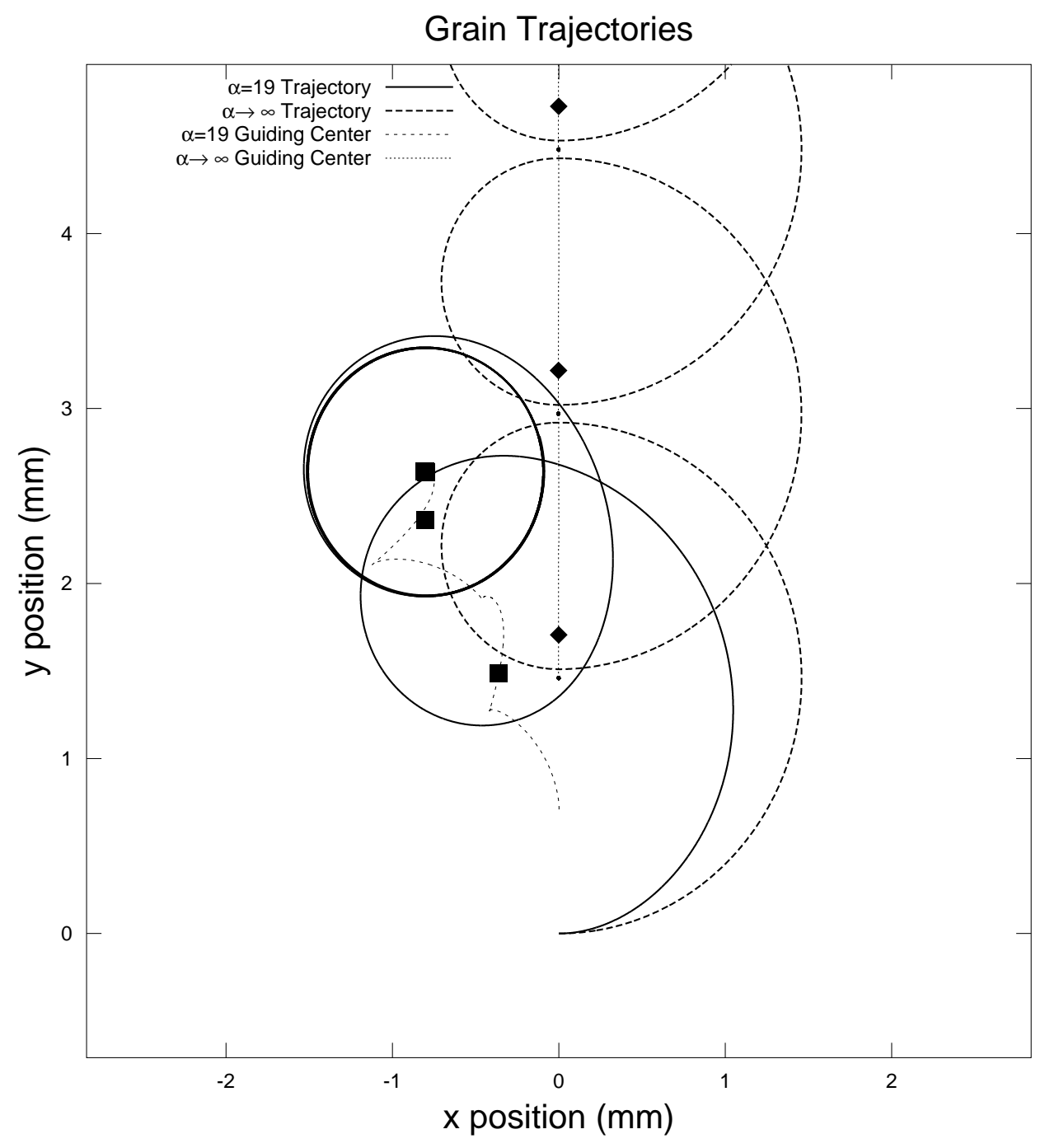

Figure 5.2: Grain trajectories for $a=0.05 \mu \mathrm{m}$. The dashed and solid lines represent the trajectories of $\tau_{g} / \tau_{c}$ ratios for $\tau_{g} / \tau_{c} \rightarrow \infty\left(n_{0}=n_{e}=n_{i}=10^{16} \mathrm{~m}^{-3}\right)$ and $\tau_{g} / \tau_{c}=19.204$ (for $n_{0}=10^{13} \mathrm{~m}^{-3}$ ) respectively. Squares and diamonds indicate the gyro-averaged guiding centers for $\tau_{g} / \tau_{c} \rightarrow \infty$ and $\tau_{g} / \tau_{c}=19.204$ respectively. 


\section{Trajectory}

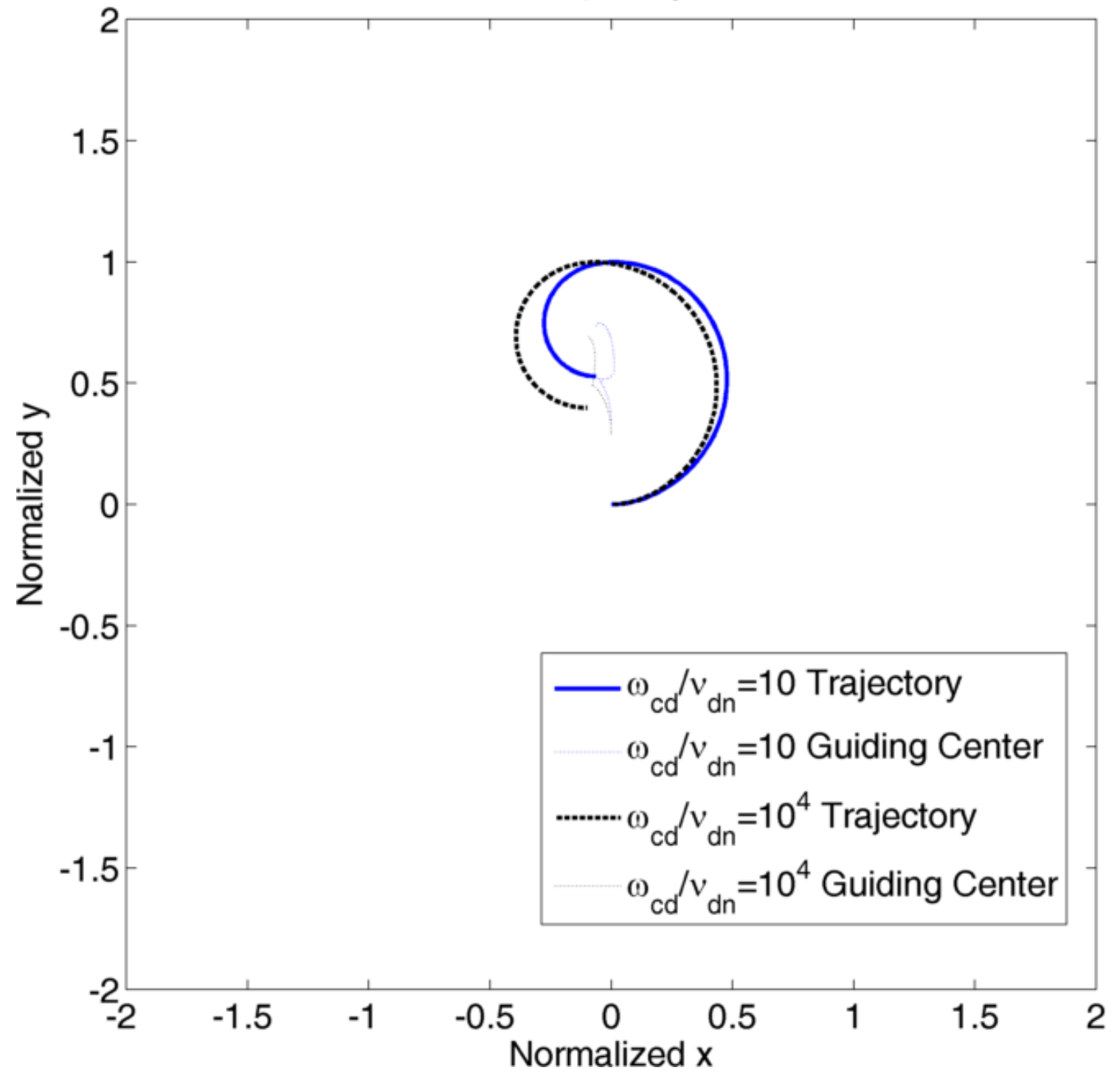

Figure 5.3: Example of a drag affected trajectory overlaid with a non-drag affected trajectory. The ratio $\omega_{c d} / \nu_{d n}$ is 10 for the drag affect trajectory and $10^{4}$ for the non-drag affected trajectory, with all other parameters equal. The drag affected trajectory has an inherent drift component along the $\hat{x}$-direction. For this plot, Argon ions are assumed, $q_{1} / q_{2}=1.74, T_{e} / T_{i}=200, a / R_{L e}=0.1$, $\lambda_{D} / a=10^{5}, \lambda_{i} / a=5, N_{D e}=10^{3}, m_{d} / m_{e} \approx 10^{12}$, and $\tau_{g} / \tau_{c} \approx 20$ (non-instantaneous charging). 

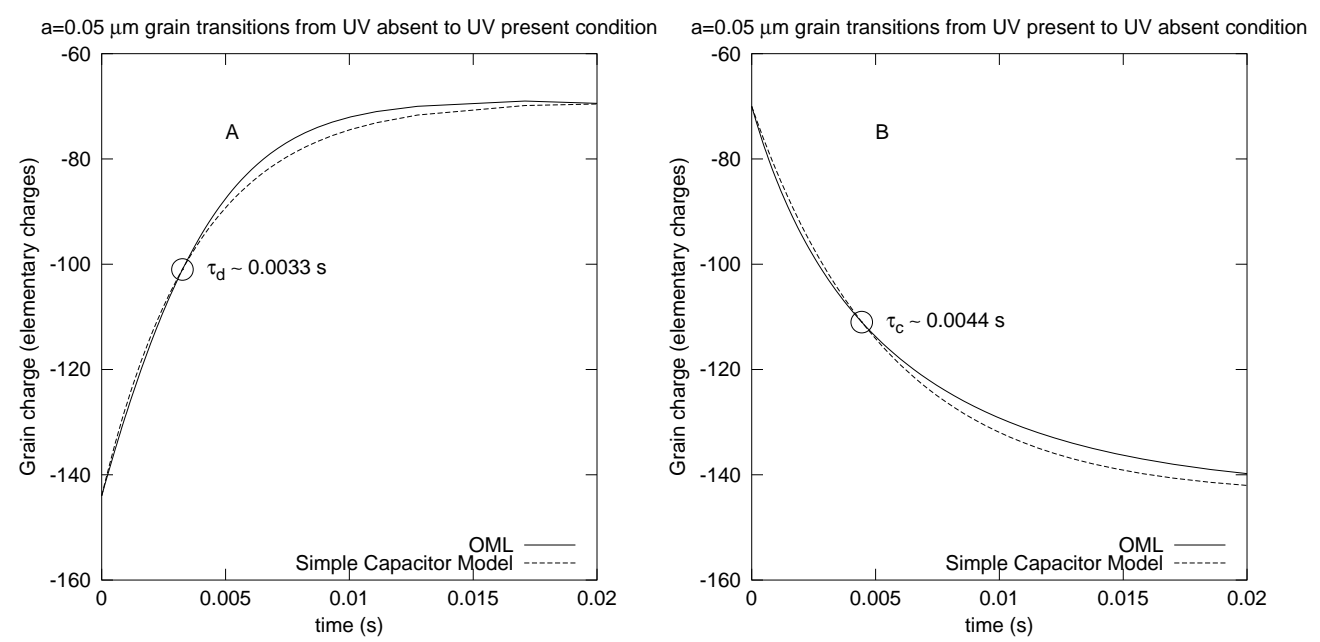

Figure 5.4: Charge evolution for $a=0.05 \mu \mathrm{m}$ grain in the OML and capacitor approximation. A: Charge evolution of a dust grain as it transitions from the UV-absent to UV-present region. The UV flux is $4 \times 10^{18} \mathrm{~m}^{-2} \mathrm{~s}^{-1}$, which yields a photo-electron current of $5.03 \times 10^{-15} \mathrm{~A}$. The capacitor model reaches $\frac{1}{\mathrm{e}}\left(q_{2}-q_{1}\right)$ at the same time as the OML charge model, although it is a different function of time. B: Charge evolution of a dust grain as it transitions from the UV-present to UV-absent region. 


\section{Trajectory}

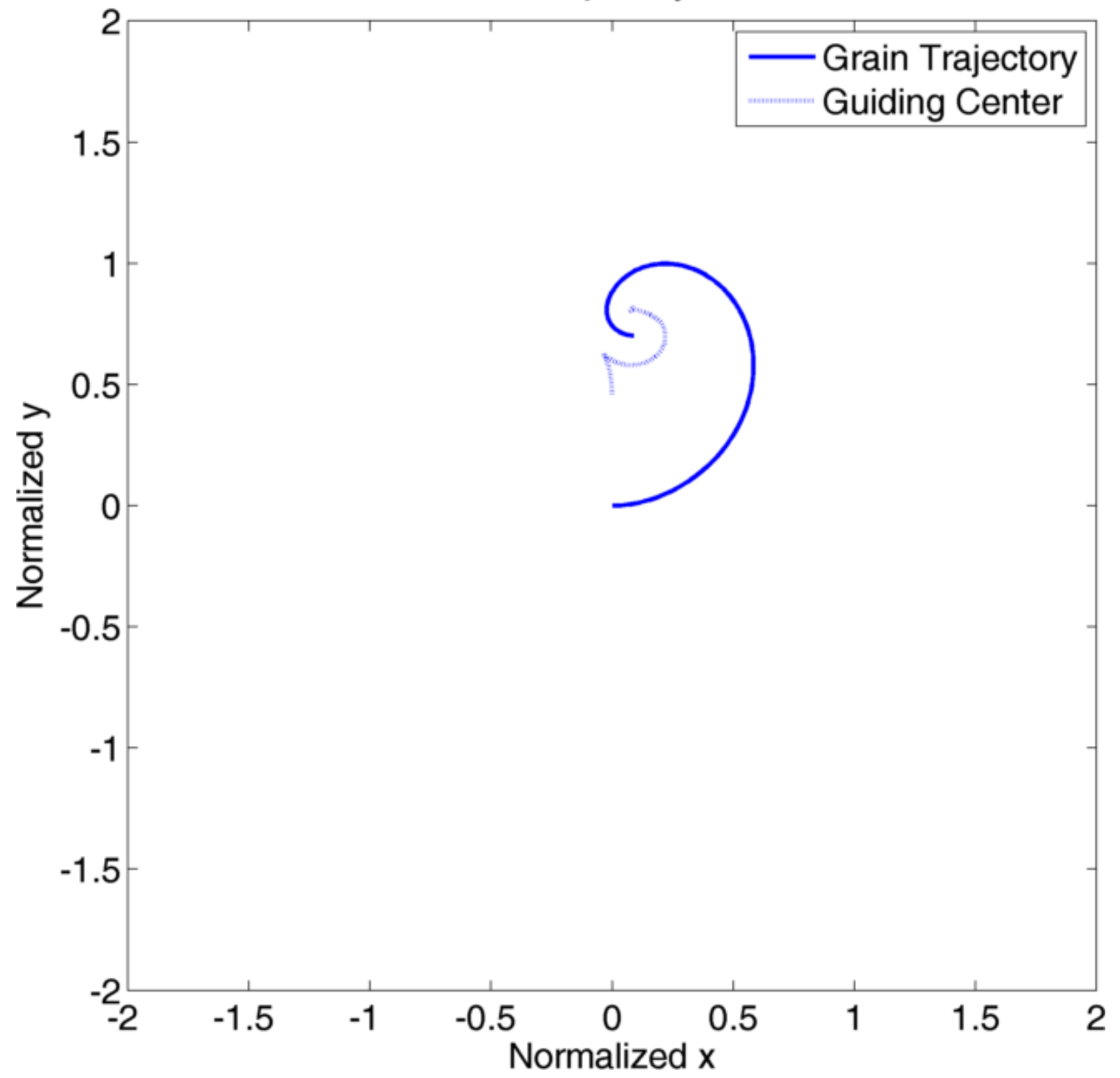

Figure 5.5: Example trajectory of a grain that starts at the origin, but does not transition back to the $x<0$ or $q_{1}$ region due to excessive drag. In this example, $\omega_{1} / \nu_{d n}=3$, so when the grain goes from $x<0$ to $x>0$ when $t \approx 0$, this ratio is barely above unity. For this plot, Argon ions are assumed, $q_{1} / q_{2}=1.74, T_{e} / T_{i}=200, a / R_{L e}=0.1, \lambda_{D} / a=10^{5}, \lambda_{i} / a=5, N_{D e}=10^{3}$, $m_{d} / m_{e}=10^{20}$, and $\tau_{g} / \tau_{c} \approx 20$ (non-instantaneous charging). 


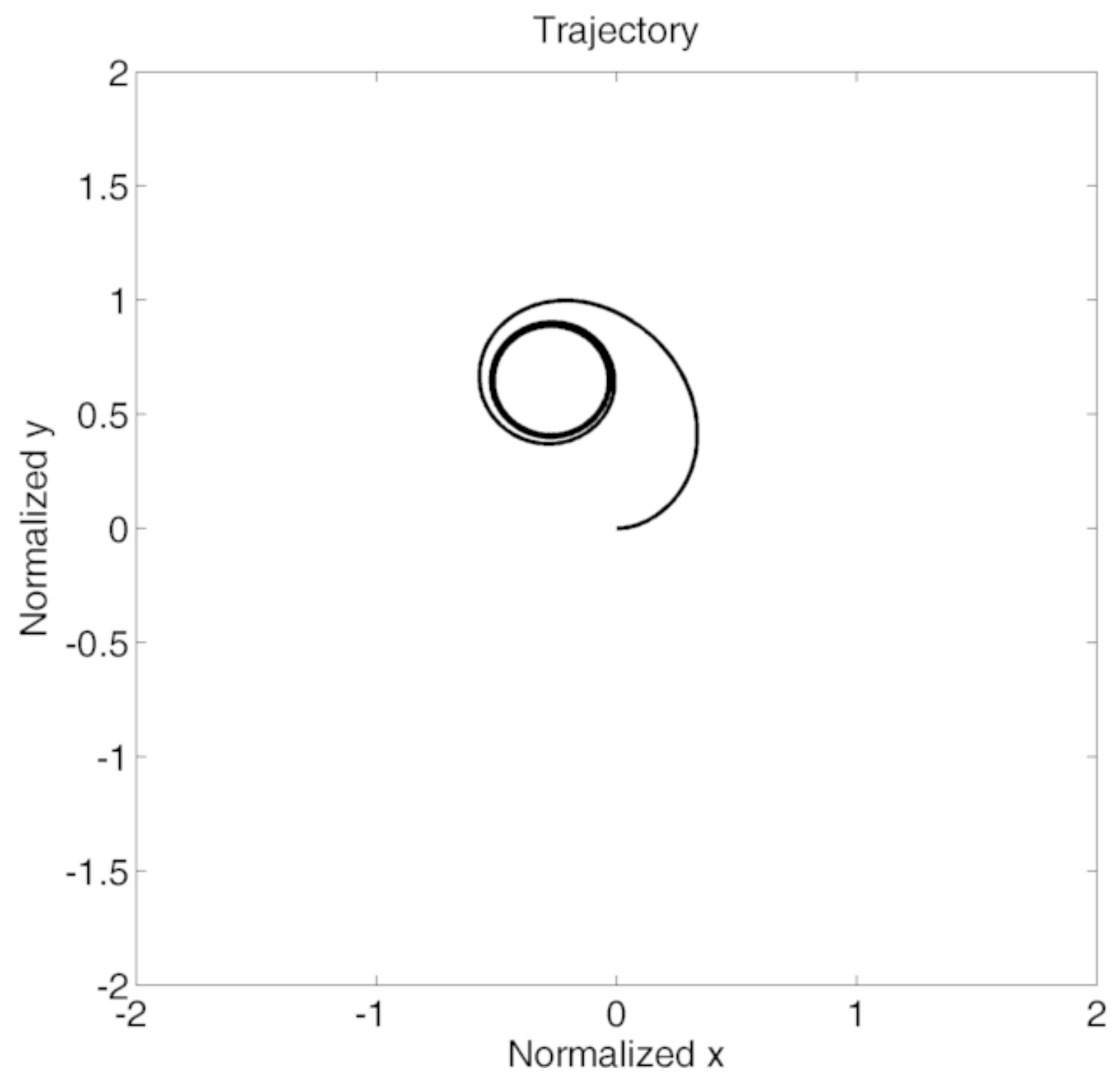

Figure 5.6: Simulated example trajectory of a grain that starts at the origin, transitions to the $x<0$ half-plane, and does not re-enter the $x>0$ region. This situation generally occurs for small values of $\tau_{g} / \tau_{c}$. Argon ions are assumed for this drag-absent simulation plot, and $a=10^{-7} \mathrm{~m}$, $\rho_{d}=90 \mathrm{~kg} \mathrm{~m}^{-3}, n_{0}=10^{14} \mathrm{~m}^{-3} T_{e}=5 \mathrm{eV}, T_{i}=0.025 \mathrm{eV}$. The dimensionless numbers for this trajectory are $q_{1} / q_{2}=1.74, T_{e} / T_{i}=200, a / R_{L e}=0.1, \lambda_{D} / a=10^{3}, \lambda_{i} / a=10^{5}, N_{D e}=10^{3}$, $m_{d} / m_{e}=10^{12}$, and $\tau_{g} / \tau_{c} \approx 20$ (non-instantaneous charging). 


\section{Trajectory}

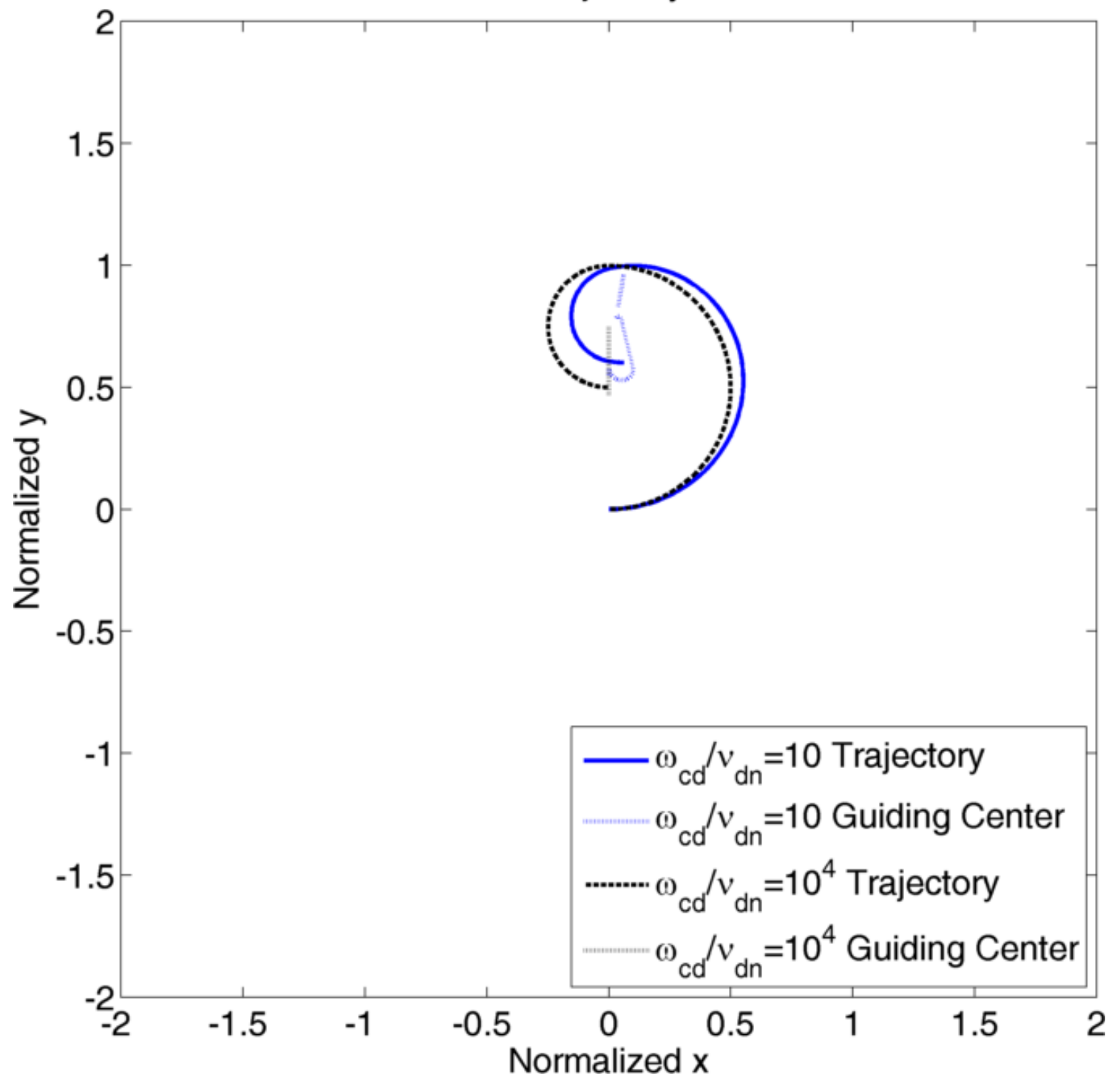

Figure 5.7: This figure shows the possibility of dust grains re-entering the $x=0$ region when drag is present. This is shown clearly in the solid blue trajectory $\omega_{c d} / \nu_{d n}=10$, while it does not occur for the drag-absent trajectory with $\omega_{c d} / \nu_{d n}=10^{4}$. Other than $\omega_{c d} / \nu_{d n}$ ratios, both cases have identical parameters; Argon ions are assumed, $q_{1} / q_{2}=2, T_{e} / T_{i}=200, a / R_{L e}=0.1, \lambda_{D} / a=10^{5}$, $\lambda_{i} / a=5, N_{D e}=10^{3}, m_{d} / m_{e}=10^{22}$, and $\log \left(\tau_{g} / \tau_{c}\right)=5.3$. When drag is present, this situation generally occurs for larger values of $\tau_{g} / \tau_{c}$. 

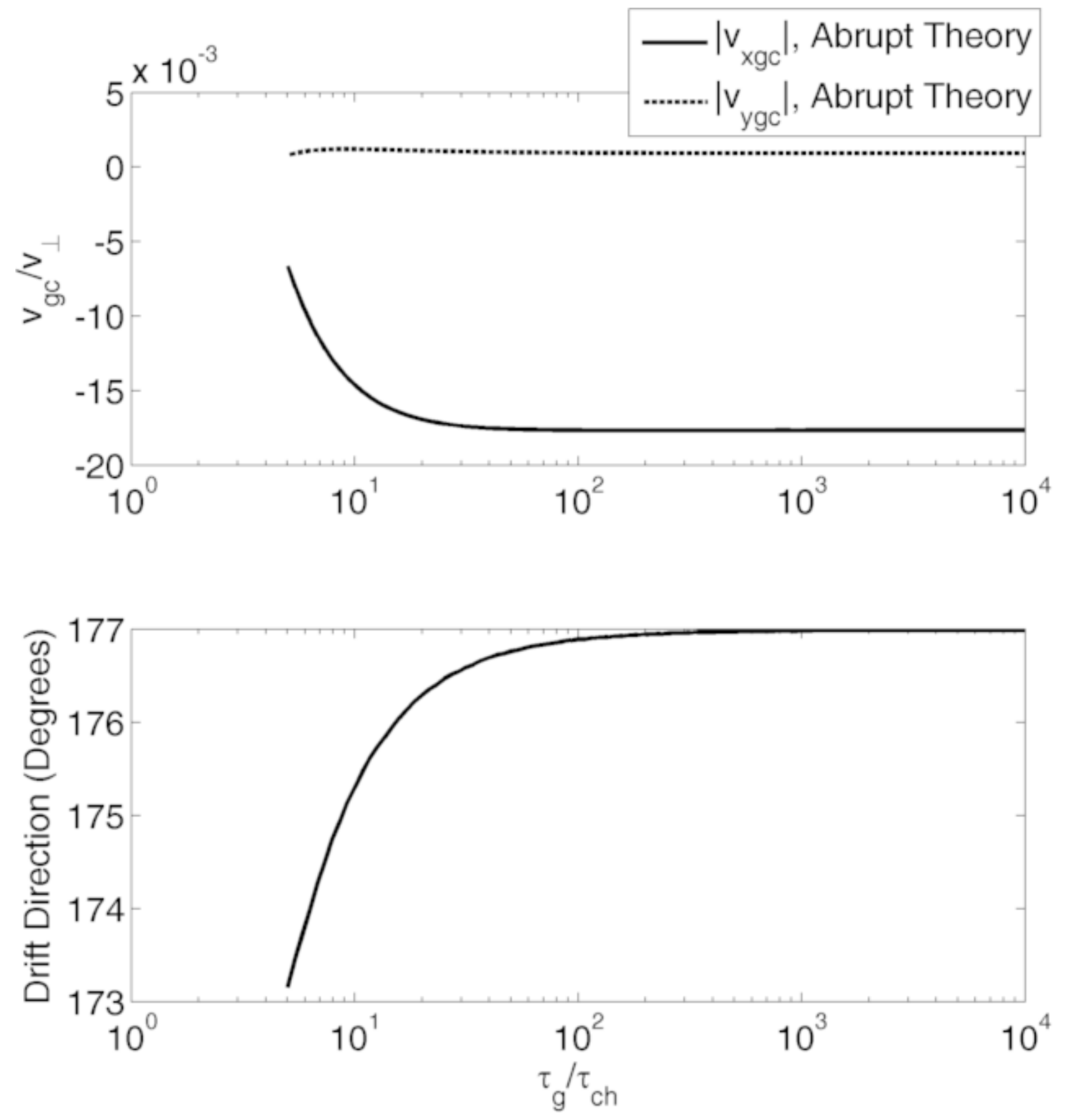

Figure 5.8: Guiding center drift magnitude dependence on the gyration parameter $\omega_{c d} / \nu_{d n}$ for the parameters $T_{e} / T_{i}=200, q_{1} / q_{2}=1.74, a / R_{L e}=0.1, \lambda_{D} / a=10^{5}, \lambda_{i} / a=5, N_{D e}=10^{3}$, $m_{d} / m_{e}=10^{12}$, and $\log \left(\tau_{g} / \tau_{c}\right)=1$. The abscissa is mislabelled; $\tau_{g} / \tau_{c}$ should be $\omega_{c d} / \nu_{d n}$. 


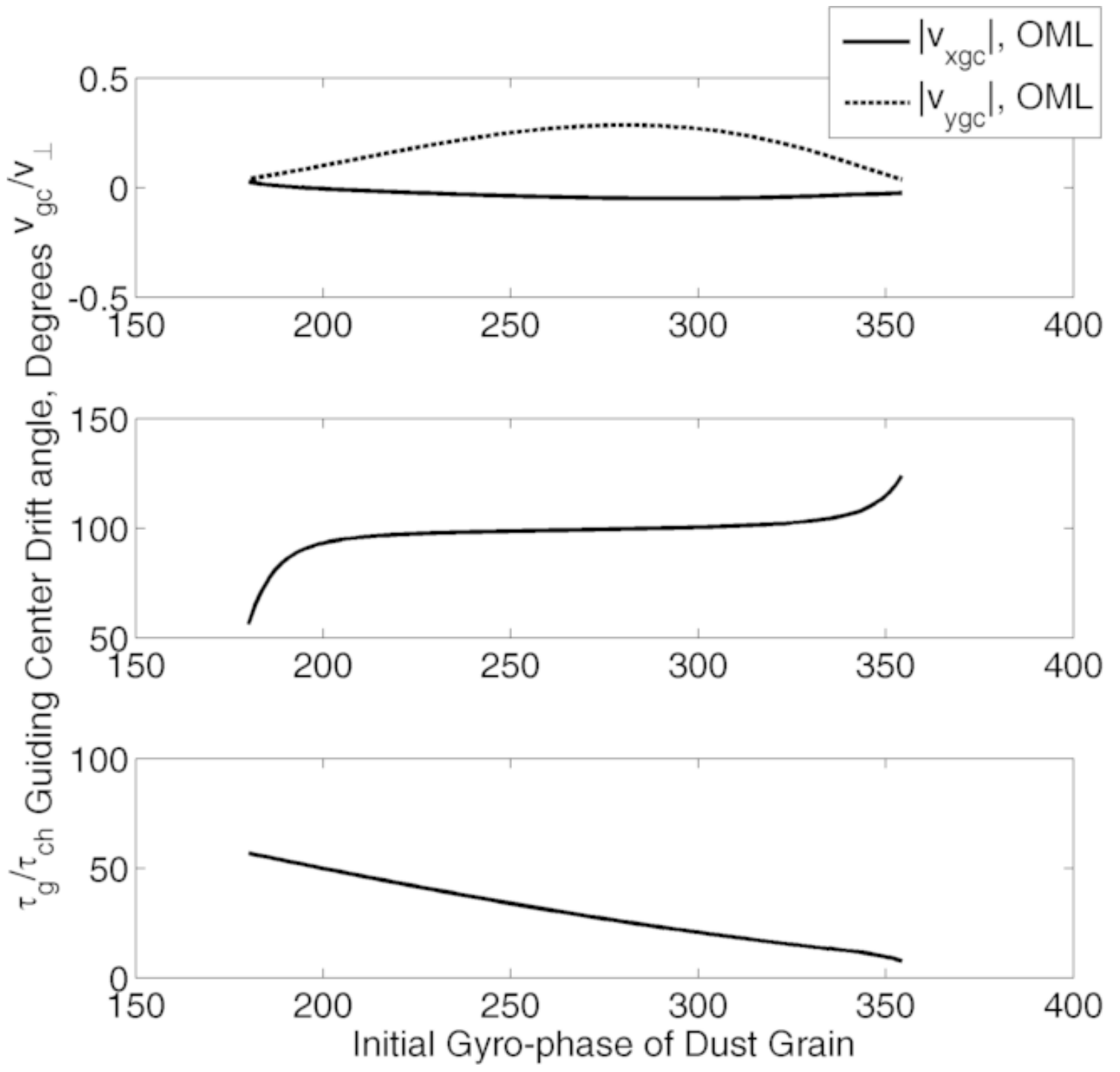

Figure 5.9: Guiding center drift magnitude and direction dependence on the initial gyro-phase angle $\theta_{0}$. In this figure, drag is absent $\left(\omega_{c d} / \nu_{d n}=10^{4}\right)$, UV illumination is the source of abrupt inhomogeneity, $q_{1} / q_{2}=2$, Argon ions are assumed, $T_{e} / T_{i}=200, a / R_{L e}=0.1, \lambda_{D} / a=10^{3}$, $\lambda_{i} / a=10^{5}, N_{D e}=2 \times 10^{4}$, and $m_{d} / m_{e}=10^{12}$. The top panel shows the guiding center drift magnitudes, the middle panel shows the guiding center drift angle relative to the $x$-axis, and the bottom panel shows the $\tau_{g} / \tau_{c}$ ratio as a function of the initial gyro-phase angle. 

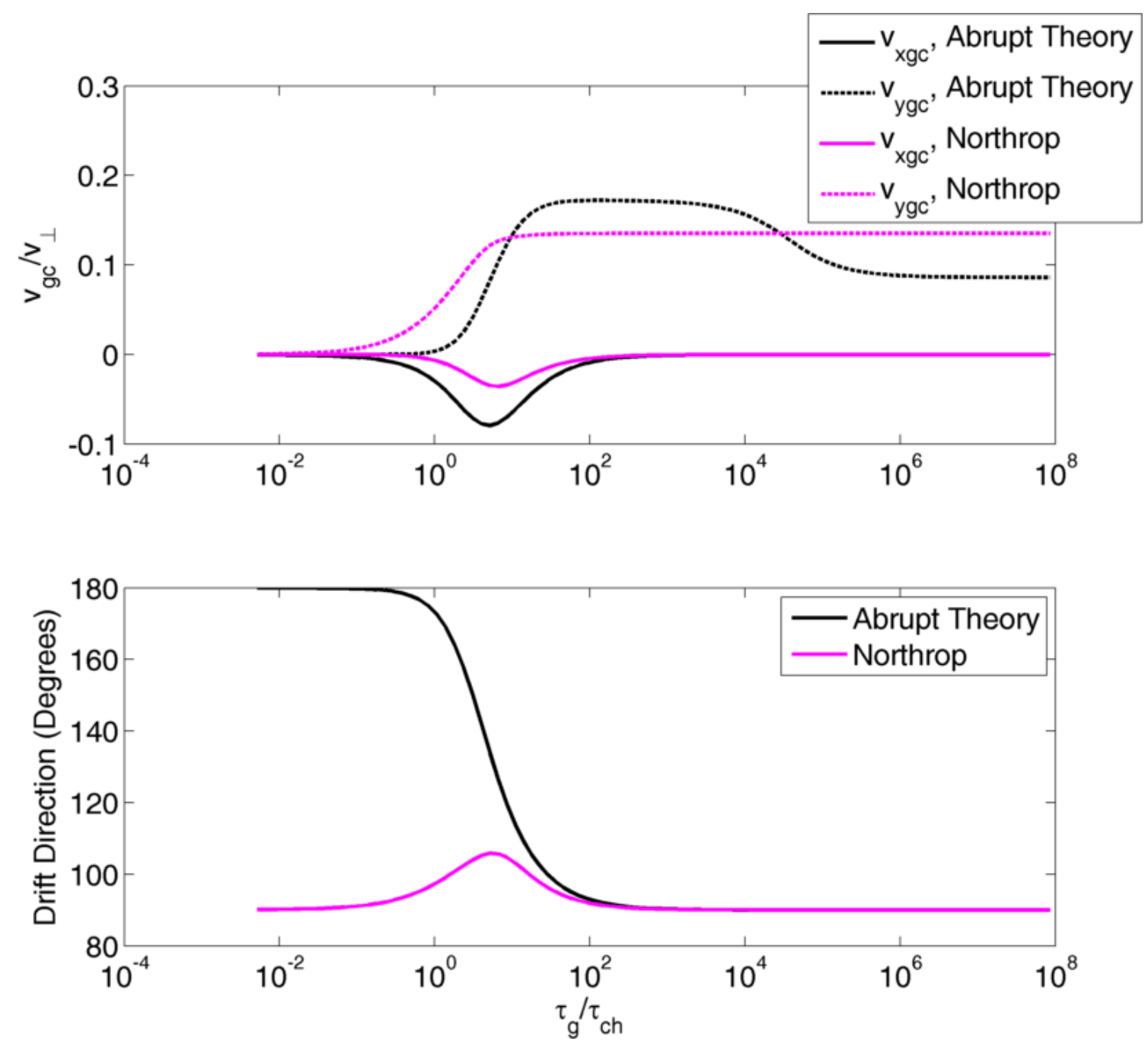

Figure 5.10: Gyro-phase drift magnitude and direction for the first gyro-cycle as a function of the ratio $\tau_{g} / \tau_{c}$ for the abrupt theory and Northrop's prediction. The absolute values of the velocities are plotted in the topmost plot, while the direction is supplied by the bottom panel. In this figure, UV illumination is the source of inhomogeneity, Argon ions are assumed, $T_{e} / T_{i}=200, a / R_{L e}=0.1$, $\lambda_{D} / a=10^{3}, \lambda_{i} / a=10^{5}, N_{D e}=2 \times 10^{4}, q_{1} / q_{2}=2$, and $10^{10} \leq m_{d} / m_{e} \leq 10^{20}$. The grain drifts in the negative $\hat{y}$-direction for $10^{-2}<\tau_{g} / \tau_{c} \leq 2$. 

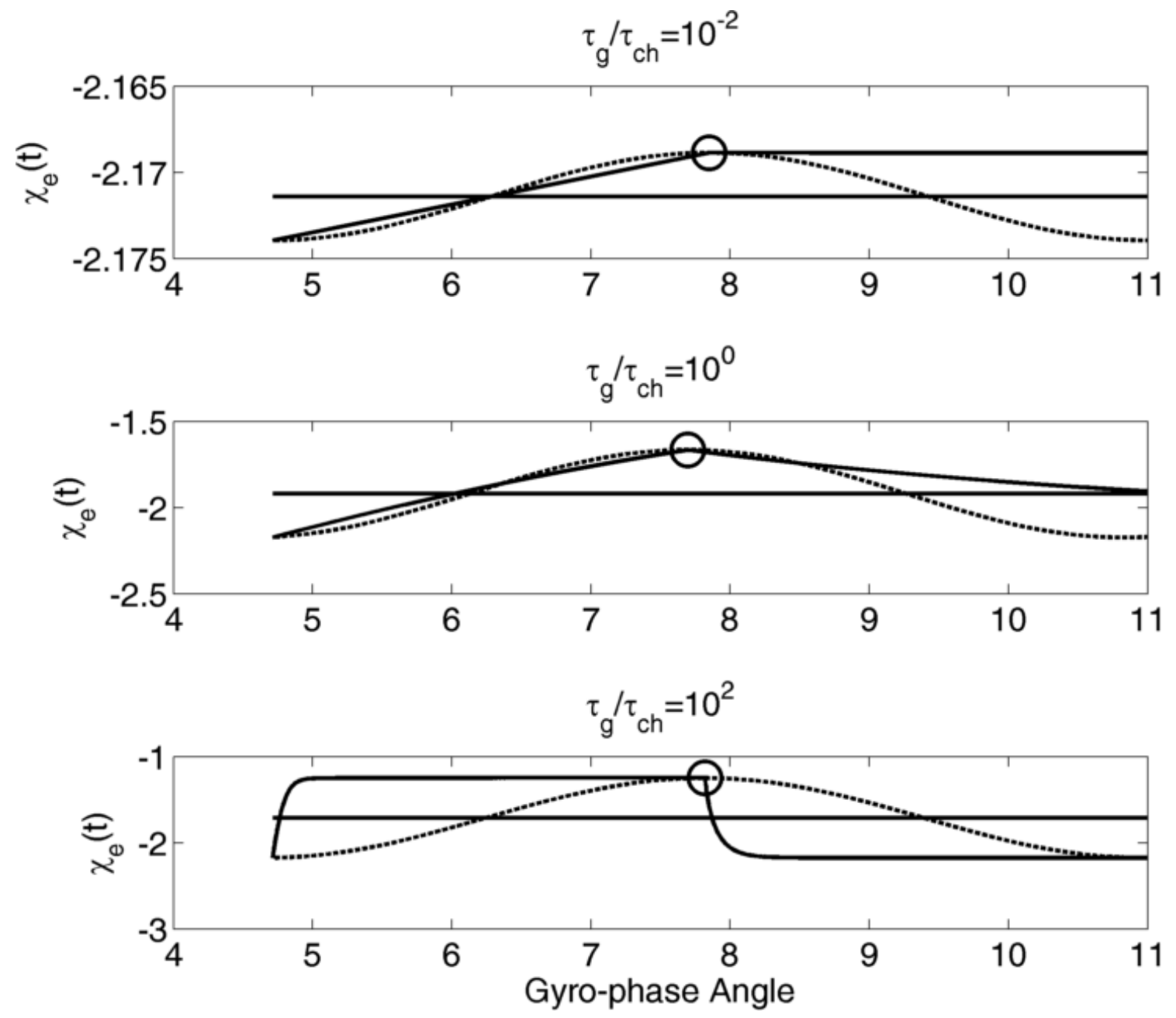

Figure 5.11: This figure shows examples of fitting a sinusoidal charge variation to $q(t)$ in order to obtain the gyro-phase drift vector using Northrop's method for the case of abrupt inhomogeneity. In this figure, the dimensionless surface potential $\chi_{e}(t)=e q(t) /\left(C_{d} k_{b} T_{e}\right)$ is shown instead of $q(t)$. Each plot shows $q(t)$ and a sinusoidal fit to a selected data point from figure 5.10. 


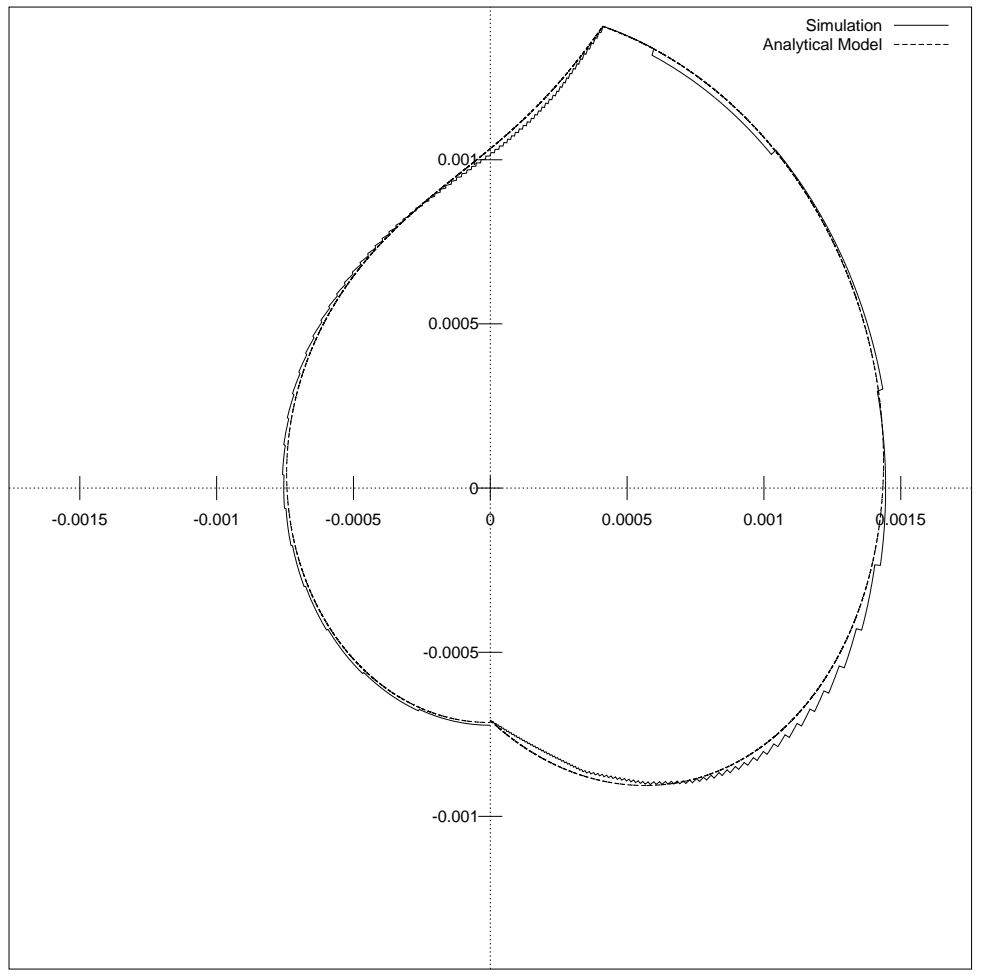

Figure 5.12: Radial distance from the origin is the gyro-radius as a function of gyro-angle in meters. The grain size is $a=0.05 \mu \mathrm{m}$. In the semi-analytical model, $q(t)$ is continuous, while for the simulations, $q(t)$ is discrete. Despite this major difference, the semi-analytical model and the simulation closely agree. The quantitiy $\frac{\mathrm{d} q}{\mathrm{~d} t}$ is discontinuous at $0^{\circ}$ and $\approx 80^{\circ}$ because the current to the grain abruptly changes at both of these phase angles. 

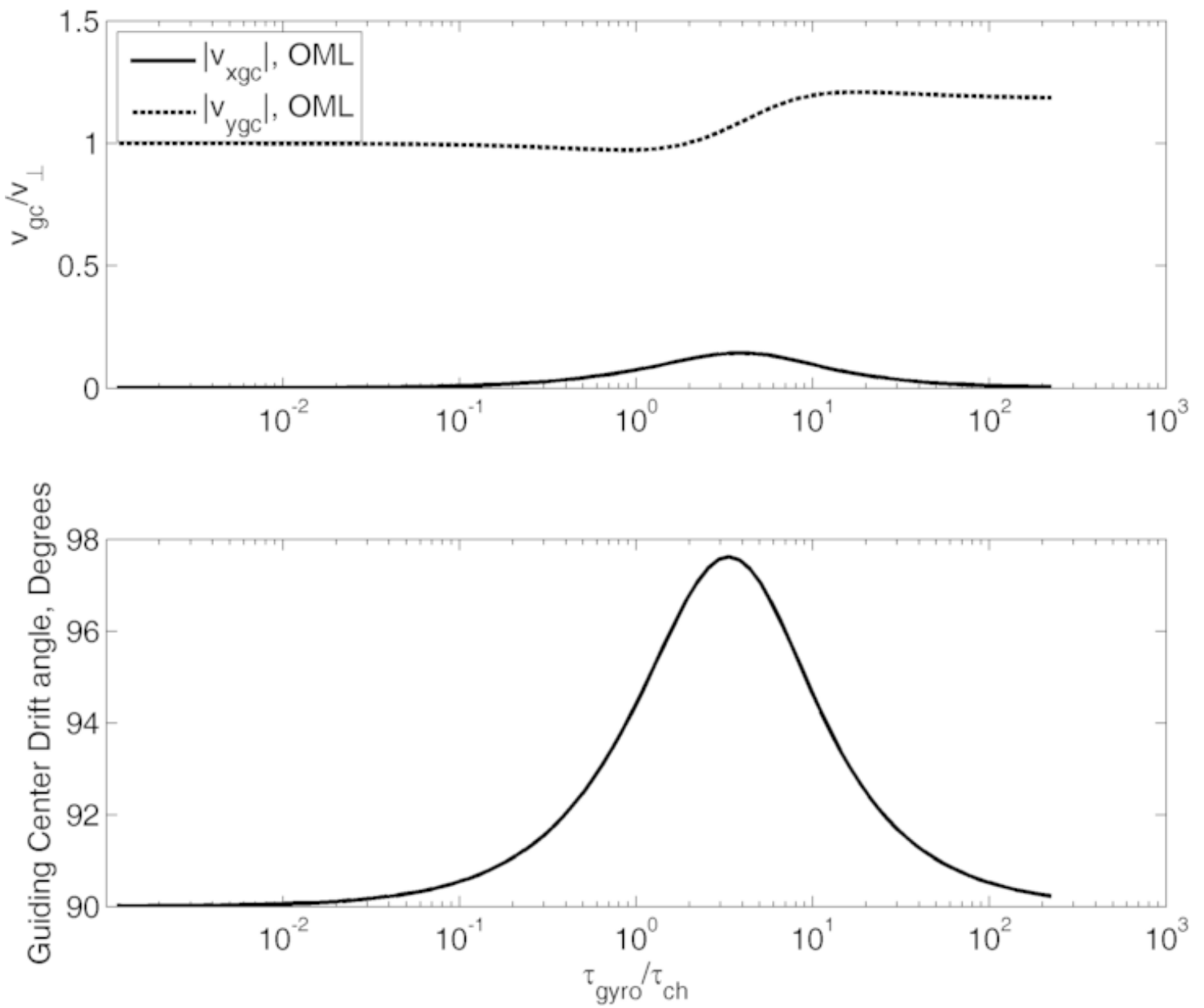

Figure 5.13: Gyro-phase drift magnitude and direction for the first gyro-cycle as a function of the ratio $\tau_{g} / \tau_{c}$ with an electric field. In this figure, drag is absent $\left(\omega_{c d} / \nu_{d n}=10^{4}\right)$, UV illumination is the source of abrupt inhomogeneity, $q_{1} / q_{2}=2$, Argon ions are assumed, $T_{e} / T_{i}=200, a / R_{L e}=0.1$, $\lambda_{D} / a=10^{3}, \lambda_{i} / a=10^{5}, N_{D e}=2 \times 10^{4}$, and $m_{d} / m_{e}$ is swept from $10^{10}$ to $10^{20}$. 

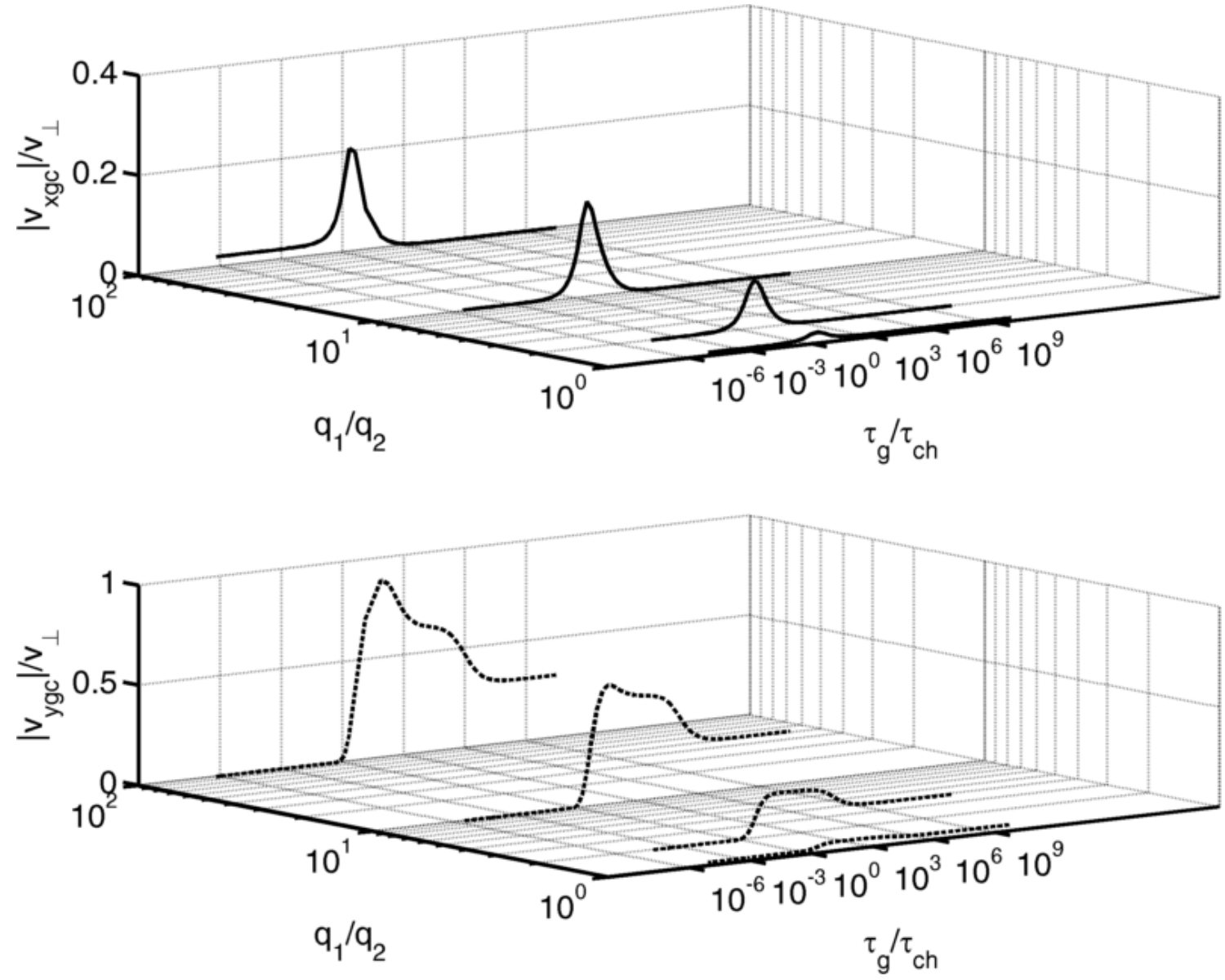

Figure 5.14: Gyro-phase drift magnitude and direction for the first gyro-cycle as a function of the ratio $\tau_{g} / \tau_{c}$ for different values of $q_{1} / q_{2}$. 

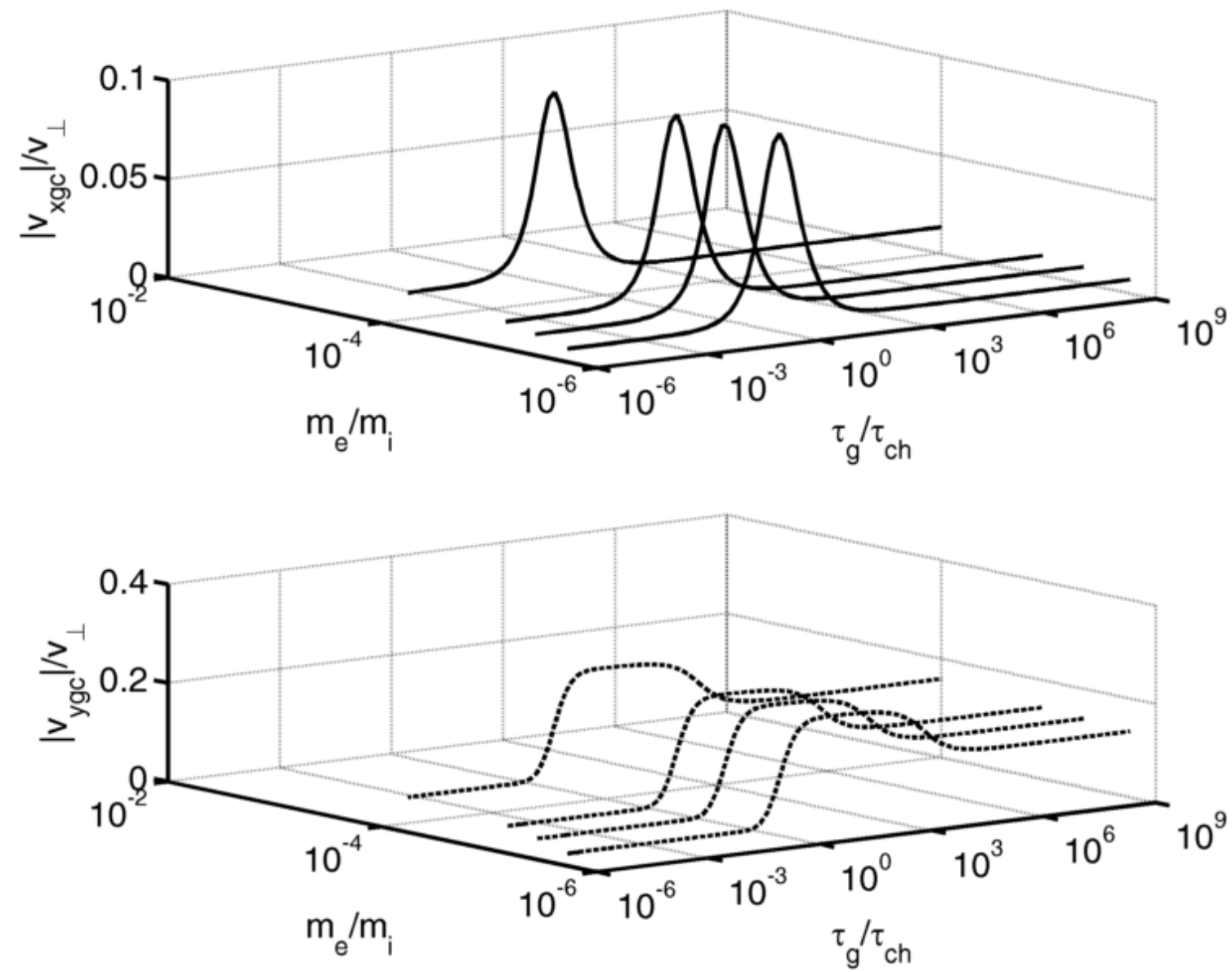

Figure 5.15: Gyro-phase drift magnitude for the first gyro-cycle as a function of the ratio $\tau_{g} / \tau_{c}$ for different values of $m_{e} / m_{i}$ when $q_{1} / q_{2}=2$ is held constant. The top panel corresponds to the guiding center velocity along the $\hat{x}$-direction and the bottom panel corresponds to the guiding center velocity along the $\hat{y}$-direction. The values of $m_{e} / m_{i}$ chosen correspond to Hydrogen, Nitrogen, Argon, and Xenon plasmas in ascending order. Evident in this figure is that all of the plots have exactly the same shape and same values, but lower values of $m_{e} / m_{i}$ allow access to smaller values of $\tau_{g} / \tau_{c}$. For this plot, $\lambda_{D} / a=10^{3}, \lambda_{i} / a=10^{5}, N_{D e}=10^{4}, a / R_{L e}=10^{-1}$, and $T_{e} / T_{i}=200$. 

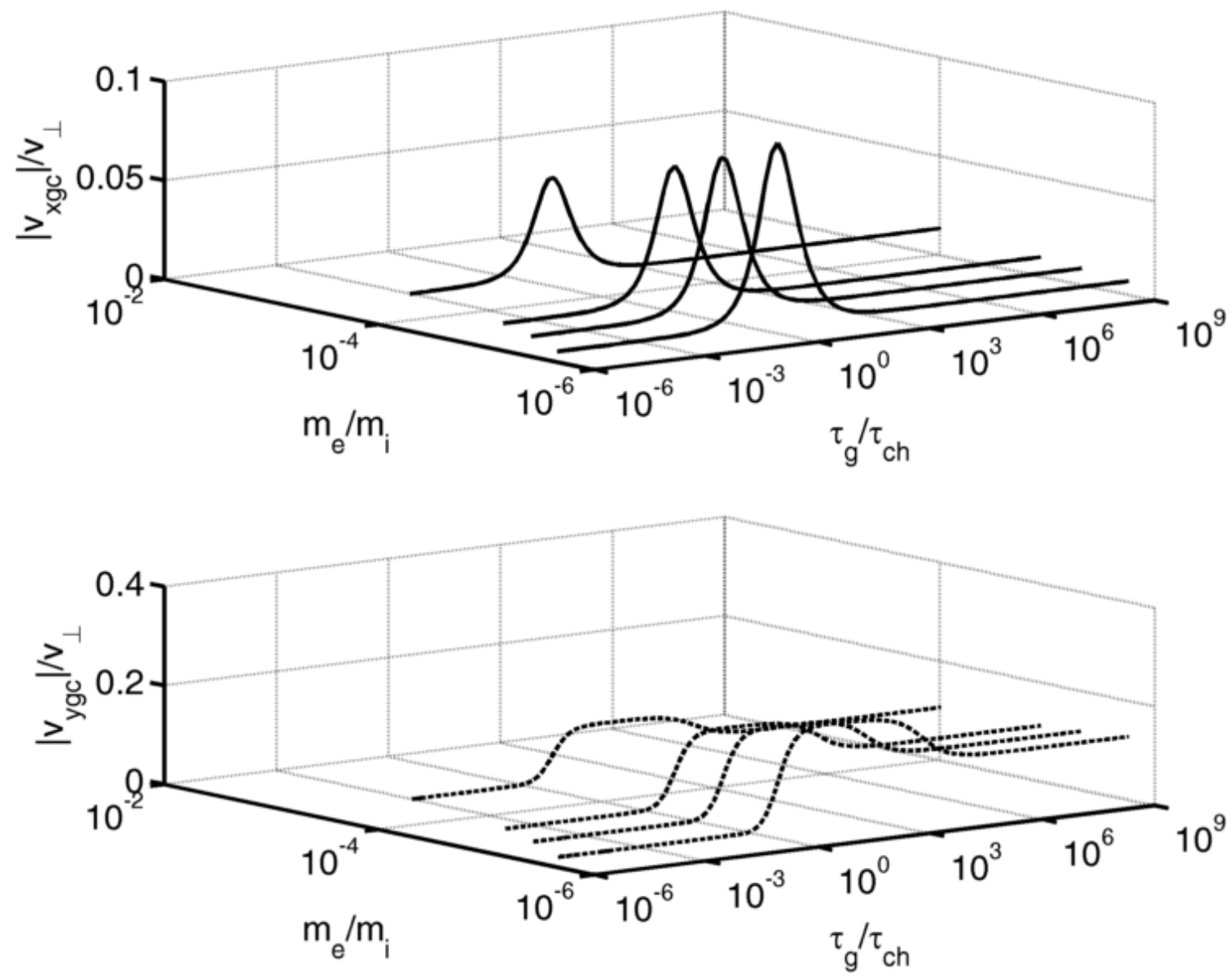

Figure 5.16: Gyro-phase drift magnitude for the first gyro-cycle as a function of the ratio $\tau_{g} / \tau_{c}$ for different values of $m_{e} / m_{i}$ when the normalized photo-current $f_{u v} /\left(n_{e} v_{\text {the }}\right)=0.25$ is held constant. The top panel corresponds to the guiding center velocity along the $\hat{x}$-direction and the bottom panel corresponds to the guiding center velocity along the $\hat{y}$-direction. The values of $m_{e} / m_{i}$ chosen correspond to Hydrogen, Nitrogen, Argon, and Xenon plasmas in ascending order, which produce $q_{1} / q_{2}$ values of $1.4,1.61,1.74$, and 1.93 respectively. Like in figure 5.15 , lower values of $m_{e} / m_{i}$ allow access to smaller values of $\tau_{g} / \tau_{c}$, but in these plots the lower values of $m_{e} / m_{i}$ also produce greater drift amplitudes. For this plot, $\lambda_{D} / a=10^{3}, \lambda_{i} / a=10^{5}, N_{D e}=10^{4}, a / R_{L e}=10^{-1}$, and $T_{e} / T_{i}=200$. 

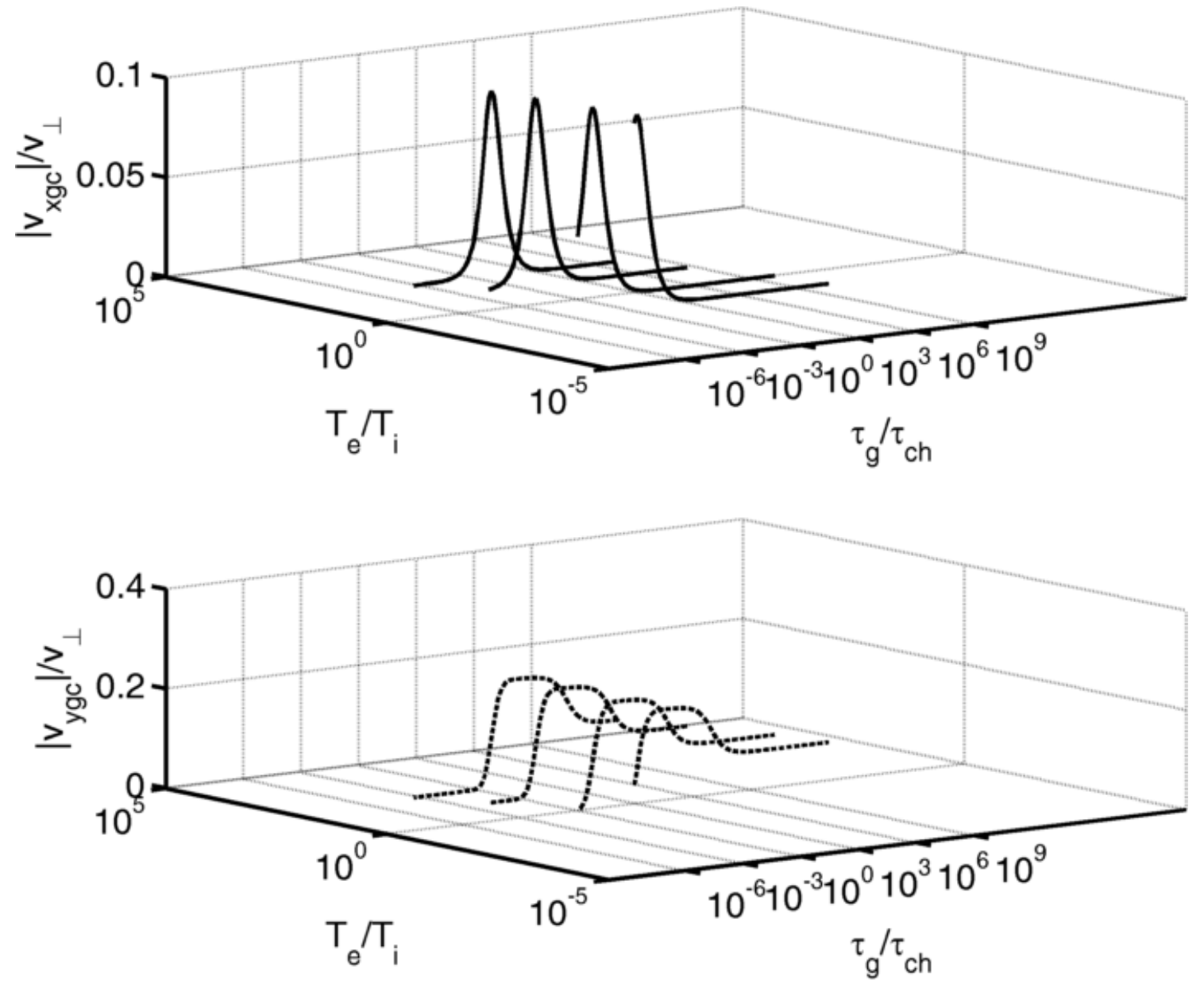

Figure 5.17: Gyro-phase drift magnitude for the first gyro-cycle as a function of the ratio $\tau_{g} / \tau_{c}$ for different values of $T_{e} / T_{i}$ when $q_{1} / q_{2}=2$ is held constant. The top panel corresponds to the guiding center velocity along the $\hat{x}$-direction and the bottom panel corresponds to the guiding center velocity along the $\hat{y}$-direction. The temperature ratios $T_{e} / T_{i}=10^{-1}, T_{e} / T_{i}=10^{0}, T_{e} / T_{i}=2 \times 10^{1}$, and $T_{e} / T_{i}=2 \times 10^{2}$ are shown in ascending order on the $\log \left(T_{e} / T_{i}\right)$ axis. Higher values of $T_{e} / T_{i}$ allow access to smaller values of $\tau_{g} / \tau_{c}$, in a much more dramatic way than seen in smaller values of $m_{e} / m_{i}$ in 5.15. For this plot, hydrogen plasma assumed, $\lambda_{D} / a=10^{3}, \lambda_{i} / a=10^{5}, N_{D e}=10^{4}$, and $a / R_{L e}=10^{-1}$. 

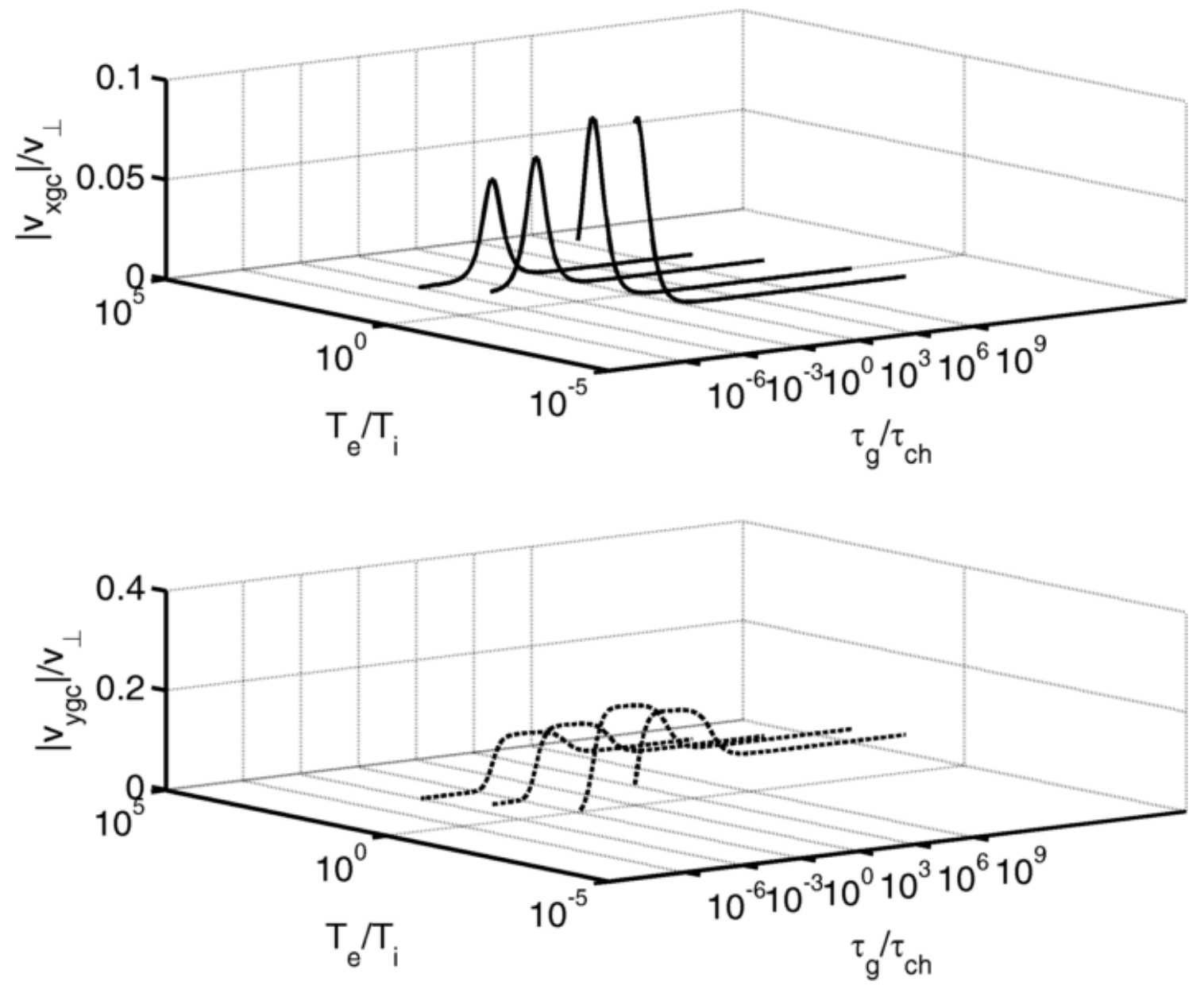

Figure 5.18: Gyro-phase drift magnitude for the first gyro-cycle as a function of the ratio $\tau_{g} / \tau_{c}$ for different values of $T_{e} / T_{i}$ when $f_{u v} /\left(n_{e} v_{t h e}\right)=0.25$ is held constant. The top panel corresponds to the guiding center velocity along the $\hat{x}$-direction and the bottom panel corresponds to the guiding center velocity along the $\hat{y}$-direction. The temperature ratios $T_{e} / T_{i}=10^{-1}, T_{e} / T_{i}=10^{0}$, $T_{e} / T_{i}=2 \times 10^{1}$, and $T_{e} / T_{i}=2 \times 10^{2}$ are shown in ascending order on the $T_{e} / T_{i}$ axis, and these temperature ratios produce $q_{1} / q_{2}$ ratios of $2,1.94,1.57$, and 1.4 respectively. Higher values of $T_{e} / T_{i}$ for constant UV photo-current allow access to smaller values of $\tau_{g} / \tau_{c}$, in a much more dramatic way than seen in smaller values of $m_{e} / m_{i}$ in figure 5.16. For this plot, hydrogen plasma assumed, $\lambda_{D} / a=10^{3}, \lambda_{i} / a=10^{5}, N_{D e}=10^{4}$, and $a / R_{L e}=10^{-1}$. 

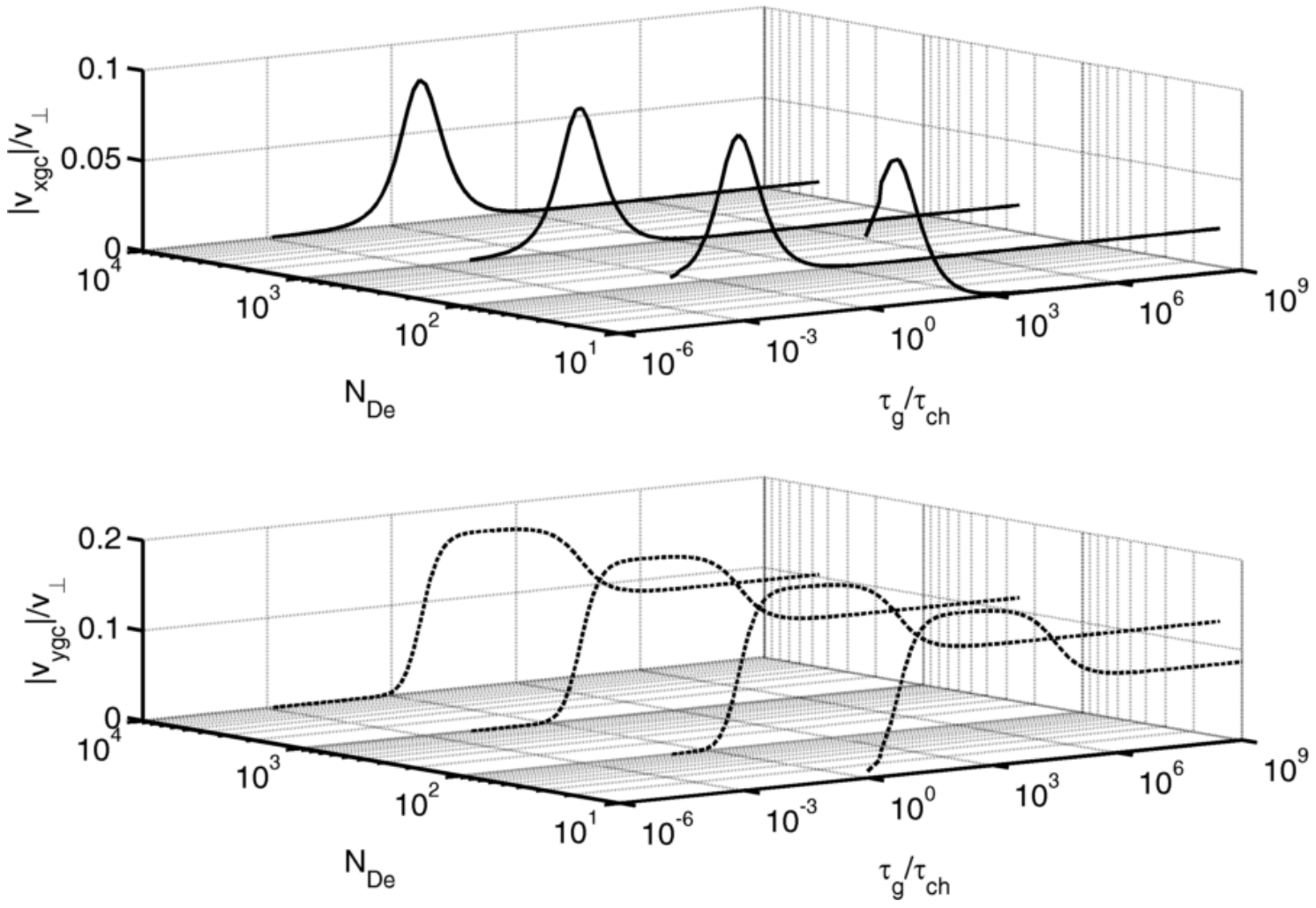

Figure 5.19: Gyro-phase drift magnitude for the first gyro-cycle as a function of the ratio $\tau_{g} / \tau_{c}$ for different values of $N_{D e}$ when $v=f_{u v} /\left(n_{e} v_{t h e}\right)=0.25$ is held constant. The top panel corresponds to the guiding center velocity along the $\hat{x}$-direction and the bottom panel corresponds to the guiding center velocity along the $\hat{y}$-direction. Higher values of $N_{D e}$ allow access to smaller values of $\tau_{g} / \tau_{c}$. For this plot, hydrogen plasma assumed, $\lambda_{D} / a=10^{3}, \lambda_{i} / a=10^{5}, T_{e} / T_{i}=200$, and $a / R_{L e}=10^{-1}$. 

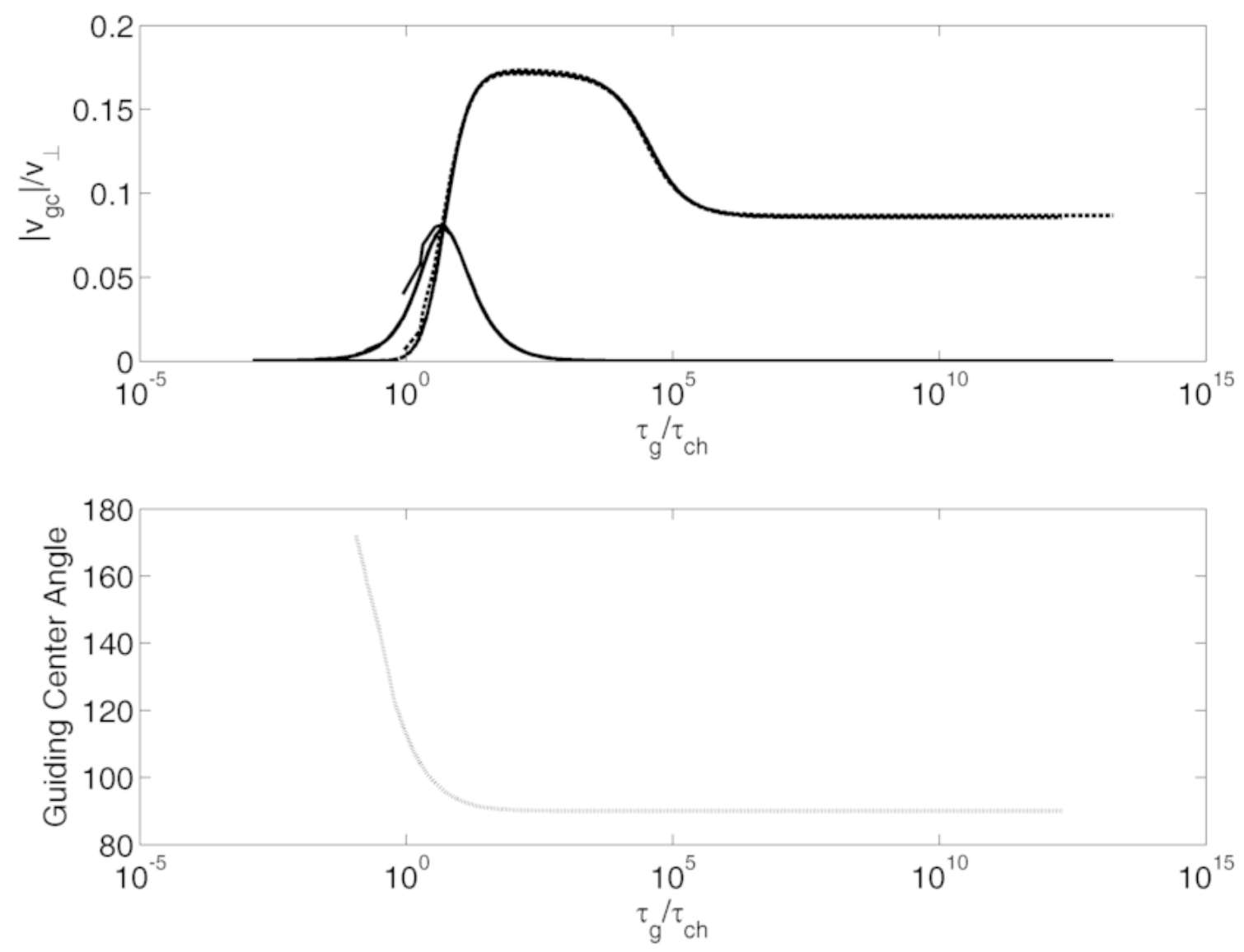

Figure 5.20: Gyro-phase drift magnitude for the first gyro-cycle as a function of the ratio $\tau_{g} / \tau_{c}$ for different values of $N_{D e}$ when $f_{u v} /\left(n_{e} v_{t h e}\right)=0.25$ is held constant. In the top panel, solid curves correspond to $\left|v_{x g c}\right|$, the guiding center velocity long the $\hat{x}$-direction, and the dashed curves correspond to $\left|v_{x g c}\right|$, the guiding center velocity along the $\hat{y}$-direction. The plots of $\left|v_{x g c}\right|$ and $\left|v_{x g c}\right|$ overlap and are identical for different values of $N_{D e}$, showing that the gyro-phase drift magnitude and direction is not affected by the $N_{D e}$ parameter. The bottom panel shows the guiding center drift angle with respect to the $x$-axis in the dust grain trajectory configuration space. The guiding center drift is calculated for electron magnetization parameter values $a / R_{L e}=10^{-2}, 10^{-1}, 10^{0}, 10^{1}$, and $10^{2}$, and the $q_{1} / q_{2}$ ratios are given by $1.74,1.74,2.57,2.57$, and 10.14 respectively. The $q_{1} / q_{2}$ ratios are increasing because the current collection regime changes at certain values of the $a / R_{L e}$ parameter. Higher values of $N_{D e}$ allow access to smaller values of $\tau_{g} / \tau_{c}$. For this plot, hydrogen plasma assumed, $\lambda_{D} / a=10^{3}, \lambda_{i} / a=10^{5}, T_{e} / T_{i}=200$, and $a / R_{L e}=10^{-1}$. 

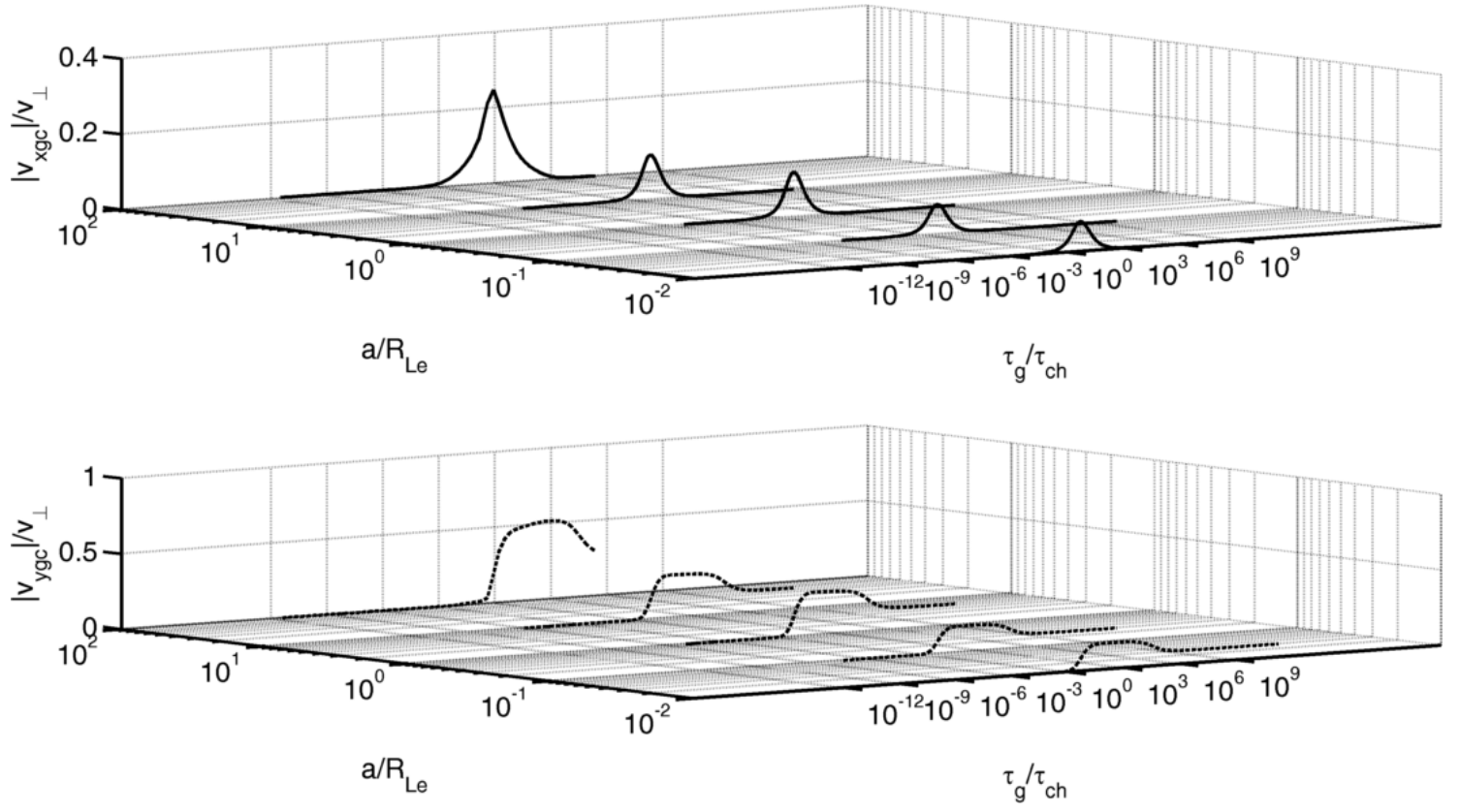

Figure 5.21: Gyro-phase drift magnitude for the first gyro-cycle as a function of the ratio $\tau_{g} / \tau_{c}$ for different values of $a / R_{L e}$ when $f_{u v} /\left(n_{e} v_{t h e}\right)=0.25$ is held constant. The top panel corresponds to the guiding center velocity along the $\hat{x}$-direction and the bottom panel corresponds to the guiding center velocity along the $\hat{y}$-direction. For this plot, Argon plasma assumed, $\omega_{c d} / \nu_{d n}=10^{4}$, $N_{D e}=10^{4}, \lambda_{D} / a=10^{3}, \lambda_{i} / a=10^{5}$, and $T_{e} / T_{i}=200$. The OML and Patacchini-Hutchinson charge models produce the same guiding center drift magnitudes for these parameters. 

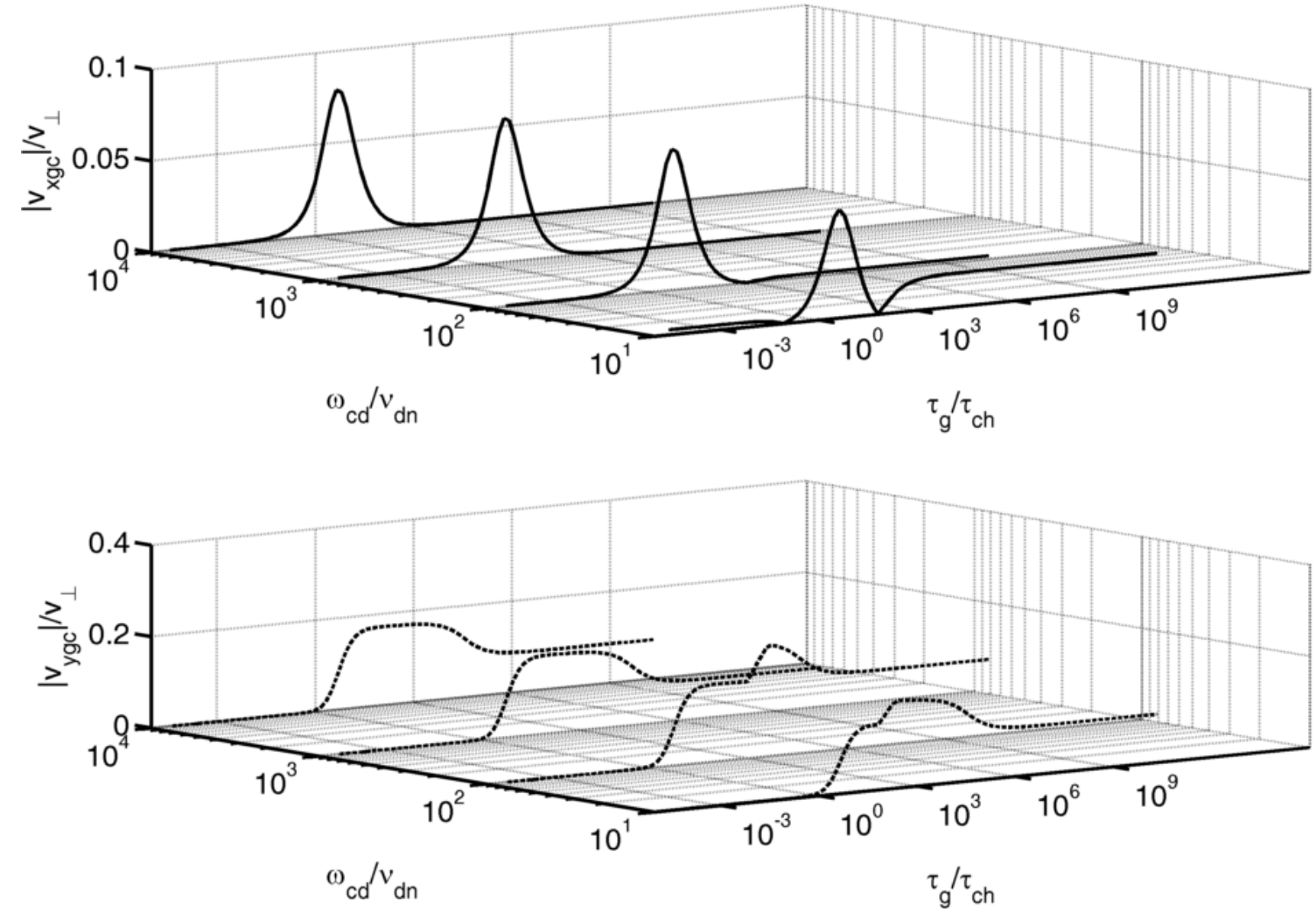

Figure 5.22: Gyro-phase drift magnitude for the first gyro-cycle as a function of the ratio $\tau_{g} / \tau_{c}$ for different values of $\omega_{c d} / \nu_{d n}$ when $f_{u v} /\left(n_{e} v_{t h e}\right)=0.25$ is held constant. The top panel corresponds to the guiding center velocity along the $\hat{x}$-direction and the bottom panel corresponds to the guiding center velocity along the $\hat{y}$-direction. For this plot, hydrogen plasma assumed, $N_{D e}=10^{4}$, $\lambda_{D} / a=10^{3}, \lambda_{i} / a=10^{5}, T_{e} / T_{i}=200$, and $a / R_{L e}=10^{-1}$. 

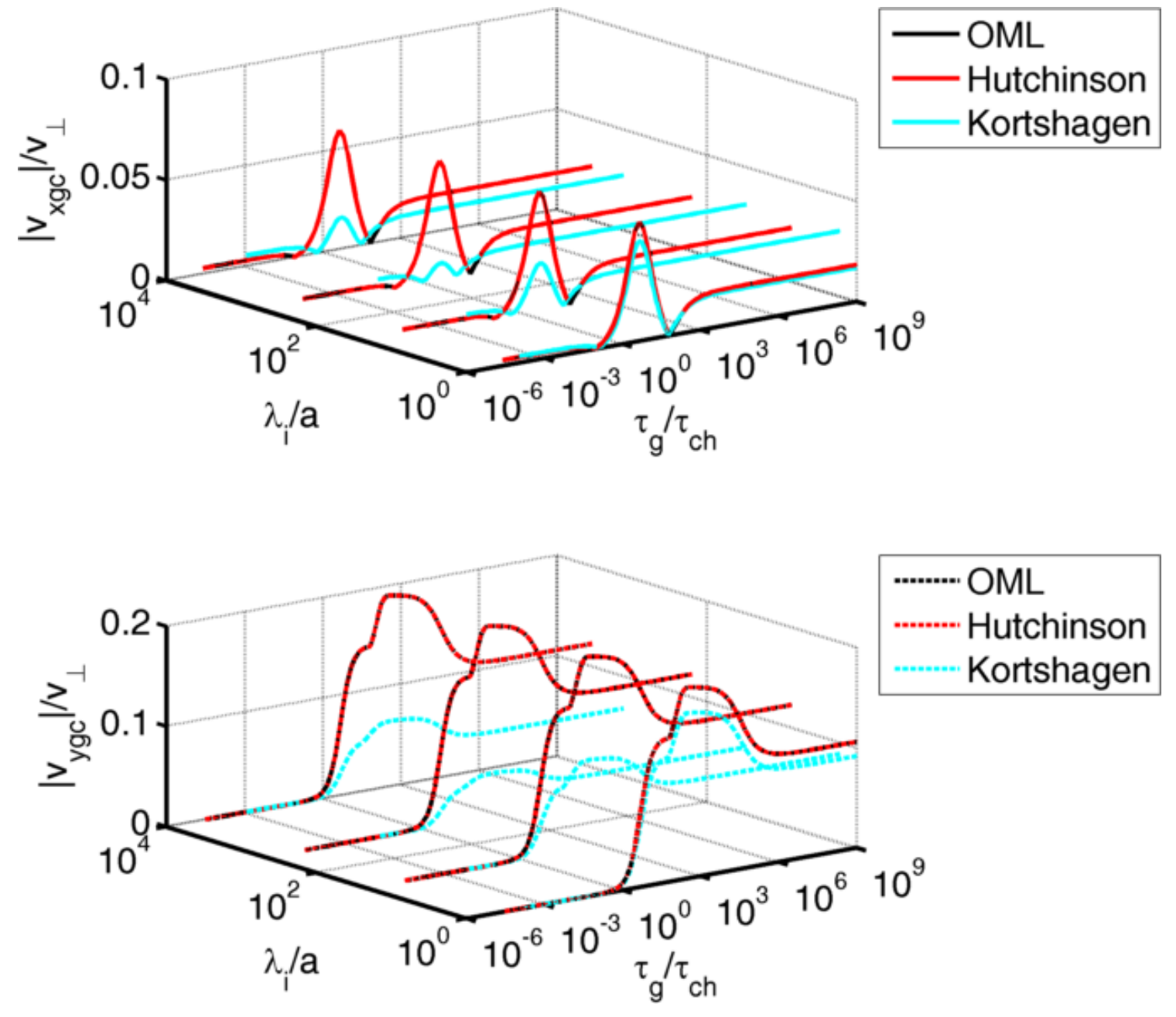

Figure 5.23: Gyro-phase magnitude and direction dependence on $\tau_{g} / \tau_{c}$ for the three different charging models for several values of the Knudsen number $\left(\lambda_{i} / a\right)$. Solid (dashed) red line corresponds to the Patacchini-Hutchinson model guiding center drift $v_{x g c}\left(v_{y g c}\right)$, solid (dashed black line corresponds to the OML model guiding center drift $v_{x g c}\left(v_{y g c}\right)$, and the solid (dashed) cyan line corresponds to the Gatti-Kortshagen model guiding center drift $v_{x g c}\left(v_{y g c}\right)$. The other parameters chosen for an Argon plasma include $f_{U V} /\left(n_{e} v_{t h e}\right)=0.25, \omega_{c d} / \nu_{d n}=10, N_{D e}=10^{4}, a / R_{L e}=0.1$, $\lambda_{D} / a=10^{3}$, and $T_{e} / T_{i}=200$. The OML and Patacchini-Hutchinson charge models predict the same guiding center drift for these parameters. 

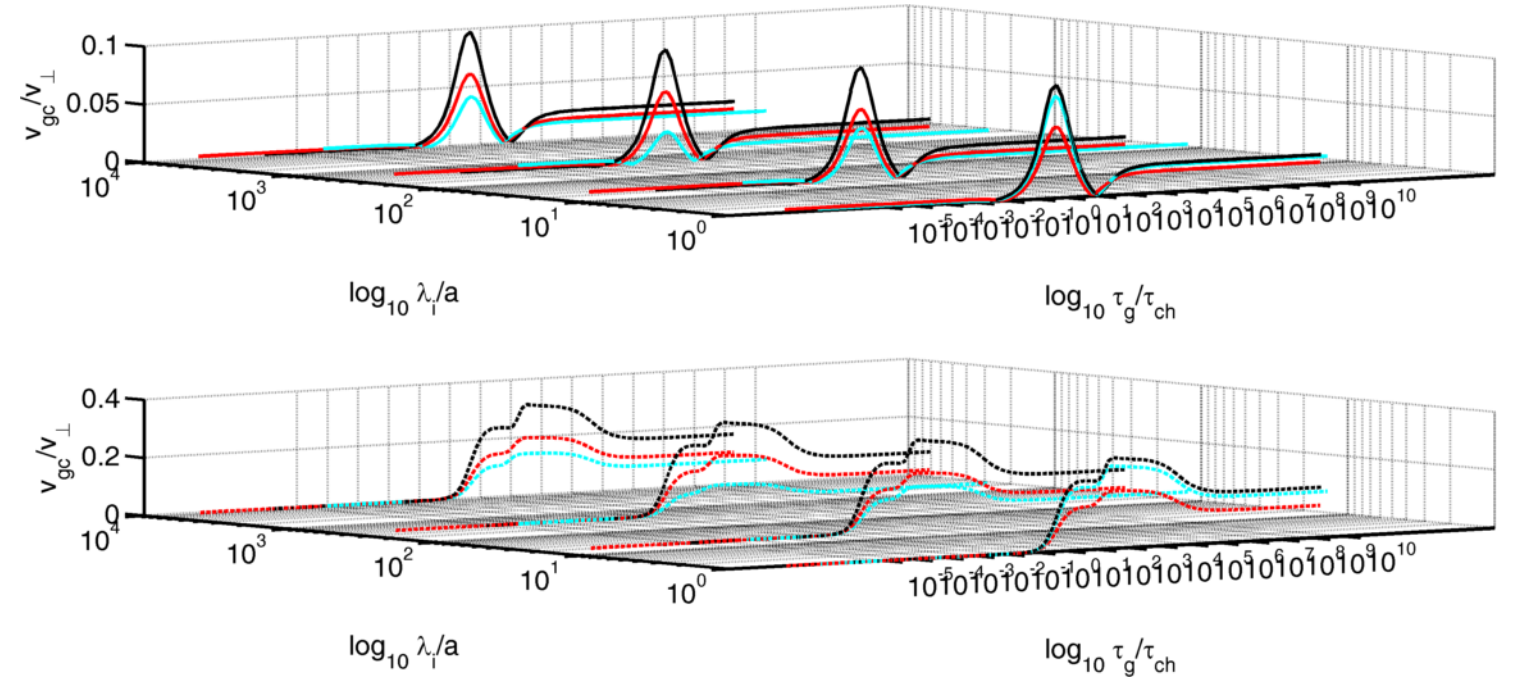

Figure 5.24: Gyro-phase magnitude and direction dependence on $\tau_{g} / \tau_{c}$ for the three different charging models for several values of the Knudsen number $\left(\lambda_{i} / a\right)$. The abscissa is $\tau_{g} / \tau_{c}, \operatorname{not} \log _{10} \frac{\tau_{g}}{\tau_{c}}$. Solid (dashed) red line corresponds to the Patacchini-Hutchinson model guiding center drift $v_{x g c}$ $\left(v_{y g c}\right)$, solid (dashed black line corresponds to the OML model guiding center drift $v_{x g c}\left(v_{y g c}\right)$, and the solid (dashed) cyan line corresponds to the Gatti-Kortshagen model guiding center drift $v_{x g c}\left(v_{y g c}\right)$. The other parameters chosen for an Argon plasma include $v=f_{U V} /\left(n_{e} v_{t h e}\right)=0.25$, $\omega_{c d} / \nu_{d n}=10, N_{D e}=10^{4}, a / R_{L e}=1.1, \lambda_{D} / a=10^{3}$, and $T_{e} / T_{i}=200$. 

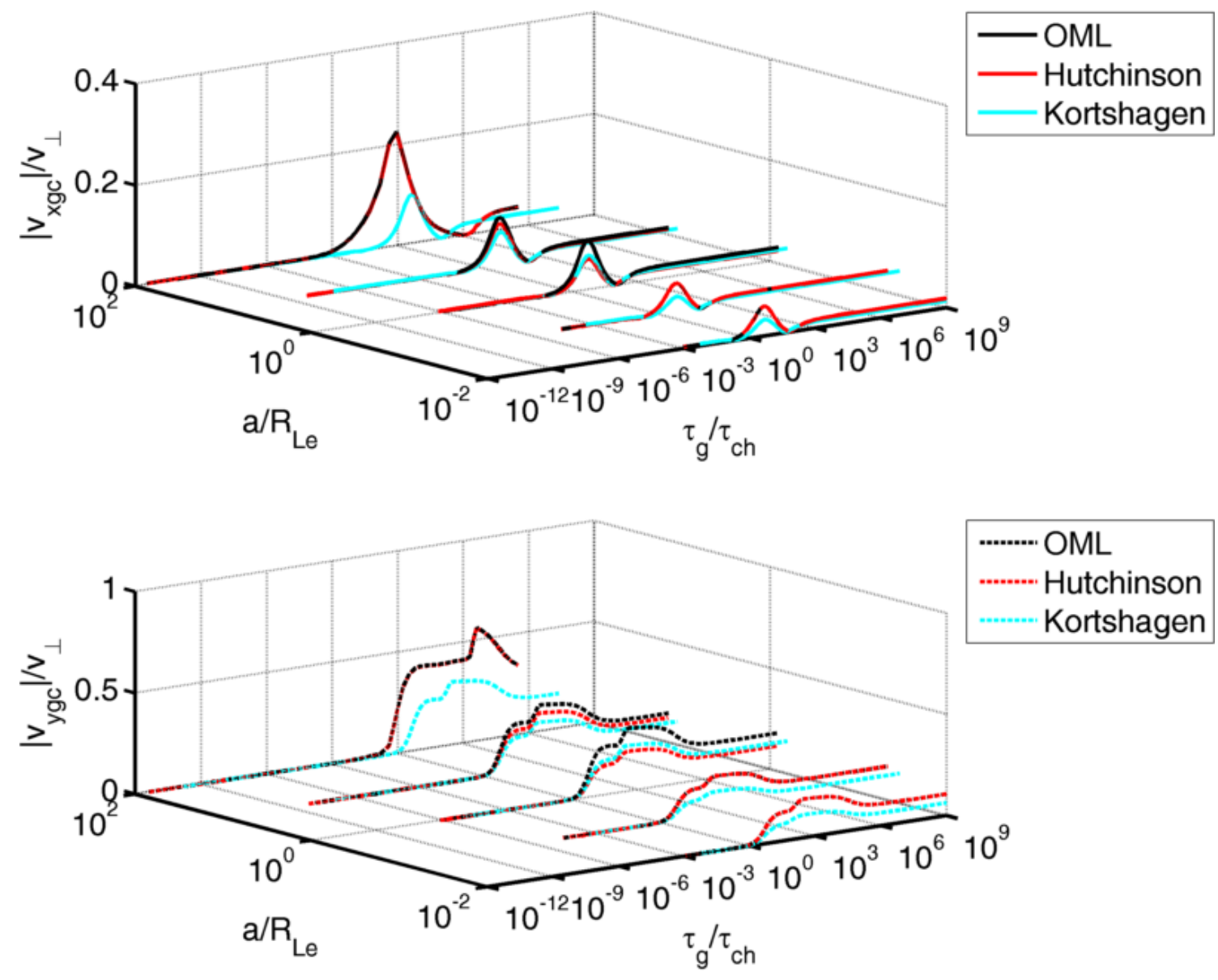

Figure 5.25: Gyro-phase magnitude and direction dependence on $\tau_{g} / \tau_{c}$ for the three different charging models for several values of $a / R_{L e}$. Solid (dashed) red line corresponds to the PatacchiniHutchinson model guiding center drift $v_{x g c}\left(v_{y g c}\right)$, solid (dashed black line corresponds to the OML model guiding center drift $v_{x g c}\left(v_{y g c}\right)$, and the solid (dashed) cyan line corresponds to the GattiKortshagen model guiding center drift $v_{x g c}\left(v_{y g c}\right)$. The other parameters chosen for an Argon plasma include $f_{U V} /\left(n_{e} v_{t h e}\right)=0.25, \omega_{c d} / \nu_{d n}=10, N_{D e}=10^{4}, \lambda_{i} / a=10^{5}, \lambda_{D} / a=10^{3}$, and $T_{e} / T_{i}=200$. The OML and Patacchini-Hutchinson charge models produce the same guiding center drift magnitudes, producing overlapping plots except for the values $a / R_{L e}=1$ and $a / R_{L e}=10$. In the limit of very large or very small values of $a / R_{L e}$, the OML and Patacchini-Hutchinson models predict the same guiding center drift magnitude. 

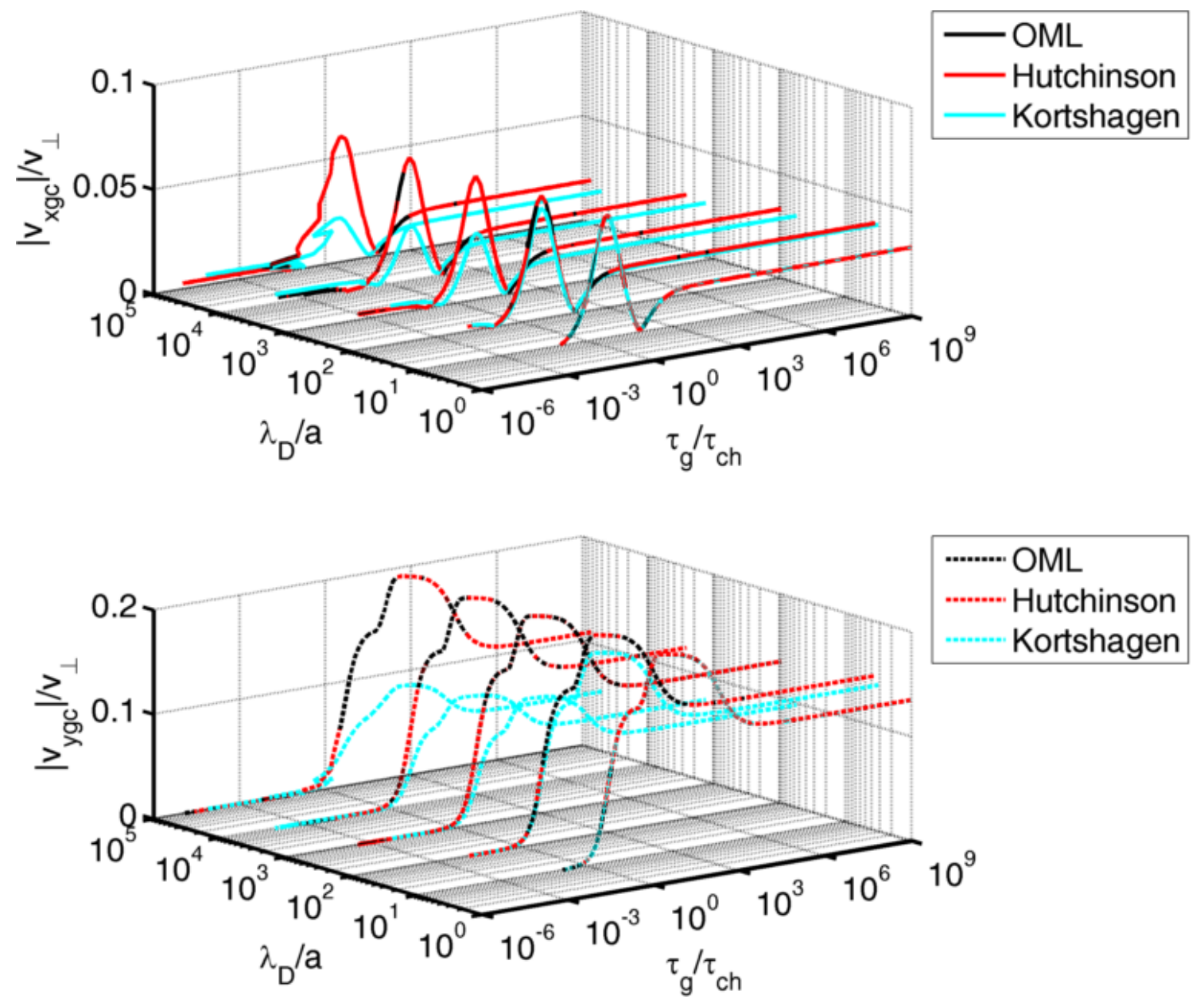

Figure 5.26: Guiding center drift magnitude dependence on $\tau_{g} / \tau_{c}$ for the three different charging models for several values of the parameter $\lambda_{D} / a$. Solid (dashed) red line corresponds to the Patacchini-Hutchinson model guiding center drift $v_{x g c}\left(v_{y g c}\right)$, solid (dashed black line corresponds to the OML model guiding center drift $v_{x g c}\left(v_{y g c}\right)$, and the solid (dashed) cyan line corresponds to the Gatti-Kortshagen model guiding center drift $v_{x g c}\left(v_{y g c}\right)$. The other parameters chosen for an Argon plasma include $v=f_{U V} /\left(n_{e} v_{t h e}\right)=0.25, \omega_{c d} / \nu_{d n}=10, N_{D e}=10^{4}, a / R_{L e}=0.1, \lambda_{i} / a=10^{5}$, and $T_{e} / T_{i}=200$. The OML and Patacchini-Hutchinson charge models predict the same guiding center drift for all parameters, and the Gatti-Kortshagen charge model predicts the same guiding center drift as the OML and Patacchini-Hutchinson models for $\lambda_{i} / \lambda_{D} \gg 1$ and $\lambda_{i} / \lambda_{D} \ll 1$. 

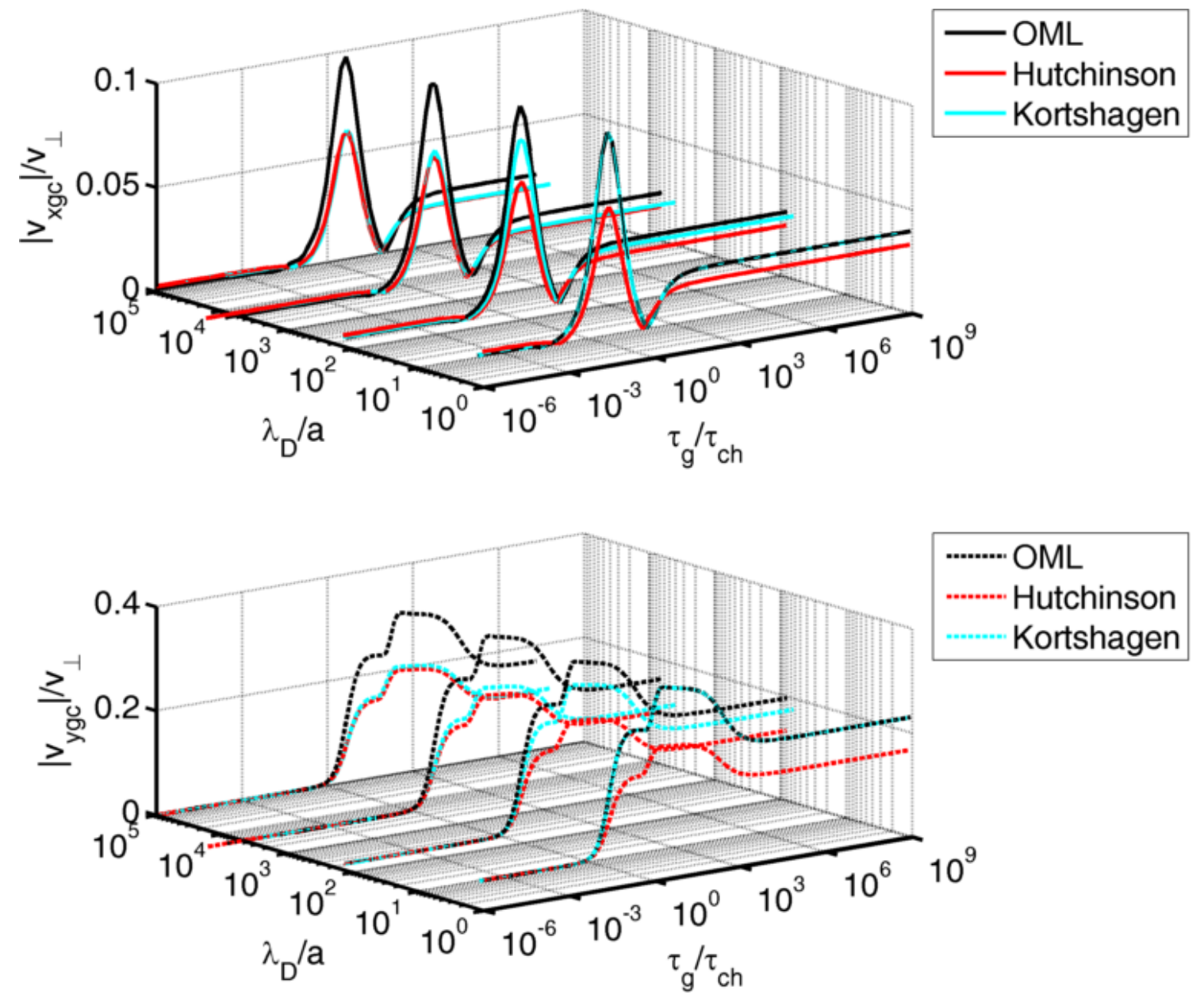

Figure 5.27: Guiding center drift magnitude dependence on $\tau_{g} / \tau_{c}$ for the three different charging models for several values of the parameter $\lambda_{D} / a$. Solid (dashed) red line corresponds to the Patacchini-Hutchinson model guiding center drift $v_{x g c}\left(v_{y g c}\right)$, solid (dashed black line corresponds to the OML model guiding center drift $v_{x g c}\left(v_{y g c}\right)$, and the solid (dashed) cyan line corresponds to the Gatti-Kortshagen model guiding center drift $v_{x g c}\left(v_{y g c}\right)$. The other parameters chosen for an Argon plasma include $v=f_{U V} /\left(n_{e} v_{t h e}\right)=0.25, \omega_{c d} / \nu_{d n}=10, N_{D e}=10^{4}, a / R_{L e}=1.1$, $\lambda_{i} / a=10^{5}$, and $T_{e} / T_{i}=200$. The OML and Patacchini-Hutchinson charge models predict the same guiding center drift for these parameters. 

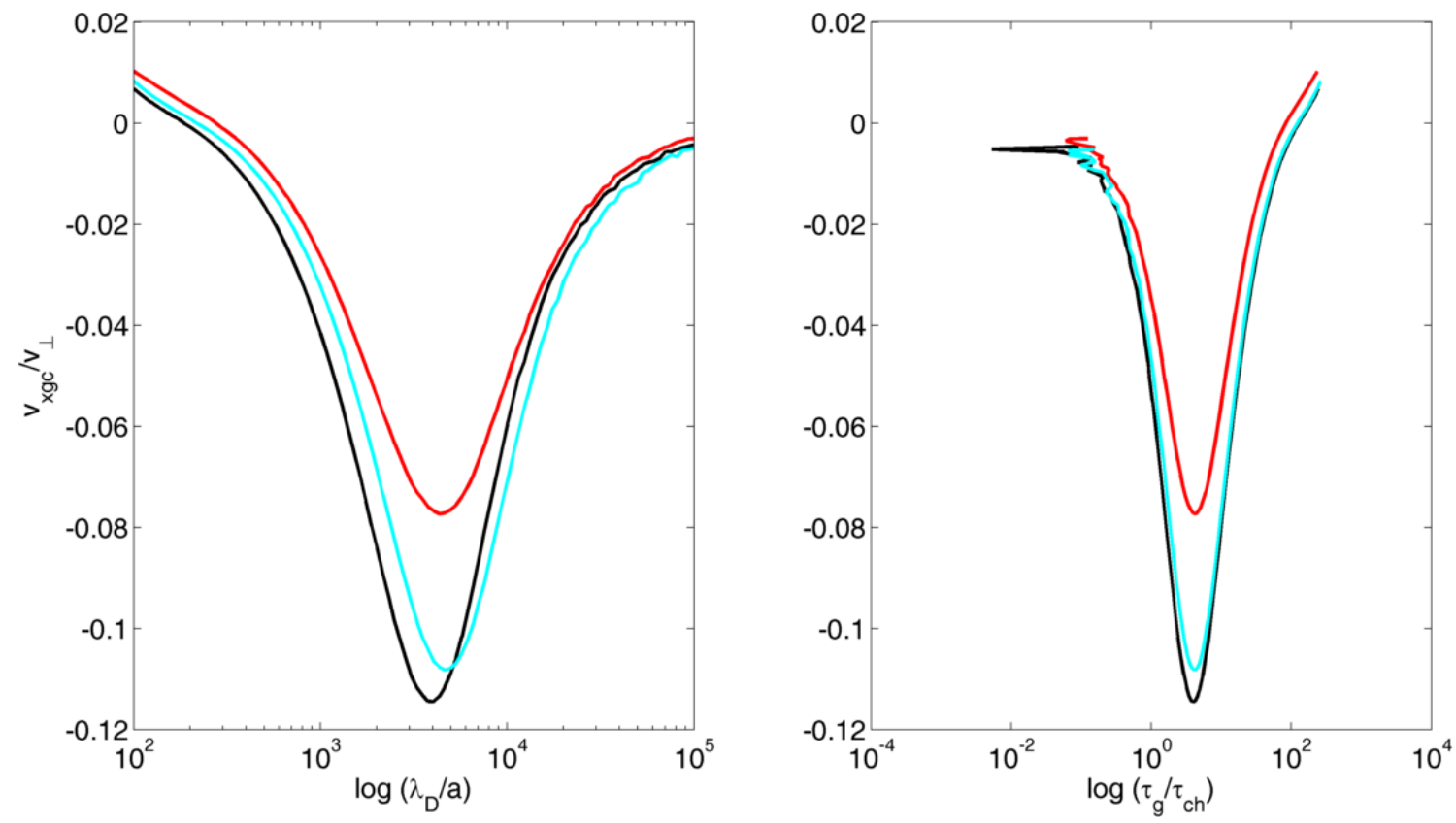

Figure 5.28: Guiding center $x$-component drift magnitude dependence on $\lambda_{D} / a$ and $\tau_{g} / \tau_{c}$ for the three different charging models as a function of the parameter $\lambda_{D} / a$. In the leftmost plot, the abscissa is actually $\lambda_{D} / a$ instead of $\log _{10} \frac{\lambda_{D}}{a}$, and in the rightmost plot, the abscissa is actually $\tau_{g} / \tau_{c}$ instead of $\log _{10} \frac{\tau_{g}}{\tau_{c}}$. Here, a grain with $a=10^{-7} \mathrm{~m}$ made out of carbon has a mass ratio $m_{d} / m_{e}=10^{13}$, and it is the variation of $\lambda_{D} / a$ that produces the $\tau_{g} / \tau_{c}$ variation of the guiding center drift. Solid red line corresponds to the Patacchini-Hutchinson model guiding center drift $v_{x g c}$, solid black line corresponds to the OML model guiding center drift $v_{x g c}$, and the solid cyan line corresponds to the Gatti-Kortshagen model guiding center drift $v_{x g c}$. The parameter $\lambda_{D} / a$ is swept while all other parameters are held constant. The other parameters chosen for an Argon plasma are $v=f_{U V} /\left(n_{e} v_{t h e}\right)=0.25, \omega_{c d} / \nu_{d n}=10, N_{D e}=10^{4}, a / R_{L e}=1.1, \lambda_{i} / a=10^{5}$, and $T_{e} / T_{i}=200$. 

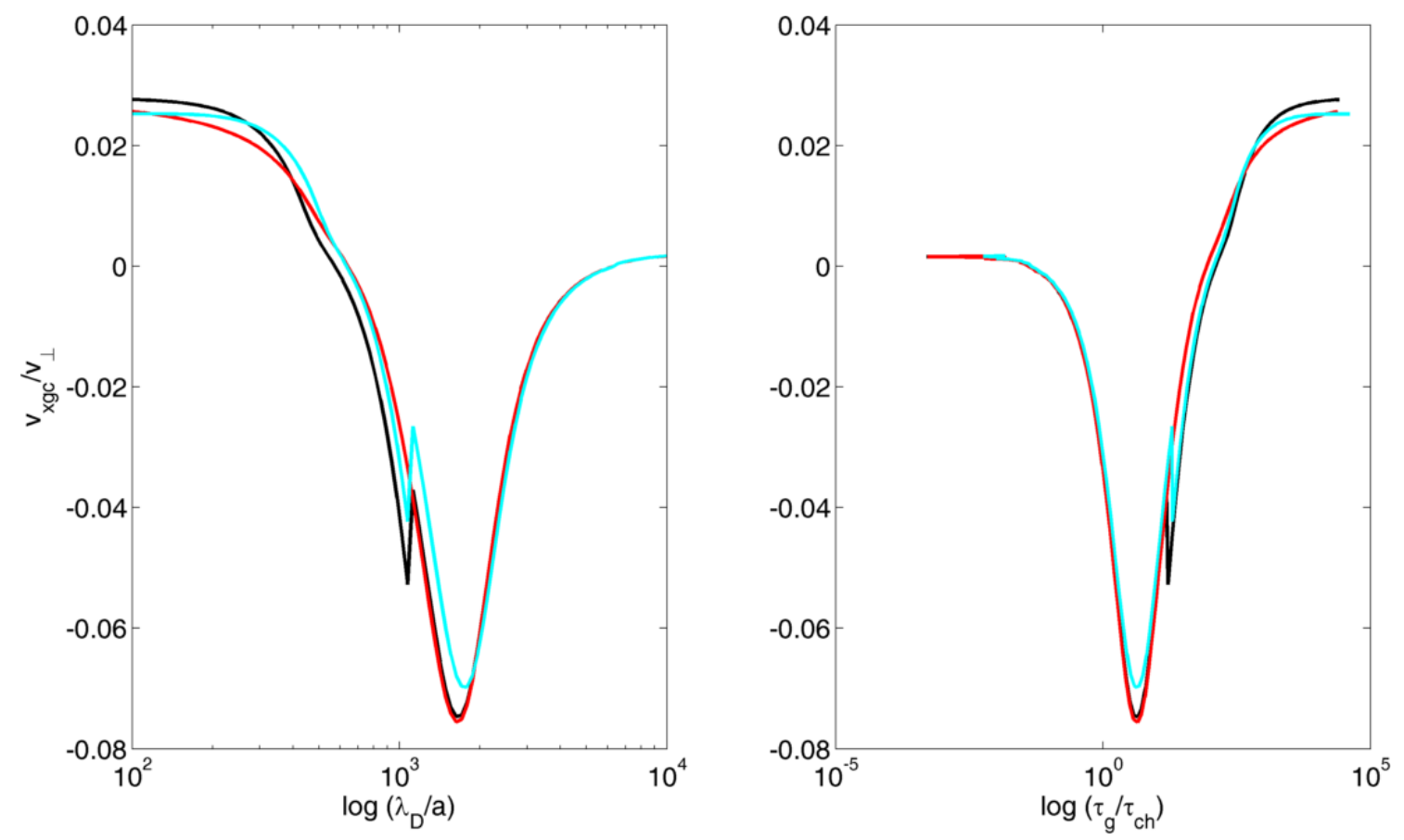

Figure 5.29: Guiding center $x$-component drift magnitude dependence on $\lambda_{D} / a$ and $\tau_{g} / \tau_{c}$ for the three different charging models for several values of the parameter $\lambda_{D} / a$. In the leftmost plot, the abscissa is actually $\lambda_{D} / a$ instead of $\log _{10} \frac{\lambda_{D}}{a}$, and in the rightmost plot, the abscissa is actually $\tau_{g} / \tau_{c}$ instead of $\log _{10} \frac{\tau_{g}}{\tau_{c}}$. Here, a grain with $10^{-7} \mathrm{~m}$ made out of carbon has a mass ratio $m_{d} / m_{e}=10^{13}$, and this is held constant while $\lambda_{D} / a$ is swept. Solid red line corresponds to the Patacchini-Hutchinson model guiding center drift $v_{x g c}$, solid black line corresponds to the OML model guiding center drift $v_{x g c}$, and the solid cyan line corresponds to the Gatti-Kortshagen model guiding center drift $v_{x g c}$. When $\lambda_{D} / a=10^{2}$, the other parameters are $a / R_{L i}=0.12, \lambda_{i} / a$, and $m_{d} / m_{e}=10^{13}$, and these parameters are swept consistently throughout the values of $\lambda_{D} / a$ shown. The other parameters chosen for an Argon plasma include $v=f_{U V} /\left(n_{e} v_{t h e}\right)=0.25, \omega_{c d} / \nu_{d n}=10$, $N_{D e}=10^{4}$, and $T_{e} / T_{i}=200$. 

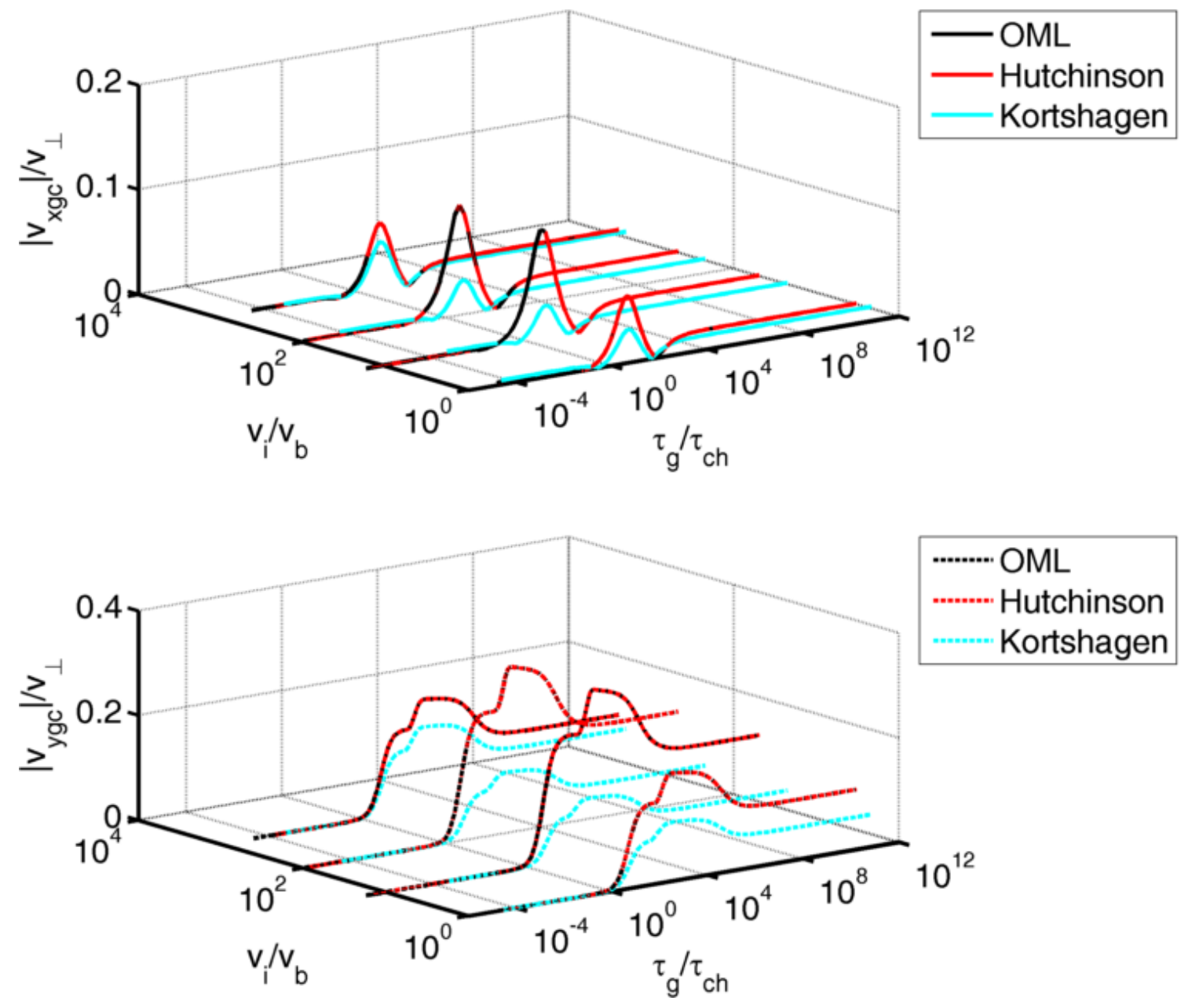

Figure 5.30: Gyro-phase magnitude and direction dependence on $\tau_{g} / \tau_{c}$ for the three different charging models with drifting Maxwellian ions for several values of the Mach number $M_{i}=v_{i} / v_{b}$, which is the flow speed normalized by the Bohm speed $v_{b}=\sqrt{\frac{k_{b} T_{e}}{m_{i}}}$. Solid (dashed) red line corresponds to the Patacchini-Hutchinson model guiding center drift $v_{x g c}\left(v_{y g c}\right)$, solid (dashed black line corresponds to the OML model guiding center drift $v_{x g c}\left(v_{y g c}\right)$, and the solid (dashed) cyan line corresponds to the Gatti-Kortshagen model guiding center drift $v_{x g c}\left(v_{y g c}\right)$. The other parameters chosen for an Argon plasma include $v=f_{U V} /\left(n_{e} v_{t h e}\right)=0.25, \omega_{c d} / \nu_{d n}=10, N_{D e}=10^{4}$, $a / R_{L e}=0.1, \lambda_{i} / a=10^{5}, \lambda_{D} / a=10^{3}, T_{e} / T_{i}=200$, and $10^{8} \leq m_{d} / m_{e} \leq 10^{22}$. The OML and Patacchini-Hutchinson charge models predict the same guiding center drift for these parameters. 

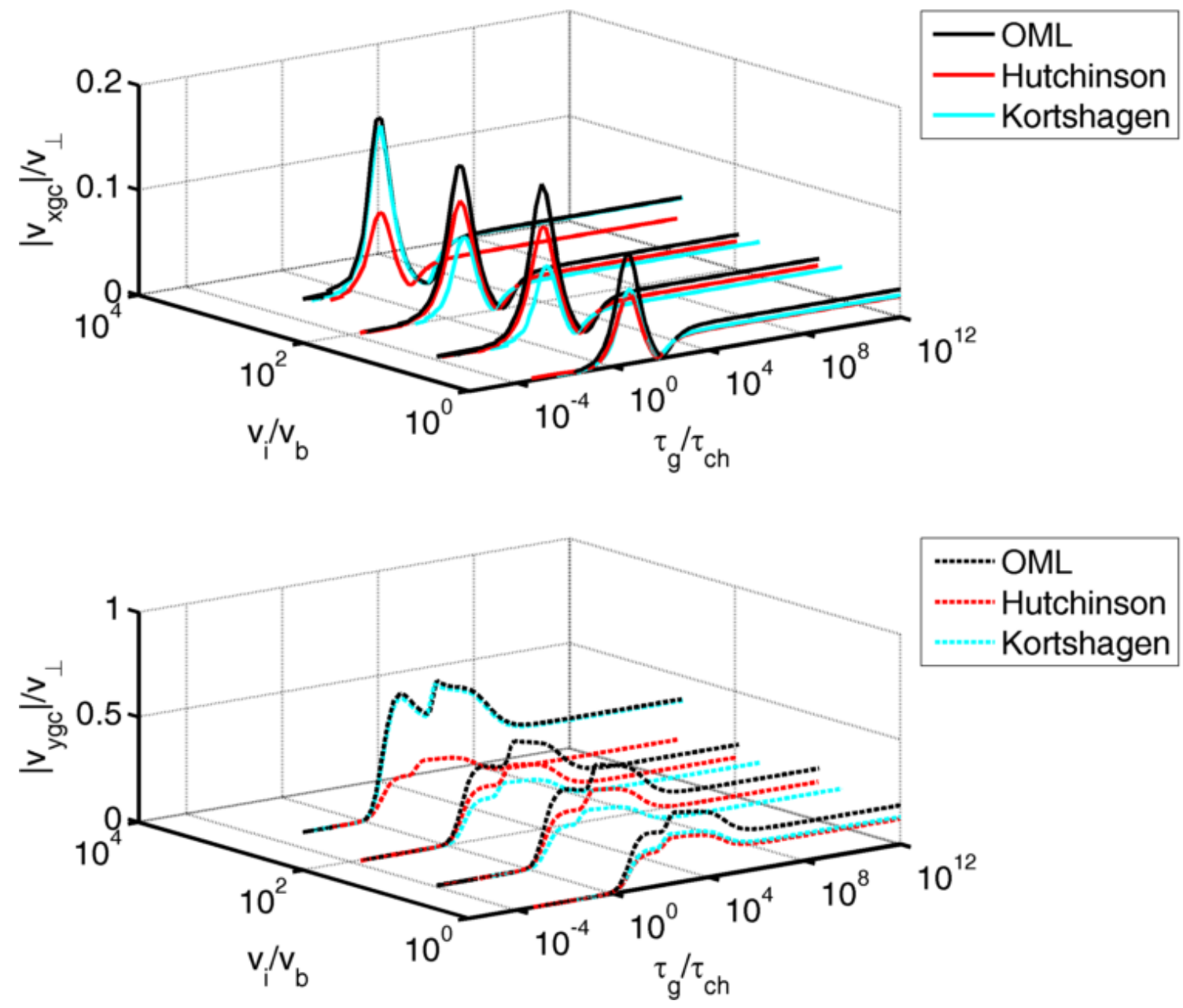

Figure 5.31: Gyro-phase magnitude and direction dependence on $\tau_{g} / \tau_{c}$ for the three different charging models with drifting Maxwellian ions for several values of the Mach number $M_{i}=v_{i} / v_{b}$, which is the flow speed normalized by the Bohm speed $v_{b}=\sqrt{\frac{k_{b} T_{e}}{m_{i}}}$. Solid (dashed) red line corresponds to the Patacchini-Hutchinson model guiding center drift $v_{x g c}\left(v_{y g c}\right)$, solid (dashed black line corresponds to the OML model guiding center drift $v_{x g c}\left(v_{y g c}\right)$, and the solid (dashed) cyan line corresponds to the Gatti-Kortshagen model guiding center drift $v_{x g c}\left(v_{y g c}\right)$. The other parameters chosen for an Argon plasma include $v=f_{U V} /\left(n_{e} v_{t h e}\right)=0.25, \omega_{c d} / \nu_{d n}=10, N_{D e}=10^{4}$, $a / R_{L e}=0.1, \lambda_{i} / a=10^{5}, \lambda_{D} / a=10^{3}, T_{e} / T_{i}=200$, and $10^{8} \leq m_{d} / m_{e} \leq 10^{22}$. 

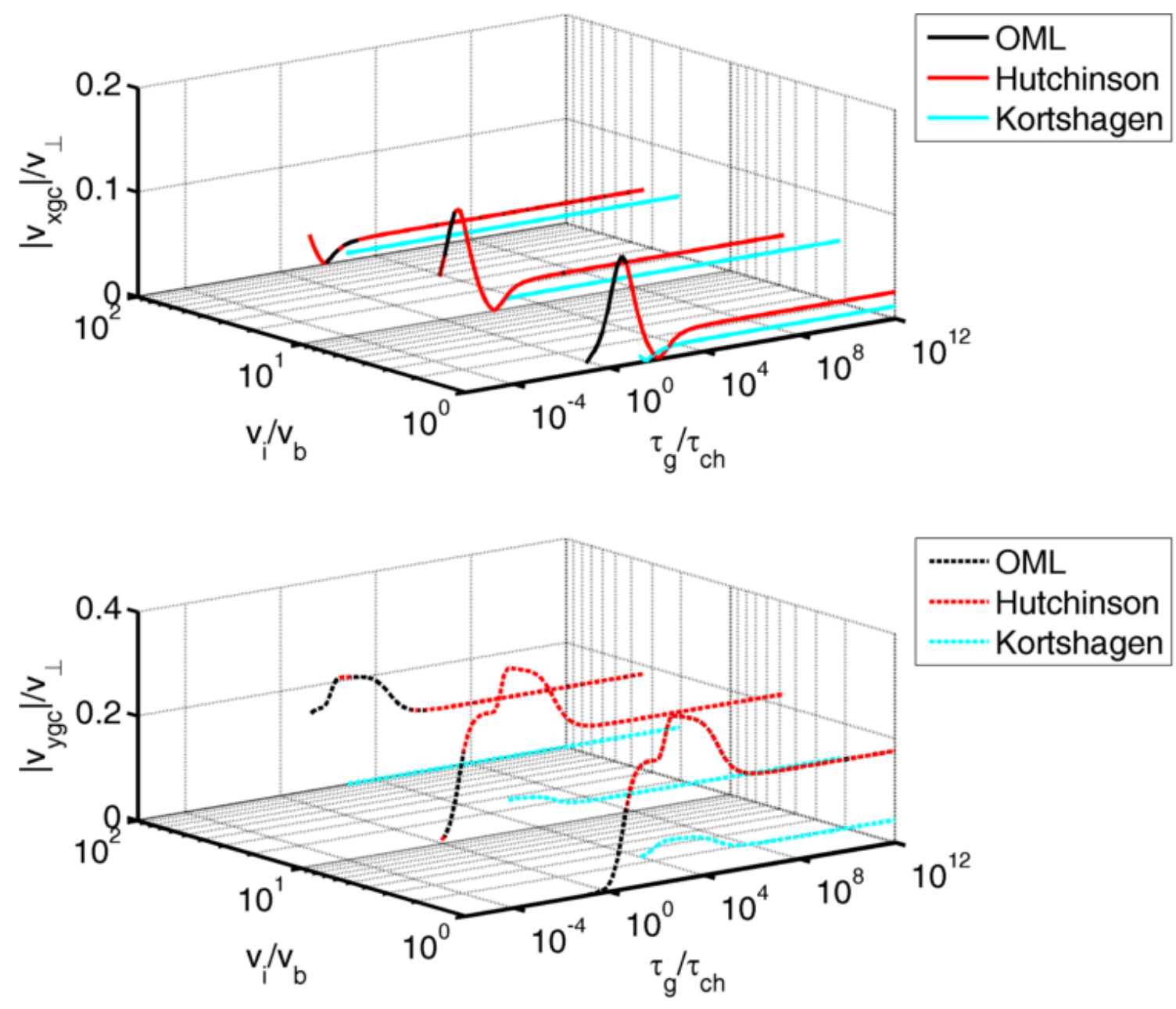

Figure 5.32: Gyro-phase magnitude and direction dependence on $\tau_{g} / \tau_{c}$ for the three different charging models with mono-energetic ions for several values of the Mach number $M_{i}=v_{i} / v_{b}$, which is the flow speed normalized by the Bohm speed $v_{b}=\sqrt{\frac{k_{b} T_{e}}{m_{i}}}$. Solid (dashed) red line corresponds to the Patacchini-Hutchinson model guiding center drift for mono-energetic (drifting Maxwellian) ions, solid (dashed black line corresponds to the OML model guiding center drift for mono-energetic (drifting Maxwellian) ions, and the solid (dashed) cyan line corresponds to the Gatti-Kortshagen model guiding center drift for mono-energetic (drifting Maxwellian) ions. The other parameters chosen for an Argon plasma include $v=f_{U V} /\left(n_{e} v_{t h e}\right)=0.25, \omega_{c d} / \nu_{d n}=10$, $N_{D e}=10^{4}, a / R_{L e}=0.1, \lambda_{i} / a=10^{5}, \lambda_{D} / a=10^{3}, T_{e} / T_{i}=200$, and $10^{8} \leq m_{d} / m_{e} \leq 10^{22}$. The OML and Patacchini-Hutchinson charge models predict the same guiding center drift for these parameters. 

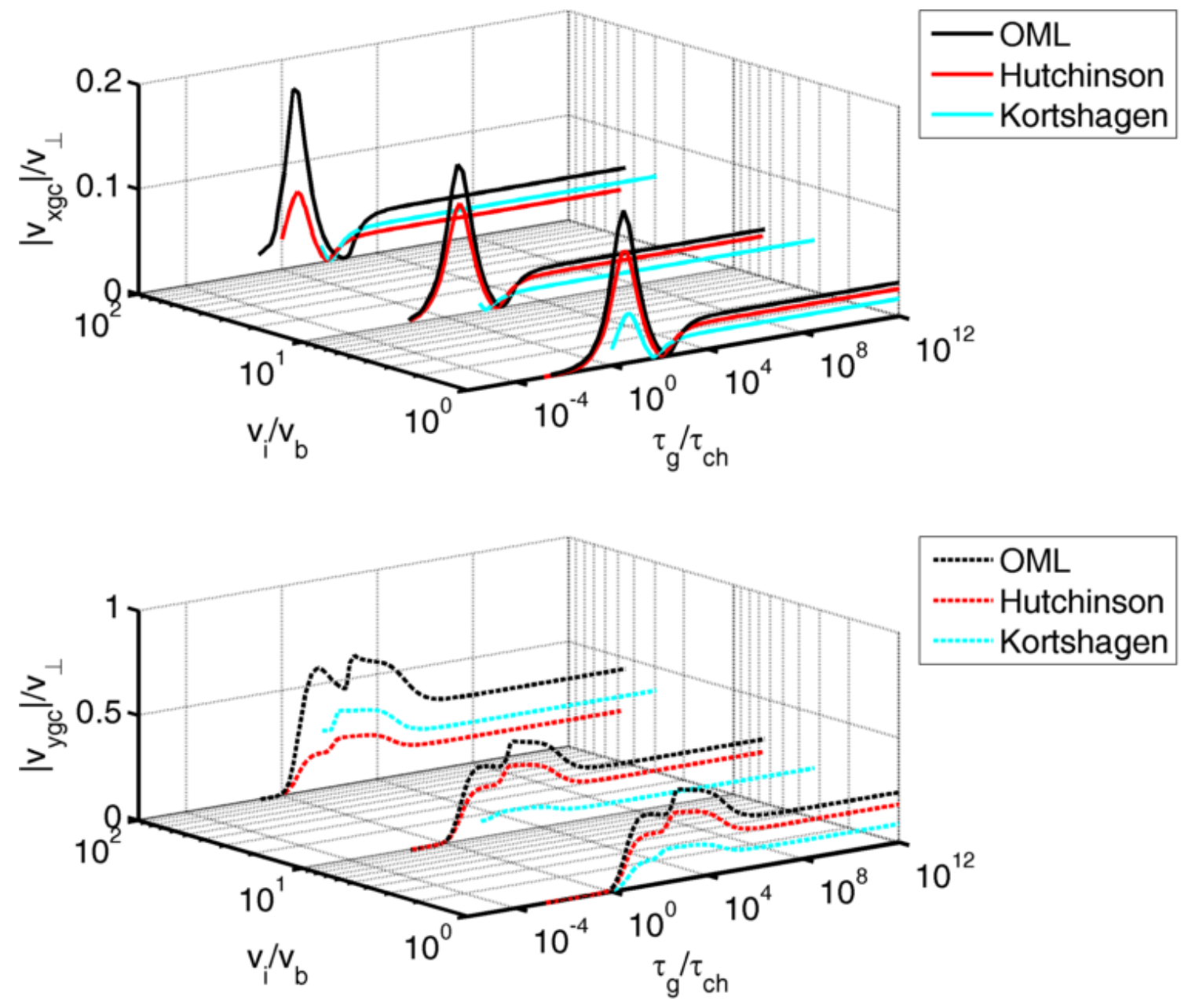

Figure 5.33: Gyro-phase magnitude and direction dependence on $\tau_{g} / \tau_{c}$ for the three different charging models with mono-energetic ions for several values of the Mach number $M_{i}=w_{i} / w_{b}$, which is the flow speed normalized by the Bohm speed $v_{b}=\sqrt{\frac{k_{b} T_{e}}{m_{i}}}$. Solid (dashed) red line corresponds to the Patacchini-Hutchinson model guiding center drift $v_{x g c}\left(v_{y g c}\right)$, solid (dashed black line corresponds to the OML model guiding center drift $v_{x g c}\left(v_{y g c}\right)$, and the solid (dashed) cyan line corresponds to the Gatti-Kortshagen model guiding center drift $v_{x g c}\left(v_{y g c}\right)$. The other parameters chosen for an Argon plasma include $v=f_{U V} /\left(n_{e} v_{t h e}\right)=0.25, \omega_{c d} / \nu_{d n}=10, N_{D e}=10^{4}$, $a / R_{L e}=0.1, \lambda_{i} / a=10^{5}, \lambda_{D} / a=10^{3}, T_{e} / T_{i}=200$, and $10^{8} \leq m_{d} / m_{e} \leq 10^{22}$. 

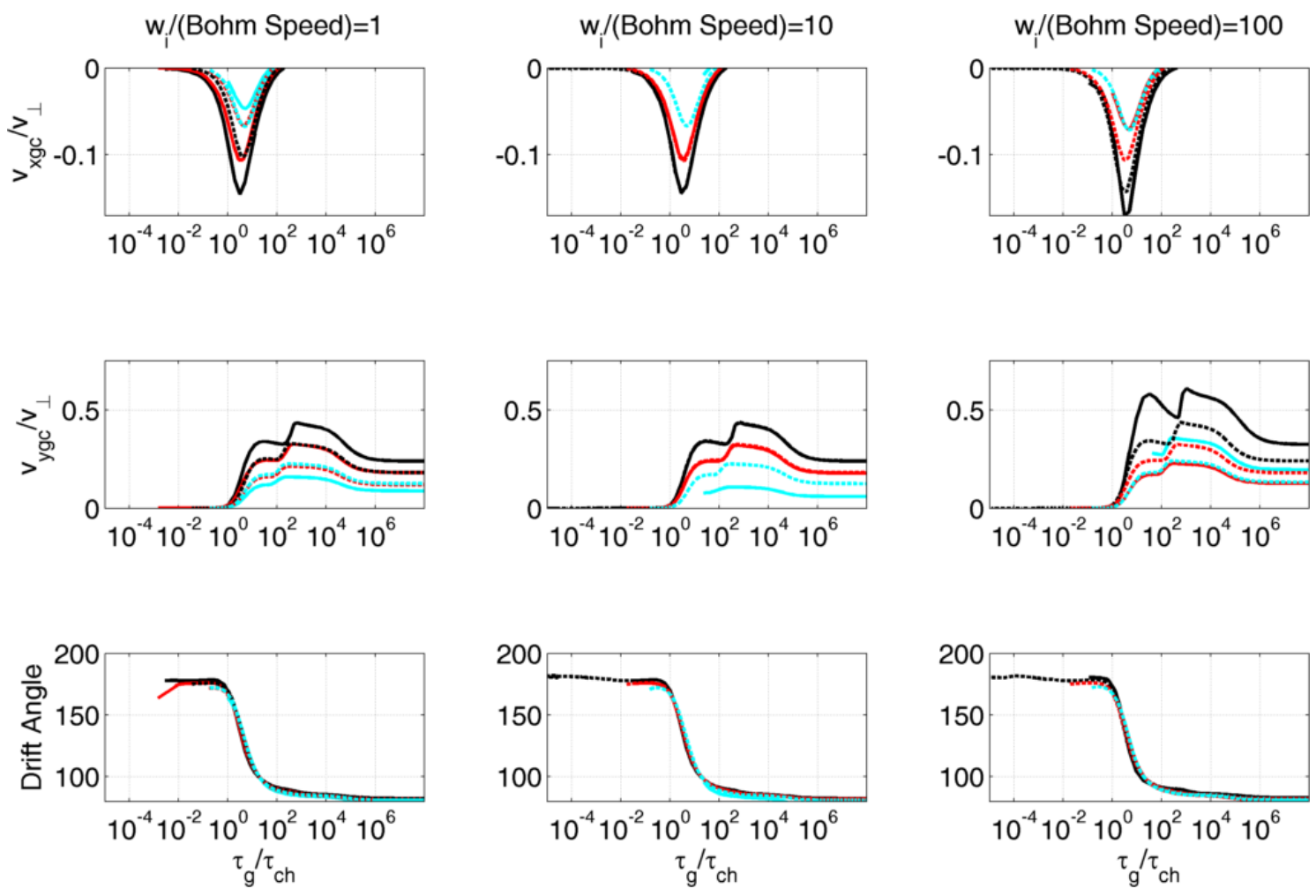

Figure 5.34: Gyro-phase magnitude and direction dependence on $\tau_{g} / \tau_{c}$ for the three different charging models for different values of the Mach number $M_{i}=v_{i} / v_{b}$, which is the flow speed normalized by the Bohm speed $v_{b}=\sqrt{\frac{k_{b} T_{e}}{m_{i}}}$. This figure shows a direct comparison between monoenergetic and drifting Maxwellian ions. All solid lines correspond with mono-energetic ions, while all dashed lines correspond to drifting Maxwellians. The colors black, red, and light blue correspond to the OML, Patacchini-Hutchinson, and Gatti-Kortshagen charge models respectively. The other parameters chosen for an Argon plasma include $f_{U V} /\left(n_{e} v_{t h e}\right)=0.25, \omega_{c d} / \nu_{d n}=10, N_{D e}=10^{4}$, $a / R_{L e}=0.1, \lambda_{i} / a=10^{5}, \lambda_{D} / a=10^{3}, T_{e} / T_{i}=200$, and $10^{8} \leq m_{d} / m_{e} \leq 10^{22}$. 

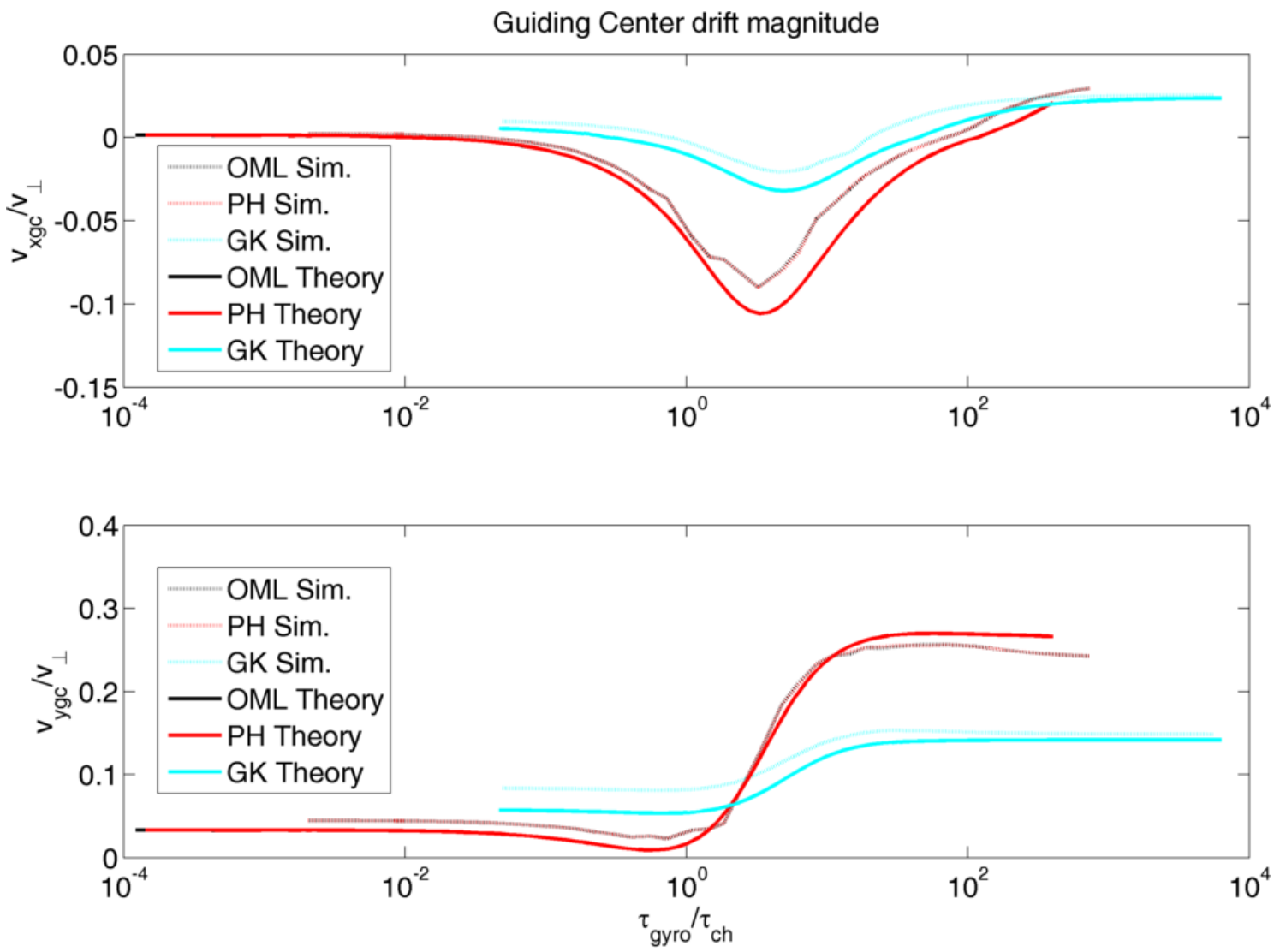

Figure 5.35: Comparison of simulation and theory results for the gyro-phase magnitude and direction dependence on $\tau_{g} / \tau_{c}$ for the three charging models. The colors black, red, and light blue correspond to the OML, Patacchini-Hutchinson, and Gatti-Kortshagen charge models respectively. Other parameters chosen for an Argon plasma include $f_{U V} /\left(n_{e} v_{t h e}\right)=0.25, \omega_{c d} / \nu_{d n} \approx 10$, drifting Maxwell-Boltzmann ions with $M_{i}=v_{i} / v_{b}=10, N_{D e}=10^{3}, a / R_{L e}=0.1, \lambda_{i} / a=10^{5}, \lambda_{D} / a=10^{3}$, $T_{e} / T_{i}=200$, and $10^{8} \leq m_{d} / m_{e} \leq 10^{13}$. The ratios of the $i n$-situ equilibrium grain charge on the shadowed side and the illuminated side of the abrupt inhomogeneity are $q_{1} / q_{2}=2.65, q_{1} / q_{2}=$ 2.65, and $q_{1} / q_{2}=1.38$ for the OML, Patacchini-Hutchinson, and Gatti-Kortshagen charge models respectively. The OML and Patacchini-Hutchinson charge models predict the same guiding center drift for these parameters. 


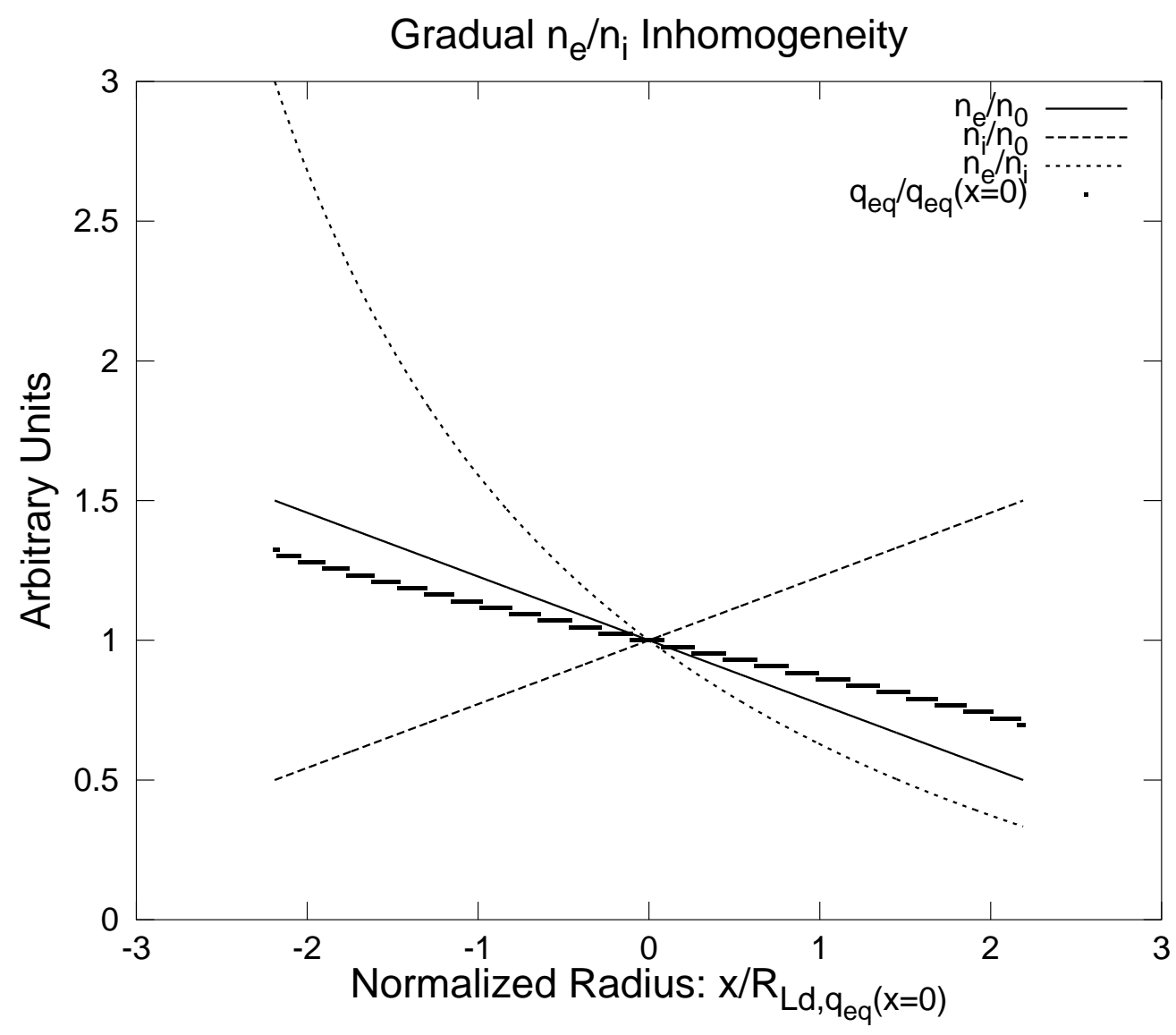

Figure 5.36: Linear profile used for simulations. All of the grain trajectories modelled using this inhomogeneity do not include the effect of the electric field that would be produced by this inhomogeneity. The electron and ion densities, $n_{e}, n_{i}$, are normalized to $n_{0}=10^{16} \mathrm{~m}^{-3}$. The ratio $n_{e} / n_{i}$ is also plotted, as is the dimensionless quantity $q_{e q}(x) / q_{e q}(x=0)$, which is proportional to the number of electrons on the grain. The discrete steps of $q_{e q}(x) / q_{e q}(x=0)$ correspond to an addition or subtraction of one electron. The abscissa is scaled to the gyro-radius corresponding to the equilibrium charge of a $0.015 \mu \mathrm{m}$ radius grain at $x=0$, which is 43 electrons $\left[R_{L d}(t=0)=0.572\right.$ $\mathrm{mm}]$. 


\section{Grain Trajectories}

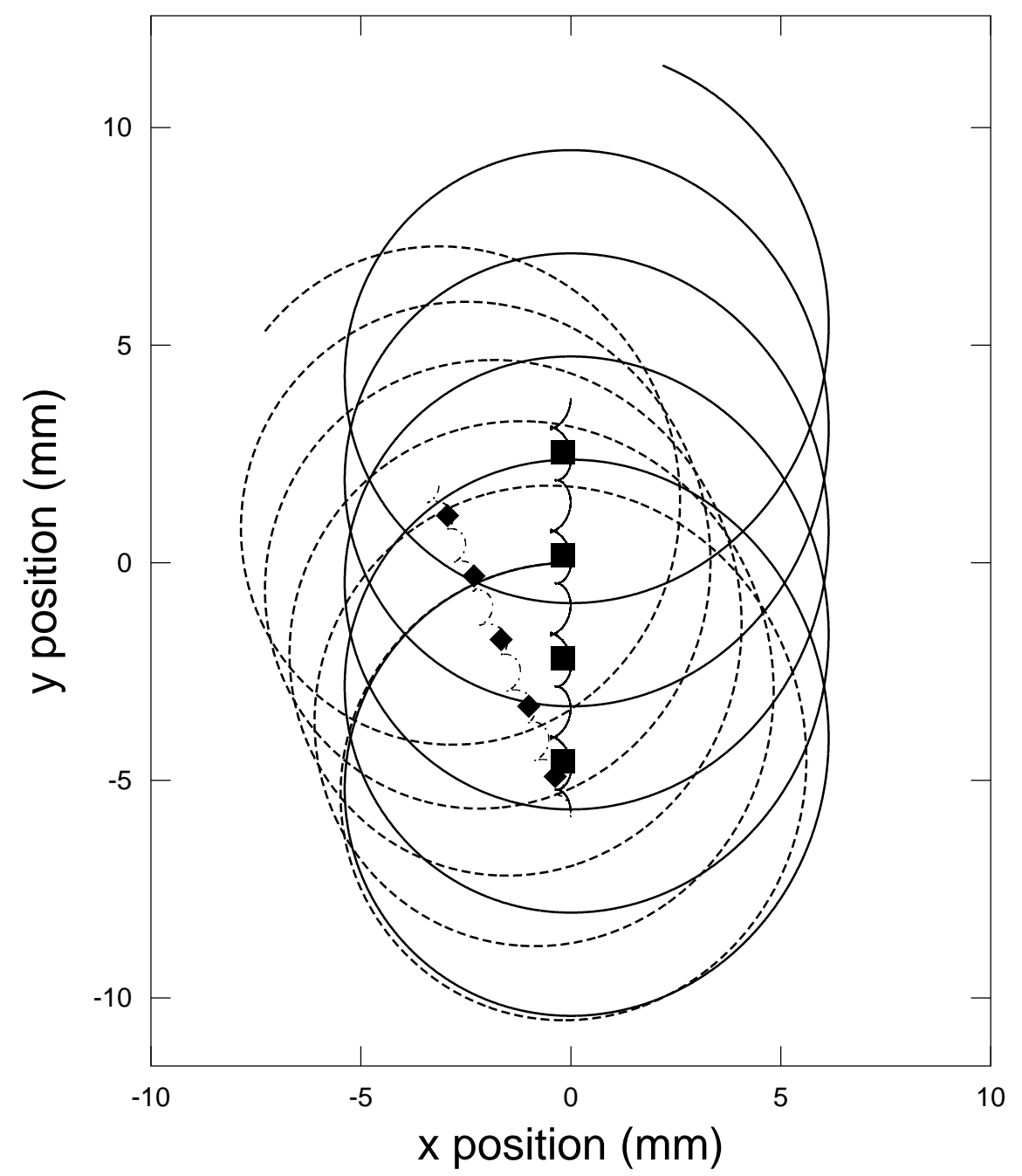

Figure 5.37: Grain trajectories for $a=0.015 \mu \mathrm{m}$ and $\boldsymbol{B}=B \hat{z}$, where $B=4 \mathrm{~T}$, using the profile from figure 5.36 and the effects of an electric field are not included in the trajectories. The dashed and solid lines correspond to a charging rate parameter of $\alpha=1$ and $\alpha=0.0105$, respectively. Squares and diamonds indicate the gyro-averaged guiding centers of the trajectories for $\alpha=1$ and $\alpha=0.0105$, respectively. The instantaneous guiding centers are represented by the solid $(\alpha=1)$ and dashed $(\alpha=0.0105)$ helical lines. 


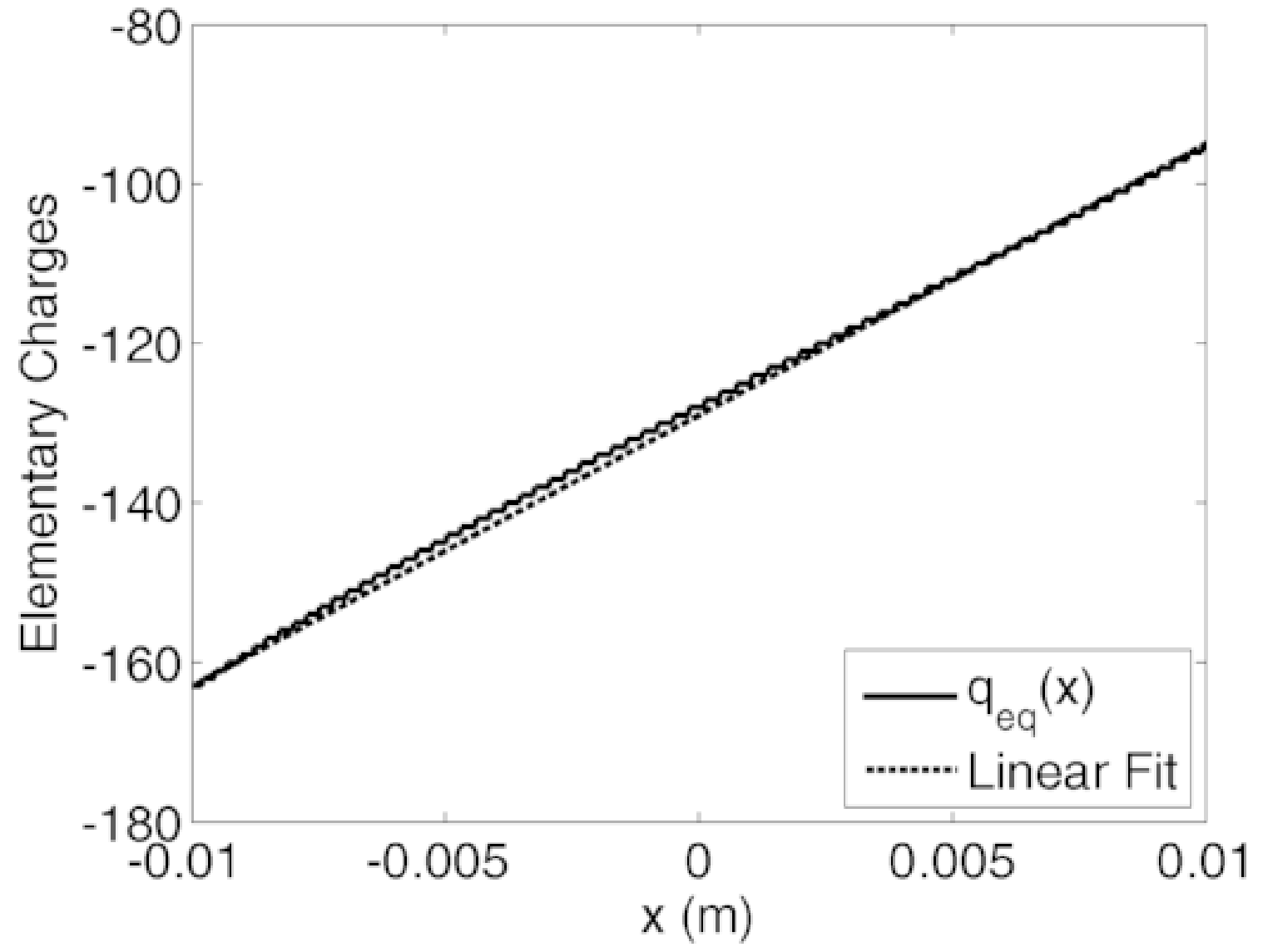

Figure 5.38: Linear fit to the in-situ equilibrium grain charge as a function of the inhomogeneous coordinate $x$. The solid line represents the in-situ equilibrium grain charge, which has a step-like appearance because it changes in increments of $1 e$. The dashed line shows the linear fit to $q_{e q}(x)$. 


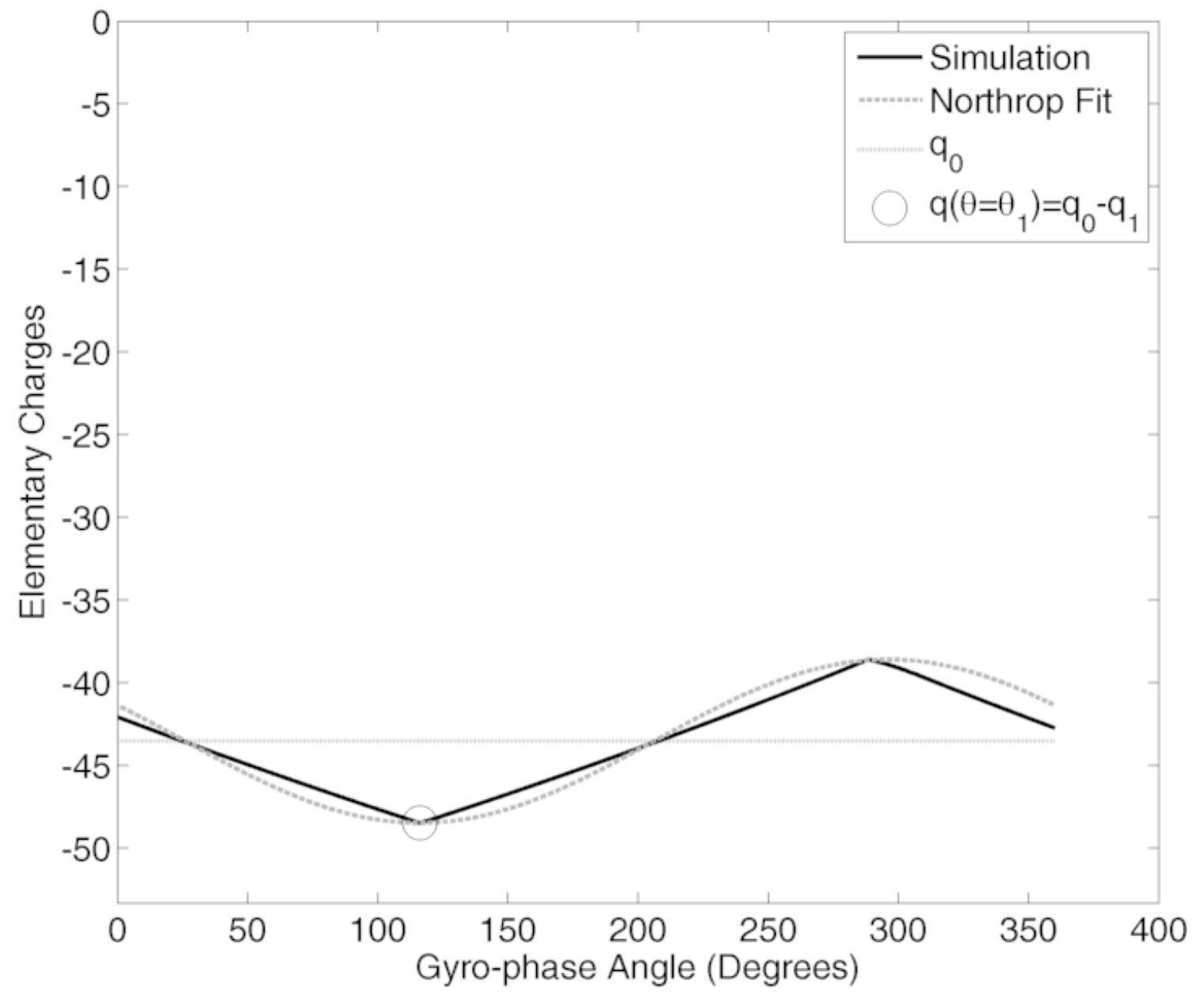

Figure 5.39: Northop fit to $q(t)=q_{0}+q_{1} \cos \left(\phi-\phi_{1}\right)$. The quantity $q_{0}$ represents the in-situ equilibrium grain charge at the gyro-averaged guiding center, and $q_{1}$ represents the amplitude of charge modulation. The solid black line shows $q(t)$, the dashed gray line shows the Northrop style fit to $q(t)$, and the circle indicates the gyro-phase angle where the grain is most negatively charged during its gyro-orbit. 


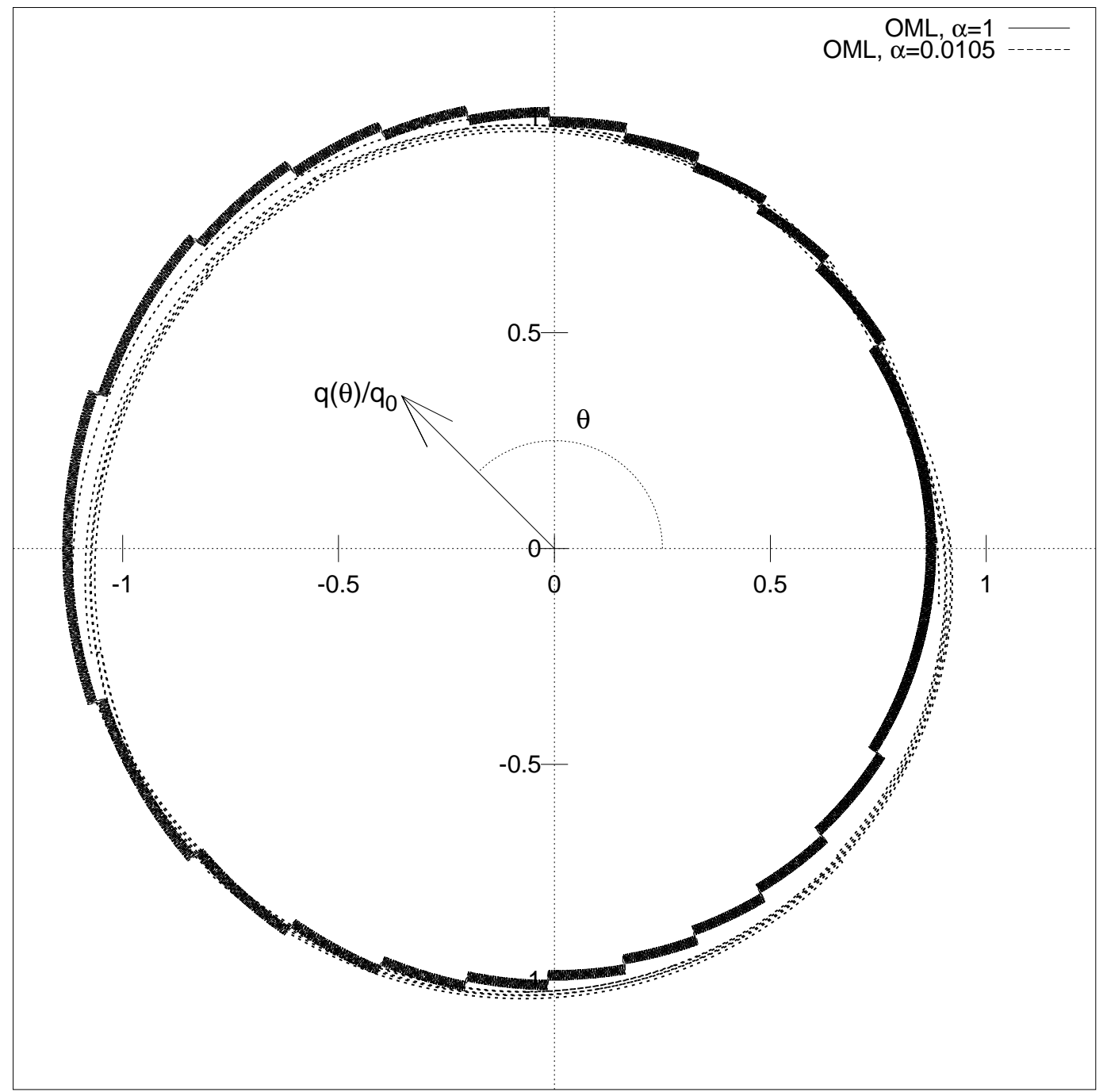

Figure 5.40: Radial distance from origin is grain charge normalized to the instantaneous in-situ equilibrium grain charge $\left(q_{0}=-43 e\right.$, in this case) as a function of gyro-angle for $\alpha=1$ (solid line) and $\alpha=0.0105$ (dashed line). In this figure, the effects of the electric field produced by the density gradient are not included. Lines appear thickened because multiple gyro-periods are displayed and gyrophase angle at which single-electron charging events occur are not unique and because the thickness reflects charge fluctuation $+1,-0$ electron. The gyro-angle $\phi=0$ refers to the $+\hat{x}$ axis here. 

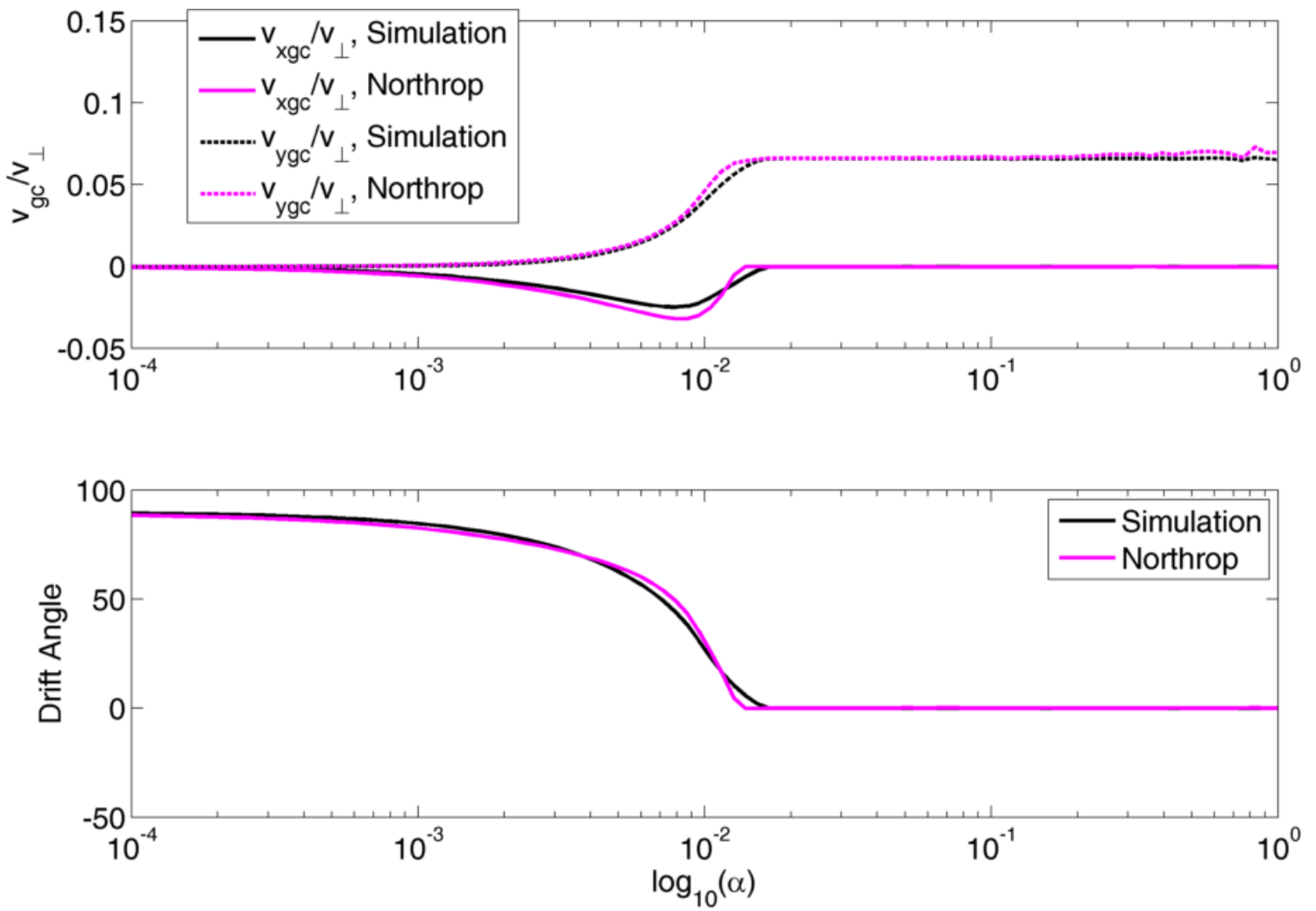

Figure 5.41: Gyro-phase drift magnitude and direction dependence on the adjustable charge-rate parameter $\alpha$. In this plot, the abscissa is actually $\alpha$ instead of $\log _{10} \alpha$. In this figure, the effects of the electric field produced by the density gradient are not included. A: The magnitude is normalized by the perpendicular velocity, $v_{\perp}=11 \mathrm{~m} / \mathrm{s}$. B: The angle $\theta_{\text {drift }}$, in degrees, is relative to the $\hat{x}_{-}$ direction. An angle of $180^{\circ}$ corresponds to a drift direction that is entirely along the $-\hat{x}$, and an angle of $90^{\circ}$ corresponds to a drift direction that is entirely along the $\hat{y}$-direction. Above $\alpha=0.02$, no gyro-phase drift occurs for this case. Below $\alpha=10^{-4}$, neither gyro-phase nor grad-q drift occurs for this case. 

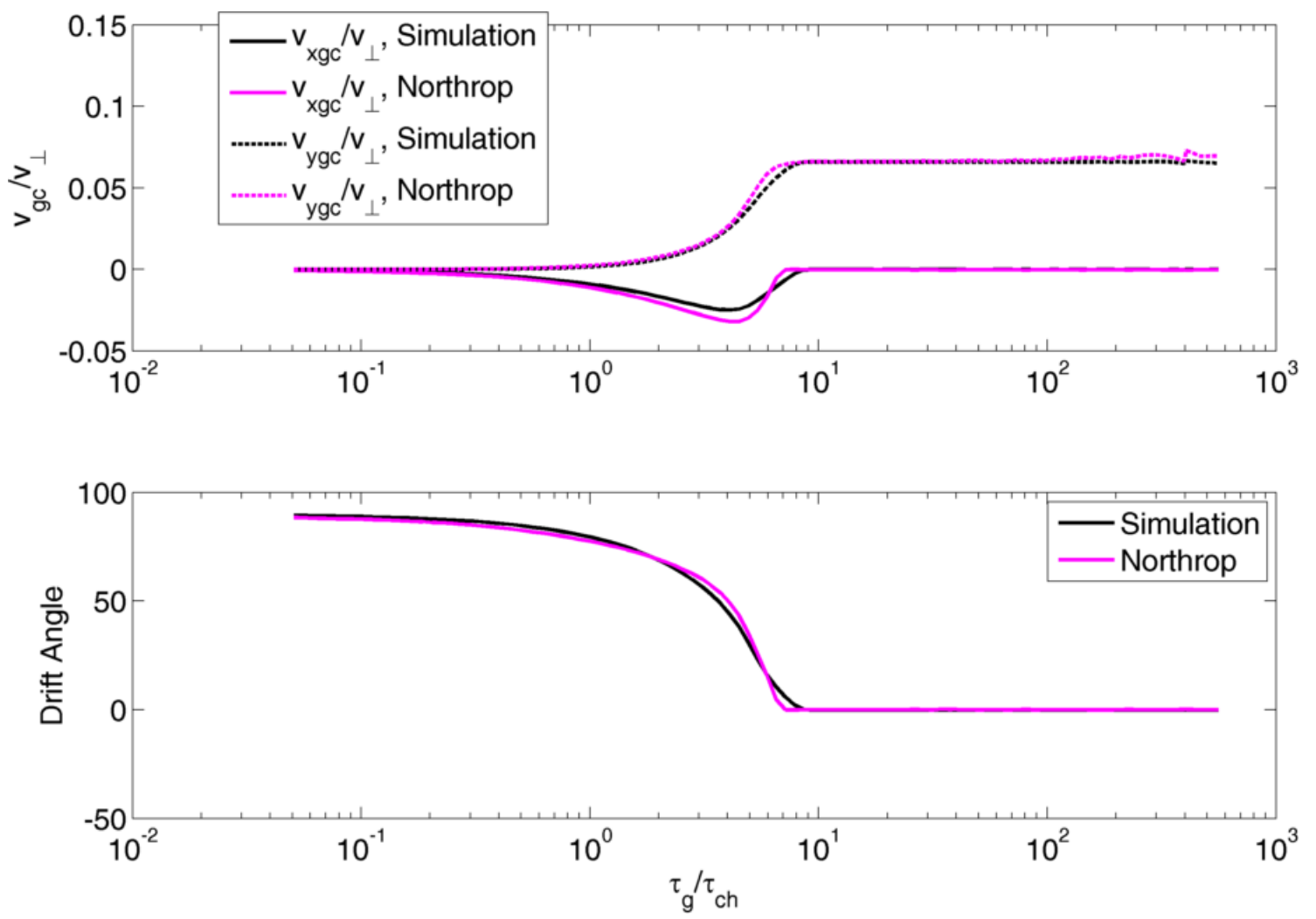

Figure 5.42: Gyro-phase drift magnitude and direction dependence on $\tau_{g} / \tau_{c}$. In this figure, the effects of the electric field produced by the density gradient are not included. This figure uses the same data as figure 5.41, but has been recast in terms of $\tau_{g} / \tau_{c}$ instead of the adjustable charge-rate parameter $\alpha$. 

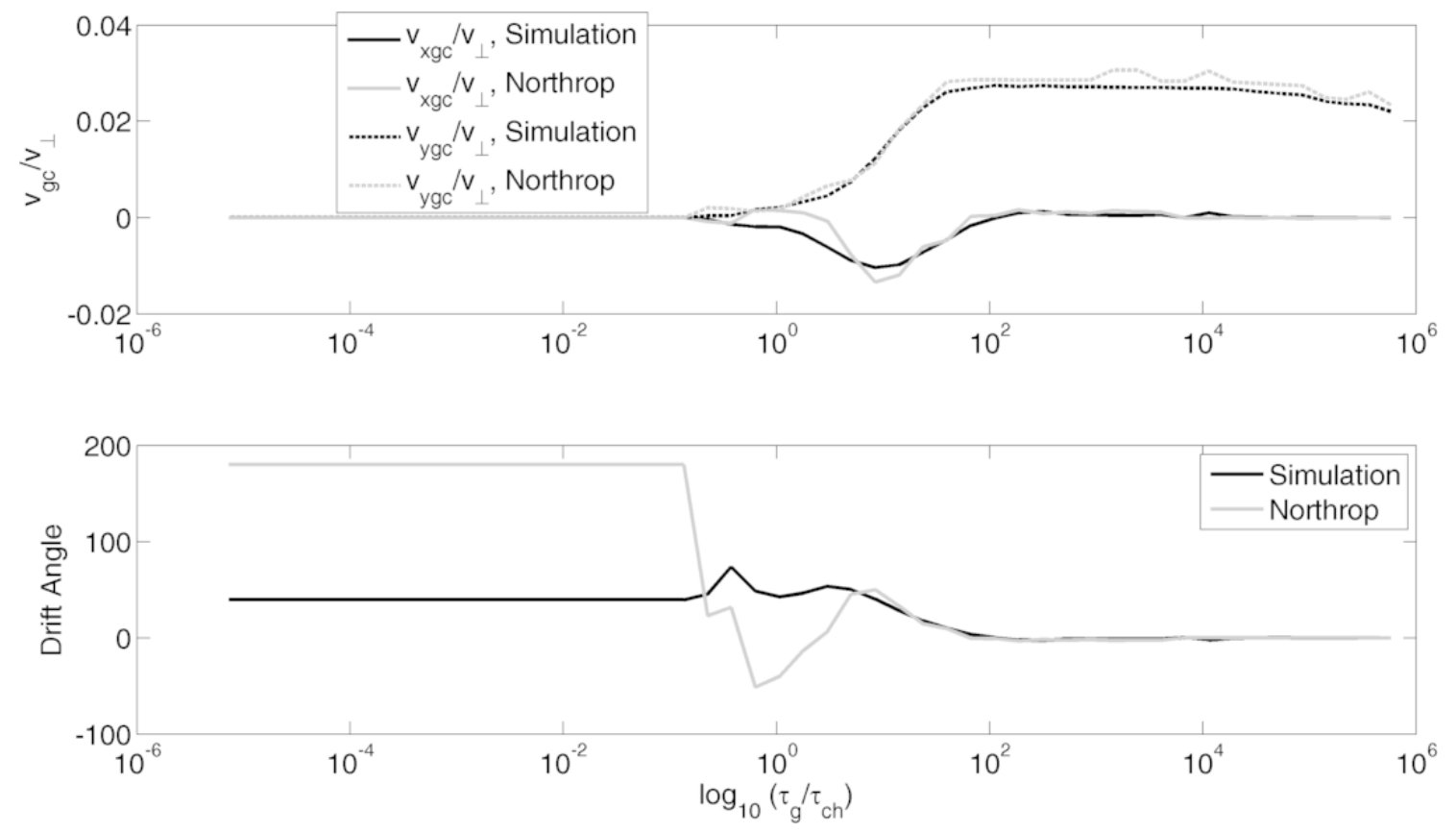

Figure 5.43: Gyro-phase drift magnitude and direction dependence on $\tau_{g} / \tau_{c}$ for $a=1.5 \times 10^{-8} \mathrm{~m}$ grain in the linear profile. In this plot, the abscissa is actually $\tau_{g} / \tau_{c}$ instead of $\log _{10} \frac{\tau_{g}}{\tau_{c}}$. In this figure, the effects of the electric field produced by the density gradient are not included. Electron and ion number density varies between $n_{0}=10^{9} \mathrm{~m}^{-3}$ and $n_{0}=10^{20} \mathrm{~m}^{-3}$ to produce $\tau_{c}$ variation. The large disparity between Northrop's theory and the simulation near $\tau_{g} / \tau_{c}<10^{-1}$ is a result from taking the arctangent of two numbers very close to zero. 

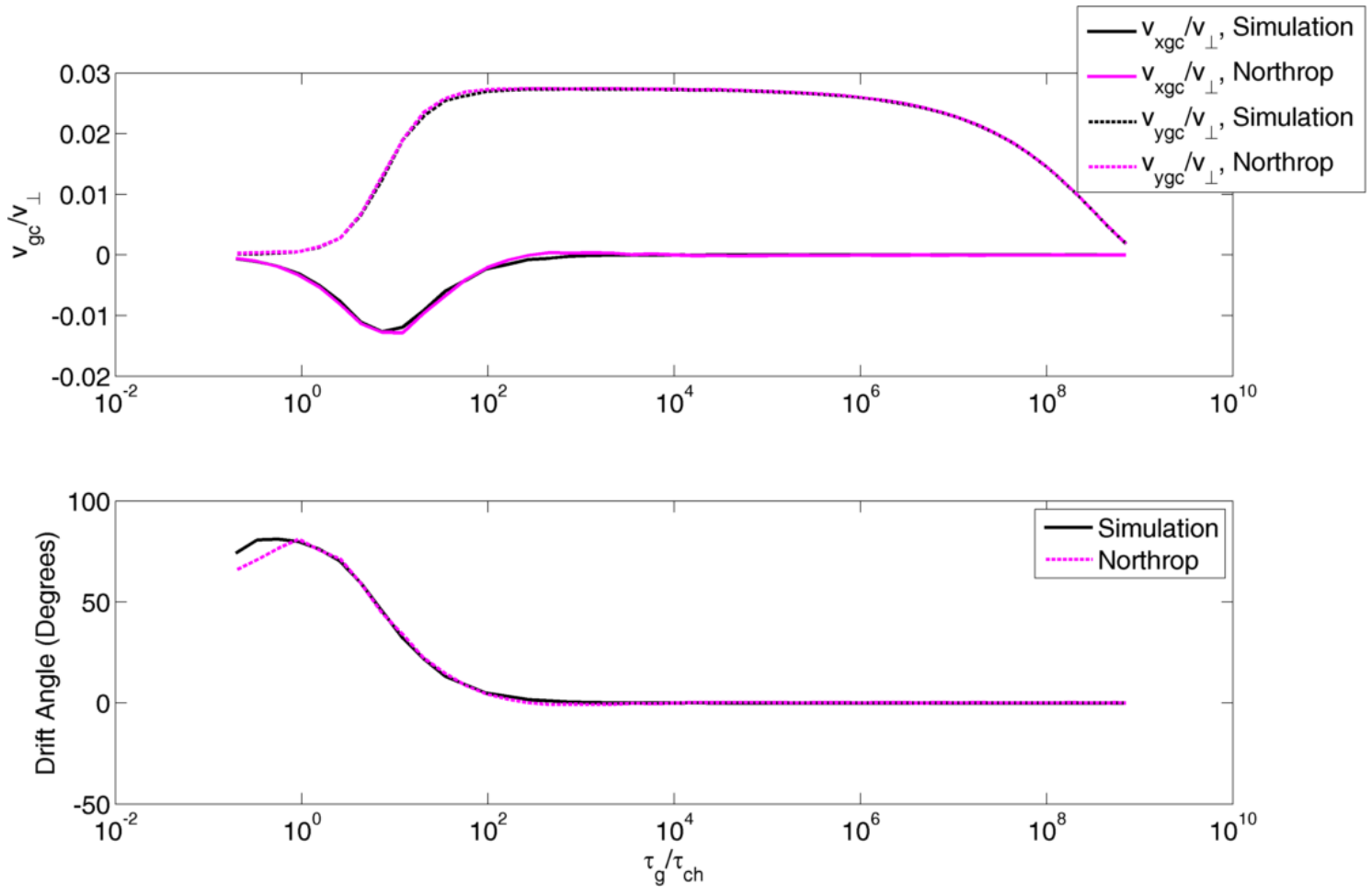

Figure 5.44: Gyro-phase drift magnitude and direction dependence on $\tau_{g} / \tau_{c}$ for $a=5 \times 10^{-7} \mathrm{~m}$ grain in the linear profile. In this figure, the effects of the electric field produced by the density gradient are not included. Electron and ion number density varies between $n_{0}=10^{9} \mathrm{~m}^{-3}$ and $n_{0}=10^{20} \mathrm{~m}^{-3}$ to produce $\tau_{c}$ variation. 

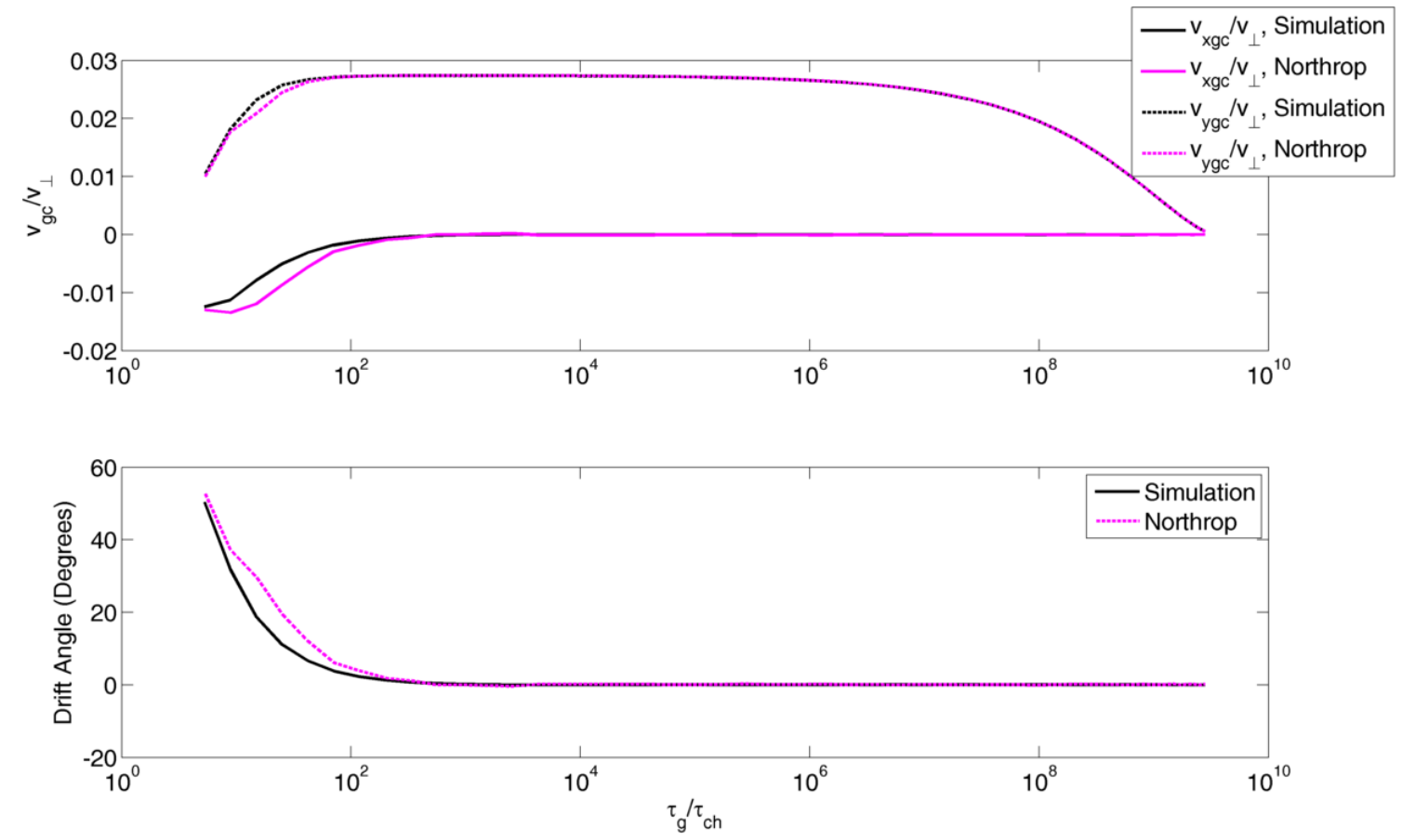

Figure 5.45: Gyro-phase drift magnitude and direction dependence on $\tau_{g} / \tau_{c}$ for $a=1.5 \times 10^{-6} \mathrm{~m}$ grain in the linear profile. In this figure, the effects of the electric field produced by the density gradient are not included. Electron and ion number density varies between $n_{0}=10^{9} \mathrm{~m}^{-3}$ and $n_{0}=10^{20} \mathrm{~m}^{-3}$ to produce $\tau_{c}$ variation. 

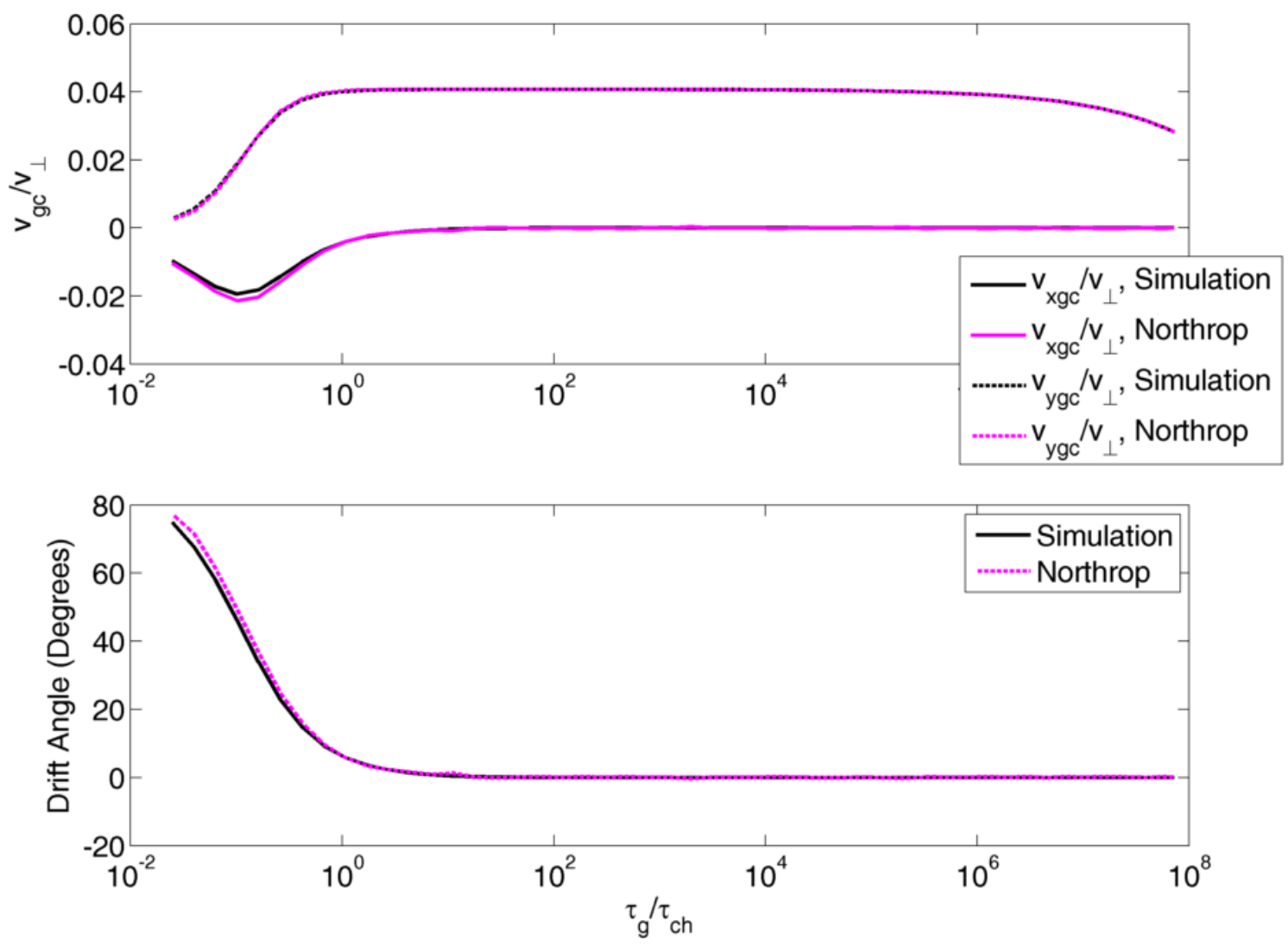

Figure 5.46: Gyro-phase drift magnitude and direction dependence on $\tau_{g} / \tau_{c}$ for $a=10 \times 10^{-6} \mathrm{~m}$ grain in the linear profile. In this figure, the effects of the electric field produced by the density gradient are not included. Electron and ion number density varies between $n_{0}=10^{9} \mathrm{~m}^{-3}$ and $n_{0}=10^{20} \mathrm{~m}^{-3}$ to produce $\tau_{c}$ variation. 


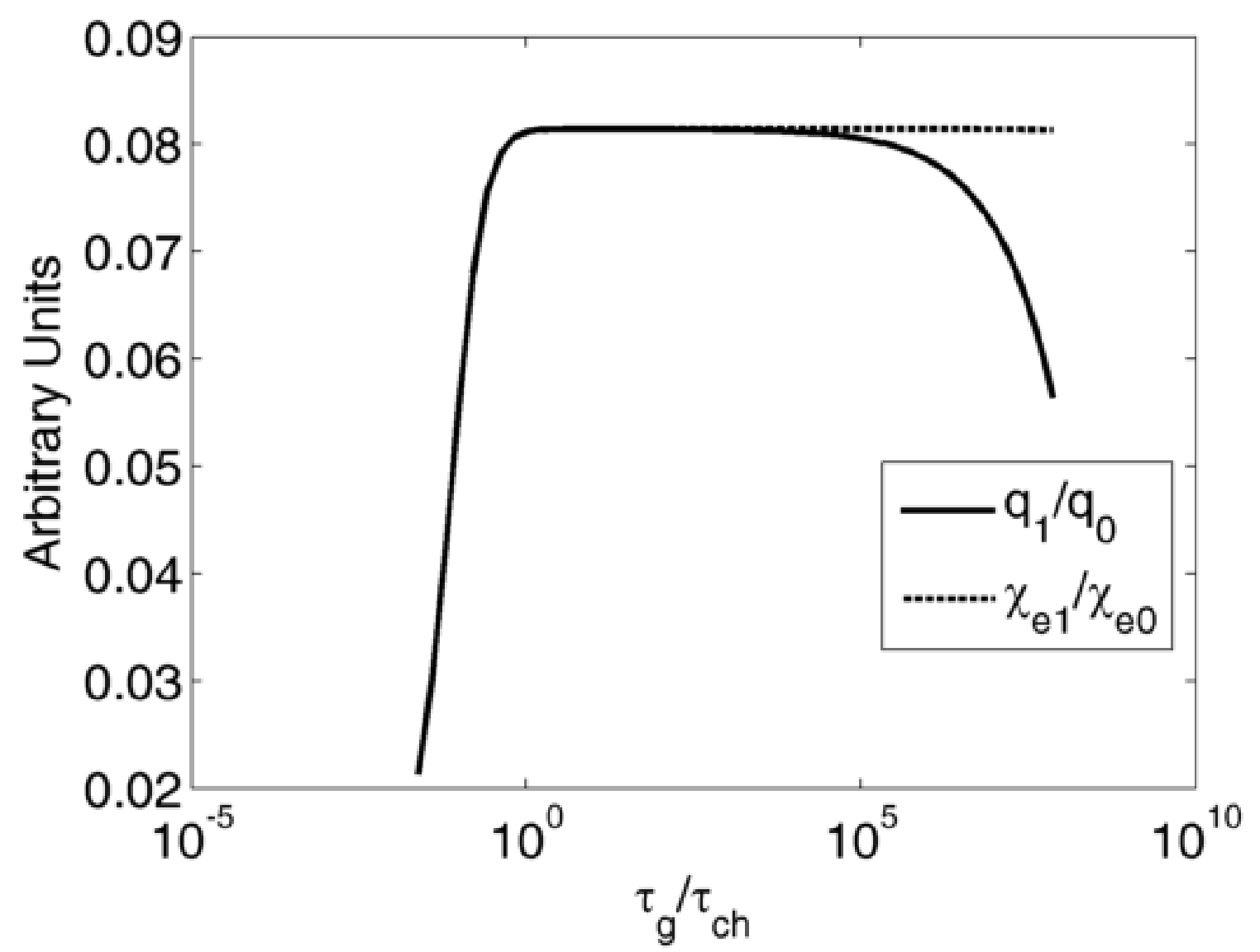

Figure 5.47: The ratio of charge variation to equilibrium charge $q_{1} / q_{0}$ dependence on $\tau_{g} / \tau_{c}$ for $a=10^{-6} \mathrm{~m}$ grain in the linear profile. This plot of $q_{1} / q_{0}$ corresponds to figure 5.46. The ratio of dimensionless surface potential variation amplitude to the $i n$-situ equilibrium dimensionless surface potential at the gyro-center $\chi_{e}^{(1)} / \chi_{0 e}$ is also shown. Electron and ion number density varies between $n_{0}=10^{9} \mathrm{~m}^{-3}$ and $n_{0}=10^{20} \mathrm{~m}^{-3}$ to produce $\tau_{c}$ variation. 


\section{Const. Charge, $\mathrm{a}=0.05 \mu \mathrm{m}$}

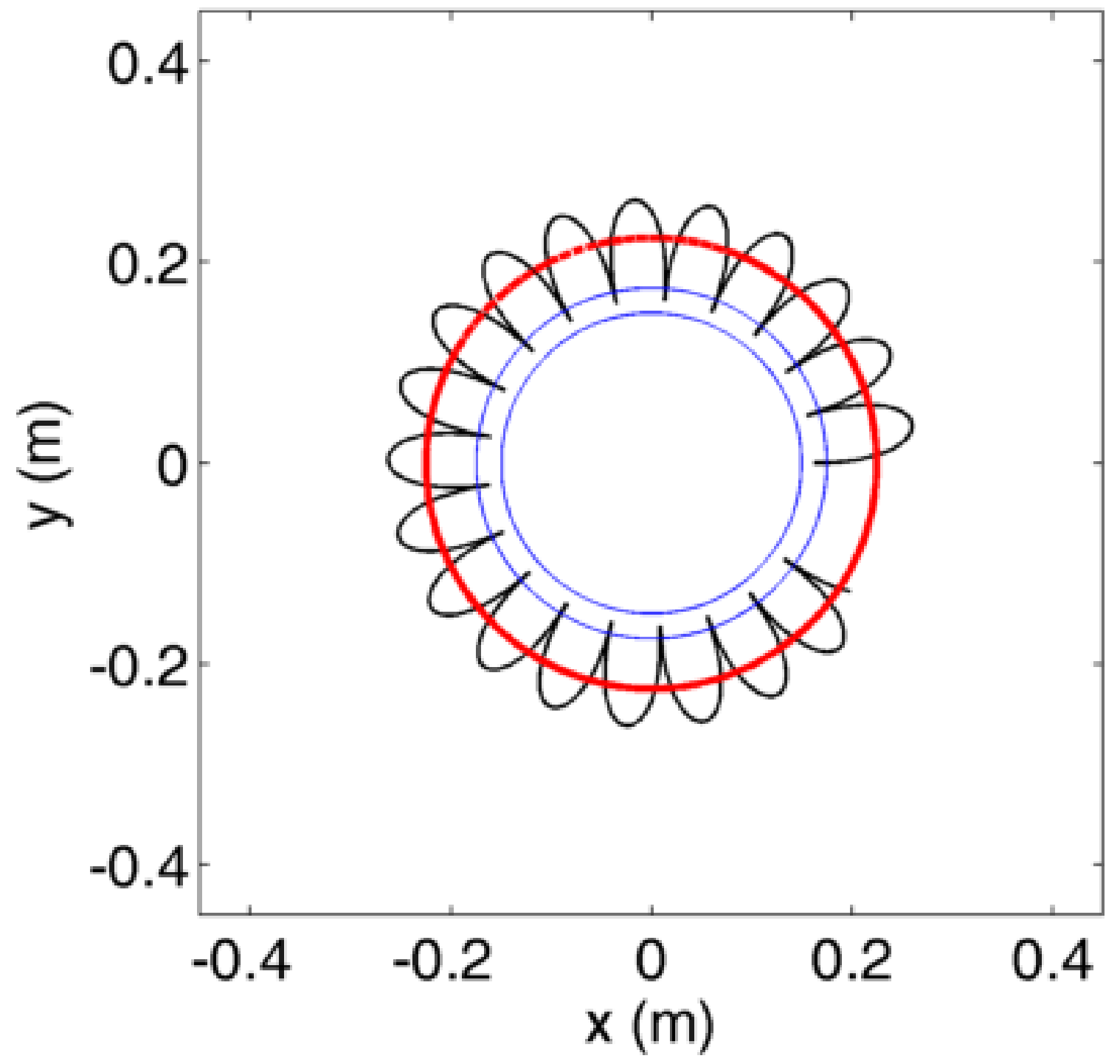

Figure 5.48: Grain trajectory in a linear-profile for drag-absent conditions in cylindrical geometry with constant charge. The coaxial blue circles correspond to the limits of the linear profile; outside this region, the plasma is quasi-neutral. The electric field is $100 \mathrm{~V} / \mathrm{m}$, directed radially-inward, and is not consistent with the density gradient. 


\section{Non-Inst. Charging, $\mathrm{a}=0.05 \mu \mathrm{m}$}

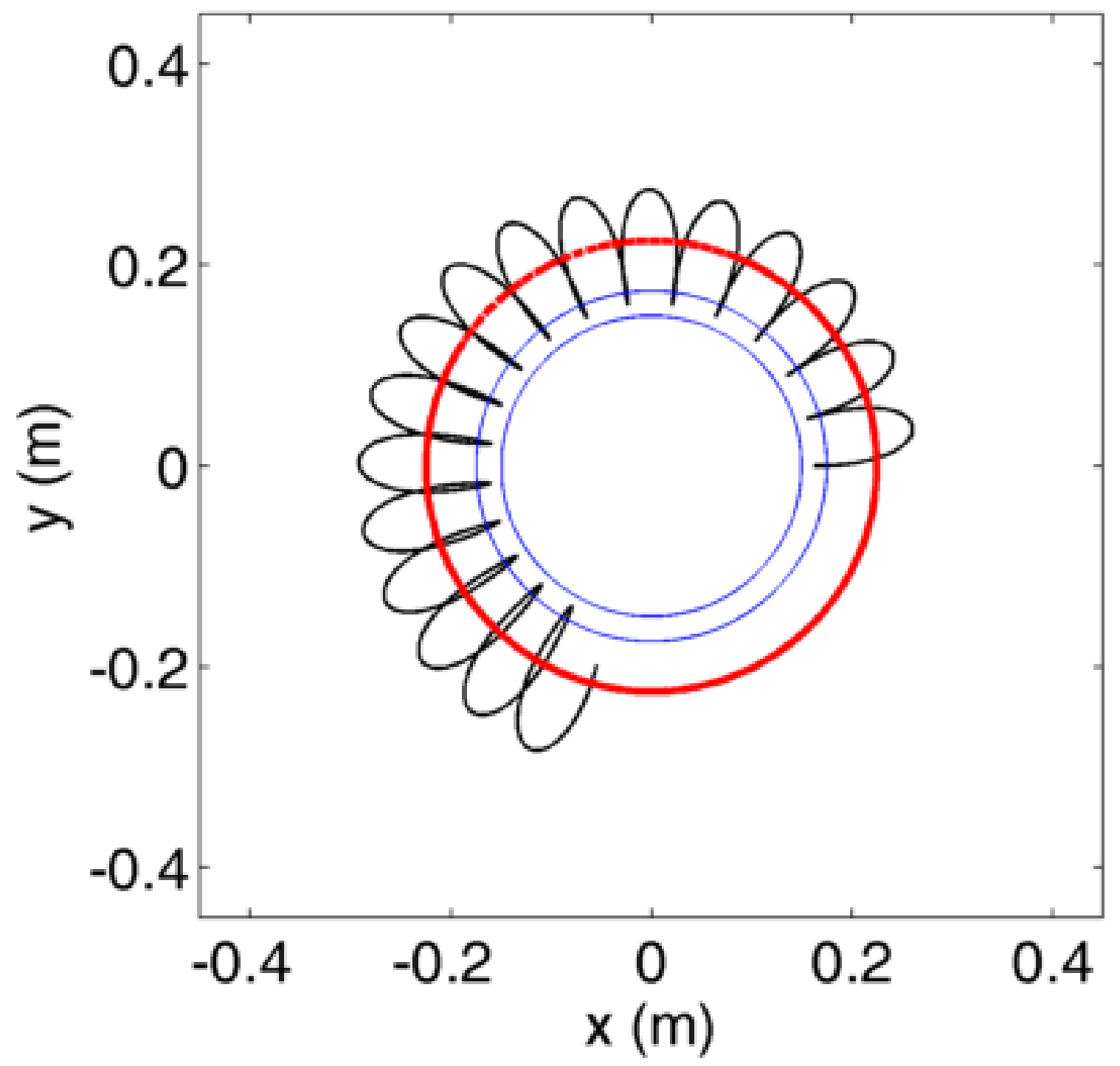

Figure 5.49: Grain trajectory in a linear-profile for drag-absent conditions in cylindrical geometry with instantaneous charging. The coaxial blue circles correspond to the limits of the linear profile; outside this region, the plasma is quasi-neutral. The electric field is $100 \mathrm{~V} / \mathrm{m}$, directed radiallyinward, and is not consistent with the density gradient. 


\section{Non-Inst. Charging, $\mathrm{a}=0.05 \mu \mathrm{m}, \mathrm{P}=1 \mathrm{mTorr}$}

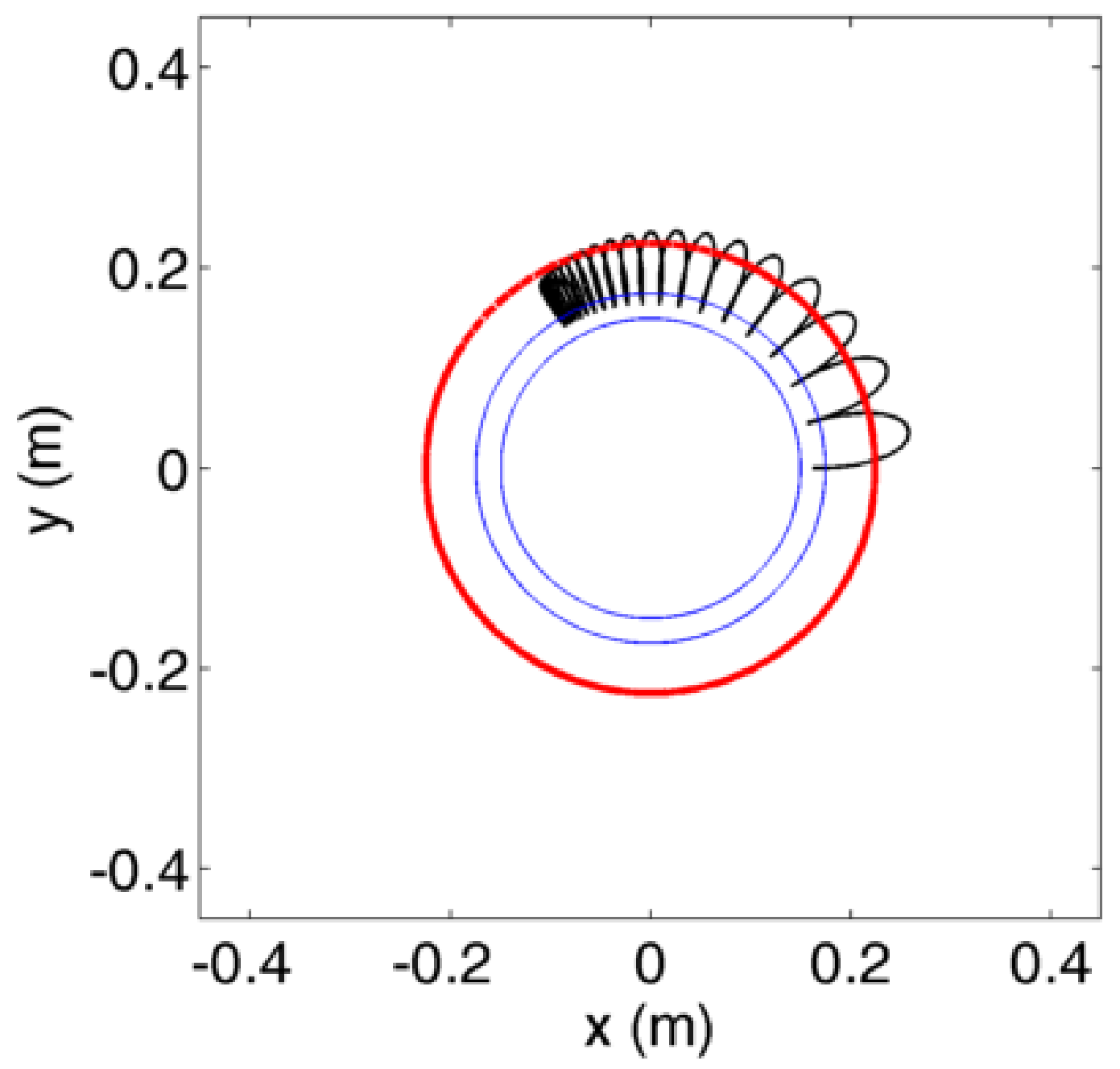

Figure 5.50: Grain trajectory in a linear-profile when drag is present in cylindrical geometry with non-instantaneous charging. The coaxial blue circles correspond to the limits of the linear profile; outside this region, the plasma is quasi-neutral. The electric field is $100 \mathrm{~V} / \mathrm{m}$, directed radiallyinward, and is not consistent with the density gradient. 

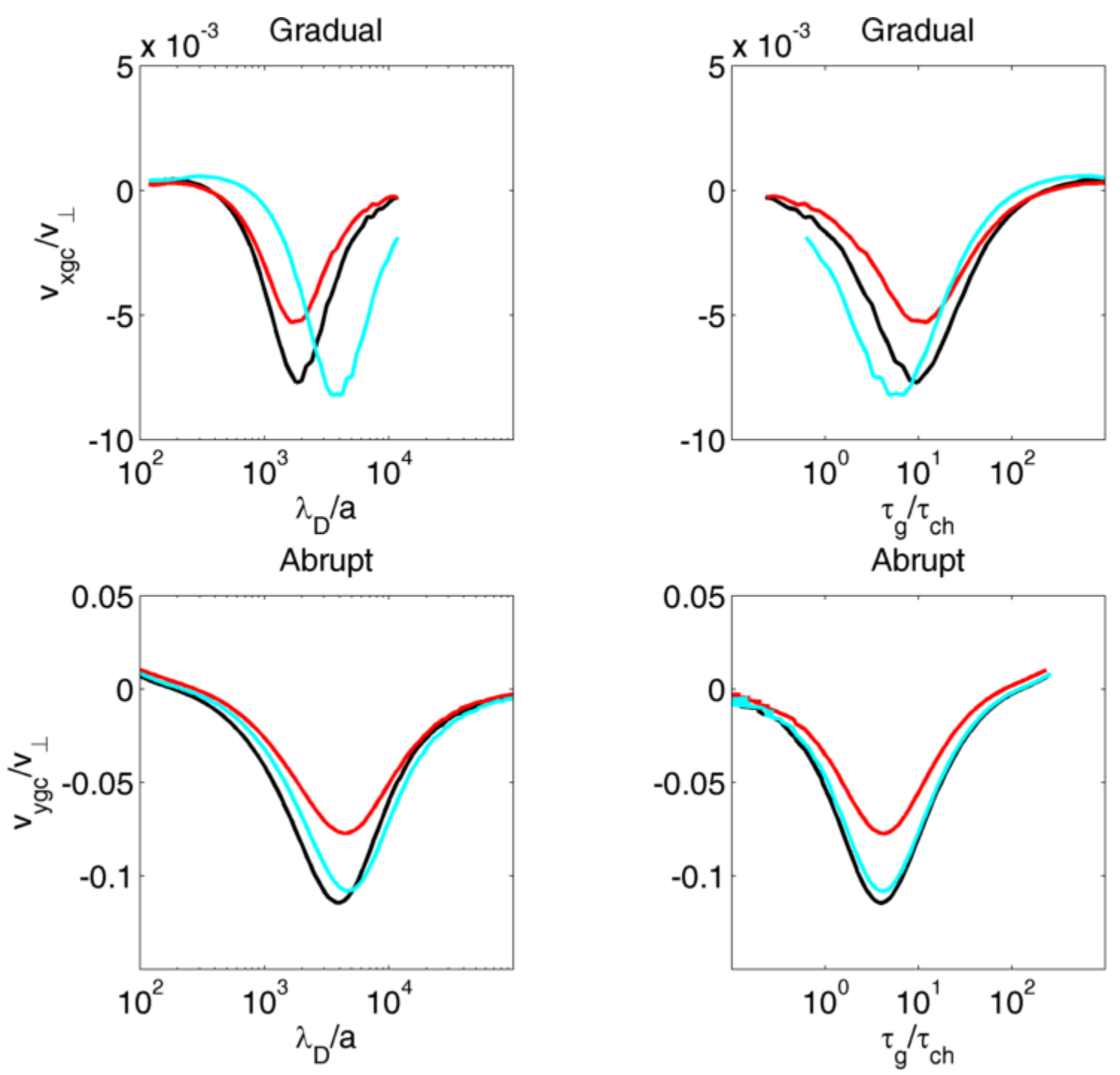

Figure 5.51: This shows a comparison between the guiding center drift for abrupt and gradual inhomogeneity for the $\lambda_{D} / a$ parameter. The data shown for the abrupt inhomogeneity use the parameters from figure 5.29. For the gradual inhomogeneity, the effects of the electric field are not included. 


\section{Spectral data}

\section{Peak wavelength $172 \mathrm{~nm}$ Radiation intensity at lamp surface $45 \mathrm{~mW} / \mathrm{cm}^{2}$ (Radiation with active cooling Generates ozone $80 \mathrm{~mW} / \mathrm{cm}^{2}$ ) \\ yes}

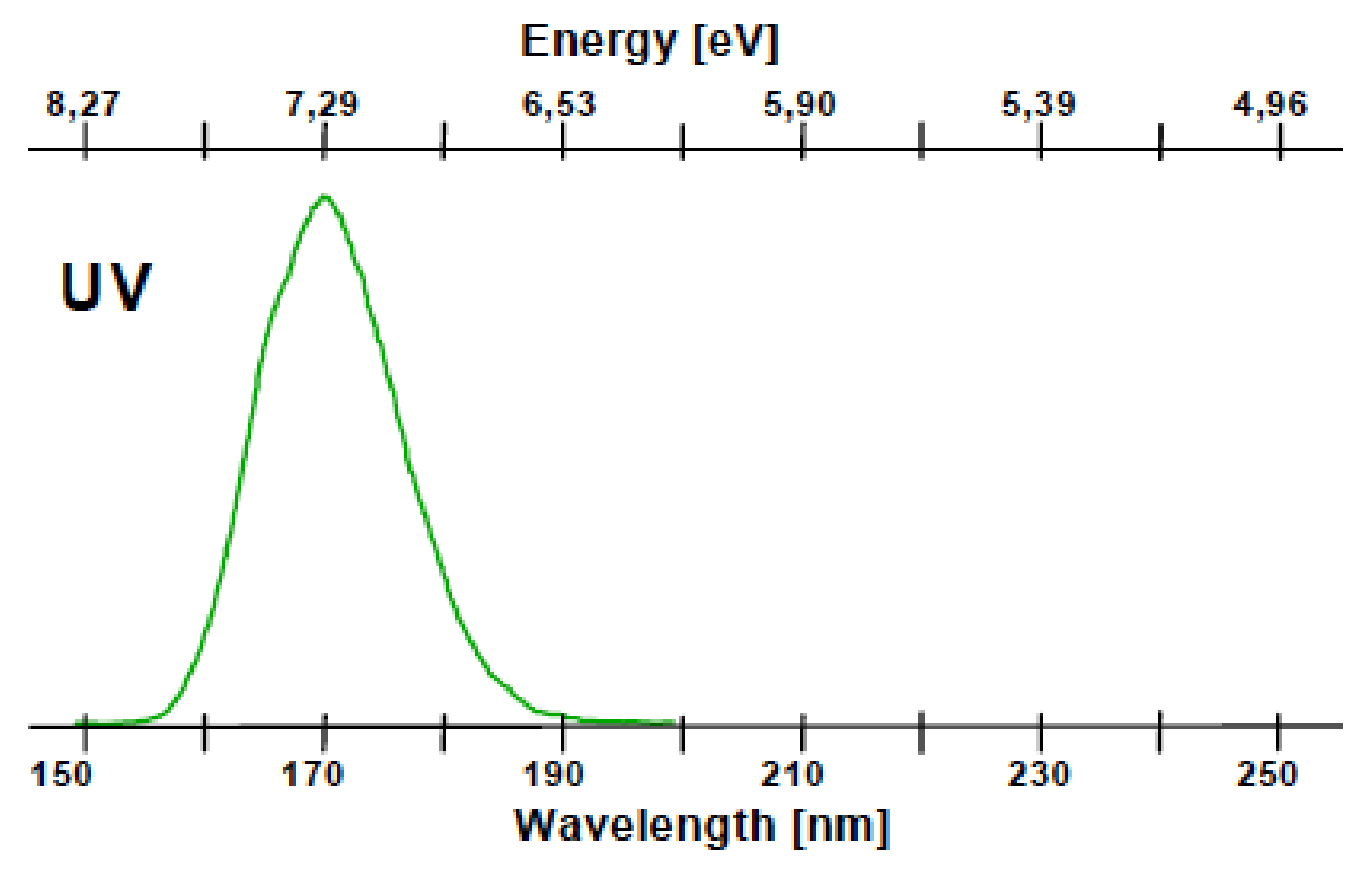

Figure 6.1: Spectrum of Osram Xeradex lamp used in the experiments of Dove et al. (2012), and used to make predictions for guiding center drift in an abrupt inhomogeneity. 


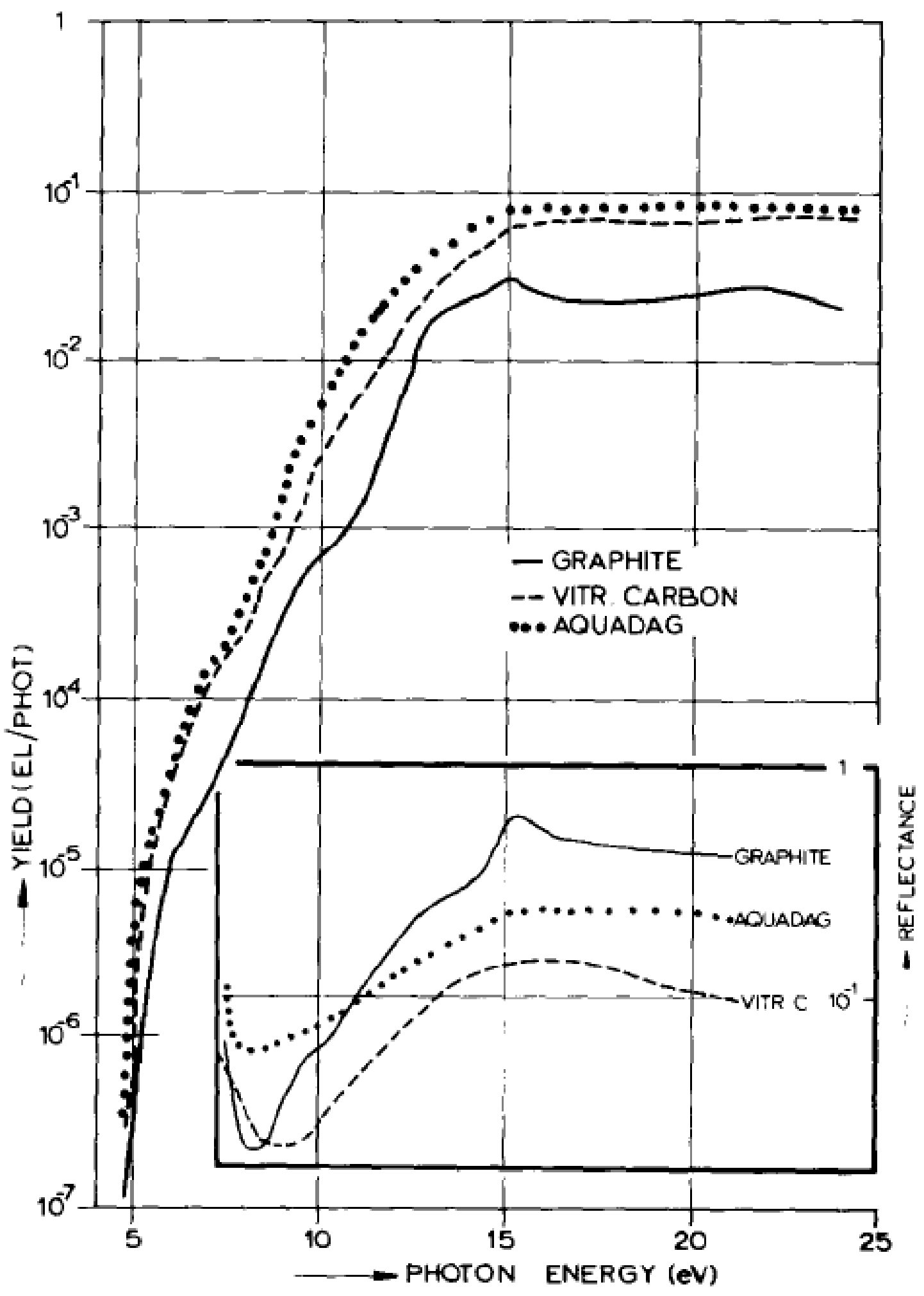

Figure 6.2: Photo-electron yield as a function of wavelength for selected Carbon allotropes. Taken from Feuerbacher and Fitton (1972). The inset shows the reflectance. 


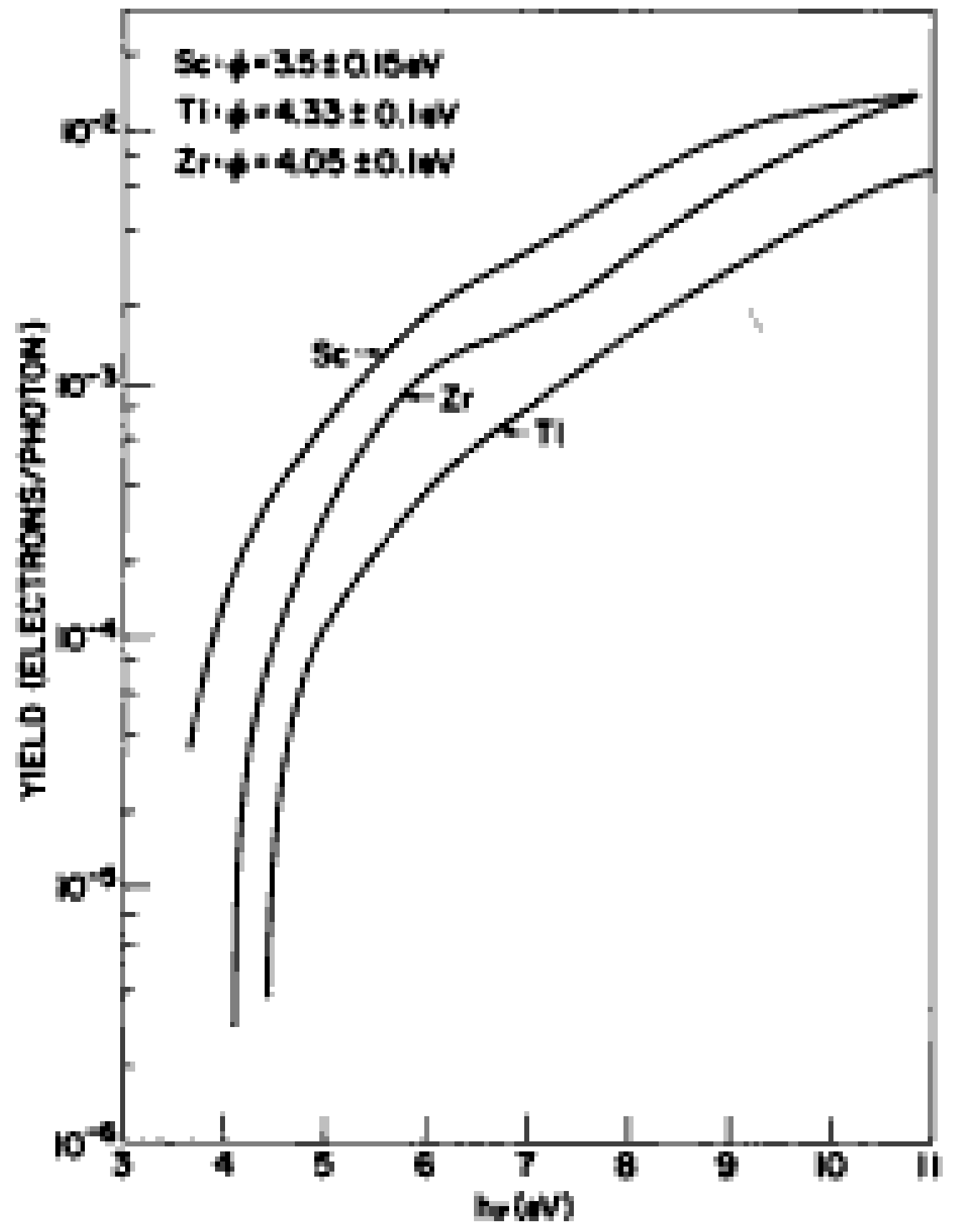

Figure 6.3: Photo-electron yield as a function of wavelength for Zirconium. Taken from Eastman (1971). 


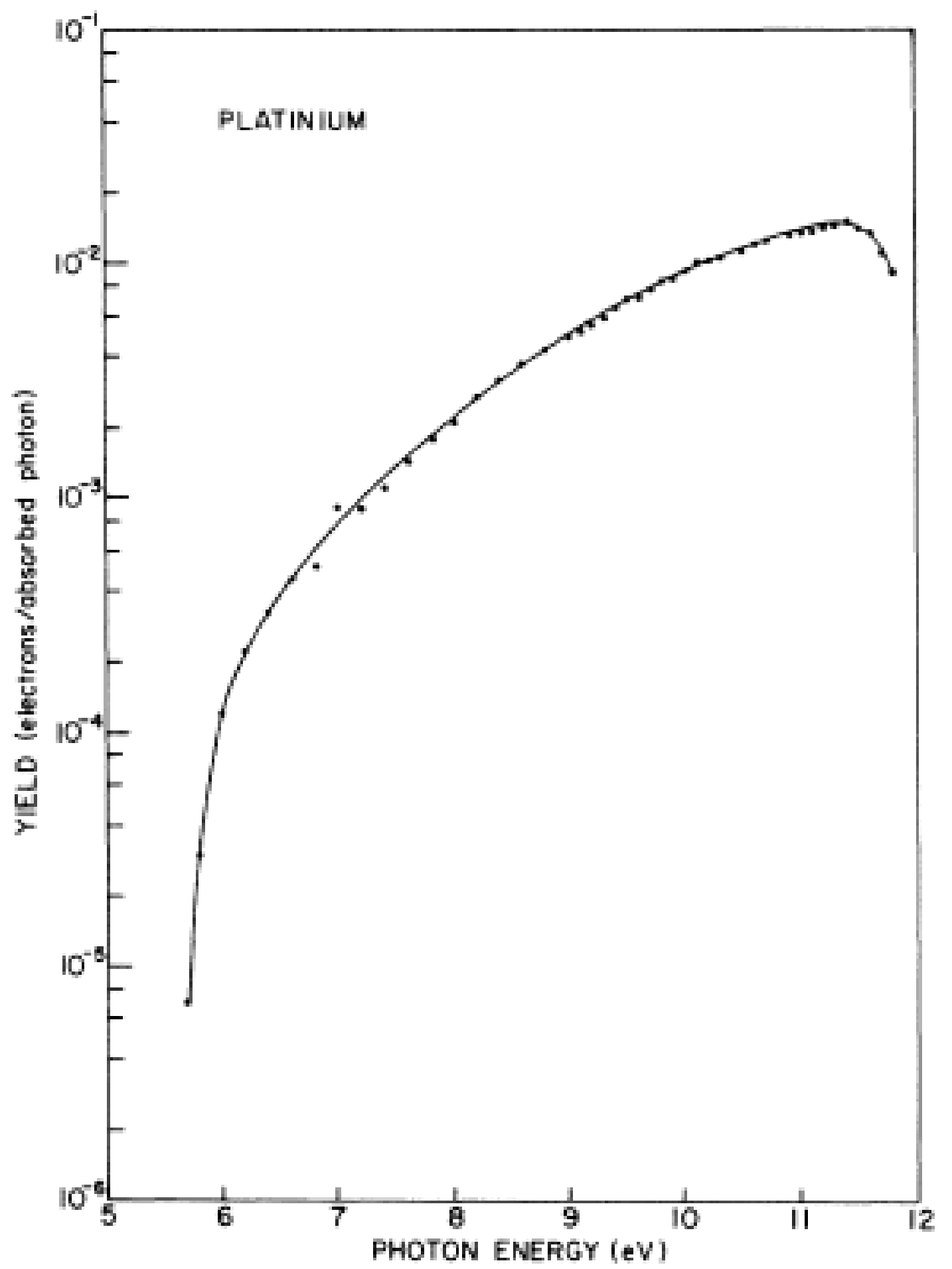

FIG. 2. Absolute spectral distribution of quantum yield of Pt.

Figure 6.4: Photo-electron yield as a function of wavelength for Platinum. Taken from Lin et al. (1971). 

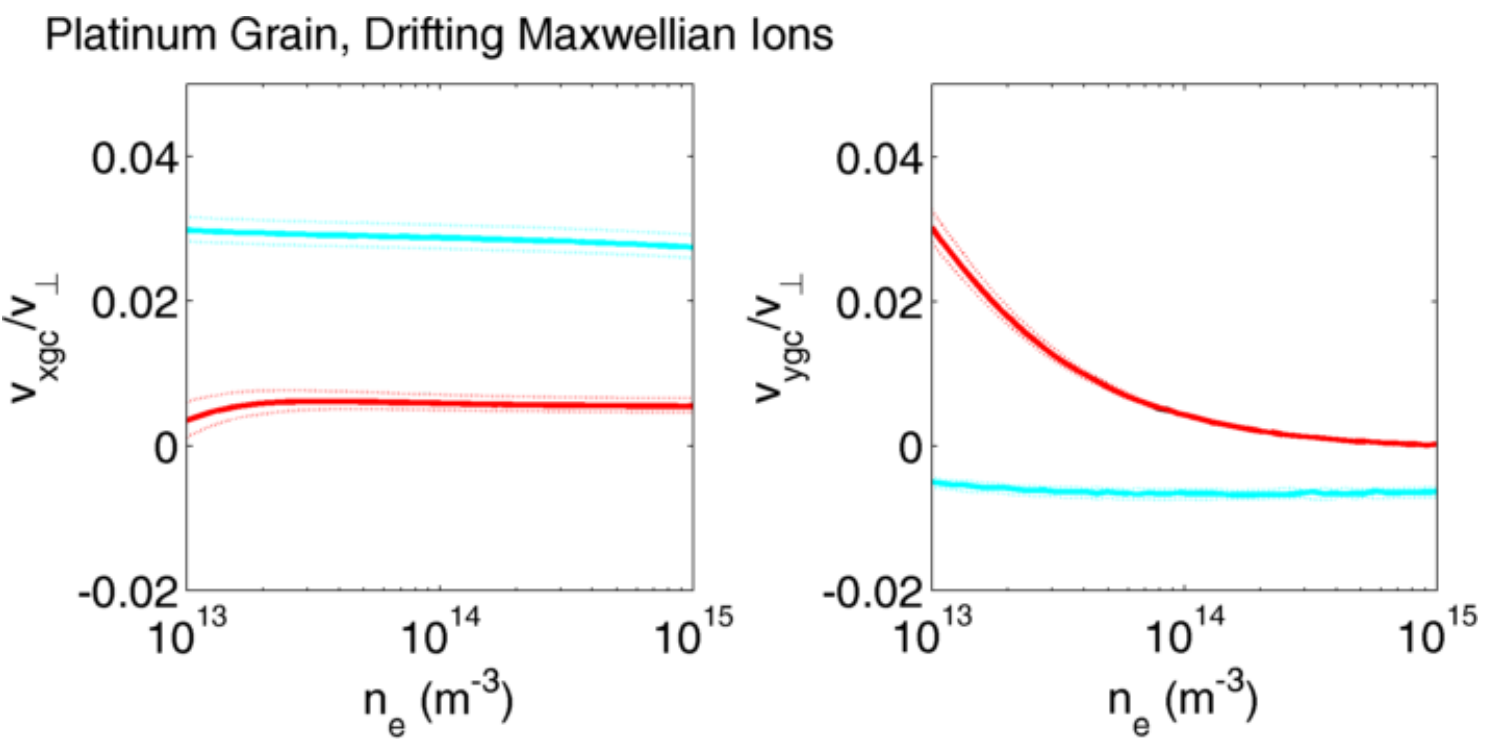

Figure 6.5: Guiding Center drift for spherical Platinum grains, assuming an initial gyro-phase of $-90^{\circ}$ and the UV spectrum shown in figure 6.1 but with 10 times as much power output. Additional parameters include $a=200 \mathrm{~nm}, T_{n}=0.025 \mathrm{eV}, T_{e}=5 \mathrm{eV}, \omega_{c d} / \nu_{d n}=4$, drifting Maxwellian ions flowing at the Bohm speed, and an Argon plasma. The solid black, red, and cyan curves correspond to the results for the OML, Patacchini-Hutchinson, and Gatti-Kortshagen charging models respectively. There is virtually no difference between the OML and Pattacchini-Hutchinson charging models. The smaller dotted lines indicate the upper and lower bounds of the guiding center drift, assuming that $T_{e}=5 \mathrm{eV}$, with an uncertainty of $\pm 0.5 \mathrm{eV}$. 


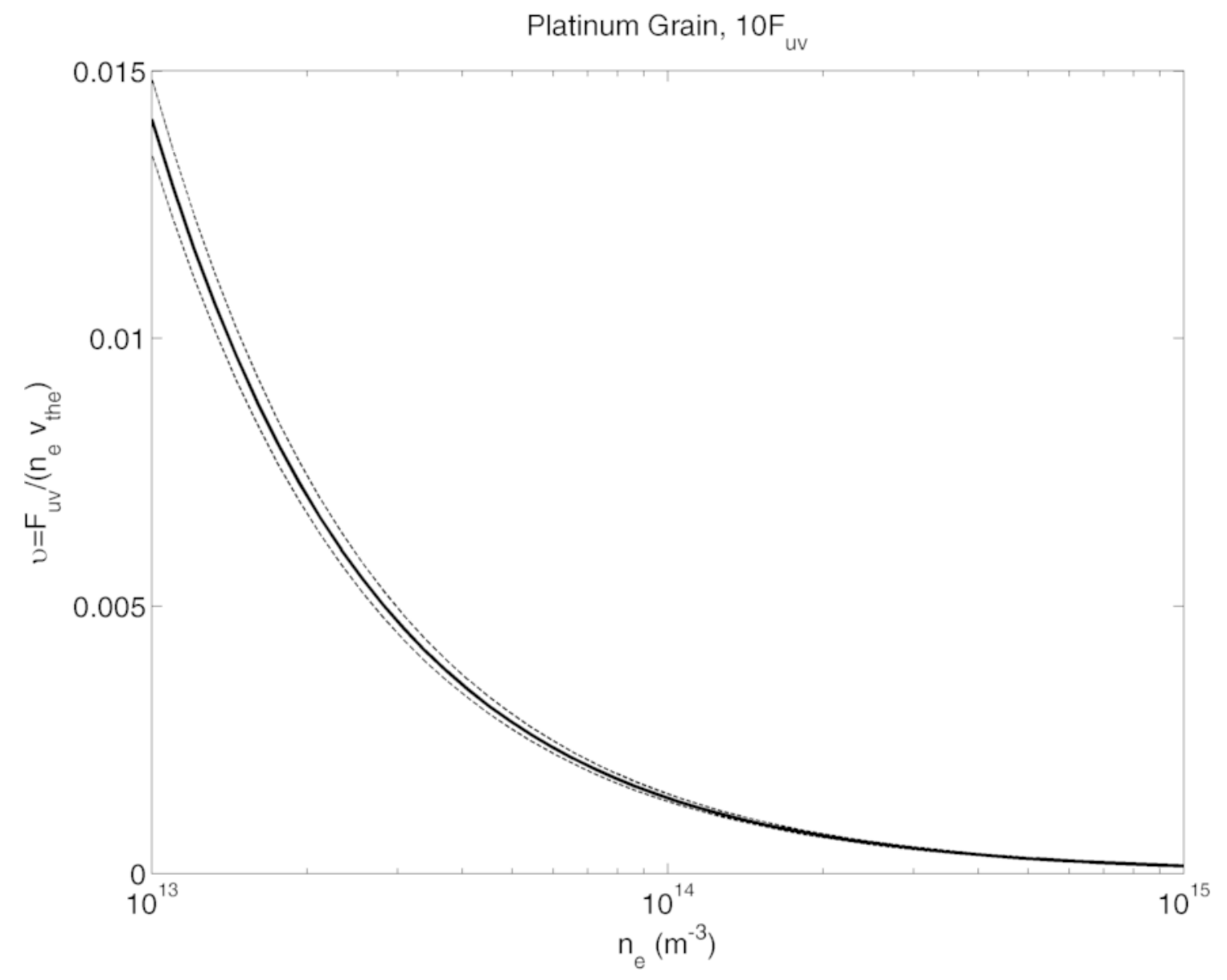

Figure 6.6: The $v=f_{u v} /\left(n_{e} v_{t h e}\right)$ parameter as a function of plasma density used in figure 6.5. The smaller dashed lines indicate the upper and lower bounds of $v$, assuming that $T_{e}=5 \mathrm{eV}$, with an uncertainty of $\pm 0.5 \mathrm{eV}$. 


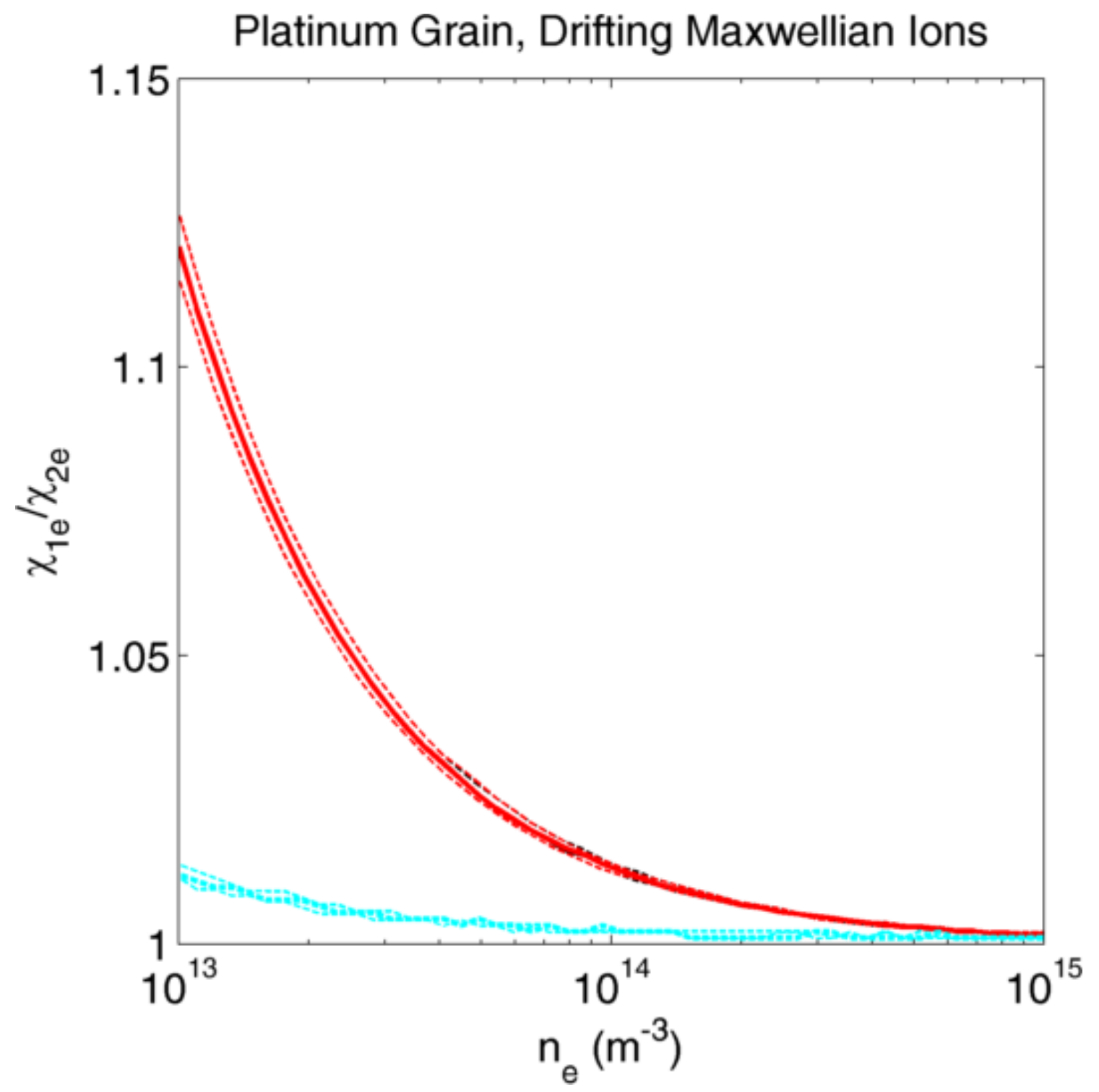

Figure 6.7: The ratio of $i n$-situ equilibrium surface potentials $\chi_{e}^{(1)} / \chi_{e}^{(2)}$ corresponding to figure 6.5. The smaller dashed lines indicate the upper and lower bounds of $\chi_{e}^{(1)} / \chi_{e}^{(2)}$, assuming that $T_{e}=5$ $\mathrm{eV}$ with an uncertainty of $\pm 0.5 \mathrm{eV}$. 

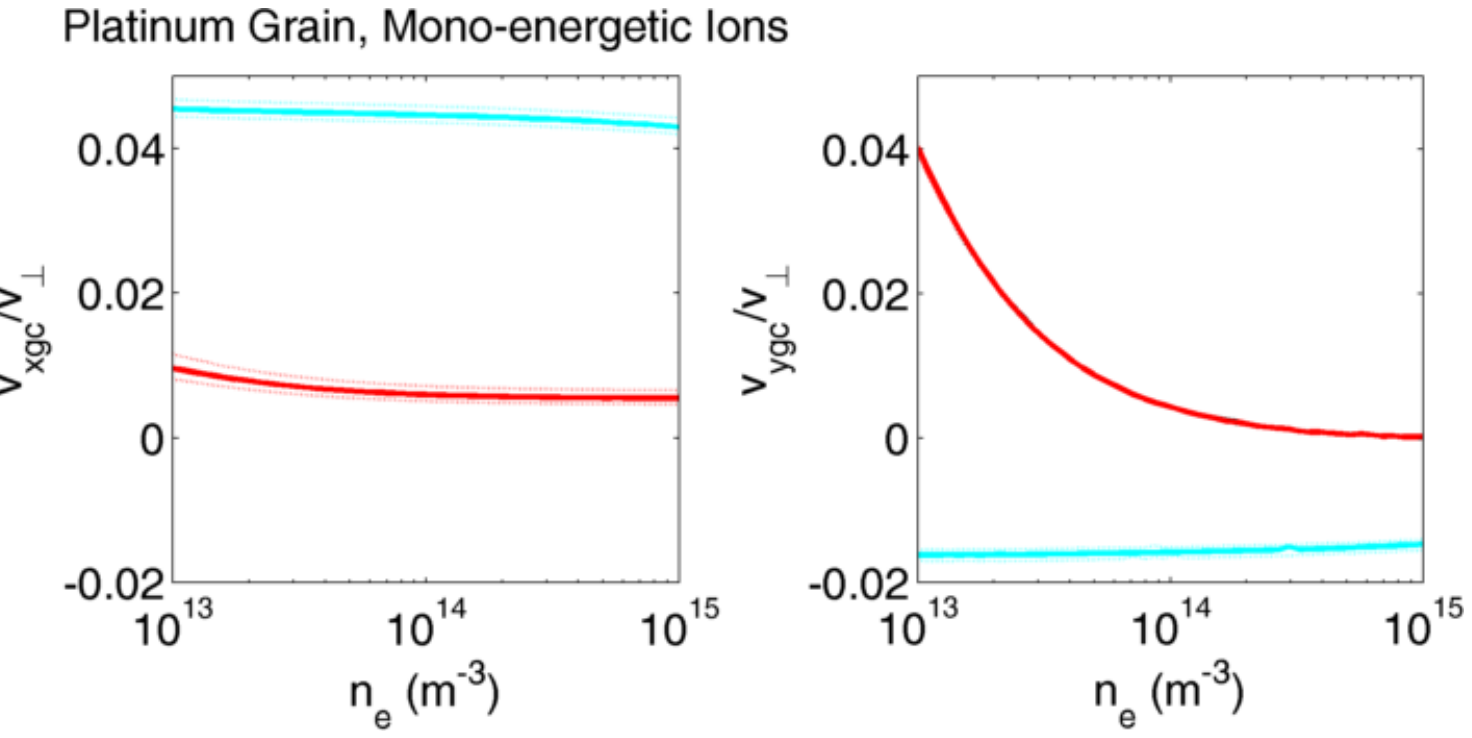

Figure 6.8: Guiding Center drift for spherical Platinum grains, assuming an initial gyro-phase of $-90^{\circ}$ and the UV spectrum shown in figure 6.1 but with 10 times as much power output. Additional parameters include $a=200 \mathrm{~nm}, T_{n}=0.025 \mathrm{eV}, T_{e}=5 \mathrm{eV}, \omega_{c d} / \nu_{d n}=4$, mono-energetic ions flowing at the Bohm speed, and an Argon plasma. The solid black, red, and cyan curves correspond to the results for the OML, Patacchini-Hutchinson, and Gatti-Kortshagen charging models respectively. There is virtually no difference between the OML and Pattacchini-Hutchinson charging models. The smaller dotted lines indicate the upper and lower bounds of the guiding center drift, assuming that $T_{e}=5 \mathrm{eV}$ with an uncertainty of $\pm 0.5 \mathrm{eV}$. 


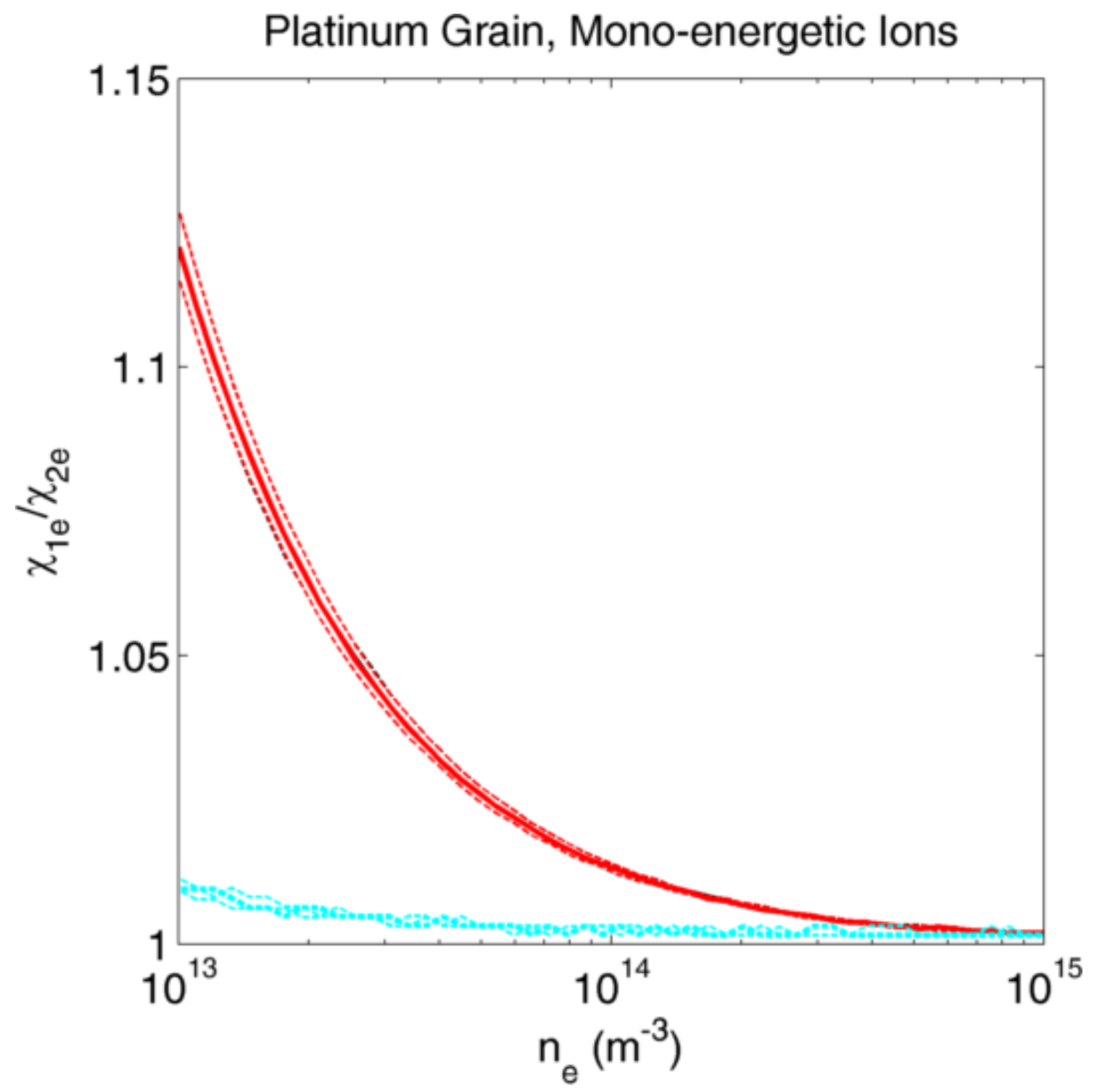

Figure 6.9: The ratio of $i n$-situ equilibrium surface potentials $\chi_{e}^{(1)} / \chi_{e}^{(2)}$ corresponding to figure 6.8. The smaller dashed lines indicate the upper and lower bounds of $\chi_{e}^{(1)} / \chi_{e}^{(2)}$, assuming that $T_{e}=5$ $\mathrm{eV}$ with an uncertainty of $\pm 0.5 \mathrm{eV}$. 

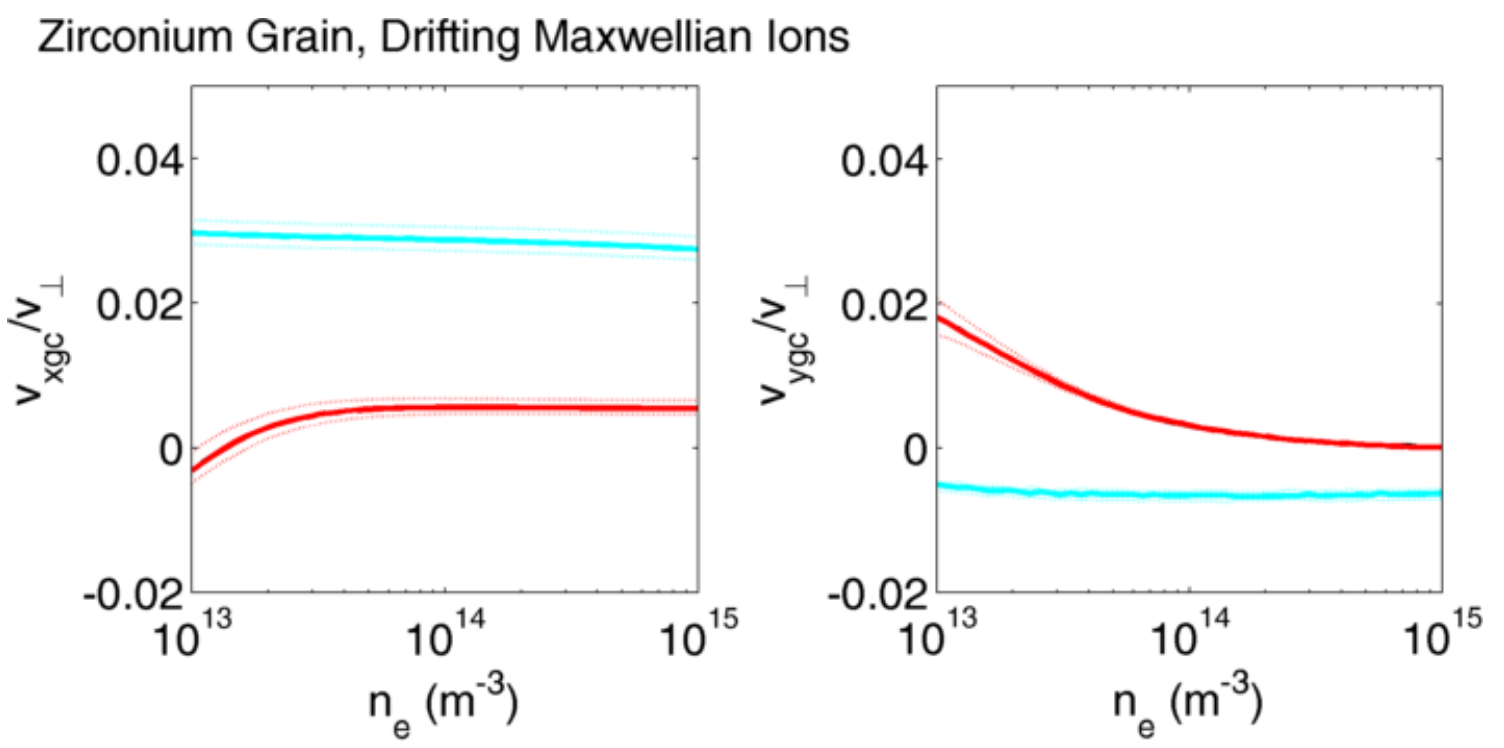

Figure 6.10: Guiding Center drift for spherical Zirconium grains, assuming an initial gyro-phase of $-90^{\circ}$ and the UV spectrum shown in figure 6.1 but with 10 times as much power output. Additional parameters include $a=200 \mathrm{~nm}, T_{n}=0.025 \mathrm{eV}, T_{e}=5 \mathrm{eV}, \omega_{c d} / \nu_{d n}=4$, drifting Maxwellian ions flowing at the Bohm speed, and an Argon plasma. The solid black, red, and cyan curves correspond to the results for the OML, Patacchini-Hutchinson, and Gatti-Kortshagen charging models respectively. There is virtually no difference between the OML and Pattacchini-Hutchinson charging models. The smaller dotted lines indicate the upper and lower bounds of the guiding center drift, assuming that $T_{e}=5 \mathrm{eV}$ with an uncertainty of $\pm 0.5 \mathrm{eV}$. 


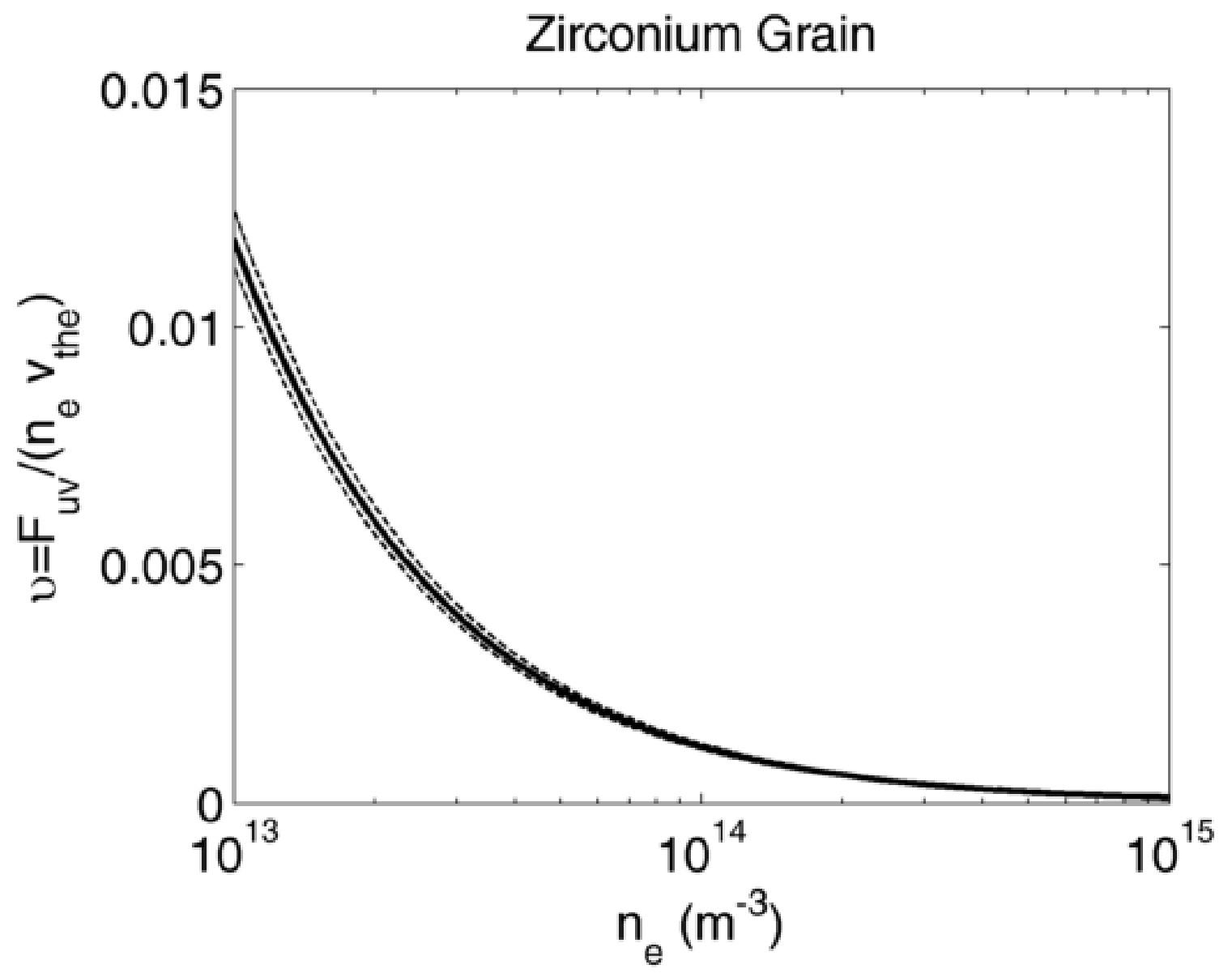

Figure 6.11: The $v=f_{u v} /\left(n_{e} v_{t h e}\right)$ parameter as a function of plasma density, used in figure 6.10. The smaller dashed lines indicate the upper and lower bounds of $v$, assuming that $T_{e}=5 \mathrm{eV}$ with an uncertainty of $\pm 0.5 \mathrm{eV}$. 


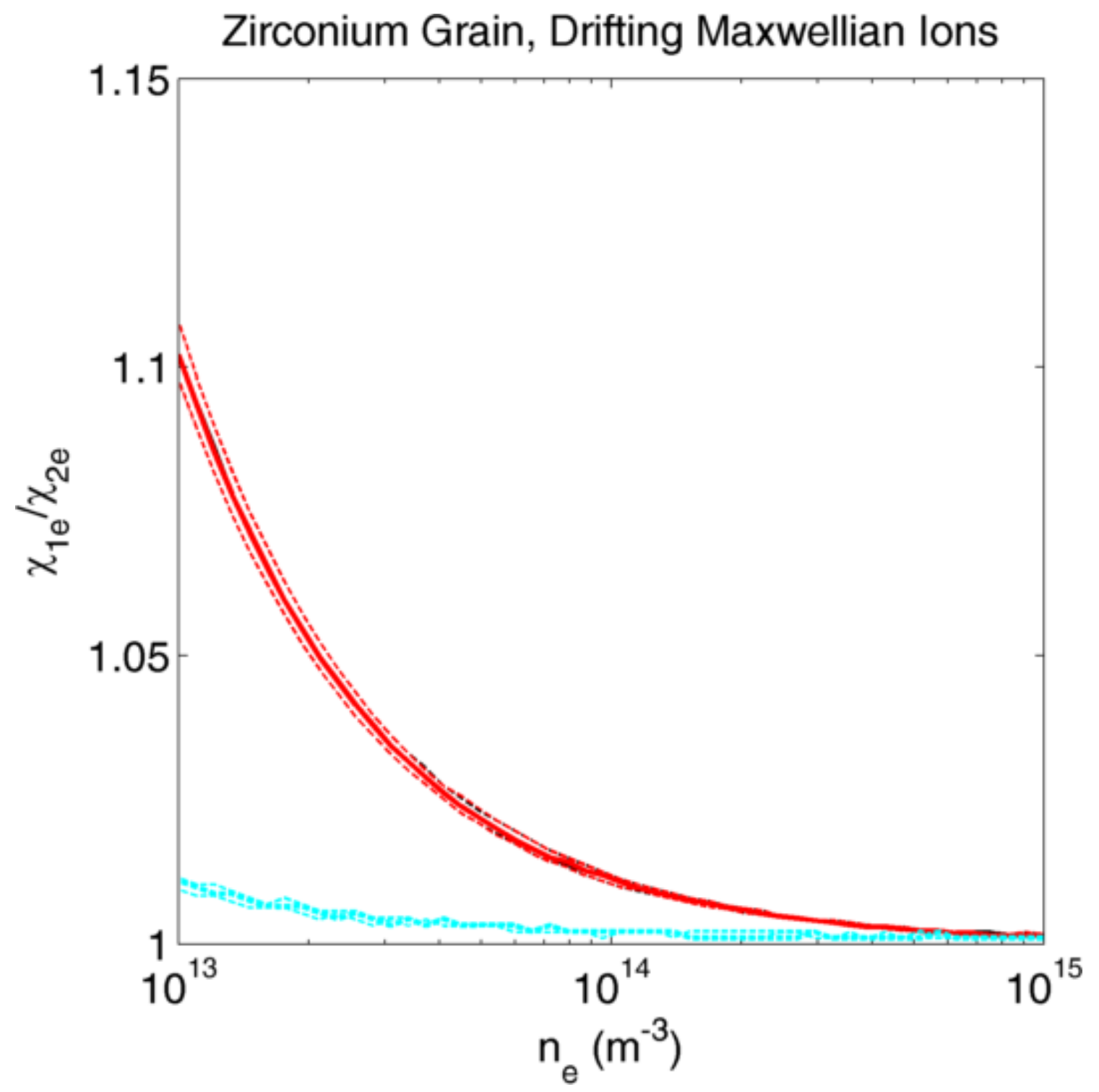

Figure 6.12: The ratio of in-situ equilibrium surface potentials $\chi_{e}^{(1)} / \chi_{e}^{(2)}$ corresponding to figure 6.10. The smaller dashed lines indicate the upper and lower bounds of $\chi_{e}^{(1)} / \chi_{e}^{(2)}$, assuming that $T_{e}=5 \mathrm{eV}$ with an uncertainty of $\pm 0.5 \mathrm{eV}$. 


\section{Zirconium Grain, Mono-energetic lons}
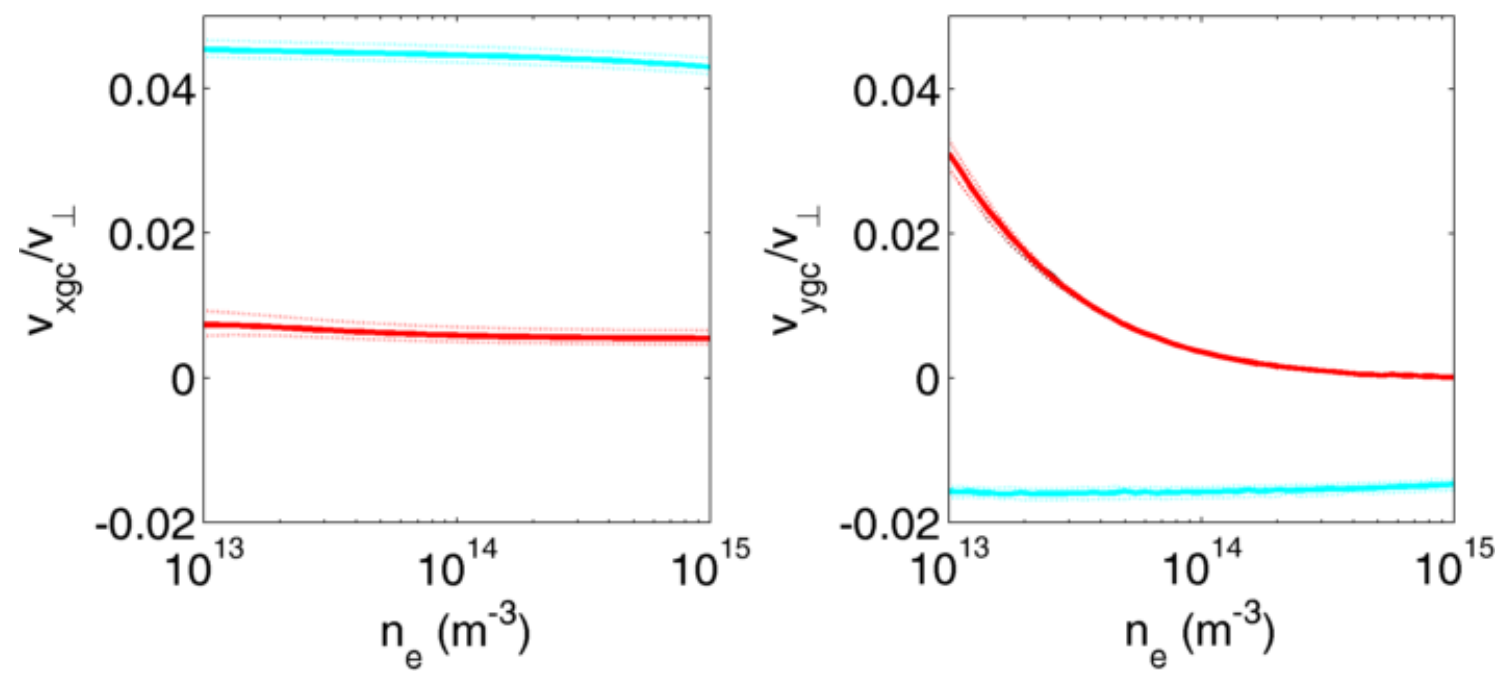

Figure 6.13: Guiding Center drift for spherical Zirconium grains, assuming an initial gyro-phase of $-90^{\circ}$ and the UV spectrum shown in figure 6.1 but with 10 times as much power output. Additional parameters include $a=200 \mathrm{~nm}, T_{n}=0.025 \mathrm{eV}, T_{e}=5 \mathrm{eV}, \omega_{c d} / \nu_{d n}=4$, mono-energetic ions flowing at the Bohm speed, and an Argon plasma. The solid black, red, and cyan curves correspond to the results for the OML, Patacchini-Hutchinson, and Gatti-Kortshagen charging models respectively. There is virtually no difference between the OML and Pattacchini-Hutchinson charging models. The smaller dashed lines indicate the upper and lower bounds of the guiding center drift, assuming that $T_{e}=5 \mathrm{eV}$ with an uncertainty of $\pm 0.5 \mathrm{eV}$. 


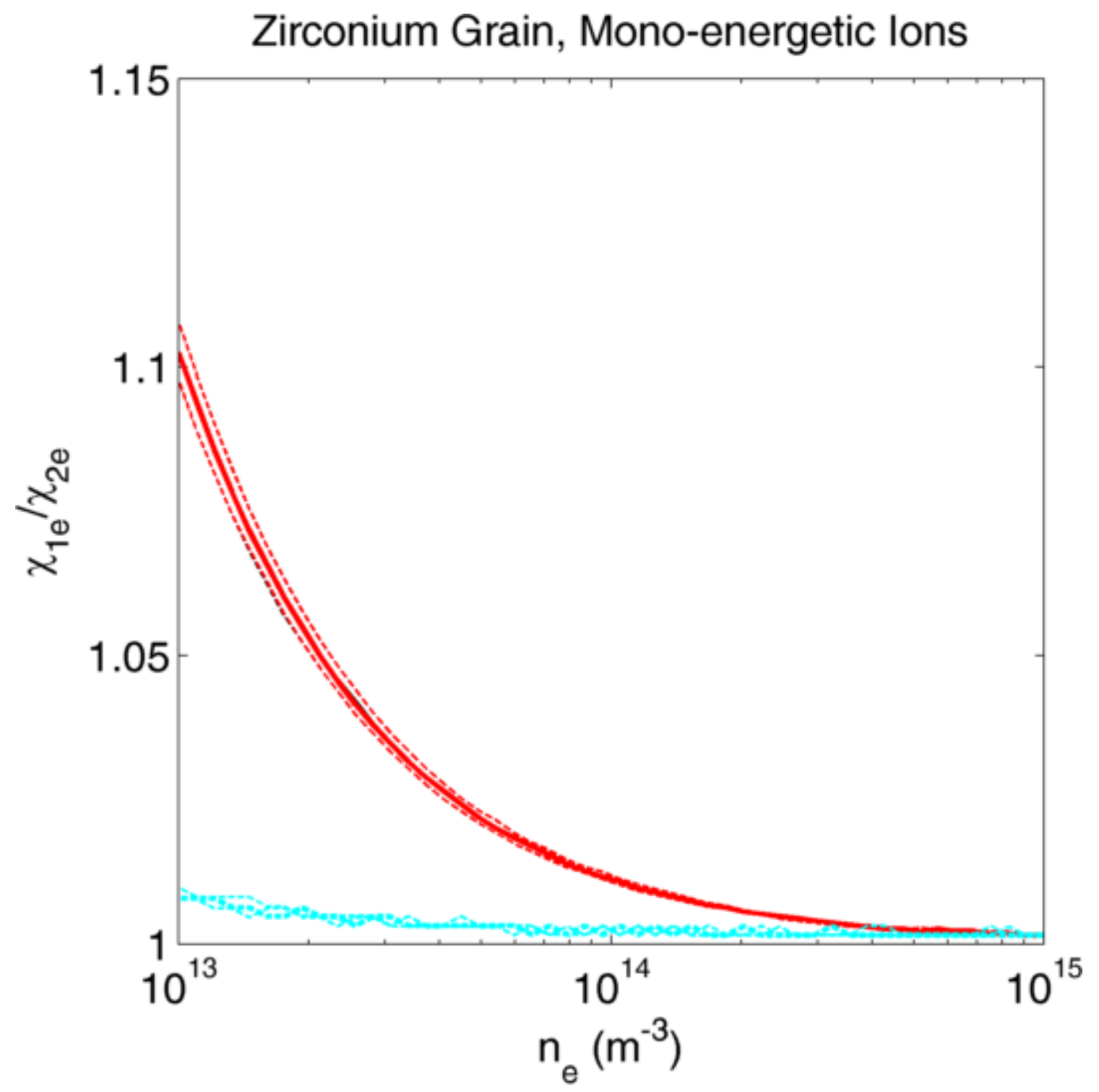

Figure 6.14: The ratio of in-situ equilibrium surface potentials $\chi_{e}^{(1)} / \chi_{e}^{(2)}$ corresponding to figure 6.13. The smaller dashed lines indicate the upper and lower bounds of $\chi_{e}^{(1)} / \chi_{e}^{(2)}$, assuming that $T_{e}=5 \mathrm{eV}$ with an uncertainty of $\pm 0.5 \mathrm{eV}$. 


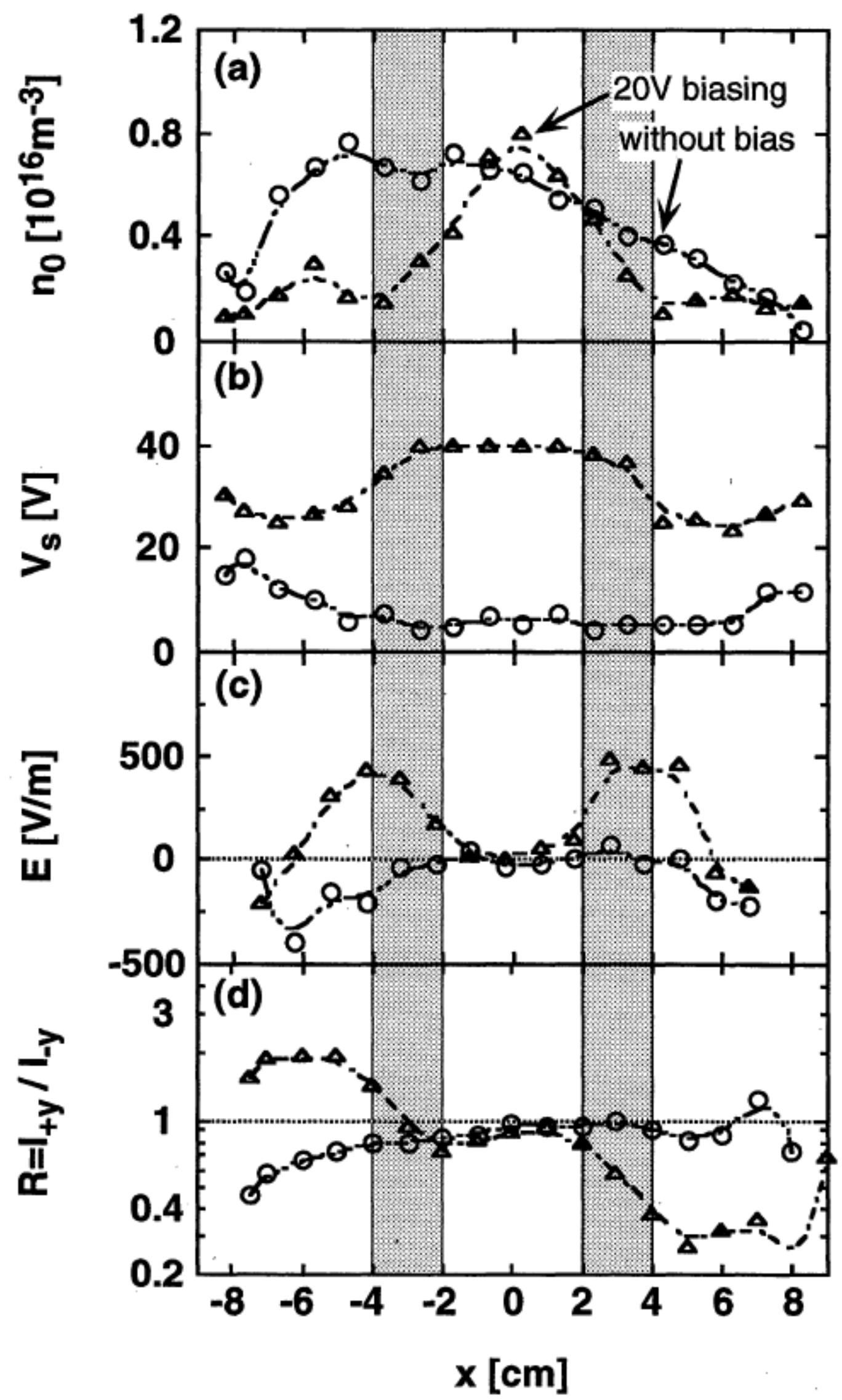

Figure 6.15: Measured profiles from an ECR plasma (Nunomura et al., 1997). 

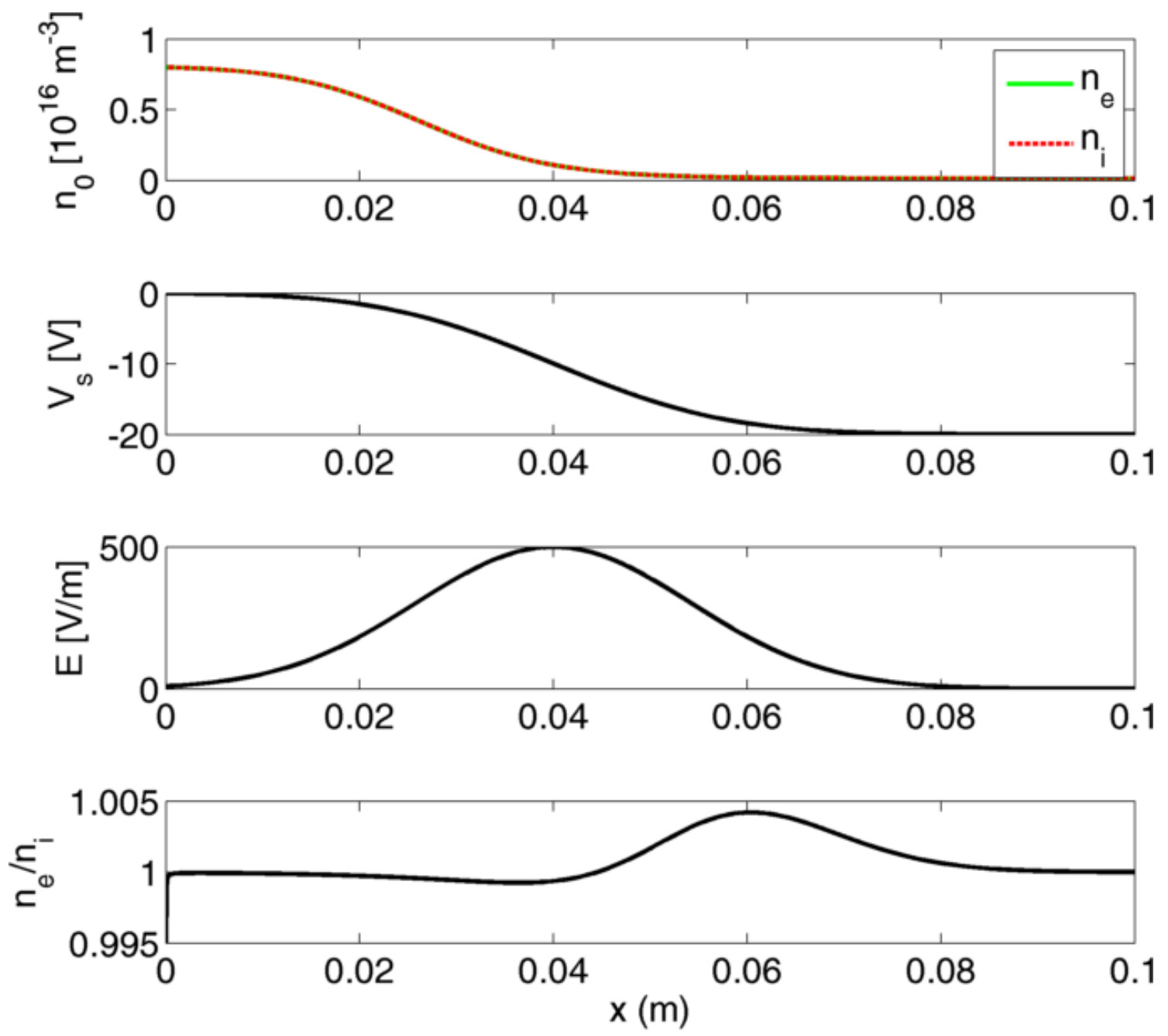

Figure 6.16: An example of an equilibrium profile assuming a gaussian electric field. In this figure, the electric field is consistent with the density gradient. Compare these modelled profiles with experimental data from figure 6.15 

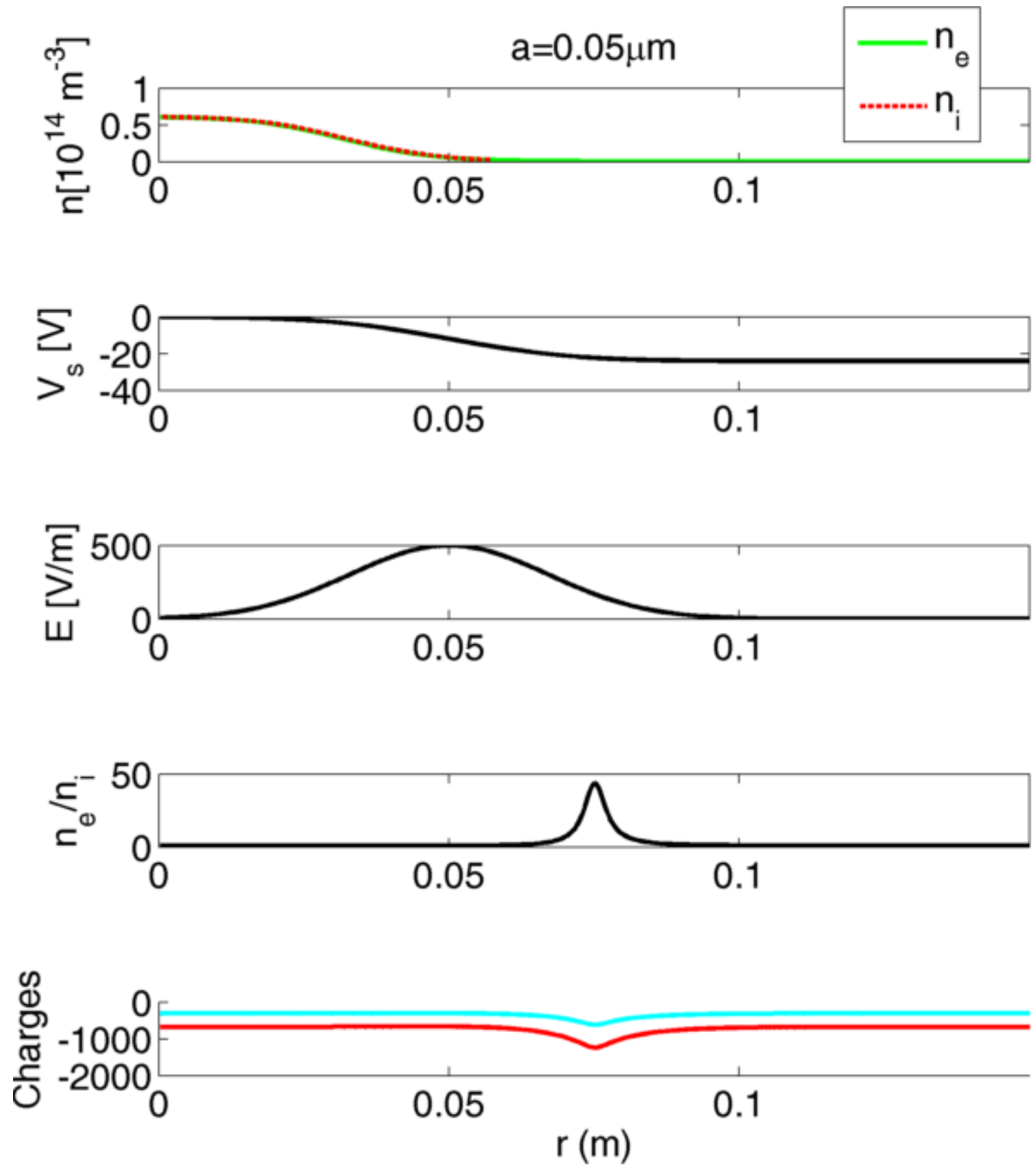

Figure 6.17: A possible equilibrium profile, assuming a gaussian electric field, using the profiles from figure 6.15 as a model. The electric field is consistent with the density gradient and it is centered at $r_{0}=5 \mathrm{~cm}$, with a FWHM of $4 \mathrm{~cm}$. In the bottom plot of this figure, the red line represents the in-situ-equilibrium charge for the Patacchini-Hutchinson and OML models, while the cyan line represents the in-situ-equilibrium charge as calculated using the Gatti-Kortshagen model. 


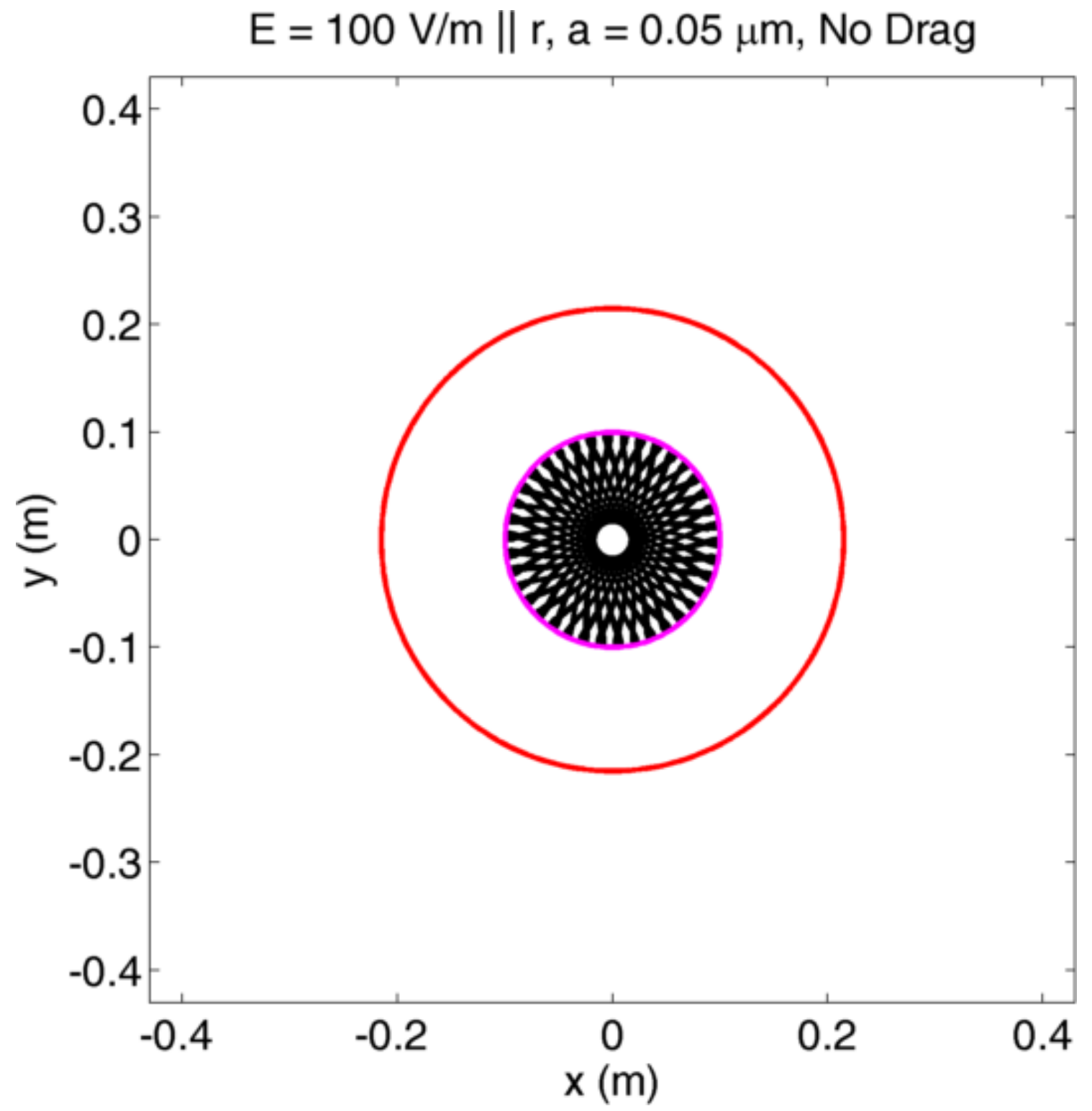

Figure 6.18: Trajectory of a zirconium grain with $a=0.05 \mu \mathrm{m}$, started at the dust thermal speed in the $x$-direction at $(x, y)=(0.1,0) \mathrm{m}$ for the profile shown in figure 6.17 ; the grain charge is kept constant at $q=-695 e$. This trajectory includes the effects of an electric field that is consistent with the density gradient. The solid red circle indicates the boundary of the vacuum vessel, the smaller, solid magenta circle indicates the uniform magnetic field region, and the black line shows the grain trajectory. 

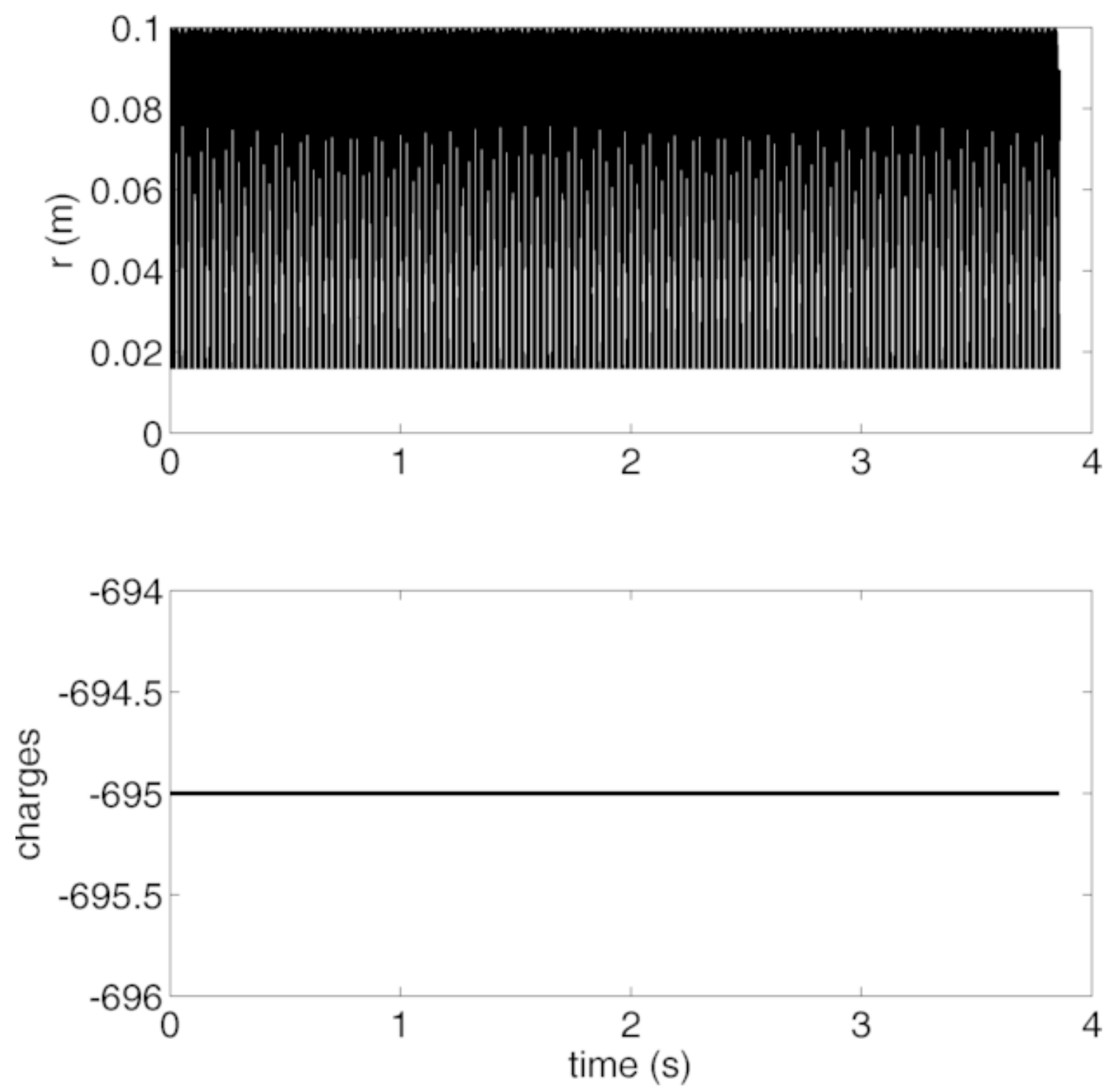

Figure 6.19: Radial excursion of the grain and grain charge corresponding to the trajectory in figure 6.18 when the grain has a constant charge $q=-695 e$. The radial excursion includes the effects of an electric field that is consistent with the density gradient. 


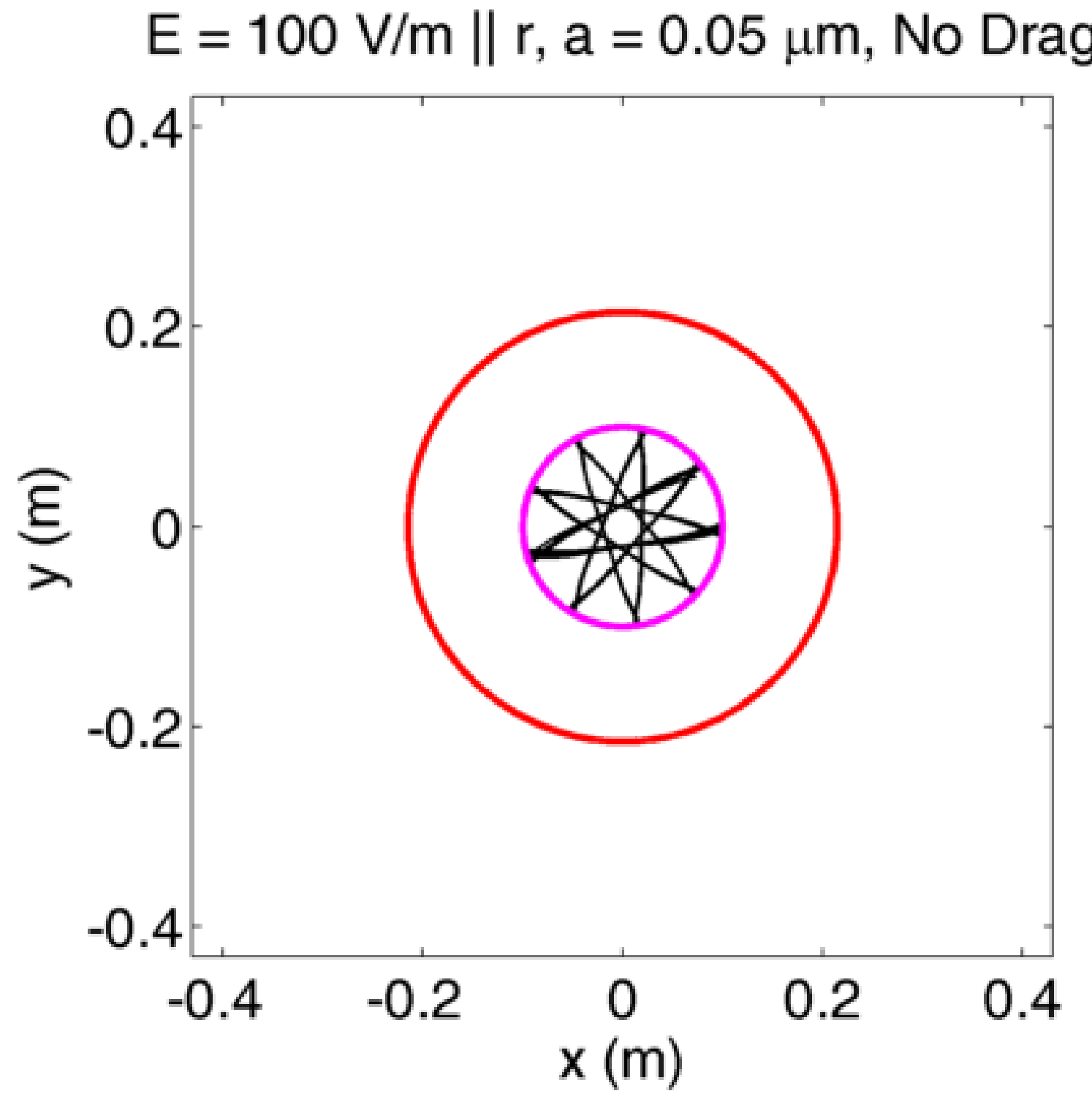

Figure 6.20: Trajectory of a zirconium grain with $a=0.05 \mu \mathrm{m}$, started at the dust thermal speed in the $x$-direction at $(x, y)=(0.1,0) \mathrm{m}$ for the profile shown in figure 6.17 and the grain is forced to charge instantaneously. The trajectory includes the effects of an electric field that is consistent with the density gradient. The solid red circle indicates the boundary of the vacuum vessel, the smaller, solid magenta circle indicates the uniform magnetic field region, and the black line shows the grain trajectory. 

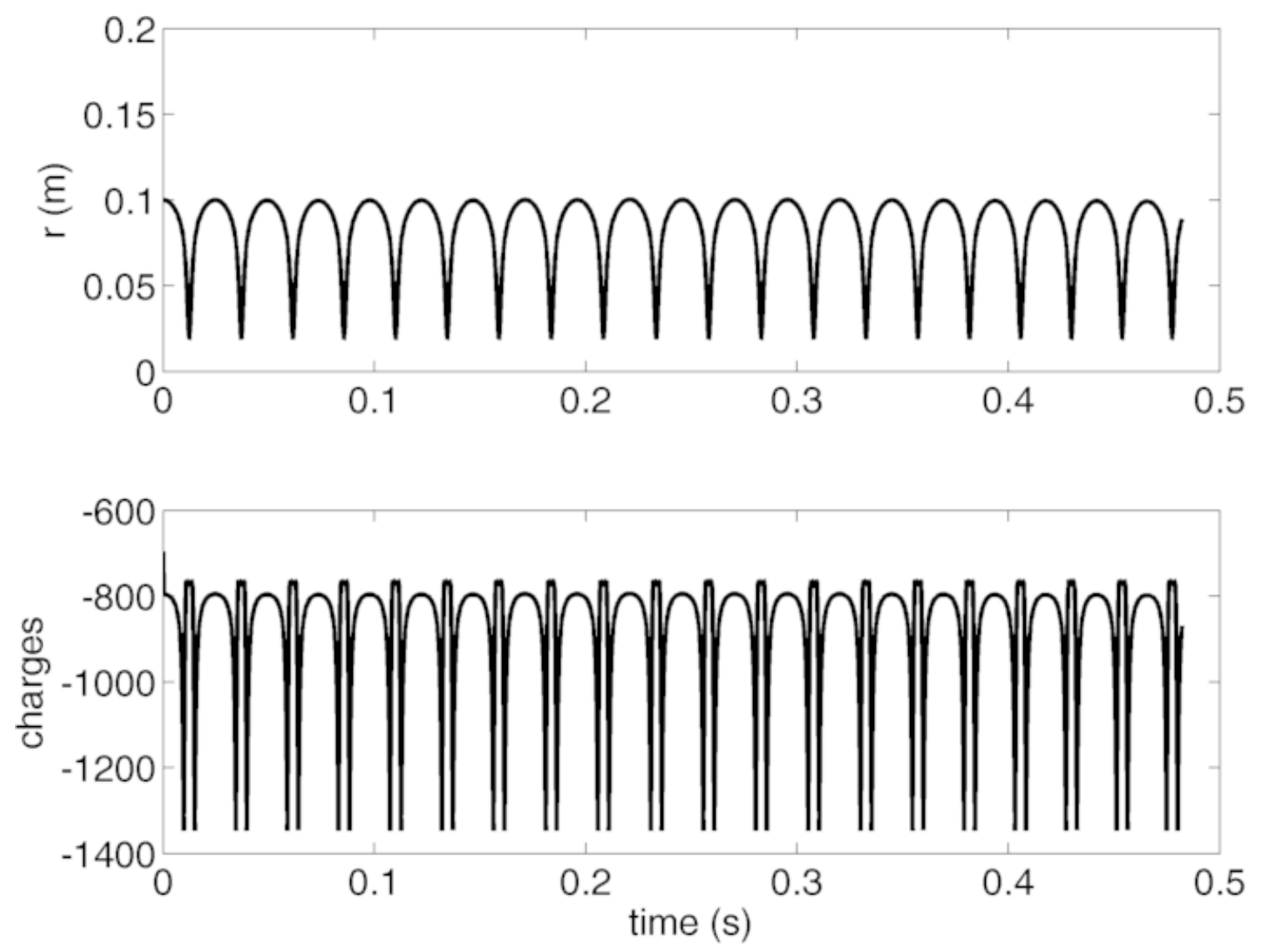

Figure 6.21: Radial excursion of the $a=0.05 \mu \mathrm{m}$ grain and grain charge corresponding to the trajectory in figure 6.20 when the grain is forced to charge instantaneously. The radial excursion includes the effects of an electric field that is consistent with the density gradient. 


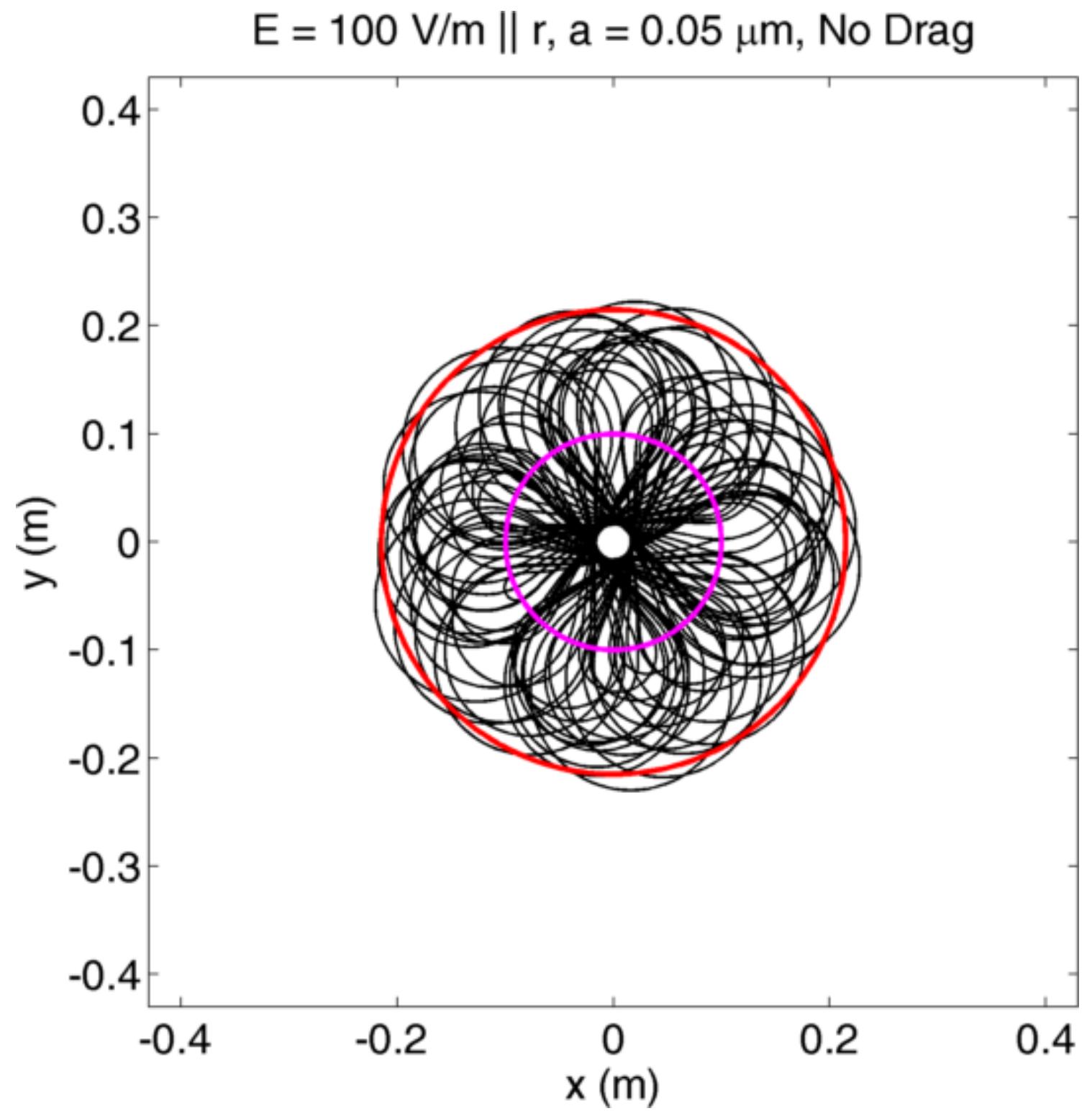

Figure 6.22: Trajectory of a zirconium grain with $a=0.05 \mu \mathrm{m}$, started at the dust thermal speed in the $x$-direction at $(x, y)=(0.1,0) \mathrm{m}$ for the profile shown in figure 6.17 using the OML model with no drag forces are present. The solid red circle indicates the boundary of the vacuum vessel, the smaller, solid magenta circle indicates the uniform magnetic field region, and the black line shows the grain trajectory. The grain charges non-instantaneously for the plasma conditions specified in figure 6.17 and the trajectory includes the effects of an electric field that is consistent with the density gradient. 

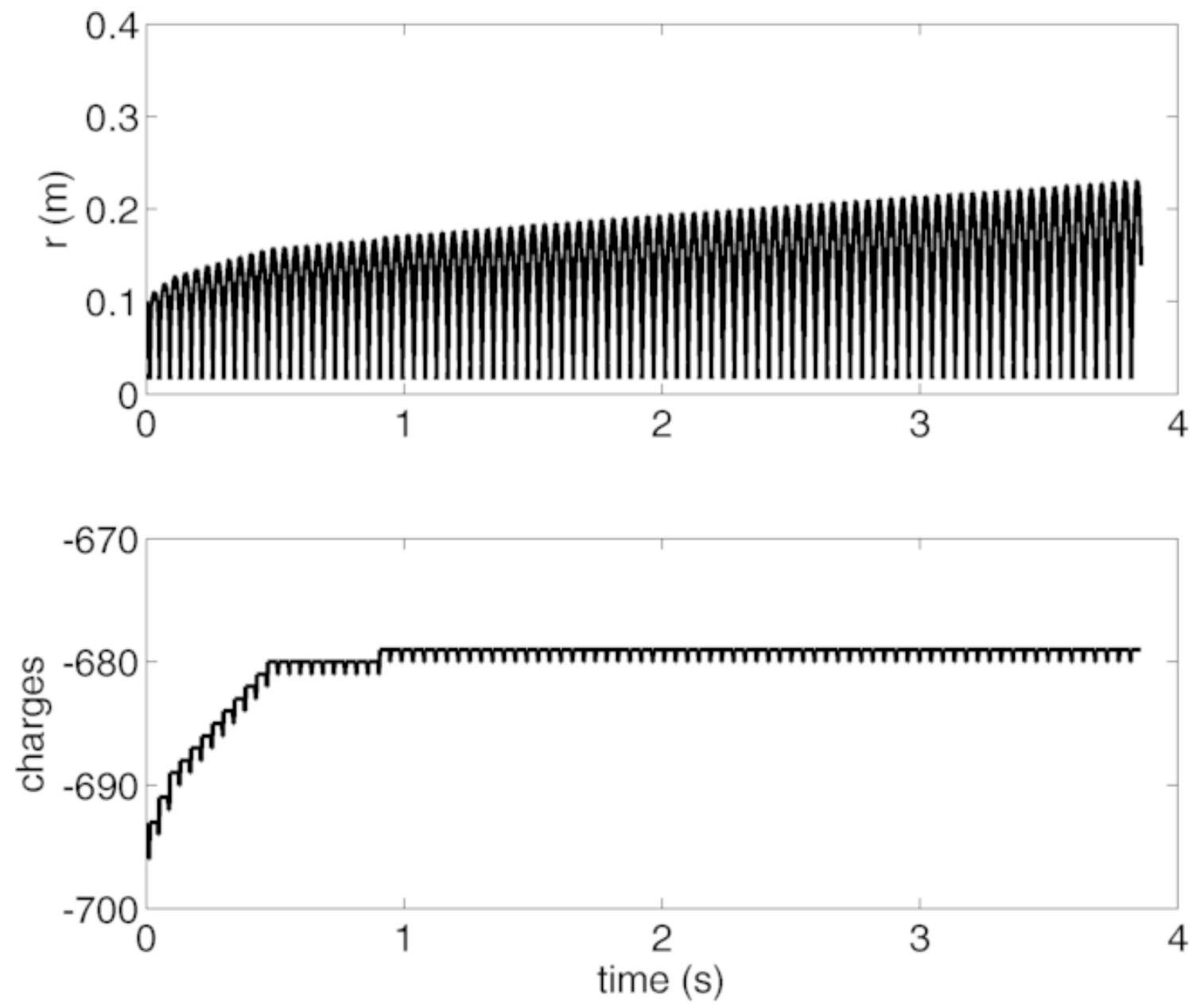

Figure 6.23: Radial excursion of the $a=0.05 \mu \mathrm{m}$ grain and grain charge corresponding to the trajectory in figure 6.22 when the grain is started with the in-situ equilibrium grain charge, using the OML model, and no drag forces are present. The grain charges non-instantaneously for the plasma conditions specified in figure 6.17 and the radial excursion includes the effects of an electric field that is consistent with the density gradient. 


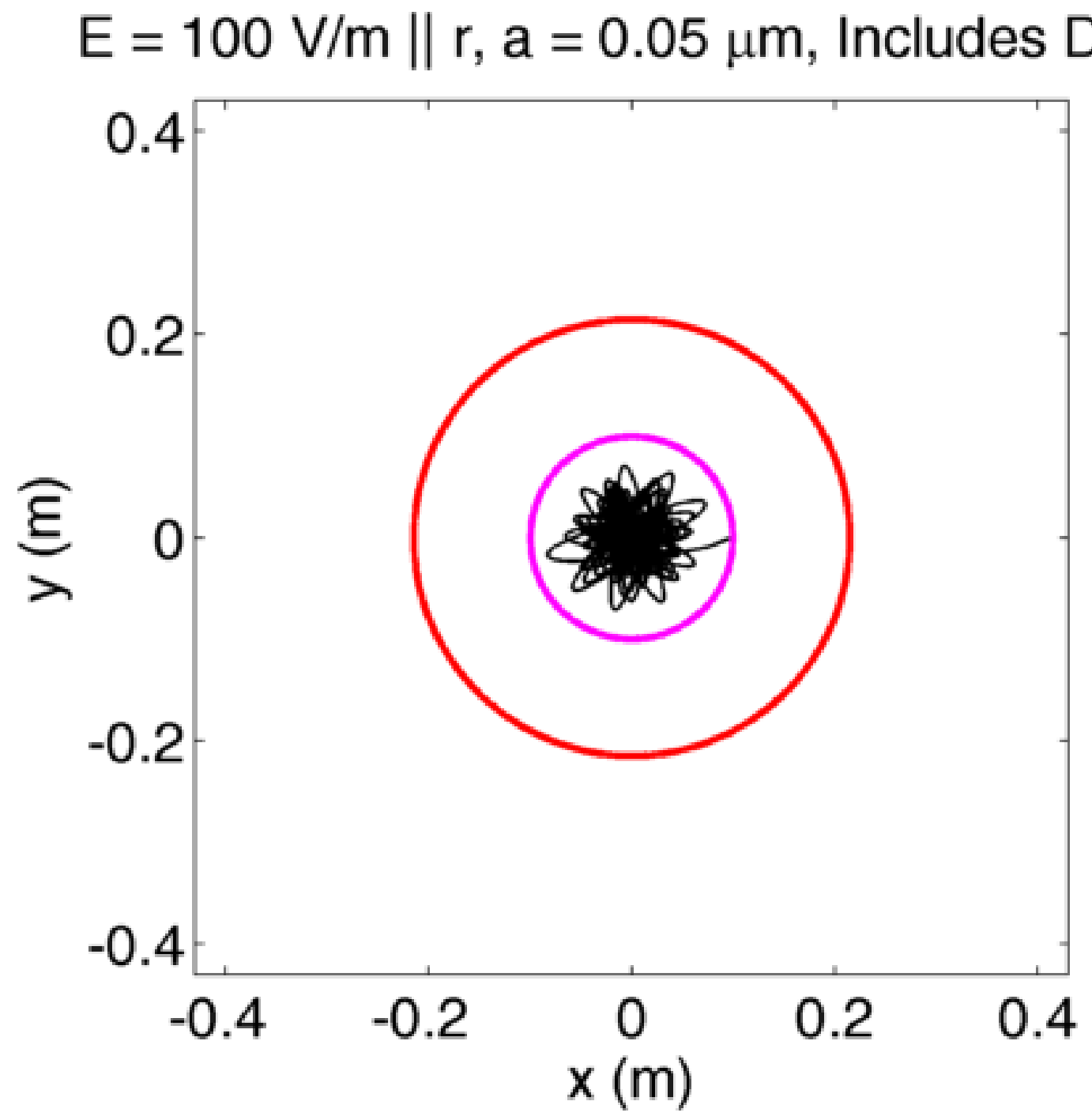

Figure 6.24: Trajectory of a zirconium grain with $a=0.05 \mu \mathrm{m}$, started at the dust thermal speed in the $x$-direction at $(x, y)=(0.1,0) \mathrm{m}$ for the profile shown in figure 6.17 using the OML model. The solid red circle indicates the boundary of the vacuum vessel, the smaller, solid magenta circle indicates the uniform magnetic field region, and the black line shows the grain trajectory. The grain charges non-instantaneously for the plasma conditions specified in figure 6.17 and the trajectory includes the effects of an electric field that is consistent with the density gradient. Neutral drag is assumed, but ion drag is not. 

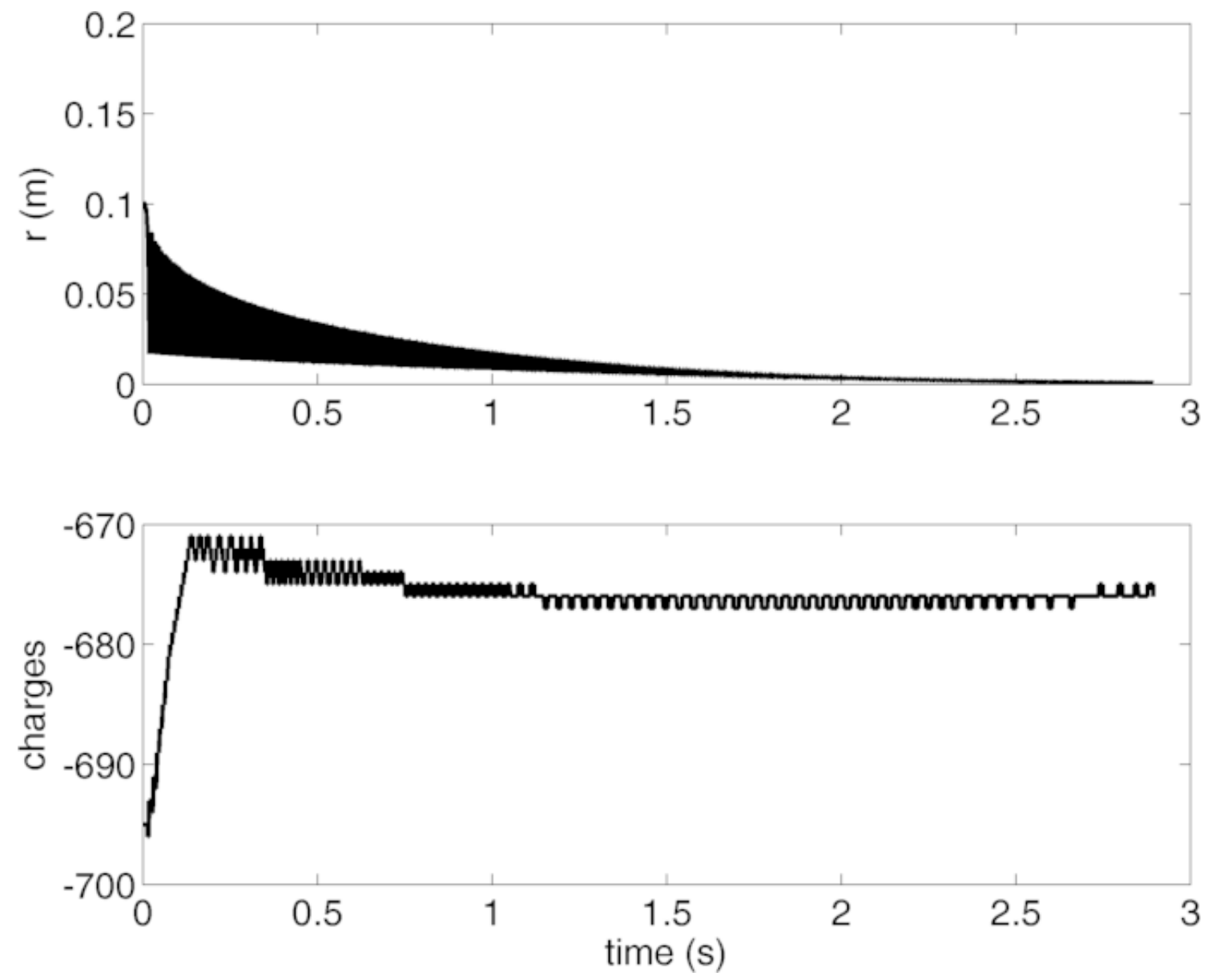

Figure 6.25: Radial excursion of the grain and grain charge corresponding to the trajectory in figure 6.24 when the $a=0.05 \mu \mathrm{m}$ grain is started with the $i n$-situ equilibrium grain charge and using the OML model. The grain charges non-instantaneously for the plasma conditions specified in figure 6.17 and the radial excursion includes the effects of an electric field that is consistent with the density gradient. Neutral drag is assumed, but ion drag is not. 

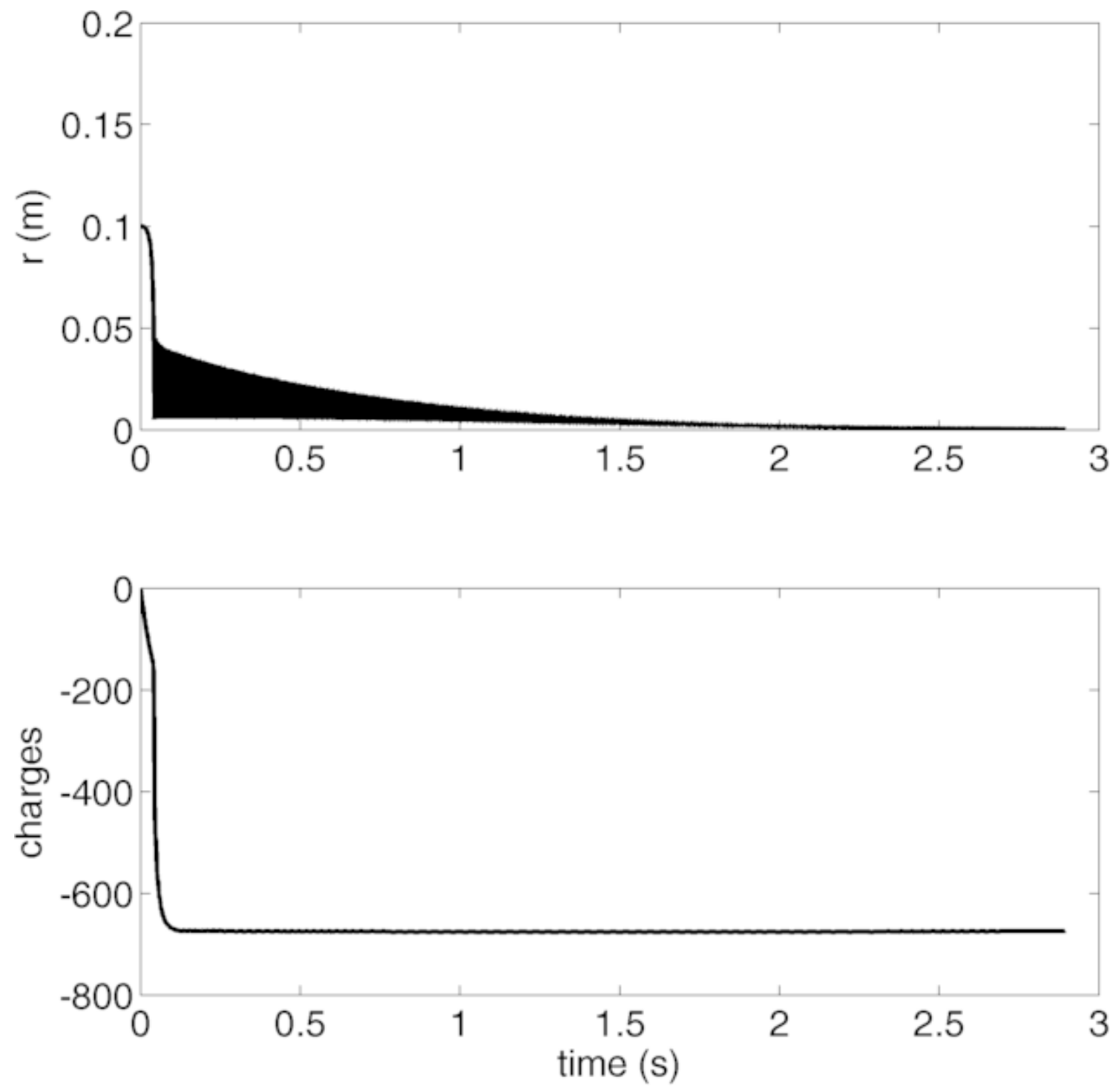

Figure 6.26: Radial excursion of the grain and grain charge corresponding to the trajectory in figure 6.24 when the $a=0.05 \mu \mathrm{m}$ grain is started with $q(t=0)=0$ and using the OML model. The grain charges non-instantaneously for the plasma conditions specified in figure 6.17 and the radial excursion includes the effects of an electric field that is consistent with the density gradient. Neutral drag is assumed, but ion drag is not. 

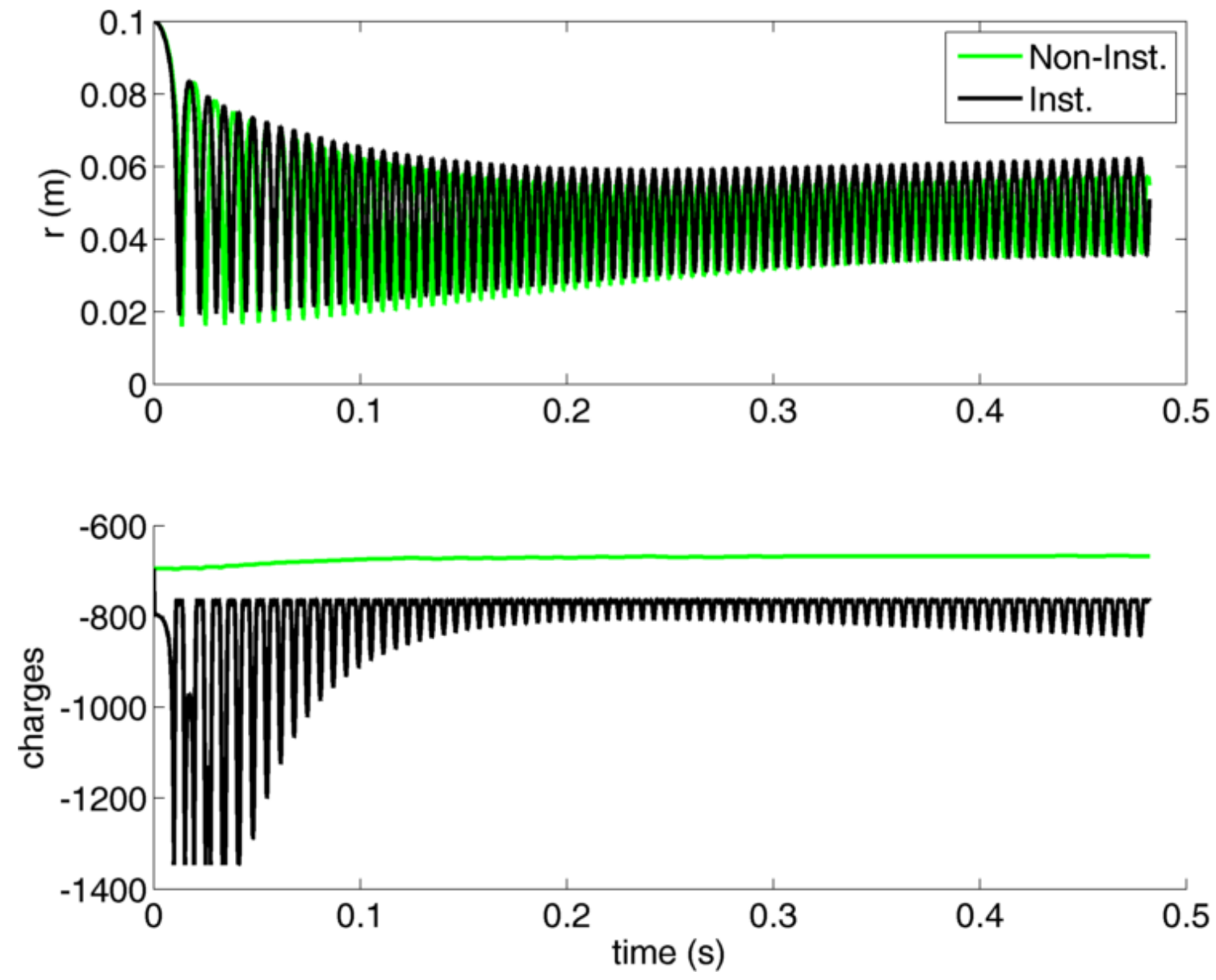

Figure 6.27: Radial excursion of the $a=0.05 \mu \mathrm{m}$ grain and grain charge is compared for instantaneous and non-instantaneous charging, using the OML model. The grain charges noninstantaneously for the plasma conditions specified in figure 6.17 and the radial excursion includes the effects of an electric field that is consistent with the density gradient. Neutral and ion drag are considered for this trajectory. 


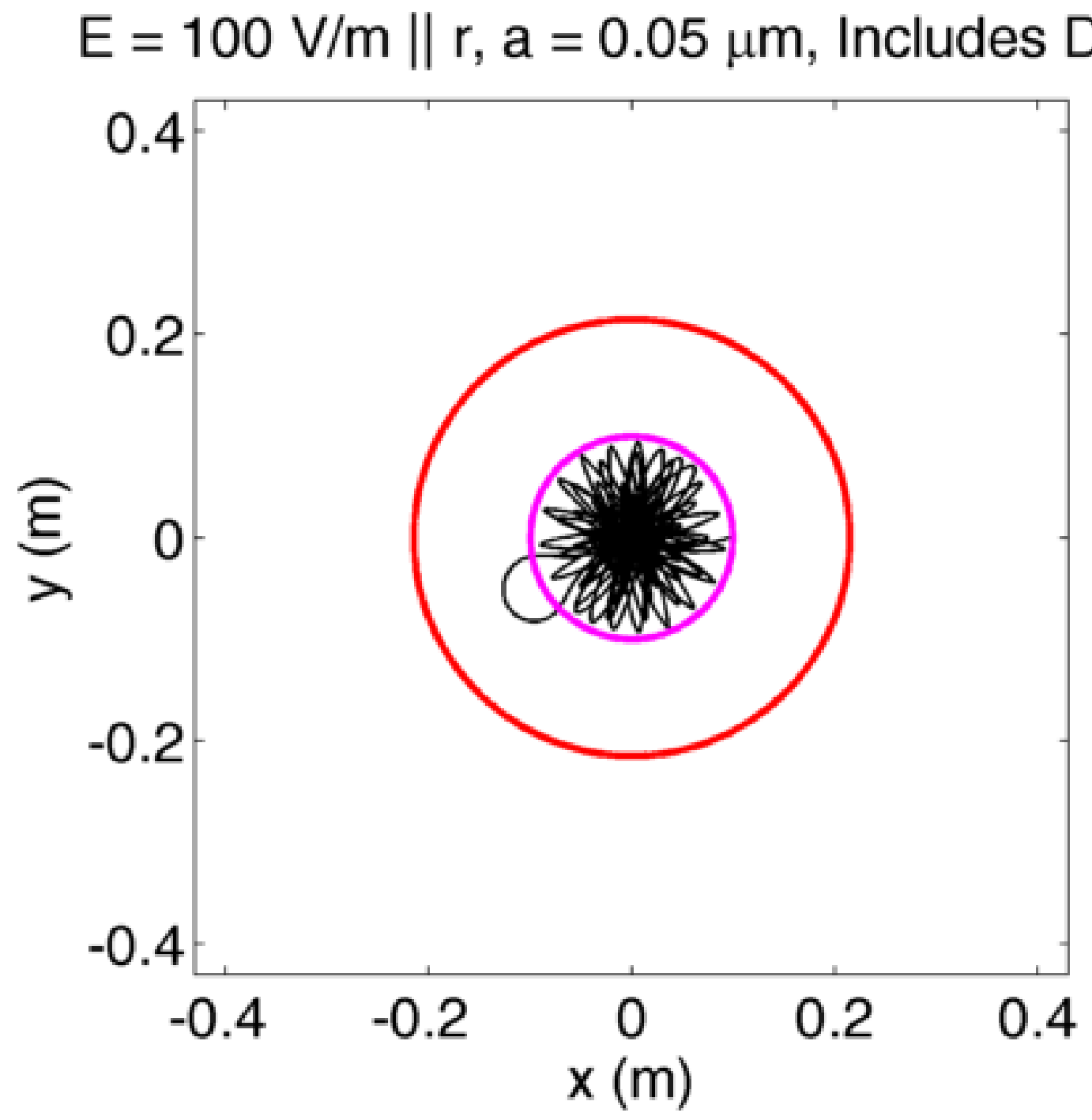

Figure 6.28: Trajectory of a zirconium grain with $a=0.05 \mu \mathrm{m}$, started at the dust thermal speed in the $x$-direction at $(x, y)=(0.1,0) \mathrm{m}$ for the profile shown in figure 6.17 using the Gatti-Kortshagen model. The solid red circle indicates the boundary of the vacuum vessel, the smaller, solid magenta circle indicates the uniform magnetic field region, and the black line shows the grain trajectory. The grain charges non-instantaneously for the plasma conditions specified in figure 6.17 and the grain trajectory includes the effects of an electric field that is consistent with the density gradient. Neutral drag is assumed, but ion drag is not. 

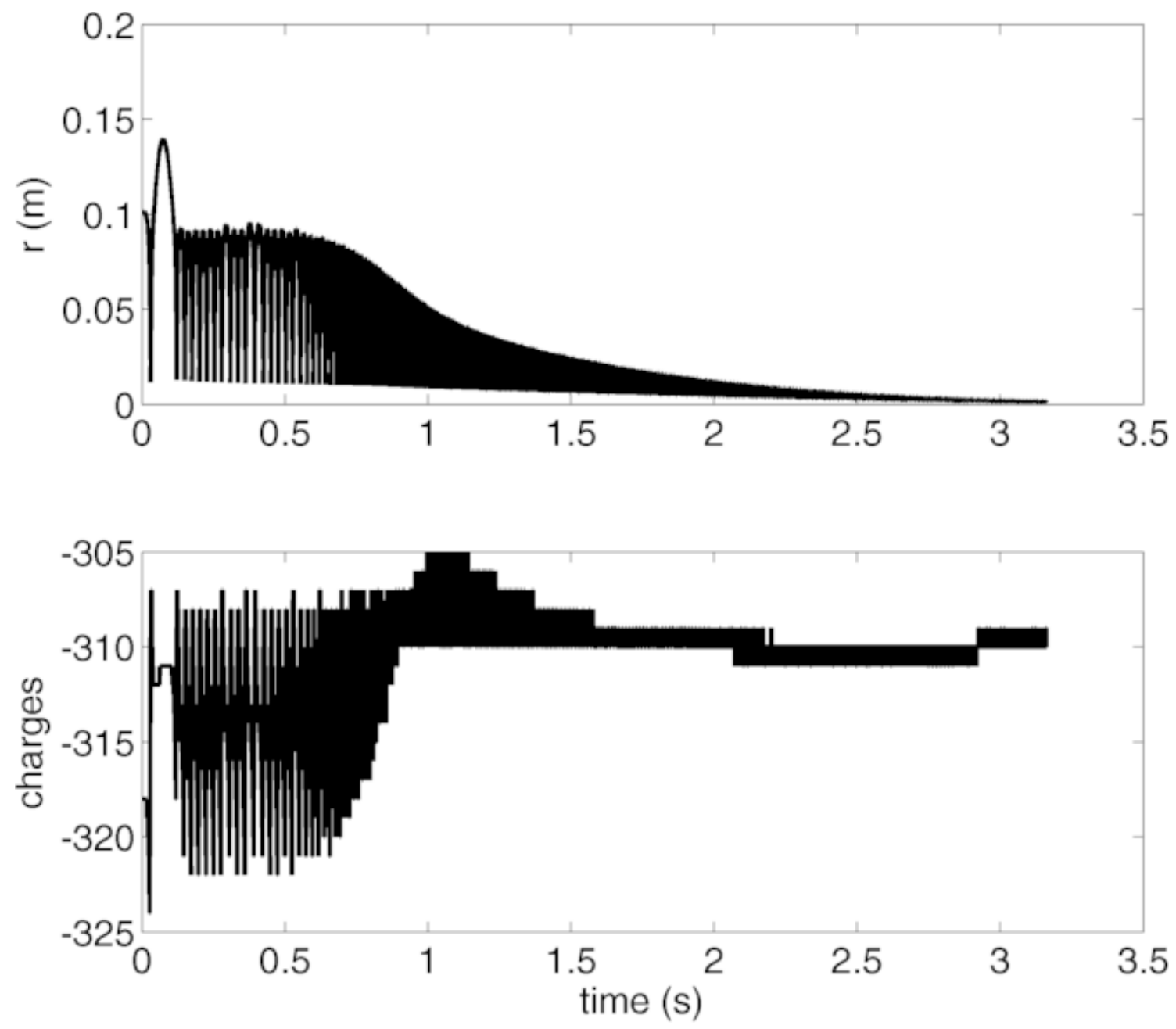

Figure 6.29: Radial excursion of the grain and grain charge corresponding to the trajectory in figure 6.28 when the grain is started with the in-situ equilibrium grain charge and using the GattiKortshagen model. The grain charges non-instantaneously for the plasma conditions specified in figure 6.17 and the radial excursion includes the effects of an electric field that is consistent with the density gradient. Neutral drag is assumed, but ion drag is not. 

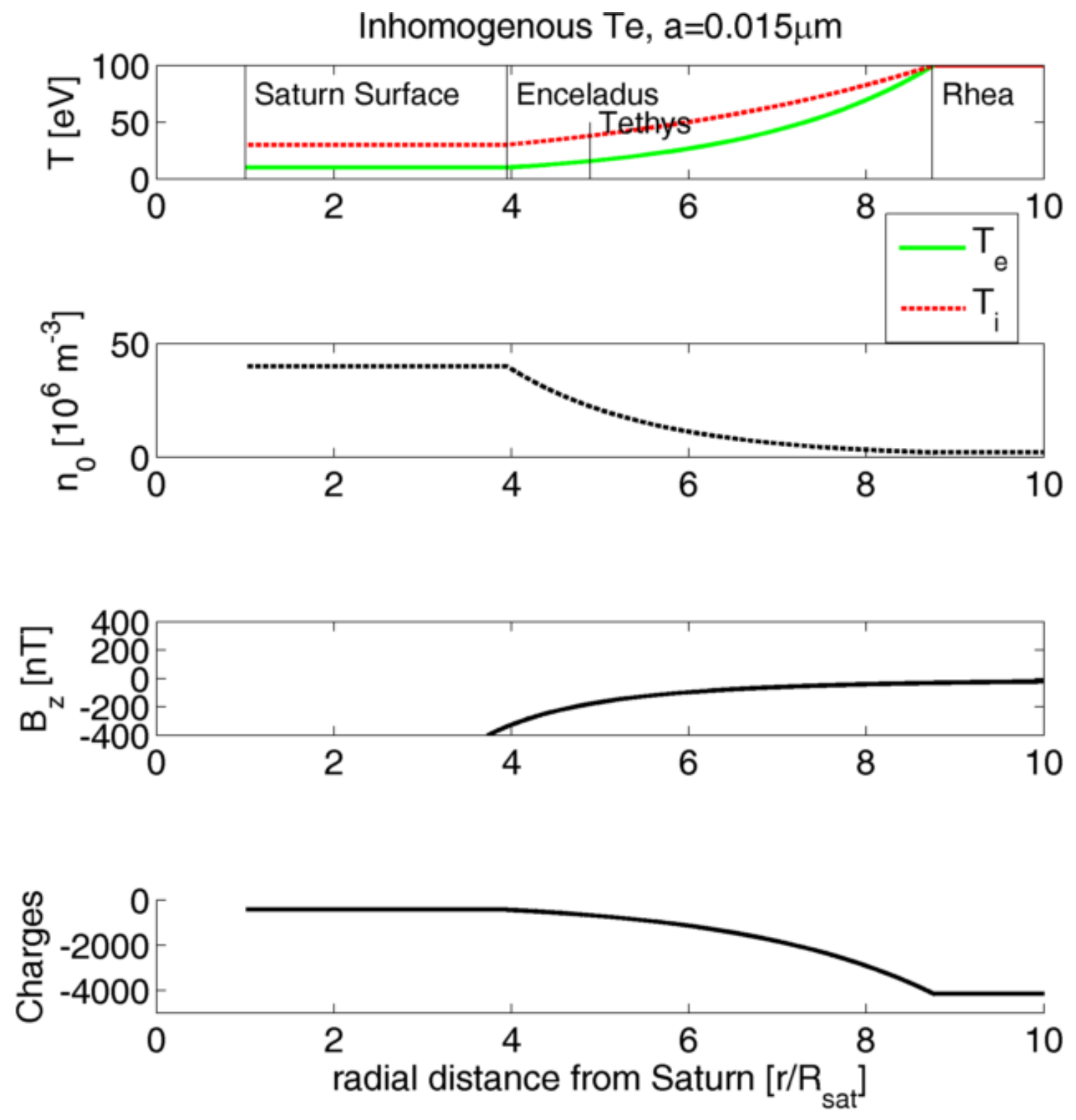

Figure 6.30: A profile of the Saturnian system to a radial distance of $10 R_{\text {sat }}$. 

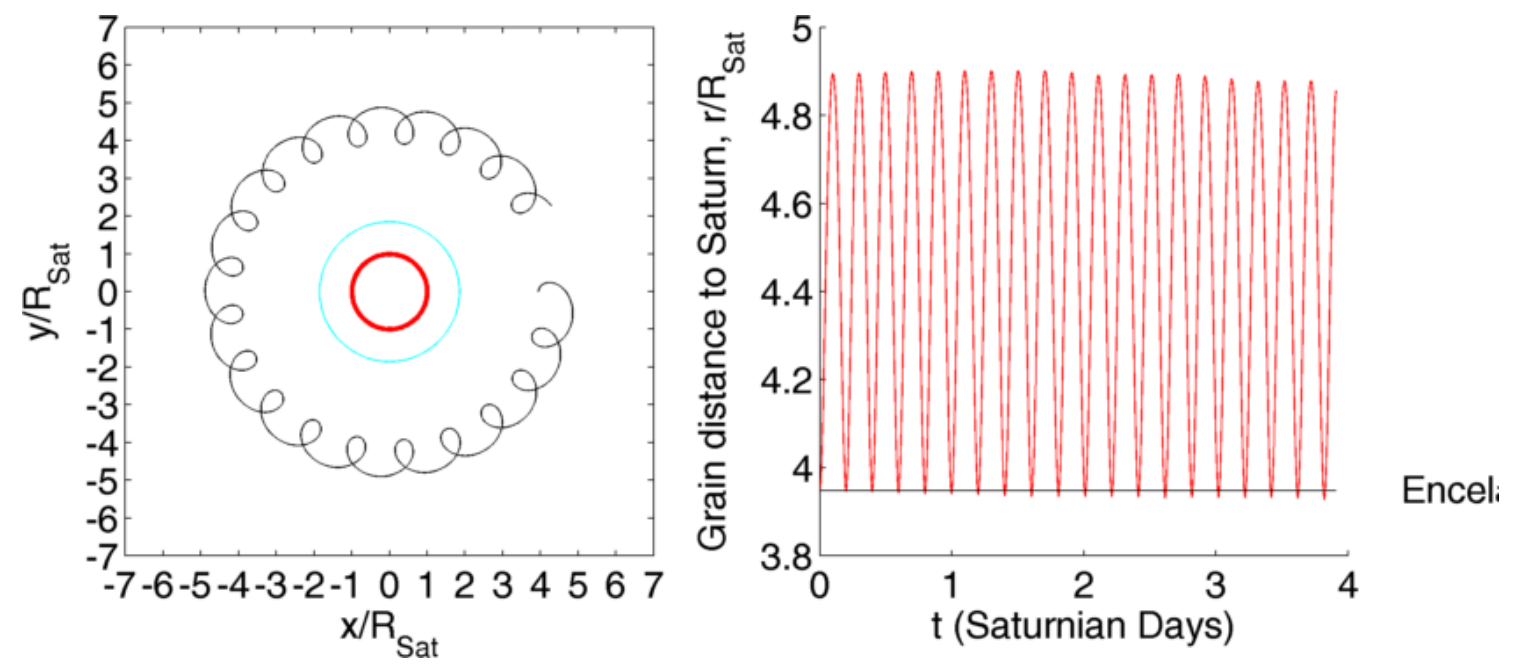

Figure 6.31: Trajectory of an $a=0.015 \mu \mathrm{m}$ grain launched from Enceladus in the co-rotating frame of Saturn when no UV is considered. In the leftmost panel, the trajectory is depicted by the solid black line, while the outer cyan circle shows the geosynchronous orbit and inner red circle shows the radial extent of Saturn's surface.
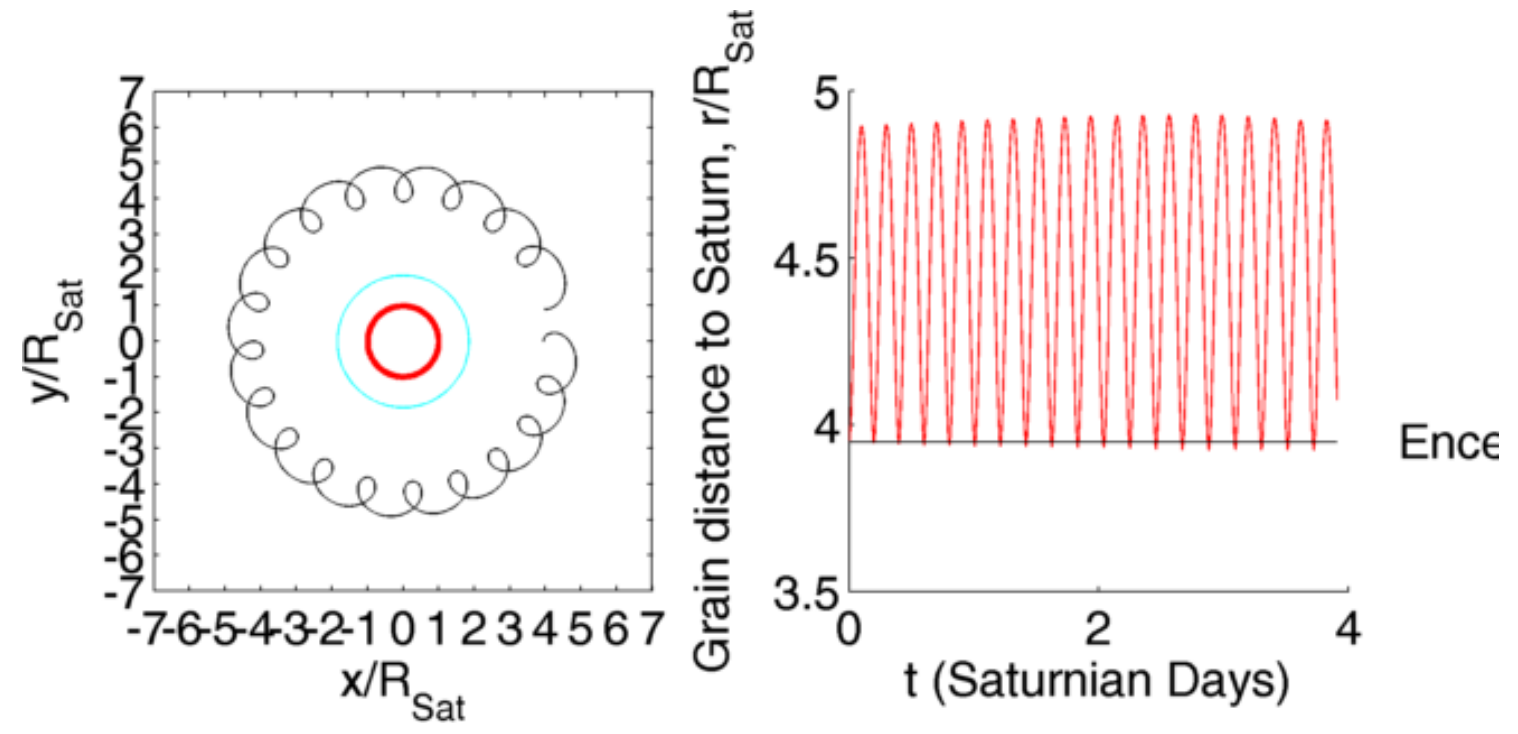

Figure 6.32: Trajectory of an $a=0.015 \mu \mathrm{m}$ grain launched from Enceladus in the co-rotating frame of Saturn when UV is considered. In the leftmost panel, the trajectory is depicted by the solid black line, while the outer cyan circle shows the geosynchronous orbit and inner red circle shows the radial extent of Saturn's surface. 

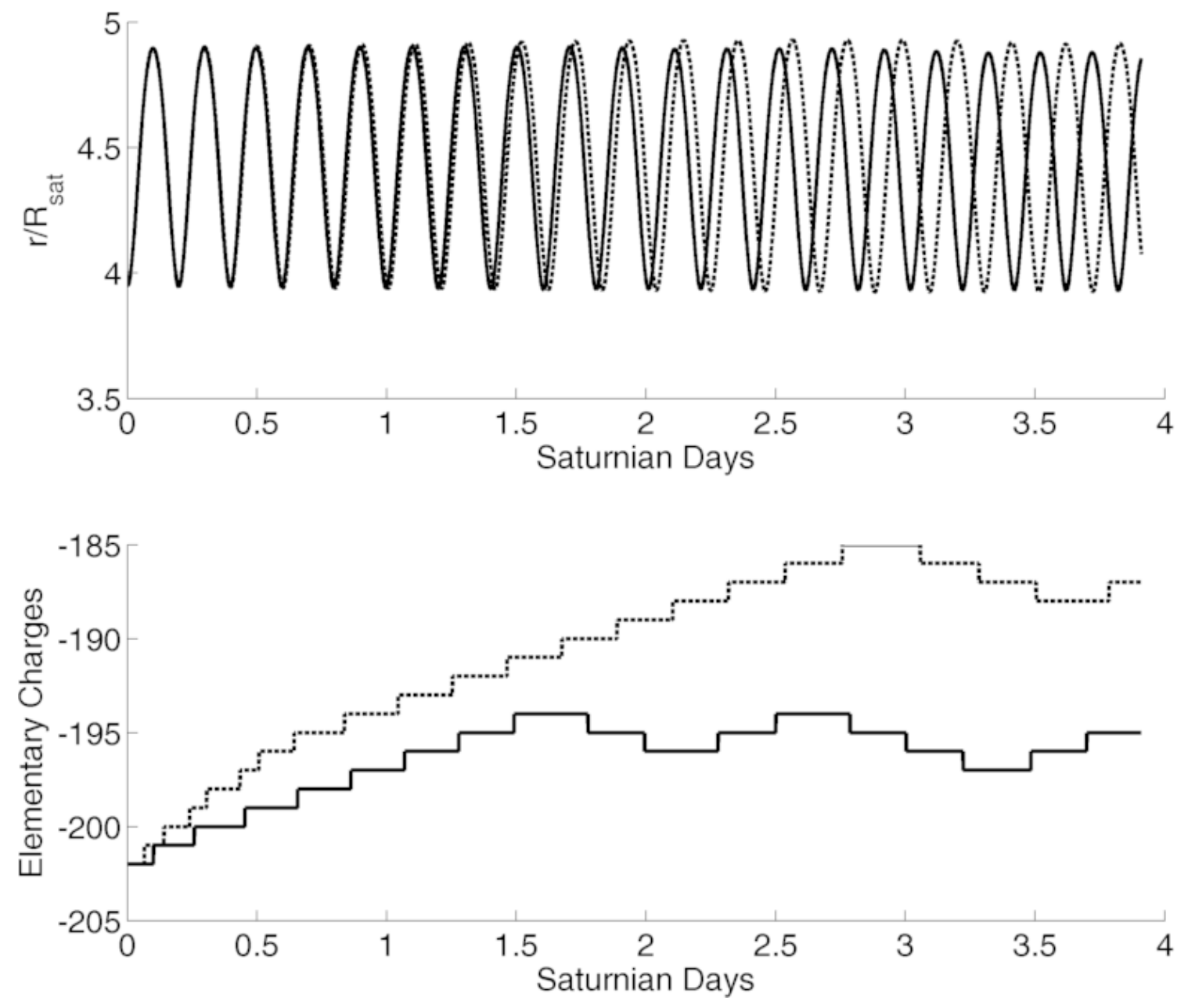

Figure 6.33: Comparison between the radial excursion and grain charge as a function of time for the trajectories from figures 6.31 and 6.32 . The solid line represents the grain trajectory when UV is not considered and the dashed line represents the grain trajectory when UV is considered. 

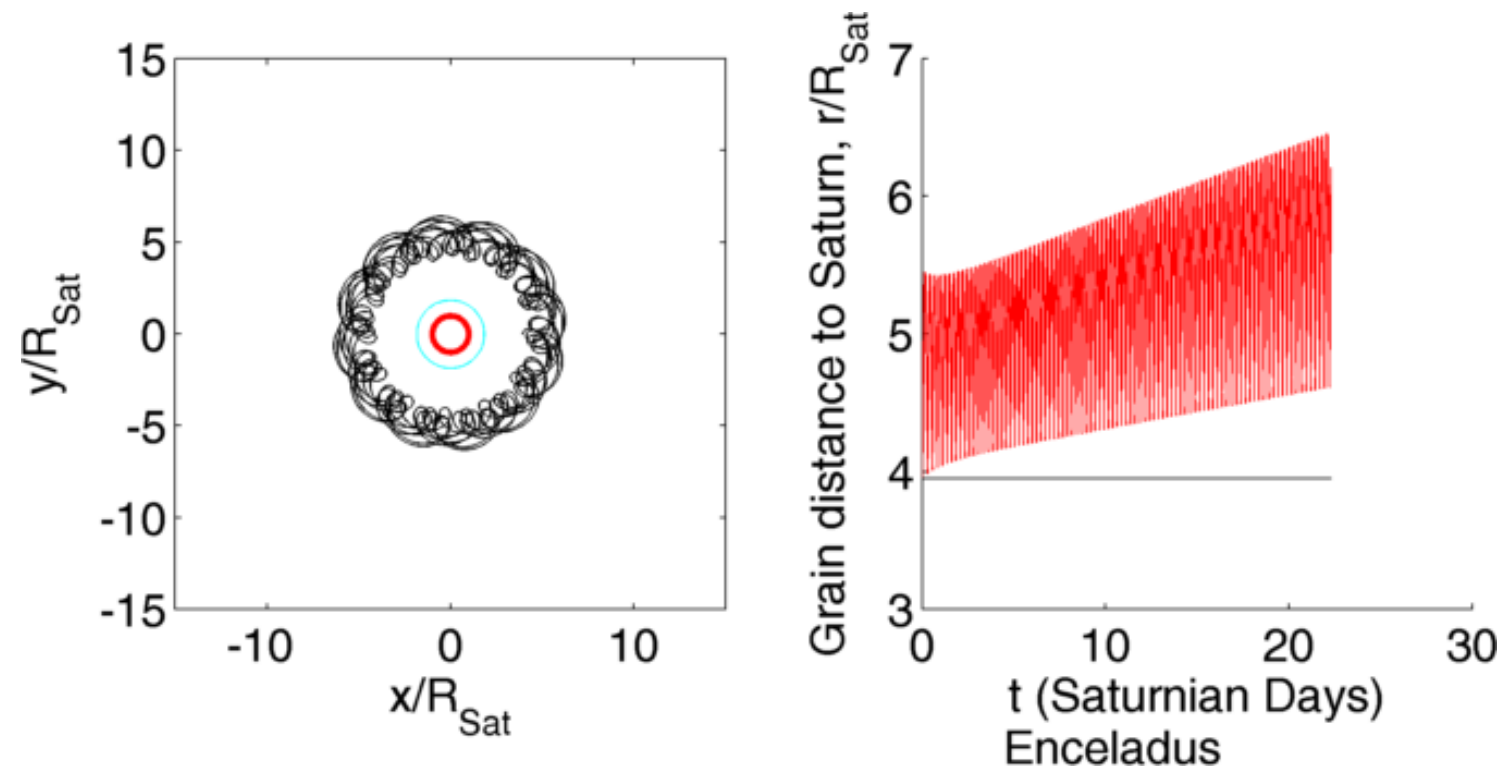

Figure 6.34: Trajectory of an $a=0.015 \mu \mathrm{m}$ grain launched from Enceladus in the co-rotating frame of Saturn when UV is considered and there is an electron temperature gradient. In the leftmost panel, the trajectory is depicted by the solid black line, while the outer cyan circle shows the geosynchronous orbit and inner red circle shows the radial extent of Saturn's surface. The rightmost panel shows the radial excursion of the dust grain. Neutral and ion drag forces are not considered for this grain trajectory.
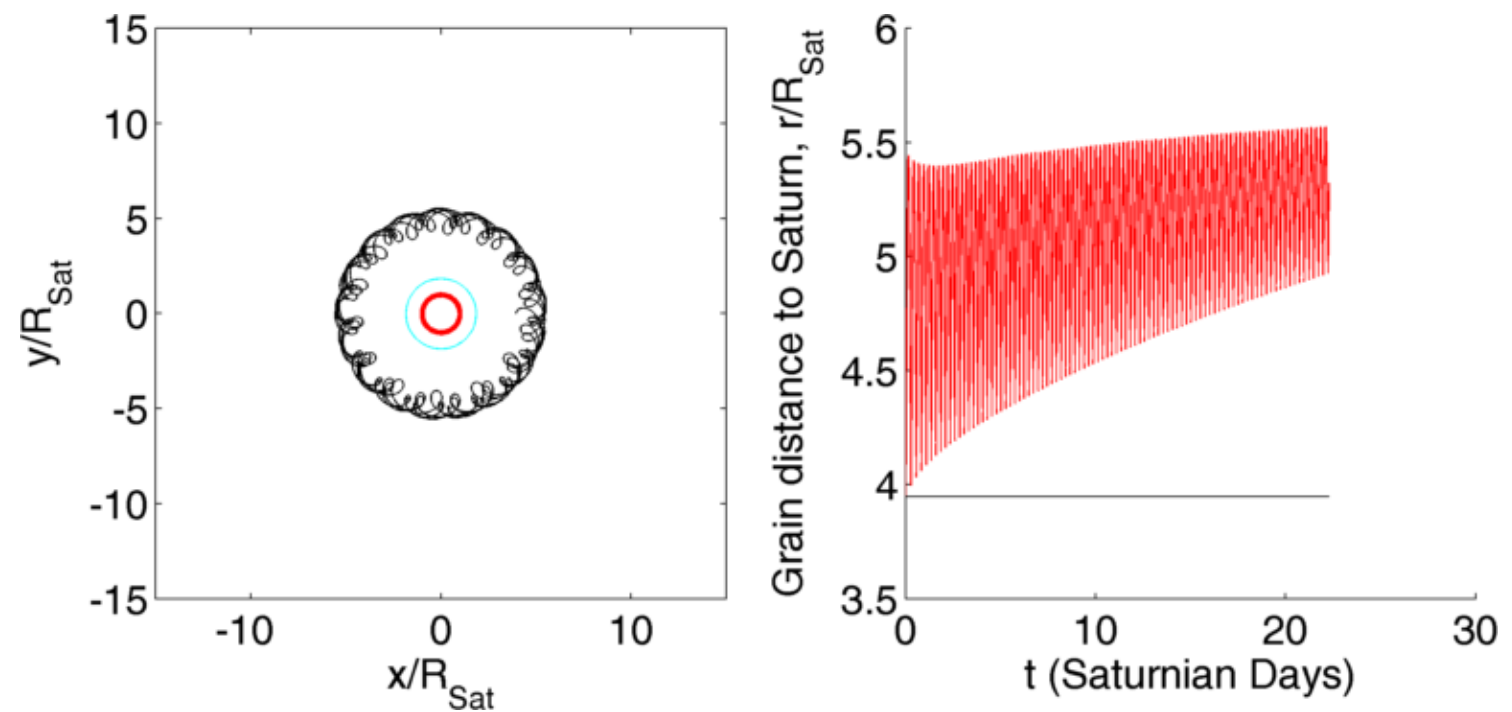

Figure 6.35: Trajectory of an $a=0.025 \mu \mathrm{m}$ grain launched from Enceladus in the co-rotating frame of Saturn when UV is considered and there is an electron and ion temperature gradient. Neutral drag force is considered for this grain trajectory. In the leftmost panel, the trajectory is depicted by the solid black line, while the outer cyan circle shows the geosynchronous orbit and inner red circle shows the radial extent of Saturn's surface. The rightmost panel shows the radial excursion of the dust grain. 

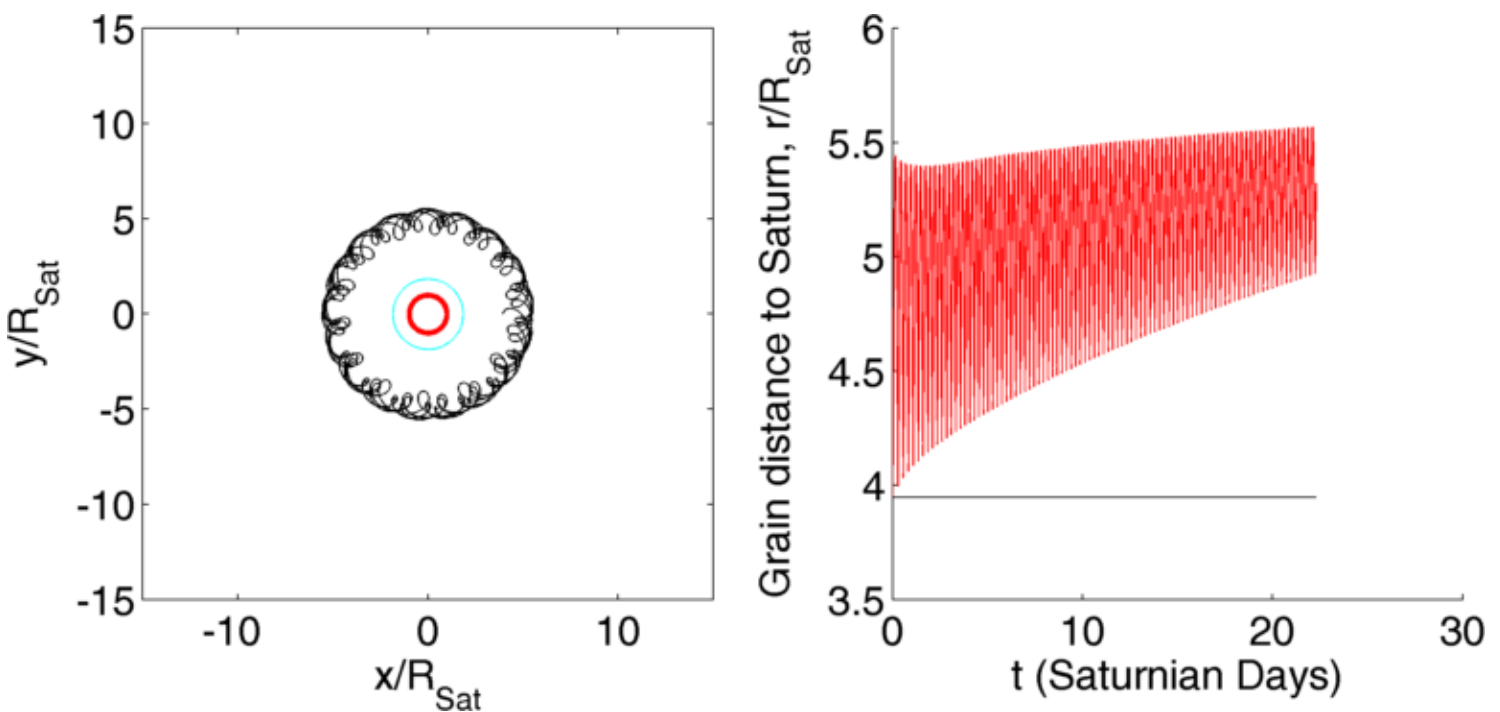

Figure 6.36: Trajectory of an $a=0.025 \mu \mathrm{m}$ grain launched from Enceladus in the co-rotating frame of Saturn when UV is considered and there is an electron and ion temperature gradient. Neutral and ion drag forces are considered for this grain trajectory. In the leftmost panel, the trajectory is depicted by the solid black line, while the outer cyan circle shows the geosynchronous orbit and inner red circle shows the radial extent of Saturn's surface. The rightmost panel shows the radial excursion of the dust grain. This figure is not noticeably different from 6.35 , which shows that the effect of ion drag on this grain trajectory is negligible. 